Nevada

Environmental

Restoration

Project

Closure Report for Corrective

Action Unit 484: Surface Debris,

Waste Sites, and Burn Area,

Tonopah Test Range, Nevada

Controlled Copy No.:

Revision: 0

September 2007

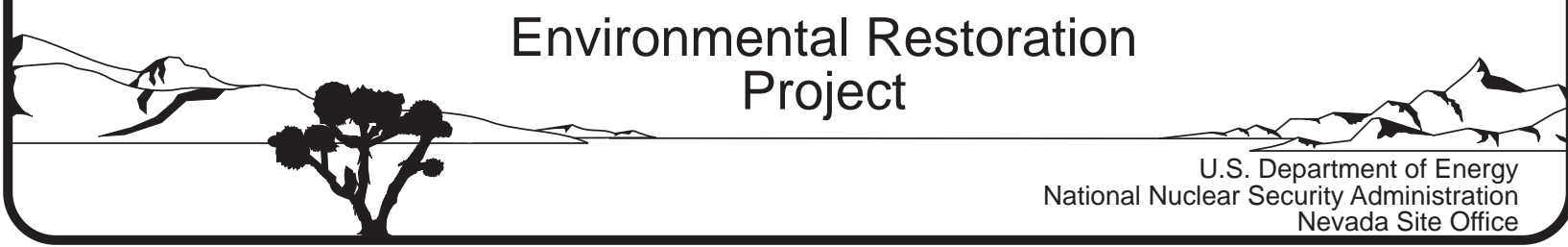




\section{DISCLAIMER}

Reference herein to any specific commercial product, process, or service by trade name, trademark, manufacturer, or otherwise, does not necessarily constitute or imply its endorsement, recommendation, or favoring by the United States Government or any agency thereof or its contractors or subcontractors.

This report has been reproduced directly from the best available copy.

Available for sale to the public from:

U.S. Department of Commerce

National Technical Information Service

5285 Port Royal Road

Springfield, VA 22161-0002

Telephone: (800) 553-6847

Fax: (703) 605-6900

E-mail: orders@ntis.gov

Online ordering: http://www.ntis.gov/ordering.htm

Available electronically at http://www.osti.gov/bridge.

Available for a processing fee to the U.S. Department of Energy and its contractors, in paper, from:

U.S. Department of Energy

Office of Scientific and Technical Information

P.O. Box 62

Oak Ridge, TN 37831-0062

Telephone: (865) 576-8401

Fax: (865) 576-5728

E-mail: reports@adonis.osti.gov 


\title{
CLOSURE REPORT FOR CORRECTIVE ACTION UNIT 484: SURFACE DEBRIS, WASTE SITES, AND BURN AREA, TONOPAH TEST RANGE, NEVADA
}

\author{
U.S. Department of Energy \\ National Nuclear Security Administration \\ Nevada Site Office \\ Las Vegas, Nevada
}

Controlled Copy No.

Revision: 0

September 2007 
THIS PAGE INTENTIONALLY LEFT BLANK 


\section{CLOSURE REPORT FOR CORRECTIVE ACTION UNIT 484: SURFACE DEBRIS, WASTE SITES, AND BURN AREA, TONOPAH TEST RANGE, NEVADA}

Approved By: SIGNATURE APPROVED

Date: $\underline{9 / 4 / 2007}$

Kevin J. Cabble

Federal Sub-Project Director

Industrial Sites Sub-Project

Approved By: SIGNATURE APPROVED

Date: $\underline{9 / 4 / 2007}$

John B. Jones

Acting Federal Project Director

Environmental Restoration Project 
THIS PAGE INTENTIONALLY LEFT BLANK 


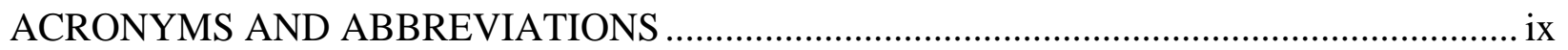

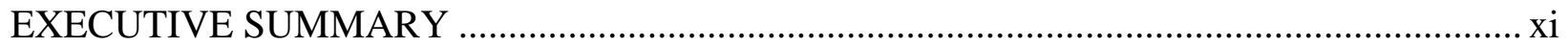

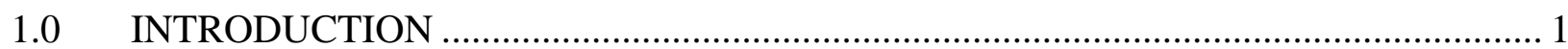

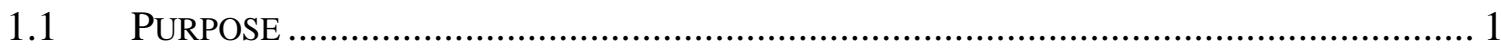

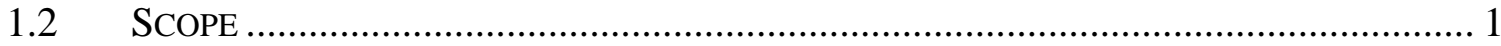

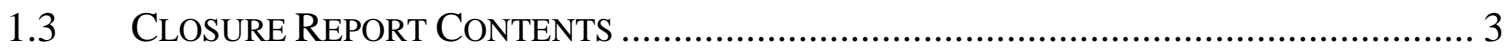

1.3.1 Data Quality Objectives........................................................................... 4

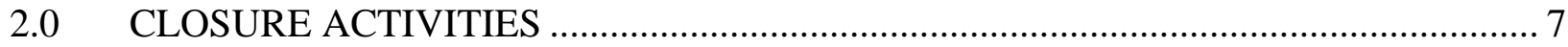

2.1 DESCRIPTION OF CORRECTIVE ACTION ACTIVITIES ............................................ 7

2.1.1 Preplanning and Site Preparation............................................................ 7

2.1.2 Closure Activities - Surface Detonation Debris Sites ................................... 7

2.1.2.1 Radiological and Geophysical Drive-Over Surveys........................... 10

2.1.2.1.1 CAS TA-52-001-TANL (NEDS Detonation Area)..........13

2.1.2.1.2 CAS TA-52-006-TAPL (DUS, “Colimbo”).....................13 13

2.1.2.1.3 CAS TA-52-004-TAAL (MPDT) ……………………..... 17

2.1.2.2 Investigation Soil Sample Collection ...............................................17

2.1.2.2.1 CAS TA-52-001-TANL (NEDS Detonation Area).......... 23

2.1.2.2.2 CAS TA-52-006-TAPL (DUS, Colimbo) ……………..... 23

2.1.2.2.3 CAS TA-52-004-TAAL (MPDT) …………………….... 23

2.1.2.3 Excavation/Removal of DU............................................................ 42

2.1.2.3.1 CAS TA-52-001-TANL (NEDS Detonation Area)......... 44

2.1.2.3.2 CAS TA-52-006-TAPL (DUS) ....................................... 44

2.1.2.3.3 CAS TA-52-004-TAAL (MPDT) ……………………. 45

2.1.3 Closure Activities - Surface-Shallow Subsurface Debris Sites................. 45

2.1.3.1 Aerial Photography and Multispectral Surveys................................... 47

2.1.3.2 Radiological and Geophysical Drive-Over Surveys............................ 50

2.1.3.2.1 CAS RG-52-007-TAML, Davis Gun Penetrator Test..... 53

2.1.3.2.2 CAS TA-52-005-TAAL, JTA DU Sites........................... 58

2.1.3.3 Exploratory Excavation and DU Removal .........................................58

2.1.3.3.1 CAS RG-52-007-TAML, Davis Gun Penetrator Test..... 58

2.1.3.3.2 CAS TA-52-005-TAAL, JTA DU Sites............................63

2.1.4 Closure Activities - Non-Impacted Surface Debris..................................... 65

2.2 DEVIATIONS FROM THE SAFER PLAN AS APPROVED ..............................................6 65

2.3 CORRECTIVE ACTION SCHEDULE AS COMPLETED ...................................................6 68

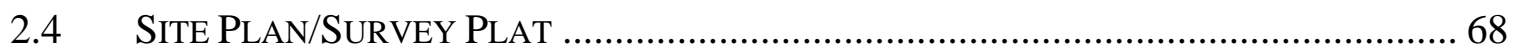

3.0 WASTE DISPOSITION ..................................................................................... 71

3.1 WASTE MINIMIZATION ……………………………………………………... 71

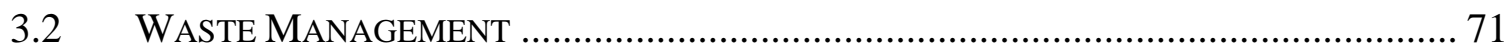

3.3 WASTE CHARACTERIZATION …………………............................................. 71

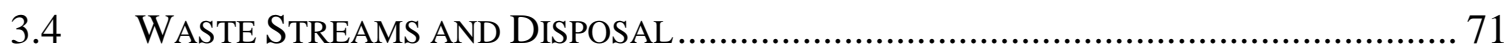

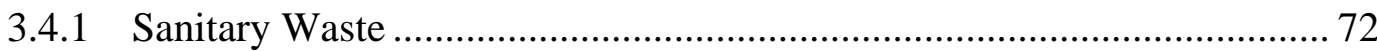

3.4.2 Low-Level Waste..................................................................................... 72

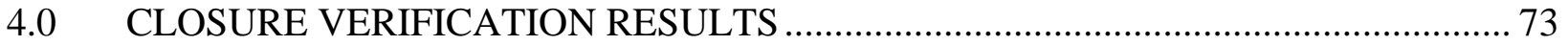




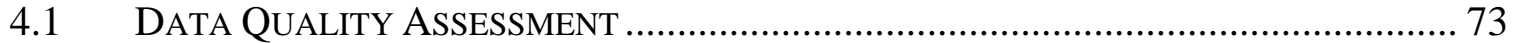

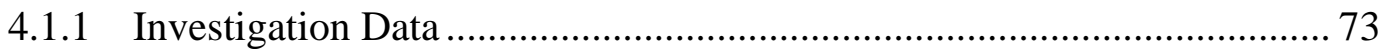

4.1.1.1 Geoprobe Analytical Data at Surface Detonation Debris Sites ......... 74

4.1.1.1.1 Data Quality Indicators ................................................... 74

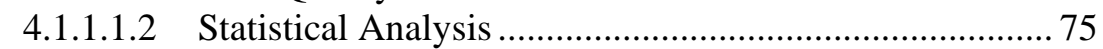

4.1.1.2 Statistical Evaluation of Davis Gun Geophysical Data ....................... 75

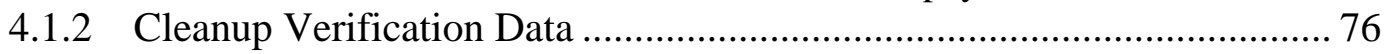

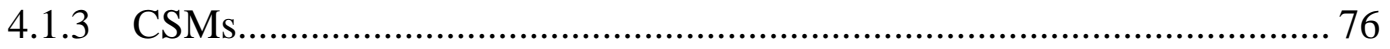

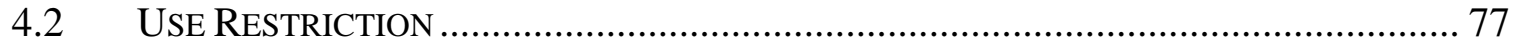

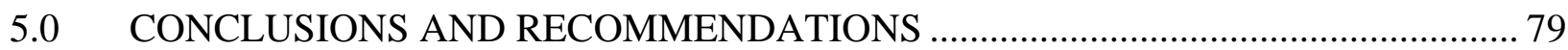

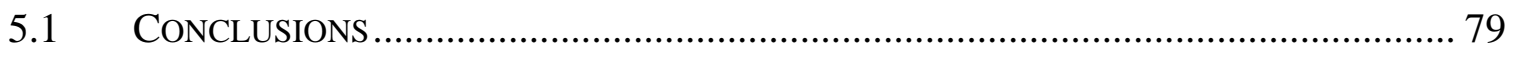

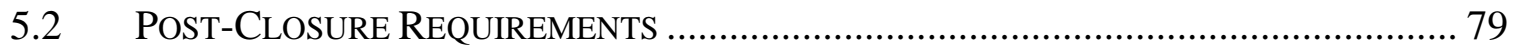

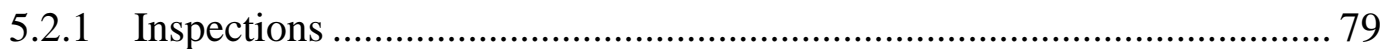

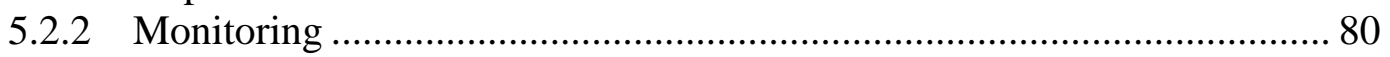

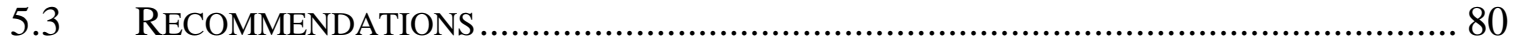

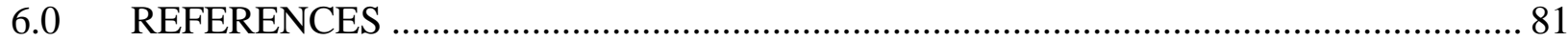

LIBRARY DISTRIBUTION LIST 


\section{LIST OF FIGURES}

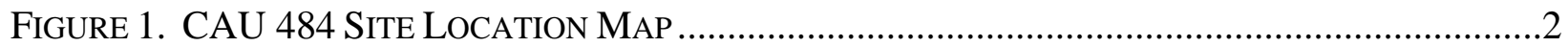

FIGURE 2. CAS TA-52-001-TANL, NEDS DETONATION AREA …..........................................9

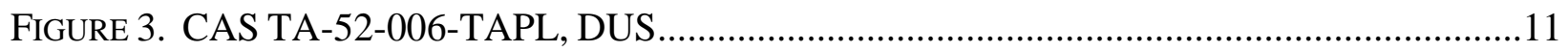

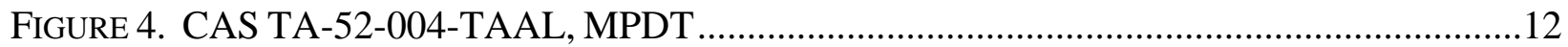

FIGURE 5. GEOPHYSICAL AND RADIOLOGICAL ANOMALIES ON NEDS LAKE ..............................14

FIGURE 6. RADIOLOGICAL ANOMALIES ON NEDS LAKE ..........................................................15

FIGURE 7. DU AND URANIUM EXCAVATIONS ON NEDS LAKE ..............................................16

FIGURE 8. DU EXCAVATIONS ON ANTELOPE LAKE.................................................................18

Figure 9. PEDRO LAKE BACKGROUND SoIL SAMPLE LOCATIONS..............................................19

FIGURE 10. CAS TA-52-001-TANL (NEDS DETONATION AREA) SOIL SAMPLE LOCATIONS.......24

FIGURE 11. CAS TA-52-006-TAPL (DUS) SOIL SAMPLE LOCATIONS.......................................30

FIGURE 12. CAS TA-52-004-TAAL (MPDT) SOIL SAMPLE LOCATIONS .....................................36

FIGURE 13. CAS RG-52-007-TAML, DAVIS GUN PENETRATOR TEST........................................46

FIGURE 14. CAS TA-52-005-TAAL, JTA DU SITES..............................................................48

Figure 15. Aerial Photograph of Pedro Lake Showing Suspected Davis Gun

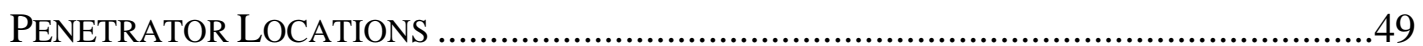

Figure 16. MultiSpectral Survey of Pedro Lake Showing Suspected Davis Gun

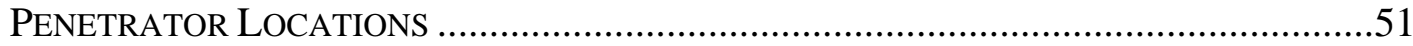

Figure 17. DEPRESSIONS AND RubBLE PILES EVIDENT AT DAVIS GUN SiTES ............................52

Figure 18. Remotely Controlled TOW Vehicle AND TOWEd ARRAY .................................54

Figure 19. Davis Gun PENETRATOR Found At Mt. HELEN .....................................................57

Figure 20. EXPLORATORY EXCAVATIONS FOR DAVIS GUN PENETRATORS ON PEDRO LAKE ........59

Figure 21. EXPLORATORY EXCAVATIONS FOR DAVIS GUN PENETRATORS ON ANTELOPE

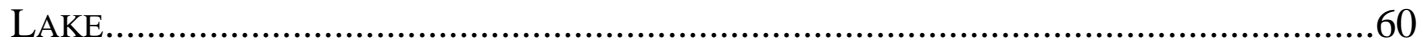

Figure 22. EXPLORATORY EXCAVATIONS FOR DAVIS Gun PENETRATORS AT Mt. HeLEN...........62

FiguRE 23. CAS TA-52-001-TANL, CONTAINMENT TANK AND STEEL STRUCTURE .....................66 


\section{LIST OF TABLES}

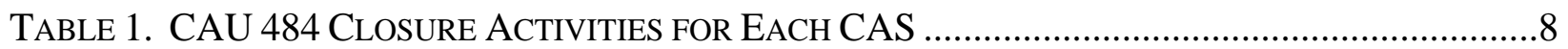

TABle 2. Soll SAmple Results for Beryllium at Pedro LaKe ................................................20

Table 3. Soil Sample Results for Uranium at Pedro LaKe ................................................21

TABLE 4. SoIl SAMPLE RESUlTS FOR BERYLLIUM AT CAS TA-52-001-TANL,

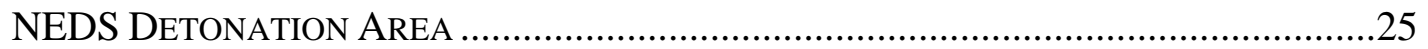

TABLE 5. SOIL SAMPLE RESULTS FOR URANIUM AT CAS TA-52-001-TANL,

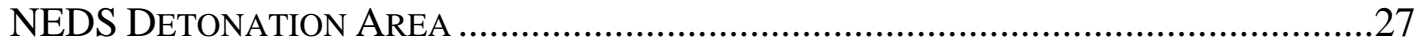

TABle 6. SoIl SAMPLE Results For BERYLLIUM at CAS TA-52-006-TAPL, DUS ...................31

TABLE 7. SoIl SAMPLE ResultS For URANIUM At CAS TA-52-006-TAPL, DUS .........................33

TABLE 8. SOIL SAMPLE RESULTS FOR BERYLLIUM AT CAS TA-52-004-TAAL, MPDT ..................37

TABLE 9. SoIl SAMPLE RESUltS FOR URANiUm AT CAS TA-52-004-TAAL, MPDT ....................39

TABLE 10. Summary of DU-EXCAVATED SoIl From SuRfaCe Detonation Debris Sites .......43

TABLE 11. Summary OF DU-EXCAVATED SOIL FROM SuRFACE-SHALlOW SUBSURFACE

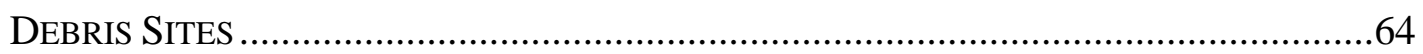

TABLE 12. CAU 484 Closure Activities SCHEDULE ............................................................69

TABLE 13. CAU 484 LLW DiSPOSITION SUMMARY ……………………..................................

\section{APPENDICES}

Appendix A. Data Quality ObJectives

APPENDiX B. SAMPle ANALyTICAL Results

APPENDIX C. WASTE DISPOSITION DOCUMENTATION

APPENDiX D. Site Closure PhOTOGRAPHS

Appendix E. Position Paper - End State Meeting for Depleted Uranium and Davis Gun TARGETS, JANUARY 2007

Appendix F. Position Paper - Initial Multi-Spectral, Electromagnetic, And RADIOMETRIC SURVEY RESULTS, JANUARY 2006

Appendix G. Position Paper - Depleted Uranium Closure In Place, August 2007

APPENDIX H. UXO REPORT

APPENDIX I. MULTISPECTRAL SURVEY REPORT

APPENDIX J. STATISTICS REPORTS

APPENDIX K. GEOPHYSICS REPORTS

ApPendiX L. DAVIS Gun TARgets TABle

APPENDIX M. RADIOLOGICAL SURVEY REPORT

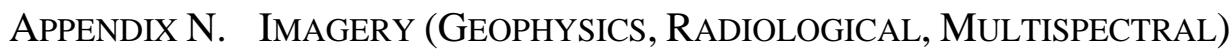

APPENDIX O. USE RESTRICTION DOCUMENTATION 


\begin{tabular}{|c|c|}
\hline bgs & below ground surface \\
\hline CAS & Corrective Action Site \\
\hline CAU & Corrective Action Unit \\
\hline $\mathrm{COC}(\mathrm{s})$ & contaminant(s) of concern \\
\hline COPC(s) & contaminant(s) of potential concern \\
\hline CR & Closure Report \\
\hline CSM & conceptual site model \\
\hline DOE & U.S. Department of Energy \\
\hline DQO & data quality objective(s) \\
\hline DU & depleted uranium \\
\hline DUS & Depleted Uranium Site \\
\hline EPA & U.S. Environmental Protection Agency \\
\hline FIDLER & field instrument for detecting low energy radiation \\
\hline FFACO & Federal Facility Agreement and Consent Order \\
\hline $\mathrm{ft}$ & foot (feet) \\
\hline $\mathrm{ft}^{3}$ & cubic foot (feet) \\
\hline gps & Global Positioning System \\
\hline JTA & Joint Test Assembly \\
\hline LLW & low-level waste \\
\hline $\mathrm{M} \& \mathrm{OC}$ & Management and Operations Contractor \\
\hline $\mathrm{mg} / \mathrm{kg}$ & milligram(s) per kilogram \\
\hline MPDT & Metal Particle Dispersion Test \\
\hline Mt. & Mount \\
\hline MTADS & multi-sensor towed array detection system \\
\hline $\mathrm{mV}$ & millivolt(s) \\
\hline NDEP & Nevada Division of Environmental Protection \\
\hline $\mathrm{NIOSH}$ & National Institute for Occupational Safety and Health \\
\hline NNSA/NSO & $\begin{array}{l}\text { U.S. Department of Energy, National Nuclear Security Administration Nevada } \\
\text { Site Office }\end{array}$ \\
\hline NNSA/NV & $\begin{array}{l}\text { U.S. Department of Energy, National Nuclear Security Administration Nevada } \\
\text { Operations Office }\end{array}$ \\
\hline
\end{tabular}




\section{ACRONYMS AND ABBREVIATIONS (continued)}

$\begin{array}{ll}\text { NTS } & \text { Nevada Test Site } \\ \text { PAL } & \text { preliminary action level } \\ \text { pCi/g } & \text { picoCuries(s) per gram } \\ \text { pCi/L } & \text { picoCuries(s) per liter } \\ \text { QA } & \text { quality assurance } \\ \text { QAPP } & \text { Quality Assurance Project Plan } \\ \text { QC } & \text { quality control } \\ \text { RMA } & \text { Radioactive Materials Area } \\ \text { RWMS } & \text { Radioactive Waste Management Site } \\ \text { SAFER } & \text { Streamlined Approach for Environmental Restoration } \\ \text { TTR } & \text { Tonopah Test Range } \\ \mu \text { g/L } & \text { micrograms per liter } \\ \text { UXO } & \text { unexploded ordnance } \\ \text { VSP } & \text { Visual Sample Plan } \\ \text { WMA } & \text { waste management area } \\ \text { yd } & \end{array}$




\section{EXECUTIVE SUMMARY}

Corrective Action Unit (CAU) 484 is identified in the Federal Facility Agreement and Consent Order (FFACO, 1996) as Surface Debris, Waste Sites, and Burn Area (TTR). CAU 484 is located at the Tonopah Test Range, Nevada, and consists of the following six Corrective Action Sites (CASs):

- CAS RG-52-007-TAML, Davis Gun Penetrator Test

- CAS TA-52-001-TANL, NEDS Detonation Area

- CAS TA-52-004-TAAL, Metal Particle Dispersion Test

- CAS TA-52-005-TAAL, Joint Test Assembly DU Sites

- CAS TA-52-006-TAPL, Depleted Uranium Site

- CAS TA-54-001-TANL, Containment Tank and Steel Structure

CAU 484 closure activities started in November 2003 and were completed in August 2007. Activities were performed in accordance with the FFACO and the Streamlined Approach for Environmental Restoration (SAFER) Plan for CAU 484 (U.S. Department of Energy, National Nuclear Security Administration Nevada Site Office, 2004b). The corrective action alternatives that were applied at each site consisted of clean closure and closure in place with best management practices.

CASs TA-52-001-TANL (NEDS Detonation Area), TA-52-004-TAAL (Metal Particle Dispersion Test), TA-52-005-TAAL (Joint Test Assembly DU Sites), and TA-52-006-TAPL (Depleted Uranium Site) were closed by locating, removing, and disposing of depleted uranium (DU) and soil that had been impacted by the DU. Drive-over radiological and geophysical surveys were conducted to locate radiological hotspots and metal fragments that could be radiologically contaminated. Soil samples were collected to assist in locating elevated radioactivity and beryllium, to determine the concentrations where radioactivity was elevated, and to confirm that excavation and removal activities had successfully reduced DU contamination to acceptable levels.

At CAS RG-52-007-TAML (Davis Gun Penetrator Test), aerial photography and multispectral surveys were conducted in addition to the drive-over radiological and geophysical surveys. The aerial surveys were performed to identify areas where historical excavations to recover penetrators were evident and to identify circular bermed areas where testing occurred. Locations where penetrators were most likely to be present were excavated at Pedro Lake, Antelope Lake, and Mount Helen. No penetrators were found at any of the excavation locations, and results of the investigation activities indicate that the penetrators were either recovered or are buried so deep as to not be a hazard to human health or the environment. However, DU was present in the soil at Antelope Lake. Although the DU locations could not be directly associated with Davis Gun penetrators, they also could not be disproved from being thus associated. DU was excavated where clean closure was feasible and closed in place where it was not, at four locations on Antelope Lake. 
CAS TA-54-001-TANL (Containment Tank and Steel Structure) was an uncontaminated site that was closed by removing the uncontaminated structures and either recycling the materials or re-using them elsewhere.

DU and radiologically-impacted soil outside of the CAS boundaries was detected because the surveys covered areas larger than the CAS boundaries. Where concentrations exceeded the action levels established in the CAU 484 SAFER Plan, these areas were also excavated and cleaned up as a best management practice.

Closure activities generated approximately 343 cubic yards of low-level waste (LLW) and approximately 40 cubic yards of sanitary waste and recyclable materials. The LLW consisted of DU and radiologically-impacted soil that was containerized and will be transported to the Nevada Test Site for disposal at the Area 5 Radioactive Waste Management Site. The recyclable materials consisted of structure debris that was size-reduced and transported to the Sandia Salvage yard in Area 3 at the TTR.

This closure report documents the activities taken to close this CAU and includes cleanup verification sample results, waste disposition paperwork, and other supporting documentation. 
This Closure Report (CR) documents closure activities for Corrective Action Unit (CAU) 484, Surface Debris, Waste Sites, and Burn Area (TTR), according to the Federal Facility Agreement and Consent Order (FFACO, 1996) and the Streamlined Approach for Environmental Restoration (SAFER) Plan for CAU 484 (U.S. Department of Energy, National Nuclear Security Administration Nevada Site Office [NNSA/NSO], 2004b). CAU 484 is located at the Tonopah Test Range (TTR), Nevada (Figure 1), and consists of the following six Corrective Action Sites (CASs):

- CAS RG-52-007-TAML, Davis Gun Penetrator Test

- $\quad$ CAS TA-52-001-TANL, NEDS Detonation Area

- CAS TA-52-004-TAAL, Metal Particle Dispersion Test (MPDT)

- CAS TA-52-005-TAAL, Joint Test Assembly (JTA) DU Sites

- CAS TA-52-006-TAPL, Depleted Uranium Site (DUS)

- CAS TA-54-001-TANL, Containment Tank and Steel Structure

\subsection{Purpose}

The purpose of this CR is to provide a summary of the completed closure activities, to document waste disposal, and to present analytical data confirming that the remediation goals were met. The closure alternatives consisted of clean closure and closure in place with implementation of best management practices.

\subsection{SCOPE}

The closure strategy for CAU 484 was as follows:

- CAS RG-52-007-TAML (Davis Gun Penetrator Test) was closed in place by investigating known Davis Gun Penetrator Test locations, identifying geophysical anomalies that were potential penetrators, and excavating DU-contaminated soil possibly associated with the Davis Gun tests. Suspected penetrator locations were excavated to identify the source of the geophysical anomalies on Antelope Lake, Pedro Lake, and at Mount (Mt.) Helen. No Davis Gun penetrator debris or DU was encountered during any of the exploratory excavations; therefore, NNSA/NSO and the Nevada Division of Environmental Protection (NDEP) agreed that no additional Davis Gun penetrator targets would be excavated or investigated. Investigating penetrators that may be present at depths greater than 10 feet (ft) below ground surface (bgs) is not feasible due to technology limitations and, at these depths, hazardous debris and/or depleted uranium (DU) does not pose a threat to human health and/or the environment. DU was found at locations not directly attributable to a Davis Gun penetrator and was excavated and clean closed where possible. Four locations on Antelope Lake were closed in place, one of which is not a CAU 484 site but which was completed as a best management practice. 

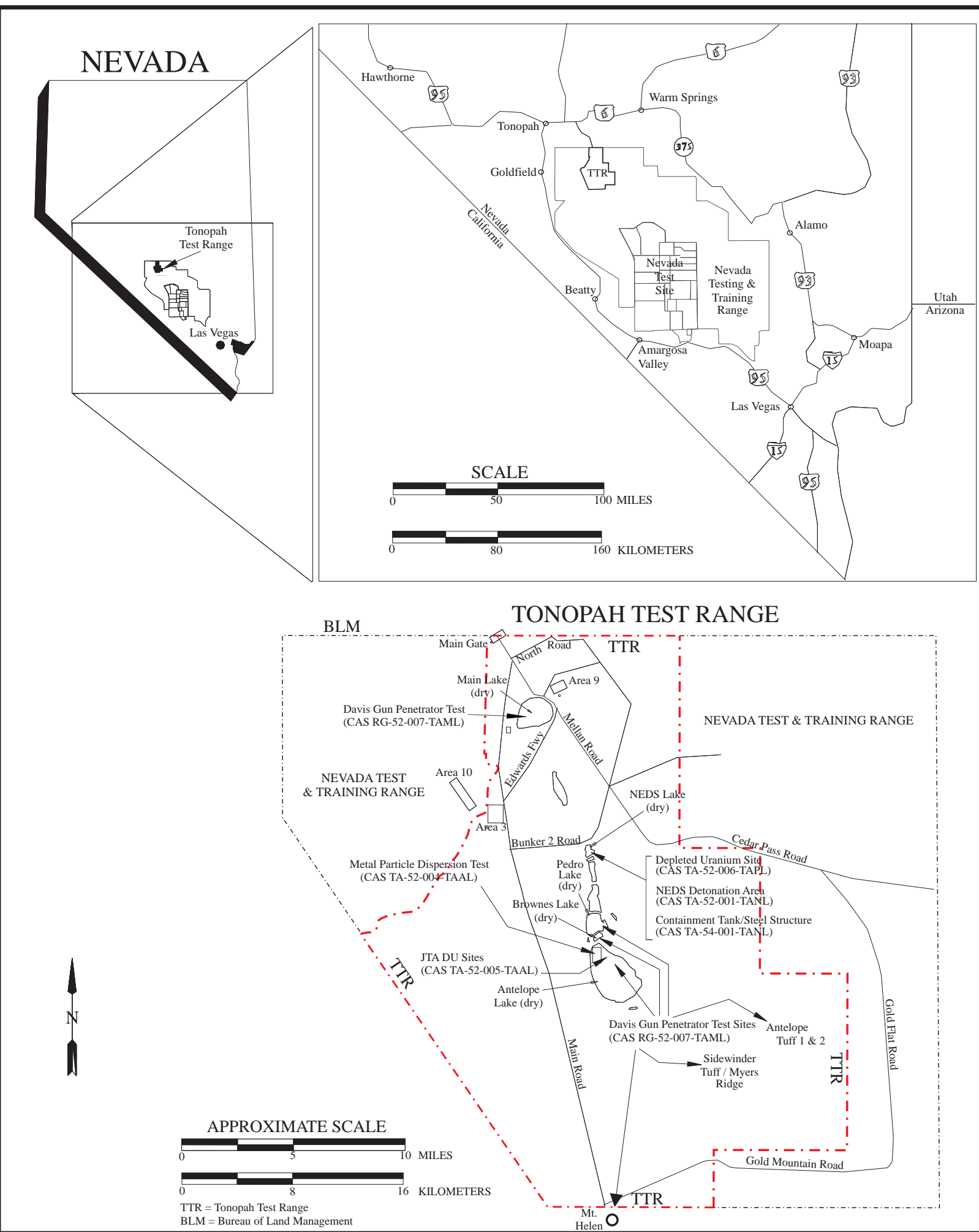

FIGURE 1

CAU 484 SiTE LOCATION MAP 
- CAS TA-52-001-TANL (NEDS Detonation Area) was clean closed by removing and disposing of DU debris and contaminated soil.

- CAS TA-52-004-TAAL (MPDT) was clean closed by removing and disposing of DU debris and contaminated soil.

- CAS TA-52-005-TAAL (JTA DU Sites) was clean closed by locating, retrieving, and disposing of three DU rings that comprise the CAS.

- CAS TA-52-006-TAPL (DUS) was clean closed by removing and disposing of DU debris and contaminated soil.

- CAS TA-54-001-TANL (Containment Tank and Steel Structure) was clean closed by removing the uncontaminated metal and wood structures and either recycling the materials or re-using them elsewhere.

\subsection{Closure Report Contents}

This CR includes the following sections:

- Section 1.0 - Introduction

- Section 2.0 - Closure Activities

- Section 3.0 - Waste Disposition

- Section 4.0 - Closure Verification Results

- Section 5.0 - Conclusions and Recommendations

- Section 6.0 - References

- Appendix A - Data Quality Objectives

- Appendix B - Sample Analytical Results

- Appendix C - Waste Disposition Documentation

- Appendix D - Site Closure Photographs

- Appendix E - Position Paper - End State Meeting for Depleted Uranium and Davis Gun Targets, January 2007

- Appendix F - Position Paper - Initial Multi-Spectral, Electromagnetic, and Radiometric Survey Results, January 2006

- Appendix G - Position Paper - Depleted Uranium Closure in Place, August 2007

- Appendix H - UXO Report

- Appendix I - Multispectral Survey Report

- Appendix J - Statistics Reports

- Appendix K - Geophysics Reports

- Appendix L - Davis Gun Targets Table 
- Appendix M - Radiological Surveys Report

- Appendix N - Imagery (Geophysical, Radiological, and Multispectral)

- Appendix O - Use Restriction Documentation

- Library Distribution List

This report was developed using information and guidance from the following documents:

- $\quad$ SAFER Plan for CAU 484 (NNSA/NSO, 2004b)

- Nevada Environmental Restoration Project Industrial Sites Quality Assurance Project Plan (QAPP) (U.S. Department of Energy, National Nuclear Security Administration Nevada Operations Office [NNSA/NV], 2002)

\subsubsection{Data Quality Objectives}

The data quality objectives (DQOs) used for closure of CAU 484 were presented in Appendix A of the SAFER Plan for CAU 484 (NNSA/NSO, 2004b) and are included as Appendix A of this report. Three conceptual site models (CSMs) were developed for CAU 484 based on process knowledge, historical information, and personnel interviews.

The first CSM, Surface-Shallow Subsurface Debris, includes CASs RG-52-007-TAML (Davis Gun Penetrator Test) and TA-52-005-TAAL (JTA DU Sites). This CSM assumes the primary source of potential contamination is discrete pieces of DU that fractured from the Davis Gun Penetrator and JTA tests. Although surface and shallow subsurface soil could be affected by DU from un-recovered penetrators and DU rings from these tests, the un-recovered penetrators and DU rings themselves were expected to be the only wastes at these sites.

The second CSM, Surface Detonation Debris, includes CASs TA-52-001-TANL (NEDS Detonation Area), TA-52-004-TAAL (MPDT), and TA-52-006-TAPL (DUS, “Colimbo”). This CSM assumes that DU and beryllium may be present as discrete fragments on the ground surface as a result of surface detonations. The assumption is that beryllium will be similarly dispersed to the DU because the beryllium and DU came from the same source and would have been dispersed in the same detonation, scattering beryllium (if present) to the same locations as DU.

The third CSM, Non-Impacted Surface Debris, addresses CAS TA-54-001-TANL (Containment Tank and Steel Structure) and assumes that the metal and wood structures that comprise the CAS are uncontaminated and can be either disposed of as construction debris or recycled.

In general, the CSMs were confirmed by closure activities. CSM confirmation and variations are identified below.

- The Surface-Shallow Subsurface Debris CSM was confirmed at CAS TA-52-005-TAAL (JTA DU Sites) by finding the three DU rings on Antelope Lake and DU-contaminated soil under the rings. One of the DU rings was found outside of the expected CAS boundary, indicating that the projectiles containing the DU rings had traveled farther than originally expected. For CAS RG-52-007-TAML (Davis Gun Penetrator Test), aerial photography and multispectral and geophysical surveys, used in conjunction with visual observations of surface features, indicated potential locations where Davis Gun Penetrators could be located. However, the only penetrator observed was a solid, inert slug (with no DU) on the ground 
surface at Mt. Helen. All penetrator excavations on Antelope and Pedro Lakes contained practice ordnance from other test events and metallic debris. Excavation activities and interviews with project personnel indicate that the penetrators were most likely recovered after the test and that, if any penetrators were not recovered, they are at such depth that they do not pose a hazard. At the locations where DU was detected, it was oxidized and present in the soil as well as being in discrete pieces. This was a deviation from the CSM and was addressed in hold point meetings with NDEP. As a best management practice, the DU was excavated and clean closed, where possible, or closed in place with use restrictions.

- The Surface Detonation Debris CSM was confirmed by locating and removing DU and debris from the CASs on NEDS Lake and Antelope Lake. DU and chemical uranium were detected, removed, and disposed. Beryllium did not exceed action levels in any of the soil samples. Further investigation, after the SAFER Plan (NNSA/NSO, 2004b) had been completed, revealed that the boundary for CAS TA-52-001-TANL (NEDS Detonation Area) was incorrectly identified and was actually approximately $700 \mathrm{ft}$ south of the original location. The boundary for TA-52-006-TAPL (DUS) was slightly modified to account for an offset dispersal of debris from the aerial detonation of the cruise missile.

- The Non-Impacted Surface Debris CSM was confirmed through removal and disposal of the surface debris and structures located at CAS TA-54-001-TANL. 
Closure Report - CAU 484

Section: Introduction

Revision: 0

Date: September 2007

THIS PAGE INTENTIONALLY LEFT BLANK 
This section details the specific activities completed during the closure of CAU 484, deviations from the CAU 484 SAFER Plan, the schedule of completed activities, and the final site plan. Photographs in Appendix D of this report document the state of the sites before corrective actions were implemented, during field work, and after completion of work.

\subsection{Description of Corrective Action Activities}

Closure activities for CAU 484 were completed according to the SAFER Plan (NNSA/NSO, 2004b) and based on other decisions made by the core decision team identified in the SAFER Plan. Closure activities consisted of aerial photography and multispectral surveys; drive-over radiological, geophysical, and unexploded ordnance (UXO) surveys; walk-over geophysical and radiological surveys; Geoprobe ${ }^{\circledR}$ investigation soil sample collection; exploratory excavations; and DU cleanup. The following sections detail the closure activities as completed. Due to the similarities of activities within each CSM, the closure activities will be presented by CSM, rather than in order of CAS number. Table 1 identifies the activities that were conducted at each CAS.

\subsubsection{Preplanning and Site Preparation}

Prior to closure activities, the following documents were prepared:

- National Environmental Policy Act Checklist

- Site-Specific Health and Safety Plan

- Field Management Plan

- NNSA/NSO Real Estate/Operations Permits

- Work control packages

\subsubsection{Closure Activities - Surface Detonation Debris Sites}

The surface detonation debris sites CSM consists of the following three CASs:

- $\quad$ CAS TA-52-001-TANL, NEDS Detonation Area, is located on the northern half of NEDS Lake. The SAFER Plan (NNSA/NSO, 2004b) shows ground zero at the northern edge of NEDS Lake; however, additional process knowledge uncovered during this investigation, along with investigation results, showed that the actual ground zero was slightly to the south of the originally identified area (Figure 2).

- CAS TA-52-006-TAPL, DUS (“Colimbo”), is located on the northern half of NEDS Lake, to the north of CAS TA-52-001-TANL. Ground zero of the test is marked on the playa surface by a survey monument; however, because the detonation was in the air, the actual area of dispersion was further to the northwest, in the direction of cruise missile travel, than originally presented in the SAFER Plan. The location of fine-particle deposition from the detonation is indicated by a circle with a 300-ft radius centered near ground zero of the 
Table 1. CAU 484 Closure ACtivities for EACH CAS

\begin{tabular}{|c|c|c|c|c|c|c|c|c|}
\hline CAS & CSM* & $\begin{array}{c}\text { AERIAL } \\
\text { PHOTOGRAPHY }\end{array}$ & $\begin{array}{l}\text { MULTI- } \\
\text { SPECTRAL } \\
\text { SURVEYS }\end{array}$ & $\begin{array}{l}\text { RADIOLOGICAL } \\
\text { SURVEYS }\end{array}$ & $\begin{array}{l}\text { GEOPHYSICAL } \\
\text { SURVEYS }\end{array}$ & $\begin{array}{l}\text { GEOPROBE } \\
\text { SAMPLING }\end{array}$ & $\begin{array}{l}\text { EXPLORATORY } \\
\text { EXCAVATION }\end{array}$ & $\begin{array}{l}\text { REMEDIAL } \\
\text { EXCAVATION/ } \\
\text { REMOVAL }\end{array}$ \\
\hline $\begin{array}{l}\text { RG-52-007-TAML } \\
\text { (Davis Gun } \\
\text { Penetrator Test) }\end{array}$ & B & $X$ & $X$ & $X$ & $X$ & & $X$ & $X$ \\
\hline $\begin{array}{l}\text { TA-52-001-TANL } \\
\text { (NEDS Detonation } \\
\text { Area) }\end{array}$ & A & $X$ & $X$ & $X$ & $X$ & $X$ & & $X$ \\
\hline $\begin{array}{l}\text { TA-52-004-TAAL } \\
\text { (MPDT) }\end{array}$ & A & $X$ & $X$ & $X$ & $X$ & $X$ & & $X$ \\
\hline $\begin{array}{l}\text { TA-52-005-TAAL } \\
\text { (JTA DU Sites) }\end{array}$ & B & $X$ & $X$ & $X$ & $X$ & & & $\mathrm{X}$ \\
\hline $\begin{array}{l}\text { TA-52-006-TAPL } \\
\text { (DUS) }\end{array}$ & A & $X$ & $X$ & $X$ & $X$ & $X$ & & $X$ \\
\hline $\begin{array}{l}\text { TA-54-001-TANL } \\
\text { (Tank \& Structures) }\end{array}$ & $\mathrm{C}$ & & & $X$ & & & & $X$ \\
\hline
\end{tabular}




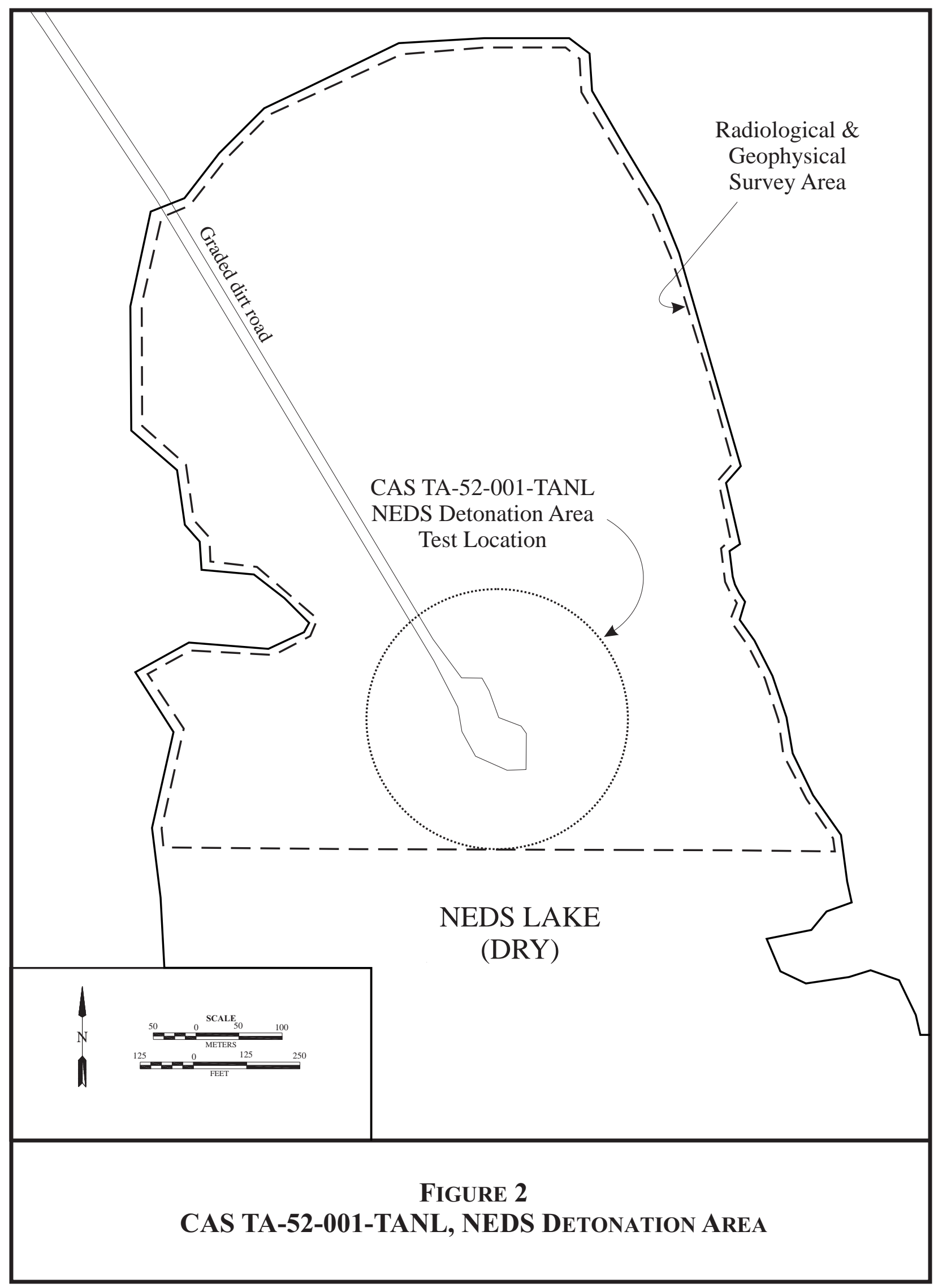


impact, is shown in Figure 3. Surface and shallow subsurface soil was sampled within a 300 -ft radius of the detonation area to access impact from fine particulates resulting from the detonation.

- $\quad$ CAS TA-52-004-TAAL, MPDT, is located in the northwest corner of Antelope Lake. The CAS is an area of approximately 2,000 ft by 3,000 ft that was bermed to keep the area from flooding after the test (NNSA/NSO, 2004b). Documentation found during the investigation confirmed the location of the detonation to be near the south end of the bermed area, and the ejecta from the detonation to have been mapped a considerable distance from the boundaries of the bermed area. Surface and shallow subsurface soil was sampled within a $300-\mathrm{ft}$ radius of the detonation area (Figure 4) to access impact from fine particulates resulting from the detonation.

Based on the CSM, these sites are generally considered to be areas where normally distributed, discrete pieces of DU debris were expected to be present on the ground surface. The sources of the debris were test articles that were detonated on or above the ground surface. Associated debris was therefore expected to be occurring at the greatest concentrations near the point source of the detonation (i.e., ground zero) and decreasing in all directions away from that point. Beryllium was associated with the test objects and, if present, was expected to be co-located with the DU debris and similarly dispersed. DU and beryllium were the contaminants of potential concern (COPCs) for these sites.

During the investigation and closure activities, it was determined that much of the DU was not in discrete pieces on the ground surface. Most of the DU encountered on NEDS and Antelope Lakes was highly oxidized, and in some cases the uranium oxide had migrated along drying/expansion cracks in the lake bed. This required re-evaluation of the CSM and the characterization/closure approach. Detailed information regarding the surface detonation debris sites CSM can be found in the SAFER Plan (NNSA/NSO, 2004b). The DQOs from that document, which present the CSM, are also included in Appendix A of this document.

\subsubsection{Radiological and Geophysical Drive-Over Surveys}

Radiological and geophysical drive-over surveys were conducted on the northern half of NEDS Lake and the northern half of Antelope Lake to map the debris and DU dispersion patterns for subsequent cleanup activities. The SAFER Plan (NNSA/NSO, 2004b) provides additional detail on the boundaries of the survey areas and the objectives, methodology, and equipment used for these surveys. Surveys were conducted from November to December 2003, and May to June 2004. The geophysical and radiological surveys were suspended in June 2004, when potentially live UXO was found on Antelope Lake; however, surveys of the Surface Detonation Debris Sites were completed before the UXO was encountered.

Geophysical results of the Surface Detonation Debris Sites was generally inconclusive and did not provide a good indication of where CAS-specific testing-related metal or associated DU (and associated beryllium, if any) might be located on the dry lake beds. Numerous activities over the history of the TTR have left metal pieces and fragments scattered over the dry lake bed surfaces, and the geophysical survey detected thousands of metallic objects, which may or may not be related to the CAU 484 CASs. An example, showing the large number of geophysical (as well 


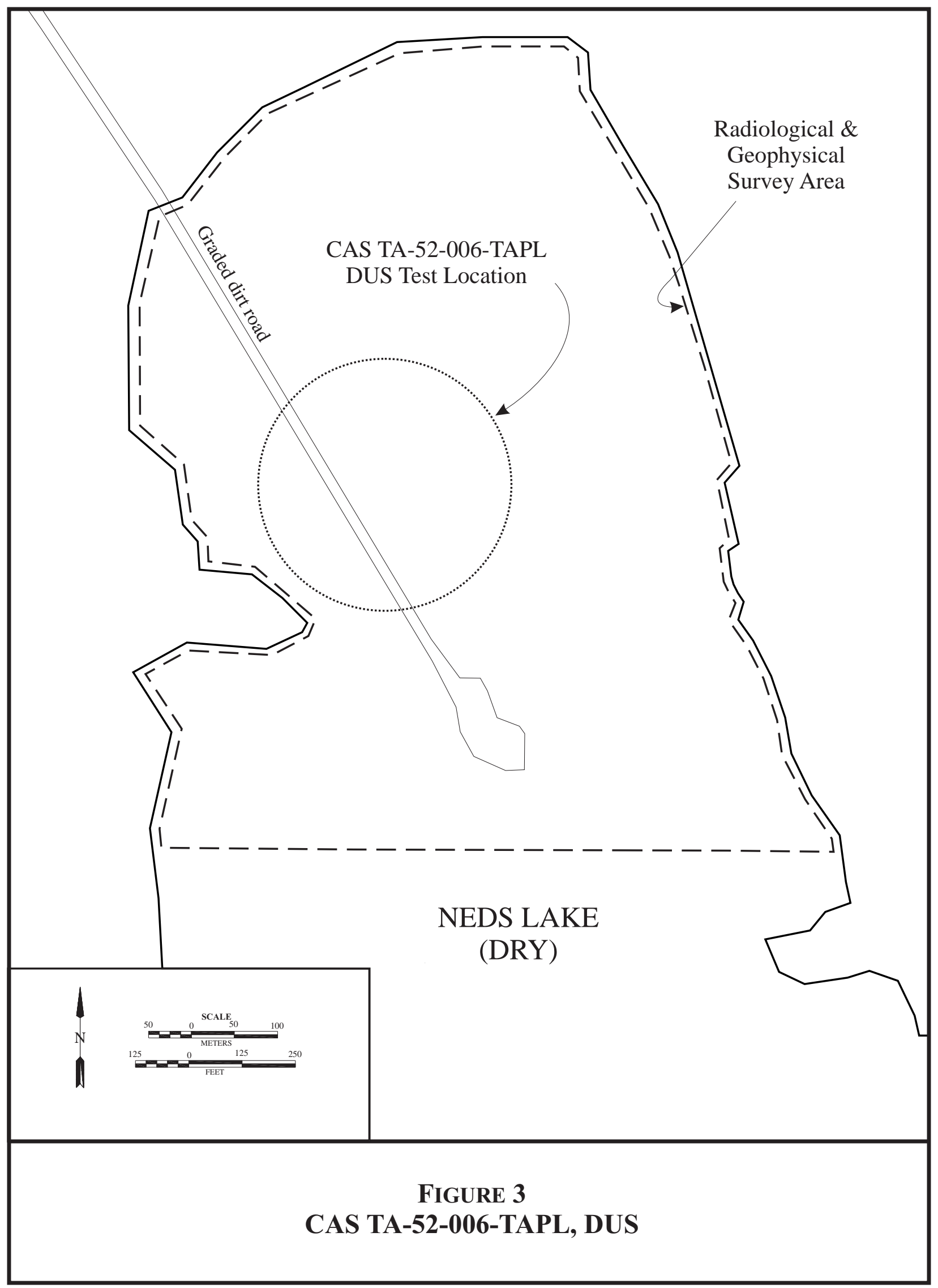




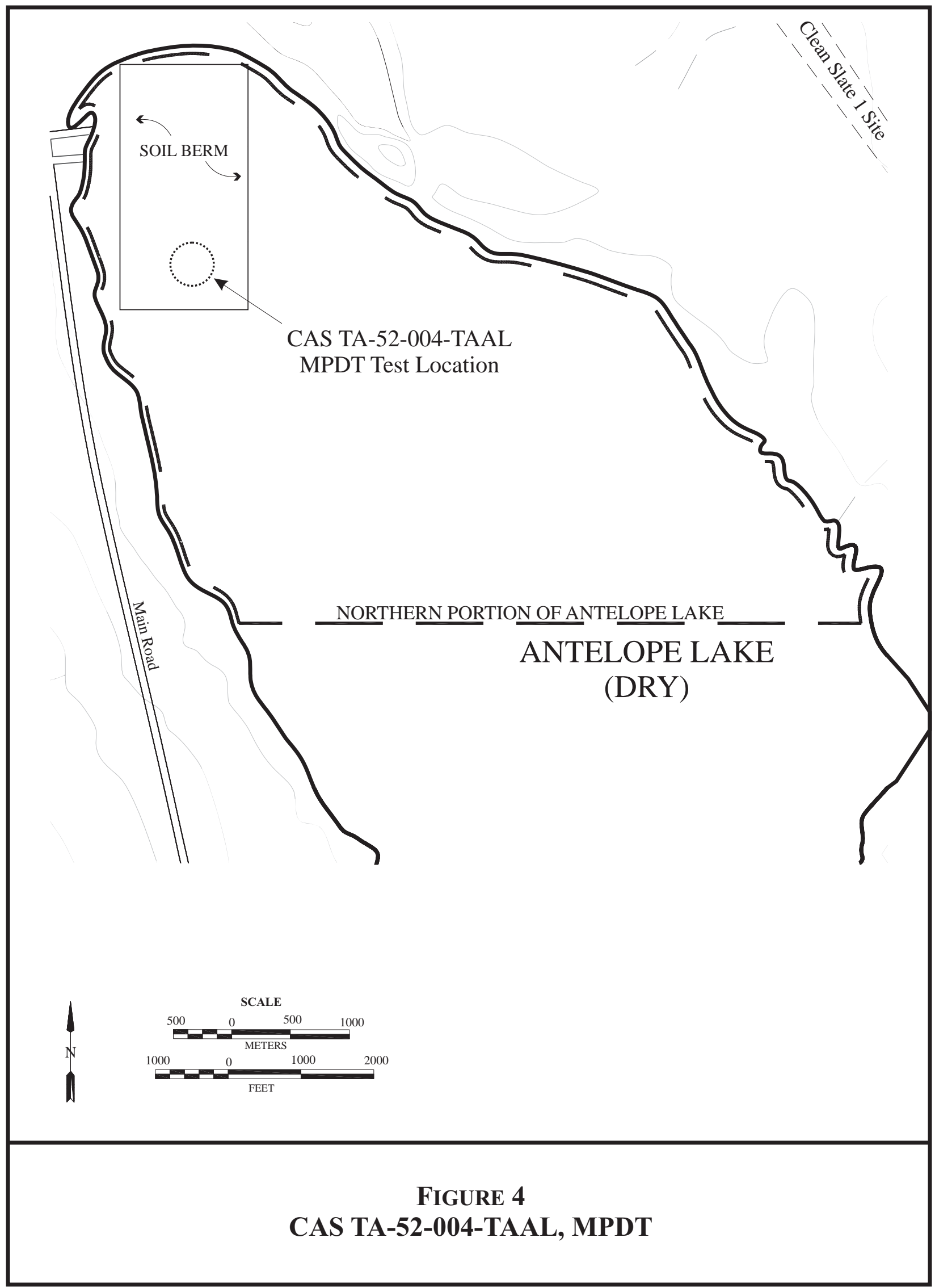


as radiological) anomalies on a portion of NEDS Lake, is provided in Figure 5. Geophysical survey reports are provided in Appendix K, and associated imagery showing the geophysical anomalies is presented in Appendix N.

Radiological survey data were processed to confirm the presence of DU, and each location was identified by a unique target number and latitude-longitude coordinate. Each DU target was evaluated and assigned a grade of A, B, C, or D, depending on the quality of the spectra and their statistical significance. Three spectral peaks and associated, elevated radioactivity were used in the rankings. For example, an "A" ranking was assigned where all three targeted spectral peaks were present, and a " $\mathrm{B}$ " ranking was assigned where only two peaks were present. From the rankings, DU was found to be present at all locations ranked "A," and at most locations ranked " $B$ " or " $C$," but not necessarily for those with a " $D$ " ranking. Locations were ranked " $D$ " if the radioactivity in the area appeared to have some statistical significance indicating possible elevated radioactivity; however, there were no discernable peaks. More details on the rankings and the radiological findings are presented in the radiological reports in Appendix $\mathrm{M}$.

A description of the radiological survey methodology and findings is presented in Appendix M. Radiological and geophysical data were compiled in a Geographic Information System database to determine areas where a DU target was associated with one or more geophysical anomalies, which may suggest more numerous DU fragments or a larger fragment of DU in the subsurface. An example showing the radiological anomalies on NEDS Lake, without the geophysical anomalies, is provided in Figure 6. Imagery of radiological and geophysical survey results is presented in Appendix N.

\subsection{CAS TA-52-001-TANL (NEDS Detonation Area)}

The radiological survey identified seven locations of DU within the 300-ft radius testing location for the NEDS Detonation Area (i.e., NEDS 3, 6, 7, 8, 10, 13, and 14) (Figure 7). Additional DU was detected at locations outside of the 300-ft areas for either the NEDS Detonation Area or the DUS. Because of the nature of the detonation tests on NEDS Lake, DU associated with the NEDS tests could not be distinguished from DU resulting from the Colimbo (DUS) detonation. Therefore, all 20 DU targets identified and confirmed to be present on NEDS Lake were ultimately remediated. During the reacquisition and cleanup activities, several DU targets were confirmed to consist of several small areas of DU instead of the one large impacted area indicated by the initial survey data.

\subsection{CAS TA-52-006-TAPL (DUS, “Colimbo”)}

The radiological survey showed two locations of DU within the $300-\mathrm{ft}$ radius testing location for the DUS (Figure 7). A Cobalt-60 radiological source (described in the SAFER Plan) was not found and is either not located on the northern portion of NEDS Lake, as was originally thought, or was removed some time in the past. Section 2.1.2.1.1 contains additional information about DU identified on NEDS Lake. 


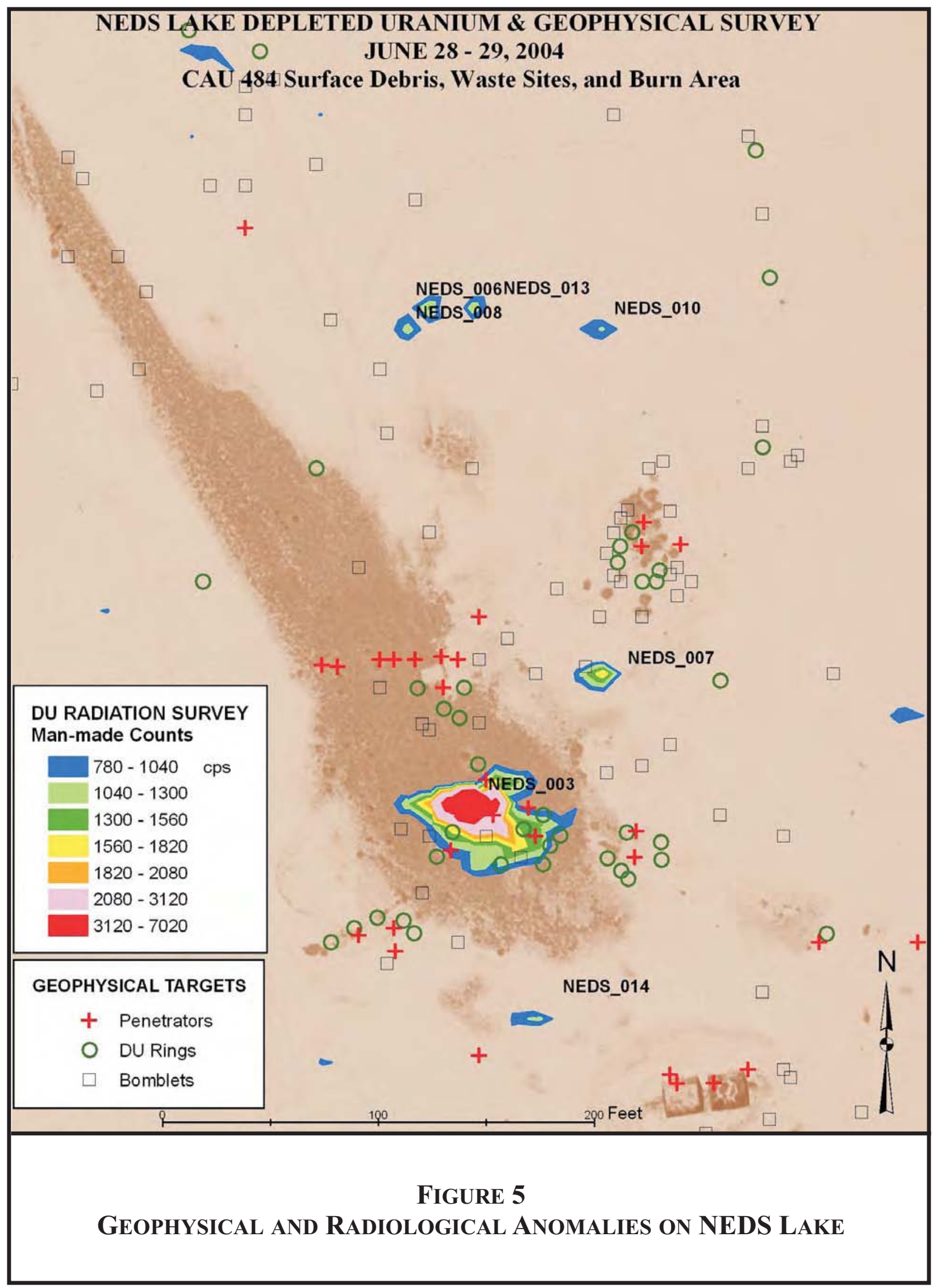




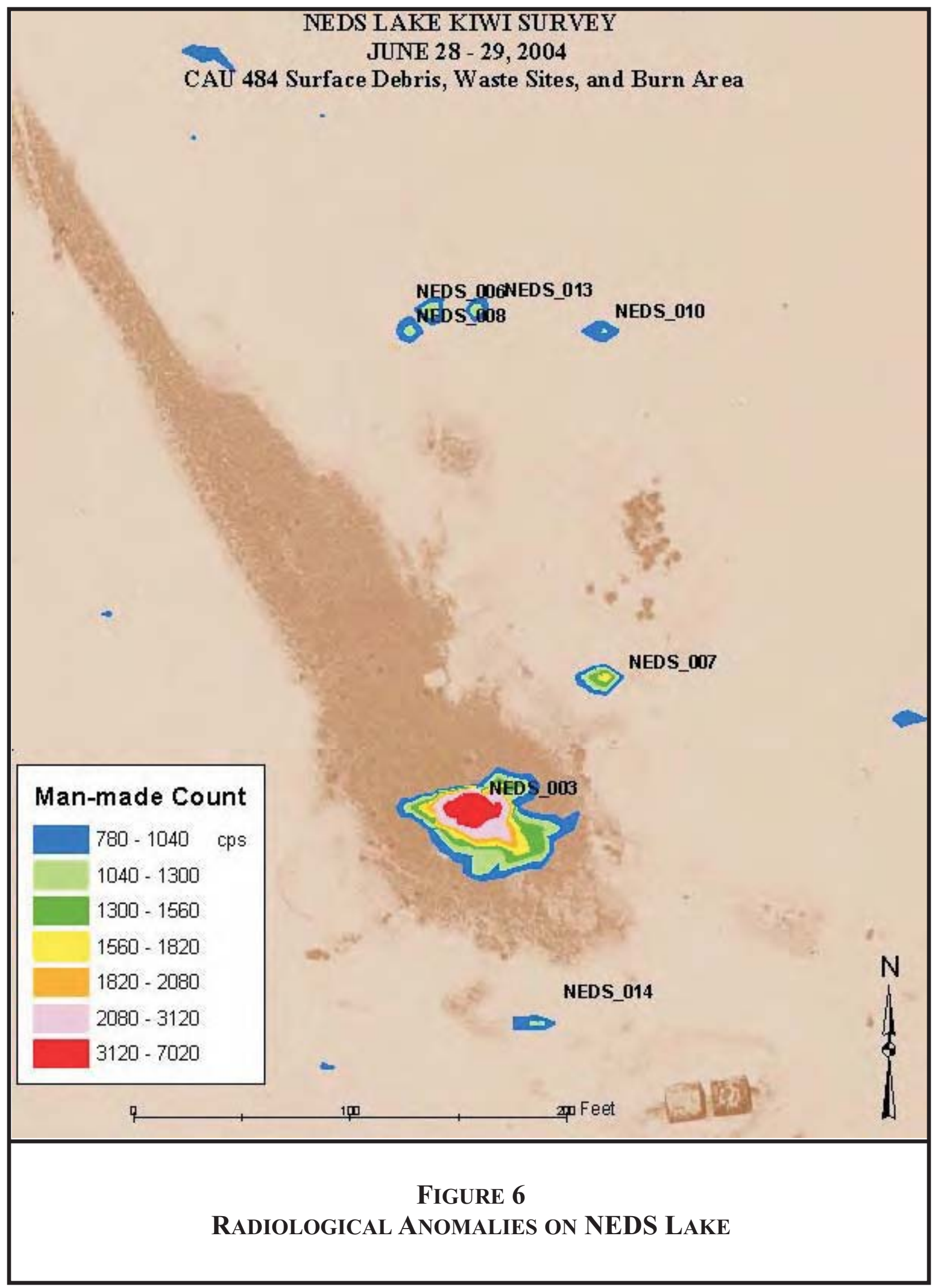




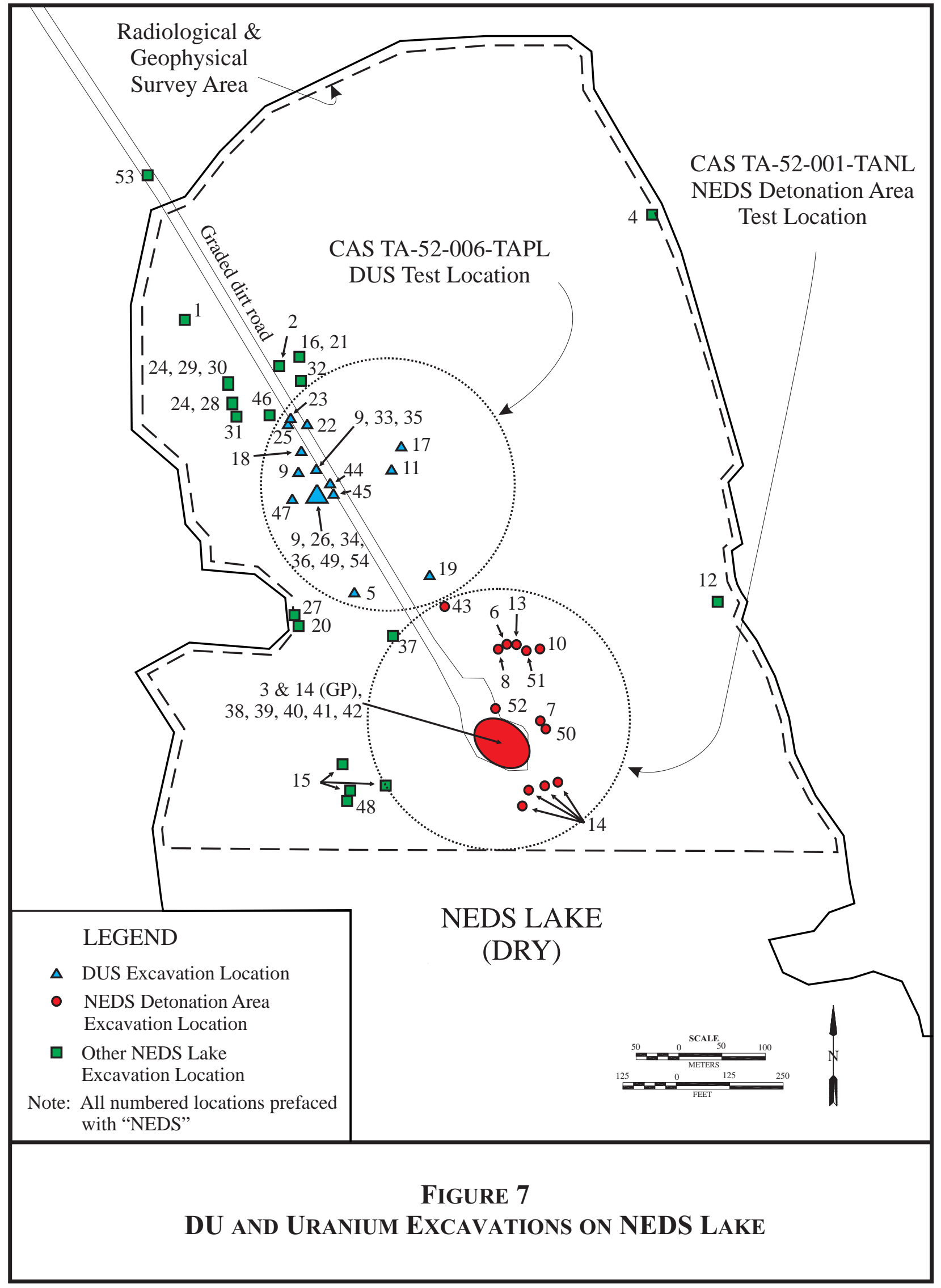




\subsection{CAS TA-52-004-TAAL (MPDT)}

The radiological survey identified numerous locations of DU within the MPDT berm, including one location where DU had substantially higher activity. All but the one "higher activity" DU locations were low-energy targets that were only slightly higher than background. The one "higher activity" location (SCA-33 in Figure 8) was within the CAS boundary. Because all of Antelope Lake was surveyed for radioactivity, the northern half intended to locate Davis Gun penetrators (CAS RG-52-007-TAML) as well as DU associated with the MPDT site, additional areas of elevated radioactivity were detected outside of the MPDT boundary. Other locations of DU identified on Figure 8 are addressed in this CR under either CAS RG-52-007-TAML (Davis Gun Penetrator Test) or CAS TA-52-005-TAAL (JTA DU Sites). All areas of DU confirmed to be present were located and remediated.

\subsubsection{Investigation Soil Sample Collection}

Soil samples were collected from within each of the Surface Detonation Debris CSM CAS boundaries to supplement the drive-over surveys and obtain additional information regarding radiological and chemical concentrations in the soil. The $300-\mathrm{ft}$ radius area identified for each location was assumed to represent the area most likely to have been impacted by fine particulates as a result of the detonation. Larger fragments were assumed to have been dispersed further from the point of detonation and outside of the area selected for sampling. Samples were collected from locations generated by Visual Sample Plan (VSP) software (Pacific Northwest National Laboratory, 2005), with input parameters designed to detect a "hot spot" with a 95-percent confidence level of detection. For that level of confidence, 36 samples were collected from within each of the three CAS boundaries, from latitude and longitude coordinates identified by the program. Assumptions used to determine the required number of samples are included in the SAFER Plan. An additional 20 samples were collected, using the same methodology, from the south end of Pedro Lake (Figure 9). The Pedro Lake samples were collected to identify the background concentrations to be expected and against which to compare the CAS sample results. The Pedro Lake location was selected because it is a lake location least likely to have been used for tests of DU or beryllium projectiles, and no evidence of DU was found during the Kiwi radiological surveys of this area.

The sample location coordinates provided by VSP were located in the field using a handheld Global Positioning System (GPS). Samples were collected from 0-0.5 ft bgs and from 1.0-1.5 ft bgs using a Geoprobe. Samples were submitted for laboratory analysis to identify concentrations of DU, chemical uranium, and beryllium. Samples were submitted for laboratory analysis following standard chain of custody and Management and Operations Contractor (M\&OC) procedures. Laboratory analytical reports are maintained in M\&OC Environmental Restoration files in Mercury, Nevada. The analytical report summaries are presented in Appendix B.

Analytical results for soil samples collected from Pedro Lake are summarized in Tables 2 and 3. Beryllium was present in all samples at low concentrations, indicating the presence of beryllium at background levels of 1.7 milligrams per kilogram $(\mathrm{mg} / \mathrm{kg})$ or less. Chemical uranium was not present above detection levels in any of the background samples. Isotopic uranium (U-234, U-235, and U-238) was present but at low concentrations, much less than the corresponding action levels. COPCs and sampling rationale are documented in the SAFER Plan 


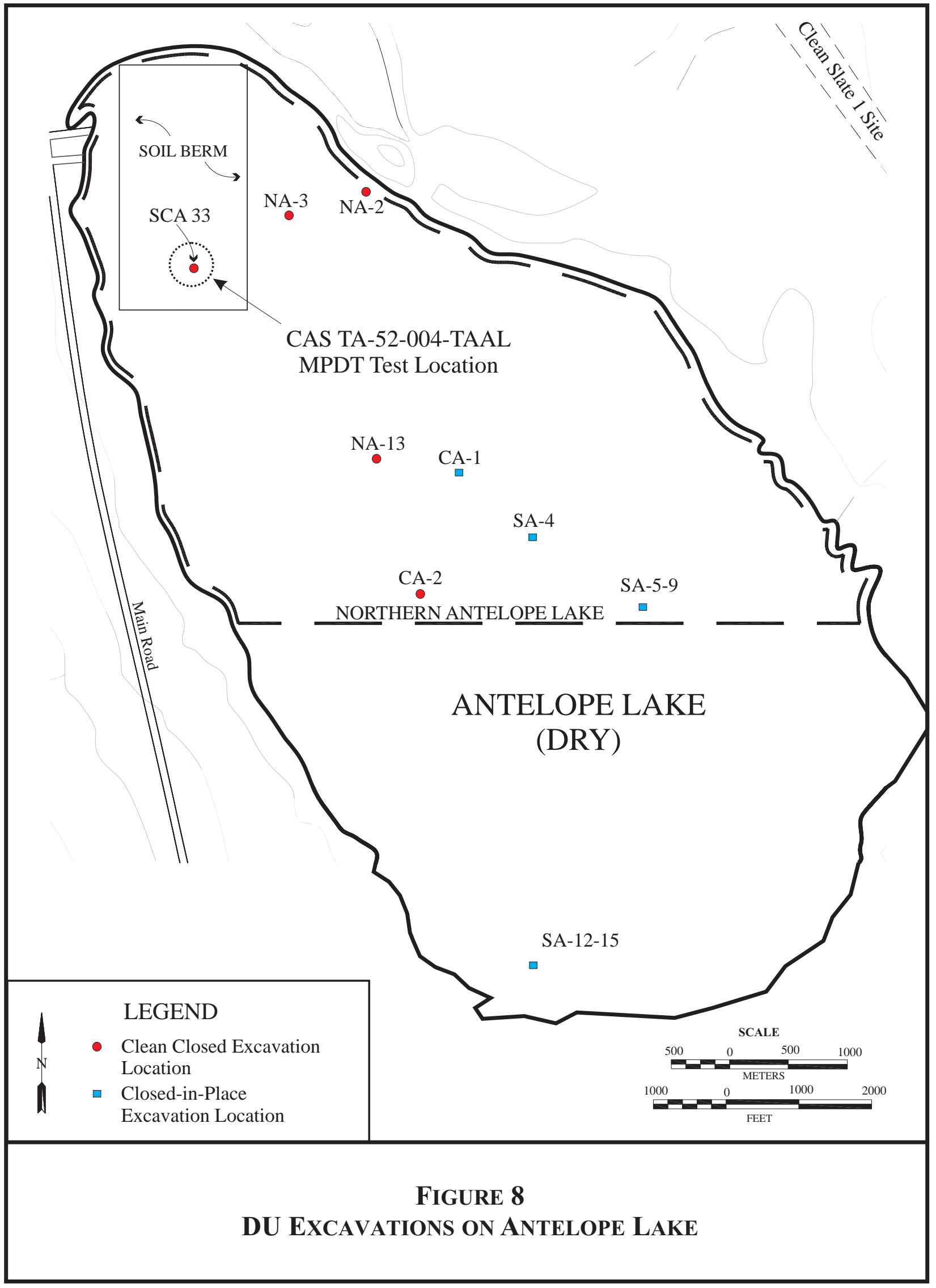




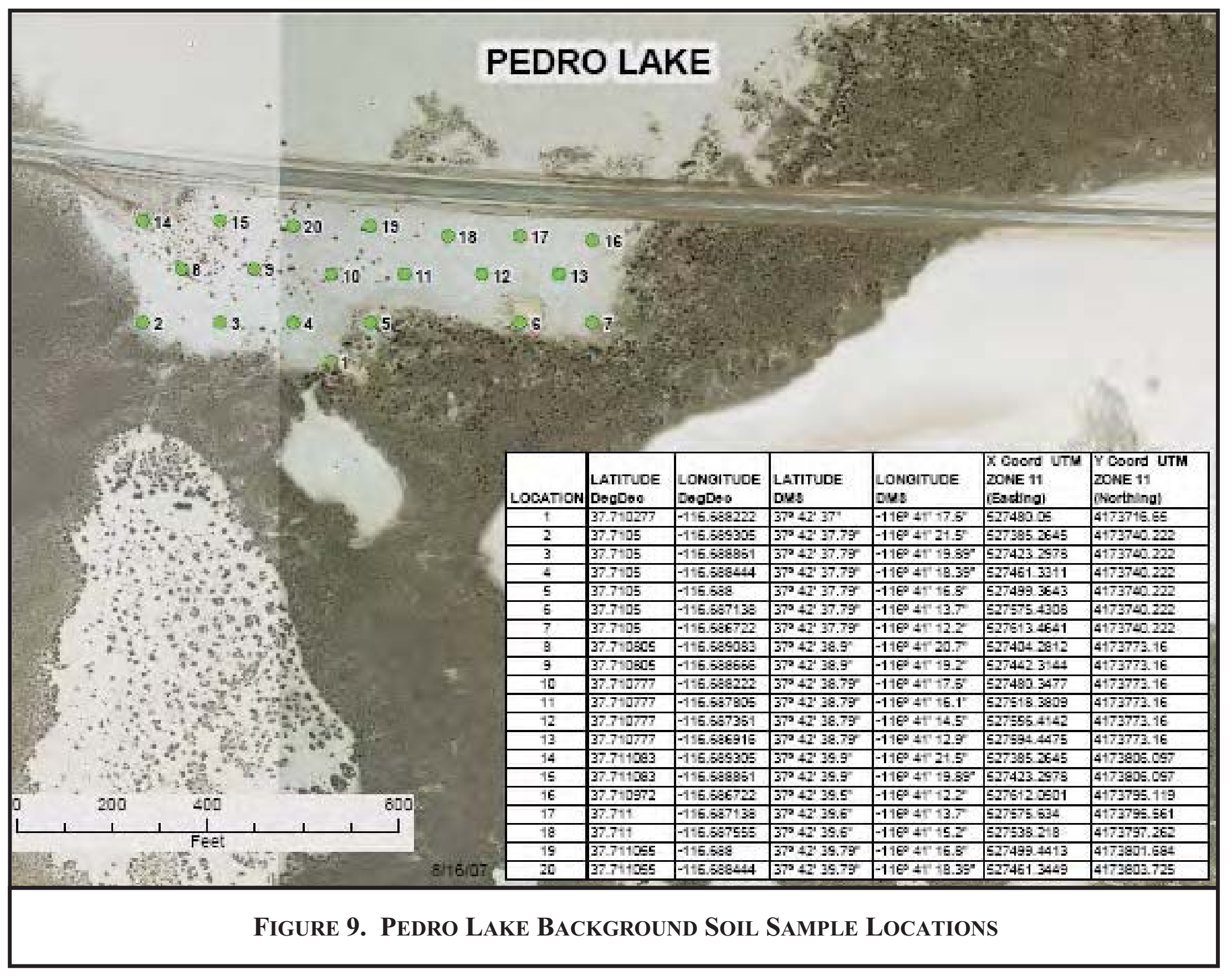


Table 2. Soil Sample Results for Beryllium at Pedro Lake

\begin{tabular}{|c|c|c|c|}
\hline \multirow{2}{*}{$\begin{array}{c}\text { SAMPLE } \\
\text { LOCATION }\end{array}$} & \multicolumn{2}{|c|}{$\begin{array}{c}\text { ANALYTICAL RESULTS } \\
(\mathrm{mg} / \mathrm{kg}) \\
\text { PAL }^{2}=1,900\end{array}$} & \multirow[b]{2}{*}{ PURPOSE } \\
\hline & $0-0.5 \mathrm{ft}$ bgs & $1.0-1.5 \mathrm{ft}$ bgs & \\
\hline PDRO-01 & 1 & 0.67 & Background \\
\hline PDRO-02 & 0.84 & 0.94 & Background \\
\hline PDRO-03 & 0.83 & 0.61 & Background \\
\hline PDRO-04 & 1.4 & 1.2 & Background \\
\hline PDRO-05 & 0.87 & 0.64 & Background \\
\hline PDRO-06 & 1 & 1.6 & Background \\
\hline PDRO-07 & 1.2 & 0.81 & Background \\
\hline PDRO-08 & 1.2 & 0.93 & Background \\
\hline PDRO-09 & 1.1 & 1.6 & Background \\
\hline PDRO-10 & 1.4 & 1.6 & Background \\
\hline PDRO-11 & 1.1 & 0.73 & Background \\
\hline PDRO-12 & 1.2 & 1.5 & Background \\
\hline PDRO-13 & 1.2 & 1.4 & Background \\
\hline PDRO-14 & 1.4 & 0.6 & Background \\
\hline PDRO-15 & 1.3 & 1 & Background \\
\hline PDRO-16 & 1.2 & 0.95 & Background \\
\hline PDRO-17 & 1.5 & 1.5 & Background \\
\hline PDRO-18 & 1.4 & 1.7 & Background \\
\hline PDRO-19 & 1.5 & 1.4 & Background \\
\hline PDRO-20 & 1.2 & 1.6 & Background \\
\hline PDRO-21 & 1.4 & 1.3 & $\mathrm{QA}^{3}$ duplicate of PDRO-17 \\
\hline PDRO-R1 & \multicolumn{2}{|c|}{$<0.2 \mu \mathrm{g} / \mathrm{L}$} & QA Equipment Rinsate Blank \\
\hline
\end{tabular}

${ }^{1}$ Quantitation limit is $0.01-0.02 \mathrm{mg} / \mathrm{kg}(0.2 \mu \mathrm{g} / \mathrm{L}$ for QA Equipment Rinsate Blank)

${ }^{2}$ PAL: preliminary action level

${ }^{3}$ QA: Quality assurance 
Table 3. Soil Sample Results for Uranium at Pedro Lake

\begin{tabular}{|c|c|c|c|c|c|}
\hline \multirow[b]{2}{*}{$\begin{array}{c}\text { SAMPLE } \\
\text { LOCATION }^{1} \\
\end{array}$} & \multicolumn{4}{|c|}{ ANALYTICAL RESULTS } & \multirow[b]{2}{*}{ PURPOSE } \\
\hline & $\begin{array}{c}\text { URANIUM } \\
\text { (mg/kg) } \\
\text { PAL = } 200 \\
\end{array}$ & $\begin{array}{c}\mathrm{U}-234 \text { (pCi/g) } \\
\text { PAL }=85.8 \\
\end{array}$ & $\begin{array}{l}\mathrm{U}-235(\mathrm{pCi} / \mathrm{g}) \\
\mathrm{PAL}=10.56\end{array}$ & $\begin{array}{c}\mathrm{U}-238(\mathrm{pCi} / \mathrm{g}) \\
\mathrm{PAL}=63 \\
\end{array}$ & \\
\hline PDRO-01A & $<0.89$ & 0.852 & 0.0292 & 0.82 & Background \\
\hline PDRO-01B & $<0.9$ & 0.883 & 0.0528 & 0.91 & Background \\
\hline PDRO-02A & $<0.86$ & 1.31 & 0.0761 & 1.22 & Background \\
\hline PDRO-02B & $<0.91$ & 1.07 & 0.0492 & 0.963 & Background \\
\hline PDRO-03A & $<0.88$ & 0.947 & 0.0388 & 0.891 & Background \\
\hline PDRO-03B & $<0.9$ & 0.823 & 0.0348 & 0.784 & Background \\
\hline PDRO-04A & $<0.91$ & 1.28 & 0.0442 & 1.17 & Background \\
\hline PDRO-04B & $<0.95$ & 1.01 & 0.0499 & 1.05 & Background \\
\hline PDRO-05A & $<0.87$ & 0.981 & 0.0592 & 0.955 & Background \\
\hline PDRO-05B & $<0.9$ & 1.69 & 0.0628 & 1.32 & Background \\
\hline PDRO-06A & $<0.9$ & 0.824 & 0.0554 & 0.807 & Background \\
\hline PDRO-06B & $<0.99$ & 1.12 & 0.0758 & 1.12 & Background \\
\hline PDRO-07A & $<0.9$ & 1.52 & 0.0651 & 1.3 & Background \\
\hline PDRO-07B & $<0.91$ & 0.883 & 0.0516 & 0.884 & Background \\
\hline PDRO-08A & $<0.91$ & 0.997 & 0.0669 & 1.04 & Background \\
\hline PDRO-08B & $<0.89$ & 0.99 & 0.0397 & 0.794 & Background \\
\hline PDRO-09A & $<0.91$ & 1.23 & 0.0666 & 1.09 & Background \\
\hline PDRO-09B & $<0.95$ & 0.956 & 0.0546 & 1.01 & Background \\
\hline PDRO-10A & $<0.96$ & 1.08 & 0.0483 & 0.952 & Background \\
\hline PDRO-10B & $<0.96$ & 1.11 & 0.0485 & 1.08 & Background \\
\hline PDRO-11A & $<0.89$ & 1.18 & 0.0552 & 1.05 & Background \\
\hline PDRO-11B & $<0.9$ & 1.14 & 0.0638 & 1.05 & Background \\
\hline PDRO-12A & $<0.91$ & 1.43 & 0.0673 & 1.17 & Background \\
\hline PDRO-12B & $<0.95$ & 1.05 & 0.0593 & 1.03 & Background \\
\hline PDRO-13A & $<0.91$ & 1.42 & 0.0658 & 1.17 & Background \\
\hline PDRO-13B & $<0.94$ & 1.28 & 0.0801 & 1.15 & Background \\
\hline PDRO-14A & $<0.97$ & 0.981 & 0.0481 & 0.988 & Background \\
\hline PDRO-14B & $<0.86$ & 0.908 & 0.0454 & 0.889 & Background \\
\hline PDRO-15A & $<0.87$ & 1.17 & 0.0562 & 0.959 & Background \\
\hline PDRO-15B & $<0.89$ & 1.13 & 0.0528 & 1.15 & Background \\
\hline PDRO-16A & $<0.9$ & 1.11 & 0.0472 & 1.01 & Background \\
\hline PDRO-16B & $<0.91$ & 1.08 & 0.0485 & 1.04 & Background \\
\hline PDRO-17A & $<0.89$ & 1.41 & 0.0647 & 1.16 & Background \\
\hline
\end{tabular}


TABle 3. Soil SAmple Results for URANiUm at Pedro Lake (CONTINUEd)

\begin{tabular}{|c|c|c|c|c|c|}
\hline \multirow{3}{*}{$\begin{array}{c}\text { SAMPLE } \\
\text { LOCATION }^{1}\end{array}$} & \multicolumn{4}{|c|}{ ANALYTICAL RESULTS } & \multirow[b]{3}{*}{ PURPOSE } \\
\hline & $\begin{array}{l}\text { URANIUM } \\
\text { (mg/kg) }\end{array}$ & $\mathrm{U}-234(\mathrm{pCi} / \mathrm{g})$ & $\mathrm{U}-235(\mathrm{pCi} / \mathrm{g})$ & U-238 (pCi/g) & \\
\hline & PAL $=200$ & PAL $=85.8$ & PAL $=10.56$ & PAL $=63$ & \\
\hline PDRO-17B & $<0.94$ & 1.2 & 0.0608 & 1.11 & Background \\
\hline PDRO-18A & $<0.91$ & 1.4 & 0.0538 & 1.2 & Background \\
\hline PDRO-18B & $<0.96$ & 1.15 & 0.0508 & 1.09 & Background \\
\hline PDRO-19A & $<0.91$ & 1.41 & 0.0705 & 1.25 & Background \\
\hline PDRO-19B & $<0.93$ & 1.31 & 0.0541 & 1.22 & Background \\
\hline PDRO-20A & $<0.89$ & 1.37 & 0.0745 & 1.19 & Background \\
\hline PDRO-20B & $<0.93$ & 1.22 & 0.0735 & 1.14 & Background \\
\hline PDRO-21A & $<0.9$ & 1.45 & 0.0733 & 1.22 & $\begin{array}{l}\text { QA duplicate of } \\
\text { PDRO-17A }\end{array}$ \\
\hline PDRO-21B & $<0.94$ & 1.21 & 0.0486 & 1.15 & $\begin{array}{l}\text { QA duplicate of } \\
\text { PDRO-17B }\end{array}$ \\
\hline PDRO-R1 ${ }^{2}$ & $<8.8 \mu \mathrm{g} / \mathrm{L}$ & $<0.0295$ & $<0.0012$ & $<0.0103$ & $\begin{array}{l}\text { QA-Equipment Rinsate } \\
\text { Blank }\end{array}$ \\
\hline
\end{tabular}

1 “A” sample collected from 0.0-0.5 ft bgs, "B” sample collected from 1.0-1.5 ft bgs.

${ }^{2}$ Quality Assurance blank in liquid units (i.e., $\mu \mathrm{g} / \mathrm{L}$ or picoCuries per liter [pCi/L]). 
(NNSA/NSO, 2004b). Several additional COPCs were identified for CAS TA-52-006-TAPL (DUS) as a result of additional investigation of Los Alamos National Laboratory records. Samples were collected to establish local background concentrations for these additional metal COPCs.

A statistical analysis of analytical data from investigation samples is provided in Appendix $\mathrm{J}$ and is summarized in Sections 4.1.1.1.2 and 4.1.1.2.

\subsection{CAS TA-52-001-TANL (NEDS Detonation Area)}

Sample locations for the NEDS Detonation Area are shown in Figure 10. Analytical results for the soil samples are summarized in Tables 4 and 5 . Beryllium was detected at all sample locations; however, concentrations were all less than $2 \mathrm{mg} / \mathrm{kg}$, less than the 1,900 $\mathrm{mg} / \mathrm{kg}$ action level for beryllium and approximately the same concentrations as the background concentrations detected at Pedro Lake. Isotopic uranium (U-234, U-235, and U-238) was detected at numerous locations; however, all concentrations were less than the associated action levels.

The chemical uranium was detected at four locations. One sample location (sample number NEDS-14B, identified as NEDS-14 [GP] in Figure 7), at $325 \mathrm{mg} / \mathrm{kg}$, exceeded the $200 \mathrm{mg} / \mathrm{kg}$ action level. This location also corresponds with ground zero of the detonation and with one of the areas of elevated radioactivity (NEDS 3) detected by the radiological drive-over survey. Uranium is a contaminant of concern (COC) for this CAS.

\subsection{CAS TA-52-006-TAPL (DUS, Colimbo)}

Sample locations for the DUS are shown in Figure 11. Analytical results for soil samples collected from the DUS are summarized in Tables 6 and 7. Beryllium was detected at all sample locations; however, concentrations were all less than $1.5 \mathrm{mg} / \mathrm{kg}$, which is less than the $1,900 \mathrm{mg} / \mathrm{kg}$ action level for beryllium and similar to the background concentrations detected at Pedro Lake. Isotopic uranium (U-234, U-235, and U-238) was detected at numerous locations; however, all isotopic uranium concentrations were less than the associated action levels. The chemical uranium was not present above action levels at any sample location.

Although analytical results of soil samples collected near the point of detonation showed no indication of contamination above action levels, the radiological drive-over survey detected areas of DU contamination that were determined to be fragments on the ground surface with limited soil contamination.

\subsection{CAS TA-52-004-TAAL (MPDT)}

Sample locations for the MPDT area are shown in Figure 12. Analytical results for soil samples collected from the MPDT area are summarized in Tables 8 and 9. Beryllium was detected at all sample locations; however, concentrations were all less than $2 \mathrm{mg} / \mathrm{kg}$, less than the 1,900 $\mathrm{mg} / \mathrm{kg}$ action level for beryllium and approximately the same concentrations as the background concentrations detected at Pedro Lake. Isotopic uranium (U-234, U-235, and U-238) was detected at numerous locations in the MPDT area; however, all concentrations were less than the associated action levels. The chemical uranium, which was detected at two locations with concentrations of 2.3 and $2.6 \mathrm{mg} / \mathrm{kg}$, also did not exceed the $200 \mathrm{mg} / \mathrm{kg}$ action level. 


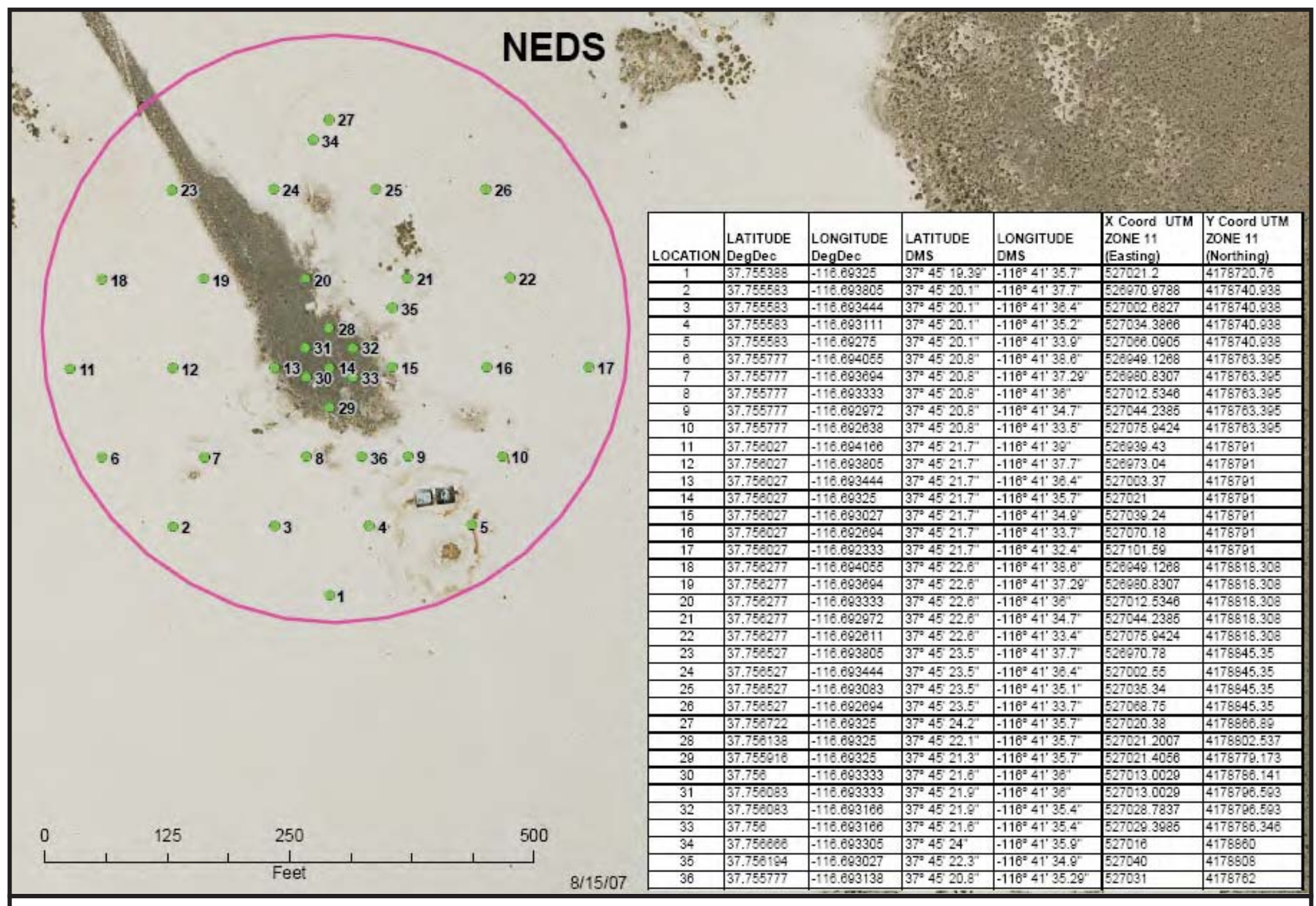

Figure 10. CAS TA-52-001-TANL (NEDS DETONATION AREA) SOIL SAMPLE LOCATIONS 
TABle 4. SoIl SAMPLE ReSUlts FOR BERYLliUM AT CAS TA-52-001-TANL, NEDS DETONATION AREA

\begin{tabular}{|c|c|c|c|}
\hline \multirow{2}{*}{$\begin{array}{c}\text { SAMPLE } \\
\text { LOCATION }\end{array}$} & \multicolumn{2}{|c|}{$\begin{array}{c}\text { ANAL YTICAL RESULTS }^{1} \\
(\mathrm{mg} / \mathrm{kg}) \\
\text { PAL }=1,900\end{array}$} & \multirow[b]{2}{*}{ PURPOSE } \\
\hline & $0-0.5 \mathrm{ft}$ bgs & $1.0-1.5 \mathrm{ft}$ bgs & \\
\hline NEDS-01 & 1.3 & 1.4 & Investigation \\
\hline NEDS-02 & 1.3 & 1.3 & Investigation \\
\hline NEDS-03 & 1.2 & 1.3 & Investigation \\
\hline NEDS-04 & 1.1 & 0.99 & Investigation \\
\hline NEDS-05 & 1.1 & 1.7 & Investigation \\
\hline NEDS-06 & 1.3 & 0.98 & Investigation \\
\hline NEDS-07 & 1.1 & 1.4 & Investigation \\
\hline NEDS-08 & 1.1 & 1.4 & Investigation \\
\hline NEDS-09 & 1.2 & 1.1 & Investigation \\
\hline NEDS-10 & 1.2 & 1.3 & Investigation \\
\hline NEDS-11 & 0.96 & 0.8 & Investigation \\
\hline NEDS-12 & 1.1 & 1.4 & Investigation \\
\hline NEDS-13 & 0.95 & 1.4 & Investigation \\
\hline NEDS-14 & 0.71 & 3.2 & Investigation \\
\hline NEDS-15 & 0.91 & 0.89 & Investigation \\
\hline NEDS-16 & 1.1 & 0.95 & Investigation \\
\hline NEDS-17 & 1.1 & 1.2 & Investigation \\
\hline NEDS-18 & 0.95 & 1.1 & Investigation \\
\hline NEDS-19 & 0.85 & 1.3 & Investigation \\
\hline NEDS-20 & 0.92 & 1.2 & Investigation \\
\hline NEDS-21 & 0.85 & 1 & Investigation \\
\hline NEDS-22 & 1 & 1 & Investigation \\
\hline NEDS-23 & 1.2 & 1.5 & Investigation \\
\hline NEDS-24 & 1.2 & 1.2 & Investigation \\
\hline NEDS-25 & 1.1 & 1.3 & Investigation \\
\hline NEDS-26 & 1 & 1.2 & Investigation \\
\hline NEDS-27 & 1.2 & 1.2 & Investigation \\
\hline NEDS-28 & 0.89 & 1.2 & Investigation \\
\hline NEDS-29 & 0.93 & 1.5 & Investigation \\
\hline NEDS-30 & 0.98 & 1.5 & Investigation \\
\hline NEDS-31 & 0.85 & 1.2 & Investigation \\
\hline NEDS-32 & 0.71 & 0.95 & Investigation \\
\hline NEDS-33 & 0.86 & 1.6 & Investigation \\
\hline
\end{tabular}


TABLE 4. SOIL SAMPLE RESUlTS FOR BERYLLIUM AT CAS TA-52-001-TANL, NEDS DETONATION AREA (CONTINUED)

\begin{tabular}{||c|c|c|c||}
\hline \multirow{2}{*}{$\begin{array}{c}\text { SAMPLE } \\
\text { LOCATION }\end{array}$} & \multicolumn{2}{|c|}{$\begin{array}{c}\text { ANALYTICAL RESULTS } \\
\text { (mg/kg) }\end{array}$} & \multirow{2}{*}{} \\
\cline { 2 - 3 } & \multicolumn{2}{|c||}{ PAL $\mathbf{1 9 0 0}$} & PURPOSE \\
\hline \hline NEDS-34 & 1.1 & 1.2 & Investigation \\
\hline NEDS-35 & 0.83 & 1.5 & Investigation \\
\hline NEDS-36 & 0.96 & 1.4 & Investigation \\
\hline NEDS-37 & 1 & 1.1 & QA Duplicate (NEDS-10) \\
\hline NEDS-38 & 0.91 & 1 & QA Duplicate (NEDS-20) \\
\hline NEDS-R1 & \multicolumn{2}{|c|}{$0.21 \mu \mathrm{g} / \mathrm{L}$} & QA Equipment Rinsate Blank \\
\hline
\end{tabular}

${ }^{1}$ Quantitation limit is $0.01-0.02 \mathrm{mg} / \mathrm{kg}(0.2 \mu \mathrm{g} / \mathrm{L}$ for QA Equipment Rinsate Blank) 
TABle 5. SoIl SAMPLE RESUltS FOR URANIUM AT CAS TA-52-001-TANL, NEDS DETONATION AREA

\begin{tabular}{|c|c|c|c|c|c|}
\hline \multirow[b]{2}{*}{$\begin{array}{c}\text { SAMPLE } \\
\text { LOCATION }^{1}\end{array}$} & \multicolumn{4}{|c|}{ ANALYTICAL RESULTS } & \multirow[b]{2}{*}{ PURPOSE } \\
\hline & $\begin{array}{c}\begin{array}{c}\text { URANIUM } \\
\text { (mg/kg) }\end{array} \\
\text { PAL = } 200\end{array}$ & $\begin{array}{c}\mathrm{U}-234(\mathrm{pCi} / \mathrm{g}) \\
\mathrm{PAL}=85.8\end{array}$ & $\begin{array}{l}\text { U-235 (pCi/g) } \\
\text { PAL = 10.56 }\end{array}$ & $\begin{array}{c}\mathrm{U}-238(\mathrm{pCi} / \mathrm{g}) \\
\mathrm{PAL}=63\end{array}$ & \\
\hline NEDS-01A & $<2.2$ & 1.78 & $<-0.0616$ & 1.02 & Investigation \\
\hline NEDS-01B & $<2.2$ & 1.19 & $<0.221$ & 1.38 & Investigation \\
\hline NEDS-02A & $<2.2$ & 1.80 & $<0.154$ & 1.24 & Investigation \\
\hline NEDS-02B & $<2.3$ & 1.03 & $<0.0563$ & 0.786 & Investigation \\
\hline NEDS-03A & $<2.1$ & 1.32 & $<-0.0827$ & 0.719 & Investigation \\
\hline NEDS-03B & $<2.2$ & $<0.773$ & $<0.0296$ & 0.633 & Investigation \\
\hline NEDS-04A & $<2.2$ & 1.66 & $<0.0432$ & 1.27 & Investigation \\
\hline NEDS-04B & $<2.2$ & $<0.770$ & $<0.194$ & $<0.665$ & Investigation \\
\hline NEDS-05A & $<2.3$ & 1.06 & $<-0.0781$ & 1.15 & Investigation \\
\hline NEDS-05B & $<2.4$ & $<0.633$ & $<0.299$ & $<0.628$ & Investigation \\
\hline NEDS-06A & $<2.1$ & $<0.418$ & $<0.096$ & 0.917 & Investigation \\
\hline NEDS-06B & $<2.2$ & 1.54 & $<0.0564$ & $<0.659$ & Investigation \\
\hline NEDS-07A & $<2.2$ & $<0.733$ & $<0.299$ & $<0.267$ & Investigation \\
\hline NEDS-07B & $<2.3$ & $<0.847$ & $<-0.211$ & $<0.714$ & Investigation \\
\hline NEDS-08A & $<2.2$ & 1.61 & $<0.149$ & $<0.673$ & Investigation \\
\hline NEDS-08B & $<2.2$ & 1.39 & $<0.0545$ & $<0.527$ & Investigation \\
\hline NEDS-09A & $<2.2$ & $<0.379$ & $<0.126$ & 0.931 & Investigation \\
\hline NEDS-09B & $<2.2$ & $<0.700$ & $<-0.139$ & $<0.419$ & Investigation \\
\hline NEDS-10A & $<2.1$ & $<0.544$ & $<-0.0526$ & 0.987 & Investigation \\
\hline NEDS-10B & $<2.2$ & 1.97 & $<0.218$ & 1.07 & Investigation \\
\hline NEDS-11A & $<2.1$ & 1.55 & 0.383 & 1.13 & Investigation \\
\hline NEDS-11B & $<2.2$ & 1.24 & $<0.0031$ & 2.32 & Investigation \\
\hline NEDS-12A & $<2.1$ & 1.51 & $<0.102$ & 1.15 & Investigation \\
\hline NEDS-12B & $<2.2$ & 1.42 & $<0.130$ & 1.46 & Investigation \\
\hline NEDS-13A & $<2.2$ & 1.83 & $<0.217$ & 1.91 & Investigation \\
\hline NEDS-13B & $<2.2$ & 1.68 & $<0.00277$ & 1.21 & Investigation \\
\hline NEDS-14A & 27.1 & 1.91 & $<-0.0713$ & 4.84 & Investigation \\
\hline NEDS-14B & $325^{2}$ & 1.96 & $<0.267$ & 8.24 & Investigation \\
\hline NEDS-15A & $<2.2$ & 1.69 & $<0.134$ & 1.79 & Investigation \\
\hline NEDS-15B & $<2.3$ & 1.89 & $<0.0775$ & 1.66 & Investigation \\
\hline NEDS-16A & $<2.2$ & 1.77 & $<0.0413$ & 1.84 & Investigation \\
\hline NEDS-16B & $<2.2$ & 1.84 & $<0.215$ & 1.87 & Investigation \\
\hline NEDS-17A & $<2.1$ & 1.72 & $<0.133$ & 0.992 & Investigation \\
\hline
\end{tabular}


TABLE 5. SoIl SAMPLE Results For URANIUM AT CAS TA-52-001-TANL, NEDS DETONATION AREA (CONTINUED)

\begin{tabular}{|c|c|c|c|c|c|}
\hline \multirow{3}{*}{$\begin{array}{c}\text { SAMPLE } \\
\text { LOCATION }^{1}\end{array}$} & \multicolumn{4}{|c|}{ ANALYTICAL RESULTS } & \multirow[b]{3}{*}{ PURPOSE } \\
\hline & $\begin{array}{l}\text { URANIUM } \\
\text { (mg/kg) }\end{array}$ & U-234 (pCi/g) & U-235 (pCi/g) & U-238 (pCi/g) & \\
\hline & PAL = 200 & PAL $=85.8$ & PAL = 10.56 & PAL $=63$ & \\
\hline NEDS-17B & $<2.2$ & 1.29 & $<-0.0181$ & 1.72 & Investigation \\
\hline NEDS-18A & $<2.1$ & 1.53 & $<-0.0334$ & 1.41 & Investigation \\
\hline NEDS-18B & $<2.3$ & 1.46 & $<0.154$ & 2.02 & Investigation \\
\hline NEDS-19A & $<2.2$ & 1.30 & $<0.0247$ & 0.786 & Investigation \\
\hline NEDS-19B & $<2.2$ & 1.99 & $<0.112$ & 1.96 & Investigation \\
\hline NEDS-20A & $<2.1$ & 1.65 & $<0.250$ & 1.33 & Investigation \\
\hline NEDS-20B & $<2.2$ & 1.69 & $<0.185$ & 1.26 & Investigation \\
\hline NEDS-21A & $<2.2$ & 1.21 & $<0.195$ & $<0.264$ & Investigation \\
\hline NEDS-21B & $<2.2$ & 3.19 & $<0.815$ & $<0.660$ & Investigation \\
\hline NEDS-22A & $<2.2$ & 2.14 & $<0.241$ & 1.36 & Investigation \\
\hline NEDS-22B & $<2.2$ & 2.00 & 0.991 & $<0.902$ & Investigation \\
\hline NEDS-23A & $<2.2$ & $<1.15$ & $<0.356$ & $<0.440$ & Investigation \\
\hline NEDS-23B & $<2.3$ & 2.76 & $<0.330$ & 1.96 & Investigation \\
\hline NEDS-24A & $<2.2$ & 2.18 & $<0.328$ & $<0.887$ & Investigation \\
\hline NEDS-24B & $<2.3$ & 2.28 & $<0.704$ & $<0.997$ & Investigation \\
\hline NEDS-25A & $<2.2$ & 1.63 & $<0.459$ & 1.11 & Investigation \\
\hline NEDS-25B & $<2.2$ & $<1.00$ & $<0.368$ & $<1.29$ & Investigation \\
\hline NEDS-26A & $<2.2$ & $<1.03$ & $<0.678$ & 1.69 & Investigation \\
\hline NEDS-26B & $<2.2$ & $<1.08$ & $<0.419$ & $<0.624$ & Investigation \\
\hline NEDS-27A & $<2.1$ & 1.24 & $<0.417$ & $<1.01$ & Investigation \\
\hline NEDS-27B & $<2.2$ & 1.54 & $<0.380$ & 0.835 & Investigation \\
\hline NEDS-28A & $<2.1$ & 2.86 & $<0.363$ & 1.54 & Investigation \\
\hline NEDS-28B & $<2.2$ & 2.73 & $<0.544$ & 1.23 & Investigation \\
\hline NEDS-29A & $<2.2$ & 2.24 & $<0.427$ & $<0.777$ & Investigation \\
\hline NEDS-29B & $<2.2$ & 1.94 & $<0.221$ & $<1.18$ & Investigation \\
\hline NEDS-30A & $<2.1$ & 2.04 & $<0.473$ & 1.21 & Investigation \\
\hline NEDS-30B & $<2.3$ & 1.81 & $<0.172$ & 0.835 & Investigation \\
\hline NEDS-31A & $<2.1$ & 3.95 & 0.955 & 1.46 & Investigation \\
\hline NEDS-31B & $<2.1$ & $<1.03$ & $<0.426$ & 2.16 & Investigation \\
\hline NEDS-32A & 48.7 & 4.45 & 0.860 & 5.71 & Investigation \\
\hline NEDS-32B & 3.4 & 14.6 & 4.10 & 7.29 & Investigation \\
\hline NEDS-33A & $<2.1$ & $<1.26$ & $<0.502$ & $<0.740$ & Investigation \\
\hline NEDS-33B & $<2.1$ & 3.49 & 1.08 & 1.59 & Investigation \\
\hline
\end{tabular}


TABLE 5. SoIl SAMPLE Results For URANIUM AT CAS TA-52-001-TANL, NEDS DETONATION AREA (CONTINUED)

\begin{tabular}{|c|c|c|c|c|c|}
\hline \multirow[b]{2}{*}{$\begin{array}{c}\text { SAMPLE } \\
\text { LOCATION }^{1}\end{array}$} & \multicolumn{4}{|c|}{ ANALYTICAL RESULTS } & \multirow[b]{2}{*}{ PURPOSE } \\
\hline & $\begin{array}{c}\text { URANIUM } \\
\text { (mg/kg) } \\
\text { PAL = } 200\end{array}$ & $\begin{array}{c}\mathrm{U}-234 \text { (pCi/g) } \\
\text { PAL = 85.8 }\end{array}$ & $\begin{array}{l}\mathrm{U}-235(\mathrm{pCi} / \mathrm{g}) \\
\text { PAL }=10.56\end{array}$ & $\begin{array}{c}\mathrm{U}-238(\mathrm{pCi} / \mathrm{g}) \\
\text { PAL }=63\end{array}$ & \\
\hline NEDS-34A & $<2.1$ & 3.32 & $<0.438$ & $<0.306$ & Investigation \\
\hline NEDS-34B & $<2.1$ & $<1.41$ & $<-0.207$ & $<0.964$ & Investigation \\
\hline NEDS-35A & $<2.1$ & 1.88 & $<0.271$ & $<0.878$ & Investigation \\
\hline NEDS-35B & $<2.1$ & 2.38 & $<0.759$ & $<1.46$ & Investigation \\
\hline NEDS-36A & $<2.2$ & 0.804 & $<0.298$ & 1.45 & Investigation \\
\hline NEDS-36B & $<2.1$ & $<1.03$ & $<1.10$ & $<0.457$ & Investigation \\
\hline NEDS-37A & $<2.2$ & 2.29 & $<0.709$ & 1.15 & $\begin{array}{l}\text { QA-Duplicate } \\
\text { (NEDS-10A) }\end{array}$ \\
\hline NEDS-37B & $<2.1$ & 3.06 & 1.51 & 2.37 & $\begin{array}{l}\text { QA-Duplicate } \\
\text { (NEDS-10B) }\end{array}$ \\
\hline NEDS-38A & $<2.1$ & 2.53 & $<0.579$ & 1.78 & $\begin{array}{l}\text { QA-Duplicate } \\
\text { (NEDS-20A) }\end{array}$ \\
\hline NEDS-38B & $<2.2$ & 1.69 & 0.919 & 1.55 & $\begin{array}{l}\text { QA-Duplicate } \\
\text { (NEDS-20B) }\end{array}$ \\
\hline NEDS-R $1^{3}$ & $<21.4 \mu \mathrm{g} / \mathrm{L}$ & $2.01 \mathrm{pCi} / \mathrm{L}$ & $<0.619 \mathrm{pCi} / \mathrm{L}$ & $\begin{array}{c}<-0.00335 \\
\mathrm{pCi} / \mathrm{L}\end{array}$ & $\begin{array}{l}\text { QA-Equipment } \\
\text { Rinsate Blank }\end{array}$ \\
\hline
\end{tabular}

1 “A” sample collected from 0.0-0.5 ft bgs, "B” sample collected from 1.0-1.5 ft bgs.

${ }^{2}$ Exceeds action level.

${ }^{3}$ Quality Assurance blank in liquid units (i.e., $\mu \mathrm{g} / \mathrm{L}$ or $\mathrm{pCi} / \mathrm{L}$ ). 


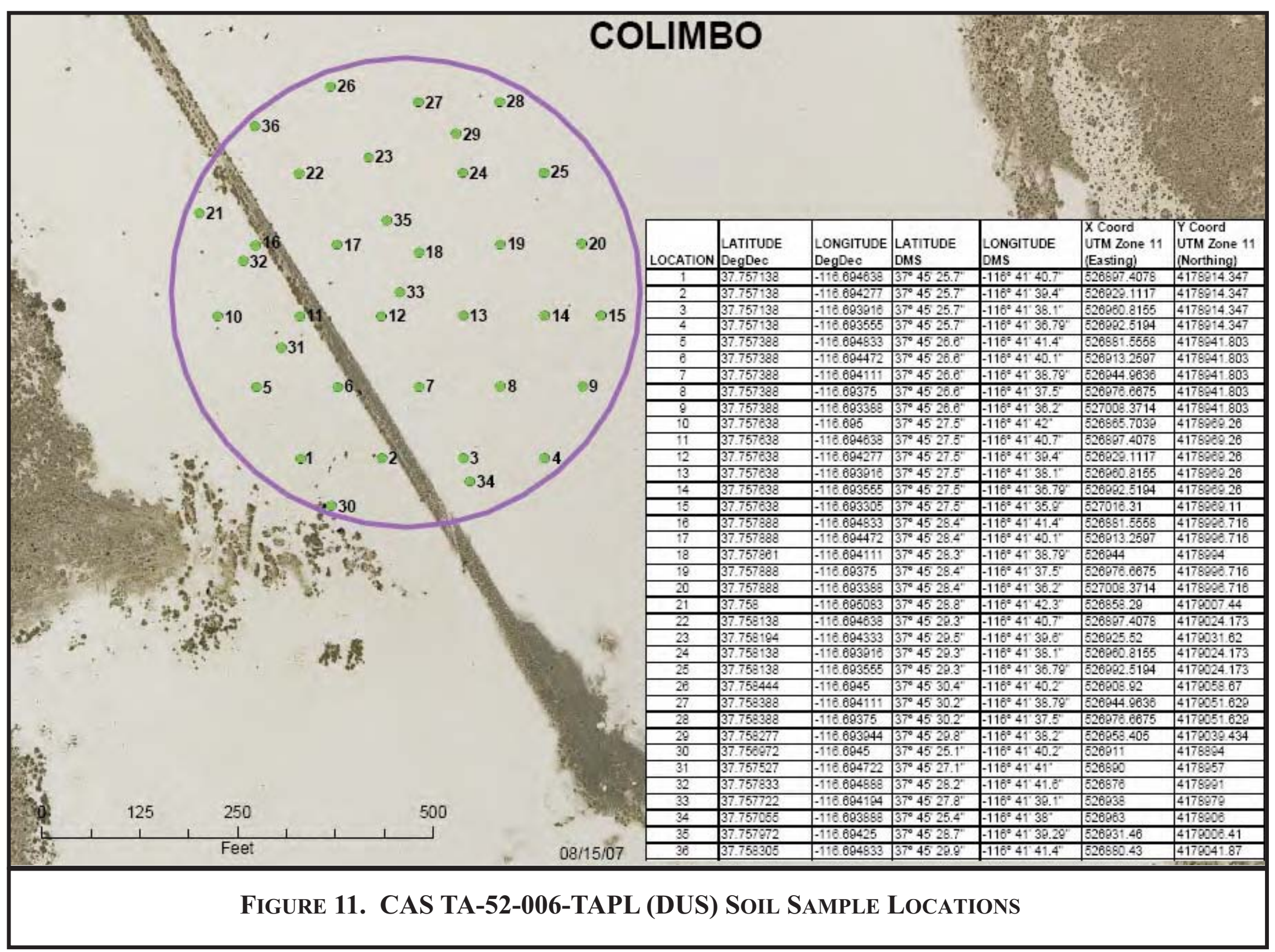


TABLE 6. SOIL SAMPLE RESULTS FOR BERYLLIUM AT CAS TA-52-006-TAPL, DUS

\begin{tabular}{|c|c|c|c|}
\hline \multirow{2}{*}{$\begin{array}{c}\text { SAMPLE } \\
\text { LOCATION }\end{array}$} & \multicolumn{2}{|c|}{$\begin{array}{c}\text { ANALYTICAL RESULTS } \\
(\mathrm{mg} / \mathrm{kg}) \\
\text { PAL }=\mathbf{1 , 9 0 0}\end{array}$} & \multirow[b]{2}{*}{ PURPOSE } \\
\hline & $0-0.5 \mathrm{ft}$ bgs & $1.0-1.5 \mathrm{ft}$ bgs & \\
\hline DUS-01 & 1.2 & 0.53 & Investigation \\
\hline DUS-02 & 1.1 & 0.94 & Investigation \\
\hline DUS-03 & 1.2 & 0.96 & Investigation \\
\hline DUS-04 & 1.1 & 1.4 & Investigation \\
\hline DUS-05 & 1.1 & 0.48 & Investigation \\
\hline DUS-06 & 1.1 & 0.57 & Investigation \\
\hline DUS-07 & 1.1 & 0.93 & Investigation \\
\hline DUS-08 & 1.3 & 0.82 & Investigation \\
\hline DUS-09 & 1.2 & 1 & Investigation \\
\hline DUS-10 & 0.99 & 0.89 & Investigation \\
\hline DUS-11 & $1.1(\mathrm{~J})^{1}$ & $0.88(\mathrm{~J})$ & Investigation \\
\hline DUS-12 & $1.2(\mathrm{~J})$ & $0.65(\mathrm{~J})$ & Investigation \\
\hline DUS-13 & $0.96(\mathrm{~J})$ & $1.2(\mathrm{~J})$ & Investigation \\
\hline DUS-14 & $1.1(\mathrm{~J})$ & $1.4(\mathrm{~J})$ & Investigation \\
\hline DUS-15 & $1.1(\mathrm{~J})$ & $1(\mathrm{~J})$ & Investigation \\
\hline DUS-16 & $1.1(\mathrm{~J})$ & $0.56(\mathrm{~J})$ & Investigation \\
\hline DUS-17 & $0.92(\mathrm{~J})$ & $0.72(\mathrm{~J})$ & Investigation \\
\hline DUS-18 & $1.3(\mathrm{~J})$ & $1.4(\mathrm{~J})$ & Investigation \\
\hline DUS-19 & $1.2(\mathrm{~J})$ & $1.1(\mathrm{~J})$ & Investigation \\
\hline DUS-20 & $1.1(\mathrm{~J})$ & $1.1(\mathrm{~J})$ & Investigation \\
\hline DUS-21 & 0.68 & 0.83 & Investigation \\
\hline DUS-22 & 1.1 & 0.51 & Investigation \\
\hline DUS-23 & 1.1 & 0.75 & Investigation \\
\hline DUS-24 & 1.1 & 0.84 & Investigation \\
\hline DUS-25 & 0.95 & 1.3 & Investigation \\
\hline DUS-26 & 0.95 & 0.61 & Investigation \\
\hline DUS-27 & 0.99 & 0.98 & Investigation \\
\hline DUS-28 & 1.1 & 1.5 & Investigation \\
\hline DUS-29 & 1.1 & 0.79 & Investigation \\
\hline DUS-30 & 0.98 & 0.53 & Investigation \\
\hline DUS-31 & 0.88 & 0.57 & Investigation \\
\hline DUS-32 & 0.94 & 0.71 & Investigation \\
\hline DUS-33 & 1 & 0.43 & Investigation \\
\hline
\end{tabular}


TABLE 6. SOIL SAMPLE RESULTS FOR BERYLLIUM AT

CAS TA-52-006-TAPL, DUS (CONTINUED)

\begin{tabular}{|c|c|c|c|}
\hline \multirow{2}{*}{$\begin{array}{c}\text { SAMPLE } \\
\text { LOCATION }\end{array}$} & \multicolumn{2}{|c|}{$\begin{array}{c}\text { ANAL YTICAL RESULTS } \\
\text { (mg/kg) }\end{array}$} \\
\cline { 2 - 3 } & $\mathbf{0 - 0 . 5} \mathbf{~ f t ~ b g s}$ & $\mathbf{1 . 0 - 1 . 5} \mathbf{f t}$ bgs & \multirow{2}{*}{ PURPOSE } \\
\hline \hline DUS-34 & 0.94 & 0.86 & Investigation \\
\hline DUS-35 & 1.1 & 0.85 & Investigation \\
\hline DUS-36 & 1.1 & 0.83 & Investigation \\
\hline DUS-37 & 0.99 & 0.88 & QA Duplicate (DUS-10) \\
\hline DUS-38 & 1.1 & 0.91 & QA Duplicate (DUS-20) \\
\hline DUS-R1 & \multicolumn{2}{|c|}{$<0.2 \mu \mathrm{g} / \mathrm{L}$} & QA Equipment Rinsate Blank \\
\hline
\end{tabular}

${ }^{1}(\mathrm{~J})$. Qualifier added because value is estimated due to interference. Quantitation limit is $0.02 \mathrm{mg} / \mathrm{kg}(0.2 \mu \mathrm{g} / \mathrm{L}$ for DUS-R1). 
TABLE 7. SoIL SAMPLE RESUltS FOR URANIUM AT CAS TA-52-006-TAPL, DUS

\begin{tabular}{|c|c|c|c|c|c|}
\hline \multirow{3}{*}{$\begin{array}{c}\text { SAMPLE } \\
\text { LOCATION }^{1} \\
\end{array}$} & \multicolumn{4}{|c|}{ ANALYTICAL RESULTS } & \multirow[b]{3}{*}{ PURPOSE } \\
\hline & \multirow{2}{*}{$\begin{array}{c}\begin{array}{c}\text { URANIUM } \\
\text { (mg/kg) }\end{array} \\
\text { PAL = } 200 \\
\end{array}$} & \multirow{2}{*}{$\begin{array}{c}\mathrm{U}-234(\mathrm{pCi} / \mathrm{g}) \\
\text { PAL = 85.8 } \\
\end{array}$} & \multirow{2}{*}{$\begin{array}{l}\mathrm{U}-235(\mathrm{pCi} / \mathrm{g}) \\
\mathrm{PAL}=10.56 \\
\end{array}$} & \multirow{2}{*}{$\begin{array}{c}\mathrm{U}-238(\mathrm{pCi} / \mathrm{g}) \\
\mathrm{PAL}=63 \\
\end{array}$} & \\
\hline & & & & & \\
\hline DUS-01A & $<2.2$ & $<0.363$ & $<0.0826$ & $<0.725$ & Investigation \\
\hline DUS-01B & $<2.2$ & 3.43 & 0.910 & $<0.922$ & Investigation \\
\hline DUS-02A & $<2.2$ & 1.54 & $<-0.0762$ & 1.93 & Investigation \\
\hline DUS-02B & $<2.3$ & 1.55 & $<-0.184$ & $<0.146$ & Investigation \\
\hline DUS-03A & $<2.2$ & $<1.31$ & $<0.544$ & $<-0.108$ & Investigation \\
\hline DUS-03B & $<2.2$ & 2.25 & 0.744 & 2.11 & Investigation \\
\hline DUS-04A & $<2.1$ & $<1.18$ & $<0.364$ & 1.56 & Investigation \\
\hline DUS-04B & $<2.2$ & 3.83 & $<0.690$ & 2.63 & Investigation \\
\hline DUS-05A & $<2.2$ & 2.40 & $<1.05$ & 1.39 & Investigation \\
\hline DUS-05B & $<2.3$ & $<0.475$ & $<-0.0677$ & $<0.247$ & Investigation \\
\hline DUS-06A & $<2.2$ & $<1.07$ & $<1.11$ & $<0.193$ & Investigation \\
\hline DUS-06B & $<2.2$ & 1.89 & 0.426 & 1.03 & Investigation \\
\hline DUS-07A & $<2.2$ & $<0.621$ & $<0.158$ & $<1.19$ & Investigation \\
\hline DUS-07B & $<2.2$ & 3.24 & 1.20 & 2.10 & Investigation \\
\hline DUS-08A & $<2.2$ & $<0.852$ & $<-0.0665$ & $<0.906$ & Investigation \\
\hline DUS-08B & $<2.2$ & $<1.51$ & $<0.653$ & $<1.21$ & Investigation \\
\hline DUS-09A & $<2.2$ & 1.80 & $<-0.244$ & $<1.04$ & Investigation \\
\hline DUS-09B & $<2.2$ & $<0.865$ & $<0.451$ & $<-0.0787$ & Investigation \\
\hline DUS-10A & $<2.2$ & 2.19 & $<0.245$ & 2.12 & Investigation \\
\hline DUS-10B & $<2.3$ & 2.33 & $<-0.187$ & $<0.596$ & Investigation \\
\hline DUS-11A & $<2.1$ & $<0.861$ & $<0.525$ & $<0.649$ & Investigation \\
\hline DUS-11B & $<2.2$ & 1.97 & $<0.122$ & $<0.786$ & Investigation \\
\hline DUS-12A & $<2.2$ & $<0.127$ & $<0.0782$ & 1.58 & Investigation \\
\hline DUS-12B & $<2.2$ & 1.85 & $<-0.0589$ & $<0.397$ & Investigation \\
\hline DUS-13A & $<2.1$ & 1.92 & $<0.518$ & $<-0.18$ & Investigation \\
\hline DUS-13B & $<2.3$ & $<1.48$ & 0.344 & 1.58 & Investigation \\
\hline DUS-14A & $<2.1$ & 2.33 & $<0.638$ & $<1.14$ & Investigation \\
\hline DUS-14B & $<2.2$ & 2.67 & $<0.0719$ & 1.57 & Investigation \\
\hline DUS-15A & $<2.2$ & $<1.63$ & $<0.758$ & $<-0.221$ & Investigation \\
\hline DUS-15B & $<2.3$ & 1.99 & $<0.157$ & $<0.405$ & Investigation \\
\hline DUS-16A & $<2.2$ & $<1.68$ & $<0.792$ & $<0.519$ & Investigation \\
\hline DUS-16B & $<2.2$ & $<1.19$ & $<-0.538$ & $<0.404$ & Investigation \\
\hline DUS-17A & $<2.1$ & $<0.968$ & 0.00 & $<0.958$ & Investigation \\
\hline
\end{tabular}


TABLE 7. SOIL SAMPLE RESUltS FOR URANIUM AT CAS TA-52-006-TAPL, DUS (CONTINUED)

\begin{tabular}{|c|c|c|c|c|c|}
\hline \multirow{3}{*}{$\begin{array}{c}\text { SAMPLE } \\
\text { LOCATION }^{1} \\
\end{array}$} & \multicolumn{4}{|c|}{ ANALYTICAL RESULTS } & \multirow[b]{3}{*}{ PURPOSE } \\
\hline & $\begin{array}{c}\text { URANIUM } \\
\text { (mg/kg) }\end{array}$ & U-234 (pCi/g) & U-235 (pCi/g) & $\mathrm{U}-238$ (pCi/g) & \\
\hline & PAL = 200 & PAL $=85.8$ & PAL = 10.56 & PAL $=63$ & \\
\hline DUS-17B & $<2.2$ & 1.60 & $<0.420$ & 1.55 & Investigation \\
\hline DUS-18A & $<2.1$ & $<1.20$ & $<0.143$ & $<0.536$ & Investigation \\
\hline DUS-18B & $<2.2$ & 2.26 & $<-0.202$ & $<0.789$ & Investigation \\
\hline DUS-19A & $<2.1$ & $<0.905$ & $<-0.049$ & $<0.849$ & Investigation \\
\hline DUS-19B & $<2.2$ & $<1.30$ & $<-0.117$ & $<1.79$ & Investigation \\
\hline DUS-20A & $<2.1$ & 1.86 & $<-0.0525$ & 1.51 & Investigation \\
\hline DUS-20B & $<2.2$ & 2.40 & $<0.473$ & $<1.35$ & Investigation \\
\hline DUS-21A & $<2.2$ & 1.6 & $<0.14$ & 2.42 & Investigation \\
\hline DUS-21B & 2.4 & $<0.421$ & $<0.308$ & $<0.457$ & Investigation \\
\hline DUS-22A & $<2.2$ & 1.17 & $<0.471$ & $<0.586$ & Investigation \\
\hline DUS-22B & $<2.2$ & $<0.790$ & $<-0.354$ & $<0.218$ & Investigation \\
\hline DUS-23A & $<2.2$ & 1.28 & $<-0.192$ & $<0.255$ & Investigation \\
\hline DUS-23B & $<2.3$ & $<0.885$ & $<0.378$ & $<0.673$ & Investigation \\
\hline DUS-24A & $<2.1$ & 1.90 & $<0.0379$ & $<0.690$ & Investigation \\
\hline DUS-24B & $<2.2$ & 0.974 & $<0.138$ & $<0.678$ & Investigation \\
\hline DUS-25A & $<2.1$ & 1.94 & $<0.179$ & 1.32 & Investigation \\
\hline DUS-25B & $<2.2$ & 2.09 & $<-0.104$ & $<0.615$ & Investigation \\
\hline DUS-26A & $<2.2$ & 1.34 & $<0.0333$ & 1.07 & Investigation \\
\hline DUS-26B & $<2.2$ & 1.07 & $<0.175$ & $<0.448$ & Investigation \\
\hline DUS-27A & $<2.2$ & 1.59 & $<-0.0178$ & $<0.590$ & Investigation \\
\hline DUS-27B & $<2.2$ & 1.18 & $<0.0457$ & $<0.861$ & Investigation \\
\hline DUS-28A & $<2.2$ & 1.05 & $<0.392$ & $<0.809$ & Investigation \\
\hline DUS-28B & $<2.3$ & 3.75 & $<0.354$ & 3.11 & Investigation \\
\hline DUS-29A & $<2.2$ & $<0.516$ & $<0.0671$ & $<0.664$ & Investigation \\
\hline DUS-29B & $<2.2$ & $<0.738$ & $<-0.116$ & $<0.969$ & Investigation \\
\hline DUS-30A & $<2.2$ & 1.22 & $<-0.209$ & $<0.706$ & Investigation \\
\hline DUS-30B & $<2.2$ & 1.44 & $<0.156$ & $<0.826$ & Investigation \\
\hline DUS-31A & $<2.2$ & 3.84 & 0.566 & 2.11 & Investigation \\
\hline DUS-31B & $<2.2$ & 5.51 & 1.01 & 1.84 & Investigation \\
\hline DUS-32A & $<2.2$ & 1.93 & $<0.435$ & 2.27 & Investigation \\
\hline DUS-32B & $<2.3$ & 2.05 & $<0.487$ & $<0.709$ & Investigation \\
\hline DUS-33A & $<2.2$ & 4.06 & $<0.535$ & 1.82 & Investigation \\
\hline DUS-33B & $<2.2$ & 2.12 & $<0.358$ & 1.45 & Investigation \\
\hline
\end{tabular}


TABLE 7. SOIL SAMPLE RESUltS FOR URANIUM AT CAS TA-52-006-TAPL, DUS (CONTINUED)

\begin{tabular}{|c|c|c|c|c|c|}
\hline \multirow{3}{*}{$\begin{array}{c}\text { SAMPLE } \\
\text { LOCATION }^{1} \\
\end{array}$} & \multicolumn{4}{|c|}{ ANALYTICAL RESULTS } & \multirow[b]{3}{*}{ PURPOSE } \\
\hline & $\begin{array}{l}\text { URANIUM } \\
\text { (mg/kg) }\end{array}$ & U-234 (pCi/g) & $\mathrm{U}-235(\mathrm{pCi} / \mathrm{g})$ & $\mathrm{U}-238(\mathrm{pCi} / \mathrm{g})$ & \\
\hline & PAL $=200$ & PAL $=85.8$ & PAL $=10.56$ & PAL $=63$ & \\
\hline DUS-34A & $<2.2$ & 2.84 & 0.892 & 1.81 & Investigation \\
\hline DUS-34B & $<2.2$ & 3.23 & 1.16 & 2.50 & Investigation \\
\hline DUS-35A & $<2.2$ & 4.10 & $<0.374$ & $<0.994$ & Investigation \\
\hline DUS-35B & $<2.3$ & 1.59 & $<-0.217$ & $<1.29$ & Investigation \\
\hline DUS-36A & $<2.2$ & 3.55 & 1.55 & 1.85 & Investigation \\
\hline DUS-36B & $<2.3$ & 2.44 & $<0.583$ & 1.18 & Investigation \\
\hline DUS-37A & $<2.1$ & 2.28 & $<0.273$ & 1.55 & $\begin{array}{l}\text { QA Duplicate } \\
\text { (DUS-10A) }\end{array}$ \\
\hline DUS-37B & $<2.3$ & 2.66 & $<0.0671$ & 1.36 & $\begin{array}{l}\text { QA Duplicate } \\
\text { (DUS-10B) }\end{array}$ \\
\hline DUS-38A & $<2.2$ & $<1.10$ & $<-0.0957$ & $<0.613$ & $\begin{array}{l}\text { QA Duplicate } \\
\text { (DUS-20A) }\end{array}$ \\
\hline DUS-38B & $<2.2$ & $<1.36$ & $<0.316$ & $<0.583$ & $\begin{array}{l}\text { QA Duplicate } \\
\text { (DUS-20B) }\end{array}$ \\
\hline DUS-R1 ${ }^{2}$ & $<21.4 \mu \mathrm{g} / \mathrm{L}$ & $<1.90 \mathrm{pCi} / \mathrm{L}$ & $<-0.32 \mathrm{pCi} / \mathrm{L}$ & $<1.08 \mathrm{pCi} / \mathrm{L}$ & $\begin{array}{l}\text { QA Equipment } \\
\text { Rinsate Blank }\end{array}$ \\
\hline
\end{tabular}

1 “A” sample collected from 0.0-0.5 ft bgs, "B” sample collected from 1.0-1.5 ft bgs.

${ }^{2}$ Quality Assurance blank in liquid units (i.e., $\mu \mathrm{g} / \mathrm{L}$ or $\mathrm{pCi} / \mathrm{L}$ ). 


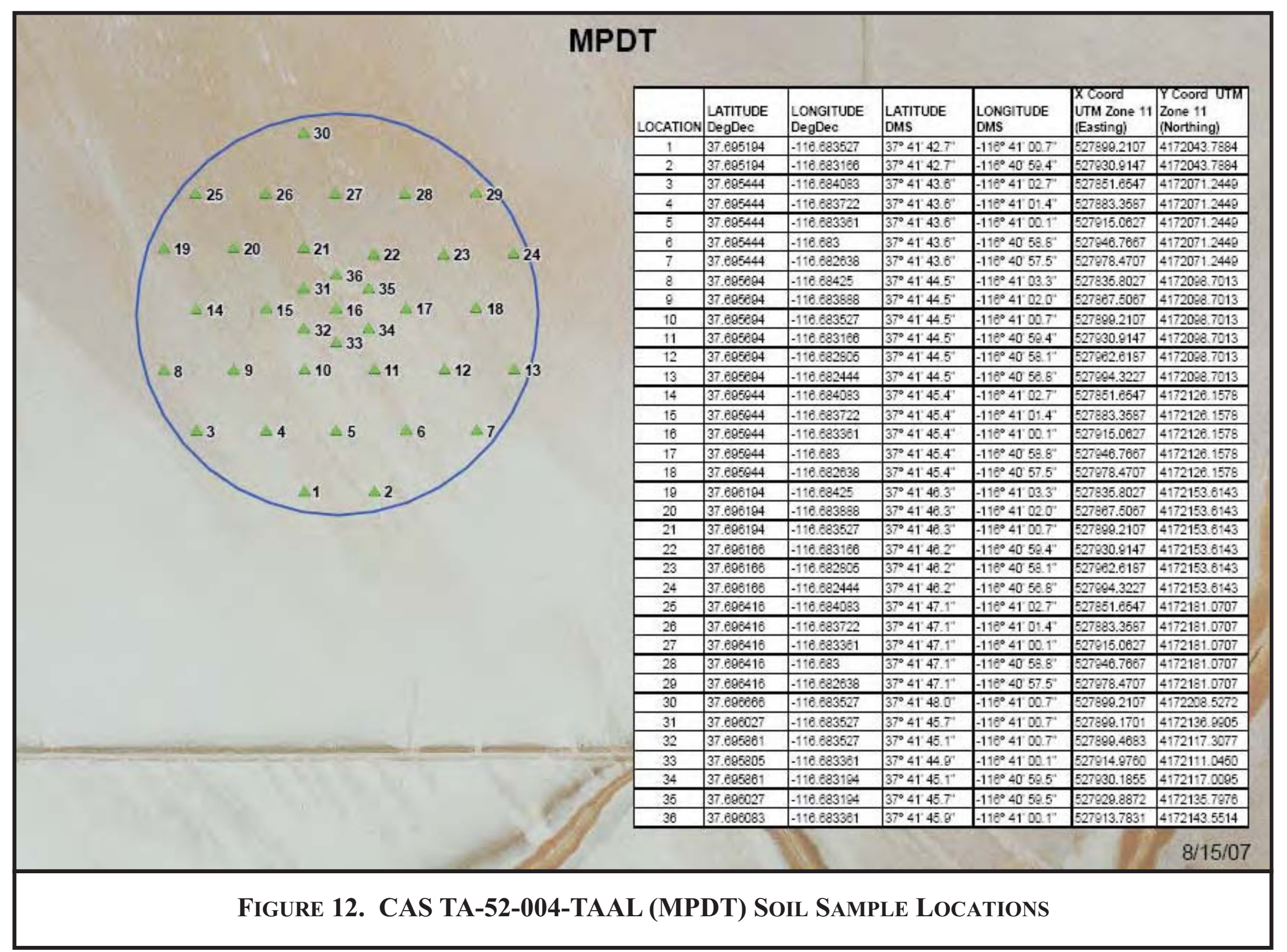


TABLE 8. SOIL SAMPLE RESUlTS FOR BERYLLIUM AT CAS TA-52-004-TAAL, MPDT

\begin{tabular}{|c|c|c|c|}
\hline \multirow{2}{*}{$\begin{array}{c}\text { SAMPLE } \\
\text { LOCATION }\end{array}$} & \multicolumn{2}{|c|}{$\begin{array}{c}\text { ANALYTICAL RESULTS }^{1} \\
(\mathrm{mg} / \mathrm{kg}) \\
\text { PAL }=\mathbf{1 , 9 0 0}\end{array}$} & \multirow[b]{2}{*}{ PURPOSE } \\
\hline & $0-0.5 \mathrm{ft}$ bgs & 1.0-1.5 ft bgs & \\
\hline MPDT-01 & 1.8 & 1.5 & Investigation \\
\hline MPDT-02 & 1.6 & 1.5 & Investigation \\
\hline MPDT-03 & 1.5 & 1.4 & Investigation \\
\hline MPDT-04 & 1.6 & 1.4 & Investigation \\
\hline MPDT-05 & 1.5 & 1.4 & Investigation \\
\hline MPDT-06 & 1.6 & 1.5 & Investigation \\
\hline MPDT-07 & 1.7 & 1.3 & Investigation \\
\hline MPDT-08 & 1.5 & 1.4 & Investigation \\
\hline MPDT-09 & 1.7 & 1.3 & Investigation \\
\hline MPDT-10 & 1.4 & 1.4 & Investigation \\
\hline MPDT-11 & 1.5 & 1.5 & Investigation \\
\hline MPDT-12 & 1.7 & 1.6 & Investigation \\
\hline MPDT-13 & 1.7 & 1.6 & Investigation \\
\hline MPDT-14 & 1.7 & 1.4 & Investigation \\
\hline MPDT-15 & 1.5 & 1.4 & Investigation \\
\hline MPDT-16 & 1.6 & 1.4 & Investigation \\
\hline MPDT-17 & 1.5 & 1.5 & Investigation \\
\hline MPDT-18 & 1.7 & 1.5 & Investigation \\
\hline MPDT-19 & 1.4 & 1.4 & Investigation \\
\hline MPDT-20 & 1.5 & 1.4 & Investigation \\
\hline MPDT-21 & 1.8 & 1.8 & Investigation \\
\hline MPDT-22 & 1.9 & 1.8 & Investigation \\
\hline MPDT-23 & 1.9 & 1.5 & Investigation \\
\hline MPDT-24 & 1.8 & 1.7 & Investigation \\
\hline MPDT-25 & 1.8 & 1.6 & Investigation \\
\hline MPDT-26 & 2 & 1.6 & Investigation \\
\hline MPDT-27 & 1.7 & 1.6 & Investigation \\
\hline MPDT-28 & 1.9 & 1.7 & Investigation \\
\hline MPDT-29 & 1.8 & 1.7 & Investigation \\
\hline MPDT-30 & 1.9 & 1.5 & Investigation \\
\hline MPDT-31 & 1.8 & 1.6 & Investigation \\
\hline MPDT-32 & 1.7 & 1.5 & Investigation \\
\hline MPDT-33 & 1.8 & 1.6 & Investigation \\
\hline
\end{tabular}


TABLE 8. SOIL SAMPLE RESULTS FOR BERYLLIUM AT

CAS TA-52-004-TAAL, MPDT (CONTINUED)

\begin{tabular}{||c|c|c|c||}
\hline \multirow{2}{*}{$\begin{array}{c}\text { SAMPLE } \\
\text { LOCATION }\end{array}$} & \multicolumn{2}{|c|}{$\begin{array}{c}\text { ANALYTICAL RESULTS } \\
\text { (mg/kg) } \\
\text { PAL = 1,900 }\end{array}$} & \multirow{2}{*}{ PURPOSE } \\
\cline { 2 - 3 } & $\mathbf{0 - 0 . 5} \mathbf{f t}$ bgs & $\mathbf{1 . 0 - 1 . 5} \mathbf{~ f t ~ b g s}$ & Investigation \\
\hline \hline MPDT-34 & 1.6 & 1.6 & Investigation \\
\hline MPDT-35 & 1.9 & 1.5 & Investigation \\
\hline MPDT-36 & 1.9 & 1.6 & QA Duplicate (MPDT-10) \\
\hline MPDT-37 & 1.7 & 1.5 & QA Duplicate (MPDT-20) \\
\hline MPDT-38 & 1.9 & 1.6 & QA Equipment Rinsate Blank \\
\hline MPDT-R1 & \multicolumn{2}{|c|}{$0.1 \mu \mathrm{g} / \mathrm{L}$} \\
\hline
\end{tabular}

${ }^{1}$ Quantitation limit is $0.01 \mathrm{mg} / \mathrm{kg}(0.1 \mu \mathrm{g} / \mathrm{L}$ for MPDT-R1). 
TABLE 9. SoIL SAMPLE RESUlts FOR URANIUM AT CAS TA-52-004-TAAL, MPDT

\begin{tabular}{|c|c|c|c|c|c|}
\hline \multirow{3}{*}{$\begin{array}{c}\text { SAMPLE } \\
\text { LOCATION }^{1} \\
\end{array}$} & \multicolumn{4}{|c|}{ ANALYTICAL RESULTS } & \multirow[b]{3}{*}{ PURPOSE } \\
\hline & $\begin{array}{c}\text { URANIUM } \\
\text { (mg/kg) }\end{array}$ & $\begin{array}{c}\mathrm{U}-234 \\
\text { (pCi/g) }\end{array}$ & U-235 (pCi/g) & U-238 (pCi/g) & \\
\hline & PAL $=200$ & PAL $=85.8$ & PAL = 10.56 & PAL $=63$ & \\
\hline MPDT-01A & $<2.2$ & 1.44 & $<0.208$ & 1.26 & Investigation \\
\hline MPDT-01B & $<2.2$ & 1.75 & $<0.0457$ & 1.55 & Investigation \\
\hline MPDT-02A & $<2.2$ & 1.35 & 0.201 & 1.62 & Investigation \\
\hline MPDT-02B & $<2.2$ & 1.46 & $<0.0539$ & 1.6 & Investigation \\
\hline MPDT-03A & $<2.2$ & 1.29 & $<0.194$ & 1.5 & Investigation \\
\hline MPDT-03B & $<2.2$ & 1.49 & $<0.0904$ & 0.959 & Investigation \\
\hline MPDT-04A & $<2.2$ & 1.61 & $<-0.0128$ & 1.32 & Investigation \\
\hline MPDT-04B & 2.3 & 1.79 & 0.391 & 1.22 & Investigation \\
\hline MPDT-05A & $<2.2$ & 1.59 & $<0.0975$ & 0.897 & Investigation \\
\hline MPDT-05B & $<2.2$ & 1.36 & $<0.17$ & 1.34 & Investigation \\
\hline MPDT-06A & $<2.2$ & 1.15 & 0.194 & 0.877 & Investigation \\
\hline MPDT-06B & $<2.2$ & 1.62 & $<0.177$ & 1.76 & Investigation \\
\hline MPDT-07A & $<2.2$ & 1.22 & $<0.037$ & 0.917 & Investigation \\
\hline MPDT-07B & $<2.2$ & 1.59 & $<0.0842$ & 1.07 & Investigation \\
\hline MPDT-08A & $<2.2$ & 2.17 & $<0.123$ & 1.13 & Investigation \\
\hline MPDT-08B & $<2.2$ & 1.42 & 0.323 & 1.55 & Investigation \\
\hline MPDT-09A & $<2.2$ & 1.22 & $<0.101$ & 1.23 & Investigation \\
\hline MPDT-09B & $<2.2$ & 1.56 & $<0.0534$ & 1.67 & Investigation \\
\hline MPDT-10A & $<2.2$ & 1.23 & 0 & 1.53 & Investigation \\
\hline MPDT-10B & $<2.2$ & 0.94 & $<0.0463$ & 1.2 & Investigation \\
\hline MPDT-11A & $<2.3$ & 1.93 & $<0.104$ & 1.53 & Investigation \\
\hline MPDT-11B & $<2.3$ & 1.27 & $<0.0447$ & 1.09 & Investigation \\
\hline MPDT-12A & $<2.3$ & 1.76 & $<0.187$ & 0.966 & Investigation \\
\hline MPDT-12B & $<2.3$ & 1.54 & 0 & 1.37 & Investigation \\
\hline MPDT-13A & $<2.3$ & 1.52 & $<0.0762$ & 0.946 & Investigation \\
\hline MPDT-13B & $<2.3$ & 1.9 & $<0.0892$ & 1.66 & Investigation \\
\hline MPDT-14A & $<2.2$ & 1.25 & $<0.0761$ & 1.06 & Investigation \\
\hline MPDT-14B & $<2.3$ & 1.61 & $<0.073$ & 1.15 & Investigation \\
\hline MPDT-15A & $<2.3$ & 1.13 & 0 & 1.27 & Investigation \\
\hline MPDT-15B & $<2.5$ & 1.31 & $<0.19$ & 1.48 & Investigation \\
\hline MPDT-16A & $<2.2$ & 1.19 & $<0.117$ & 1.2 & Investigation \\
\hline MPDT-16B & $<2.3$ & 1.74 & $<0.172$ & 1.67 & Investigation \\
\hline MPDT-17A & $<2.2$ & 1.36 & $<0.0966$ & 1.67 & Investigation \\
\hline
\end{tabular}


TABle 9. SoIL SAMPle Results for URANiUM at CAS TA-52-004-TAAL, MPDT (CONTINUED)

\begin{tabular}{|c|c|c|c|c|c|}
\hline \multirow{3}{*}{$\begin{array}{c}\text { SAMPLE } \\
\text { LOCATION }^{1} \\
\end{array}$} & \multicolumn{4}{|c|}{ ANALYTICAL RESULTS } & \multirow[b]{3}{*}{ PURPOSE } \\
\hline & $\begin{array}{c}\text { URANIUM } \\
\text { (mg/kg) }\end{array}$ & $\begin{array}{c}\mathrm{U}-234 \\
(\mathrm{pCi} / \mathrm{g})\end{array}$ & U-235 (pCi/g) & $\mathrm{U}-238$ (pCi/g) & \\
\hline & PAL $=200$ & PAL $=85.8$ & PAL $=10.56$ & PAL $=63$ & \\
\hline MPDT-17B & $<2.3$ & 1.6 & $<0.184$ & 0.955 & Investigation \\
\hline MPDT-18A & $<2.3$ & 1.66 & 0.288 & 0.942 & Investigation \\
\hline MPDT-18B & $<2.3$ & 1.78 & $<0.204$ & 1.35 & Investigation \\
\hline MPDT-19A & $<2.2$ & 1.05 & $<0.0931$ & 1.7 & Investigation \\
\hline MPDT-19B & $<2.3$ & 1.67 & $<0.0982$ & 0.873 & Investigation \\
\hline MPDT-20A & $<2.2$ & 1.41 & $<0.0491$ & 1.18 & Investigation \\
\hline MPDT-20B & $<2.3$ & 2.18 & 0.386 & 1.08 & Investigation \\
\hline MPDT-21A & $<2.3$ & 0.928 & 0.27 & 1.63 & Investigation \\
\hline MPDT-21B & $<2.3$ & 1.84 & $<0.125$ & 1.23 & Investigation \\
\hline MPDT-22A & $<2.3$ & 1.3 & 0.14 & 1.03 & Investigation \\
\hline MPDT-22B & $<2.3$ & 1.42 & $<0.0955$ & 1.01 & Investigation \\
\hline MPDT-23A & $<2.3$ & 1.92 & $<0.0268$ & 1.19 & Investigation \\
\hline MPDT-23B & $<2.3$ & 1.28 & $<0.176$ & 1.37 & Investigation \\
\hline MPDT-24A & $<2.3$ & 1.4 & $<0.0304$ & 1.32 & Investigation \\
\hline MPDT-24B & $<2.3$ & 1.32 & $<0.123$ & 0.762 & Investigation \\
\hline MPDT-25A & $<2.2$ & 1.53 & 0 & 1.55 & Investigation \\
\hline MPDT-25B & $<2.3$ & 1.09 & $<0.044$ & 1.06 & Investigation \\
\hline MPDT-26A & $<2.2$ & 1.08 & $<0.0166$ & 1.09 & Investigation \\
\hline MPDT-26B & $<2.3$ & 1.71 & $<0.086$ & 1.11 & Investigation \\
\hline MPDT-27A & $<2.2$ & 1.66 & $<0.0472$ & 1.45 & Investigation \\
\hline MPDT-27B & $<2.3$ & 1.1 & $<0.234$ & 1.05 & Investigation \\
\hline MPDT-28A & $<2.3$ & 1.58 & $<0.174$ & 1.43 & Investigation \\
\hline MPDT-28B & $<2.3$ & 1.5 & $<0.138$ & 1.02 & Investigation \\
\hline MPDT-29A & $<2.2$ & 1.3 & 0.145 & 1.47 & Investigation \\
\hline MPDT-29B & $<2.3$ & 1.67 & 0.144 & 1.55 & Investigation \\
\hline MPDT-30A & 2.6 & 1.36 & $<0.0883$ & 1.6 & Investigation \\
\hline MPDT-30B & $<2.3$ & 1.16 & $<0.142$ & 1.15 & Investigation \\
\hline MPDT-31A & $<2.2$ & 1.59 & $<0.0787$ & 1.15 & Investigation \\
\hline MPDT-31B & $<2.3$ & 1.77 & 0.131 & 1.33 & Investigation \\
\hline MPDT-32A & $<2.2$ & 1.21 & $<0.0701$ & 1.22 & Investigation \\
\hline MPDT-32B & $<2.2$ & 1.45 & $<0.0371$ & 1.5 & Investigation \\
\hline MPDT-33A & $<2.2$ & 1.52 & $<0.0725$ & 1.12 & Investigation \\
\hline MPDT-33B & $<2.2$ & 1.28 & $<0.165$ & 1.48 & Investigation \\
\hline
\end{tabular}


TABLE 9. SoIL SAMPLE Results For URANiUm AT

CAS TA-52-004-TAAL, MPDT (CONTINUED)

\begin{tabular}{|c|c|c|c|c|c|}
\hline \multirow{3}{*}{$\begin{array}{c}\text { SAMPLE } \\
\text { LOCATION }^{1}\end{array}$} & \multicolumn{4}{|c|}{ ANALYTICAL RESULTS } & \multirow[b]{3}{*}{ PURPOSE } \\
\hline & $\begin{array}{l}\text { URANIUM } \\
\text { (mg/kg) }\end{array}$ & $\begin{array}{c}\mathrm{U}-234 \\
(\mathrm{pCi} / \mathrm{g})\end{array}$ & U-235 (pCi/g) & U-238 (pCi/g) & \\
\hline & PAL $=200$ & PAL $=85.8$ & PAL = 10.56 & PAL $=63$ & \\
\hline MPDT-34A & $<2.2$ & 1.33 & $<0.139$ & 1.34 & Investigation \\
\hline MPDT-34B & $<2.2$ & 1.81 & $<0.0924$ & 1.37 & Investigation \\
\hline MPDT-35A & $<2.2$ & 1.62 & 0 & 1.49 & Investigation \\
\hline MPDT-35B & $<2.2$ & 1.39 & $<-0.00438$ & 1.06 & Investigation \\
\hline MPDT-36A & $<2.2$ & 1.58 & $<-0.0044$ & 0.949 & Investigation \\
\hline MPDT-36B & $<2.3$ & 1.96 & $<0.132$ & 1.34 & Investigation \\
\hline MPDT-37A & $<2.2$ & 1.35 & $<0.0931$ & 1.49 & $\begin{array}{l}\text { QA Duplicate } \\
\text { (MPDT-10A) }\end{array}$ \\
\hline MPDT-37B & $<2.2$ & 1.43 & $<0.0925$ & 1.32 & $\begin{array}{l}\text { QA Duplicate } \\
\text { (MPDT-10B) }\end{array}$ \\
\hline MPDT-38A & $<2.2$ & 1.47 & $<0.0322$ & 0.996 & $\begin{array}{l}\text { QA Duplicate } \\
\text { (MPDT-20A) }\end{array}$ \\
\hline MPDT-38B & $<2.2$ & 1.67 & 0.148 & 1.46 & $\begin{array}{l}\text { QA Duplicate } \\
\text { (MPDT-20B) }\end{array}$ \\
\hline MPDT-R1 ${ }^{2}$ & $<21.4 \mu \mathrm{g} / \mathrm{L}$ & $\begin{array}{c}<0.0977 \\
\mathrm{pCi} / \mathrm{L}\end{array}$ & $<0.0177 \mathrm{pCi} / \mathrm{L}$ & $<0.0751 \mathrm{pCi} / \mathrm{L}$ & $\begin{array}{l}\text { QA Equipment } \\
\text { Rinsate Blank }\end{array}$ \\
\hline
\end{tabular}

1 “A” sample collected from 0.0-0.5 ft bgs, "B” sample collected from 1.0-1.5 ft bgs.

${ }^{2}$ Quality Assurance blank in liquid units (i.e., $\mu \mathrm{g} / \mathrm{L}$ or pCi/L). 
Although analytical results showed no indication of contamination above action levels, the radiological drive-over survey detected one location within the MPDT bermed area with DU contamination. This contamination was found to be a DU fragment on the ground surface with limited soil contamination.

\subsubsection{Excavation/Removal of DU}

Data collected to date were reviewed and confirmed on the ground (i.e., "ground-truthed") prior to beginning excavation activities. To do this, the Kiwi vehicle, which is configured with eight sodium iodide gamma detectors in four pods, was used to re-acquire the areas where DU was originally detected. The Kiwi vehicle had been used to perform the original radiological surveys. Handheld radiological screening instruments (Field Instrument for Detecting Low Energy Radiation [FIDLER] and/or Electra ${ }^{\circledR}$ ) were then used to confirm locations of elevated radioactivity and to support DU cleanup.

DU fragments and contaminated soil were removed and excavated from both NEDS Lake and Antelope Lake. Most of the DU was on the ground surface or at shallow depths in the soil; however, it was present at some locations on Antelope Lake to depths of nearly $10 \mathrm{ft}$ bgs. DU contamination at CAS TA-52-001-TANL (NEDS Detonation Area) test location was found to consist of impacted debris and contaminated soil interlayered with clean fill. This was consistent with interviews with project personnel who recalled clean fill being added to the site after each test to maintain a "clean" working surface. Within the NEDS Detonation Area test location, one area approximately 6 inches deep with a diameter of approximately $30 \mathrm{ft}$ was excavated (NEDS 3). Within this 30-ft diameter area, one deeper excavation (approximately $4 \mathrm{ft}$ wide by $3 \mathrm{ft}$ deep), appeared to be a location where NEDS-related debris was buried.

Where DU was present at the ground surface or at shallow depths (to about $1 \mathrm{ft}$ bgs), it was removed and/or excavated using hand tools. Deeper contamination was excavated using heavy equipment. Table 10 identifies locations where DU debris and contaminated soil were excavated and the approximate volume of each excavation. DU and contaminated soil was placed in soft-sided containers or B-25 boxes and managed in a radioactive materials area (RMA) until disposal (Section 3.0).

The initial approach to verify clean closure using handheld radiological survey instruments assumed that only DU fragments and minor quantities of impacted soil were present. Because investigation activities showed some areas of impact to exceed 1 cubic foot $\left(\mathrm{ft}^{3}\right)$ of contaminated soil and DU debris, the CSM for these areas was not upheld and re-evaluation of the closure approach was required. A sampling plan to justify the number and location of samples was prepared and submitted to NDEP for review in December 2006. The sampling plan (Appendix E) was approved and implemented. Samples were collected to verify clean closure of the sites, from locations selected within each excavation based on results of handheld instruments. Handheld instruments were used to select sample locations so that samples would be collected from those portions of the excavation with the highest residual radioactivity. This provides a level of confidence that contamination would be detected in the soil samples if it is still present above action levels.

Subsequent evaluation of the verification data confirmed that using handheld instruments to guide sample collection was not a valid approach for CAU 484. The handheld instruments that 
TABLE 10. SUMMARY OF DU-EXCAVATED SOIL FROM SURFACE DETONATION DEBRIS SITES

\begin{tabular}{|c|c|c|c|}
\hline $\begin{array}{c}\text { EXCAVATION } \\
\text { LOCATION } \\
\end{array}$ & $\begin{array}{c}\text { VOLUME } \\
\text { EXCAVATED } \\
\end{array}$ & $\begin{array}{c}\text { EXCAVATION } \\
\text { LOCATION } \\
\end{array}$ & $\begin{array}{c}\text { VOLUME } \\
\text { EXCAVATED } \\
\end{array}$ \\
\hline NEDS 1 & $<1 \mathrm{ft}^{3}$ & NEDS 29 & $<1 \mathrm{ft}^{3}$ \\
\hline NEDS 2 & $<1 \mathrm{ft}^{3}$ & NEDS 30 & $<1 \mathrm{ft}^{3}$ \\
\hline NEDS 3 / NEDS-14 (GP) & $62 \mathrm{yd}^{3}$ & NEDS 31 & $<1 \mathrm{ft}^{3}$ \\
\hline NEDS 4 & $6 \mathrm{yd}^{3}$ & NEDS 32 & $<1 \mathrm{ft}^{3}$ \\
\hline NEDS 5 & $1 \mathrm{ft}^{3}$ & NEDS 33 & $<1 \mathrm{ft}^{3}$ \\
\hline NEDS 6 & $<1 \mathrm{ft}^{3}$ & NEDS 34 & $<1 \mathrm{ft}^{3}$ \\
\hline NEDS 7 & $1 \mathrm{ft}^{3}$ & NEDS 35 & $<1 \mathrm{ft}^{3}$ \\
\hline NEDS 8 & $<1 \mathrm{ft}^{3}$ & NEDS 36 & $<1 \mathrm{ft}^{3}$ \\
\hline NEDS 9 & $1 \mathrm{ft}^{3}$ & NEDS 37 & $<1 \mathrm{ft}^{3}$ \\
\hline NEDS 10 & $<1 \mathrm{ft}^{3}$ & NEDS 38 & $<1 \mathrm{ft}^{3}$ \\
\hline NEDS 11 & $<1 \mathrm{ft}^{3}$ & NEDS 39 & $<1 \mathrm{ft}^{3}$ \\
\hline NEDS 12 & $<1 \mathrm{ft}^{3}$ & NEDS 40 & $<1 \mathrm{ft}^{3}$ \\
\hline NEDS 13 & $<1 \mathrm{ft}^{3}$ & NEDS 41 & $<1 \mathrm{ft}^{3}$ \\
\hline NEDS 14 & $<1 \mathrm{ft}^{3}$ & NEDS 42 & $<1 \mathrm{ft}^{3}$ \\
\hline NEDS 15 & $<1 \mathrm{ft}^{3}$ & NEDS 43 & $<1 \mathrm{ft}^{3}$ \\
\hline NEDS 16 & $<1 \mathrm{ft}^{3}$ & NEDS 44 & $<1 \mathrm{ft}^{3}$ \\
\hline NEDS 17 & $<1 \mathrm{ft}^{3}$ & NEDS 45 & $<1 \mathrm{ft}^{3}$ \\
\hline NEDS 18 & $<1 \mathrm{ft}^{3}$ & NEDS 46 & $<1 \mathrm{ft}^{3}$ \\
\hline NEDS 19 & $<1 \mathrm{ft}^{3}$ & NEDS 47 & $<1 \mathrm{ft}^{3}$ \\
\hline NEDS 20 & $<1 \mathrm{ft}^{3}$ & NEDS 48 & $<1 \mathrm{ft}^{3}$ \\
\hline NEDS 21 & $<1 \mathrm{ft}^{3}$ & NEDS 49 & $<1 \mathrm{ft}^{3}$ \\
\hline NEDS 22 & $<1 \mathrm{ft}^{3}$ & NEDS 50 & $<1 \mathrm{ft}^{3}$ \\
\hline NEDS 23 & $<1 \mathrm{ft}^{3}$ & NEDS 51 & $<1 \mathrm{ft}^{3}$ \\
\hline NEDS 24 & $<1 \mathrm{ft}^{3}$ & NEDS 52 & $<1 \mathrm{ft}^{3}$ \\
\hline NEDS 25 & $<1 \mathrm{ft}^{3}$ & NEDS 53 & $<1 \mathrm{ft}^{3}$ \\
\hline NEDS 26 & $<1 \mathrm{ft}^{3}$ & NEDS 54 & $<1 \mathrm{ft}^{3}$ \\
\hline NEDS 27 & $<1 \mathrm{ft}^{3}$ & \multirow{2}{*}{ SCA 33} & \multirow{2}{*}{$6 \mathrm{yd}^{3}$} \\
\hline NEDS 28 & $<1 \mathrm{ft}^{3}$ & & \\
\hline
\end{tabular}


were used at CAU 484 were designed to detect alpha/beta radiation, which is easily shielded by a thin covering of soil. Also, the ability to obtain representative results in soil samples is highly dependent on collecting representative and homogeneous samples. The presence of one fragment of DU, which is undetectable with handheld survey instruments, can highly skew the analytical results. Instead, an approach presented in the SAFER Plan (NNSA/NSO, 2004b) and subsequent hold point meetings was implemented. This approach used the Kiwi to guide DU cleanup and to verify and document cleanup effectiveness. The Kiwi sodium iodide detectors proved to be effective for reacquiring the location of previously identified DU and for verifying cleanup effectiveness. Use of the Kiwi was deemed sufficient to show clean closure for locations accessible to the Kiwi vehicle; therefore, cleanup verification sample collection was only needed for those locations that were inaccessible to the Kiwi vehicle. Clean closure was confirmed using the Kiwi for all of the sites in this CSM. See Appendix M for additional detail on use of the Kiwi and the radiological data collected.

\subsection{CAS TA-52-001-TANL (NEDS Detonation Area)}

In November 2006, DU and uranium-contaminated soil were excavated from within the NEDS Detonation Area (Figure 7). All areas had been identified by the drive-over radiological survey. One of those areas (NEDS 3) was also identified as a subsurface hot spot by analytical results from the Geoprobe soil sampling (NEDS-14B). Handheld radiological instruments (FIDLERs and/or Electras) were used to verify that the elevated radioactivity had been removed, and cleanup verification soil samples were collected from areas where more than $1 \mathrm{ft}^{3}$ of soil had been removed.

After the November 2006, sampling effort, cleanup verification sample analytical results showed that DU and chemical uranium were still present above action levels at several locations. From April to June 2007, excavation on NEDS Lake continued until clean closure could be verified by the Kiwi. Cleanup verification results, as confirmed by the Kiwi, are presented in Appendix M.

Upon completion of excavation activities on NEDS Lake, approximately 68 cubic yards ( $\mathrm{yd}^{3}$ ) of DU and impacted soil had been removed and containerized in B-25 boxes and soft-sided containers. The waste will be transported to the Nevada Test Site (NTS) and disposed of in the Area 5 Radioactive Waste Management Site (RWMS) in September 2007.

\subsection{CAS TA-52-006-TAPL (DUS)}

In November 2006, DU and associated contaminated soil were excavated from within the DUS (Figure 7). All areas had been identified by the drive-over radiological survey. Handheld radiological instruments (FIDLERs and/or Electras) were used to verify that the elevated radioactivity had been removed. Soil samples were collected from areas where more than $1 \mathrm{ft}^{3}$ of soil had been removed, and sample results again indicated that DU was still present above action levels.

From April to June 2007, additional Kiwi radiological surveys were conducted on NEDS Lake to locate DU that was previously not found and to verify the effectiveness of previous DU cleanup. Previously excavated locations were determined to contain residual DU contamination, and several locations previously not found were re-acquired and confirmed to contain DU 
contamination. All areas containing DU were cleaned up to background conditions and verified using the Kiwi. Cleanup verification results, as confirmed by the Kiwi, showed that the elevated radioactivity had been successfully removed from these locations. Results are presented in Appendix M.

Several of the locations on NEDS Lake that were originally identified as single, large DU targets during the initial Kiwi surveys were later confirmed to be multiple, small individual fragments of DU. This change is depicted in the Kiwi survey figures (Appendix N) by a greater number of DU targets than identified during the initial survey. This difference is due to slower survey speeds and a lower detection height used for the verification surveys.

Waste was containerized with the other radioactive waste from NEDS Lake (see Section 2.1.2.3.1).

\subsection{CAS TA-52-004-TAAL (MPDT)}

In November 2006, one area was excavated to remove DU and associated contaminated soil from within the MPDT Detonation Area (SCA-33 in Figure 8). This area had been identified by the drive-over radiological survey. Other areas were also excavated from the northern half of Antelope Lake to remove DU and contaminated soil that was detected by the drive-over radiological survey (Figure 8). The Kiwi and handheld radiological instruments (FIDLERs and/or Electras) were used to verify that the elevated radioactivity had been removed. Other areas that were excavated from the northern half of Antelope Lake to remove DU and contaminated soil are documented under CAS RG-52-007-TAML (Davis Gun Penetrator Test).

Waste from SCA-33 was containerized with other radioactive waste from Antelope Lake and managed in an RMA until disposition. Waste disposition is documented in Section 3.0.

\subsubsection{Closure Activities - Surface-Shallow Subsurface Debris Sites}

The surface-shallow subsurface debris sites CSM consists of the following two CASs:

- CAS RG-52-007-TAML, Davis Gun Penetrator Test, consists of locations on Main Lake, Pedro Lake, Brownes Lake, Antelope Lake, Antelope Tuff Target 1, Antelope Tuff Target 2, Sidewinder Tuff, Myers Ridge, and Mt. Helen (Figure 13). Details regarding the Davis Gun Penetrator Tests can be found in the CAU 484 SAFER Plan (NNSA/NSO, 2004b). As stated in the DQOs for CAU 484 (Appendix A), there is evidence that all penetrators were recovered completely intact. It is highly unlikely that test components were left at the sites where the experiments were conducted because an unrecovered penetrator would be considered a test failure, and no failures for Davis Gun tests have been reported.

Survey documentation confirmed that Mt. Helen is not within the permit boundary for the TTR and is located on the Nevada Test and Training Range 76. It is unknown how this site was selected and approved for Sandia testing of the Davis Gun penetrators; however, it is known that the site was selected because of its geology (good surface exposure of welded tuff) and was used for penetrator testing. Mt. Helen was the area selected and used for the earliest series of penetrator tests at tuff locations from the early 1970s to the late 1970s or early 1980s. The survey monument at the Mt. Helen site is dated 1968. At or about the early 1980s, Davis Gun tests at tuff locations were moved from Mt. Helen to the Antelope Tuff 


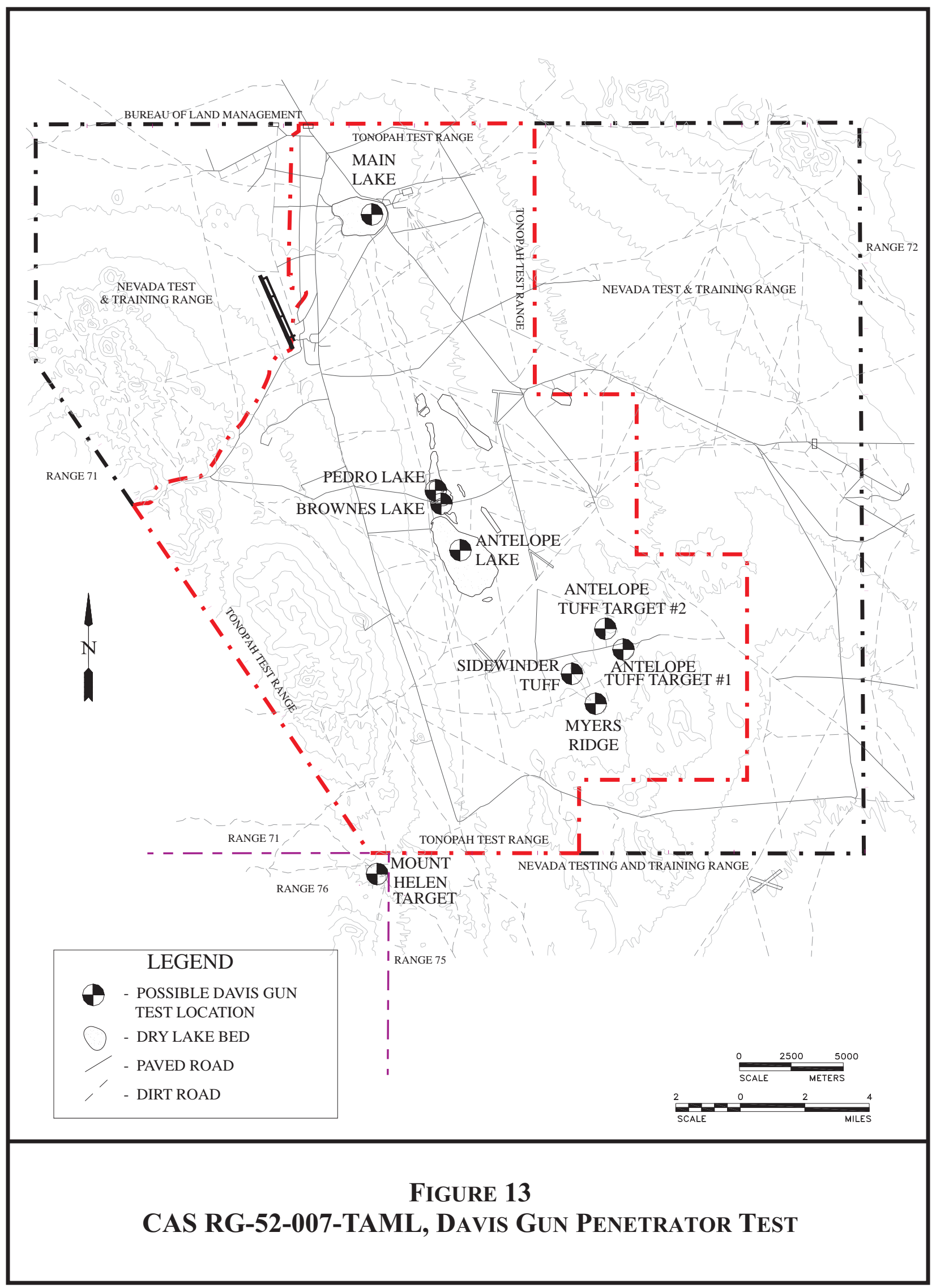


Target 1 location. The reasoning for this move is not known, but testing at Mt. Helen was discontinued after the relocation of operations. Only the areas where testing activities obviously took place were included in the scope for geophysical and radiological surveys. Based on the findings of the CAU 484 investigation, the remaining geophysical anomalies will not be investigated or removed.

- $\quad$ CAS TA-52-005-TAAL, JTA DU Site, is located on Antelope Lake (Figure 14) and consists of three DU rings that could not be located after projectile testing from Area 9 to Antelope Lake. The three rings were expected to be found on the northern half of Antelope Lake. The three rings were found and were removed.

These are sites where DU and/or system components, if present, were expected to have been released to the ground surface or to have penetrated the shallow subsurface. Any DU was expected to be present in discrete pieces and, based on process knowledge and technology limitations, at depths of $10 \mathrm{ft}$ bgs or less. The CSM was confirmed to be incorrect in that DU, which was originally present in discrete pieces, has oxidized and contaminated the surrounding soil. Some DU was also encountered in the shallow subsurface on Antelope Lake.

Detailed information regarding the surface-shallow subsurface debris sites CSM can be found in the SAFER Plan (NNSA/NSO, 2004b). The DQOs from that document, which present the CSM, are also included in Appendix A of this document.

\subsubsection{Aerial Photography and Multispectral Surveys}

Aerial photography and multispectral surveys were conducted over the entire footprint of CAU 484, including locations where Davis Gun Penetrator Tests (CAS RG-52-007-TAML) were conducted: Main Lake, Pedro Lake, Brownes Lake, NEDS Lake, Antelope Lake, Antelope Tuff Targets 1 and 2, Sidewinder Tuff, Myers Ridge, and Mt. Helen. Davis Gun tests were conducted throughout the entire footprint of each test location. Aerial photographs and multispectral survey data were reviewed along with data collected later in the investigation to identify locations where Davis Gun projectiles may have impacted the surface.

On the dry lakes/playas, where soil was historically bermed in a circle around Davis Gun impact locations to keep the area from flooding, circular features that could be berms were identified as suspect areas and targeted for additional investigation. Disturbed areas within the berms were suspected locations of where excavation or drilling may have been performed to recover penetrators. Many circular features are still present and were discernable in the aerial photography and multispectral imagery.

The aerial photographs provided a better indication of features related to potential penetrator locations on the playas (see Figure 15 for an example). Evidence of many historical testing locations could not be identified on aerial photographs because the testing areas had been largely "erased” by wave and wind action on the dry lake bed. Multispectral surveys were helpful because different colors on the multispectral surveys indicate different soil types, including areas of disturbed ground. Circular areas with a different soil type on the surface provide an indication of disturbance, where soil from a greater depth may have been excavated and brought to the surface, as would have occurred by excavating a Davis Gun penetrator, or where soil may have been brought in to berm an area, as would have occurred when protecting a Davis Gun penetrator 


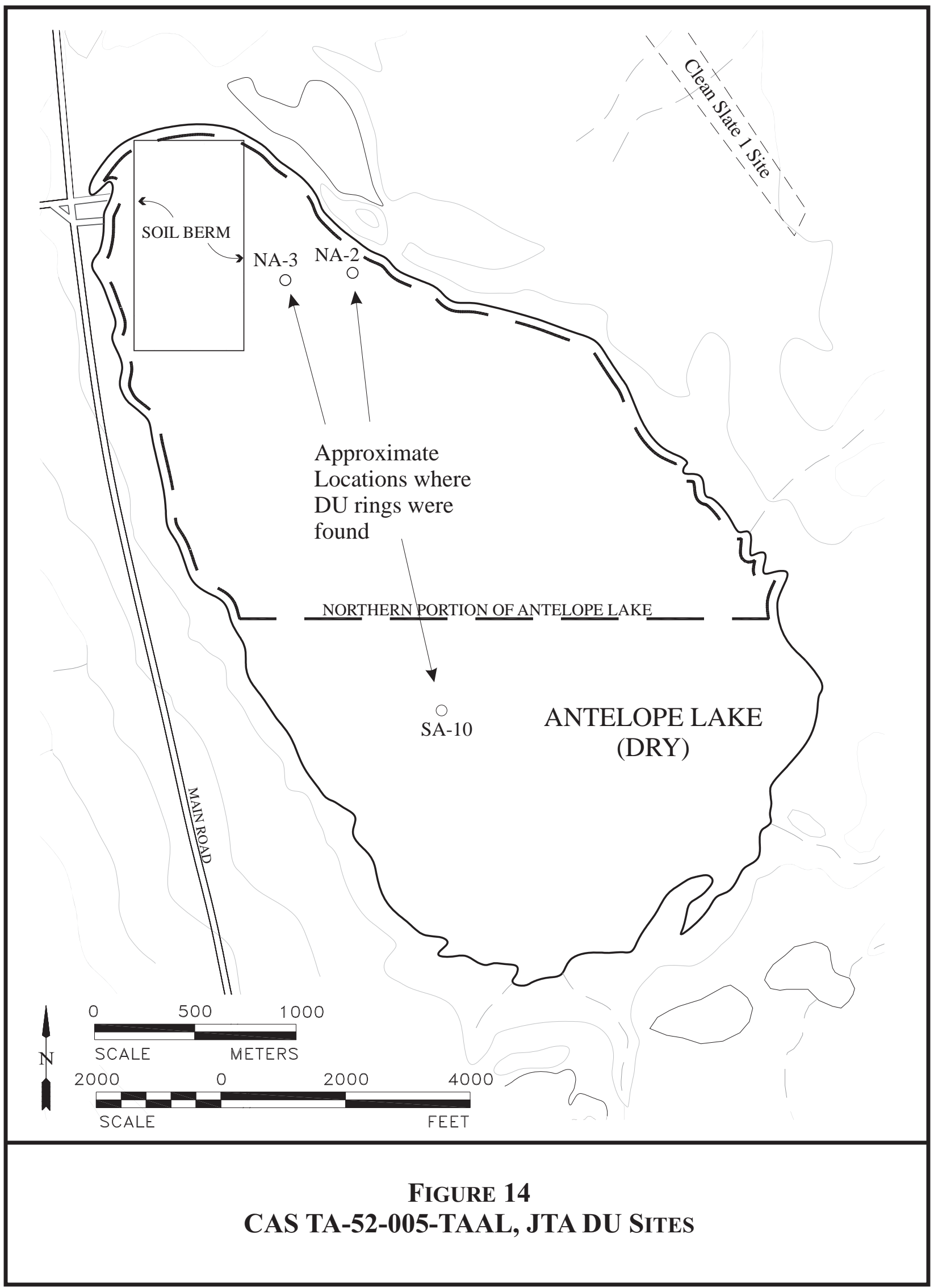




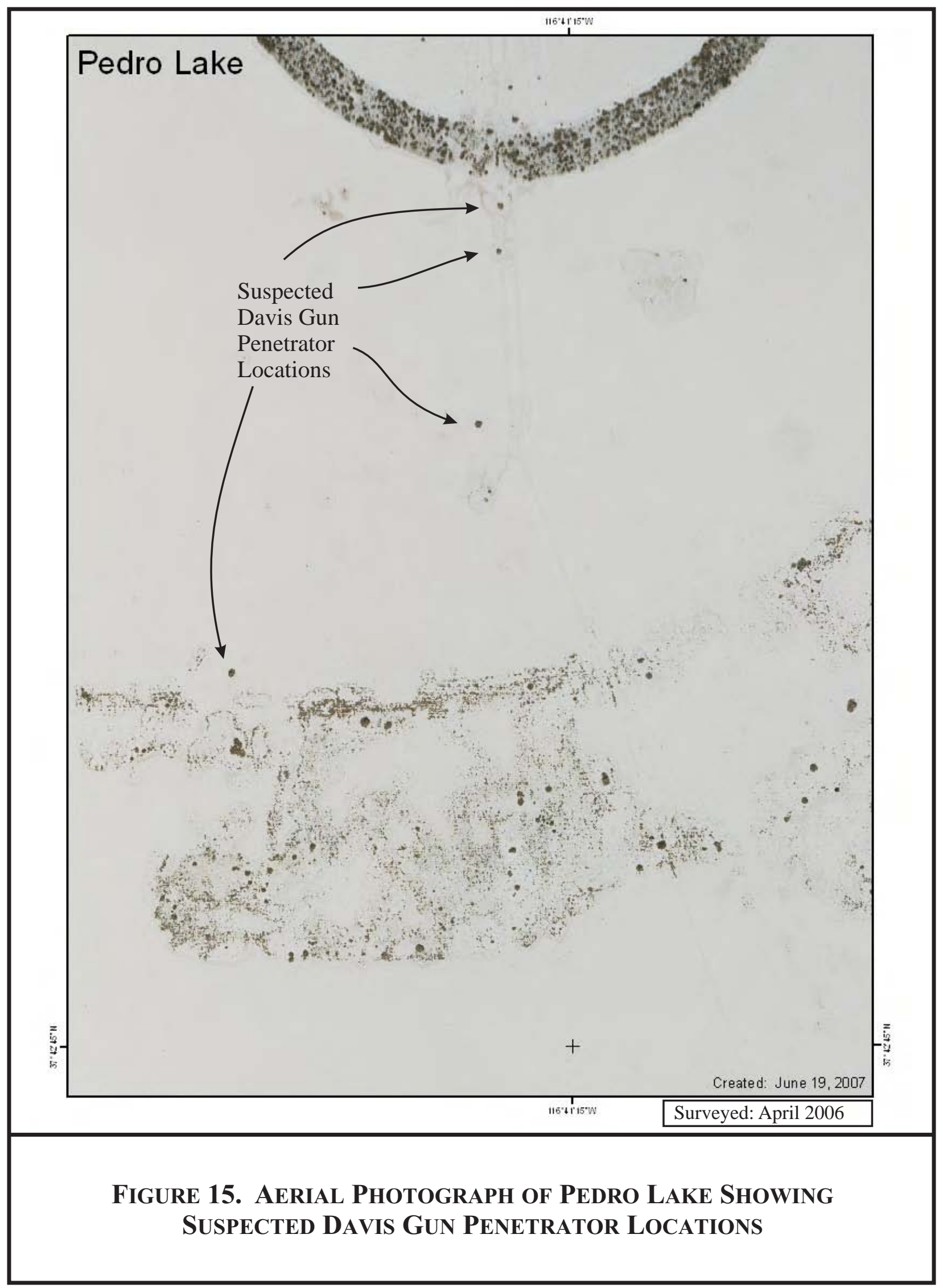


target location from flooding. An example of the multispectral survey results for the playa locations is provided in Figure 16. Appendix $\mathrm{N}$ contains imagery with the multispectral survey results.

For the tuff locations, depressions and rubble piles are indicators of potential penetrator entrance and recovery locations (Figure 17). Depressions in the tuff with little or no rubble nearby were identified as areas where projectiles may have entered the earth but were not recovered, whereas depressions with associated rubble piles were more likely representative of recovered projectiles. Historical documentation also confirms that debris piles usually correspond to a test recovery or attempted recovery. Aerial photographs were helpful in identifying the areas that had been disturbed by activity; however, neither aerial photographs nor multispectral survey results were helpful in identifying potential penetrator locations at the tuff sites because individual depressions or rubble piles were generally indistinguishable from the topography.

Results from these surveys were generally used together with other survey results (e.g., radiological and geophysical surveys, ground-truthing, etc.) and not independent of other input. Aerial and multispectral survey results did not help in locating any of the DU rings associated with CAS TA-52-005-TAAL (JTA DU Site) due to the size and nature of the test article.

\subsubsection{Radiological and Geophysical Drive-Over Surveys}

Radiological and geophysical drive-over surveys were performed on Brownes Lake, Pedro Lake, NEDS Lake, and Antelope Lake. All areas received nearly 100 percent survey coverage (see survey path coverage figure in attached imagery disk, Appendix N). The data were used to help identify locations where Davis Gun penetrators (or resulting debris) may be present on the surface and in the shallow subsurface, and to assist in the location of the CAS TA-52-005-TAAL DU rings on Antelope Lake. The surveys were conducted using the same methodology and instrumentation as described in Section 2.1.2.1.

Radiological and geophysical surveys were conducted from November through December 2003, and May through June 2004. During the surveys in 2003 and 2004, survey areas were visually inspected for explosive hazards by a UXO technician prior to survey personnel and equipment entering the area. The radiological and geophysical surveys were suspended in June 2004, when UXO was found on Antelope Lake. The remaining radiological and geophysical surveys for approximately 500 acres of CAU 484, on the north half of Antelope Lake, was completed from June to September 2006 using an unmanned, pre-programmed, autonomously controlled survey vehicle.

Prior to resuming radiological and geophysical surveys, geophysical "prove-outs” were conducted on Brownes Lake and at a tuff location to identify the expected geophysical signatures (instrument responses) to the various targets for the two geologic environments of interest. Prove-outs were conducted at a tuff location that had no history of Davis Gun activity, to the north of Antelope Tuff Target 1, to determine the expected signatures of different targets at tuff locations. Prove-outs were also conducted on Brownes Lake to identify the corresponding geophysical signatures on a playa (i.e., dry lake bed). Brownes Lake was selected because it was found to be relatively free of geophysical anomalies during the earlier geophysical survey.

To conduct a prove-out, objects were buried to represent: (a) metallic objects similar to the DU rings of CAS TA-52-005-TAAL (JTA DU Sites), (b) Davis Gun projectile-shaped objects, and 


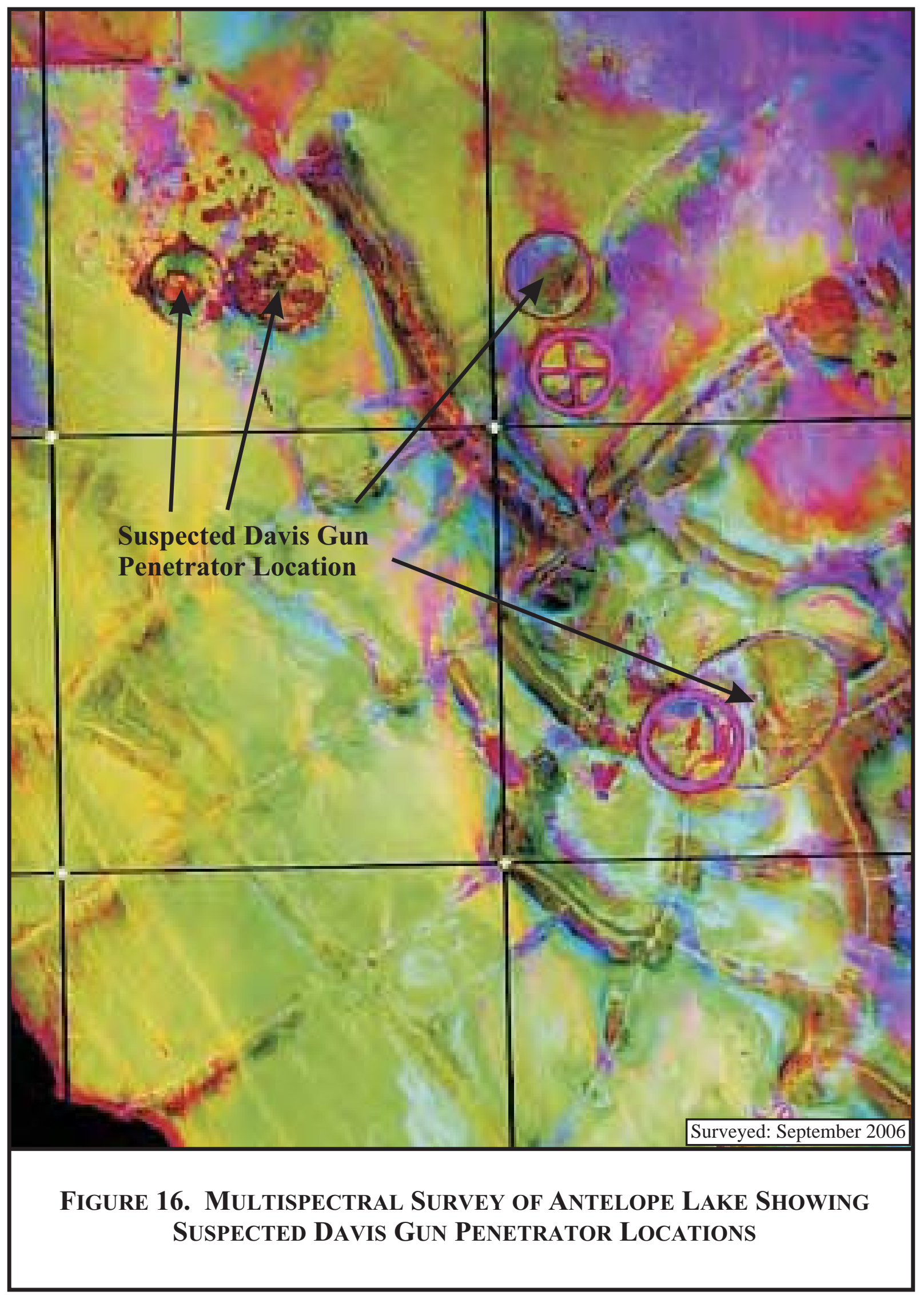




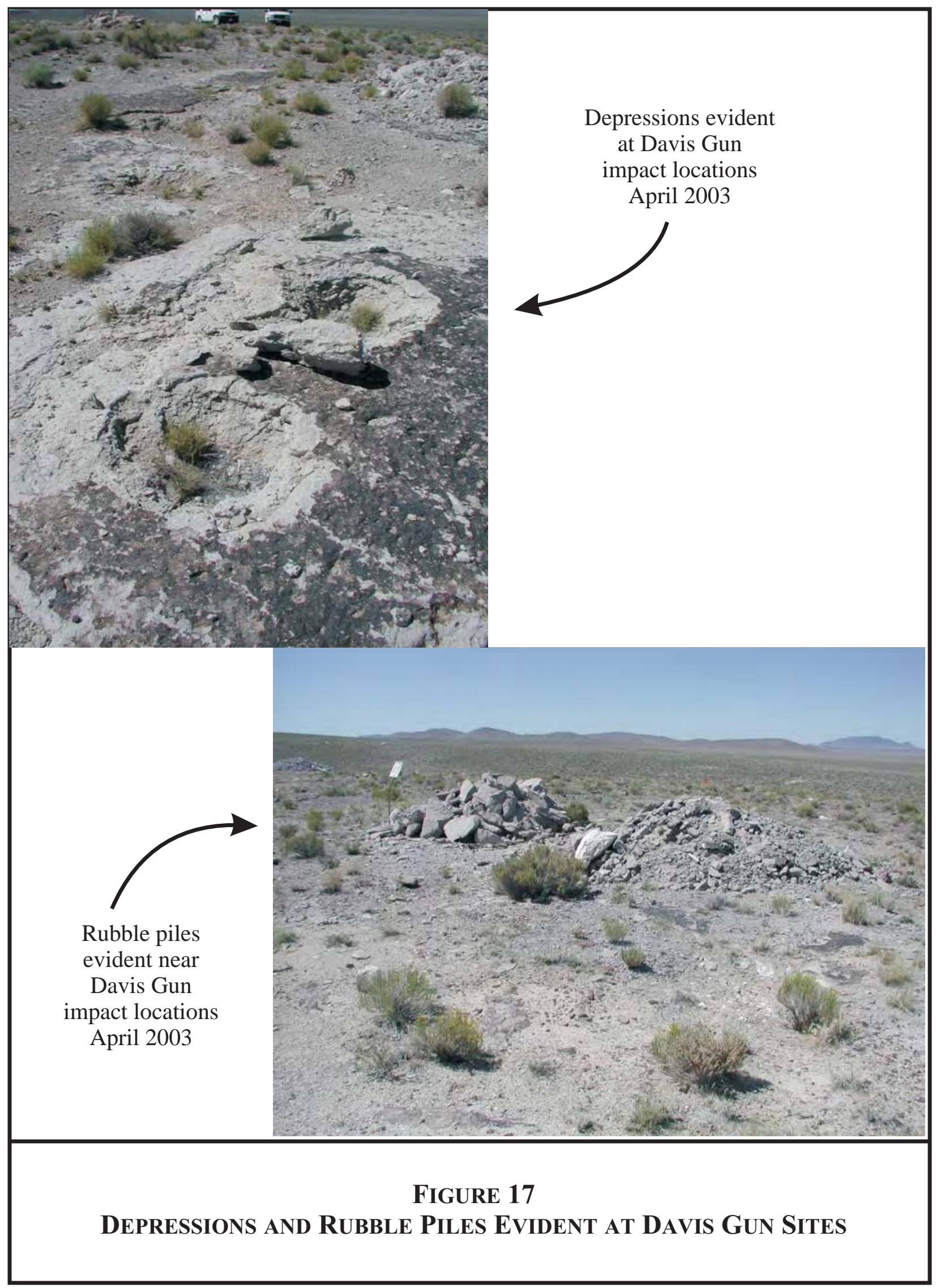


(c) various bomblets. (Bomblet data was also collected for CAU 408 at this time and will be presented in the CAU 408 CR.) The objects were buried at different depths and orientations.

For the geophysical prove-out on Brownes Lake, two objects similar to the Davis Gun projectiles were buried at several different depths and orientation. For the geophysical prove-out at the tuff site, two objects similar to Davis Gun projectiles were buried at depths of 6.5 and $9 \mathrm{ft}$ bgs. A geophysical survey was then conducted over the prove-out areas. Results of the geophysical surveys over the prove-out areas were used to identify expected geophysical signatures for CAU 484-related objects.

After the prove-outs were completed, geophysical and radiological surveys of the remainder of Antelope Lake and of the Davis Gun tuff sites continued. For safety reasons, because of the potential for live UXO, the portion of Antelope Lake that had not previously been surveyed was completed using an autonomously operated robotic tractor (also known as a "towbot") that towed the geophysical and radiological arrays (Figure 18). Upon completion of these surveys, all of Antelope Lake was covered by radiological and geophysical surveys; however, only the northern half of Antelope Lake is included in CAU 484 (see the SAFER Plan [NNSA/NSO, 2004b] for details on the boundaries of the CAU 484 survey area). The southern half of the lake was surveyed for CAU 408, results of which will be presented in the CAU 408 CR (not yet published).

The tuff areas consisted of uneven terrain over which the robotic tractor could not be used, with a greater likelihood of damaging the fragile equipment than of obtaining useable data. Instead, an all-terrain vehicle was used to pull a smaller geophysical array over the tuff areas. Instead of the EM61-MKII system (which was used on the playas), a multi-sensor towed array detection system (MTADS), similar to the EM61-MKII but with a narrower detection width, was used. As with the MKII system, the MTADS is a high-sensitivity time-domain electromagnetic instrument capable of detecting both ferrous (steel) and non-ferrous (aluminum, copper, brass, etc.) metals. The MTADS has a higher transmitter moment and overlapping coils, which makes it more effective at mapping large metal items buried at depth (e.g., Davis Gun penetrators). Radiological surveys were performed on select target locations using manually-operated handheld instruments at the tuff areas.

Appendix $\mathrm{N}$ contains a full set of images showing the geophysical survey results and target locations.

\subsection{CAS RG-52-007-TAML, Davis Gun Penetrator Test}

Data (geophysical, multispectral, and radiological) collected through 2005 (before the geophysical prove-outs were conducted) were reviewed to identify potential Davis Gun penetrator locations on the playas, results of which are presented in a position paper provided in Appendix F. As a result of this review, fourteen locations on Pedro Lake and twenty locations on Antelope Lake were selected for further investigation. No locations on Brownes Lake were selected for further investigation because available data showed no clear indication of a Davis Gun site with associated DU or buried projectiles.

A site walkdown with an EM61 push cart system was performed to confirm the location of the selected locations on Antelope and Pedro Lakes and to confirm that the corresponding geophysical anomaly resulted from a large, deep source, rather than from smaller, shallow 


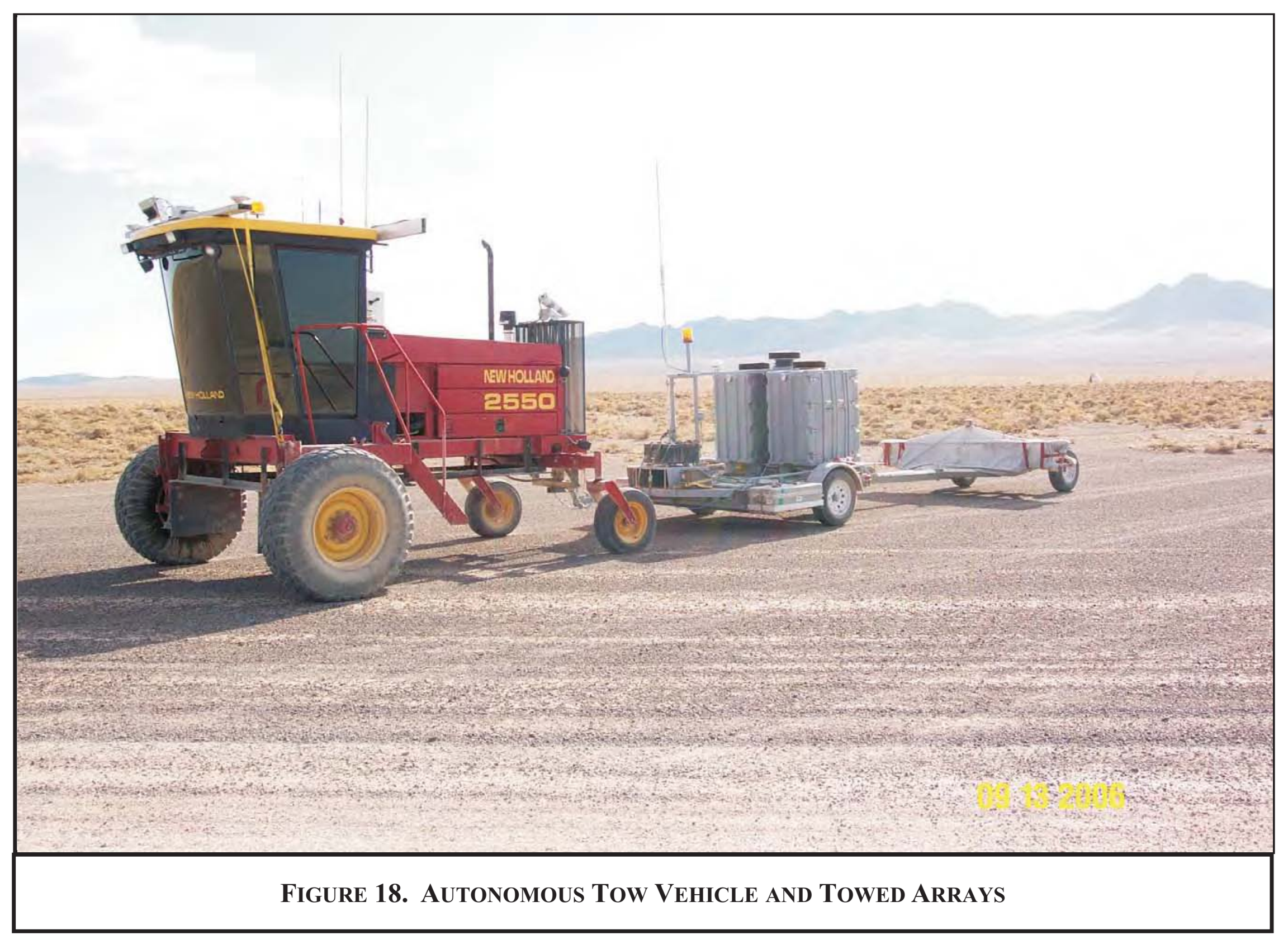


debris. Locations were selected for excavation based on the following criteria. On Antelope Lake, excavation locations were predominantly selected based on the response of the geophysical equipment. Where the response of the EM61 indicated smaller, shallow subsurface metal, those locations were removed from the list of potential excavation sites.

On Pedro Lake, circular features were evident. Excavation locations were selected based on the presence of circular features where a significant, deep geophysical anomaly was also present. Additional locations on Pedro Lake were selected (1) within a bermed area, where a large, deep anomaly was identified through geophysical data processing, and (2) at another large, deep geophysical anomaly identified through the multispectral survey where soil was visibly mounded. Results of the exploratory excavations can be found in Section 2.1.3.3.1.

Results of the geophysical prove-out on Brownes Lake indicated that projectiles buried deeper than $10 \mathrm{ft}$ bgs would not be detectable with the existing technology. Deep (yet less than $10 \mathrm{ft}$ bgs) projectile-like objects provided a geophysical response greater than 10 millivolts (mV) and greater than 5 meters in diameter. The intensity of the reading (in $\mathrm{mV}$ ) was not a good indication of Davis Gun-type projectiles because the electromagnetic intensity was found to be similar for shallow metallic objects from other sources, which were numerous at the sites. Because the deeper buried object (from the prove-out) produced a larger diameter geophysical response, the physical size of the signature (a minimum of 5 meters) was used as the primary indicator that a geophysical response greater than $10 \mathrm{mV}$ represented the presence of a Davis Gun projectile.

Results from the geophysical surveys were compared against the prove-out criteria to identify potential Davis Gun projectile locations. For the playas, the geophysical signature, compared with the prove-out, met the criteria for a potential Davis Gun projectile at hundreds of locations (see Appendices K, L, and N):

- Pedro Lake: 134 targets

- Antelope Lake: 732 targets

- Brownes Lake: 15 targets

- Main Lake: under water and was not surveyed.

All of these targets were not investigated after the prove-out results were obtained. Excavation activities at Pedro and Antelope Lakes had begun before the geophysical survey results were compared against the geophysical prove-outs. Those excavation locations were selected based on results of the geophysical surveys that had been completed in 2003 and 2004 (see results in Section 2.1.3.3.1) and other data that were available.

After the comparison with the prove-outs, the only area that was critically evaluated for additional investigation was the southern 500 acres on the north half of Antelope Lake. This is where the geophysical and radiological surveys had not previously been completed, and is the only area where additional drive-over geophysical and radiological data were collected after the discontinuation of these surveys in 2004. This area had not previously been evaluated because the data for this area were not previously available. For this area, geophysical results were compared against aerial photographs and multispectral surveys and field-checked using the handheld EM61 to verify that the anomaly represented a deep target (rather than shallow surface 
debris). Results were used to select two additional excavation locations most representative of a potential penetrator location.

The other hundreds of locations were not investigated further because excavation activities at the most likely locations for a Davis Gun projectile showed that the anomalies were representative of practice ordnance and other scrap metal, and not Davis Gun projectiles. This confirmed the CSM expectation that Davis Gun projectiles were recovered at the time of the test. See Section 2.1.3.3.1 for more details on the Davis Gun investigation of the playas.

For tuff locations, the geophysical prove-out at the tuff site showed that the deeper object provided a response greater than $15 \mathrm{mV}$ and approximately 5 meters in diameter. Again, the intensity of the reading (in $\mathrm{mV}$ ) did not provide a good indication of whether the resulting geophysical signature was for Davis Gun projectiles or of surface or shallow buried metallic objects from other sources which, again, were numerous in the areas. Figure 19 shows an inert Davis Gun penetrator that was found on Mt. Helen.

For the tuff sites, there was some additional evaluation of the locations where the geophysical signature met the criteria for a potential Davis Gun projectile. A site walkdown with an EM61 push cart system was performed, as well as a visual inspection of each penetrator location that was originally identified. On the basis of this follow-up investigation, many of the original locations were disqualified from being Davis Gun projectile locations because of the presence of surface metal (e.g., stakes, piles of nails, metal cans) that met the Davis Gun geophysical criteria, or the absence of any ground disturbance indicating that a penetrator may have impacted the site. The following provides the initial number of potential Davis Gun penetrator locations, along with the reduced number after ground-truthing at each tuff site:

- Antelope Tuff Target 1: 74 targets, reduced to 8 targets

- Antelope Tuff Target 2: 98 targets, reduced to 21 targets

- Sidewinder Tuff: 33 targets, reduced to 17 targets

- Myers Ridge: 76 targets, reduced to 12 targets

- Mt. Helen: 8 targets, reduced to 5 targets

Geophysical survey results indicating potential Davis Gun projectile locations (as well as the other types of targets) are provided on the imagery in Appendix N. Appendices K and L contain this information for all of the sites where geophysical surveys were conducted and include tables that identify the geographic location, the type of target, and estimated depth of burial. Davis Gun projectiles were the only targets in the tuff areas. Additional non-penetrator geophysical anomalies were identified at the Davis Gun tuff sites locations. These anomalies were not considered to be related to CAU 484 and were not investigated.

The radiological survey results were unsuccessful in identifying locations of potentially intact, buried penetrators at any of the Davis Gun sites. Buried penetrators containing DU cannot be detected at the surface with existing technology. Additionally, based on process knowledge, any penetrators that are still present on the playas should not contain DU and are likely much deeper than $10 \mathrm{ft}$ bgs.

Because a penetrator impacting the lithified tuff might fragment or leave small pieces at the entry point on impact, potential impact locations (depressions and holes) and/or associated rubble piles were screened using handheld equipment to identify elevated radioactivity. It was expected that, 


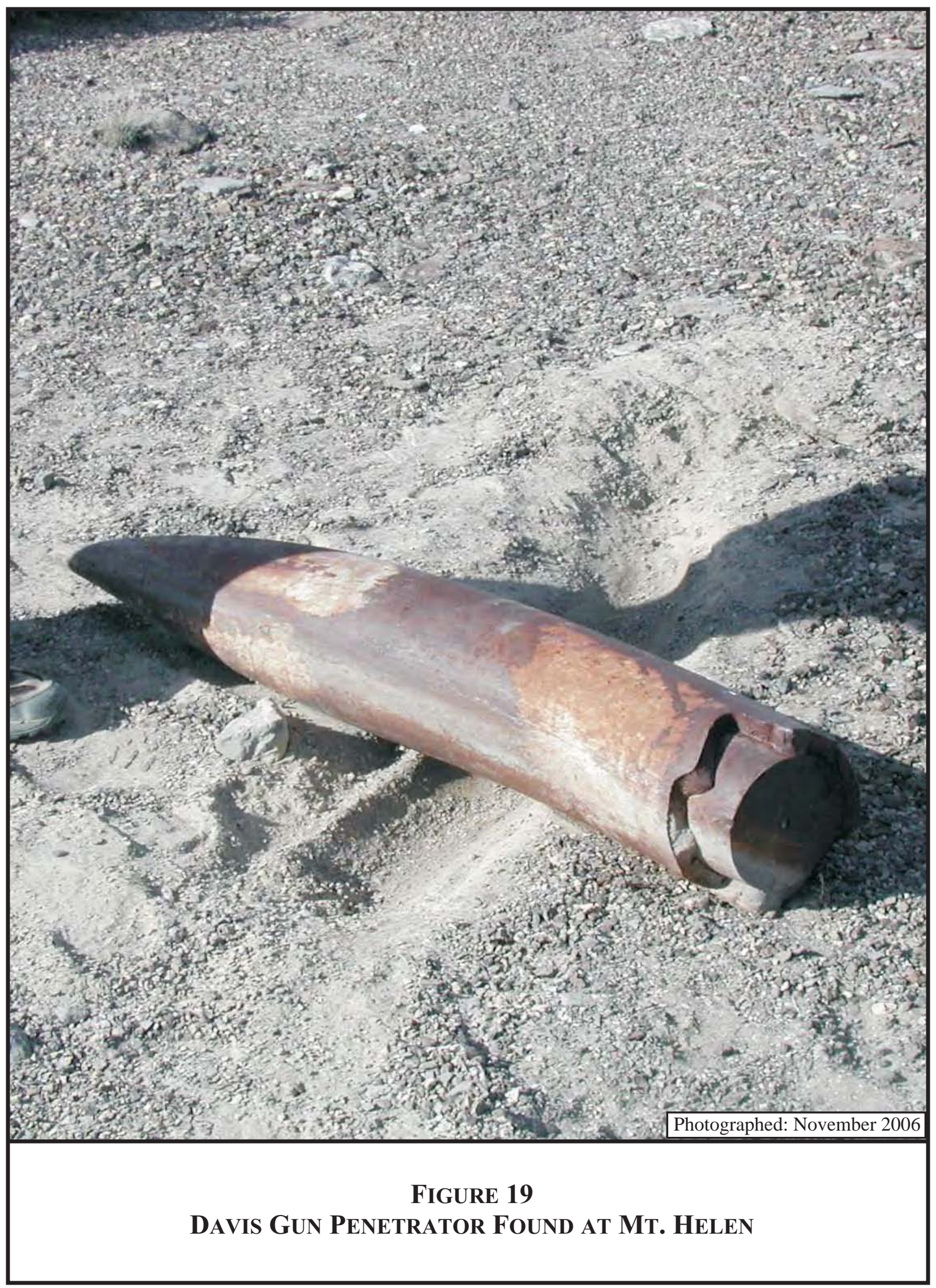


if DU was associated with penetrators at the tuff sites, there would be elevated radioactivity at or near the impact site and/or associated rubble piles where penetrators were recovered. Handheld instruments were used to screen the depression, holes (if present), and surrounding rubble for DU that may have fragmented off of the penetrator on impact or during recovery. Sixty-three depressions were screened in this manner, and radioactivity above background concentrations was not detected at any of these locations.

Radiological surveys completed over the north half of Antelope Lake identified numerous locations where DU was present at or near the ground surface. All of the locations were within or near known, historical Davis Gun penetrator test areas; however, it could not be confirmed if the residual DU was related to Davis Gun penetrator testing activities. Radiological survey results were used in conjunction with geophysical data to identify locations on the north half of Antelope Lake (the southern half will be addressed with CAU 408) where DU fragments or contaminated soil were present, for further investigation and cleanup.

The radiological results provided information regarding the location of DU fragments and contaminated soil on the ground surface or in the shallow subsurface, while the geophysical survey results were considered more valuable for locating projectiles, if present.

\subsection{CAS TA-52-005-TAAL, JTA DU Sites}

Similar to the CASs in the Surface Detonation Debris Sites CSM, geophysical results did not provide a good indication of the location of the JTA DU rings because of the relatively small size of the rings and the large number of metallic objects on Antelope Lake. Therefore, all of the DU rings were found based on the radiological results. Excavation areas for DU cleanup are identified in Figure 8. Two of the DU rings were found on the north half of Antelope Lake, at radiological target locations NA-2 and NA-3. The third DU ring was found on the south end of Antelope Lake, within the footprint of CAU 408 (Bomblet Target Area), at radiological target location SA-10. The presence of the DU ring on south Antelope Lake required the CSM to be modified to reflect that the DU ring had been projected further than originally anticipated by the test, beyond the original boundary of CAU 484. The approximate location of where all three rings were found is shown on Figure 14.

\subsubsection{Exploratory Excavation and DU Removal}

Results from the geophysical and radiological surveys were compared against aerial photography and multispectral surveys to better define the targets of interest (i.e., Davis Gun projectiles and DU rings).

\subsection{CAS RG-52-007-TAML, Davis Gun Penetrator Test}

Based on an evaluation of the data collected through 2005, Pedro and Antelope Lakes were excavated at locations where Davis Gun penetrators were determined most likely to be present. Five locations were excavated on Pedro Lake (Figure 20) and six locations were excavated on Antelope Lake (all but the southernmost two in Figure 21). At the five locations on Pedro Lake, three practice bombs (locations IM13, PD2, and PD3) and scrap metal (locations IM7 and PL_253) were uncovered. On Antelope Lake, numerous items were uncovered and consisted of 


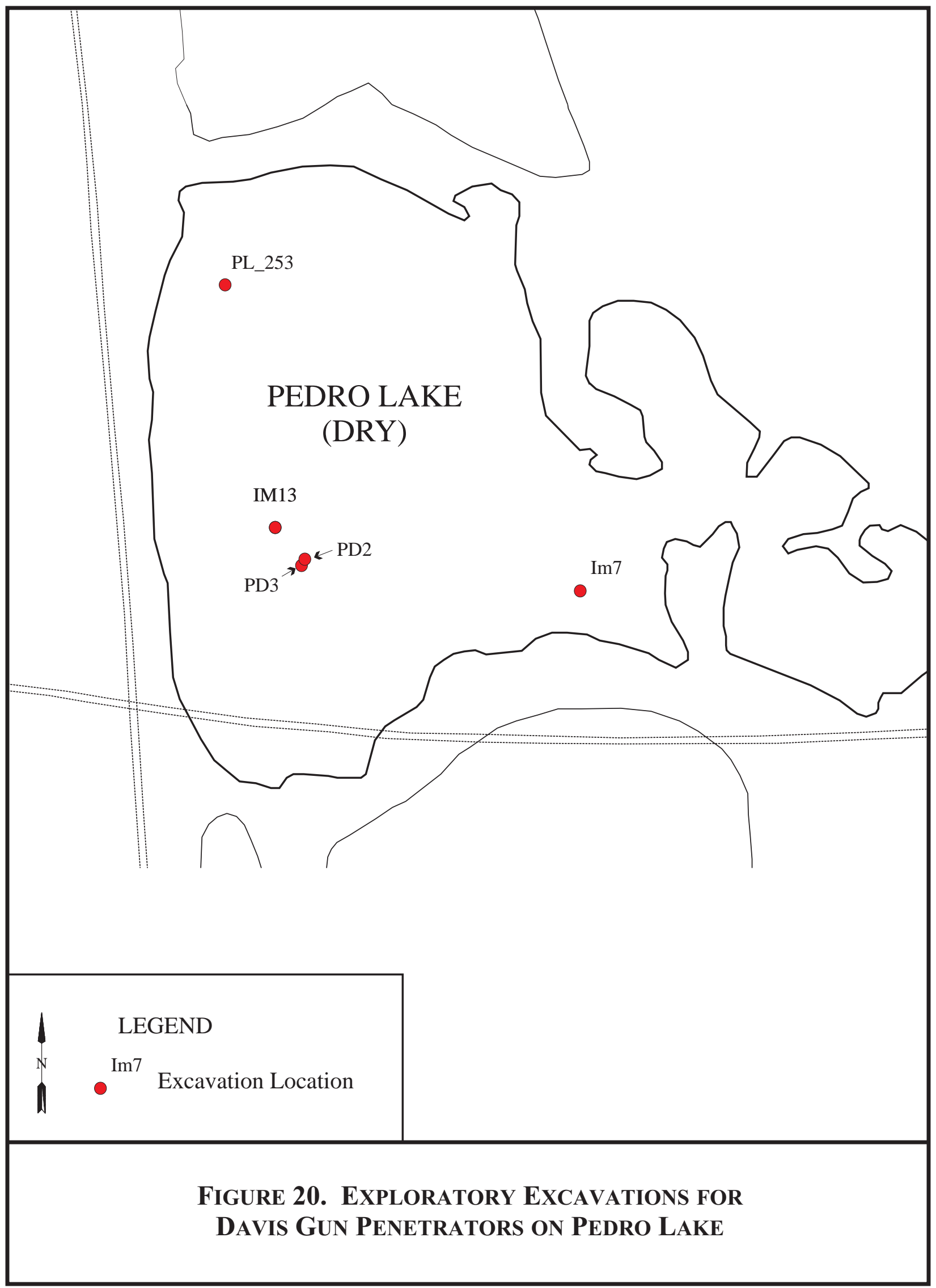




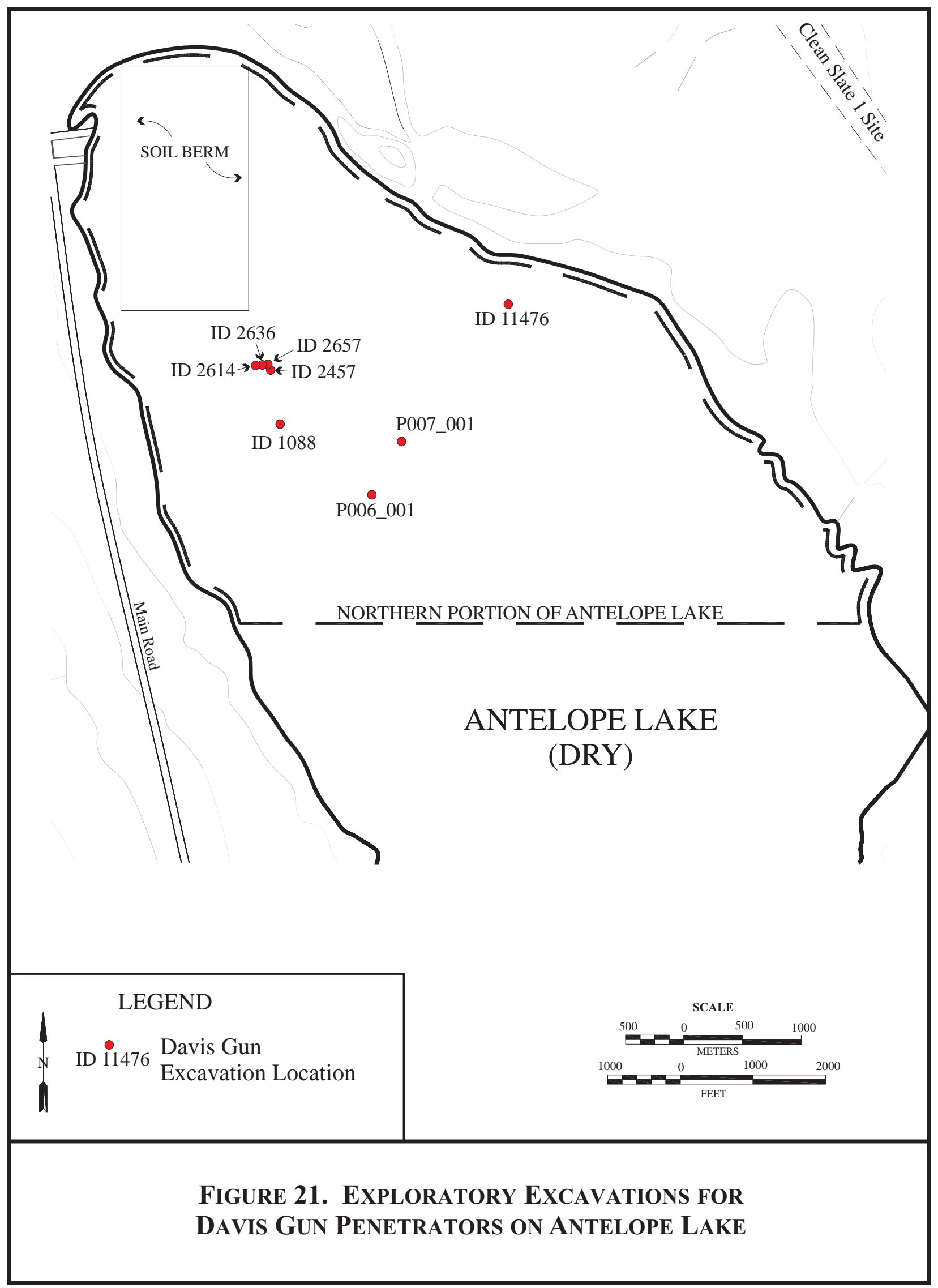


non-Davis Gun-related practice ordnance, metal plate, buried concrete slabs, and scrap metal. Excavation activities were temporarily halted in October 2006, when two objects appearing to be UXO were uncovered, and resumed in November 2006. No Davis Gun penetrators were found at any of the excavation locations. A report containing the results of the UXO removal activities is provided in Appendix $\mathrm{H}$.

Excavation was not completed at all of the locations identified as potential locations for Davis Gun penetrators because no Davis Gun penetrators were found at those locations deemed most likely to contain a penetrator. Further excavation was temporarily put on hold pending a meeting of the primary CAU 484 decision makers. The preliminary geophysical results were presented to NDEP at a hold point/status meeting in November 2006. A technical position paper (Appendix E) presented at that meeting provided the following recommendations and justifications for remaining Davis Gun activities:

- For the playas, two more excavations were proposed in the southernmost portion of north Antelope Lake (P006_001 and P007_001 in Figure 21). These were in the area that was covered in the 2006 geophysical survey. They were considered to be the most likely penetrator locations based on geophysical results and were both located inside circular berms. Discontinuation of excavation for Davis Gun penetrators on the playas was proposed if no penetrators were found at these locations. This was proposed because of the potential to uncover UXO and other non-Davis Gun-related debris, which is all that had been found to date.

- For the tuff sites, two locations where data indicate Davis Gun projectiles would most likely be present on Mt. Helen would be excavated to retrieve Davis Gun penetrators, if present. The two anomalies that yielded the best geophysical signature that corresponded to the geophysical prove-out were selected at Mt. Helen. Mt. Helen is the oldest of the tuff locations and was used during the early 1970s to early 1980s. Environmental issues were not a paramount concern during this time frame; therefore, this location had the best potential for containing residual Davis Gun penetrator debris and/or DU. Of the five Davis Gun targets at Mt. Helen, PH01_001 and PH01_002 had the highest mV readings, the largest diameter geophysical expressions, and were located in very close proximity to visible surface depressions. As such, these two locations were targeted for excavation. The paper proposed discontinuation of the investigation of penetrator anomalies at other tuff locations if the investigation at Mt. Helen did not yield Davis Gun penetrator-related debris or DU.

The technical position paper was approved and the plan was implemented. In November 2006, the remaining two locations on Antelope Lake were excavated (southernmost on Figure 21). In June 2007, Mt. Helen locations PH01_001 and PH01_002 (Figure 22) were excavated to investigate the source of the geophysical anomalies and to identify whether Davis Gun projectiles were present. Excavation at all locations revealed only inert, metallic debris. No Davis Gun penetrators were unearthed.

In addition to the exploratory excavations, six locations on Antelope Lake were excavated to remove DU as a part of the Davis Gun closure activities (Figure 8, locations NA-13, SA-4, SA-5-9, CA-1, CA-2, and SA-12-15). Two areas (CA-1 and CA-2), identified to contain large fragments of DU on the ground surface prior to conducting the radiological and geophysical surveys, were fenced and posted as contamination areas and no subsequent investigation was 


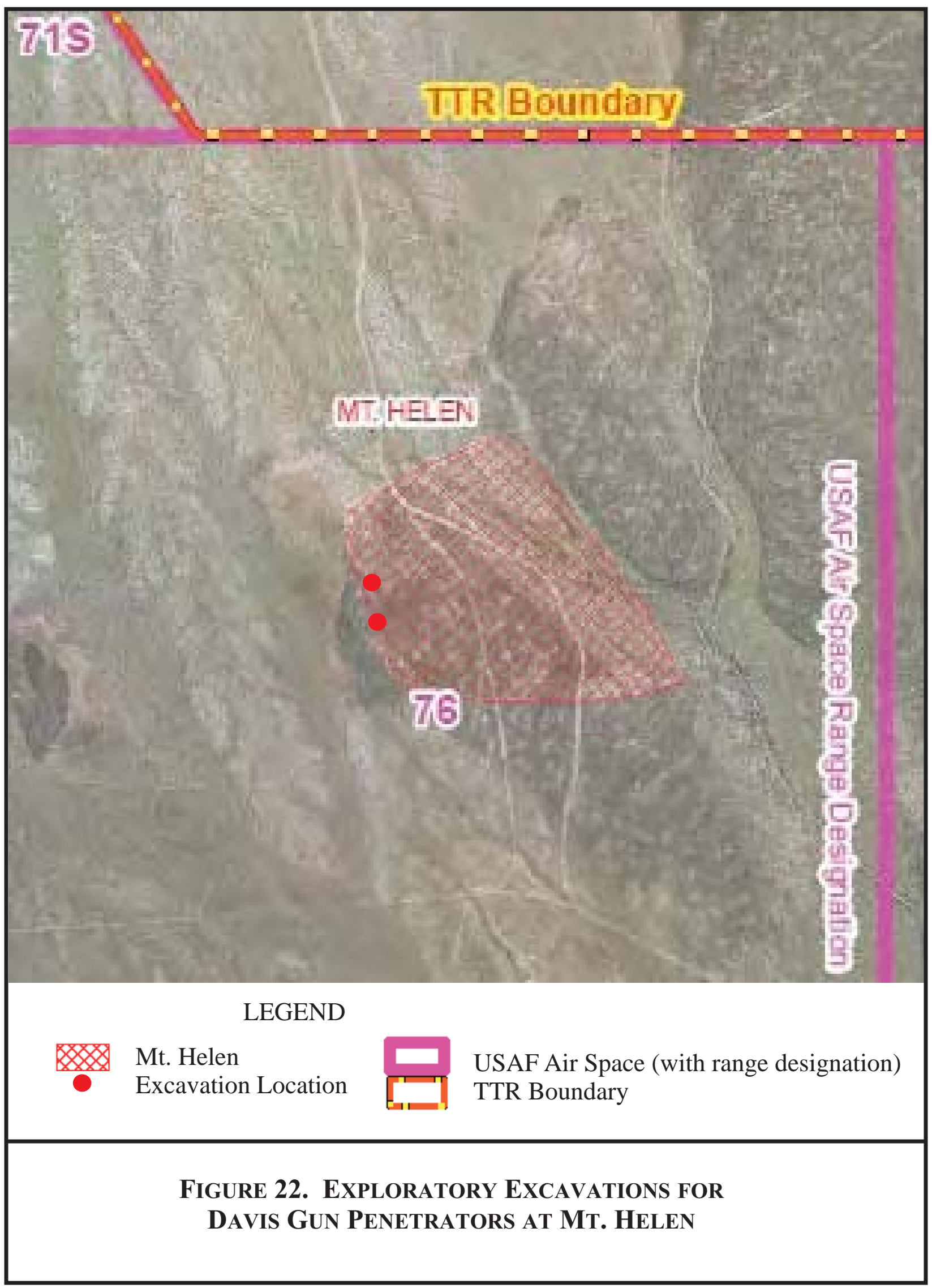


conducted over these areas. Instead, they were addressed at the same time as the other DU-contaminated areas on Antelope Lake. SA-12-15 is actually located in CAU 408; however, because the nature of the contamination and the field effort required to effect closure are the same at this location as for CAU 484, DU was excavated and the site was closed in place. This was a more efficient process, as the equipment was already available at the site, and the exposure hazard was removed earlier than would have occurred with CAU 408 field activities. DU was excavated and removed, and clean closure was verified using field-screening methods. Table 11 identifies locations where DU debris and contaminated soil were excavated, the approximate volume of each excavation, and whether the location was clean closed or if it has been closed in place.

Clean closure was verified using the Kiwi equipment. After excavation, the Kiwi vehicle was stopped over the excavation and the resulting radiological results were obtained and processed to determine whether clean closure had been attained. At location CA-1, DU-contaminated soil was excavated from an area of approximately $15 \mathrm{ft} \times 20 \mathrm{ft}$, to a depth of $6 \mathrm{ft}$ bgs. It was impossible to drive the Kiwi vehicle over this area; therefore, soil was scraped from the excavation surfaces, placed on plastic, and screened using the Kiwi vehicle on the ground surface level (see photo in Appendix D). The methodology to verify clean closure is described in detail in Appendix M.

All but four locations on Antelope Lake were clean closed. Locations CA-1, SA-4, SA-5-9, and SA-12-15 have been closed in place with use restrictions. At three of the four locations, the volume of DU-contaminated soil greatly exceeds that identified in the CSM, which assumes hot spots with small volumes of contamination. At the fourth location (SA-4), UXO and other debris not included in CAU 484 is present. NDEP was contacted and the hold point was resolved by implementing closure in place of these four sites. Surface contamination was excavated to depths of $0.5 \mathrm{ft}$ bgs or deeper to remove related exposure hazards. The excavations were then backfilled with clean, native fill material and slightly mounded so that water will run off these areas. The position paper describing the proposed actions, which have since been completed, is presented in Appendix G. Appendix M contains details on the cleanup verification methodology and results. A use restriction has been implemented for each of the four sites and is provided in Appendix O. Post-closure requirements are described in Section 5.0.

\subsection{CAS TA-52-005-TAAL, JTA DU Sites}

The JTA DU rings were recovered during the DU cleanup of Antelope Lake, details of which are covered under the MPDT CAS. Two of the rings (NA-2 and SA-10 in Figure 14) were on the ground surface. These rings were retrieved and containerized with the other DU waste from Antelope Lake. The third ring was found approximately 6 inches bgs at NA-3. It also was excavated, and the ring and surrounding impacted soil was containerized with the other DU waste from Antelope Lake. Table 11 identifies locations where DU rings and associated contaminated soil was excavated and the approximate volume of each excavation.

CAS TA-52-005-TAAL was clean closed upon removal of the three DU rings and associated, impacted soil. Cleanup verification was confirmed using photographic documentation and the Kiwi equipment. 

TABLE 11. Summary OF DU-EXCAVATED SOIL FROM SURFACE-SHALLOW
SUBSURFACE DEBRIS SiTES

\begin{tabular}{|c|c|c|}
\hline EXCAVATION LOCATION & $\begin{array}{c}\text { VOLUME } \\
\text { EXCAVATED }\end{array}$ & $\begin{array}{c}\text { CLEAN CLOSURE } \\
\text { ATTAINED? }\end{array}$ \\
\hline \hline NA-2 & $<1 \mathrm{yd}^{3}$ & Yes \\
\hline NA-3 & $<1 \mathrm{yd}^{3}$ & Yes \\
\hline NA-13 & $14 \mathrm{yd}^{3}$ & Yes \\
\hline CA-1 & $98 \mathrm{yd}^{3}$ & No \\
\hline CA-2 & $7 \mathrm{yd}^{3}$ & Yes \\
\hline SA-4 & $14 \mathrm{yd}^{3}$ & No \\
\hline SA-5-9 & $98 \mathrm{yd}^{3}$ & Yes \\
\hline SA-10 (JTA DU ring on south end of & $1 \mathrm{ft}^{3}$ & No \\
\hline Antelope Lake) & 36 & SA-12-15 ${ }^{*}$ \\
\hline
\end{tabular}

* SA-12-15 is on the south end of Antelope Lake and is not within the boundary of CAU 484 (it is within the boundary of CAU 408). Because the DU at this location was similar in nature to the other Antelope Lake DU contamination, the DU at this location was excavated, and remaining subsurface DU has been use-restricted with the three use-restricted areas of CAS RG-52-007-TAML, Davis Gun Penetrator Test. 


\subsubsection{Closure Activities - Non-Impacted Surface Debris}

This CSM consisted of one CAS, TA-54-001-TANL, Containment Tank and Steel Structure. This CAS consisted of three structures of non-impacted waste/debris on the north end of NEDS Lake (Figure 23). All structures associated with this CAS were surveyed to verify that radiological contamination was less than the free-release limit established in the NV/YMP Radiological Control Manual (NNSA/NSO, 2004a). The structures were size-reduced and transported to the Sandia Salvage yard in Area 3 at the TTR. Appendix D provides photographs of the site before and after structure removal.

\subsection{DEVIATIONS FROM THE SAFER PlAN AS APPROVED}

Several deviations from the CAU 484 SAFER Plan (NNSA/NSO, 2004b) were necessary, as described below.

- Surface Detonation Debris Sites:

- $\quad$ The CSM assumed that DU would exist as discrete fragments on the ground surface; however, DU was found on the ground surface and in the soil to a depth of approximately $10 \mathrm{ft}$ bgs. Also, DU was found as metal fragments and as oxide, and the DU oxide had dispersed into the surrounding soil. The initial approach to verifying clean closure using field survey methods was therefore changed to collecting cleanup verification samples where more than $1 \mathrm{ft}^{3}$ of soil was removed (see sampling technical paper in Appendix E). This approach was later modified to consist of verification using the Kiwi sodium iodide detectors (a field survey method) for excavations accessible to the Kiwi survey vehicle, and collecting cleanup verification soil samples for excavations that were not accessible to the Kiwi vehicle.

- $\quad$ CAS RG-52-007-TAML, Davis Gun Penetrator Test:

- Geophysical and radiological surveys were not conducted on Main Lake because it was covered with water. A decision was made to postpone investigation of Main Lake until such time that investigation results indicated that if Davis Gun penetrators were present at other playa locations, an investigation strategy would be developed for Main Lake. If no penetrators were located on other playas, then Main Lake would also be considered closed.

- All Davis Gun penetrator geophysical targets were not investigated because no Davis Gun-related debris or DU was encountered in the 13 excavations that were completed on Antelope and Pedro Lakes, and because six of the geophysical anomalies that were investigated turned out to be UXO. The geophysical surveys, aerial photography, and multispectral surveys indicated that these 13 locations were those where Davis Gun penetrators were most likely to be located. In absence of finding penetrators at these locations, the other anomalies most likely contain bombs, UXO, or other scrap metal, as was found at the 13 locations. As part of the conditions stated in the Davis Gun position paper (Appendix E), a confidence level was calculated using the targets identified during the investigation and the findings of the excavations on Antelope and Pedro Lakes. 


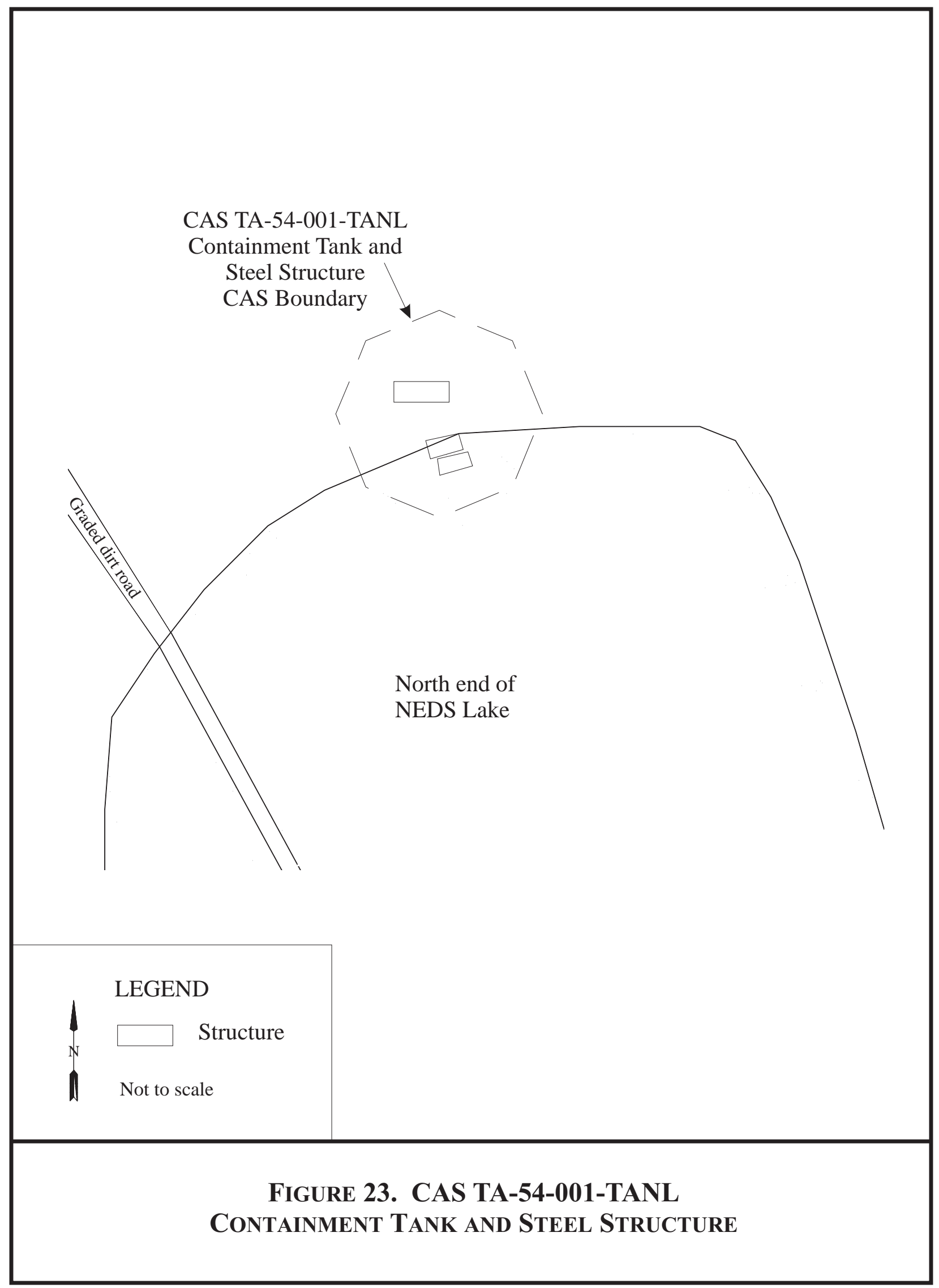


The calculations and support documentation are included in the statistical analysis report located in Appendix J.

- Radiological surveys at the volcanic tuff locations were completed using handheld instrumentation instead of the Kiwi. Handheld instrumentation can identify the presence of elevated radioactivity; however, it cannot specifically identify the presence of DU as can the Kiwi sodium iodide detectors. The Kiwi was not used due to the rough terrain at the tuff sites, which prevented acquisition of useable data from the Kiwi equipment. The survey data of the tuff sites that was collected during the investigation was evaluated by radiological control personnel and determined to be consistent with background conditions of the area.

- $\quad$ NEDS and Antelope Lakes: DU was encountered outside of expected/known areas of dispersion.

- $\quad$ On NEDS Lake, DU dispersion extended outside of the preliminary anticipated area identified in the SAFER Plan for the NEDS Detonation Area and the DUS. The only known tests on NEDS Lake are the NEDS and Colimbo (DUS) Tests, and the DU debris that was found was consistent with dispersal from these detonations; therefore, the DU cleaned up on NEDS Lake is not outside the bounds of CAU 484.

- $\quad$ The DU encountered on Antelope Lake can be grouped into two categories: (1) fragments with limited contaminated soil on the playa surface, and (2) larger, oxidized debris disseminated on the playa surface and in the shallow subsurface soil. The DU fragments on the ground surface are consistent with a surface detonation, which could be related to dispersion from the MPDT, and possible subsequent movement of fragments on the playa surface due to weather/physical forces. Documentation on this test indicates that debris was dispersed approximately 1,500 ft from the point of detonation. The source of the larger oxidized areas of DU on the playa surface and shallow subsurface is unknown. Most of the areas with DU are in or near areas where historical Davis Gun penetrator tests were known to have been conducted, even though the relation of the DU to these tests is not known. In a May 7, 2007, meeting, NNSA/NSO decided that areas of DU on Antelope Lake that are within or near areas of Davis Gun testing would be cleaned up within the scope of CAU 484.

- Antelope Lake: DU contamination was closed in place at four locations because it was technically not feasible to continue excavation of the buried DU. This decision was made in a July 30, 2007, hold point meeting with NDEP. The following actions were taken based on the rationale presented at the meeting, as described in the position paper presented in Appendix G, and as summarized below.

- At three locations (CA-1, SA-5-9, and SA-12-15), DU contamination exceeds the expected CSM volume limitations for small-volume hot spots. From 6 to $95 \mathrm{yd}^{3}$ have been excavated at each location, and DU contamination is still present. Although the vertical extent of the contamination is unknown, it is unlikely that contamination will impact the ground water based on the relative immobility of DU in soil and a depth to groundwater of approximately $300 \mathrm{ft}$ bgs, based on depth to water at water well on the TTR. The lateral extent of surface DU has been successfully excavated to depths of 0.5 to $3 \mathrm{ft}$ bgs to remove the potential for exposure to DU. 
- $\quad$ At SA-4, UXO and debris unrelated to CAU 484 were exposed while excavating DU contamination. The excavation was at a depth of $2 \mathrm{ft}$ bgs when excavation was suspended for the hold point. To provide for worker protection as well as to remove the potential for exposure to DU, the lateral extent of remaining surface DU contamination was excavated to a depth of $0.5 \mathrm{ft}$ bgs.

\subsection{Corrective ACtion Schedule as COMPLETED}

Closure activities for CAU 484 started in November 2003 and were completed in August 2007. Details of the schedule are provided in Table 12.

\subsection{Site Plan/Survey Plat}

CAS RG-52-007-TAML, Davis Gun Penetrator Test, was closed in place with administrative controls (i.e., use restrictions were implemented). Figures showing the locations of the surveyed points delineating the use-restricted areas are provided in Appendix $\mathrm{O}$ of this report. 
Table 12. CAU 484 Closure Activities Schedule

\begin{tabular}{|c|c|c|c|c|}
\hline ACTIVITY & CAS & LOCATION & START DATE & END DATE \\
\hline Aerial Photography and Multispectral Surveys & RG-52-007-TAML (Davis Gun) & All Davis Gun sites & \multicolumn{2}{|c|}{$\begin{array}{c}\text { September 21, } 2003 \\
\text { April 29, } 2006 \\
\text { July 15, } 2006 \\
\end{array}$} \\
\hline \multirow{4}{*}{$\begin{array}{l}\text { Radiological and Geophysical Drive-Over } \\
\text { Surveys }\end{array}$} & RG-52-007-TAML (Davis Gun) & Brownes Lake & \multirow{4}{*}{$\begin{array}{l}\text { November } 2003 \\
\text { and } \\
\text { May } 2004\end{array}$} & \multirow{4}{*}{$\begin{array}{c}\text { December } 2003 \\
\text { and } \\
\text { June } 2004\end{array}$} \\
\hline & RG-52-007-TAML (Davis Gun) & Pedro Lake & & \\
\hline & $\begin{array}{l}\text { RG-52-007-TAML (Davis Gun) } \\
\text { TA-52-001-TANL (NEDS) } \\
\text { TA-52-006-TAPL (DUS) }\end{array}$ & NEDS Lake & & \\
\hline & $\begin{array}{l}\text { RG-52-007-TAML (Davis Gun) } \\
\text { TA-52-004-TAAL (MPDT) } \\
\text { TA-52-005-TAAL (JTA DU Sites) }\end{array}$ & $\begin{array}{l}\text { Antelope Lake } \\
\text { (north) }\end{array}$ & & \\
\hline \multirow{3}{*}{$\begin{array}{l}\text { Investigation Soil Sample Collection (using } \\
\text { Geoprobe) }\end{array}$} & $\begin{array}{l}\text { TA-52-001-TANL (NEDS) } \\
\text { TA-52-006-TAPL (DUS) }\end{array}$ & NEDS Lake & \multirow{3}{*}{ August 2006} & \multirow{3}{*}{ September 2006} \\
\hline & TA-52-004-TAAL (MPDT) & Antelope Lake & & \\
\hline & None - Background determination & Pedro Lake & & \\
\hline \multirow{2}{*}{ Geophysical Prove-Outs } & Playa & Browne Lake & \multirow{2}{*}{ May 2006} & \multirow{2}{*}{ July 2006} \\
\hline & Tuff & Tuff site & & \\
\hline \multirow{2}{*}{$\begin{array}{l}\text { Radiological and Geophysical Remote Drive- } \\
\text { Over Surveys }\end{array}$} & $\begin{array}{l}\text { RG-52-007-TAML (Davis Gun) } \\
\text { TA-52-005-TAAL (JTA DU Sites) }\end{array}$ & $\begin{array}{l}\text { Antelope Lake } \\
\text { (south) }\end{array}$ & \multirow[t]{2}{*}{ August 2006} & \multirow[t]{2}{*}{ August 2006} \\
\hline & RG-52-007-TAML (Davis Gun) & Davis Gun tuff sites & & \\
\hline \multirow{2}{*}{ Exploratory Excavation } & RG-52-007-TAML (Davis Gun) & Pedro Lake & \multirow{2}{*}{ October 2006} & \multirow{2}{*}{ November 2006} \\
\hline & RG-52-007-TAML (Davis Gun) & Antelope Lake & & \\
\hline
\end{tabular}


Table 12. CAU 484 Closure Activities Schedule (Continued)

\begin{tabular}{|c|c|c|c|c|}
\hline ACTIVITy & CAS & LOCATION & START DATE & END DATE \\
\hline \multirow{2}{*}{$\begin{array}{l}\text { UXO Removal (Exploratory Excavation } \\
\text { Locations) }\end{array}$} & RG-52-007-TAML (Davis Gun) & Pedro Lake & \multirow{2}{*}{ February 2007} & \multirow{2}{*}{ February 2007} \\
\hline & RG-52-007-TAML (Davis Gun) & Antelope Lake & & \\
\hline \multirow[b]{2}{*}{ Excavation/Removal of DU, Phase 1} & TA-52-001-TANL (NEDS) & NEDS Lake & \multirow[b]{2}{*}{ October 2006} & \multirow[b]{2}{*}{ November 2006} \\
\hline & $\begin{array}{l}\text { TA-52-004-TAAL (MPDT) } \\
\text { TA-52-005-TAAL (JTA DU Sites) }\end{array}$ & Antelope Lake & & \\
\hline \multirow{2}{*}{ Excavation/Removal of DU, Phase 2} & TA-52-001-TANL (NEDS) & NEDS Lake & \multirow{2}{*}{ April 2007} & \multirow{2}{*}{ August 2007} \\
\hline & TA-52-004-TAAL (MPDT) & Antelope Lake & & \\
\hline Exploratory Excavation & RG-52-007-TAML (Davis Gun) & Mt. Helen & June 2007 & June 2007 \\
\hline Structure removal and disposal & $\begin{array}{l}\text { TA-54-001-TANL (Containment } \\
\text { Tank and Steel Structure) }\end{array}$ & $\begin{array}{l}\text { NEDS Lake, north } \\
\text { end }\end{array}$ & June 2007 & June 2007 \\
\hline \multirow[b]{2}{*}{ Waste disposal } & $\begin{array}{l}\text { TA-52-001-TANL (NEDS) } \\
\text { TA-52-006-TAPL (DUS) }\end{array}$ & NEDS Lake waste & \multirow{2}{*}{\multicolumn{2}{|c|}{ September 2007 (in process) }} \\
\hline & $\begin{array}{l}\text { TA-52-004-TAAL (MPDT) } \\
\text { TA-52-005-TAAL (JTA DU Sites) } \\
\text { RG-52-007-TAML (Davis Gun) }\end{array}$ & $\begin{array}{l}\text { Antelope Lake } \\
\text { waste }\end{array}$ & & \\
\hline
\end{tabular}




\subsection{WASTE DISPOSITION}

This section describes the waste streams generated during closure activities and their final disposition.

\subsection{WASTE MinimizATion}

Waste minimization practices were applied where possible. Structures associated with CAS TA-54-001-TANL (Containment Tank and Steel Structure) were size-reduced and transported to the Sandia Salvage Yard in Area 3 at the TTR, from which they will either be re-used or recycled.

\subsection{WASTE MANAgEMENT}

All waste was managed according to applicable federal and state regulations, U.S. Department of Energy orders, and M\&OC procedures. Waste management areas (WMAs) were established throughout the project, as needed. All WMAs were identified with appropriate signs and boundaries to restrict unauthorized access. The WMAs were inspected on a monthly basis, or as otherwise required, to ensure that all containers were intact, not leaking, and not exceeding storage duration times. Applicable WMAs were posted as RMAs whenever radiological waste was stored in the area. Upon removal of radiologically impacted waste, the RMAs will be surveyed and de-posted.

Waste containers were purchased either new or reconditioned. All containers were inspected prior to use to verify that they were in good condition (e.g., no leaks, rust, or dents), lined or made of material that would not react with the waste, and met U.S. Department of Transportation requirements. The containers remained closed while stored unless waste was being added. Containers were also handled in such a manner that the integrity of the container was not compromised. Appropriate labels were affixed, and relevant information was marked on the containers with an indelible marker. All information was legible and clearly visible.

\subsection{WASTE Characterization}

Waste streams were characterized according to industry standards and M\&OC company procedures. Laboratory samples were collected, sealed with a custody seal, cooled to 4 degrees Celsius, and logged on a chain of custody. Waste was also screened for radiological contamination using handheld radiological survey instruments (FIDLERs and/or Electras).

\subsection{WASTE STREAMS AND DisposAL}

Waste streams generated during closure activities at CAU 484 consisted of low-level waste (LLW) and sanitary waste. Waste generation and disposition is summarized in Table 13 and discussed in detail in the following sections. Waste disposition documentation is maintained in the NTS M\&OC project files in Mercury, Nevada. 


\subsubsection{Sanitary Waste}

Sanitary waste from cleanup activities was generated by UXO removal. All waste was screened to verify that radiological contamination was less than the free-release limit established in the NV/YMP Radiological Control Manual (NNSA/NSO, 2004a). Inert ordnance debris recovered from Pedro and Antelope Lakes during the UXO clearance (see Appendix $\mathrm{H}$ for a report of the cleanup activities) was transported to and disposed of at the Air Force Range 71 UXO debris staging area.

\subsubsection{Low-Level Waste}

A total of approximately $343 \mathrm{yd}^{3}$ of LLW was generated during closure activities.

Radiologically impacted soil and debris were packaged in B-25 boxes and soft-sided containers and stored at TTR in an RMA. The waste profile has been completed and the waste has been approved for disposal in the Area 5 RWMS at the NTS. Table 13 provides a summary of the LLW generated during CAU 484 closure activities. The waste is expected to be transported to the NTS and disposed of in Fiscal Year 2008.

TABLE 13. CAU 484 LLW Disposition SUMMARY

\begin{tabular}{|c|c|c|c|c|}
\hline $\begin{array}{l}\text { WASTE } \\
\text { STREAM }\end{array}$ & LOCATION & CAS & $\begin{array}{l}\text { VOLUME } \\
\qquad\left(\mathbf{y d}^{3}\right)\end{array}$ & DISPOSITION \\
\hline \multirow{5}{*}{ LLW } & NEDS Lake & $\begin{array}{l}\text { TA-52-001-TANL } \\
\text { NEDS Detonation Area }\end{array}$ & 68 & $\begin{array}{l}\text { NTS } \\
\text { Area } 5 \\
\text { RWMS }\end{array}$ \\
\hline & & TA-52-006-TAPL, DUS & & \\
\hline & \multirow{3}{*}{$\begin{array}{l}\text { Antelope } \\
\text { Lake }\end{array}$} & $\begin{array}{l}\text { TA-52-004-TAAL, } \\
\text { MPDT }\end{array}$ & \multirow{3}{*}{275} & \multirow{3}{*}{$\begin{array}{l}\text { NTS, } \\
\text { Area } 5 \\
\text { RWMS }\end{array}$} \\
\hline & & $\begin{array}{l}\text { RG-52-007-TAML, Davis } \\
\text { Gun Penetrator Test }\end{array}$ & & \\
\hline & & $\begin{array}{l}\text { TA-52-005-TAAL, } \\
\text { JTA DU Sites }\end{array}$ & & \\
\hline
\end{tabular}




\subsection{CLOSURE VERIFICATION RESULTS}

Closure verification was confirmed through radiological surveys using the drive-over Kiwi equipment. Results show that COCs above the action levels have been removed from all but four of the excavation locations. Cleanup verification via radiological survey (i.e., use of the Kiwi) is documented in the radiological results provided in Appendix M.

\subsection{Data Quality Assessment}

Accurate and defensible analytical data were collected to verify that waste was properly characterized, managed, and disposed of and that verification samples adequately confirm closure for CAU 484 closure activities. Data were also processed to verify that samples collected during investigation activities (i.e., Geoprobe sample results for the Surface Detonation Debris Sites) met the applicable QA criteria. The following sections describe the QA/quality control (QC) procedures, data validation process, and a reconciliation of the CSM with actual findings during CAU 484 closure activities. More detail on the QA/QC procedures for CAU 484 can be found in the SAFER Plan for CAU 484 (NNSA/NSO, 2004b) and the Industrial Sites QAPP (NNSA/NV, 2002).

All samples were collected with disposable polyethylene scoops and placed in appropriately labeled sample containers secured with custody seals. Samples were labeled with a unique sample number, placed on ice, and transported under a chain of custody. Standard QA/QC samples were collected (i.e., one blind duplicate and one equipment blank per twenty samples). Samples were analyzed by certified offsite contract laboratories. Analytical results were validated at the laboratory using stringent QA/QC procedures, including matrix spike/matrix spike duplicates, spiked surrogate recovery analysis, verification of analytical results, and data quality indicator requirements. Detailed information regarding the QA/QC program requirements can be found in the Industrial Sites QAPP (NNSA/NV, 2002).

Data validation was performed according to the Industrial Sites QAPP (NNSA/NV, 2002) which is based on the U.S. Environmental Protection Agency (EPA) functional guidelines for data quality (EPA, 1994 and 1999). Data were reviewed to ensure that samples were appropriately processed and analyzed, and that the results are valid. All sample data were internally validated at the Tier I and Tier II levels.

\subsubsection{Investigation Data}

During investigation of the Surface Detonation Debris Sites, soil samples were collected using the Geoprobe and submitted for laboratory analysis. Results of these analyses were used in conjunction with other data (i.e., geophysical and radiological survey results) to locate areas where COCs were present. This section provides information regarding the QA/QC evaluation of these data. 


\subsubsection{Geoprobe Analytical Data at Surface Detonation Debris Sites}

\subsection{Data Quality Indicators}

All of the Geoprobe data met the data quality indicators required by the DQOs (precision, accuracy, sensitivity, completeness, comparability, and representativeness) and were useable for making decisions except as noted below:

- Precision - The $20 \%$ relative percent difference was exceeded for lithium and thallium samples at the DUS and for uranium isotopes at other sites; however, all of the sample concentrations were orders of magnitude less than the action levels such that the data were useable for making a decision. Results from most of the duplicate samples were near or less than the detection limit, which is where the relative percent difference calculations are subject to such results and have little impact on the value of the data quality.

- Accuracy - Laboratory recoveries were inadequate for all niobium analyse,s and all niobium data were rejected. Niobium is not a typical analyte, and a standard method for detecting the concentration of niobium has not been identified in the Test Methods for Evaluating Solid Waste, SW-846 (EPA, 1996). All other samples met this data quality criterion.

- Completeness - This criterion was met for all critical analytes and for all non-critical analytes other than niobium. The presence of niobium was intended to be a potential indicator that DU would be present and is not considered a hazard itself. Niobium has no established action level in the Region 9, EPA preliminary remediation goals (EPA, 2004) and is not listed as a chemical hazard by the U.S. Department of Health and Human Services, National Institute for Occupational Safety and Health (NIOSH) in the NIOSH Pocket Guide to Chemical Hazards (NIOSH, 2005). Because the DU was adequately detected and remediated, the inability to adequately identify the concentration of niobium is not considered a failure in completing the objectives of the CAU 484 closure.

An additional evaluation of the analytical data was completed to verify that a sufficient number of samples were collected to characterize the site within the level of confidence and methodologies identified in the SAFER Plan. The plan was prepared using the Visual Sampling Plan (VSP) software in conjunction with sample number calculations based on the EPA guidance discussed in the SAFER Plan. The VSP "Hot Spot" identification module was used with the input parameters of 95 percent probability of locating an elliptical hot spot with a semi-major axis of 30 feet and a height to width ratio of 0.8 oriented at an unknown angle. The software applied a triangular grid sampling layout and calculated the actual sampling coordinates for each of the sampling locations. The probability and hot spot detection parameters were based on the assumptions stated in the SAFER Plan. The samples were collected for the purpose of identifying an aerial dispersion area, and not to identify the small hot spots that were present because of individual particles and small radioactive metal pieces, as was detected by the radiological surveys.

For the evaluation of characterization data, the same methodologies and assumptions used in the SAFER Plan were used. The initial calculations used to estimate the number of samples needed for characterization used existing data for beryllium and U-238 collected from a similar detonation site at the NTS. A 95 percent confidence and an error of 20 percent were used. The resulting calculation estimated that 36 samples would be needed. For the verification calculation, only U-238 data was used since beryllium was not confirmed at any of the sites. All 
U-238 data from each of the compliance sampling locations (i.e., DUS, NEDS, and MPDT) were pooled and normalized to adjust for a lognormal distribution. Data was analyzed using the Grubbs test for outliers, and three locations from the NEDS test area were determined to be outliers and were excluded from the data analysis. Based on this analysis, the number of samples required to characterize each site is less than 36. The 36 samples collected at each location provides a confidence level that approaches 99 percent with an associated error of less than 10\% and a statistical significance of 0.01 . In summary, the DUS, NEDS, and MPDT sites have been adequately characterized.

\subsection{Statistical Analysis}

Statistical evaluations of the Geoprobe investigation data were performed to compare and evaluate the different data sets. The statistical evaluations include results regarding comparability of data collected from the two different depths at each site, comparisons of characterization data with local background concentrations, and other evaluations. Results of the statistical analysis are provided in Appendix J-1 and are summarized below:

- Surface versus subsurface concentrations - There is no statistically significant difference in uranium concentrations between samples collected from the surface (i.e., 0.0-0.5 ft bgs) and those collected from the subsurface (i.e., 1.0-1.5 ft bgs) with the exception of one hot spot in the center of the NEDS Detonation Debris Site. There are some differences for the other analytes.

- Investigation versus background concentrations - There were some differences between background concentrations and the individual site concentrations at all locations; however, there were no differences between concentrations of mercury and thallium at the DUS.

- Investigation versus action level - The action level for one analyte was exceeded at only one location, the uranium hot spot at the NEDS site.

- Probability of concentration exceeding the action level - The probability of any random point at the site exceeding the action level is extremely low (i.e., less than 0.000001), except for the uranium hot spot at the NEDS site.

\subsubsection{Statistical Evaluation of Davis Gun Geophysical Data}

Statistical evaluations of the Davis Gun geophysical data were performed to compare and evaluate the different data sets. Results of the statistical analysis are provided in Appendix J-2. Results indicate that pursuing alternate options to excavating all of the potential targets would be more prudent than to excavate the extremely large number of targets that would be necessary to get a reasonable degree of assurance (i.e., the 95 percent confidence level) that all of the DU and/or Davis Gun penetrators have been identified. This supports the closure alternatives that were undertaken, which took measures to identify and conduct closer investigation of those sites where penetrators or DU were most likely present, rather than excavating all of the anomalies that were identified. 


\subsubsection{Cleanup Verification Data}

Date: September 2007

Cleanup verification was confirmed using the Kiwi vehicle sodium iodide detectors. The results of this field detection method are direct readings of the remaining radioactivity at the cleanup locations. Data are post-processed to evaluate the spectral quality and confirm if radiological count measurements exceed the threshold of standard deviations from the mean. Technical evaluation of the Kiwi system has documented that three standard deviations from average background is the lowest threshold where the presence of depleted uranium can be quantified. All but four locations on Antelope Lake (i.e., CA-1, SA-4, SA-5-9, and SA-12-15) were verified clean closed. Results of cleanup verification using this method are described in more detail in Appendix M.

\subsubsection{CSMs}

Three CAU 484 CSMs were developed and were presented in Appendix A of the approved SAFER Plan for CAU 484 (NNSA/NSO, 2004b). This information is also included in Appendix A of this document. All CSMs were confirmed and verified by closure activities with the following exceptions:

- $\quad$ Surface Detonation Debris Sites:

- $\quad$ The CSM assumed that DU would exist as discrete fragments on the ground surface; however, DU was found on the ground surface and in the surrounding soil to a depth of as much as $10 \mathrm{ft}$ bgs. Also, DU was found as metal fragments and as oxide, and the DU oxide had dispersed into the surrounding soil. Cleanup was completed and verified using the Kiwi sodium iodide detectors.

- $\quad$ CAS RG-52-007-TAML, Davis Gun Penetrator Test:

- Geophysical and radiological surveys were not conducted on Main Lake because it was covered with water. A decision was made to postpone investigation of Main Lake until such time that investigation results indicated that if Davis Gun penetrators were present at other playa locations, an investigation strategy would be developed for Main Lake. If no penetrators were located on other playas, then Main Lake would also be considered closed. No penetrators were located on the other playas.

- All Davis Gun penetrator geophysical targets were not investigated because no Davis Gun related debris or DU was encountered in the 13 excavations that were completed on Antelope and Pedro Lakes, and because six of the geophysical anomalies that were investigated turned out to be UXO rather than Davis Gun penetrators.

- $\quad$ NEDS and Antelope Lakes: DU was encountered outside of expected/known areas of dispersion.

- On NEDS Lake, DU dispersion extended outside of the anticipated area identified in the SAFER Plan for the NEDS Detonation Area and the DUS. The only known tests on NEDS Lake are the NEDS and Colimbo (DUS) Tests, and the DU debris that was found was consistent with dispersal from these detonations; therefore, the DU cleaned up on NEDS Lake is not outside the bounds of CAU 484. 
- $\quad$ The DU encountered on Antelope Lake can be grouped into two categories: (1) fragments with limited contaminated soil on the playa surface, and (2) larger, oxidized debris disseminated on the playa surface and in the shallow subsurface. The DU fragments on the ground surface are consistent with a surface detonation, which could be related to dispersion from the MPDT. The source of the larger oxidized areas of DU on the playa surface and shallow subsurface is unknown; however, the areas with DU are in or near areas where historical Davis Gun penetrator tests were known to have been conducted, even though the relation of the DU to these tests is not known. In a May 7, 2007, meeting, NNSA/NSO decided that areas of DU on Antelope Lake that are within or near areas of Davis Gun testing would be cleaned up within the scope of CAU 484. Cleanup was completed and verified using the Kiwi sodium iodide detectors for excavations accessible to the Kiwi survey vehicle, and collecting cleanup verification soil samples for excavations that were not accessible to the Kiwi vehicle.

\subsection{USE RESTRICTION}

A use restriction has been implemented for CAS RG-52-007-TAML, Davis Gun Penetrator Test. Four locations on Antelope Lake were use-restricted for remaining subsurface DU contamination. Surface contamination was excavated to remove DU and DU-contaminated soil shallower than $0.5 \mathrm{ft}$ bgs. Excavated areas have been filled with clean, native fill and slightly mounded to prevent ponding of rainwater. Each of the four areas has been posted with four use restriction signs to warn against intrusive activity. "Underground Radiological Material” signs have also been posted at each location. The CAU Land-Use Restriction Information form and figures showing the locations of the surveyed points delineating the use-restricted areas are provided in Appendix O. This information has been submitted to the U.S. Air Force at the TTR. Annual site inspections will be performed to verify that the signs are in good repair and that the use restriction has been maintained. Details on the post-closure requirements for this CAS are included in Section 5.2. 
Closure Report - CAU 484

Section: Closure Verification

Revision: 0

Date: September 2007

THIS PAGE INTENTIONALLY LEFT BLANK 


\subsection{CONCLUSIONS AND RECOMMENDATIONS}

\subsection{CONCLUSIONS}

The following site closure activities were performed at CAU 484 and are documented in this CR:

- CAS RG-52-007-TAML (Davis Gun Penetrator Test) was closed in place at four locations on Antelope Lake where clean closure was not possible. DU debris and contaminated soil that were detected on the ground surface at Antelope Lake was excavated, packaged, and will be disposed of as LLW. At the four locations, extent of DU contamination was extensive and/or complicated by the presence of non-CAU $484 \mathrm{UXO}$ and debris. These four locations were excavated to remove surface contamination, covered with clean fill that has been mounded to provide for water runoff, and closed in place with a use restriction. Numerous investigation activities were also undertaken to locate Davis Gun penetrators or associated debris. No Davis Gun penetrator debris or DU was encountered during any of the exploratory excavations made to locate the penetrators; therefore, NNSA and NDEP agreed that no additional Davis Gun penetrator targets would be excavated or investigated. Investigating penetrators that may be present at depths greater than $10 \mathrm{ft}$ bgs is not feasible due to technology limitations and, at these depths, hazardous debris and/or DU does not pose a threat to human health and/or the environment.

- CAS TA-52-001-TANL (NEDS Detonation Area) was clean closed by removing and disposing of DU debris and contaminated soil.

- CAS TA-52-004-TAAL (MPDT) was clean closed by removing and disposing of DU debris and contaminated soil.

- CAS TA-52-005-TAAL (JTA DU Sites) was clean closed by locating, retrieving, and disposing of three DU rings, which comprise the CAS, and associated, contaminated soil.

- CAS TA-52-006-TAPL (DUS) was clean closed by removing and disposing of DU debris, DU, and uranium-contaminated soil.

- CAS TA-54-001-TANL (Containment Tank and Steel Structure) was clean closed by removing the uncontaminated structures and either recycling the materials or re-using them elsewhere.

\subsection{Post-Closure REQuirements}

Results of all inspections in a given year will be documented in the annual combined post-closure report for the TTR. This report will include a discussion of inspections and observations, and copies of the site inspection checklists. This report will be submitted to the NDEP annually or as otherwise agreed to with the NDEP.

\subsubsection{Inspections}

Inspections will be performed semi-annually for the first year post-closure, after which they will be performed annually. Inspections will consist of visual observations to verify that the underground radioactive material area and UR warning signs are in place and readable and that 
the UR is maintained. The interior of each of the UR areas will also be inspected to confirm that there have been no disturbances. Any repairs or maintenance will be documented in writing at the time of the repair. A Post-Closure Inspection Checklist will be completed to document the results of the inspection and to describe repairs that were performed since the previous inspection.

\subsubsection{Monitoring}

No monitoring other than visual inspections will be required for CAU 484.

\subsection{RECOMMENDATIONS}

Since closure activities for CAU 484 have been completed following the NDEP-approved SAFER Plan (NNSA/NSO, 2004b) or as otherwise documented in this report, NNSA/NSO requests the following:

1. Provision of a Notice of Completion from NDEP to NNSA/NSO for the closure of CAU 484.

2. Transfer of CAU 484 from Appendix III to Appendix IV, Closed CAUs, of the FFACO (FFACO, 1996). 


\subsection{REFERENCES}

EPA, see U.S. Environmental Protection Agency.

Federal Facility Agreement and Consent Order, 1996 (as amended August 2006). Agreed to by the state of Nevada; the U.S. Department of Energy, Environmental Management; the U.S. Department of Defense; and the U.S. Department of Energy, Legacy Management.

FFACO, see Federal Facility Agreement and Consent Order.

NIOSH, see U.S. Department of Health and Human Services, Centers for Disease Control and Prevention, National Institute for Occupational Safety and Health.

NNSA/NSO, see U.S. Department of Energy, National Nuclear Security Administration Nevada Site Office.

NNSA/NV, see U.S. Department of Energy, National Nuclear Security Administration Nevada Operations Office.

Pacific Northwest National Laboratory, 2005. Visual Sample Plan Software, Version 4.6. Prepared for the U.S. Department of Energy under Contract DE-AC05-76RL01830.

U.S. Department of Energy, National Nuclear Security Administration Nevada Operations Office, 2002. Nevada Environmental Restoration Project Industrial Sites Quality Assurance Project Plan, Nevada Test Site, Nevada. DOE/NV--372-REV.3. Las Vegas, NV.

U.S. Department of Energy, National Nuclear Security Administration, Nevada Site Office, 2004a. NV/YMP Radiological Control Manual. DOE/NV/11718-079 Rev. 5. Las Vegas, NV.

U.S. Department of Energy, National Nuclear Security Administration Nevada Site Office, 2004b. Streamlined Approach for Environmental Restoration Plan for Corrective Action Unit 484: Surface Debris, Waste Sites, and Burn Area, Tonopah Test Range, Nevada. DOE/NV--975. Las Vegas, NV.

U.S. Department of Health and Human Services, Centers for Disease Control and Prevention, National Institute for Occupational Safety and Health, 2005. NIOSH Pocket Guide to Chemical Hazards. NIOSH Publication No. 2005-149. Washington, D.C.

U.S. Environmental Protection Agency, 1994. Guidance for the Data Quality Objectives Process. EPA QA/G-4. Washington D.C.

U.S. Environmental Protection Agency, 1996. Test Methods for Evaluating Solid Waste Physical/Chemical Methods, SW-846, 3rd Edition. Washington, D.C. 


\subsection{REFERENCES (continued)}

U.S. Environmental Protection Agency, 1999. Contract Laboratory Program National Functional Guidelines for Organic Data Review. EPA540/R-99/008. Washington D.C.

U.S. Environmental Protection Agency, 2004. Region IX Preliminary Remediation Goals. San Francisco, CA. 


\section{APPENDIX A*}

\section{DATA QUALITY OBJECTIVES}

* As presented and published in Appendix A1 of the approved Streamlined Approach for Environmental Restoration Plan for Corrective Action Unit 484: Surface Debris, Waste Sites, and Burn Area, Tonopah Test Range, Nevada. 2004. DOE/NV--975. Las Vegas, NV. 
Closure Report - CAU 484

Section: Appendix A

Revision: 0

Date: September 2007

THIS PAGE INTENTIONALLY LEFT BLANK 


\section{ACRONYMS AND ABBREVIATIONS}

BN Bechtel Nevada

CAI Corrective Action Investigation

CAS Corrective Action Site

CAU Corrective Action Unit

COC Contaminants of concern

COPC Contaminants of potential concern

CSM Conceptual Site Model

$\mathrm{CV} \quad$ Coefficient of variation

DOE U.S. Department of Energy

DOE/NV U.S. Department of Energy, Nevada Operations Office

DQO Data Quality Objective(s)

DU Depleted uranium

EPA U.S. Environmental Protection Agency

FFACO Federal Facility Agreement and Consent Order

$\mathrm{ft} \quad$ foot (feet)

$\mathrm{ft}^{2} \quad$ square feet

$\mathrm{ft}^{3} \quad$ cubic feet

IT International Technology

JTA Joint Test Assembly

$\mathrm{m} \quad$ meter(s)

$\mathrm{m}^{2} \quad$ square meters

$\mathrm{m}^{3} \quad$ cubic meters

MPDT Metal Particle Dispersion Test

NCRP National Council on Radiation Protection and Measurements

NDEP Nevada Division of Environmental Protection

NEDS Nonviolent Explosive Destruct System

NNSA/NSO U.S. Department of Energy, National Nuclear Security Administration Nevada Site Office

PRG Preliminary remediation goal

QA/QC Quality Assurance/Quality Control

SAFER Streamlined Approach for Environmental Restoration

TTR Tonopah Test Range 
SAFER PLAN - CAU 484

Section: Appendix A1

Revision: 0

Date: May 2004

THIS PAGE INTENTIONALLY LEFT BLANK 


\section{APPENDIX A \\ DATA QUALITY OBJECTIVES FOR \\ CORRECTIVE ACTION UNIT 484: SURFACE DEBRIS, WASTE SITES, AND BURN AREA, TONOPAH TEST RANGE, NEVADA}

\section{Presentation of Known Data Related to Corrective Action Unit 484}

The information presented in this worksheet is based on historical data generated from preliminary assessment activities for Corrective Action Unit (CAU) 484 at the Tonopah Test Range (TTR). The Data Quality Objective (DQO) worksheet follows the U.S. Environmental Protection Agency (EPA) DQO guidance outline (EPA, 2000b). The steps systematically build on the data acquired during preliminary assessment work and background research. Copies of the preliminary assessment work are retained in the CAU 484 project files.

Members of the Planning Team (* indicates members present at the DQO meeting):

1. U.S. Department of Energy, National Nuclear Security Agency Nevada Site Office (NNSA/NSO) Janet Appenzeller-Wing

Kevin Cabble*

2. Bechtel Nevada (BN)

Ronald Jackson*

Reed Poderis*

Dudley Emer*

Kevin Campbell

3. Nevada Division of Environmental Protection (NDEP)

Clem Goewert*

4. Core Decision Team

Janet Appenzeller-Wing (NNSA/NSO)

Kevin Cabble (NNSA/NSO)

Brad Jackson (BN)

5. Primary Decision Makers

Janet Appenzeller- Wing (NNSA/NSO)

Kevin Cabble (NNSA/NSO) 


\subsection{STATE THE PROBLEM (Step 1)}

gain a sufficient understanding to define the problem.

The general location, nature, and extent of the CAU 484 corrective action sites (CASs) is understood; however, additional information is needed to verify that the existing information is adequate, confirm the existence of contamination and/or waste, and/or verify previously completed cleanup activities. Information will be used to close these CASs under the Streamlined Approach to Environmental Restoration (SAFER) process.

\subsection{CAS Specific Information}

CAU 484, Surface Debris, Waste Sites, and Burn Area, is located at the TTR and consists of the following six CASs:

- CAS RG-52-007-TAML, Davis Gun Penetrator Test,

- CAS TA-52-001-TANL, NEDS Detonation Area,

- CAS TA-52-004-TAAL, Metal Particle Dispersion Test,

- CAS TA-52-005-TAAL, Joint Test Assembly DU Sites,

- CAS TA-52-006-TAPL, Depleted Uranium Site, and

- CAS TA-54-001-TANL, Containment Tank and Steel Structure.

The following sections describe the CASs in CAU 484 and the conceptual site models (CSMs) that apply to each CAS.

\subsubsection{CAS RG-52-007-TAML, Davis Gun Penetrator Test}

CAS RG-52-007-TAML, Davis Gun Penetrator Test, consists of potentially 9 locations at TTR and the Nevada Testing and Training Range (NTTR) and is identified within the Federal Facilities Agreement and Consent Order (FFACO) as Depleted Uranium (DU) Surface Debris. The potential locations where Davis Gun Penetrator tests were conducted include Antelope Tuff Target 1, Antelope Tuff Target 2, Myers Ridge, Sidewinder Tuff Target, Antelope Lake, Main Lake, Pedro Lake, Brownes Lake, and Mt. Helen (Mt. Helen was apparently erroneously reported as Mellan Hill in some documentation; no Davis Gun tests are known to have been conducted at Mellan Hill.)

The Davis Gun is a recoilless rifle used by Sandia National Laboratories to fire artillery projectiles into soil, rock, and concrete targets. According to information collected previously, the penetrators used at TTR were usually inert with the occasional use of DU as ballast. At least one test included a plutonium pit in a penetrator and was recovered intact and returned to the test customer. Historical evidence indicates that one penetrator was possibly lost in 1976 when a penetrator was reported as falling into a fracture that opened on Antelope Lake after a heavy rain. The penetrator could not be subsequently located and it is unknown if the lost penetrator contained DU or if the event actually occurred.

Release of DU is not a likely scenario at Davis Gun test locations, as there is evidence that all penetrators were recovered completely intact. The purpose of Davis Gun tests was to evaluate delivery system packages and a primary goal of the experiments was to recover the test components. Therefore, it is highly unlikely that test components were left at the sites where the 
experiments were conducted. An unrecovered penetrator would be considered a test failure and no failures for Davis Gun tests have been reported. Areas where Davis Gun tests occurred will be investigated as necessary and the appropriate corrective actions will be taken.

\subsubsection{CAS TA-52-001-TANL, NEDS Detonation Area}

The NEDS (Nonviolent Explosive Destruct System) Detonation Area is a test site located on the northern half of NEDS Lake and is identified within the FFACO as DU Surface Debris. The tests were performed in 1974 and involved exploding mock nuclear warheads using high explosive to test containment. Depleted uranium and/or beryllium were used as components within the mock warheads. Although the area was cleaned and the majority of debris was recovered after the tests, the possibility remains that residual DU and/or beryllium may remain on the playa surface in the area of the detonations. The area where the NEDS test occurred will be investigated and the appropriate corrective actions will be taken.

\subsubsection{CAS TA-52-004-TAAL, Metal Particle Dispersion Test}

The Metal Particle Dispersion Test (MPDT) is located within the northwest corner of Antelope Lake and is identified within the FFACO as DU Surface Debris. The MPDT is the site of a 1987 experiment in which a W-82 mock nuclear artillery shell was detonated with high explosive to test dispersal patterns. Depleted uranium and/or beryllium were used to simulate the dispersal of radioactive materials. The location of the test is documented and a recovery operation took place after the test to map the dispersion of the particles. A soil berm was also constructed around the area of the test. Residual radiological materials may remain within the bermed area depending on the thoroughness of the cleanup completed in 1987.

\subsubsection{CAS TA-52-005-TAAL, Joint Test Assembly DU Sites}

The Joint Test Assembly (JTA) DU Sites are located within the northern portion of Antelope Lake and are identified within the FFACO as DU Surface Debris. The JTA experiments were conducted in 1987 and involved firing four W-33 mock nuclear artillery shells from a 155-millimeter howitzer. The projectiles were fired from Area 9 toward Antelope Lake. The artillery rounds reportedly contained DU rings for additional reaction mass. Interviews of project personnel indicate that at up to three of the rings were never recovered despite a 2-square mile search of the impact area on the northern portion of Antelope Lake. For this reason, DU associated with the JTA experiments may be present on the surface or shallow subsurface of the northern portion of Antelope Lake. This site is being investigated to determine if any materials from the JTA tests remain on Antelope Lake.

\subsubsection{CAS TA-52-006-TAPL, Depleted Uranium Site}

The Depleted Uranium Site is located on NEDS Lake and is identified within the FFACO as DU Surface Debris. This site is the result of the 1985 Colimbo test in which a fully configured W-81 cruise missile was detonated above the surface of NEDS Lake. Recovery and cleanup operation was conducted to remove components and debris related to the test. However, there may be residual DU and/or beryllium as well as an unconfirmed sealed cobalt radiological source remaining on the playa surface. The location of the detonation is well documented and the area will be investigated to determine if residual components of the Colimbo test remain at the site. 


\subsubsection{CAS TA-54-001-TANL, Containment Tank and Steel Structure}

Several large pieces of debris associated with NEDS Lake operations are located at the north end of the lake. The structures are believed to have been used during the NEDS tests and have been previously evaluated and determined not to be contaminated. The tank and structure is considered non-impacted housekeeping waste/debris and will be cleaned up accordingly. A wood and steel structure associated with CAU 410 is also present at this location and may be removed.

The CAS has been incorrectly described as containing a burn area. CAS TA-54-001-TANL was originally described and thought to have been a location for burning rocket fuel. However, burning of rocket fuel was determined not to have occurred at this location and the CAS name was changed to "Containment Tank and Steel Structure." The "containment tank" is actually designed to be used as a shelter by personnel during some type of testing activity. The use of the steel structure is unknown. However, both the containment tank and steel structure were used at different locations on the TTR and have been subsequently moved to their present location. A burn site is located on NEDS lake but it is related to training exercises conducted by TTR security forces and is not part of CAU 484.

\subsection{Develop/Refine the Conceptual Model}

Available information from which the conceptual models are based was derived from site process knowledge, historical background information, site analysis, and personnel interviews relative to the activities related to the CAS. Three CSMs have been developed for CAU 484 .

\subsubsection{Surface-Shallow Subsurface Debris CSM}

This CSM addresses areas where DU and/or system components may have been released to the ground surface or may have penetrated the shallow subsurface. A graphical depiction of the CSM is shown in Figure 1 of this appendix. Based on the types of testing activities conducted at the locations included in this CSM, if DU is detected it will be present in discrete pieces. Based on process knowledge and technology limitations, shallow subsurface is defined as being a depth of $3 \mathrm{~m}$ (10 feet [ft]) or less below ground surface. The surface and shallow subsurface will be evaluated using geophysical methods. The following sites are included within the SurfaceShallow Subsurface Debris CSM:

\subsubsection{CAS RG-52-007-TAML, Davis Gun Penetrator Test}

The locations of the Davis Gun Penetrator Test sites are primarily identified on lake beds by evidence of a circular berm (constructed to prevent storm water run-on during penetrator recovery operations) and, at locations not on lake beds, by evidence of an excavation and associated rock spoils. Small piles of rock fragment are commonly present adjacent to targets located in areas where tests were conducted in the tuff. Known or possible test locations include Antelope Tuff Target 1, Antelope Tuff Target 2, Myers Ridge, Sidewinder Tuff Target, Antelope Lake, Main Lake, Pedro Lake, Brownes Lake, and Mt. Helen. Based on the documentation, it is unlikely that any unrecovered penetrators or fragments remain below the surface. However, the CAS is listed as potential surface contamination because it is known that the DU that was occasionally used as ballast in the penetrator projectiles. These areas are not located within known radiological control areas and contaminants of potential concern (COPC) are not expected. Depleted uranium was used as ballast within some penetrators but all penetrators were 
reported as being recovered intact. It is unknown if the penetrator reported lost in a crack on Antelope Lake contained DU or if it was actually lost.

\subsubsection{CAS TA-52-005-TAAL, Joint Test Assembly DU Sites}

The exact location of the Joint Test Assembly (JTA) tests is unknown. Based on available documentation, the apparent target area for the JTA tests is northern half of Antelope Lake. Three DU rings were reported lost on Antelope Lake. Although a large search was conducted for the missing material after the tests, the lost depleted rings were never located. If the DU rings are located on Antelope Lake, they are not located within known radiological control areas and COPC other than DU are not expected.

\subsubsection{Surface Detonation Debris CSM}

This CSM addresses CASs where some type of dispersion test was conducted which resulted in the release of DU, beryllium, and system components to the ground surface (Figure 2 of this appendix). Based on the types of testing activities that resulted in potential contamination at the sites within this CSM, DU, beryllium, and system components will consist of discrete pieces of metal and/or debris on the ground surface. Impacted soil associated with the waste at these sites is assumed to be minimal and only associated with the DU and/or beryllium fragments. Although no subsurface debris is suspected at these sites, the shallow subsurface will be evaluated using geophysical techniques to confirm the CSM. Documentation indicates that cleanup activities were conducted following each of the tests performed at these sites. However, previously completed cleanup of DU at the sites within this CSM has not been verified, and will be evaluated during the planned characterization activities. The following sites are included within the Surface Detonation Debris CSM:

\subsubsection{CAS TA-52-001-TANL, NEDS Detonation Area}

The NEDS tests took place on the northern half of NEDS Lake. However, the exact test location(s) are not known. Based on the documentation, DU and beryllium fragments were recovered at the completion of the NEDS tests. The potential for DU and/or beryllium fragments on the surface of NEDS Lake is possible and will be evaluated during the site investigation. Buried debris is unlikely due to the nature of the tests. The CAS is listed as potential surface contamination due to the DU and/or beryllium that may have been used in the test units. These areas are not located within known radiological control areas, and COPC other than beryllium and/or DU are not expected.

\subsubsection{CAS TA-52-004-TAAL, Metal Particle Dispersion Test}

The location of the MPDT is within a bermed area on the north end of Antelope Lake. Depleted uranium and/or beryllium were used to simulate the dispersal of fissionable radioactive materials. Based on the documentation available, it is unlikely that appreciable amounts of unrecovered DU and/or beryllium fragments remain within the bermed area. The site is described as potential surface contamination area and some amounts of DU and/or beryllium may remain on the surface. Due to the nature of the test and the recovery effort completed as part of the experiment, it is unlikely that any buried debris relating to the MPDT is present. While the bermed area is not formally posted as a radiological area, signs have been placed at the site indicating that the area is a soil contamination area. COPC other than beryllium and/or DU are not expected. 
The location of the Colimbo Test is marked by a brass at-grade monument on the northern half of NEDS Lake. Documentation indicated that it is unlikely that any large unrecovered fragments remain on the surface. This CAS includes possible surface debris which may be DU and/or beryllium. The area of this CAS is not located within a known radiological control area and COPC other than beryllium and/or DU are not expected.

\subsubsection{Non-Impacted Surface Debris CSM}

This CSM addresses CAS TA-54-001-TANL (Containment Tank and Steel Structure) which consists of non-impacted structures on the ground surface. Figure 3 of this appendix shows this CSM. The structures will be screened to confirm they are not radiologically impacted and the materials will be removed for disposal or recycling.

\subsection{Secondary Conceptual Site Models for CAU 484}

The conditions under the secondary CSMs for CAU 484 are considered less likely than the conditions outlined in the primary CSMs. No information has been identified that suggests conditions outside the primary model are present. The secondary CSMs for CAU 484 are similar to the primary model with one or more of the following exceptions:

- Potentially buried debris may be deeper than anticipated. This poses a situation where site conditions may exceed the technical limitations of available geophysical methods.

- Surface debris that is expected to be non-impacted is determined to be impacted.

- A primary assumption is that DU, if present, will be in discrete pieces and that there will be minimal soil impact. If significant areas of DU-impacted soil are present the CSM will be reevaluated and the investigation-closure strategy may be revised as necessary.

- Areas with suspected surface debris are determined to extend into the subsurface.

- A CAS can not be located due to unavailability of information, technology limitations, or other causes. Closure of the site will proceed following the precedent established by CAU 495 where the site was promoted to FFACO Appendix IV with the caveat that the site would be reopened for investigation if additional information was found in the future (NDEP, 1999).

\subsection{Potential Hold Points}

During closure activities, certain conditions affecting the project schedule and budget may require decisions prior to continuing work. Primary hold/decision points that may occur during the CAU 484 SAFER process have been identified and include the review of data for the selection of potential site locations for further investigation and the review of analytical data.

The results of geophysical and radiological surveys will be reviewed and presented to the NNSA/NSO and/or the NDEP. Three potential results are possible and will be evaluated for further action. The potential results include the following: a distinct target representing the site will be located, several potential targets will be located, or no potential targets will be identified. If a single target is identified, the data will be reviewed and a decision will be made concerning the type and nature of investigation required. If multiple potential targets are identified, a decision will be made concerning which targets are to be investigated and the nature of investigation required. If no targets or potential targets are identified for a specific site the alternative CSM will be evaluated for additional areas of site investigation or closure by no 
further action using the CAU 495 scenario (caveat that the CAU may be closed but will be reopened if additional information is found in the future) (NDEP, 1999).

An additional hold/decision point occurs during the investigation when results of soil sampling and laboratory analysis for beryllium and DU screening results will be reviewed with the NNSA/NSO and/or the NDEP to confirm the cleanup and/or closure approach. Throughout the investigation/closure process, data will be collected, evaluated, and presented to NNSA/NSO for review and input.

In addition to the previously discussed hold/decision points, work may be temporarily suspended until the issue can be satisfactorily resolved if any of the following unexpected conditions occur:

- Conditions outside the scope of work are encountered such as large amounts of unexploded ordinance,

- Radiological screening yields results which require an upgrade in procedures to continue survey work in specific areas,

- Elevated beryllium levels are found in soil samples,

- Elevated levels of additional COPCs are found that were not originally identified as being present at the sites,

- Encountering unexpected conditions including waste and/or contamination,

- Out-of-scope work activities are required due to the detection of other contaminants of concern (COC) that would require re-evaluating a disposal pathway, such as with hazardous or low level waste,

- Unsafe conditions or work practices posing a threat to personnel, equipment, or the environment, not originally documented in the Site Specific Health and Safety Plan, are encountered,

- Other technical factors are encountered that require the preparation of a Record of Technical Change to the approved SAFER Plan.

\subsection{IDENTIFY THE DECISION (Step 2)}

Identify what questions the study will attempt to resolve, and what actions may result.

\subsection{Develop Decision Statements}

A SAFER Plan will be prepared based on the currently available process knowledge, historical information, geophysical data, and radiological survey data. The CASs within CAU 484 may be divided into sites where the general location and nature of debris/contamination is known, and sites where the location has not been confirmed and the nature of the debris/contamination is known.

Sites where the general location and nature of debris/contamination are known include the following CASs:

- CAS TA-52-001-TANL, NEDS Detonation Area

- CAS TA-52-004-TAAL, Metal Particle Dispersion Test

- CAS TA-52-006-TAPL, Depleted Uranium Site

- CAS RG-52-007-TAML, Davis Gun Penetrator Test (Antelope Tuff Target 1, Antelope Tuff Target 2, Myers Ridge, Sidewinder Tuff Target, Mt. Helen) 
Sites where the location has not been confirmed and the nature of the debris/contamination is known include the following CASs:

- CAS TA-52-005-TAAL, Joint Test Assembly DU Sites

- CAS RG-52-007-TAML, Davis Gun Penetrator Test

- (Antelope Lake, Main Lake, Pedro Lake, Brownes Lake, Antelope Tuff, Myers Ridge, Sidewinder Tuff, and Mt. Helen)

For CASs where the location has not been confirmed, historical information, interviews, site conditions, and geophysical and radiological data will be evaluated and a potential target location will be identified for each CAS, if possible. Potential target information will be presented to NNSA/NSO for concurrence. Selected targets will be addressed during the Corrective Action Investigation (CAI). Findings of the CAI will be evaluated with the NNSA/NSO and the corrective action approach will be confirmed and documented. Sites with impacted materials will be either clean closed by removing the impacted material, or closed in place. Clean closure will be verified by conducting a radiological survey for DU, and verification soil samples will be collected if beryllium is identified as a COC.

\subsection{Decision Statements}

Decision I - Is waste present and/or is contamination present above action levels within a CAS? A COPC that is detected at a concentration exceeding action levels will be considered a COC. A COC is defined as a site-related constituent that exceeds the screening criteria or is detected during surface radiological surveys. Depleted uranium will be considered a COC if it is detected during surface surveys.

Decision II - If waste is present and/or contamination is present above action levels or screening levels, has the lateral and vertical extent been determined and is it technically feasible to remove the waste/contamination? (If technically feasible all CASs containing waste/contamination will be clean closed.) If potentially buried debris can not be located the site will be closed using the CAU 495 model (no further action unless additional information is identified in the future) (NDEP, 1999).

\subsection{Alternative Actions to the Decision}

If a COPC is not present, further assessment of that COPC in the CAS is not required. If a COC is present, resolve Decision II. The alternative for Decision II is: "If the extent and migration of a COC is defined in both the lateral and vertical directions, further assessment of the CAS is not required. If the extent of a COC is not defined, reevaluate site conditions and collect additional samples.”

Sites with insufficient information and/or data will be evaluated for additional data collection or a "no further action" alternative. If the "no further action" alternative is selected, the approach previously approved by NDEP for CAS 495 (Unconfirmed Joint Test Assembly (JTA) Sites) will be implemented (NDEP, 1999). This approach will allow closure of unconfirmed sites with no land/site use restrictions and no post-closure monitoring. However, should additional evidence become available in the future which indicates the location for the site, the site should be reopened to continue the investigation. 


\subsection{IDENTIFY THE INPUTS TO THE DECISION (Step 3)}

Identify the information that needs to be obtained and the measurements that need to be taken to resolve the decision statement. This step identifies the information needed and sources of information, the basis for establishing action levels, and sampling and analysis methods that can meet the data requirements.

\subsection{Identify the information inputs needed and resolve the decision.}

In order to confirm the site CSM and to determine the nature and extent of contamination, data must be collected and analyzed following the following three criteria: 1 . Survey data must be collected in areas containing impacted debris and/or contamination; 2. Samples will be collected from areas most likely to be contaminated by beryllium (samples may be analyzed for beryllium and depleted uranium); and 3. The data must be adequate to detect COC. Investigation and closure verification data for DU will be collected using field-screening methods, and beryllium cleanup will be verified through sample collection and analysis. Soil samples may be collected to verify closure activities at depleted uranium-impacted sites.

In order to determine if a COC (e.g., beryllium, depleted uranium) is present at a particular CAS, sample data must be collected and analyzed by two criteria: 1 . Samples must be collected in areas most likely to be contaminated; and 2. The requested analyses must be sufficient to detect any contamination present within the samples. The only COCs identified for CASs within CAU 484 are DU and beryllium. Depleted uranium characterization will be completed using field survey and screening instruments, and sample collection for laboratory analysis. For sites with beryllium as a COC, the contamination will be associated with DU (due to the nature of the release).

In order to confirm the extent of contamination, data must be collected and analyzed using a data collection method adequate to detect the COC. Samples will be collected based on radiological and geophysical surveys, field observations, and field screening results. Samples will be collected from areas expected not to be impacted by the COC so that the impacted area can be bounded and defined.

Biasing factors to support the determination of the nature and extent criteria include:

- Radiological surveys,

- Geophysical surveys,

- Documented process knowledge on source and location of release,

- Field observations,

- Experience and data from investigations of similar sites,

- Professional judgment, and

- Field screening results.

\section{Quantitative Data}

Quantitative data measures the quantity or amount of a characteristic or component within the population of interest. These data require the highest level of quality assurance/quality control (QA/QC) in collection and measurement systems because the intended use of the data is to resolve primary decisions, and/or to verify that closure standards have been met. Laboratory analytical data are generally considered quantitative. 


\section{Semiquantitative Data}

Semiquantitative data indirectly measure the quantity or amount of a characteristic or component. Inferences are drawn about the quantity or amount of a characteristic or component because a correlation has been shown to exist between the indirect measurement and the results from a quantitative measurement. The QA/QC requirements on semiquantitative collection and measurement systems are high but may not be as rigorous as a quantitative measurement system. Semiquantitative data contribute to decision making but are not sued alone to resolve primary decisions. Field-screening data are generally considered semiquantitative. The data are often used to guide investigations toward quantitative data collection.

\section{Qualitative Data}

Qualitative data identify or describe the characteristics or components of the population of interest. The QA/QC requirements are the least rigorous on data collection methods and measurement systems. The intended use of the data is for information purposes, to refine conceptual models, and to guide investigations rather than resolve primary decisions. This measurement of quality is typically assigned to historical information and data where QA/QC may be highly variable or not known. Professional judgment is often used to generate qualitative data.

\section{Hold Points}

Hold points will be designed into the investigation and closure activities for CAU 484. Hold points are designed to allow decision makers to review the existing data and decide which of the available options are most suitable. Hold points include the review of geophysical and radiological data and field observations for selection of targets for investigation, selection of corrective actions. The major hold points for this project have been identified and are discussed in Section 1.4

\subsection{List Types of Contaminants of Potential Concern and Affected Media}

It is expected that DU and beryllium fragments may be found at several locations on the dry lake surfaces (Surface Detonation Debris CSM). The most likely locations where these materials may be found are within the following CASs:

- TA-52-001-TANL, NEDS Detonation Area

- TA-52-004-TAAL, Metal Particle Dispersion Test

- TA-52-006-TAPL, Depleted Uranium Site

Depleted uranium and/or inert debris may be found on the surface and/or shallow subsurface at several locations on the dry lake and other testing locations on the TTR (Surface-Shallow Subsurface Debris CSM). The most likely locations where these materials may be found are within the following CASs:

- $\quad$ RG-52-007-TAML, Davis Gun Penetrator Test

- $\quad$ TA-52-005-TAAL, Joint Test Assembly DU Sites

\subsection{Identify Potential Sampling Approaches and Appropriate Analytical Methods}

The sampling techniques and analytical methods identified below will be used to resolve the decision rules and confirm the nature and extent of contamination at each CAS. 


\section{Geophysical Surveys}

Electromagnetic surveys will be used to determine the presence and lateral extent of areas containing surface or near-surface metallic objects/debris. Geophysical surveys followed standard procedures for the instrumentation used. The initial geophysical method used to survey CAS locations will be surveys with a metal detector, however additional geophysical methods may be used as needed to better refine the CAS boundaries and/or locations.

\section{Radiological Survey}

Radiological surveys will be used to determine the presence and lateral extent of radiological contamination. The initial survey will be completed using a truck mounted gamma ray spectrometer system reporting, as a minimum, gross counts for meta-stable Protactinium-234. Additional radiological survey methods may be used as needed to complete the corrective action investigation. Data will be collected and reduced daily in order to provide data in near real-time for QA and survey operational decisions. The initial survey will be conducted at an instrument height of 71 centimeters (28 inches) above the ground surface with an expected sensitivity of 10 milliCuries (approximately 30 grams or 1.5 cubic centimeters of DU). The instrument height may be lowered to approximately 35.5 centimeters (14 inches) above the ground surface for an expected sensitivity of 1 milliCurie (approximately 3 grams or 0.15 cubic centimeters of DU).

\section{Radiological Field Screening}

Based on site conditions and available data, field-screening activities may be conducted for alpha and beta/gamma radiation. A handheld radiological survey instrument or method may be used, based on the possibility that radiologically contaminated soil/debris may be present. If determined appropriate, on-site gamma spectrometry may also be used to screen samples.

Field screening techniques may be used during the Decision I and II sampling activities. These field-screening techniques will provide semiquantitative data that can be used to guide potential confirmatory sampling and waste management activities.

\section{Soil Sampling}

Hand sampling, auguring, direct push, excavation, drilling, or other appropriate sampling methods may be used to collect soil samples. Sample collection and handling activities will only be conducted in accordance with approved procedures. Soil samples will be collected (from CASs within the Surface Detonation Debris CSM) from biased and random locations after potential target areas have been determine from the geophysical and radiological survey data.

\subsection{DEFINE THE STUDY BOUNDARIES (Step 4)}

Specify the time periods and spatial area to which decisions will apply. Determine when and where data should be collected. The purpose of this step is to define the target population of interest, specify the spatial and temporal features of that population that are pertinent for decision making, determine practical constraints on data collection, and define the scale of decision making relevant to target populations for Decision I and Decision II. 


\subsubsection{Define the Geographic Area Within Which all Decisions Must Apply}

CAU 484 has been defined based on the historical data collected during previous investigations. The area of the surveys will include Brownes Lake, Pedro Lake, the northern half of Antelope Lake, and the northern half of NEDS Lake. Former test locations at Myers Ridge, Sidewinder Tuff, and Mt. Helen are also included in CAU 484.

\subsubsection{Specify the Characteristics that Define the Population of Interest}

The population of interest is all of the survey areas. The debris may include material found on the surface or buried near-surface, as identified by geophysical and radiological methods and/or visual inspection of the survey area.

\subsection{Define the Time Frame of the Decision}

\subsubsection{Determine the Time Frame to Which the Study Data Apply}

The study data should be relevant with the length of time allowed for by the SAFER process under the FFACO agreement. The decisions will be based on the documentation and data collection activities planned for 2004 and combined with the planned surveys to determine the proper recommendations for each of the CASs.

\subsubsection{Determine When to Collect Data}

Data collection activities are scheduled to begin in fiscal year 2004 and closure activities will be completed after approval of the final SAFER Plan. Data will be collected at times that meet the security and safety constraints of the TTR site, and at times when weather conditions that allow adequate site access and safe working conditions. A tentative schedule of activities for the completion of CAU 484 is presented in the SAFER Plan.

Radiological screening will be used to detect radiation above free-release criteria before any debris is removed off-site. There will be no other data collection, unless staining or other evidence of the presence of COPC or potential environmental impact is visibly detected and is determined to be related to CAU 484.

\subsubsection{Define Relevant Time Constraints}

- The final SAFER Plan is due to the U.S. Department of Energy, National Nuclear Security Administration Nevada Site Office by May 10, 2004.

- The FFACO deadline for the SAFER Plan is September 30, 2004.

- Fieldwork will be tentatively scheduled to begin during fiscal year 2005.

\subsection{Identify Any Practical Constraints on Data Collection}

1. Approval of the SAFER Plan and the DQO process by the NDEP.

2 Equipment access and mobility at the TTR.

3. Meteorological events that may impact fieldwork activities.

4. Health and safety of workers.

5. Operational/Security issues at the TTR.

6. Unforeseen conditions including unexploded ordnance and other unsafe working conditions. 


\subsection{DEVELOP A DECISION RULE (Step 5)}

Define the parameter of interest, specify the action level, and integrate the previous DQO inputs into a single statement that describes the logical basis for choosing among alternative actions. This step integrates outputs from the previous steps, with the inputs developed in this step into a decision rule ('If..., then...") statement. This decision rule describes the conditions under which possible alternative actions would be chosen.

\subsection{Decision Rule}

Decision I - If waste is present and/or contamination is present above action levels, then the horizontal and vertical extent will be determined.

Decision II - If waste or contamination is present above action levels, and it is technically feasible to clean close the site, then the site will be clean closed.

If contamination is inconsistent with the CSM or extends beyond the identified CAS boundaries, work will be suspended and the investigation strategy will be reevaluated. If contamination is consistent with the CSM and is within CAS boundaries, the decision will be to define its extent.

\subsection{Specify the Action Level or Preliminary Action Level for the Decision}

Sufficient process knowledge and site surveys exist to support the CSMs. Beryllium and DU are the only COPCs within CAU 484. The most recently approved preliminary remediation goal (PRG) for industrial soils will be used as the action level for beryllium (EPA, 2002). As any DU detected at the sites will likely be in the form of discrete pieces, a hot-spot cleanup approach will be used. Depleted uranium pieces detected during the surveys will be removed as localized hotspots from which the DU and the soil immediately surrounding it will be removed for disposal. Closure will be verified using field survey methods. An approach similar to that used to characterize, remove, and verify cleanup of DU at CAU 425 will be utilized at CAU 484 (NNSA/NSO, 2003). Soil samples may be collected to confirm depleted uranium concentrations and to verify closure activities. Depleted uranium analytical results will be compared to the recently approved action level of 60 picoCuries per gram which is based on a 15 mrem per year dose limit described in Nation Council on Radiation Protection report number 129 (NCRP 1999).

\subsection{SPECIFY TOLERABLE LIMITS ON DECISION ERRORS (Step 6)}

Define the decision makers' tolerable decision error rates based on a consideration of the consequences of making an incorrect decision.

\subsection{Decision Errors}

False Negative (Rejection) Decision Error (Is waste/contamination determined not to be present when it actually is present?)

False Positive Decision Error (Is waste/contamination determined to be present when it is actually not present?) 


\subsection{COPC Models}

Models have been developed to characterize the COPCs for CASs within CAU 484. The models contain assumptions and statistical methodologies as appropriate to achieve the investigation/closure objectives. Table 1 summarizes the COPCs expected at CAU 484.

TABLE 1 - CAU 484 WASTE AND COPC DESCRIPTION

\begin{tabular}{|c|c|c|c|c|c|}
\hline \multirow{2}{*}{ CAS } & \multirow{2}{*}{$\begin{array}{c}\text { CAS } \\
\text { Description }\end{array}$} & \multicolumn{2}{|c|}{\begin{tabular}{|c|}
$\begin{array}{c}\text { Contaminants of } \\
\text { Potential Concern }\end{array}$ \\
\end{tabular}} & \multirow{2}{*}{$\begin{array}{c}\text { Impacted/Non } \\
\text {-Impacted } \\
\text { Debris }\end{array}$} & \multirow{2}{*}{$\begin{array}{l}\text { Contaminant/Waste } \\
\text { Description }\end{array}$} \\
\hline & & \begin{tabular}{|l|} 
Depleted \\
Uranium
\end{tabular} & Beryllium & & \\
\hline \begin{tabular}{|l} 
RG-52-007- \\
TAML
\end{tabular} & \begin{tabular}{|c|} 
Davis Gun \\
Penetrator Test
\end{tabular} & Unknown & N/A & Unknown & $\begin{array}{l}\text { DU/inert debris on the } \\
\text { ground surface or } \\
\text { shallow subsurface }\end{array}$ \\
\hline $\begin{array}{l}\text { TA-52-001- } \\
\text { TANL }\end{array}$ & $\begin{array}{c}\text { NEDS } \\
\text { Detonation } \\
\text { Area } \\
\end{array}$ & Yes & Yes & N/A & $\begin{array}{l}\text { DU and beryllium } \\
\text { dispersed on the ground } \\
\text { surface }\end{array}$ \\
\hline $\begin{array}{l}\text { TA-52-004- } \\
\text { TAAL }\end{array}$ & \begin{tabular}{|c|} 
Metal Particle \\
Dispersion \\
Test
\end{tabular} & Yes & Yes & N/A & $\begin{array}{l}\text { DU and beryllium } \\
\text { dispersed on the ground } \\
\text { surface }\end{array}$ \\
\hline \begin{tabular}{|l} 
Ta-52-005- \\
TAAL
\end{tabular} & \begin{tabular}{|c|} 
Joint Test \\
Assembly DU \\
Sites \\
\end{tabular} & Yes & N/A & N/A & $\begin{array}{l}\text { DU rings on the ground } \\
\text { surface or shallow } \\
\text { subsurface }\end{array}$ \\
\hline \begin{tabular}{|l} 
TA-52-006- \\
TAPL
\end{tabular} & $\begin{array}{c}\text { Depleted } \\
\text { Uranium Site } \\
\text { (Colimbo) }\end{array}$ & Yes & Yes & Unknown* & $\begin{array}{l}\text { DU and beryllium } \\
\text { dispersed on the ground } \\
\text { surface } \\
\text { *An unconfirmed sealed } \\
\text { cobalt radiological } \\
\text { source may be present } \\
\text { at the site }\end{array}$ \\
\hline $\begin{array}{l}\text { TA-54-001- } \\
\text { TANL }\end{array}$ & \begin{tabular}{|c|}
$\begin{array}{c}\text { Tank and Steel } \\
\text { Structure }\end{array}$ \\
\end{tabular} & N/A & N/A & Yes & $\begin{array}{l}\text { Debris on the ground } \\
\text { surface }\end{array}$ \\
\hline
\end{tabular}

\subsubsection{Beryllium Statistical Model}

Statistical model applies to potential beryllium contamination within the Surface Detonation Debris CSM sites. The EPA document Guidance for Choosing a Sampling Design for Environmental Data Collection (EPA, 2000a) defines the methodology suggested to determine the sufficient number of samples to be collected to ensure a 95 percent confidence level in the COPC concentration. This methodology has been used to determine the number of sampling locations required at sites potentially impacted by beryllium in CAU 484. The number of samples required may be determined using the following equation:

$\mathrm{n}=\mathrm{t}_{\alpha}{ }^{2}(\mathrm{CV})^{2} / \mathrm{p}^{2}$ 
Where:

As the CV increases at a set margin of error, the number of samples required increases. When the variability is low relative to the mean of the data, the $\mathrm{CV}$ is also low. However, as the variability in the population begins to increase relative to the mean of the data, the CV will increase and the number of required samples will increase if characterization of the site at a 95\% confidence level and a set margin of error is desired. The value of $t \alpha^{2}$ increases as the number of samples decreases. Therefore, t $\alpha 2$ is inversely proportional to the number of samples collected. The minimum number of samples required to ensure a 95-percent confidence level is also directly proportional to the coefficient of variation $(\mathrm{CV})$ in the concentration of the COPCs. The $\mathrm{CV}$ is a quantification of the amount of internal fluctuation in the concentration from sample to sample. It is an absolute measure of the amount of internal variation in the concentration data and does not, to a first approximation, depend on the number of samples collected. The margin of error may be obtained by dividing the precision wanted by the known or anticipated mean concentration of the specific COPC. Upon completion of the soil sampling effort, the data obtained for the COCs is reviewed. It can then be determined if an adequate number of samples were collected with respect to the margin of error and confidence selected during the planning process. This determination is completed by calculating the CV using the data obtained during the study. The standard deviation of the concentration for a COC is divided by the mean concentration to calculate the CV. This CV may be higher or lower than the CV selected during the planning process. Using this $\mathrm{CV}$ value, the same equation is used to determine the required number of samples based on the actual CV for the study. If this second value for " $n$ " is less than or equal to the number of samples collected during the study, then the site has been characterized for extent of COCs within the limits of confidence and error stated. If the second value for " $n$ " is significantly greater, then additional sampling is necessary, or an adjustment to the margin of error or confidence level should be considered. If the collection of additional samples is deemed necessary, the data that has been generated may be used to plan for a more efficient and cost-effective re-sampling of the site. Areas of the site where higher than anticipated variabilities were obtained may be segregated from areas of lower variability (stratified design). A recalculation of the number of samples required to characterize each strata should then be completed and resampling may proceed.

Beryllium and isotopic uranium analytical data collected from CAU 529 (Area 25 Contaminated Materials) CAS 25-23-17 (Contaminated Wash) will be used to calculate an estimate of the number of samples needed for CAU 484. Soil samples for beryllium and DU analysis will be collected concurrently; therefore, the most conservative CV within the CAU 529 beryllium and DU data will be used to estimate the number of samples to be collected at the Surface Detonation Debris Sites in CAU 484. CAU 529 has been selected because contamination at this site was the result of a detonation of a test article containing similar material to that used during testing activities at the Surface Detonation Debris sites within CAU 484. Analytical data from samples collected at CAU 484 sites will be statistically analyzed and evaluated to confirm a sufficient number of samples have been collected and data meets project objectives. 
Due to the nature of the event (detonation of a test article from a point source) beryllium concentrations are assumed to be normally distributed on the ground surface with the greatest concentration occurring at and near the point source and decreasing in all directions away from the point source. The sampling plan will include a combination of biased and random sample locations. Random sample locations will be selected using a grid and a random number generator. Surface and shallow subsurface soil samples will be collected to document the lateral and vertical extent of beryllium contamination. If beryllium is detected above the PRG in any sample, step-out sample locations will be established and additional samples will be collected for laboratory analysis.

\subsubsection{Depleted Uranium Model}

The DU model applies to sites within the Surface Detonation Debris and Surface-Shallow Subsurface Debris CSMs. Antelope, Brownes, Pedro and NEDS Lake beds will be surveyed using geophysical and radiological equipment with a coverage exceeding 90 percent of the study area.

\subsubsection{Surface Detonation Debris}

Due to the nature of the event (detonation of a test article from a point source) DU debris is assumed to be normally distributed as discrete pieces on the ground surface, with the greatest concentration occurring at and near the point source of detonation and decreasing in all directions away from the point source. Conditions similar to those encountered at CAU 529 CAS 25-23-17 (Contaminated Wash) are expected at sites within the surface detonation debris CSM. The source of contamination is similar in that events at all locations are related to the detonation of a test article containing uranium and beryllium. Uranium detected during the investigation of CAU 529 CAS 25-23-17 (Contaminated Wash) consisted of discrete pieces as is expected at the sites within CAU 484. Locations containing DU within this CSM will be identified and verified using field survey methods. While field screening will be the primary tool for confirming removal of depleted uranium, soil samples may be collected to verify depleted uranium concentrations and closure objectives. Soil sampling will be based on the methods described for the beryllium statistical model. Cleanup of DU will be based on the approach discussed in Section 5.2.

\subsubsection{Surface-Shallow Subsurface Debris}

Depleted uranium at sites within this CSM may contain debris and/or intact test articles on the ground surface or shallow subsurface. Depleted uranium present at these sites is assumed to consist of discrete pieces and may only have impacted the soil/media in contact with the DU. Locations containing DU within this CSM will be identified and verified using field survey methods. Cleanup will be based on the approach discussed in section 5.2.

\subsection{Confirmation of Corrective Actions}

Sites selected for clean closure will require the collection of data to support and verify that corrective actions are complete. Beryllium analytical data collected during the CAI will be used as input to the equation presented in Section 6.2.1 to determine the minimum number of samples required to verify the closure objectives. Beryllium verification sample results will be compared to the most current PRG at the time of the cleanup. As any DU detected is assumed to be discrete pieces, the pieces and associated soil will be removed and clean closure of sites 
impacted with DU will be verified using field-survey methods (similar to DU cleanup completed at CAU 425 [NNSA/NSO, 2003]).

\subsection{OPTIMIZE THE DESIGN (Step 7)}

Evaluate information from the previous steps and generate alternative data collection designs. Choose the most resource-effective design that meets all DQOs. This section presents an overview of the resource-effective strategy planned to obtain the data required to meet the project DQOs developed in previous steps. As additional data or information is obtained, this step will be reevaluated and refined, if necessary, to reduce uncertainty and increase the confidence that the SAFER CAI has met its intended goals.

\subsection{Develop General Sampling and Analysis Design Alternatives}

Assumptions for the beryllium statistical model are presented in Section 6.2.1. Data collected during the investigation of a similar corrective action site will be used to make an estimation of the number of samples that need to be collected at sites within the surface detonation debris conceptual site model. This is necessary since no analytical data are available from the sites within CAU 484 on which to make a determination. After the CAU 484 CAI is complete, the beryllium and/or depleted uranium data will be analyzed to confirm that sufficient samples were collected to characterize the site at the 95-percent confidence level.

A combination of biased and random samples will be collected to verify the horizontal and vertical extent of potential beryllium contamination for sites within the Surface Detonation Debris CSM. One sample will be collected from the central area of the detonation and one sample will be collected from four locations some distance from the central area of the detonation. Additional random samples will be collected as indicated by the output from the equation presented in section 6.2.1. Additional samples may be collected as needed to fill data gaps and to provide data needed to complete the investigation/closure.

Areas on the dry lake beds containing DU will be initially surveyed with a coverage exceeding 90 percent using the Kiwi equipment. This phase of the CAI will be to confirm the locations of CASs within the CAU. Sites identified during this phase to be associated with CAU 484 will be further investigated using field screening methodologies.

\subsection{CAU 484 Closure Decision Process}

Hold/decision points have been included in the process to allow critical data to be reviewed by the parties prior to proceeding with the closure activities. A primary hold point for the CAU 484 closure activities will be the review of geophysical and radiological survey data and the selection of locations representing sites belonging to CAU 484. Due to the broadness of the initial surveys and the historical activities that have been conducted on the dry lake beds at TTR, there is a potential for identifying sites containing waste and/or contamination that are not associated with CAU 484. Only sites determined to be associated with CAU 484 will be addressed within the corrective actions described in these data quality objectives. 


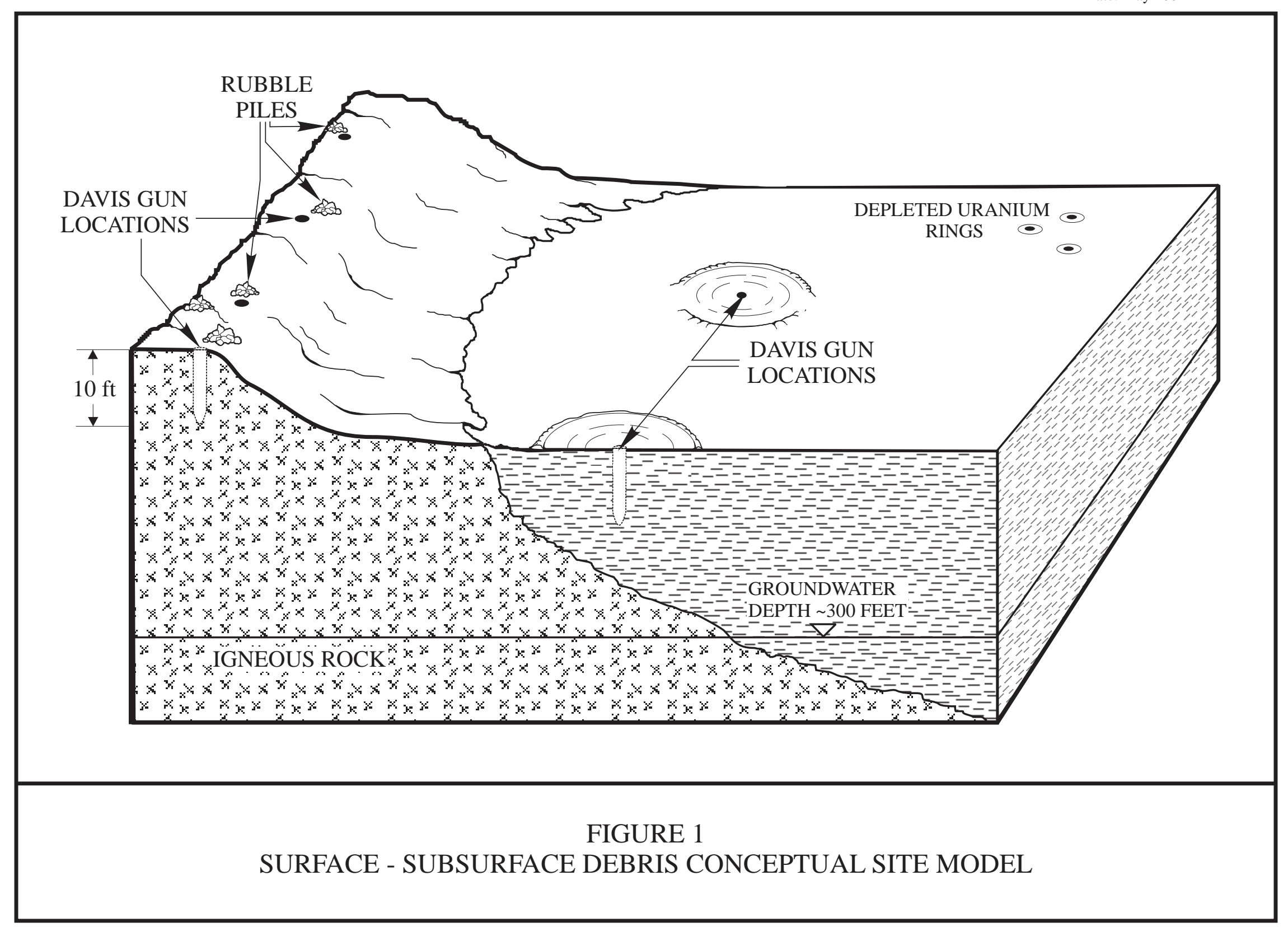




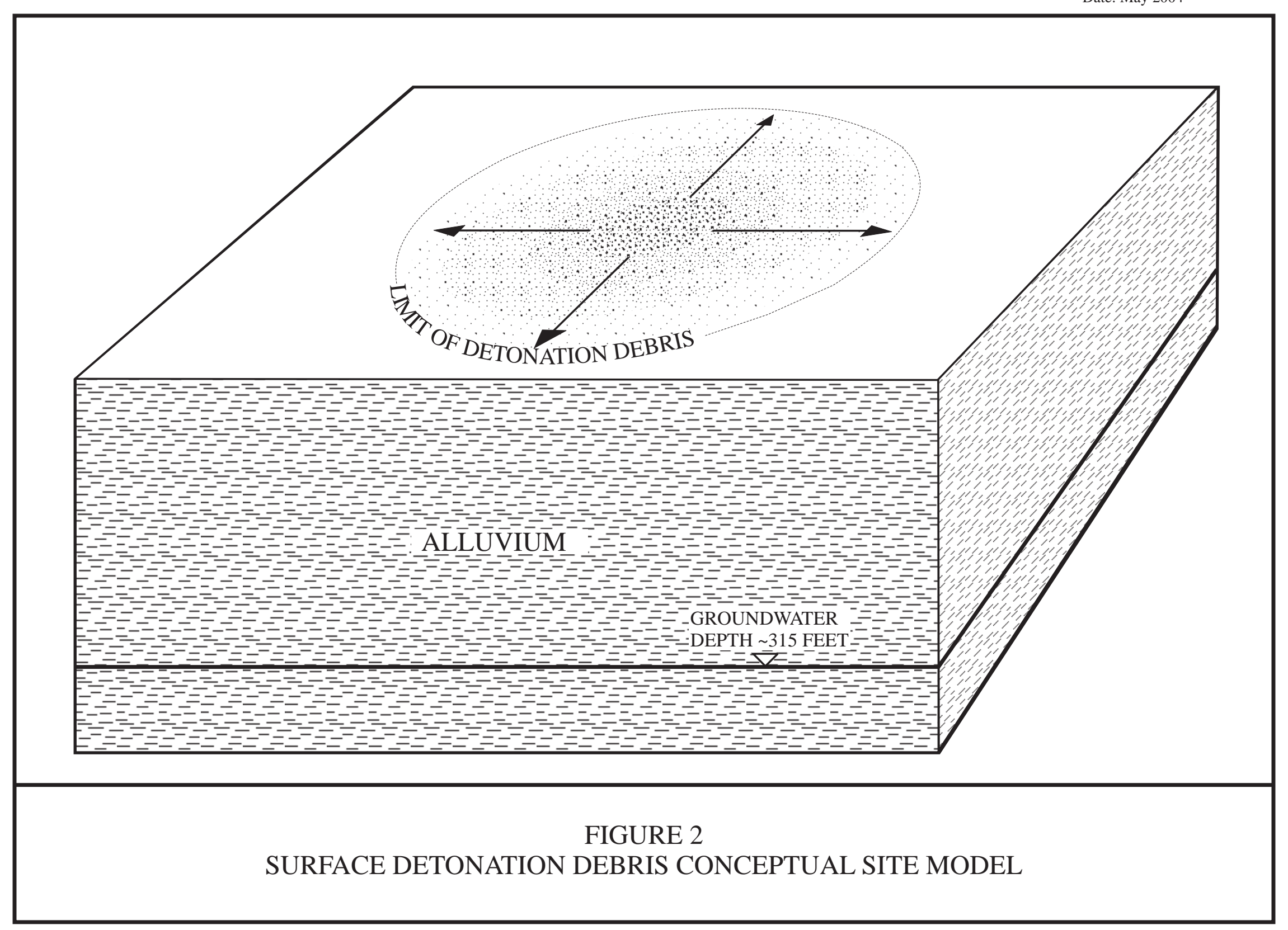




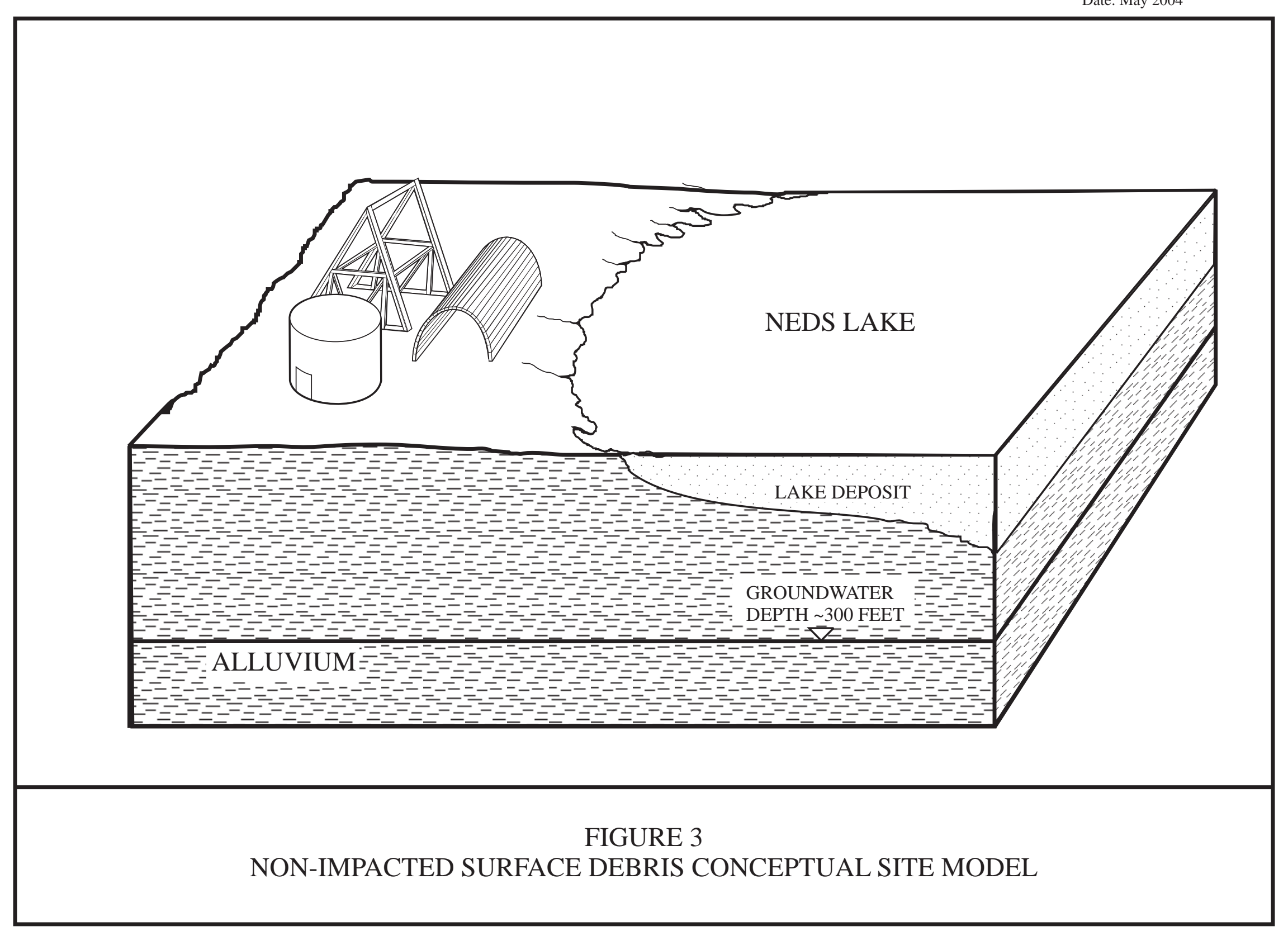




\section{REFERENCES}

EPA, see U.S. Environmental Protection Agency.

NCRP, see National Council on Radiation Protection and Measurements.

NDEP, see Nevada Division of Environmental Protection.

NNSA/NSO, see U.S. Department of Energy, National Nuclear Security Administration Nevada Site Office

National Council on Radiation Protection and Measurements. 1999. Recommended Screening Limits for contaminated Surface Soil and Review of Factors Relevant to Site-Specific Studies. NCRP Report No. 129. National Council on Radiation Protection and Measurement, Bethesda, MD.

Nevada Division of Environmental Protection. 1999. Letter to Runore Wycoff, U.S. Department of Energy Nevada Operations Office. Notice of Completion for Corrective Action Unit 495, Unconfirmed Joint Test Assembly (JTA) Sites, TTR. July 14. Carson City, NV.

U.S. Department of Energy, National Nuclear Security Administration Nevada Site Office. 2003. Closure Report for Corrective Action Unit 425: Area 9 Main Lake Construction Debris Disposal Area, Tonopah Test Range, Nevada, DOE/NV--875. Las Vegas, NV.

U.S. Environmental Protection Agency. 2000a. Guidance for Choosing a Sampling Design for Environmental Data Collection (EPA QA/G-5), Office of Environmental Information, Washington, D.C.

U.S. Environmental Protection Agency. 2000b. Guidance for the Data Quality Objective Process, EPA QA/G-4, Washington, D.C.

U.S. Environmental Protection Agency. 2002. Region IX Preliminary Remediation Goals (PRGs), San Francisco, CA. 
SAFER PLAN - CAU 484

Section: Appendix A1

Revision: 0

Date: May 2004

THIS PAGE INTENTIONALLY LEFT BLANK 


\section{APPENDIX B}

\section{SAMPLE ANALYTICAL RESULTS}

Analytical laboratory summary reports are provided for the following sample analytical results:

Investigation (Geoprobe ${ }^{\circledR}$ ) soil sample analytical results for:

a. Pedro Lake, Background

b. CAS TA-52-001-TANL, NEDS Detonation Area

c. CAS TA-52-006-TAPL, Depleted Uranium Site

d. CAS TA-52-004-TAAL, Metal Particle Dispersion Test

This appendix contains the analytical data summary reports, and not the complete data analytical packages. Page numbers reflect the location of the summary report within the complete package and not their location within this report (i.e., whatever page numbers are shown on the pages within Appendix B do not apply to their location within the appendix). 


\title{
INVESTIGATION (GEOPROBE $\left.{ }^{\circledR}\right)$ SOIL SAMPLE ANALYTICAL RESULTS \\ PEDRO LAKE
}

\author{
Chemical Data
}


1 INORGANIC ANALYSES DATA SHEET
EPA SAMPLE NO.

\section{A}

Contract: 60052

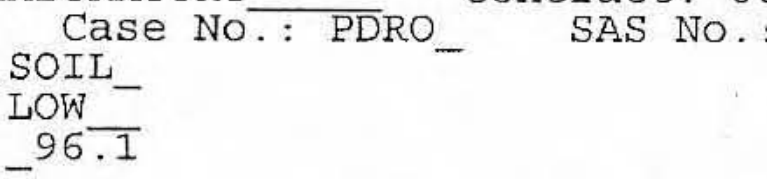

SAS NO. :

Lab Sample ID: 0608L795-001

Date Received: 08/24/06
Lab Code: LVLI

Matrix (soil/wat \% Solids:

Concentration Units (ug/L or $\mathrm{mg} / \mathrm{kg}$ dry weight): MG/KG

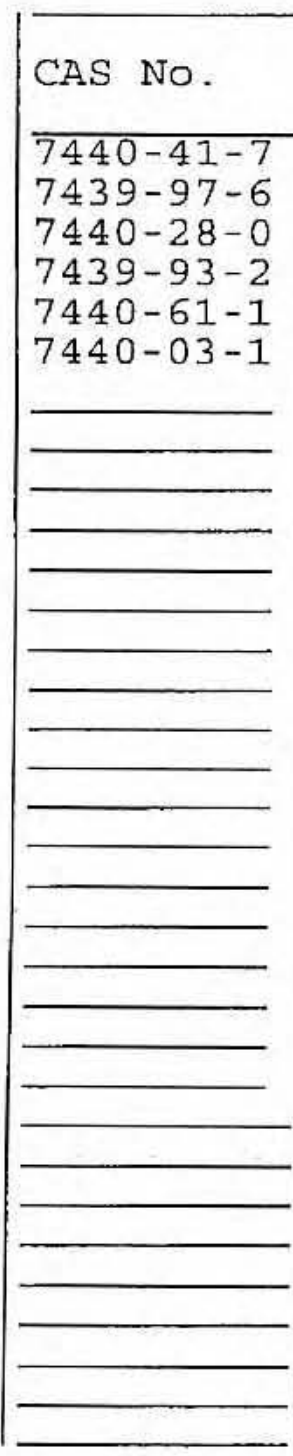

Color Before:

Color After:

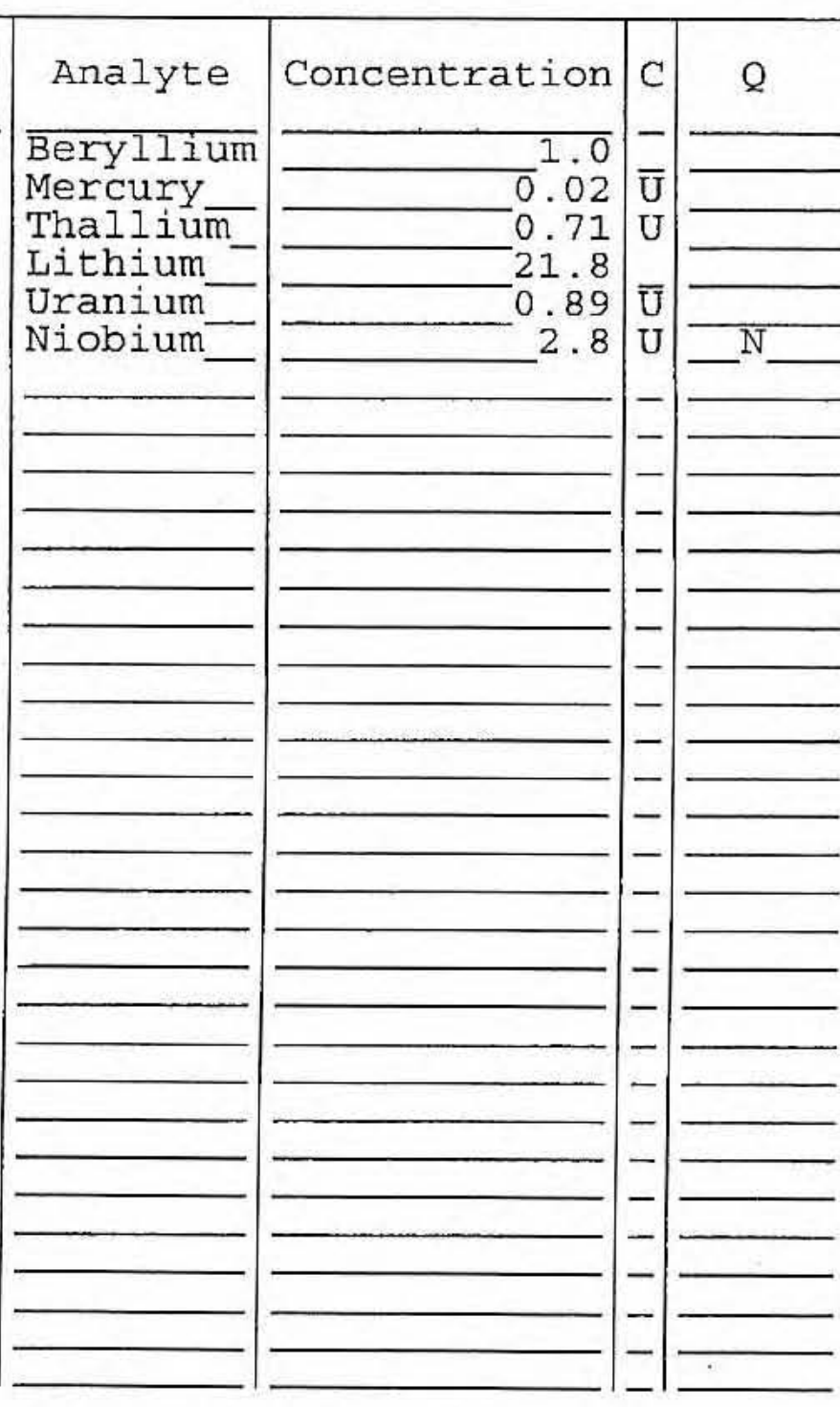

Clarity Before:

Clarity After:

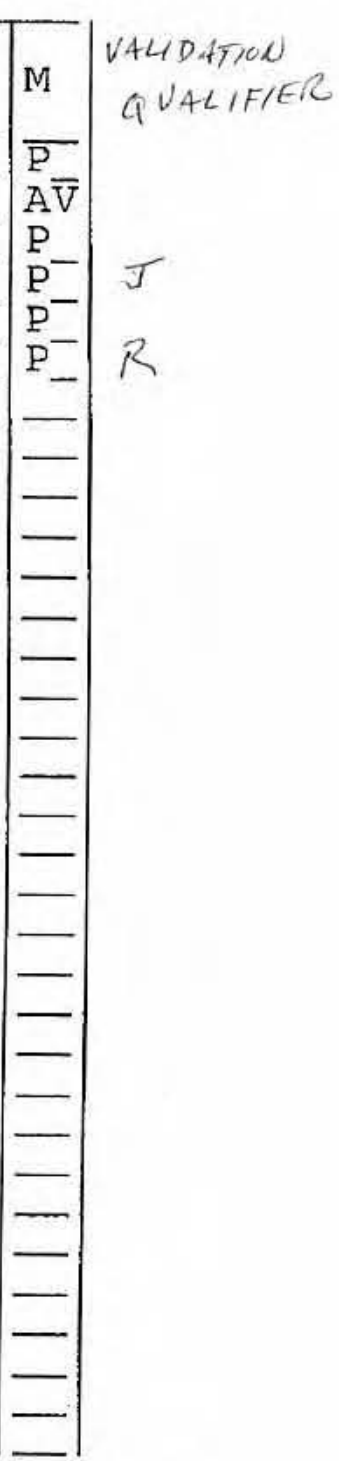

Texture:

Artifacts:

Comments :

PDRO- 01A 
Lab Name: LIONVILIE_LABORATORY Lab Code: LVLI Matrix (soil/water): SOII Level (low/med): \% Solids : LOW $-93 \overline{.6}$
Contract: 60052 SAS NO. : SDG NO : V2734 Lab Sample ID: 0608L795-0002
O1B

Date Received: 08/24/06

Concentration Units (ug/L or mg/kg dry weight) : MG/KG

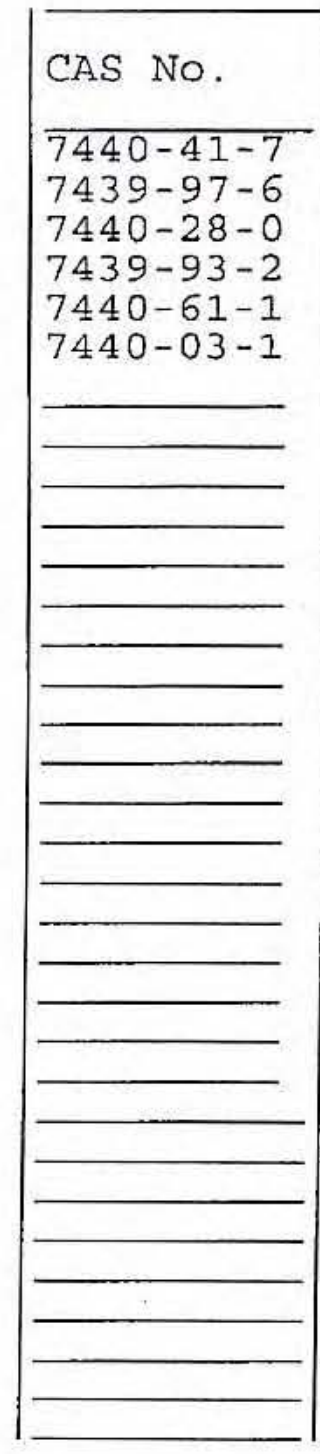

Color Before:

Color After:

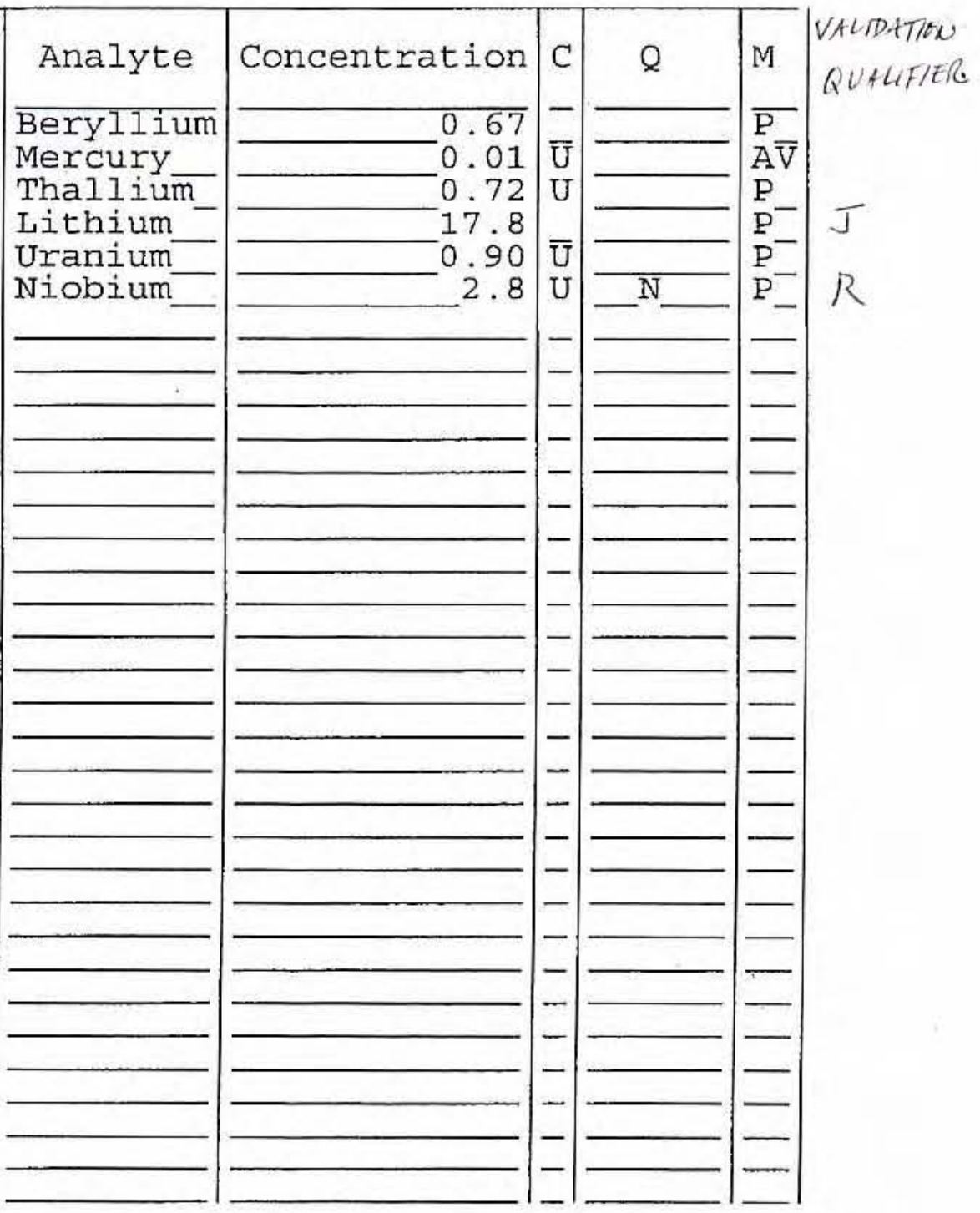

Clarity Before: Clarity After:
Texture: Artifacts :

Comments:

PDRO-O1B 
1

INORGANIC ANALYSES DATA SHEET
Lab Name: LIONVILLE_LABORATORY

Lab Code: LVLI Matrix (soil/water): SOIL Level (low/med): Solids :
Contract: 60052

SAS NO. :

SDG NO.: V2734

Lab Sample ID: 0608L795-0̄03

Date Received: 08/24/06

Concentration Units (ug/L or $\mathrm{mg} / \mathrm{kg}$ dry weight): MG/KG

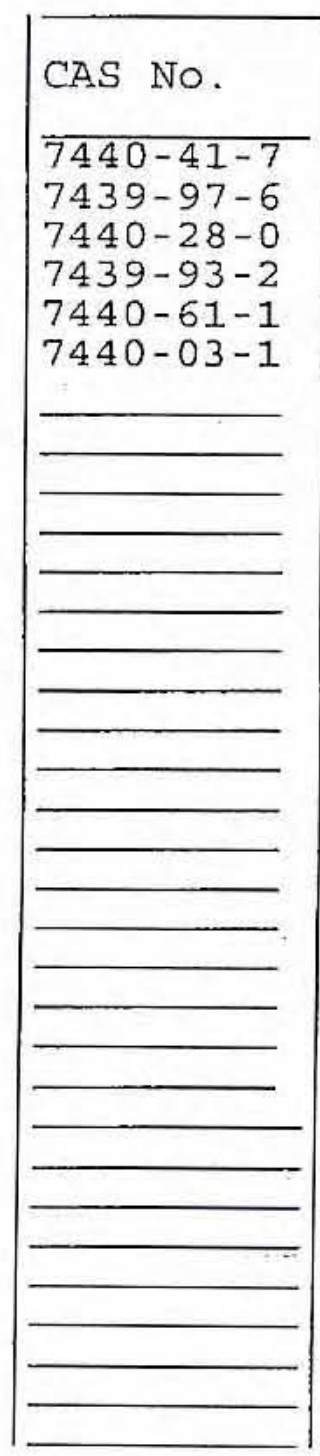

Color Before: Color After:

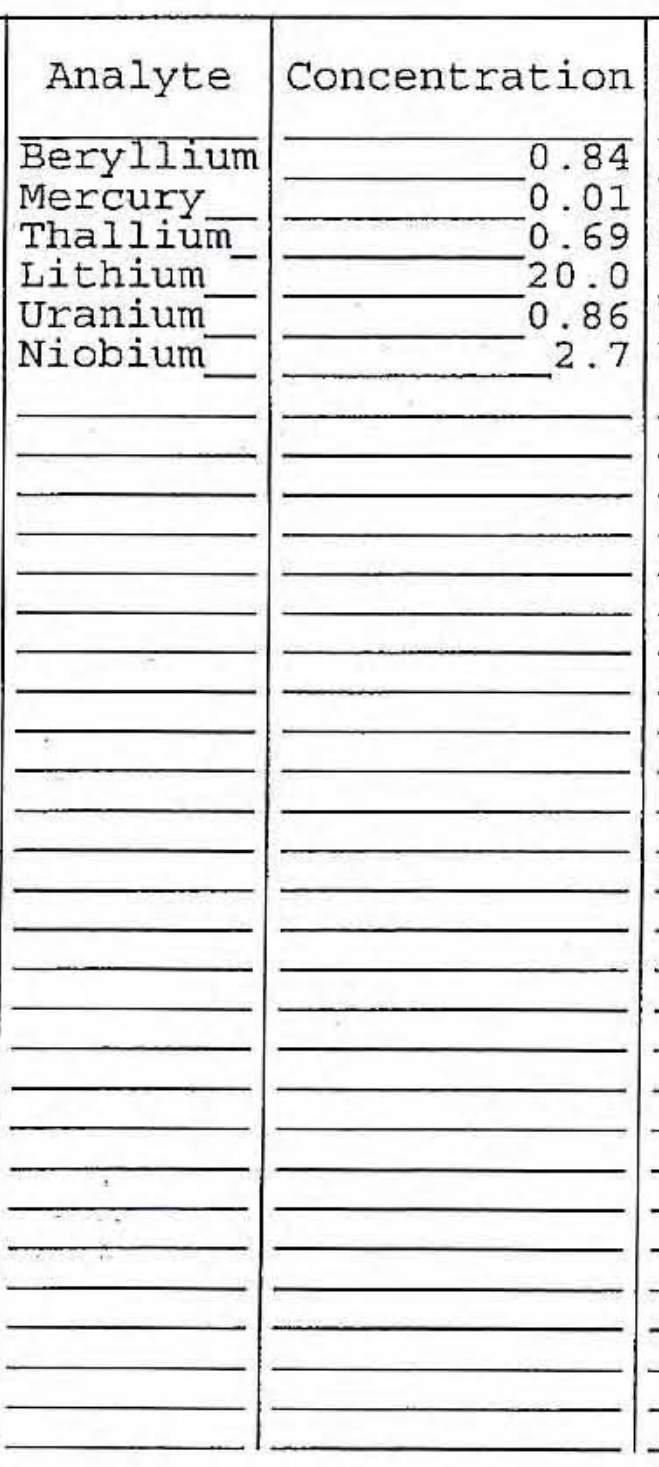

Clarity Before: Clarity After:

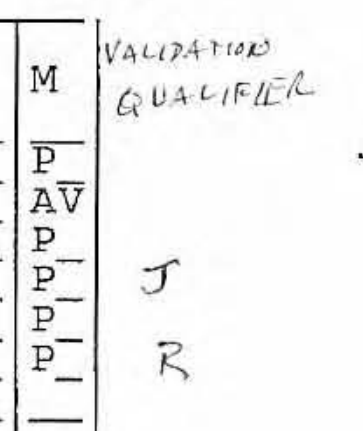

Comments:

PDRO-02A 
1

\section{INORGANIC ANALYYSES DATA SHEET}

EPA SAMPLE NO.

\section{$\mathrm{O} 2 \mathrm{~B}$}

Contract: 60052

SAS No.:

Lab Sample ID: 0608I795-004

Date Received: 08/24/06
Lab Name: IION
Lab Code: IVLI

Matrix (soil/water): soIL

Level (low/med): \% Solids:

$$
\text { LOW }
$$

Concentration Units (ug/L or $\mathrm{mg} / \mathrm{kg}$ dry weight): MG/KG

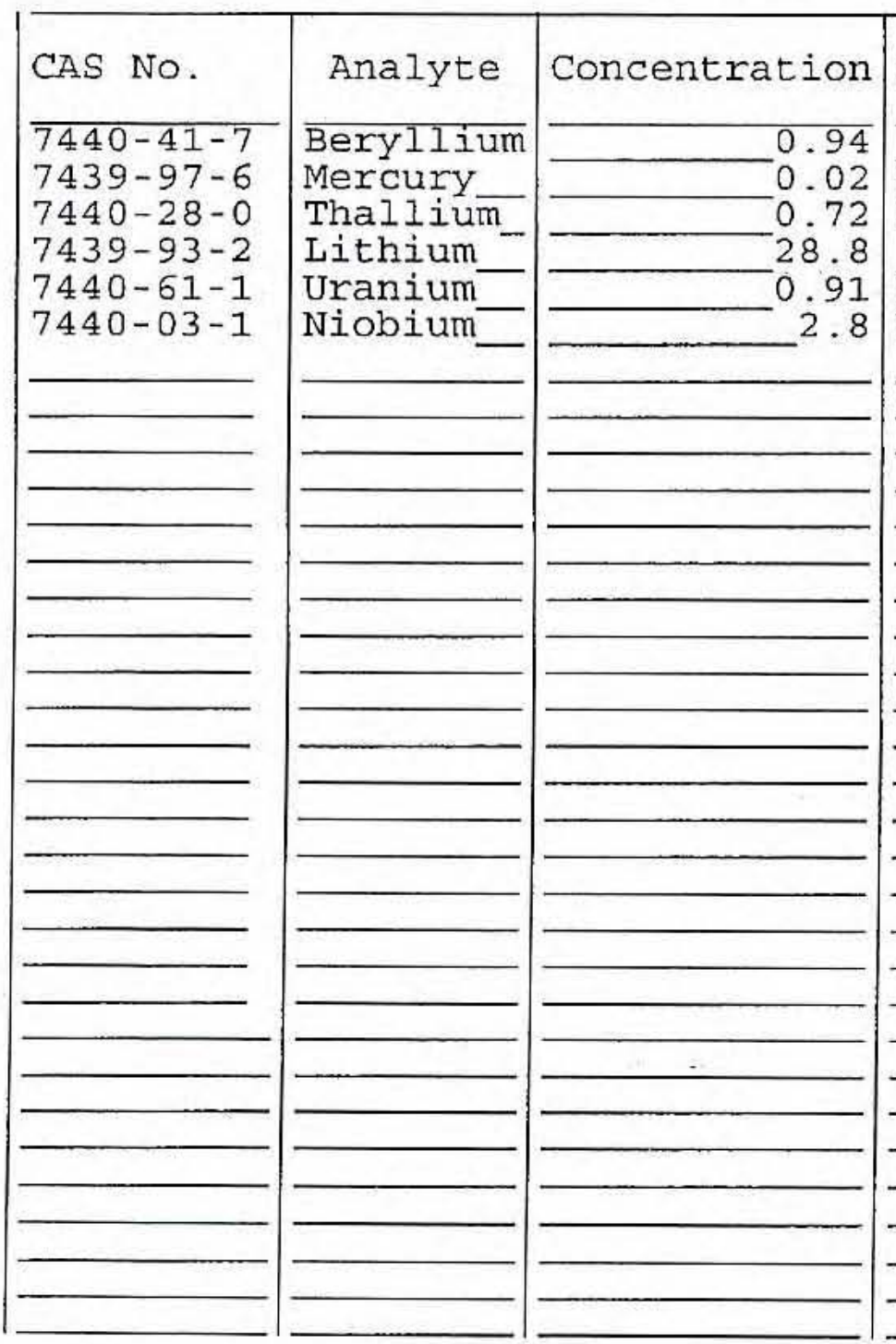

Clarity Before: Clarity After:

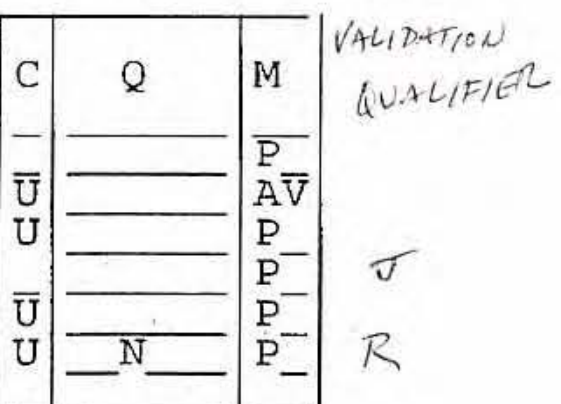

Color Before: Color After:

Comments:

PDRO-02B

Texture:

Artifacts: 
1 INORGANIC ANALYSES DATA SHEET
Lab Name: LIONVILLE_LABORATORY

Lab Code: LVLI

Matrix (soil/water): Level (low/med): Solids :
Contract: 60052 Case NO.: PDRO_ SAS NO.: SOIL_
LOW
96.6
EPA SAMPLE NO.

$$
\text { 03A }
$$

SDG NO.: V2734

Lab Sample ID: 0608L795-005

Date Received: 08/24/06

Concentration Units (ug/L or $\mathrm{mg} / \mathrm{kg}$ dry weight): MG/KG

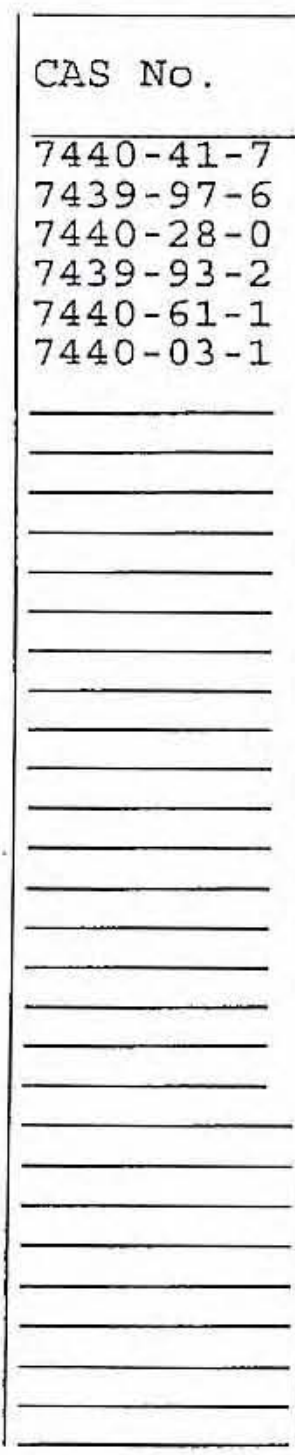

Color Before:

Color After:

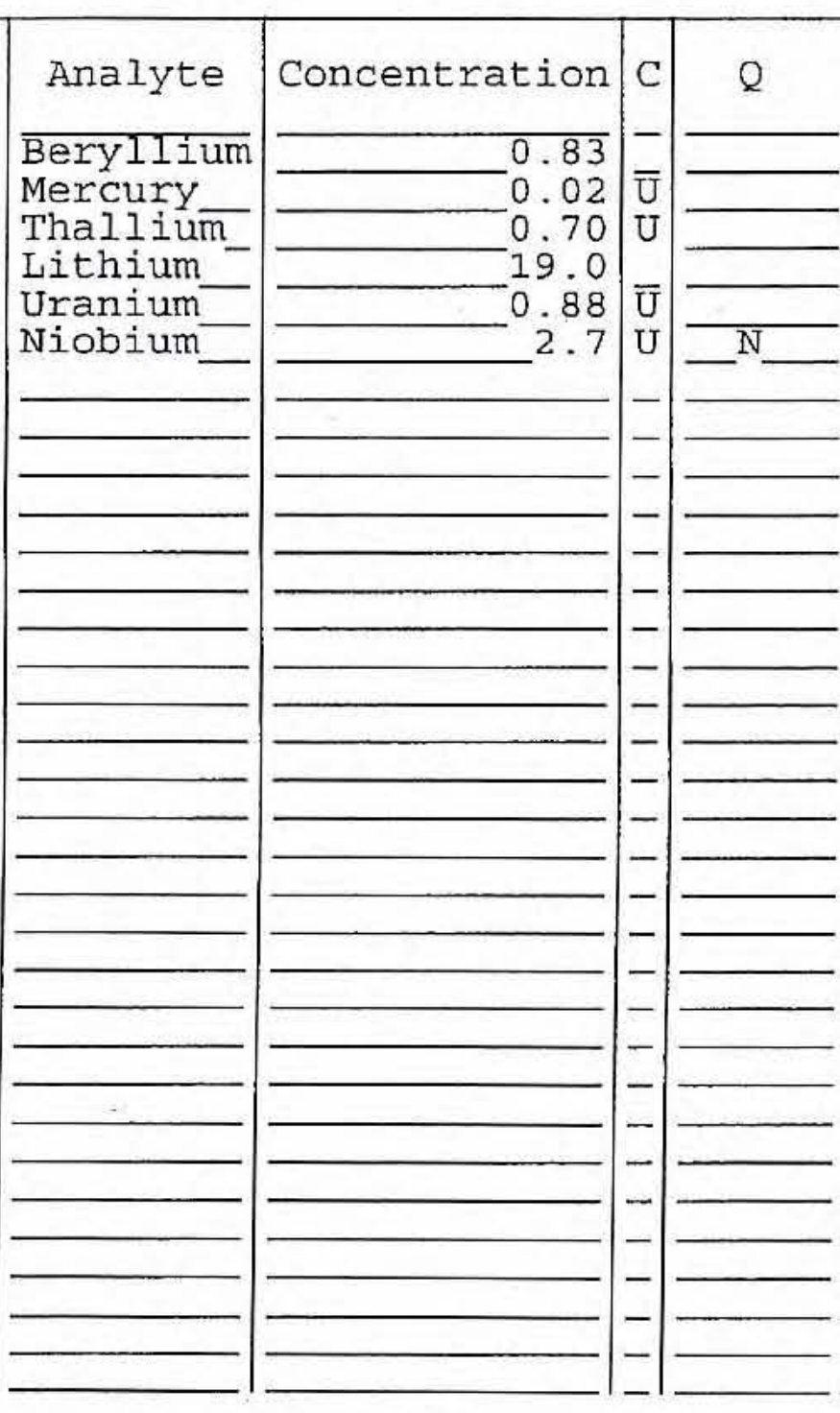

Clarity Before:

Clarity After:

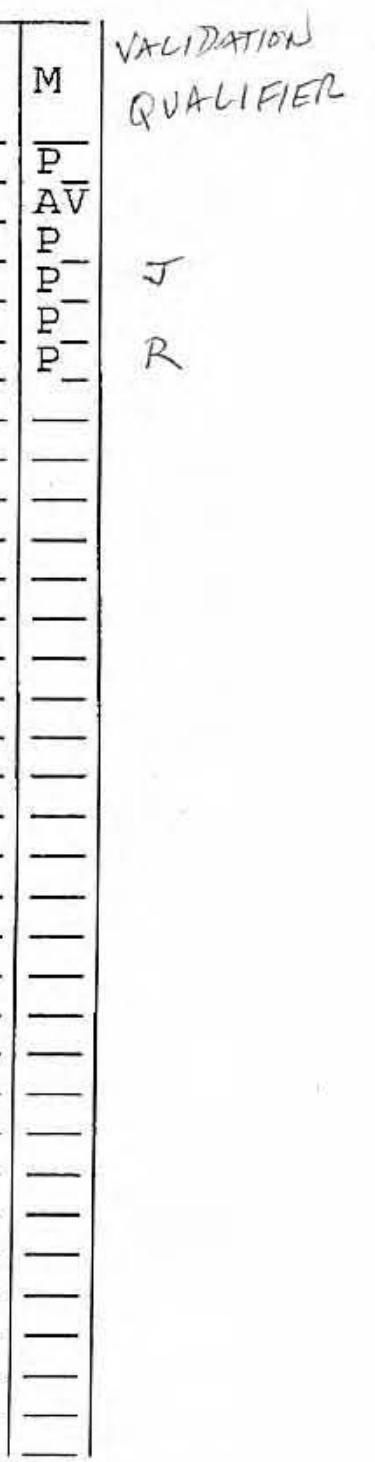

Texture:

Artifacts:

Comments :

PDRO-03A 


\section{U.S. EPA}

\section{INORGANIC ANALYSES DATA SHEET}

EPA SAMPLE NO.

Lab Name: LIONVILLE_LABORATORY

Lab Code: LVLI Matrix (soil/water): SOIL Level (low/med): LOW \% Solids:
Contract: 60052

SAS NO. :

SDG NO.: V2734

Lab Sample ID: 0608L795-0̄06

$-94 \overline{.1}$

Date Received: 08/24/06

Concentration Units (ug/L or $\mathrm{mg} / \mathrm{kg}$ dry weight) : MG/KG

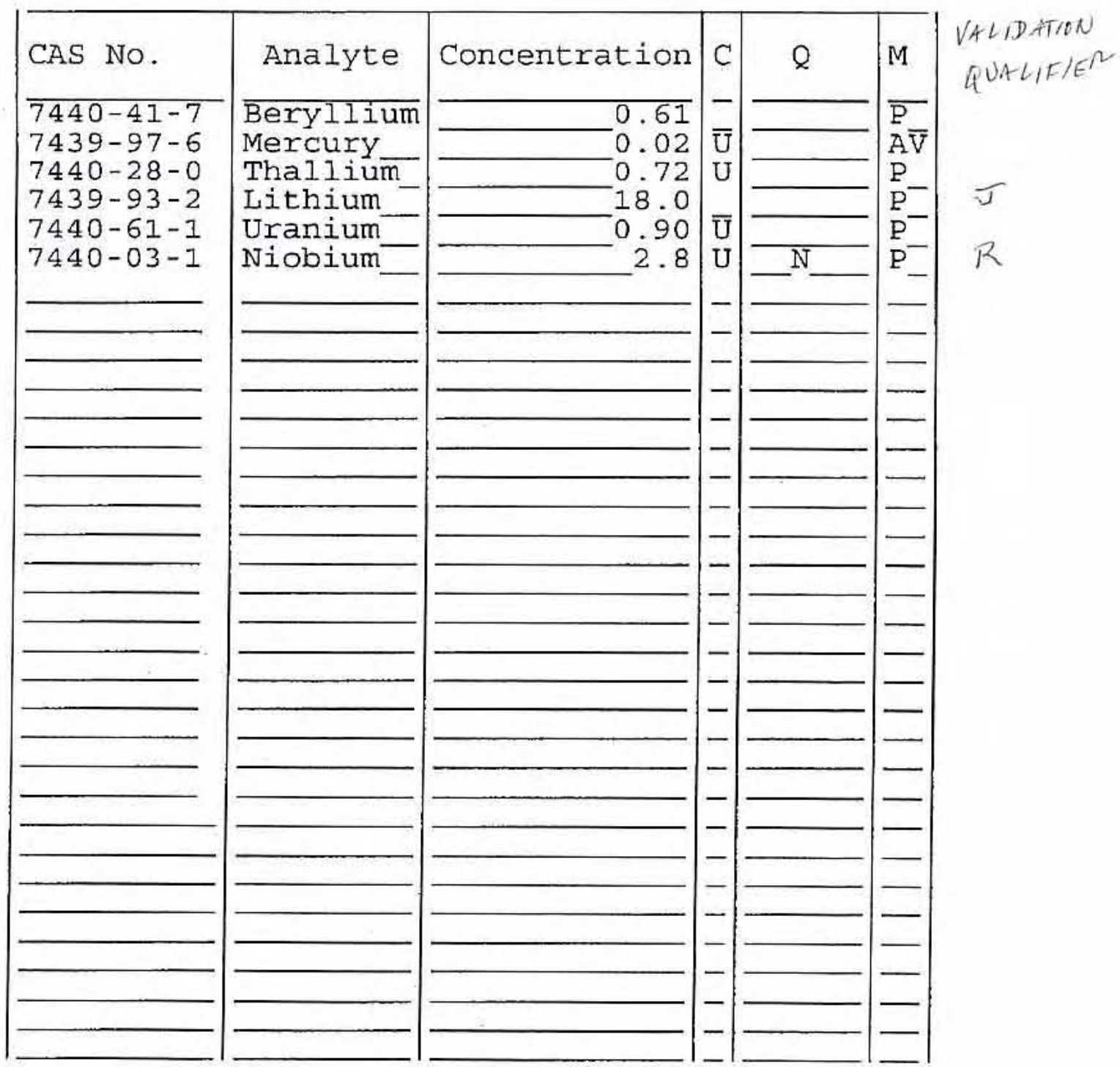

Color Before: Color After:
Clarity Before: Clarity After:
Texture: Artifacts :

Comments :

PDRO-O3B 
1

INORGANIC ANALYSES DATA SHEET
EPA SAMPLE NO.

$04 \mathrm{~A}$

Contract: 60052

SAS No. :

Case No.: PDRO

Lab Code: LVLI

Matrix (soil/water) : SOIL

Level (low/med):

$\div$ Solids:

LOW
-94.4

Lab Sample ID: 0608L795-0̄07

Date Received: 08/24/06

Concentration Units (ug/L or $\mathrm{mg} / \mathrm{kg}$ dry weight): MG/KG

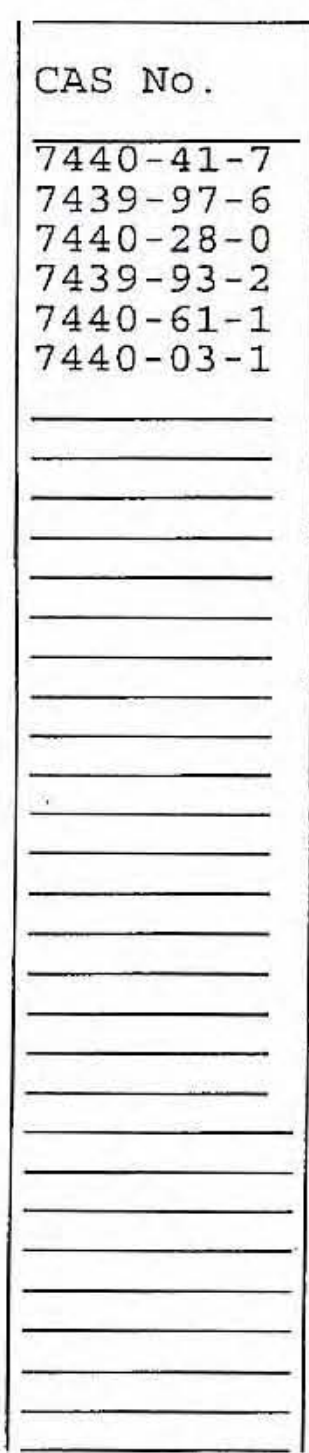

Color Before:

Color After:

Comments :

PDRO-04A

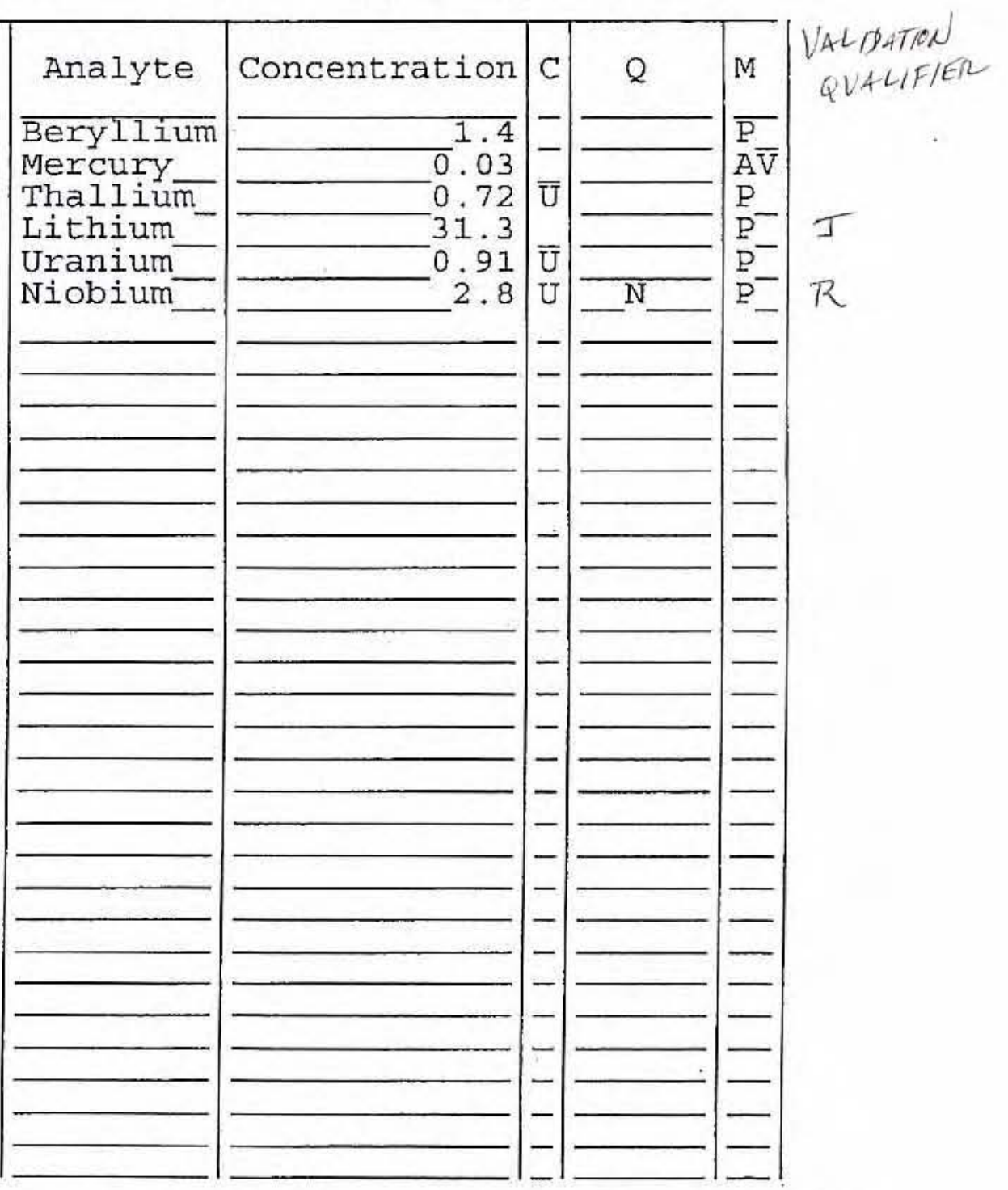

Clarity Before:

Texture:

Artifacts: 
EPA SAMPLE NO.

\section{O4B}

Contract: 60052

SAS NO.:

Lab Sample ID: 0608L795-008

Date Received: 08/24/06
Lab Name: LIONVILLE_LABORATORY

Matrix (soil/water): SoIL

Level (low/med): LOW

Solids :

Concentration Units (ug/L or $\mathrm{mg} / \mathrm{kg}$ dry weight) : MG/KG

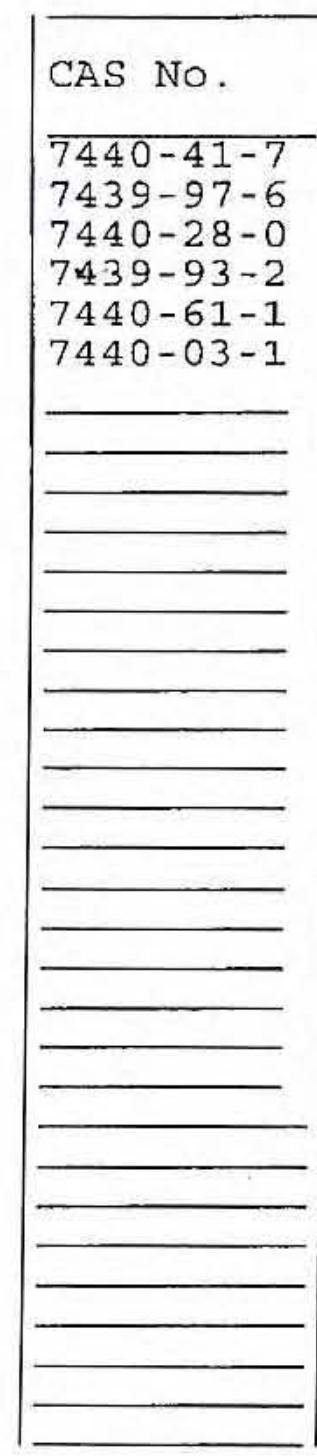

Color Before:

Color After:

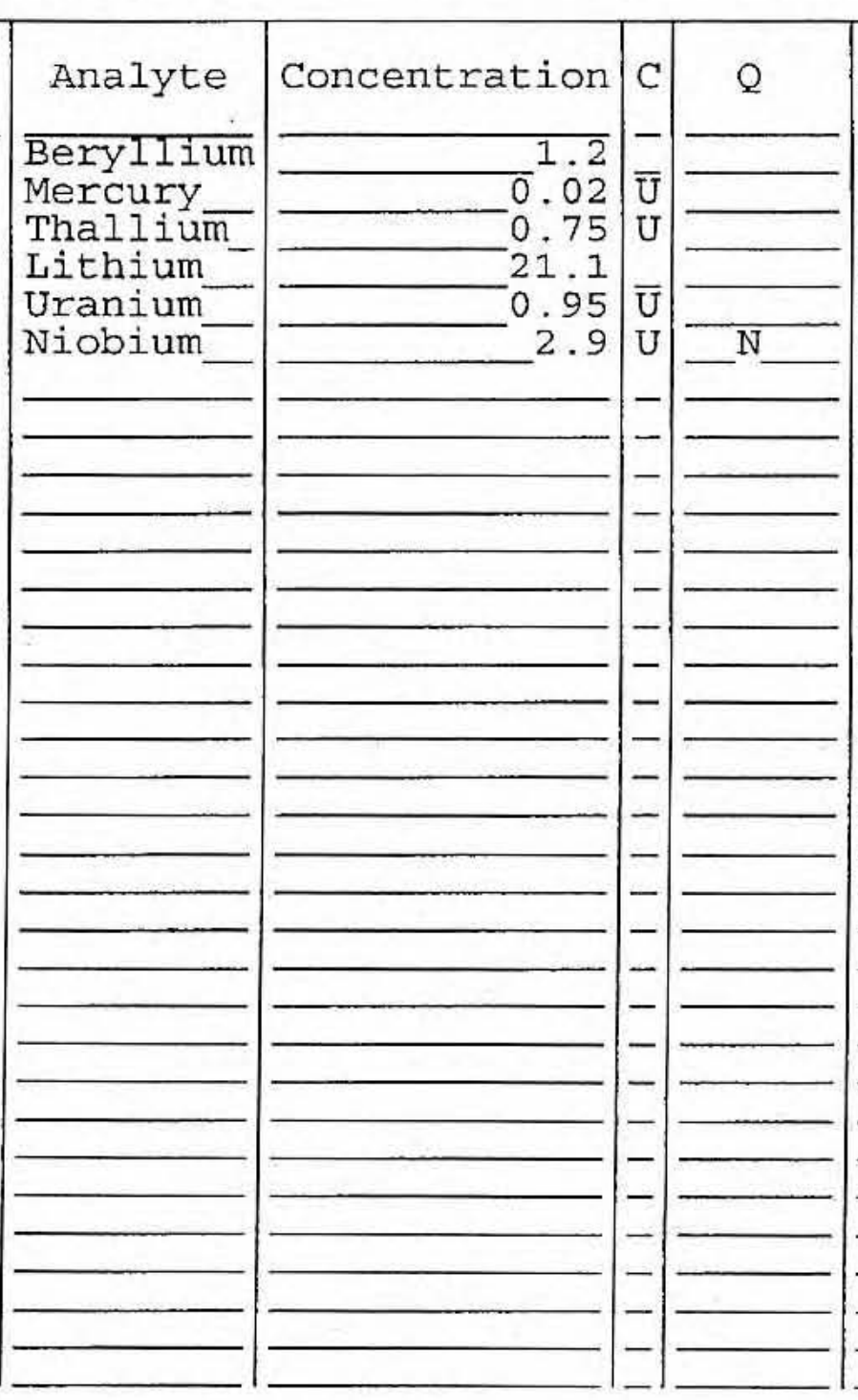

Clarity Before: Clarity After:

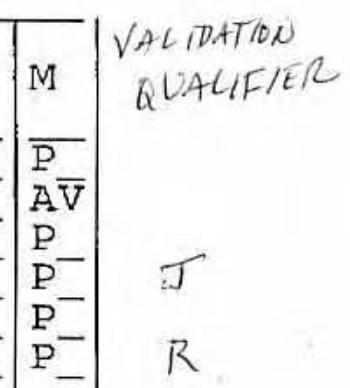

Texture:

Artifacts:

Comments :

PDRO-04B 
1

INORGANIC ANALYSES DATA SHEET
Lab Name: LIONVILLE_LABORATORY

Lab Code: LVLI Matrix (soil/water): SoIL_ Level (low/med) : 믐 Solids:
Contract: 60052

SAS NO.:

SDG No.: V2734

$$
05 \mathrm{~A}
$$

Lab Sample ID: 0608L795-0009

Date Received: 08/24/06

Concentration Units (ug/L or $\mathrm{mg} / \mathrm{kg}$ dry weight): MG/KG

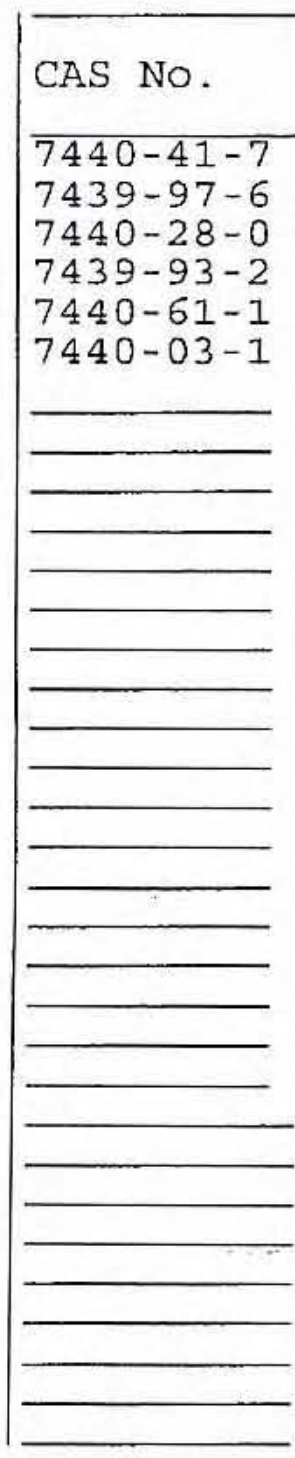

$7440-28-0$

$7439-93-2$

$7440-61-1$

$7440-03-1$

Color Before:

Color After:

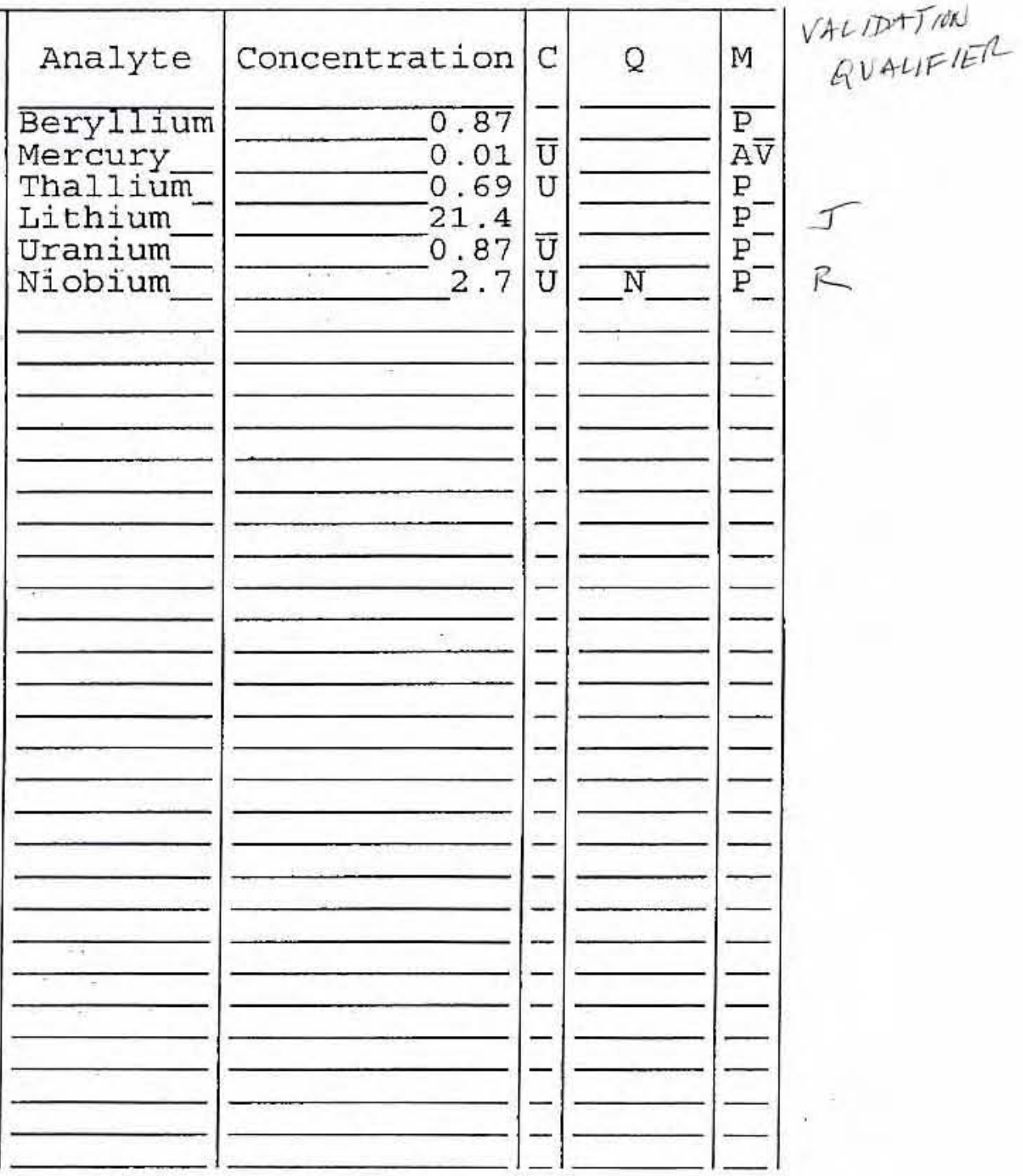

Clarity Before: Clarity After:
Texture:

Artifacts:

Comments :

PDRO - O5A 
1 INORGANIC ANALYSES DATA SHEET
Lab Name: LIONVILLE LABORATORY

Lab Code: LVLI

Matrix (soil/water): Level (low/med): Solids:

SOIL

LOW
Contract: 60052

SAS NO. :

Lab Sample ID: 06081795-0̄10
EPA SAMPLE NO.

05B

Date Received: 08/24/06

Concentration Units (ug/L or $\mathrm{mg} / \mathrm{kg}$ dry weight): MG/KG

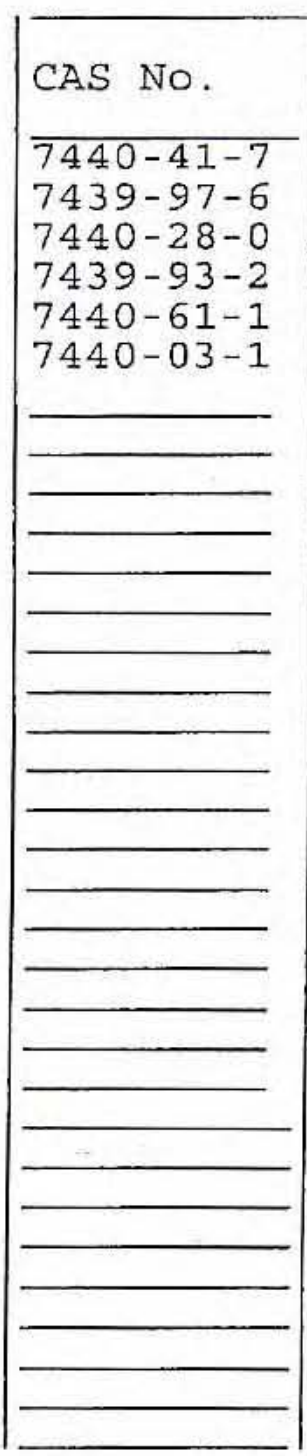

Color Before:

Color After:

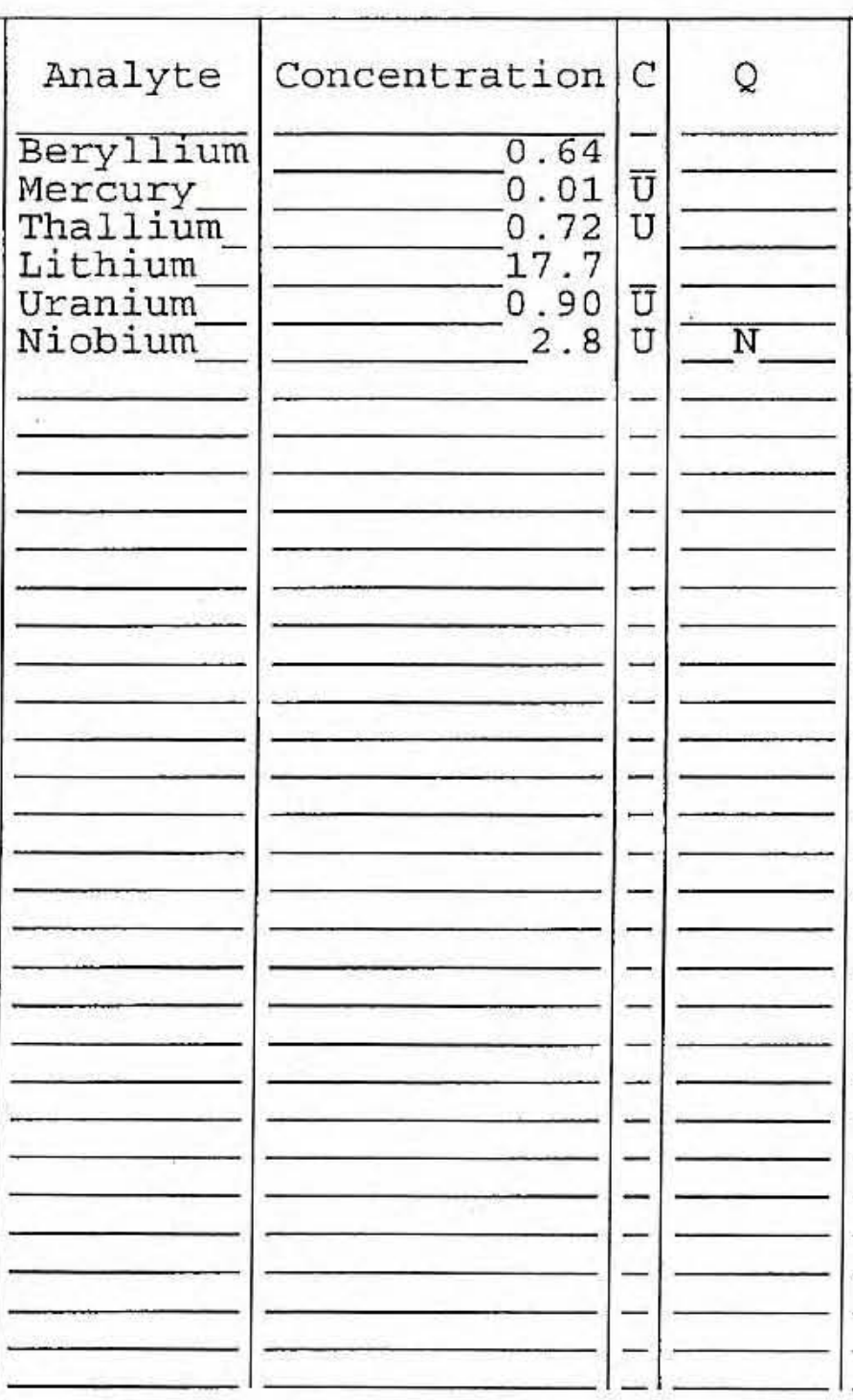

Clarity Before:

Clarity After:

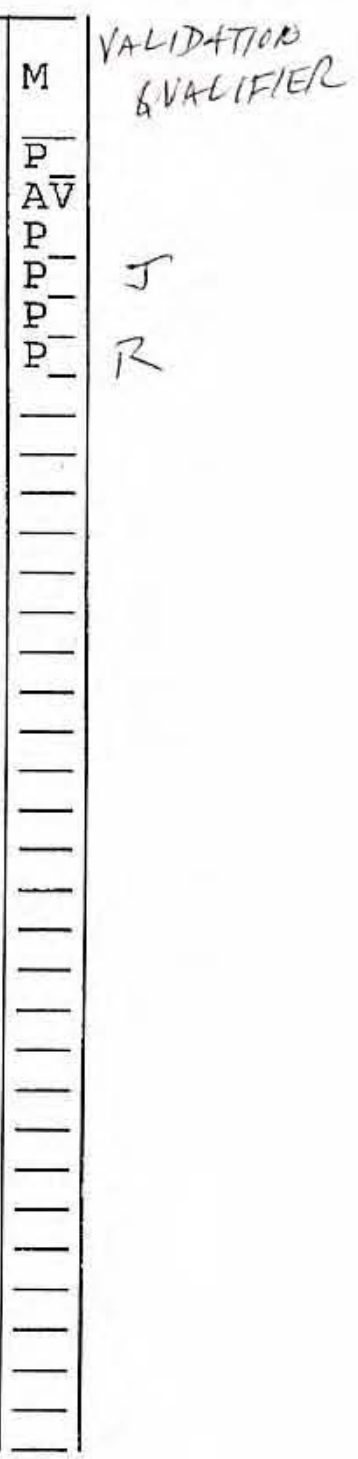

Texture:

Artifacts:

Comments:

PDRO-05B 
Lab Name: LIONVILLE_LABORATORY

Lab Code: LVLI

Matrix (soil/water): SOIL

Level (low/med):

: Solids:

LOW

$-94 \overline{.7}$
Contract: 60052

SAS NO.:

Lab Sample ID: 0608L795-0̄11
$06 \mathrm{~A}$

SDG NO.: V2734

Date Received: 08/24/06

Concentration Units (ug/L or $\mathrm{mg} / \mathrm{kg}$ dry weight): $\mathrm{MG} / \mathrm{KG}$

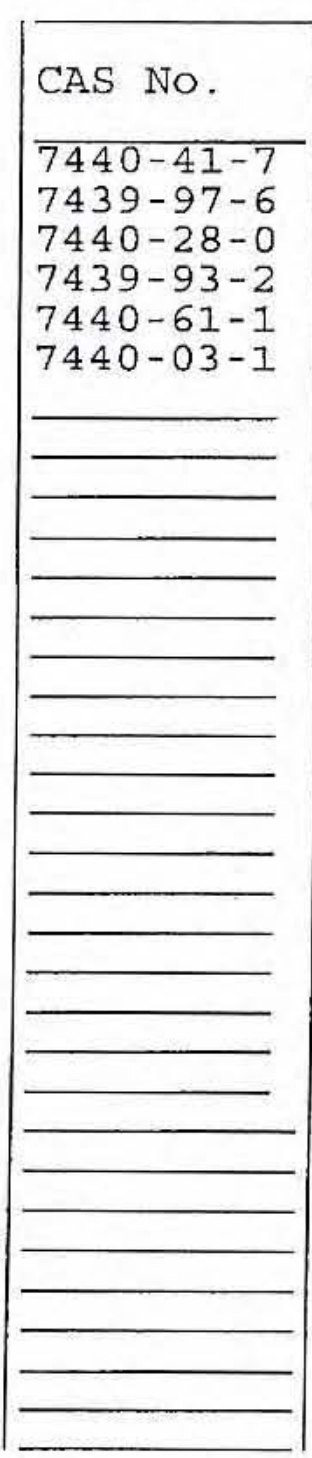

Color Before:

Color After:

Comments :

PDRO-06A

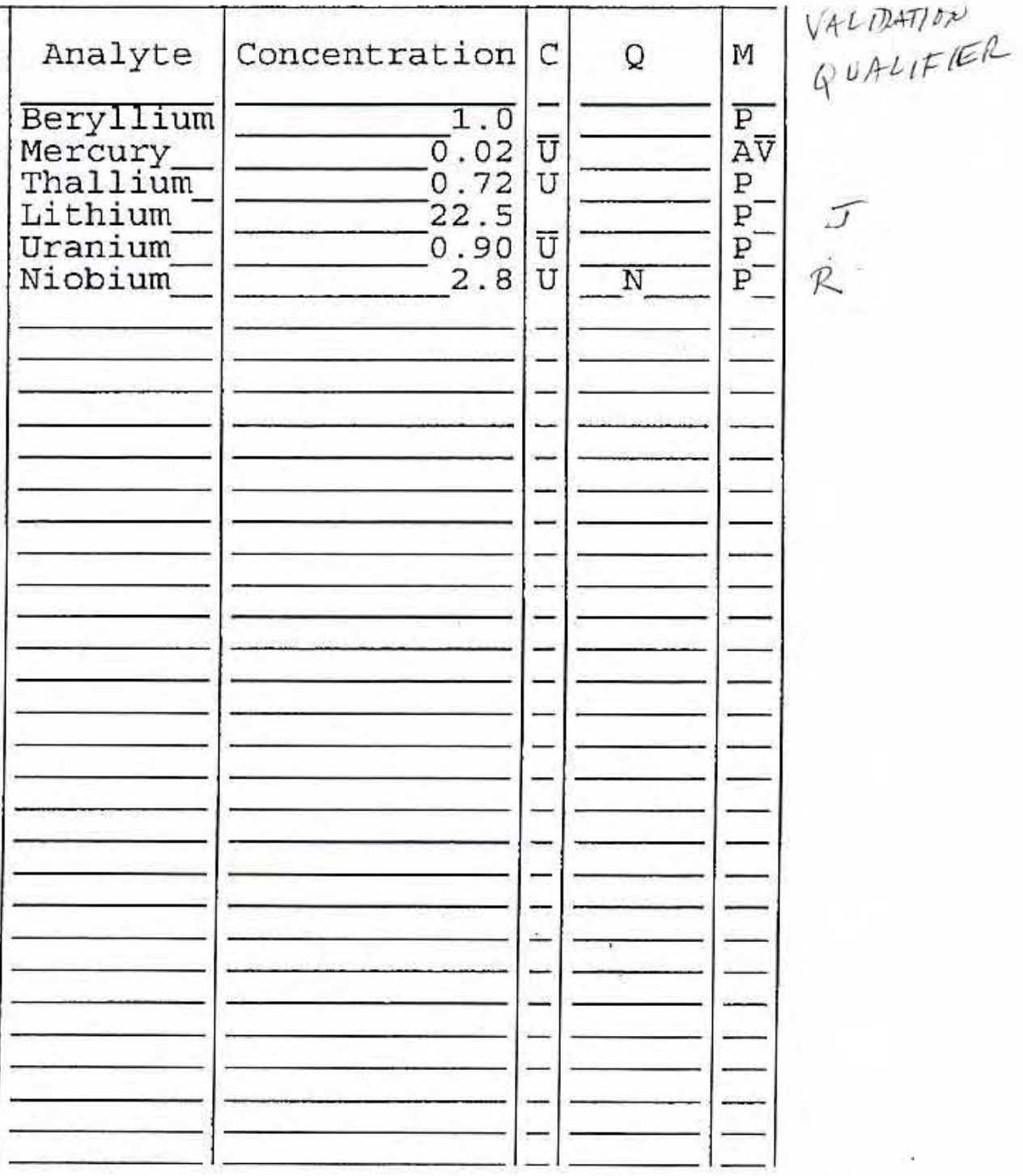

Clarity Before:

Texture: Artifacts : 


\section{1}

INORGANIC ANALYSES DATA SHEET
Lab Name: LIONVILLE LABORATORY

Lab Code: LVLI

Matrix (soil/water): SoIL

Level (low/med):

Solids:
Contract: 60052

$$
\text { LOW - }
$$

SAS NO.:

SDG NO.: V2734

Lab Sample ID: 0608L795-0̄12

Date Received: 08/24/06

Concentration Units (ug/L or $\mathrm{mg} / \mathrm{kg}$ dry weight): MG/KG

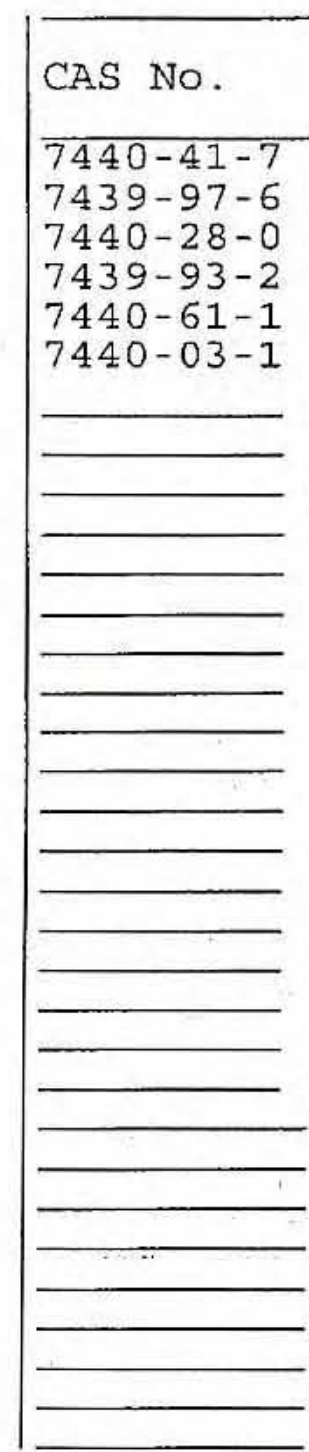

Color Before:

Color After:

Comments :

PDRO-06B

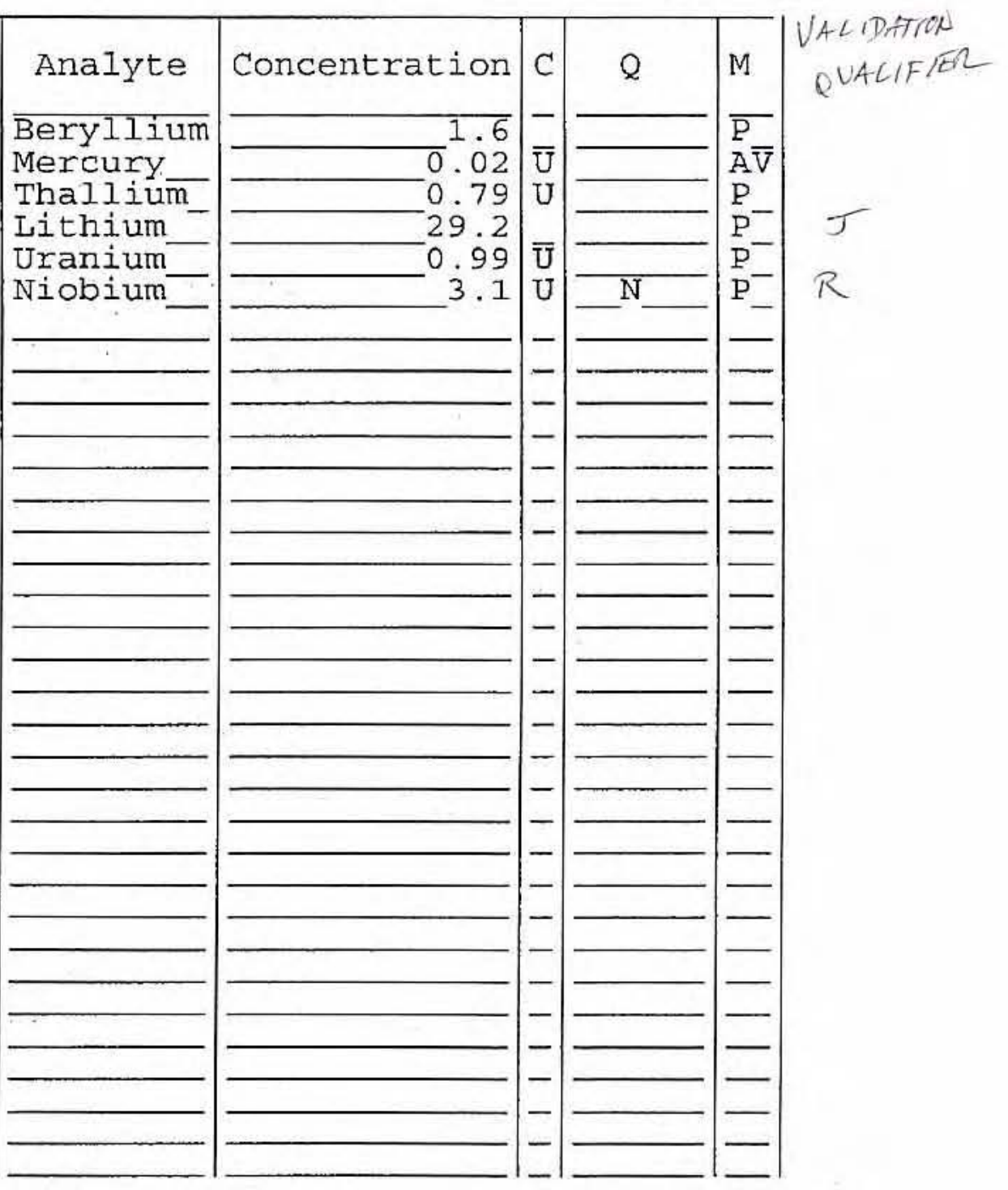

Clarity Before:

Clarity After:
Texture:

Artifacts : 


\section{1 \\ INORGANIC ANALYSES DATA SHEET}

Lab Name: LIONVILLE LABORATORY

Lab Code: LVLI

Matrix (soil/water): SOIL

Level (low/med): LOW

\% Solids :

$-95 \overline{.0}$

Case No.: PDRO
Contract: 60052

SAS NO.:

SDG NO.: V2734

Lab Sample ID: 0608L795-0̄13

Date Received: 08/24/06

Concentration Units (ug/L or $\mathrm{mg} / \mathrm{kg}$ dry weight): MG/KG

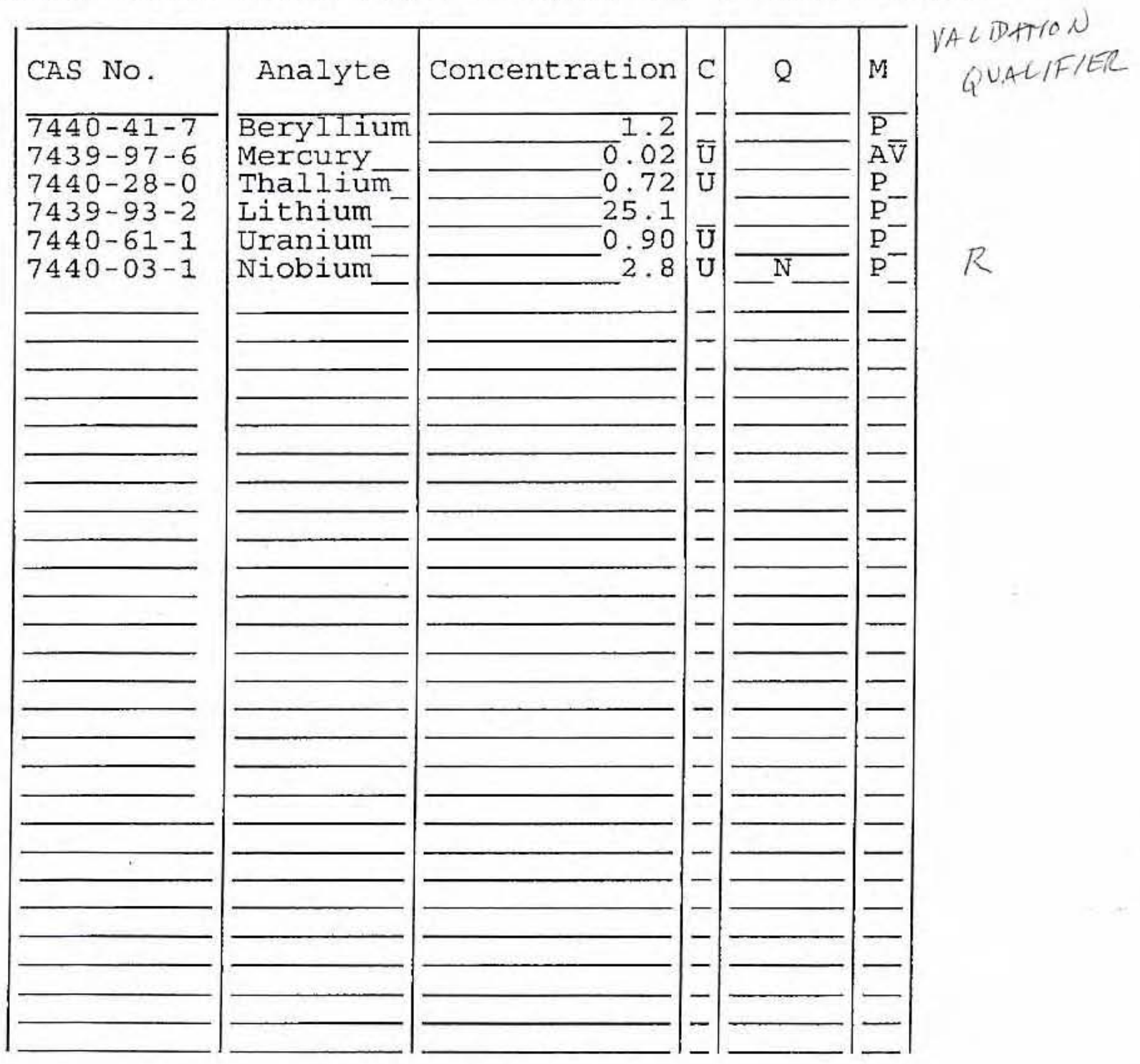

Color Before:

Color After:

EPA SAMPLE NO.

07A

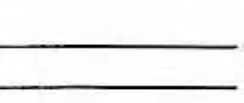

Clarity Before:

Clarity After:
Texture:

Artifacts :

Comments:

PDRO-07A 
1

INORGANIC ANALYSES DATA SHEET
EPA SAMPLE NO.

$$
07 \mathrm{~B}
$$

Contract: 60052

SDG No.: V2734

Case No.: PDRO_ SAS No.

Lab Sample ID: 0608L795-0̄14

Date Received: 08/24/06
Lab Name: LION
Lab Code: LVLI

Level (low/med) : Solids:

LOW -
$\quad 94 \overline{.3}$

Concentration Units (ug/L or $\mathrm{mg} / \mathrm{kg}$ dry weight): MG/KG

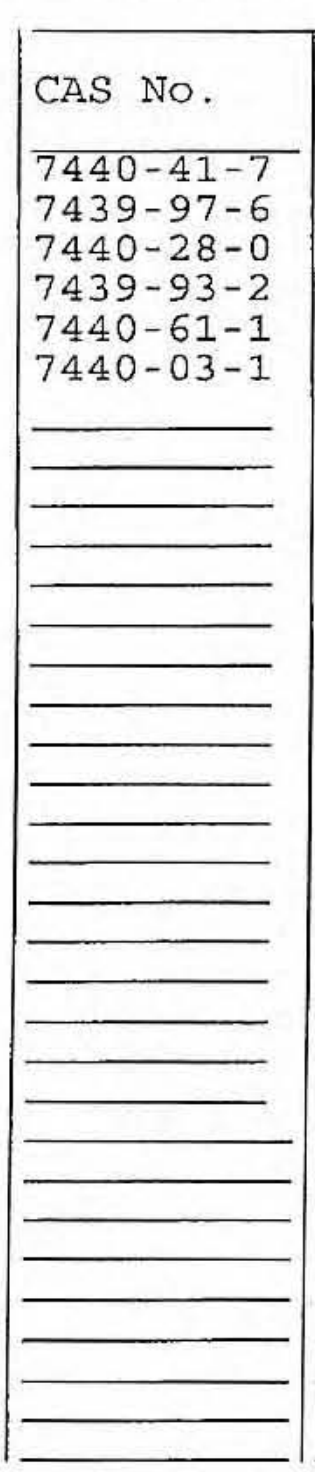

Color Before:

Color After:

Comments :

PDRO-07B

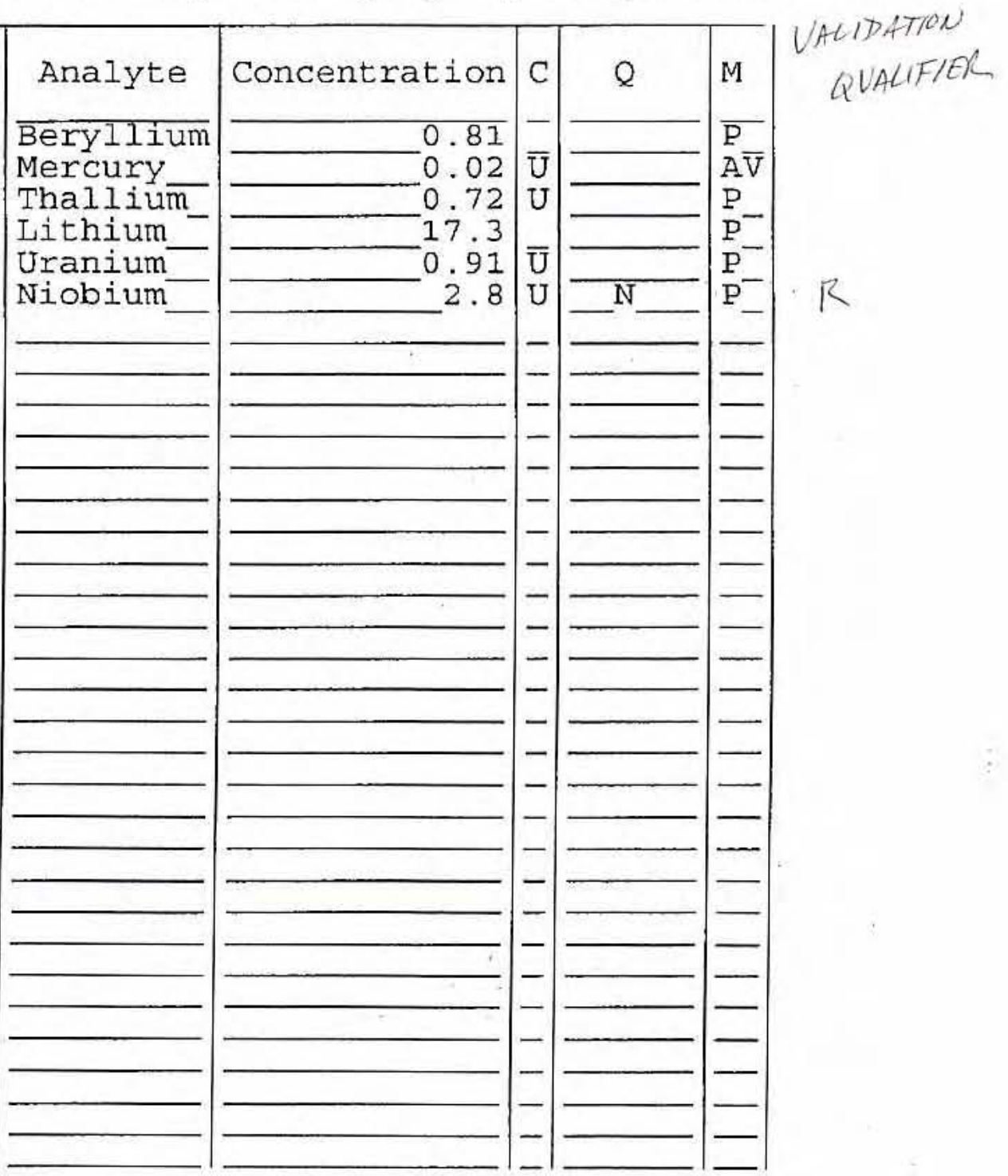

Clarity Before: Clarity After:
Texture:

Artifacts: 
1 INORGANIC ANALYSES DATA SHEET
EPA SAMPLE NO.

$08 \mathrm{~A}$

SDG NO.: V2734

60052

Lab Sample ID: 06் 0 L795-0̄15

Date Received: 08/24/06 $\begin{array}{ll}\text { Matrix (soil/water): SOIL } & \text { LOW } \\ \text { Level (low/med): } & \text { Low }\end{array}$

$\begin{array}{ll} & \\ & 94 . \overline{0}\end{array}$

Contract: 6005
Case NO.: PDRO_ SAS NO.:

Concentration Units (ug/L or $\mathrm{mg} / \mathrm{kg}$ dry weight): $\mathrm{MG} / \mathrm{KG}$

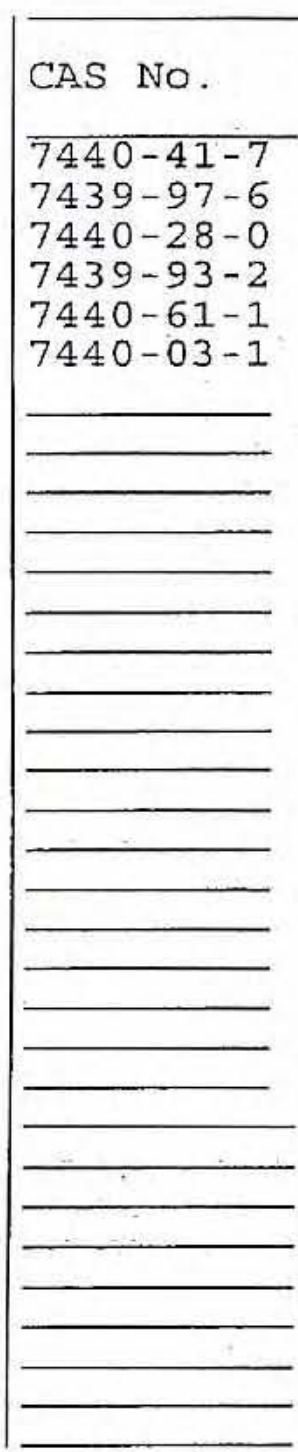

Color Before:

Color After:
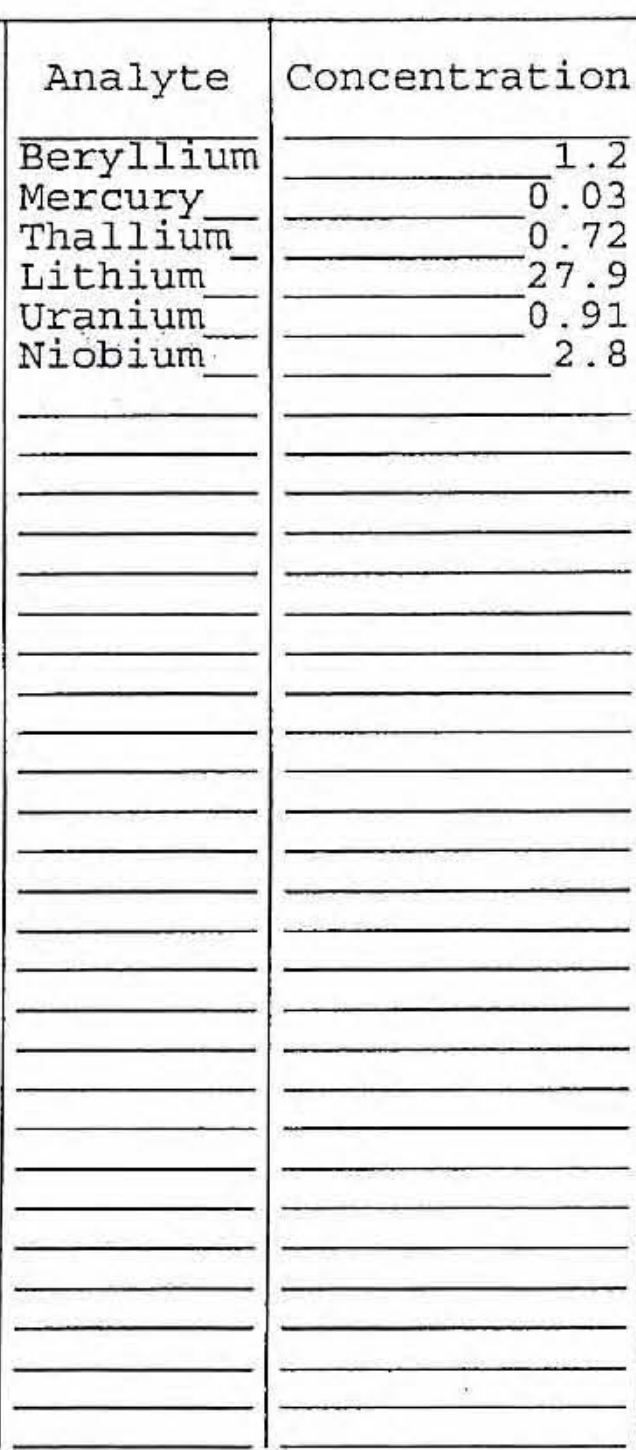

$\mathrm{C}$
-
-
$\mathrm{U}$
$\overline{\mathrm{U}}$
$\mathrm{U}$
-
-
-
-
-
-
-
-
-
-
-
-
-
-

Clarity Before:

Clarity After:

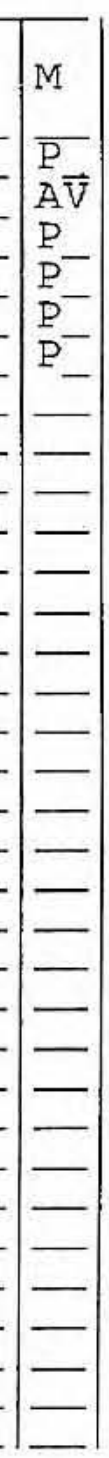

$R$

SADATION

Q VALIFIER

Comments:

PDRO - 08A

Texture:

Artifacts : 
1

\section{INORGANIC ANALYSES DATA SHEET}

EPA SAMPLE NO.

\section{$08 \mathrm{~B}$}

Contract: 60052

SAS NO. :

Lab Sample ID: 06்08L795-016

Date Received: 08/24/06
Lab Name: LIONVILLE_LABORATORY

Lab Code: LVLI Matrix (soil/water): SoIL Level (low/med): Solids :

$$
\begin{array}{r}
\text { LOW } \\
-94.4
\end{array}
$$

Concentration Units (ug/L or $\mathrm{mg} / \mathrm{kg}$ dry weight): MG/KG

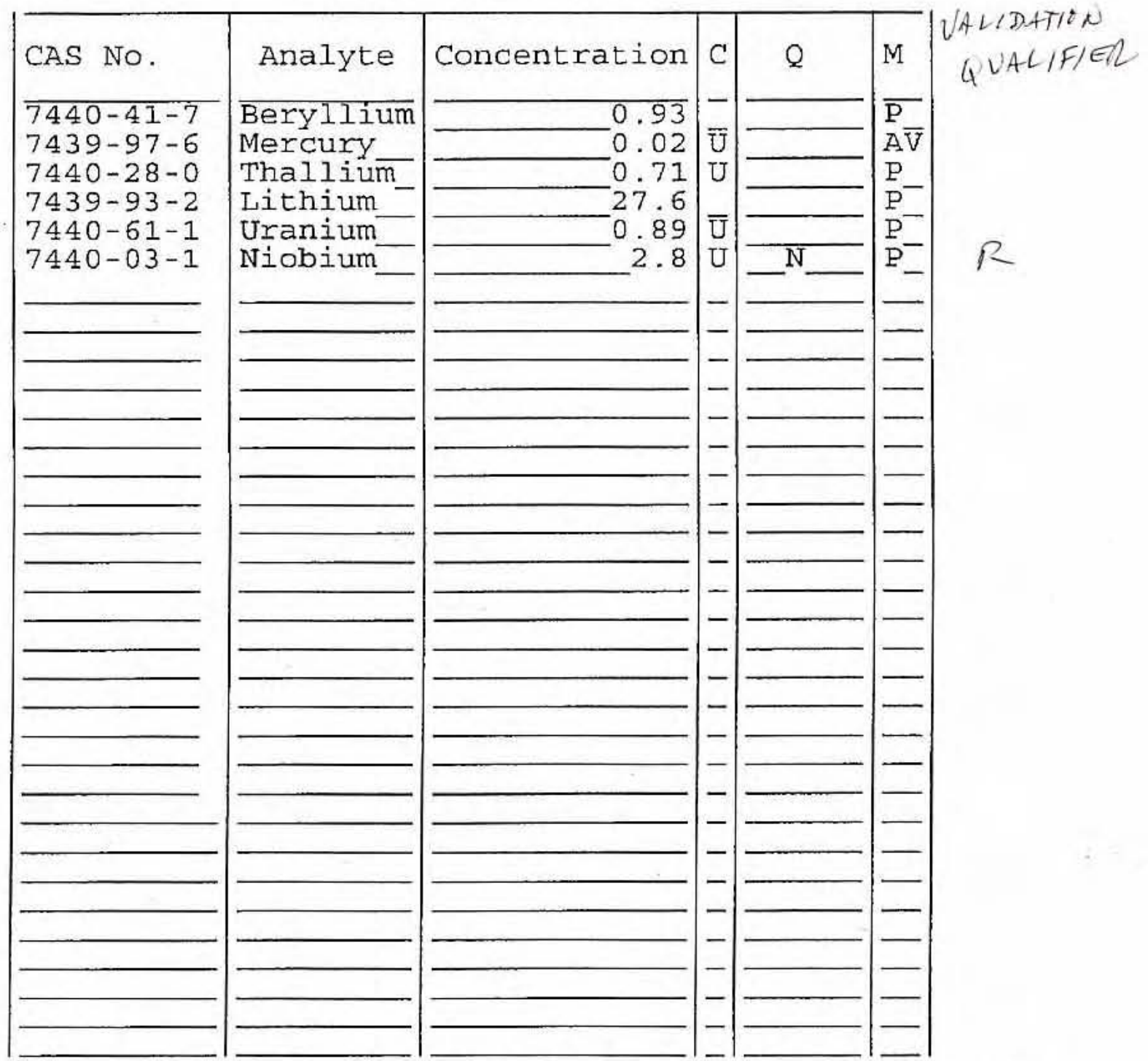

Clarity Before:

Clarity After:
Texture:

Artifacts :
Color Before:
Color After:

Comments :

PDRO-08B 
1 INORGANIC ANALYSES DATA SHEET
EPA SAMPLE NO.

$$
09 A
$$

SDG NO.: V2734

SAS NO. : Lab Sample ID: 0608L795-0̄17 Date Received: 08/24/06
Lab Name: LION
Lab Code: LVLI Matrix (soil/water): SOIL Level (low/med): LOW Solids:
Contract: 60052

$$
- 9 3 \longdiv { . 7 }
$$

Concentration Units (ug/L or $\mathrm{mg} / \mathrm{kg}$ dry weight) : $\mathrm{MG} / \mathrm{KG}$

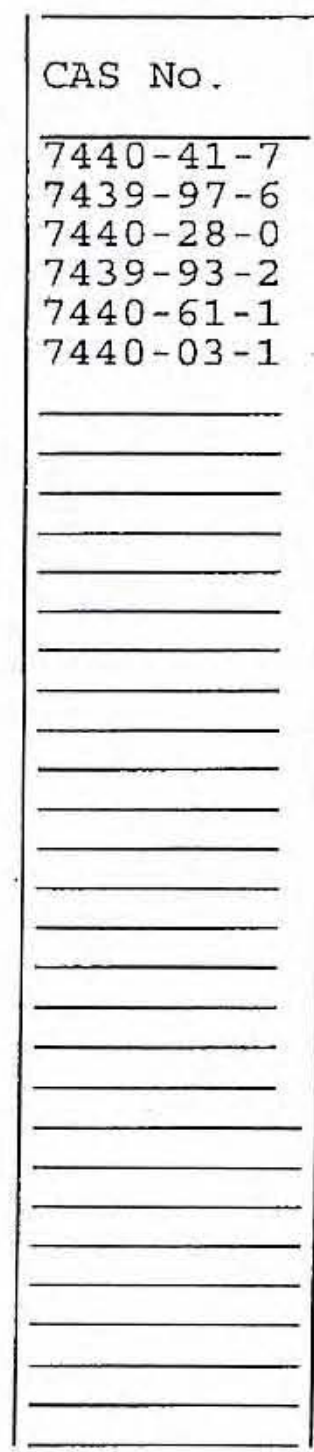

Color Before:

Color After:

Comments :

PDRO-09A

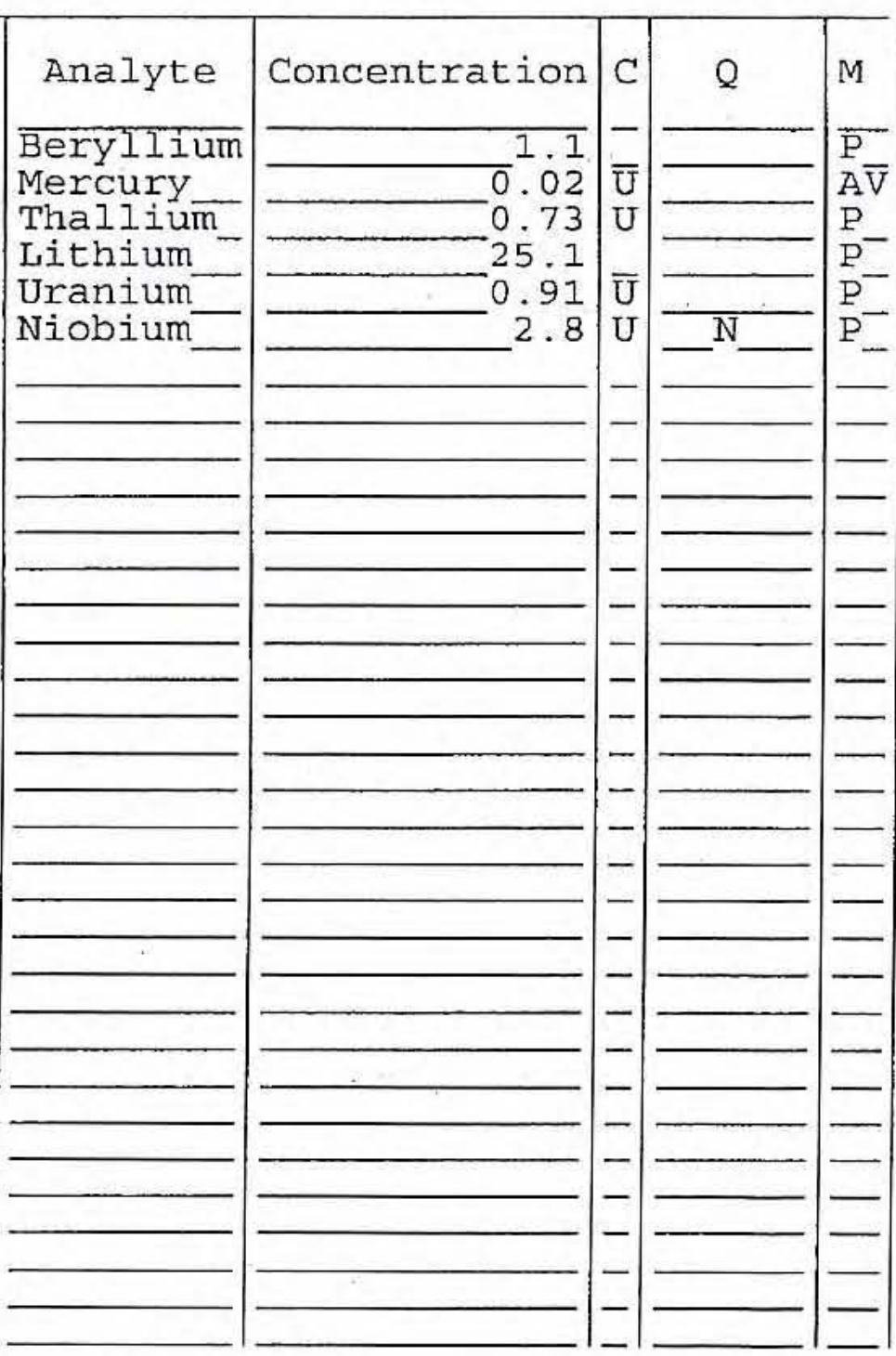

Clarity Before: Clarity After:
VACIDATION GUA LIFER

$R$
Texture: Artifacts: 
1

INORGANIC ANALYSES DATA SHEET
EPA SAMPLE NO.

$$
\text { O9B }
$$

SDG NO.: V2734

Lab Sample ID: 0608L795-0̄18

Date Received: 08/24/06
Lab Name: LIONVILLE_LABORATORY

Lab Code: LVLI

Matrix (soil/water):

Level (low/med):

\% Solids:
Case NO.: PDRO

$$
\begin{array}{r}
\text { LOW } \\
-88 . \overline{8}
\end{array}
$$

Contract: 60052 SAS NO. :

Concentration Units (ug/L or $\mathrm{mg} / \mathrm{kg}$ dry weight): MG/KG

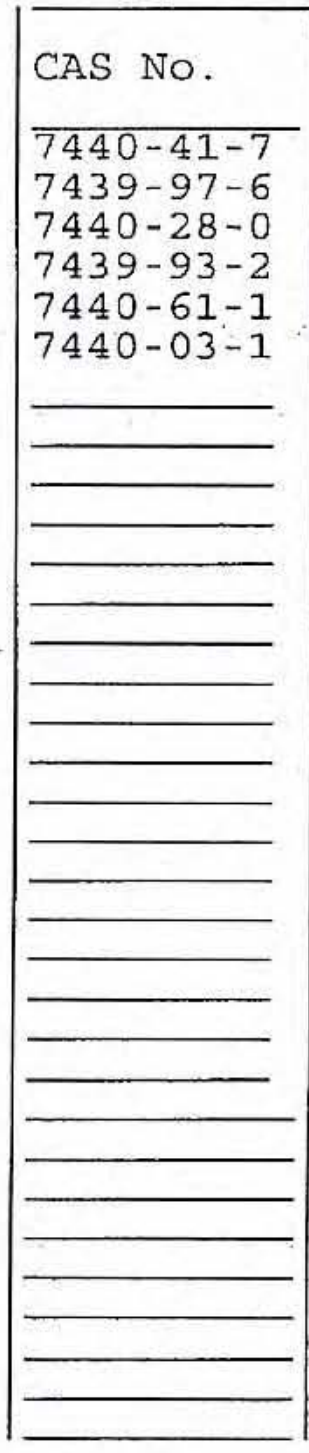

Color Before:

Color After:

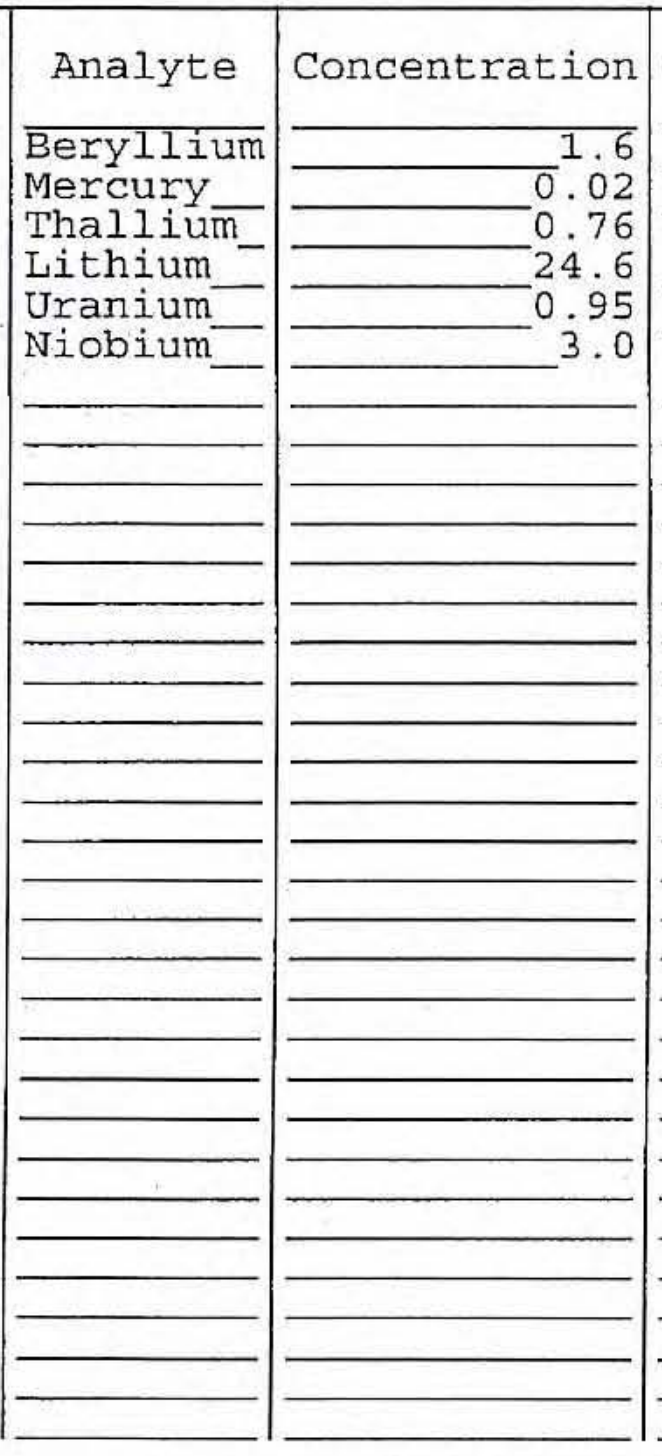

Clarity Before: Clarity After:

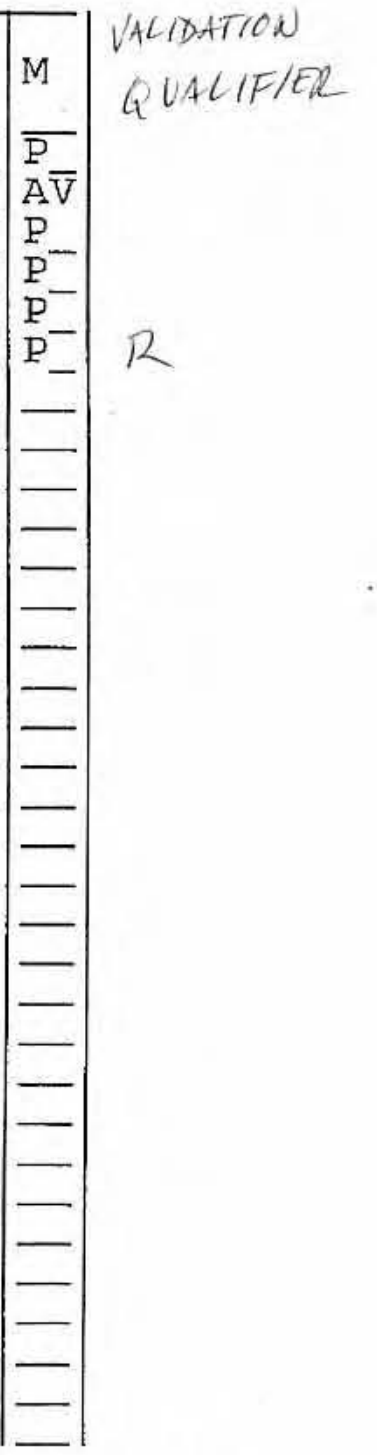

Texture: Artifacts:

Comments :

PDRO-09B 
1

INORGANIC ANALYSES DATA SHEET
Lab Name: LIONVILLE_LABORATORY

Lab Code: IVLI

Matrix (soil/water): SoIL Level (low/med): : Solids:

LOW
88.6
Contract: 60052

SAS NO. :

SDG No.: V2734
EPA SAMPLE NO.

\section{$10 \mathrm{~A}$}

Date Received: 08/24/06

Concentration Units (ug/L or $\mathrm{mg} / \mathrm{kg}$ dry weight) : MG/KG

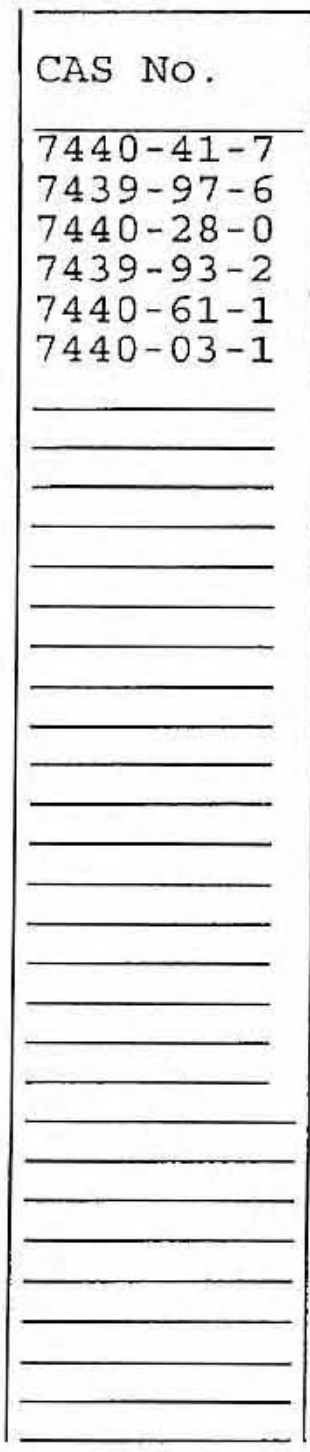

$7440-61-1$

7440-03-1

Color Before:

Color After:

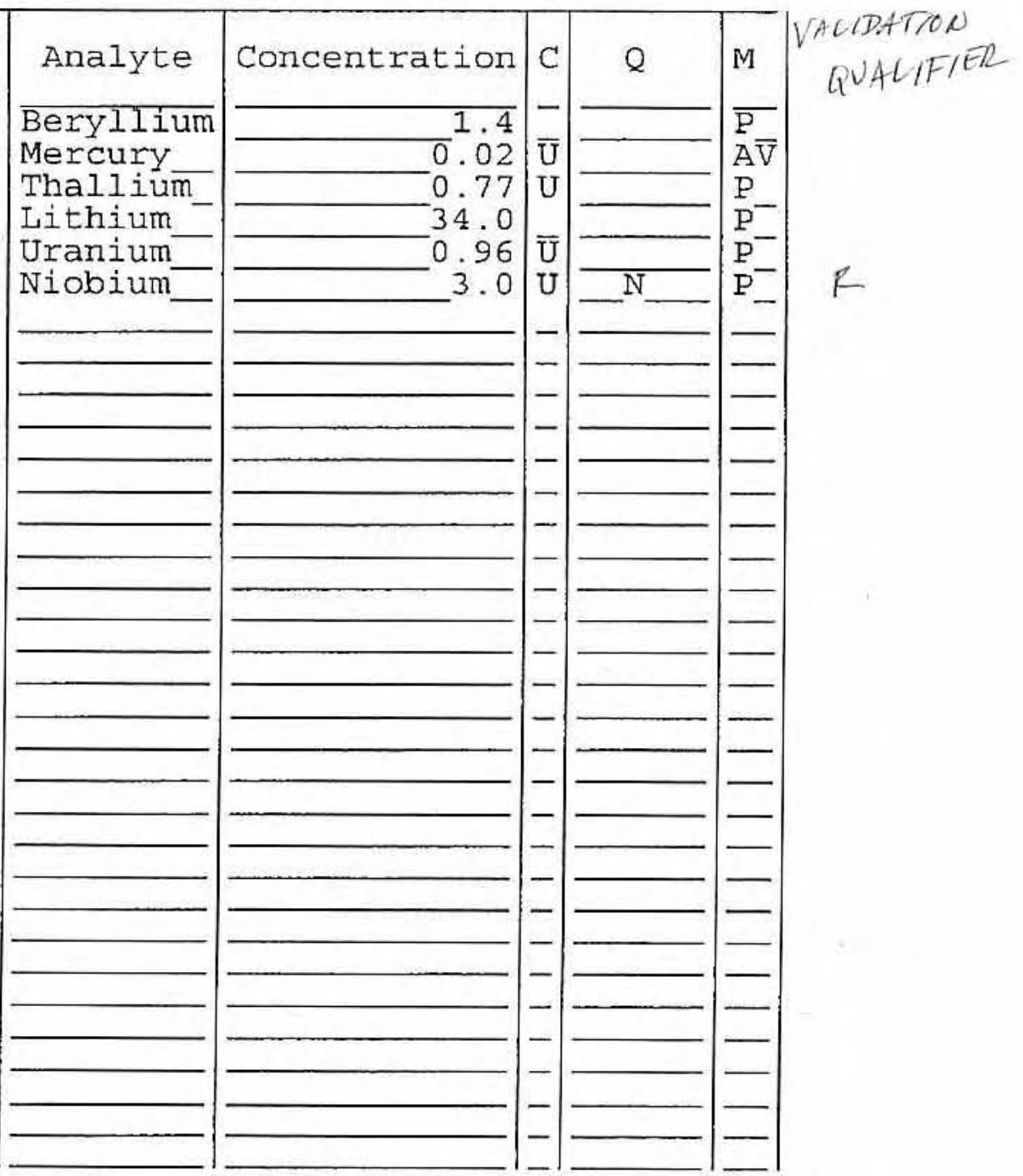

Clarity Before: Clarity After:
Texture:

Artifacts :

Comments :

PDRO-10A 
EPA SAMPLE NO.

Lab Name: LIONVILLE LABORATORY

Lab Code: LVLI

Matrix (soil/water): SoIL

Level (low $/ \mathrm{med}$ ):

$\div$ Solids:
Contract: 60052

SAS NO.:
$10 B$

SDG NO.: V2734

Lab Sample ID: 0608L795-ס̄20 Date Received: 08/24/06

Concentration Units (ug/L or $\mathrm{mg} / \mathrm{kg}$ dry weight): MG/KG

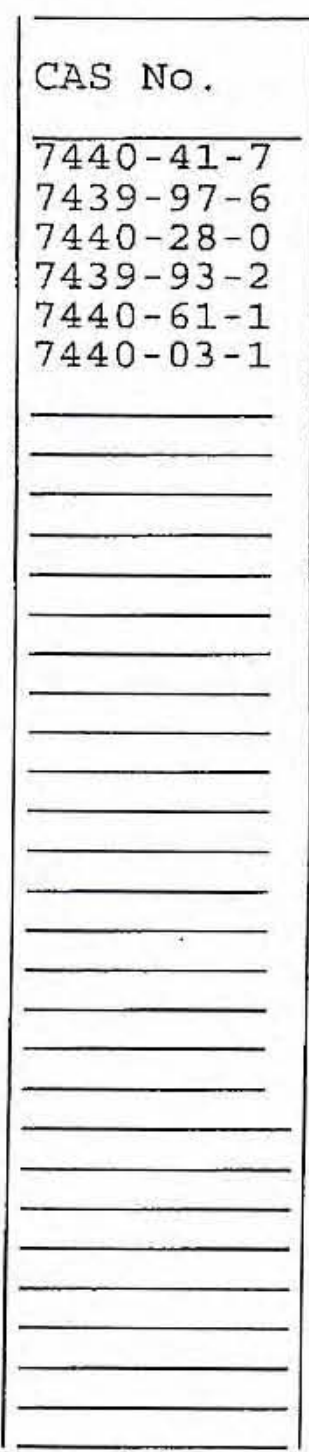

Color Before:

Color After:

Comments :

PDRO-10B

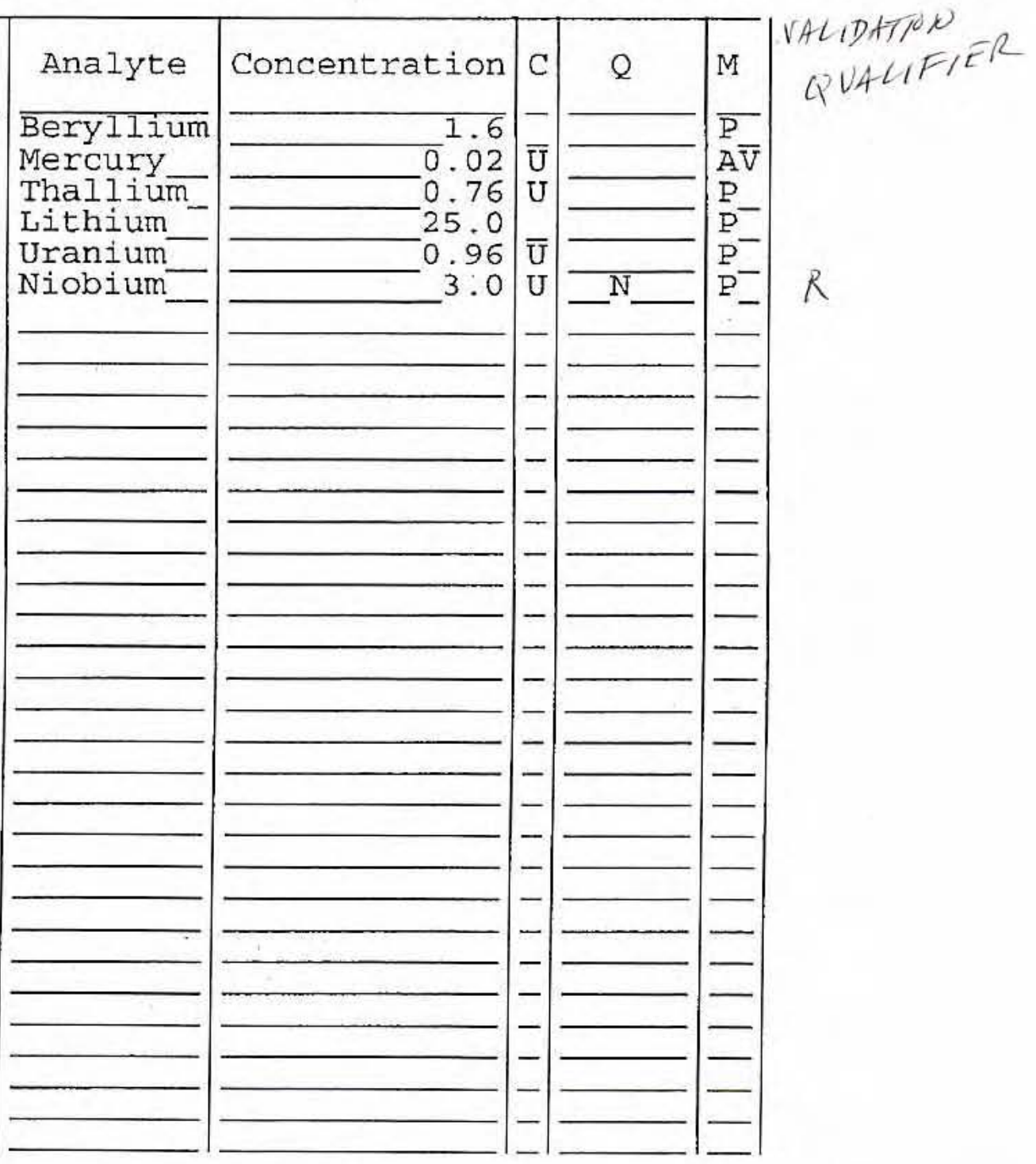

Clarity Before:

Clarity After:
Texture:

Artifacts: 


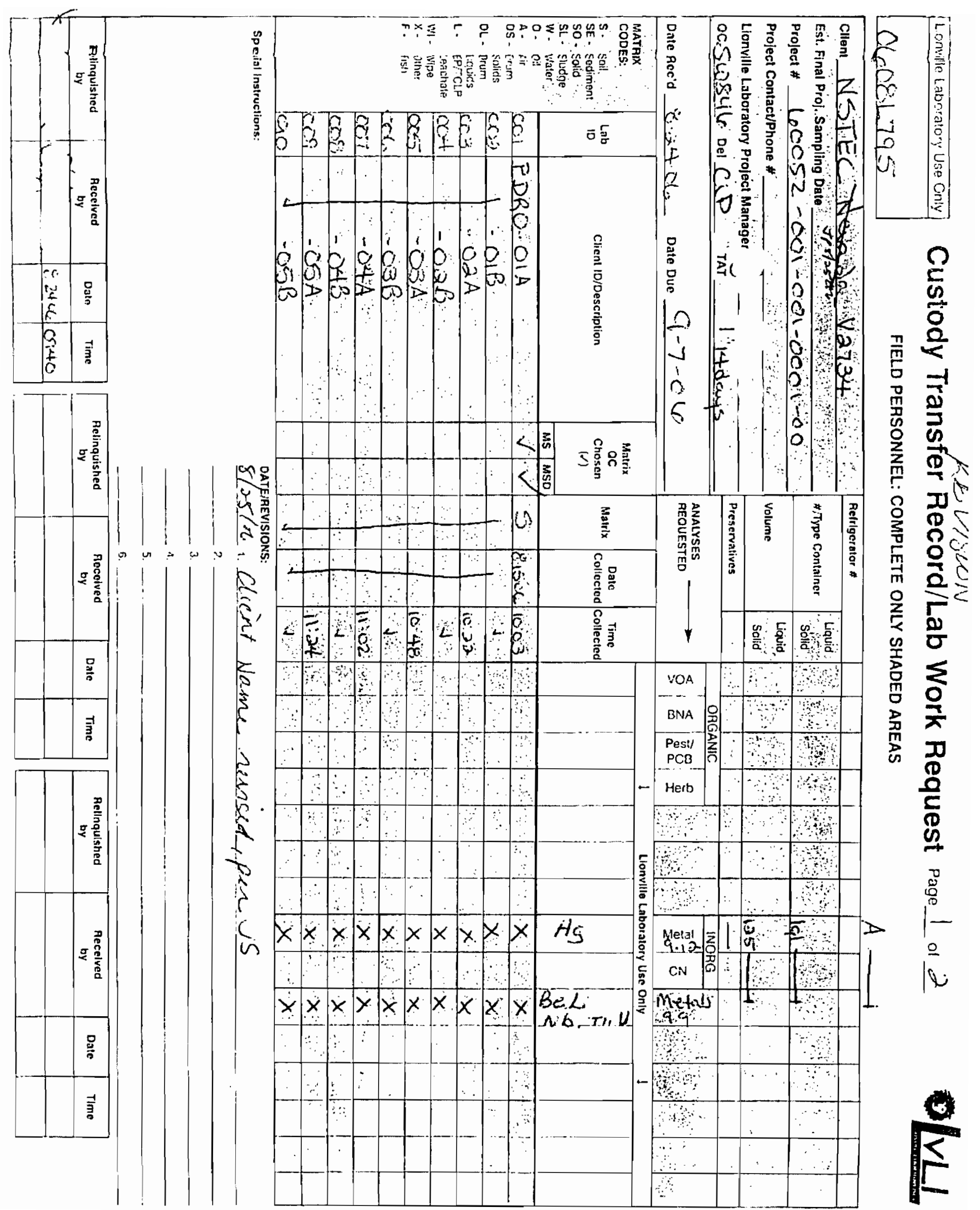




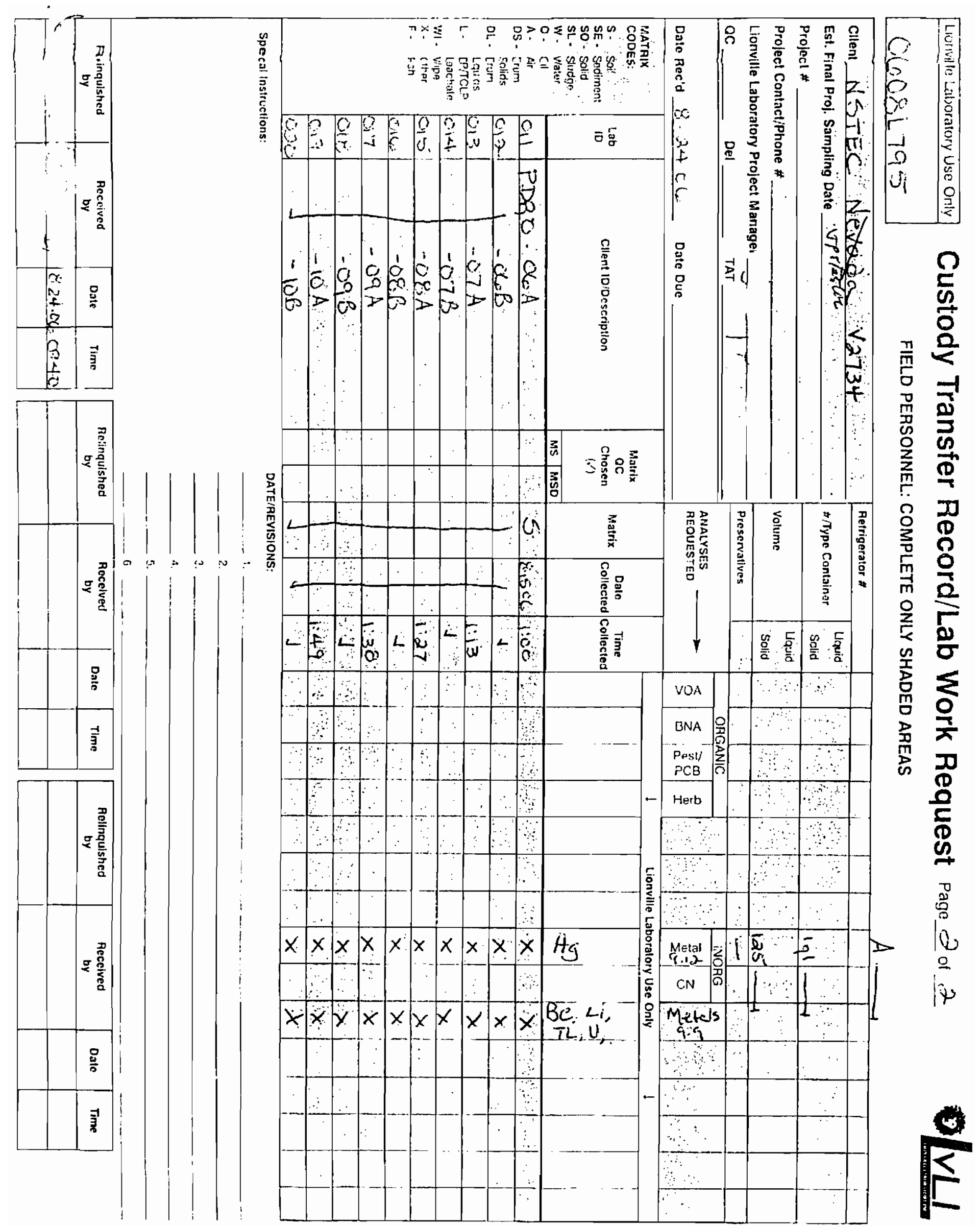




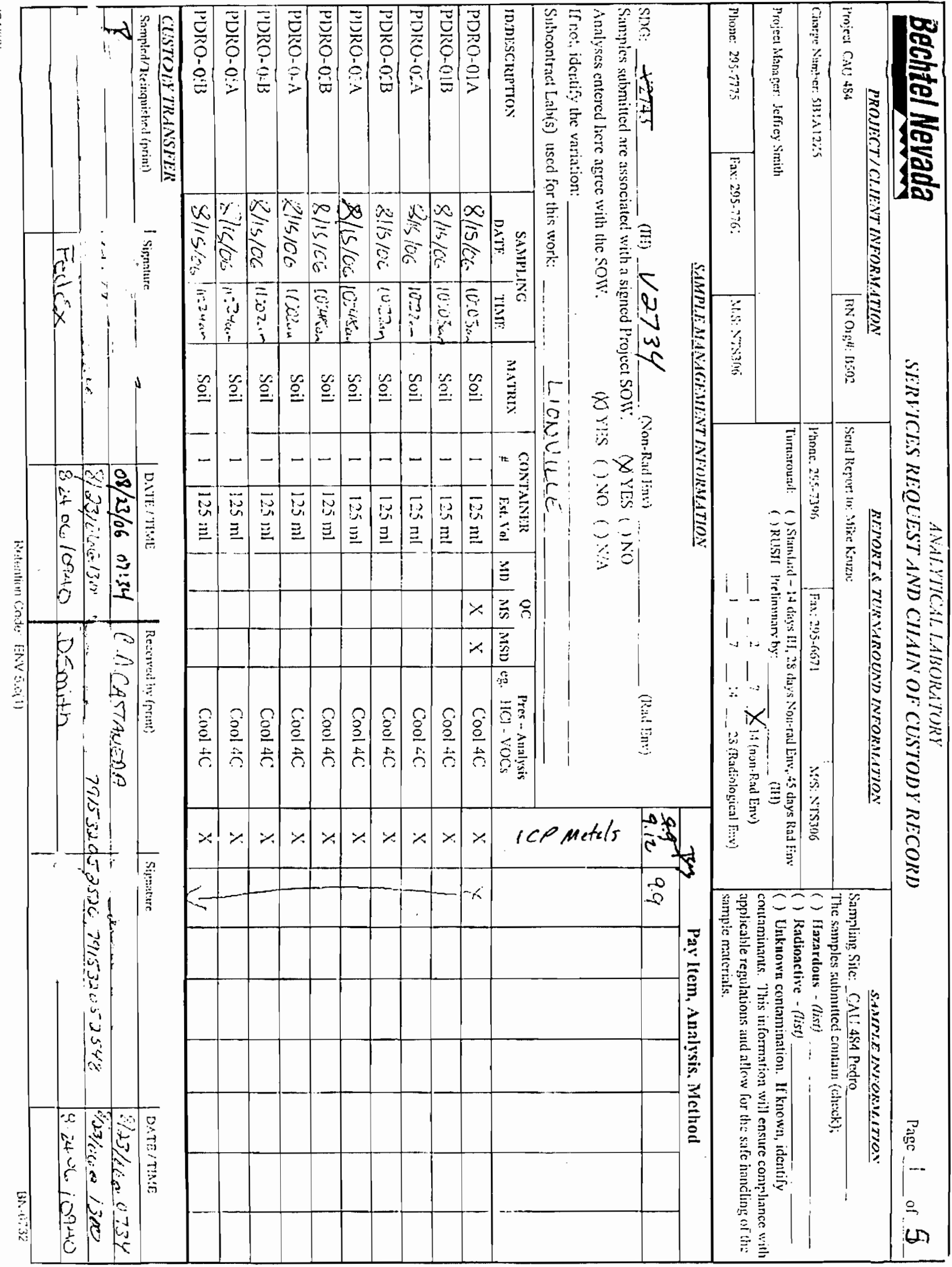




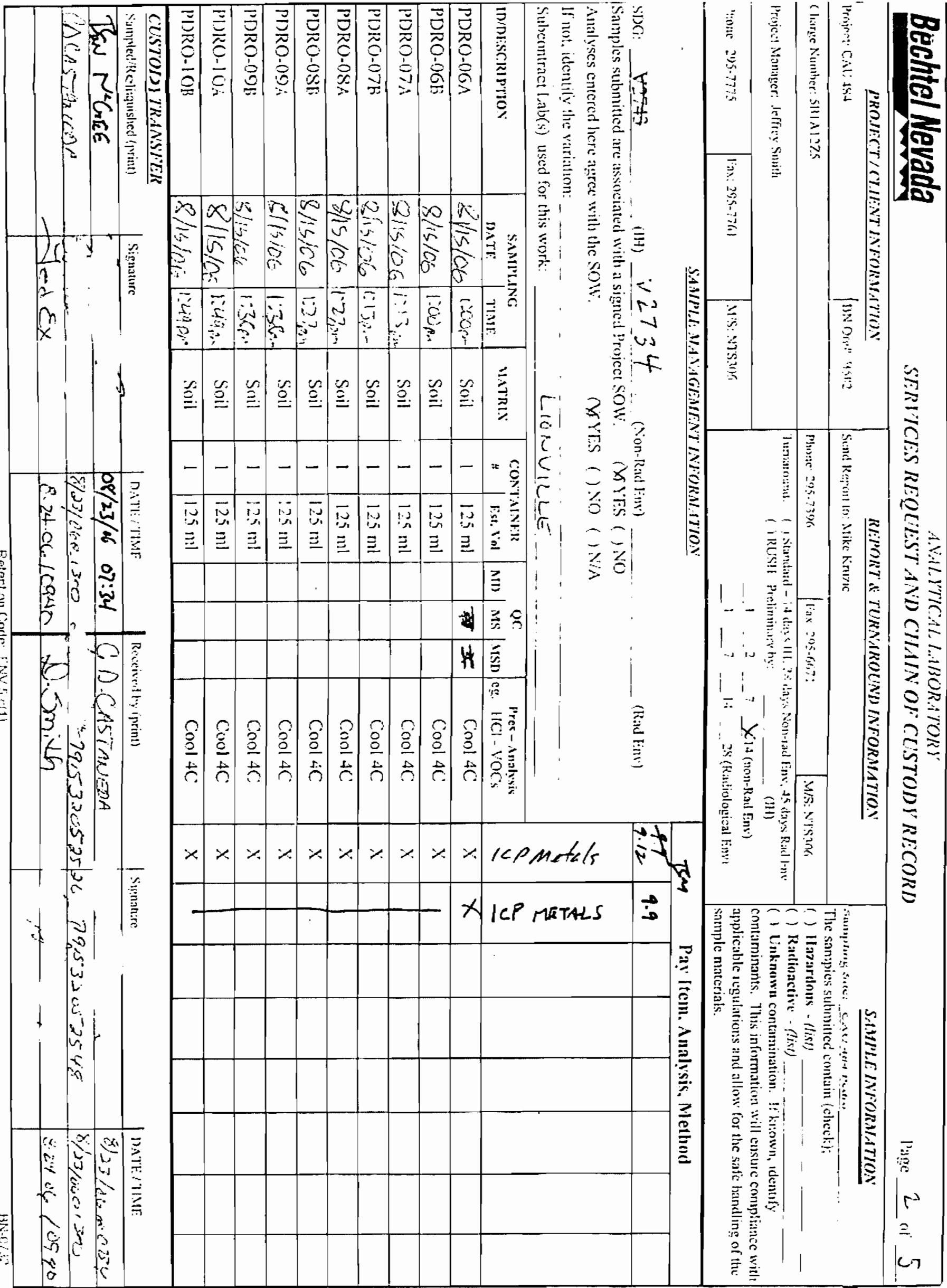


1 INORGANIC ANALYSES DATA SHEET
EPA SAMPLE NO.

$11 \mathrm{~A}$

Contract: 60052

SAS NO. :

Lab Sample ID : 0608L79 $\overline{6-0} 01$ SOIL LOW
-92.9

Date Received: 08/24/06

$$
-92 \overline{9}
$$

Lab Code: LVLI Matrix (soil/wa Level

Concentration Units (ug/L or $\mathrm{mg} / \mathrm{kg} \mathrm{dry}$ weight): $\mathrm{MG} / \mathrm{KG}$

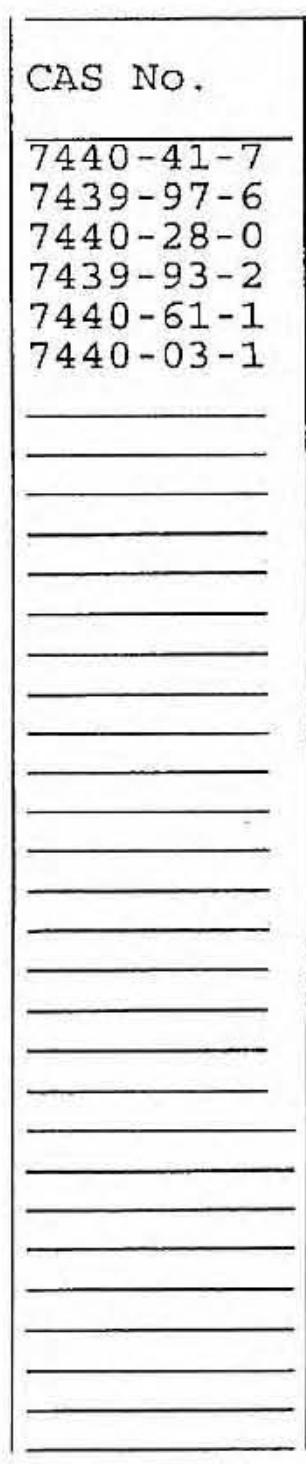

Color Before: Color After:

Comments:

PDRO-11A

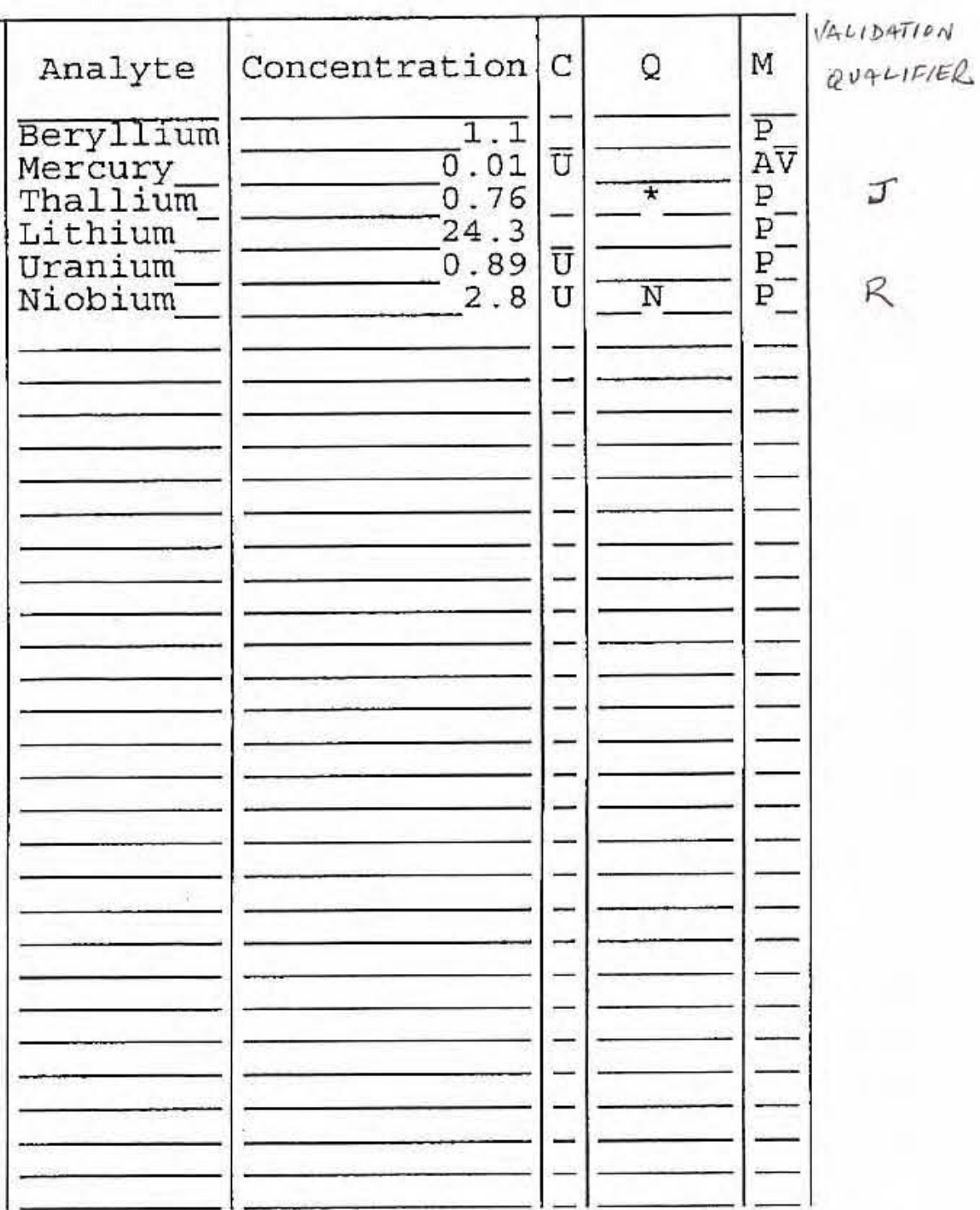

Clarity Before: Clarity After:

Texture:

Artifacts: 
1 INORGANIC ANALYSES DATA SHEET
Lab Name: LIONVILLE LABORATORY

Lab Code: LVLI

Matrix (soil/water): SoIL

Level (low $/ \mathrm{med}$ ) :

Solids:

$$
\begin{array}{r}
\text { LOW }- \\
-92.5
\end{array}
$$

Contract: 60052

SAS No. :

SDG NO.: 11A

$11 \mathrm{~B}$

Lab Sample ID: $0608179 \overline{6-002}$

Date Received: 08/24/06

Concentration Units (ug/L or $\mathrm{mg} / \mathrm{kg}$ dry weight): $\mathrm{MG} / \mathrm{KG}$

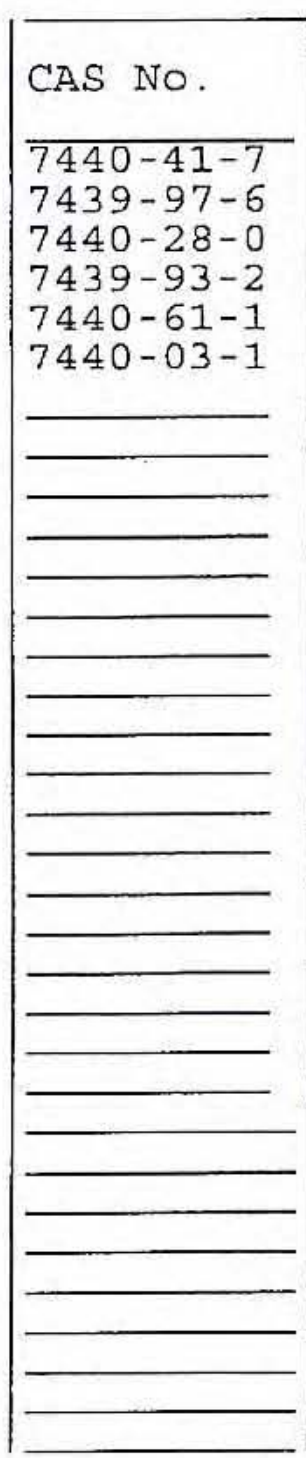

Color Before:

Color After:

Comments:

PDRO-11B

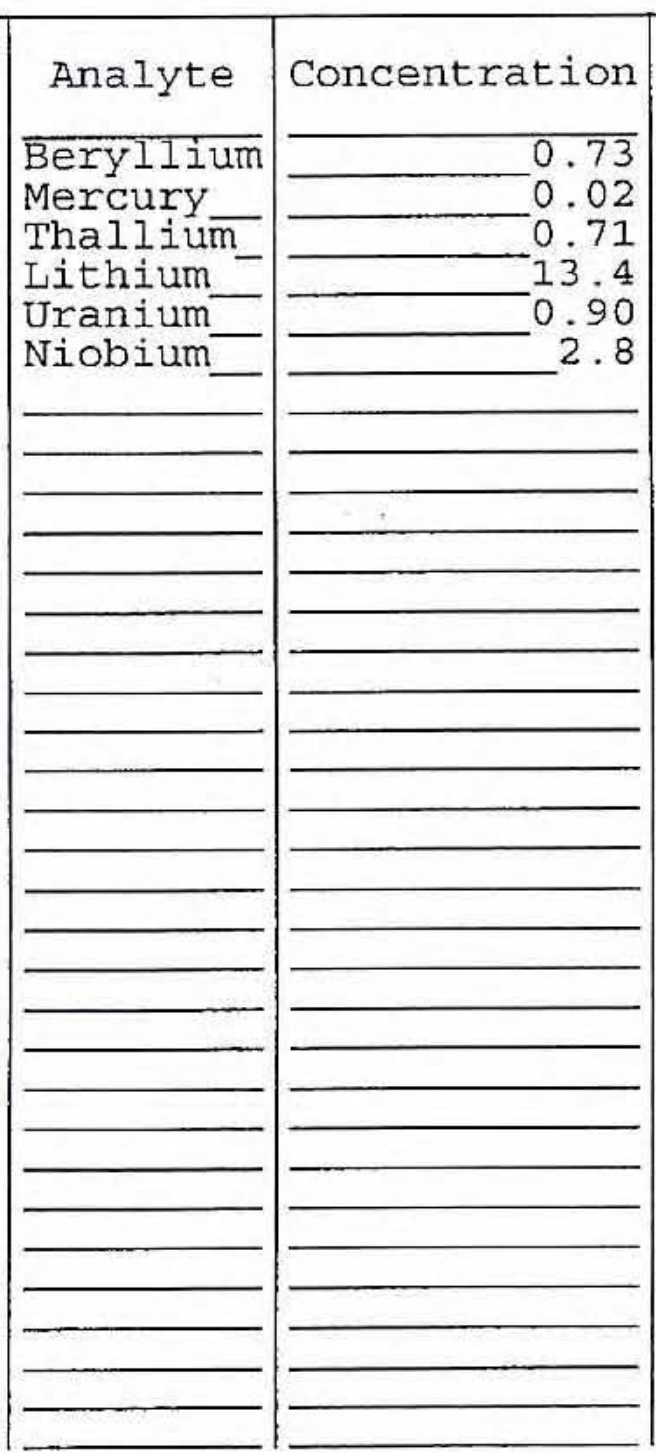

$\mathrm{C}$
-
$\overline{\mathrm{U}}$
$\mathrm{U}$

Clarity Before: Clarity After:
VALIDATION QUAUIFIER

J

$R$
Texture:

Artifacts: 
Lab Name: LIONVILLE_LABORATORY

\section{Lab Code: LVLI}

Matrix (soil/water): SOIL Level (low/med): LOW Solids:

$91 \overline{0}$
Contract: 60052

SAS NO. :

\section{$12 \mathrm{~A}$}

SDG NO.: $11 \mathrm{~A}$

Lab Sample ID : 0608L79 $\overline{6-0} 03$

Concentration Units (ug/L or $\mathrm{mg} / \mathrm{kg}$ dry weight): MG/KG

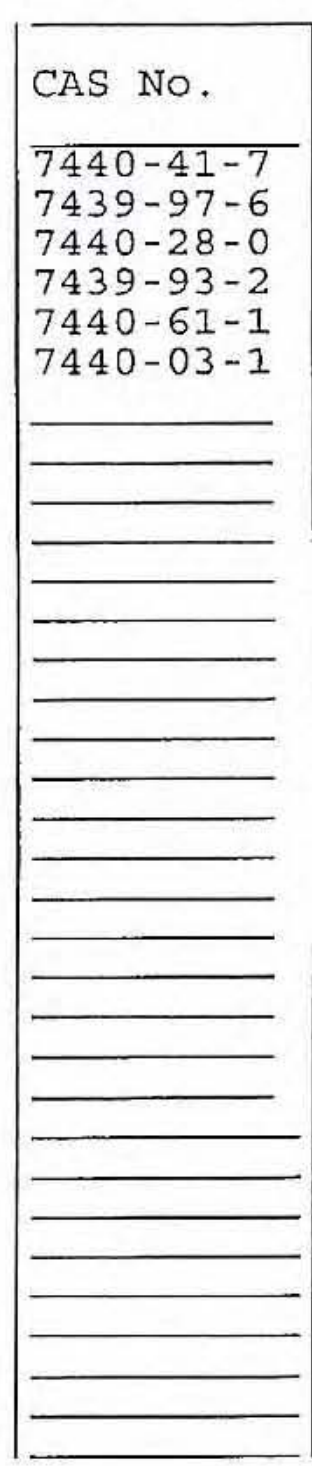

Color Before:

Color After:

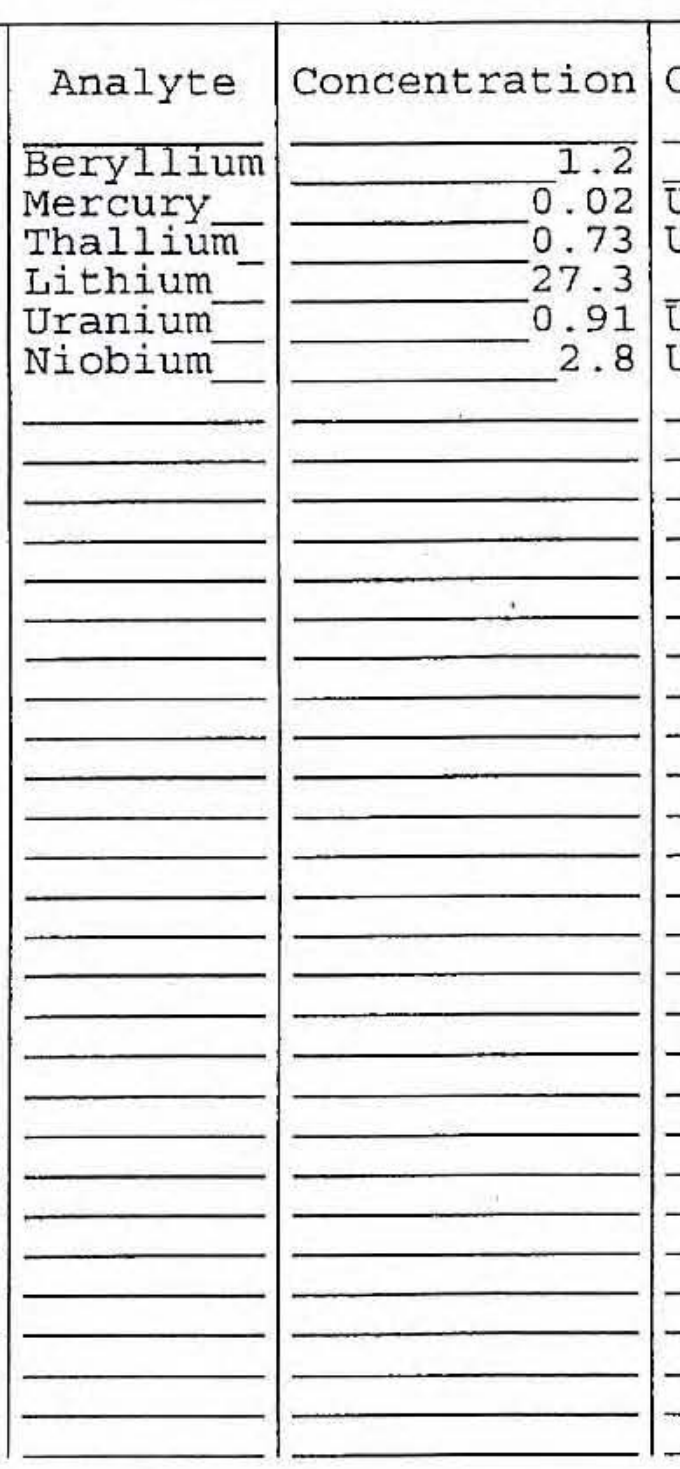

Clarity Before: Clarity After:

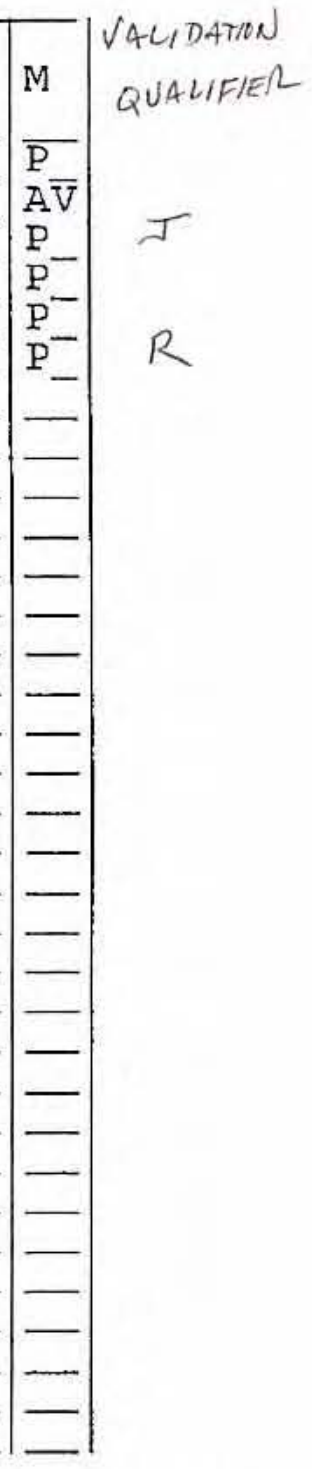

Texture: Artifacts:

Comments:

PDRO-12A 
Lab Name: LIONVILLE LABORATORY

Lab code: LVLI

Matrix (soil/water): soIL

Level (low/med):

Solids :

$$
\text { LOW - } 88 \overline{.1}
$$

Contract: 60052

SAS NO. :

$12 \mathrm{~B}$

SDG NO.: 11A

Lab Sample ID: 0608L79

Date Received: 08/24/06

Concentration Units (ug/L or $\mathrm{mg} / \mathrm{kg} \mathrm{dry}$ weight): $\mathrm{MG} / \mathrm{KG}$

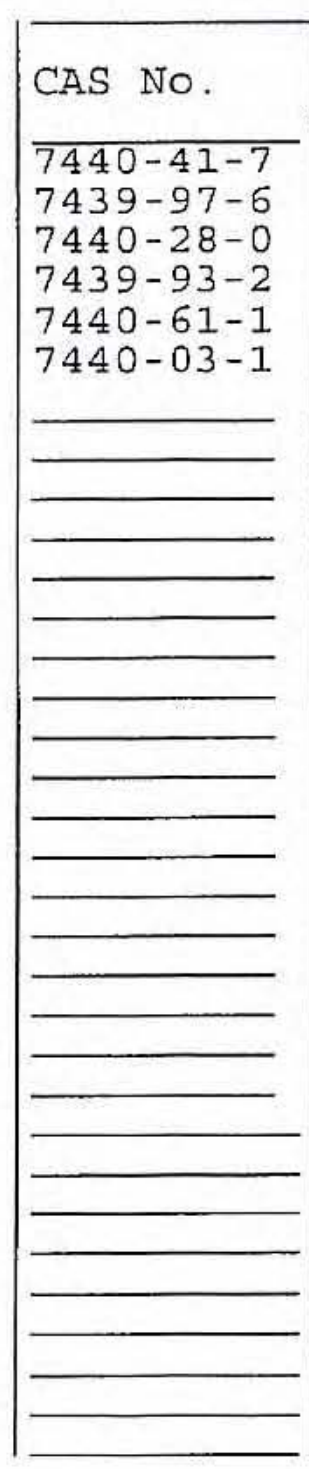

Color Before:

Color After:

Comments :

PDRO $-12 B$

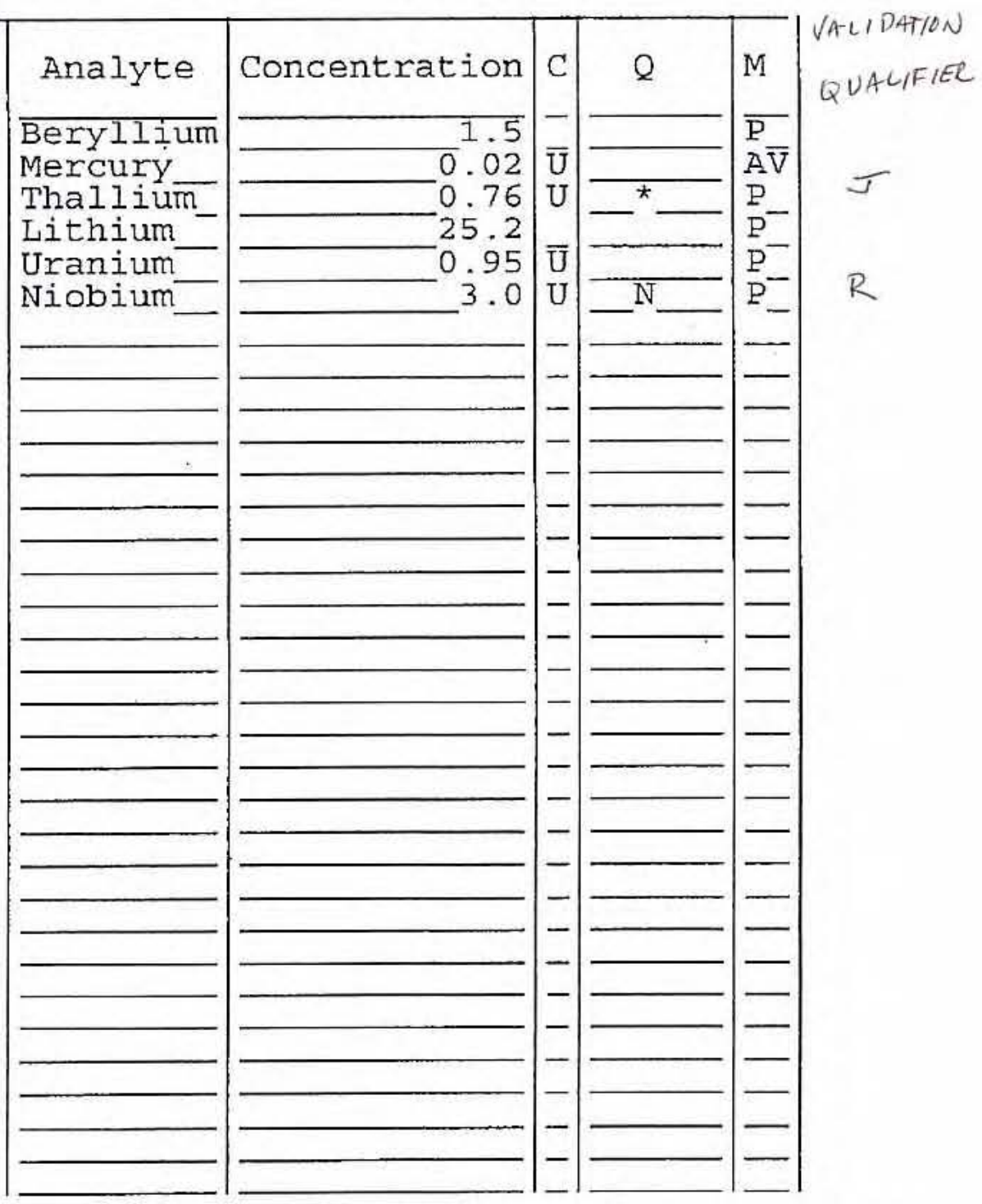

Clarity Before: Clarity After:
Texture:

Artifacts: 
1 INORGANIC ANALYSES DATA SHEET
Lab Name: LIONVILLE_LABORATORY

Lab Code: LVLI Matrix (soil/water): SoIL Level (low/med): \% Solids:
Contract: 60052

SAS NO.:

Lab Sample ID: 0608L79 $\overline{6-0} 05$

Date Received: 08/24/06

Concentration Units (ug/L or $\mathrm{mg} / \mathrm{kg}$ dry weight): MG/KG

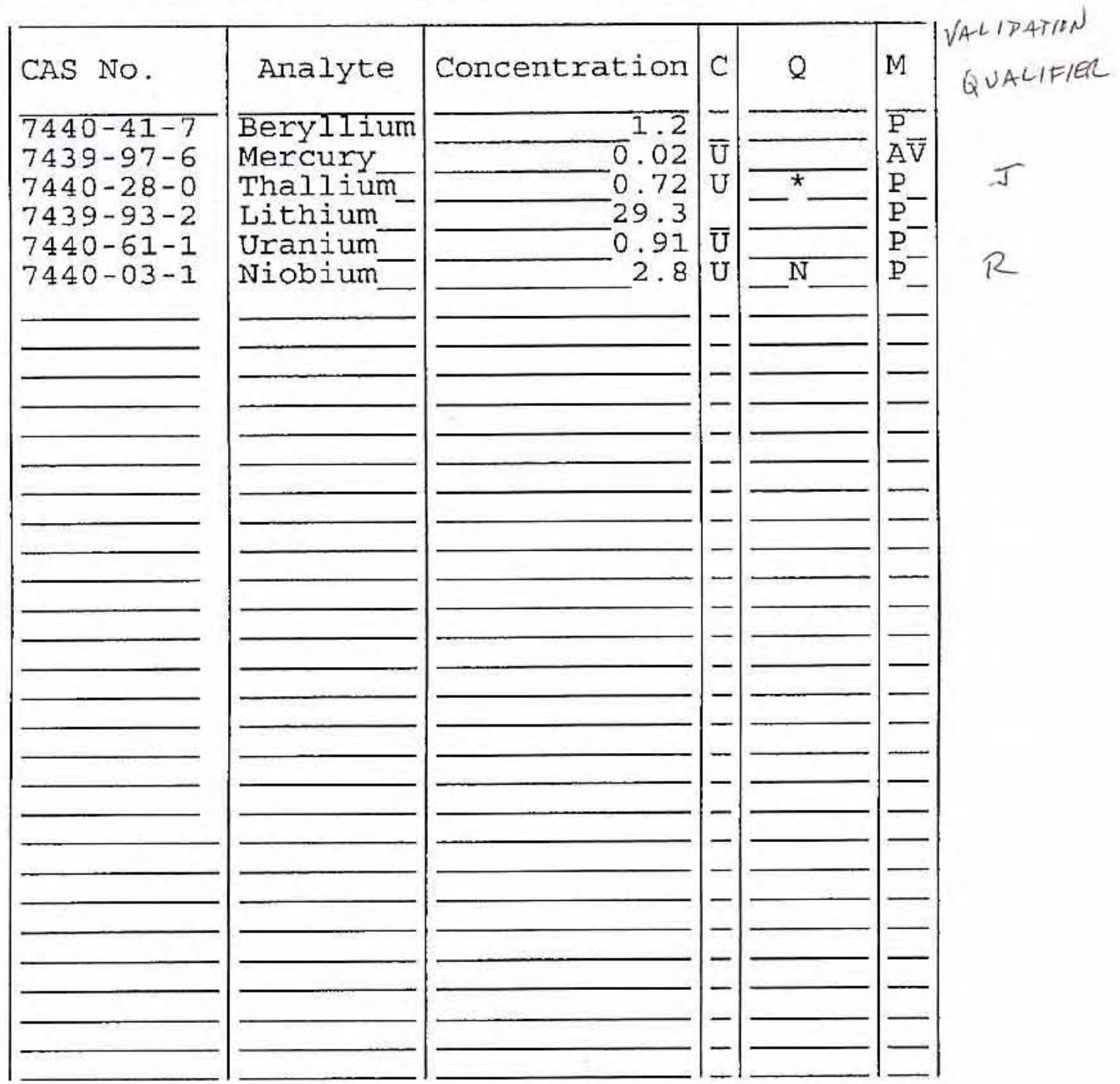

Color Before: Color After:
Clarity Before:

Clarity After:
Texture:

Artifacts :

Comments :

PDRO-13A 
1

INORGANIC ANALYSES DATA SHEET
EPA SAMPLE NO.

\section{$13 B$}

Contract: 60052

SAS No. :

SDG No.: 11A

Case No.: PDRO

Lab Sample ID : 0608L79 $\overline{6-0} 06$

Date Received: 08/24/06 $\begin{array}{ll}\text { Lab Code: LVLI } & \text { Cas } \\ \text { Matrix (soil/water): } & \text { soIL } \\ \text { Level (low/med): } & \text { LOW } \\ \% \text { Solids: } & -89.4\end{array}$

Concentration Units (ug/L or $\mathrm{mg} / \mathrm{kg}$ dry weight): MG/KG

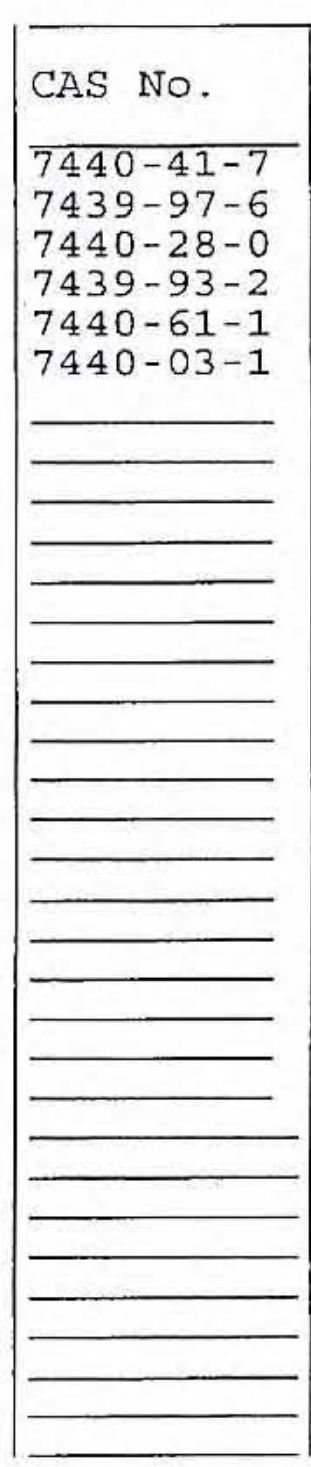

Color Before:

Color After:

Comments:

PDRO-13B

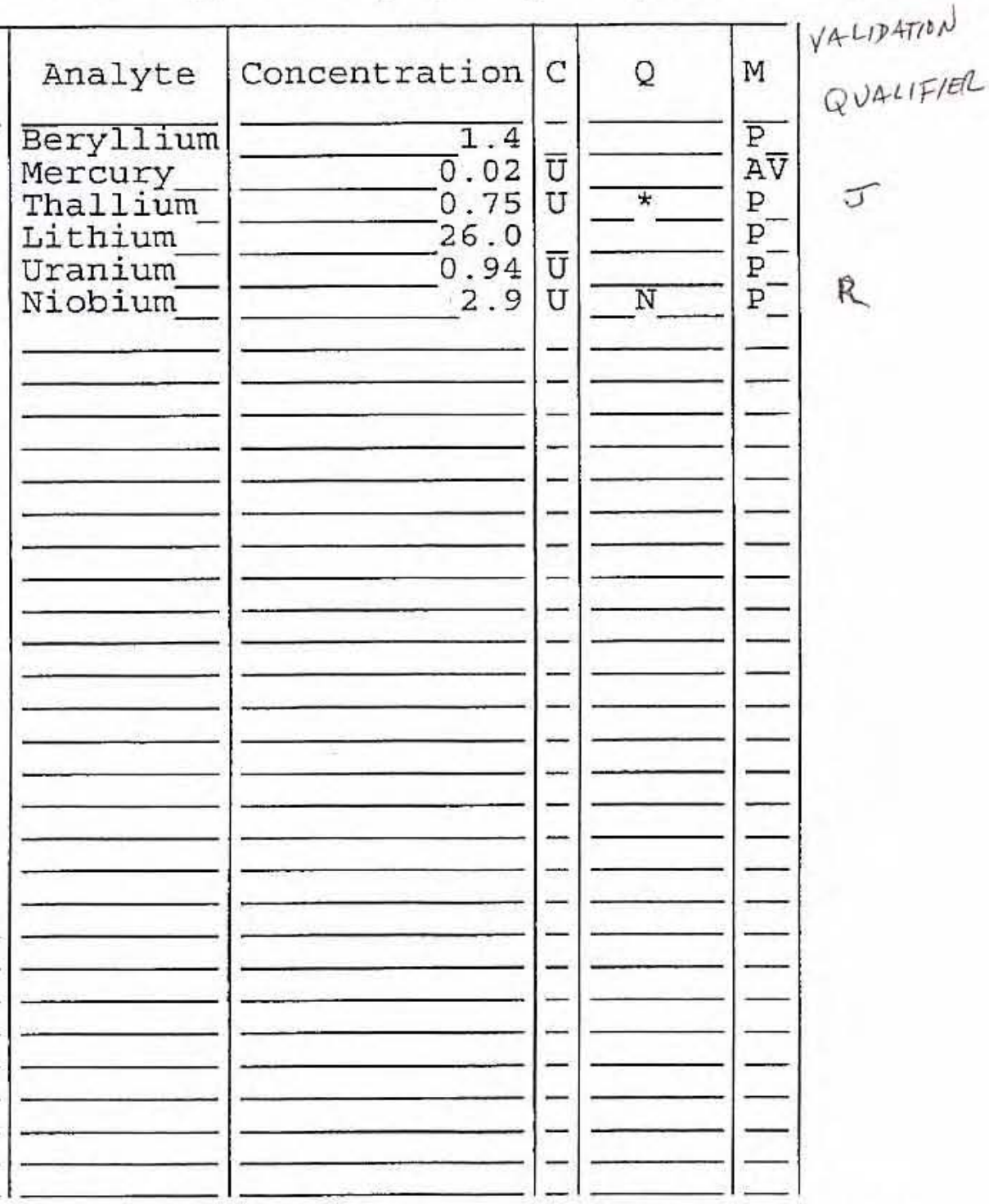

Clarity Before:

Clarity After:
Texture:

Artifacts : 
1

INORGANIC ANALYSES DATA SHEET
EPA SAMPLE NO.

$14 \mathrm{~A}$

SDG NO.: 11A

SAS NO.:

Lab Sample ID: 0608L79 $\overline{6-0} 07$

Date Received: $08 / 24 / 06$
Lab Name: LIONVILLE LABORATORY

Lab Code: LVLI Matrix (soil/water): SoIL Level (low/med): $\div$ Solids:

\section{LOW \\ $86 \overline{.2}$}

Contract: 60052

Case NO.: PDRO

$$
-
$$

Concentration Units (ug/L or $\mathrm{mg} / \mathrm{kg}$ dry weight): MG/KG

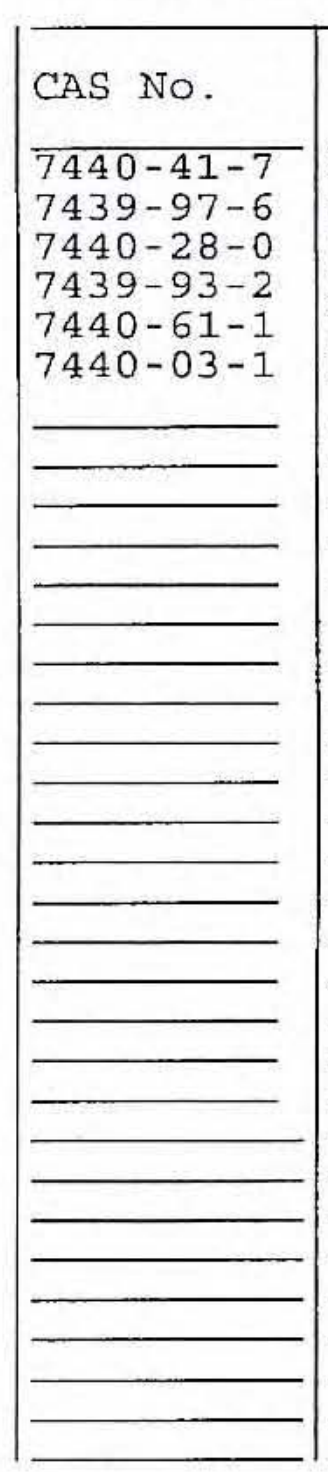

Color Before:

Color After:

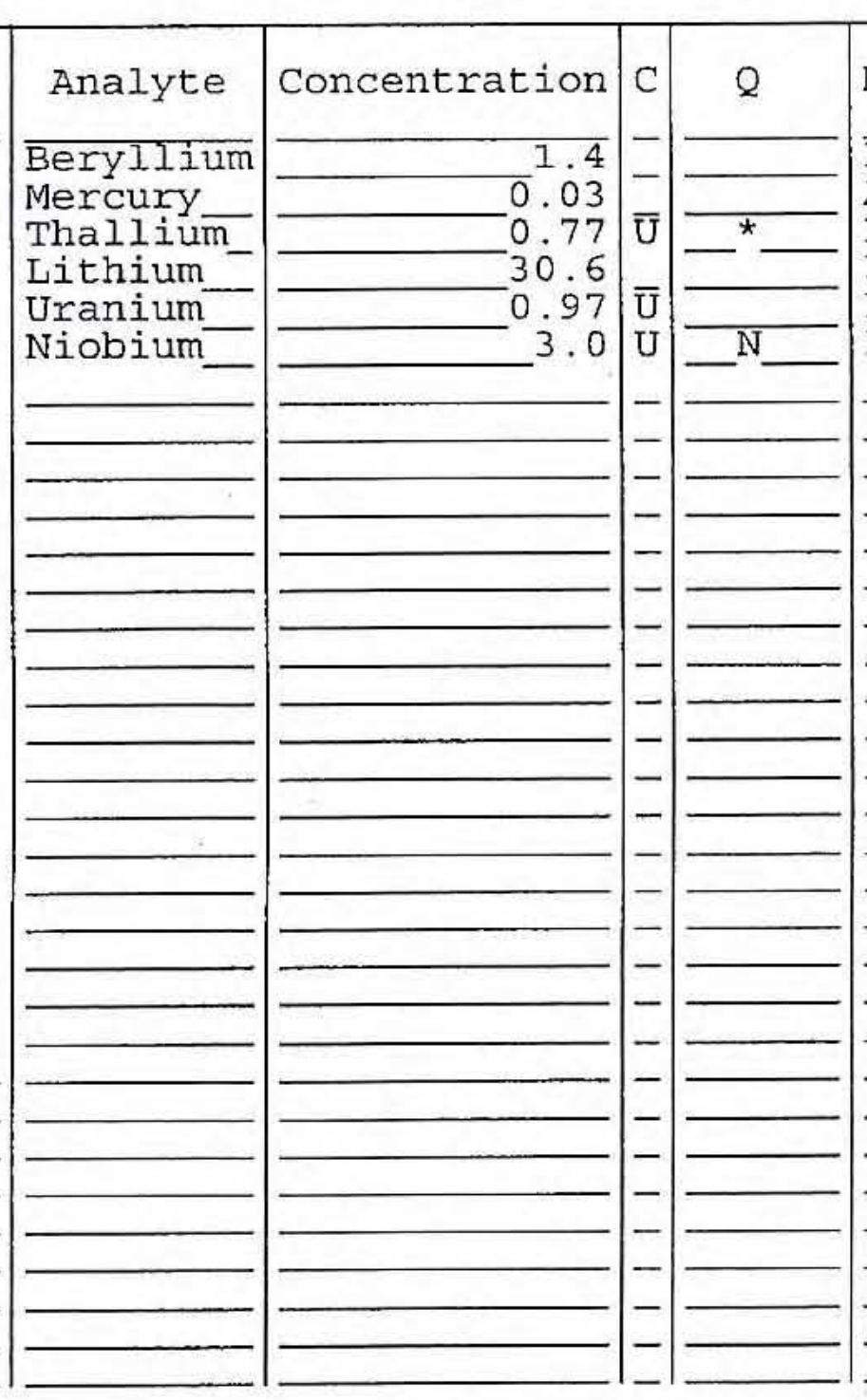

Clarity Before: Clarity After:

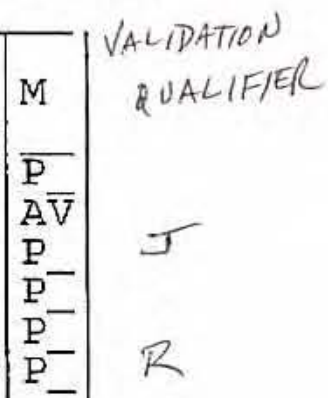

Texture:

Artifacts:

Comments:

PDRO-14A 
1

INORGANIC ANALYSES DATA SHEET
EPA SAMPLE NO.

\section{$14 B$}

Contract: 60052

Lab Sample ID: 06்08I79

Date Received: 08/24/06
Lab Name: LION
Lab Code: LVLI Matrix (soil/wat
Level (low/med) \% Solids :
SAS NO. :
Case No.: PDRO SOIL_ LOW

SDG NO.: $11 \mathrm{~A}$ :

Concentration Units (ug/L or $\mathrm{mg} / \mathrm{kg}$ dry weight): MG/KG

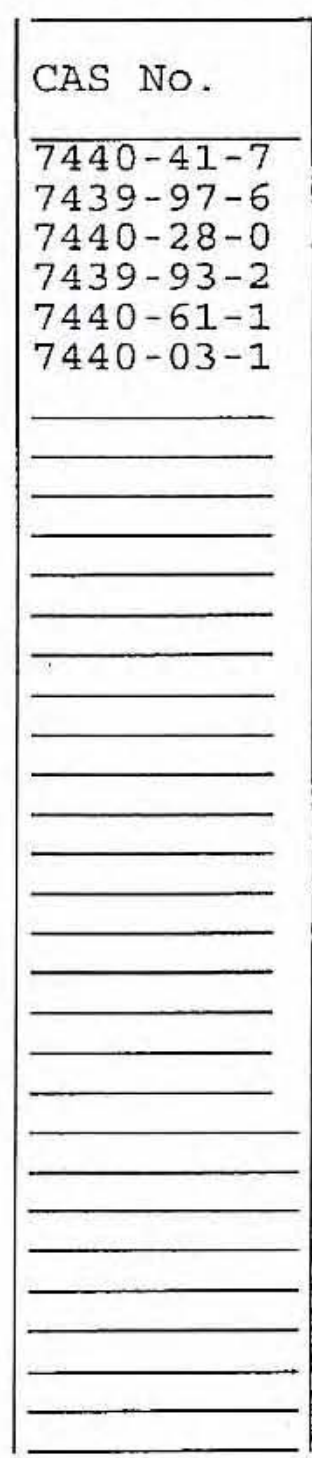

Color Before: Color After:

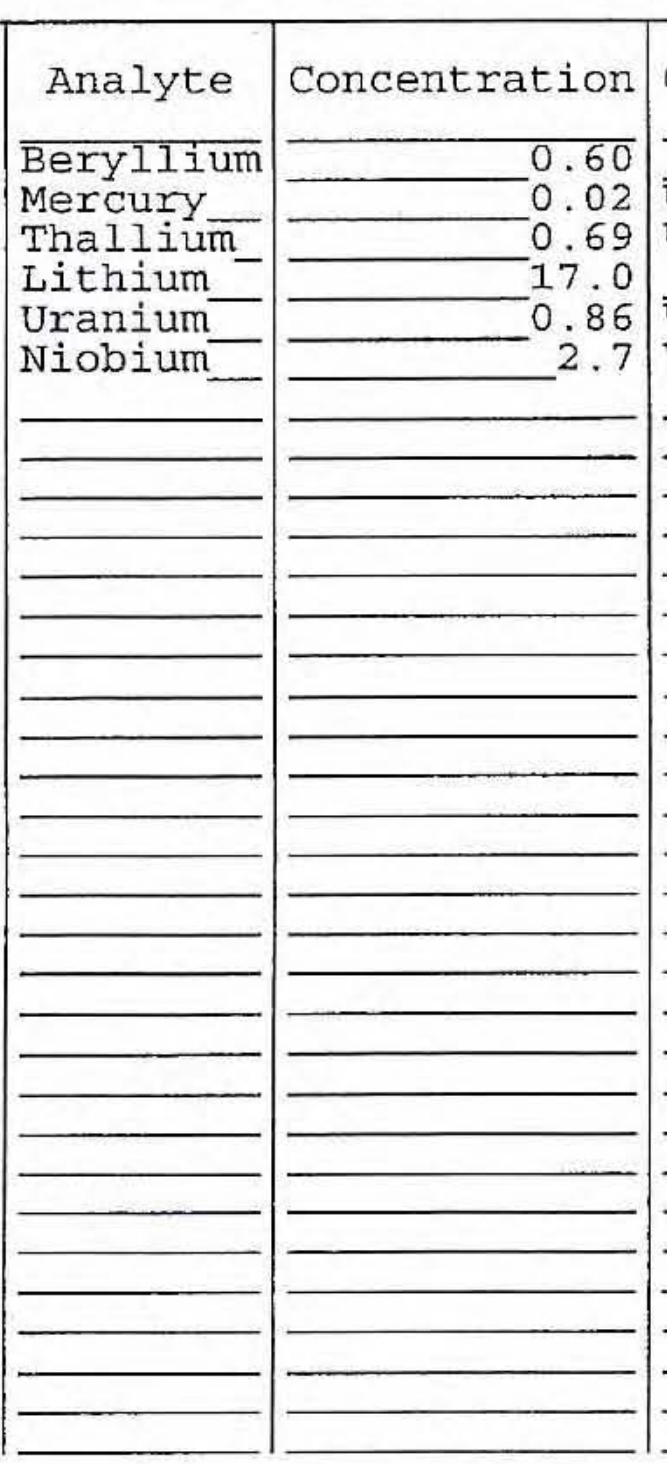

Clarity Before: Clarity After:

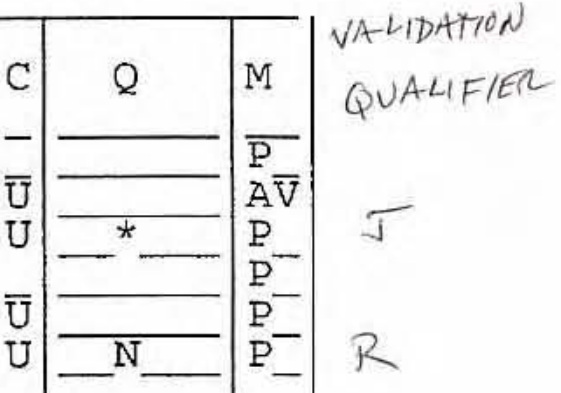

Comments :

PDRO-14B 


\section{1}

INORGANIC ANALYSES DATA SHEET
EPA SAMPLE NO.

\section{$15 \mathrm{~A}$}

Contract: 60052

SAS NO.:

SDG NO : : 11A

Lab Sample ID: $0608479 \overline{6-0} 09$

Date Received: 08/24/06
Lab Code: LVLI

Matrix (soil/wat
Level (low/med):

Level
Case NO.: PDRO

$$
\begin{aligned}
& \text { SOIL } \\
& \text { LOW- } \\
& -95.0
\end{aligned}
$$

Concentration Units (ug/L or $\mathrm{mg} / \mathrm{kg}$ dry weight): $\mathrm{MG} / \mathrm{KG}$

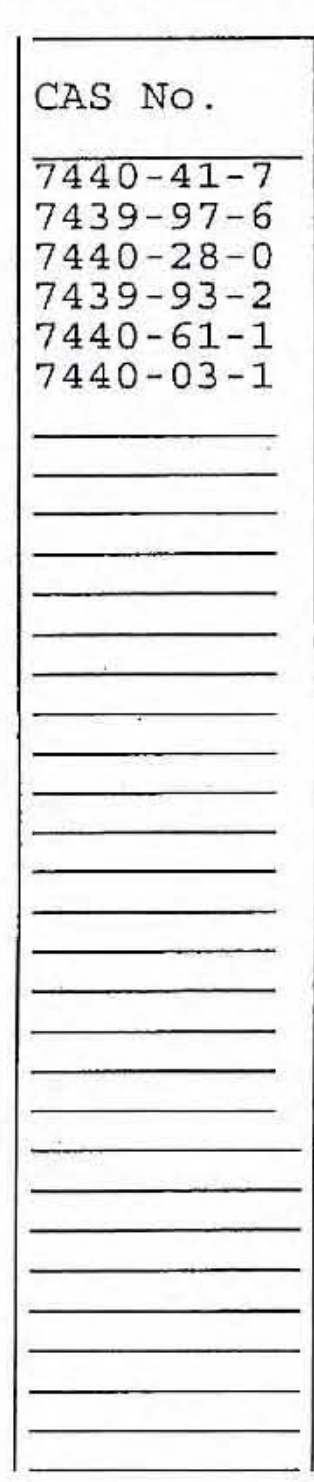

Color Before: Color After:

Comments :

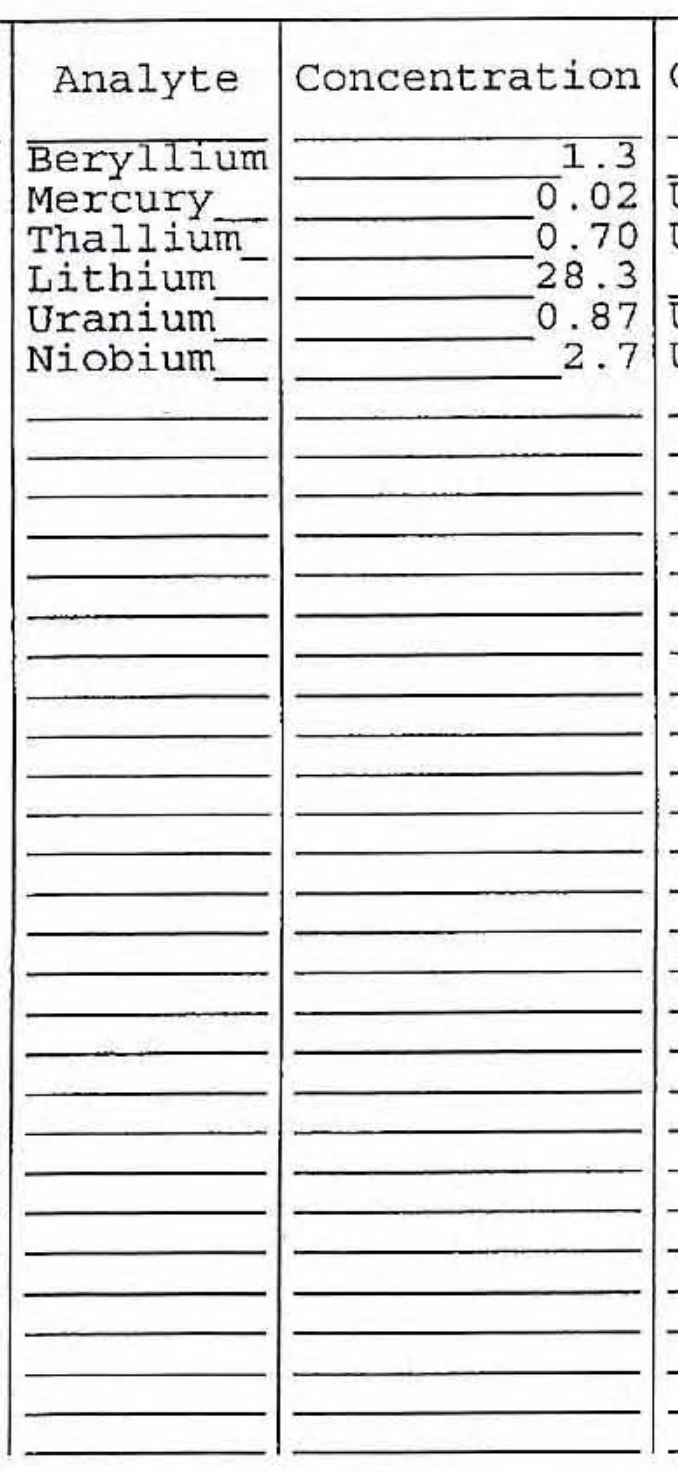

Clarity Before: Clarity After:

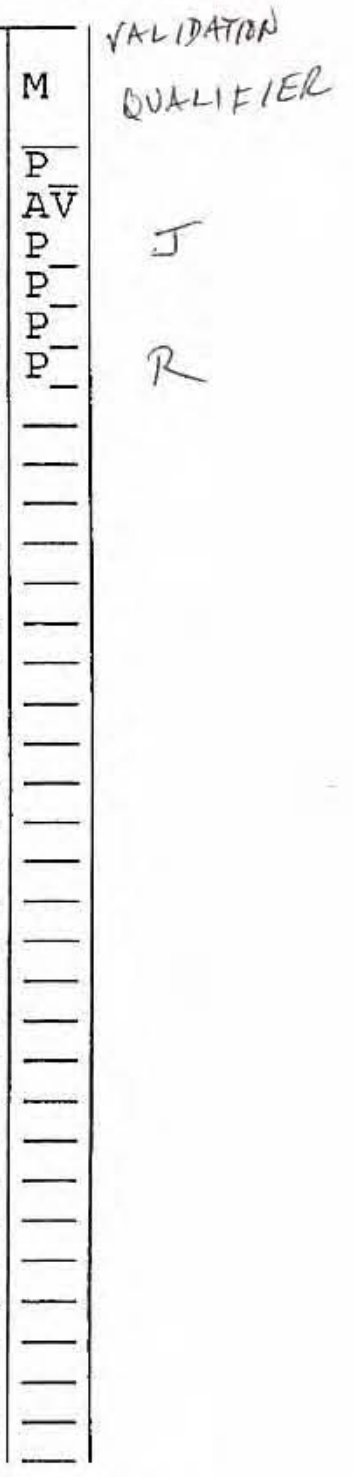

Texture: Artifacts: PDRO-15A 
1 INORGANIC ANALYSES DATA SHEET
EPA SAMPLE NO.

\section{$15 B$}

SDG NO.: 11A

Lab sample ID : $0608 \mathrm{~L} 79 \overline{6-0} 10$

Date Received: 08/24/06
Lab Name: LION

Matrix (soil/wa $\div$ Solids :

Contract: 60052

SAS No.

\section{Case No.: PDRO}

LOW -92.7

Concentration Units (ug/L or $\mathrm{mg} / \mathrm{kg}$ dry weight): MG/KG

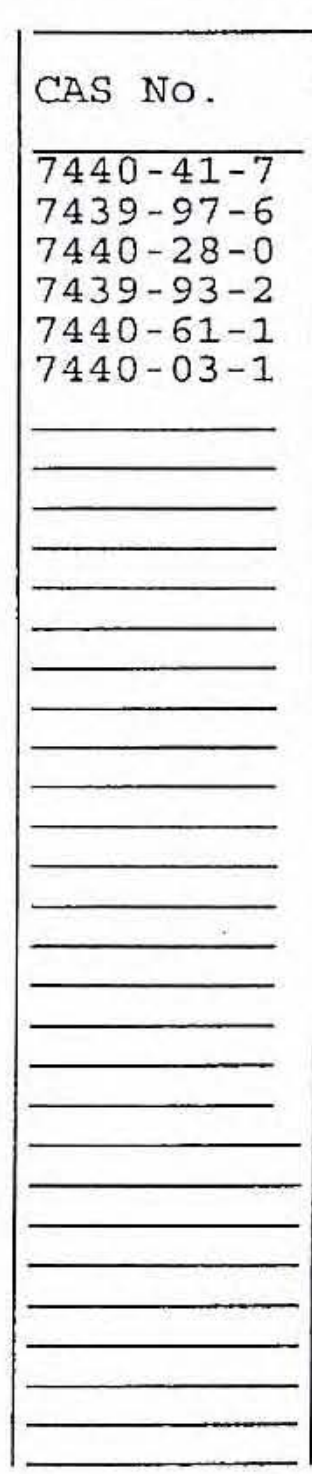

Color Before: Color After:

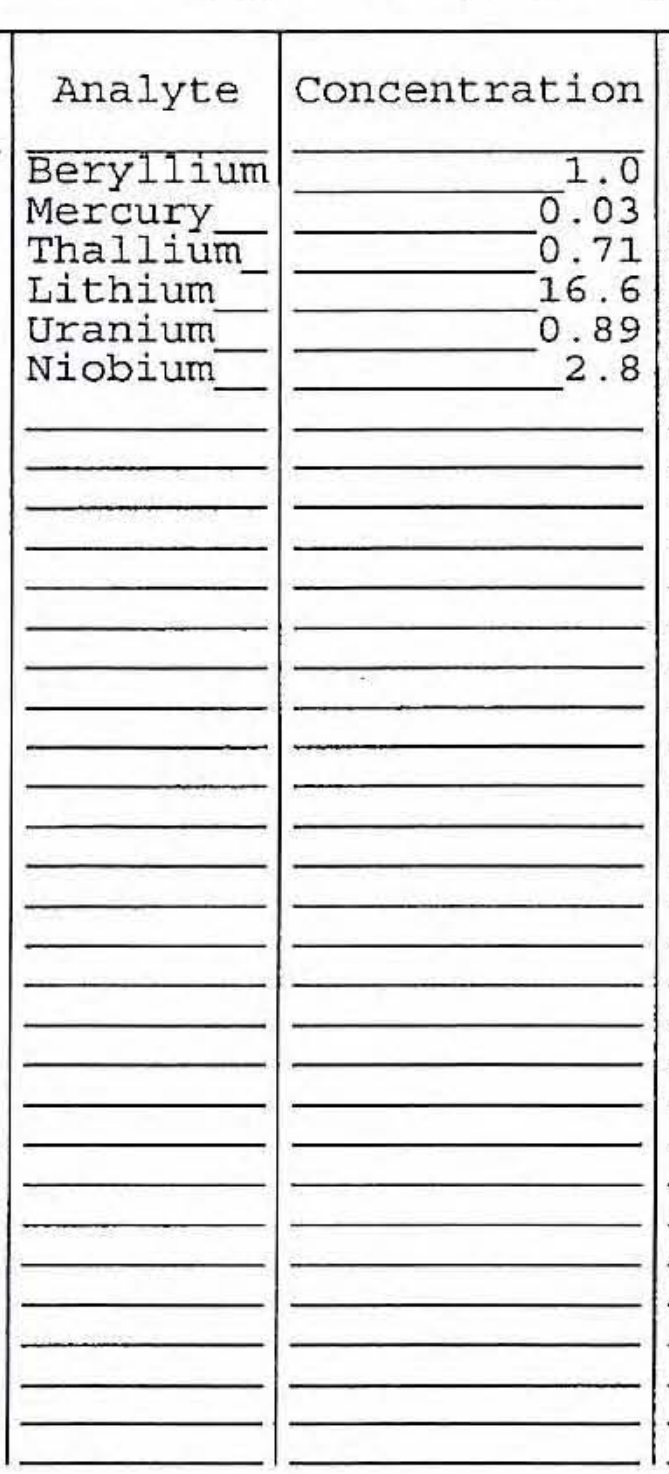

Clarity Before: Clarity After:

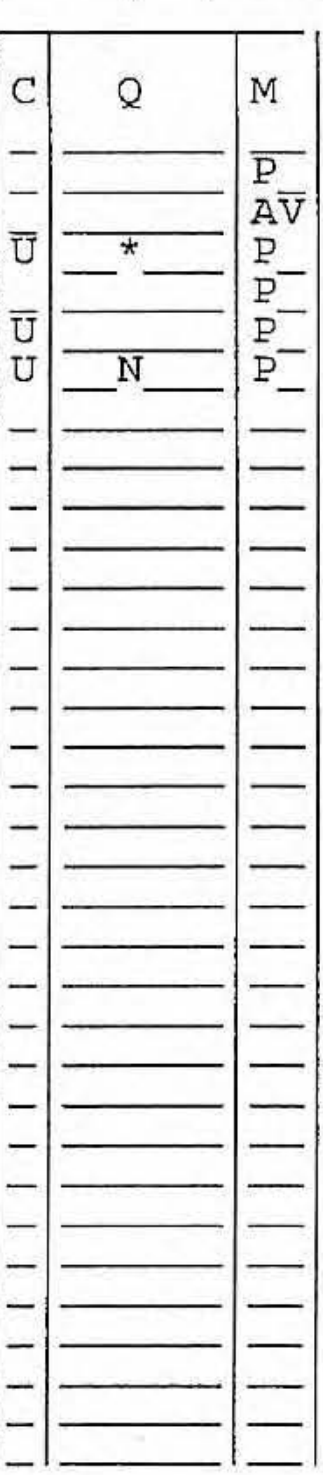

Texture: Artifacts:

Comments :

PDRO-15B 


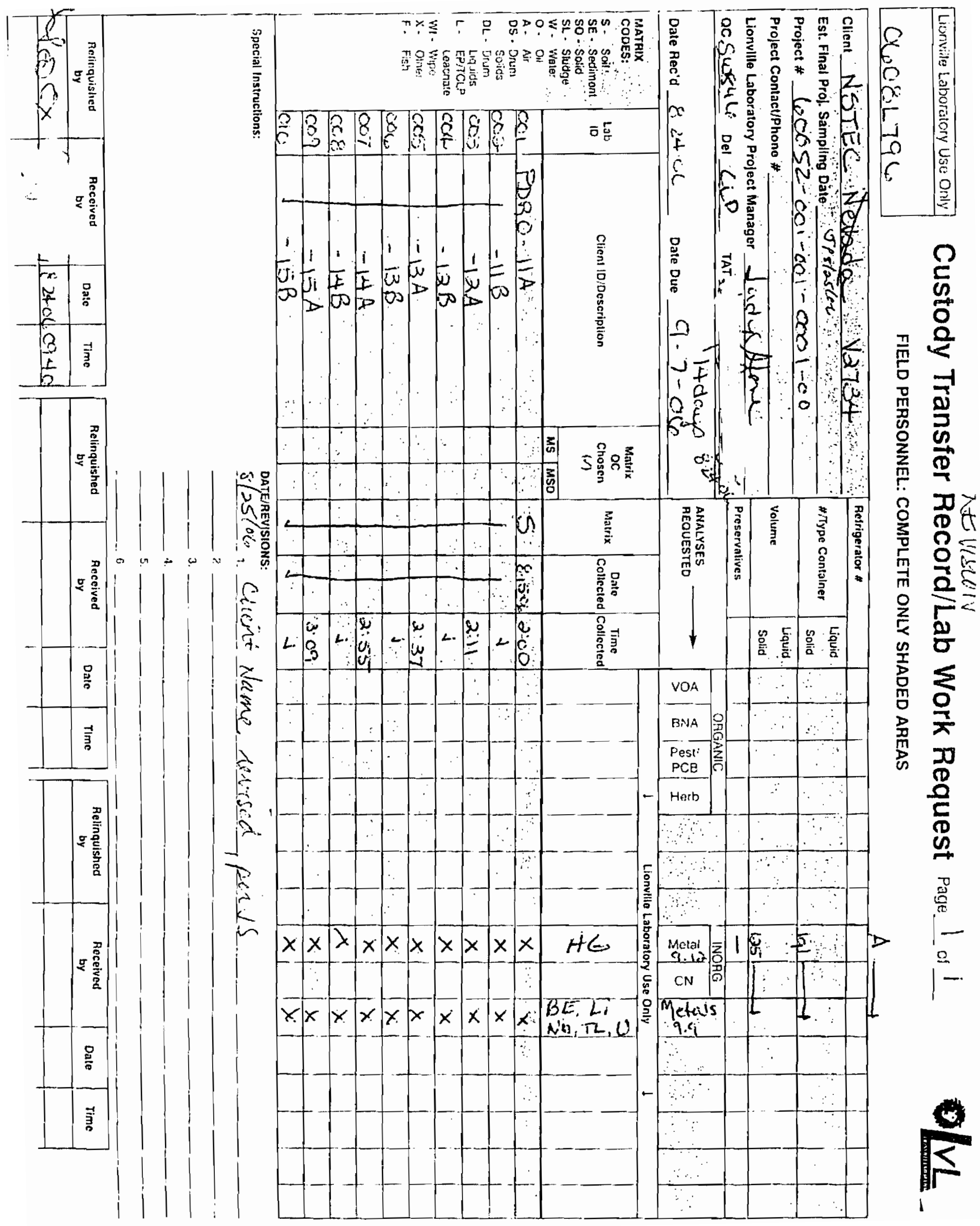




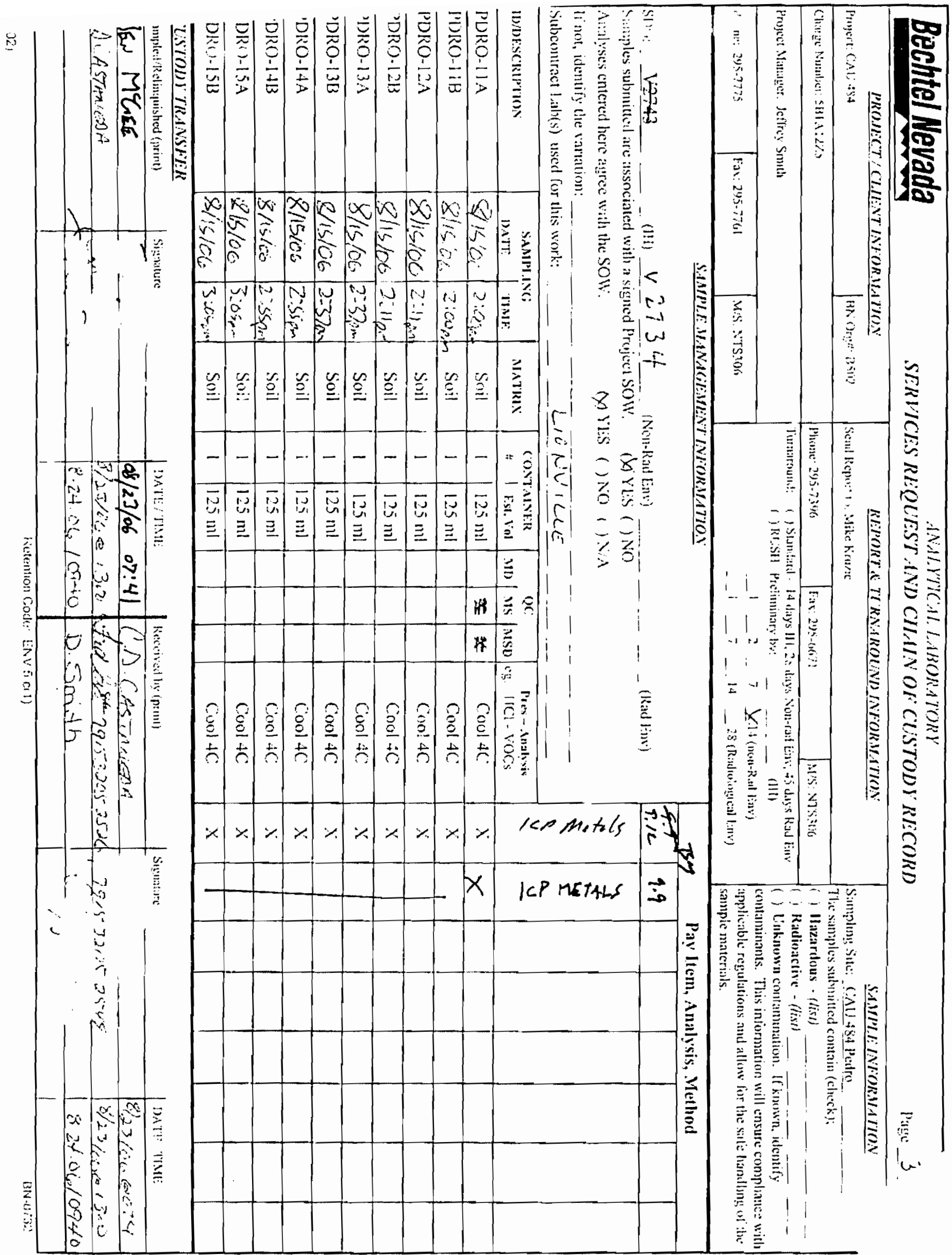


1

INORGANIC ANALYSES DATA SHEET
EPA SAMPLE NO.

$16 \mathrm{~A}$

Contract: 60052

SAS NO.:

SDG No.: V2734

Lab Sample ID: 0608L797-001

Date Received: 08/24/06
Lab Name: LIONT
Lab Code: LVLI Matrix (soil/water): SoIL Level (low/med): LOW 음 Solids:
I.
Case

Concentration Units (ug/L or $\mathrm{mg} / \mathrm{kg}$ dry weight): MG/KG

\begin{tabular}{l}
\hline CAS NO. \\
$\overline{7440-41-7}$ \\
$7439-97-6$ \\
$7440-28-0$ \\
$7439-93-2$ \\
$7440-61-1$ \\
$7440-03-1$ \\
\hline $\bar{\square}$ \\
\hline \\
\hline \\
\hline
\end{tabular}

Color Before:
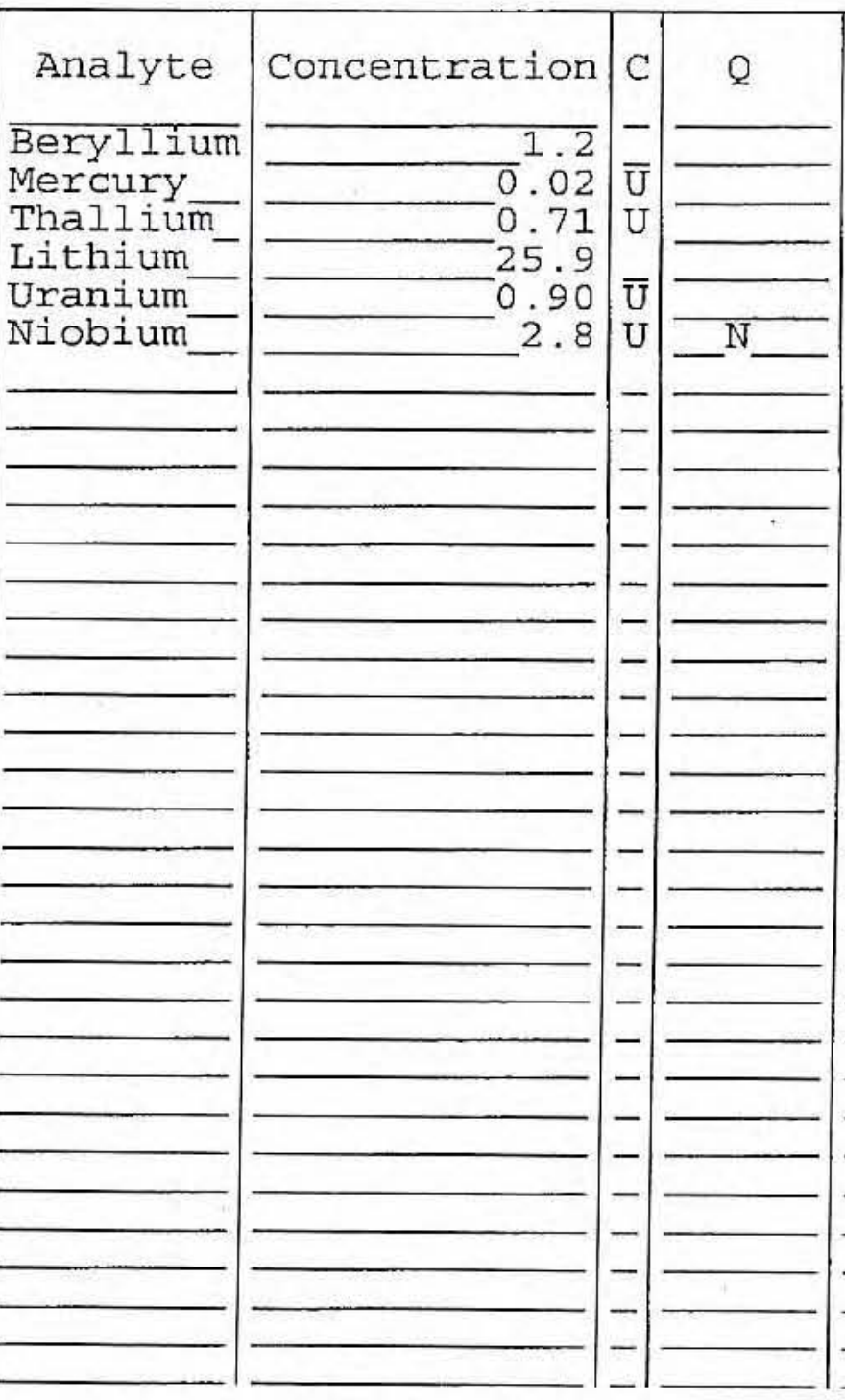

Clarity Before:

Clarity After:

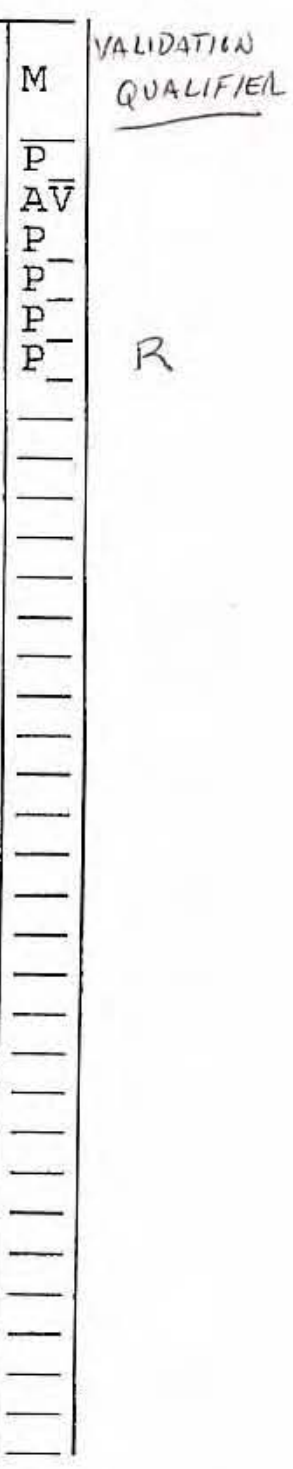

Texture: Artifacts:

Comments:

PDRO-16A 


\section{U.S. EPA}

1

INORGANIC ANALYSES DATA SHEET
EPA SAMPLE NO.

\section{$16 \mathrm{~B}$}

SDG NO.: V2734

Lab Sample ID: 0608I797-0̄02
SAS NO.:

Date Received: 08/24/06

Contract: 60052
Lab Nane: IIONVILLE_LABORATORY

Matrix (soil/water): SoIL Level (low/med):

\% Solids:
LOW

$-92 . \overline{0}$

Concentration Units (ug/L or $\mathrm{mg} / \mathrm{kg}$ dry weight): MG/KG

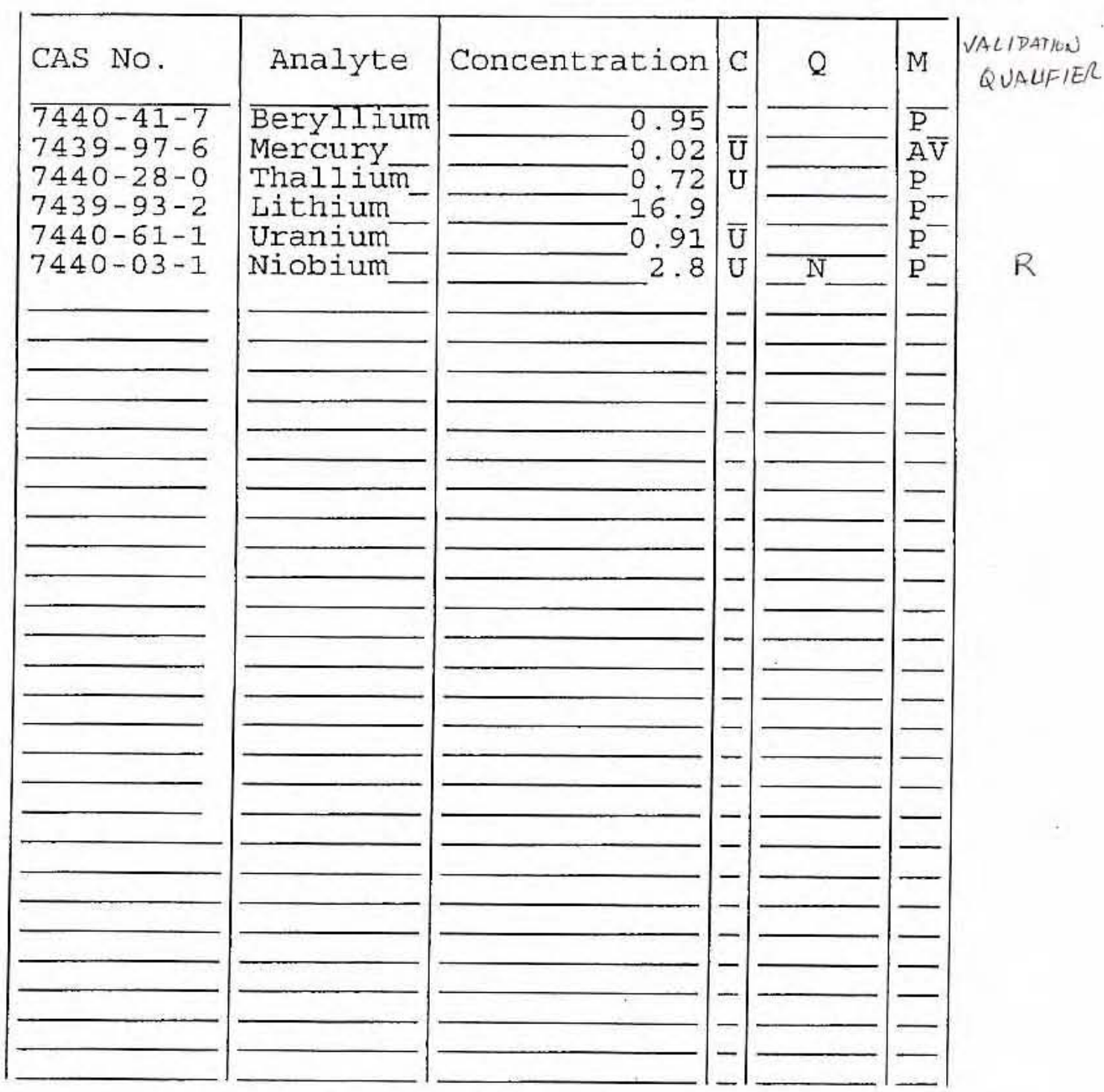

Color Before:

Color After:
Clarity Before:

Clarity After:
Texture:

Artifacts:

Comments :

PDRO-16B 
1 INORGANIC ANALYSES DATA SHEET
EPA SAMPLE NO.

$17 \mathrm{~A}$

Contract: 60052

SAS No. :

Case NO.: PDRO

SOIL
LOW
-93.5

Lab Sample ID: 0608L797-0003

Date Received: 08/24/06

Concentration Units (ug/L or $\mathrm{mg} / \mathrm{kg}$ dry weight) : MG/KG

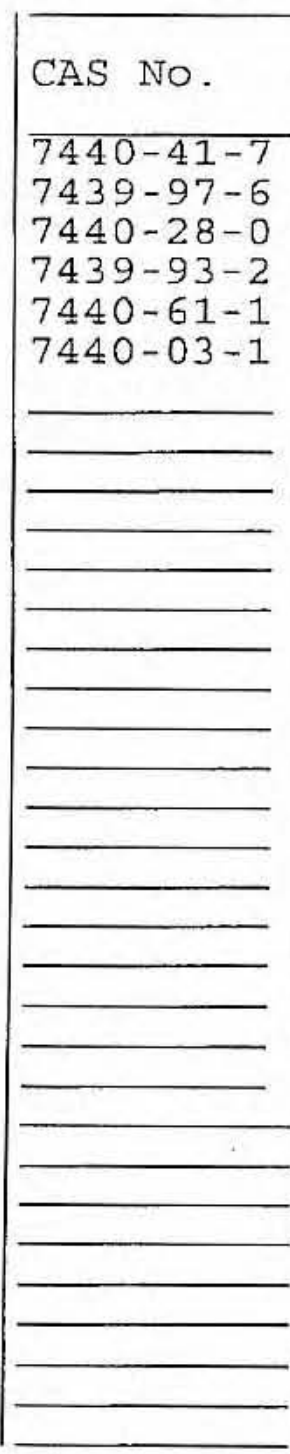

Color Before:

Color After:

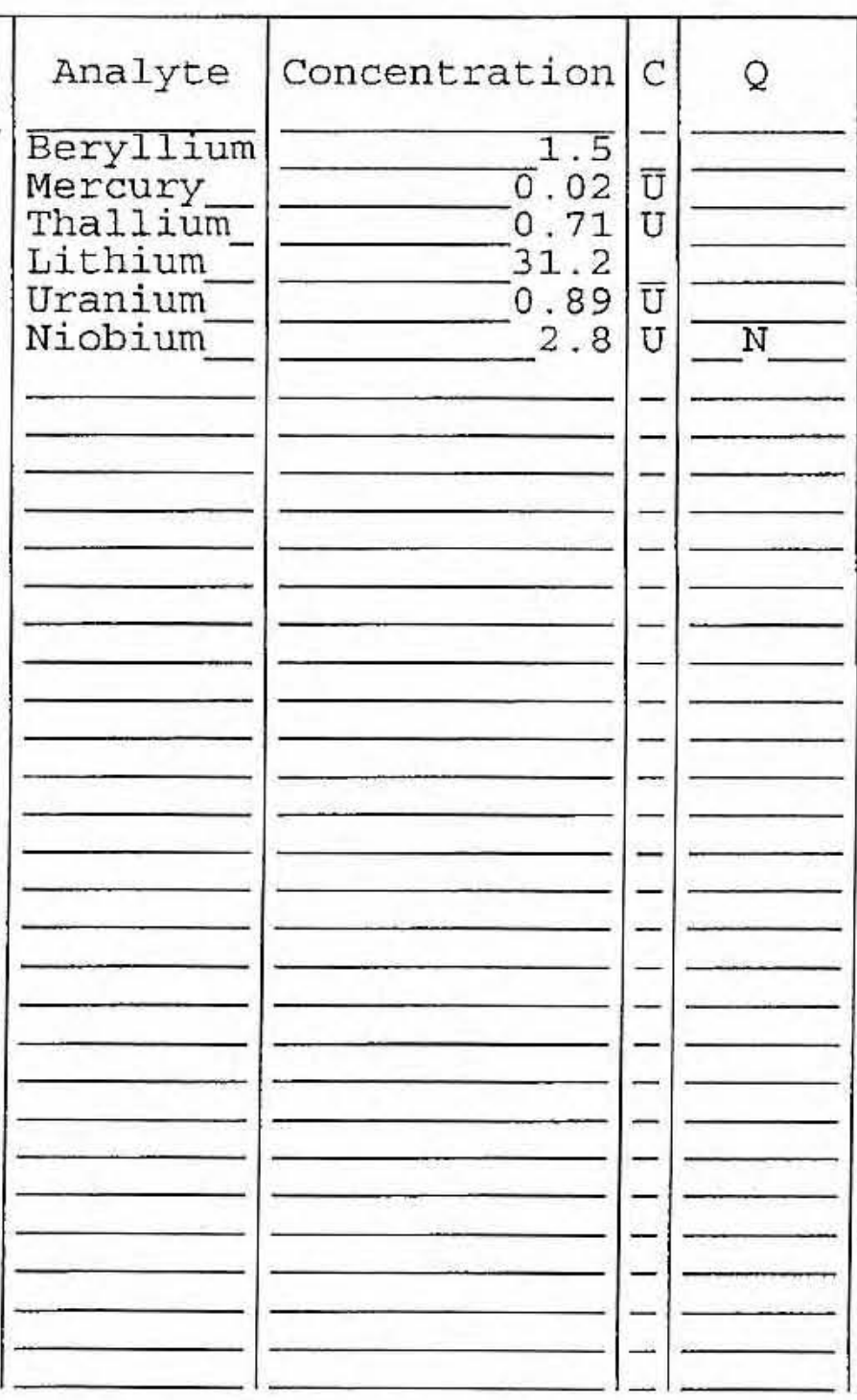

Clarity Before:

Clarity After:

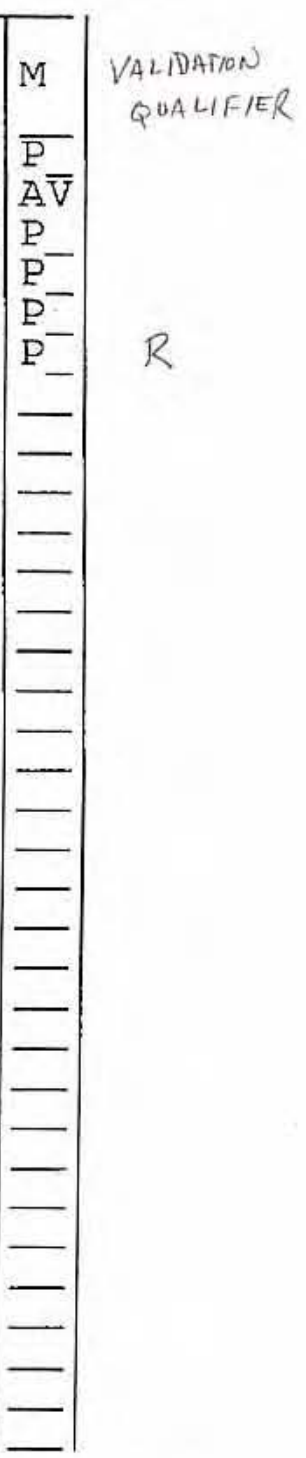

Texture:

Artifacts:

Comments:

PDRO-17A 
1 INORGANIC ANALYSES DATA SHEET
EPA SAMPLE NO.

\section{$17 \mathrm{~B}$}

Contract: 60052

Lab Sample ID: 0608L797-004
Lab Code: LVLI

Matrix (soil/water) Level (low/med): \% Solids:
SAS NO.: SOIL LOW

$-88 \overline{\cdot 8}$

Case NO.: PDRO

$$
8.8
$$

Concentration Units (ug/L or $\mathrm{mg} / \mathrm{kg}$ dry weight) : MG/KG
Color Before: Color After:

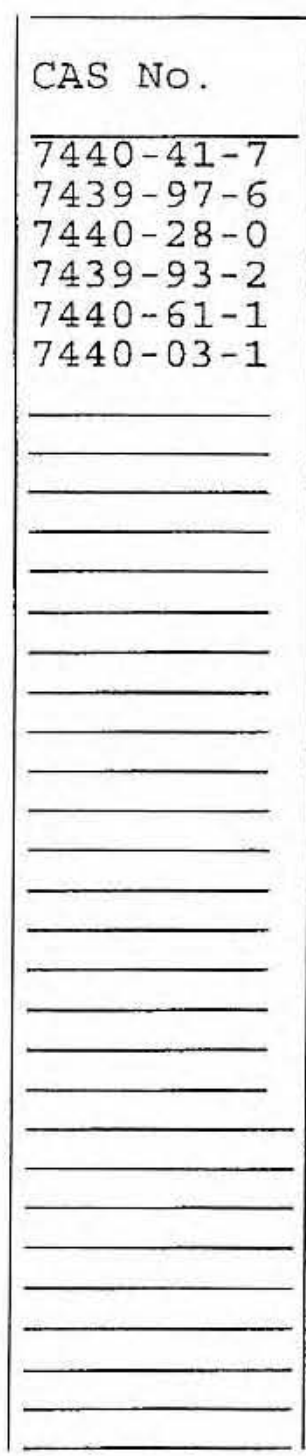

Clarity Before: Clarity After:
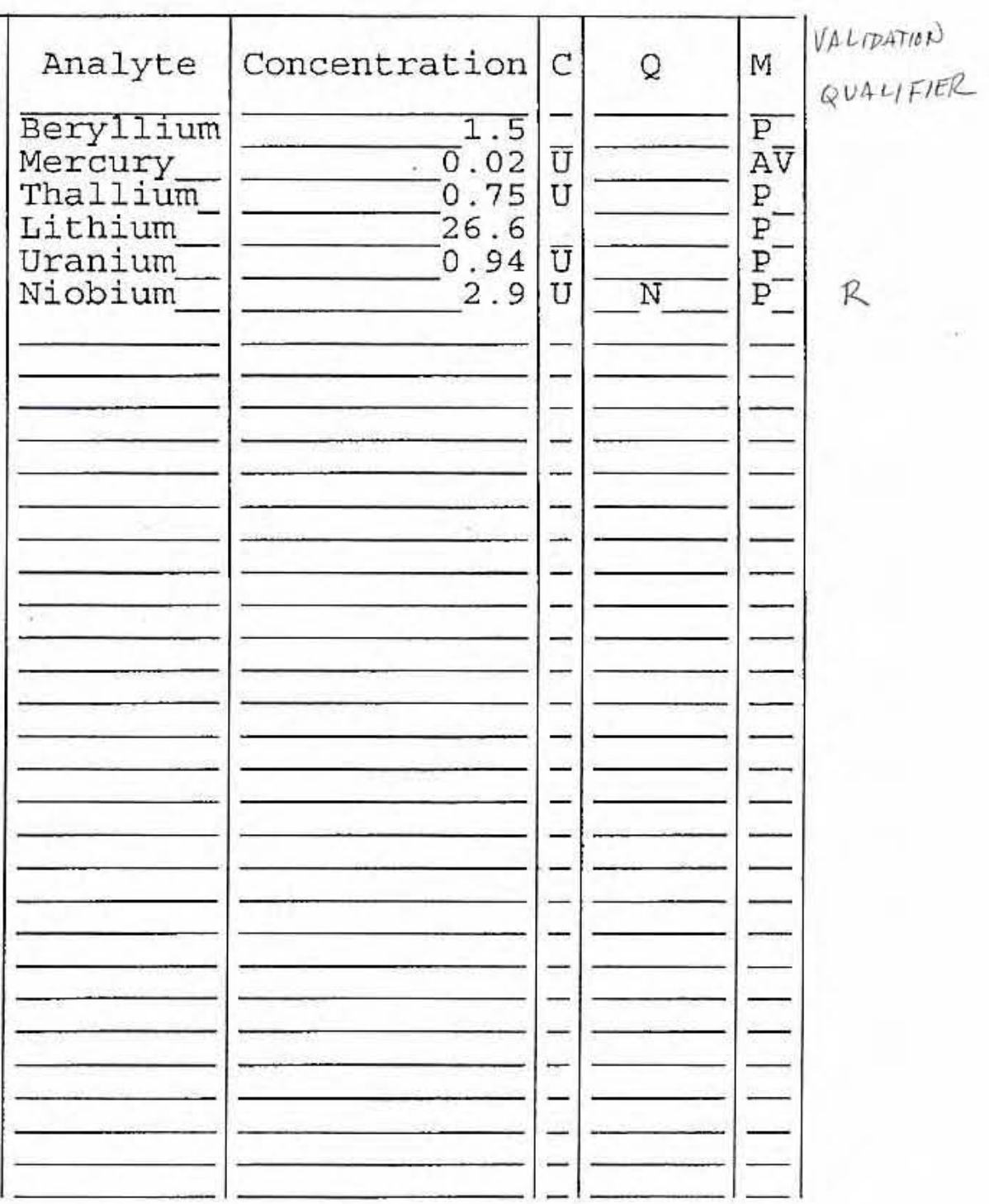

Comments:

PDRO- I 7 B

Texture:

Artifacts: 


\section{1}

INORGANIC ANALYSES DATA SHEET
EPA SAMPLE NO.

$18 \mathrm{~A}$

Contract: 60052

SAS NO.:

SDG NO.: V2734

Lab Sample ID: 0608L797-0̄05

Date Received: 08/24/06

RA
Cas
IL_-
W

RATORY
Matrix (soil/water): SOIL
Level (low/med):

$\begin{array}{ll}\text { Matrix (soil/water): SOII } & \text { LOW } \\ \text { Level (low/med): } & \text { Low }\end{array}$

Lab Name: LION
Lab Code: LVII

\%olids:

$$
-
$$

Concentration Units (ug/L or mg/kg dry weight): MG/KG

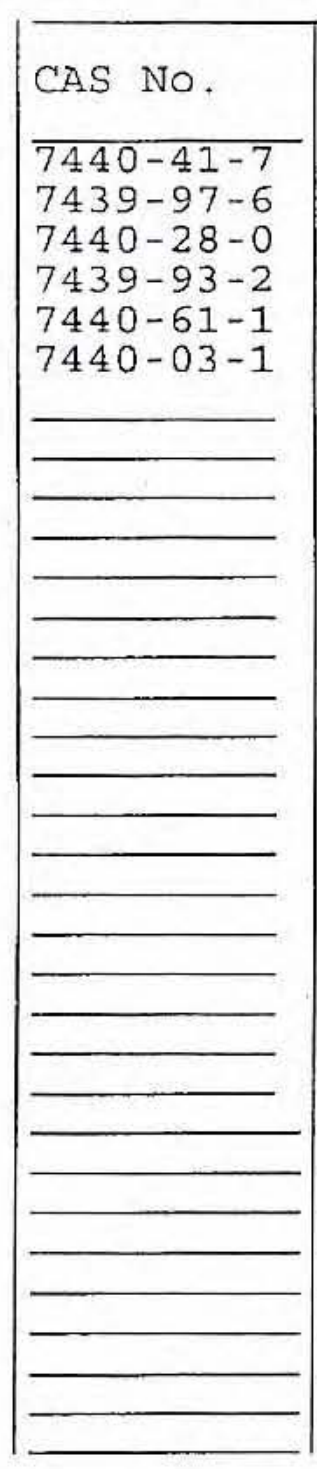

Color Before: Color After:

Comments :

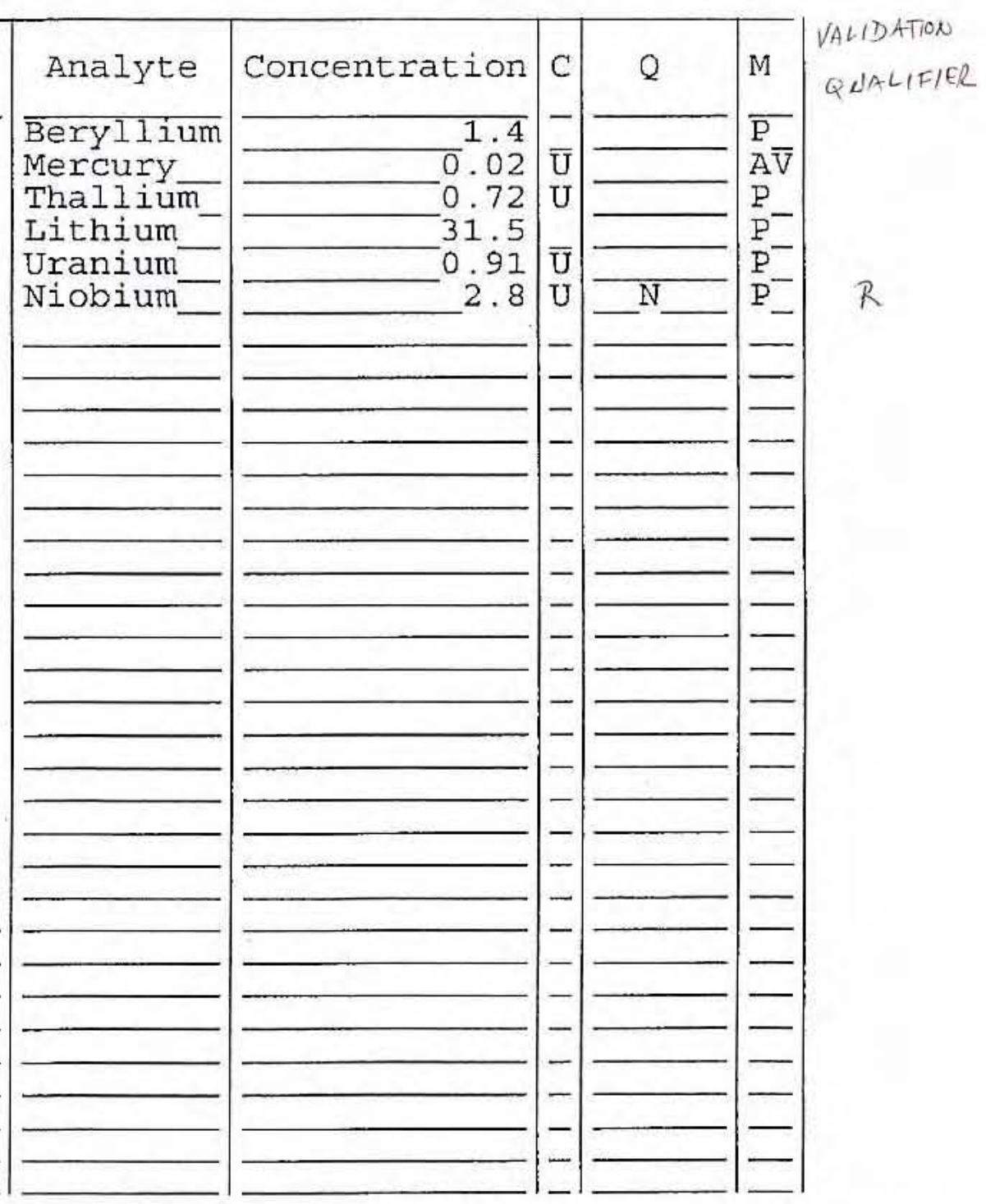

Clarity Before: Clarity After:
Texture: Artifacts :

\section{PDRO-18A}


1

INORGANIC ANALYYSES DATA SHEET
EPA SAMPLE NO.

\section{B}

Contract: 60052

SAS NO.:

SDG NO.: V2734

Lab Sample ID: 0608L797-006

Date Received: 08/24/06

Lab Code: LVII
Matrix (soil/water): SoII
Level (low/med):

$\begin{array}{ll}\text { Level (low/med) : } & \text { LOW } \\ \text { \% Solids: } & \quad 87.5\end{array}$

Concentration Units (ug/L or $\mathrm{mg} / \mathrm{kg}$ dry weight): MG/KG

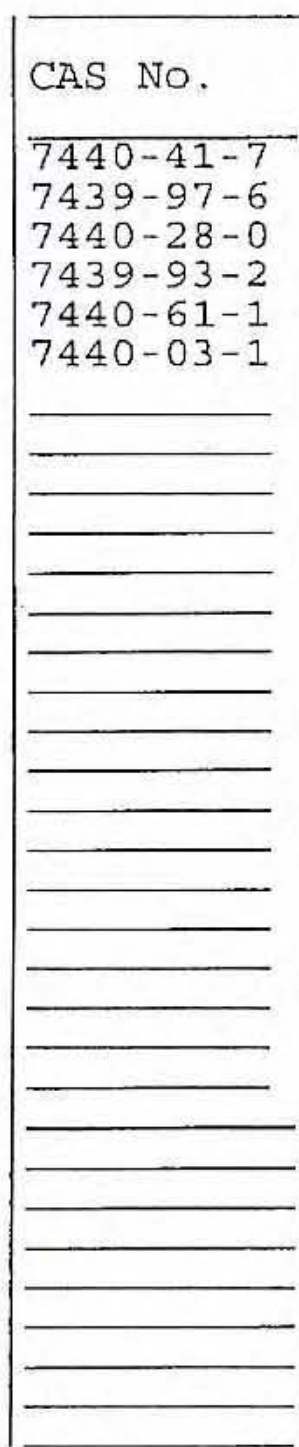

Color Before:

Color After:

Comments :

PDRO-18B

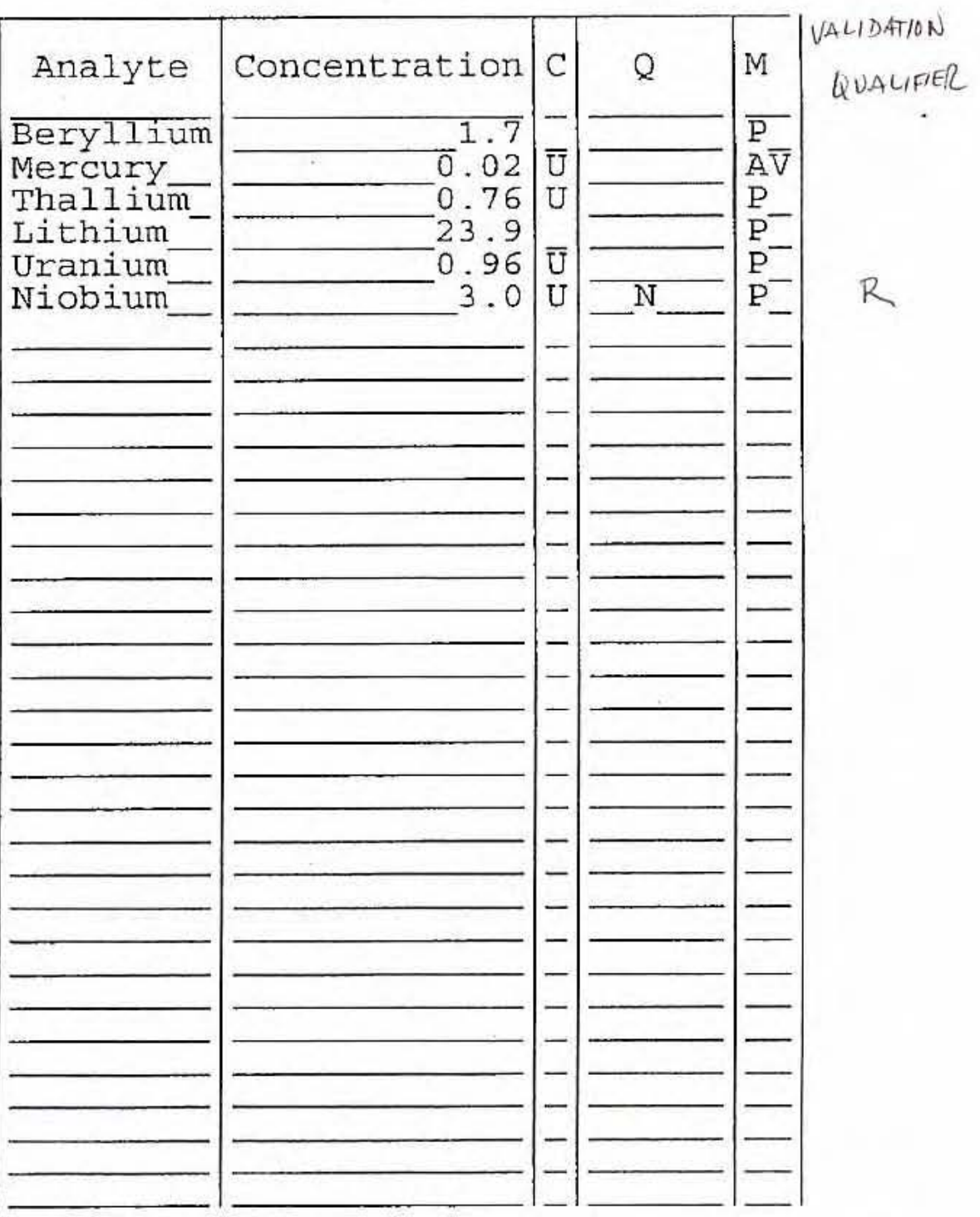

Clarity Before: Clarity After:

Texture:

Artifacts: 
EPA SAMPLE NO.

$19 \mathrm{~A}$

Lab Name: LIONVILLE_LABORATORY_Contract: 60052

Lab Code: LVLI - Case No.: PDRO_ SAS No.: Matrix (soil/water): SoIL

Level (low/med): LOW

\% Solids:
SDG NO.: V2734

Lab Sample ID: 0608L797-0̄07

Date Received: 08/24/06

Concentration Units (ug/L or $\mathrm{mg} / \mathrm{kg}$ dry weight): MG/KG

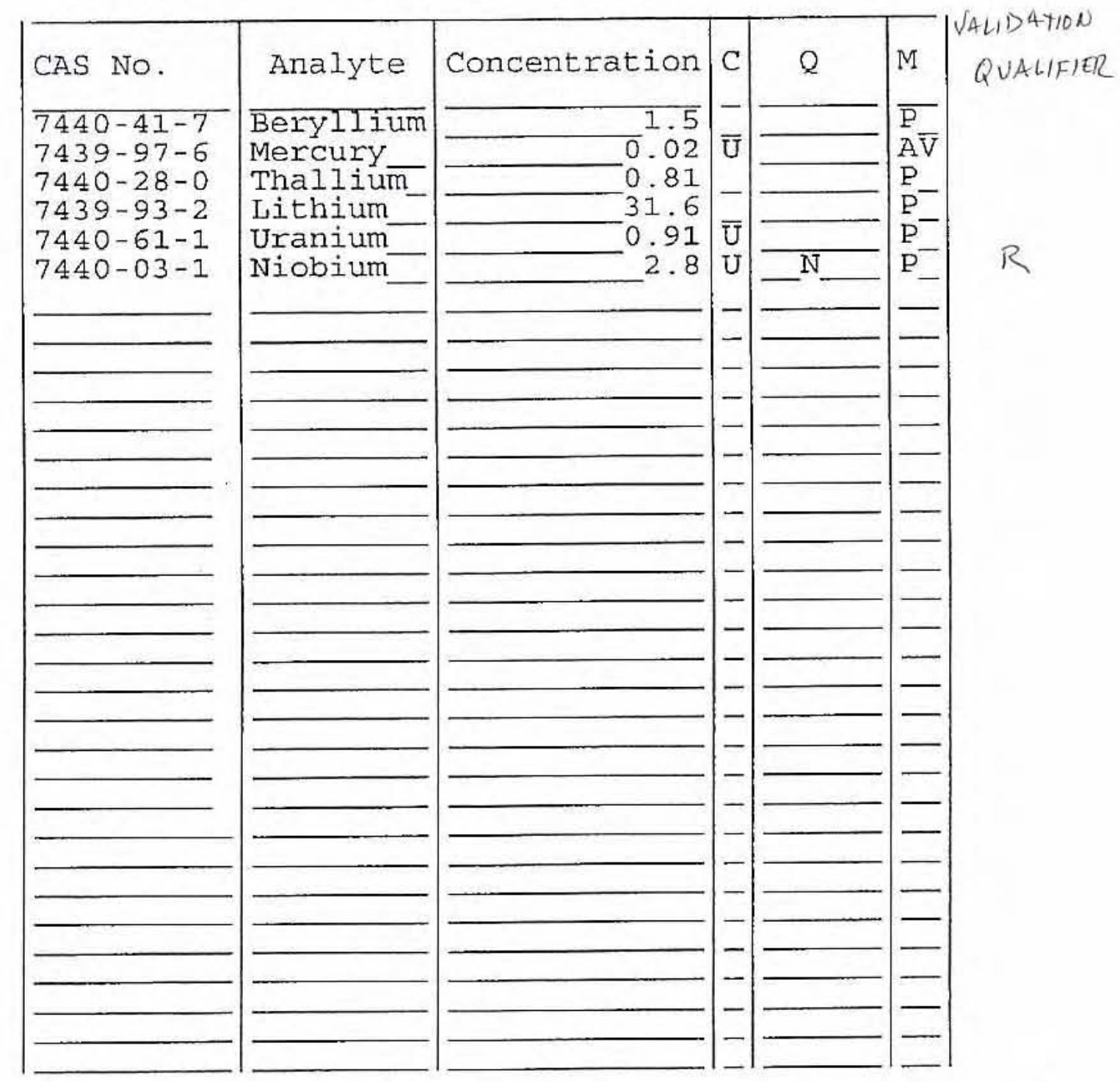

Color Before:

Color After:
Clarity Before:

Clarity After:
Texture:

Artifacts:

Comments:

PDRO-19A 


\section{1}

INORGANIC ANALYSES DATA SHEET
EPA SAMPLE NO.

$19 B$
Lab Name: LIONVILLE_LABORATORY

Lab Code: LVLI

Matrix (soil/water) : SOIL

Level (low/med):

$\%$ Solids:
Contract: 60052

SAS No.:
Lab Sample ID: 0608L797-008

Date Received: 08/24/06

Concentration Units (ug/L or $\mathrm{mg} / \mathrm{kg}$ dry weight): MG/KG

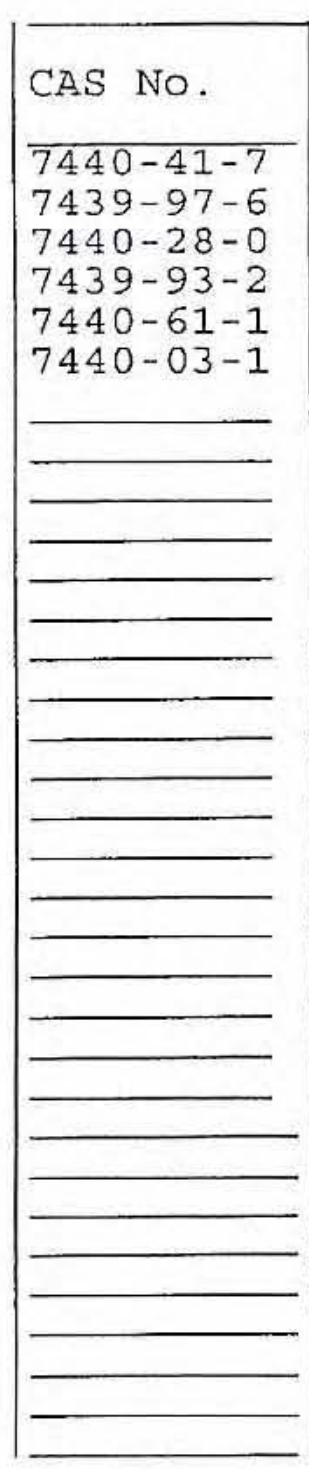

Color Before:

Color After:

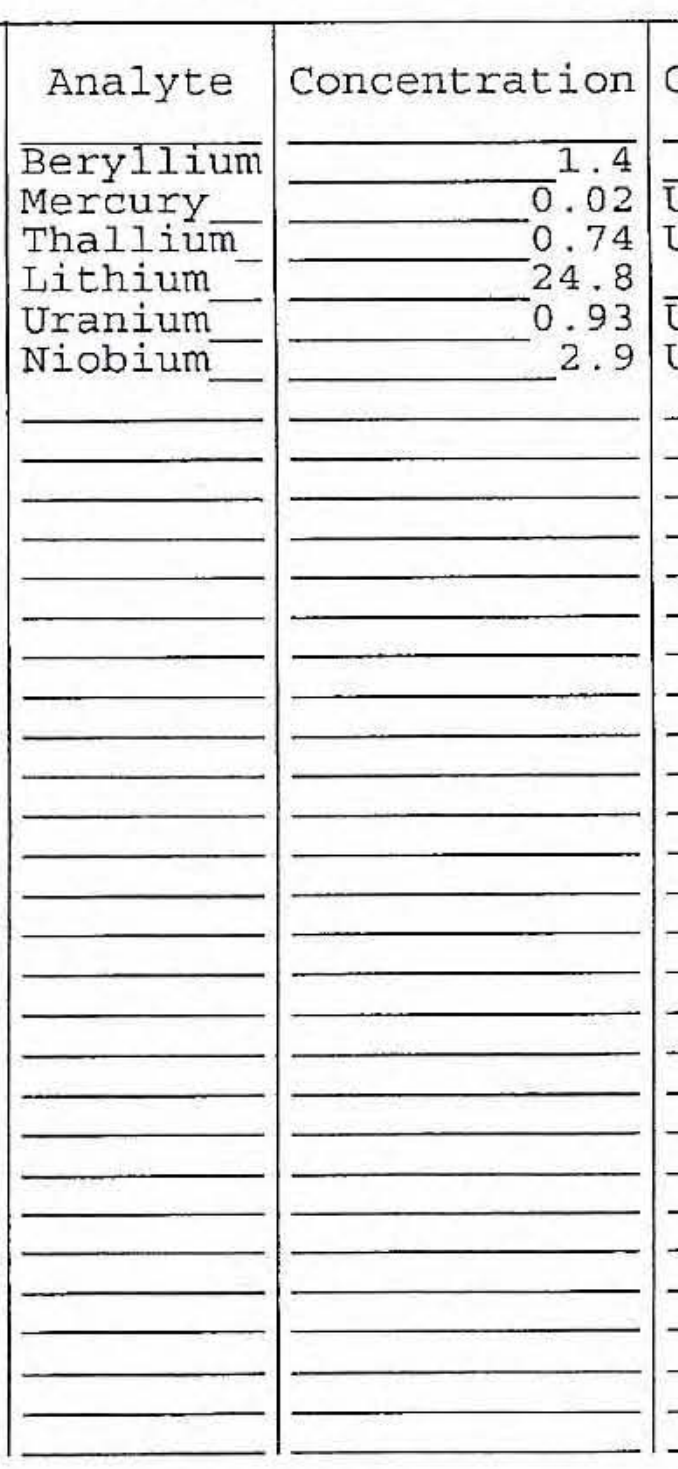

Clarity Before: Clarity After:

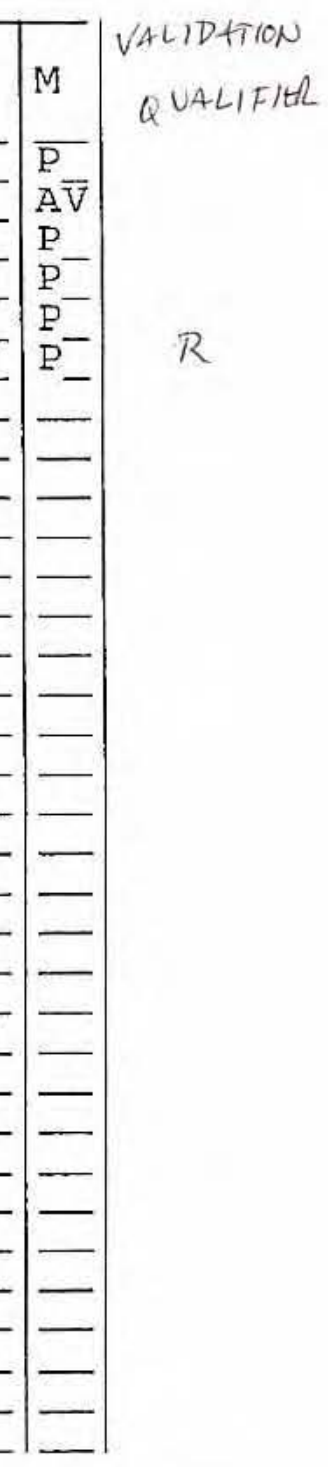

Texture:

Artifacts:

Comments :

PDRO-19B 
1

INORGANIC ANALYSES DATA SHEET
EPA SAMPLE NO.

$$
20 \mathrm{~A}
$$

SDG NO.: V2734

SAS No. :

$$
\begin{aligned}
& \text { Cas } \\
& \text { SOIL- } \\
& \text { LOW }
\end{aligned}
$$$$
-93 \overline{.8}
$$

Contract: 60052

Lab Sample ID: 0608L797-0̄09

Date Received: 08/24/06 Level (lor
$\%$ Solids:

Concentration Units (ug/L or $\mathrm{mg} / \mathrm{kg}$ dry weight): MG/KG

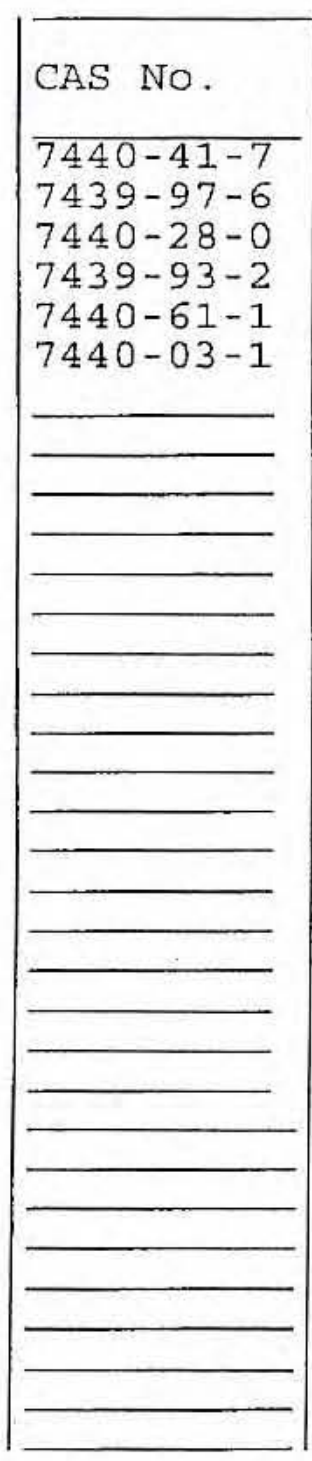

Color Before:

Color After:

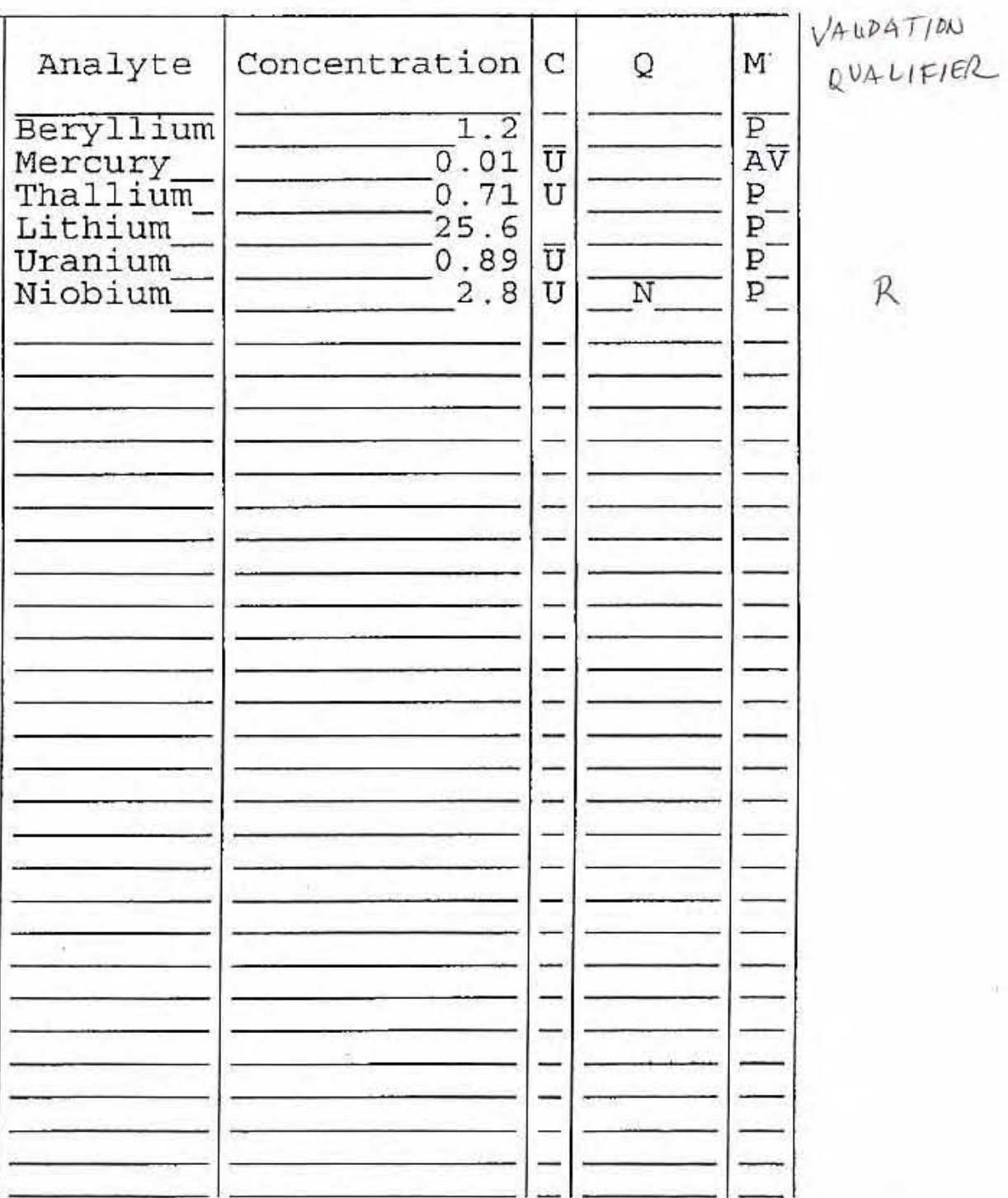

Clarity Before:

Clarity After:
Texture:

Artifacts :

Comments :

PDRO-2OA 
1 INORGANIC ANALYSES DATA SHEET
EPA SAMPLE NO.

$$
20 B
$$

Contract: 60052

SAS NO. :

Lab Sample ID: 06081797-̄̄10

Date Received: 08/24/06
Lab Name: LION
Lab Code: LVLI

Matrix (soil/water):

Level (low/med) :

$\div$ Solids:

\section{Case No.: PDRO}

$$
\begin{array}{r}
\text { LOW- } \\
-90.0
\end{array}
$$

Concentration Units (ug/L or $\mathrm{mg} / \mathrm{kg}$ dry weight): MG/KG

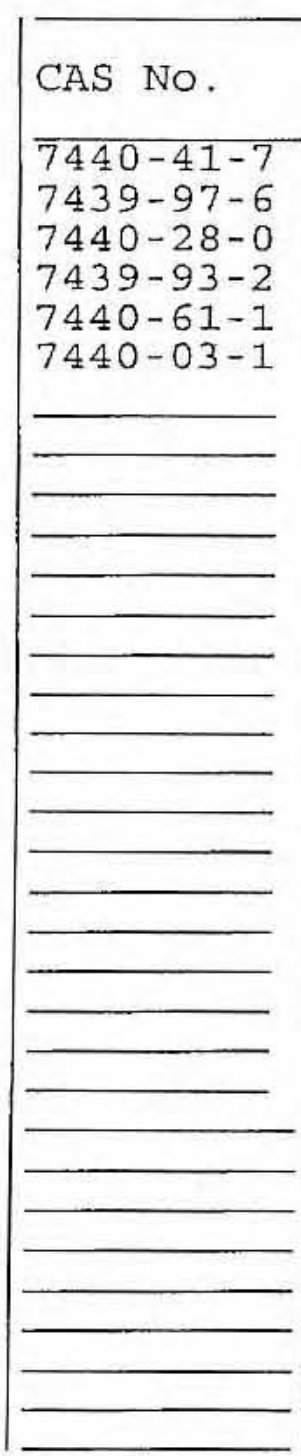

Color Before:

Color After:
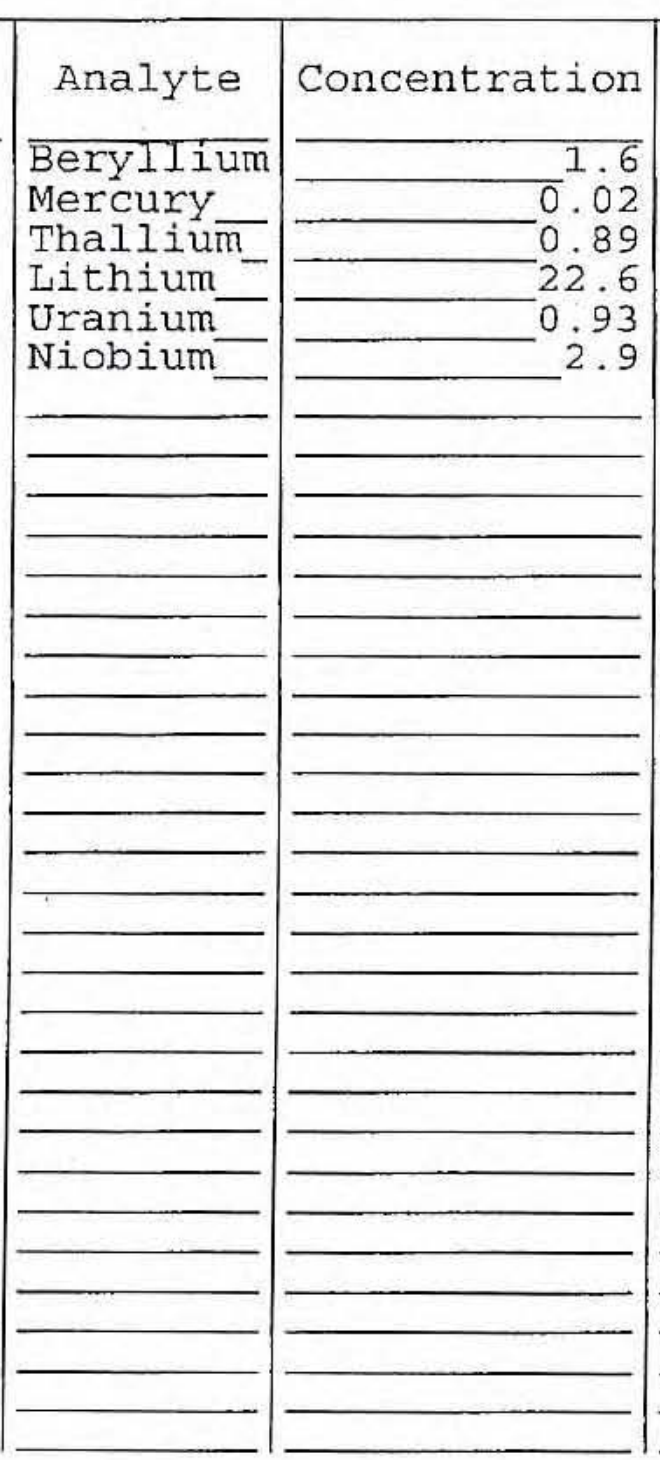

C
-
U
-
$-U$
U
-
-
-
-
-
-
-
-
-
-
-

Clarity Before:

Clarity After:

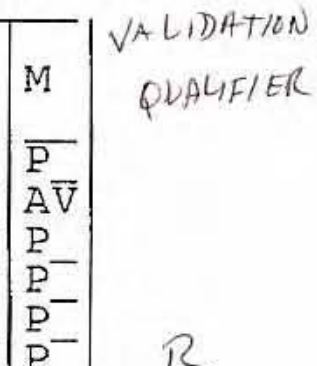

N

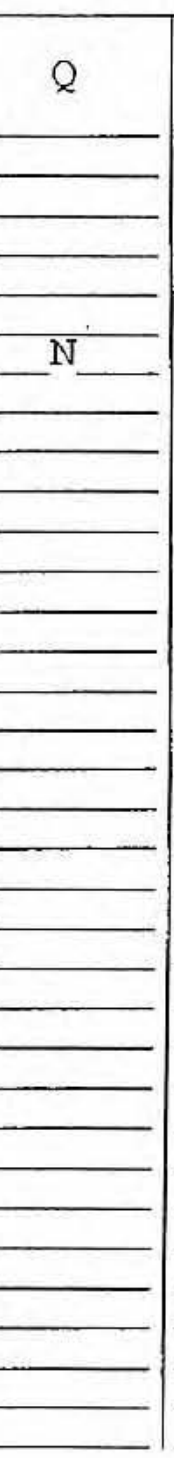

Texture:

Artifacts:

Comments :

PDRO-2OB 
1

INORGANIC ANALYSES DATA SHEET
EPA SAMPLE NO.

$$
21 \mathrm{~A}
$$

Contract: 60052 SAS NO.:

Lab Sample ID : 06்08L797-0̄11

Date Received: 08/24/06
Lab Name: IIONVILLE_LABORATORY

Lab Code: LVLI Matrix (soil/water): SoIL_ Level (low/med): \% Solids:

$$
\text { LOW }-93.6
$$

Concentration Units (ug/L or $\mathrm{mg} / \mathrm{kg}$ dry weight): MG/KG

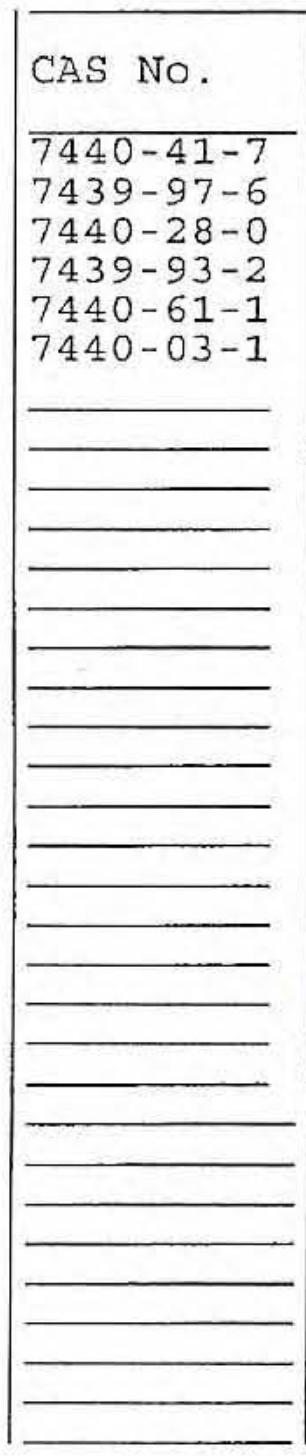

Color Before: Color After:
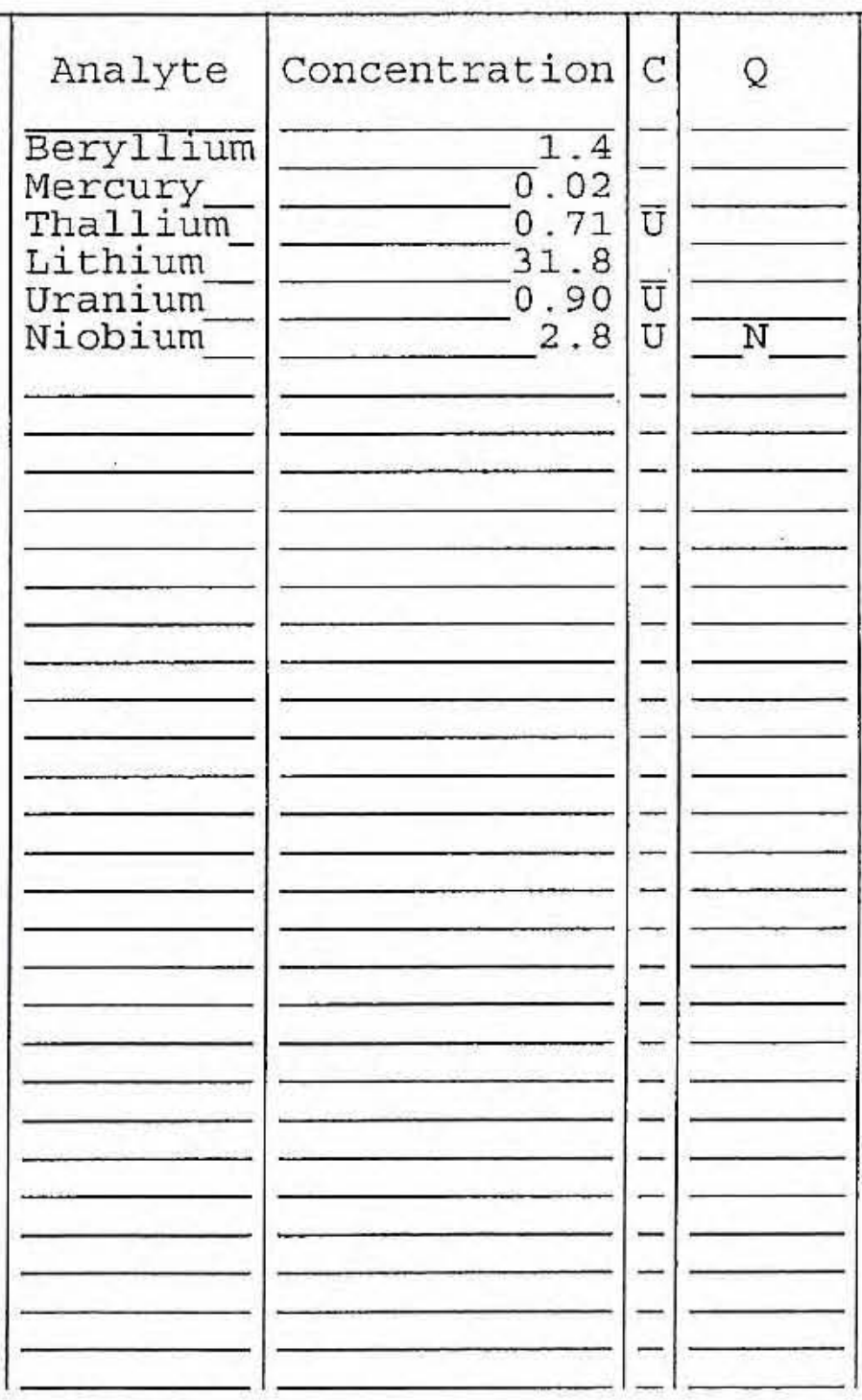

Clarity Before: Clarity After:

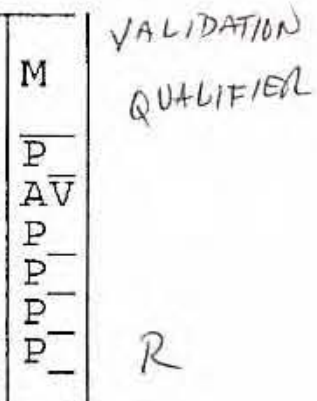

Comments:

PDRO-21A 
Lab Name: LIONVILLE LABORATORY

Lab Code: LVLI

Matrix (soil/water) :

Level (Iow/med):

\% Solids:
Contract: 60052

Case No.: PDRO_ SAS NO. SOIL -
LOW $\overline{88.4}$
$21 B$

Lab Sample ID: 0608L797-̄̄12

Date Received: $08 / 24 / 06$

Concentration Units (ug/L or $\mathrm{mg} / \mathrm{kg}$ dry weight): MG/KG

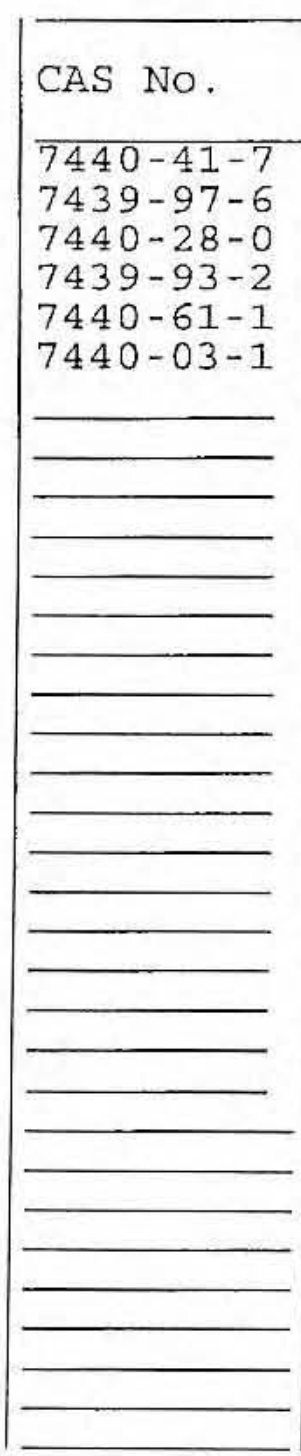

Color Before:

color After:

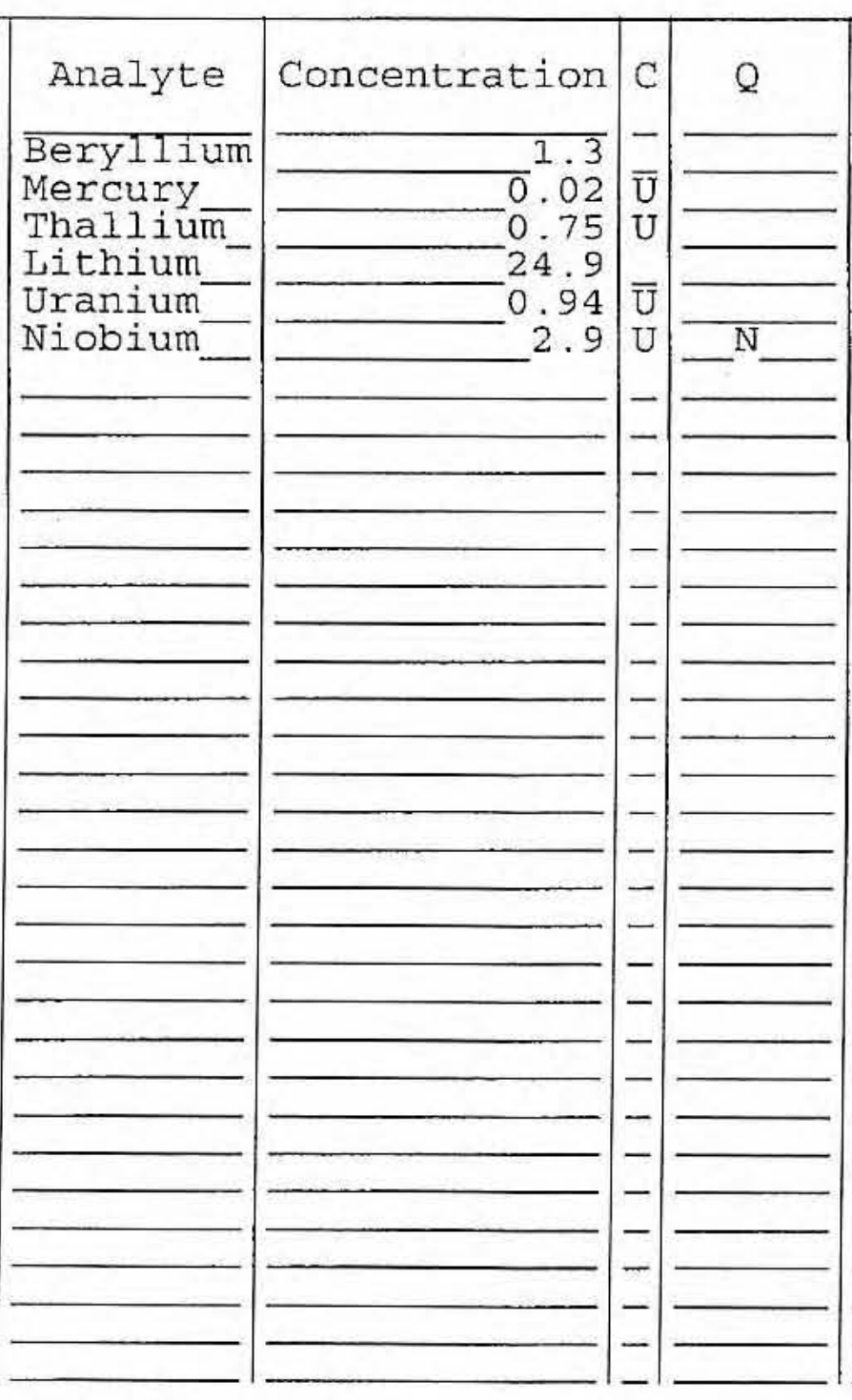

Clarity Before:

Clarity After:

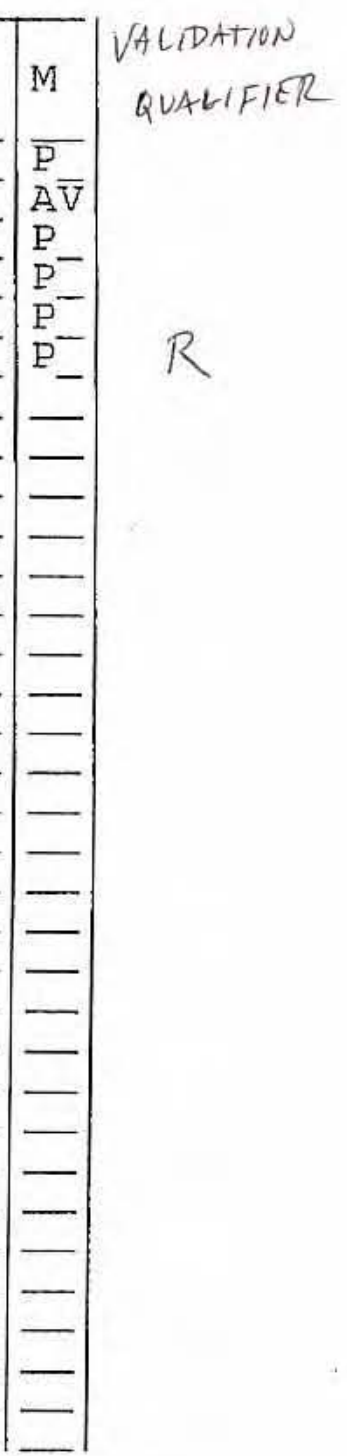

Texture:

Artifacts:

Comments:

PDRO-21B 


\section{U.S. EPA}

1

INORGANIC ANALYSES DATA SHEET
EPA SAMPLE NO.

\section{R1}

SDG NO.: V2734 60052

Lab Sample ID: 0608L797-0̄13
Lab Narne: LION
Lab Code: IVLI Matrix (soil/wat
Level (Low/med) : Solids:
SAS NO.: LOW

$0 \overline{0}$

Case No.: PDRO WATER$$
\text { - }
$$

Concentration Units (ug/L or $\mathrm{mg} / \mathrm{kg}$ dry weight): UG/L_

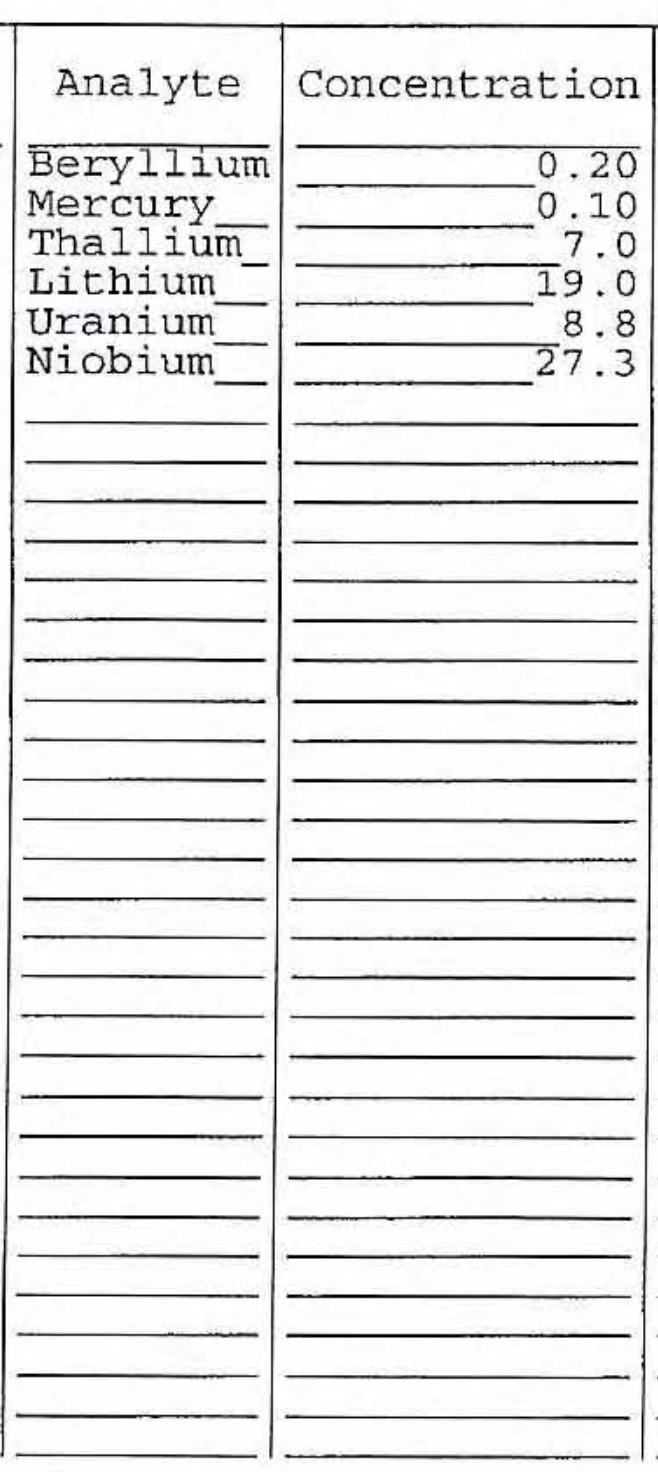

Clarity Before: Clarity After:

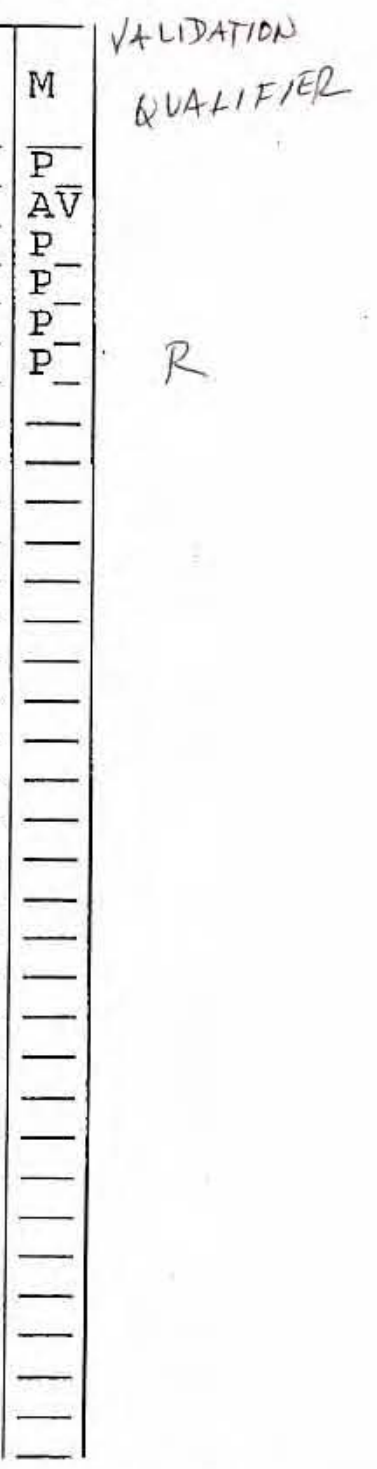

Texture: Artifacts : 


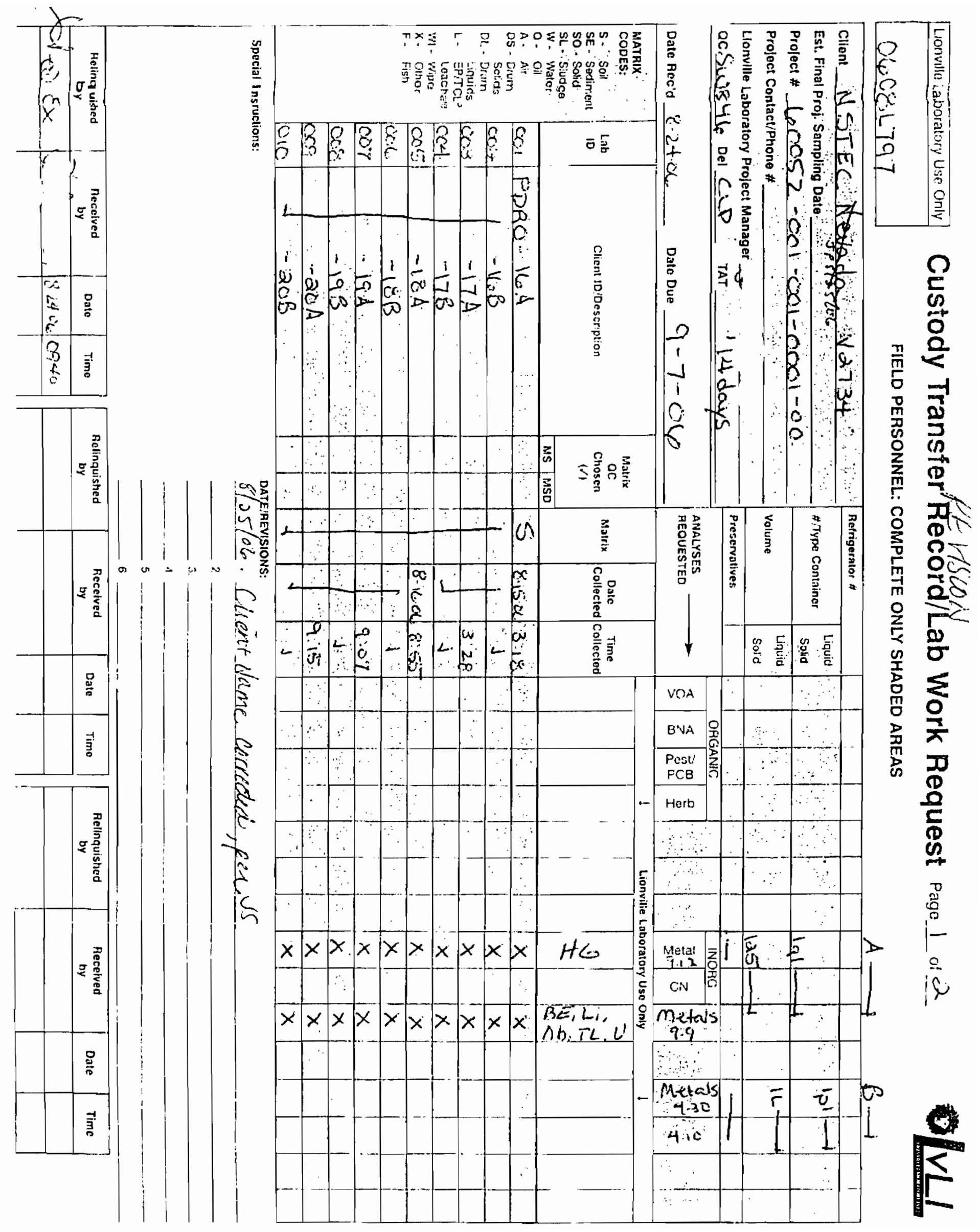




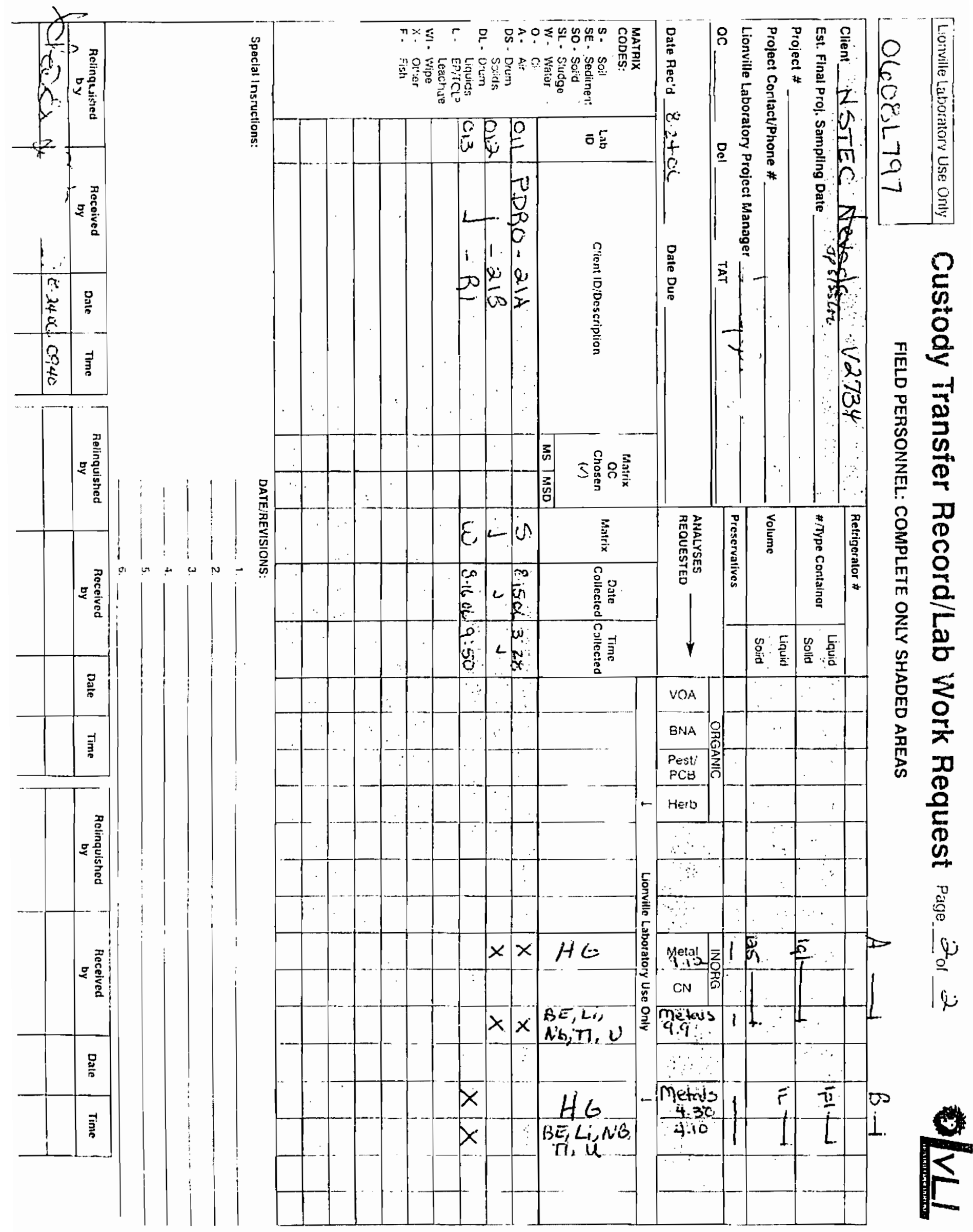




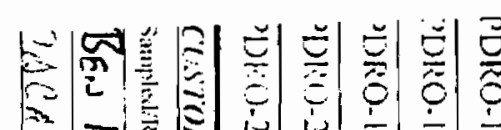

(2) 7

$y$

ती

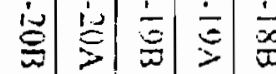

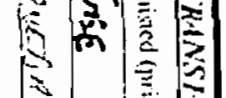

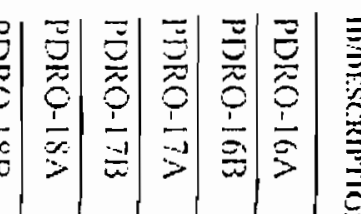

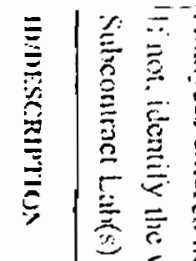

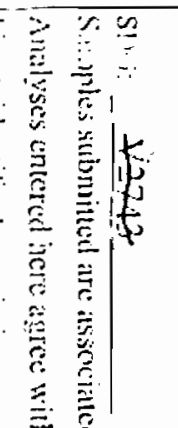

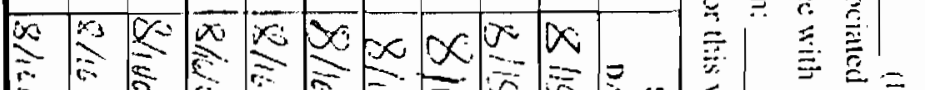

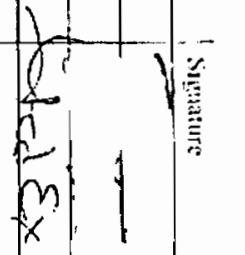

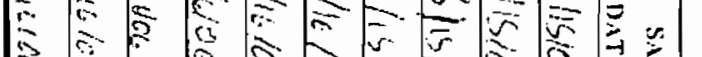

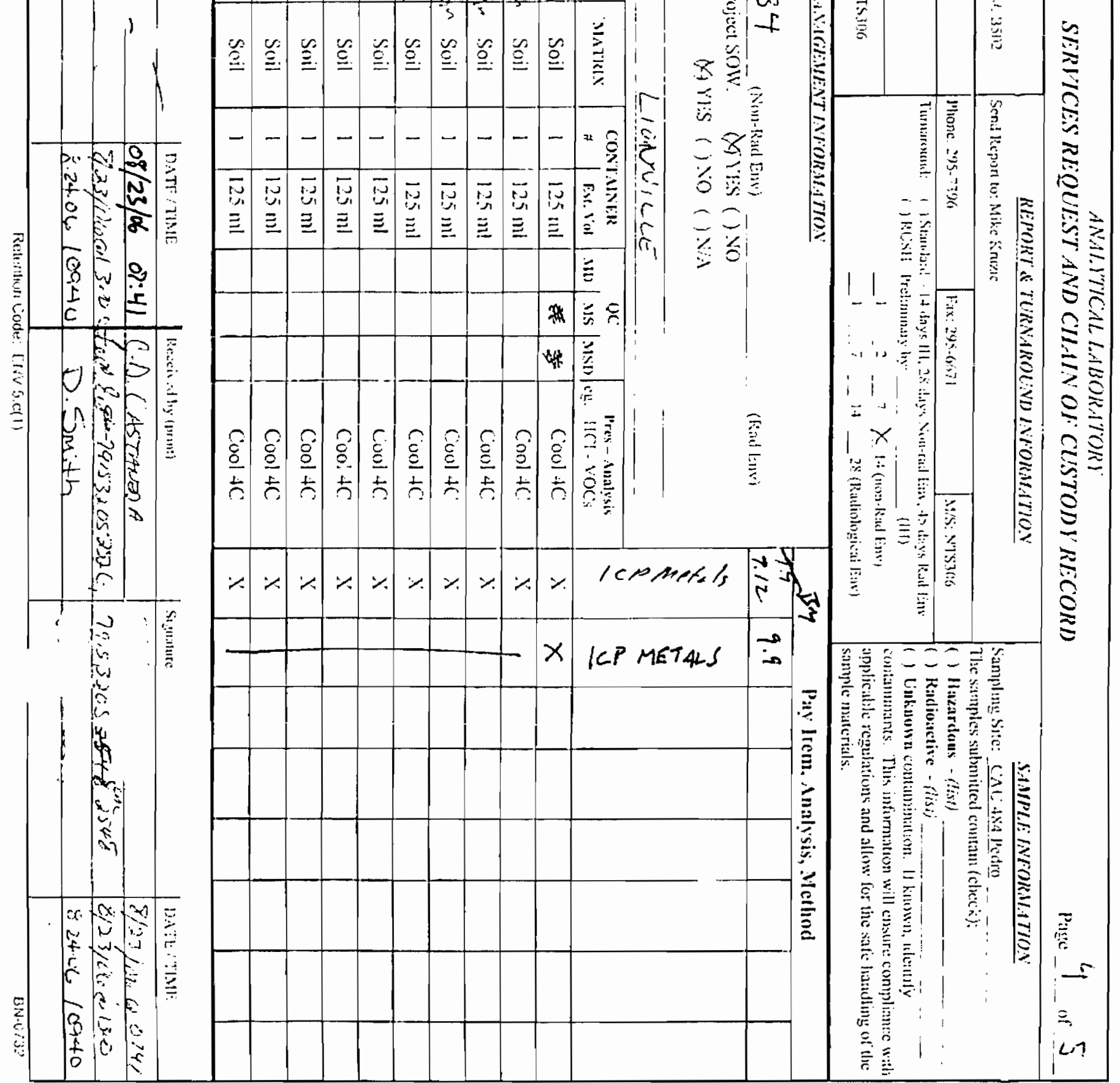

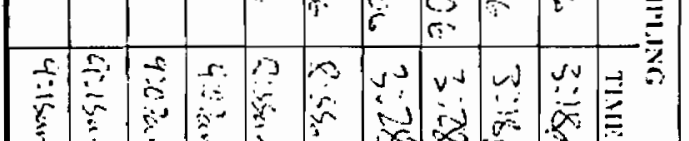

实产

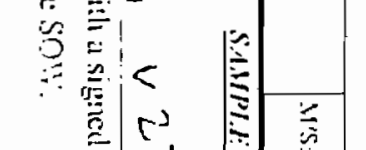

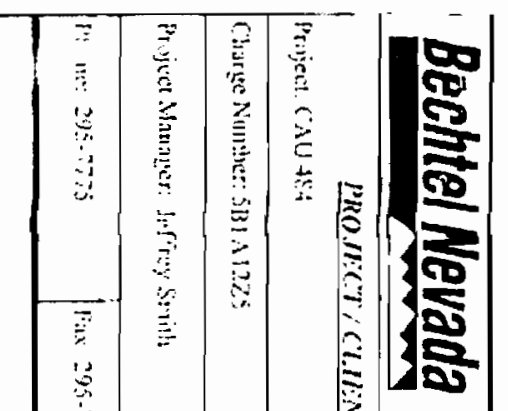




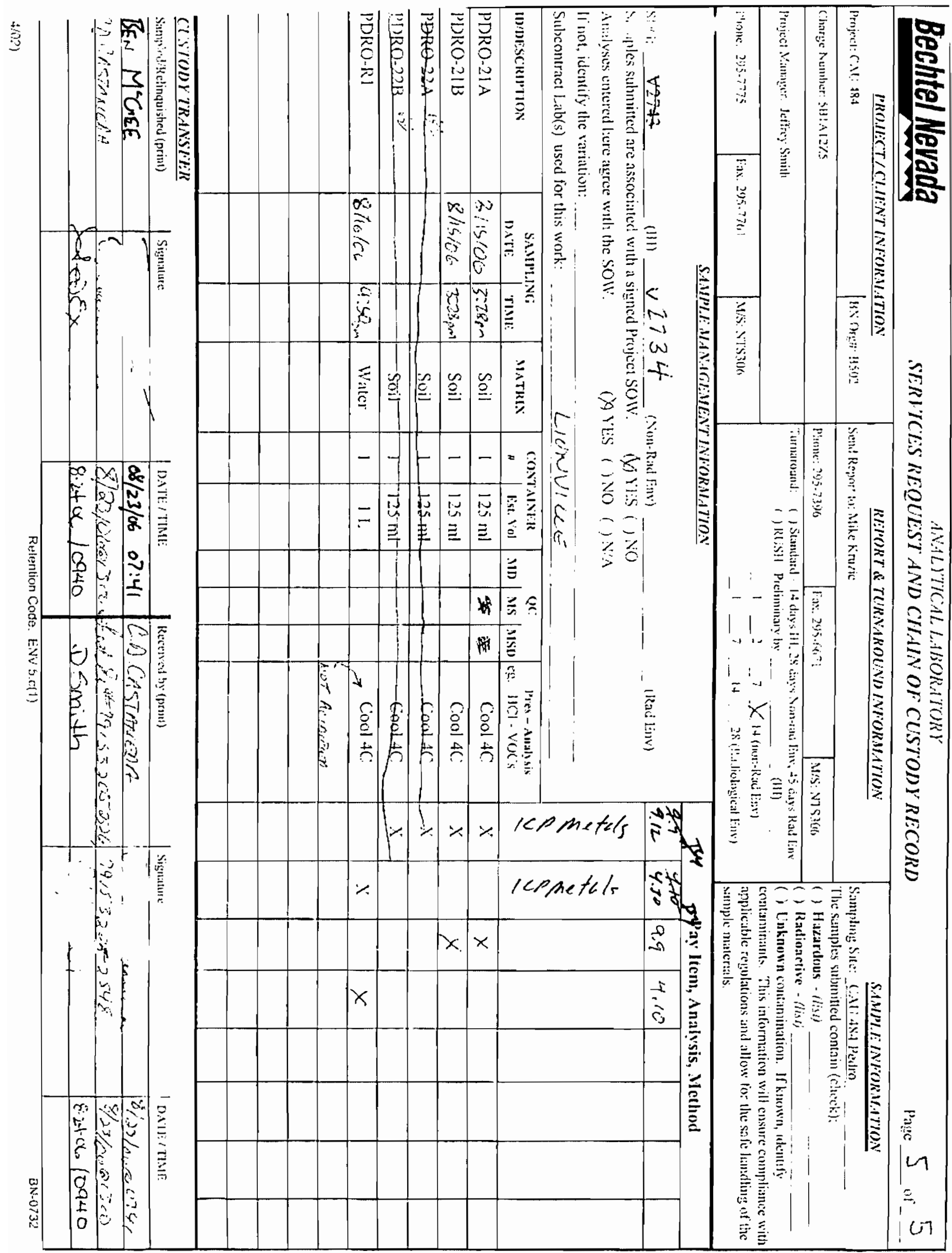




\title{
INVESTIGATION (GEOPROBE ${ }^{\circledR}$ ) SOIL SAMPLE ANALYTICAL RESULTS PEDRO LAKE
}

\author{
Radiological Data
}




\section{Isotopic Uranium By Alpha Spectroscopy Sample Results Summary}

Client Name: National Sercurity Technologies, LLC Client Project Name: CAU 484 Client Project Number: V2735
Laboratory Name: Paragon Analytics PAI Work Order: 0608181
Page: 1 of 15

Reported on: Wednesday, September 20, 2006 10:11:26 AM

\begin{tabular}{|c|c|c|c|c|c|c|c|c|c|c|}
\hline $\begin{array}{c}\text { Lab } \\
\text { Sample ID }\end{array}$ & Client Sample ID & $\begin{array}{l}\text { Sample } \\
\text { Type }\end{array}$ & Nuclide & Result $+1-2 \mathrm{~s}$ TPU & MDC & Units & Matrix & Prep Batch & $\begin{array}{c}\text { Date } \\
\text { Analyzed }\end{array}$ & Flags \\
\hline $0608181-1$ & PDRO-01A & Sample & U-233/234 & $8.52 \mathrm{E}-01+1-1.53 \mathrm{E}-01$ & $1.13 \mathrm{E}-02$ & $\mathrm{pCi} / \mathrm{g}$ & SOIL & AS060901-1 & $9 / 9 / 2006$ & \\
\hline $0608181-1$ & PDRO-01A & Sample & U-235 & $2.92 \mathrm{E}-02+/-1.33 \mathrm{E}-02$ & $8.34 \mathrm{E}-03$ & $\mathrm{pC} / \mathrm{g}$ & SOIL & AS060901-1 & $9 / 9 / 2006$ & \\
\hline $0608181-1$ & PDRO-01A & Sample & U-238 & $8.20 E-01+/-1.47 E-01$ & $1.02 \mathrm{E}-02$ & $\mathrm{pCi} / \mathrm{g}$ & SOIL & AS060901-1 & 9/9/2006 & \\
\hline 0608181.2 & PDRO.01B & Sample & U-233/234 & $8.83 \mathrm{E}-01+1-1.56 \mathrm{E}-01$ & $1.50 \mathrm{E}-02$ & $\mathrm{pCi} / \mathrm{g}$ & SOIL & AS060901-1 & $9 / 9 / 2006$ & \\
\hline $0608181-2$ & PDRO-01B & Sample & U-235 & $5.28 \mathrm{E}-02+1-1.78 \mathrm{E}-02$ & $7.43 \mathrm{E}-03$ & $\mathrm{pCi} / \mathrm{g}$ & SOIL & ASO60901-1 & 9/9/2006 & \\
\hline $0608181-2$ & PDRO-01B & Sample & U.238 & $9.10 \mathrm{E}-01+1-1.60 \mathrm{E}-01$ & $1.25 \mathrm{E}-02$ & $\mathrm{pCi} / \mathrm{g}$ & SOIL & AS060901-1 & 9/9/2006 & \\
\hline 0608181.3 & PDRO-02A & Sample & U-233/234 & $1.31 E+00+/-2.23 E-01$ & $9.53 E-03$ & $\mathrm{pCi} / \mathrm{g}$ & SOIL & AS060901-1 & 9/9/2006 & \\
\hline 0608181.3 & PDRO-02A & Sample & U.235 & $7.61 \mathrm{E}-02+/-2.18 \mathrm{E}-02$ & $2.86 \mathrm{E}-03$ & $\mathrm{pCi} / \mathrm{g}$ & SOIL & AS060901-1 & 9/9/2006 & \\
\hline $0608181-3$ & PDRO-02A & Sample & U-238 & $1.22 \mathrm{E}+00+1-2.08 \mathrm{E}-01$ & $7.45 \mathrm{E}-03$ & $\mathrm{pCl} / \mathrm{g}$ & SOIL & AS060901-1 & $9 / 9 / 2006$ & \\
\hline
\end{tabular}

\section{Comments:}

\section{Data Package ID: UR0608181-1}

\section{Qualifiers/Flags:}

$U$ - Result is less than the sample specific MDC

LT - Result is less than Requested MDC, greater than sample specific MDC

Y1 - Chemical Yield is in control at 100-110\% Quantitative Yield is assumed

2. Chemical Yield outside default limils

$M$ - The requested MDC was not met

M3 - The requested MDC was not met, but the reported activity is greater than the reported MDC

\section{Abbreviations}

TPU - Total Propagated Uncertainty (see PAI SOP 743)

MDC - Minimum Detectable Concentration (see PAI SOP 709

BDL - Below Detection Lim

Dati rinted: Wednesday, September 20, 2006 


\section{Isotopic Uranium By Alpha Spectroscopy Sample Results Summary}

Client Name: National Sercurity Technologies, LLC Client Project Name: CAU 484 Client Project Number: V2735
Laboratory Name: Paragon Analytics PAl Work Order: 0608181
Page: 2 of 15

Reported on: Wednesday, September 20,2006 10:11:26 AM

\begin{tabular}{|c|c|c|c|c|c|c|c|c|c|c|}
\hline $\begin{array}{c}\text { Lab } \\
\text { Sample ID }\end{array}$ & Client Sample ID & $\begin{array}{c}\text { Sample } \\
\text { Type }\end{array}$ & Nuclide & Result $+1-2$ s TPU & MDC & Units & Matrix & Prep Batch & $\begin{array}{c}\text { Date } \\
\text { Analyzed }\end{array}$ & Flags \\
\hline $0608181-4$ & PDRO-02B & Sample & $U-233 / 234$ & $1.07 E+00+1-1.84 E-01$ & $5.79 \mathrm{E}-03$ & $\mathrm{pCi} / \mathrm{g}$ & SOIL & AS060901-1 & $9 / 9 / 2006$ & \\
\hline $0608181-4$ & PDRO-02B & Sample & U.235 & $4.92 \mathrm{E}-02+1-1.63 \mathrm{E}-02$ & $2,78 \mathrm{E}-03$ & $\mathrm{pCi} / \mathrm{g}$ & SOIL & AS060901-1 & $9 / 9 / 2006$ & \\
\hline $0608181-4$ & PDRO-02B & Sample & U.238 & $9.63 \mathrm{E}-01+/-1.66 \mathrm{E}-01$ & $5.79 \mathrm{E}-03$ & $\mathrm{pCi} / \mathrm{g}$ & SOIL & AS060901-1 & 9/9/2006 & \\
\hline $0608181-5$ & PDRO-03A & Sample & $U-233 / 234$ & $9.47 \mathrm{E}-01+/-1.62 \mathrm{E}-01$ & $8.02 E-03$ & $\mathrm{pCi} / \mathrm{g}$ & SOIL & AS060901-1 & 9/9/2006 & \\
\hline $0608181-5$ & PDRO-03A & Sample & U.235 & $3.88 \mathrm{E}-02+/-1.40 \mathrm{E}-02$ & $6.58 \mathrm{E}-03$ & $\mathrm{pCi} / \mathrm{g}$ & SOIL & AS060901-1 & $9 / 9 / 2006$ & \\
\hline $0608181-5$ & PDRO-03A & Sample & U-238 & $8.91 \mathrm{E}-01+/-1.54 \mathrm{E}-01$ & $8.90 \mathrm{E}-03$ & $\mathrm{pCi} / \mathrm{g}$ & SOIL & AS060901-1 & $9 / 9 / 2006$ & \\
\hline $0608181-6$ & PDRO-03B & Sample & U-233/234 & 8.23E-01+/-1.43E-01 & $2.34 \mathrm{E}-03$ & $\mathrm{pCl} / \mathrm{g}$ & SOIL & AS060901-1 & 9/9/2006 & \\
\hline $0608181-6$ & PDRO-03B & Sample & U-235 & $3.48 \mathrm{E}-02+1-1.33 \mathrm{E}-02$ & $6.75 \mathrm{E}-03$ & $\mathrm{pCi} / \mathrm{g}$ & SOIL & AS060901-1 & $9 / 9 / 2006$ & \\
\hline $0608181-6$ & PDRO-03B & Sample & U-238 & $7.84 \mathrm{E}-01+1-1.37 \mathrm{E}-01$ & $7.15 \mathrm{E}-03$ & $\mathrm{pCi} / \mathrm{g}$ & SOIL & AS060901-1 & $9 / 9 / 2006$ & \\
\hline
\end{tabular}

\section{Comments:}

\section{Data Package ID: UR0608181-1}

\footnotetext{
Qualifiers/Flags:

$U$ - Result is less tran the sample specific MDC.

LT - Result is less than Requested MDC, greater than sample specific MDC

Y1 - Chemical Yield is in control at $100-110 \%$, Quantitative Yield is assumed.

$Y 2$. Chemical Yield outside default limits

$M$ - The requested MDC was not met

M3 - The requested MDC was not met, but the reported activity is greater than the reported MDC.
}

Abbreviations

TPU - Total Propagated Uncertainty (see PAI SOP 743)

MDC - Minimum Detectable Concentration (see PAI SOP 709)

BDL - Below Detection Limit 


\section{Isotopic Uranium By Alpha Spectroscopy Sample Results Summary}

Client Name: National Sercurity Technologies, LLC Client Project Name: CAU 484 Client Project Number: V2735
Laboratory Name: Paragon Analytics PAI Work Order: 0608181
Page: 3 of 15

Reported on: Wednesday, September 20, 2006 10:11:26 AM

\begin{tabular}{|c|c|c|c|c|c|c|c|c|c|c|}
\hline $\begin{array}{c}\text { Lab } \\
\text { Sample ID }\end{array}$ & Client Sample ID & $\begin{array}{l}\text { Sample } \\
\text { Type }\end{array}$ & Nuclide & Result +/- $2 \mathrm{~s}$ TPU & MDC & Units & Matrix & Prep Batch & $\begin{array}{c}\text { Date } \\
\text { Analyzed }\end{array}$ & Flags \\
\hline $0608181-7$ & PDRO-04A & Sample & U-233/234 & $1.28 \mathrm{E}+00+1-2.18 \mathrm{E}-01$ & $1.49 \mathrm{E}-02$ & $\mathrm{pCi} / \mathrm{g}$ & SOIL & ASO60901-1 & $9 / 9 / 2006$ & \\
\hline $0608181-7$ & PDRO-04A & Sample & U-235 & $4.42 \mathrm{E}-02+/-1.58 \mathrm{E}-02$ & $9,86 \mathrm{E}-03$ & $\mathrm{pCi} / \mathrm{g}$ & SOIL & AS060901-1 & $9 / 9 / 2006$ & \\
\hline 0608181.7 & PDRO-04A & Sample & U-238 & $1.17 \mathrm{E}+00+/-2.00 \mathrm{E}-01$ & $7.28 \mathrm{E}-03$ & $\mathrm{pCi} / \mathrm{g}$ & SOIL & AS060901-1 & $9 / 9 / 2006$ & \\
\hline $0608181-8$ & PDRO-04B & Sample & U-233/234 & $1.01 E+00+/-1.73 E-01$ & $9.13 E-03$ & $\mathrm{pCi} / \mathrm{g}$ & SOIL & ASO60901-1 & 9/9/2006 & \\
\hline $0608181-8$ & PDRO-04B & Sample & U-235 & $4.99 \mathrm{E}-02+/-1.65 \mathrm{E}-02$ & $6.75 \mathrm{E}-03$ & $\mathrm{pCi} / \mathrm{g}$ & SOIL & AS060901-1 & $9 / 9 / 2006$ & \\
\hline 0608181.8 & PDRO-04B & Sample & U-238 & $1.05 E+00+/-1.80 E-01$ & $5.73 \mathrm{E}-03$ & $\mathrm{pCi} / \mathrm{g}$ & SOIL & AS060901-1 & $9 / 9 / 2006$ & \\
\hline $0608181-9$ & PDRO-05A & Sample & $\mathrm{U}-233 / 234$ & $9.81 \mathrm{E}-01+/-1.68 \mathrm{E}-01$ & $1.16 \mathrm{E}-02$ & $\mathrm{pCi} / \mathrm{g}$ & SOIL & ASO60901-1 & $9 / 9 / 2006$ & \\
\hline $0608181-9$ & PDRO-05A & Sample & U.235 & $5.92 \mathrm{E}-02+1-1.80 \mathrm{E}-02$ & $2.67 \mathrm{E}-03$ & $\mathrm{pCi} / \mathrm{g}$ & SOIL & AS060901-1 & 9/9/2006 & \\
\hline $0608181-9$ & PDRO-05A & Sample & $U-238$ & $9.55 \mathrm{E}-01+/-1.64 \mathrm{E}-01$ & $8.89 \mathrm{E}-03$ & $\mathrm{pCi} / \mathrm{g}$ & SOIL & AS060901-1 & 9/9/2006 & \\
\hline
\end{tabular}

\section{Comments:}

\section{Data Package ID: UR0608181-1}

\section{Qualifiers/Flags:}

$v$ - Result is less than the sample specific MDC

LT - Result is less than Requested MDC, greater than sample specific MDC.

$\mathrm{Y1}$ - Chemical Yield is in control at $100-110 \%$. Quantitative Yield is assumed

Y2 - Chemical Yield outside default limits.

$M$ - The requested $M D C$ was not met

M3 - The requested MDC was not met, but the reported activity is greater than the reported MDC 


\section{Isotopic Uranium By Alpha Spectroscopy Sample Results Summary}

Client Name: National Sercurity Technologies, LLC Client Project Name: CAU 484 Client Project Number: V2735
Laboratory Name: Paragon Analytics PAl Work Order: 0608181
Page: 4 of 15

Reported on: Wednesday, September 20, 2006 10:11:26 AM

\begin{tabular}{|c|c|c|c|c|c|c|c|c|c|c|}
\hline $\begin{array}{c}\text { Lab } \\
\text { Sample ID }\end{array}$ & Client Sample ID & $\begin{array}{l}\text { Sample } \\
\text { Type }\end{array}$ & Nuclide & Result + $/-2$ s TPU & MDC & Units & Matrix & Prep Batch & $\begin{array}{c}\text { Date } \\
\text { Analyzed }\end{array}$ & Flags \\
\hline $0608181-10$ & PDRO-05B & Sample & U.233/234 & $1.69 \mathrm{E}+00+/-2.84 \mathrm{E}-01$ & $1.28 \mathrm{E}-02$ & $\mathrm{pCi} / \mathrm{g}$ & SOIL & AS060901-1 & $9 / 9 / 2006$ & \\
\hline $0608181-10$ & PDRO.05B & Sample & U-235 & $6.28 \mathrm{E}-02+/-1.94 \mathrm{E}-02$ & $9.85 \mathrm{E}-03$ & $\mathrm{pCi} / \mathrm{g}$ & SOIL & AS060901-1 & 9/9/2006 & \\
\hline $0608181-10$ & PDRO-05B & Sample & U-238 & $1.32 \mathrm{E}+00+/-2.24 \mathrm{E}-01$ & $9.30 \mathrm{E}-03$ & $\mathrm{pCi} / \mathrm{g}$ & SOIL & AS060901-1 & $9 / 9 / 2006$ & \\
\hline $0608181-11$ & PDRO-06A & Sample & U-233/234 & $8.24 E-01+/-1.42 E-01$ & $5.32 \mathrm{E}-03$ & $\mathrm{pCi} / \mathrm{g}$ & SOIL & AS060901-1 & 9/9/2006 & \\
\hline $0608181-11$ & PDRO-06A & Sample & U-235 & $5.54 \mathrm{E}-02+1-1.69 \mathrm{E}-02$ & $2.55 \mathrm{E}-03$ & $\mathrm{pCi} / \mathrm{g}$ & SOIL & ASO60901-1 & 9/9/2006 & \\
\hline $0608181-11$ & PDRO-06A & Sample & $U-238$ & 8.07E-01+/-1.39E-01 & $6.62 \mathrm{E}-03$ & $\mathrm{pCi} / \mathrm{g}$ & SOIL & AS060901-1 & 9/9/2006 & \\
\hline $0608181-12$ & PDRO-06B & Sample & $\mathrm{U}-233 / 234$ & $1.12 \mathrm{E}+00+/-1.91 \mathrm{E}-01$ & $1.14 \mathrm{E}-02$ & $\mathrm{pCi} / \mathrm{g}$ & SOIL & AS060901-1 & $9 / 9 / 2006$ & \\
\hline $0608181-12$ & PDRO-06B & Sample & U-235 & $7.58 \mathrm{E}-02+1-2.20 \mathrm{E}-02$ & $1.17 \mathrm{E}-02$ & $\mathrm{pCi} / \mathrm{g}$ & SOIL & AS060901-1 & 9/9/2006 & \\
\hline $0608181-12$ & PDRO-06B & Sample & $\mathrm{U}-238$ & $1.12 \mathrm{E}+00+1-1.91 \mathrm{E}-01$ & $9.18 \mathrm{E}-03$ & $\mathrm{pCi} / \mathrm{g}$ & SOIL & AS060901-1 & $9 / 9 / 2005$ & \\
\hline
\end{tabular}

\section{Comments:}

\section{Data Package ID: UR0608181-1}

Qualifiers/Flags:

$U$ - Result is less than the sample specific MDC.

LT - Result is less than Requested MDC, greater than sample specific MDC.

$Y_{i}$ - Chemical Yield is in control at $100.110 \%$ Quantitative Yield is assumed

Y2 - Chemical Yield outside default limits

$M$ - The requested $M D C$ was not me:

M3 - The requested MDC was not met, but the reported activity is greater than the reported MDC.
Abbreviations:

TPU - Total Propagated Uncertainty (see PAI SOP 743)

MDC - Minimum Detectabie Concentration (see PAI SOP 709)

BDL - Below Detection Limit 


\section{Isotopic Uranium By Alpha Spectroscopy Sample Results Summary}

Client Name: National Sercurity Technologies, LLC Client Project Name: CAU 484 Client Project Number: V2735
Laboratory Name: Paragon Analytics PAI Work Order: 0608181
Page: 5 of 15

Reported on: Wednesday, September 20,2006 10:11:26 AM

\begin{tabular}{|c|c|c|c|c|c|c|c|c|c|c|}
\hline $\begin{array}{l}\text { Lab } \\
\text { Sample ID }\end{array}$ & Client Sample ID & $\begin{array}{l}\text { Sample } \\
\text { Type }\end{array}$ & Nuclide & Result +/- 2 s TPU & MDC & Units & Matrix & Prep Batch & $\begin{array}{c}\text { Date } \\
\text { Analyzed }\end{array}$ & Flags \\
\hline $0608181-13$ & PDRO-07A & Sample & U-233/234 & $1.52 E+00+1-2.59 E-01$ & $9.06 \mathrm{E}-03$ & $\mathrm{pCi} / \mathrm{g}$ & SOIL & ASO60901-1 & $9 / 11 / 2006$ & \\
\hline $0608181-13$ & PDRO-07A & Sample & U-235 & $6.51 \mathrm{E}-02+1-2.03 \mathrm{E}-02$ & $7.43 \mathrm{E}-03$ & pCitg & SOIL & ASO60901-1 & $9 / 11 / 2006$ & \\
\hline $0608181-13$ & PDRO-07A & Sample & U. 238 & $1.30 E+00+/-2.23 E-01$ & $7.87 \mathrm{E}-03$ & pCilg & SOIL & ASO60901-1 & $9 / 11 / 2006$ & \\
\hline $0608181-14$ & PDRO-07B & Sample & U-233/234 & $8.83 \mathrm{E}-01+/-1.51 \mathrm{E}-01$ & $9.01 \mathrm{E}-03$ & $\mathrm{pCi} / \mathrm{g}$ & SOIL & AS060901-1 & $9 / 9 / 2006$ & \\
\hline $0608181-14$ & PDRO-07B & Sample & U-235 & $5.16 \mathrm{E}-02+1-1.66 \mathrm{E}-02$ & $9.75 \mathrm{E}-03$ & $\mathrm{pCi} / \mathrm{g}$ & SOIL & AS060901-1 & $9 / 9 / 2006$ & \\
\hline $0608181-14$ & PDRO-07B & Sample & U-238 & $8.84 \mathrm{E}-01+/-1.51 \mathrm{E}-01$ & $7.46 \mathrm{E}-03$ & $\mathrm{pCi} / \mathrm{g}$ & SOIL & AS050901-1 & $9 / 9 / 2006$ & \\
\hline $0608181-15$ & PDRO-08A & Sample & U-233/234 & $9.97 \mathrm{E}-01+/-1.79 \mathrm{E}-01$ & $1.20 \mathrm{E}-02$ & $\mathrm{pCi} / \mathrm{g}$ & SOIL & AS060901-1 & $9 / 10 / 2006$ & \\
\hline $0608181-15$ & PDRO-08A & Sample & U.235 & $6.69 \mathrm{E}-02+/-2.21 \mathrm{E}-02$ & $8.85 E-03$ & $\mathrm{pCi} / \mathrm{g}$ & SOIL & AS060901-1 & $9 / 10 / 2006$ & \\
\hline $0608181-15$ & PDRO-08A & Sample & U.238 & $1.04 \mathrm{E}+00+/ .1 .86 \mathrm{E}-01$ & $1.08 \mathrm{E}-02$ & $\mathrm{pCl} / \mathrm{g}$ & SOIL & AS060901-1 & $9 / 10 / 2006$ & \\
\hline
\end{tabular}

Comments:

Data Package ID: UR0608181-1

\section{Qualifiers/Flags:}

$U$ - Result is less than the sample specific MDC

LT - Result is less than Requested MDC, greater than sample specific MDC

Y1 - Chemical Yield is in control at $100-110 \%$. Quantitative Yield is assumed.

2 - Chemical Yield outside default limits.

$M$ - The requested $M D C$ was not met

M3 - The requested MDC was not met, but the reported activity is greater than the reported MDO 


\section{Isotopic Uranium By Alpha Spectroscopy Sample Results Summary}

Client Name: National Sercurity Technologies, LLC Client Project Name: CAU 484 Client Project Number: V2735
Laboratory Name: Paragon Analytics PAI Work Order: 0608181
Page: 6 of 15

Reported on: Wednesday, September 20, 2006 10:11:26 AM

\begin{tabular}{|c|c|c|c|c|c|c|c|c|c|c|}
\hline $\begin{array}{c}\text { Lab } \\
\text { Sample ID }\end{array}$ & Client Sample ID & $\begin{array}{l}\text { Sample } \\
\text { Type }\end{array}$ & Nuclide & Result +/- $2 \mathrm{~s}$ TPU & MDC & Units & Matrix & Prep Batch & $\begin{array}{c}\text { Date } \\
\text { Analyzed }\end{array}$ & Flags \\
\hline $0608181-16$ & PDRO-08B & Sample & U.233/234 & $9.90 \mathrm{E}-01+/-1.71 \mathrm{E}-01$ & $1.97 \mathrm{E}-02$ & $\mathrm{pCi} / \mathrm{g}$ & SOIL & AS060901-1 & $9 / 10 / 2006$ & \\
\hline $0608181-16$ & PDRO-08B & Sample & U-235 & $3.97 \mathrm{E}-02+/-1.50 \mathrm{E}-02$ & $1.00 \mathrm{E}-02$ & pCirg & SOIL & AS060901-1 & $9 / 10 / 2006$ & \\
\hline $0608181-16$ & PDRO-08B & Sample & U-238 & $7.94 \mathrm{E}-01+/-1.39 \mathrm{E}-01$ & $1.41 \mathrm{E}-02$ & $\mathrm{pCi} / \mathrm{g}$ & SOIL & ASO60901-1 & $9 / 10 / 2006$ & \\
\hline $0608181-17$ & PDRO-09A & Sample & U-233/234 & $1.23 E+00+/-2.07 E-01$ & $1.27 \bar{E}-02$ & $\mathrm{pCi} / \mathrm{g}$ & SOIL & AS060901-1 & $9 / 10 / 2006$ & \\
\hline $0608181-17$ & PDRO-09A & Sample & U-235 & $6.66 \mathrm{E}-02+1-1.93 \mathrm{E}-02$ & $6.30 E-03$ & pCi/g & SOIL & AS060901-1 & $9 / 10 / 2006$ & \\
\hline $0608181-17$ & PDRO-09A & Sample & U-238 & $1.09 E+00+/-1.85 E-01$ & $1.06 \mathrm{E}-02$ & pCi/g & SOIL & AS060901-1 & 9/10/2006 & \\
\hline $0608181-18$ & PDRO-09B & Sample & U-233/234 & $9.56 \mathrm{E}-01+/-1.66 \mathrm{E}-01$ & $9.64 \mathrm{E}-03$ & $\mathrm{pCi} / \mathrm{g}$ & SOIL & AS060901-1 & $9 / 10 / 2006$ & \\
\hline $0608181-18$ & PDRO-09B & Sample & U-235 & $5.46 E-02+/-1.77 E-02$ & $2.90 \mathrm{E}-03$ & pCi/g & SOIL & AS060901-1 & $9 / 10 / 2006$ & \\
\hline $0608181-18$ & PDRO-09B & Sample & U.238 & $1.01 E+00+/-1.74 E-01$ & $7.54 \mathrm{E}-03$ & pCilg & SOIL & ASO60901-1 & $9 / 10 / 2006$ & \\
\hline
\end{tabular}

Comments:

\section{Data Package ID: UR0608181-1}

\section{Qualifiers/Flags:}

$U$ - Result is less than the sample specific MDC.

LT - Result is less than Requested MDC. greater than sample specfic MDC.

$\mathrm{Y} 1$. Chemical Yield is in control at $100-110 \%$. Quantitative Yietd is assumed.

$Y_{2}$. Chemical Yield outside default limits.

$M$ - The requested $M D C$ was not met

Abbreviations:

TPU - Total Propagated Uncertainty (see PAI SOP 743)

M.DC - Minimum Detectable Concentration (see PAI SOP 709)

M3 - The requested MDC was not met, but the reported activity is greater than the reported MDC

BDL-Below Detection Limit

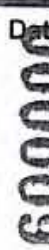




\section{Isotopic Uranium By Alpha Spectroscopy Sample Results Summary}

Client Name: National Sercurity Technologies, LLC Client Project Name: CAU 484 Client Project Number: V2735
Laboratory Name: Paragon Analytics PAI Work Order: 0608181
Page: 7 of 15

Reported on: Wednesday, September 20, 2006 10:11:27 AM

\begin{tabular}{|c|c|c|c|c|c|c|c|c|c|c|}
\hline $\begin{array}{c}\text { Lab } \\
\text { Sample ID }\end{array}$ & Client Sample ID & $\begin{array}{c}\text { Sample } \\
\text { Type }\end{array}$ & Nuclide & Result $+/-2 \mathrm{~s}$ TPU & MDC & Units & Matrix & Prep Batch & $\begin{array}{c}\text { Date } \\
\text { Analyzed }\end{array}$ & Flags \\
\hline $0608181-19$ & PDRO-10A & Sample & U-233/234 & $1.08 \mathrm{E}+00+1-1.86 \mathrm{E}-01$ & $6.07 \mathrm{E}-03$ & $\mathrm{pCi} / \mathrm{g}$ & SOIL & AS060901-1 & $9 / 10 / 2006$ & \\
\hline $0608181-19$ & PDRO-10A & Sample & U-235 & $4.83 \mathrm{E}-02+/-1.64 \mathrm{E}-02$ & $2.91 E-03$ & $\mathrm{pCi} / \mathrm{g}$ & SOIL & AS060901-1 & $9 / 10 / 2006$ & \\
\hline $0608181-19$ & PDRO-10A & Sample & $U-238$ & $9.52 \mathrm{E}-01+/-1.65 \mathrm{E}-01$ & $6.07 \mathrm{E}-03$ & pCi/g & SOIL & AS060901-1 & $9 / 10 / 2006$ & \\
\hline 0608181.20 & PDRO-10B & Sample & $U-233 / 234$ & $1.11 \mathrm{E}+00+1-1.90 \mathrm{E}-01$ & $8.50 E-03$ & $\mathrm{pCi} / \mathrm{g}$ & SOIL & AS060901-1 & $9 / 10 / 2006$ & \\
\hline $0608181-20$ & PDRO-10B & Sample & U.235 & $4.85 \mathrm{E}-02+1-1.64 \mathrm{E}-02$ & $6.97 \mathrm{E}-03$ & $\mathrm{pCi} / \mathrm{g}$ & SOIL & ASO60901-1 & $9 / 10 / 2006$ & \\
\hline $0608181-20$ & PDRO-10B & Sample & U.238 & $1.08 \mathrm{E}+00+1-1.85 \mathrm{E}-01$ & $9.44 \mathrm{E}-03$ & $\mathrm{pCi} / \mathrm{g}$ & SOIL & ASO60901-1 & $9 / 10 / 2006$ & \\
\hline $0608181-21$ & PDRO-11A & Sample & U-233/234 & $1.18 \mathrm{E}+00+/-2.10 \mathrm{E}-01$ & $1.87 \mathrm{E}-02$ & $\mathrm{pCi} / \mathrm{g}$ & SOIL & AS060901.2 & $9 / 18 / 2006$ & \\
\hline 0608181.21 & PDRO-11A & Sample & $\mathrm{U}-235$ & $5.52 \mathrm{E}-02+1-1.95 \mathrm{E}-02$ & $3.65 \mathrm{E} \cdot 03$ & pCilg & SOIL & ASO60901-2 & $9 / 18 / 2006$ & \\
\hline $0608181-21$ & PDRO-11A & Sample & U.238 & $1.05 E+00+/-1.88 E-01$ & $7.61 \mathrm{E}-03$ & $\mathrm{pCi} / \mathrm{g}$ & SOIL & AS060901-2 & $9 / 18 / 2006$ & \\
\hline
\end{tabular}

\section{Comments:}

\section{Data Package ID: UR0608181-1}

\section{Qualifiers/Flags:}

$U$ - Result is less than the sample specific MDC

LT - Result is less than Requested MDC, greater than sample specific MDC.

$Y_{1}$-Chemical Yield is in control at $100-110 \%$ Quarititative Yield is assumed.

Y2 - Chemical Yield outside default timits

$M$ - The requested $M D C$ was not met

Abbreviations:

TPU - Total Propagated Uncertainty (see PAI SOP 743)

MOC - Minimum Detectable Concentration (see PAI SOP 709)

M3 - The requested MDC was not met, but the reported activity is greater than the reported MDC.

BDL - Beiow Detection Limit

Dale? rinted: Wednesday, September 20, 2006

$\Leftrightarrow$ 


\section{Isotopic Uranium By Alpha Spectroscopy Sample Results Summary}

Client Name: National Sercurity Technologies, LLC Client Project Name: CAU 484 Client Project Number: V2735
Laboratory Name: Paragon Analytics

PAI Work Order: 0608181
Page: 8 of 15

Reported on: Wednesday, September 20, 2006

\begin{tabular}{|c|c|c|c|c|c|c|c|c|c|c|}
\hline $\begin{array}{c}\text { Lab } \\
\text { Sample ID }\end{array}$ & Client Sample ID & $\begin{array}{c}\text { Sample } \\
\text { Type }\end{array}$ & Nuclide & Result + $/$ - 2 s TPU & MDC & Units & Matrix & Prep Batch & $\begin{array}{c}\text { Date } \\
\text { Analyzed }\end{array}$ & Flags \\
\hline $0508181-22$ & PDRO-11B & Sample & U-233/234 & $1.14 \mathrm{E}+00+/-1.97 \mathrm{E}-01$ & $9.87 \mathrm{E}-03$ & pCilg & SOIL & AS060901-2 & $9 / 9 / 2006$ & \\
\hline $0608181-22$ & PDRO-11B & Sample & U-235 & $6.38 \mathrm{E}-02+1-1.98 \mathrm{E}-02$ & $7.29 \mathrm{E}-03$ & $\mathrm{pCi} / \mathrm{g}$ & SOIL & AS060901-2 & $9 / 9 / 2006$ & \\
\hline $0608181-22$ & PDRO-11B & Sample & U-238 & $1.05 \mathrm{E}+00+1-1.83 \mathrm{E}-01$ & $6.20 \mathrm{E}-03$ & pCilg & SOIL & AS060901-2 & $9 / 9 / 2006$ & \\
\hline $0608181-23$ & PDRO-12A & Sample & U-233/234 & $1.43 \mathrm{E}+00+/-2,42 \mathrm{E}-01$ & $1.17 \mathrm{E}-02$ & $\mathrm{pCl} / \mathrm{g}$ & SOIL & ASO50901-2 & $9 / 9 / 2006$ & \\
\hline $0608181-23$ & PDRO-12A & Sample & U-235 & $6.73 \mathrm{E}-02+/-2.05 \mathrm{E}-02$ & $1.30 \mathrm{E}-02$ & $\mathrm{pCi} / \mathrm{g}$ & SOIL & AS060901-2 & $9 / 9 / 2006$ & \\
\hline $0608181-23$ & PDRO-12A & Sample & $U-238$ & $1.17 \mathrm{E}+00+/-1.99 \mathrm{E}-01$ & $9.71 \mathrm{E}-03$ & $\mathrm{pCi} / \mathrm{g}$ & SOIL & AS060901-2 & $9 / 9 / 2006$ & \\
\hline 0608181.24 & PDRO-12B & Sample & U-233/234 & $1.05 E+00+1-1.79 E-01$ & $1,04 \mathrm{E}-02$ & $\mathrm{pCi} / \mathrm{g}$ & SOIL & ASO60901-2 & $9 / 9 / 2006$ & \\
\hline $0608181-24$ & PDRO-12B & Sample & U-235 & $5.93 \mathrm{E}-02+/-1.85 \mathrm{E}-02$ & $9.45 \mathrm{E}-03$ & $\mathrm{pCi} / \mathrm{g}$ & SOIL & ASO60901-2 & $9 / 9 / 2006$ & \\
\hline $0608181-24$ & PDRO-12B & Sample & $U-238$ & $1.03 \mathrm{E}+00+1-1.77 \mathrm{E}-01$ & $8.93 \mathrm{E}-03$ & $\mathrm{pCi} / \mathrm{g}$ & SOIL & AS060901-2 & 9/9/2006 & \\
\hline
\end{tabular}

\section{Comments:}

\section{Data Package ID: UR0608181-1}

\section{Qualifiers/Flags:}

$U \cdot$ Result is less than the sample specific MDC

LT - Result is less than Requested MDC, greater than sample specific MDC.

$\mathrm{Y}_{1}$ - Chemical Yield is in controt at 100-110\%, Quantitative Yield is assumed

Y2 - ChemicakYYield outside default limits

$M$ - The requested MDC was not met

Abbreviations

TPU - Total Propagated Uncertainty (see PAI SOP 743)

MDC - Minimum Detectable Concentration (see PAI SOP 709)

$\mathrm{M}^{3}$-The requested MDC was not mot, but the reported activity is greater than the reported MDC

BDL - Below Detection Limit

Printed: Wednesday, September 20, 2006




\section{Isotopic Uranium By Alpha Spectroscopy Sample Results Summary}

Client Name: National Sercurity Technologies, LLC Client Project Name: CAU 484 Client Project Number: V2735
Laboratory Name: Paragon Analytics

PAI Work Order: 0608181
Page: 9 of 15

Reported on: Wednesday, September 20,2006 10:11:27 AM

\begin{tabular}{|c|c|c|c|c|c|c|c|c|c|c|}
\hline $\begin{array}{c}\text { Lab } \\
\text { Sample ID }\end{array}$ & Client Sample ID & $\begin{array}{l}\text { Sample } \\
\text { Type }\end{array}$ & Nuclide & Result + $1-2$ s TPU & MDC & Units & Matrix & Prep Batch & $\begin{array}{c}\text { Date } \\
\text { Analyzed }\end{array}$ & Flags \\
\hline $0608181-25$ & PDRO-13A & Sample & U-233/234 & $1.42 \mathrm{E}+00+/-2.42 \mathrm{E}-01$ & $9.78 \mathrm{E}-03$ & pCi/g & SOIL & AS060901-2 & $9 / 9 / 2006$ & \\
\hline $0608181-25$ & PDRO-13A & Sample & U-235 & $6.58 \mathrm{E}-02+1-2.03 \mathrm{E}-02$ & $9.00 \mathrm{E}-03$ & pCirg & SOIL & AS060901-2 & 9/9/2006 & \\
\hline $0608181-25$ & PDRO-13A & Sample & U. 238 & $1.17 \mathrm{E}+00+/-2.03 \mathrm{E}-01$ & $9.78 \mathrm{E}-03$ & $\mathrm{pCi} / \mathrm{g}$ & SOIL & ASO60901.2 & $9 / 9 / 2006$ & \\
\hline $0608181-26$ & PDRO-13B & Sample & U-233/234 & $1.28 \mathrm{E}+00+/-2.18 \mathrm{E}-01$ & 8.52E-03 & pCirg & SOIL & AS060901-2 & $9 / 9 / 2006$ & \\
\hline $0608181-26$ & PDRO-13B & Sample & U-235 & $8.01 E-02+1-2.26 E-02$ & $6.99 \mathrm{E}-03$ & $\mathrm{pCi} / \mathrm{g}$ & SOIL & ASO60901-2 & $9 / 9 / 2006$ & \\
\hline $0608181-26$ & PDRO-13B & Sample & U.238 & $1.15 E+00+/-1.98 E-01$ & $7.40 \mathrm{E}-03$ & pCirg & SOIL & AS060901-2 & 9/9/2006 & \\
\hline $0608181-27$ & PDRO-14A & Sample & U-233/234 & $9.81 \mathrm{E}-01+/-1.69 \mathrm{E}-01$ & $1.68 \mathrm{E}-02$ & $\mathrm{pCi} / \mathrm{g}$ & SOIL & ASO60901-2 & $9 / 10 / 2006$ & \\
\hline $0608181 \cdot 27$ & PDRO-14A & Sample & U-235 & $4.81 \mathrm{E}-02+/-1.66 \mathrm{E}-02$ & $1.15 \mathrm{E}-02$ & $\mathrm{pCi} / \mathrm{g}$ & SOIL & AS060901-2 & $9 / 10 / 2006$ & \\
\hline $0608181-27$ & PDRO-14A & Sample & U-238 & $9.88 \mathrm{E}-01+/-1.70 \mathrm{E}-01$ & 8.95E-03 & $\mathrm{pCi} / \mathrm{g}$ & SOIL & ASO60901-2 & $9 / 10 / 2006$ & \\
\hline
\end{tabular}

\section{Comments:}

\section{Data Package ID: UR0608181-1}

Qualifiers/Flags:

$U$ - Result is less than the sample specific MDC.

LT - Resuit is less than Requested MDC, greater than sample specific MDC.

$Y 1$ - Chemical Yield is in control at $100-110 \%$. Quantitative Yield is assumed

Y2 - Chemical Yield outside defautl limits.

$M$ - The requested $M D C$ was not met

Abbreviations:

TPU - Total Propagated Uncertainty (see PAI SOP 743)

MDC - Minimum Detectable Concentration (see PAI SOP 709)

M3 - The requested MDC was not met, but the reported activity is greater than the reported MDC

BDL - Below Detection Limit 


\section{Isotopic Uranium By Alpha Spectroscopy Sample Results Summary}

Client Name: National Sercurity Technologies, LLC Client Project Name: CAU 484 Client Project Number: V2735
Laboratory Name: Paragon Analytics PAI Work Order: 0608181
Page: 10 of 15

Reported on: Wednesday, September 20, 2006 10:11:27 AM

\begin{tabular}{|c|c|c|c|c|c|c|c|c|c|c|}
\hline $\begin{array}{c}\text { Lab } \\
\text { Sample ID }\end{array}$ & Client Sample ID & $\begin{array}{c}\text { Sample } \\
\text { Type }\end{array}$ & Nuclide & Result $+1-2$ s TPU & MDC & Units & Matrix & Prep Batch & $\begin{array}{c}\text { Date } \\
\text { Analyzed }\end{array}$ & Flags \\
\hline $0608181-28$ & PDRO-14B & Sample & U-233/234 & $9.08 \mathrm{E}-01+1-1.57 \mathrm{E}-01$ & $1.10 \mathrm{E}-02$ & pCi/g & SOIL & AS060901-2 & $9 / 10 / 2006$ & \\
\hline $0608181-28$ & PDRO-14B & Sample & U-235 & $4.54 \mathrm{E}-02+/-1.53 \mathrm{E}-02$ & $2.67 \mathrm{E}-03$ & $\mathrm{pCi} / \mathrm{g}$ & SOIL & AS060901-2 & $9 / 10 / 2006$ & \\
\hline $0608181-28$ & PDRO-14B & Sample & U-238 & $8.89 \mathrm{E}-01+1-1.53 \mathrm{E}-01$ & $8.00 E-03$ & $\mathrm{pCi} / \mathrm{g}$ & SOIL & AS060901.2 & $9 / 10 / 2006$ & \\
\hline $0608181-29$ & PDRO-15A & Sample & U-233/234 & $1.17 \mathrm{E}+00+/-1.99 \mathrm{E}-01$ & $8.84 E-03$ & $\mathrm{pCitg}$ & SOIL & AS060901-2 & $9 / 10 / 2006$ & \\
\hline $0608181-29$ & PDRO-15A & Sample & U-235 & $5.62 \mathrm{E}-02+/-1.75 \mathrm{E}-02$ & $6.53 \mathrm{E}-03$ & $\mathrm{pCi} / \mathrm{g}$ & SOIL & AS060901-2 & $9 / 10 / 2006$ & \\
\hline $0608181-29$ & PDRO-15A & Sample & $U-238$ & $9.59 \mathrm{E}-01+/-1.64 \mathrm{E}-01$ & $5.55 \mathrm{E}-03$ & pCilg & SOIL & AS060901-2 & $9 / 10 / 2006$ & \\
\hline $0608181-30$ & PDRO-15B & Sample & U-233/234 & $1.13 E+00+/-1.95 E-01$ & $9.66 \mathrm{E} \cdot 03$ & $\mathrm{pCi} / \mathrm{g}$ & SOIL & AS060901-3 & $9 / 12 / 2006$ & \\
\hline 0608181.30 & PDRO-15B & Sample & $U-235$ & $5.28 \mathrm{E}-02+/-1.75 \mathrm{E}-02$ & $7.13 \mathrm{E}-03$ & $\mathrm{pCi} / \mathrm{g}$ & SOIL & ASO60901-3 & $9 / 12 / 2006$ & \\
\hline $0608181-30$ & PDRO-15B & Sample & U-238 & 1.15E+00 +/- $1.97 \mathrm{E}-01$ & $6.06 \mathrm{E}-03$ & $\mathrm{pCi} / \mathrm{g}$ & SOIL & AS060901-3 & $9 / 12 / 2006$ & \\
\hline
\end{tabular}

\section{Comments:}

\section{Data Package ID: UR0608181-1}

\section{Qualifiers/Flags:}

$U$ - Result is less than the sample specific MDC

$L T$ - Result is less than Requested MDC, greater than sample specific MDC

Y1 - Chemical Yield is in control at 100-110\%. Quantitative Yield is assumed

Y2 - Chernical Yield outside default timits

$M$ - The requested MDC was not met

Abbreviations:

TPU - Total Propagated Uncertainty (see PAI SOP 743)

MDC - Minimum Detectable Concentration (see PAI SOP 709)

M3 - The requested MDC was not met, but the reported activity is greater than the reported MDC

BDL - Below Detection Limit

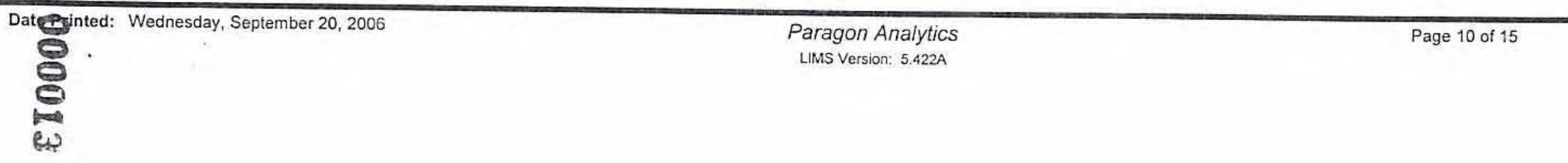




\section{Isotopic Uranium By Alpha Spectroscopy Sample Results Summary}

Client Name: National Sercurity Technologies, LLC Client Project Name: CAU 484 Client Project Number: V2735
Laboratory Name: Paragon Analytics PAI Work Order: 0608181
Page: 11 of 15

Reported on: Wednesday, September 20, 2006 10:11:27 AM

\begin{tabular}{|c|c|c|c|c|c|c|c|c|c|c|}
\hline $\begin{array}{l}\text { Lab } \\
\text { Sample ID }\end{array}$ & Client Sample ID & $\begin{array}{l}\text { Sample } \\
\text { Type }\end{array}$ & Nuclide & Result $+1-2$ s TPU & MDC & Units & Matrix & Prep Batch & $\begin{array}{c}\text { Date } \\
\text { Analyzed }\end{array}$ & Flags \\
\hline $0608181-31$ & PDRO-16A & Sample & U.233/234 & $1.11 E+00+/-1.90 E-01$ & $1.06 \mathrm{E}-02$ & pCirg & SOIL & AS060901-3 & $9 / 12 / 2006$ & \\
\hline $0608181-31$ & PDRO-16A & Sample & $U-235$ & $4.72 E-02+/-1.62 E-02$ & $9.61 \mathrm{E}-03$ & pCirg & SOIL & AS060901-3 & $9 / 12 / 2006$ & \\
\hline $0608181-31$ & PDRO-16A & Sample & U-238 & $1.01 E+00+/-1.73 E-01$ & $9.07 E-03$ & $\mathrm{pCi} / \mathrm{g}$ & SOIL & AS060901-3 & $9 / 12 / 2006$ & \\
\hline $0608181-32$ & PDRO-16B & Sample & $U-233 / 234$ & $1.08 \mathrm{E}+00+/-1.88 \mathrm{E}-01$ & $9.81 \mathrm{E}-03$ & $\mathrm{pCi} / \mathrm{g}$ & SOIL & AS060901-3 & $9 / 12 / 2006$ & \\
\hline $0608181-32$ & PDRO-16B & Sample & U.235 & $4.85 \mathrm{E}-02+/-1.69 \mathrm{E}-02$ & $9.02 \mathrm{E}-03$ & $\mathrm{pCi} / \mathrm{g}$ & SOIL & AS060901-3 & $9 / 12 / 2006$ & \\
\hline $0608181-32$ & PDRO-16B & Sample & U-238 & $1.04 \mathrm{E}+00+/-1.81 \mathrm{E}-01$ & $9.81 \mathrm{E}-03$ & $\mathrm{pCi} / \mathrm{g}$ & SOIL & AS060901-3 & $9 / 12 / 2006$ & \\
\hline $0608181-33$ & PDRO-17A & Sample & U.233/234 & $1.41 E+00+1-2.40 E-01$ & $8.86 \mathrm{E}-03$ & pCìg & SOIL & AS060901-3 & $9 / 12 / 2006$ & \\
\hline $0608181-33$ & PDRO-17A & Sample & U-235 & $6.47 \mathrm{E}-02+1-2.00 \mathrm{E}-02$ & $7.26 \mathrm{E}-03$ & $\mathrm{pCi} / \mathrm{g}$ & SOIL & AS060901-3 & $9 / 12 / 2006$ & \\
\hline $0608181-33$ & PDRO-17A & Sample & U-238 & $1.16 \mathrm{E}+00+1-1.99 \mathrm{E}-01$ & $7.69 \mathrm{E}-03$ & $\mathrm{pCi} / \mathrm{g}$ & SOIL & AS060901-3 & $9 / 12 / 2006$ & \\
\hline
\end{tabular}

\section{Comments:}

\section{Data Package ID: UR0608181-1}

\section{Qualifiers/Flags:}

$U$ - Result is less than the sample specific MDC.

LT - Result is less than Requested MOC, greater than sample specific MDC

Y1 - Chemical Yield is in control at 100-110\%. Quantitative Yield is assumed

Y2 - Chemical Yield outside default limits.

$M$ - The requested $M D C$ was not met

M3 - The requested MDC was not met, but the reported activity is greater than the reported MDC
Abbreviations:

TPU - Total Propagated Uncerlainty (see PAI SOP 743)

MDC - Minimum Detectable Concentration (see PAI SOP 709)

BDL - Below Detection Limit

Dategragon Analytics
uMs Version: $5.422 \mathrm{~A}$




\section{Isotopic Uranium By Alpha Spectroscopy Sample Results Summary}

Client Name: National Sercurity Technologies, LLC Client Project Name: CAU 484 Client Project Number: V2735
Laboratory Name: Paragon Analytics PAI Work Order: 0608181
Page: 12 of 15

Reported on: Wednesday, September 20, 2006 10:11:27 AM

\begin{tabular}{|c|c|c|c|c|c|c|c|c|c|c|}
\hline $\begin{array}{c}\text { Lab } \\
\text { Sample ID }\end{array}$ & Client Sample ID & $\begin{array}{c}\text { Sample } \\
\text { Type }\end{array}$ & Nuclide & Resuit +/- 2 s TPU & MDC & Units & Matrix & Prep Batch & $\begin{array}{c}\text { Date } \\
\text { Analyzed }\end{array}$ & Flags \\
\hline $0608181-34$ & PDRO-17B & Sample & U.233/234 & $1.20 \mathrm{E}+00+/-2.14 \mathrm{E}-01$ & $1.24 \mathrm{E}-02$ & pCi/g & SOIL & AS060901-3 & $9 / 12 / 2006$ & \\
\hline 0608181.34 & PDRO-17B & Sample & U.235 & $6.08 \mathrm{E}-02+j-2.11 \mathrm{E}-02$ & $9.13 \mathrm{E}-03$ & pCirg & SOIL & AS060901-3 & $9 / 12 / 2006$ & \\
\hline $0608181-34$ & PDRO-17B & Sample & $U-238$ & $1.11 E+00+/-1.99 E-01$ & $1.11 \mathrm{E}-02$ & $\mathrm{pCi} / \mathrm{g}$ & SOIL & AS060901-3 & $9 / 12 / 2006$ & \\
\hline 0608181.35 & PDRO-18A & Sample & U. $233 / 234$ & $1.40 \mathrm{E}+00+1-2.38 \mathrm{E}-01$ & $1.94 \mathrm{E}-02$ & $\mathrm{pClig}$ & SOIL & AS060901-3 & $9 / 12 / 2006$ & \\
\hline $0608181-35$ & PDRO-18A & Sample & U-235 & $5.38 \mathrm{E}-02+/-1.77 \mathrm{E}-02$ & $9.90 \mathrm{E}-03$ & pCilg & SOIL & AS060901-3 & $9 / 12 / 2006$ & \\
\hline $0608181-35$ & PDRO-18A & Sample & $U-238$ & $1.20 \mathrm{E}+00+/-2.05 \mathrm{E}-01$ & $1.39 \mathrm{E}-02$ & $\mathrm{pCi} / \mathrm{g}$ & SOIL & ASO60901-3 & $9 / 12 / 2006$ & \\
\hline $0608181-36$ & PDRO-18B & Sample & U-233/234 & 1.15E+00 +/-1.97E-01 & $1.36 \mathrm{E}-02$ & pCiig & SOIL & AS060901-3 & $9 / 12 / 2006$ & \\
\hline $0608181-36$ & PDRO-18B & Sample & U-235 & $5.08 \mathrm{E}-02+/-1.67 \mathrm{E}-02$ & $6.72 \mathrm{E}-03$ & $\mathrm{pCi} / \mathrm{g}$ & SOIL & AS060901.3 & $9 / 12 / 2006$ & \\
\hline $0608181-36$ & PDRO-18B & Sample & U-238 & $1.09 \mathrm{E}+00+1-1.87 \mathrm{E}-01$ & $1.13 \mathrm{E}-02$ & $\mathrm{pCi} / \mathrm{g}$ & SOIL & AS060901-3 & $9 / 12 / 2006$ & \\
\hline
\end{tabular}

\section{Comments:}

\section{Data Package ID: UR0608181-1}

\section{Qualifiers/Flags:}

$U$ - Result is less than the sample specific MDC

LT - Result is less than Requested MDC, greater than sample specific MDC

Y1 - Chemical Yield is in control at $100-110 \%$ Quantitative Yield is assumed

Y2. Chemical Yield outside default limits.

$M$ - The requested $M D C$ was not met

M3 - The requested MDC was not met, but the reported activity is greater than the reported MDC
Dafeprinted: Wednesday, September 20, 2006

:
Abbreviations

TPU - Total Propagated Uncertainty (see PAI SOP 743)

MDC - Minimum Detectabie Concentration (see PAI SOP 709)

BDL - Below Detection Lim: 


\section{Isotopic Uranium By Alpha Spectroscopy Sample Results Summary}

Client Name: National Sercurity Technologies, LLC Client Project Name: CAU 484 Client Project Number: V2735
Laboratory Name: Paragon Analytics

PAI Work Order: 0608181
Page: 13 of 15

Reported on: Wednesday, September 20,2006 10:11:27 AM

\begin{tabular}{|c|c|c|c|c|c|c|c|c|c|c|}
\hline $\begin{array}{c}\text { Lab } \\
\text { Sample ID }\end{array}$ & Client Sample ID & $\begin{array}{l}\text { Sample } \\
\text { Type }\end{array}$ & Nuclide & Result +1- $2 \mathrm{~s}$ TPU & MDC & Units & Matrix & Prep Batch & $\begin{array}{c}\text { Date } \\
\text { Analyzed }\end{array}$ & Flags \\
\hline 0608181.37 & PDRO-19A & Sample & U-233/234 & $1.41 E+00+/-2.47 E-01$ & $1.20 \mathrm{E}-02$ & pCilg & SOIL & AS060901-3 & $9 / 12 / 2006$ & \\
\hline $0608181-37$ & PDRO-19A & Sample & U.235 & $7.05 \mathrm{E}-02+1-2.26 \mathrm{E}-02$ & $3.61 \mathrm{E}-03$ & $\mathrm{pCi} / \mathrm{g}$ & SOIL & AS060901-3 & $9 / 12 / 2006$ & \\
\hline $0608181-37$ & PDRO-19A & Sample & U.238 & $1.25 E+00+/-2.21 E-01$ & $9.37 \mathrm{E}-03$ & $\mathrm{pCi} / \mathrm{g}$ & SOIL & AS060901-3 & $9 / 12 / 2006$ & \\
\hline $0608181-38$ & PDRO-19B & Sample & U.233/234 & $1.31 E+00+/-2.23 E-01$ & $6.00 \mathrm{E}-03$ & $\mathrm{pCi} / \mathrm{g}$ & SOIL & AS060901-3 & $9 / 12 / 2006$ & \\
\hline $0608181-38$ & PDRO-19B & Sample & U-235 & $5.41 \mathrm{E}-02+/-1.75 \mathrm{E}-02$ & $2.87 \mathrm{E}-03$ & pCi/g & SOIL & AS060901-3 & $9 / 12 / 2006$ & \\
\hline $0608181-38$ & PDRO-19B & Sample & U.238 & $1.22 \mathrm{E}+00+1-2.08 \mathrm{E}-01$ & $6.00 \mathrm{E}-03$ & $\mathrm{pCi} / \mathrm{g}$ & SOIL & AS060901-3 & $9 / 12 / 2006$ & \\
\hline $0608181-39$ & PDRO-20A & Sample & U.233/234 & $1.37 E+00+/-2.33 E-01$ & $8.48 \mathrm{E}-03$ & $\mathrm{pCi} / \mathrm{g}$ & SOIL & ASO60901-3 & $9 / 12 / 2006$ & \\
\hline $0608181-39$ & PDRO-20A & Sample & U.235 & $7.45 \mathrm{E}-02+/-2.15 \mathrm{E}-02$ & $6.95 \mathrm{E}-03$ & pCitg & SOIL & AS060901-3 & $9 / 12 / 2006$ & \\
\hline $0608181-39$ & PDRO-20A & Sample & U. 238 & $1.19 E+00+/-2.03 E-01$ & $9.42 E-03$ & $\mathrm{pCi} / \mathrm{g}$ & SOIL & AS060901-3 & $9 / 12 / 2006$ & \\
\hline
\end{tabular}

\section{Comments:}

\section{Data Package ID: UR0608181-1}

Qualifiers/Flags:

U - Result is tess than the sample specific MDC.

LT - Result is less than Requested MOC, greater than sample specific MDC

$\mathrm{Y1}$ - Chemical Yield is in control at $100-110 \%$.Quantiative Yield is assumed.

Y2 - Chemical Yield outside default limits

$M$ - The requested $M D C$ was not met

Abbreviations:

TPU - Totat Propagated Uncertainty (see PAI SOP 743)

MDC - Minimum Detectable Concentration (see PAI SOP 709)

M3 - The requested MDC was not met, but the reported activity is greater than the reported MDC.

BDL - Below Detection Limit 


\section{Isotopic Uranium By Alpha Spectroscopy Sample Results Summary}

Client Name: National Sercurity Technologies, LLC Client Project Name: CAU 484 Client Project Number: V2735
Laboratory Name: Paragon Analytics PAI Work Order: 0608181
Page: 14 of 15

Reported on: Wednesday, September 20, 2006 10:11:27 AM

\begin{tabular}{|c|c|c|c|c|c|c|c|c|c|c|}
\hline $\begin{array}{c}\text { Lab } \\
\text { Sample ID }\end{array}$ & Client Sample ID & $\begin{array}{l}\text { Sample } \\
\text { Type }\end{array}$ & Nuclide & Result $+1-2 \mathrm{~s}$ TPU & MDC & Units & Matrix & Prep Batch & $\begin{array}{c}\text { Date } \\
\text { Analyzed }\end{array}$ & Flags \\
\hline $0608181-40$ & PDRO-2OB & Sample & U-233/234 & $1.22 \mathrm{E}+00+/-2.08 \mathrm{E}-01$ & $2.48 \mathrm{E}-03$ & $\mathrm{pCl} / \mathrm{g}$ & SOIL & ASO60901-3 & $9 / 12 / 2006$ & \\
\hline $0608181-40$ & PDRO-20B & Sample & U-235 & $7.35 \mathrm{E}-02+1-2.16 \mathrm{E}-02$ & $7.17 \mathrm{E}-03$ & pCi'g & SOIL & ASO60901-3 & $9 / 12 / 2006$ & \\
\hline $0608181-40$ & PDRO-2OB & Sample & $U-238$ & $1.14 \mathrm{E}+00+/-1.96 \mathrm{E}-01$ & $7.59 \mathrm{E}-03$ & $\mathrm{pCi} / \mathrm{g}$ & SOIL & AS060901-3 & $9 / 12 / 2006$ & \\
\hline $0608181-41$ & PDRO-21A & Sample & $U-233 / 234$ & $1.45 \mathrm{E}+00+/-2.48 \mathrm{E}-01$ & $1.58 \mathrm{E}-02$ & $\mathrm{pCi} / \mathrm{g}$ & SOIL & ASO60901-3 & $9 / 12 / 2006$ & \\
\hline $0608181-41$ & PDRO-21A & Sample & U-235 & $7.33 \mathrm{E}-02+/-2.20 \mathrm{E}-02$ & $1.05 \mathrm{E}-02$ & pCig & SOIL & ASO60901-3 & $9 / 12 / 2006$ & \\
\hline $0608181-41$ & PDRO-21A & Sample & U.238 & $1.22 E+00+/-2.10 E-01$ & $7.73 \mathrm{E}-03$ & $\mathrm{pCi} / \mathrm{g}$ & SOIL & ASO60901-3 & $9 / 12 / 2006$ & \\
\hline $0608181-42$ & PDRO-21B & Sample & U-233/234 & $1.21 E+00+/-2.07 E-01$ & $9.25 \mathrm{E} \cdot 03$ & $\mathrm{pCi} / \mathrm{g}$ & SOIL & ASO60901-3 & $9 / 12 / 2000$ & \\
\hline $0608181-42$ & PDRO-21B & Sample & U-235 & $4.86 \mathrm{E}-02+1-1.63 \mathrm{E}-02$ & $6.83 \mathrm{E}-03$ & $\mathrm{pCl} / \mathrm{g}$ & SOIL & ASO60901-3 & $9 / 12 / 2006$ & \\
\hline $0608181-42$ & PDRO-21B & Sample & U-238 & $1.15 \mathrm{E}+00+/-1.96 \mathrm{E}-01$ & $5.81 \mathrm{E}-03$ & $\mathrm{pCi} / \mathrm{g}$ & SOIL & ASO60901-3 & $9 / 12 / 2006$ & \\
\hline
\end{tabular}

Comments:

\section{Data Package ID: UR0608181-1}

\section{Qualifiers/Flags:}

$U$ - Result is less than the sample specific MDC.

LT - Result is less than Requested MDC, greater than sample specific MDC

Y1 1 Cैhemical Yield is in control at $100-110 \%$. Quantitative Yield is assumed

Y2 - Chemical Yield outside default imits

$M$ - The requested $M D C$ was not me:

M3 - The requested MDC was not met, but the reported activity is greater than the reported MDC.
Abbreviations:

TPU - Total Propagated Uncertainty (see PAI SOP 743)

MDC - Minimum Detectable Concentration (see PAI SOP 709)

BDL - Below Detection Limit

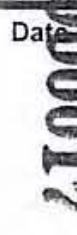




\section{Isotopic Uranium By Alpha Spectroscopy Sample Results Summary}

Client Name: National Sercurity Technologies, LLC Client Project Name: CAU 484 Client Project Number: V2735
Laboratory Name: Paragon Analytics PAI Work Order: 0608181
Page: 15 of 15

Reported on: Wednesday, September 20, 2006 10:11:27 AM

\begin{tabular}{|c|c|c|c|c|c|c|c|c|c|c|}
\hline $\begin{array}{c}\text { Lab } \\
\text { Sample ID }\end{array}$ & Client Sample ID & $\begin{array}{l}\text { Sample } \\
\text { Type }\end{array}$ & Nuclide & Result + $/$ - 2 s TPU & MDC & Units & Matrix & Prep Batch & $\begin{array}{c}\text { Date } \\
\text { Analyzed }\end{array}$ & Flags \\
\hline $0608181-43$ & PDRO-R1 & Sample & U-233/234 & $2.95 \mathrm{E}-02+/-2.67 \mathrm{E}-02$ & $3.86 \mathrm{E}-02$ & $p \mathrm{Cin}$ & WATER & ASO60828-4 & $8 / 30 / 2006$ & $u$ \\
\hline 0608181.43 & PDRO-R1 & Sample & U-235 & $1.20 \mathrm{E}-03+/-1.83 \mathrm{E}-02$ & 2.85E-02 & $\mathrm{pCi} / 1$ & WATER & ASO60828-4 & $8 / 30 / 2006$ & $u$ \\
\hline $0608181-43$ & PDRO-R1 & Sample & U.238 & 1.03E-02 +/- $1.87 E-02$ & $3,47 \mathrm{E}-02$ & $\mathrm{pCin}$ & WATER & AS060828-4 & $8 / 30 / 2006$ & u \\
\hline
\end{tabular}

Comments:

Data Package ID: UR0608181-1
Qualifiers/Flags:
(1) - Result is less than the sample specific MDC.
LT - Result is less than Requested MDC, greater than sample speciric MDC.
Y1. Chemical Yield is in control at $100-110 \%$. Quantitative Yield is assumed
Y2 - Chemical Yield outside default limits
$M$ - The requested MDC was not met
M3 - The requested MDC was not met, but the reported activity is greater than the reported MDC

Abbreviations

TPU - Total Propagated Uncertainty (see PAI SOP 743)

MDC - Minimum Detectable Concentration (see PAI SOP 709)

BDL - Below Detection Limit

Date Printed: Wednesday, September 20, 2006 


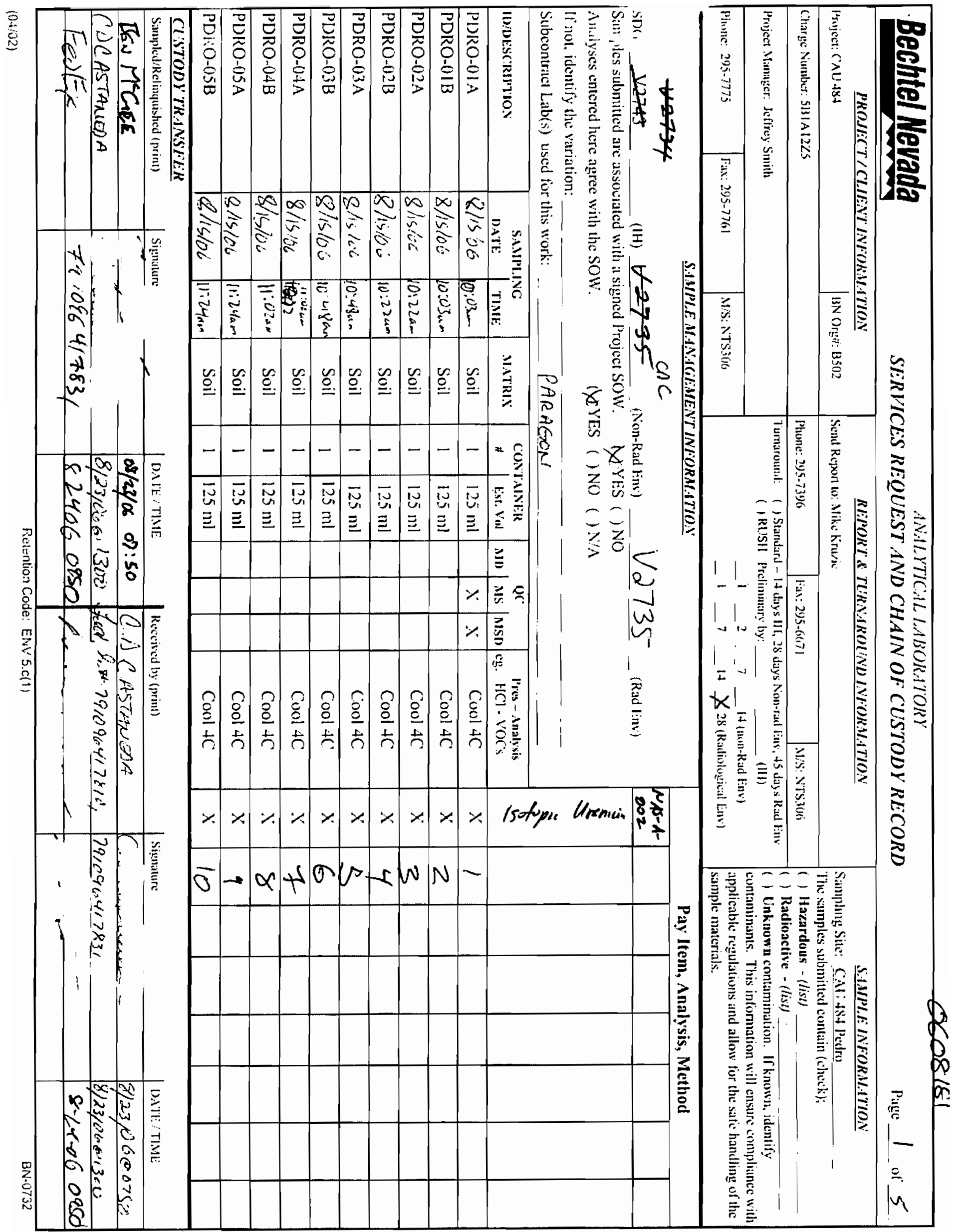




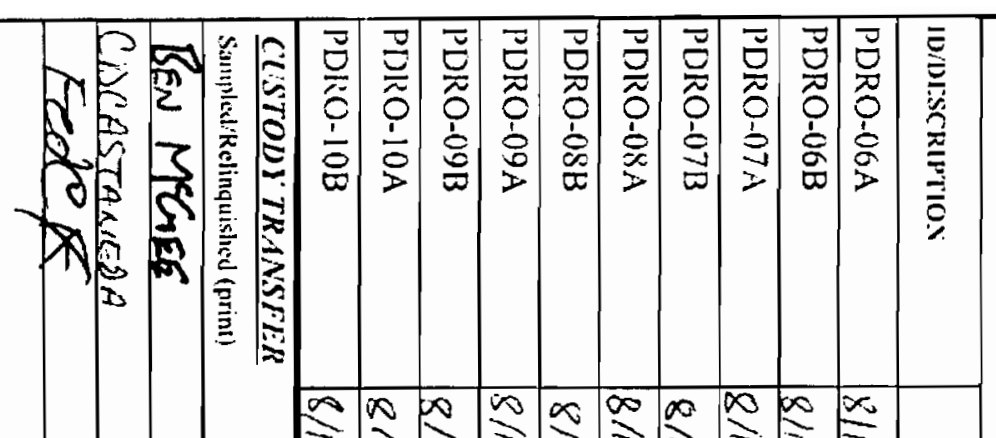

$\infty \infty_{\infty}^{\infty} \infty \infty^{\infty} \infty \infty^{\infty} \infty \infty^{\infty}$

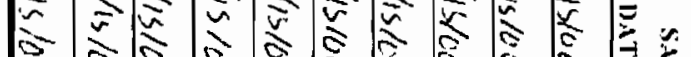

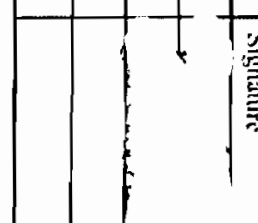

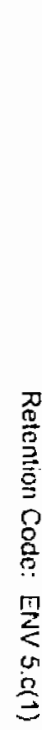

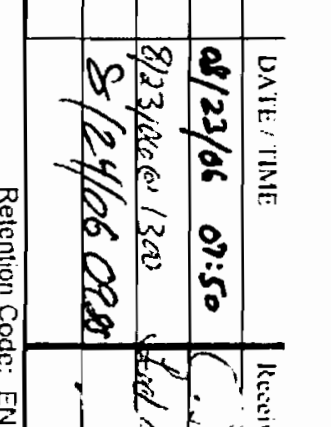

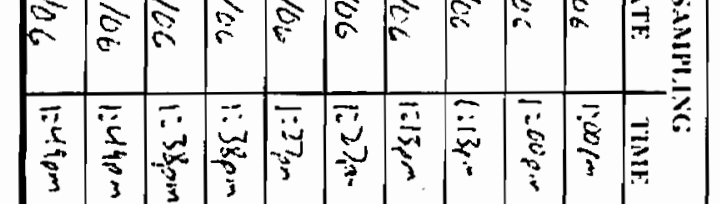

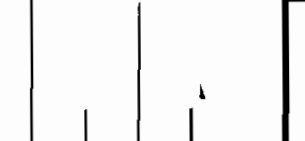

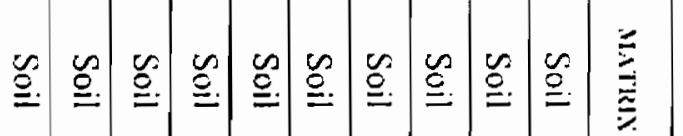

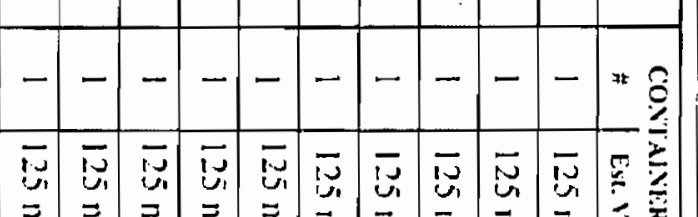

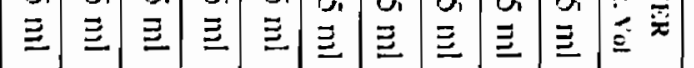

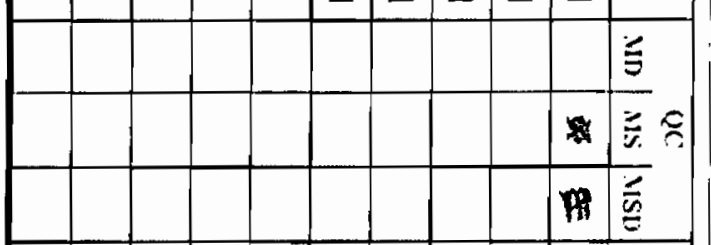

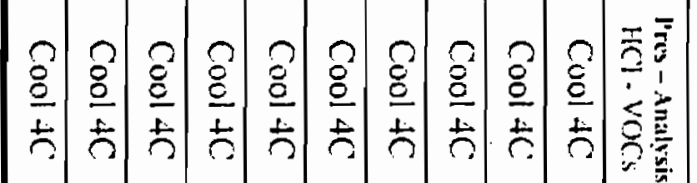

$\times \times \times \times \times \times \times 15 \times 4$

$s=\bar{a} \bar{\sigma} \bar{v}=\bar{w}=$

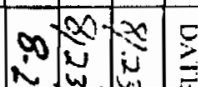

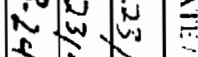

*

鿷

$0 \approx \Omega$

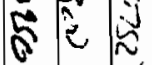

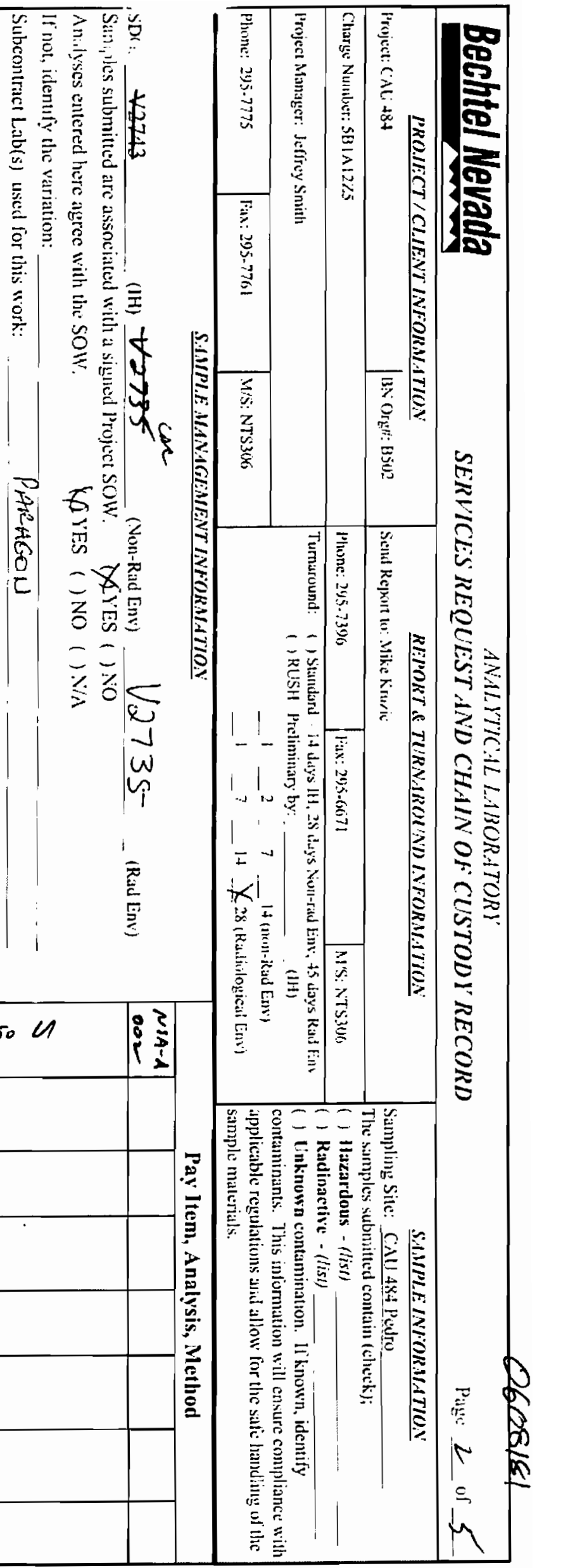


$\stackrel{\mathscr{C}}{\ddot{E}}$ $x 3$

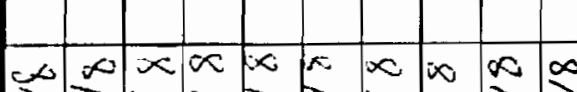

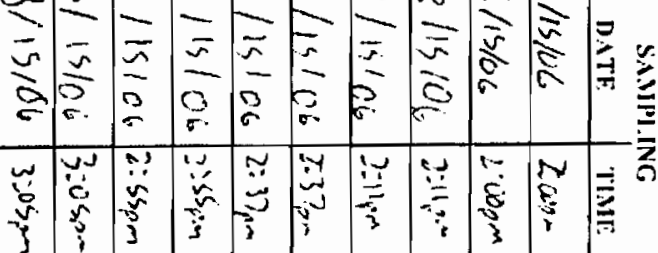
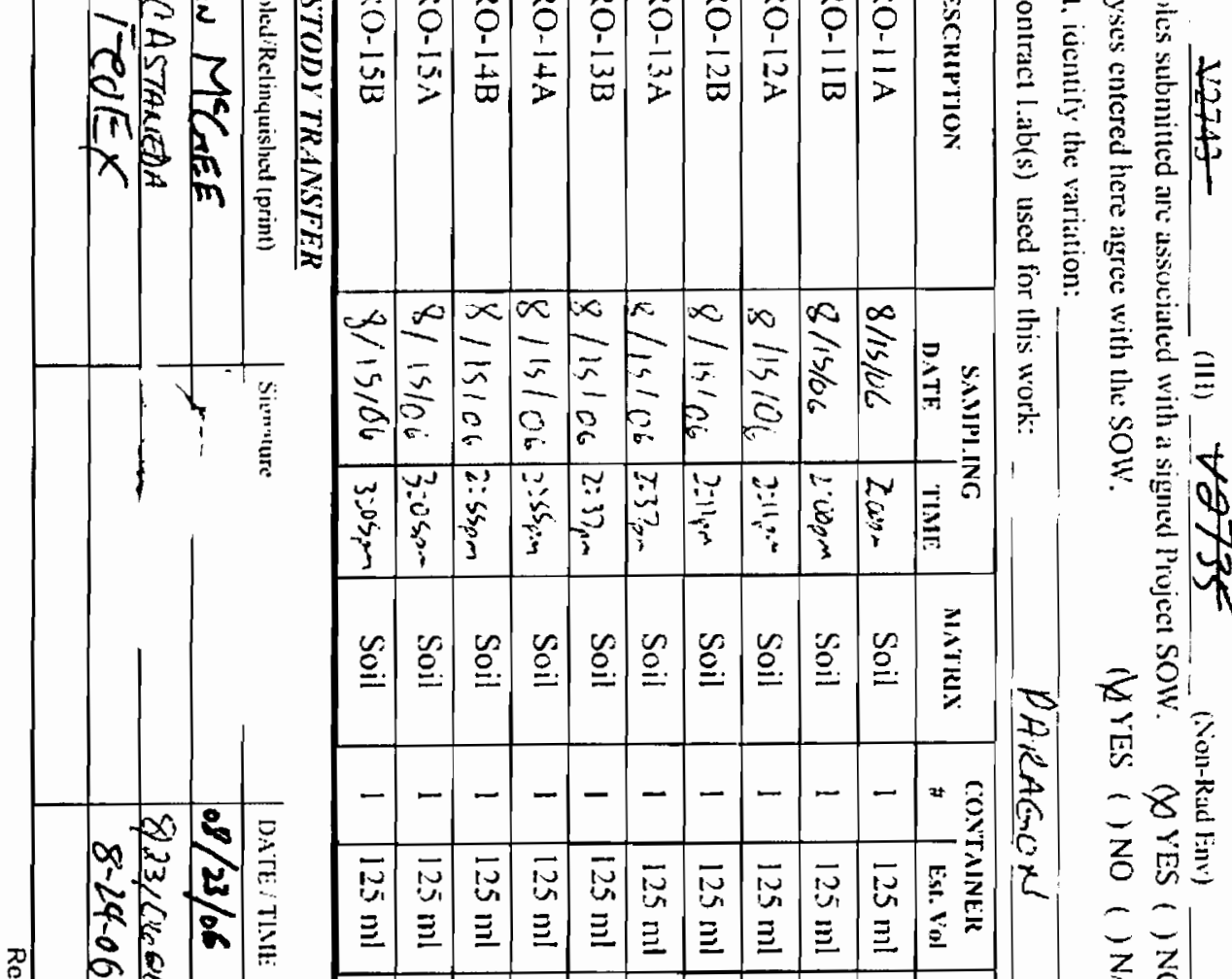

害

悹

三

番

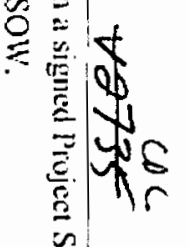

$\nabla \overrightarrow{2}$

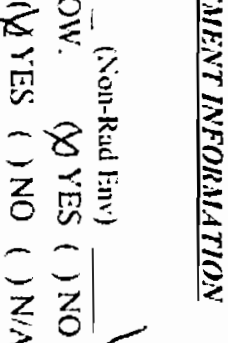

总余

$40 \pi$

S 5

霖

梠

23

B

5

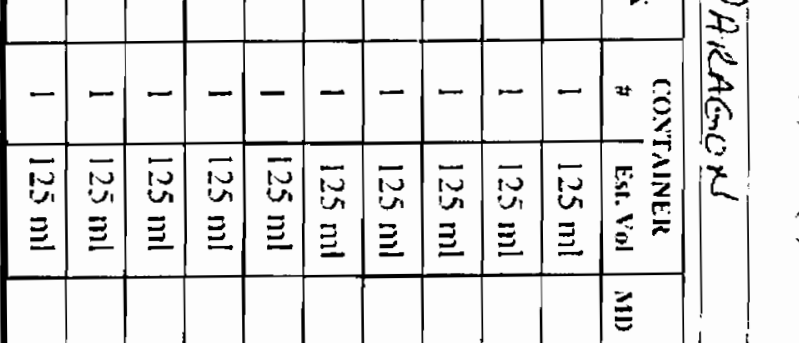
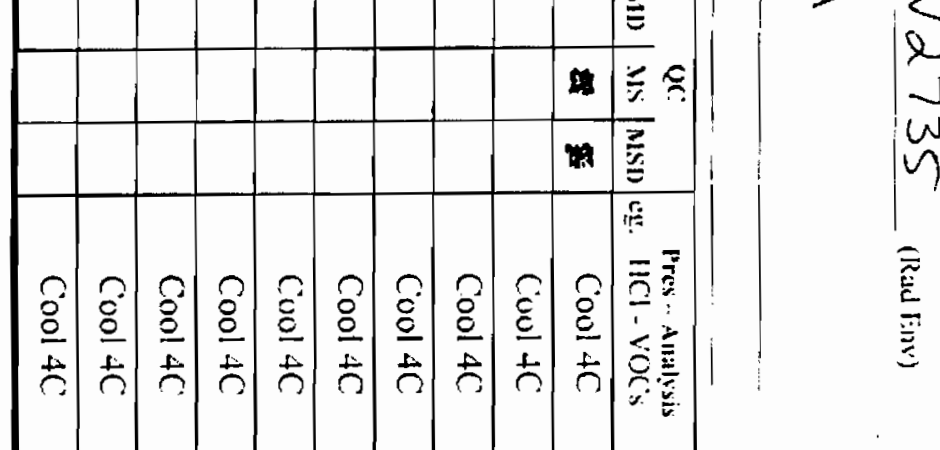

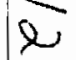

w

w

$x$

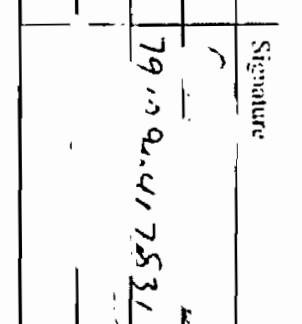

$$
\times \times \times \times \times \times \times \times 150 \mathrm{U} .
$$

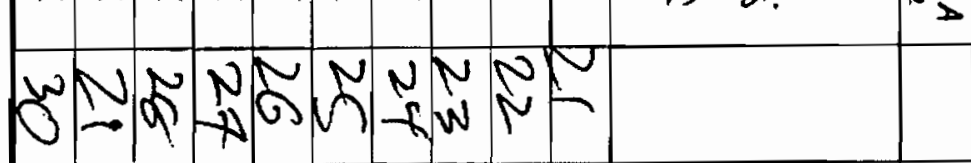

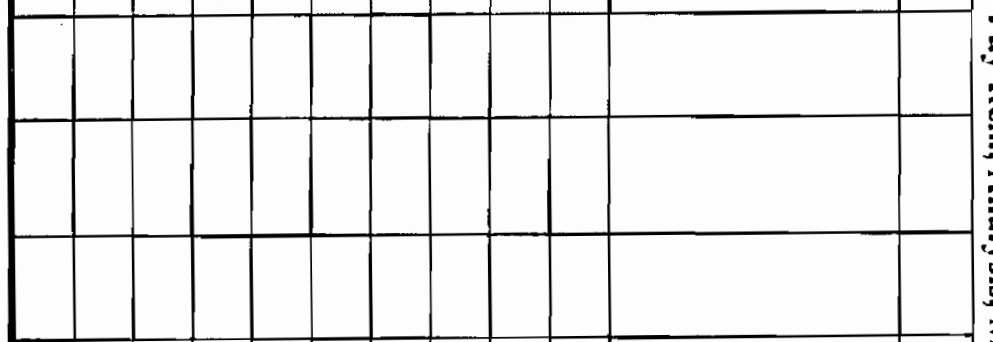

$a \sqrt{2} x^{2}$

远

की 

Q $\rightarrow$

$$
\text { : }
$$

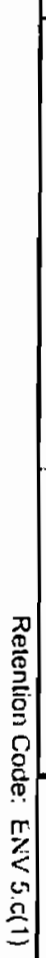

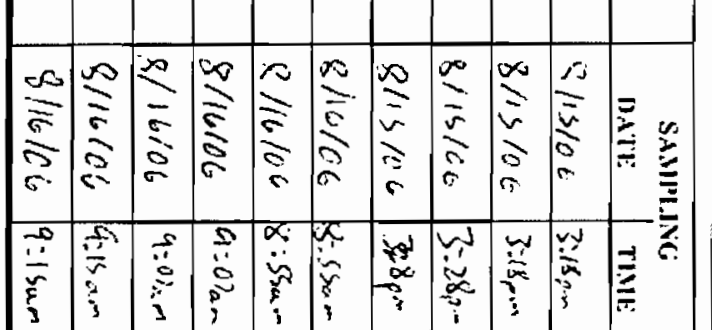

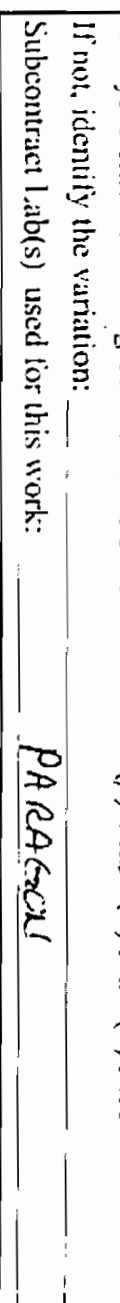

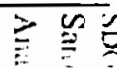

त

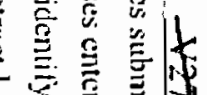

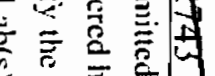

产亭离

音嵒

北要

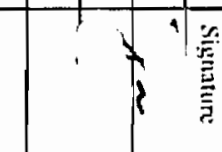

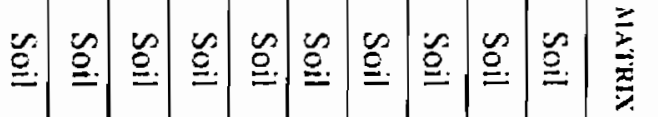

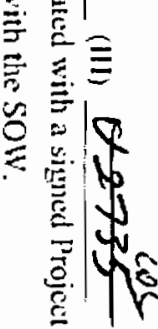

$\varangle \stackrel{2}{2}$

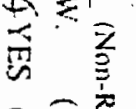

$\infty$ i \&

实类

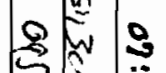

$\stackrel{\ddot{0}}{\ddot{*}}$

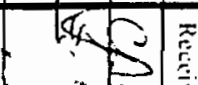

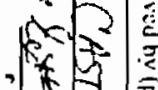

这

$\therefore 8$

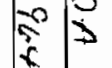

$\leqslant$

in

$\therefore$

$\because$

$\sqrt{\infty}$

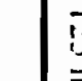

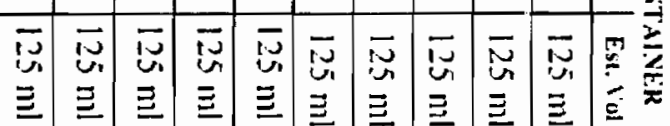

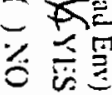

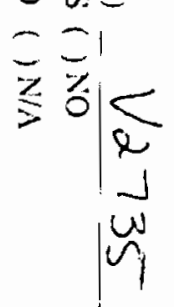

x内 $\frac{\frac{2}{5}}{2}$

\&)

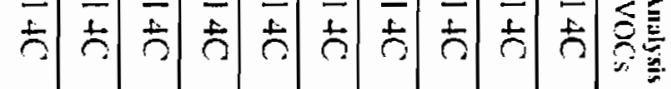

彥

i.

$\times \times \times \times \times \times \times \times \times \times 150.4$

$2 \frac{1}{3}$

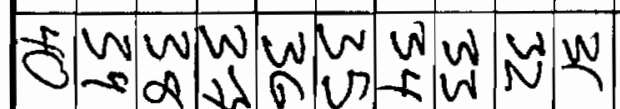

$\infty \times \frac{\infty}{\infty} \equiv$

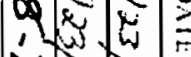

SE

率

a 0

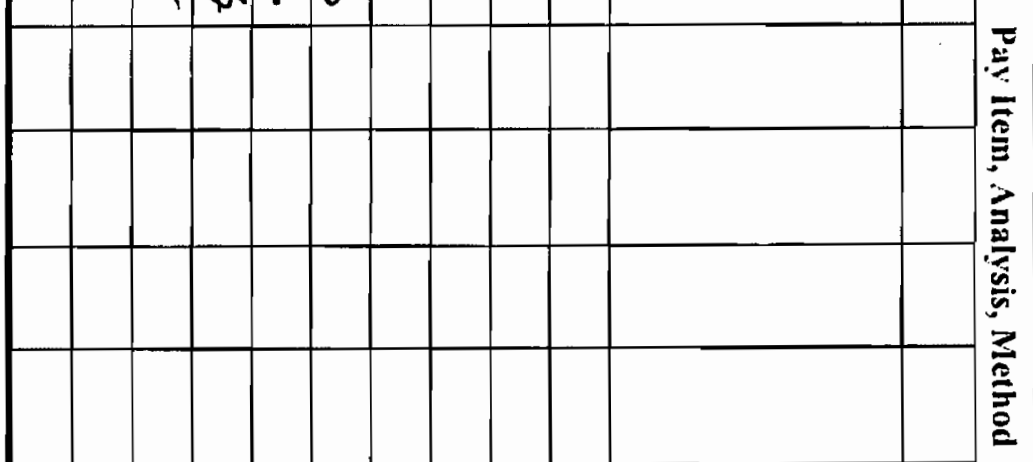

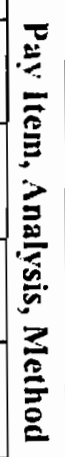

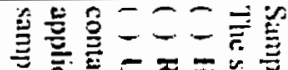

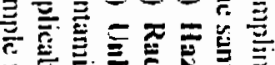

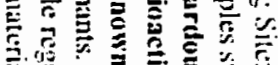

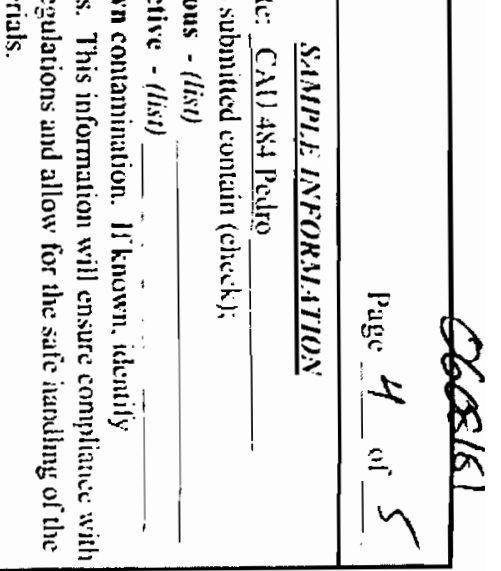




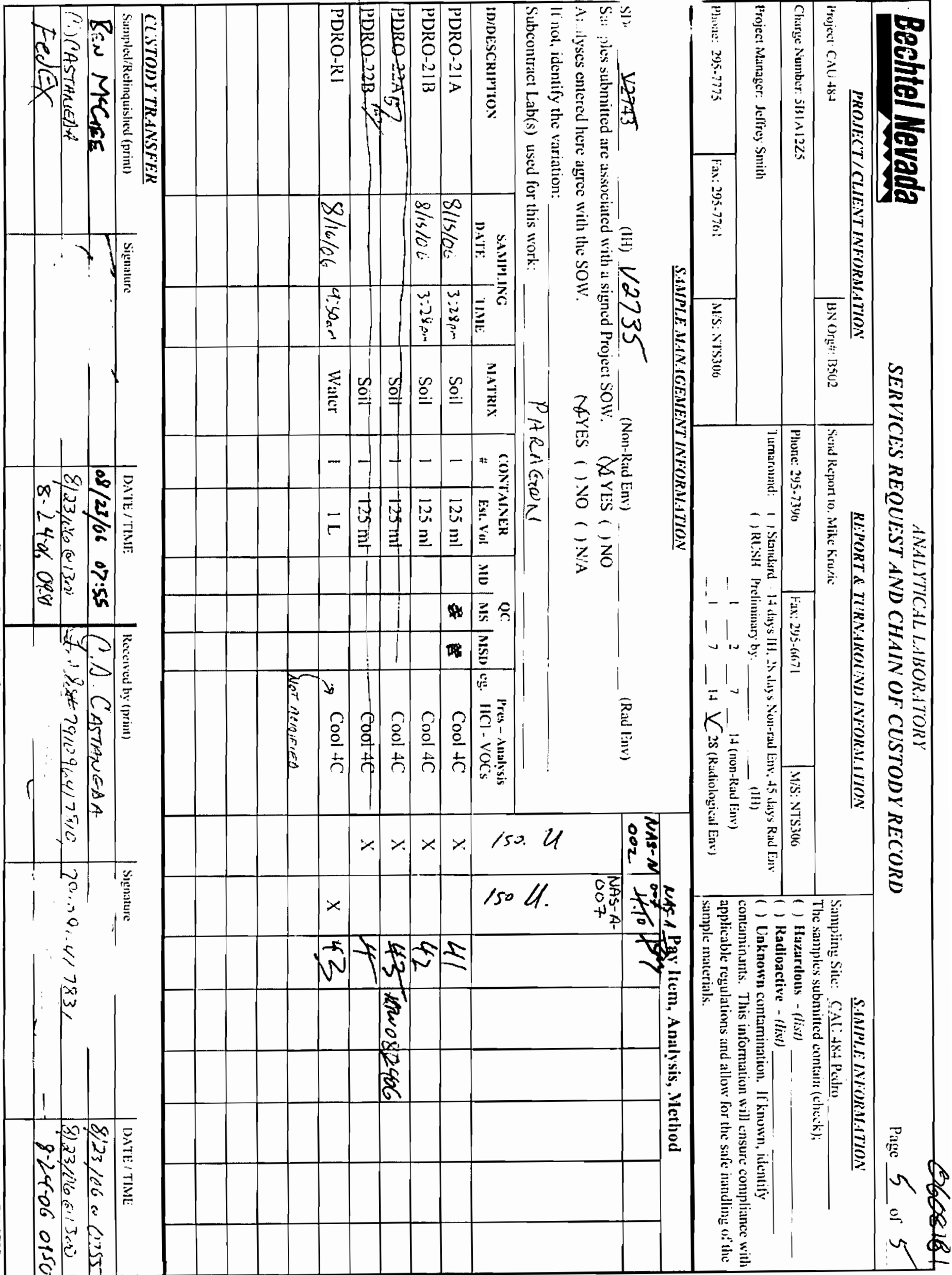


Lab Name: LIONVILLE_LABORATORY

Lab Code: LVLI

Matrix (soil/water): SoIL

Level (low/med): LOW

음 Solids :
Contract: 60052

SAS NO.:

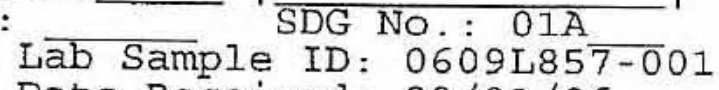

$-94 \overline{.7}$
$01 \mathrm{~A}$

Date Received: 09/01/06

Concentration Units (ug/L or $\mathrm{mg} / \mathrm{kg}$ dry weight): MG/KG

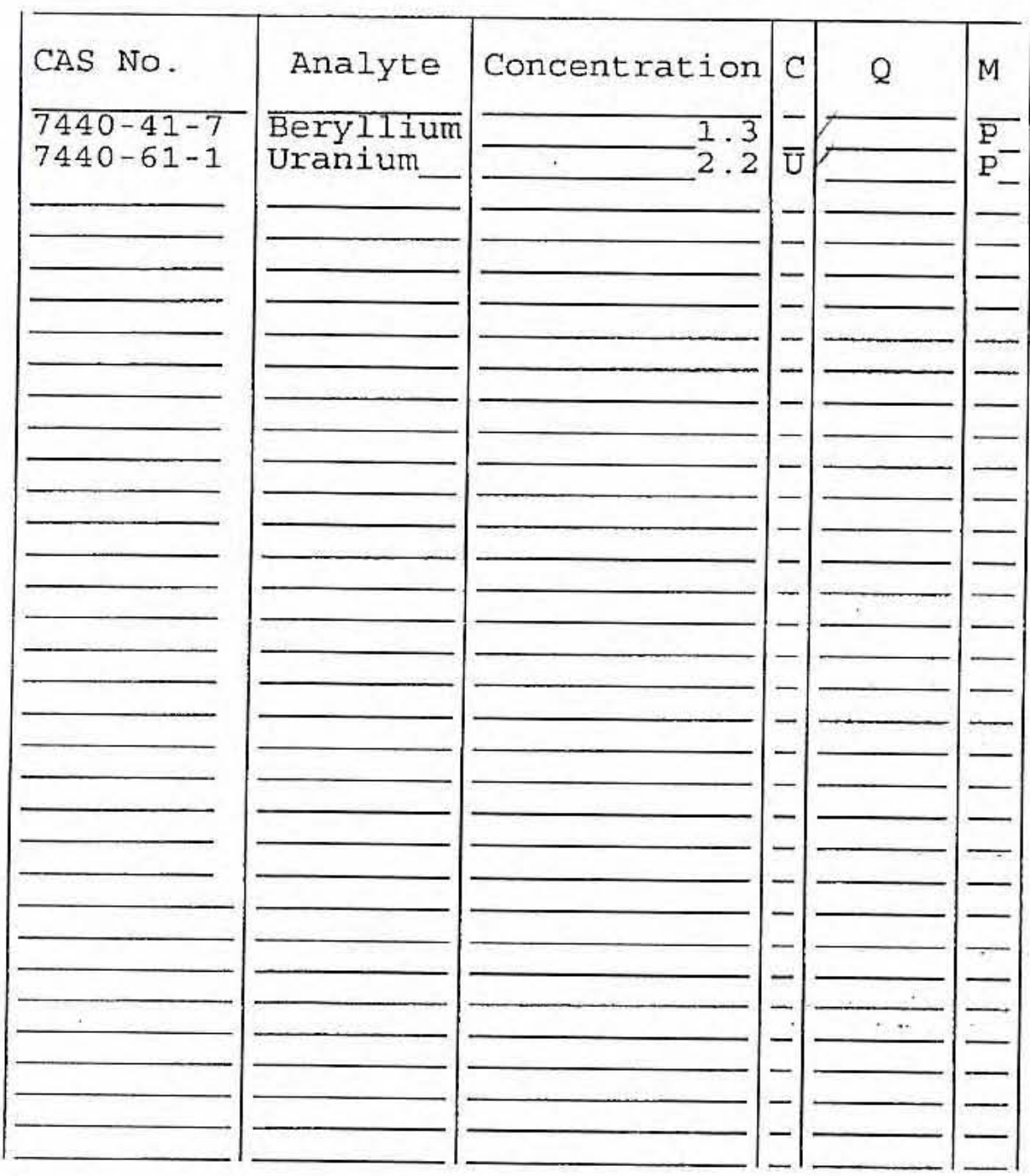

Color Before: Color After:

\section{Comments :}

NEDS-01A
Clarity Before:

Clarity After:
Texture:

Artifacts: 


\title{
INVESTIGATION (GEOPROBE ${ }^{\circledR}$ ) SOIL SAMPLE ANALYTICAL RESULTS
}

CAS TA-52-001-TANL NEDS DETONATION AREA

\author{
Chemical Data
}


1

INORGANIC ANALYSES DATA SHEET
Lab Name: LIONVILLE LABORATORY

Lab Code: LVLI Case No.: NEDS

Level (low/med): $\div$ Solids :

$$
\begin{aligned}
& \text { SOIL } \\
& \text { LOW - } \\
& \text { - } 90 . \overline{6}
\end{aligned}
$$

Contract: 60052 SAS NO. :
EPA SAMPLE NO.

$01 B$

SDG NO.: $01 \mathrm{~A}$

Lab Sample ID: 0609L857-002

Date Received: 09/01/06

Concentration Units (ug/L or $\mathrm{mg} / \mathrm{kg}$ dry weight): MG/KG

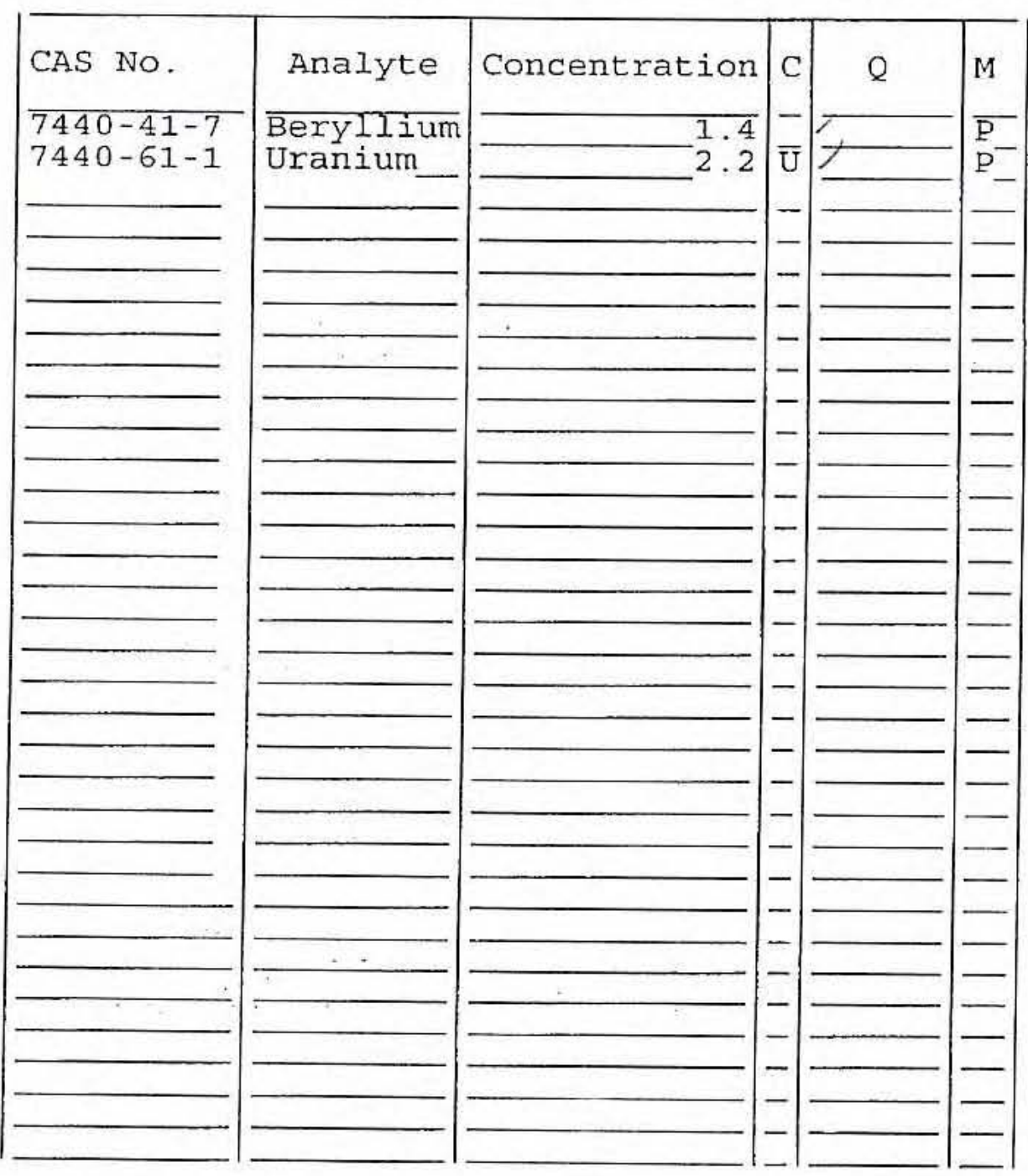

Color Before: Color After: Comments :
Clarity Before:

Clarity After:
Texture:

Artifacts : 


\section{INORGANIC ANALYSES DATA SHEET}

Iab Name: LIONVILLE LABORATORY Lab Code: LVLI Matrix (soil/water): SoIL Level (low/med): LOW $\%$ Solids:
EPA SAMPLE NO.

\section{$02 \mathrm{~A}$}

SDG NO.: 01A

SAS NO.:

0052

Lab Sample ID: 0609L857-003

Date Received: 09/01/06

Concentration Units (ug/L or $\mathrm{mg} / \mathrm{kg}$ dry weight) : MG/KG

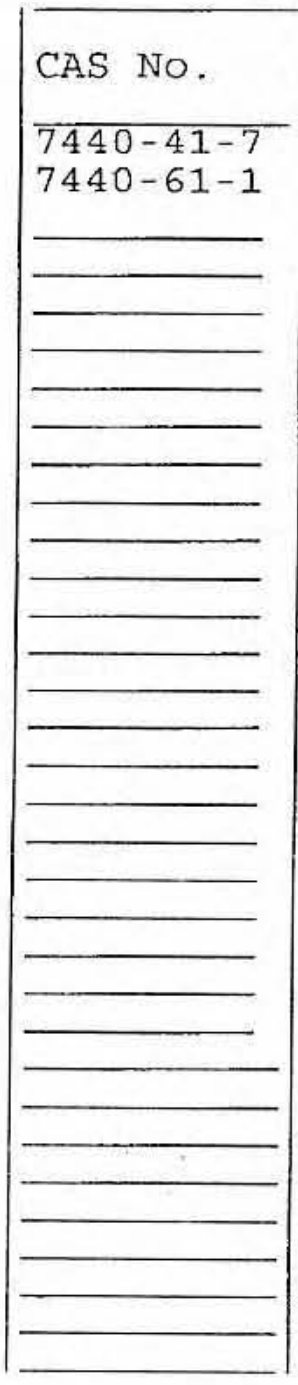

Color Before: Color After:

Comments :

NEDS-02A

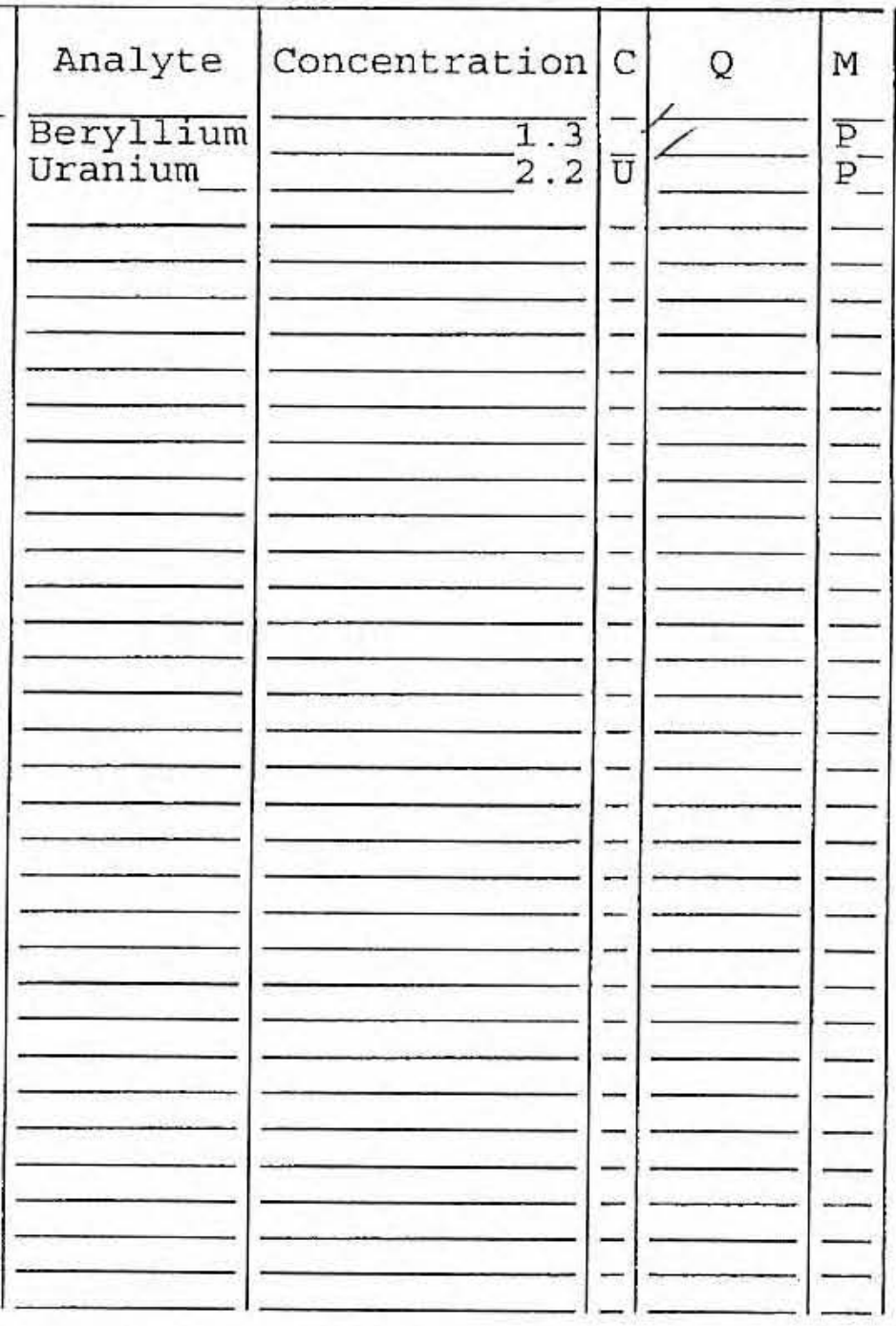

Clarity Before:

Clarity After:
Texture:

Artifacts : 
U.S. EPA

\section{INORGANIC ANALYSES DATA SHEET}

EPA SAMPLE NO.

Lab Name: LIONVILLE LABORATORY

Lab Code: LVLI Matrix (soil/water) : SOIL Level (low/med): LOW $\%$ Solids:

$$
-88 \overline{.2}
$$

\section{$02 \mathrm{~B}$}

Contract: 60052

SAS NO.:

Iab Sample ID: 0609L857-004

Date Received: 09/01/06

Concentration Units (ug/L or $\mathrm{mg} / \mathrm{kg}$ dry weight): MG/KG
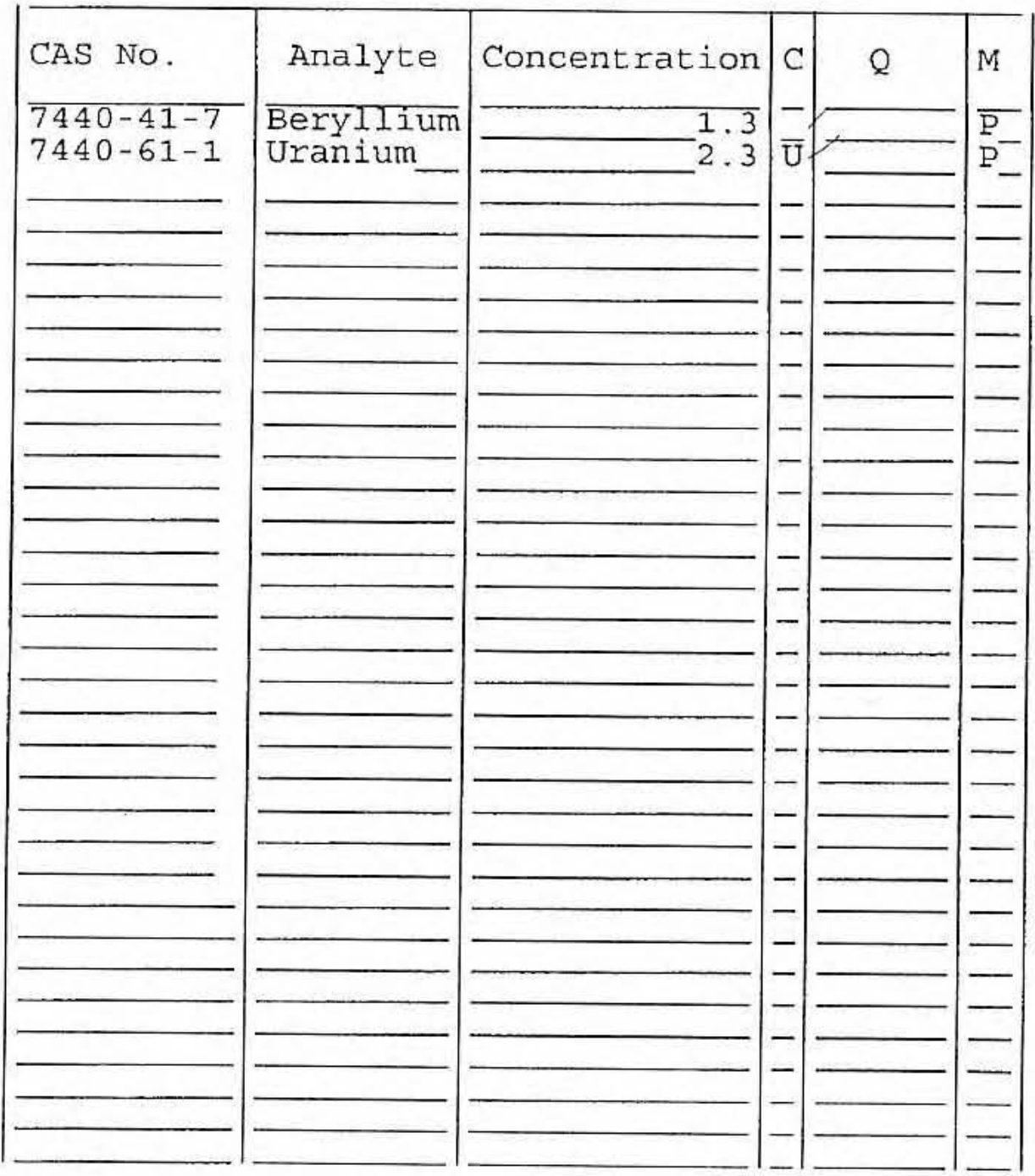

Color Before:

Color After:

Clarity Before:

Clarity After:

Texture:

Artifacts :

Comments:

NEDS - 02B 
EPA SAMPLE NO.

$$
\text { 03A }
$$

Lab Name: LIONVILLE LABORATORY Lab Code: LVLI Matrix (soil/water): SoIL Level (low/med): LOW \% Solids:

Contract: 60052

SAS NO. :

Lab Sample ID: 0609L85 $\overline{7-0} 05$

Date Received: 09/01/06

Concentration Units (ug/L or $\mathrm{mg} / \mathrm{kg}$ dry weight) : MG/KG

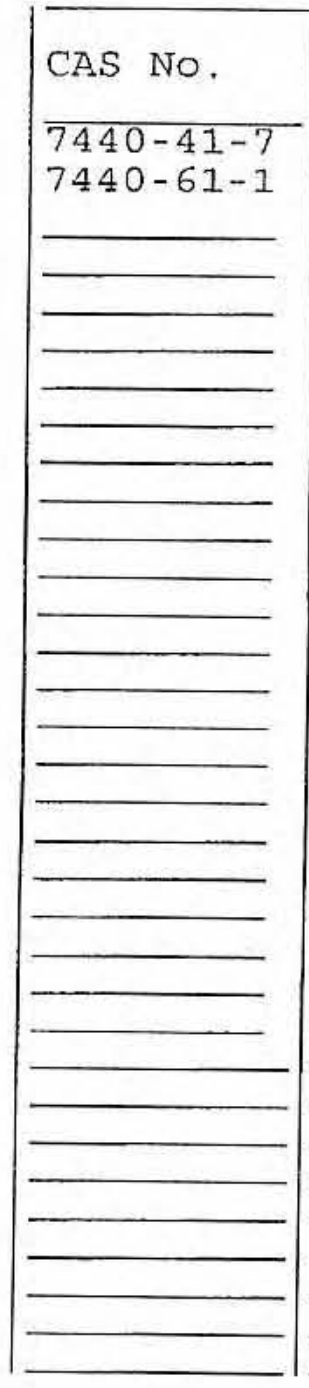

Color Before: Color After: Comments :

NEDS - 03A

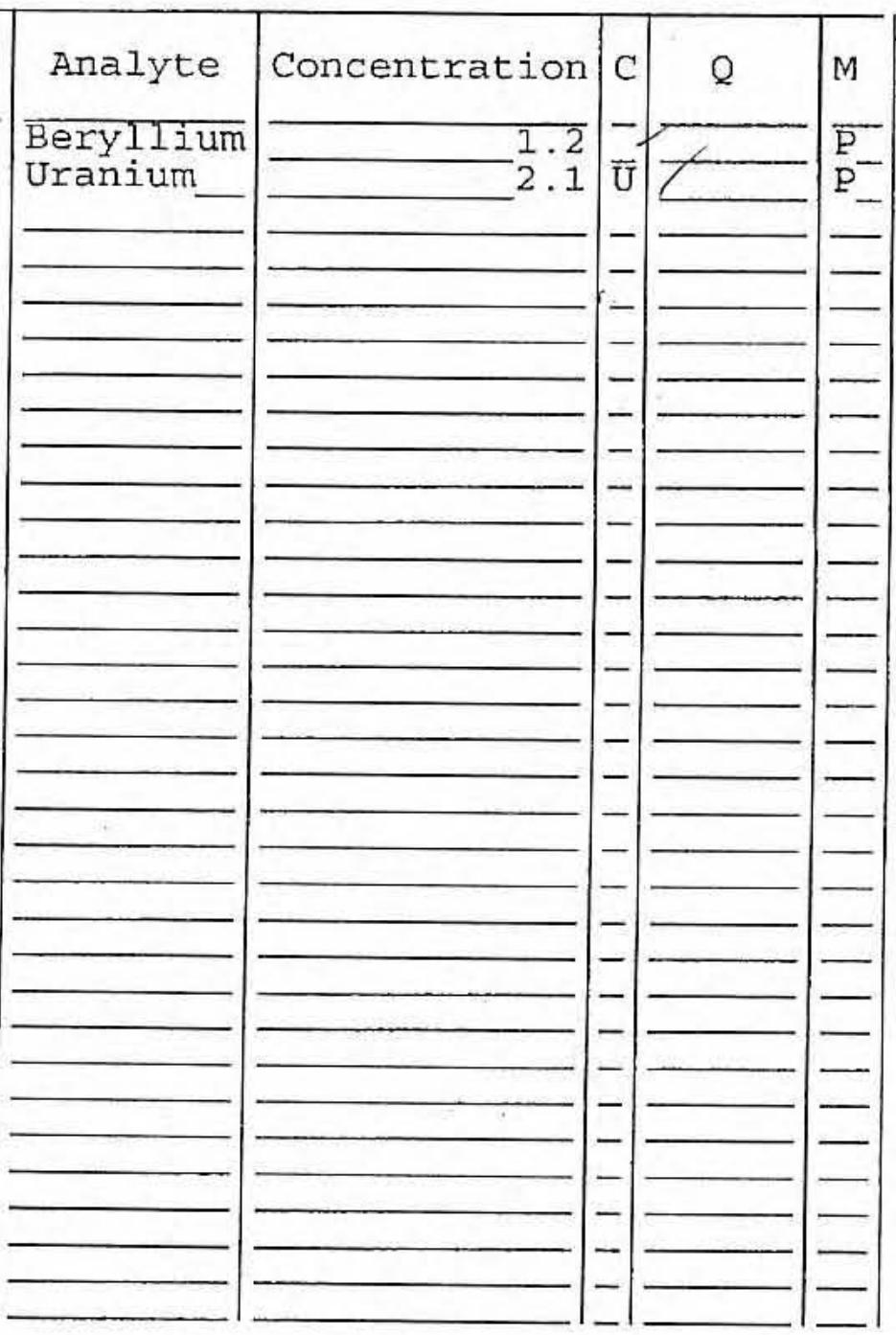

Clarity Before: Clarity After:
Texture: Artifacts:

\section{FORM I - IN}


U.S. EPA

1

INORGANIC ANALYSES DATA SHEET
EPA SAMPLE NO.

03B

Contract: 60052

SAS No.:

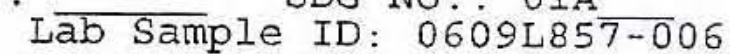

Date Received: 09/01/06
Lab Name: LIONVILLE_LABORATORY

Lab Code: LVLI

Matrix (soil/water): SOIL

Level (low $/ \mathrm{med}$ ) :

solids:
LOW

$-91 \overline{.9}$

Concentration Units (ug/L or $\mathrm{mg} / \mathrm{kg}$ dry weight): MG/KG

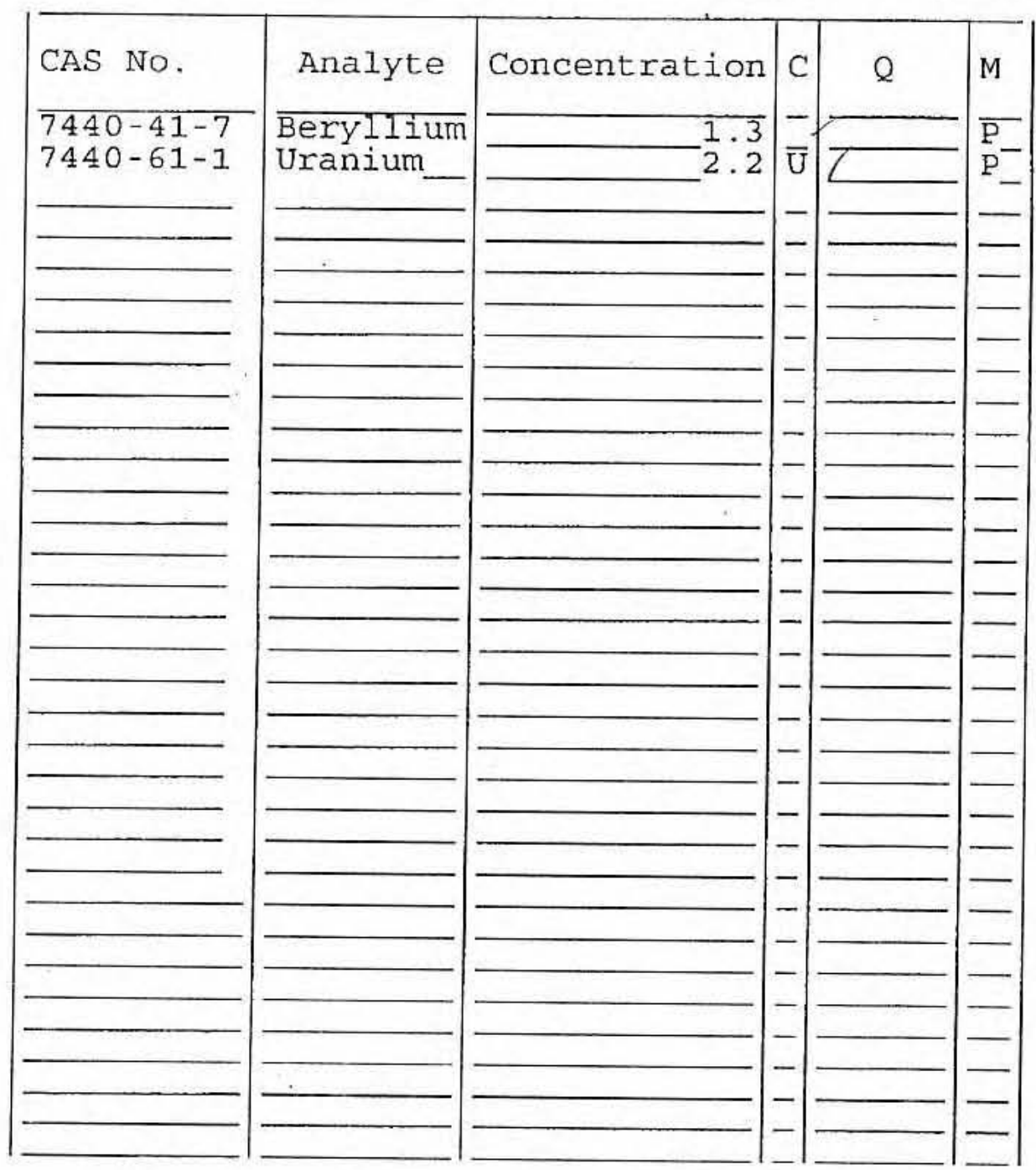

Color Before: Color After:

Comments :

NEDS-03B
Clarity Before:

Clarity After:
Texture:

Artifacts: 
U.S. EPA

INORGANIC ANAIYYSES DATA SHEET

EPA SAMPLE NO.

Lab Name: LIONVILLE_LABORATORY

Lab Code: LVLI

Matrix (soil/water): SOIL

Level (low/med): LOW

$\div$ Solids:

$$
-93 . \overline{6}
$$

$04 \mathrm{~A}$

Contract: 60052

SAS NO.:

SDG NO.: 01A

Lab Sample ID: 0609L857-007

Date Received: 09/01/06

Concentration Units (ug/L or $\mathrm{mg} / \mathrm{kg}$ dry weight): $\mathrm{MG} / \mathrm{KG}$

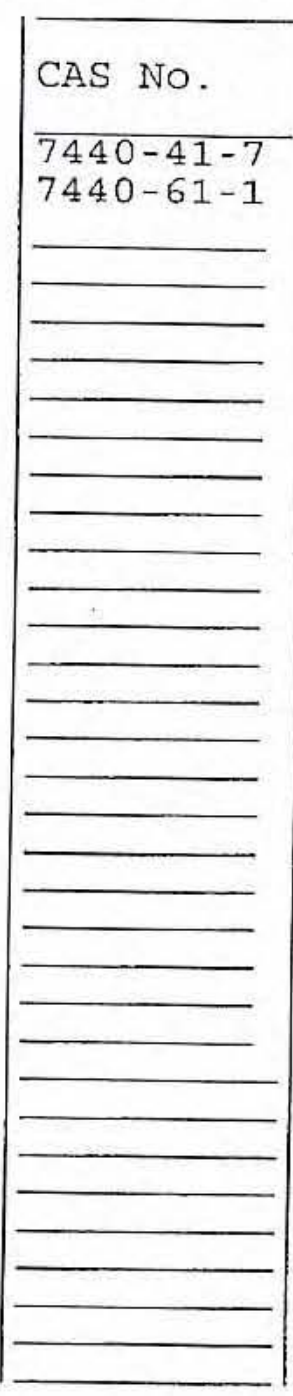

Color Before:

Color After:

Comments :

NEDS - 04A

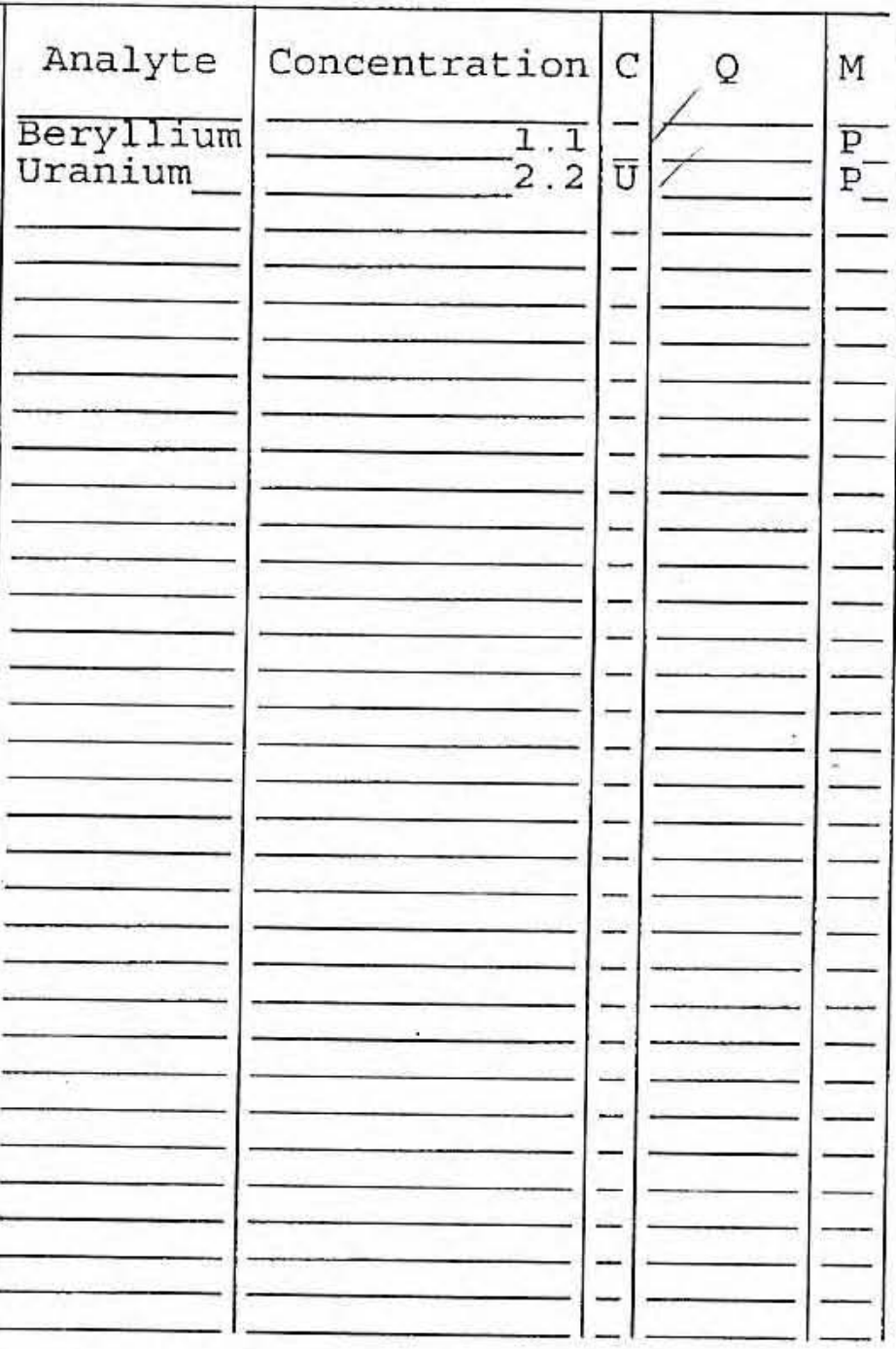

Clarity Before:

Clarity After:
Texture: Artifacts : 
Lab Name: LIONVILLE_LABORATORY

Lab Code: LVLI

Matrix (soil/water): SOIL

Level (low/med):

\% Solids:

LOW

$90 \overline{.4}$
Contract: 60052
SAS NO.:

Lab Sample ID: 06091857-008

Date Received: 09/01/06

Concentration Units (ug/L or $\mathrm{mg} / \mathrm{kg}$ dry weight): MG/KG

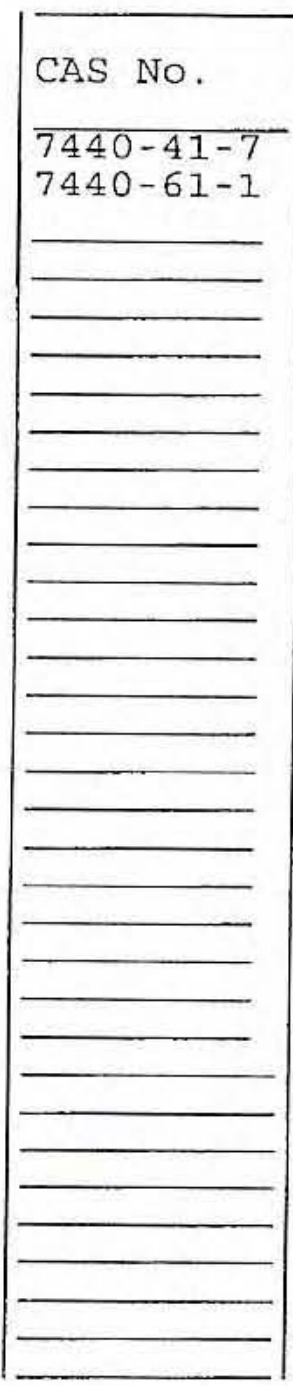

Color Before: Color After:

Comments :

NEDS - OAB

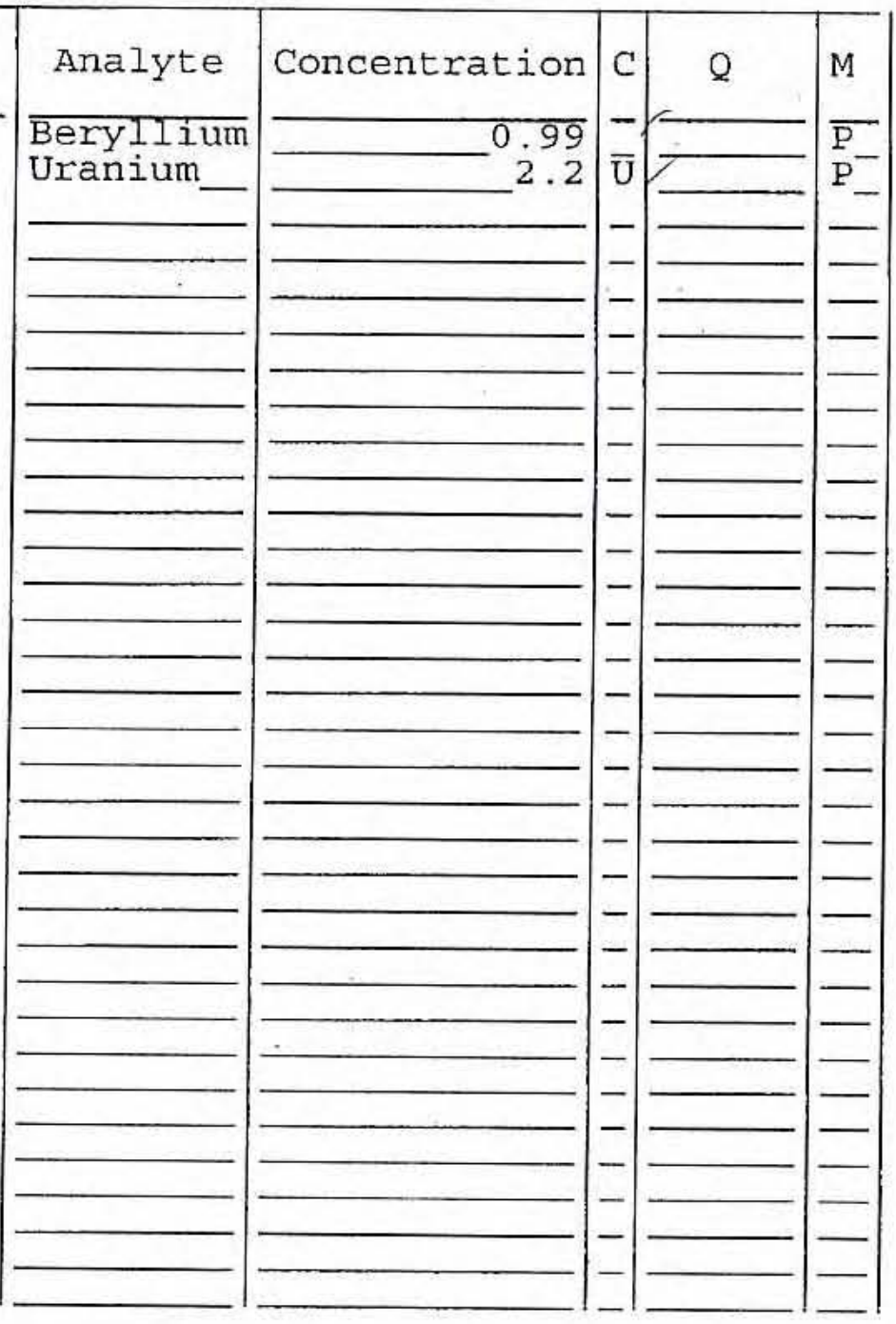

Clarity Before:

Clarity After:
Texture: Artifacts : 


\section{U.S. EPA}

1 INORGANIC ANALYSES DATA SHEET
EPA SAMPLE NO.

\section{$05 \mathrm{~A}$}

Lab Name: LIONVILLE_LABORATORY

Lab Code: IVLI

Matrix (soil/water): SOIL

Level (low/med) :

$\div$ Solids:
Contract: 60052

SAS NO.:

SDG NO.: 01A

$$
\text { LOW - }
$$

Lab Sample ID: 0609L85 $\overline{7-0} 09$

Date Received: 09/01/06

Concentration Units (ug/L or $\mathrm{mg} / \mathrm{kg}$ dry weight): MG/KG

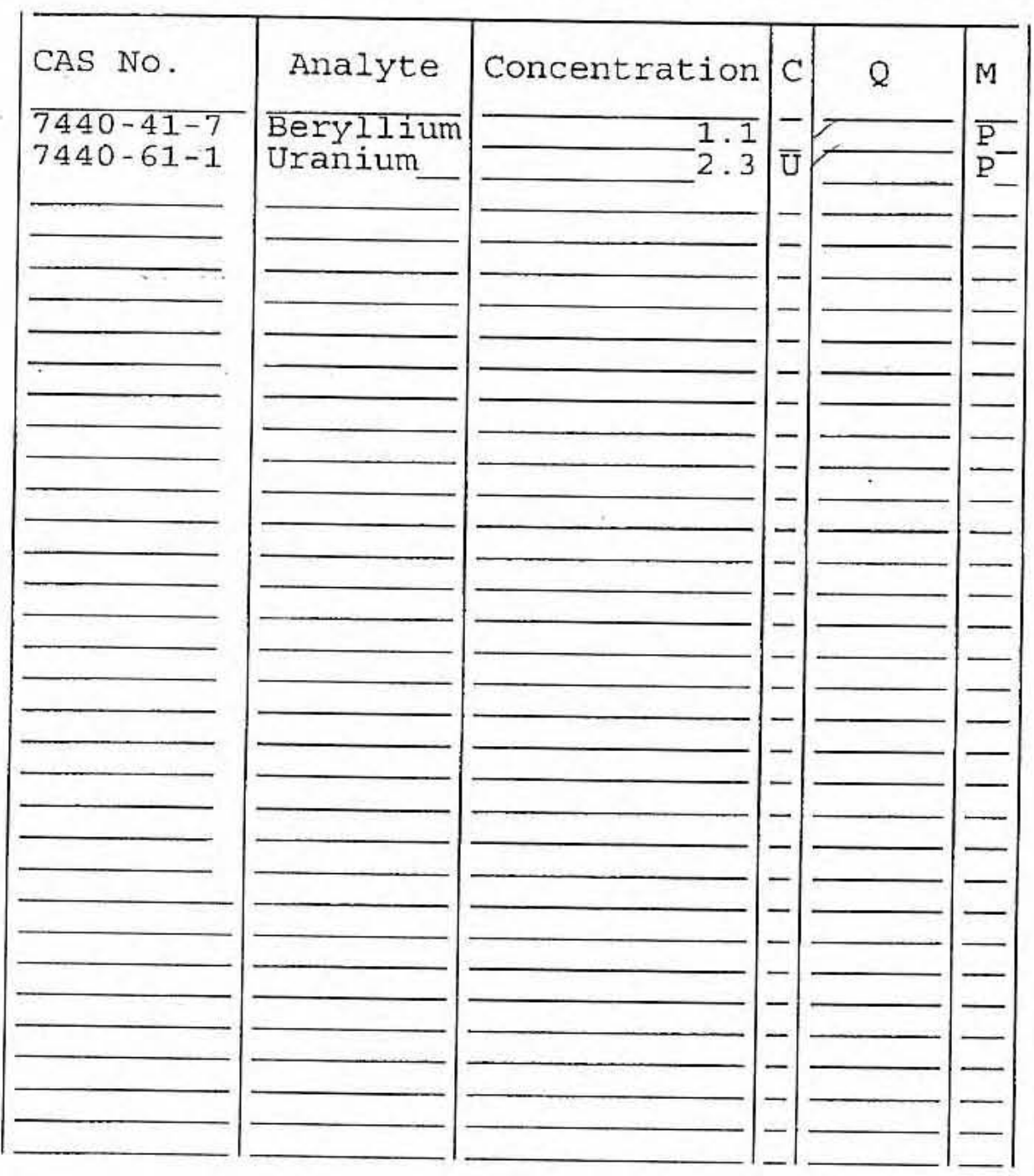

Color Before: Color After:

Comments :

NEDS - 05A
Clarity Before:

Clarity After:
Texture:

Artifacts: 


\section{U.S. EPA}

1 INORGANIC ANALYSES DATA SHEET
EPA SAMPLE NO.

05B

Contract: 60052

SAS NO.:

SDG NO.: 01A

Lab Sample ID: 0609L857-010

Date Received: 09/01/06
Lab Name: LIONVILLE_LABORATORY

Matrix (soil/water): SOIL

Level. (low/med):

$\div$ Solids:

$$
\begin{array}{r}
\text { LOW } \\
-85.8
\end{array}
$$

Concentration Units (ug/L or $\mathrm{mg} / \mathrm{kg}$ dry weight): MG/KG

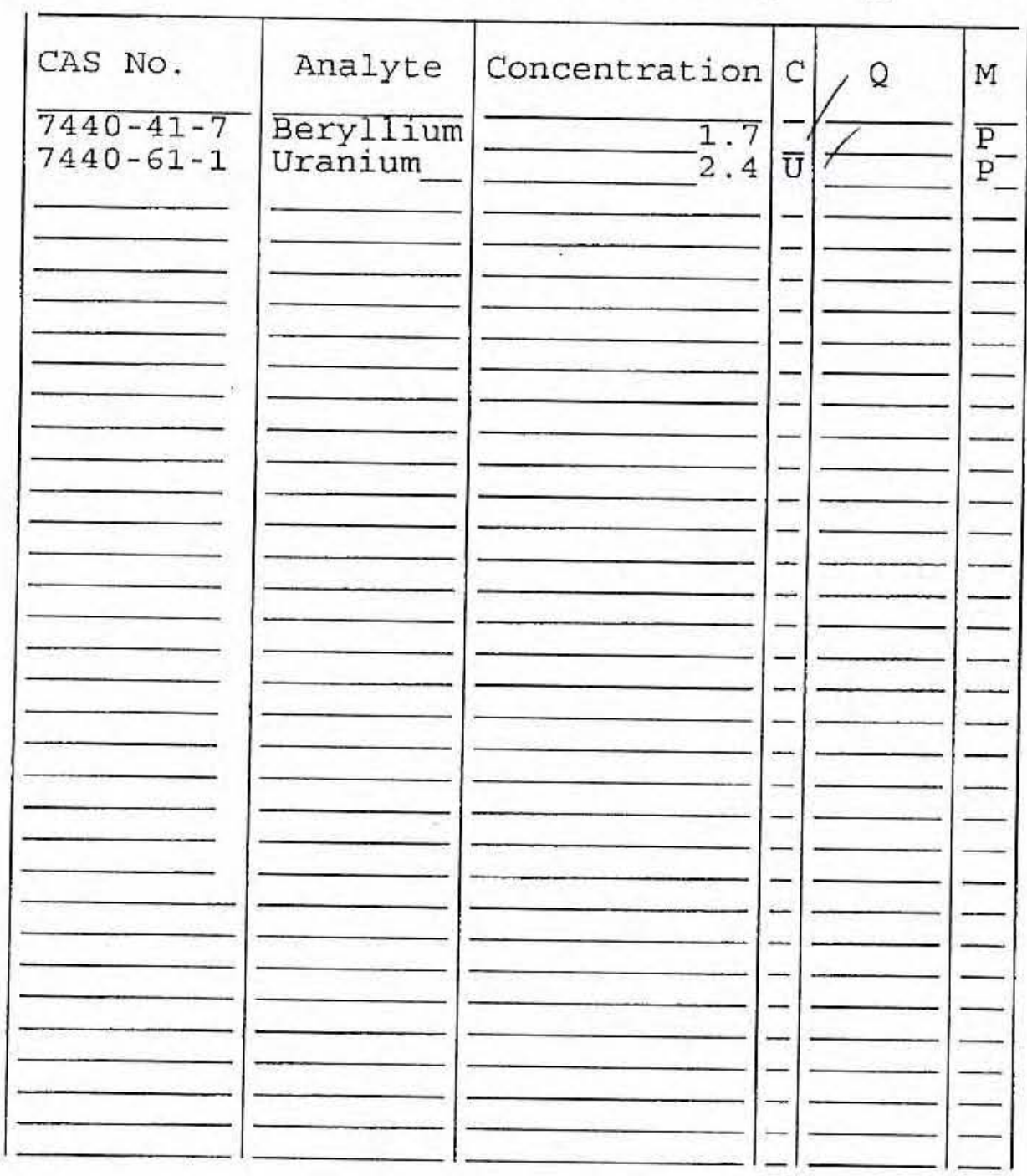

Clarity Before: Clarity After:
Texture:

Artifacts:

Comments :

NEDS - 05B 


\section{U.S. EPA}

\section{INORGANIC ANALYSES DATA SHEET}

EPA SAMPLE NO.

Lab Name: LIONVILLE_LABORATORY

Contract: 60052

\section{A}

Lab Code: LVLI

Matrix (soil/water): SoIL

Level (low/med) :

\% Solids :

LOW
-95.4

SAS No.:

Lab Sample ID: 0609L85 $\overline{7-0} 11$

Date Received: 09/01/06

Concentration Units (ug/L or $\mathrm{mg} / \mathrm{kg}$ dry weight): $\mathrm{MG} / \mathrm{KG}$

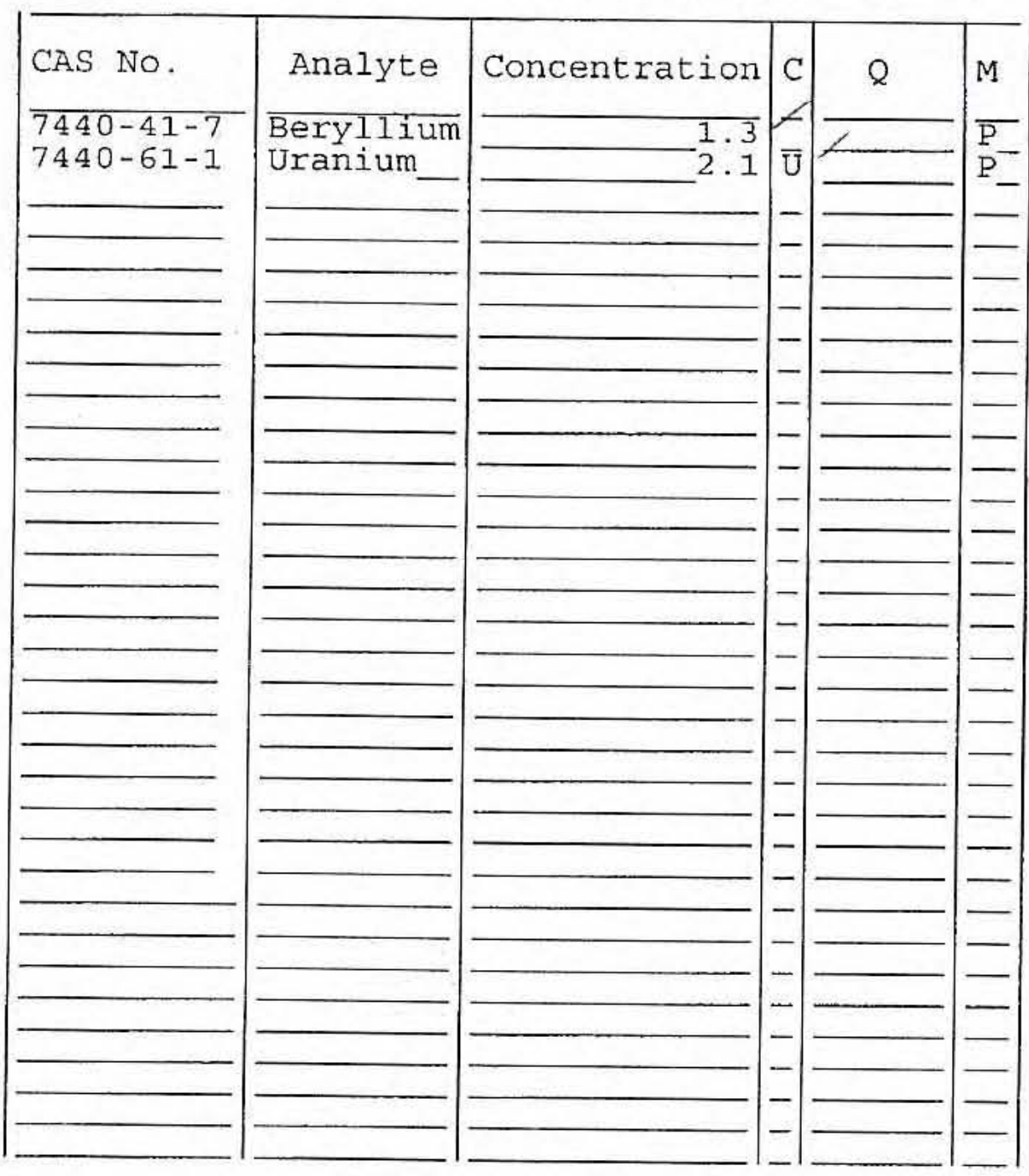

Color Before:

Color After:

Comments :

NEDS-06A
Clarity Before:

Clarity After:
Texture:

Artifacts : 
EPA SAMPLE NO.

$$
06 B
$$

Lab Name: LIONVILLE_IABORATORY

Lab Code: LVLI Matrix (soil/water): SoIL

Level (low/med):

: Solids:
Contract: 60052

SAS No.:

Lab Sample ID: 0609L85 $\overline{7-0} 12$

Date Received: 09/01/06

Concentration Units (ug/L or $\mathrm{mg} / \mathrm{kg}$ dry weight): MG/KG

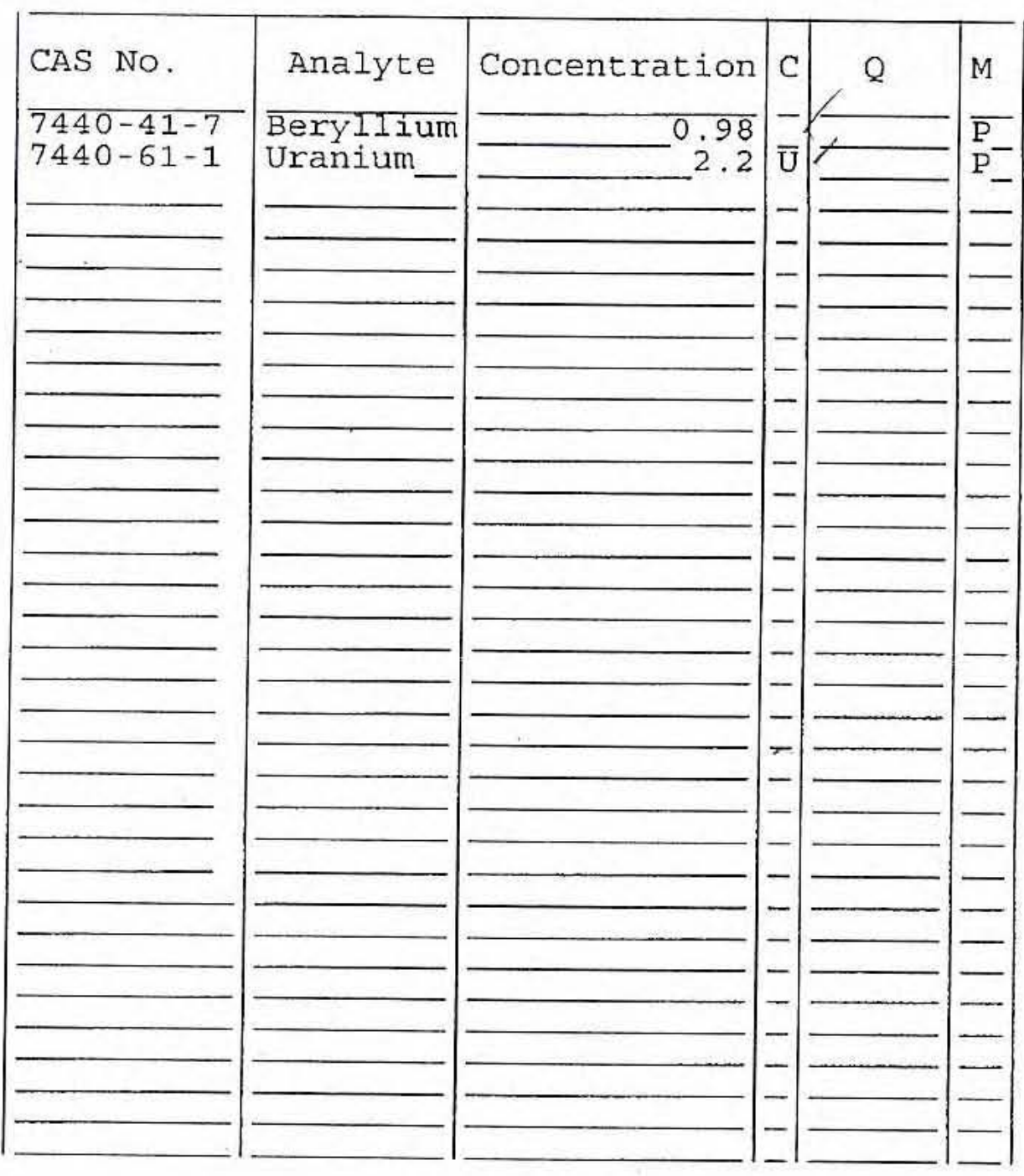

Color Before:

Color After:

Comments :

NEDS-06B
Clarity Before:

Clarity After:
Texture:

Artifacts: 
U.S. EPA

1

INORGANIC ANALYSES DATA SHEET
EPA SAMPLE NO.

$$
07 \mathrm{~A}
$$

Lab Name: LIONVILLE_LABORATORY

Lab Code: LVLI

Matrix (soil/water):

Level (low/med) :

$\div$ Solids:

Contract: 60052

SAS NO.:
Case No.: NEDS

$$
\begin{aligned}
& \text { SOIL } \\
& \text { LOW- } \\
& \text { - } 93 . \overline{3}
\end{aligned}
$$

SDG No : $01 \mathrm{~A}$

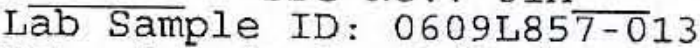

Date Received: 09/01/06

Concentration Units (ug/L or $\mathrm{mg} / \mathrm{kg}$ dry weight): MG/KG

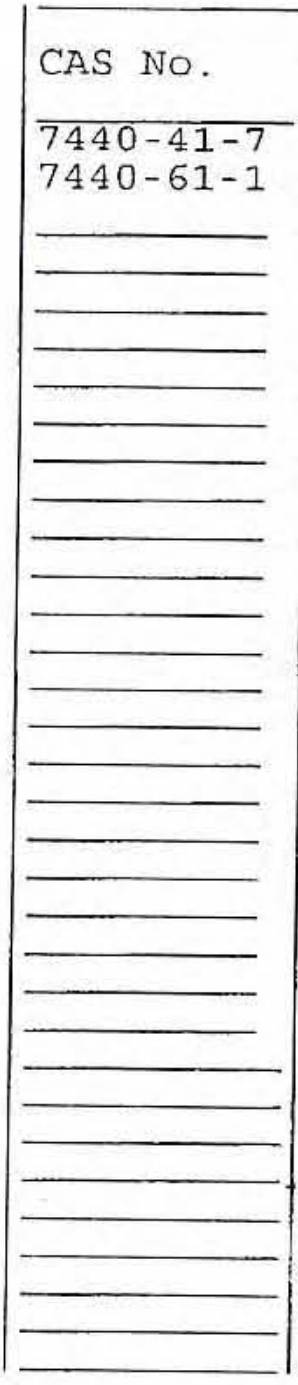

Color Before:

Color After:

Comments:

NEDS - 07A

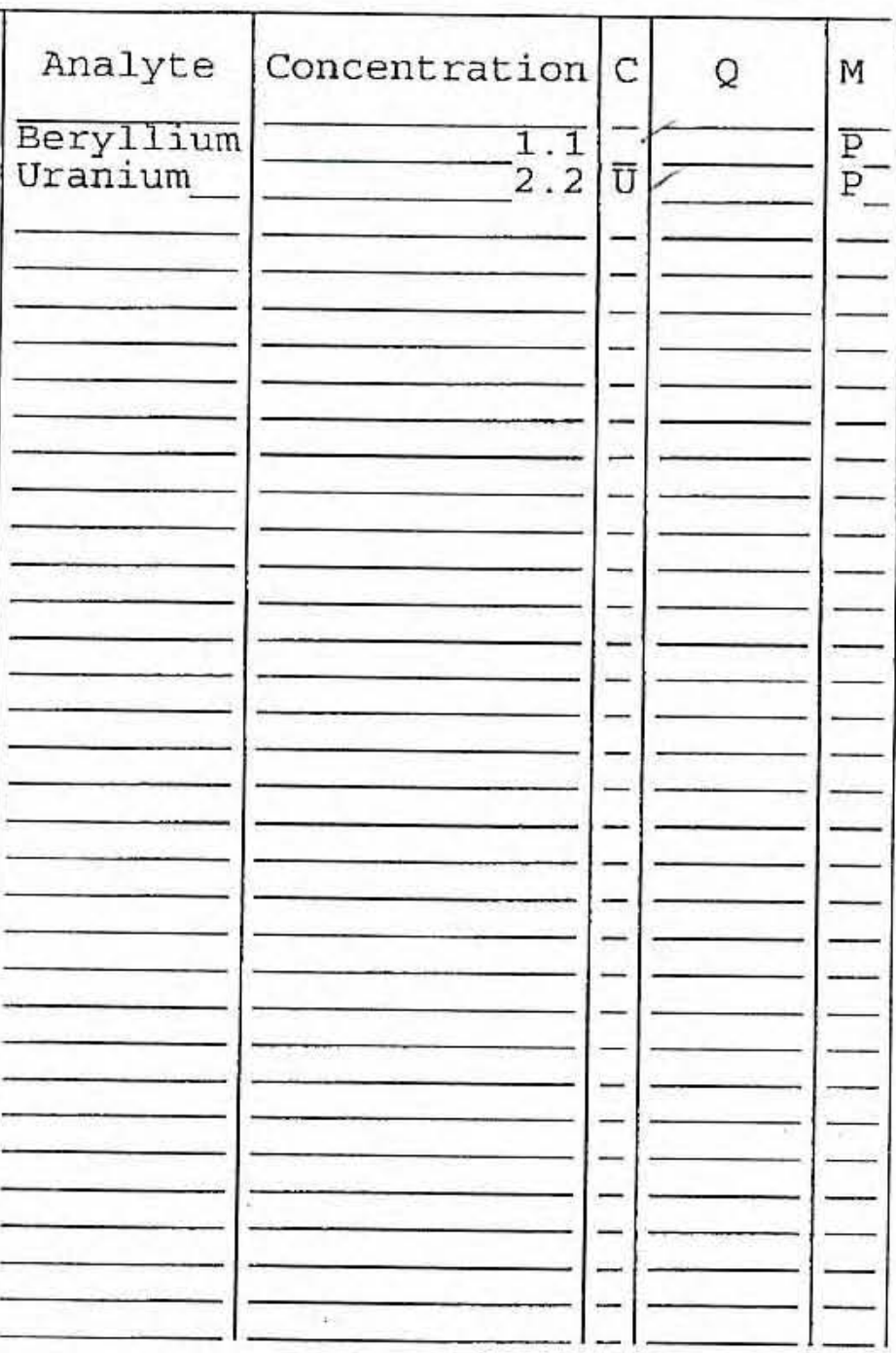

Clarity Before:

Clarity After:
Texture: Artifacts: 


\section{U.S. EPA}

1

INORGANIC ANALYSES DATA SHEET
EPA SAMPLE NO.

$$
07 \mathrm{~B}
$$

Lab Name: LIONVIILE LABORATORY

Contract: 60052

Case No.: NEDS_ SAS No.:

Matrix (soil/water): SOIL

Level (low/med):

: Solids:

LOW

Lab Sample ID: 0609L857-014

Date Received: 09/01/06

Concentration Units (ug/L or $\mathrm{mg} / \mathrm{kg}$ dry weight) : MG/KG
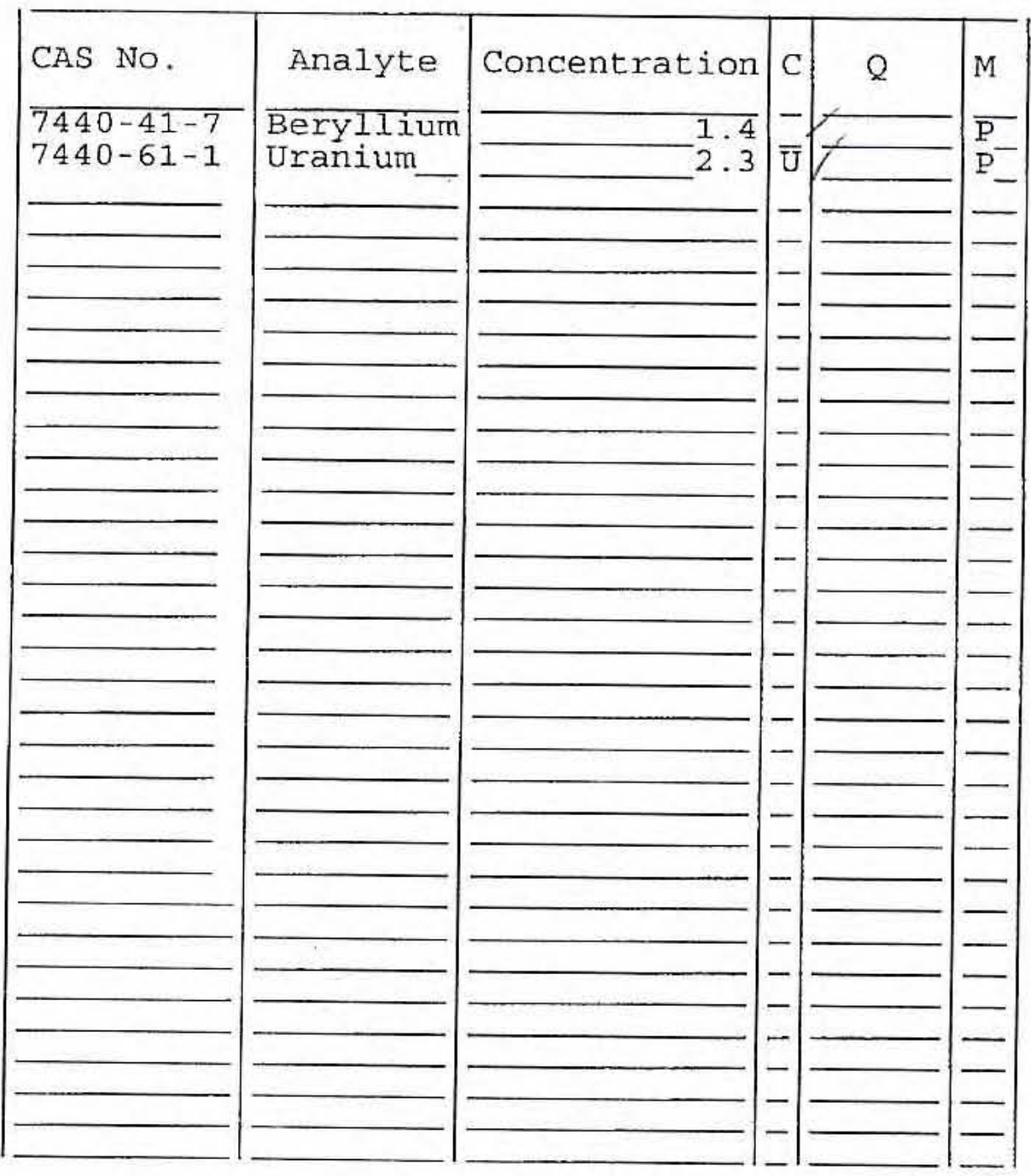

Color Before:

Clarity Before:

Clarity After:

Texture:

Artifacts :

\section{Comments :}

NEDS - $07 B$ 
EPA SAMPLE NO.

08A

Lab Name: LIONVILLE_LABORATORY

Lab Code: LVLI

Matrix (soil/water): SoIL

Level (low $/ \mathrm{med})$ : LOW

$\div$ Solids:
Contract: 60052

SAS NO.:

SDG NO.: $01 \mathrm{~A}$

Lab Sample ID: 0609L85 $\overline{7-0} 15$

Date Received: 09/01/06

Concentration Units (ug/L or $\mathrm{mg} / \mathrm{kg}$ dry weight): MG/KG

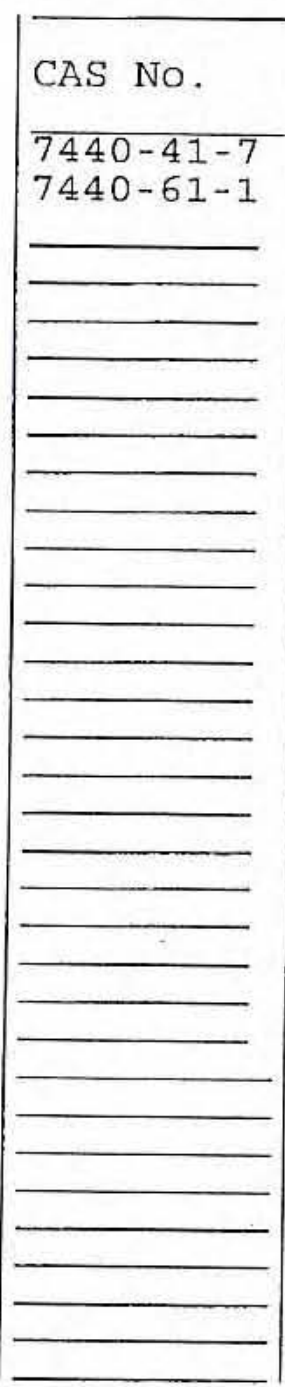

Color Before:

Color After:

Comments :

NEDS - 08A

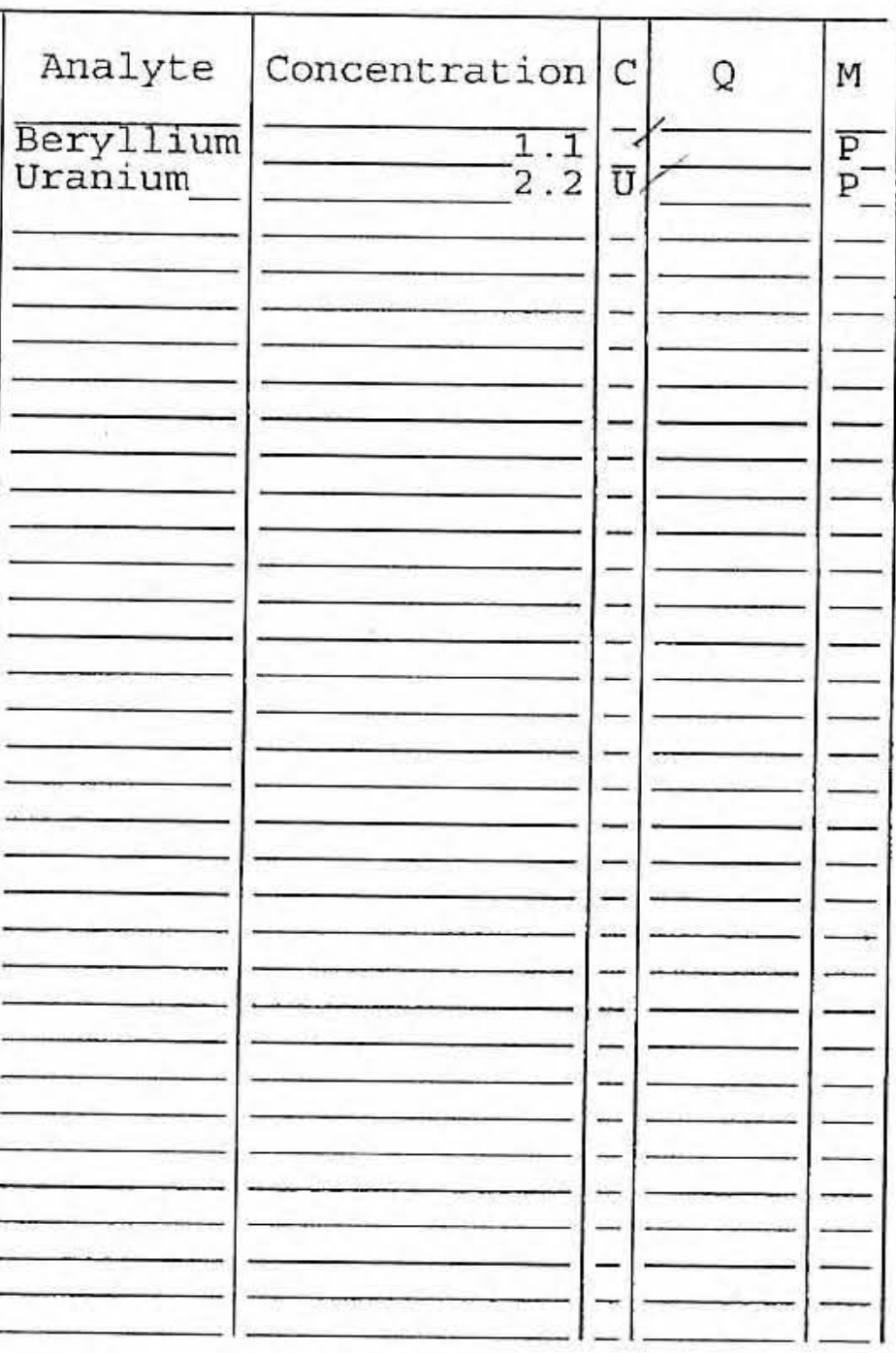

Clarity Before:

Clarity After:
Texture:

Artifacts : 


\section{INORGANIC ANALYSES DATA SHEET}

Lab Name: LIONVILLE LABORATORY

Lab Code: LVLI

Matrix (soil/water): SOIL

Level (low/med): LOW

$\div$ Solids:

Concentration Units (ug/L or $\mathrm{mg} / \mathrm{kg}$ dry weight) : MG/KG

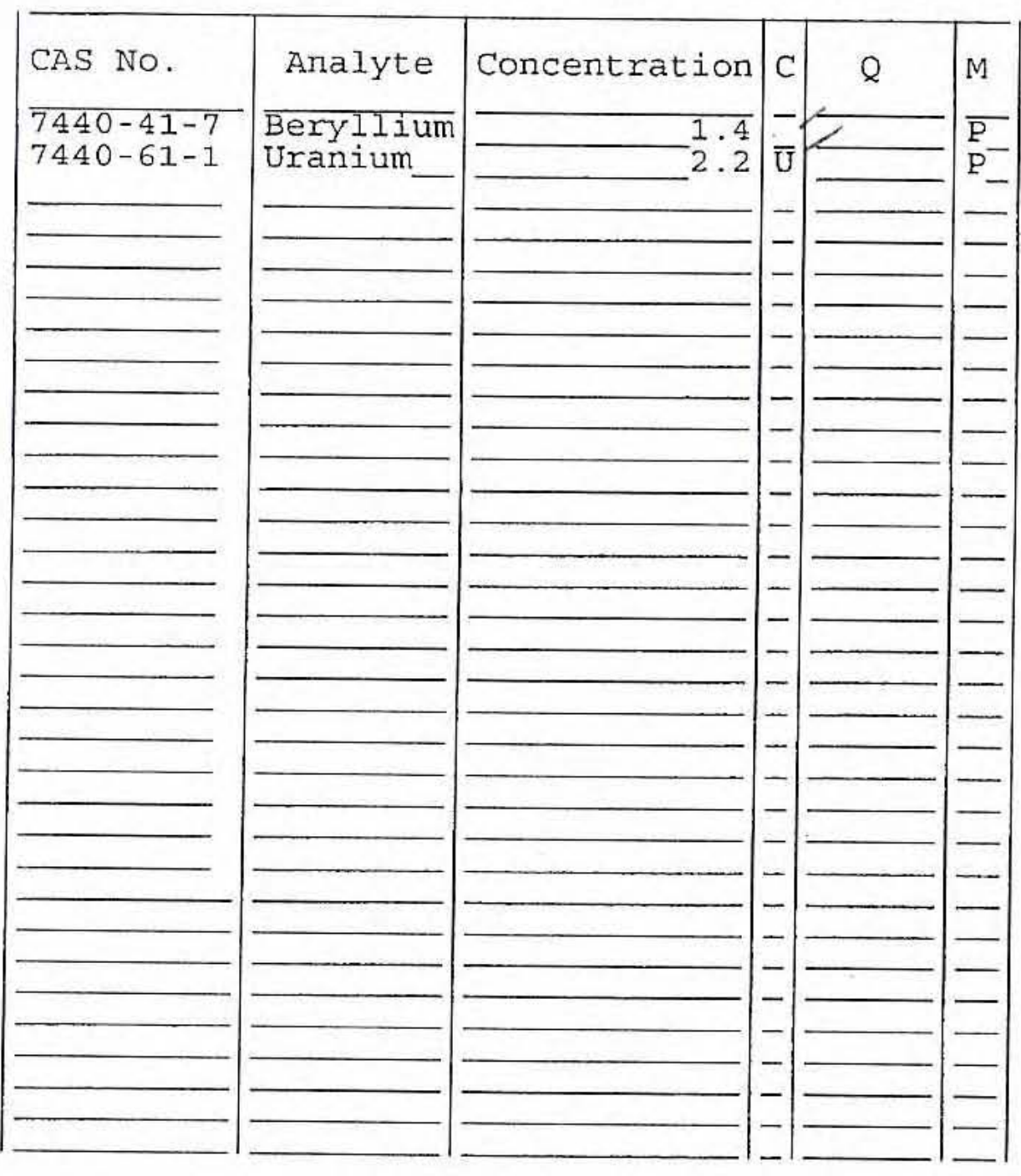

Color Before:

Color After:

Comments:

NEDS-08B
Contract: 60052

SAS NO. :

SDG NO.: 01A

Lab Sample ID: 0609L857-016

Date Received: 09/01/06
EPA SAMPLE NO.

$$
08 B
$$

Clarity Before:

Clarity After:

Texture:

Artifacts: 
1

INORGANIC. ANALYSES DATA SHEET
EPA SAMPLE NO.

$$
\text { O9A }
$$

Contract: 60052

SAS NO. : Case No.: NEDS

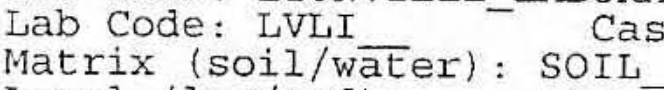
Level (low/med): LOW 음 Solids :
SDG NO.: 01A

Lab Sample ID: 0609L85 7-017

Date Received: 09/01/06

\section{Concentration Units (ug/L or $\mathrm{mg} / \mathrm{kg}$ ary weight) : MG/KG}

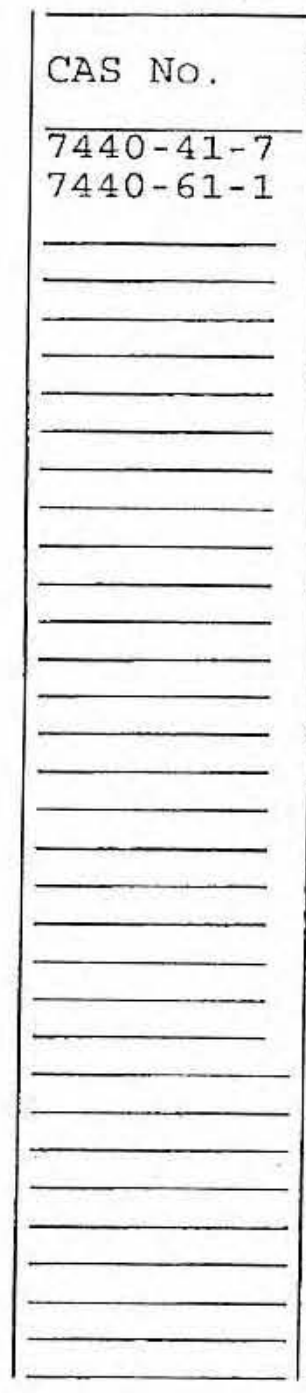

Color Before: Color After:

Comments :

NEDS - 09A

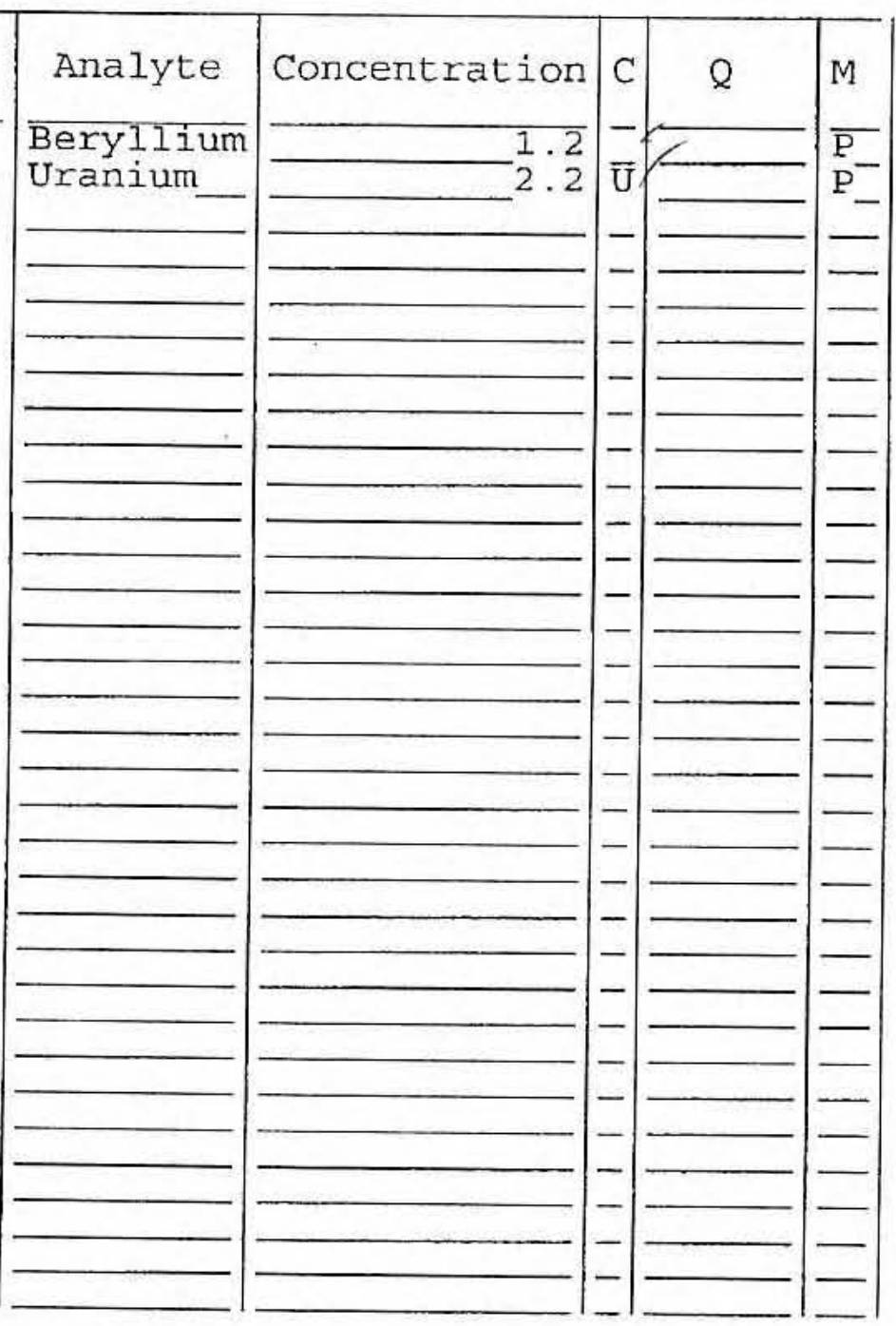

Clarity Before: Clarity After:
Texture: Artifacts: 
U.S. EPA

INORGANIC ANALYSES DATA SHEET.

EPA SAMPLE NO.

Lab Name: LIONVILLE_LABORATORY

Lab Code: LVII

Case NO.: NEDS

Matrix (soil/water): SoIL

Level (low/med):

$\div$ Solids:

LOW

$-92 \overline{2}$
Contract: 60052

SAS NO. :

Lab Sample ID: 0609L857-018

Date Received: 09/01/06

Concentration Units (ug/L or $\mathrm{mg} / \mathrm{kg}$ ary weight): MG/KG

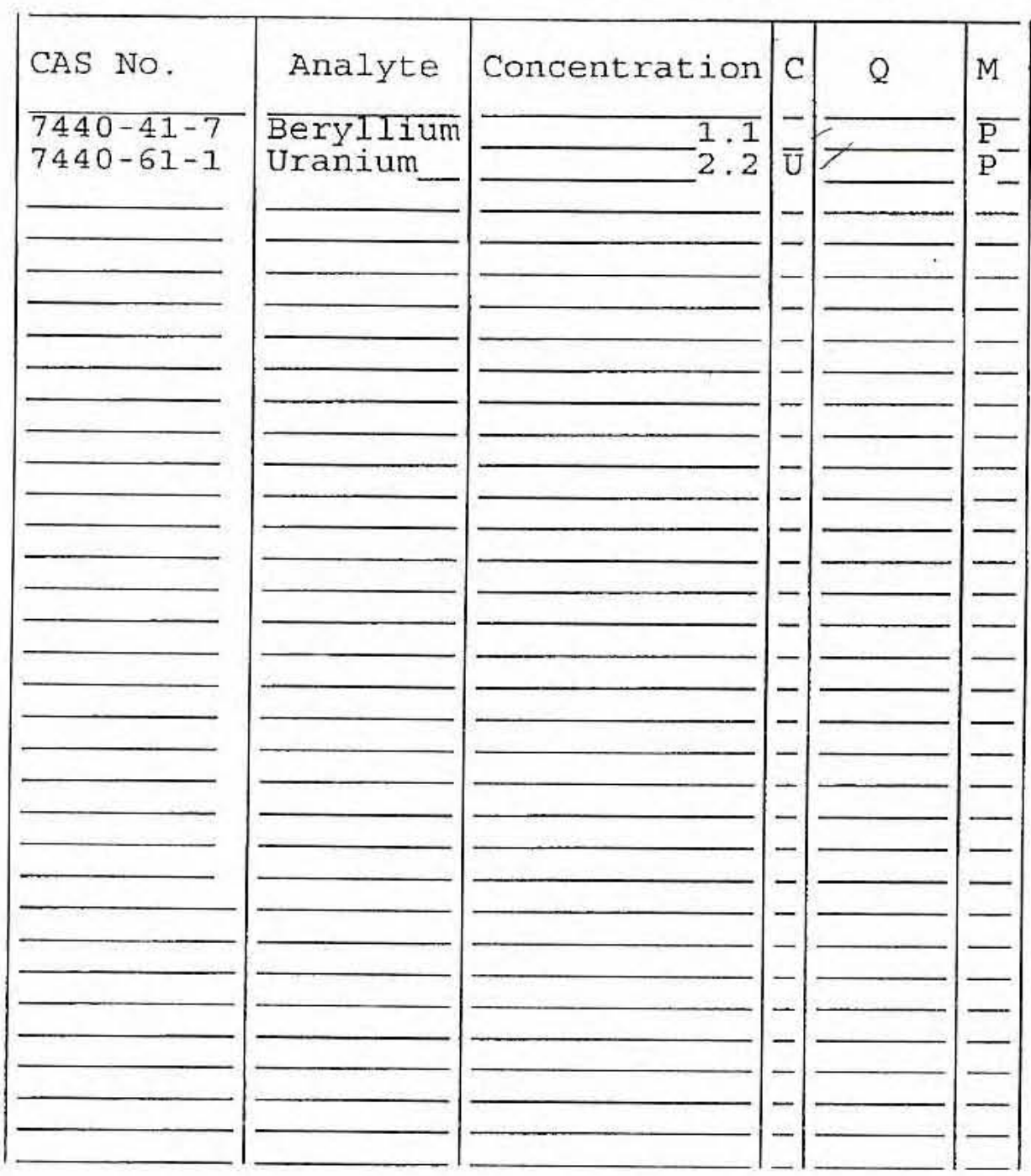

Color Before:

Color After:

Comments:

NEDS-09B
Clarity Before:

Clarity After:
Texture:

Artifacts : 


\section{INORGANIC ANALYSES DATA SHEET}

Lab Name: LIONVILLE LABORATORY

Lab Code: LVLI

Matrix (soil/water): SoIL

Level (low/med): LOW

\% Solids:
1

EPA SAMPLE NO.

$$
010 \mathrm{~A}
$$

Contract: 60052

SAS NO.:

SDG NO.: 01A

Lab Sample ID: 0609L857-019

Date Received: 09/01/06

Concentration Units (ug/L or $\mathrm{mg} / \mathrm{kg}$ dry weight) : $\mathrm{MG} / \mathrm{KG}$

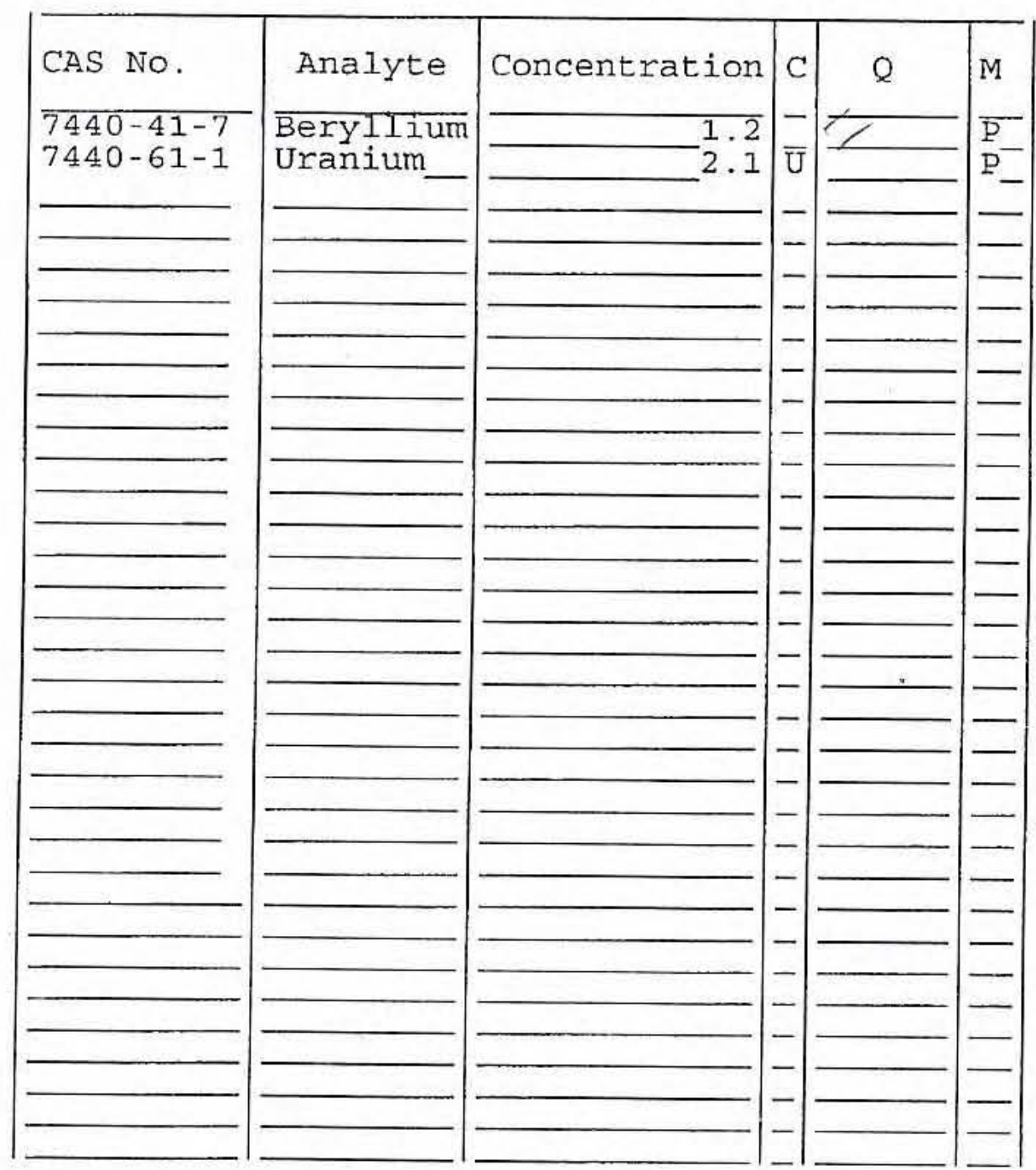

Color Before: Color After: Comments:

NEDS-OIOA
Clarity Before:

Clarity After:
Texture:

Artifacts: 
EPA SAMPLE NO.

$$
010 B
$$

Lab Name: LIONVILLE_LABORATORY

Lab Code: LVLI

Matrix (soil/water): Soll

Level (low/med): LOW

\% Solids:
Contract: 60052

SAS NO.:

Lab Sample ID: 0609L85 $\overline{7-0} 20$

Date Received: 09/01/06

Concentration Units (ug/L or $\mathrm{mg} / \mathrm{kg}$ dry weight): $\mathrm{MG} / \mathrm{KG}$

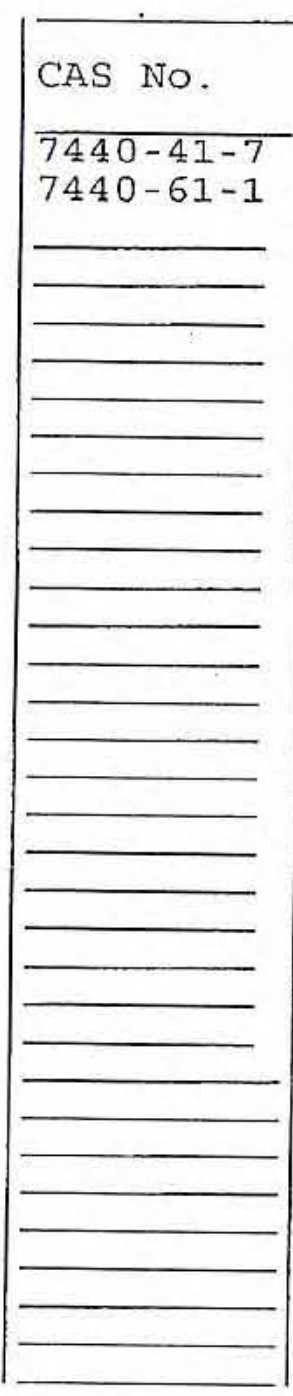

Color Before:

Color After:

Comments:

NEDS - O $10 B$

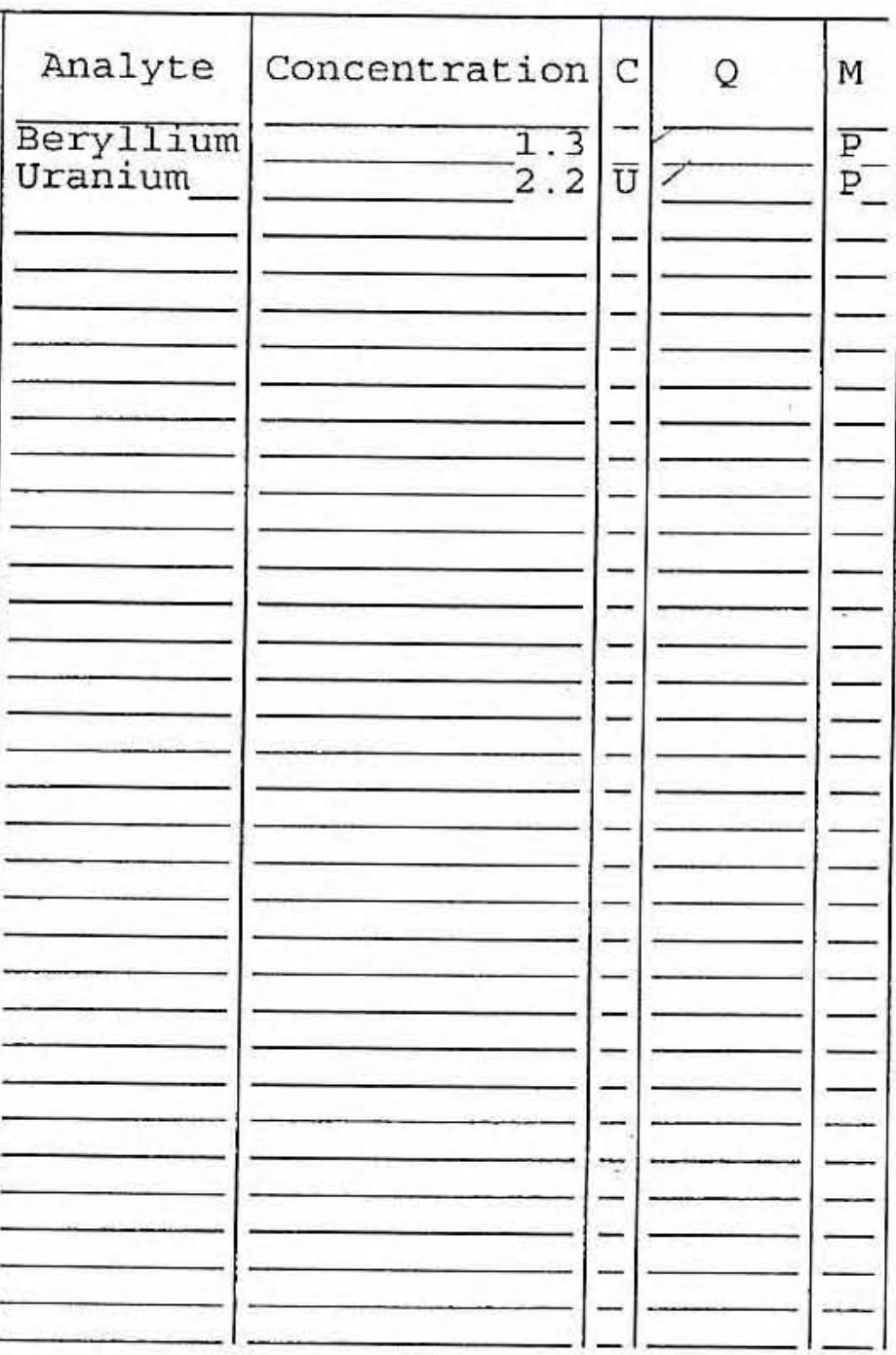

Clarity Before: Clarity After:
Texture: Artifacts: 


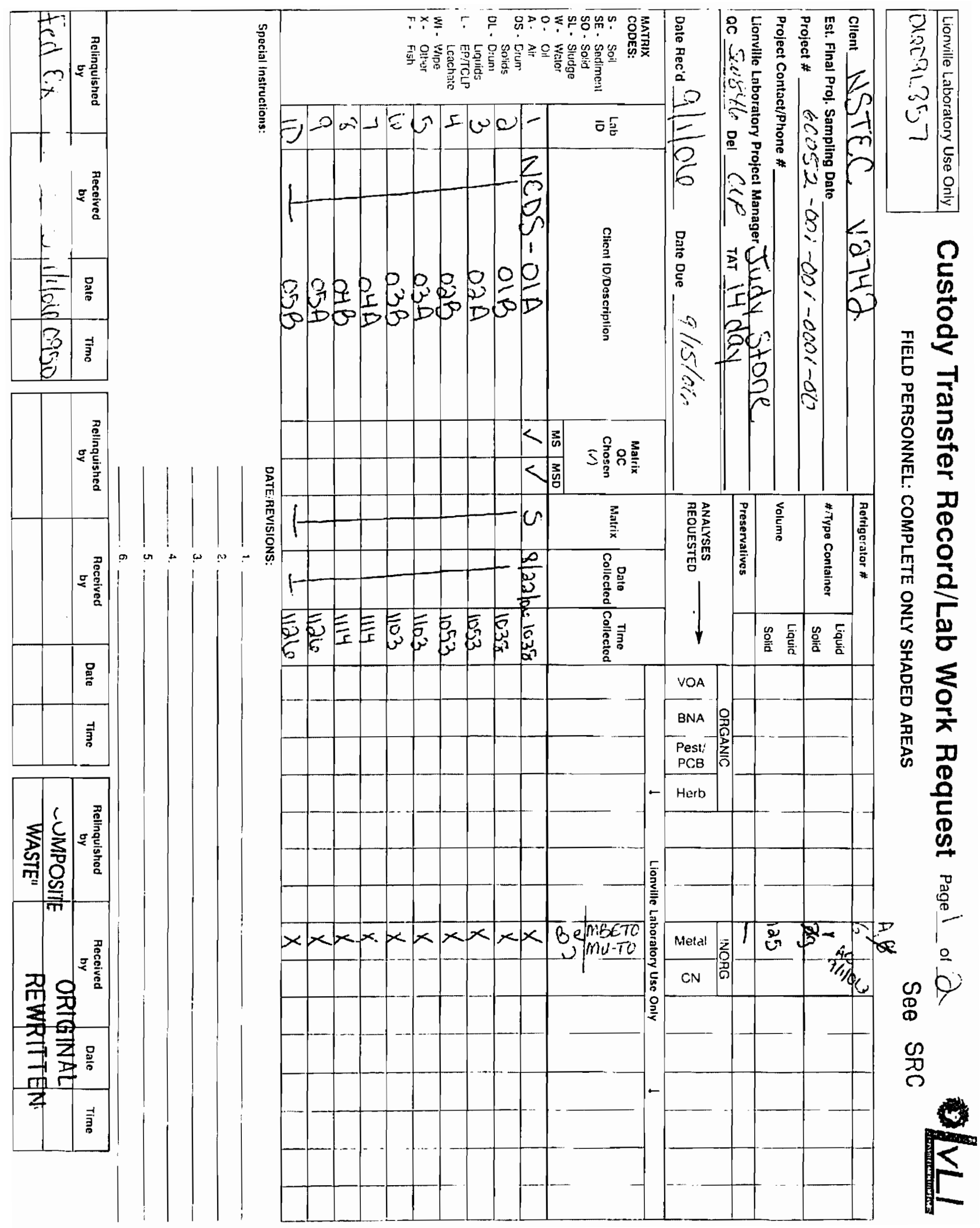




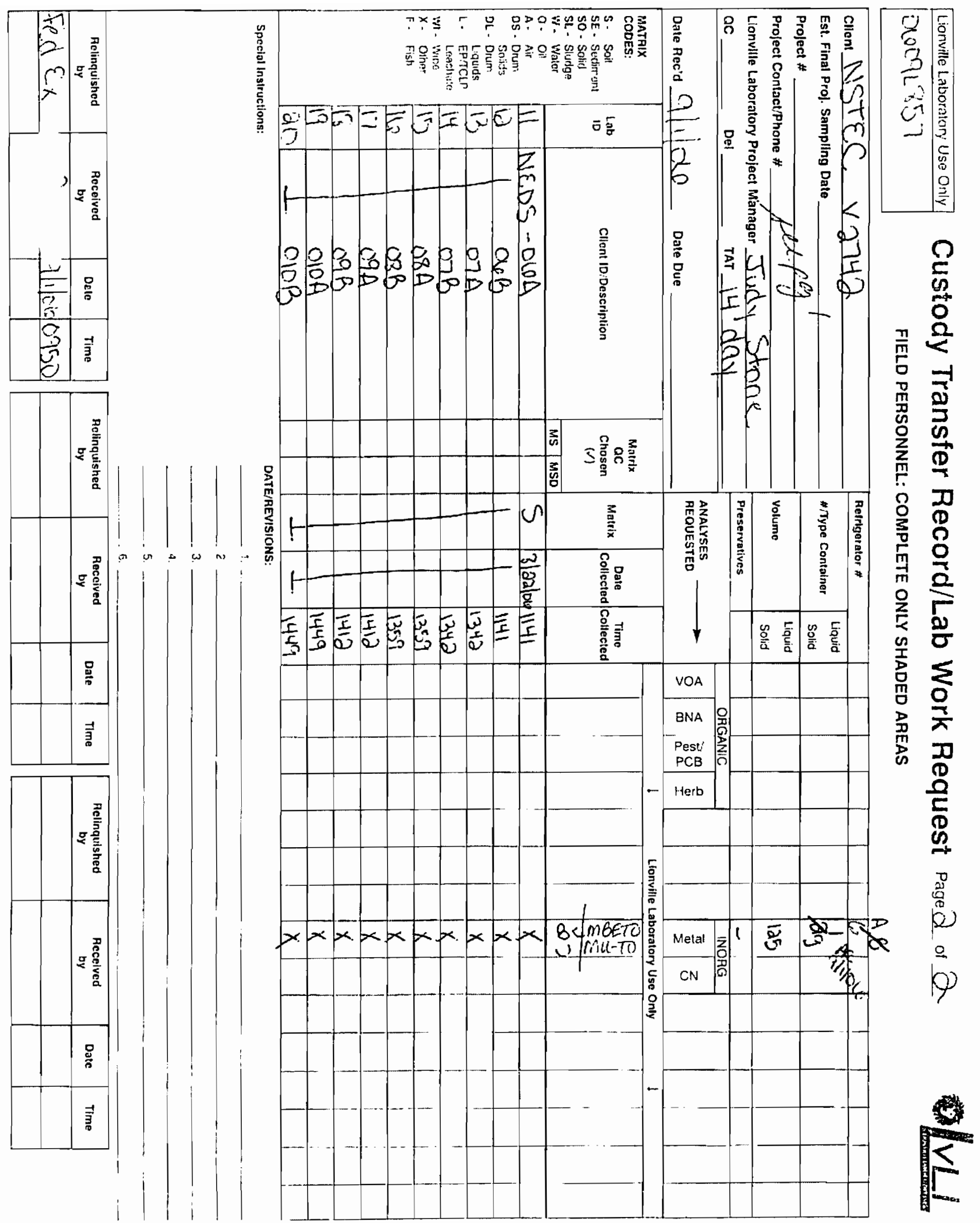




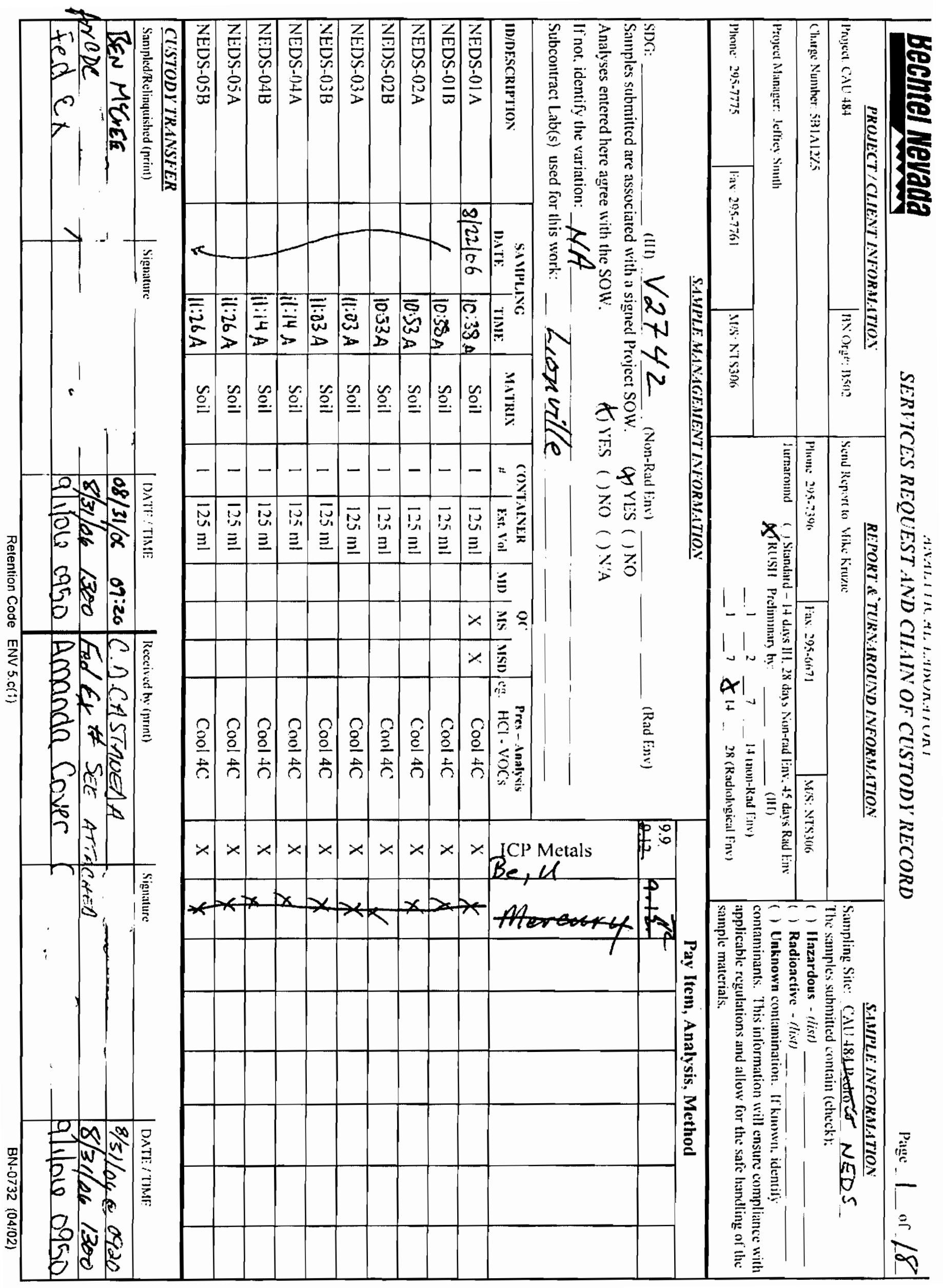




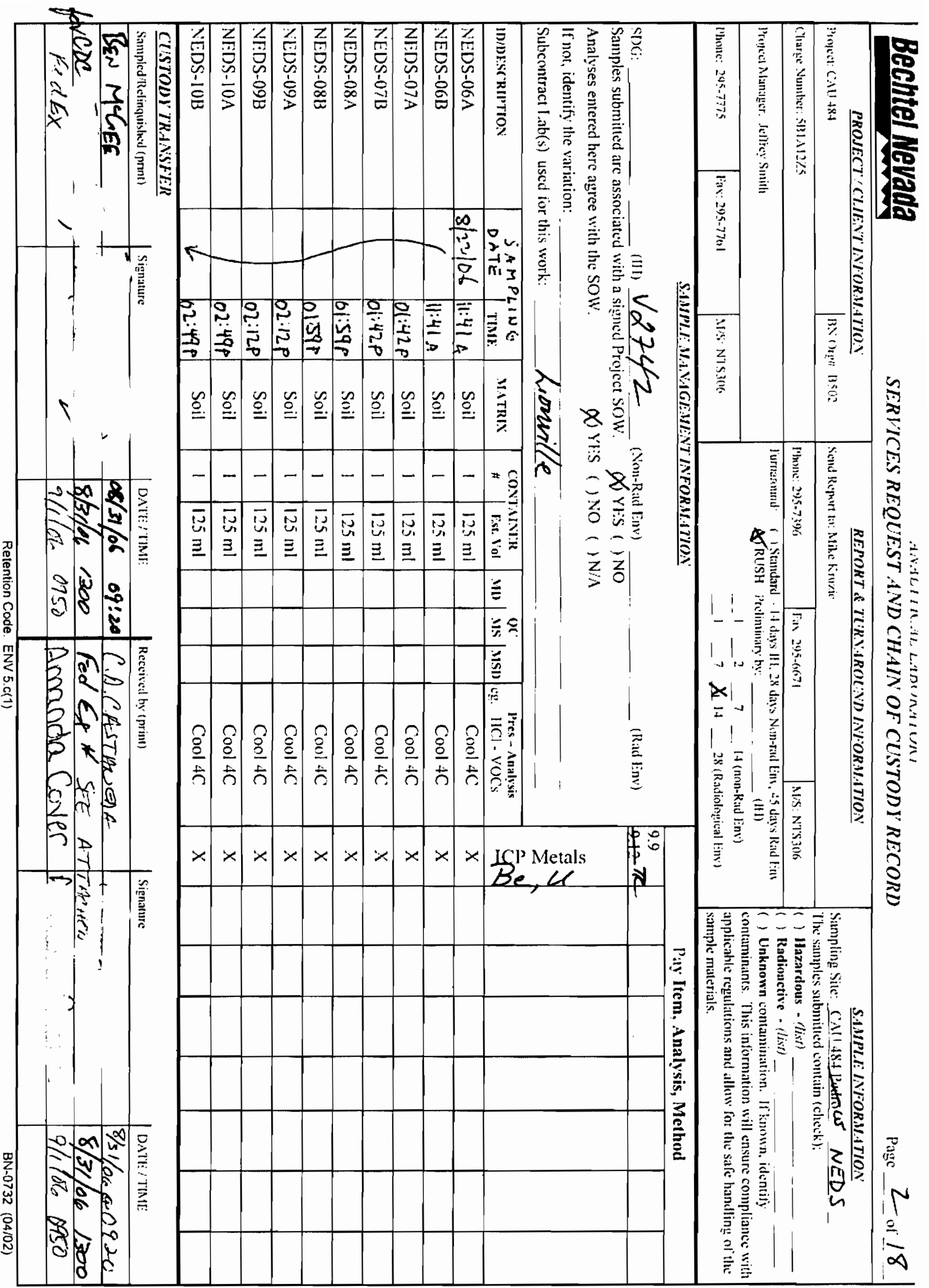


EPA SAMPLE NO.

\section{$11 \mathrm{~A}$}

Lab Name: LIONVILLE LABORATORY Lab Code: LVLI Matrix (soil/water): Soll Level (low/med): LOW o Solids :
Contract : 60052

SAS NO.:

Lab Sample ID: 0609L858-001

Date Received: 09/01/06

Concentration Units (ug/L or $\mathrm{mg} / \mathrm{kg}$ dry weight) : MG/KG

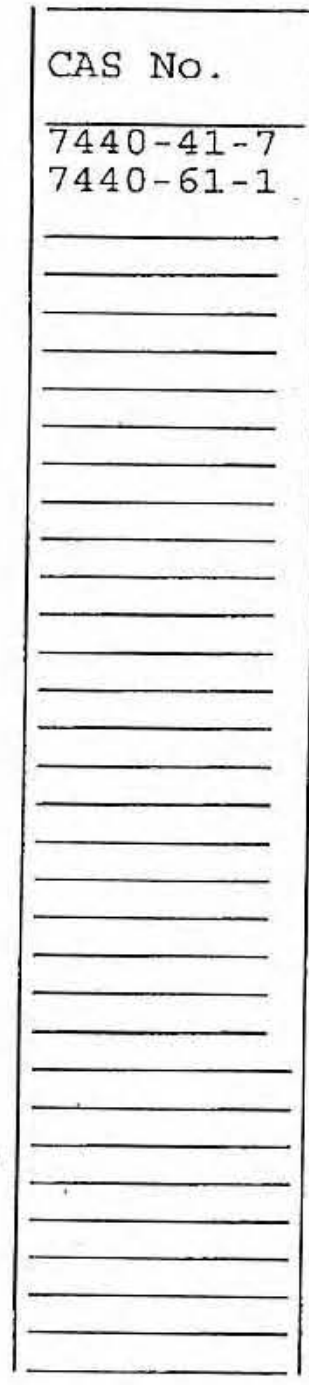

Color Before: Color After: Comments:

NEDS - 11A

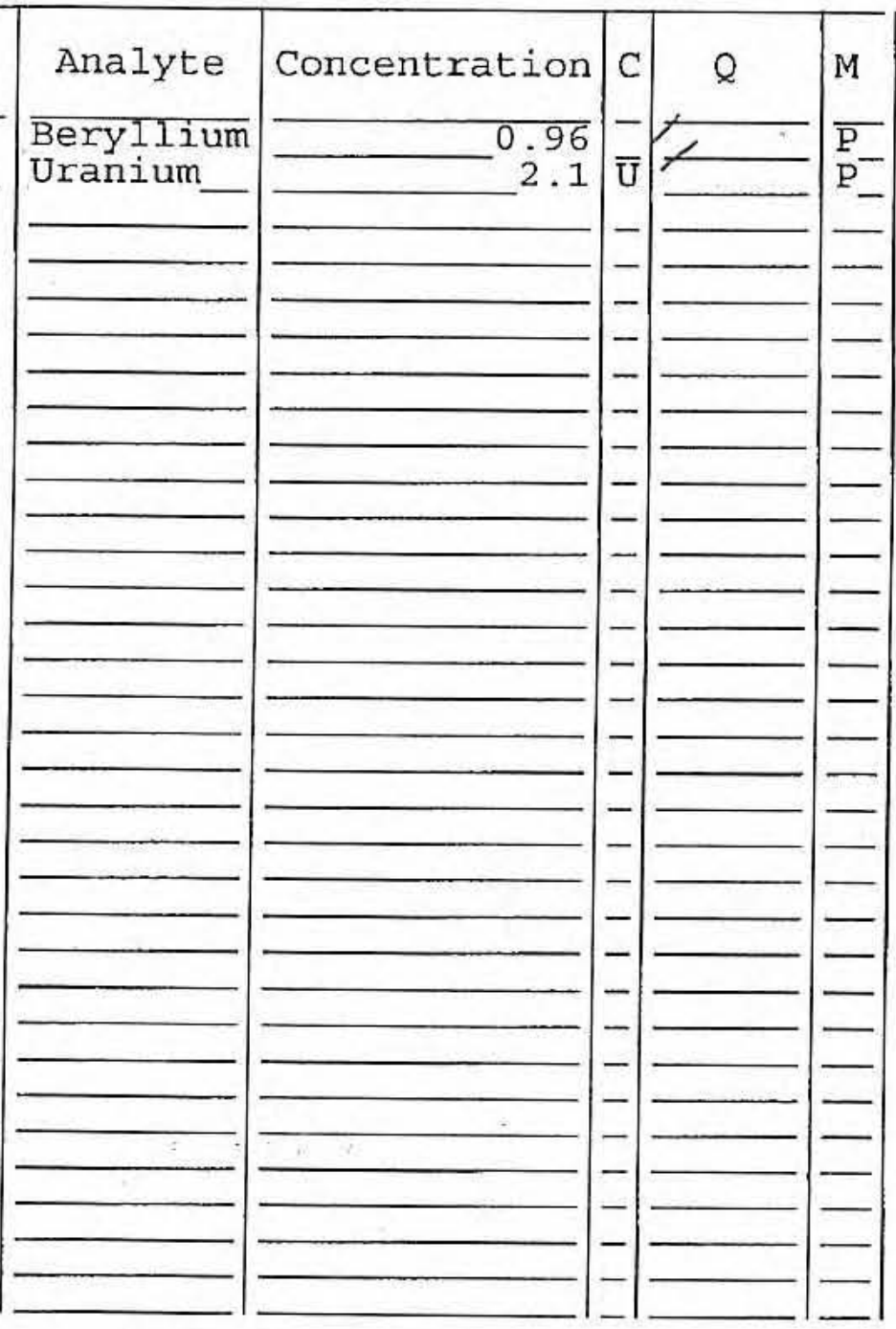

Clarity Before: Clarity After:
Texture: Artifacts : 


\section{U.S. EPA.}

\section{INORGANIC ANALYSES DATA SHEET}

EPA SAMPLE NO.

Lab Name: LIONVILLE_LABORATORY Lab Code: LVLI Matrix (soil/water): SoIL Level (low/med) : \% Solids :
Contract: 60052

SAS No.:

$11 \mathrm{~B}$

Lab Sample ID: 0609L858-0002

Date Received: 09/01/06

Concentration Units (ug/L or $\mathrm{mg} / \mathrm{kg}$ dry weight): MG/KG

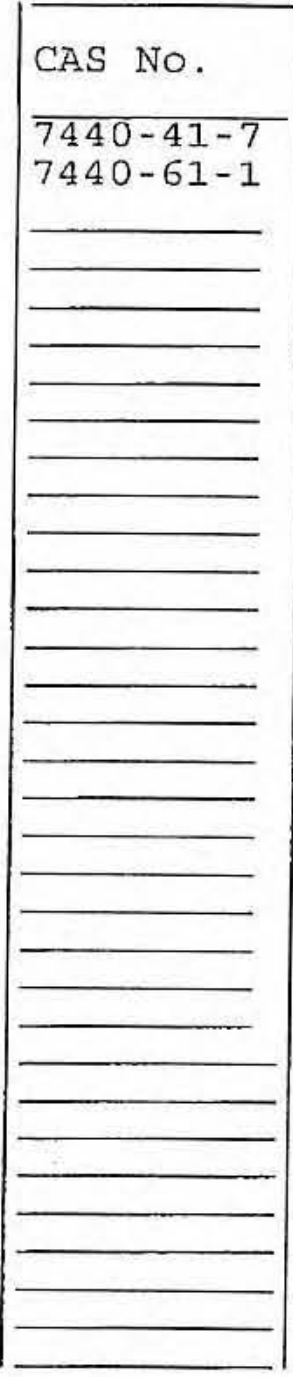

Color Before: Color After: Comments :

NEDS - 11B

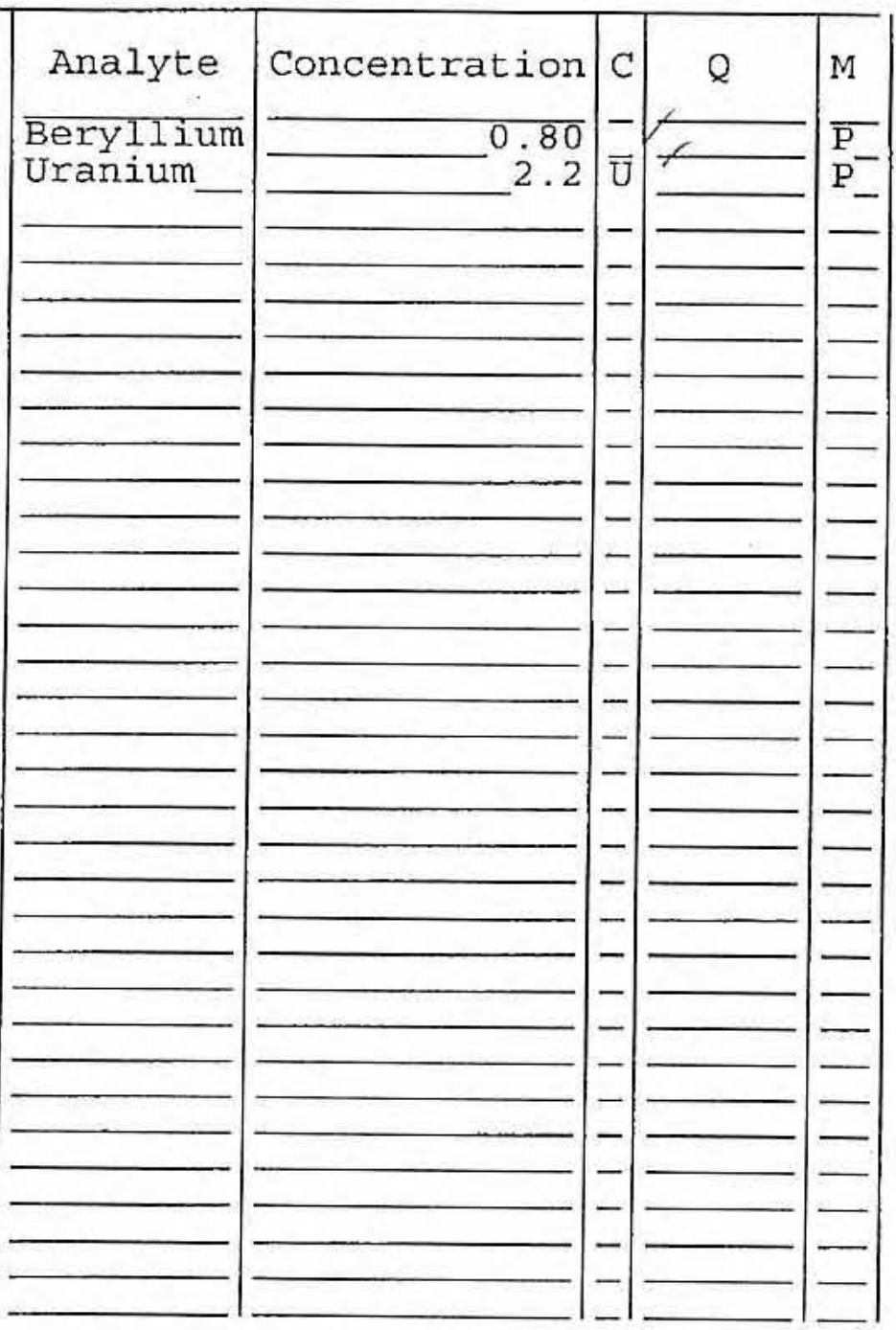

Clarity Before: Clarity After:
Texture: Artifacts: 


\section{U.S. EPA}

1

INORGANIC ANALYSES DATA SHEET
EPA SAMPLE NO.

$12 \mathrm{~A}$

Lab Name: LIONVILLE_LABORATORY Contract: 60052

Case No.: NEDS

Matrix (soil/water): SOIL

Level (low/med): LOW

$\div$ Solids :
SAS NO.:

Lab Sample ID

$-95 \overline{.2}$
Date Received: 09/01/06

Concentration Units (ug/L or $\mathrm{mg} / \mathrm{kg}$ dry weight): MG/KG

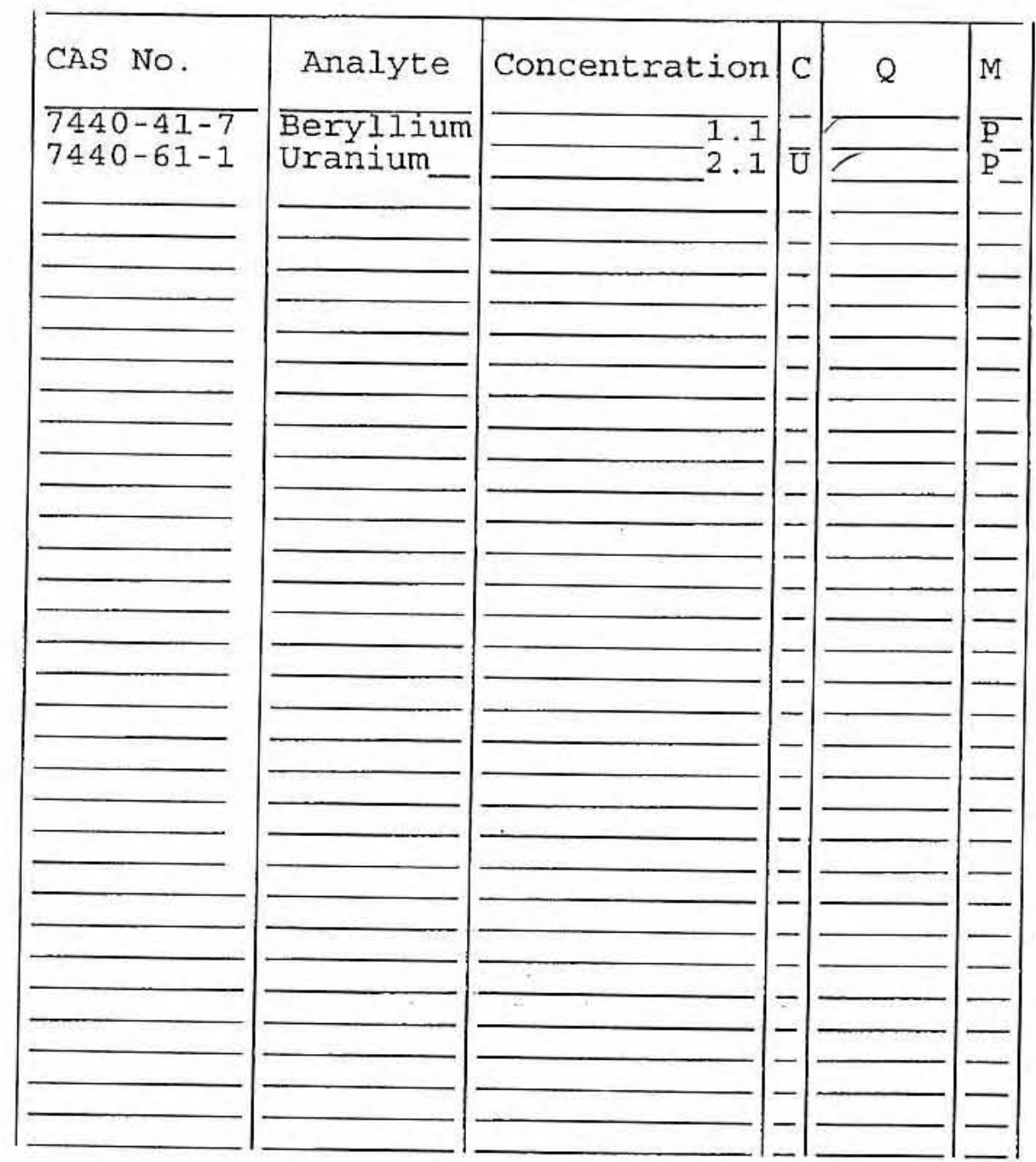

Zolor Before:

zolor After:

Clarity Before:

Clarity After:
Texture:

Artifacts:

Jomments :

NEDS - I2A 
U.S. EPA

INORGANIC ANALÝSES DATA SHEET

EPA SAMPLE NO.

Lab Name: LIONVILLE_LABORATORY

Lab Code: LVLI

Matrix (soil/water): Level (low/med) :

\% Solids:
Contract: 60052

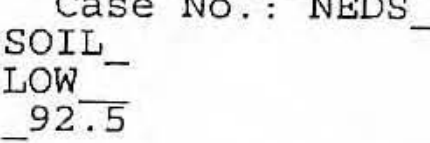

\section{$12 \mathrm{~B}$}

SDG NO.: V2742

Lab Sample ID: 0609L858-0̄04

Date Received: 09/01/06

Concentration Units (ug/L or $\mathrm{mg} / \mathrm{kg}$ dry weight) : MG/KG

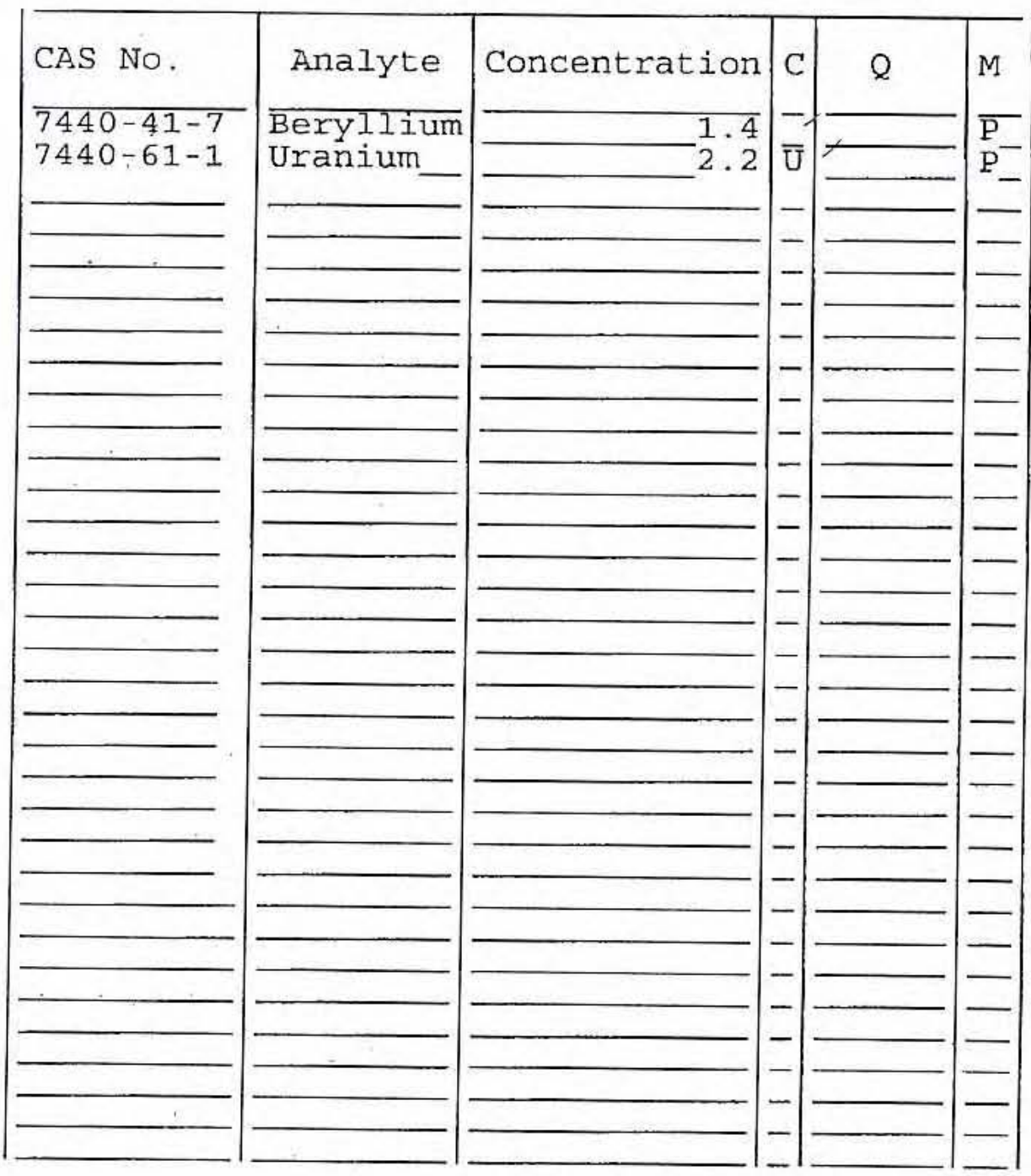

Color Before:

Color After:

Jomments :

NEDS - i $2 \mathrm{~B}$
Clarity Before:

Clarity After:
Texture:

Artifacts: 
U.S. EPA

1 INORGANIC ANALYSES DATA SHEET
Lab Name: LIONVILLE_LABORATORY

Lab Code: LVLI

Matrix (soil/water): SOIL

Level (low/med):

\% Solids :
Contract: 60052 SAS No.:
EPA SAMPLE NO.

$13 \mathrm{~A}$

SDG NO.: V2742

Lab Sample ID: 0609L858-005

Date Received: 09/01/06

Concentration Units (ug/I or $\mathrm{mg} / \mathrm{kg}$ dry weight): MG/KG

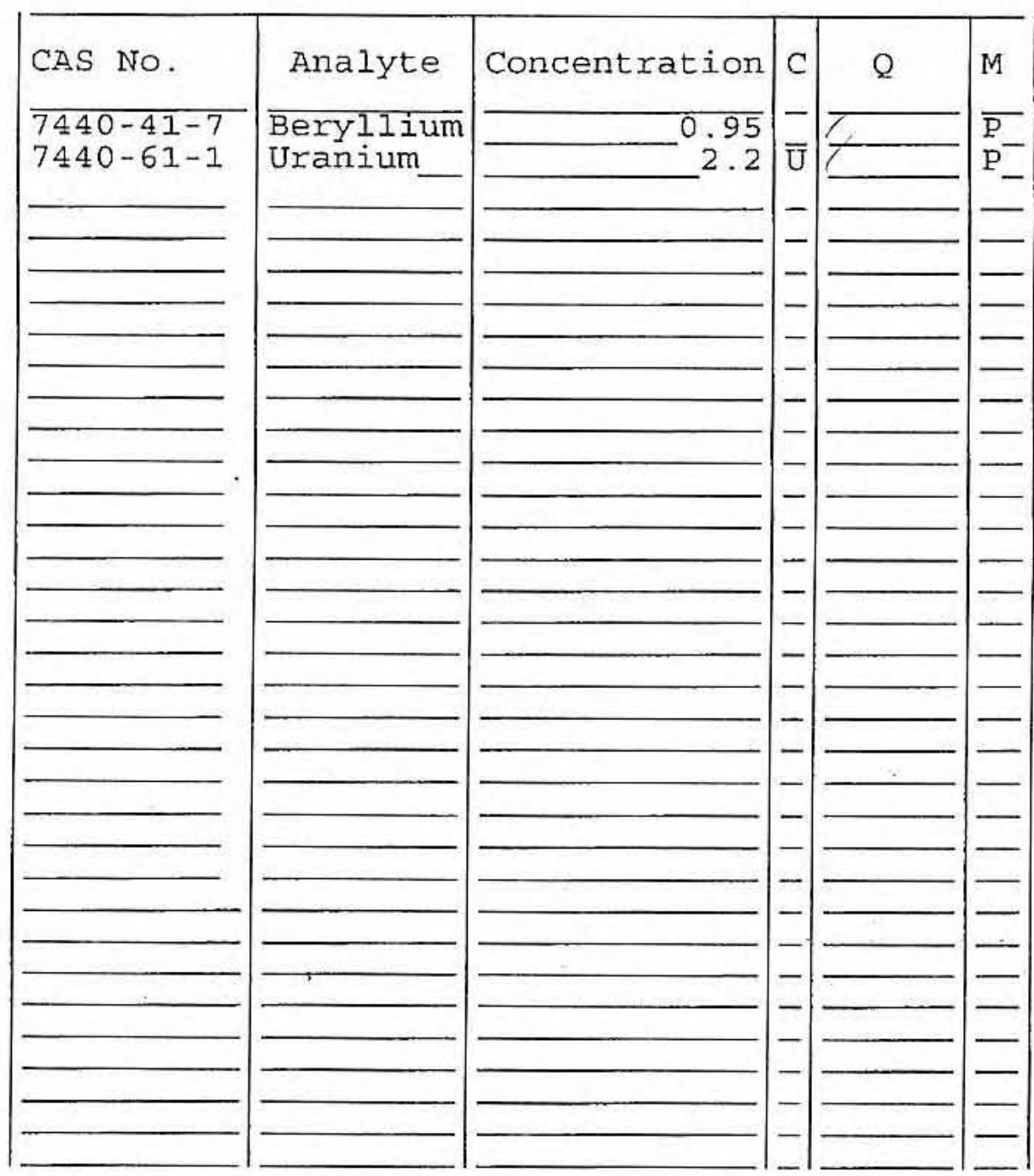

Color Before:

Clarity Before:

Texture:

Color After:

Clarity After:

Artifacts:

Comments :

NEDS - 13A. 
EPA SAMPLE NO.

Lab Name: IIONVILLE LABORATORY

Lab Code: LVLI Matrix (soil/water): SoIL Level (low/med): LOW \% Solids: _ $\quad$ : $2 \overline{.0}$
$13 B$

Contract: 60052

SAS NO. :

Lab Sample ID: 0609L858-006

Date Received: 09/01/06

Concentration Units (ug/L or $\mathrm{mg} / \mathrm{kg}$ dry weight): $\mathrm{MG} / \mathrm{KG}$

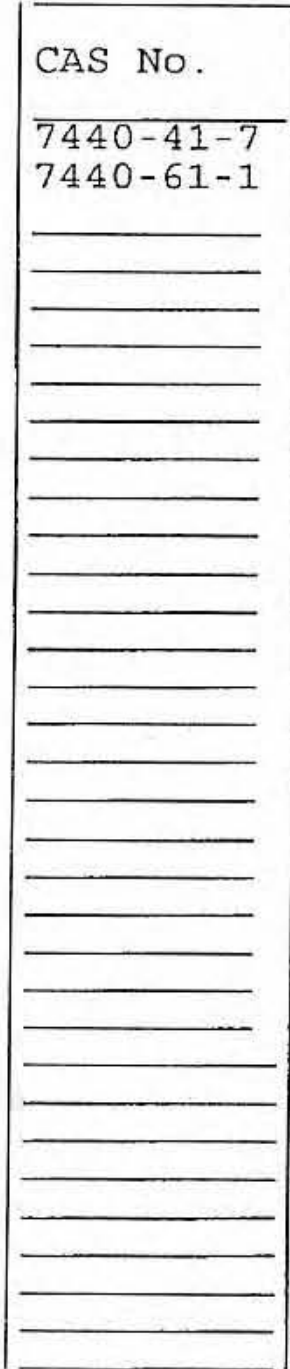

Color Before:

Color After:

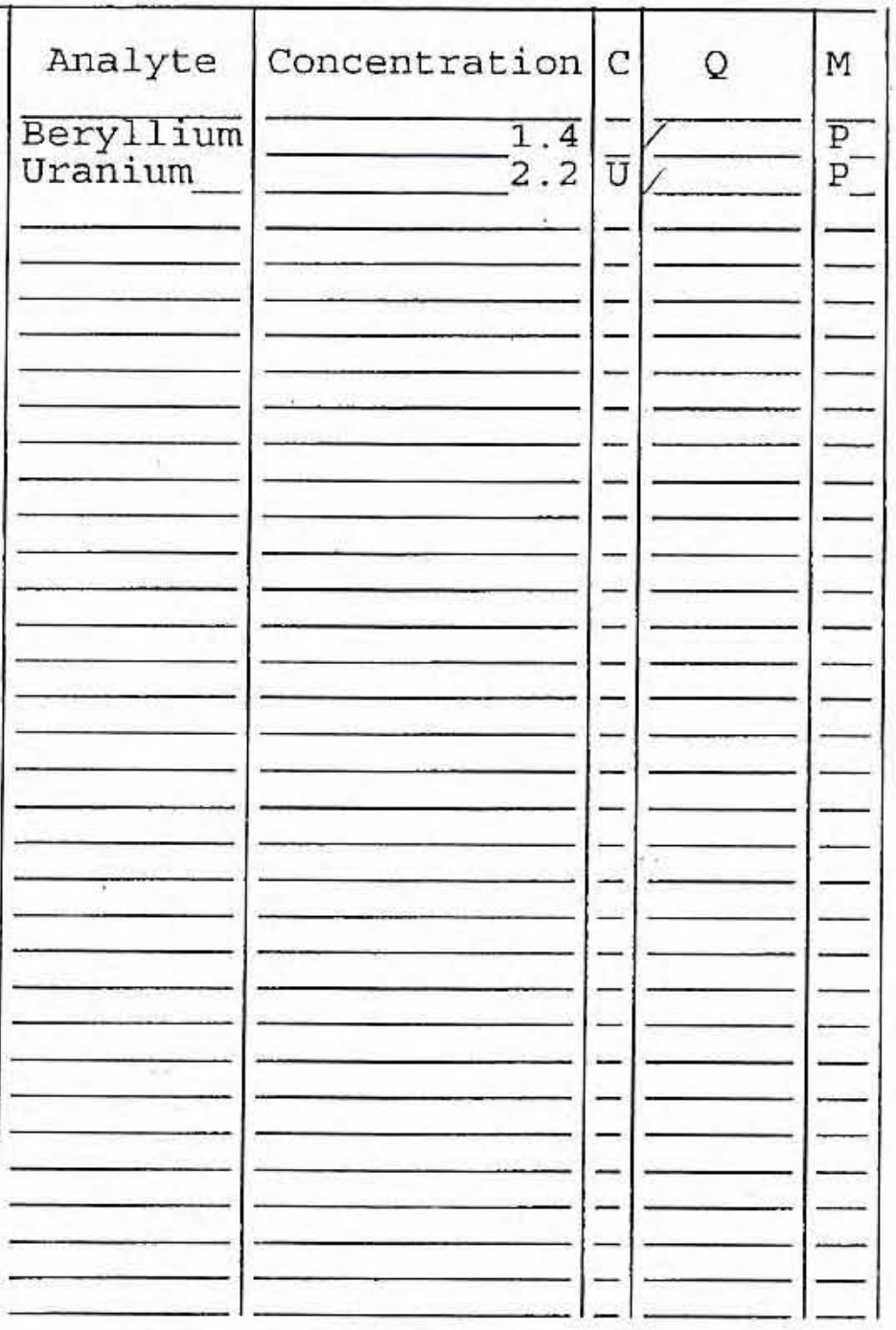

Clarity Before: Clarity After:
Texture:

Artifacts :

Comments :

NEDS - 13B 
1

INORGANIC ANALYSES DATA SHEET

Iab Name: LIONVILLE LABORATORY

Lab Code: LVLI

Matrix (soil/water)

Level (low $/ \mathrm{med}$ ):

$\%$ Solids :
LABORATORY
Case NO.: NEDS
: SOIL_

Contract: 60052

LOW

$-98 \overline{.9}$

SAS NO.:
EPA SAMPLE NO.

\section{$14 \mathrm{~A}$}

SDG NO.: V2742

Lab Sample ID: 0609L858-0007

Date Received: 09/01/06

Concentration Units (ug/L or $\mathrm{mg} / \mathrm{kg}$ dry weight): MG/KG

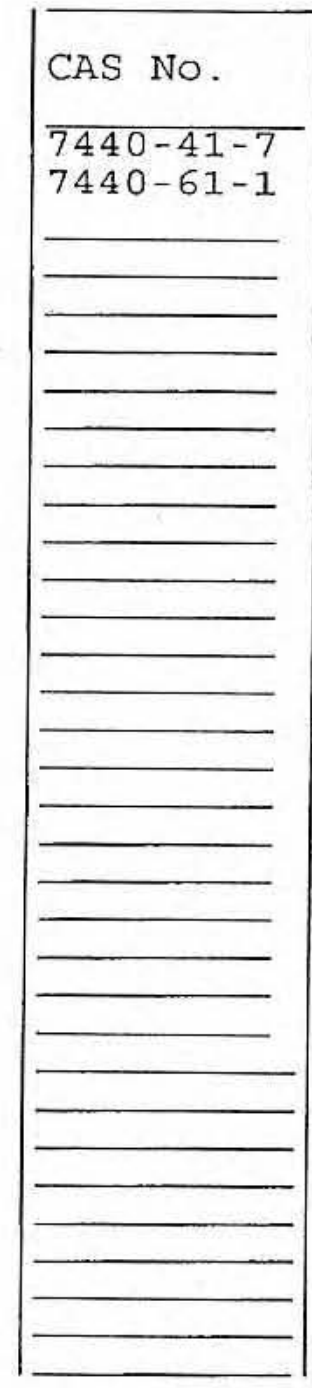

Color Before:

Color After:

Comments :

NEDS - 14A

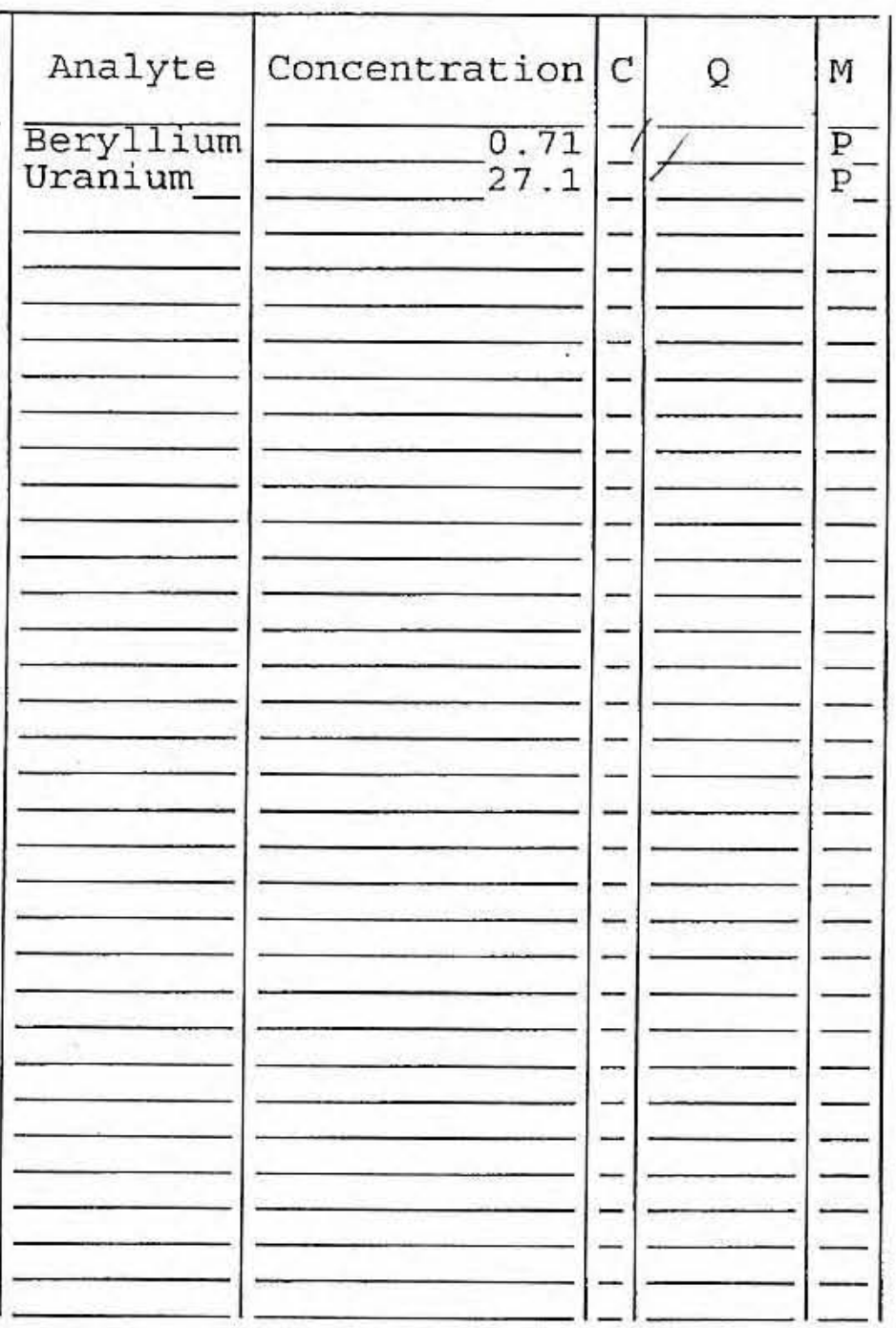

Clarity Before: Clarity After:
Texture: Artifacts: 
EPA SAMPLE NO.

$$
14 B
$$

Lab Name: LIONVILLE LABORATORY

Lab Code: LVLI Matrix (soil/water): SOIL Level (low/med): $\div$ Solids:
Contract: 60052

SAS NO.:

SDG NO : V2742

Lab Sample ID: 0609L858-̄008

Date Received: 09/01/06

Concentration Units (ug/L or $\mathrm{mg} / \mathrm{kg}$ dry weight): MG/KG

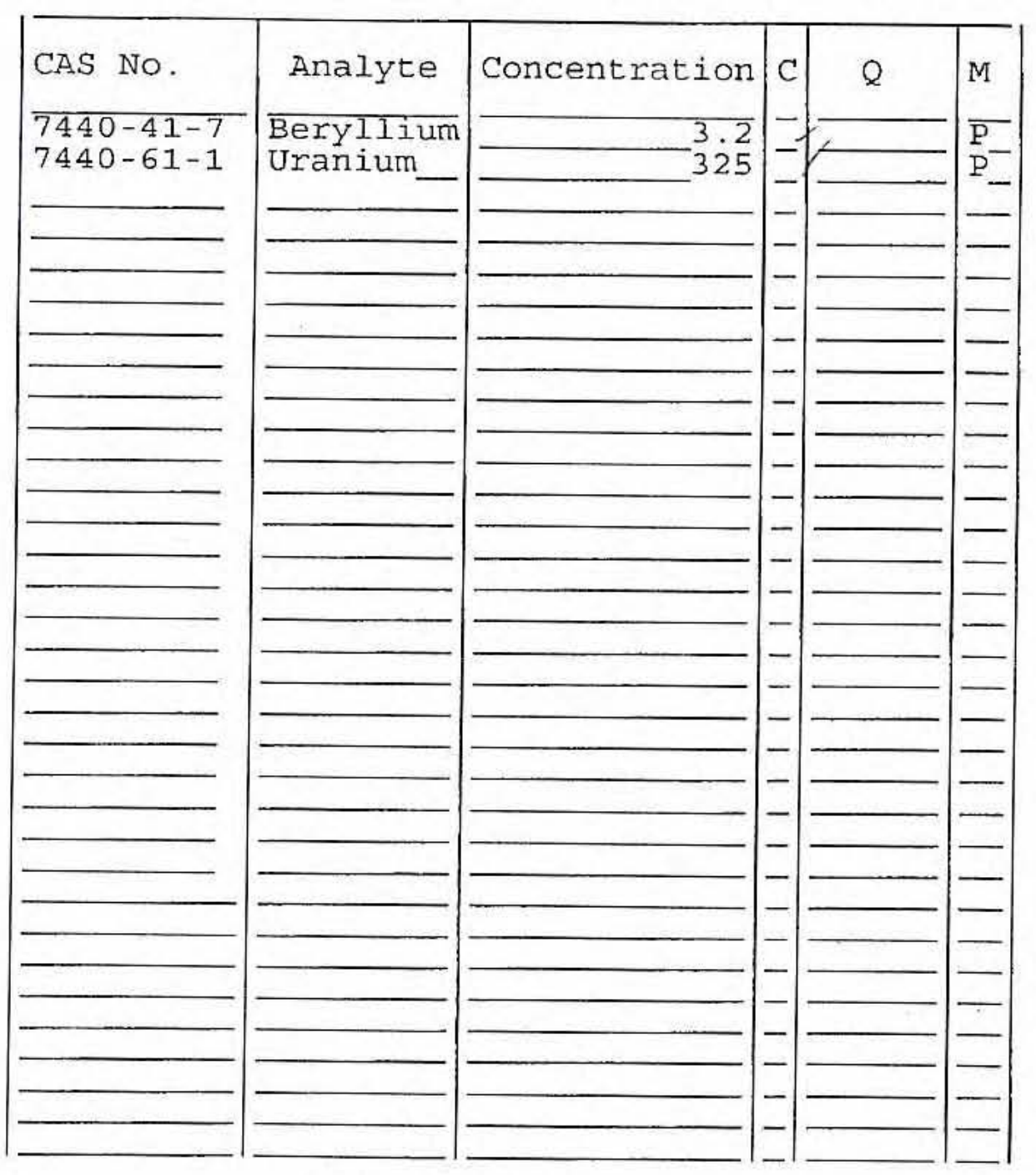

Color Before: Color After:

Comments :
Clarity Before:

Clarity After:
Texture: Artifacts: 
EPA SAMPLE NO.

$$
15 \mathrm{~A}
$$

Lab Name: LIONVILIE LABORATORY

Lab Code: LVLI Matrix (soil/water): SOIL_ Level (low/med): : Solids:
Contract: 60052

SAS NO.:

SDG NO.: V2742

Lab Sample ID: 0609L858-0̄09

Date Received: 09/01/06

Concentration Units (ug/L or $\mathrm{mg} / \mathrm{kg}$ dry weight): MG/KG

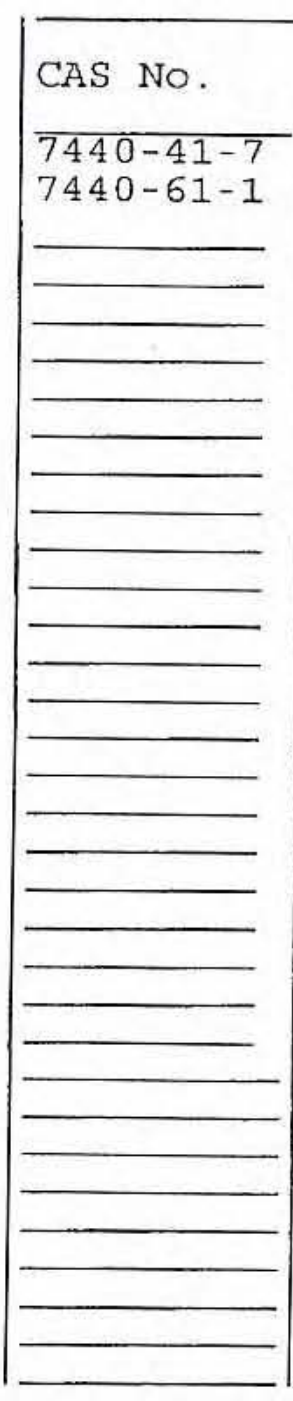

Color Before: Color After:

Comments :

NEDS - 15A

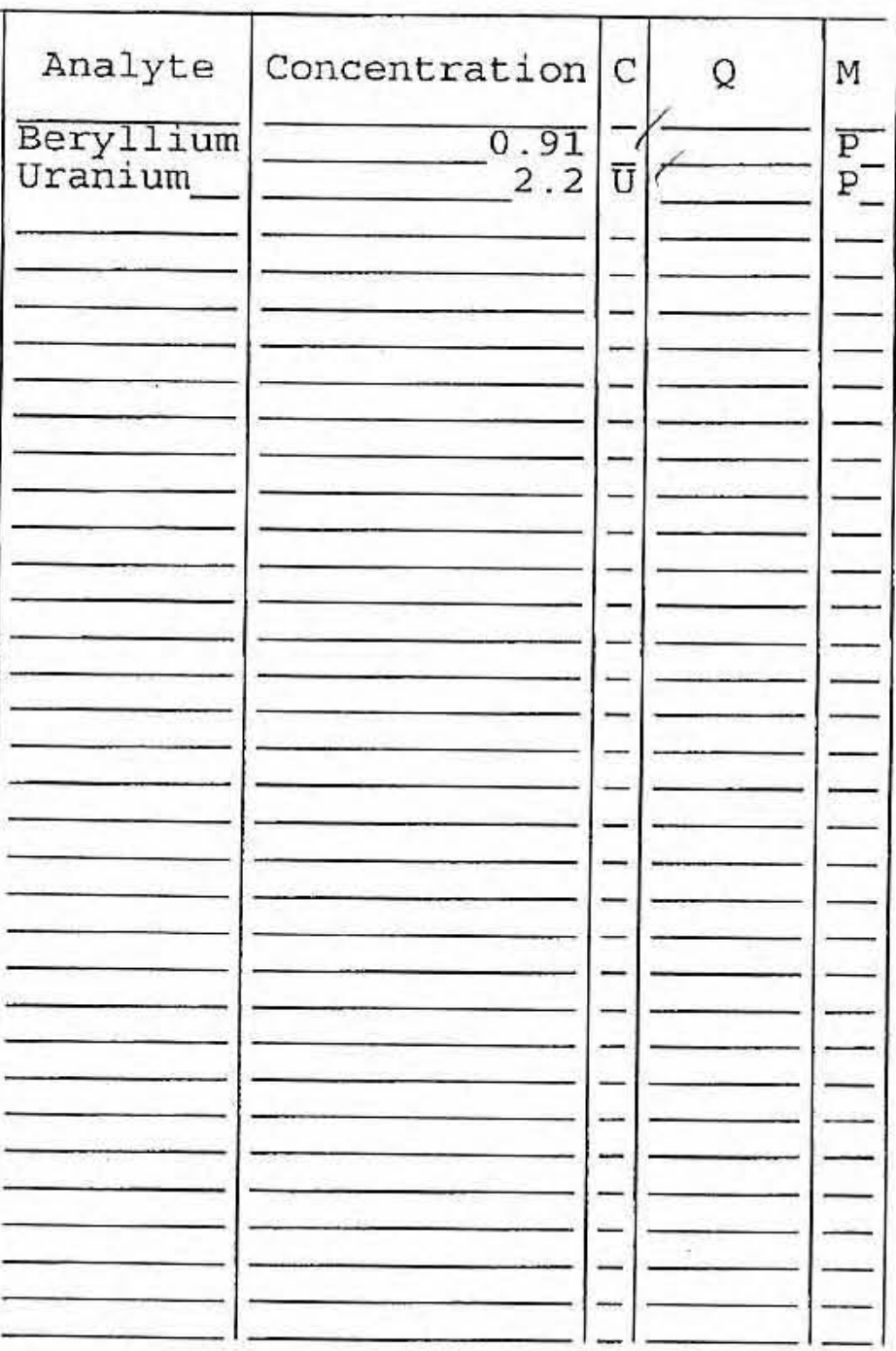

Clarity Before: Clarity After:
Texture : Artifacts : 
1

INORGANIC ANALYSES DATA SHEET
Lab Name: LIONVILLE LABORATORY

Lab Code: LVLI Matrix (soil/water): SoIL Level. (low/med): 음 Solids :

LOW
Contract: 60052

SAS NO.:

SDG NO.: V2742

Lab Sample ID: 0609L858-0̄10

$-90 \overline{2}$
$15 B$

EPA SAMPLE NO.

Date Received: 09/01/06

Concentration Units (ug/L or $\mathrm{mg} / \mathrm{kg}$ dry weight): $\mathrm{MG} / \mathrm{KG}$

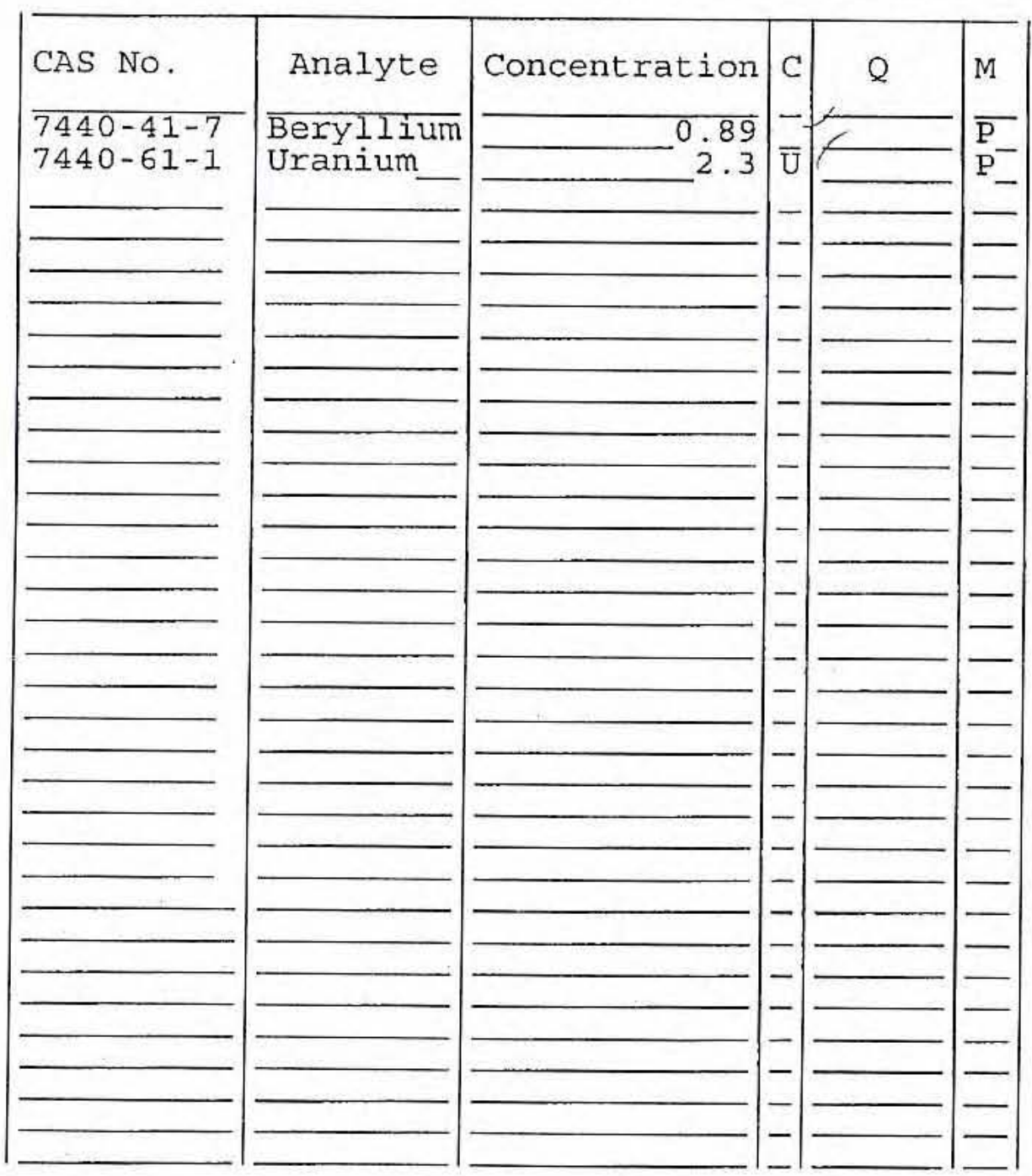

Color Before:

Color After:

Comments:

NEDS $-15 B$
Clarity Before:

Clarity After:
Texture: Artifacts: 


\section{INORGANIC ANALYSES DATA SHEET}

Lab Name: LIONVILLE_LABORATORY

Lab Code: LVLI

Matrix (soil/water): SoIL

Level (low/med):

$\div$ Solids :

\section{EPA. SAMPLE NO.}

\section{$16 \mathrm{~A}$}

Contract: 60052

SAS NO.:

Lab Sample ID: $0609 \mathrm{~L} 0858-011$

LOW

Date Received: 09/01/06

Concentration Units (ug/L or $\mathrm{mg} / \mathrm{kg}$ dry weight): MG/KG

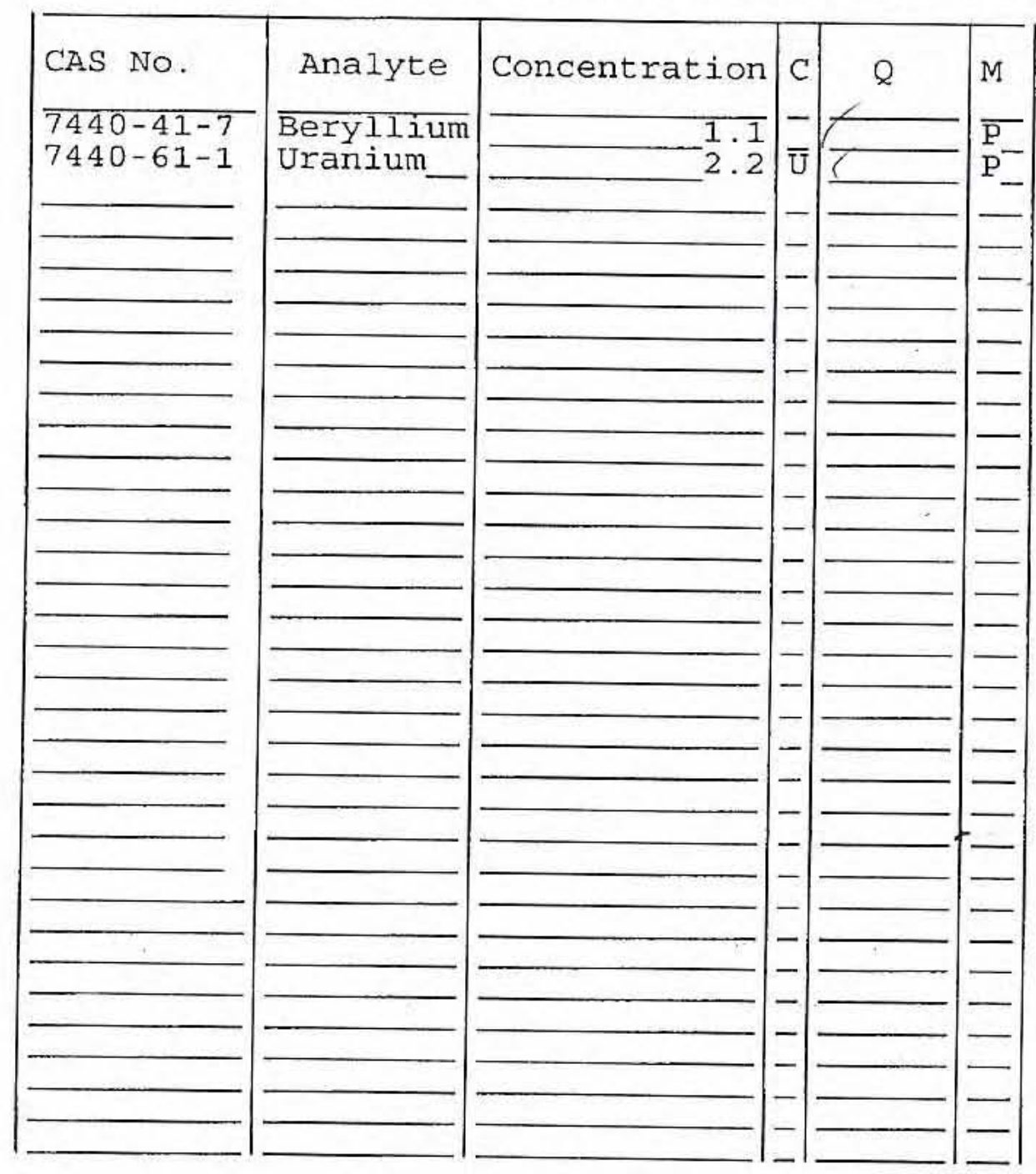

Color Before: Color After:

Comments :

NEDS - . 16A
Clarity Before:

Clarity After:
Texture:

Artifacts : 


\section{INORGANIC ANALYSES DATA SHEET}

Lab Name: LIONVILLE_LABORATORY

Lab Code: LVLI Matrix (soil/water): SOIL

Level. (low/med): LOW

\% Solids :
EPA SAMPLE NO.

\section{$16 \mathrm{~B}$}

Contract: 60052

SAS NO. :

Lab Sample ID:

Date Received: 09/01/06

Concentration Units (ug/L or $\mathrm{mg} / \mathrm{kg}$ dry weight): $\mathrm{MG} / \mathrm{KG}$

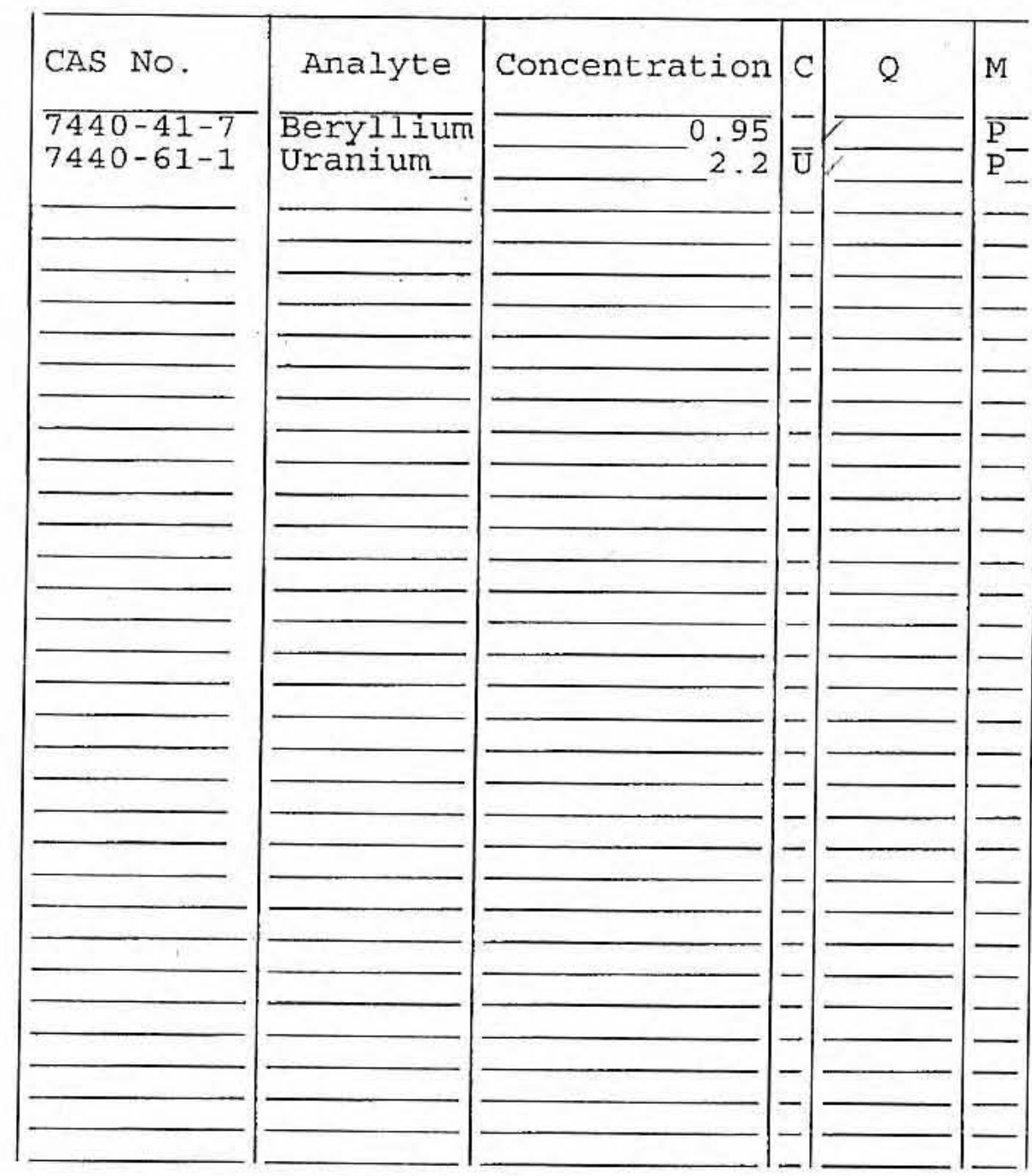

Color Before:

Color After:

Clarity Before:

Clarity After:

Texture:

Artifacts:

Comments :

NEDS - 16B

FORM I - IN 
1

INORGANIC ANALYSES DATA SHEET
EPA SAMPLE NO.

\section{A}

Lab Name: LIONVILLE_LABORATORY_Contract: 60052

Lab Code: LVLI

Matrix (soil/water): SOIL
Level (low/med):

$\begin{array}{ll}\text { Level (low/med) : } & \text { LOW } \\ \text { \% Solids: } & 94 . \overline{3}\end{array}$

SAS NO.:

Lab Sample ID: 0609L858-013

Date Received: 09/01/06

Concentration Units (ug/L or $\mathrm{mg} / \mathrm{kg}$ dry weight): MG/KG

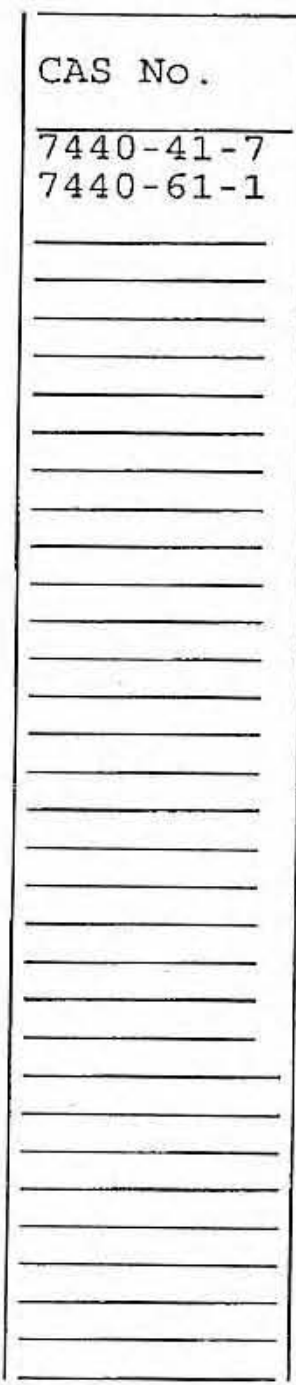

Color Before:

Color After:
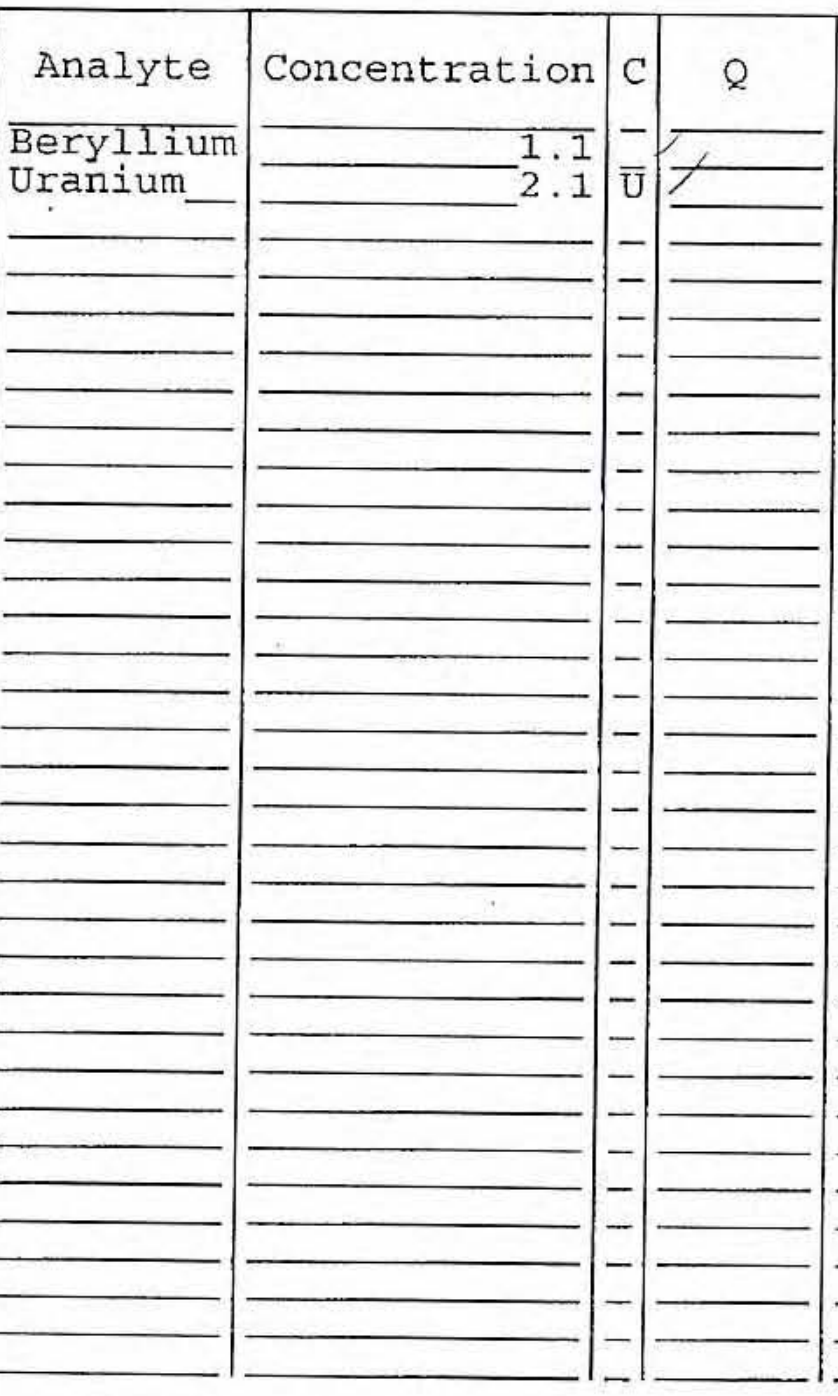

Clarity Before:

Clarity After:

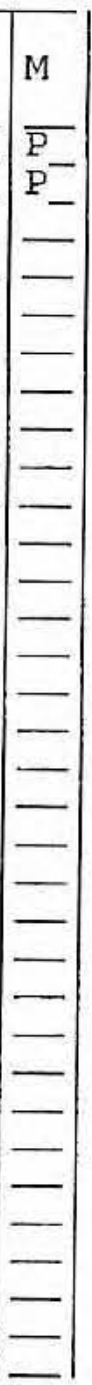

Texture:

Artifacts :

Comments :

NEDS - 17A 
1

INORGANIC ANALYSES DATA SHEET
EPA SAMPLE NO.

$17 \mathrm{~B}$
Lab Name: LIONVILLE LABORATORY

Lab Code: LVLI Matrix (soil/water): soIL Level (low/med): LOW $\%$ Solids:
Contract: 60052

SAS No. :

SDG NO.: V2742

Lab Sample ID: 0609L858-0̄14

Date Received: 09/01/06

Concentration Units (ug/L or $\mathrm{mg} / \mathrm{kg}$ dry weight): MG/KG

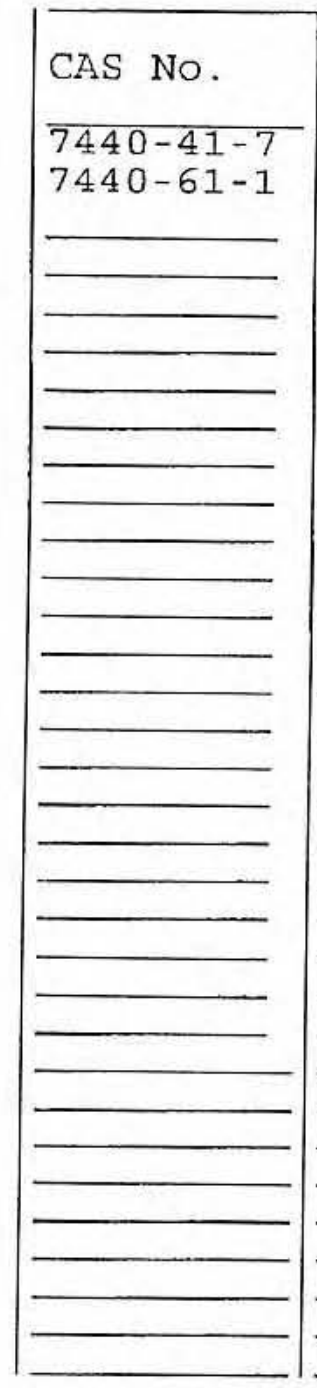

Color Before:

Color After:

comments :

NEDS - 17B

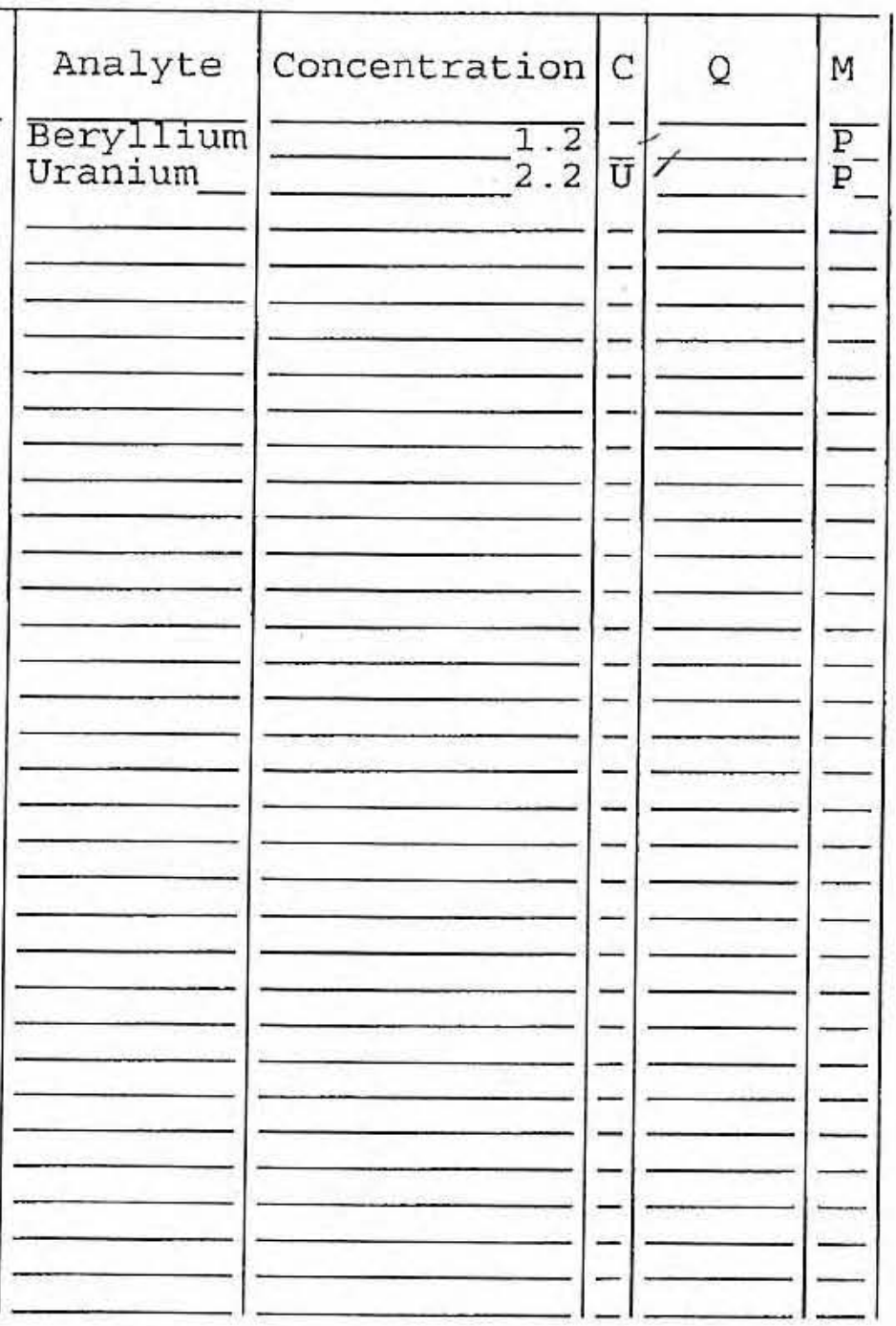

Clarity Before: Clarity After:
Texture: Artifacts: 


\section{U.S. EPA.}

\section{INORGANIC ANALYSES DATA SHEET}

EPA SAMPLE NO.

$18 \mathrm{~A}$

Lab Name: LIONVILLE_LABORATORY_Contract: 60052

Lab Code: LVLI

Matrix (soil/water): SoIL

Level (low/med): LOW

음 Solids :
SAS NO.:

Lab Sample ID: 0609L858-0̄15

Date Received: 09/01/06

Concentration Units (ug/L or $\mathrm{mg} / \mathrm{kg}$ dry weight): $\mathrm{MG} / \mathrm{KG}$

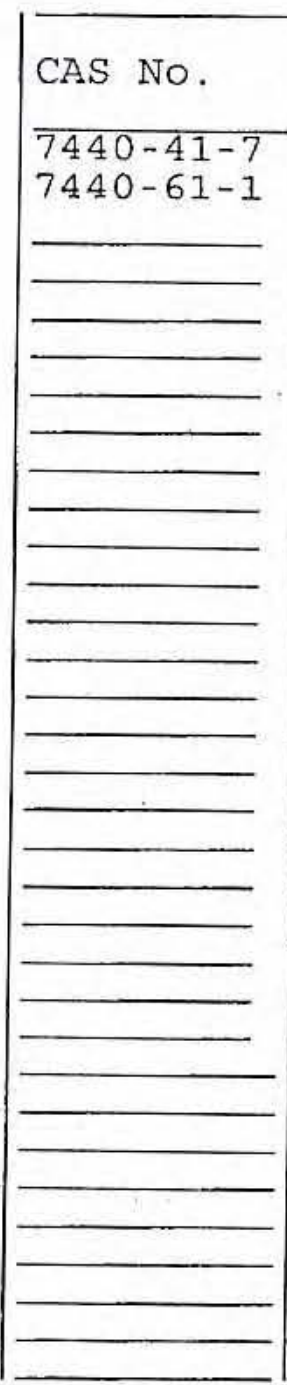

Color Before: Color After:

Comments :

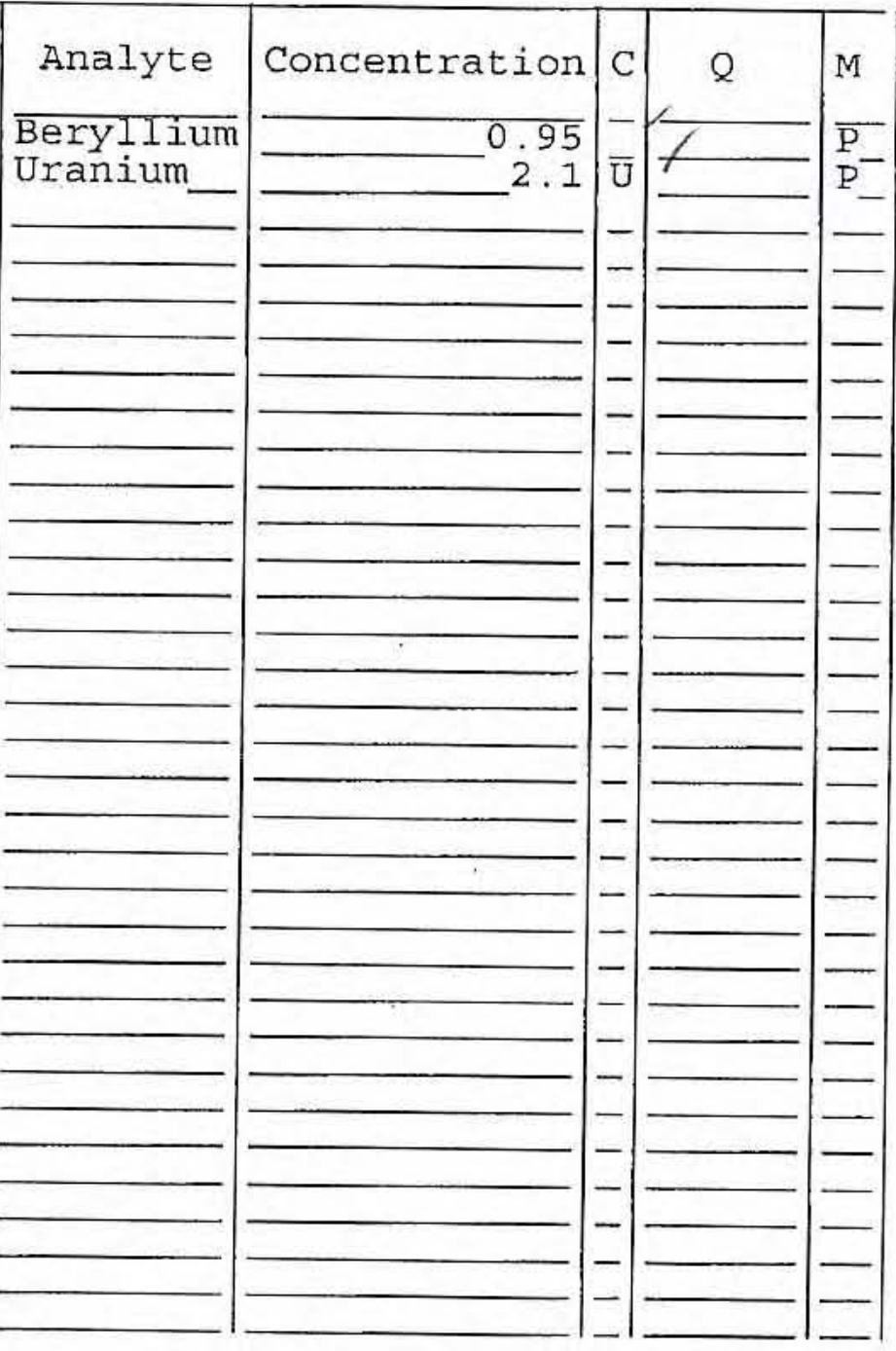

Clarity Before: Clarity After:
Texture:

Artifacts:

NEDS - 18A 
Lab Name: LIONVILLE_LABORATORY Lab Code: LVLI Matrix (soil/wa Level (low/med):

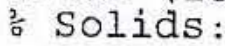

Contract: 60052

SAS NO. :

Case No.: NEDS

\section{$18 \mathrm{~B}$}

SDG NO.: V2742

Lab Sample ID: 0609L858-0̄16

Date Received: 09/01/06

Concentration Units (ug/L or $\mathrm{mg} / \mathrm{kg}$ dry weight): MG/KG

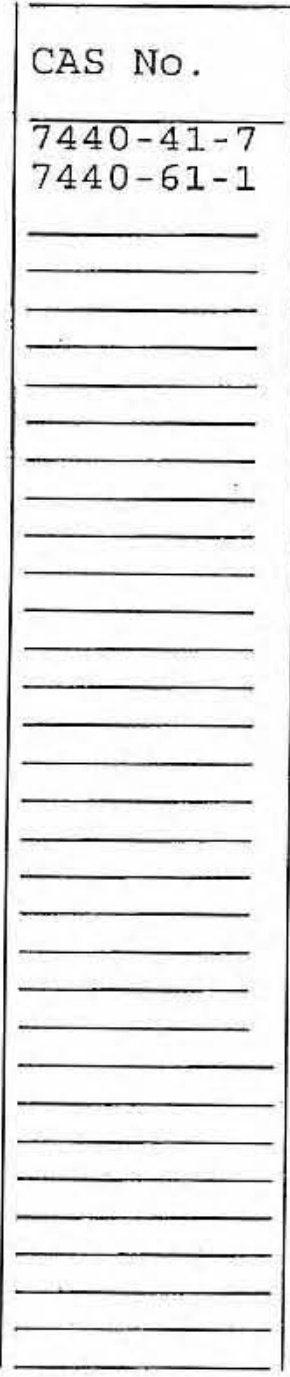

Iolor Before:

zolor After:

Zomments :

NEDS - 18B

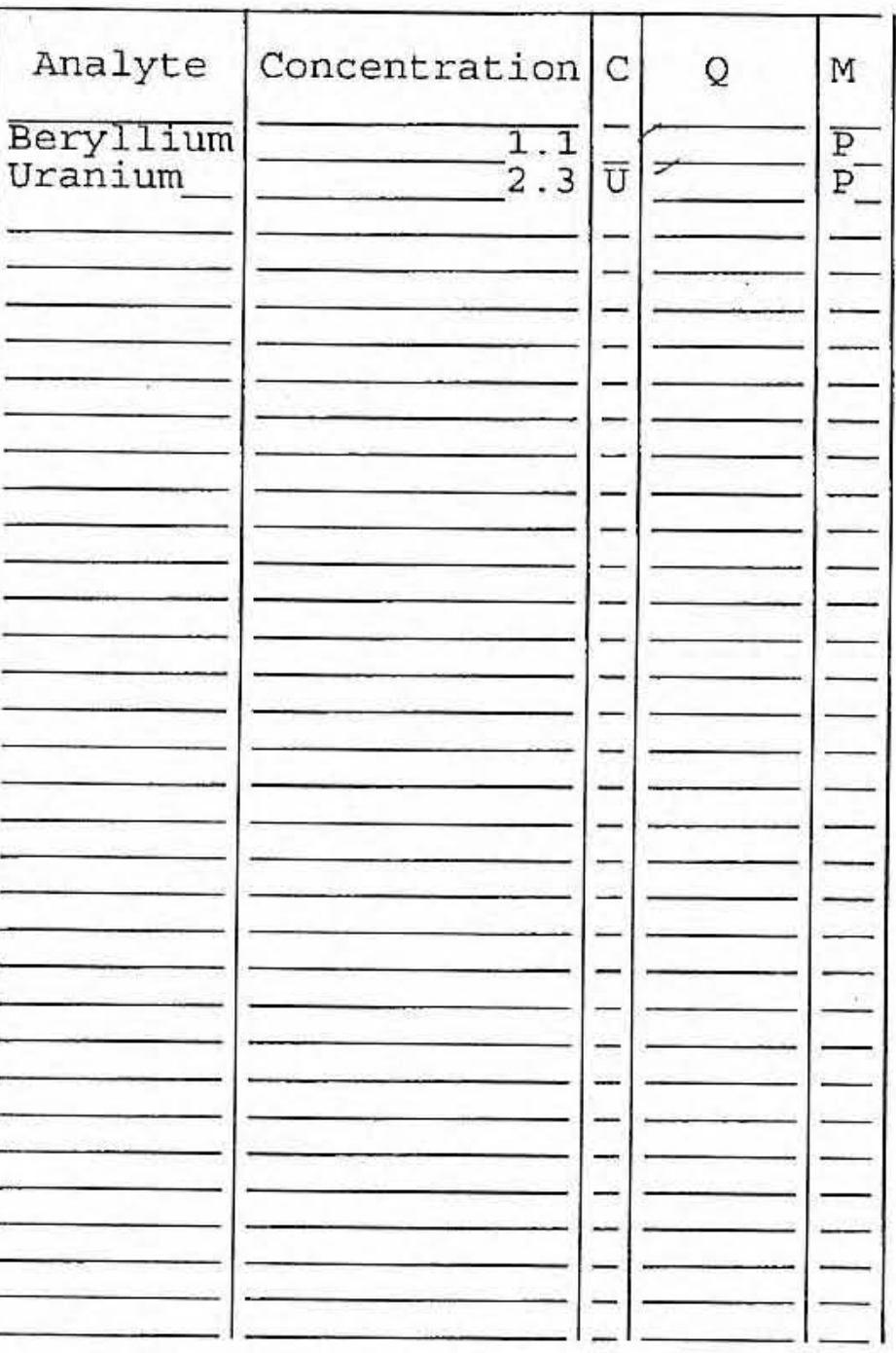

Clarity Before: Clarity After:
Texture:

Artifacts : 


\section{U.S. EPA}

1

INORGANIC ANALYSES DATA SHEET
EPA SAMPLE NO.

\section{$19 A$}

Lab Name: LIONVILLE LABORATORY

Lab Code: LVLI

Matrix (soil/water): SOIL

Level (low/med) :

$\circ$ Solids :
Contract: 60052

SAS No.:

Lab Sample ID: 0609L858-0̄17 LOW $-92 \overline{8}$

Date Received: 09/01/06

Concentration Units (ug/L or $\mathrm{mg} / \mathrm{kg}$ dry weight): $\mathrm{MG} / \mathrm{KG}$

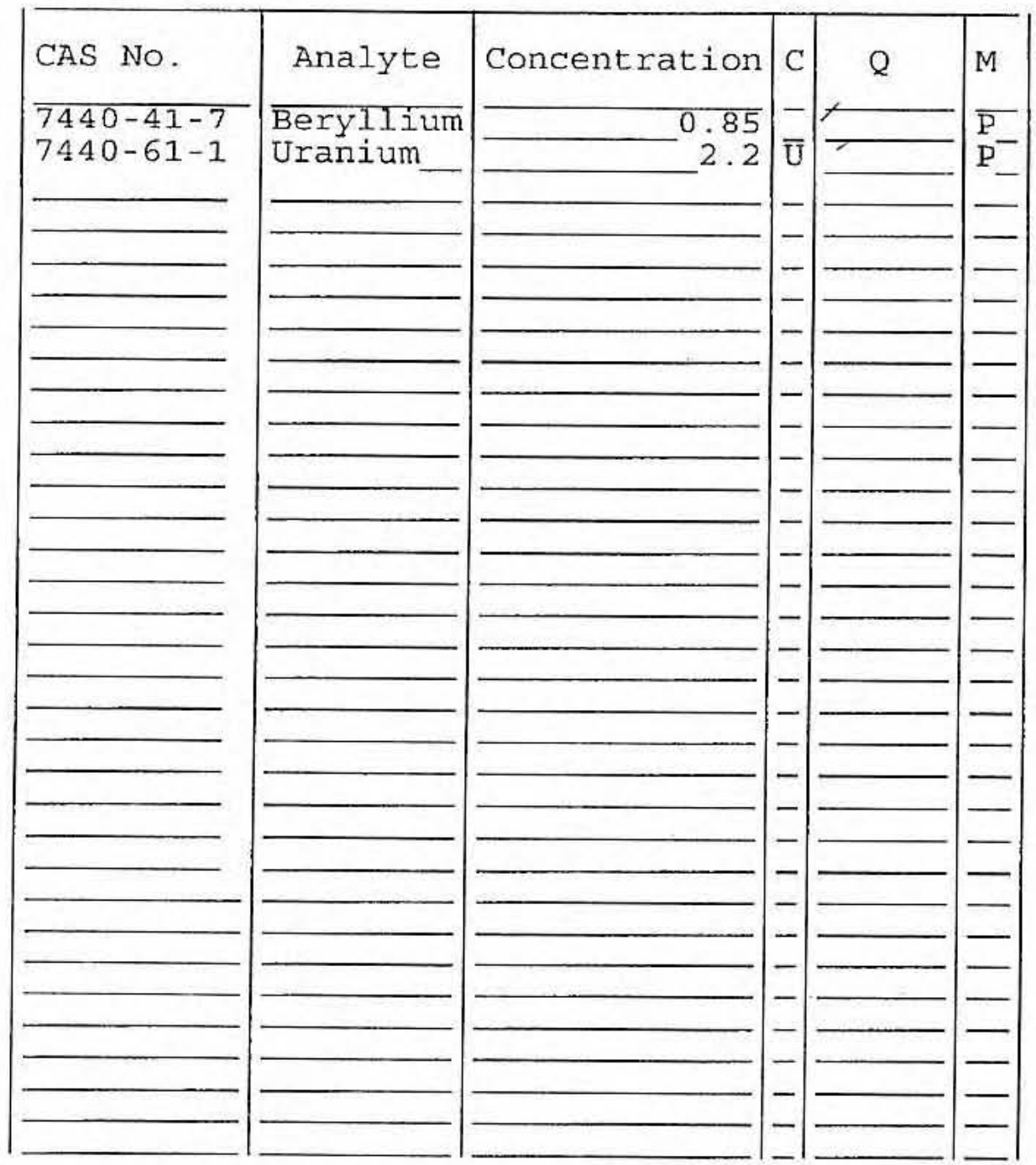

Jolor Before:

Dolor After:
Clarity Before:

Clarity After:
Texture:

Artifacts :

Jomments :

NEDS - 19A 
U.S. EPA

INORGANIC ANALYSES DATA SHEET

EPA SAMPLE NO.

Lab Name: LIONVILLE_LABORATORY

Lab Code: LVLI

Matrix (soil/water): SOIL

Level (low/med):

LOW

Contract: 60052

$19 B$

: Solids:

SAS No.:

Lab Sample ID: 0609L858-0̄18

Date Received: 09/01/06

Concentration Units (ug/L or $\mathrm{mg} / \mathrm{kg}$ dry weight): MG/KG

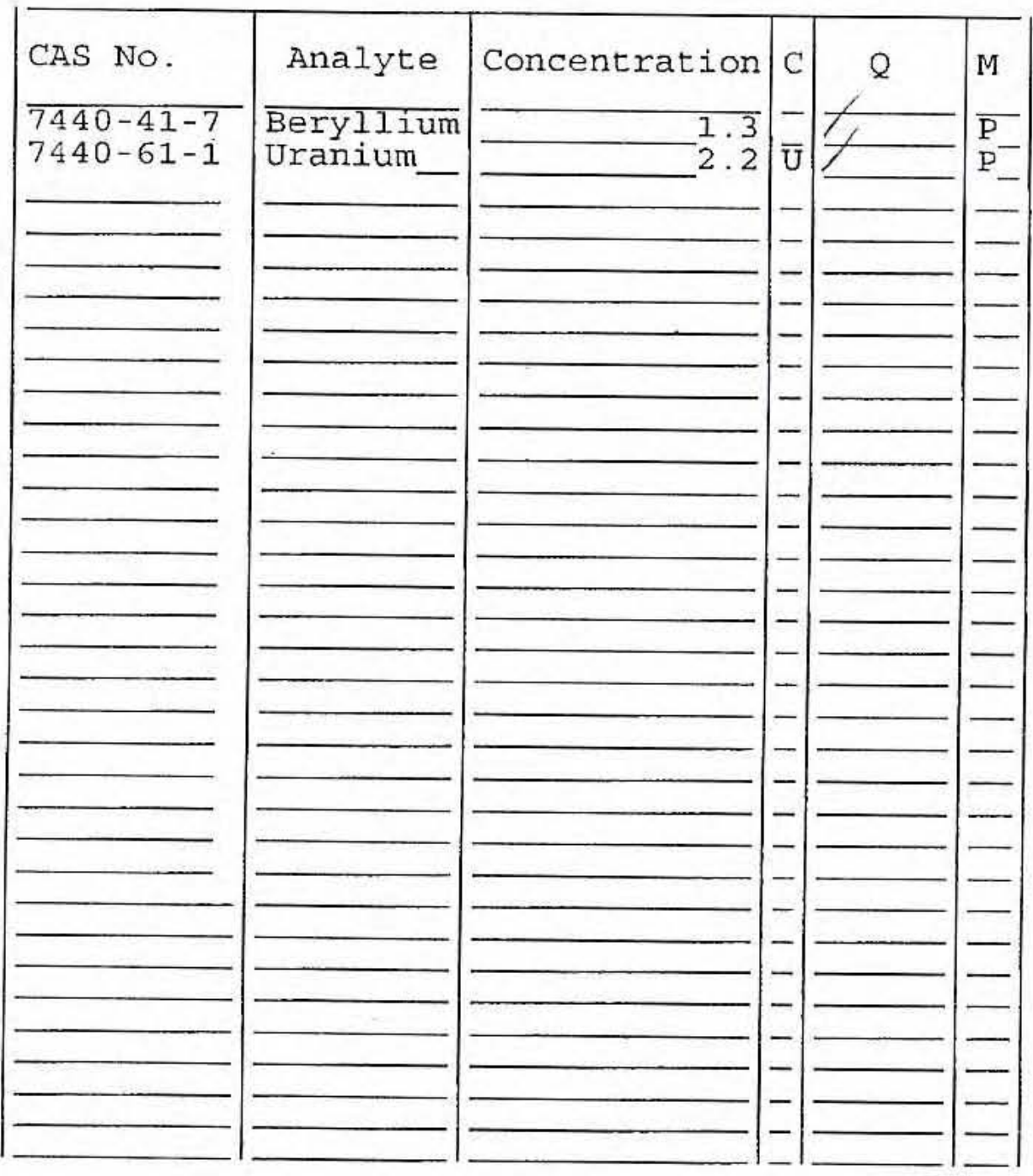

Color Before:

Color After:

Comments:

NEDS-19B
Clarity Before:

Clarity After:
Texture:

Artifacts: 


\section{U.S. EPA}

\section{INORGANIC ANALYSES DATA SHEET}

EPA SAMPLE NO.

\section{$20 \mathrm{~A}$}

SDG NO.: V2742

Lab Name: LIONVILLE_LABORATORY Contract: 60052

Lab Code: LVLI

Matrix (soil/water): SoIL

Level (low/med): LOW

\% Solids: $\quad-96 \overline{.0}$

SAS No. :

Lab Sample ID: 0609L858-̄̄19

Date Received: 09/01/06

Concentration Units (ug/L or $\mathrm{mg} / \mathrm{kg}$ dry weight): MG/KG

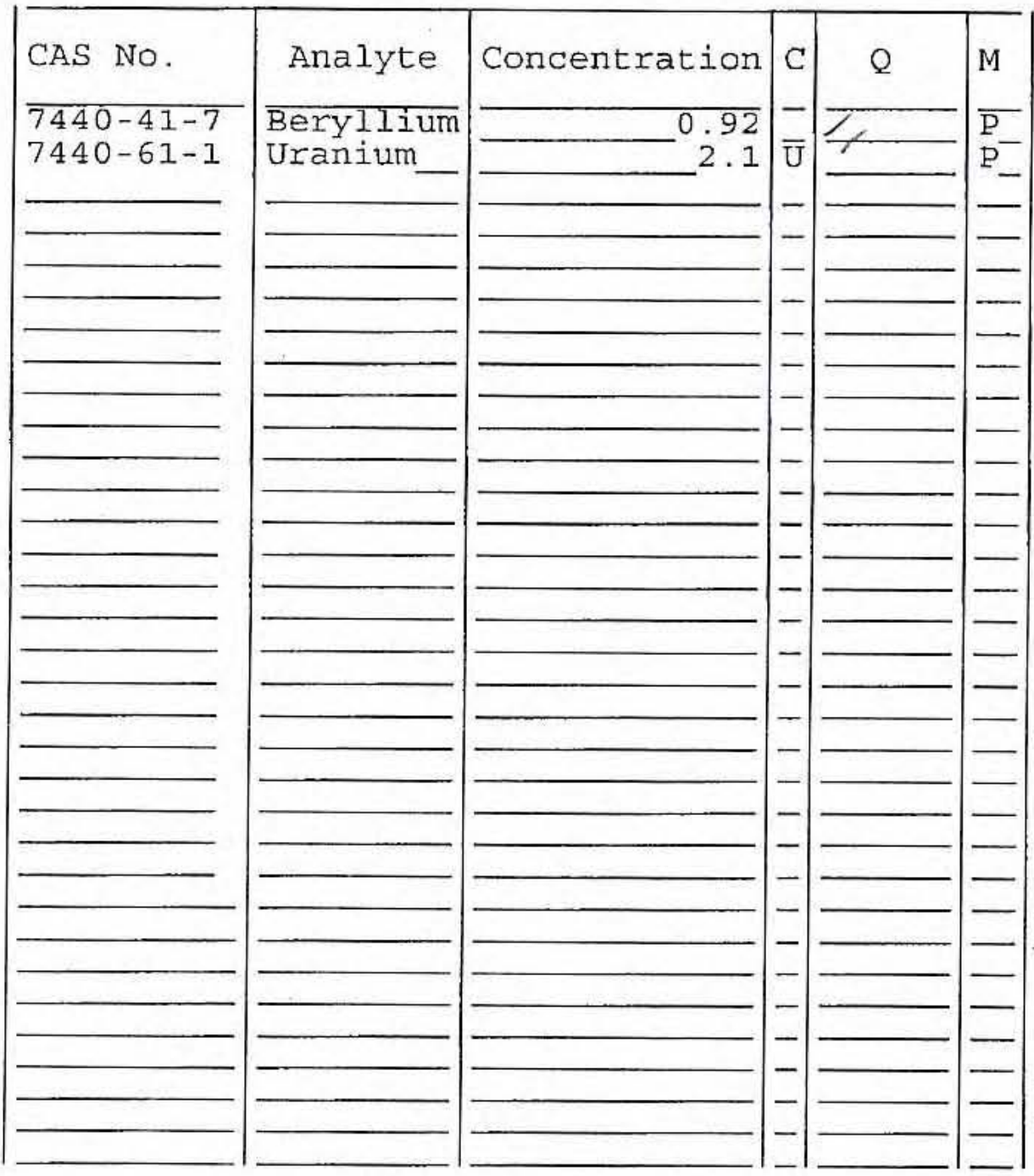

Color Before:

Clarity Before:

Clarity After:

Texture:

Artifacts:

Comments :

NEDS - 2OA 
EPA SAMPLE NO.

$$
20 \mathrm{~B}
$$

Lab Name: LIONVILLE_LABORATORY

Lab Code: LVLI

$\begin{array}{ll}\text { Matrix (soil/water): SOIL } & \text { LOW } \\ \text { Level (low/med): } & \text { LOW }\end{array}$

\% Solids :
Contract: 60052

SAS NO.:

SDG NO.: V2742

Lab Sample ID: 0609L858-0̄20

Date Received: 09/01/06

Concentration Units (ug/L or $\mathrm{mg} / \mathrm{kg}$ dry weight): MG/KG

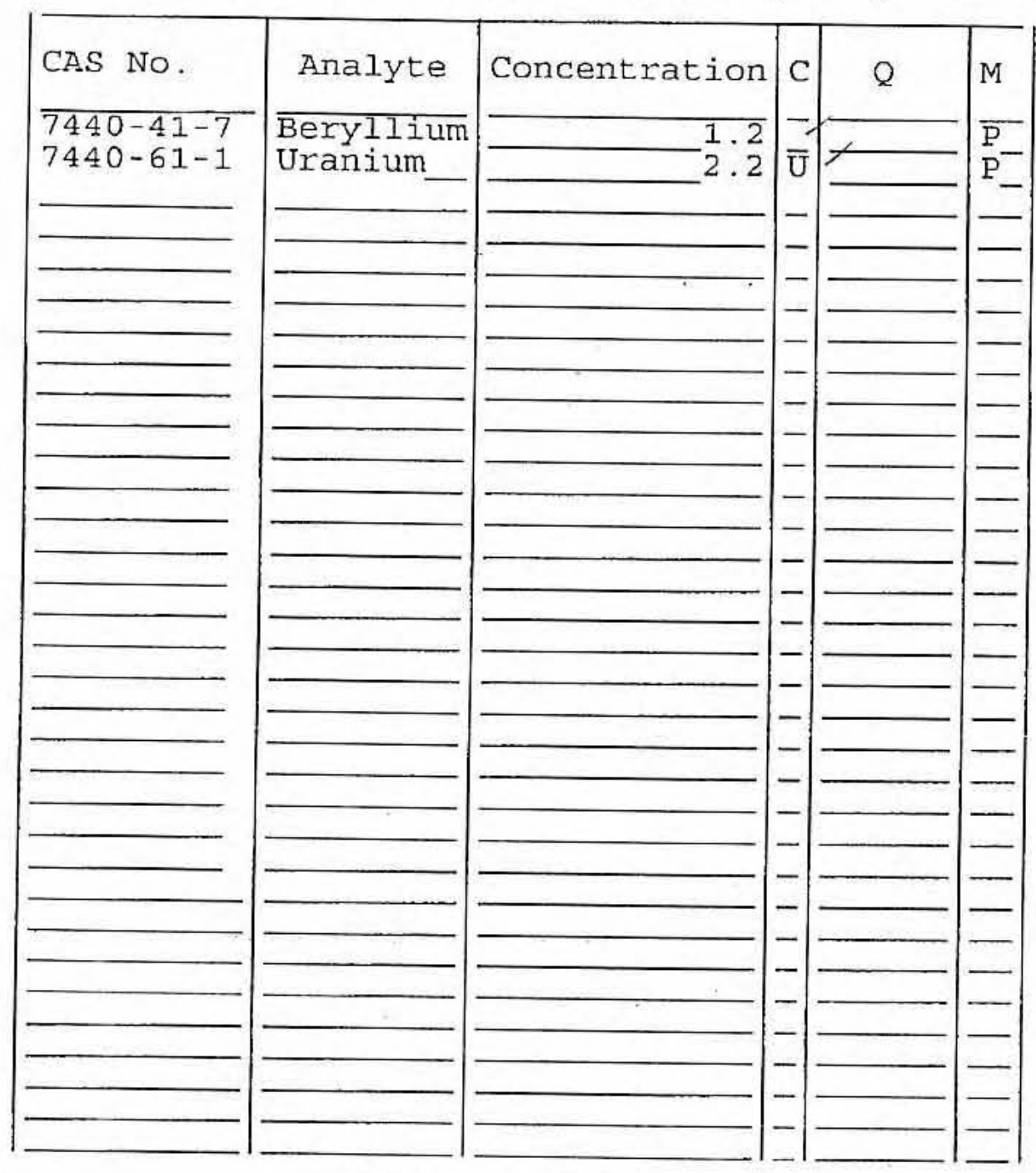

Color Before: Color After:

Comments :

NEDS $-2 O B$
Clarity Before:

Clarity After:
Texture:

Artifacts : 


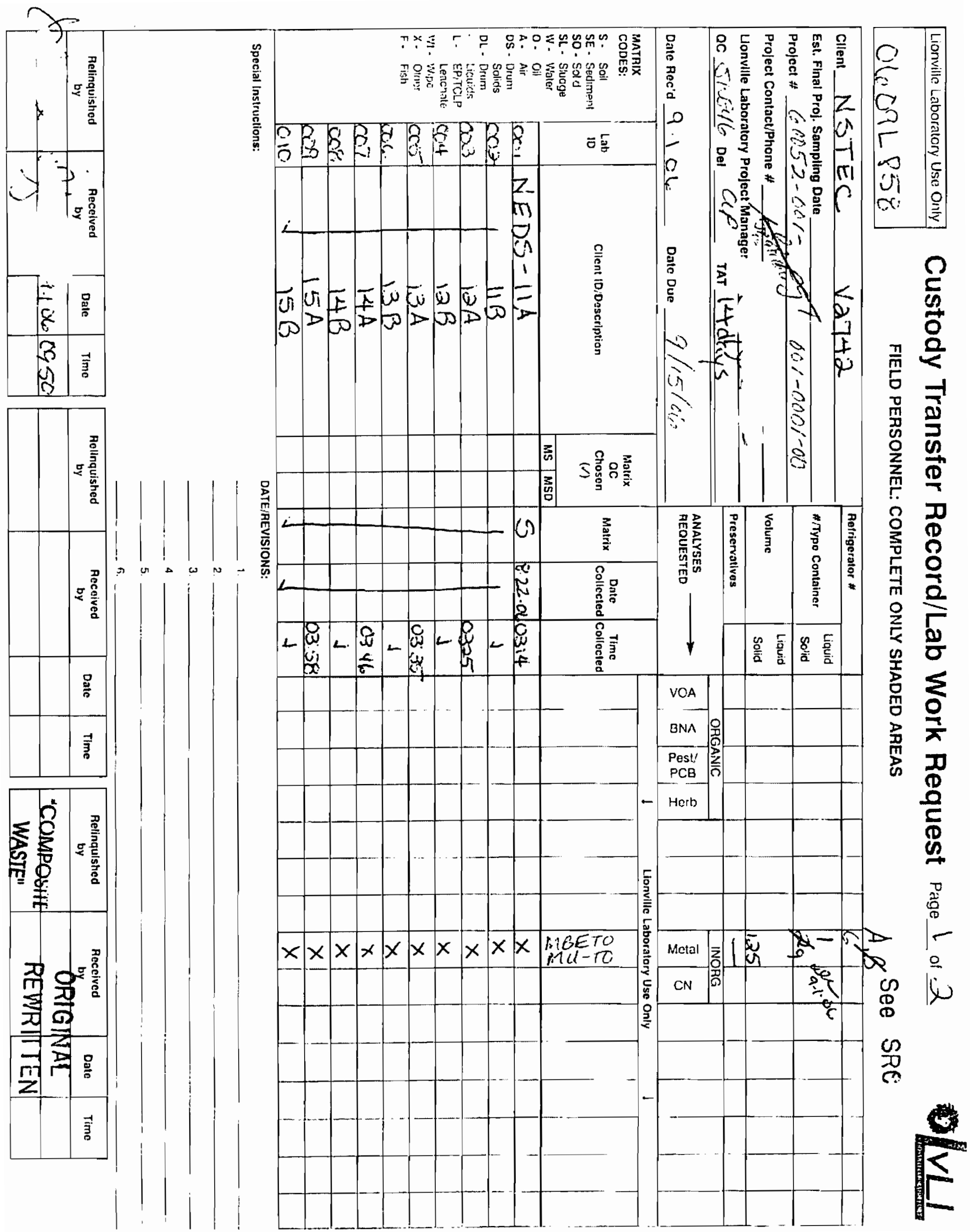




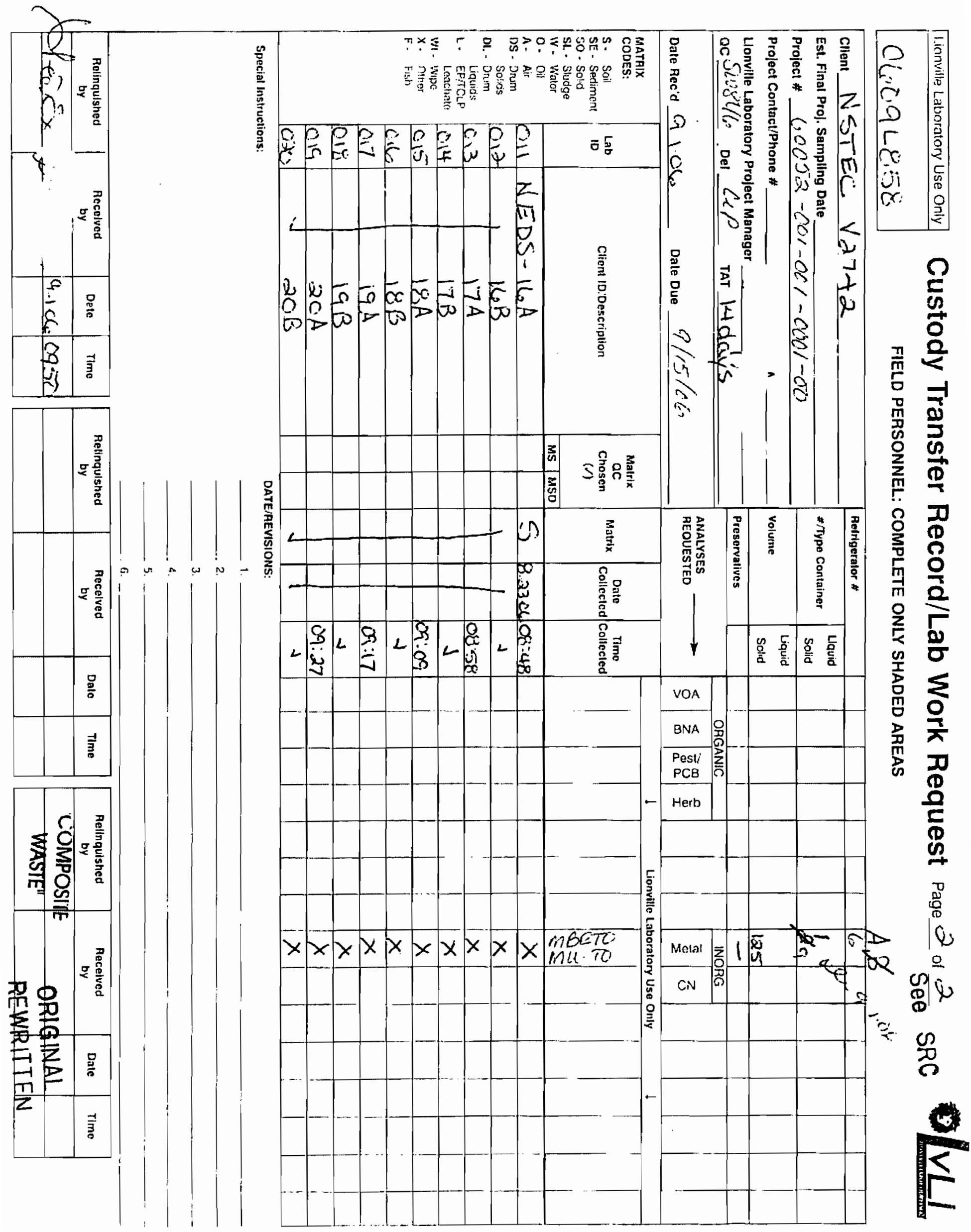




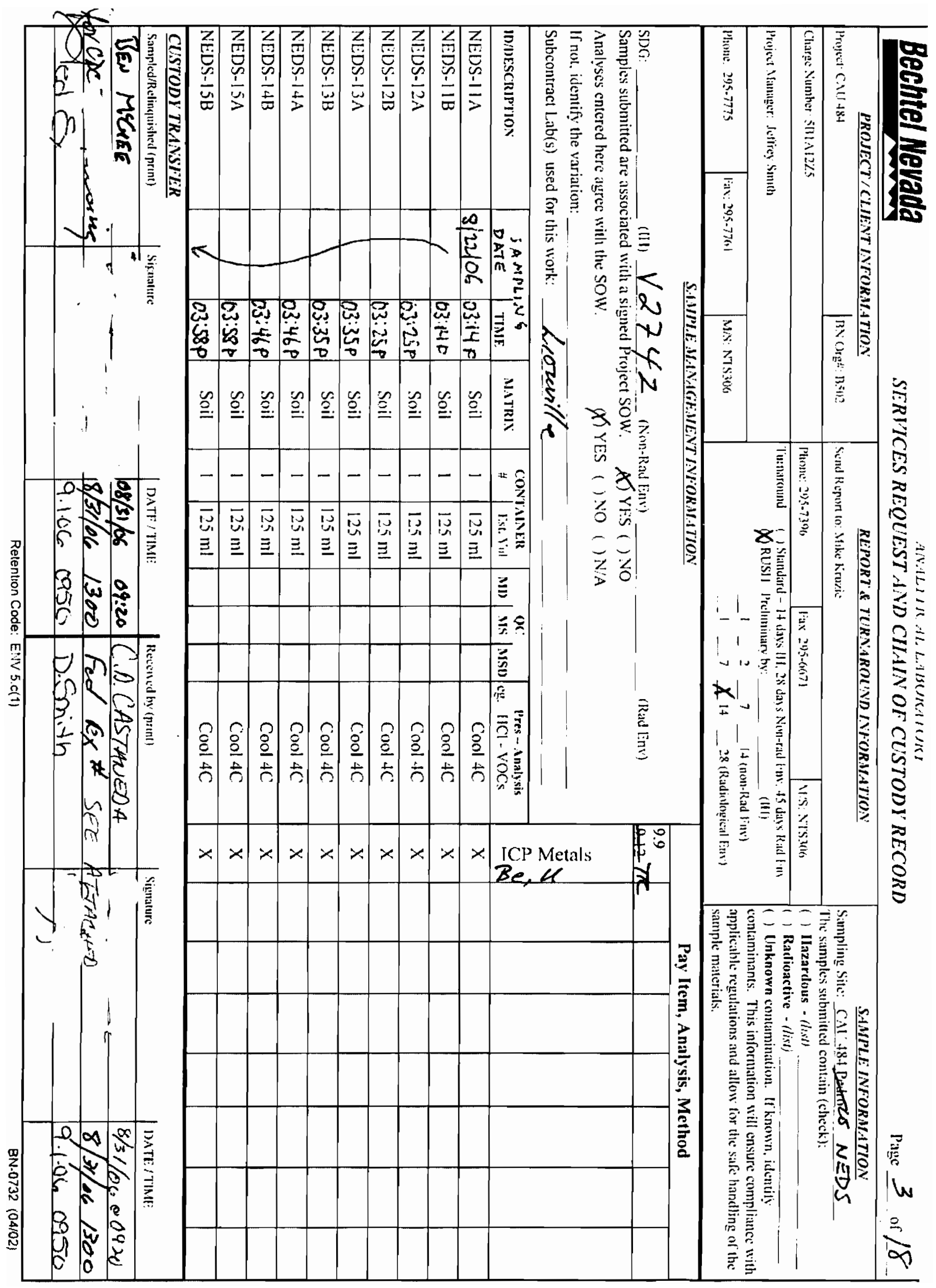




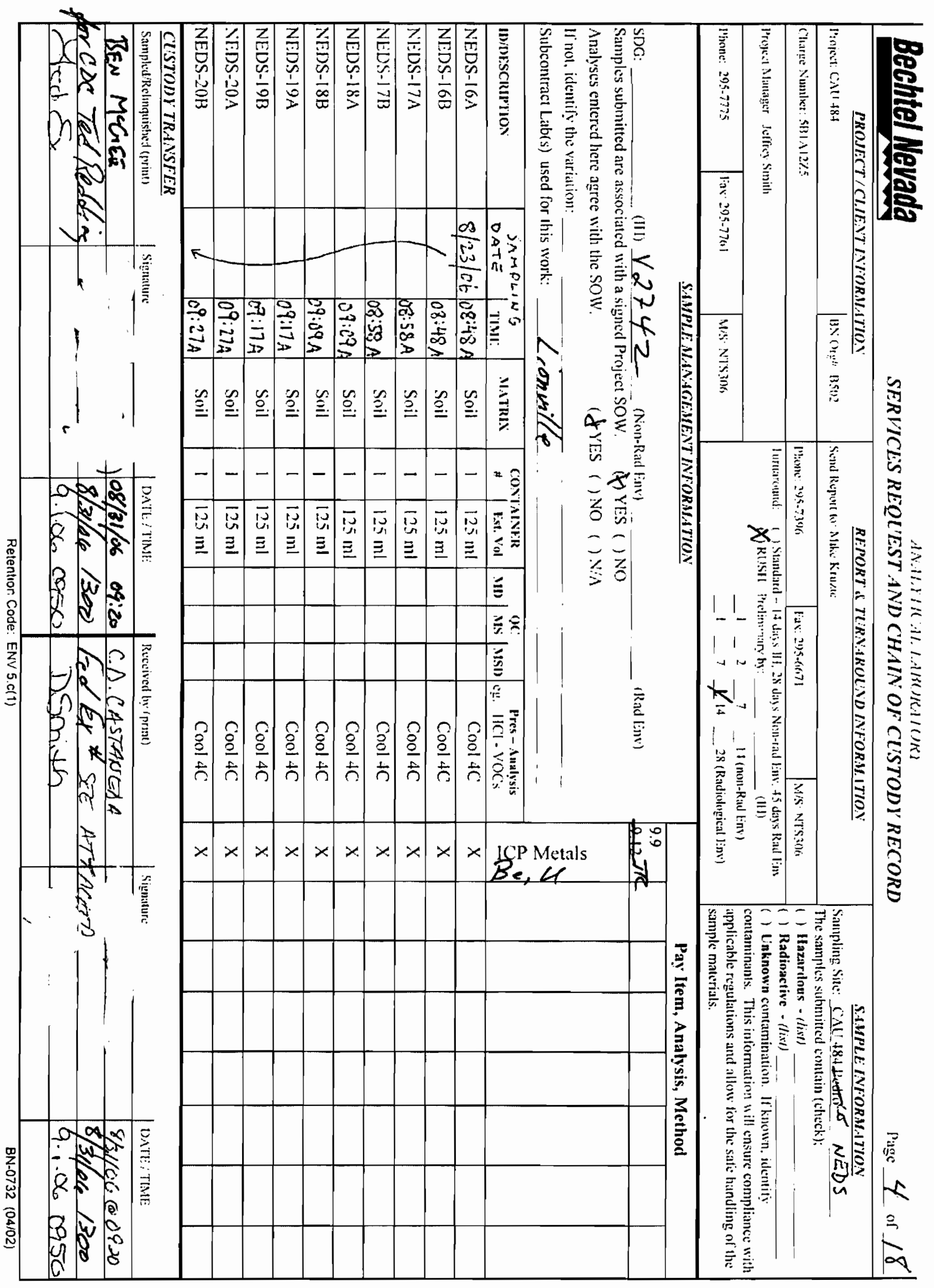




\section{U.S. EPA}

\section{INORGANIC ANALYSES DATA SHEET}

EPA SAMPLE NO.

Lab Name: LIONVILLE_LABORATORY

Lab Code: LVLI

Contract: 60052

$21 \mathrm{~A}$

Matrix (soil/water): Level. (low/med):

\% Solids:

Case No.: NEDS_ SAS No.: SOIL
LOW-
$\quad 93 . \overline{3}$
SDG NO.: V2742

Lab Sample ID: 0609L859-0̄01

Date Received: 09/01/06

Concentration Units (ug/L or $\mathrm{mg} / \mathrm{kg}$ dry weight): MG/KG

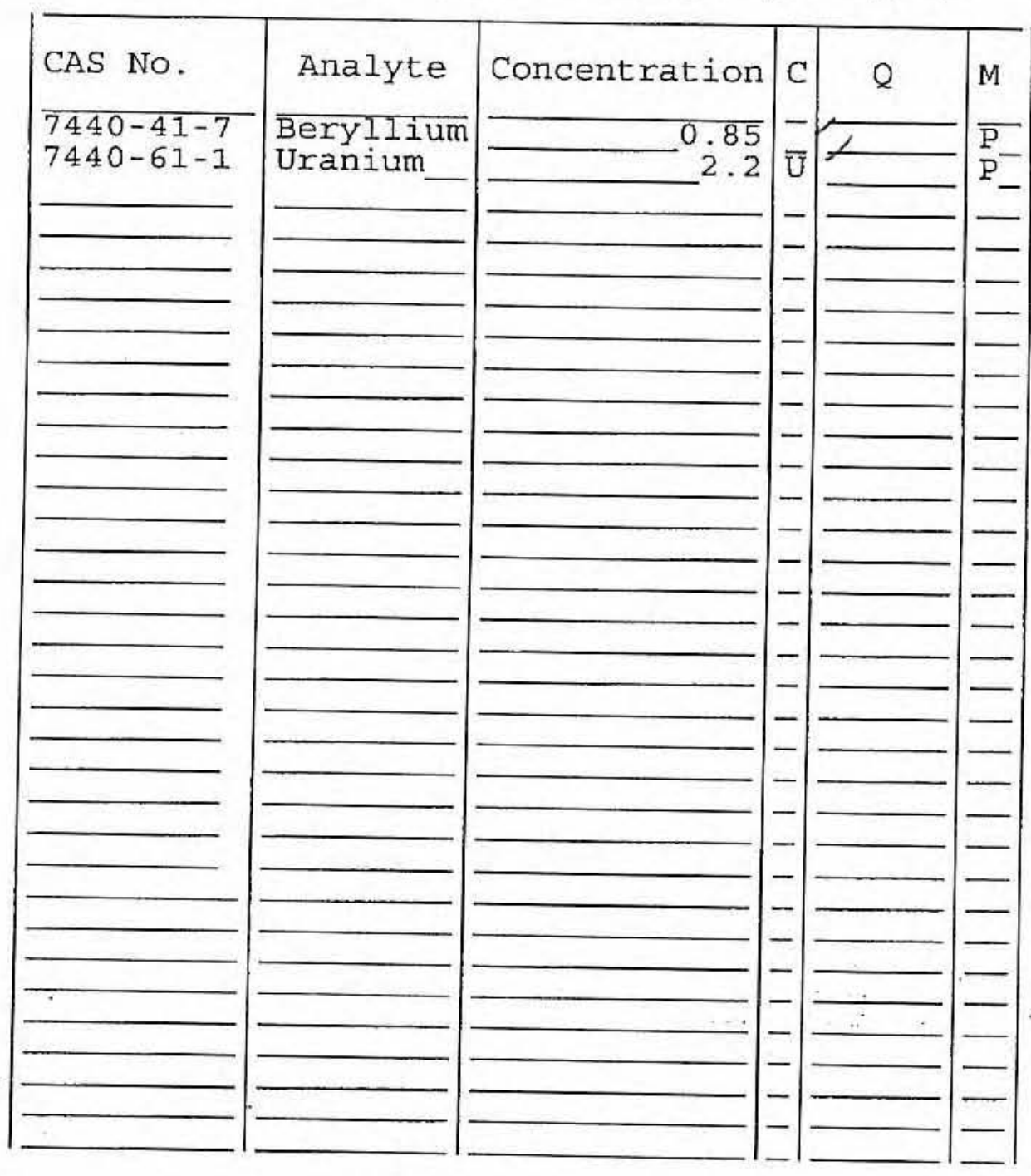

Jolor Before: zolor After: Jomments : NEDS $-21 \mathrm{~A}$
Clarity Before: Clarity After:
Texture: Artifacts: 
1 INORGANIC ANALYSES DATA SHEET
EPA SAMPLE NO.

$$
21 B
$$

SDG NO.: V2742

Lab Sample ID: 0609L859-0̄02

Date Received: 09/01/06

Lab Name: LIONVILLE_LABORATORY_ Contract: 60052

Matrix (soil/water): SOIL

Level (low/med): LOW

\% Solids:

$$
-92 \overline{1}
$$

Concentration Units (ug/L or $\mathrm{mg} / \mathrm{kg}$ dry weight): $\mathrm{MG} / \mathrm{KG}$

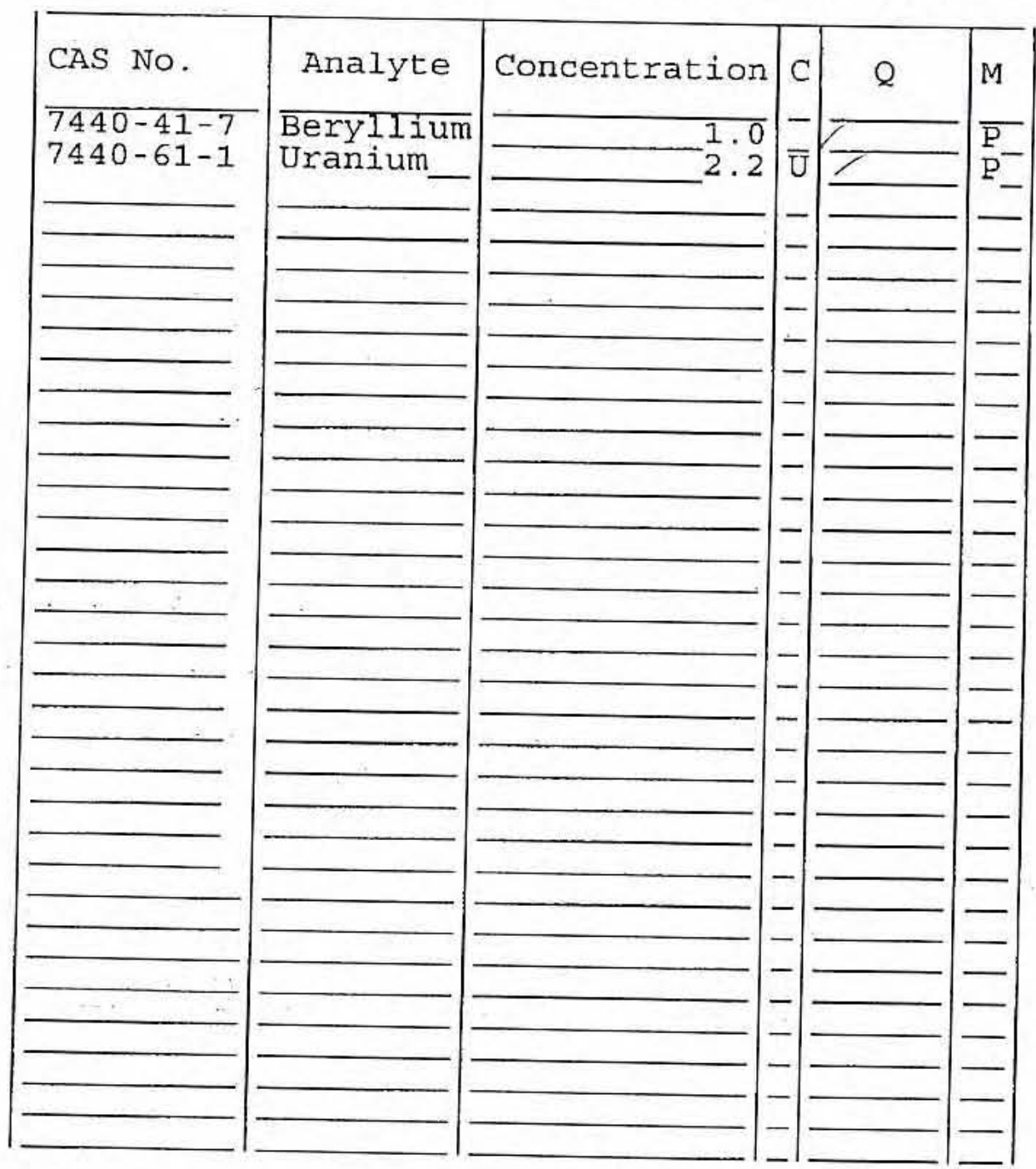

Color Before: Zolor After:

Jomments :
Clarity Before:

Clarity After:
Texture:

Artifacts : 
1

INORGANIC ANALYSES DATA SHEET
EPA SAMPLE NO.

$22 \mathrm{~A}$

Contract: 60052

SAS No.

SDG No.: V2742

Lab Code: LVLI

Matrix (soil/water): SOIL

Level (low/med): LOW

\% Solids :
Lab Sample ID: 06்09L859-0003

Date Received: 09/01/06

Concentration Units (ug/L or $\mathrm{mg} / \mathrm{kg}$ dry weight): MG/KG

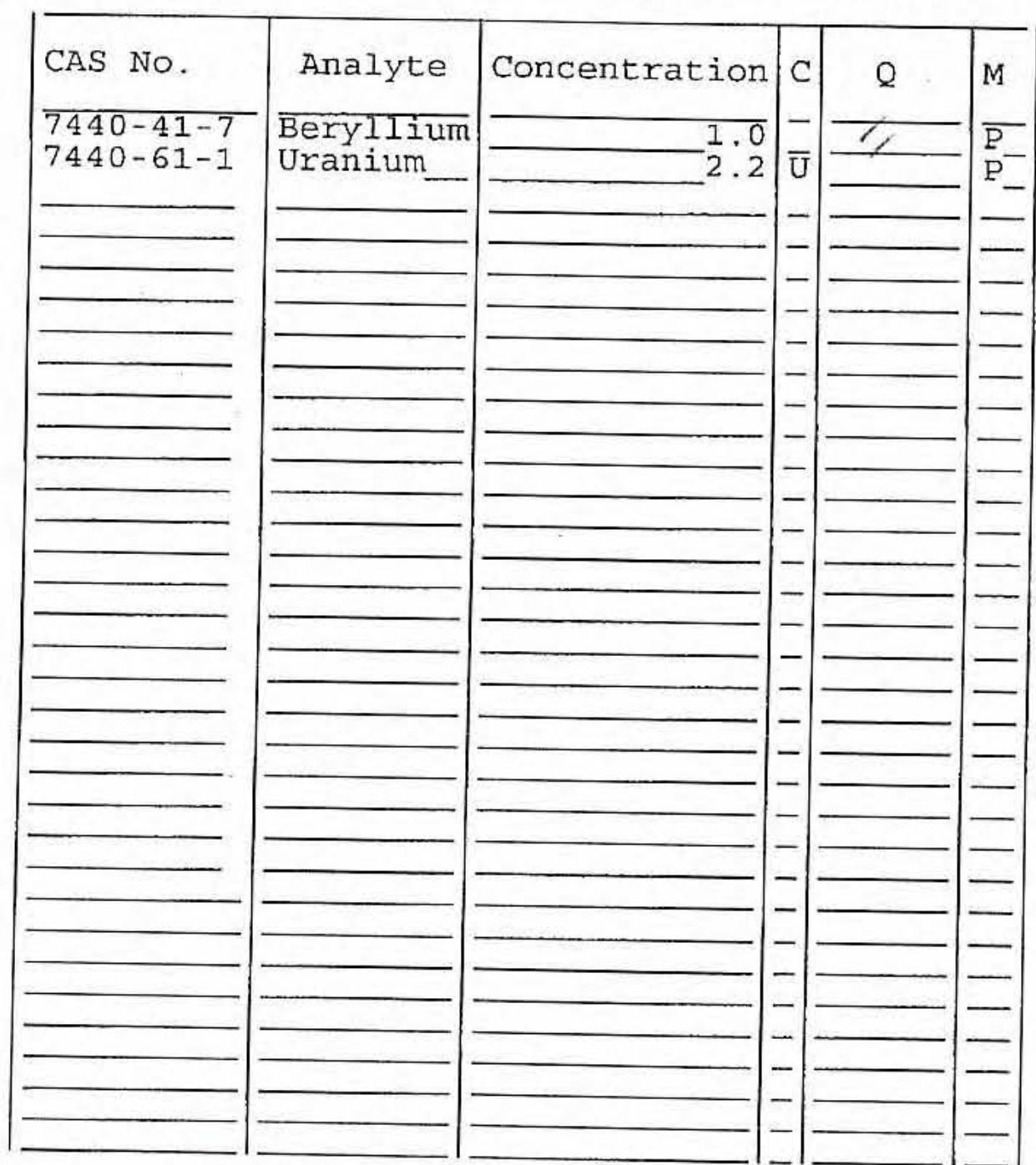

Color Before: Color After:

Comments :

NEDS - 22A
Clarity Before:

Clarity After:
Texture:

Artifacts: 
Lab Name: IIONVILLE_LABORATORY Lab Code: LVLI Matrix (soil/water): SoIL Level (low/med): LOW \% Solids:

$-90 \overline{.9}$
Contract: 60052

SAS No.:

Lab Sample ID: 0609L859-004

Date Received: 09/01/06

Concentration Units (ug/L or $\mathrm{mg} / \mathrm{kg}$ dry weight): MG/KG

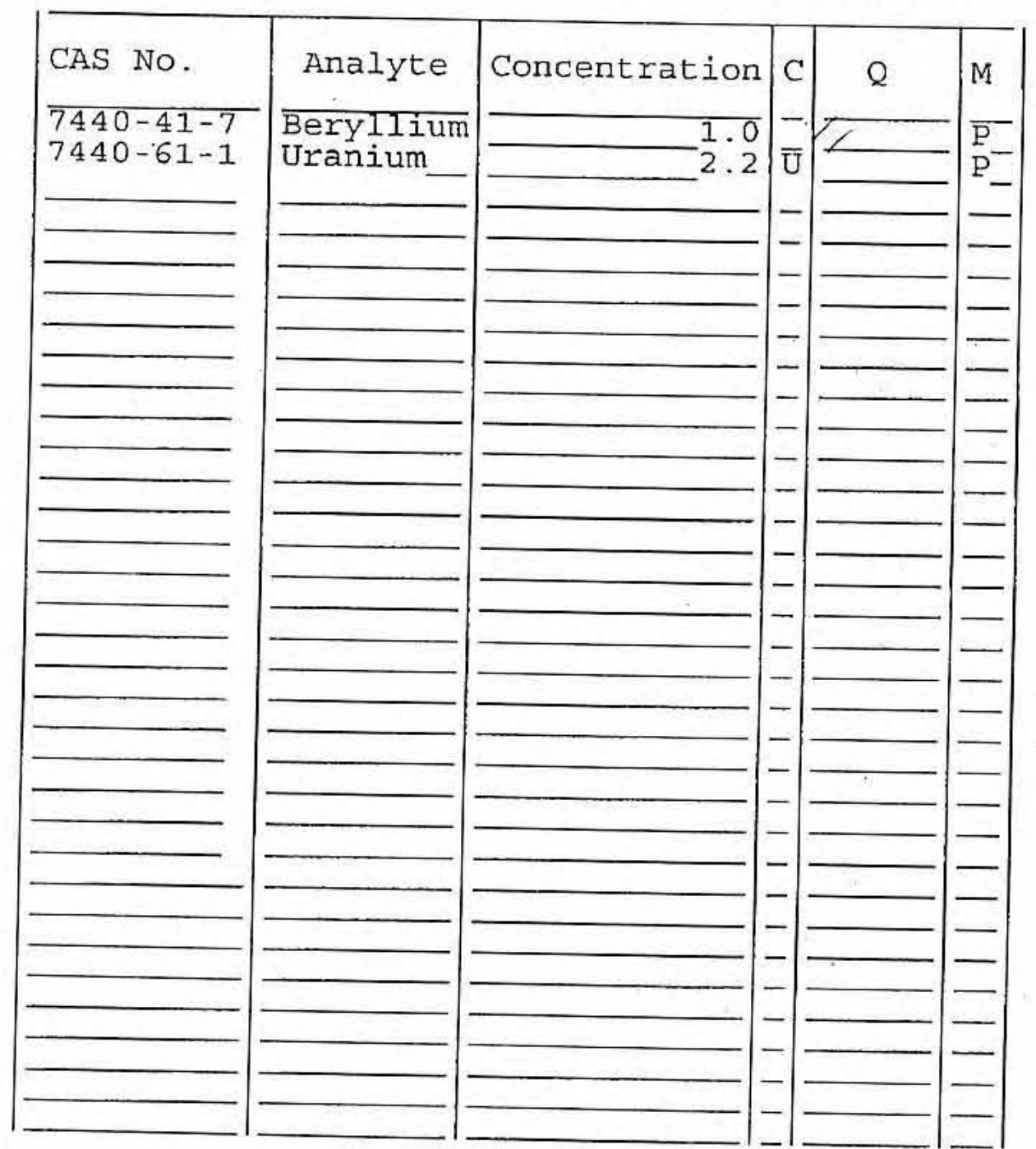

Color Before: Jolor After: Iomments : NEDS - 22B
Clarity Before:

Clarity After:
Texture: Artifacts : 
Lab Name: LIONVILLE_LABORATORY

Lab Code: LVLI

Matrix (soil/water): SoIL

Level. (low/med):

SAS NO.:

Iab Sample I

Date Received: 09/01/06

Concentration Units (ug/L or $\mathrm{mg} / \mathrm{kg}$ dry weight): MG/KG

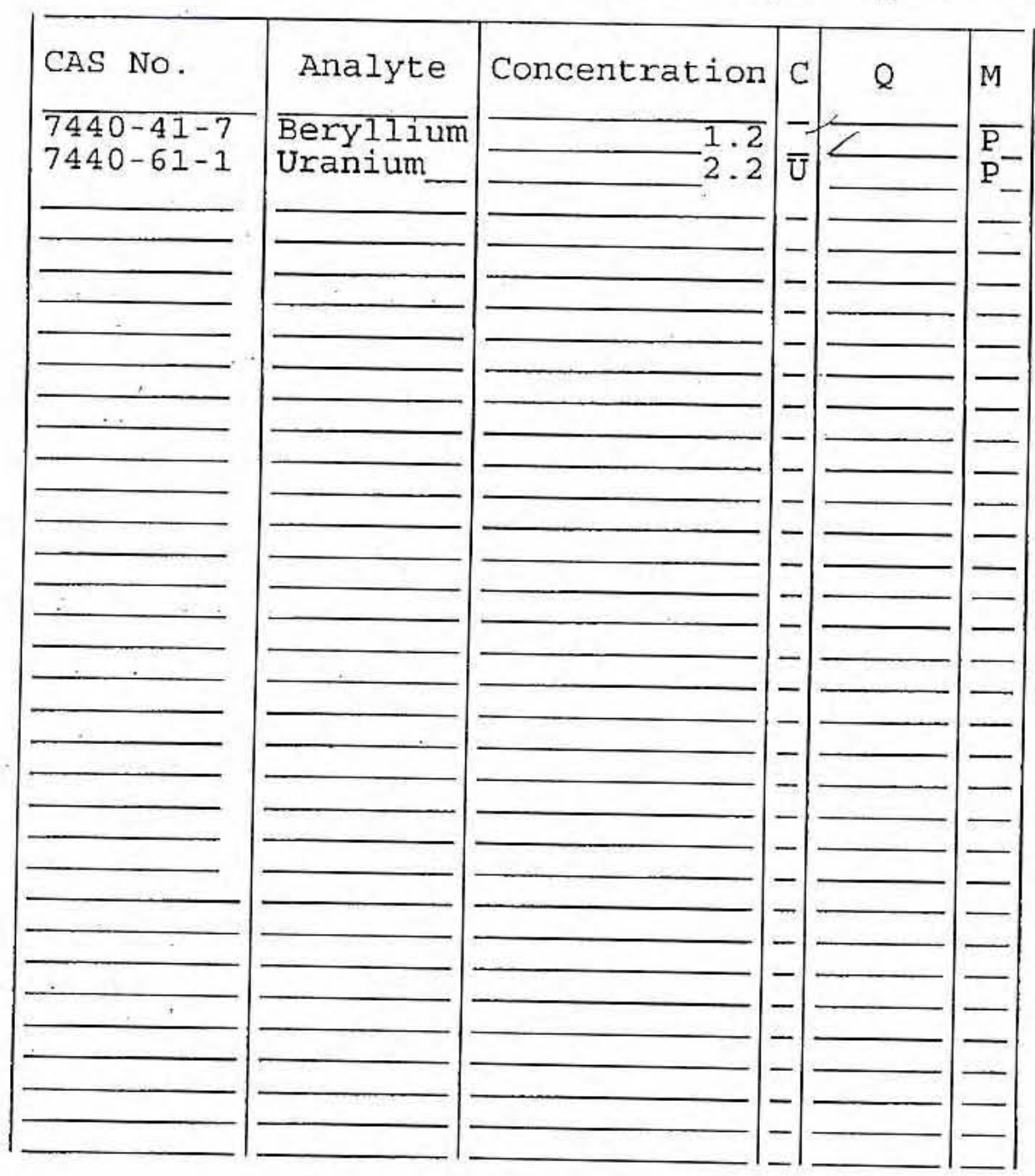

Zolor Before: zolor After:

Jomments:

NEDS - 23A
Clarity Before:

Clarity After:
Texture:

Artifacts :

\section{FORM I - IN}


EPA SAMPLE NO.

Lab Name: LIONVILLE_LABORATORY

Lab Code: IVLI

Matrix (soil/water): SoIL

Level (low/med):

: Solids:

$$
\begin{aligned}
& \text { SOIL_ } \\
& \text { LOW- } \\
& -90.7
\end{aligned}
$$

$23 \mathrm{~B}$

Contract: 60052

SAS NO.:

SDG NO.: V2742

Lab Sample ID: 0609L859-006

Date Received: 09/01/06

Concentration Units (ug/L or $\mathrm{mg} / \mathrm{kg}$ dry weight) : MG/KG

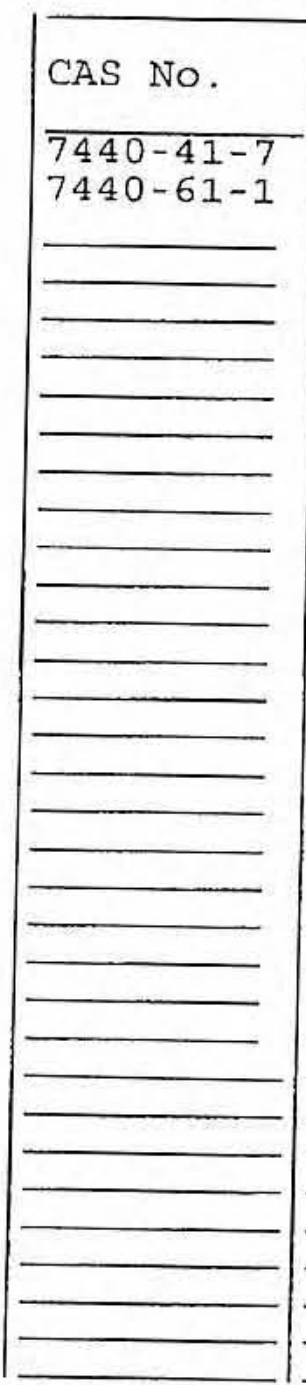

Jolor Before: Iolor After:

Zomments:

NEDS - 23B

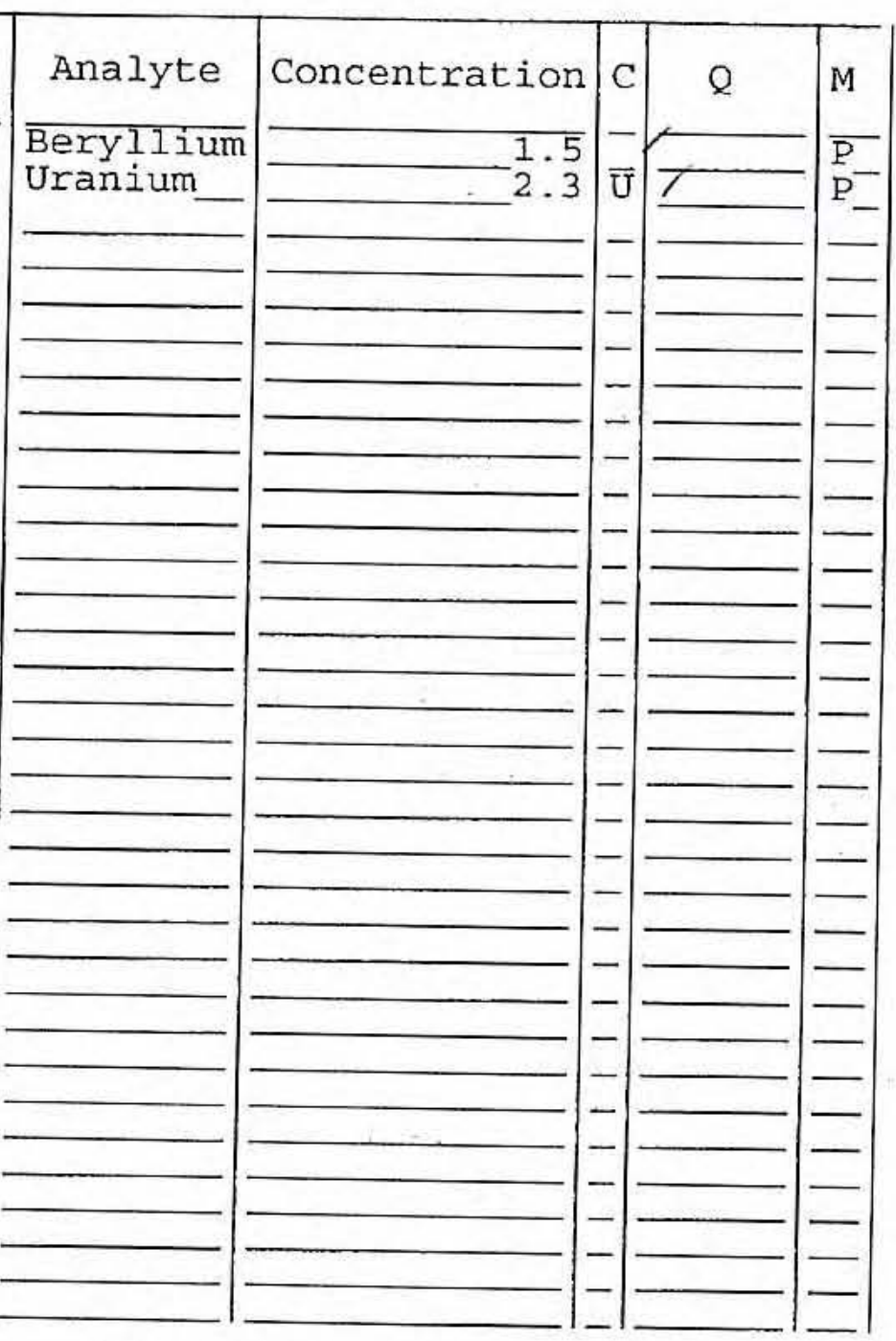

Clarity Before: Clarity After:
Texture: Artifacts: 


\section{U.S. EPA}

\section{INORGANIC ANALYSES DATA SHEET}

EPA SAMPLE NO.

Lab Name: LIONVILLE_LABORATORY

$$
24 \mathrm{~A}
$$

Lab Code: LVLI Matrix (soil/water): SOIL

Level (low/med) :

Case No.: NEDS

Contract: 60052

\% Solids :

$$
\begin{array}{r}
\text { LOW }- \\
-94.6
\end{array}
$$

SAS NO. :

SDG No.: V2742

Lab Sample ID: 0609L859-0̄07

Date Received: 09/01/06

Concentration Units (ug/L or $\mathrm{mg} / \mathrm{kg}$ dry weight): MG/KG
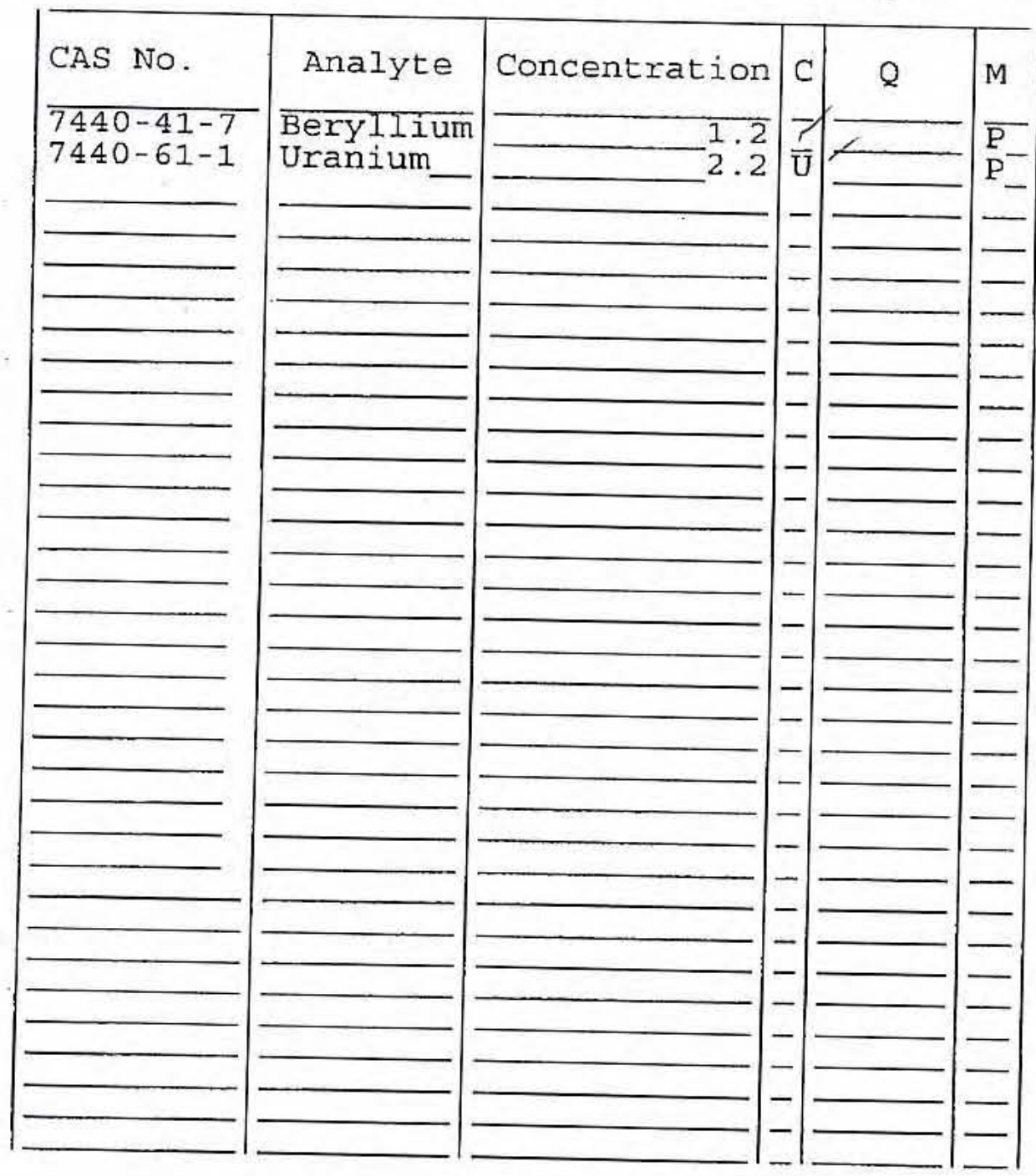

Jolor Before: Lolor After:

Clarity Before: Clarity After:
Texture:

Artifacts:

\section{Jomments :}

NEDS - 24A 


\section{U.S. EPA}

\section{INORGANIC ANALYSES DATA SHEET}

EPA SAMPLE NO.

Lab Name: LIONVILLE_LABORATORY 24B

Lab Code: IVLI

Matrix (soil/water) : SoIL

Level (low/med):

$\div$ Solids :
LOW

$-90 . \overline{5}$
Contract: 60052

SAS NO.:

Lab Sample ID: 0609L859-0̄08

Date Received: 09/01/06

Concentration Units (ug/L or mg/kg dry weight): MG/KG

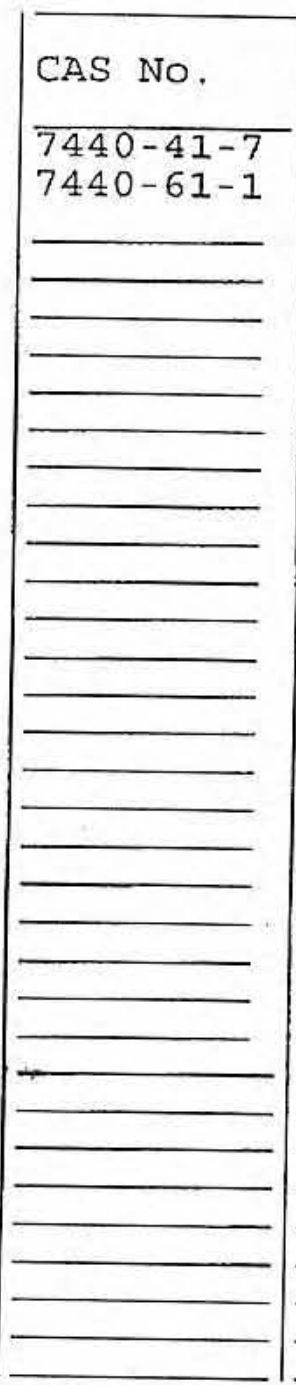

Dolor Before: Jolor After:

\section{Jomments:}

NEDS - 24B

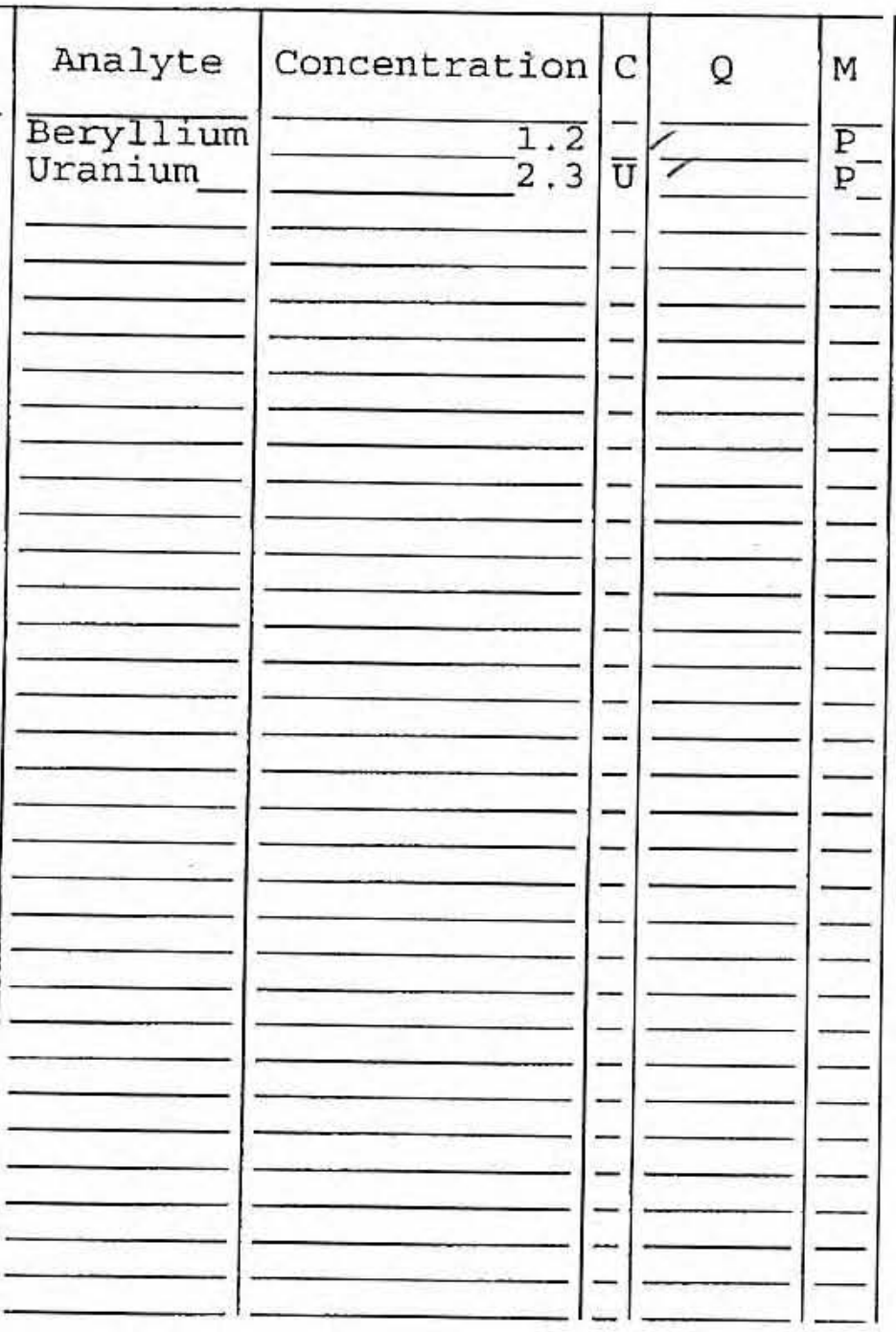

Clarity Before:

Clarity After:

Artifacts : 
U.S. EPA

\section{INORGANIC ANALYYSES DATA SHEET}

EPA SAMPLE NO.

Lab Name: LIONVILLE_LABORATORY

Lab Code: LVII

Matrix (soil/water): SoIL

Level. (low/med):

\% Solids:

\section{LOW}

$94 \overline{.1}$

$25 A$

Contract: 60052

SAS No.:

SDG NO.: V2742

Lab Sample ID: 0609L859-009

Date Received: 09/01/06

Concentration Units (ug/L or $\mathrm{mg} / \mathrm{kg}$ ary weight): MG/KG

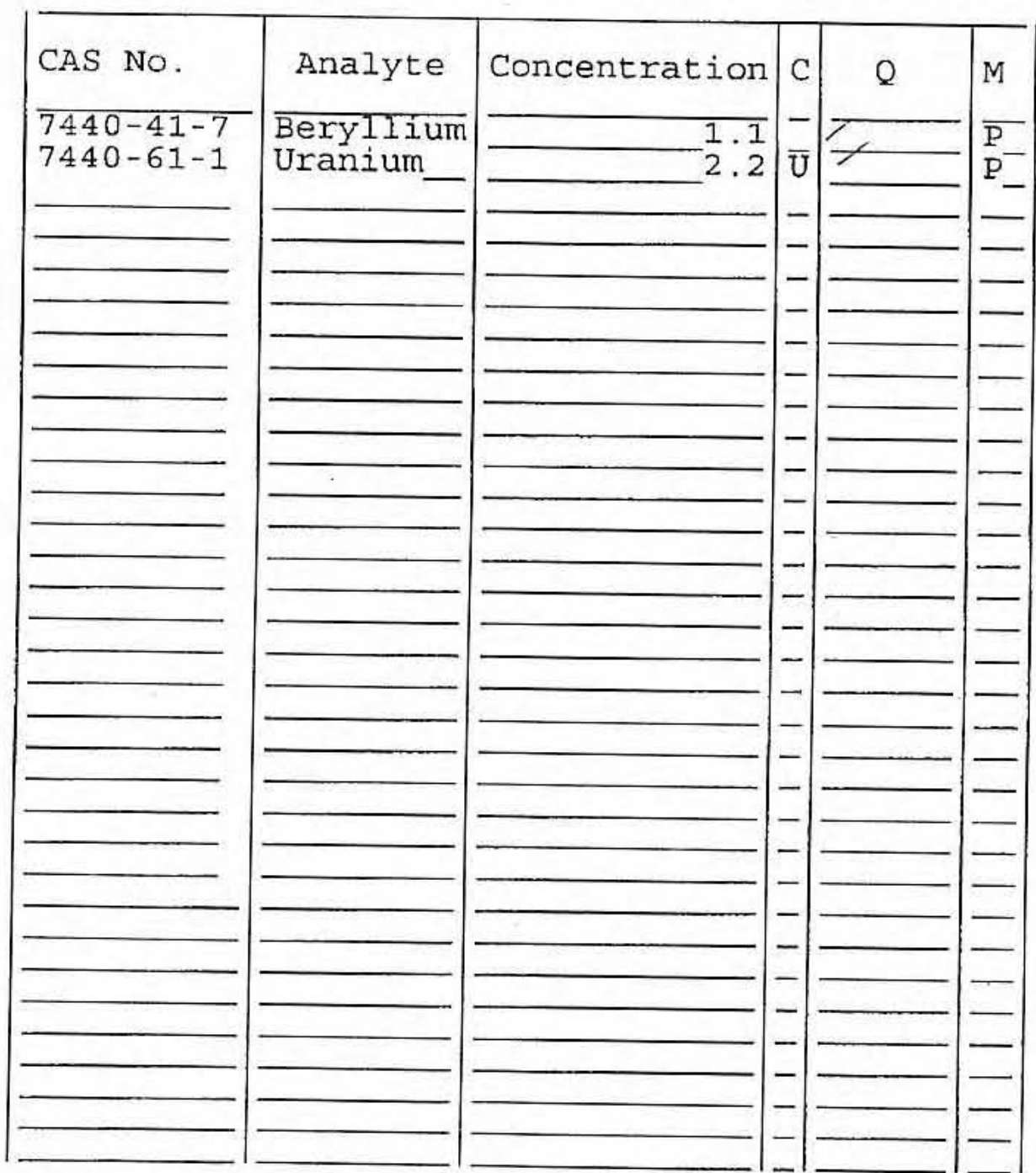

Color Before:

Color After:

Clarity Before:

Clarity After:
Texture:

Artifacts :

\section{Comments :}

NEDS - 25A 
EPA SAMPLE NO.

Lab Name: LIONVILLE_LABORATORY

Lab Code: LVLI

Matrix (soil/water):

Level. (low/med):

$\%$ Solids:
Contract: 60052

SAS NO.:

SOIL
LOW
$92 . \overline{7}$

$25 B$

SDG NO.: V2742

Lab Sample ID: 0609L859-0̄10

Date Received: 09/01/06

Concentration Units (ug/L or $\mathrm{mg} / \mathrm{kg}$ dry weight): MG/KG

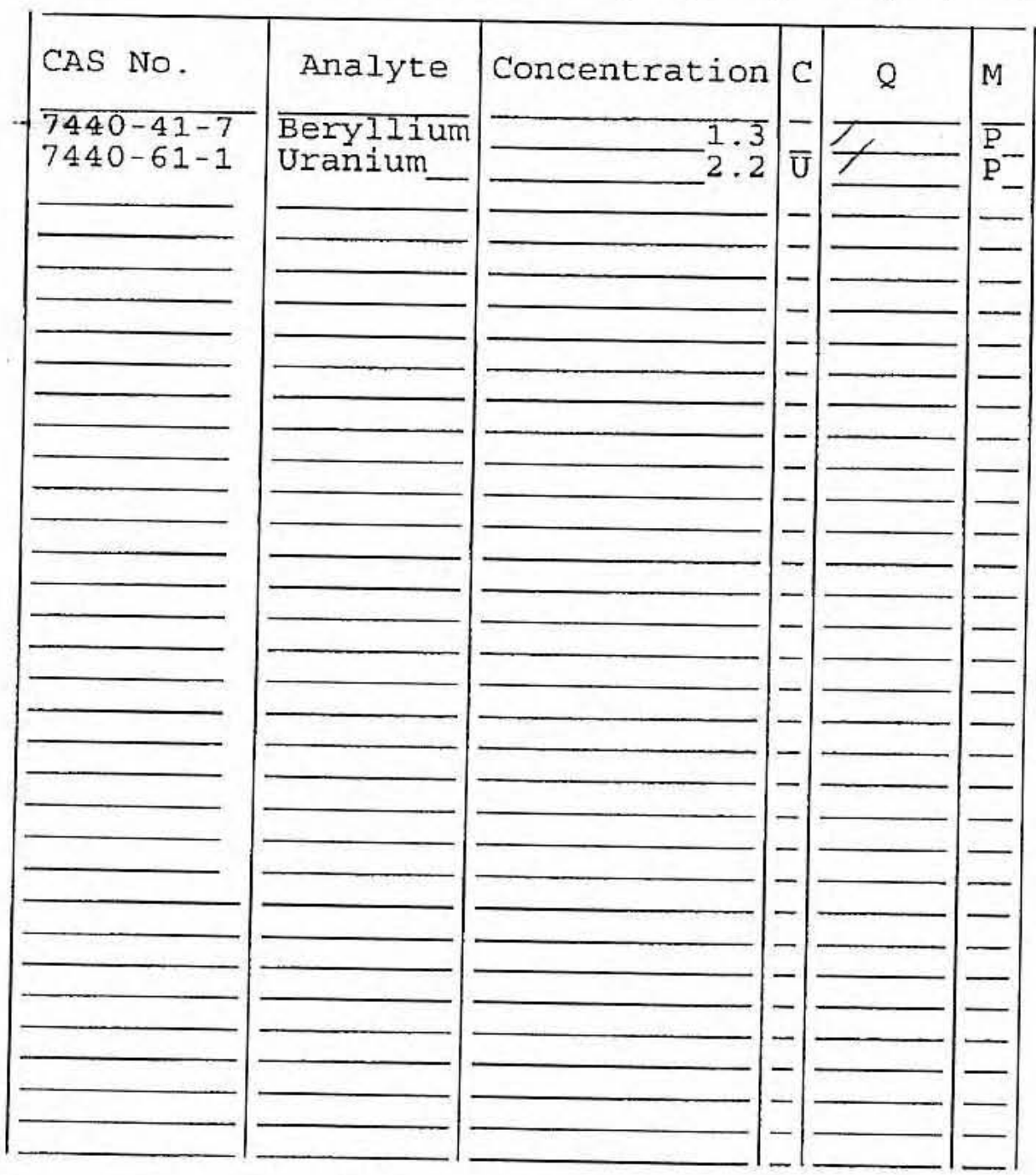

zolor Before: zolor After: Jomments:
Clarity Before: Clarity After:
Texture:

Artifacts: 
Lab Name: LIONVILLE_LABORATORY

Lab Code: LVLI

Matrix (soil/water): SoIL

Level. (low/med):

$\div$ Solids:
Contract: 60052

SAS NO.:

$$
\begin{array}{r}
\text { LOW } \\
-94 . \overline{3}
\end{array}
$$

SDG No.: V2742

Lab Sample ID: 0609L859-0̄11

Date Received: 09/01/06

Concentration Units (ug/L or $\mathrm{mg} / \mathrm{kg}$ dry weight) : MG/KG

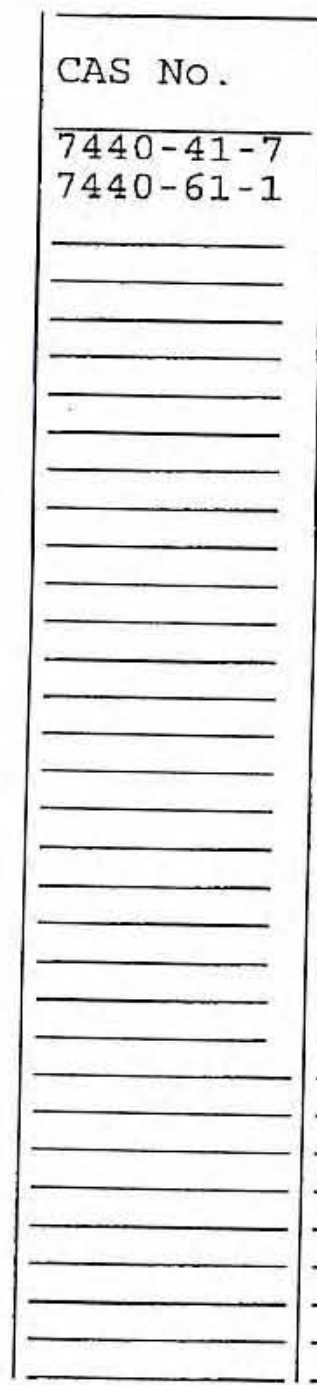

Color Before: Color After:

Comments:

NEDS - 26A

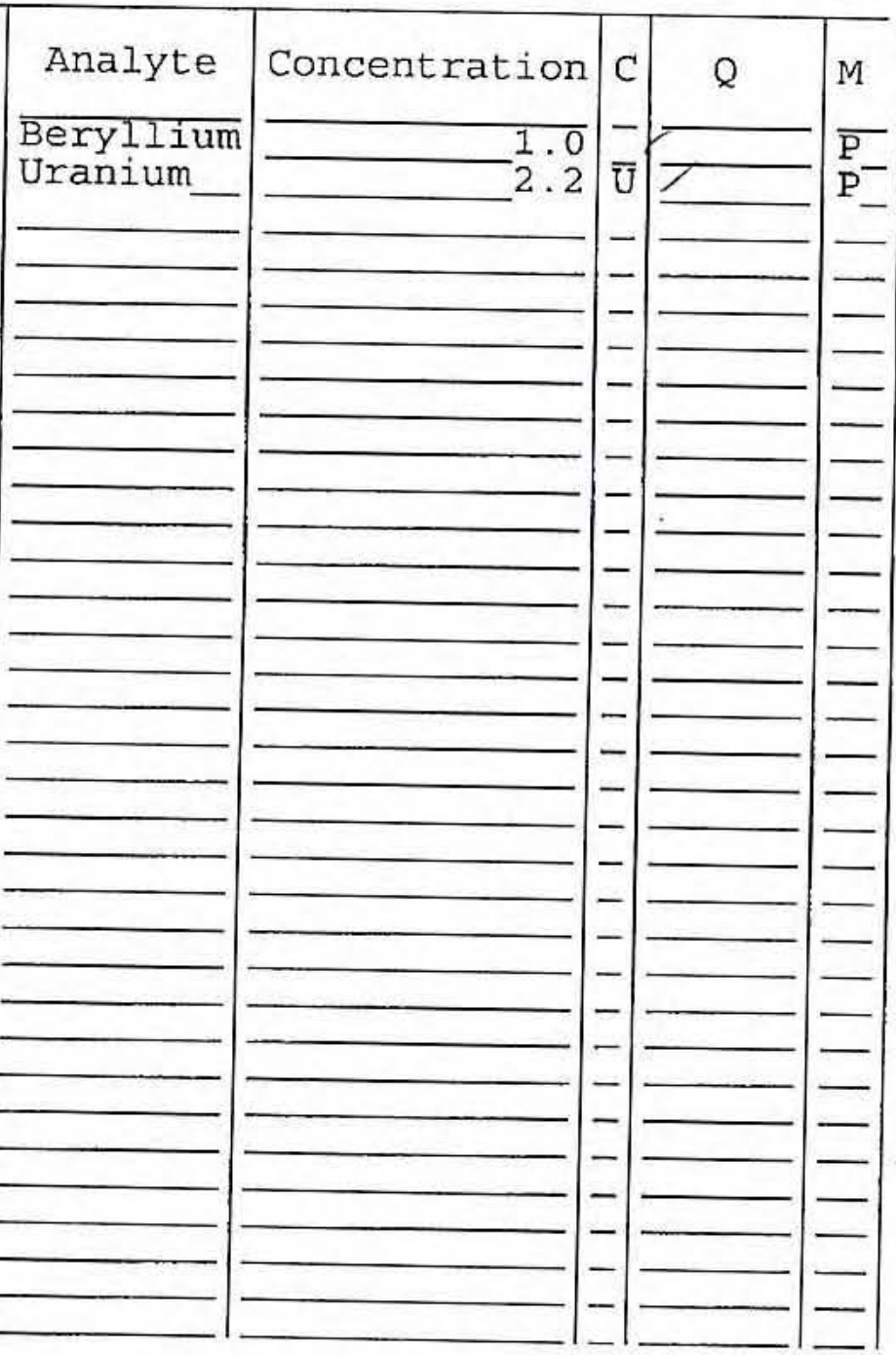

Clarity Before: Clarity After:
Texture:

Artifacts :

\section{FORM I - IN}


U.S. EPA

INORGANIC ANALY Y SES DATA SHEET

EPA SAMPLE NO.

Lab Name: LIONVILLE_LABORATORY

Lab Code: LVLI

Matrix (soil/water): soIL

Level. (low/med): LOW

\% Solids:

$-93 \overline{.2}$

Contract: 60052

\section{$26 B$}

SDG NO : V V2742

Lab Sample ID: 0609L859-0̄12

Date Received: 09/01/06

Concentration Units (ug/L or $\mathrm{mg} / \mathrm{kg}$ dry weight): MG/KG

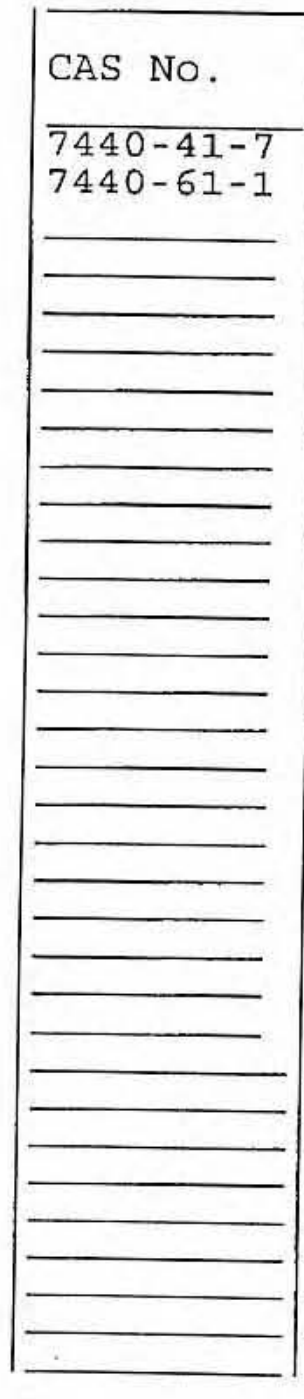

Solor Before:

Dolor After:

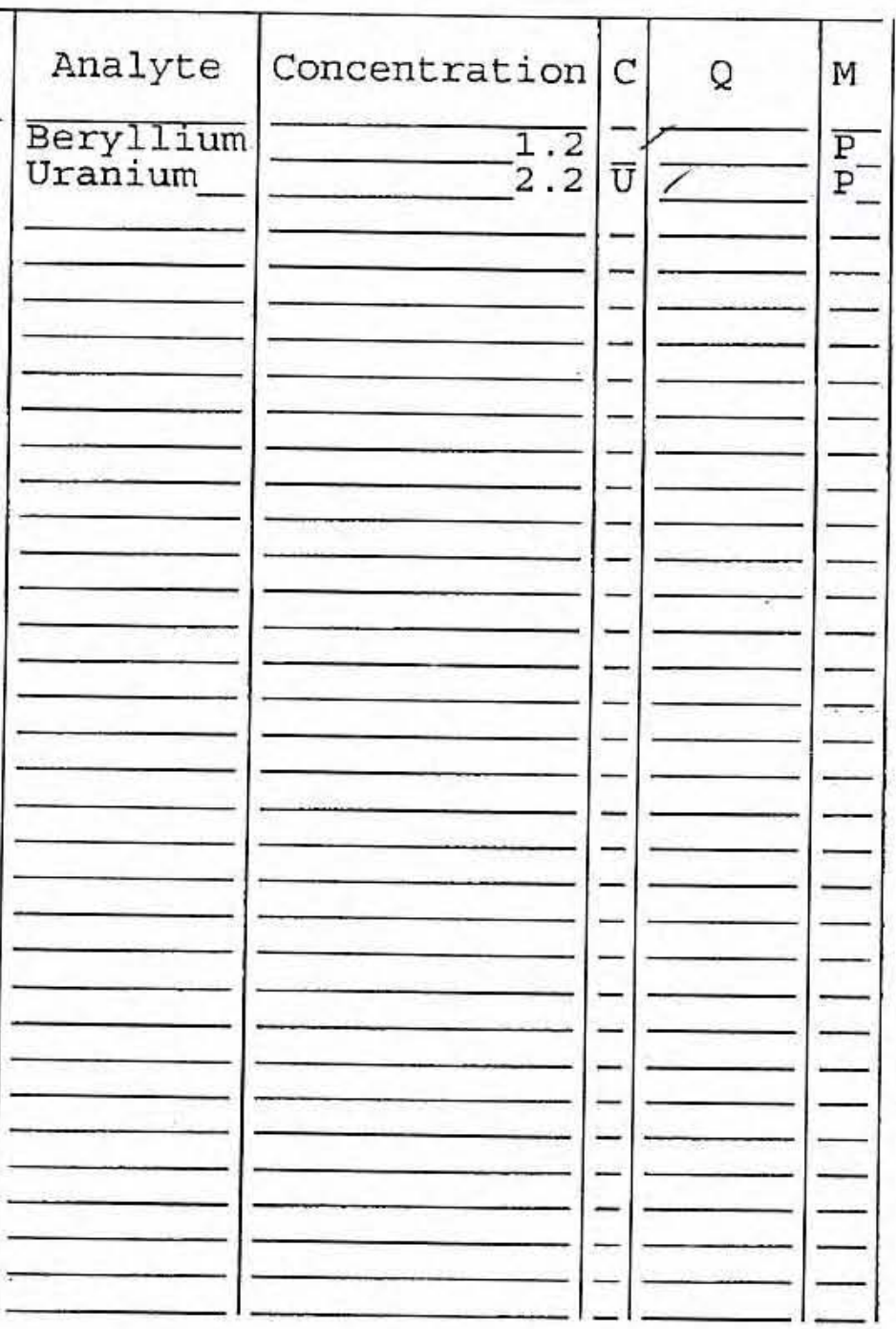

Clarity Before:

Clarity After:

Texture:

Artifacts :

Zomments :

NEDS $-26 B$ 
EPA SAMPLE NO.

$27 \mathrm{~A}$

Lab Name: LIONVILLE LABORATORY

Lab Code: LVLI

Matrix (soil/water): SoIL

Level (low/med) :

음 Solids :

Contract: 60052

SAS NO.:

SDG No.: V2742

Date Received: 09/01/06

$$
\text { LOW - }
$$

Lab Sample ID: 0609L859-0̄13

Concentration Units (ug/L or $\mathrm{mg} / \mathrm{kg}$ dry weight): MG/KG

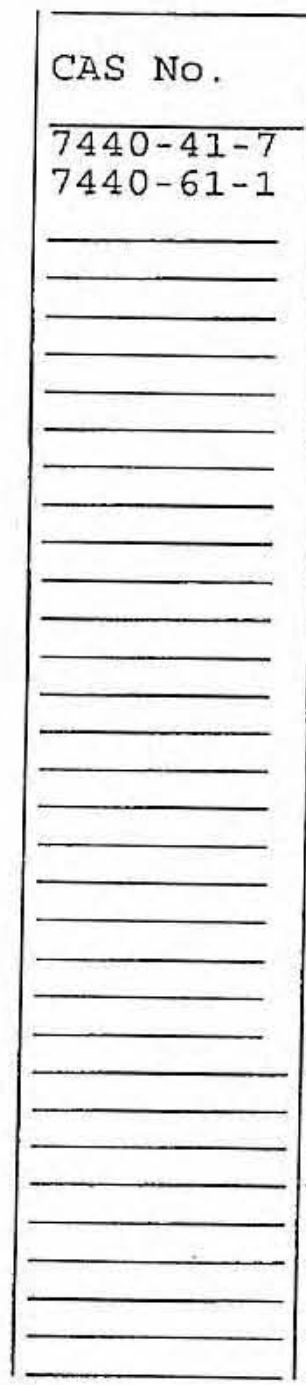

Folor Before:

Jolor After:

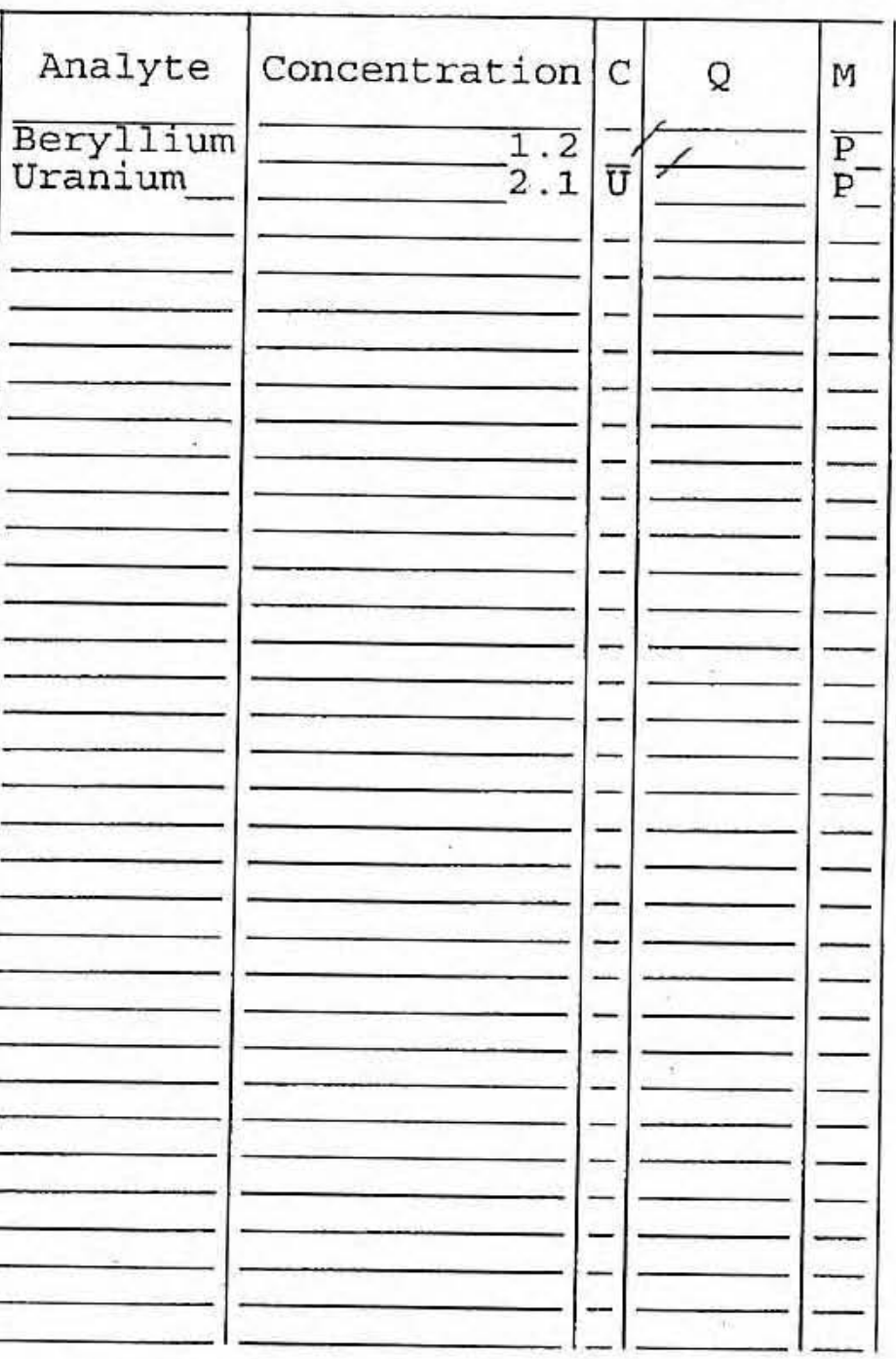

Clarity Before:

Clarity After:

Texture:

Artifacts:

\section{Zomments :}

NEDS - 27A 


\section{U.S. EPA}

1

INORGANIC ANALYSES DATA SHEET
EPA SAMPLE NO.

\section{$27 \mathrm{~B}$}

Lab Name: LIONVILLE_LABORATORY

Contract: 60052

Case No.: NEDS_ SAS No.:

Lab Code: LVLI

Matrix (soil/water): SoIL

Level (low/med) :

\% Solids :

Concentration Units (ug/L or $\mathrm{mg} / \mathrm{kg}$ dry weight): MG/KG

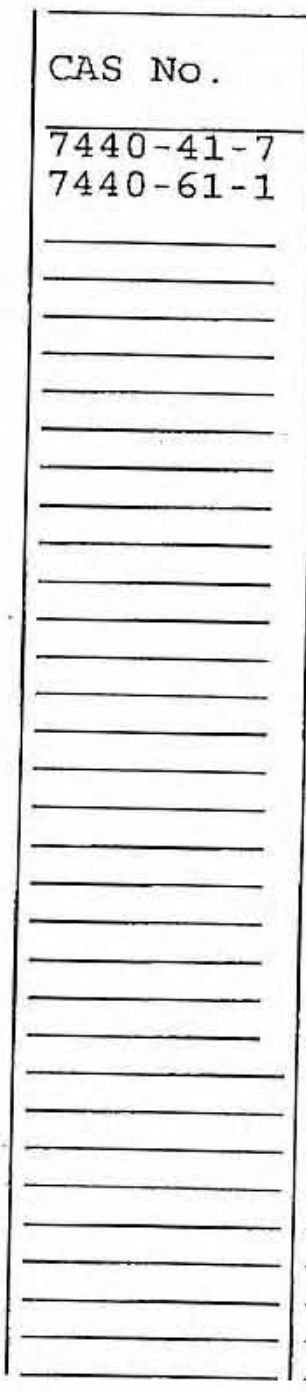

Jolor Before: Zolor After:

Jomments :

NEDS - 27B

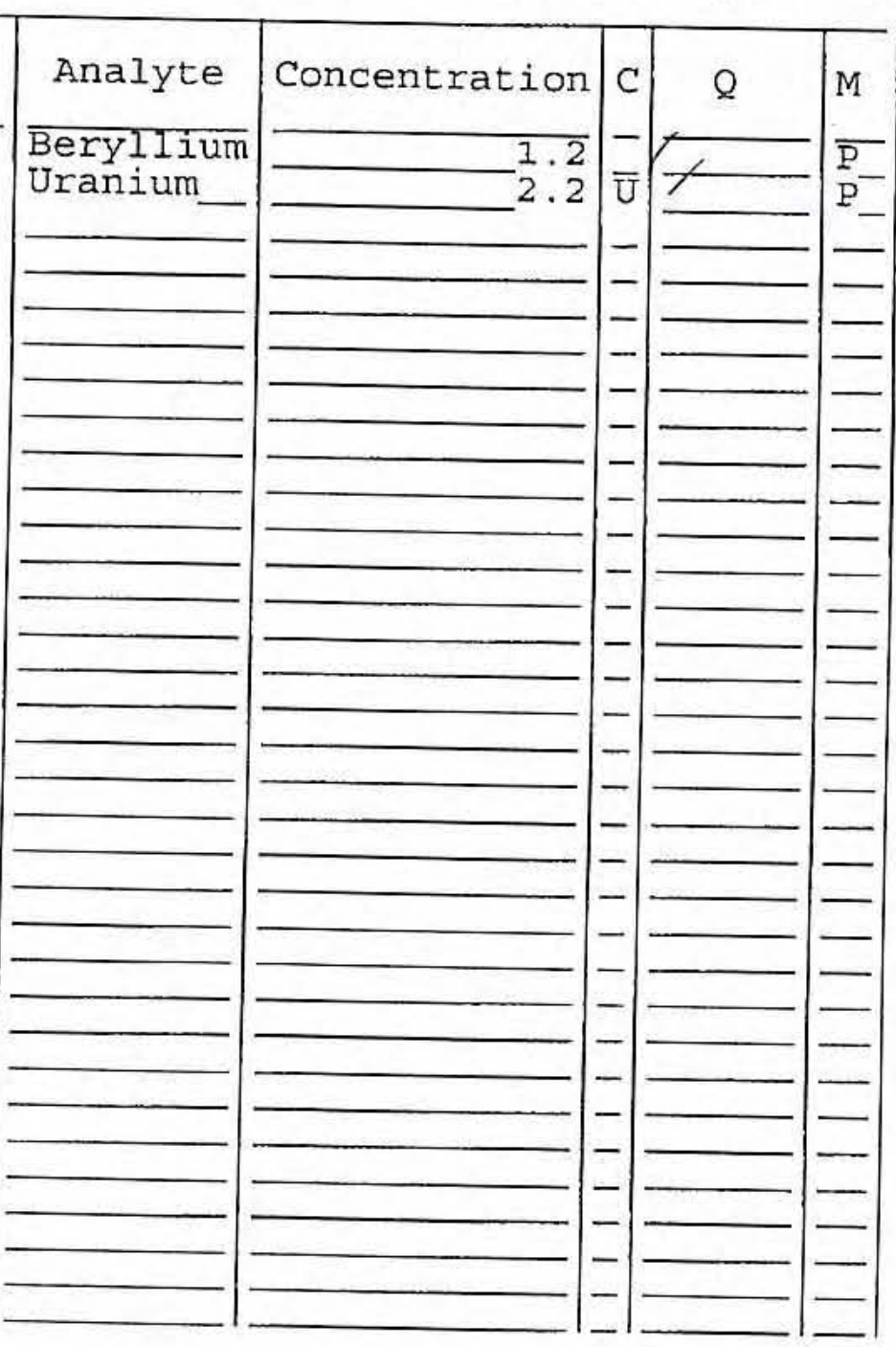

Clarity Before: Clarity After:

Texture: Artifacts : 
EPA SAMPLE NO.

$28 \mathrm{~A}$

Lab Name: LIONVILLE_LABORATORY

Lab Code: LVLI

Matrix (soil/water): SOII

Level. (low/med):

\% Solids :
Contract: 60052

$$
\begin{aligned}
& \text { LOW - } \\
& -96.9
\end{aligned}
$$

SDG No.: V2742

Lab Sample ID: 0609L859-0̄15

Date Received: 09/01/06

Concentration Units (ug/L or $\mathrm{mg} / \mathrm{kg}$ dry weight): MG/KG

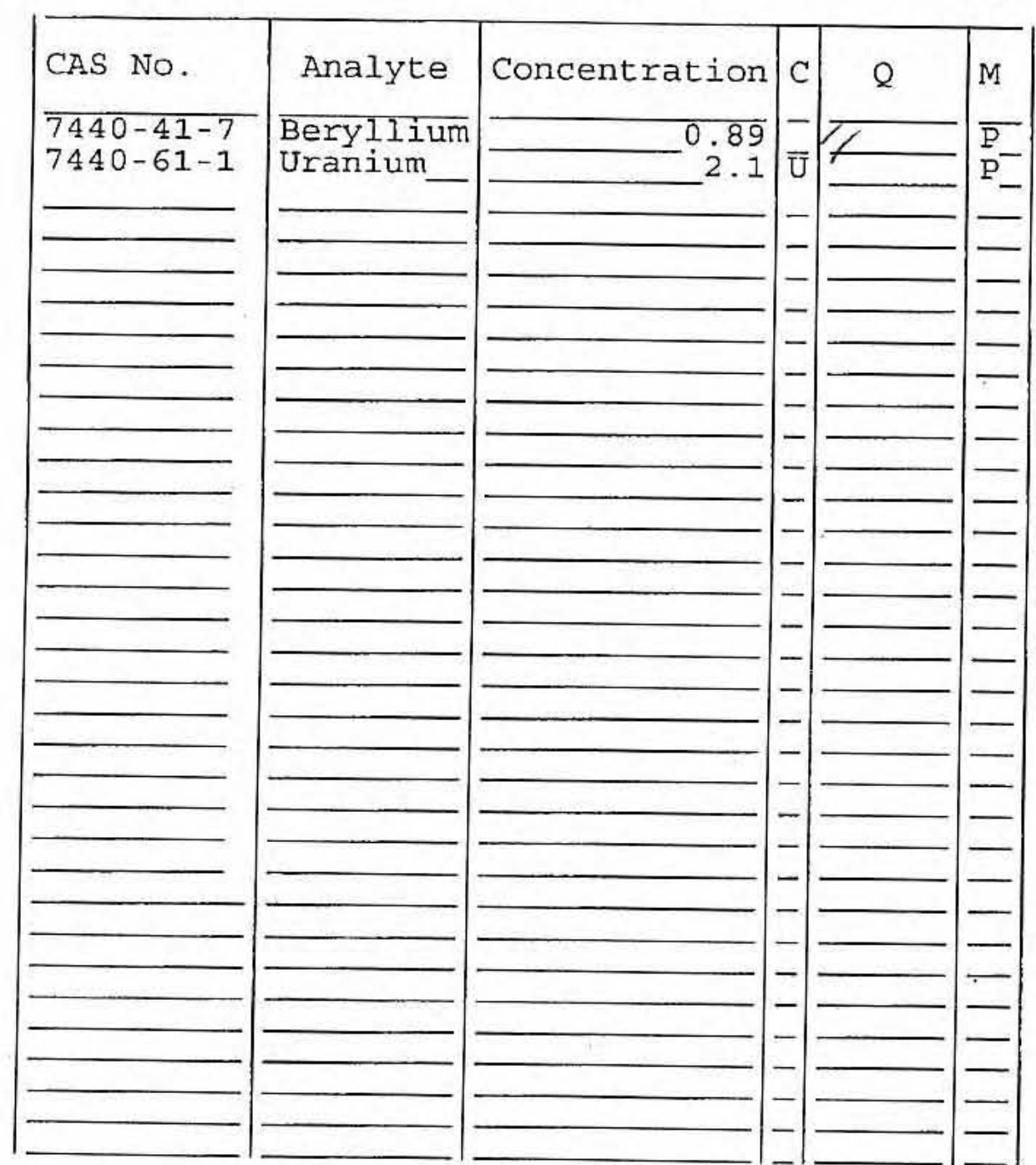

Color Before: Color After:

Jomments:

NEDS - 28A
Clarity Before:

Clarity After:
Texture:

Artifacts : 


\section{U.S. EPA}

\section{INORGANIC ANALYSES DATA SHEET}

EPA SAMPLE NO.

Lab Name: LIONVILLE_LABORATORY

Lab Code: LVLI

Matrix (soil/water): SOIL

Level. (low/med):

: Solids : LOW
-93.9
Contract: 60052

SAS NO.:

SDG NO : V2742

Lab Sample ID: 0609L859-0̄16

Date Received: 09/01/06

Concentration Units (ug/L or $\mathrm{mg} / \mathrm{kg}$ dry weight): MG/KG

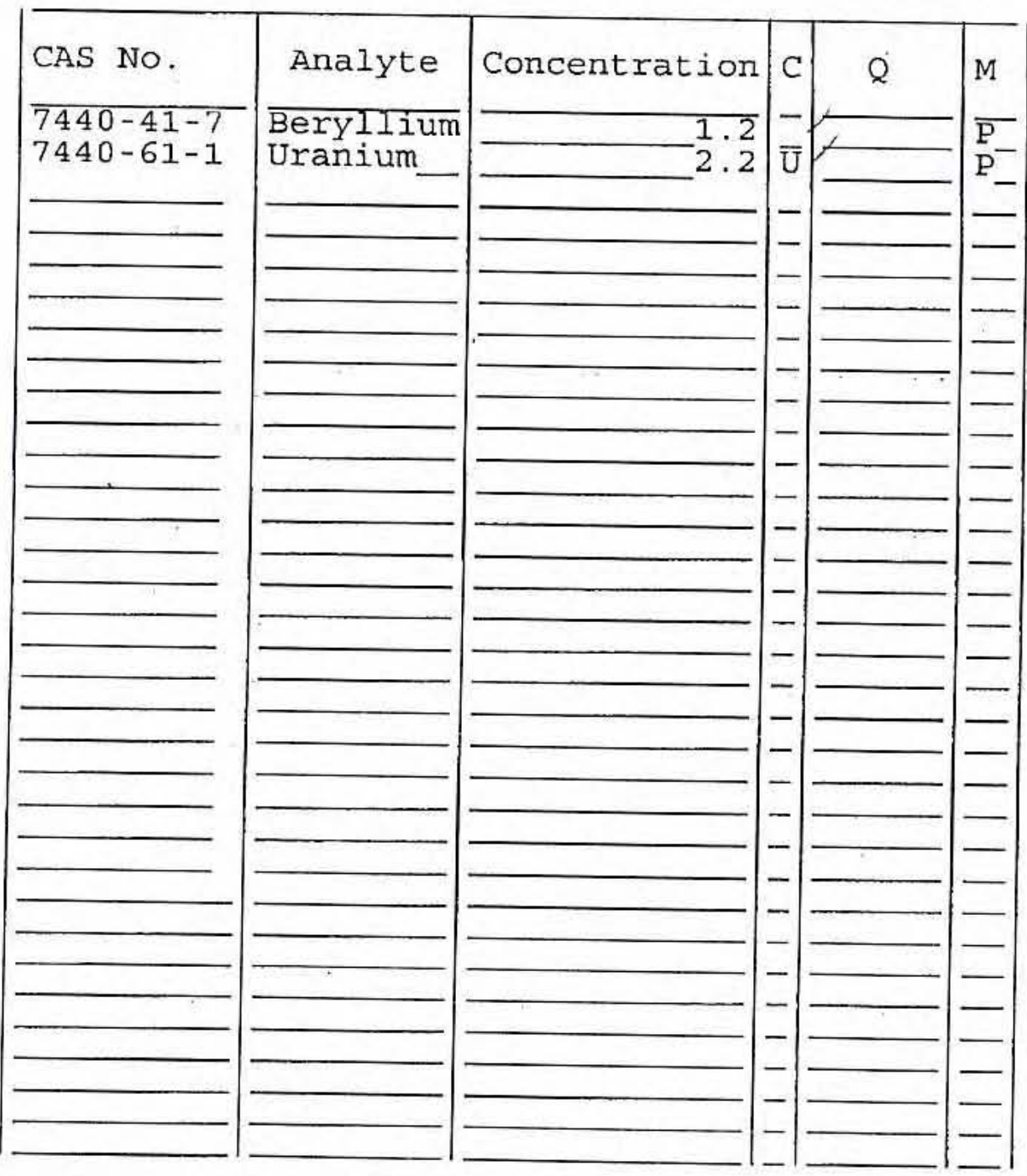

Color Before: Color After:

\section{Comments :}

NEDS $-28 B$
Clarity Before:

Clarity After;
Texture:

Artifacts: 
EPA SAMPLE NO.

29 A

Lab Name: LIONVILLE_LABORATORY_ Contract: 60052

Lab Code: LVLI

Matrix (soil/water): SoIL

Level (low/med): LOW

\% Solids:
SAS No. :

Lab Sample ID: $0609 \mathrm{~L}$ : $859-\overline{0} 17$

Date Received: 09/01/06

Concentration Units (ug/L or $\mathrm{mg} / \mathrm{kg}$ dry weight): MG/KG

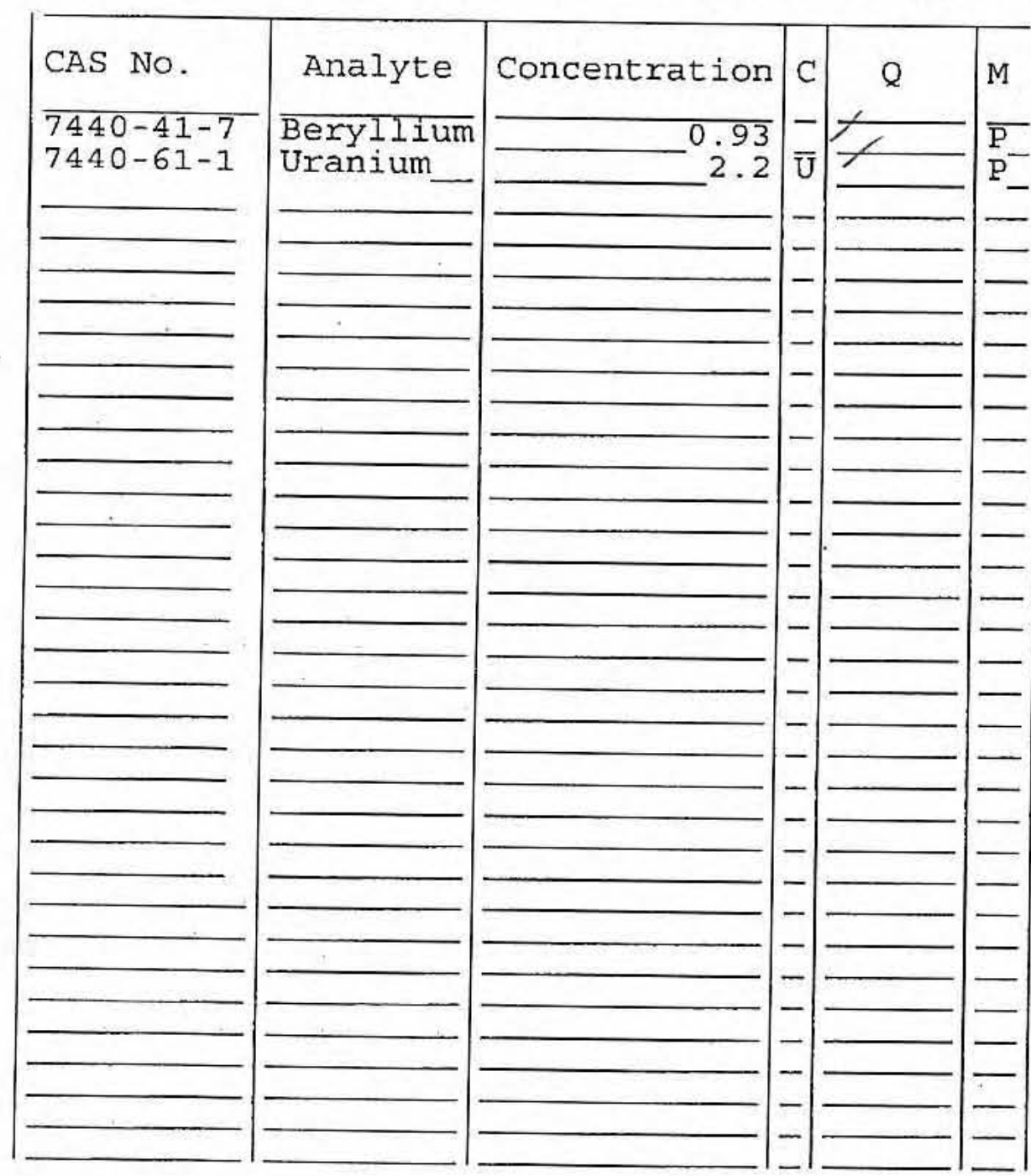

Color Before: Dolor After:

Comments:

NEDS-29A
Clarity Before:

Clarity After:
Texture:

Artifacts: 
Lab Name: LIONVILLE_LABORATORY

Lab Code: LVLI

Matrix (soil/water): SOIL

Level (low/med):

\% Solids :

$$
\text { LOW - }
$$$$
-91 . \overline{9}
$$

\section{$29 B$}

SDG NO.: V2742

SAS NO.:

Lab Sample ID: 0609L859-0̄18

Date Received: 09/01/06

Concentration Units (ug/L or $\mathrm{mg} / \mathrm{kg}$ dry weight): MG/KG

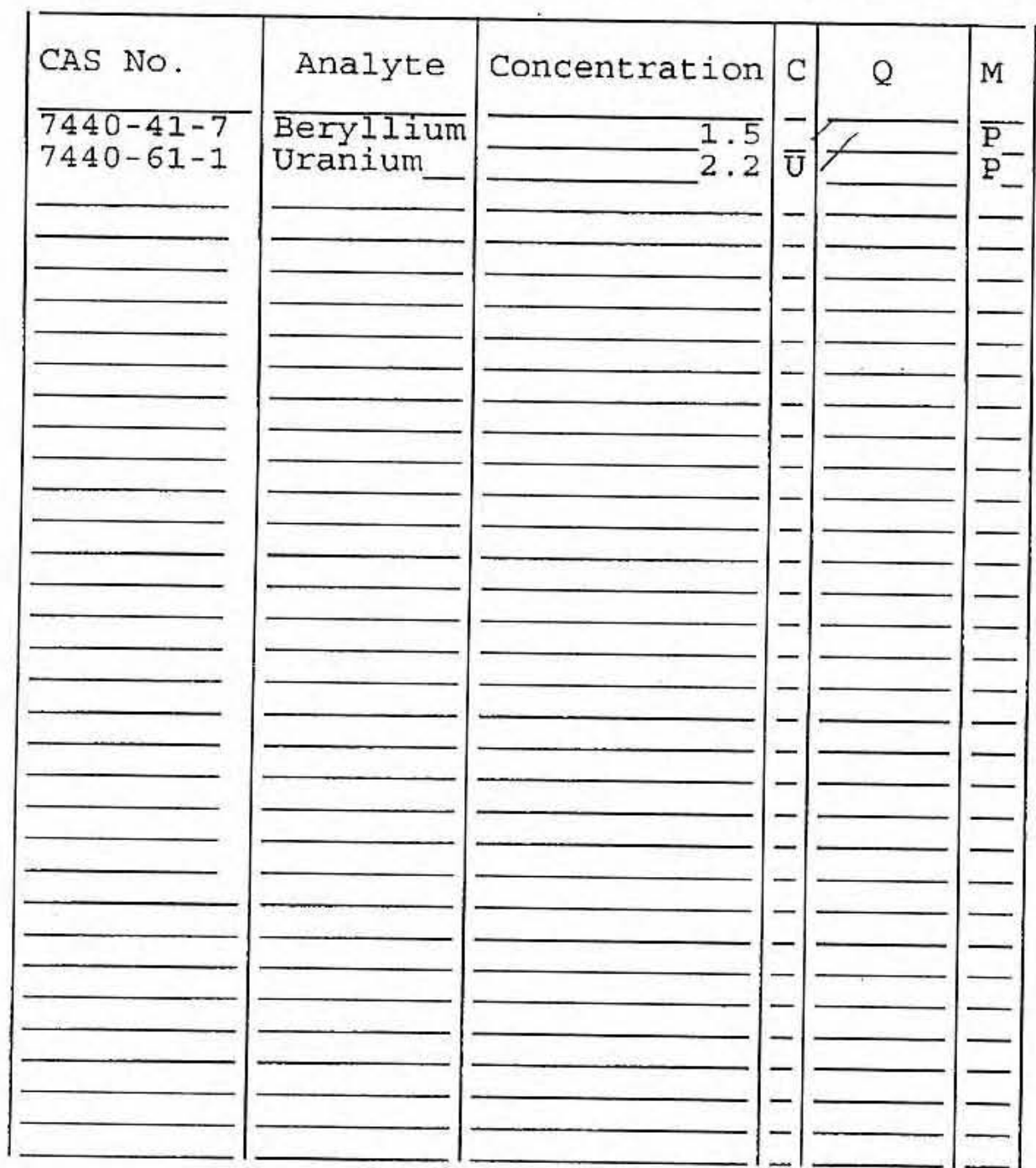

Color Before: Color After:

Comments :

NEDS - 29B
Clarity Before:

Clarity After:
Texture:

Artifacts: 


\section{INORGANIC ANALYSSES DATA SHEET}

EPA SAMPLE NO.

$$
30 \mathrm{~A}
$$

Lab Name: LIONVILLE_LABORATORY

Lab Code: LVLI

Matrix (soil/water) : soIL

Level (low/med): LOW

$\div$ Solids :
Contract: 60052

SAS NO.:

SDG NO.: V2742

Lab Sample ID: 0609L859-0̄19

Date Received: 09/01/06

Concentration Units (ug/L or $\mathrm{mg} / \mathrm{kg}$ dry weight): MG/KG

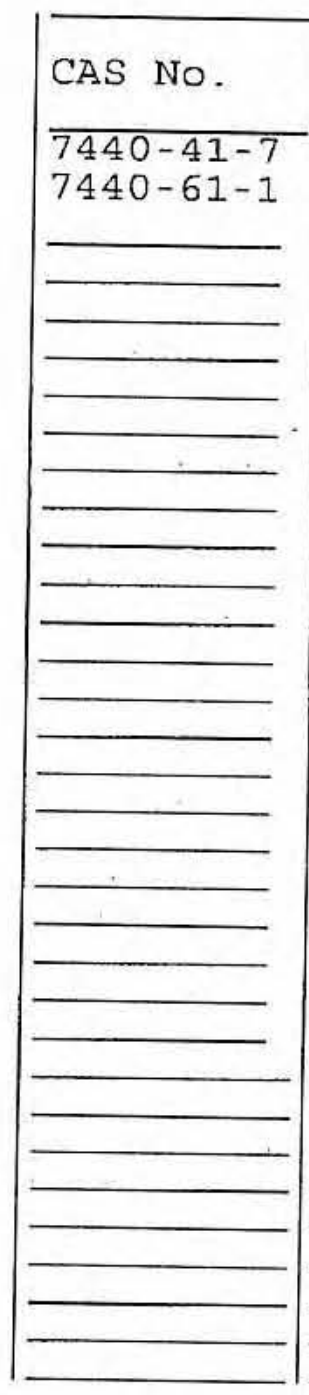

Zolor Before: Jolor After: zomments :

NEDS - 30A

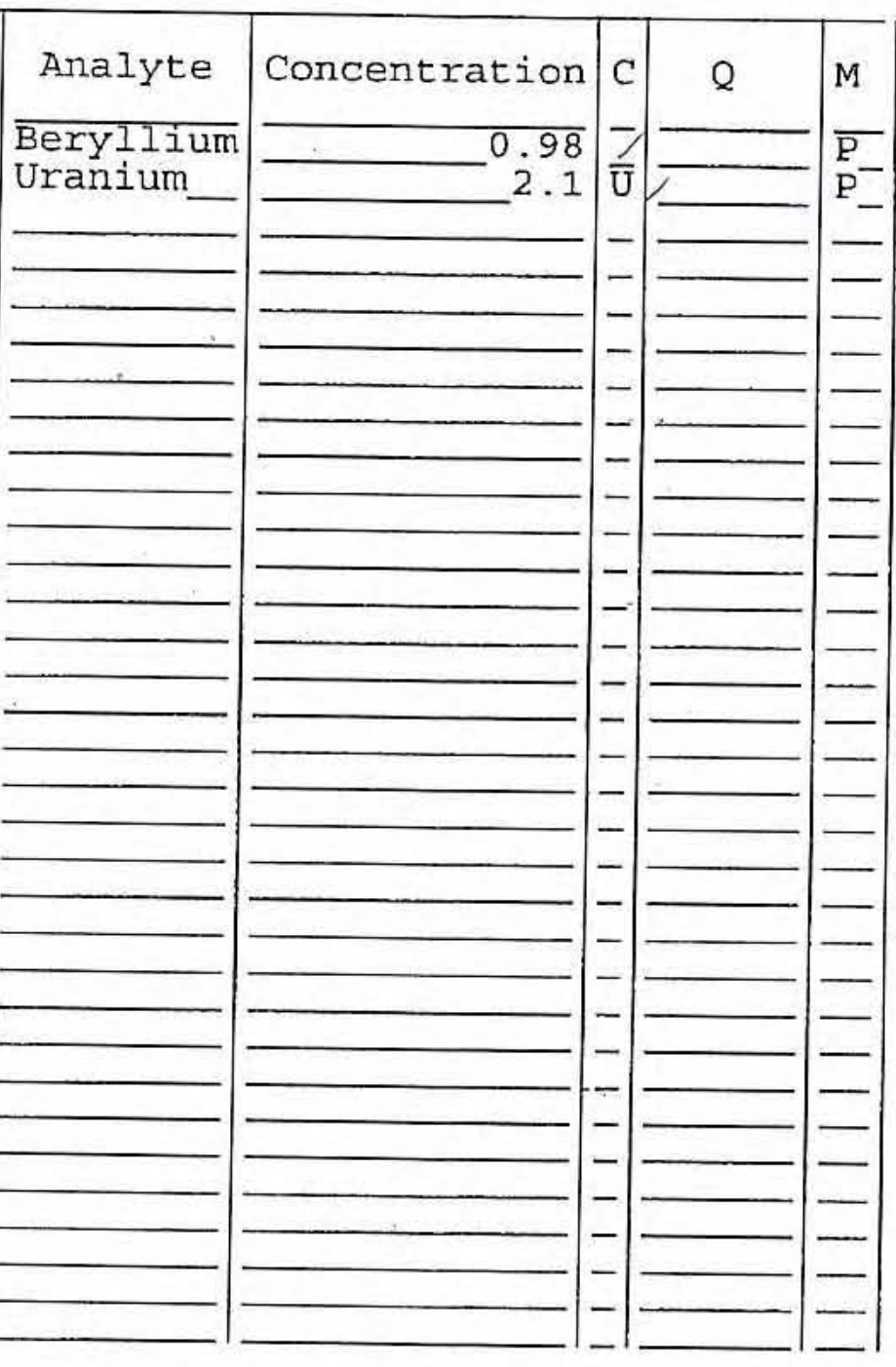

Clarity Before: Clarity After:
Texture: Artifacts: 
Lab Name: LIONVILLE_LABORATORY

Lab Code: LVLI

Matrix (soil/water): SolL

Level (low/med): LOW

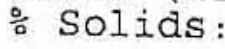

LOW

$30 B$

SDG NO.: V2742

SAS NO.:

Late Received: 09/01/06

Concentration Units (ug/L or $\mathrm{mg} / \mathrm{kg}$ dry weight): MG/KG

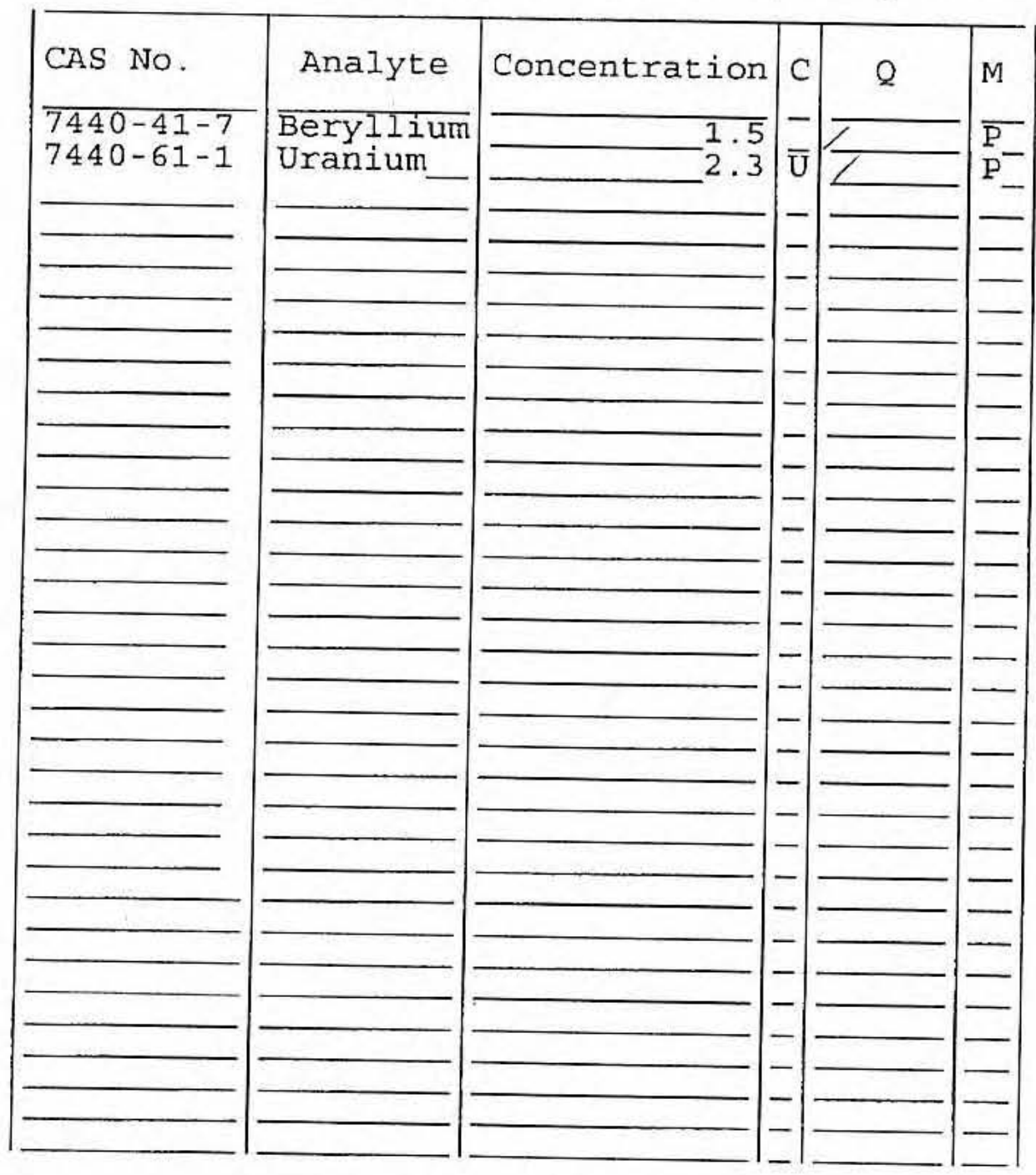

Zolor Before: Jolor After:

Jomments:

NEDS - 3OB
Clarity Before:

Clarity After:
Texture:

Artifacts : 


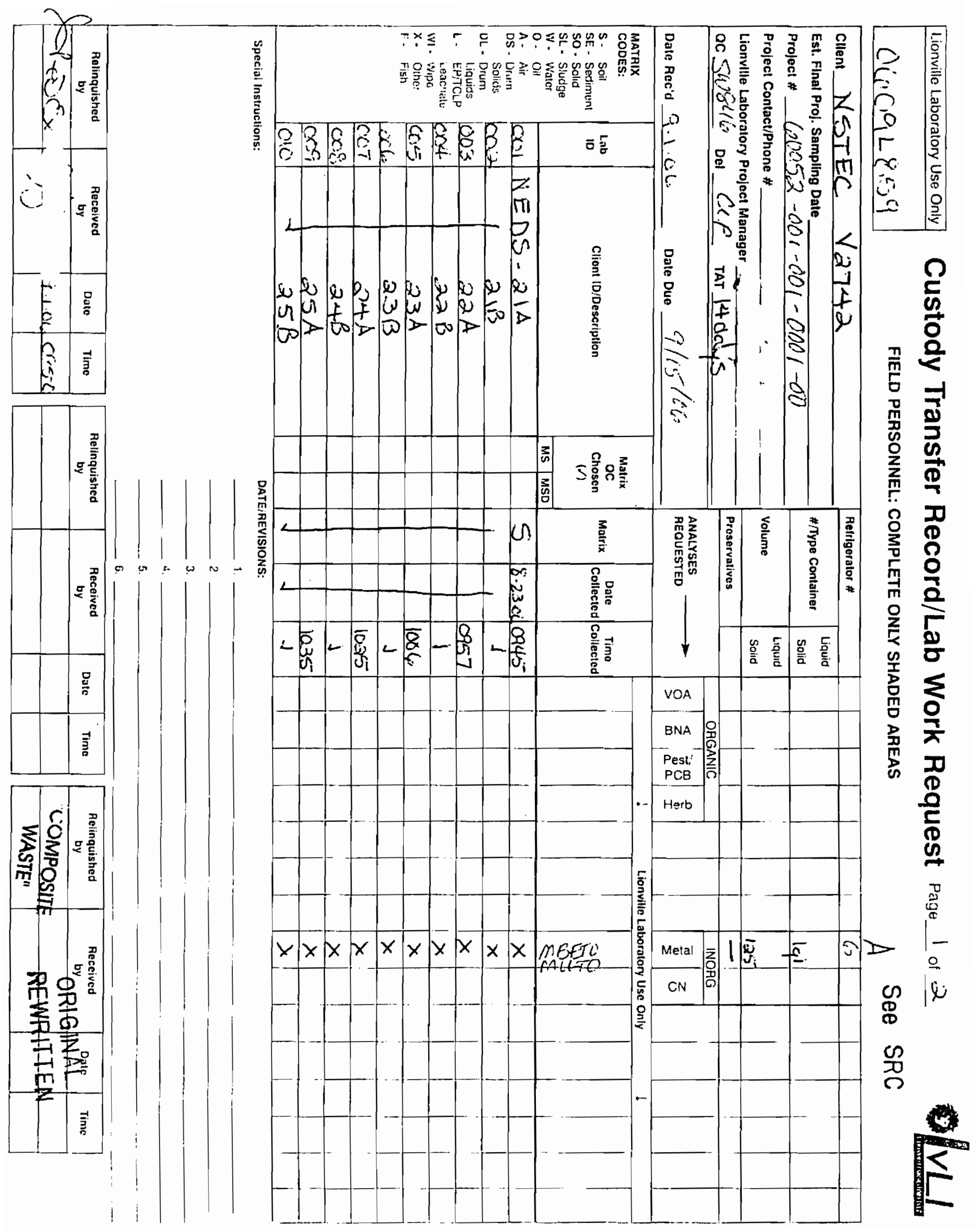




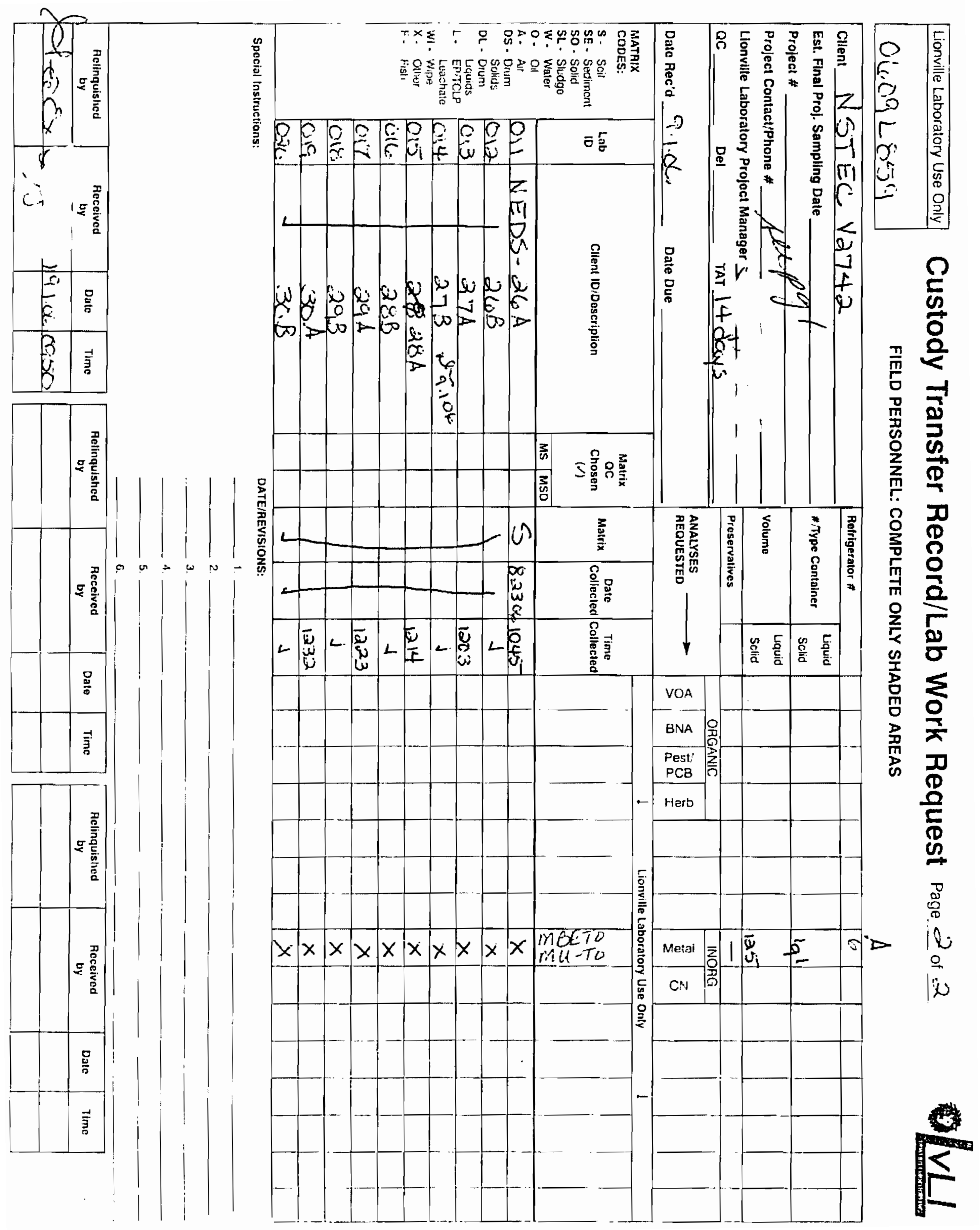




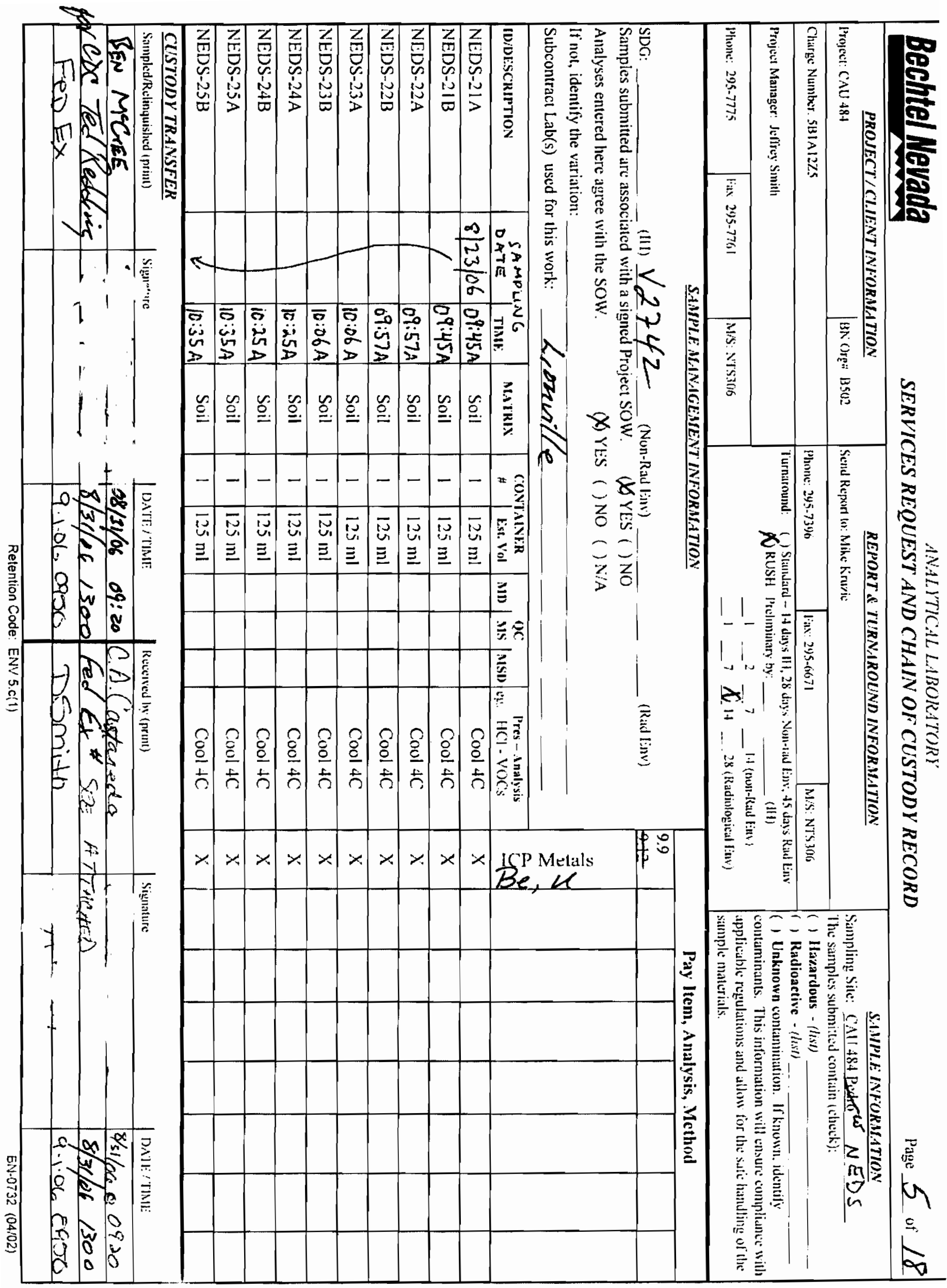




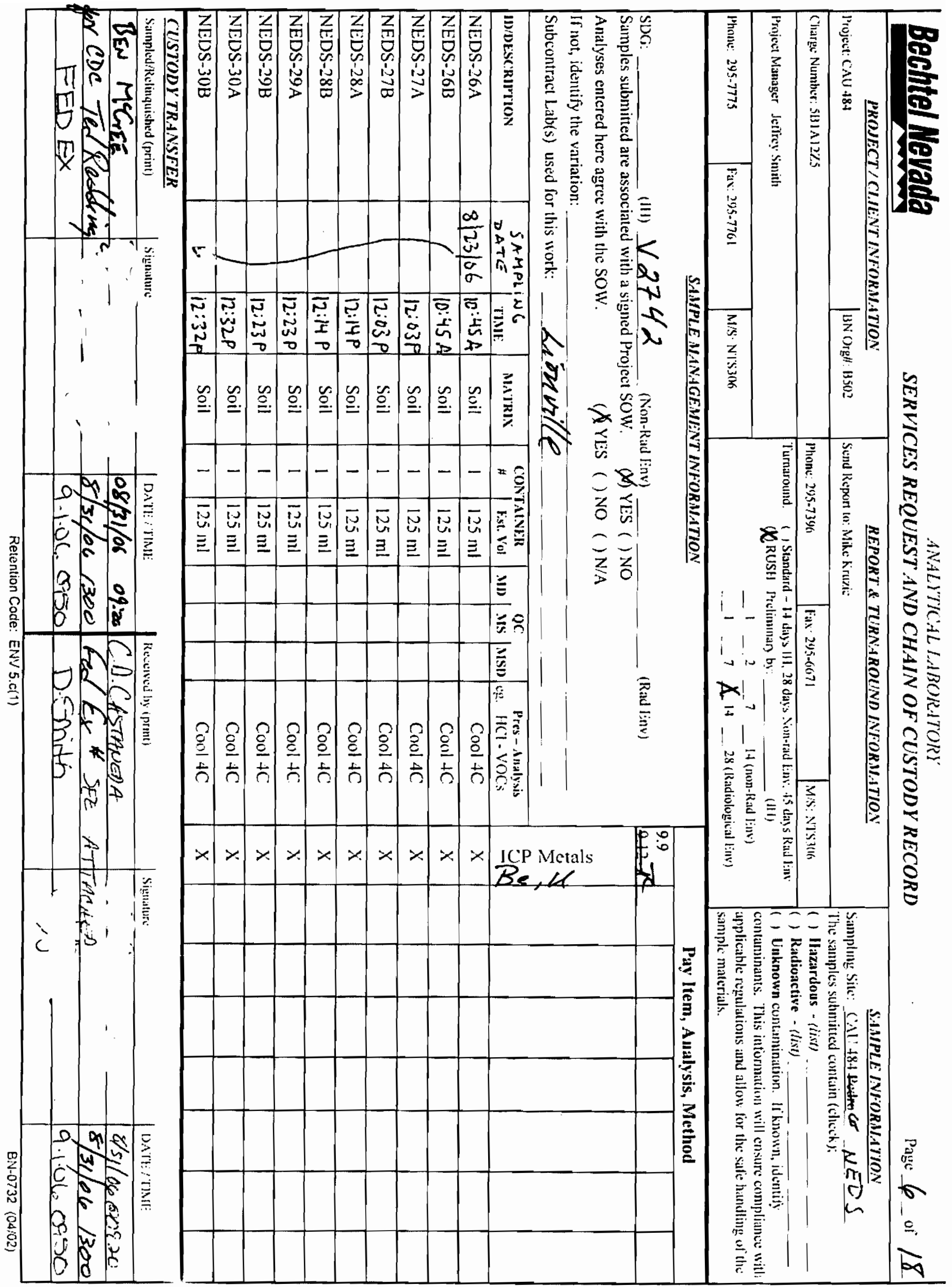


Lab Name: LIONVILLE_LABORATORY

Lab Code: LVLI

Matrix (soil/water): SoIL

Level (low/med): LOW

\% Solids:

\section{$31 \mathrm{~A}$}

SDG NO.: V2742

Lab Sample ID: 0609L860-̄01

Date Received: 09/01/06

Concentration Units (ug/L or $\mathrm{mg} / \mathrm{kg}$ dry weight) : MG/KG

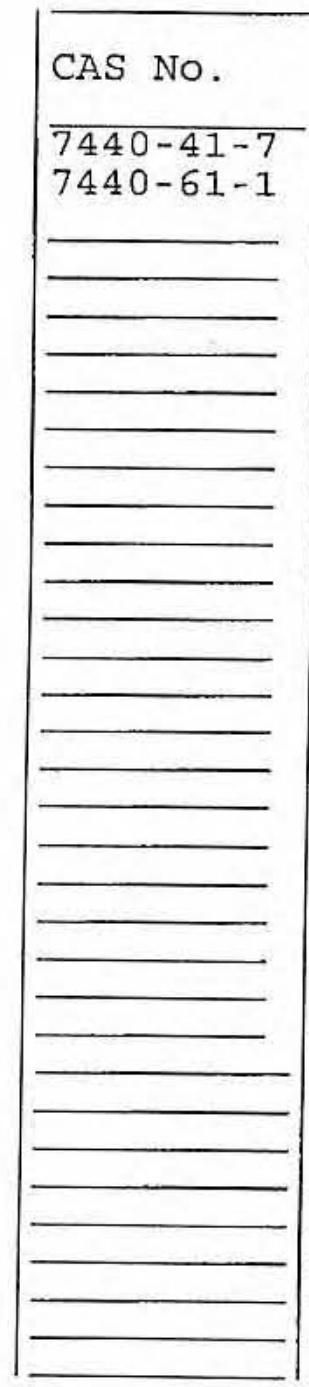

Color Before: Color After:

\section{Comments :}

NEDS $-31 A$

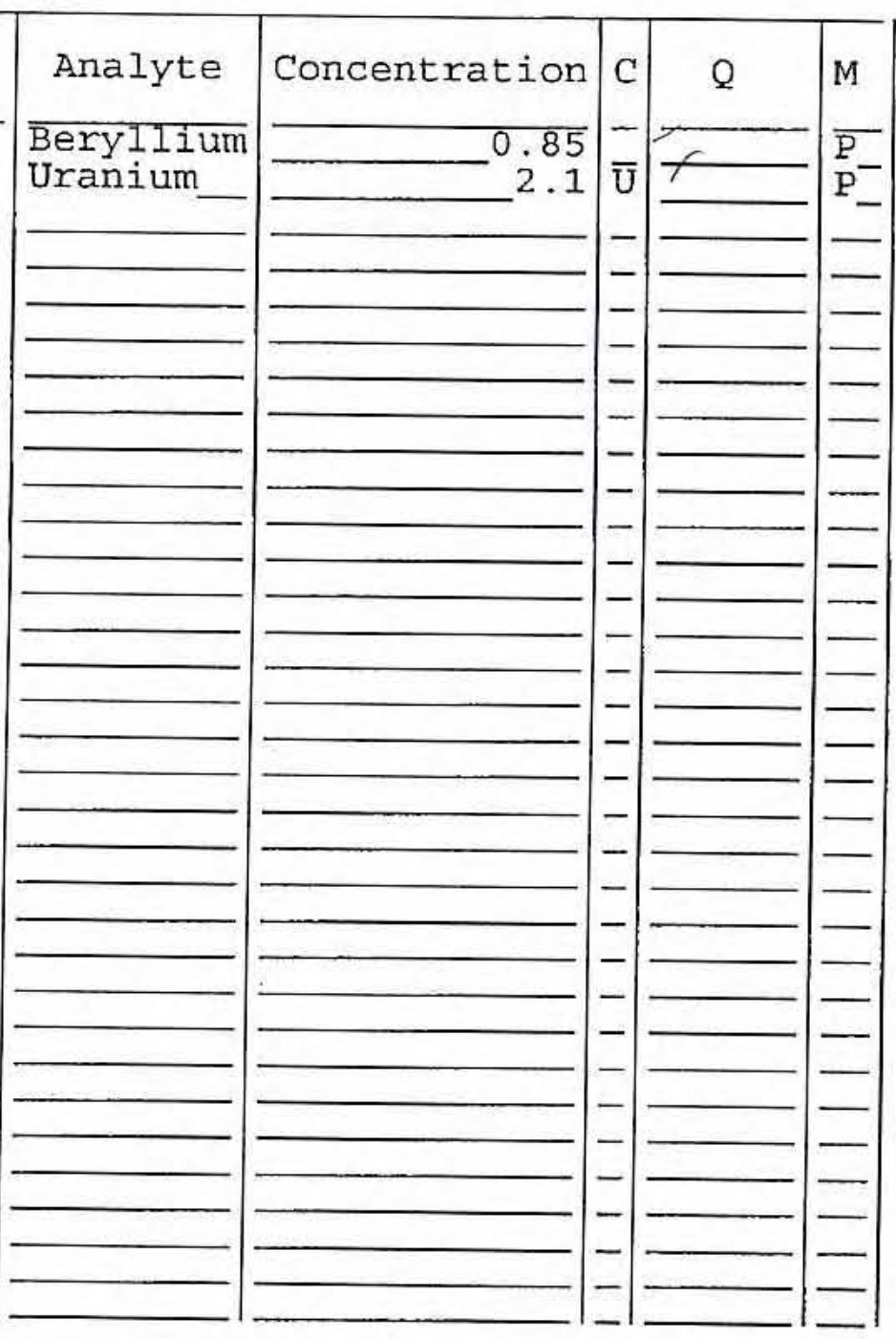

Clarity Before:

Clarity After:
Texture:

Artifacts : 


\section{U.S. EPA}

1

INORGANIC ANALYSES DATA SHEET
EPA SAMPLE NO.

\section{$31 \mathrm{~B}$}

Lab Name: LIONVILLE_LABORATORY

Contract: 60052

Lab Code: LVLI

Matrix (soil/water): SOIL

Level. (low/med): LOW

\% Solids :
SAS NO.:

Lab Sample ID: 0609L860-002

Date Received: 09/01/06

Concentration Units (ug/L or $\mathrm{mg} / \mathrm{kg}$ dry weight): $\mathrm{MG} / \mathrm{KG}$

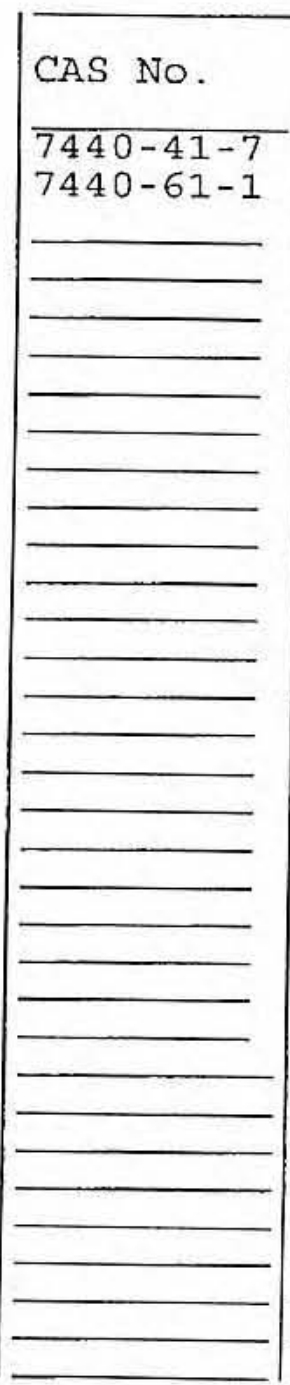

Color Before: Color After:

Comments:

NEDS - $31 B$

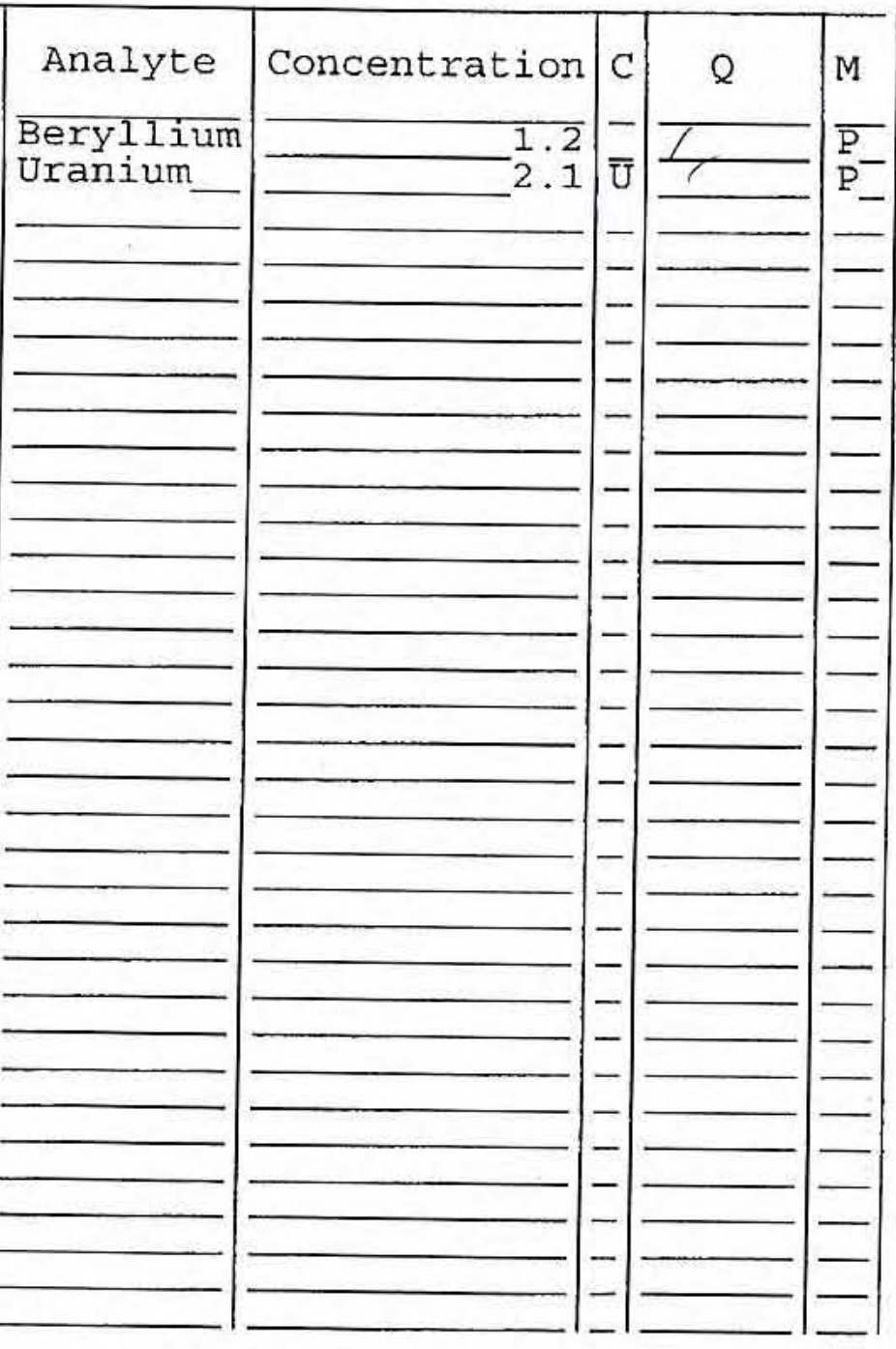

Clarity Before:

Clarity After:
Texture:

Artifacts: 


\section{INORGANIC ANALYSES DATA SHEET}

Lab Name: LIONVILIE_LABORATORY

Lab Code: LVLI

Matrix (soil/water): SoII

Level. (low/med): LOW

\% Solids :
EPA SAMPLE NO.

$32 \mathrm{~A}$

Contract: 60052

SAS No.:

Lab Sample ID: 0609L860-003

Date Received: 09/01/06

Concentration Units (ug/L or $\mathrm{mg} / \mathrm{kg}$ dry weight): MG/KG

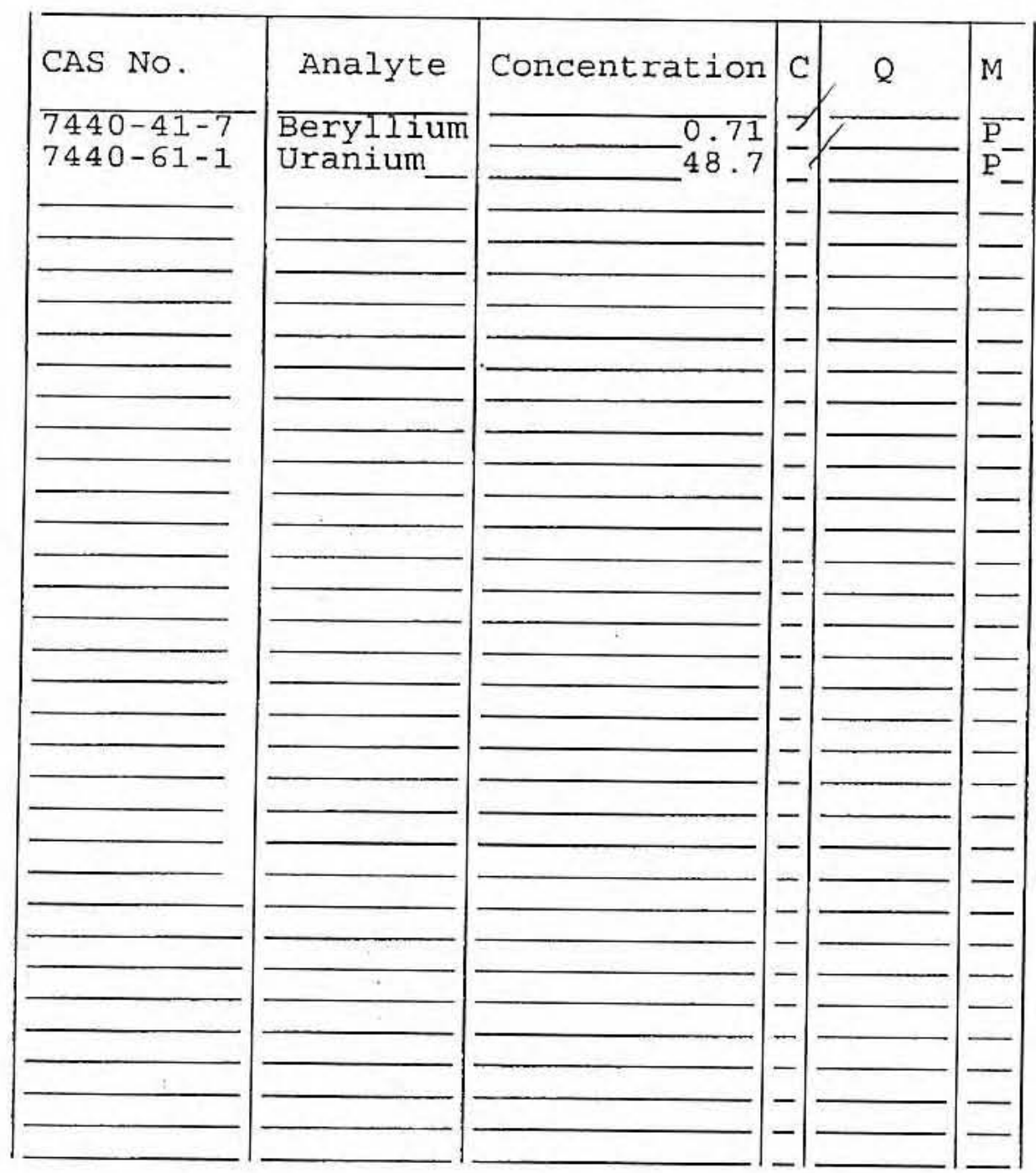

Color Before:

Clarity Before:

Clarity After:

Texture:

Artifacts:

\section{Comments :}

NEDS - 32A 
U.S. EPA

INORGANIC ANALY̛SSES DATA SHEET

EPA SAMPLE NO.

Lab Name: LIONVILLE_LABORATORY

Lab Code: LVLI Matrix (soil/water): SoIL Level (low/med): \% Solids:

\section{Contract: 60052}

SAS NO. : LOW $-94 \overline{.1}$

\section{$32 \mathrm{~B}$}

SDG NO.: V2742

Lab Sample ID: 0609L860-0̄04

Date Received: 09/01/06

Concentration Units (ug/L or $\mathrm{mg} / \mathrm{kg}$ dry weight): MG/KG

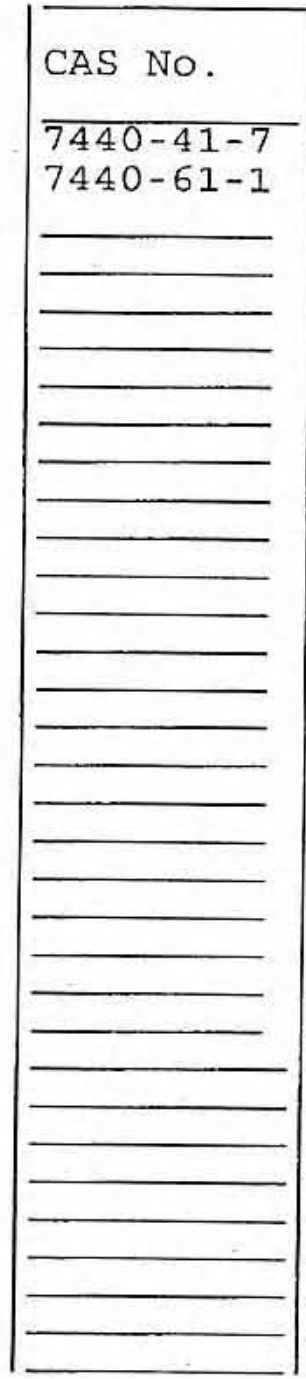

Color Before: Color After:

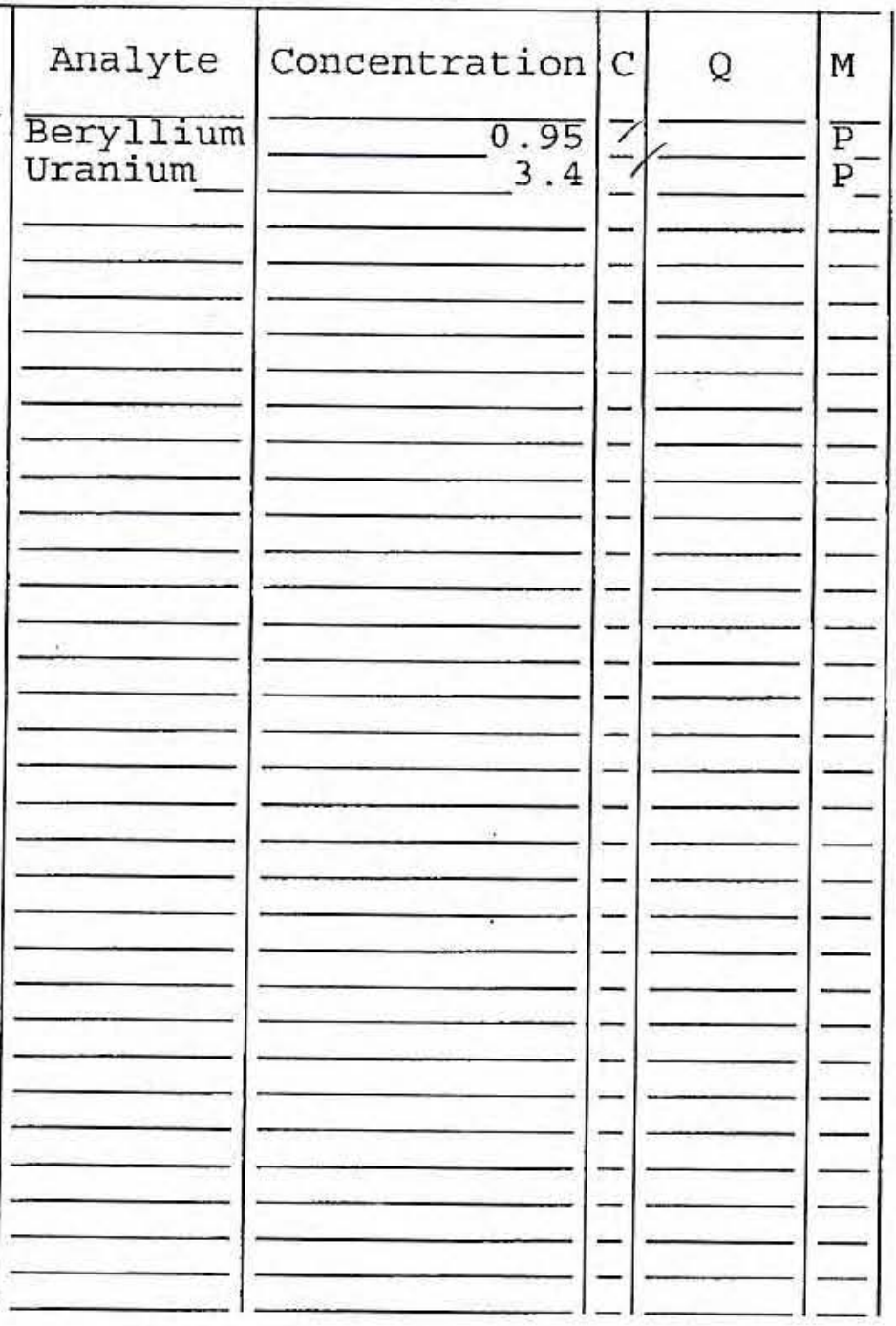

Clarity Before: Clarity After:
Texture: Artifacts :

\section{Comments :}

NEDS - 32B 


\section{INORGANIC ANALYSES DATA SHEET}

Lab Name: LIONVILLE_LABORATORY

Lab Code: LVLI

Matrix (soil/water) : SoIL

Level (low/med) :

$\div$ Solids:
Contract: 60052
EPA SAMPLE NO.

\section{$33 \mathrm{~A}$}

SDG NO.: V2742

Lab Sample ID: 0609L860-̄05

Date Received: 09/01/06

Concentration Units (ug/L or $\mathrm{mg} / \mathrm{kg} \mathrm{dry}$ weight): MG/KG

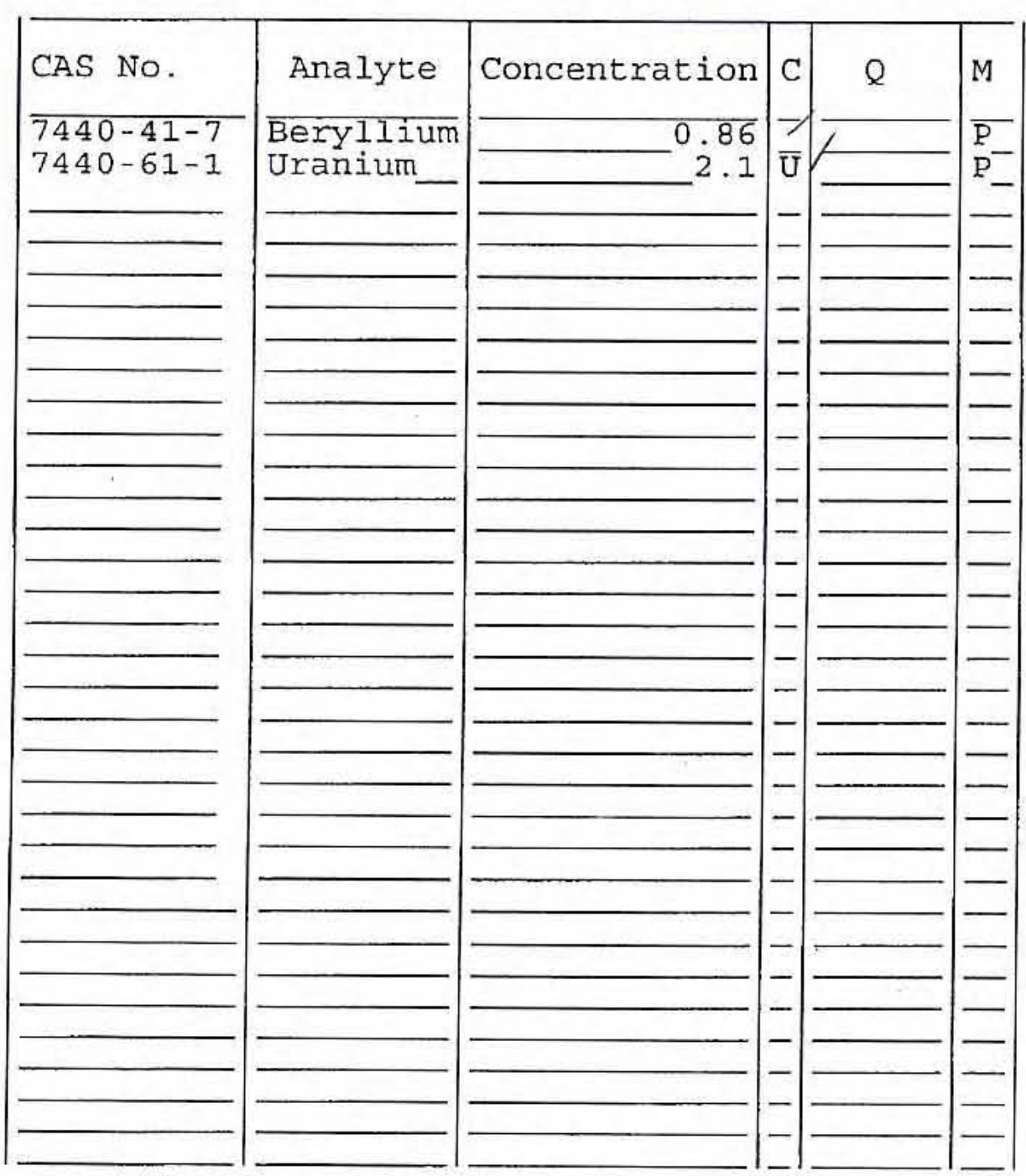

Color Before:

Color After:

Comments :

NEDS - 33A
Clarity Before:

Clarity After:
Texture:

Artifacts: 


\section{U.S. EPA}

\section{1}

INORGANIC ANALYSES DATA SHEET
EPA SAMPLE NO.

$33 \mathrm{~B}$

Contract: 60052

SAS No.:

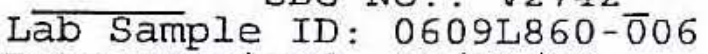

Date Received: 09/01/06

Level (low/med): $\quad$ LOW

\% Solids: $\quad-93 \overline{.4}$

Concentration Units (ug/L or $\mathrm{mg} / \mathrm{kg}$ dry weight): MG/KG

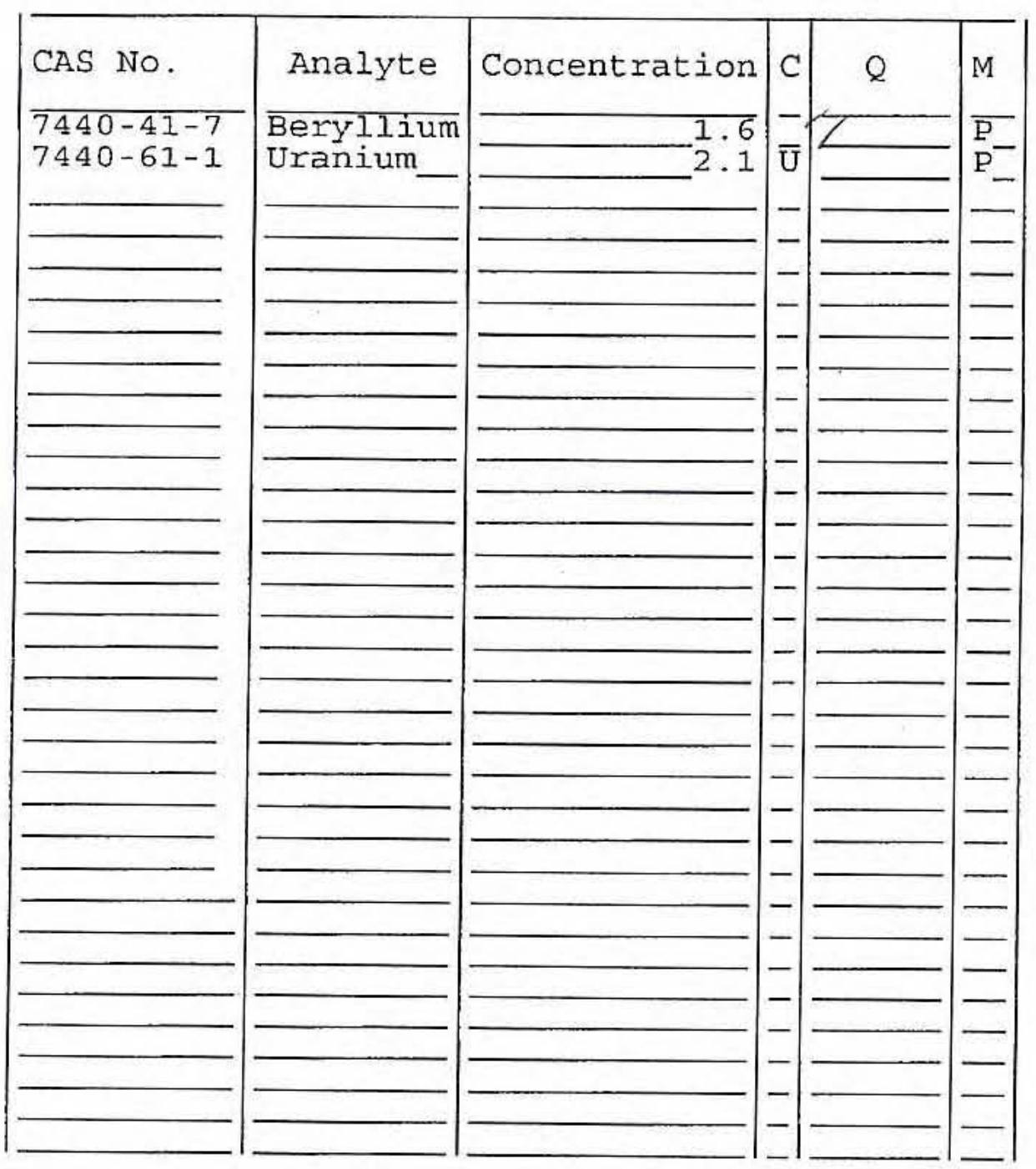

Color Before:

Color After:

Comments :

NEDS - 33B
Clarity Before:

Clarity After:
Texture:

Artifacts: 
1 INORGANIC ANALYSES DATA SHEET
EPA SAMPLE NO.

$34 \mathrm{~A}$

Lab Name: LIONVILLE_LABORATORY_Contract: 60052

Lab Code: LVLI

Matrix (soil/water): SOIL

Level (low/med): LOW

o Solids:
SDG No.: V2742

SAS NO.:

Lab Sample ID: 0609L860-̄007

Date Received: 09/01/06

Concentration Units (ug/L or $\mathrm{mg} / \mathrm{kg}$ dry weight): MG/KG

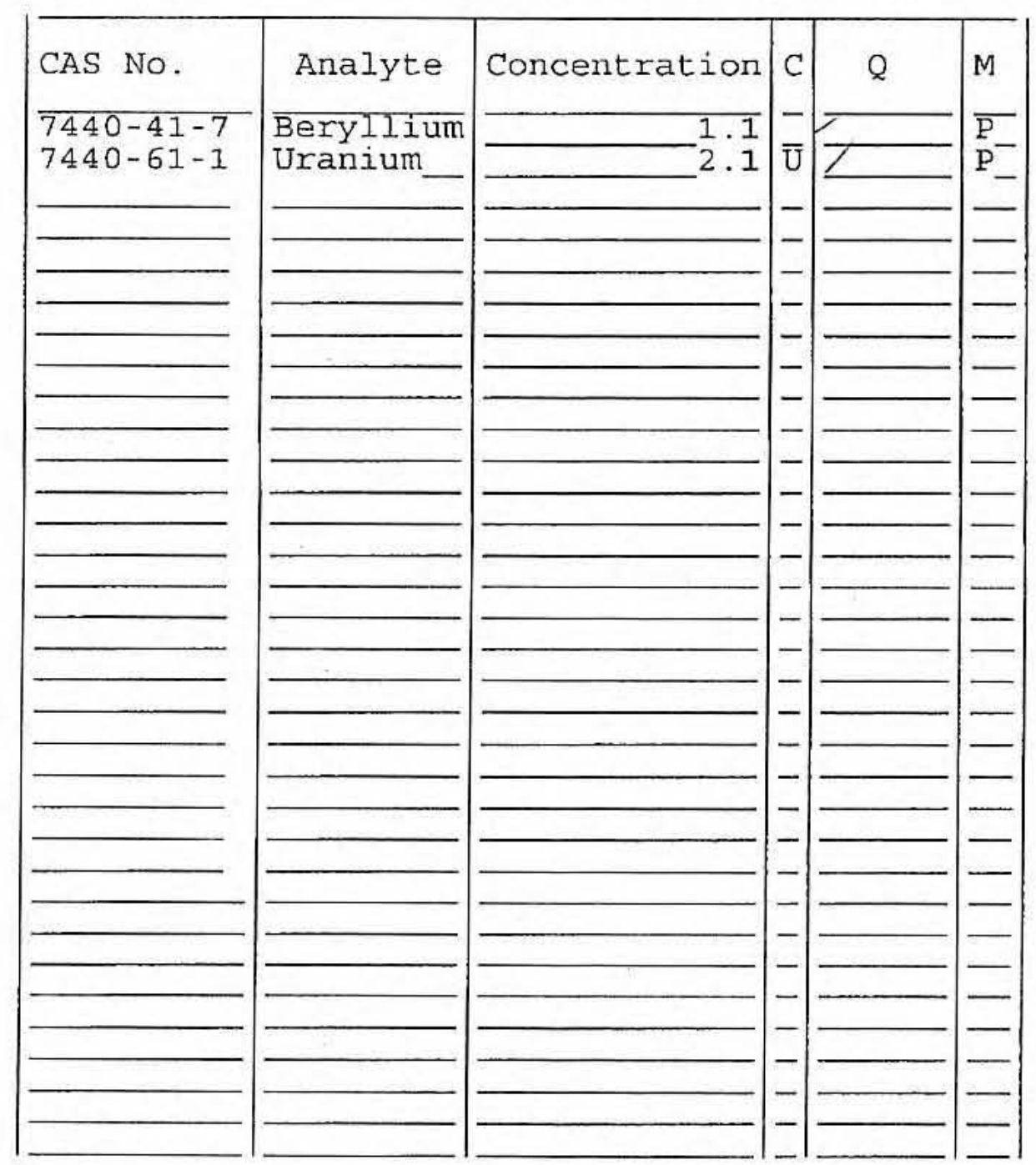

Color Before:

Color After:

Jomments:

NEDS - 34A
Clarity Before:

Clarity After:
Texture:

Artifacts: 
Lab Name: LIONVILIE_LABORATORY

Lab Code: LVLI

Matrix (soil/water): SOIL

Level (low/med):

\% Solids:

LOW
$-92 \cdot \overline{8}$
Case No.: NEDS_ SAS No.:
EPA SAMPLE NO.

$34 \mathrm{~B}$

SDG No.: V2742

Lab Sample ID: 0609L860-008

Date Received: 09/01/06

Concentration Units (ug/L or $\mathrm{mg} / \mathrm{kg}$ dry weight): $\mathrm{MG} / \mathrm{KG}$

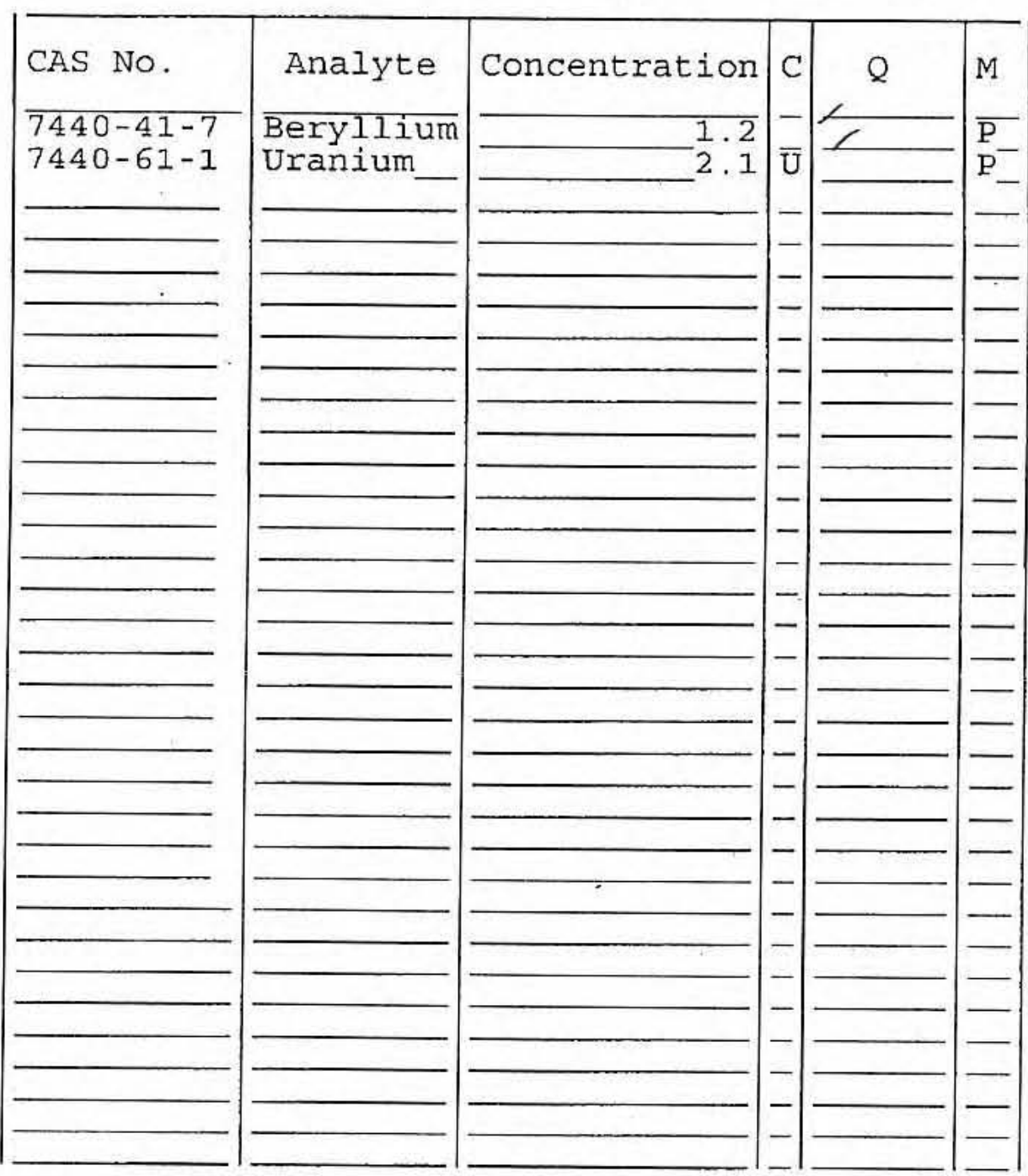

Color Before:

Color After:

\section{Comments :}

NEDS - 34B
Clarity Before:

Clarity After:
Texture:

Artifacts: 


\section{INORGANIC ANALYSES DATA SHEET}

Lab Name: LIONVILLE_LABORATORY

Lab Code: LVLI

Matrix (soil/water): SoIL

Level. (low/med): LOW

$\div$ Solids :
EPA SAMPLE NO.

$35 \mathrm{~A}$

Contract: 60052

SAS No. :

Lab Sample ID: 06094860-0̄09

Date Received: 09/01/06

Concentration Units (ug/L or $\mathrm{mg} / \mathrm{kg}$ dry weight) : MG/KG

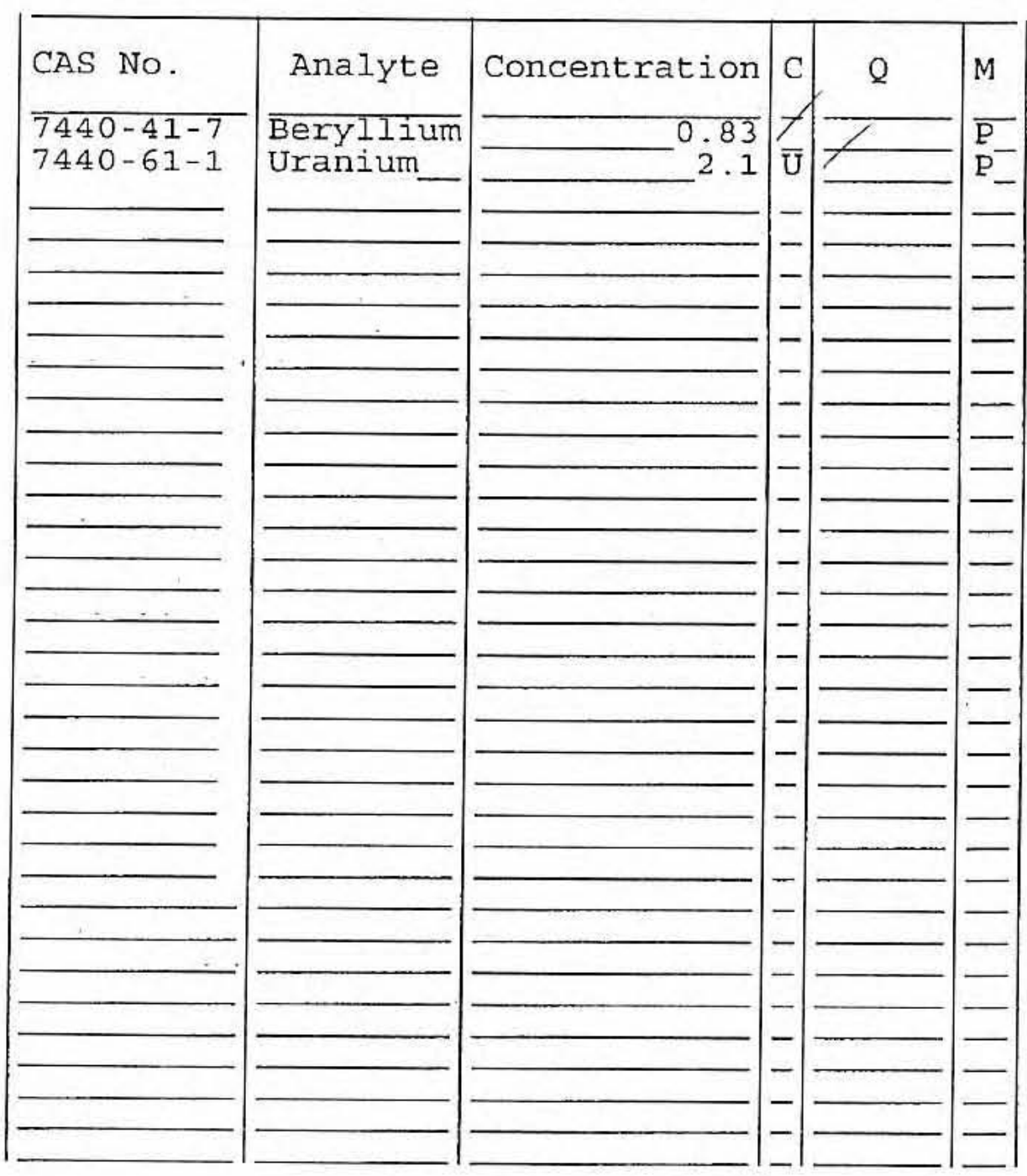

Color Before:

Color After:

Comments:

NEDS $-35 A$
Clarity Before:

Clarity After:
Texture:

Artifacts : 
U.S. EPA

\section{1}

INORGANIC ANALYSES DATA SHEET
EPA SAMPLE NO.

$35 B$

Contract: 60052

SAS NO. :

Case No:: NEDS SOIL_

LOW
Lab Sample ID: 0609L860-0̄10

Date Received: 09/01/06

Level (low

Concentration Units (ug/L or $\mathrm{mg} / \mathrm{kg}$ dry weight): MG/KG

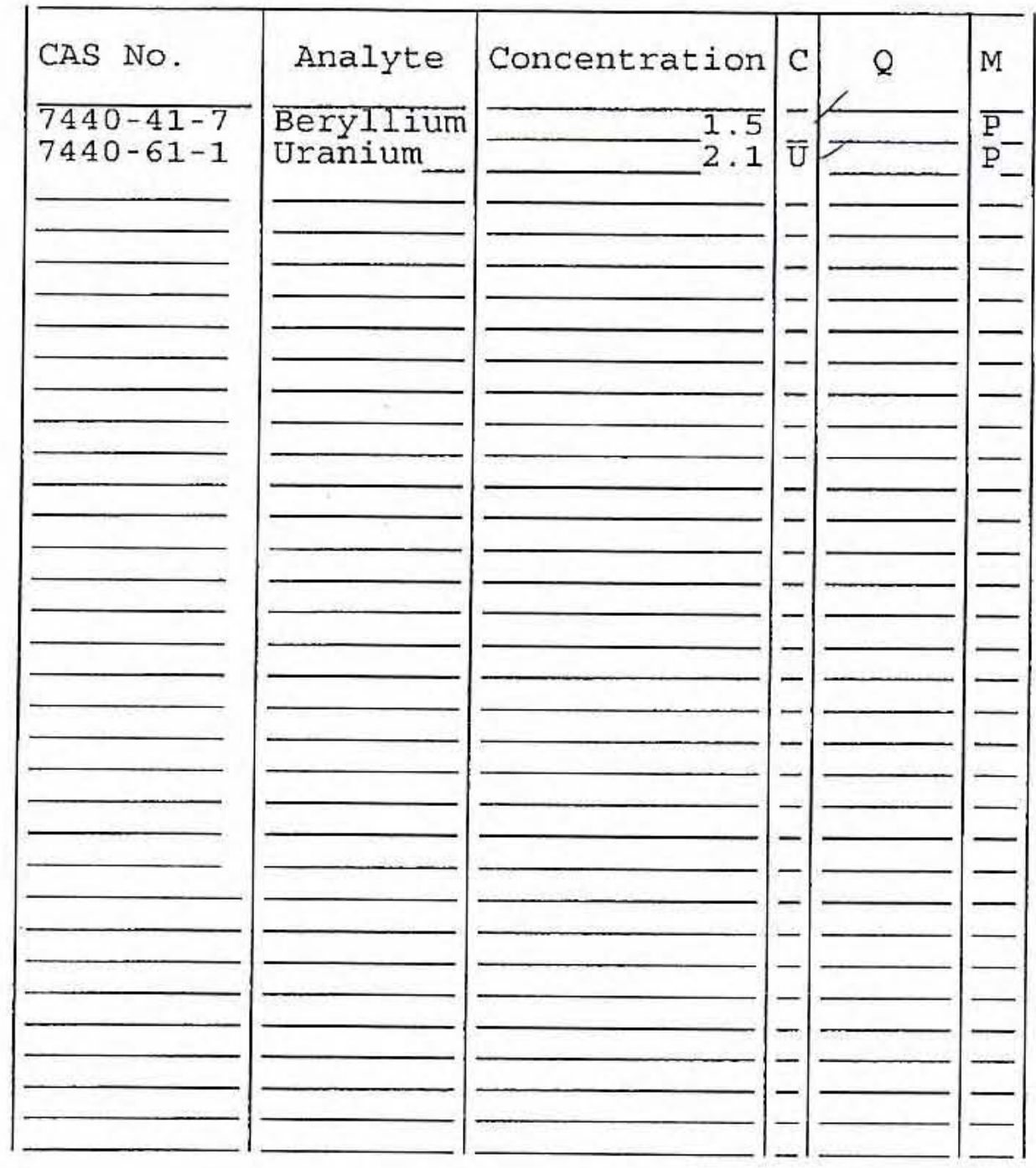

Color Before:

Color After:

Comments :

NEDS - 35B
Clarity Before:

Clarity After:
Texture:

Artifacts : 


\section{U.S. EPA}

1

INORGANIC ANALYSES DATA SHEET
EPA SAMPLE NO.

$36 \mathrm{~A}$

Lab Name: LIONVILLE_LABORATORY_Contract: 60052

Lab Code: LVLI

Matrix (soil/water): SOIL

Level (low/med):

\% Solids:
LOW

$-92 \overline{9}$
SAS No.:

SDG No.: V2742

Lab Sample ID: 0609L860-0̄11

Date Received: 09/01/06

Concentration Units (ug/L or $\mathrm{mg} / \mathrm{kg}$ dry weight): MG/KG

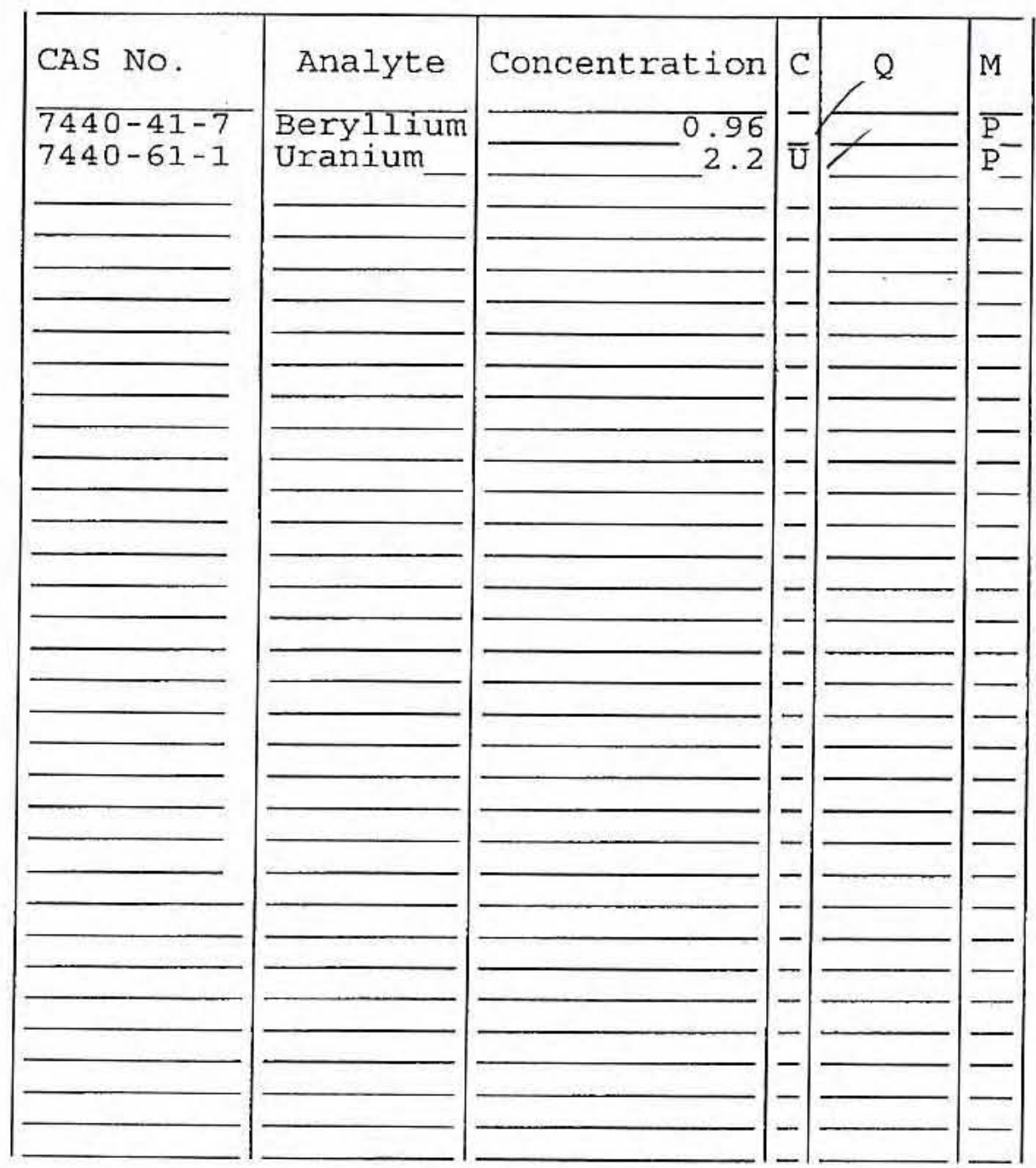

Color Before:

Color After:

\section{Comments:}

NEDS $-36 \mathrm{~A}$
Clarity Before:

Clarity After:
Texture:

Artifacts : 


\section{U.S. EPA}

\section{1}

INORGANIC ANALYSES DATA SHEET
EPA SAMPLE NO.

\section{$36 \mathrm{~B}$}

Lab Name: LIONVILLE LABORATORY Contract: 60052

Lab Code: LVLI Matrix (soil/water): SoIL Level (low/med): LOW : Solids: $\quad-92 . \overline{5}$

SAS NO.:

SDG No.: V2742

Lab Sample ID: 0609L860-0̄12

Date Received: 09/01/06

Concentration Units (ug/L or $\mathrm{mg} / \mathrm{kg}$ dry weight): MG/KG

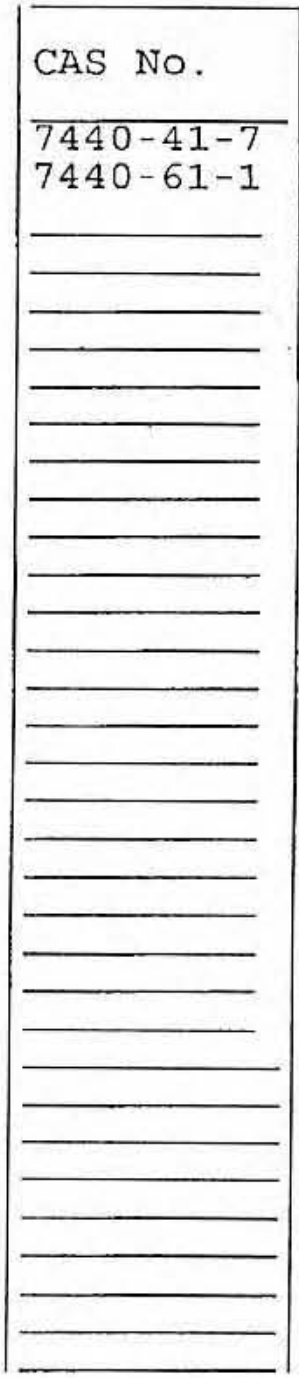

Color Before:

Color After:

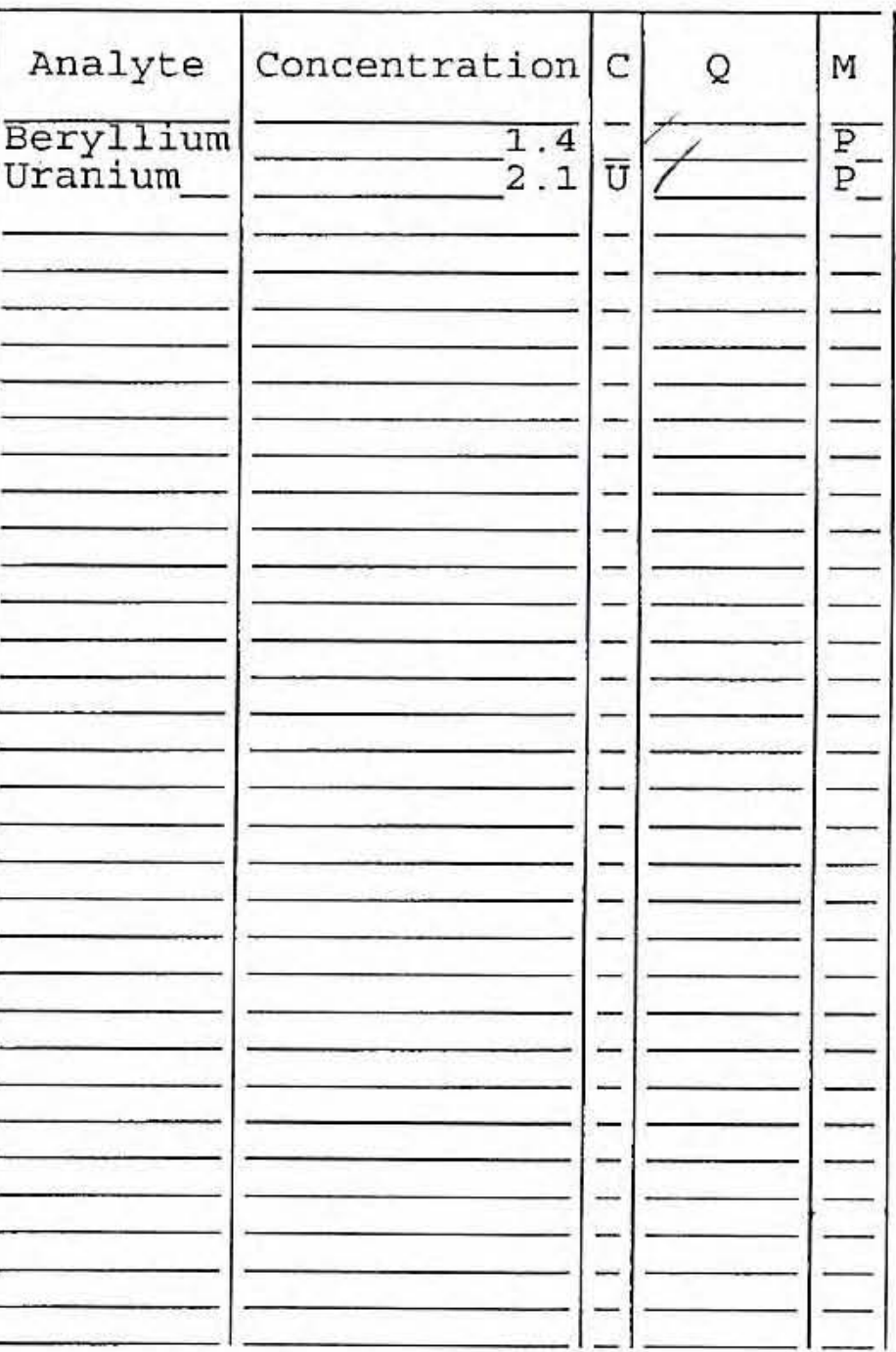

Clarity Before:

Clarity After:

Texture:

Artifacts:

Comments :

NEDS - 36B 
EPA SAMPLE NO.

$37 \mathrm{~A}$

Lab Name: LIONVILLE_LABORATORY_Contract: 60052

Lab Code: LVLI

Matrix (soil/water)

Level (low/med) :

: Solids:
Case No.: NEDS_ SAS No.

SOIL_
LOW -
-91.4

SDG No: V2742

Lab Sample ID: 06091860-0̄13

Date Received: 09/01/06

Concentration Units (ug/L or $\mathrm{mg} / \mathrm{kg}$ dry weight): MG/KG

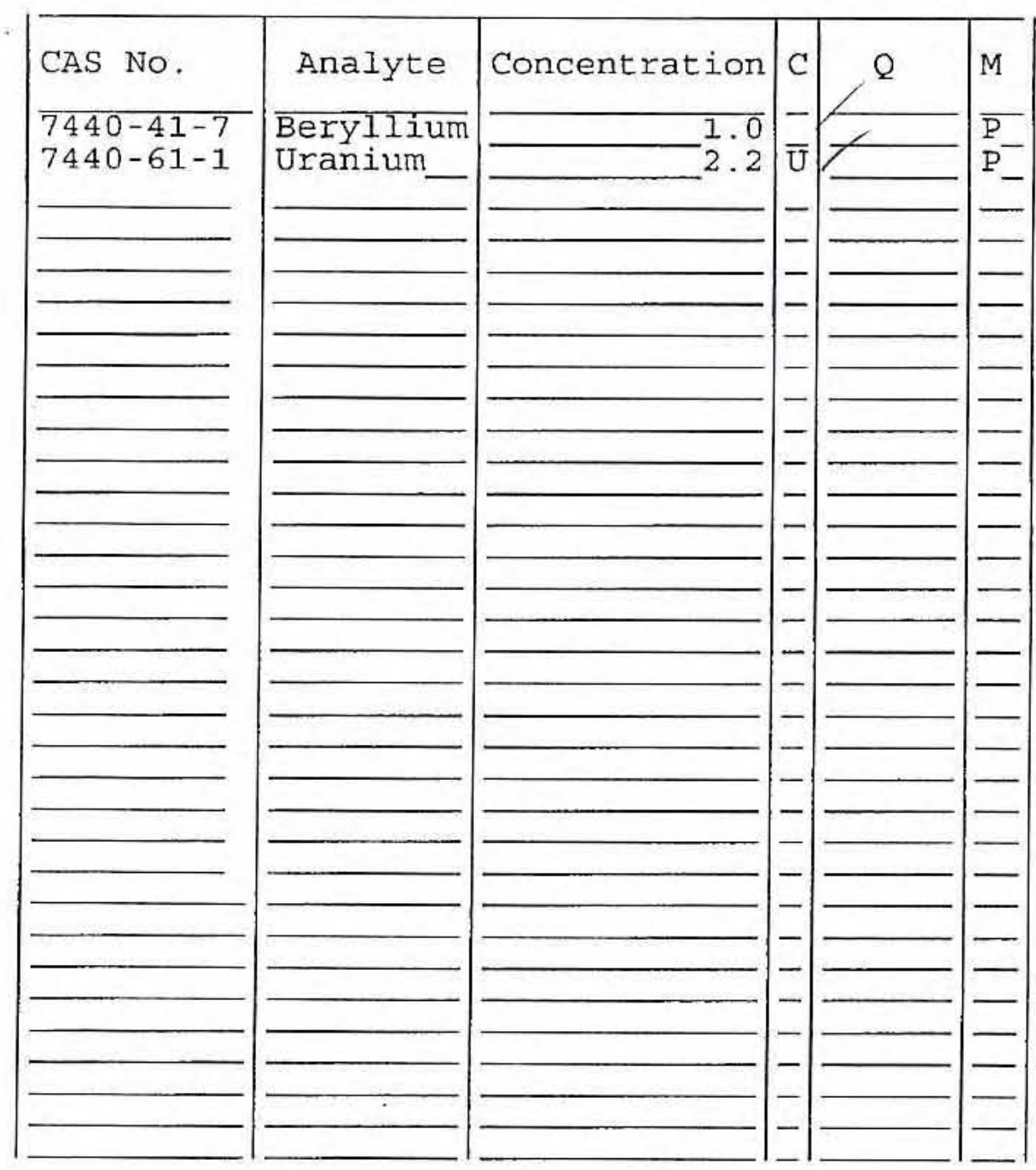

Color Before:

Color After:

\section{Comments :}

NEDS - 37A
Clarity Before:

Clarity After:
Texture:

Artifacts: 
U.S. EPA

1

INORGANIC ANALYSES DATA SHEET
EPA SAMPLE NO.

\section{$37 \mathrm{~B}$}

Contract: 60052

Lab Name: LIONVILLE_LABORATORY

Lab Code: LVLI

Matrix (soil/water): SoIL

Level (low/med):

\% Solids:
SAS No. :

SDG No.: V2742 LOW $-93 . \overline{8}$
Lab Sample ID: 0609L860-0̄14

Date Received: 09/01/06

Concentration Units (ug/L or mg/kg dry weight): MG/KG

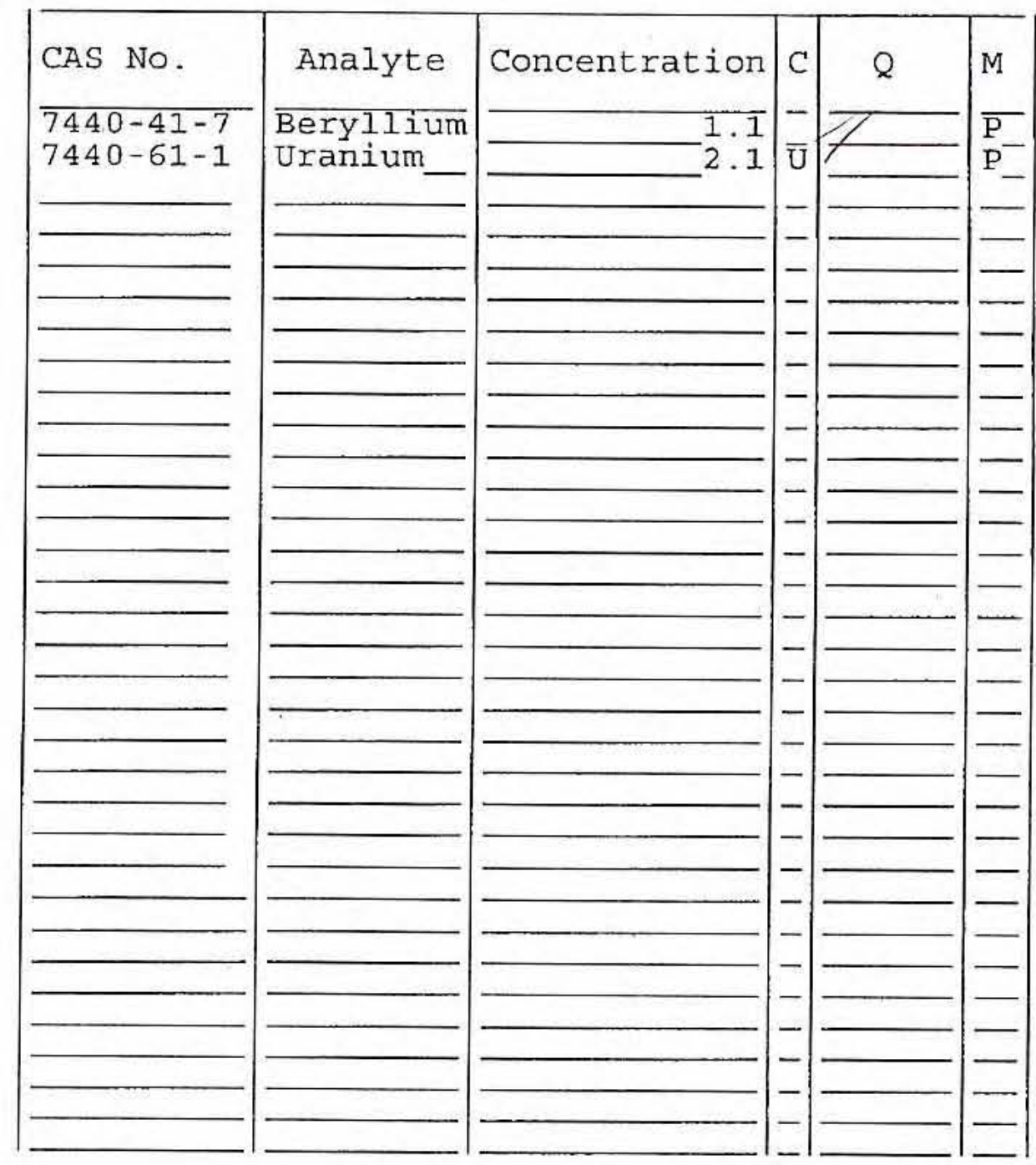

Color Before:

Color After:

Comments :

NEDS $-37 B$
Clarity Before:

Clarity After:
Texture:

Artifacts: 
1 INORGANIC ANALYSES DATA SHEET
EPA SAMPLE NO.

$38 \mathrm{~A}$

Contract: 60052

SAS No.:

SDG No.: V2742

Date Received: 09/01/06
Lab Name: LIONVILLE_LABORATORY

Lab Code: LVLI

Matrix (soil/water): SOIL

Level (low/med):

: Solids :
LOW

$-96.1$
Case No.: NEDS I

\section{Concentration Units (ug/L or $\mathrm{mg} / \mathrm{kg}$ dry weight): MG/KG}

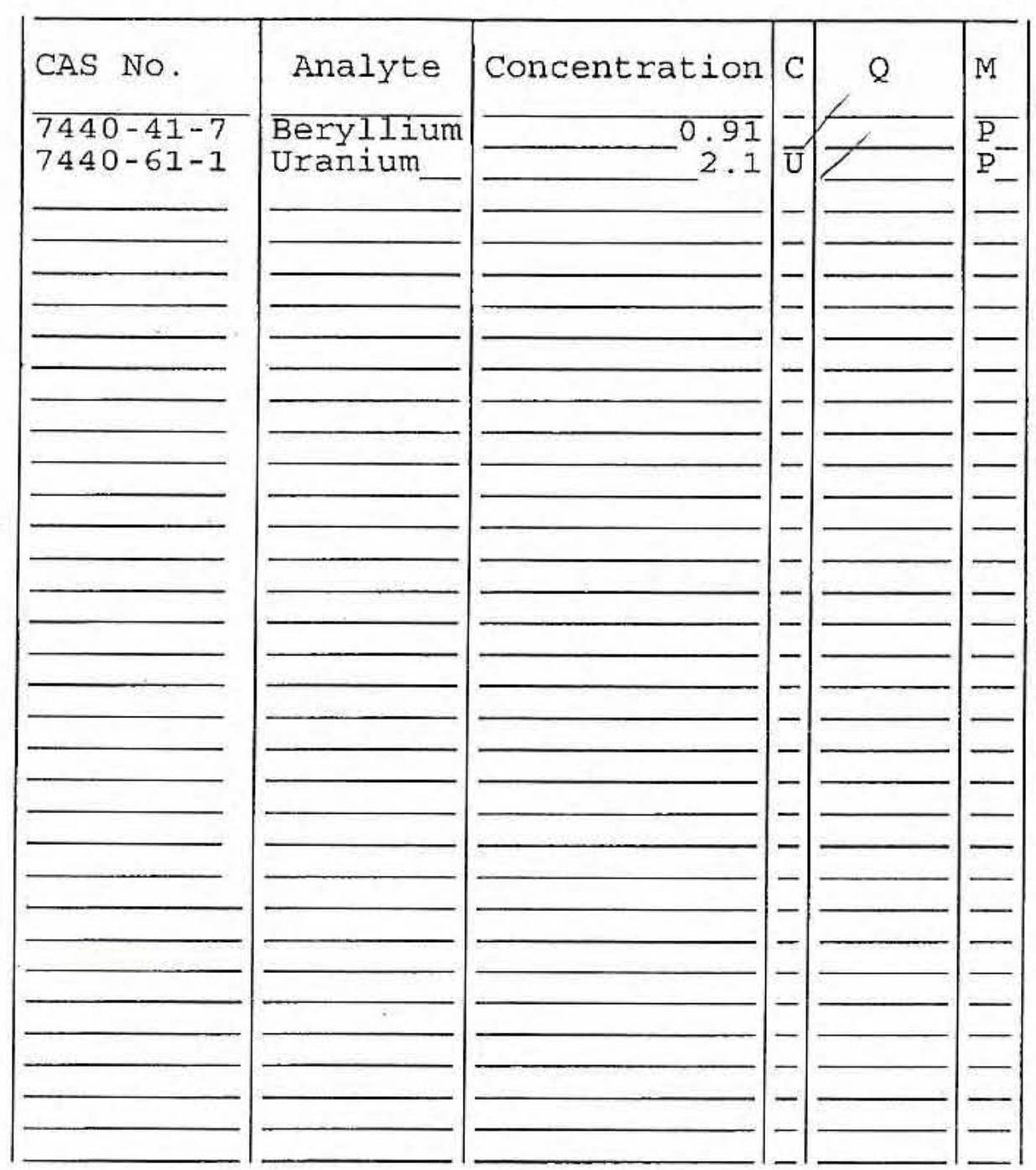

Color Before:

Color After:

\section{Comments :}

NEDS - 38A
Clarity Before:

Clarity After:
Texture:

Artifacts: 
EPA SAMPLE NO.

$38 \mathrm{~B}$

Lab Name: LIONVILLE_LABORATORY

Lab Code: LVLI

Matrix (soil/water) : SoIL

Level (low/med):

: Solids :
Contract: 60052

SAS NO.:

$$
\begin{array}{r}
\text { LOW } \\
-92.1
\end{array}
$$

Lab Sample ID: 0609L860-0̄16

Date Received: 09/01/06

Concentration Units (ug/L or $\mathrm{mg} / \mathrm{kg}$ dry weight) : MG/KG

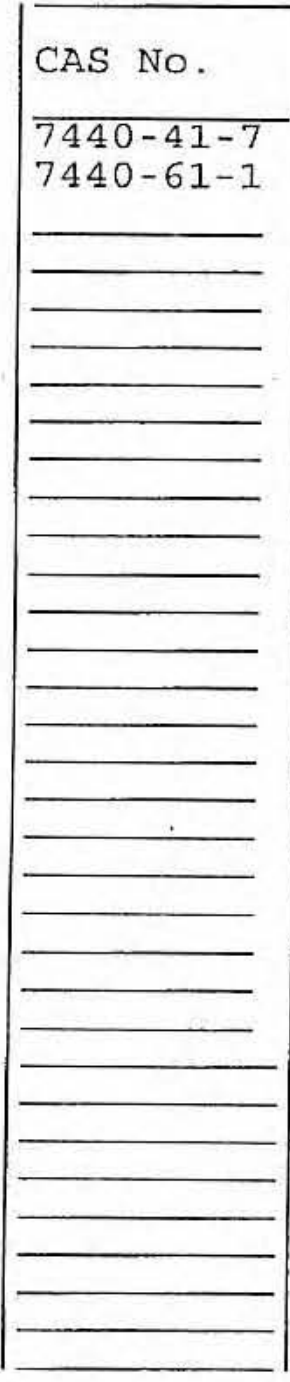

Color Before:

Color After:

Comments :

NEDS - 38B

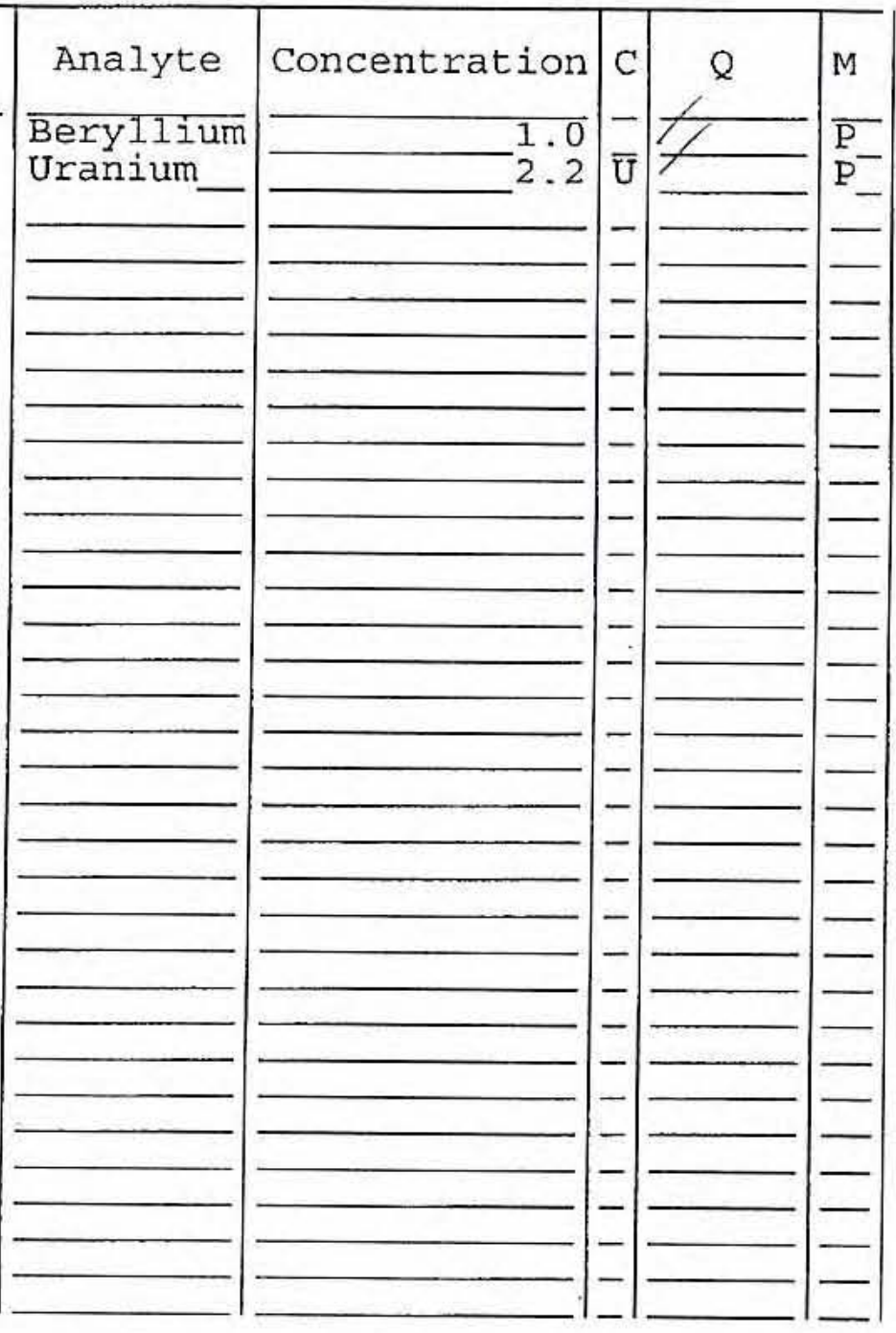

Clarity Before:

Clarity After:
Texture:

Artifacts : 
1 INORGANIC ANALYSES DATA SHEET
EPA SAMPLE NO.

R1.

Contract: 60052

SAS NO. :

SDG No.: V2742

Lab Code: LVLI IL_LABORATORY
Case No.: NEDS

Matrix (soil/water): WATER

Level (low/med): LOW

\% Solids :
LOW
Lab Sample ID: 060்9L860-0̄17

Date Received: 09/01/06

Concentration Units (ug/L or $\mathrm{mg} / \mathrm{kg}$ dry weight): UG/L_

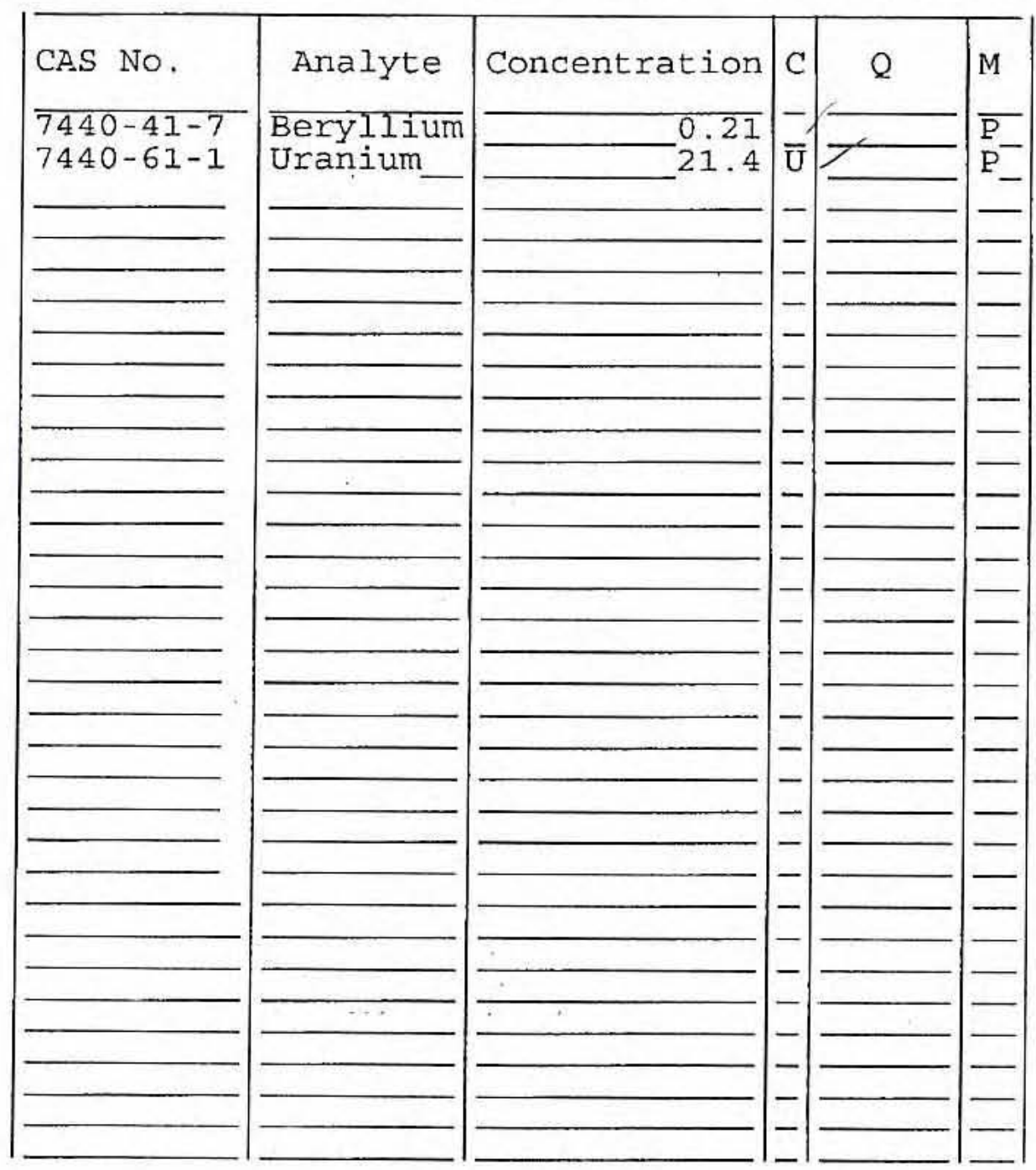

Color Before:

Color After:

Comments :

NEDS - R 1
Clarity Before:

Clarity After:
Texture:

Artifacts: 


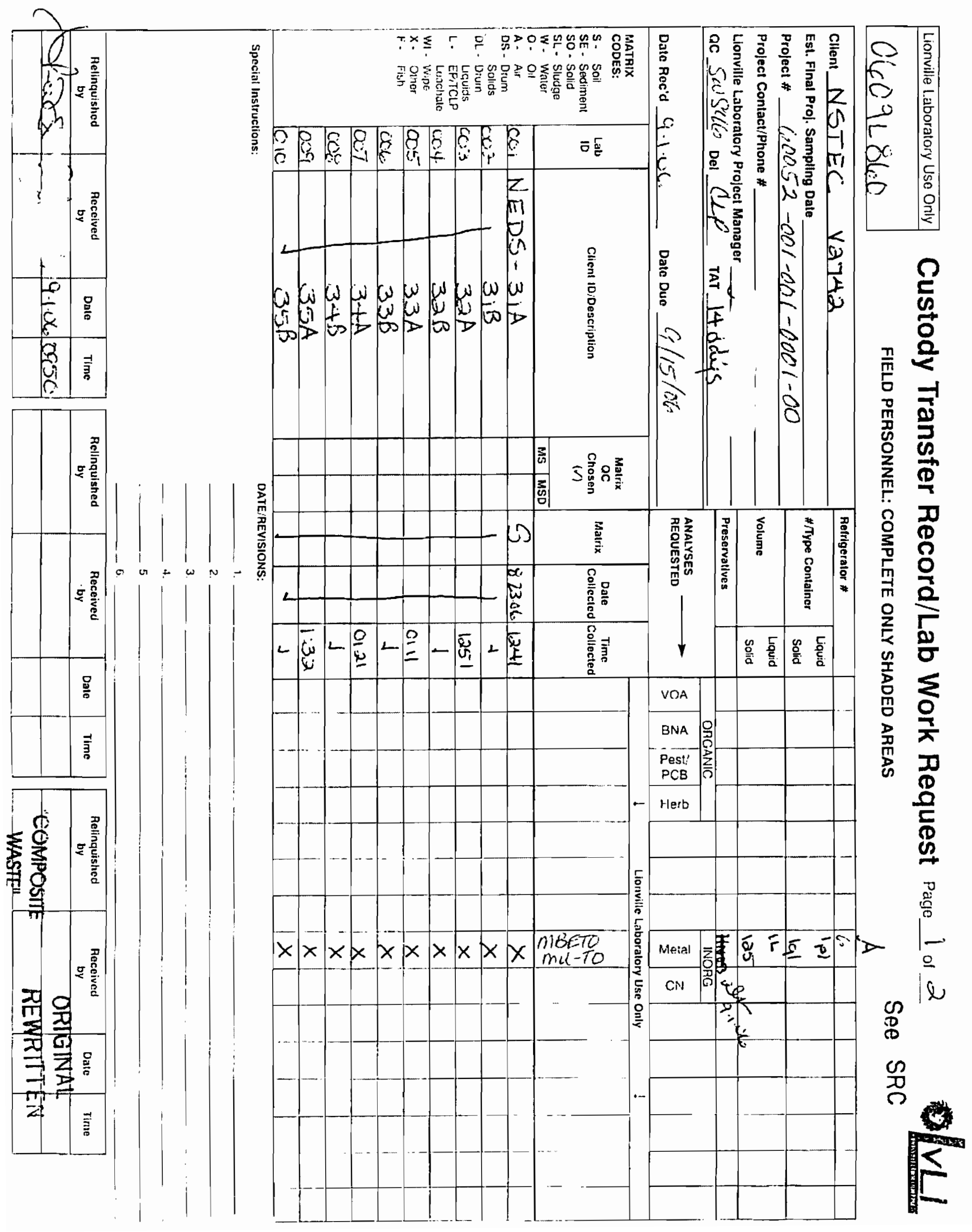




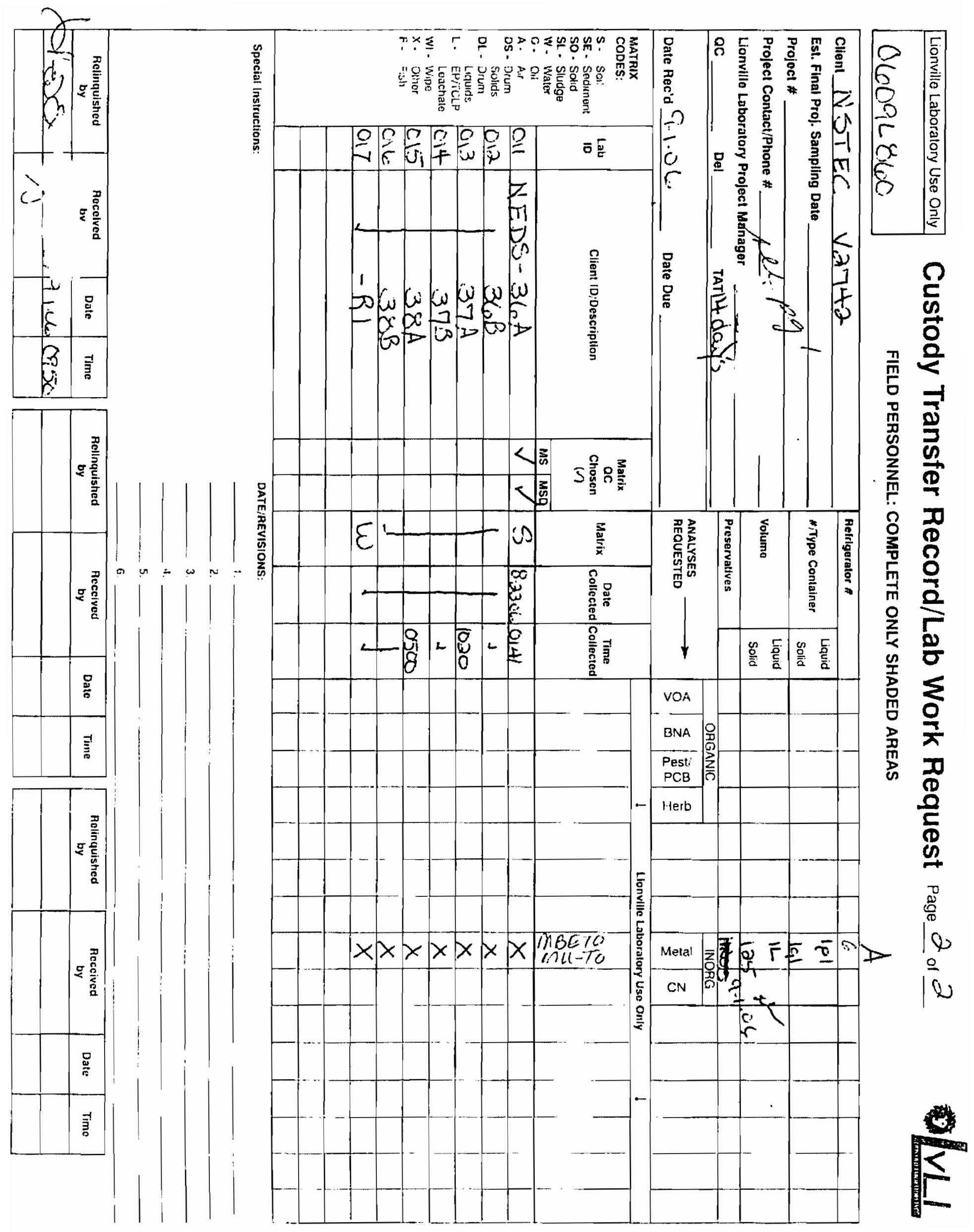




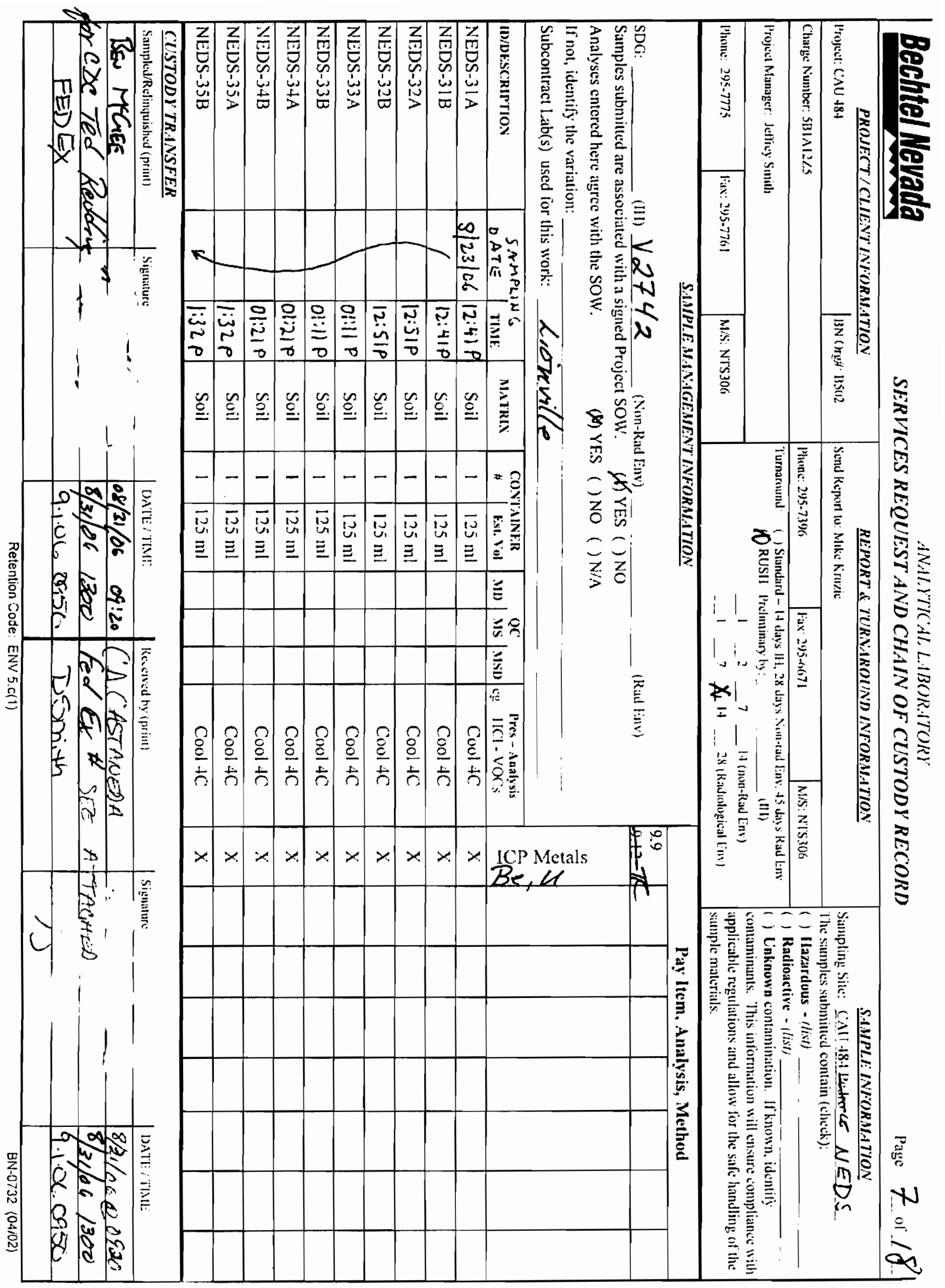




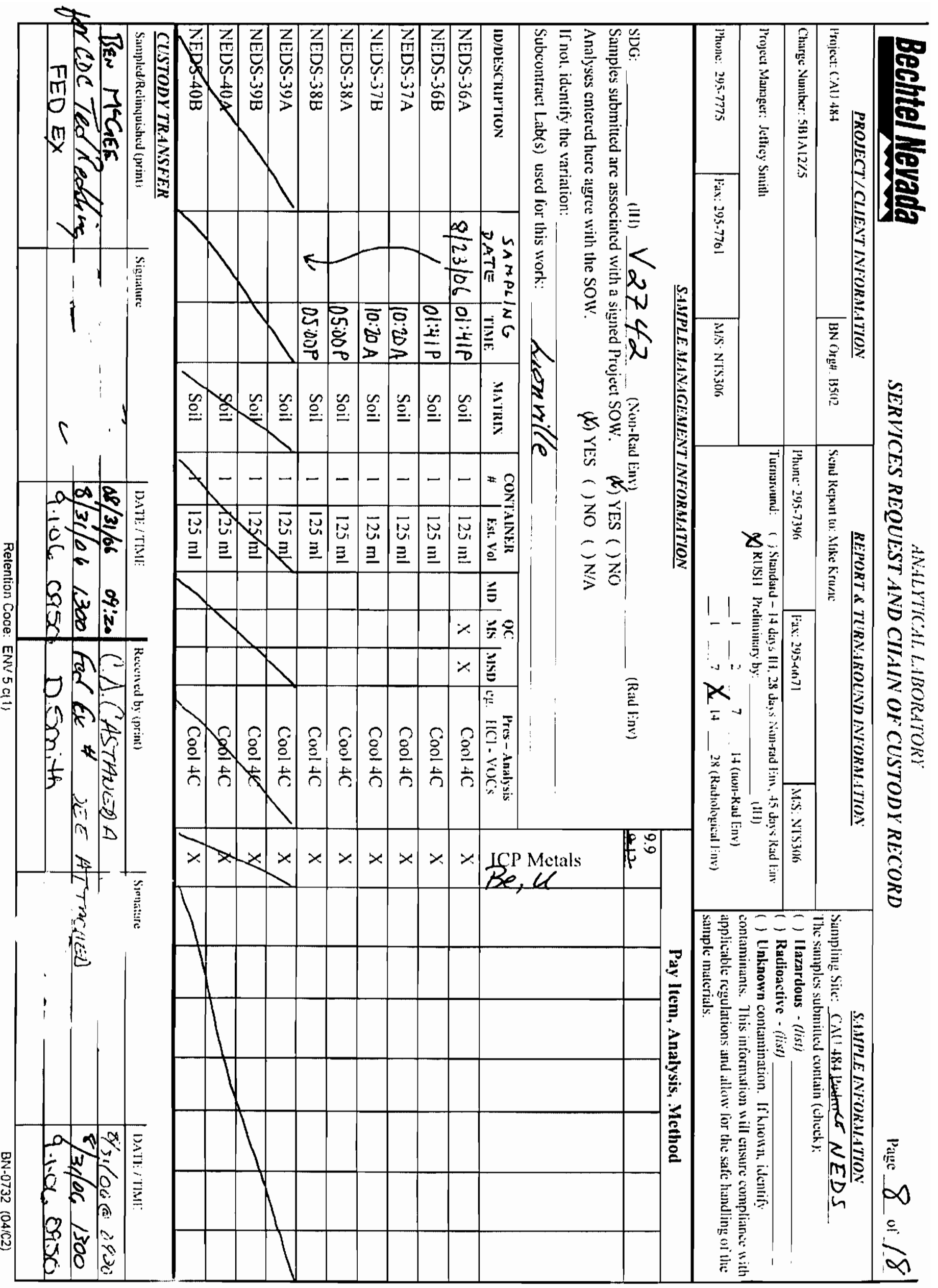




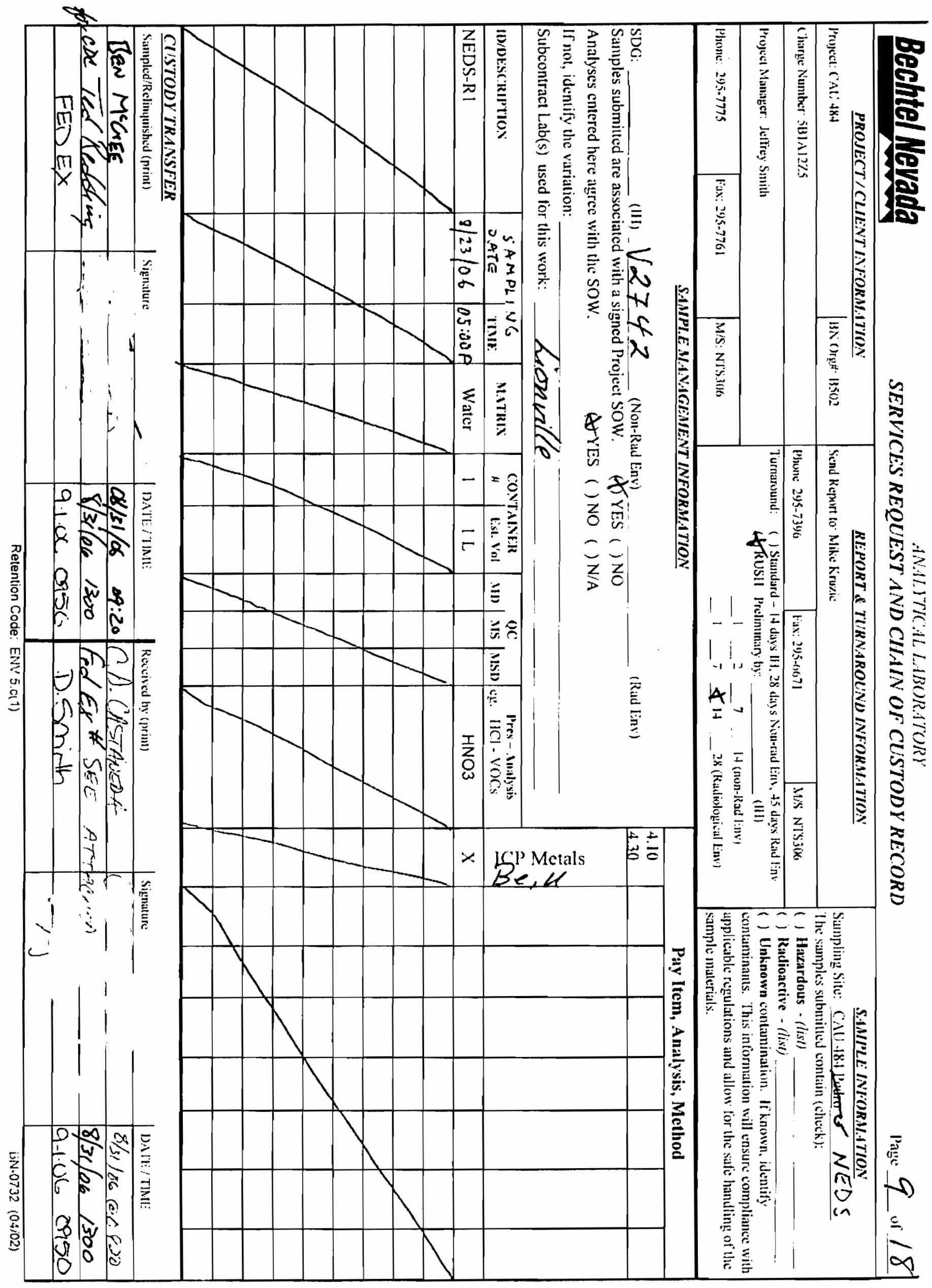




\title{
INVESTIGATION (GEOPROBE ${ }^{\circledR}$ ) SOIL SAMPLE ANALYTICAL RESULTS
}

\author{
CAS TA-52-001-TANL \\ NEDS DETONATION AREA
}

\author{
Radiological Data
}




\title{
GENERAL ENGINEERING LABORATORIES, LLC \\ 2040 Savage Hoad Charleston SC 29407 - (843) 556-8171 - www.gel.com
}

\section{Certificate of Analysis}

\author{
Company: Bechtel Nevada Corp. \\ Address: 2621 Losee Road \\ M/S NTS273 \\ North Las Vegas, Nevada 89030-4134 \\ Contact: $\quad$ Mr. Ted Redding \\ Project: Environmental Rad Analysis
}

Report Date: September 20, 2006

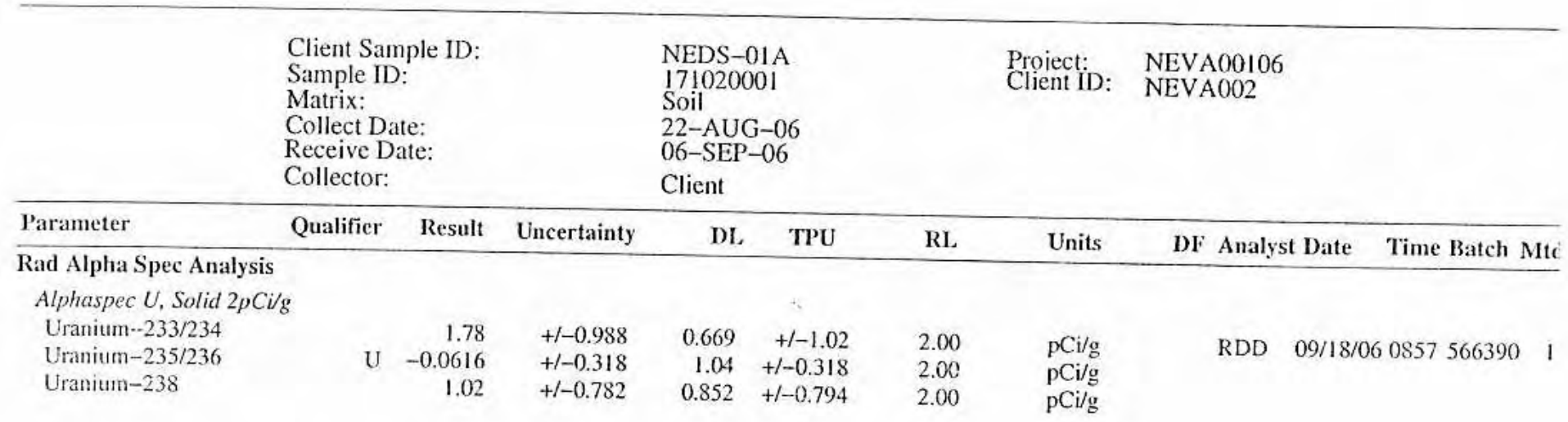

The following Prep Methods were performed

\begin{tabular}{llllll}
\hline Method & Description & Analyst & Date & Time & Prep Batch \\
\hline Dry Soil Prep & Dry Soil Prep GL-RAD-A-021 & AXP2 & $09 / 07 / 06$ & 1221 & 565878
\end{tabular}

The following Analytical Methods were performed

Method Description

\begin{tabular}{llcc} 
Surrogate/Tracer recovery & Test & Recovery\% & Acceptable Linits \\
\hline Uranium-232 & Alphaspec U, Solid 2pCi/g & 80 & $(25 \%-125 \%)$
\end{tabular}

\section{Notes:}

The Qualifiers in this report are defined as follows :

* A quality control analyte recovery is outside of specified acceptance criteria

$<$ Result is less than value reported

$>$ Result is greater than value reported

A The TIC is a suspected aldol-condensation product

$B$ Target analyte was detected in the associated blank

$\mathrm{BD}$ Results are either below the MDC or tracer recovery is low

C Analyte has been confirmed by GC/MS analysis

D Results are reported from a diluted aliquot of the sample

$\mathrm{H}$ Analytical holding time was exceeded

$\mathrm{J}$ Value is estimated

N/A Spike recovery limits do not apply. Sample concentration exceeds spike concentration by $4 X$ or more

$\mathrm{R}$ Sample results are rejected

$\mathrm{U}$ Analyte was analyzed for, but not detected above the MDL, MDA, or LOD.

UI Gamma Spectroscopy-Uncertain identification 


\section{GENERAL ENGINEERING LABORATORIES, LLC \\ 2040 Savage Road Charleston SC 29407 - (843) 556-8171 - www.gel.com}

\section{Certificate of Analysis}

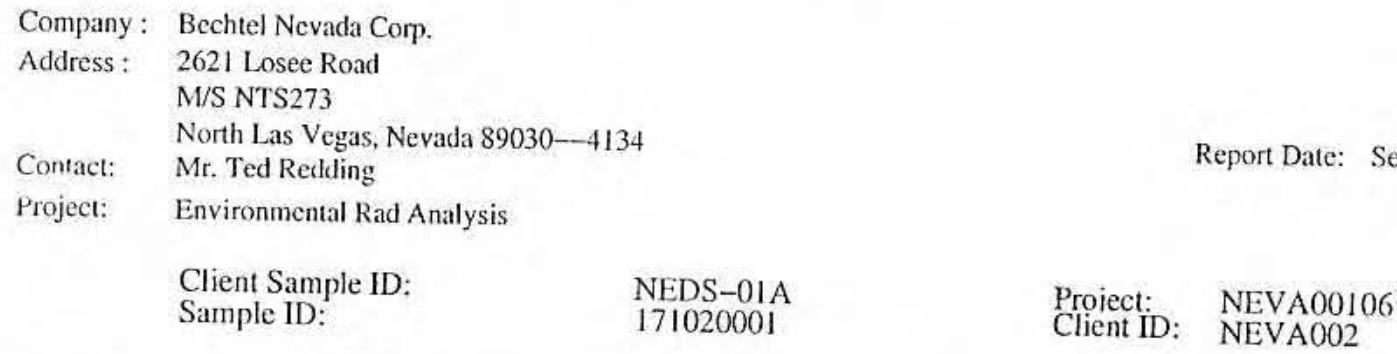

X Consult Case Narrative, Data Summary package, or Project Manager concerning this qualifier

Y QC Samples were not spiked with this compound

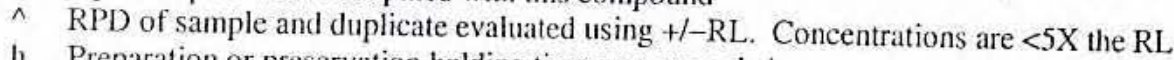

h Preparation or preservation holding time was exceeded

The above sample is reported on a dry weight basis. 


\title{
GENERAL ENGINEERING LABORATORIES, LLC \\ 2040 Savage Road Charleston SC 29407 - (843) 556-8171 - www.gel.com
}

\section{Certificate of Analysis}

\author{
Company: Bechtel Nevada Corp. \\ Address : 2621 Losee Road \\ M/S NTS273 \\ North Las Vegas, Nevada 89030—4134 \\ Contact: $\mathrm{Mr}$ Ted Reddig \\ Project: Enyironmental Rad Analysis \\ Report Date: September 20, 2006 \\ Client Sample ID: \\ 171020002 \\ Collect Date: $\quad$ 22-AUG-06 \\ Receive Date: $\quad$ 06-SEP-06 \\ Collector: \\ Client

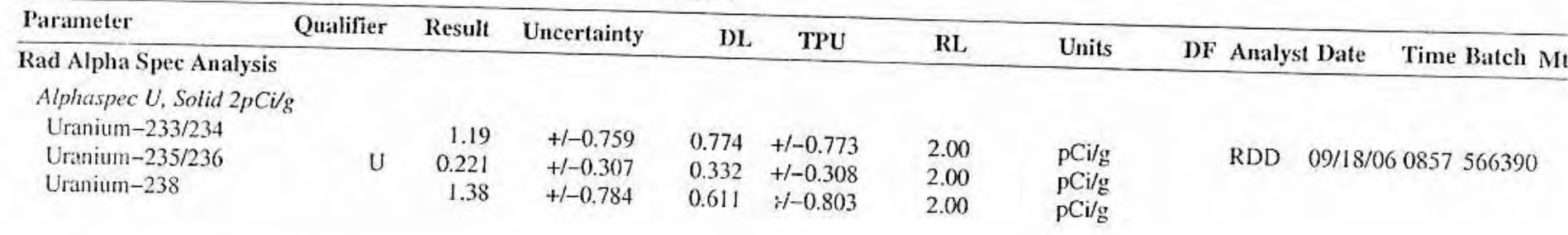 \\ The following Prep Methods were performed

\begin{tabular}{llllll}
\hline Method & Description & Analyst & Date & Time & Prep Batch \\
\hline Dry Soil Prep & Dry Soil Prep GL-RAD-A-021 & AXP2 & $09 / 07 / 06$ & 1221 & 565878
\end{tabular} \\ The following Analytical Methods were performed

\begin{tabular}{ll} 
Method & Description \\
\hline & DOE EML HASL-300, U-02-RC Modified
\end{tabular}

\begin{tabular}{llcc} 
Surrogate/Tracer recovery & Test & Recovery $\%$ & Acceptable Limits \\
\hline Uranium-232 & Alphaspec U, Solid 2pCi/g & 98 & $(25 \%-125 \%)$
\end{tabular} \\ Notes: \\ The Qualifiers in this report are defined as follows: \\ * A quality control analyte recovery is outside of specified acceptance criteria \\ $<$ Result is less than value reported \\ $>$ Result is greater than value reported \\ A The TIC is a suspected aldol-condensation product \\ B Target analyte was detected in the associated blank \\ BD Results are either below the MDC or tracer recovery is low \\ C Analyte has been confirmed by GC/MS analysis \\ D Results are reported from a diluted aliquot of the sample \\ $\mathrm{H}$ Analytical holding time was exceeded \\ J Value is estimated \\ N/A Spike recovery limits do not apply. Sample concentration exceeds spike concentration by $4 X$ or more
$R$ Sample results are rejected \\ $\mathrm{U}$ Analyte was analyzed for, but not detected above the MDL, MDA, or LOD. \\ UI Gamma Spectroscopy--Uncertain identification \\ $X$ Consult Case Narrative, Data Summary package, or Project Manager concerning this qualifier
}




\section{GENERAL ENGINEERING LABORATORIES, LLC \\ 2040 Savage Road Charleston SC 29407 - (813) 556-8171 - www.gel.com}

\section{Certificate of Analysis}

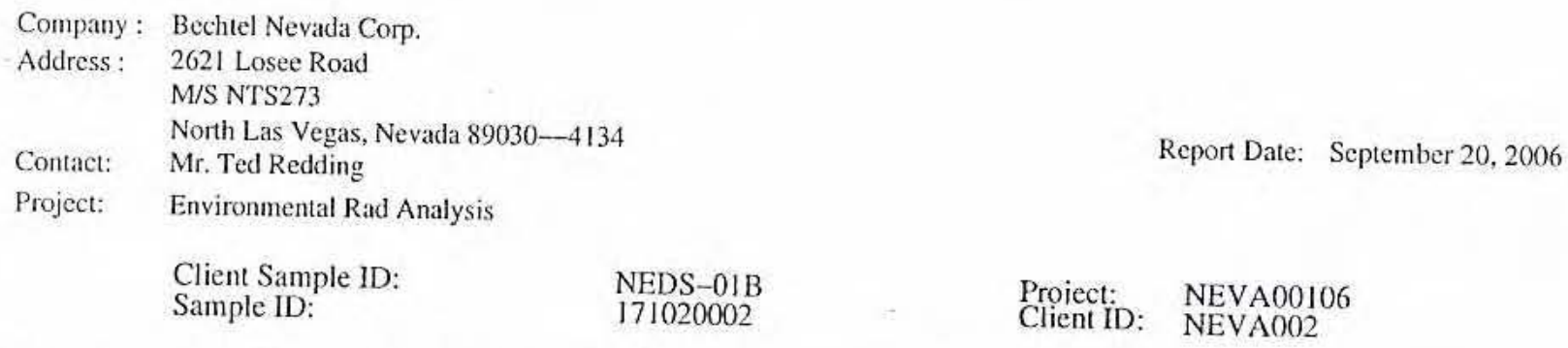

Y QC Samples were not spiked with this compound

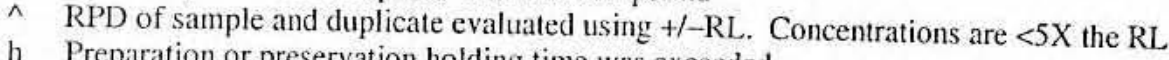

h Preparation or preservation holding time was exceeded

The above sample is reported on a dry weight basis. 


\section{GENERAL ENGINEERING LABORATORIES, LLC \\ 2040 Savage Road Charleston SC 29407 - (843) 556-8171 . www.gel.com}

\section{Certificate of Analysis}

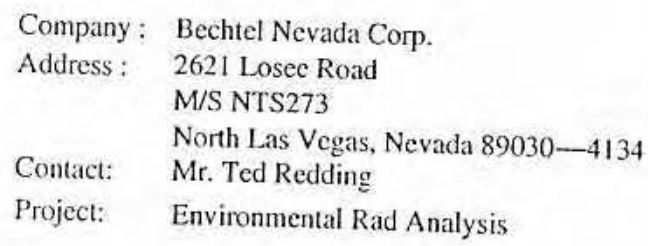

\begin{tabular}{|c|c|c|c|c|c|c|c|c|c|c|}
\hline Parameter & Qualifier & Result & Uncertainty & DL & TPU & RL & Units & DF & Analyst Date & Time Batch Mt \\
\hline $\begin{array}{l}\text { Rad Alpha Spec Ana } \\
\text { Alphaspec U, Solid }\end{array}$ & & & & & & & & & & \\
\hline $\begin{array}{l}\text { Uranium-233/234 } \\
\text { Uranium-235/236 } \\
\text { Uranium-238 }\end{array}$ & $\mathrm{U}$ & $\begin{array}{r}1.80 \\
0.154 \\
1.24\end{array}$ & $\begin{array}{l}+/-0.980 \\
+/-0.347 \\
+/-0.826\end{array}$ & $\begin{array}{l}0.967 \\
0.733 \\
0.927\end{array}$ & $\begin{array}{r}+/-1.01 \\
+/-0.347 \\
+/-0.842\end{array}$ & $\begin{array}{l}2.00 \\
2.00 \\
2.06\end{array}$ & $\begin{array}{l}\mathrm{pCi} / \mathrm{g} \\
\mathrm{pCi} / \mathrm{g} \\
\mathrm{pCi} / \mathrm{g}\end{array}$ & & RDD $\quad 09 / 18 / 0$ & 60857566390 \\
\hline
\end{tabular}

The following Prep Methods were performed

\begin{tabular}{lllllll}
\hline Method & Description & Analyst & Date & Time & Prep Batch \\
\cline { 2 - 5 } Dry Soil Prep & Dry Soil Prep GL-RAD-A-021 & AXP2 & $09 / 07 / 06$ & 1221 & 565878
\end{tabular}

The following Analytical Methods were performed

$\begin{array}{ll}\text { Method } & \text { Description } \\ 1 & \text { DOE EML HASL-300, U-02-RC Modified }\end{array}$

\begin{tabular}{llcc} 
Surrogate/Tracer recovery & Test & Recovery $\%$ & Acceptable Limits \\
\hline Uranium-232 & Alphaspec U, Solid 2pCi/g & 99 & $(25 \%-125 \%)$
\end{tabular}

Notes:

The Qualifiers in this report are defined as follows :

* A quality control analyte recovery is outside of specified acceptance criteria

$<$ Result is less than value reporied

$>$ Result is greater than value reported

A The TIC is a suspected aldol-condensation product

B Target analyte was detected in the associated blank

$\mathrm{BD}$ Results are either below the MDC or tracer recovery is low

C Analyte has been confirmed by GC/MS analysis

D Results are reported from a diluted aliquot of the sample

$\mathrm{H}$ Analytical holding time was exceeded

$\mathrm{J}$ Value is estimated

N/A Spike recovery limits do not apply. Sample concentration exceeds spike concentration by $4 \mathrm{X}$ or more
R Sample results are rejected

U Analyte was analyzed for, but not detected above the MDL, MDA, or LOD.

UI Gamma Spectroscopy-Uncertain identification

$X$ Consult Case Narrative, Data Summary package, or Project Manager concerning this qualifier 


\section{GENERAL ENGINEERING LABORATORIES, LLC \\ 2040 Savage Road Charleston SC 29407 - (843) 556-8171 - www.gel.corn}

\section{$\underline{\text { Certificate of Analysis }}$}

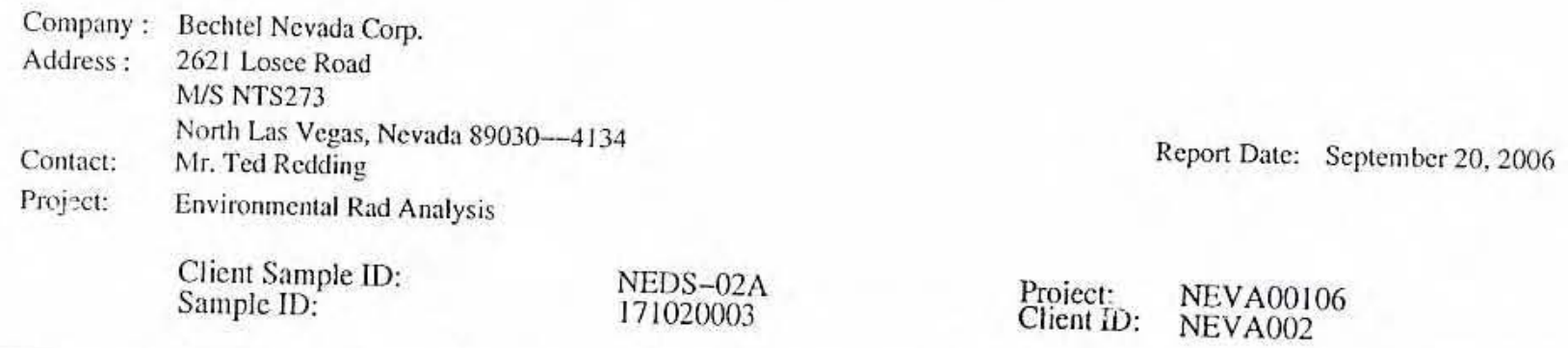

DL TPU

RL

Y QC Samples were not spiked with this compound

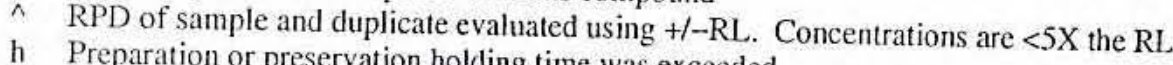

$h$ Preparation or preservation holding time was exceeded

The arove sample is reported on a dry weight basis. 


\section{GENERAL ENGINEERING LABORATORIES, LLC \\ 2040 Savage Road Charleston SC 29407 - (843) 556-8171 - www.gel.cum}

\section{Certificate of Analysis}

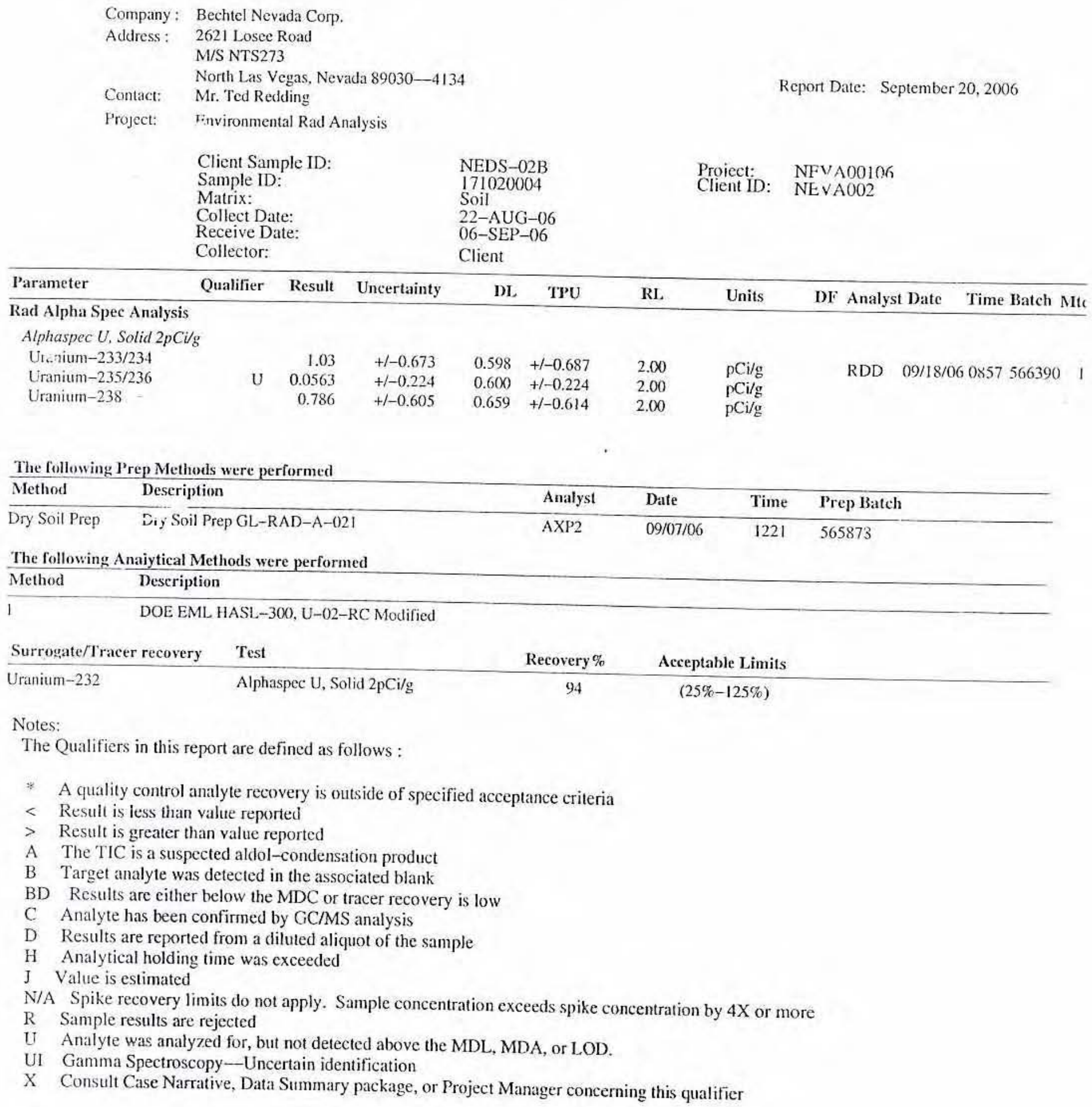




\section{GENERAL ENGINEERING LABORATORIES, LLC \\ 2040 Savage Road Charleston SC 29407 - (843) 556-8171 - www.gel.com}

\section{Certificate of Analysis}

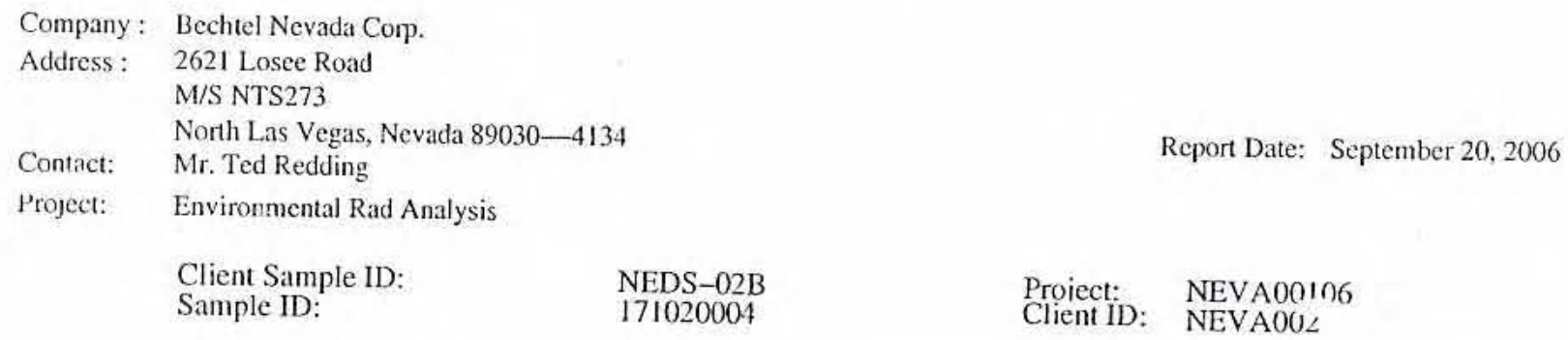

DL TPU

RI,

Units

Y QC Samples were not spiked with this compound

$\hat{A}$ RPT of sample and duplicate evaluated using $+/-$ RL. Concentrations are $<5 X$ the RL

$\mathrm{h}$ Preparation or preservation holding time was exceeded

The above sample is reported on a dry weight basis. 


\section{GENERAL ENGINEERING LABORATORIES, LLC \\ 2040 Savage Road Charleston SC 29407 - (843) 556-8171 - www.gel.com}

\section{Certificate of Analysis}

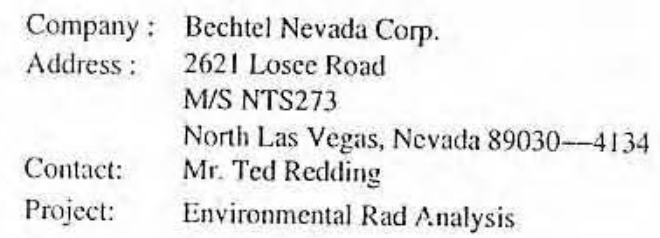

Report Date: September 20,2006

Proiect: NEVA00106

Client ID: NEVA002

\begin{tabular}{|c|c|c|c|c|c|c|c|c|c|c|c|}
\hline Parameter & Qualifier & Result & Uncertainty & DL & TPU & RL & Units & $\mathrm{DF}$ & Analyst & Date & Time Batch Mt \\
\hline $\begin{array}{l}\text { Rad Alpha Spec Ana } \\
\text { Alphaspec U. Solid } 2\end{array}$ & & & & & & & & \multirow{2}{*}{\multicolumn{2}{|c|}{ RDD }} & \multirow{2}{*}{\multicolumn{2}{|c|}{$09 / 18 / 060857566390$}} \\
\hline $\begin{array}{l}\text { Uranium-233/234 } \\
\text { Uranium-235/236 } \\
\text { Jranium-238 }\end{array}$ & U & $\begin{array}{r}1.32 \\
-0.0827 \\
0.719\end{array}$ & $\begin{array}{l}+/-0.781 \\
+/-0.244 \\
+/-0.601\end{array}$ & $\begin{array}{l}0.634 \\
0.701 \\
0.699\end{array}$ & $\begin{array}{l}+1-0.799 \\
+/-0.244 \\
+-0.608\end{array}$ & $\begin{array}{l}2.00 \\
2.00 \\
2.00\end{array}$ & $\begin{array}{l}\mathrm{pCi} / \mathrm{g} \\
\mathrm{pCi} / \mathrm{g} \\
\mathrm{pCi} / \mathrm{g}\end{array}$ & & & & \\
\hline
\end{tabular}

The following Prep Methods were performed

\begin{tabular}{llllll}
\hline Method & Description & Analyst & Date & Time & Prep Batch \\
\hline Dry Soil Prep & Dry Soil Prep GL-KAD-A-021 & AXP2 & $09 / 07 / 06$ & 1221 & 565878
\end{tabular}

The followirg Analytical Methods were performed

\begin{tabular}{ll} 
Method Description \\
\hline DOE EML HASL-300, U-02-RC Modified
\end{tabular}

\begin{tabular}{llcc} 
Surrogate/Tracer recovery & Test & Recovery $\%$ & Acceptable Limits \\
\hline Uranium-232 & Alphaspec U, Solid 2pCi/g & 102 & $(25 \%-125 \%)$
\end{tabular}

Notes:

The Qualifiers in this report are defined as follows :

* A quality control analyte recovery is outside of specified acceptance criteria

$<$ Result is less than value reported

$>$ Result is greater than value reported

A The TIC is a suspected aldol-condensation product

$B$ Target analyte was detected in the associated blank

$\mathrm{BD}$ Results are either below the MDC or tracer recovery is low

C Analyte has been confirmed by GC/MS analysis

D Results are reported from a diluted aliquot of the sample

$\mathrm{H}$ Analytical holding time was exceeded

$\mathrm{J}$ Value is estimated

N/A Spike recovery limits do not apply. Sample concentration exceeds spike concentration by $4 X$ or more
R Sample results are rejected

U Analyte was analyzed for, but not detected above the MDL, MDA, or LOD.

UI Gamma Spectroscopy-Uncertain identification

X Consult Case Narrative, Data Summary package, or Project Manager concerning this qualifier 


\section{GENERAL ENGINEERING LABORATORIES, LLC}

2040 Savage Road Charleston SC 29407 - (843) 556-8171 - www.gel.com

\section{$\underline{\text { Certificate of Analysis }}$}

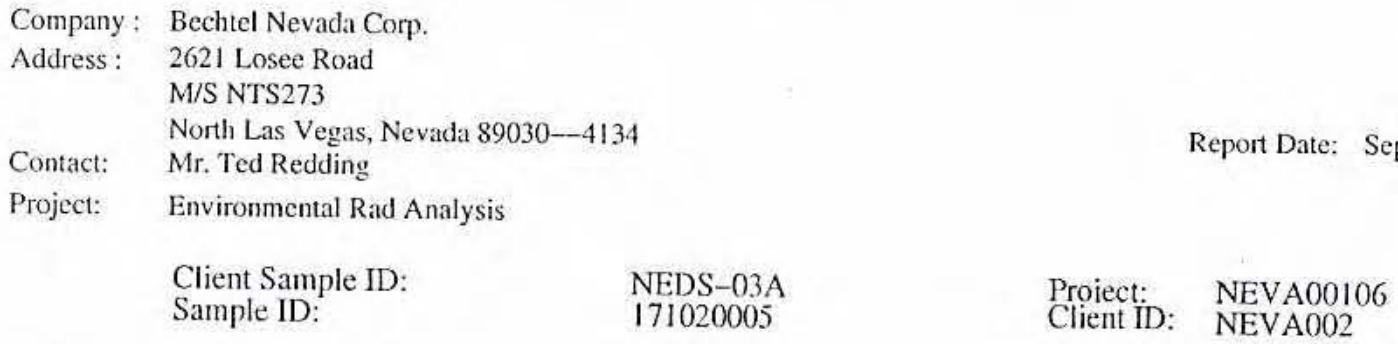

DL TPU

RI.

Units

DF Analyst Date

Time Batch Mt

Y QC Samples were not spiked with this compound

$\wedge$ RPD of sample and duplicate evaluated using $+/-R L$. Concentrations are $<5 X$ the RL

h Preparation or preservation holding time was exceeded

The above sample is reported on a dry weight basis. 


\section{GENERAL ENGINEERING LABORATORIES, LLC \\ 2040 Savage Road Charleston SC 29407 - (843) 556-8171 - www.gel.com}

\section{Certificate of Analysis}

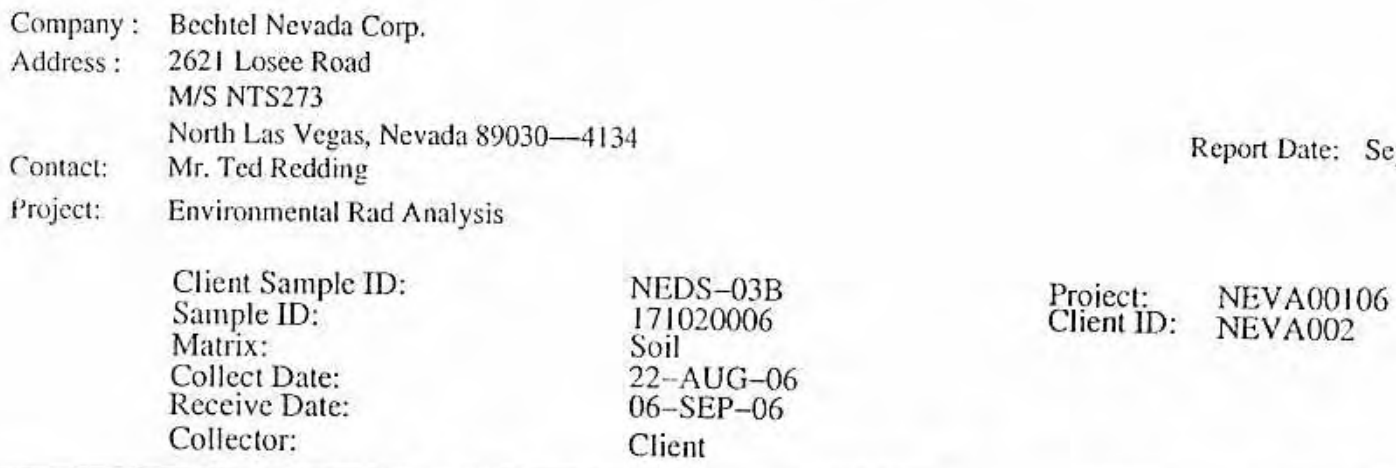

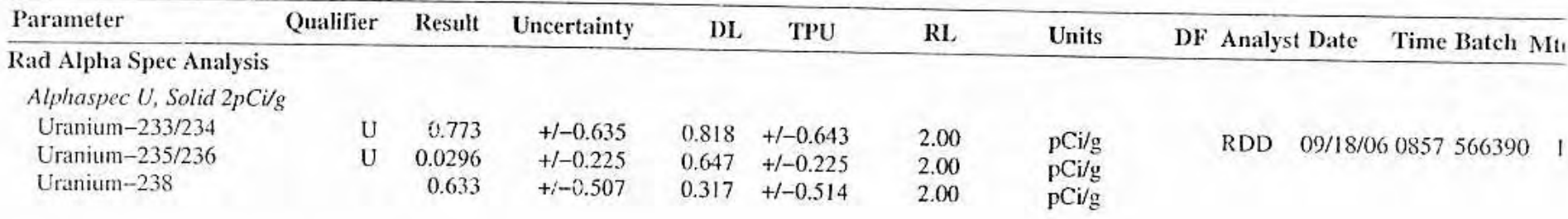

The following l'rep Methods were performed

\begin{tabular}{llllll}
\hline Method & Description & Analyst & Date & Time & Prep Batch \\
\hline Dry Soil Prep & Dry Soil Prep GL-RAD-A-021 & AXP2 & $09 / 07 / 06$ & 1221 & 565878
\end{tabular}

The following Analytical Methods were performed

\begin{tabular}{ll} 
Method & Description \\
\hline DOE EML HASL-300, U-02-RC Modified
\end{tabular}

\begin{tabular}{llcc} 
Surrogate/Tracer recovery & Test & Recovery \% & Acceptable Limits \\
\hline Uranium-232 & Alphaspec U, Solid 2pCi/g & 93 & $(25 \%-125 \%)$
\end{tabular}

Notes:

The Qualifiers in this report are defined as follows :

* A quality control analyte recovery is outside of specified acceptance criteria

$<$ Resuit is less than value reported

$>$ Result is greater than value reported

A The TIC is a suspected aldol-condensation product

B Target analyte was detected in the associated blank

$\mathrm{BD}$ Results are either below the MDC or tracer recovery is low

C Analyte has been confirmed by GC/MS analysis

D Results are reported from a diluted aliquot of the sample

$\mathrm{H}$ Analytical holding time was exceeded

$\mathrm{J}$ Value is estimated

N/A Spike recovery limits do not apply. Sample concentration exceeds spike concentration by $4 \mathrm{X}$ or more

$R$ Sample results are rejected

U Analyte was analyzed for, but not detected above the MDL, MDA, or LOD.

UI Gamma Spectroscopy--Uncertain identification

$\mathrm{X}$ Consult Case Narrative, Data Summary package, or Project Manager concerning this qualifier 


\section{GENERAL ENGINEERING LABORATORIES, LLC}

2040 Savage Road Charleston SC 29407 - (843) 556-8171 - www.gel.com

\section{Certificate of Analysis}

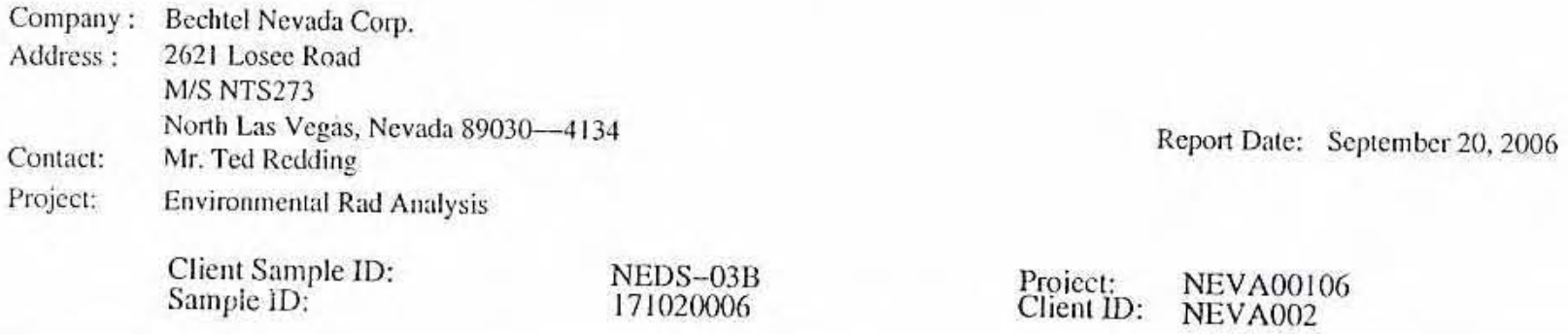

Y QC Samples were not spiked with this compound

$\wedge$ RPD of sample and duplicate evaluated using $+/-$ RL. Concentrations are $<5 X$ the RL

h Preparation or preservation holding time was exceeded

The above sample is reported on a dry weight basis. 


\section{GENERAL ENGINEERING LABORATORIES, LLC \\ 2040 Savage Road Charleston SC 29407 - (843) 556-8i71 - www.gel.com}

\section{Certificate of Analysis}

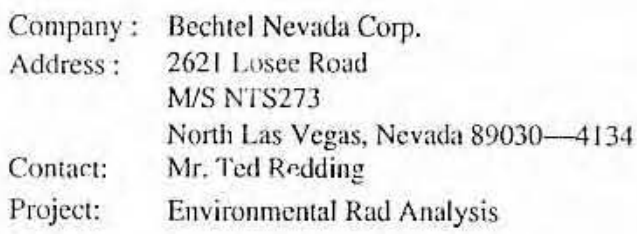

Report Date: September 20, 2006

Proiect:
Client ID: NEVA00106

\begin{tabular}{|c|c|c|c|c|c|c|c|c|c|c|c|}
\hline Parameter & Qualilier & Result & Uncertainty & DI. & TPU & $\mathrm{RL}$ & Units & DF & Analyst & t Date & Time Batch Mlt \\
\hline \multicolumn{12}{|c|}{ Rad Alpha Spec Analysis } \\
\hline \multicolumn{12}{|c|}{ Alphaspec $U$, Solid $2 p C i / g$} \\
\hline Uranium-233/234 & & 1.66 & $\begin{array}{r}+1-1.06 \\
+1-0.328\end{array}$ & 1.08 & $+1-1.08$ & 2.00 & $\mathrm{pCi} / \mathrm{g}$ & & $\mathrm{RDD}$ & $09 / 18 / 0$ & $60857566390 \quad 1$ \\
\hline $\begin{array}{l}\text { Uranium-235/236 } \\
\text { Uranium-238 }\end{array}$ & $\mathrm{U}$ & $\begin{array}{r}0.0432 \\
1.27\end{array}$ & $\begin{array}{l}+1-0.328 \\
+1-0.913\end{array}$ & $\begin{array}{l}0.943 \\
0.940\end{array}$ & $\begin{array}{l}+1-0.328 \\
+i-0.932\end{array}$ & $\begin{array}{l}2.00 \\
2.00\end{array}$ & $\begin{array}{l}\mathrm{pCi} / \mathrm{g} \\
\mathrm{pCi} / \mathrm{g}\end{array}$ & & & & \\
\hline
\end{tabular}

The following Prep Methods were performed

\begin{tabular}{llllll}
\hline Method & Description & Analyst & Date & Time & Prep Batch \\
\hline Dry Soil Prep & Dry Soil Prep GL-RAD-A-021 & AXP2 & $09 / 07 / 06$ & 1221 & 565878
\end{tabular}

The following Analytical Methods were performeo

$\begin{array}{ll}\text { Method Description } \\ 1 & \text { DOE EML HASL-300, U-02-RC Modified }\end{array}$

\begin{tabular}{llcc} 
Surrogate/Tracer recovery & Test & Recovery\% & Acceptable Limits \\
\hline Uranium-232 & Alphaspec U, Solid 2pCi/g & 74 & $(25 \%-125 \%)$
\end{tabular}

Notes:

The Qualifiers in this report are defined as follows :

* A quality control analyte recovery is outside of specified acceptance criteria

$<$ Resuit is less than value reported

$>$ Result is greater than value reported

A The TIC is a suspected aldol-condensation product

B Target analyte was detected in the associated blank

BD Results are either below the MDC or tracer recovery is low

C Analyte has been confirmed by GC/MS analysis

D Results are reported from a diluted aliquot of the sample

$\mathrm{H}$ Analytical holding time was exceeded

$\mathrm{J}$ Value is estimated

N/A Spike recovery limits do not apply. Sample concentration exceeds spike concentration by $4 \mathrm{X}$ or more

$\mathrm{R}$ Sample results are rejected

U Analyte was analyzed for, but not detected above the MDL, MDA, or LOD.

UI Gamma Spectroscopy--Uncertain identification

$\mathrm{X}$ Consult Case Narrative, Data Summary package, or Project Manager concerning this qualifier 


\section{GENERAL ENGINEERING LABORATORIES, LLC \\ 2040 Savage Foad Charleston SC 29407 - (843) 556-8171 - www.gel.com}

\section{Certificate of Analysis}

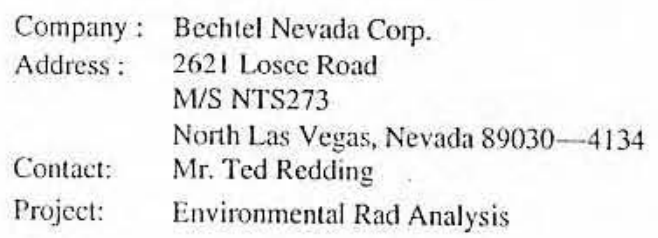

Client Sample ID: Sample ID:

NEDS-04A

171020007

Proiect:

Client ID:

Report Date: September 20, 2006

Parameter Qualifier

Result Uncertainty

DL TPU

RL

Units

DF Analyst Date

Time Batch Mt

Y QC Samples were not spiked with this compound

$\hat{R}$ RPD of sample and duplicate evaluated using $+/-\mathrm{RL}$. Concestrations are $\angle 5 \mathrm{X}$ the RL

$\mathrm{h}$ Preparation or preservation holding time was exceeded

The above sample is reported on a dry weight basis. 


\section{GENERAL ENGINEERING LABORATORIES, LLC \\ 2040 Savage Road Charleston SC 29407 - (843) 556-8171 - www.gel.com}

\section{Certificate of Analysis}

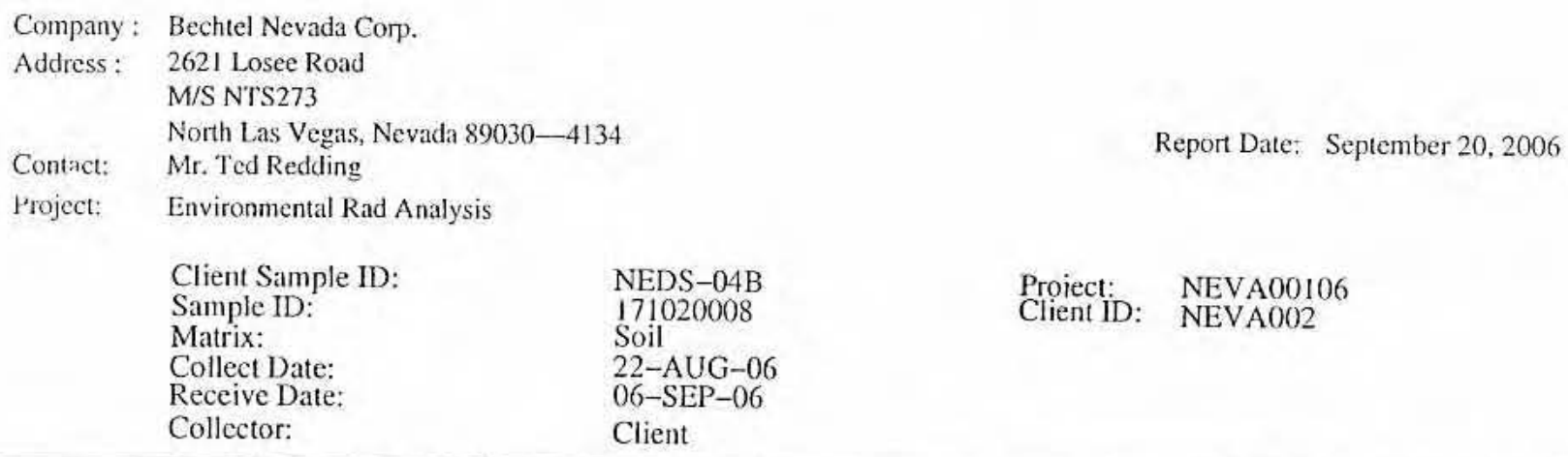

\begin{tabular}{|c|c|c|c|c|c|c|c|c|c|c|}
\hline Parameter & Qualifier & Result & Uncertainty & $\mathrm{DL}$ & TPU & RL & Units & DF & Analyst Date & Time Batch Mt \\
\hline \multicolumn{11}{|c|}{ Rad Alpha Spec Analysis } \\
\hline \multicolumn{11}{|c|}{ Alphaspec $U$, Solid $2 p C i g$} \\
\hline Uranium-233/234 & $\mathrm{U}$ & 0.770 & $+1-0.632$ & 0.815 & $+1-0.640$ & 2.00 & $\mathrm{pCi} / \mathrm{g}$ & & RDD $\quad 09 / 18 /($ & 60857566390 \\
\hline Uranium-235/236 & $\mathrm{U}$ & 0.194 & $+1-0.439$ & 0.884 & $+1-0.440$ & 2.00 & $\mathrm{pCi} / \mathrm{g}$ & & & \\
\hline Uranium-238 & $u$ & 0.665 & $+1-0.597$ & 0.815 & $+1-0.604$ & 2.00 & $\mathrm{pCi} / \mathrm{g}$ & & & \\
\hline
\end{tabular}

The foilowing Prep Methods were performed

\begin{tabular}{llllll}
\hline Method & Description & Analyst & Date & Time & Prep Batch \\
\hline Dry Soil Prep & Dry Soil Prep GL-RAD-A-021 & AXP2 & $09 / 0 / / 06$ & 1221 & 565878
\end{tabular}

The following Analytical Methods were performed

\begin{tabular}{ll} 
Method Description \\
\hline DOE EML HASL-300, U-02-RC Modified
\end{tabular}

\begin{tabular}{llcc} 
Surrogate/Tracer recovery & Test & Recovery\% & Acceptable Limits \\
\hline Uranium-232 & Alphaspec U, Solid 2pCi/g & 88 & $(25 \%-125 \%)$
\end{tabular}

Notes:

The Qualifiers in this report are defined as follows :

* A quality control analyte recovery is outside of specified acceptance criteria

$<$ Result is less than value reported

$>$ Result is greater than value reported

A The TIC is a suspected aldol-condensation product

B Target analyte was detected in the associated blank

$\mathrm{BD}$ Results are either below the MDC or tracer recovery is low

C Analyte has been confirmed by GC/MS analysis

D Results are reported from a diluted aliquot of the sample

$\mathrm{H}$ Analytical holding time was exceeded

$\mathrm{J}$ Value is estimated

N/A Spike recovery limits do not apply. Sample concentration exceeds spike concentration by $4 X$ or more

$\mathrm{R}$ Sample results are rejected

U Analyte was analyzed for, but not detected above the MDL, MDA, or LOD.

UI Gamma Spectroscopy-Uncertain identification

X Consult Case Narrative, Data Summary package, or Project Manager concerning this qualifier 


\section{GENERAL ENGINEERING LABORATORIES, LLC}

2040 Savage Road Chaileston SC 29407 - (843) 556-8171 - www.gel.com

\section{Certificate of Analysis}

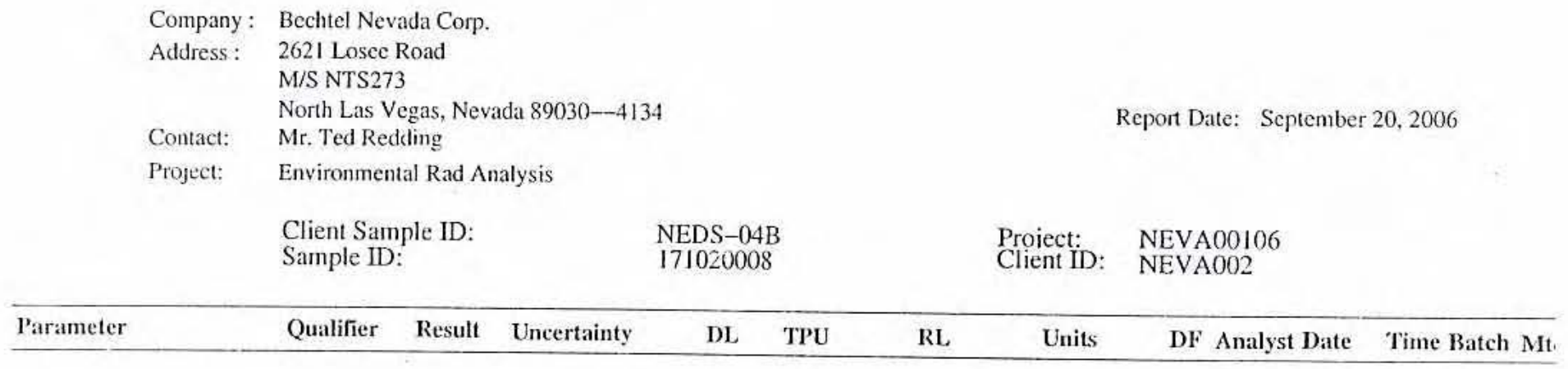

Y QC Samples were not spiked with this compound

$\wedge$ RPD of sample and duplicate evaluated using $+/-$ RL. Concentrations are $<5 X$ tite RL

h Preparation or preservation holding time was exceeded

The above sample is reported on a dry weight basis. 


\section{GENERAL ENGINEERING LABORATORIES, LLC \\ 2040 Savage Road Charleston SC 29407 - (843) 556-8171 - www.gel.com}

\section{Certificate of Analysis}

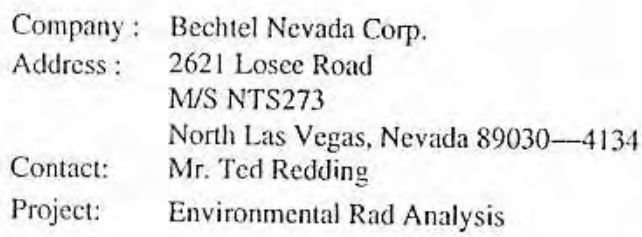

NEDS-05A
171020009
Soil
22-AUG-06
$06-S E P-06$
Client

Proiect:

NEVA002

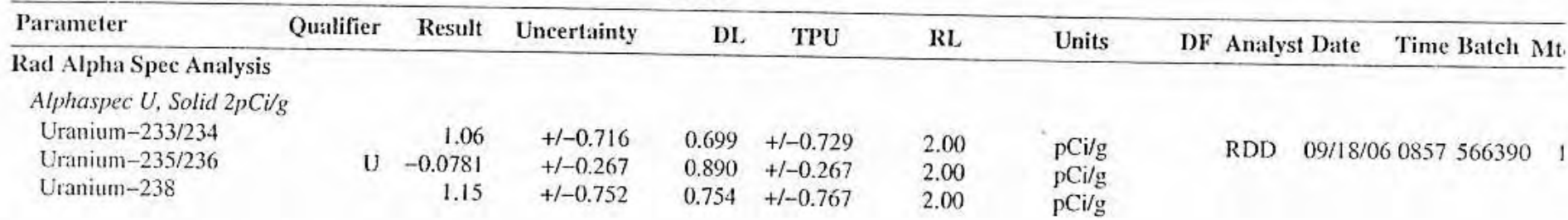

The following l'rep Methods were performed

\begin{tabular}{llllll}
\hline Method & Description & Analyst & Date & Time & Pren Batch \\
\hline Dry Soil Prep & Dry Soil Prep GL-RAD-A-021 & AXP2 & $09 / 07 / 06$ & 1221 & 565878
\end{tabular}

The following Analytical Methods were performed

Method Description

1 DOE EML HASL-300, U-02-RC Modified

\begin{tabular}{llcc} 
Surrogate/Tracer recovery & Test & Recovery \% & Acceptable Limits \\
\hline Uranium-232 & Alphaspec U, Solid 2pCi/g & 97 & $(25 \%-125 \%)$
\end{tabular}

Notes:

The Qualifiers in this report are defined as follows :

* A quality control analyte recovery is outside of specified acceptance criteria

$<$ Result is less than value reported

$>$ Result is greater than value reported

A The TIC is a suspected aldol-condensation product

$B$ Target analyte was detected in the associated blank

BD Results are either below the MDC or tracer recovery is low

C Analyte has been confirmed by GC/MS analysis

D Results are reported from a diluted aliquot of the sample

$\mathrm{H}$ Analytical holding time was exceeded

$\mathrm{J}$ Value is estimated

N/A Spike recovery limits do not apply. Sample concentration exceeds spike concentration by $4 X$ or more
R Sample results are rejected

U Analyte was analyzed for, but not detected above the MDL, MDA, or LOD.

UI Gamma Spectroscopy-Uncertain identification

X Consult Case Narrative, Data Summary package, or Project Manager concerning this qualifier 


\section{GENERAL ENGINEERING LABORATORIES, LLC \\ 2040 Savage Road Charleston SC 29407 - (843) 556-8171 - www.gel.com}

\section{Certificate of Analysis}

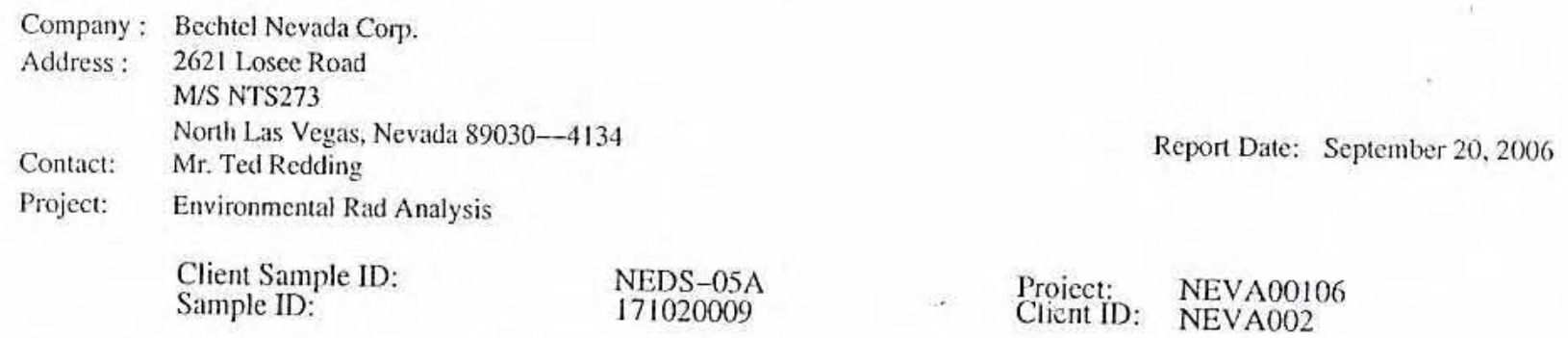

Y QC Samples were not spiked with this compound

$A$ RPD of sample and duplicate evaluated using $+/-R L$. Concentrations are $<5 X$ the $R L$

h Preparation or preservation holding time was exceeded

The above sample is reported on a dry weight basis. 


\section{GENERAL ENGINEERING LABORATORIES, LLC}

2040 Savage Road Charleston SC 29407 - (843) 556-8171 - www.géi.cum

\section{Certificate of Analysis}

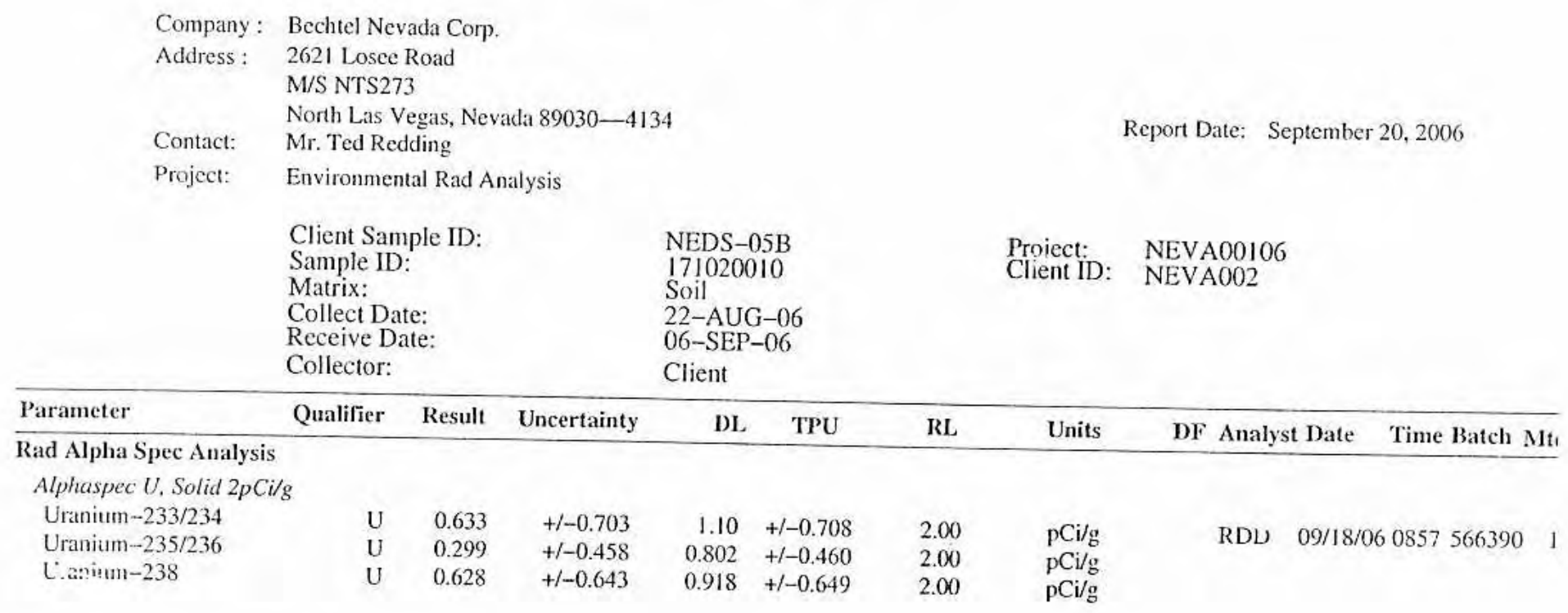

The following Prep Methods were performed

\begin{tabular}{llllll}
\hline Method & Description & Analyst & Date & Time & Prep Batch \\
\cline { 2 - 5 } D.y Sinep & Dry Soil Prep GL-RAD-A-021 & AXP2 & $09 / 07 / 06$ & 1221 & 565878
\end{tabular}

The following Analytical Methods were performed

Method Description

I DOE EML HASL-300, U-02-RC Modified

\begin{tabular}{llcc} 
Surrogate/Tracer recovery & Test & Recovery \% & Acceptable Limits \\
\hline Uranium-232 & Alphaspec U, Solid $2 \mathrm{pCi} / \mathrm{g}$ & 87 & $(25 \%-125 \%)$
\end{tabular}

Notes:

The Qualifiers in this report are defined as follows :

* A quality control analyte recovery is outside of specified acceptance criteria

$<$ Restilt is less than value reported

$>$ Result is greater than value reported

A The TIC is a suspected aldol-condensation product

$\mathrm{B}$ Target analyte was detected in the associated blank

$\mathrm{BD}$ Results are either below the MDC or tracer recovery is low

C Analyte has been confirmed by GC/MS analysis

D Results are reported from a diluted aliquot of the sample

$\mathrm{H}$ Analytical holding time was exceeded

$\mathrm{J}$ Value is estimated

N/A Spike recovery limits do not apply. Sample concentration exceeds spike concentration by $4 X$ or more
$R$ Sample results are rejected

U Analyte was analyzed for, but not detected above the MDL, MDA, or LOD.

UI Gamma Spectroscopy--Uncertain identification

$X$ Consult Case Narrative, Data Summary package, or Project Manager concerning this qualifier 


\section{GENERAL ENGINEERING LABORATORIES, LLC \\ 2040 Savage Road Charleston SC 29407 - (843) 556-8171 - www.gol.com}

\section{Certificate of Analysis}

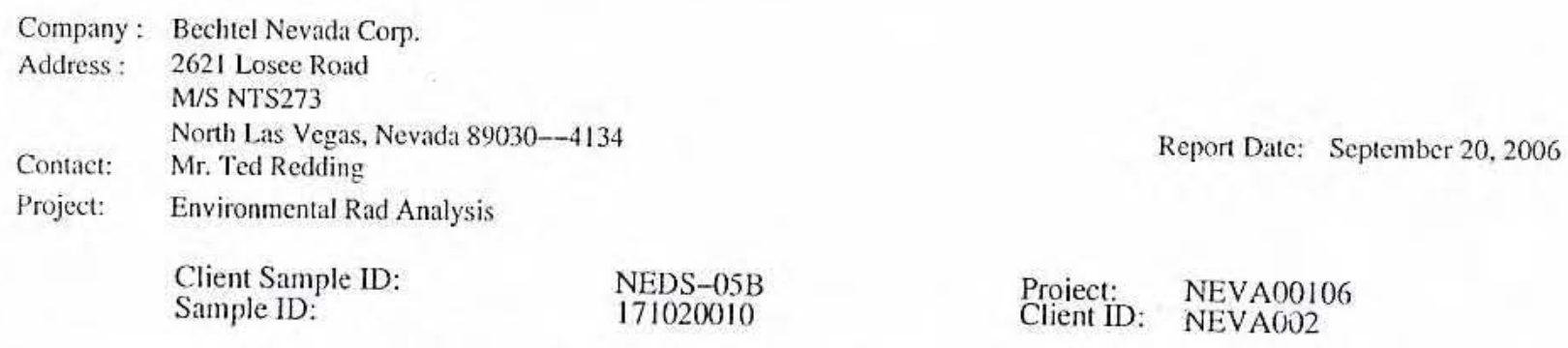

Y QC Samples were not spiked with this compound

$\wedge \quad$ RPD of sample and duplicate evaluated using $+/-$ RL. Concentrations are $<5 X$ the RL

$\mathrm{h}$ Preparation or preservation holding time was exceeded

The above sample is reported on a dry weight basis. 


\section{GENERAL ENGINEERING LABORATORIES, LLC \\ 2040 Savage Road Charleston SC 29407 - (843) 556-8171 - www.gel.com}

\section{Certificate of Analysis}

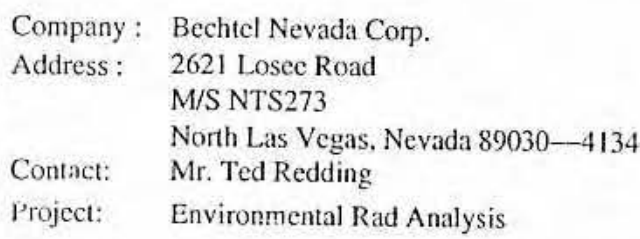

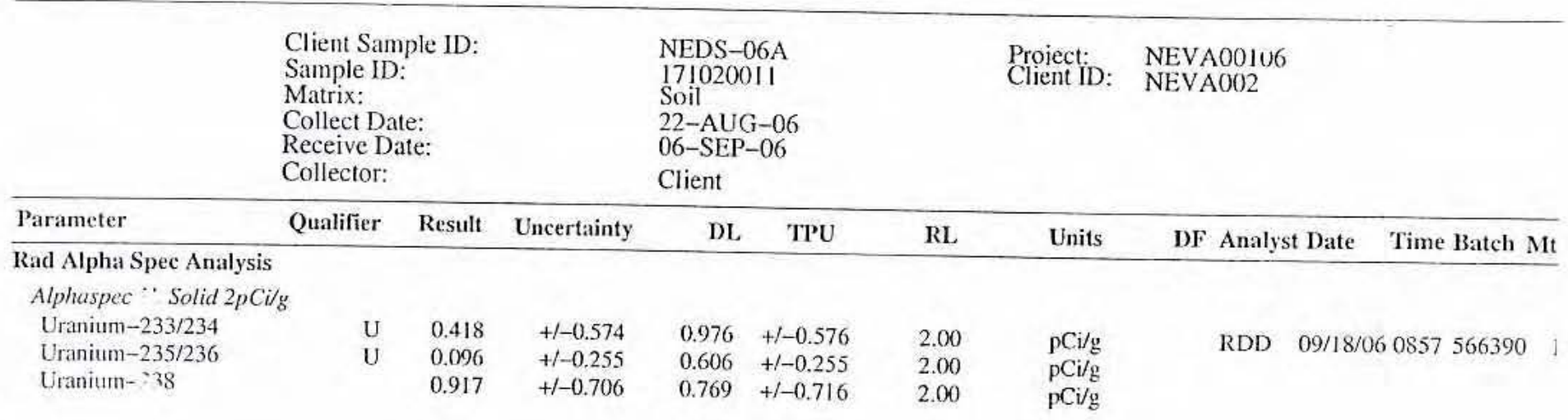

The following Prep Methods were performed

\begin{tabular}{llllll}
\hline Method & Descriptio: & Analyst & Date & Time & Prep Batch \\
\hline Dry Soil Prep & Dry Soil Prep GL-RAD-A-021 & AXP2 & $09 / 07 / 06$ & 1221 & 565878
\end{tabular}

The following Analytical Methods were performed

\begin{tabular}{ll} 
Method Description \\
\hline DOE EML HASL-300, U-02-RC Modified
\end{tabular}

\begin{tabular}{llcc} 
Surrogate/Tracer recovery & Test & Recovery\% & Acceptable Limits \\
\hline Uranium-232 & Alphaspec U, Solid 2pCi/g & 91 & $(25 \%-125 \%)$
\end{tabular}

Notes:

The Qualifiers in this report are defined as follows:

* A quality control analyte recovery is outside of specified acceptance criteria

$<$ Result is less than value reported

$>$ Result is greater than value reported

A The TIC is a suspected aldol-condensation product

B Target analyte was detected in the associated blank

$\mathrm{BD}$ Results are either below the MDC or tracer recovery is low

C Analyte has been confirmed by GC/MS analysis

D Results are reported from a diluted aliquot of the sample

$\mathrm{H}$ Analytical holding time was exceeded

$\mathrm{J}$ Value is estimated

N/A Spike recovery limits do not apply. Sample concentration exceeds spike concentration by $4 X$ or more

$R$ Sample results are rejected

$\mathrm{U}$ Analyte was analyzed for, but not detected above the MDL, MDA, or LOD.

Ui Gamma Spectroscopy--Uncertain identification 


\section{GENERAL ENGINEERING LABORATORIES, LLC \\ 2040 Savage Road Charleston SC 29407 - (843) 556-8171 - www.gel.com}

\section{$\underline{\text { Certificate of Analysis }}$}

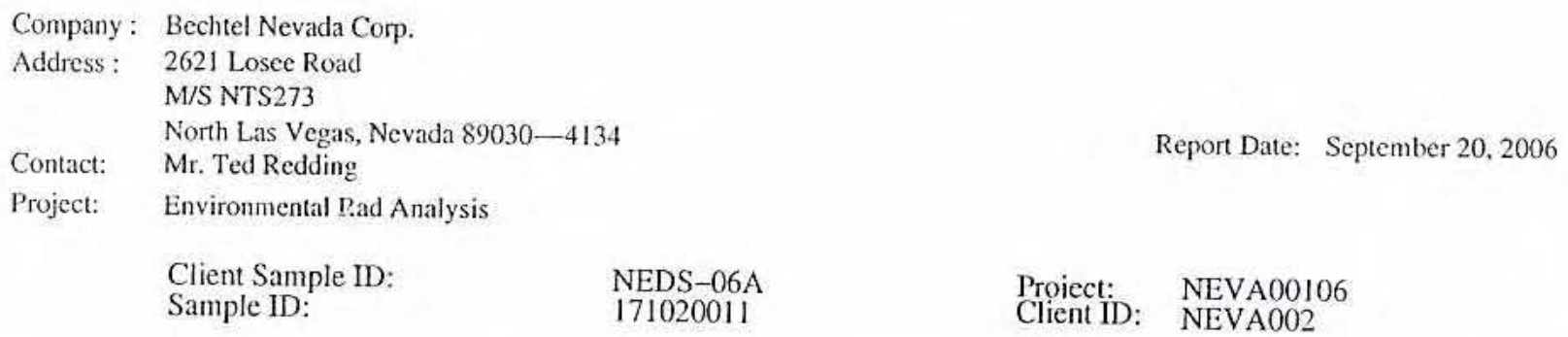

X Consult Case Narrative, Data Summary package, or Project Manager concerning this qualifier

Y QC Sampl were not spiked with this compound

$\wedge \quad \mathrm{RPD}$ of sample and duplicate evaluated using $+1-\mathrm{RL}$. Concentrations are $<5 \mathrm{X}$ the RL

h Preparation or preservation holding time was exceeded

The above samp is is reported on a dry weight basis. 


\section{GENERAL ENGINEERING LABORATORIES, LLC}

2040 Savage Road Charleston SC 29407 - (843) 556-8171 - www.gel.com

\section{Certificate of Analysis}

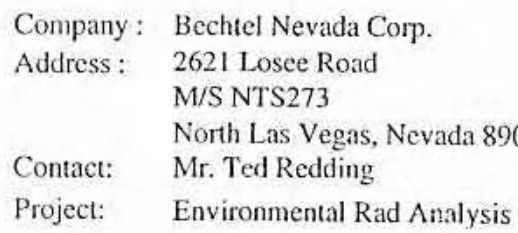

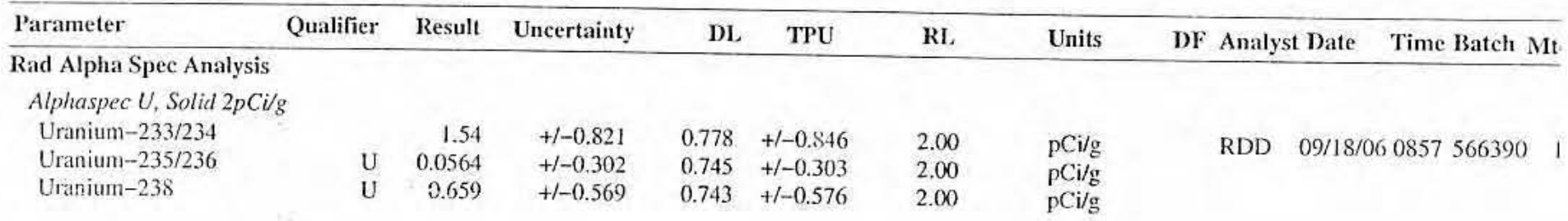

The following Prep Methods were performed

\begin{tabular}{llllll}
\hline Method & Description & Analyst & Date & Time & Prep Batch \\
\hline Dry Soil Prep & Dry Soil Prep GL-RAD-A-021 & AXP2 & $09 / 07 / 06$ & 1221 & 565878
\end{tabular}

The following Analytical Methods were performed

Method Description $\quad$ DOE EML HASL-300, U-02-RC Modified

\begin{tabular}{llcc} 
Surrogate/Tracer recovery & Test & Recovery $\%$ & Acceptable Limits \\
\hline Uranium-232 & Alphaspec U, Solid 2pCi/g & 90 & $(25 \%-125 \%)$
\end{tabular}

Notes:

The Cialifiers in this report are defined as follows :

* A quality control analyte recovery is outside of specified acceptance criteria

$<$ Result is less than value reported

$>$ Result is greater than value reported

A The TIC is a suspected aldol-condensation product

B Target analyte was detected in the associated blank

$\mathrm{BD}$ Results are either below the MDC or tracer recovery is low

C Analyte has been confirmed by GC/MS analysis

D Results are reported from a diluted aliquot of the sample

$\mathrm{H}$ Analytical holding time was exceeded

$\mathrm{J}$ Value is estimated

N/A Spike recovery limits do not apply. Sample concentration exceeds spike concentration by $4 \mathrm{X}$ or more

$\mathrm{R}$ Sample results are rejected

U Analyte was analyzed for, but not detected above the MDL, MDA, or LOD.

UI Gamma Spectroscopy--Uncertain identification

X Consult Case Narrative, Dati Summary package, or Project Manager concerning this qualifier 


\section{GENERAL ENGINEERING LABORATORIES, LLC}

2040 Savage Road Charleston SC 29407 - (843) 556-8171 - www.gel.com

\section{Certificate of Analysis}

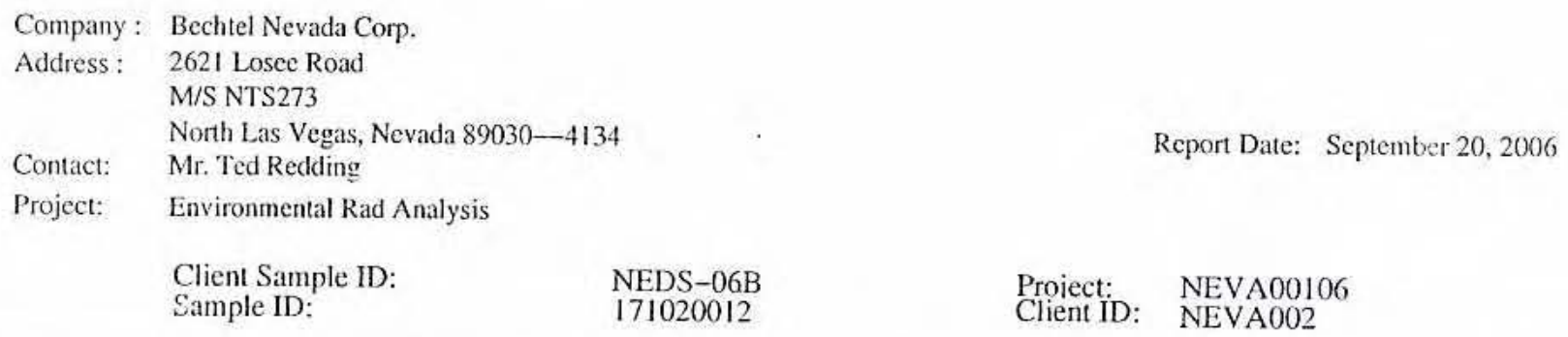

Y QC Samples were not spiked with this compound

$\wedge$ RPD of sample and duplicate evaluated using $+/-$ RL. Concentrations are $<5 X$ the RL

$\mathrm{h}$ Preparation or preservation holding time was exceeded

The above sample is reported on a dry weight basis. 


\section{GENERAL ENGINEERING LABORATORIES, LLC \\ 2040 Savage Road Charleston SC 29407 - (843) 556-8171 - www.gel.com}

\section{Certificate of Analysis}

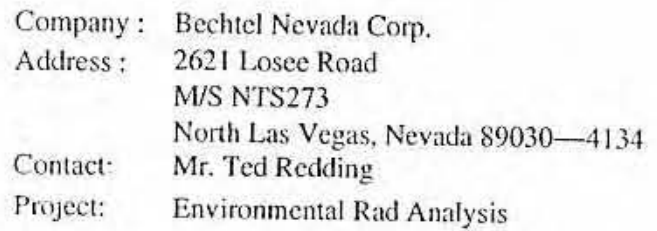

Report Date: September 20, 2006

NEDS-07A
$17 ! 020013$
Soil
22-AUG-06
06-SEP-06
Client

Proiect: NEVA00I06
Client ID: NEVA002

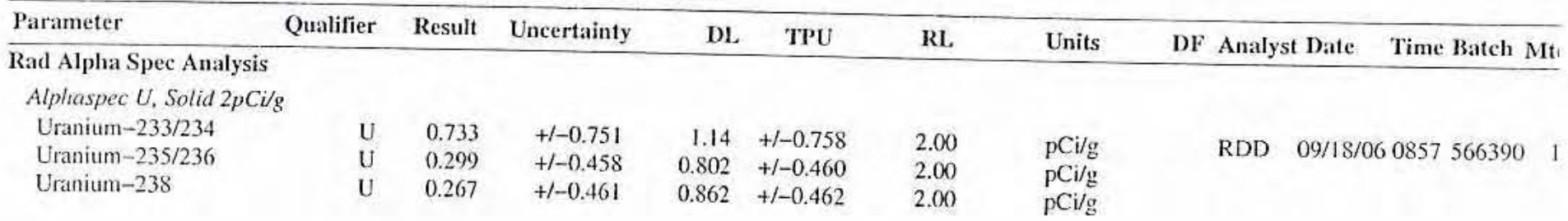

The following Prep Methods were performed

\begin{tabular}{llllll}
\hline Method & Description & Analyst & Date & Time & Prep Batch \\
\hline Dry Soil Prep & Dry Soil Prep GL-RAD-A-021 & AXP2 & $09 / 07 / 06$ & 1221 & 565878
\end{tabular}

The following Analytical Methods vere performed

Method Description

I DOE EML HASL-300, U-02-RC Modified

\begin{tabular}{llcc} 
Surrogate/Tracer recovery & Test & Recovery \% & Acceptable Limits \\
\hline Uranium-232 & Alphaspec U, Solid 2pCi/g & 79 & $(25 \%-125 \%)$
\end{tabular}

Notes:

The Qualifiers in this repori are defined as follows :

* A quality control analyte recovery is outside of specified acceptance criteria

< Resuli is less than valie reported

$>$ Result is greater than value reported

A The TIC is a suspected aldol-condensation product

B Target analyte was detected in the associated blank

$\mathrm{BD}$ Results are either below the MDC or tracer recovery is low

C Analyte has been confirmed by GC/MS analysis

D Results are reported from a diluted aliquot of the sample

H Analytical holding time was exceeded

$\mathrm{J}$ Value is estimated

N/A Spike recovery limits do not apply. Sample concentration exceeds spike concentration by $4 X$ or more
R Sample results are rejected

U Analyte was analyzed for, but not detected above the MDL, MDA, or LOD.

UI Gamma Spectroscopy-Uncertain identification

X Consult Case Narrative, Data Summary package, or Project Manager concerning this qualifier 


\section{GENERAL ENGINEERING LABORATORIES, LLC \\ 2040 Savage Road Charleston SC 29407 - (843) 556-8171 - www.gel.com}

\section{Certificate of Analysis}

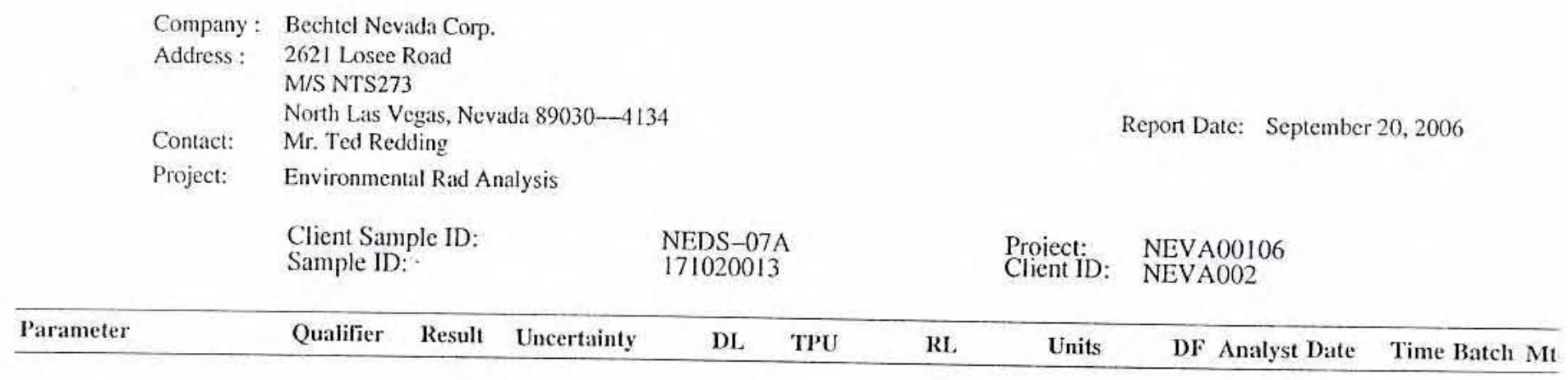

Y QC Samples were not spiked with this compound

$\hat{R}$ RPD of sample and duplicate evaluated using $+/-\mathrm{KL}$. Concentrations are $<5 \mathrm{X}$ the RL

h Preparation or preservation holding time was exceeded

The above sample is reported on a dry weight basis. 


\section{GENERAL ENGINEERING LABORATORIES, LLC \\ 2040 Savage Poad Charleston SC 29407 - (843) 556-8171 - www.gel.com}

\section{Certificate of Analysis}

\author{
Company: Bechtel Nevada Corp. \\ Address : 2621 Losee Road \\ M/S NTS273 \\ North Las Vegas, Nevada $89030-4134$ \\ Contact: $\quad$ Mr. Ted Redding \\ Project: Environmental Rad Analysis
}

Report Date: September 20, 2006

$\begin{array}{ll}\text { Client Sample ID: } & \text { NEDS-07B } \\ \text { Sample ID: } & 171020014 \\ \text { Matrix: } & \text { Soil } \\ \text { Collect Date: } & 22-A U G-06 \\ \text { Receive Date: } & 06-S E P-06 \\ \text { Collector: } & \text { Client }\end{array}$

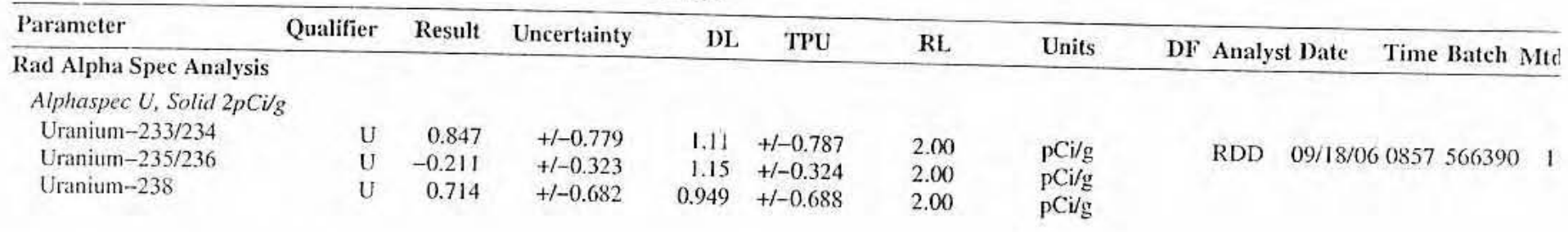

The following Prep Methods were performed

\begin{tabular}{llllll}
\hline Method & Description & Analyst & Date & Time & Prep Batch \\
\hline Dry Soil Prep & Dry Soil Prep GL-RAD-A-021 & AXF2 & $09 / 07 / 06$ & 1221 & 565878
\end{tabular}

The following Analytical Methods were performed

\begin{tabular}{ll} 
Method & Description \\
\hline & DOE EML HASL-300, U-02-RC Modified
\end{tabular}

\begin{tabular}{llcc} 
Surrogate/Tracer recovery & Test & Recovery \% & Acceptable Limits \\
\hline Uranium-232 & Alphaspec U, Solid 2pCi/g & 92 & $(25 \%-125 \%)$
\end{tabular}

Notes:

The Qualifiers in this report are defined as follows :

* A quality control analyte recovery is outside of specified acceptance criteria

$<$ Result is less than value reported

$>$ Result is greater than value reported

A The TIC is a suspected aldol-condensation product

B Target analyte was detected in the associated blank

$\mathrm{BD}$ Results are either below the MDC or tracer recovery is low

C Analyte has been confirmed by GC/MS analysis

D Results are reported from a diluted aliquot of the sample

$\mathrm{H}$ Analytical holding time was exceeded

J Value is estimated

N/A Spike recovery limits do not apply. Sample concentration exceeds spike concentration by $4 \mathrm{X}$ or more
$\mathrm{R}$ Sample results are rejected

$\mathrm{U}$ Analyte was analyzed for, but not detected above the MDL, MDA, or LOD.

UI Gamma Spectroscopy-Uncertain identification

$X$ Consult Case Narrative, Data Summary package, or Project Manager concerning this qualificr 


\section{GENERAL ENGINEERING LABORATORIES, LLC \\ 2040 Savage Road Charleston SC 29407 - (843) 556-8171 - www.gel.com}

\section{Certificate of Analysis}

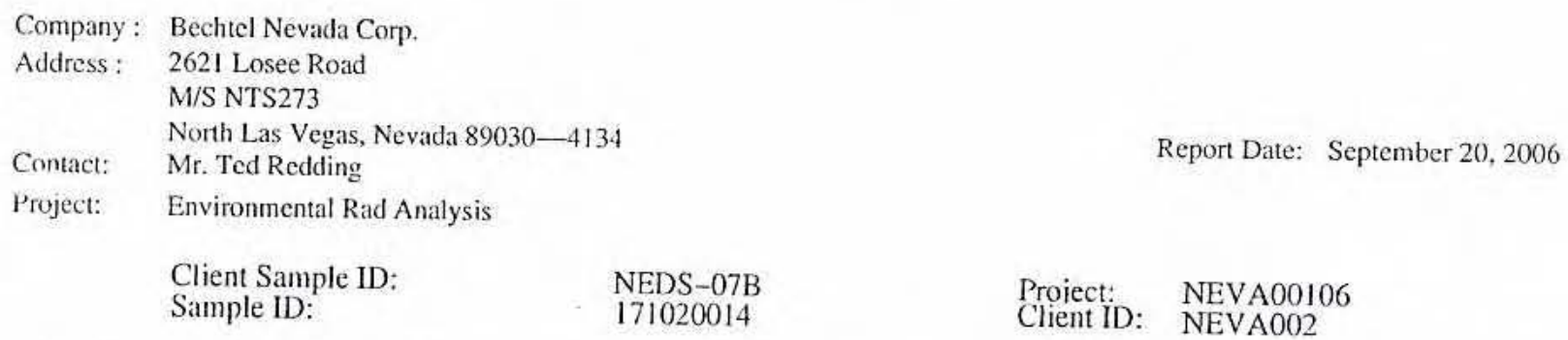

Y QC Samples were not spiked with this compound

$\wedge$ RPD of sample and duplicate evaluated using $+/-$ RL. Concentrations are $<5 X$ the RL

h Preparation or preservation holding time was exceeded

The above sample is reported on a dry weight basis. 


\section{GENERAL ENGINEERING LABORATORIES, LLC
2040 Savage Road Charleston SC $29407-(843) 556-8171-10$ \\ 2040 Savage Road Charleston SC 29407 - (843) 556-8171 - www.gel.com}

\section{Certificate of Analysis}

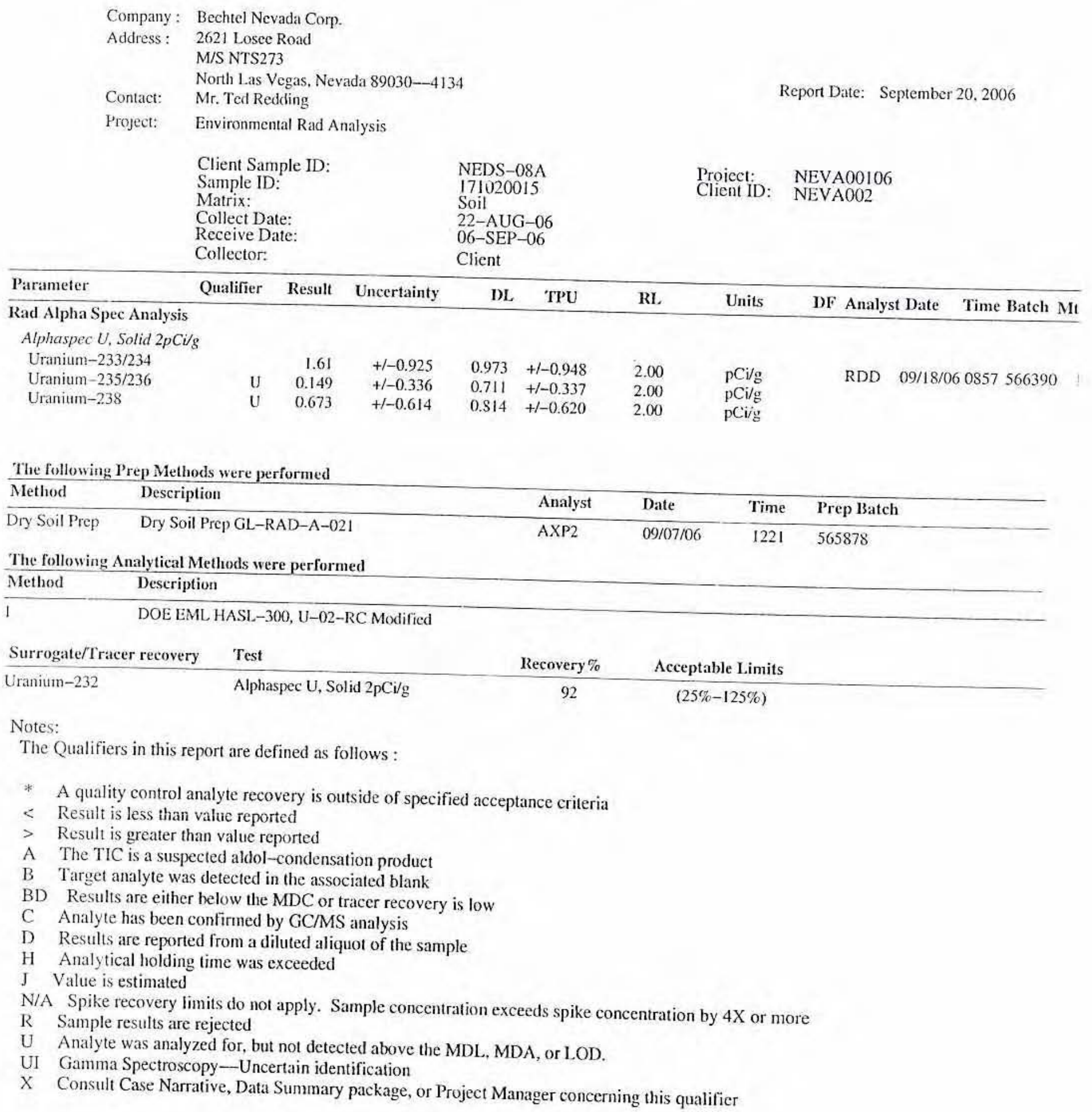




\section{GENERAL ENGINEERING LABORATORIES, LLC \\ 2040 Savage Road Charleston SC 29407 - (843) 556-8171 - www.gel.com}

\section{Certificate of Analysis}

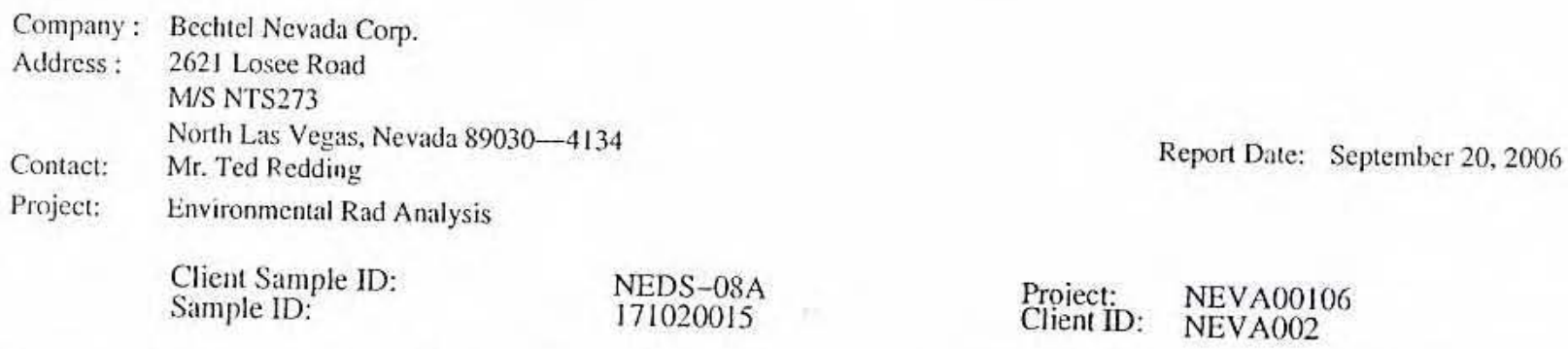

DL TPU

RL.

Units DF Analyst Date

Time Batch Mt

Y QC Samples were not spiked with this compound

$\wedge \quad \mathrm{RPD}$ of sample and duplicate evaluated using $+1-\mathrm{RL}$. Concentrations are $<5 \mathrm{X}$ the RL

h Preparation or preservation holding time was exceeded

The above sample is reported on a dry weight basis. 


\section{GENERAL ENGINEERING LABORATORIES, LLC \\ 2040 Savage Road Charleston SC 29407 - (843) 556-8171 - www.gel.com}

\section{$\underline{\text { Certificate of Analysis }}$}

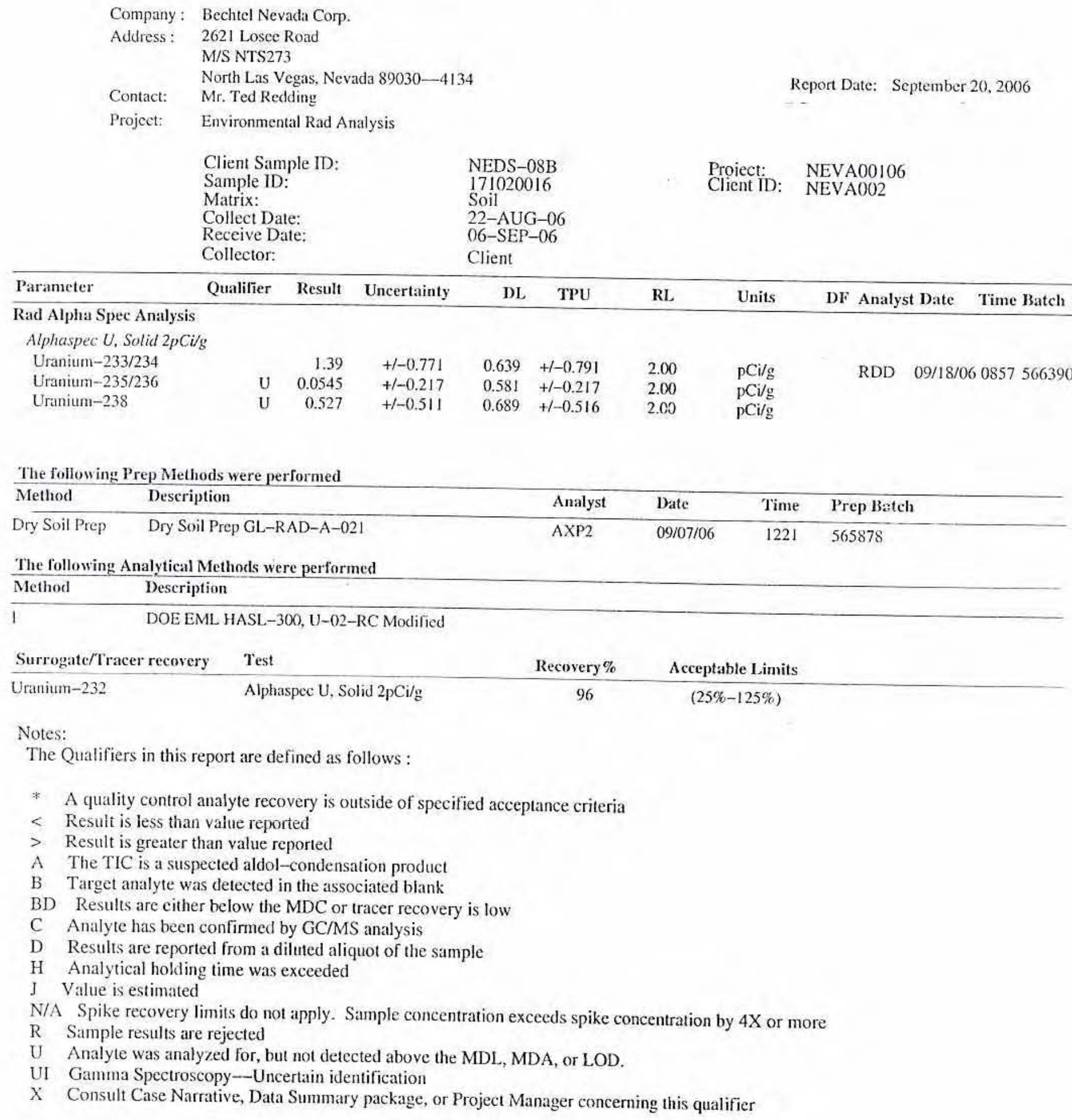




\section{GENERAL ENGINEERING LABORATORIES, LLC \\ 2040 Savage Road Charleston SC 29407 - (843) 556-8171 - www.gel.com}

\section{Certificate of Analysis}

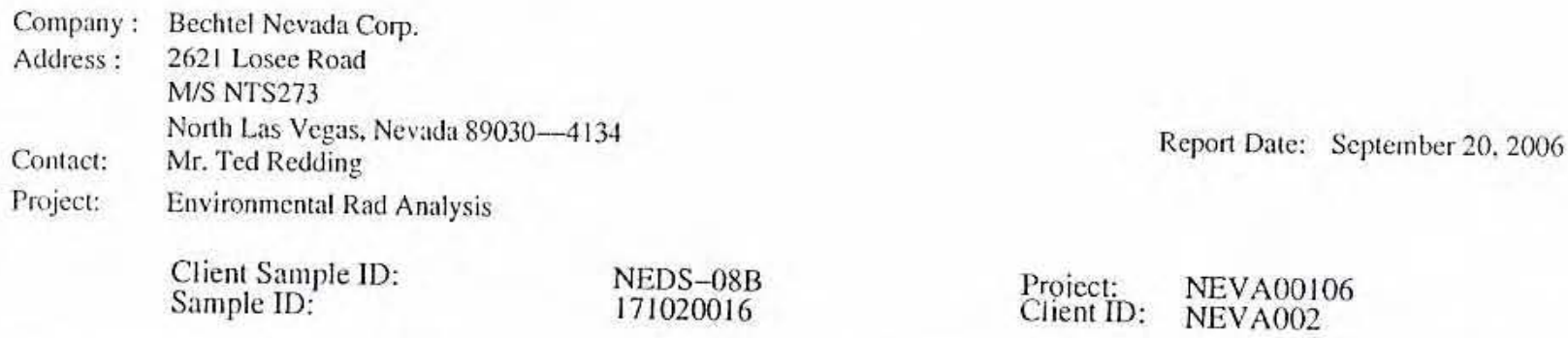

Y QC Samples were not spiked with this compound

$\hat{R}$ RPD of sample and duplicate evaluated using $+/-\mathrm{RL}$. Concentrations are $<5 \mathrm{X}$ the RL

$\mathrm{h}$ Preparation or preservation holding time was exceeded

The above sample is reported on a dry weight basis. 


\section{GENERAL ENGINEERING LABORATORIES, LLC \\ 2040 Savage Road Charleston SC 29407 - (843) 556-8171 - www.gel.com}

\section{Certificate of Analysis}

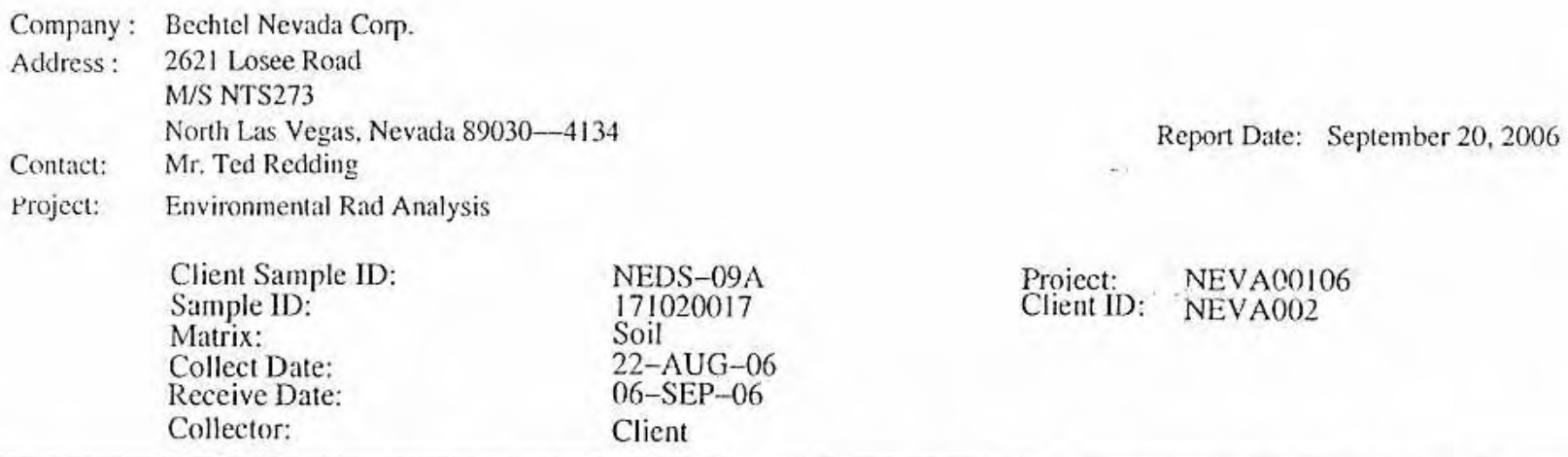

\begin{tabular}{|c|c|c|c|c|c|c|c|c|c|c|}
\hline Parameter & Qualifier & Result & Uncertainty & $\mathrm{DL}$ & TPU & RL & Units & DF & Analyst Date & Time Batch Mt \\
\hline \multicolumn{11}{|c|}{ Rad Alpha Spec Analysis } \\
\hline \multicolumn{11}{|c|}{ Alphaspec $U$, Solid $2 p C i / g$} \\
\hline Uranium-233/234 & $\mathrm{U}$ & 0.379 & $+/-0.534$ & 0.926 & $+1-0.536$ & 2.00 & $\mathrm{pCi} / \mathrm{g}$ & & RDD $09 / 18 / 0$ & 60857566390 \\
\hline Uranium-235/236 & U & 0.126 & $+1-0.283$ & 0.599 & $+1-0.284$ & 2.00 & $\mathrm{pCi} / \mathrm{g}$ & & & \\
\hline Uranium-2?8 & & 0.931 & $+/-0.610$ & 0.542 & $+/-0.622$ & 2.00 & $\mathrm{pCi} / \mathrm{g}$ & & & \\
\hline
\end{tabular}

The following Prep Methods were performed

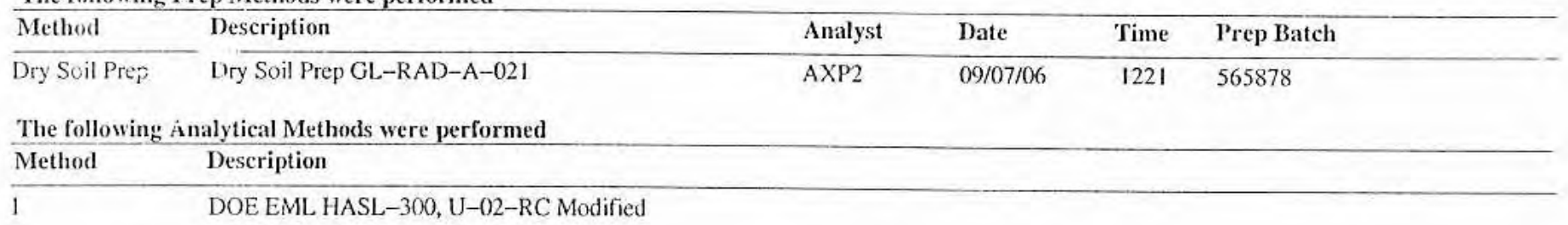

\begin{tabular}{llcc} 
Surrogate/Tracer recovery & Test & Recovery\% & Acceptable Limits \\
\hline Uranium-232 & Alphaspec U, Solid 2pCi/g & 87 & $(25 \%-125 \%)$
\end{tabular}

Notes:

The Qualifiers in this report are defined as follows :

* A quality control analyte recovery is outside of specified acceptance criteria

$<$ Result is less than value reported

$>$ Result is greater than value reported

A The TIC is a suspected aldol-condensation product

B Target analyte was detected in the associated blank

$\mathrm{BD}$ Results are either below the MDC or tracer recovery is low

C Analyte has been confirmed by GC/MS analysis

D Results are reported from a diluted aliquot of the sample

$\mathrm{H}$ Analytical holding time was exceeded

$\mathrm{J}$ Value is estimated

N/A Spike recovery limits do not apply. Sample concentration exceeds spike concentration by $4 X$ or more

R Sample results are rejected

U Analyte was analyzed for, but not detected above the MDL, MDA, or LOD.

UI Gamma Spectroscopy--Uncertain identification

$\mathrm{X}$ Consult Case Narrative, Data Summary package, or Project Manager concerning this qualifier 


\section{GENERAL ENGINEERING LABORATORIES, LLC \\ 2040 Savage Road Charleston SC 29407 - (843) 556-8171 - www.gel.comi}

\section{Certificate of Analysis}

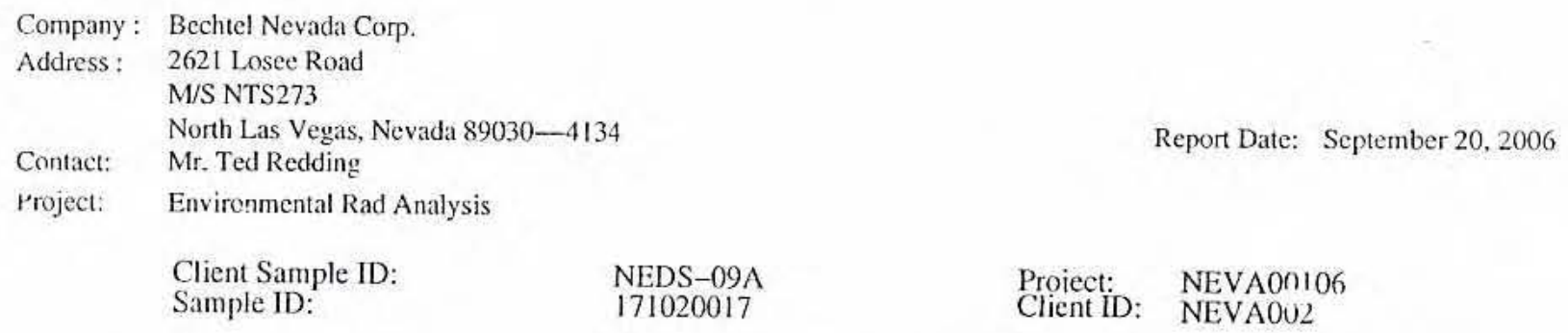

Y QC Samples were not spiked with this compound

$\wedge \quad$ RF $P$ of sample and duplicate evaluated using $+/-R L$. Concentrations are $<5 X$ the $R L$

h Preparation or preservation holding time was exceeded

The above sample is reported on a dry weight basis. 


\section{GENERAL ENGINEERING LABORATORIES, LLC \\ 2040 Savage Road Charleston SC 29407 - (843) 556-8171 - www.gel.com}

\section{Certificate of Analysis}

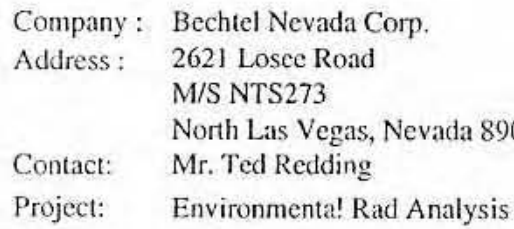

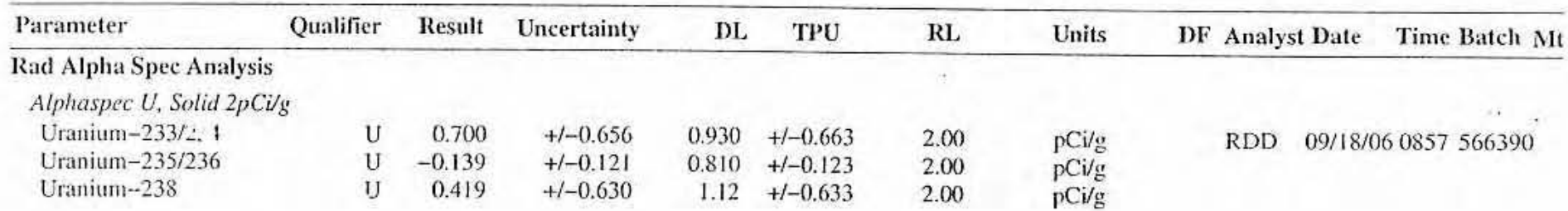

The following Prep Methods were performed

\begin{tabular}{llllll}
\hline Method & Description & Analyst & Date & Time & Prep Batch \\
\hline Dry Soil Prep & Dry Soil Ptep GiL-RAD-A-021 & AXP2 & $09 / 07 / 06$ & 1221 & 565878
\end{tabular}

The following Analytical Methods were performed

\begin{tabular}{ll} 
Method Description \\
\hline DOE EML HASL-300, U-02-RC Modified
\end{tabular}

\begin{tabular}{llcc} 
Surrogate/Tracer recovery & Test & Recovery\% & Acceptable Limits \\
\hline Uranium-232 & Alphaspec U, Solid 2pCi/g & 87 & $(25 \%-125 \%)$
\end{tabular}

Notes:

The Qualifiers in this report are defined as follows :

* A quality control analyte recovery is outside of specified acceptance criteria

$<$ Result is less than value reported

$>$ Result is greater than value reported

A The TIC is a suspected aldol-condensation product

B Target analyte was detected in the associated blank

$\mathrm{BD}$ Rrisults are either below the MDC or tracer recovery is low

C Analyte has been confirmed by GC/MS analysis

D Results are reported from a diluted aliquot of the sample

$\mathrm{H}$ Analytical holding time was exceeded

$\mathrm{J} \quad$ Value is estimated

N/A Spike recovery limits do not apply. Sample concentration exceeds spike concentration by $4 X$ or more

R Sample results are rejected

U Analyte was analyzed for, but not detected above the MDL, MDA, or LOD.

UI Gamma Spectroscopy-Uncertain identification

X Consult Case Narrative, Data Summary package, or Project Manager concerning this qualifier 


\section{GENERAL ENGINEERING LABORATORIES, LLC}

2040 Savage Road Charleston SC 29407 - (843) 556-8171 - www.gel.com

\section{Certificate of Analysis}

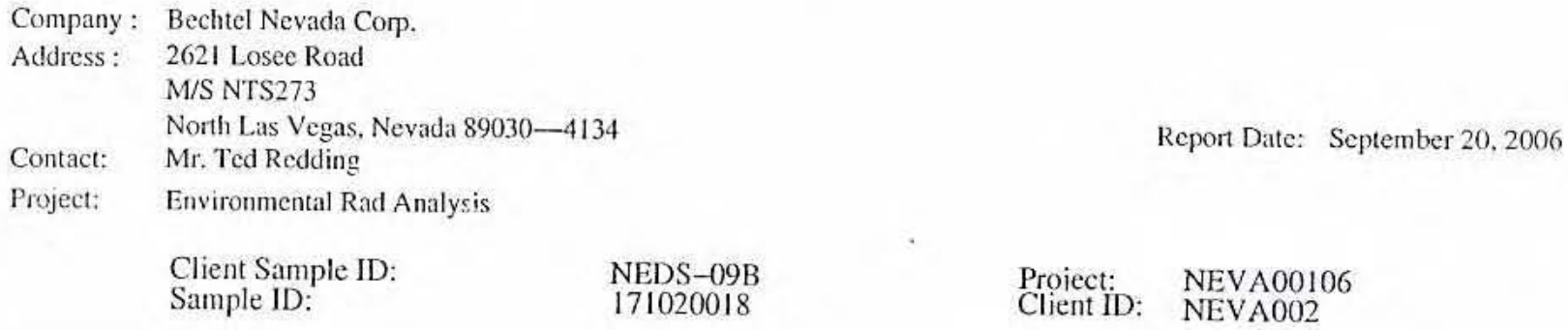

\begin{tabular}{lllllll}
\hline Parameter Qualifier Result Uncertainty DL TPU RL & Units & DF Analyst Date Time Batch Mt
\end{tabular}

Y QC Samples were not spiked with this compound

$\wedge \quad$ RPD of sample and (it:plicate evaluated using $+/-R L$. Concentrations are $<5 X$ the RL

h Preparation or preservation holding time was exceeded

The above sample is reported on a dry veight basis. 


\section{GENERAL ENGINEERING LABORATORIES, LLC \\ 2040 Savage Road Charleston SC 29407 - (843) 556-8171 - www.gel.com}

\section{Certificate of Analysis}

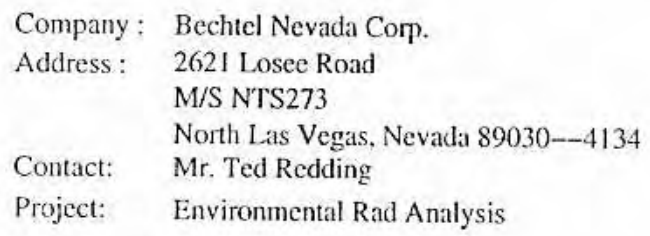

Client Sample ID:

sample ID:

NEDS-10A

$17 ! 020019$

Matrix:

Soil

Collect Date:

Receive Date:

Collector:

22-AUG-06

06-SEP-06

Client

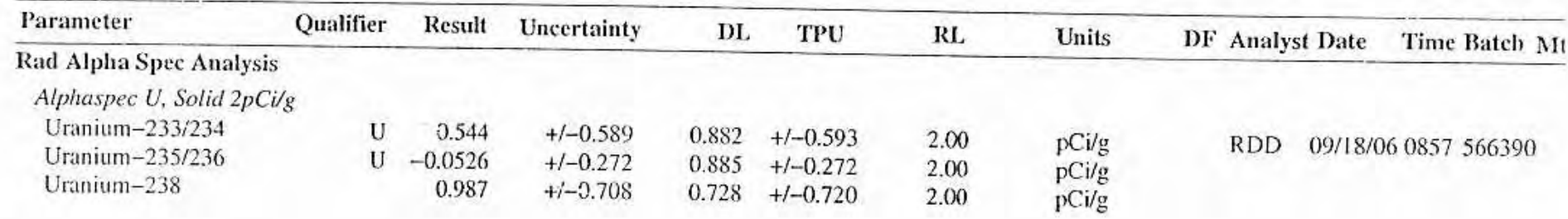

The following l'rep Methods were performed

\begin{tabular}{llllll}
\hline Method & Description & Analyst & Date & Time & Prep Batch \\
\hline Dry Soil Prep & Dry Soil Prep GL-RAD-A-021 & AXP2 & $09 / 07 / 06$ & 1221 & 565878
\end{tabular}

The following Analytical Methods were performed

\begin{tabular}{ll} 
Method & Description \\
\hline & DOE EML. HASL-300, U-02-RC Modified
\end{tabular}

\begin{tabular}{llcc} 
Surrogate/Tracer recovery & Test & Recovery\% & Acceptable Limits \\
\hline Uranium-232 & Alphaspec U, Solid 2pCi/g & 95 & $(25 \%-125 \%)$
\end{tabular}

Notes:

The Qualifiers in this report are defined as follows :

* A quality control analyte recovery is outside of specified acceptance criteria

$<$ Result is less than value reported

$>$ Result is greater than value reported

A The TIC is a suspected aldol-condensation product

B Target analyte was detected in the associated blank

BD Results are either below the MDC or tracer recovery is low

C Analyte has been confirmed by GC/MS analysis

D Results are reported from a diluted aliquot of the sample

$\mathrm{H}$ Analytical holding time was exceeded

$J$ Value is estimated

N/A Spike recovery limits do not apply. Sample concentration exceeds spike concentration by $4 X$ or more

$\mathrm{R}$ Sample results are rejected

$\mathrm{U}$ Analyte was analyzed for, but not detected above the MDL, MDA, or LOD.

UI Gamma Spectroscopy--Uncertain identification

$\mathrm{X}$ Consult Case Narrative, Data Summary package, or Project Manager concerning this qualifier 


\section{GENERAL ENGINEERING LABORATORIES, LLC \\ 2040 Savage Road Charleston SC 29407 - (843) 556-8171 - www.gel.com}

\section{Certificate of Analysis}

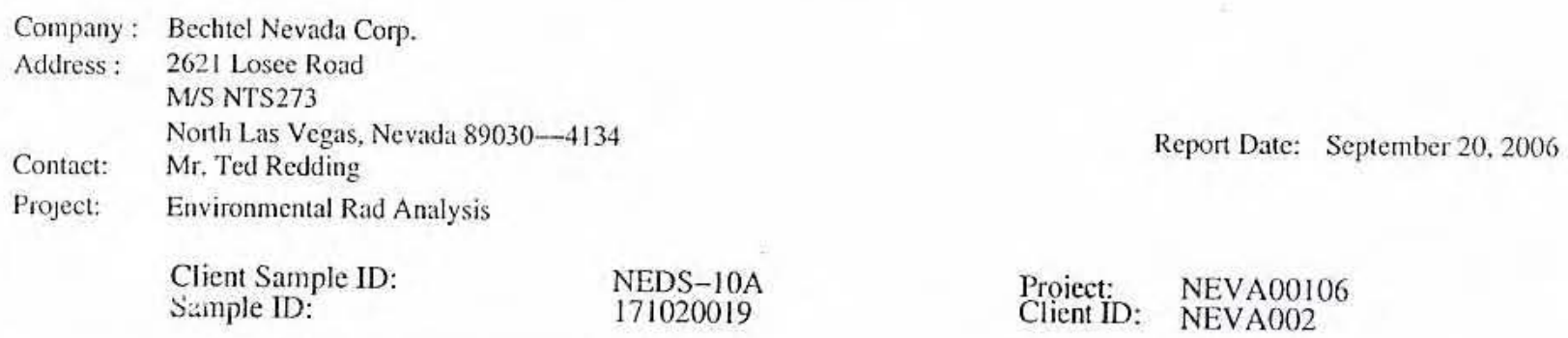

Y QC Samples were not spiked with this compound

$\wedge$ RPD of sample and duplicate evaluated using $+/-$ RL. Concentrations are $<5 X$ the RL

$\mathrm{h}$ Preparation or preservation holding time was exceeded

The above sample is reported on a dry weight basis. 


\section{GENERAL ENGINEERING LABORATORIES, LLC}

2040 Savage Road Charleston SC 29407 - (843) 556-8171 - www.gel.com

\section{Certificate of Analysis}

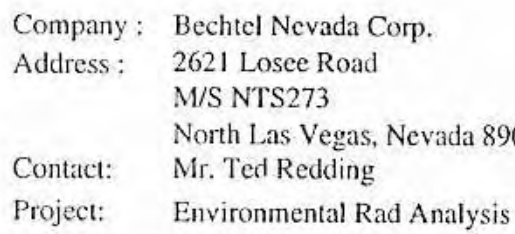

NEDS-10B
171020020
Soil
$22-A U G-06$
$06-S E P-06$
Client

Proiect: NEVA00106

Client ID: NEVA002

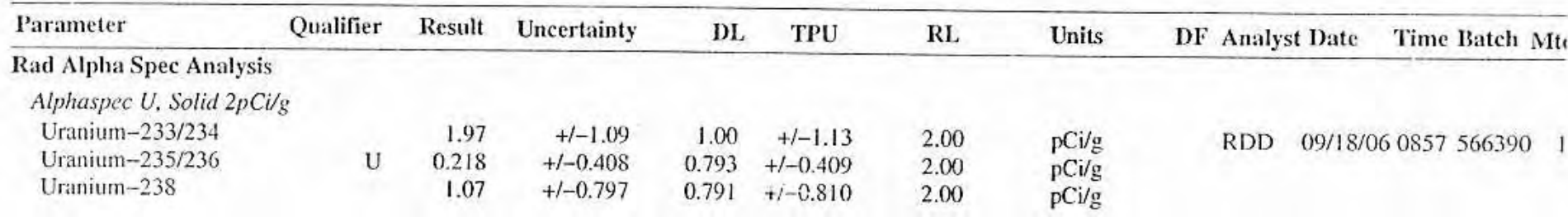

The following Prep Methods were performed

\begin{tabular}{llllll}
\hline Method & Description & Analyst & Date & Time & Prep Batch \\
\hline Dry Soil Prep & Dry Soil Prep GL-RAD-A-021 & AXP2 & $09 / 07 / 06$ & 1221 & 565878
\end{tabular}

The following Analytical Methods were performed

Method Description

1 DOE EML HASL-300, U-02-RC Modified

\begin{tabular}{llcc} 
Surrogate/Tracer recovery & Test & Recovery $\%$ & Acceptable Limits \\
\hline Uranium-232 & Alphaspec U, Solid 2pCi/g & 91 & $(25 \%-125 \%)$
\end{tabular}

Notes:

The Qualifiers in this report are defined as follows:

* A quality control analyte recovery is outside of specified acceptance criteria

$<$ Result is less than value reported

$>$ Result is greater than value reported

A The TIC is a suspected aldol-condensation product

B Target analyte was detected in the associated blank

BD Results are either below the MDC or tracer recovery is low

C Analyte has been confirmed by GC/MS analysis

D Results are reported from a diluted aliquot of the sample

$\mathrm{H}$ Analytical holding time was exceeded

$\mathrm{J}$ Value is estimated

N/A Spike recovery limits do not apply. Sample concentration exceeds spike concentration by $4 \mathrm{X}$ or more

R Sample results are rejected

U Analyte was analyzed for, but not detected above the MDL, MDA, or LOD.

UI Gamma Spectroscopy-Uncertain identification

X Consult Case Narrative, Data Summary package, or Project Manager concerning this qualifier 


\section{GENERAL ENGINEERING LABORATORIES, LLC \\ 2040 Savage Road Charleston SC 29407 - (343) 556-8171 - www.gel.com}

\section{Certificate of Analysis}

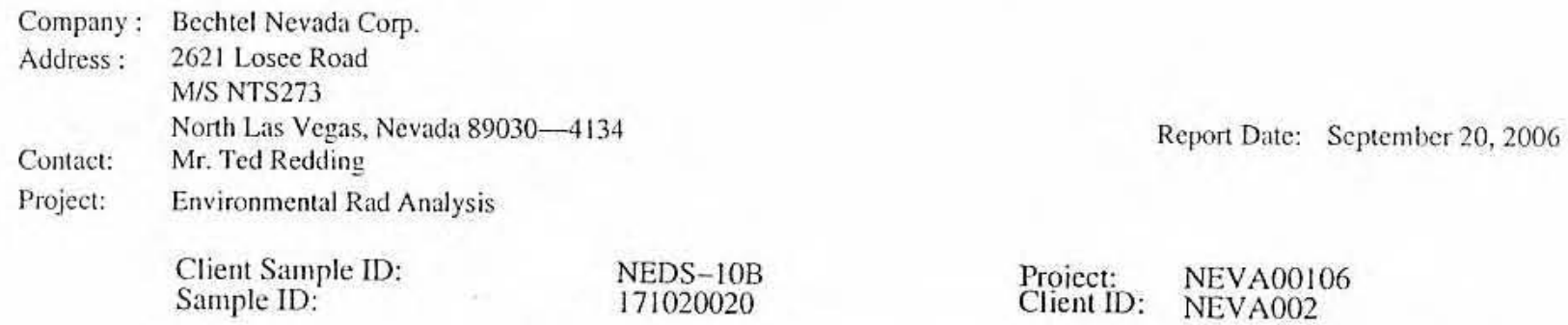

Y QC Samples were not spiked with this compound

A RPD of sample and duplicate evaluated using $+/-\mathrm{RL}$. Concentrations are $<5 \mathrm{X}$ the RL

h Preparation or preservation holding time was exceeded

The above sample is reported on a dry weight basis. 


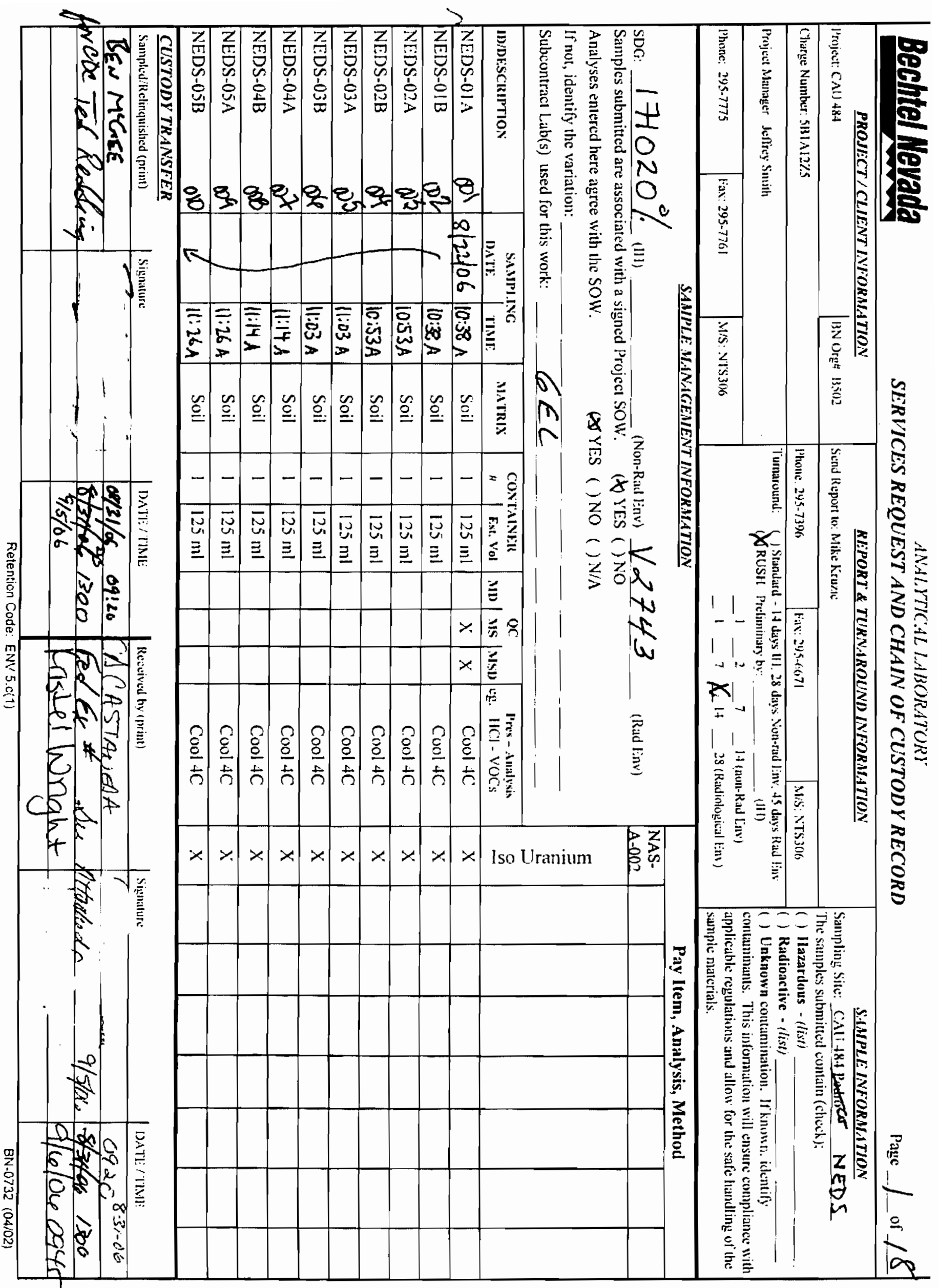




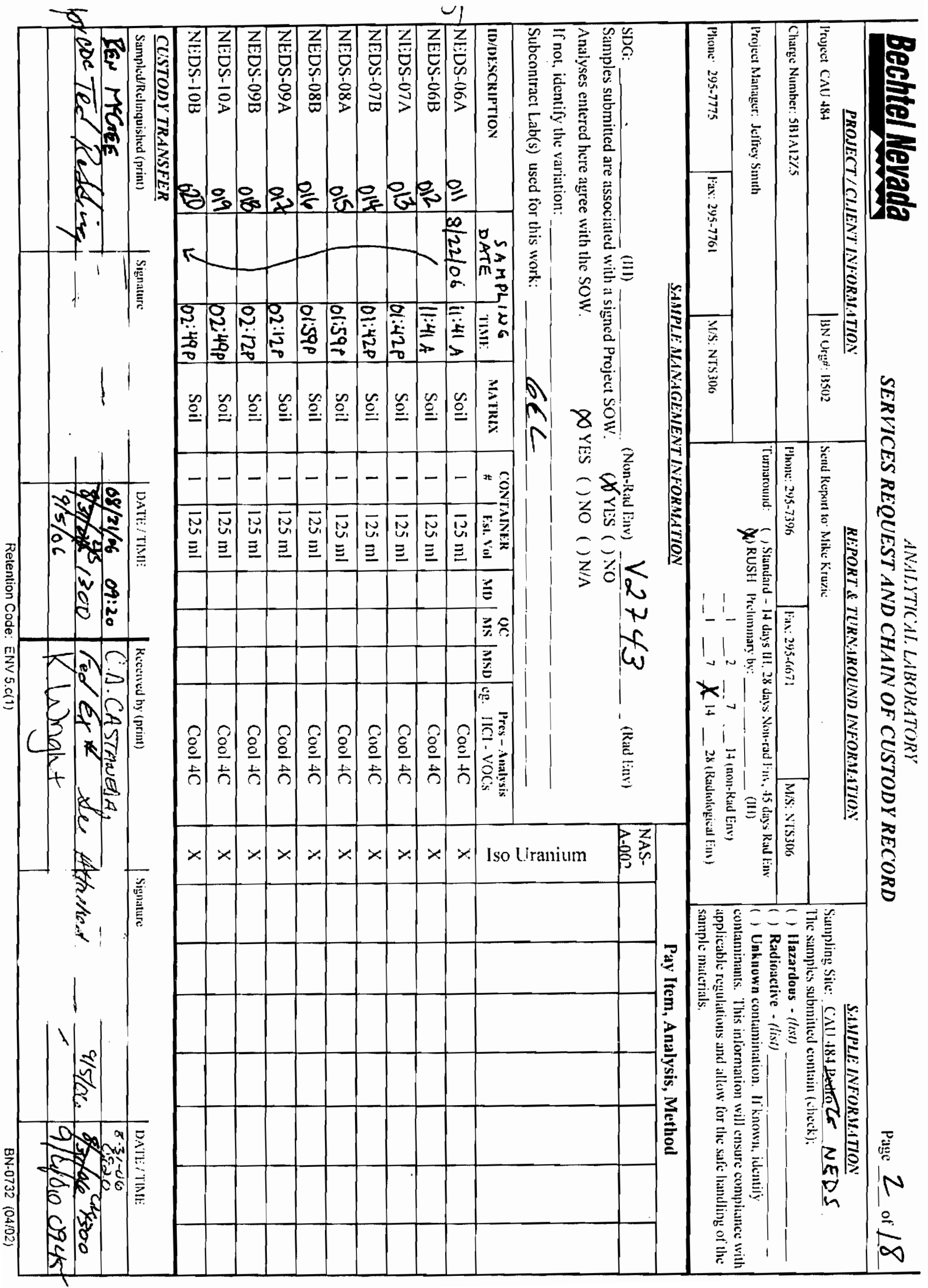




\section{GENERAL ENGINEERING LABORATORIES, LLC \\ 2040 Savage Road Charleston SC 29407 - (843) 556-8171 - www.gel.com}

\section{Certificate of Analysis}

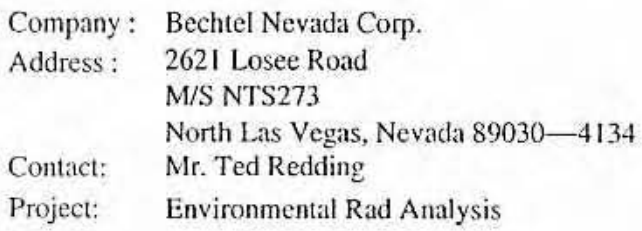

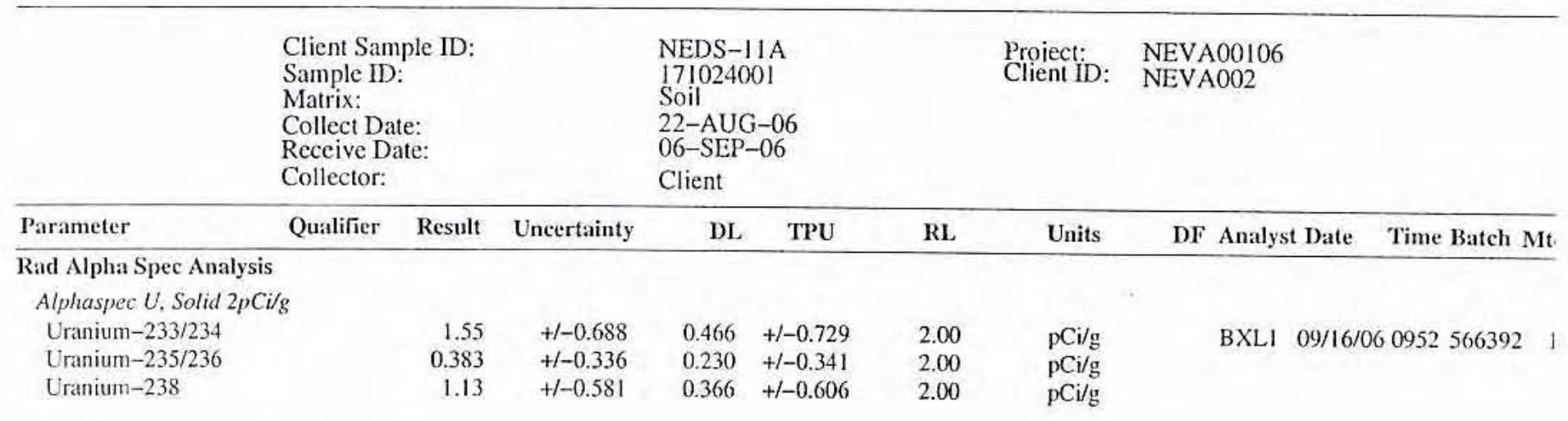

The following Prep Methods were performed

\begin{tabular}{llllll}
\hline Nethod & Description & Analyst & Date & Time & Prep Batel: \\
\hline Dry Soil Prep & Dry Soil Prep GL-RAD-A-021 & LXM2 & $09 / 07 / 06$ & 1708 & 565882
\end{tabular}

The following Analytical Methods were performed

\begin{tabular}{ll} 
Method & Description \\
\hline 1 & DOE EML HASL-300, U-02-RC Modified
\end{tabular}

\begin{tabular}{llcc} 
Surrogate/Tracer recovery & Test & Recovery \% & Acceptable Limits \\
\hline Uranium-232 & Alphaspec U, Solid 2pCi/g & 74 & $(25 \%-125 \%)$
\end{tabular}

Notes:

The Qualifiers in this report are defined as follows :

* A quality control analyte recovery is outside of specified acceptance criteria

$<$ Result is less than value reported

$>$ Result is greater than value reported

A The TIC is a suspected aldol-condensation product

B Target analyte was detected in the associated blank

BD Results are either below the MDC or tracer recovery is low

C Analyte has been confirmed by GC/MS analysis

D Results are reported from a diluted aliquot of the sample

$\mathrm{H}$ Analytical holding time was exceeded

$\mathrm{J}$ Value is estimated

N/A Spike recovery limits do not apply. Sample concentration exceeds spike concentration by $4 \mathrm{X}$ or more

$\mathrm{R}$ Sample results are rejected

U Analyte was analyzed for, but not detected above the MDL, MDA, or LOD.

UI Gamma Spectroscopy--Uncertain identification 


\section{GENERAL ENGINEERING LABORATORIES, LLC}

2040 Savage Road Charleston SC 29407 - (843) 550-8171 - www.gel.com

\section{$\underline{\text { Certificate of Analysis }}$}

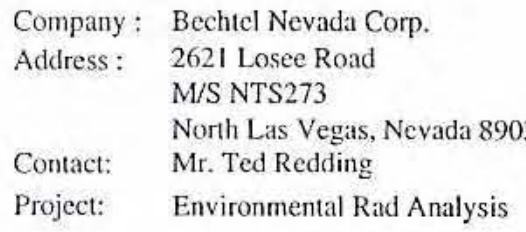

\begin{tabular}{llllllll}
\hline Parameter & Qualilier & Result & Uncertainty & DL & TPU & RL & Units \\
\hline
\end{tabular}

$\mathrm{X}$ Consult Case Narrative, Data Summary package, or Project Manager concerning this qualifier

Y QC Samples were not spiked with this compound

$\wedge$ RPD of sample and duplicate evaluated using $+/-$ RL. Concentrations are $<5 X$ the RL

h Preparation or preservation holding time was exceeded

The above sample is reported on a dry weight basis. 


\section{GENERAL ENGINEERING LABORATORIES, LLC \\ 2040 Savage Road Charleston SC 29407 - (843) 556-8171 - www.gel.com}

\section{Certificate of Analysis}

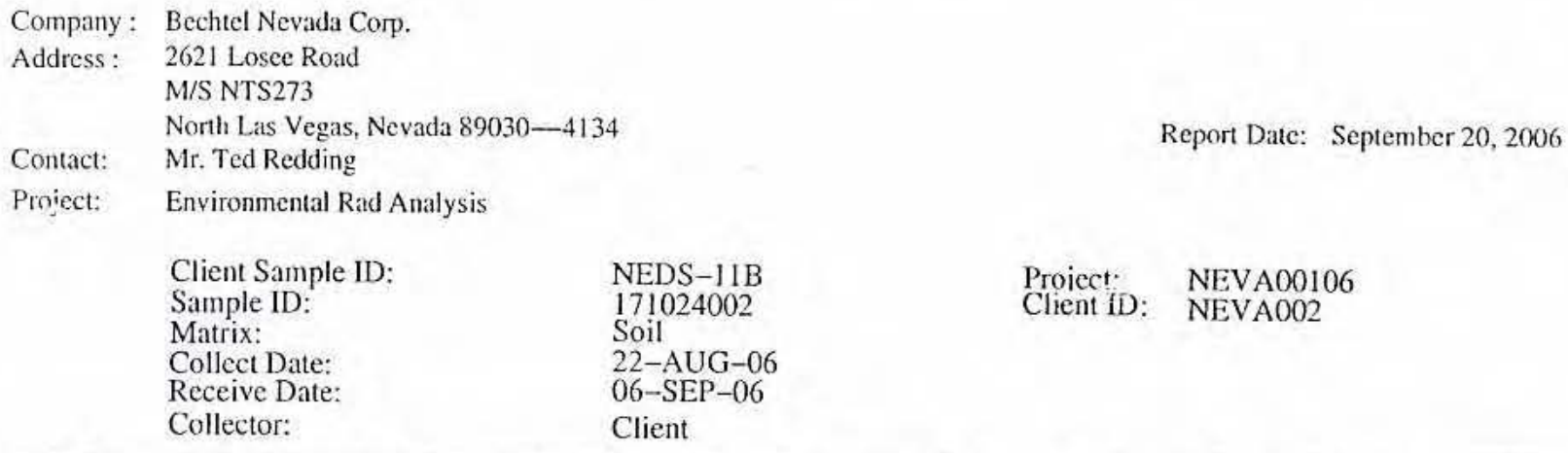

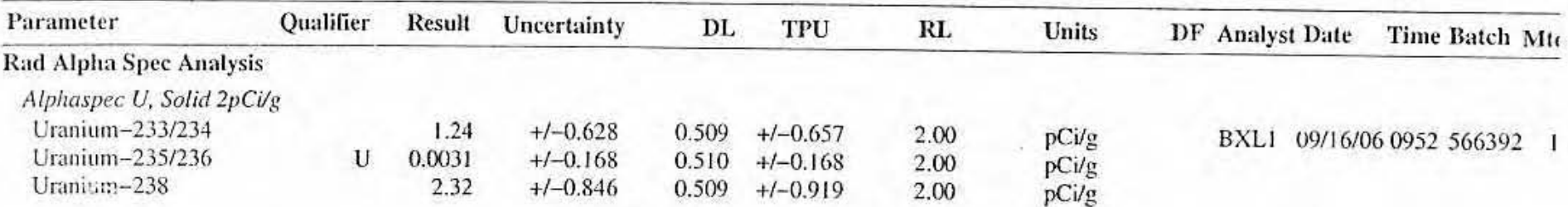

The following Prep Methods were performed

\begin{tabular}{llllll}
\hline Method & Description & Analyst & Date & Time & Prep Batch \\
\cline { 2 - 6 } Dryt Pricp & Dry Soil Prep GL-RAD-A-021 & LXM2 & $09 / 07 / 06$ & 1708 & 565882
\end{tabular}

The following Analytical Methods were performed

\begin{tabular}{ll} 
Method & Description \\
\hline & DOE EML HASL-300, U-02-RC Modified
\end{tabular}

\begin{tabular}{llcc} 
Surrogate/Tracer recovery & Test & Recovery\% & Acceptable Limits \\
\hline Uranium-232 & Alphaspec U, Solid 2pCi/g & 86 & $(25 \%-125 \%)$
\end{tabular}

Notes:

The Qualifiers in this report are defined as follows :

* A quality control analyte recovery is outside of specified acceptance criteria

$<$ Result is less than value reported

$>$ Result is greater than value reported

A The TIC is a suspected aldol-condensation product

B Target analyte was detected in the associated blank

BD Results are either below the MDC or tracer recovery is low

C Analyte has been confirmed by GC/MS analysis

D Results are reported from a diluted aliquot of the sample

H Analytical holding time was exceeded

$\mathrm{J}$ Value is estimated

N/A Spike recovery limits do not apply. Sample concentration exceeds spike concentration by $4 X$ or more

R Sample results are rejected

U Analyte was analyzed for, but not detected above the MDL, MDA, or LOD.

UI Gamma Spectroscopy--Uncertain identification

X Consult Case Narrative, Data Summary package, or Project Manager concerning this qualifier 


\section{GENERAL ENGINEERING LABORATORIES, LLC \\ 2040 Savage Road Charleston SC 29407 - (843) 556-8171 - www.gei.com}

\section{Certificate of Analysis}

Company: Bechtel Nevada Corp.

Address: 2621 Losee Road

M/S NTS273

North Las Vegas, Nevada 89030--4134

Contact: Mr. Ted Redding

Report Date: September 20,2006

Project: Environmental Rad Analysis

Client Sample ID: NEDS-11B

Sample ID: $\quad 171024002$

Proiect: NEVA00106

Client ID: NEVAO02

Parameter

Qualifier

Result Uncertainty

DI TPU

RL

Units

DF Analyst Date Time Batch Mti

Y QC Samples were not spiked with this compound

$\wedge$ RPD of sample and duplicate evaluated using $+/-\mathrm{RL}$. Concentrations are $<5 \mathrm{X}$ the RL

$\mathrm{h}$ Preparation or preservation holding time was exceeded

The above sample is reported on a dry weight basis. 


\section{GENERAL ENGINEERING LABORATORIES, LLC
2040 Savage Road Charleston SC 29407 - (843) 556-8171 - www.gel.com}

\section{Certificate of Analysis}

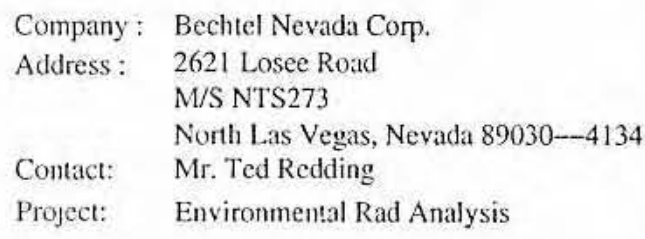

$\begin{array}{llll}\text { Client Sample ID: } & \text { NEDS-12A } & \text { Proiect: } & \text { NEVA00106 } \\ \text { Sample ID: } & 171024003 & \text { Client ID: NEVA002 } \\ \text { Matrix: } & \text { Soil } & & \\ \text { Collect Date: } & \text { 22-AUG-06 } & \\ \text { Receive Date: } & 06-\text { SEP-06 } & \\ \text { Collector: } & \text { Client } & \end{array}$

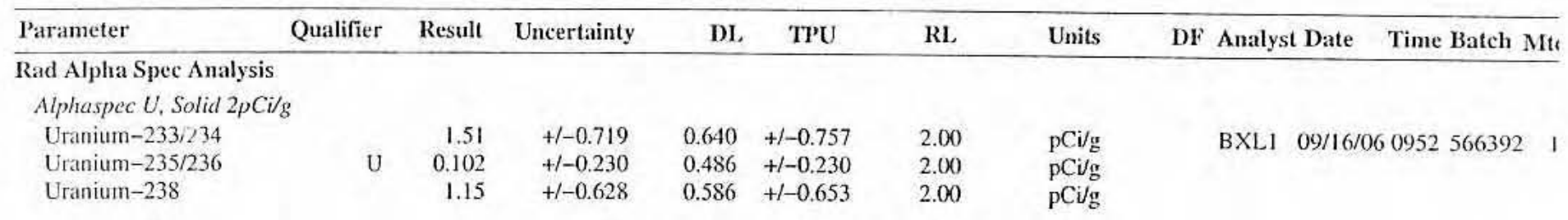

The following Prep Methods were performed

\begin{tabular}{llllll}
\hline Method & Description & Analyst & Date & Time & Prep Batch \\
\hline Dry Soil Prep & Dry Soil Pícö GL-RAD-A-021 & LXM2 & $09 / 07 / 06$ & 1708 & 565882
\end{tabular}

The following Analytical Methods were performed

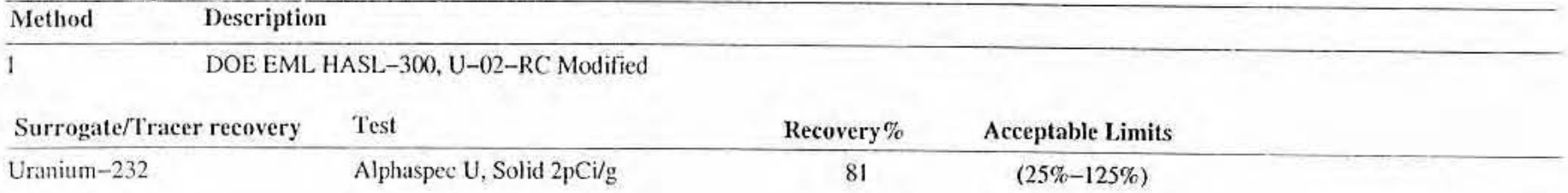

Notes:

The Qualifiers in this report are defined as follows :

* A quality control analyte recovery is outside of specified acceptance criteria

$<$ Result is less than value reported

$>$ Result is greater than value reported

A The TIC is a suspected aldol-condensation product

B Target analyte was detected in the associated blank

BD Results are either below the MDC or tracer recovery is low

C Arialyte has been confirmed by GC/MS analysis

D Results are reported from a diluted aliquot of the sample

$\mathrm{H}$ Analytical holding time was exceeded

$\mathrm{J}$ Value is estimated

N/A Spike recovery limits do not apply. Sample concentration exceeds spike concentration by $4 X$ or more

$\mathrm{R}$ Sample results are rejected

U Analyte was analyzed for, but not detected above the MDL, MDA, or LOD.

UI Gamma Spectroscopy--Uncertain identification

X Consult Case Narrative, Data Summary package, or Project Manager concerning this qualifier 


\section{GENERAL ENGINEERING LABORATORIES, LLC}

2040 Savage Road Charleston SC 29407 - (843) 556-8171 - www.gel.com

\section{Certificate of Analysis}

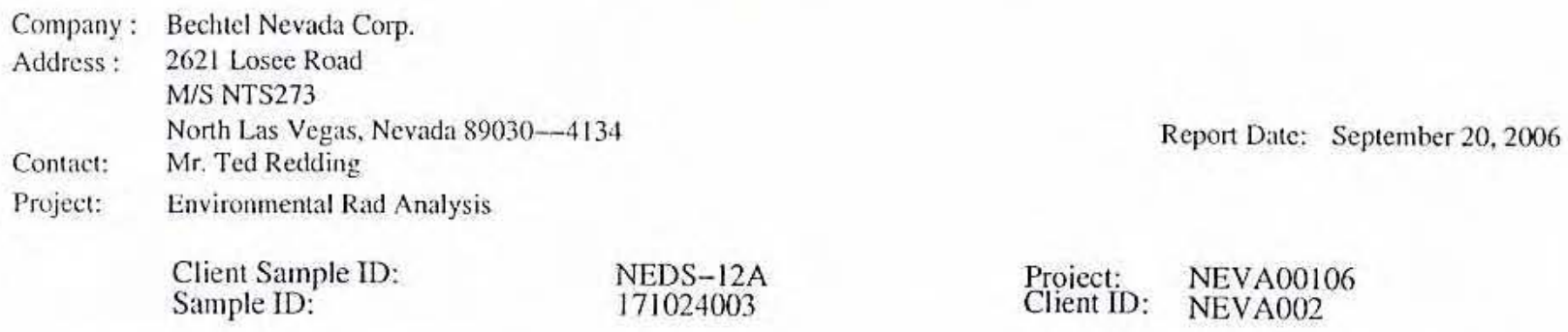

RI.

Units

DF Analyst Date

Time Batch Mts

Y QC Samples were not spiked with this compound

$\wedge$ RPD of sample and duplicate evaluated using $+/-$ RL. Concentrations are $\angle 5 X$ the RL

h Preparation or preservation holding time was exceeded

The above sample is reported on a dry weight basis. 


\section{GENERAL ENGINEERING LABORATORIES, LLC}

2040 Savage Road Charleston SC 29407 - (843) 556-8171 - www.gel.com

\section{Certificate of Analysis}

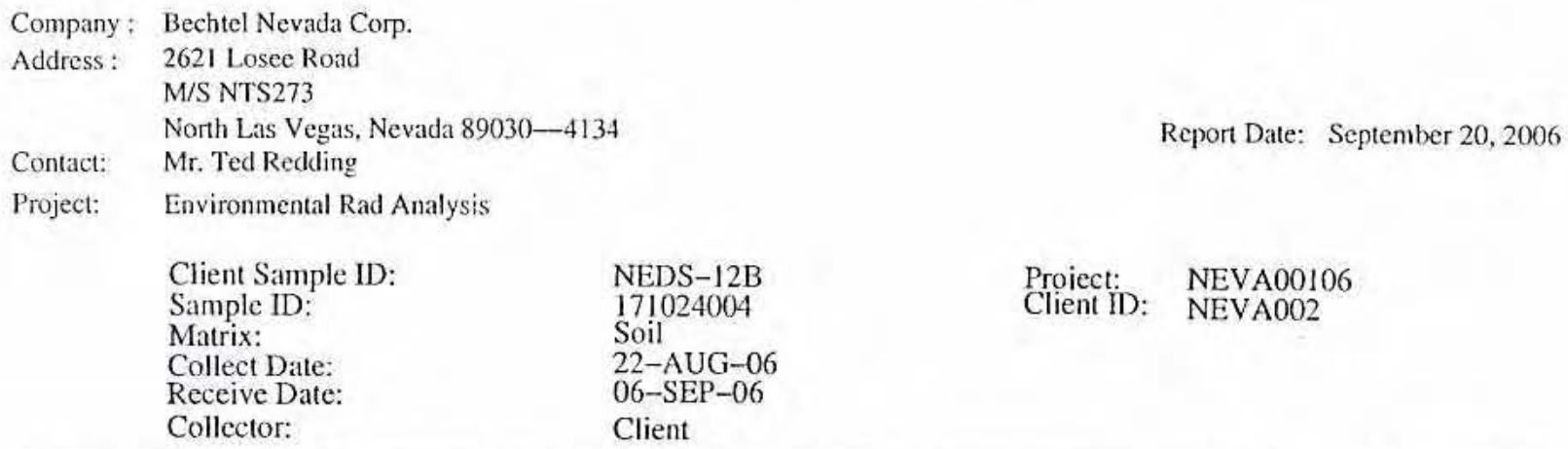

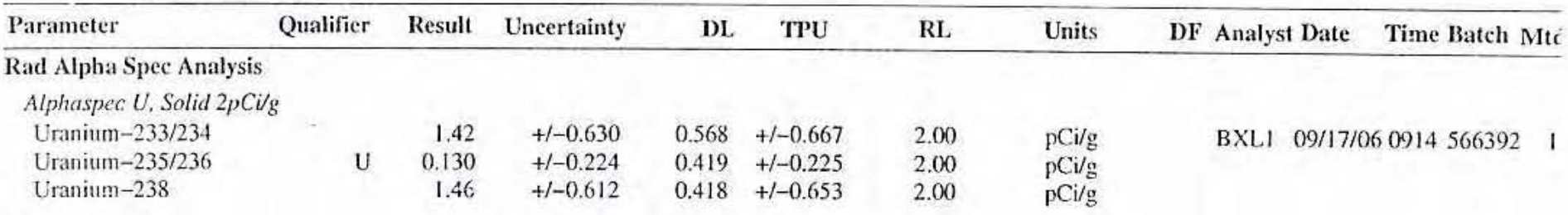

The following Prep Methods were performed

\begin{tabular}{llllll}
\hline Method & Description & Analyst & Date & Time & Prep Batch \\
\hline Dry Soil Prep & Dry Soil Prep GL-RAD-A-021 & LXM2 & $09 / 07 / 06$ & 1709 & 565882
\end{tabular}

The following Analytical Methods were performed

\begin{tabular}{llcc}
\hline Method & & \\
\hline 1 & Description & & \\
DOE EML HASL-300, U-02-RC Modified & \\
Surrogate/Tracer recovery & Test & Recovery \% & Acceptable Limits \\
\hline Uranium-232 & Alphaspec U, Solid 2pCi/g & 87 & $(25 \%-125 \%)$
\end{tabular}

Notes:

The Qualifiers in this report are defined as follows :

* A quality control analyte recovery is outside of specified acceptance criteria

$<$ Result is less than value reported

$>$ Result is greater than value reported

A The TIC is a suspected aldol-condensation product

B Target analyte was detected in the associated blank

$\mathrm{BD}$ Results are either below the MDC or tracer recovery is low

C Analyte has been confirmed by GC/MS analysis

D Results are reported from a diluted aliquot of the sample

H Analytical holding time was exceeded

$\mathrm{J}$ Value is estimated

N/A Spike recovery limits do not apply. Sample concentration exceeds spike concentration by $4 \mathrm{X}$ or more

$\mathrm{R}$ Sample results are rejected

U Analyte was analyzed for, but not detected above the MDL, MDA, or LOD.

UI Gamma Spectroscopy--Uncertain identification

X Consult Case Narrative, Data Summary package, or Project Manager concerning this qualifier 


\section{GENERAL ENGINEERING LABORATORIES, LLC}

2040 Savage Road Charleston SC 29407 - (843) 556-8171 - www.gel.com

\section{Certificate of Analysis}

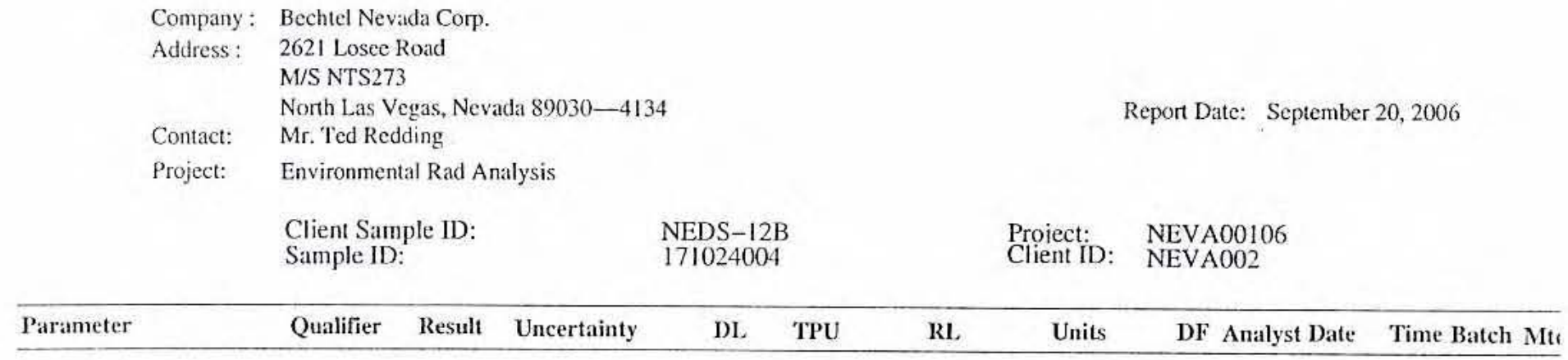

Y QC Samples were not spiked with this compound

$\wedge$ RPD of sample and duplicate evaluated using $+/-\mathrm{RL}$. Concentrations are $<5 \mathrm{X}$ the $\mathrm{RL}$

$\mathrm{h}$ Preparation or preservation holding time was exceeded

The above sample is reported on a dry weight basis. 


\section{GENERAL ENGINEERING LABORATORIES, LLC}

2040 Savage Road Charleston SC 29407 - (843) 556-8171 - www.gel.com

\section{$\underline{\text { Certificate of Analysis }}$}

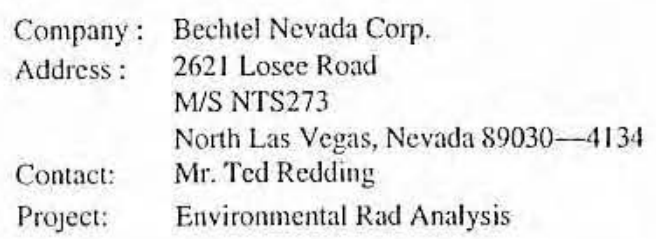

Client Sample ID:

Sample ID:

Matrix:

Collect Date:

Receive Date:

Collector:

NEDS-13A
171024005
Soil
22-AUG-06
06-SEP-06
Client

Proiect: NEVA00106

Client ID: NEVA002

Qualilier Result Uncertainty

DL TPU

RI.

Units

DF Analyst Date

Time Batch Mt

Rad Alpha Spec Analysis

Alphaspec U. Solid $2 p \mathrm{Ci} / \mathrm{g}$

Uranium-233/234

\begin{tabular}{|c|c|c|c|c|c|c|}
\hline & 1.83 & $+1-0.760$ & 0.541 & $+1-0.817$ & 2.00 & $\mathrm{pCi}$ \\
\hline & 0.217 & $+/-0.314$ & 0.542 & $+1-0.316$ & 2.00 & $\mathrm{Ci}$ \\
\hline & 1.91 & $+1-0.775$ & 0.541 & $+1-0.835$ & 2.00 & $\mathrm{pC}$ \\
\hline
\end{tabular}

Uranium-235/236

$1.91+1-0.775$

$0.542+1-0.316$

2.00

$\mathrm{pCi} / \mathrm{g}$

BXL1 09/17/060914 566392

Uranium-238

The following Prep Methods were performed

\begin{tabular}{llllll}
\hline Method & Description & Analyst & Date & Time & Prep Batch \\
\hline Dry Soil Prep & Dry Soil Prep GL-RAD-A-021 & LXM2 & $09 / 07 / 06$ & I709 & 565882
\end{tabular}

The following Analytical Methods were performed

\begin{tabular}{ll} 
Method & Description \\
\hline DOE EML HASL-300, U-02-RC Modified
\end{tabular}

\begin{tabular}{llcc} 
Surrogate/Tracer recovery & Test & Recovery\% & Acceptable Limits \\
\hline Urauium-232 & Alphaspec U, Solid 2pCi/g & 84 & $(25 \%-125 \%)$
\end{tabular}

Notes:

The Qualifiers in this report are defined as follows:

* A quality control analyte recovery is outside of specified acceptance criteria

$<$ Result is less than value reported

$>$ Result is greater than value reported

A The TIC is a suspected aldol-condensation product

B Target analyte was detected in the associated blank

$\mathrm{BD}$ Results are either below the MDC or tracer recovery is low

C Analyte has been confirmed by GC/MS analysis

D Results are reported from a diluted aliquot of the sample

$\mathrm{H}$ Analytical holding time was exceeded

$\mathrm{J}$ Value is estimated

N/A Spike recovery limits do not apply. Sample concentration exceeds spike concentration by $4 X$ or more

$\mathrm{R}$ Sample results are rejected

U Analyte was analyzed for, but not detected above the MDL, MDA, or LOD.

UI Gamma Spectroscopy--Uncertain identification

$\mathrm{X}$ Consult Case Narrative, Data Summary package, or Project Manager concerning this qualifier 


\section{GENERAL ENGINEERING LABORATORIES, LLC \\ 2040 Savage Road Charleston SC 29407 - (843) 556-8171 - www.gel.com}

\section{Certificate of Analysis}

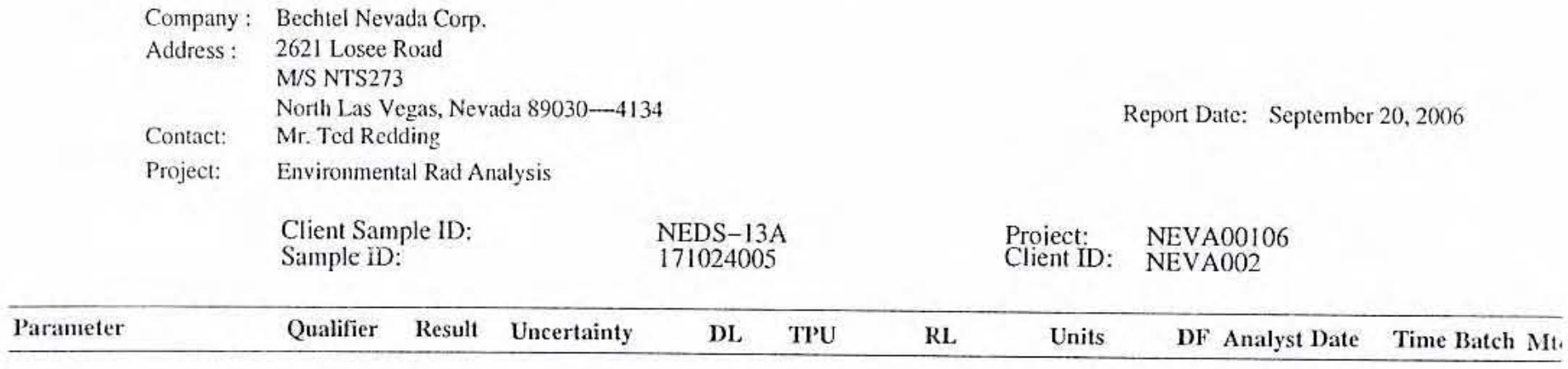

Y QC Samples were not spiked with this compound

A RPD of sample and duplicate evaluated using $+/-$ RL. Concentrations are $<5 \mathrm{X}$ the RL

$h$ Preparation or preservation holding time was exceeded

The above sample is reported on a dry weight basis. 


\section{GENERAL ENGINEERING LABORATORIES, LLC \\ 2040 Savage Road Charleston SC 29407 - (843) 556-8171 - www.gel.com}

\section{Certificate of Analysis}

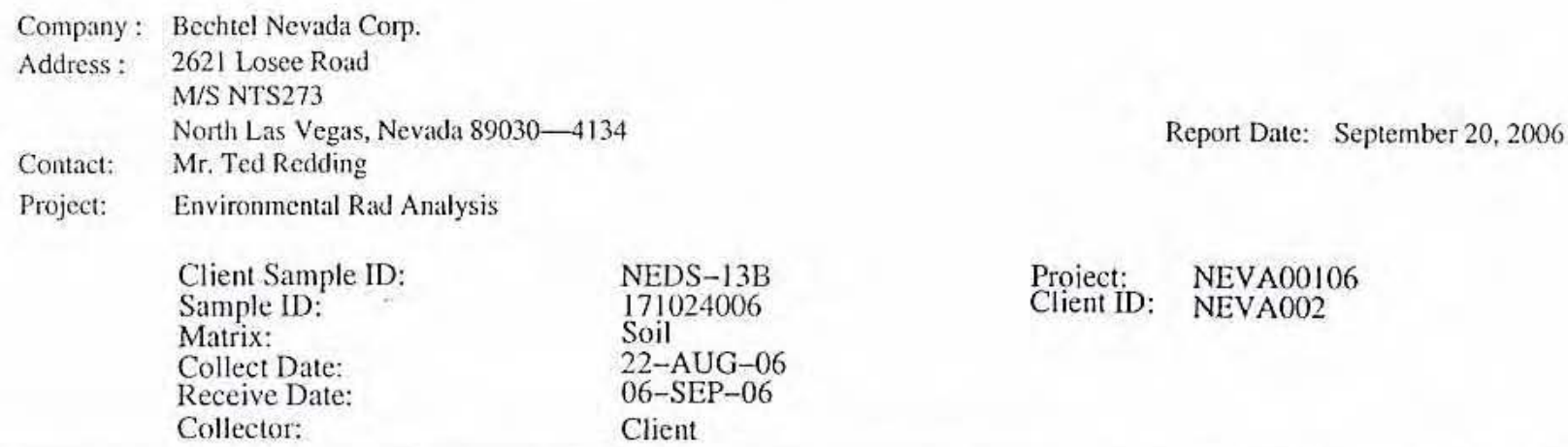

\begin{tabular}{|c|c|c|c|c|c|c|c|c|c|c|}
\hline Parameter & Qualifier & Result & Uncertainty & DL & TPU & RI & Units & DF & Analyst Date & Time Batch Mt \\
\hline \multicolumn{11}{|c|}{ Rad Alpha Spec Analysis } \\
\hline \multicolumn{11}{|c|}{ Alphaspec U. Solid $2 p C i / g$} \\
\hline Uranium-233/234 & & 1.68 & $+1-0.680$ & 0.422 & $+1-0.731$ & 2.00 & $\mathrm{pCi} / \mathrm{g}$ & & BXLI 09/17/ & 060914566392 \\
\hline Uranium-235/236 & $\mathrm{U}$ & 0.00277 & $+1-0.151$ & 0.457 & $+1-0.151$ & 2.00 & $\mathrm{pCi} / \mathrm{g}$ & & & \\
\hline Uranium-238 & & 1.21 & $+1-0.596$ & 0.511 & $+1-0.627$ & 2.00 & $\mathrm{pCi} / \mathrm{g}$ & & & \\
\hline
\end{tabular}

The following Prep Methods were performed

\begin{tabular}{llllll}
\hline Method & Description & Analyst & Date & Time & Prep Batch \\
\hline Dry Soil Prep & Dry Soil Prep GL-RAD-A-021 & LXiM2 & $09 / 07 / 06$ & 1709 & 565882
\end{tabular}

The following Analytical Methods were performed

\begin{tabular}{|c|c|c|c|}
\hline Method Descriptio & & & \\
\hline DOE EMI & ASL-300, U-02-RC Modified & & \\
\hline Surrogate/Tracer recovery & Test & Recovery\% & Acceptable I imits \\
\hline Uranium-232 & Alphaspec U, Solid 2pCi/g & 77 & $(25 \%-125 \%)$ \\
\hline
\end{tabular}

Notes:

The Qualifiers in this report are defined as follows :

* A quality control analyte recovery is outside of specified acceptance criteria

$<$ Result is less than value reported

$>$ Result is greater than value reported

A The TIC is a suspected aldol-condensation product

B Target analyte was detected in the associated blank

$\mathrm{BD}$ Results are either below the MDC or tracer recovery is low

C Analyte has been confirmed by GC/MS analysis

D Results are reported from a diluted aliquot of the sample

$\mathrm{H}$ Analytical holding time was exceeded

$\mathrm{J}$ Value is estimated

N/A Spike recovery limits do not apply. Sample concentration exceeds spike concentration by $4 \mathrm{X}$ or more

$\mathrm{R}$ Sample results are rejected

U Analyte was analyzed for, but not detected above the MDL, MDA, or L.OD.

UI Gamma Spectroscopy-Uncertain identification

$\mathrm{X}$ Consult Case Narrative, Data Summary package, or Project Manager concerning this qualifier 


\section{GENERAL ENGINEERING LABORATORIES, LLC \\ 2040 Savage Road Charleston SC 29407 - (843) 556-8171 - www.gel.com}

\section{Certificate of Analysis}

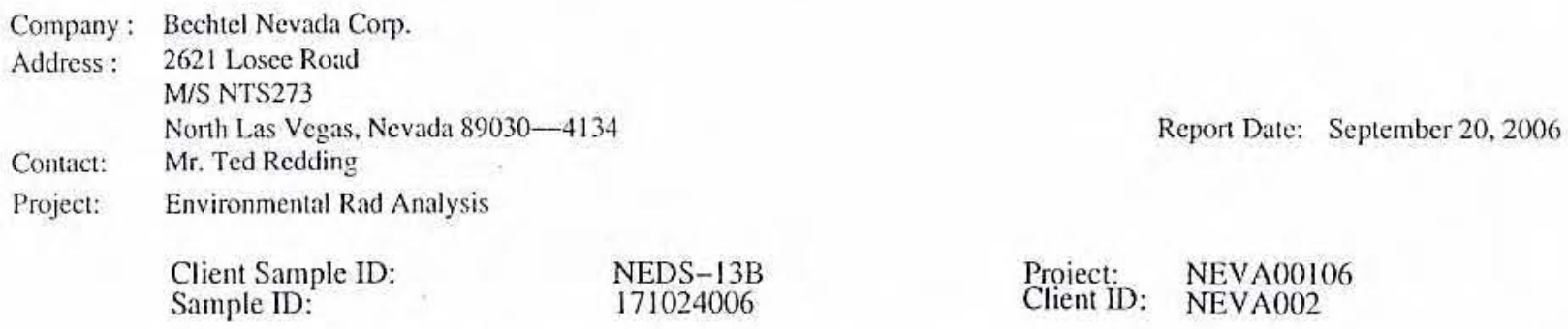

Y QC Samples were not spiked with this compound

$\wedge$ RPD of sample and duplicate evaluated using $+/-$ RL. Concentrations are $<5 X$ the RL

$\mathrm{h}$ Preparation or preservation holding time was exceeded

The above sample is reported on a dry weight basis. 


\section{GENERAL ENGINEERING LABORATORIES, LLC \\ 2040 Savage Road Charleston SC 29407 - (843) 556-8171 - www.gel.com}

\section{Certificate of Analysis}

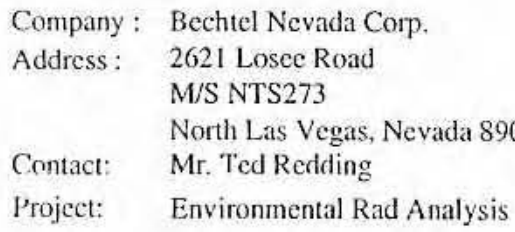

\begin{tabular}{|c|c|c|c|c|c|c|c|c|c|c|}
\hline Parameter & Qualifier & Result & Uncertainty & DL & TPU & RL & Units & DF & Analyst Date & Time Batch Mt \\
\hline \multicolumn{11}{|c|}{ Rad Alpha Spec Analysis } \\
\hline \multicolumn{11}{|c|}{ Alphaspec U, Solid $2 p \mathrm{Ci} / \mathrm{g}$} \\
\hline $\begin{array}{l}\text { Uranium-233/234 } \\
\text { Uranium-235/236 } \\
\text { Uranium-238 }\end{array}$ & $\mathrm{U}$ & $\begin{array}{r}1.91 \\
-0.0713 \\
4.84\end{array}$ & $\begin{array}{r}+1-0.758 \\
+/-0.0699 \\
+/-1.19\end{array}$ & $\begin{array}{l}0.519 \\
0.489 \\
0.574\end{array}$ & $\begin{array}{r}+1-0.819 \\
+1-0.0708 \\
+1-1.43\end{array}$ & $\begin{array}{l}2.00 \\
2.00 \\
2.00\end{array}$ & $\begin{array}{l}\mathrm{pCi} / \mathrm{g} \\
\mathrm{pCi} / \mathrm{g} \\
\mathrm{pCi} / \mathrm{g}\end{array}$ & & BXLI $\quad 09 / 17 / 0$ & 60914566392 \\
\hline
\end{tabular}

The following Prep Methods were performed

\begin{tabular}{|c|c|c|c|c|c|}
\hline Method & Description & Analyst & Date & Time & Prep Batch \\
\hline Dry Soil Prep & Dry Soil Prep GL-RAD-A-021 & LXM2 & $09 / 07 / 06$ & 1709 & 565882 \\
\hline \multicolumn{6}{|c|}{ The following Analytical Methods were performed } \\
\hline Method & Description & & & & \\
\hline 1 & DOE EML HASL-300, U-02-RC Modified & & & & \\
\hline Surrogate/Tr & er recovery & Recovery \% & \multicolumn{2}{|c|}{ Acceptable Limits } & \\
\hline Uranium-232 & Alphaspec U, Solid $2 \mathrm{pCi} / \mathrm{g}$ & 80 & \multicolumn{2}{|c|}{$(25 \%-125 \%)$} & \\
\hline
\end{tabular}

Notes:

The Qualifiers in this report are defined as follows :

* A quality control analyte recovery is outside of specified acceptance criteria

$<$ Result is less than value reported

$>$ Result is greater than value reported

A The TIC is a suspected aldol-condensation product

B Target analyte was detected in the associated blank

BD Results are either below the MDC or tracer recovery is low

C Analyte has been confirmed by GC/MS analysis

D Results are reported from a diluted aliquot of the sample

$\mathrm{H}$ Analytical holding time was exceeded

$\mathrm{J}$ Value is estimated

N/A Spike recovery limits do not apply. Sample concentration exceeds spike concentration by $4 X$ or more

R Sample results are rejected

U Analyte was analyzed for, but not detected above the MDL, MDA, or LOD.

UI Gamma Spectroscopy-Uncertain identification

X Consult Case Narrative, Data Summary package, or Project Manager concerning this qualifier 


\section{GENERAL ENGINEERING LABORATORIES, LLC \\ 2040 Savage Road Charleston SC 29407 - (643) 556-8171 - www.gel.com}

\section{Certificate of Analysis}

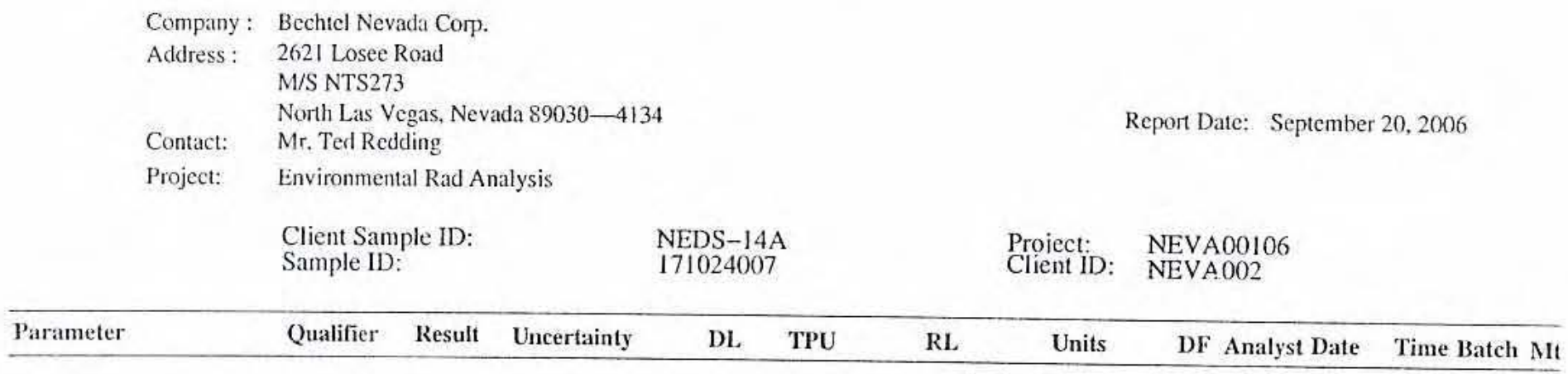

Y QC Samples were not spiked with this compound

$\wedge$ RPD of sample and duplicate evaluated using $+/-R L$. Concentrations are $\angle 5 X$ the $R L$

$\mathrm{h}$ Preparation or preservation holding time was exceeded

The above sample is reported on a dry weight basis. 


\section{GENERAL ENGINEERING LABORATORIES, LLC}

2040 Savage Road Charleston SC 29407 - (843) 556-6i71 - www.gel.com

\section{Certificate of Analysis}

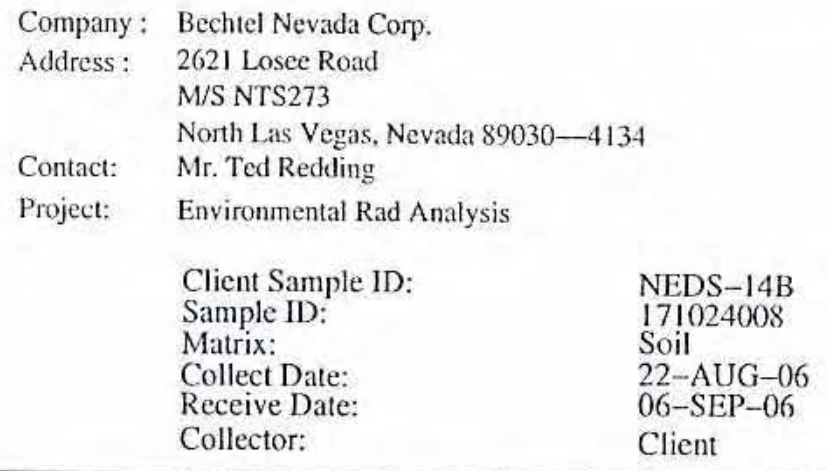

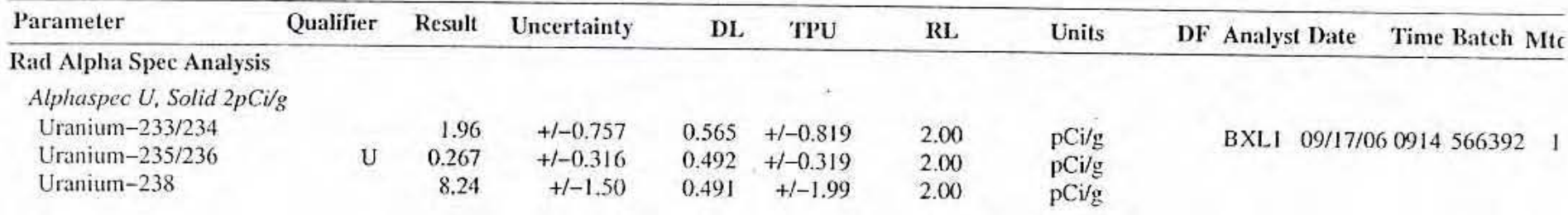

The following Prep Methods were performed

\begin{tabular}{llllll}
\hline Method & Description & Analyst & Date & Time & Prep Batcl. \\
\hline Dry Soil Prep & Dry Soil Prep GL-RAD-A-021 & LXM2 & $09 / 07 / 06$ & 1709 & 565882
\end{tabular}

The following Analytical Methods were performed

\begin{tabular}{ll}
\hline Method & Description \\
\hline DOE EML HASL-300, U-02-RC Modified
\end{tabular}

\begin{tabular}{llcc} 
Surrogate/Tracer recovery & Test & Recovery \% & Acceptable Limits \\
\hline Uranium-232 & Alphaspec U, Solid 2pCi/g & 96 & $(25 \%-125 \%)$
\end{tabular}

Notes:

The Qualifiers in this report are defined as follows :

* A quality control analyte recovery is outside of specified acceptance criteria

$<$ Result is less than value reported

$>$ Result is greater than value reported

A The TIC is a suspected aldol-condensation product

B Target analyte was detected in the associated blank

BD Results are either below the MDC or tracer recovery is low

C Analyte has been confirmed by GC/MS analysis

D Results are reported from a diluted aliquot of the sample

$\mathrm{H}$ Analytical holding time was exceeded

$\mathrm{J}$ Value is estimated

N/A Spike recovery limits do not apply. Sample concentration exceeds spike concentration by $4 X$ or more

$\mathrm{R}$ Sample results are rejected

U Analyte was analyzed for, but not detected above the MDL, MDA, or LOD.

UI Gamma Spectroscopy--Uncertain identification

X Consult Case Narrative, Data Summary package, or Project Manager concerning this qualifier 


\section{GENERAL ENGINEERING LABORATORIES, LLC}

2040 Savage Road Charleston SC 29407 - (843) 556-8171 - www.gel.com

\section{Certificate of Analysis}

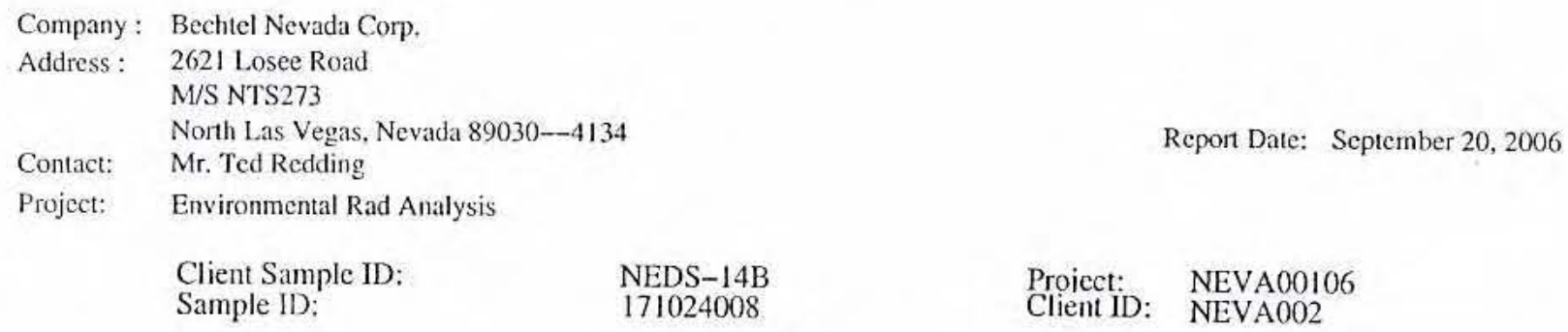

RL

Units

DF Analyst Date

Time Batch Mtc

Y QC Samples were not spiked with this compound

$\wedge$ RPD of sample and duplicate evaluated using $+1-$ RL. Concentrations are $<5 X$ the RL

h Preparation or preservation holding time was exceeded

The above sample is reported on a dry weight basis. 


\section{GENERAL ENGINEERING LABORATORIES, LLC \\ 2040 Savage Road Charleston SC 29407 - (843) 556-6171 - www.gei.com}

\section{Certificate of Analysis}

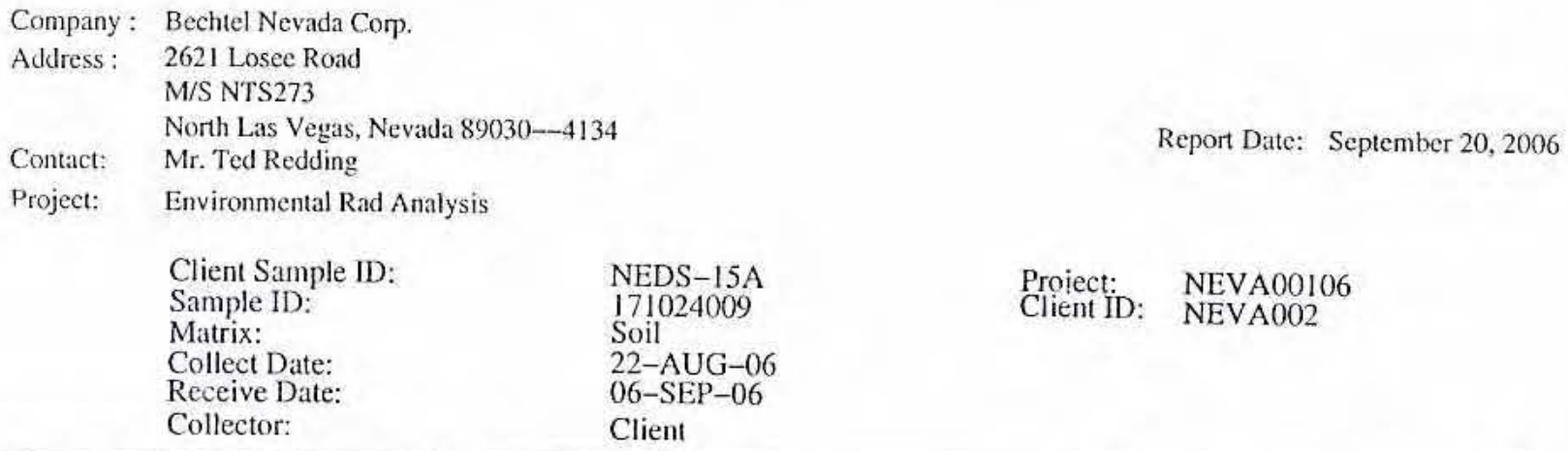

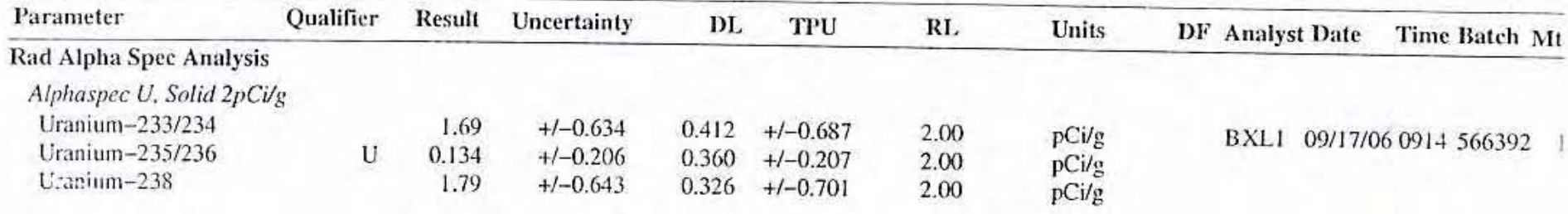

The following Prep Methods were performed

\begin{tabular}{|c|c|c|c|c|c|c|}
\hline Method & \multicolumn{2}{|c|}{ Description } & Analyst & Date & Time & Prep Batch \\
\hline Dry üñl Prep & \multicolumn{2}{|c|}{ Dry Soil Prep GL-RAD-A-02I } & LXM2 & $09 / 07 / 06$ & 1709 & 565882 \\
\hline \multicolumn{7}{|c|}{ The following Analytical Methods were performed } \\
\hline Method & \multicolumn{6}{|c|}{ Description } \\
\hline 1 & \multicolumn{6}{|c|}{ DOE EML HASL-300, U-02-RC Modified } \\
\hline Surrogate/Tr & r recovery & Test & Recovery \% & \multicolumn{3}{|c|}{ Acceptable Limits } \\
\hline Uranium-232 & & Alphaspec U, Solid 2pCi/g & 95 & \multicolumn{3}{|c|}{$(25 \%-125 \%)$} \\
\hline
\end{tabular}

Notes:

The Qualifiers in this report are defined as follows:

* A quality control analyte recovery is outside of specified acceptance criteria

$<$ Result is less than value reported

$>$ Result is greater than value reported

A The TIC is a suspected aldol-condensation product

B Target analyte was detected in the associated blank

BD Results are either below the MDC or tracer recovery is low

C Analyte has been confirmed by GC/MS analysis

D Results are reported from a diluted aliquot of the sample

$\mathrm{H}$ Analytical holding time was exceeded

$\mathrm{J}$ Value is estimated

N/A Spike recovery limits do not apply. Sample concentration exceeds spike concentration by $4 \mathrm{X}$ or more

$\mathrm{R}$ Sample results are rejected

UT Analyte was analyzed for, but not detected above the MDL, MDA, or LOD.

UI Gamma Spectroscopy--Uncertain identification

X Consult Case Narrative, Data Summary package, or Project Manager concerning this qualifier 


\section{GENERAL ENGINEERING LABORATORIES, LLC}

2040 Savage Road Charleston SC 29407 - (843) 556-8171 - www.yei.com

\section{Certificate of Analysis}

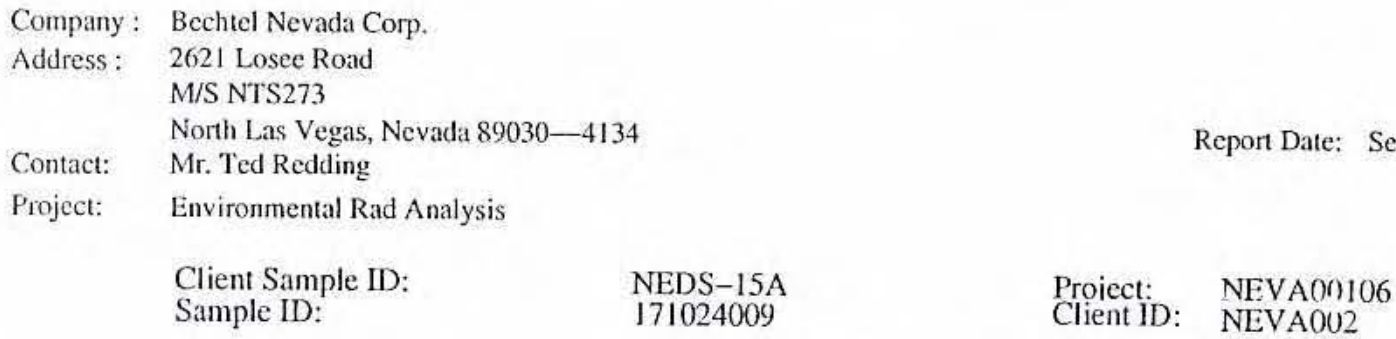

DI TPU

RL

Units

DF Analyst Date Time Batch Mt.

Y QC Samples were not spiked with this compound

$\wedge$ RPD of sample and duplicate evaluated using $+/-$ RL. Concentrations are $<5 X$ the $R L$

h Preparation or preservation holding time was exceeded

The above sample is reported on a dry weight basis. 


\section{GENERAL ENGINEERING LABORATORIES, LLC \\ 2040 Savage Road Charleston SC 29407 - (843) 556-8171 - www.gel.com}

\section{Certificate of Analysis}

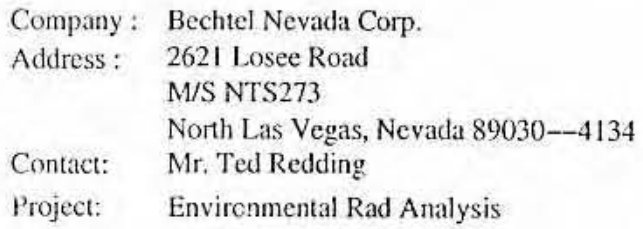

Report Date: September 20,2006

NEDS-15B
171024010
Soil
22-AUG-06
06-SEP-06
Client

Proiect: NEVAOn!06

Client ID: NEVAOU2

\begin{tabular}{|c|c|c|c|c|c|c|c|c|c|c|}
\hline Parameter & Qualifier & Result & Uncertainty & $\mathrm{DL}$ & TPU & $\mathrm{RL}$ & Units & DF & Analyst Date & Time Batch Mt \\
\hline \multicolumn{11}{|c|}{ Rad Alpha Spec Analysis } \\
\hline \multicolumn{11}{|c|}{ Alphaspec U. Solid $2 p C i / g$} \\
\hline $\begin{array}{l}\text { Uranium } 233 / 234 \\
\text { Uranium-235/236 } \\
\text { Uranium-238 }\end{array}$ & $\mathrm{U}$ & $\begin{array}{r}1.89 \\
0.0775 \\
1.66\end{array}$ & $\begin{array}{l}+1-0.696 \\
+-0.175 \\
+1-0.641\end{array}$ & $\begin{array}{l}0.540 \\
0.370 \\
0.446\end{array}$ & $\begin{array}{l}+1-0.748 \\
+1-0.175 \\
+1-0.684\end{array}$ & $\begin{array}{l}2.00 \\
2.00 \\
2.00\end{array}$ & $\begin{array}{l}\mathrm{pCi} / \mathrm{g} \\
\mathrm{pCi} / \mathrm{g} \\
\mathrm{pCi} / \mathrm{g}\end{array}$ & & BXLI $09 / 17 / 0$ & 60914566392 \\
\hline
\end{tabular}

The following Prep Methods were performed

\begin{tabular}{llllll}
\hline Method & Description & Analyst & Date & Time & Prep Batch \\
\hline Dry Soil Prep & Dry Soil Prep GL-RAD-A-021 & LXM2 & $09 / 07 / 06$ & 1709 & 565882
\end{tabular}

The following Analyticai Methods were performed

\begin{tabular}{ll} 
Method & Description \\
\hline 1 & DOE EML HASL-300, U-02-RC Modified
\end{tabular}

\begin{tabular}{llcc} 
Surrogate/Tracer recovery & Test & Recovery \% & Acceptable Limits \\
\hline Uranium-232 & Alphaspec U, Solid 2pCi/g & 97 & $(25 \%-125 \%)$
\end{tabular}

Notes:

The Qualifiers in this report are defined as follows :

* A quality control analyte recovery is outside of specified acceptance criteria

$<$ Result is less than value reported

$>$ Result is greater than value reported

A The TIC is a suspected aldol-condensation product

B Target analyte was delected in the associated blank

$\mathrm{BD}$ Results are either below the MDC or tracer recovery is low

C. Analyte has been confirmed by GC/MS analysis

D Results are reported from a diluted aliquot of the sample

$\mathrm{H}$ Analytical holding time was exceeded

$\mathrm{J}$ Value is estimated

N/A Spike recovery limits do not apply. Sample concentration exceeds spike concentration by $4 X$ or more

$\mathrm{R}$ Sample results are rejected

U Analyte was analyzed for, but not detected above the MDL, MDA, or LOD.

U1 Gamma Spectroscopy--Uncertain identification

X Consult Case Narrative, Data Summary package, or Project Manager concerning this qualifier 


\section{GENERAL ENGINEERING LABORATORIES, LLC}

2040 Savage Road Charleston SC 29407 - (843) 556-8171 - www.gel.com

\section{Certificate of Analysis}

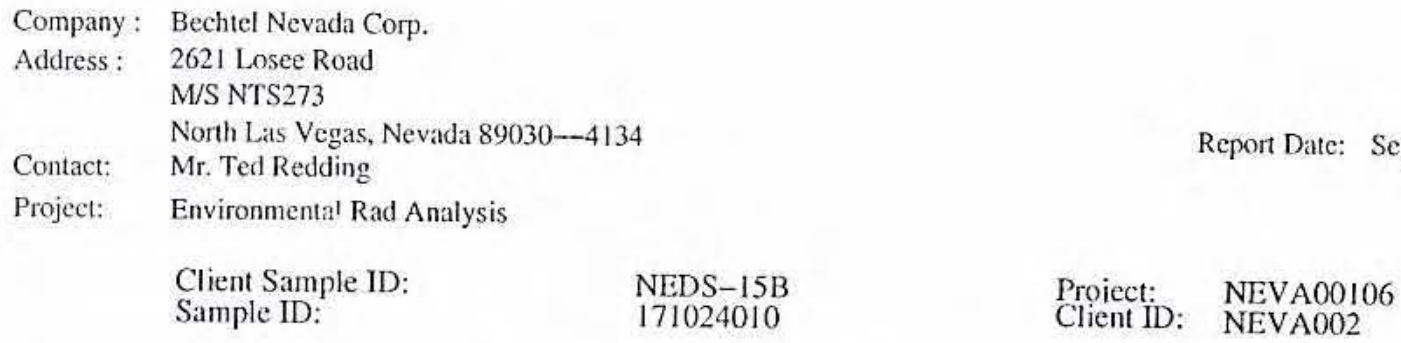

\begin{tabular}{llllllll}
\hline Parameter Qualifier Result Uncertainty & DL & TPU & RL & Units & DF Analyst Date Time Batch Mt
\end{tabular}

Y QC Samples were not spiked with this compound

$\wedge \quad$ RPD of sample and duplicate evaluated using $+/-$ RL. Concentrations are $<5 X$ the $R L$

h Preparation or preservation holding time was exceeded

The above sample is reported on a dry weight basis. 


\section{GENERAL ENGINEERING LABORATORIES, LLC \\ 2040 Savage Road Charleston SC 29407 - (843) 556-8171 - www.gel.com}

\section{Certificate of Analysis}

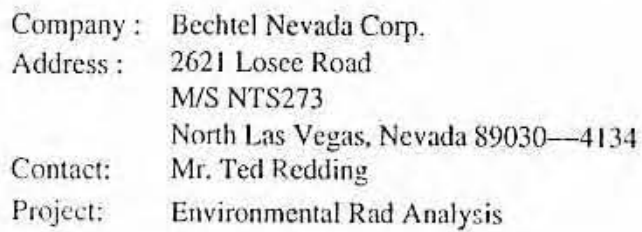

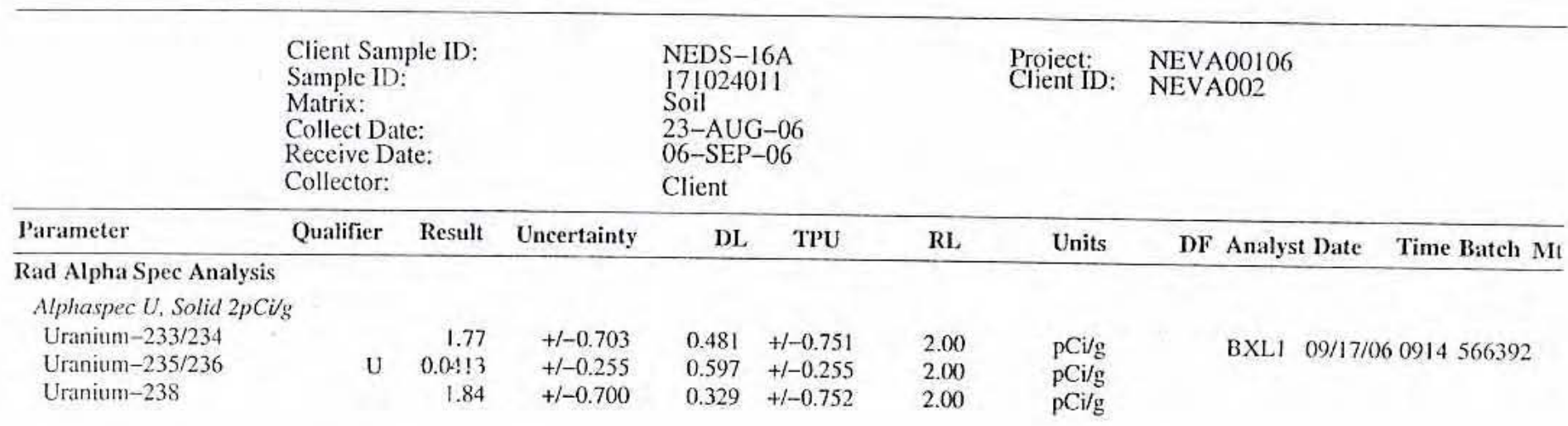

The following Prep Methods were performed

\begin{tabular}{llllll}
\hline Method & Description & Analyst & Date & Time & Prep Batch \\
\hline Dry Soil Prep & Dry Soil Prep GL-RAD-A-021 & LXM2 & $09 / 07 / 06$ & 1709 & 565882
\end{tabular}

The following Analytical Methods were performed

\begin{tabular}{ll} 
Method & Description \\
\hline DOE EML HASL-300, U-02-RC Modified
\end{tabular}

\begin{tabular}{llcc} 
Surrogate/Tracer recovery & Test & Recovery \% & Acceptable Limits \\
\hline Uranium-232 & Alphaspec U, Solid 2pCi/g & 90 & $(25 \%-125 \%)$
\end{tabular}

Notes:

The Quaufiers in this report are defined as follows :

* A quality control analyte recovery is outside of specified acceptance criteria

$<$ Result is less than value reported

$>$ Result is greater than value reported

A The TIC is a suspected aldol-condensation product

B Target analyte was detected in the associated blank

BD Results are either below the MDC or tracer recovery is low

C Analyte has been confirmed by GC/MS analysis

D Results are reported from a diluted aliquot of the sample

$\mathrm{H}$ Analytical holding time was exceeded

$\mathrm{J}$ Value is estimated

N/A Spike recovery limits do not apply. Sample concentration exceeds spike concentration by $4 X$ or more

$\mathrm{R}$ Sample results are rejected

U Analyte was analyzed for, but not detected above the MDL, MDA, or LOD.

UI Gamma Spectroscopy--Uncertain identification 


\section{GENERAL ENGINEERING LABORATORIES, LLC \\ 2040 Savage Road Charleston SC 29407 - (843) 556-8171 - www.gel.com}

\section{$\underline{\text { Certificate of Analysis }}$}

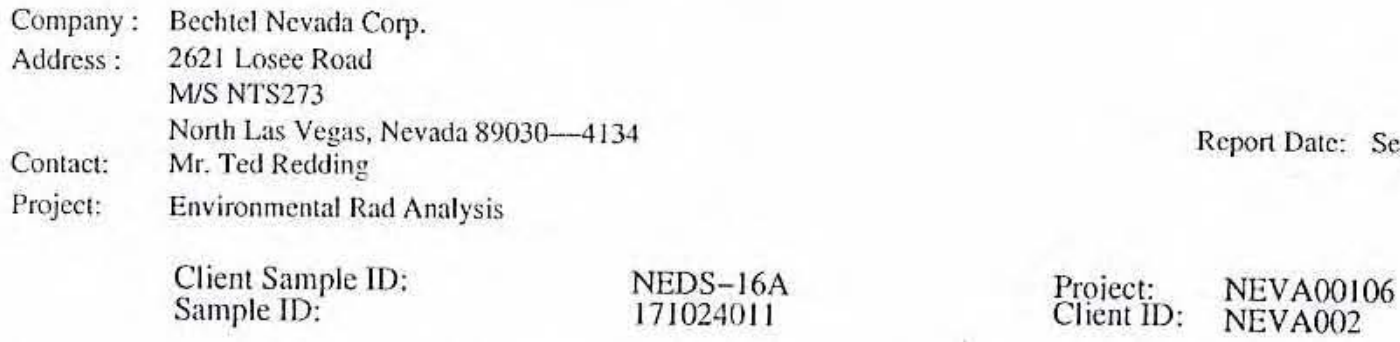

RL.

Units

DF Analyst Date

Time Batch Mt

$X$ Consult Case Narrative, Data Summary package, or Project Manager concerning this qualifier

Y QC Samples were not spiked with this compound

$\wedge$ RPD of sample and duplicate evaluated using $+/-\mathrm{RL}$. Concentrations are $<5 \mathrm{X}$ the RL

h Preparation or preservation holding time $w /$ as exceeded

The above sample is reported or a dry weight basis. 


\section{GENERAL ENGINEERING LABORATORIES, LLC \\ 2040 Savage Road Charleston SC 29407 - (843) 556-8171 - www.gel.com}

\section{Certificate of Analysis}

\begin{tabular}{|c|c|c|c|c|c|c|c|c|c|}
\hline \multirow{4}{*}{$\begin{array}{l}\text { Company: } \\
\text { Address : }\end{array}$} & \multicolumn{9}{|c|}{ Bechtel Nevada Corp. } \\
\hline & \multicolumn{9}{|c|}{2621 Losee Road } \\
\hline & \multicolumn{9}{|c|}{$\mathrm{M} / \mathrm{S}$ NTS273 } \\
\hline & \multicolumn{7}{|c|}{$\begin{array}{l}\text { North Las Vegas, Nevada 89030-4134 } \\
\text { Mr. Ted Redding }\end{array}$} & \multicolumn{2}{|c|}{ Report Date: September 20,2006 } \\
\hline \multirow[t]{4}{*}{ Project: } & \multicolumn{9}{|c|}{ Environmental Rad Analysis } \\
\hline & \multirow{2}{*}{\multicolumn{3}{|c|}{$\begin{array}{l}\text { Client Sample ID: } \\
\text { Sample ID: } \\
\text { Matrix: } \\
\text { Collect Date: } \\
\text { Receive Date: } \\
\text { Collector: }\end{array}$}} & \multirow{2}{*}{\multicolumn{3}{|c|}{$\begin{array}{l}\text { NEDS-16B } \\
171024012 \\
\text { Soil } \\
\text { 23-AUG-06 } \\
\text { 06-SEP-06 } \\
\text { Client }\end{array}$}} & \multirow[t]{2}{*}{$\begin{array}{l}\text { Proiect: } \\
\text { Client ID: }\end{array}$} & \multirow{2}{*}{\multicolumn{2}{|c|}{$\begin{array}{l}\text { NEVA00106 } \\
\text { NEVA002 }\end{array}$}} \\
\hline & & & & & & & & & \\
\hline & Qualifier & Result & Uncertainty & DL & TPU & RL & Units & DF Analyst Date & Time Batch Mt \\
\hline \multicolumn{10}{|c|}{ Spec Analysis } \\
\hline $\begin{array}{l}\text { U. Solid } 2 p C i \\
233 / 234 \\
235 / 236 \\
238\end{array}$ & $\mathrm{U}$ & $\begin{array}{r}1.84 \\
0.215 \\
1.87\end{array}$ & $\begin{array}{l}+/-0.667 \\
+/-0.243 \\
+/-0.665\end{array}$ & $\begin{array}{l}0.372 \\
0.338 \\
0.292\end{array}$ & $\begin{array}{l}+/-0.718 \\
+/-0.245 \\
+/-0.718\end{array}$ & $\begin{array}{l}2.00 \\
2.00 \\
2.00\end{array}$ & $\begin{array}{l}\mathrm{pCi} / \mathrm{g} \\
\mathrm{pCi} / \mathrm{g} \\
\mathrm{pCi} / \mathrm{g}\end{array}$ & \multicolumn{2}{|c|}{ BXLI $09 / 17 / 060914566392 \quad 1$} \\
\hline
\end{tabular}

The following Prep Methods were performed

\begin{tabular}{llllll}
\hline Method & Description & Analyst & Date & Time & Prep Batch \\
\hline Dry Soil Prep & Dry Soil Prep GL-RAD-A-021 & LXM2 & $09 / 07 / 06$ & 1709 & 565882
\end{tabular}

The following Analytical Methods were performed

\begin{tabular}{ll} 
Method & Description \\
\hline 1 & DOE EML HASL-300, U-02-RC Modified
\end{tabular}

\begin{tabular}{llcc} 
Surrogate/Tracer recovery & Test & Recovery \% & Acceptable Limits \\
\hline Uramium-232 & Alphaspec U, Solid 2pCi/g & 97 & $(25 \%-125 \%)$
\end{tabular}

Notes:

The Qualifiers in this report are defined as follows :

* A quality control analyte recovery is outside of specified acceptance criteria

$<$ Result is less than value reported

$>$ Result is greater than value reported

A The TIC is a suspected aldol-condensation product

B Target analyte was detected in the associated blank

$\mathrm{BD}$ Results are either below the MDC or tracer recovery is low

C Analyte has been confirmed by GC/MS analysis

D Results are reported from a diluted aligutot of the sample

$\mathrm{H}$ Analytical holding time was exceeded

$\mathrm{J}$ Value is estimated

N/A Spike recovery limits do not apply. Sample concentration exceeds spike concentration by $4 X$ or more

R Sample results are rejected

U Analyte was analyzed for, but not detected above the MDL, MDA, or LOD.

UI Gamma Spectroscopy-Uncertain identification

X Consult Case Narrative, Data Summary package, or Project Manager concerning this qualifier 


\section{GENERAL ENGINEERING LABORATORIES, LLC}

2040 Savage Road Charleston SC 29407 - (843) 556-8171 - www.gel.com

\section{Certificate of Analysis}

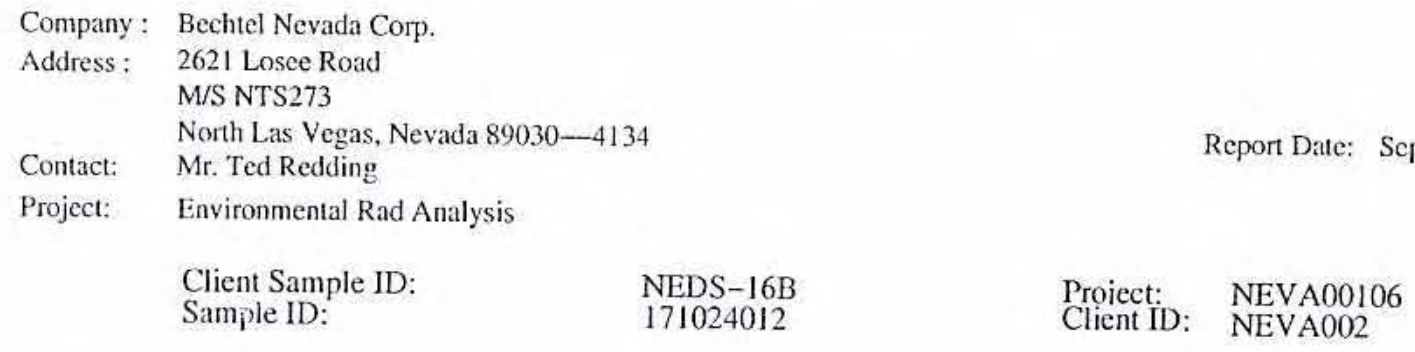

Y QC Samples were not spiked with this compound

$\wedge$ RPD of sample and duplicate evaluated using $+/-$ RL. Concentrations are $<5 \mathrm{X}$ the RL

$\mathrm{h}$ Preparation or preservation holding time was exceeded

The above sample is reported on a dry weight basis. 


\section{GENERAL ENGINEERING LABORATORIES, LLC \\ 2040 Savage Road Charleston SC 29407 - (843) 556-8171 - www.gel.com}

\section{Certificate of Analysis}

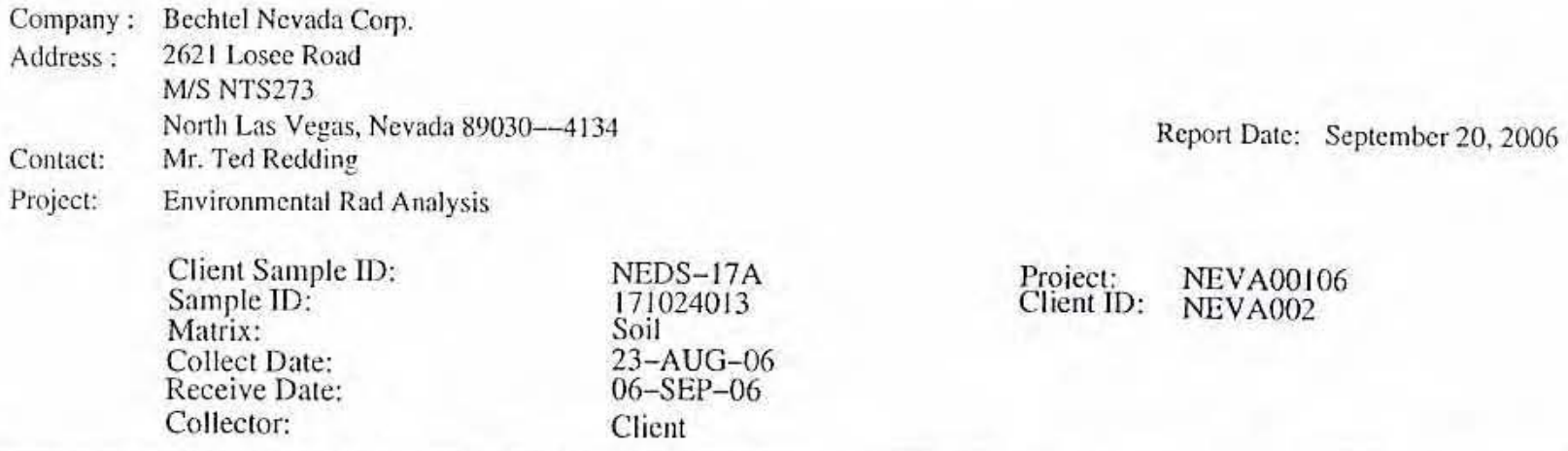

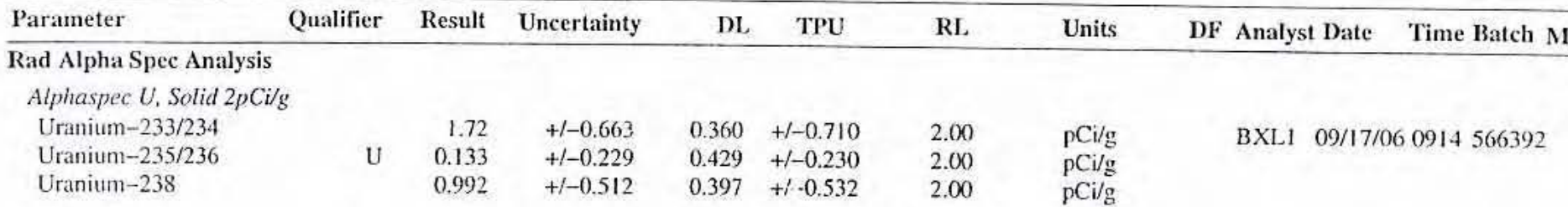

The following Prep Methods were performed

\begin{tabular}{|c|c|c|c|c|c|}
\hline Method & Description & Analyst & Date & Time & Prep Batch \\
\hline Dry Soil Prep & Dry Soil Prep GL-RAD-A-021 & LXM2 & $09 / 07 / 06$ & 1709 & 565882 \\
\hline \multicolumn{6}{|c|}{ The following Analytical Methods were performed } \\
\hline Method & Description & & & & \\
\hline 1 & DOE EML HASL-300, U-02-RC Modified & & & & \\
\hline Surrogate/ $\mathrm{Tr}$ & r recovery & Recovery\% & \multicolumn{2}{|c|}{ Acceptable Limits } & \\
\hline Uranium-232 & Alphaspec U, Solid 2pCi/g & 94 & \multicolumn{2}{|c|}{$(25 \%-125 \%)$} & \\
\hline
\end{tabular}

Notes:

The Qualifiers in this report are defined as follows :

* A quality control analyte recovery is outside of specified acceptance criteria

$<$ Result is less than value reported

$>$ Result is greater than value reported

A The TIC is a suspected aldol-condensation product

B Target analyte was detected in the associated blank

$\mathrm{BD}$ Results are either below the MDC or traces recovery is low

C Analyte has been confirmed by GC/MS analysis

D Results are reported from a diluted aliquot of the sample

$\mathrm{H}$ Analytical holding time was exceeded

J Value is estimated

N/A Spike recovery limits do not apply. Sample concentration exceeds spike concentration by $4 \mathrm{X}$ or more

R Sample results are rejected

U Analyte was analyzed for, but not detected above the MDL, MDA, or LOD.

UI Gamma Spectroscopy--Uncertain identification

$X$ Consult Case Narrative, Data Summary package, or Project Manager concerning this qualifier 


\section{GENERAL ENGINEERING LABORATORIES, LLC \\ 2040 Savage Road Charleston SC 29407 - (843) 556-8171 - www.gel.com}

\section{Certificate of Analysis}

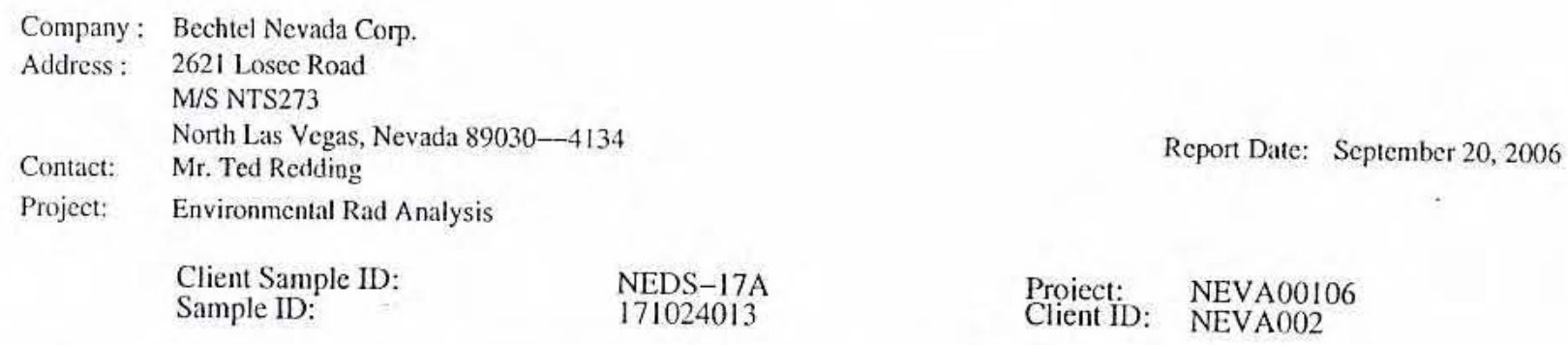

Y QC Samples were not spiked with this compound

$\wedge$ RPD of sample and duplicate evaluated using $+/-\mathrm{RL}$. Concentrations are $<5 \mathrm{X}$ the RL

$h$ Preparation or preservation holding time was exceeded

The above sample is reported on a dry weight basis. 


\title{
GENERAL ENGINEERING LABORATORIES, LLC \\ 2040 Savage Hoad Charleston SC 29407 - (843) 556-8171 - www.gel.com
}

\section{Certificate of Analysis}

\author{
Company: Bechtel Nevada Corp. \\ Address : 2621 Losee Road \\ M/S NTS273 \\ North Las Vegas, Nevada 89030--4134 Report Date: September 20, 2006 \\ Contact: Mr. Ted Redding \\ Project: Environmental Rad Analysis

\begin{tabular}{|c|c|c|c|}
\hline Client Sample ID: & NEDS - 17B & Project: & NEVA00106 \\
\hline Sample ID: & $17 ! 024014$ & Client ID: & NEVA002 \\
\hline $\begin{array}{l}\text { Matrix: } \\
\text { Collect Date: }\end{array}$ & $\begin{array}{l}\text { Soil } \\
23-A U G-06\end{array}$ & & \\
\hline Receive Date: & $06-\mathrm{SEP}-06$ & & \\
\hline Collector: & Client & & \\
\hline
\end{tabular}

\begin{tabular}{|c|c|c|c|c|c|c|c|c|c|c|c|}
\hline Parameter & Qualifier & Result & Uncertainty & $\mathrm{DL}$ & TPU & RL & Units & DF & Analyst Date & Time Batch $\mathrm{M}$ & Mitc \\
\hline \multicolumn{12}{|c|}{ Rad Alpha Spec Analysis } \\
\hline \multicolumn{12}{|c|}{ Alphaspec U. Solid $2 p C i / g$} \\
\hline Uranium-233/234 & & 1.29 & $+1-0.690$ & 0.552 & +-0.721 & 2.00 & $\mathrm{pCi} / \mathrm{g}$ & & BXL1 $09 / 17 /$ & 60914566392 & 1 \\
\hline Uranium-235/236 & $\mathrm{U}$ & -0.0181 & $+1-0.202$ & 0.636 & $+1-0.202$ & 2.00 & $\mathrm{pCi} / \mathrm{g}$ & & & & \\
\hline Uranium-238 & & 1.72 & $+/-0.797$ & 0.596 & $+1-0.844$ & 2.00 & $\mathrm{pCi} / \mathrm{g}$ & & & & \\
\hline
\end{tabular}

The following Prep Methods were performed

\begin{tabular}{llllll}
\hline Method & Description & Analyst & Date & Time & Prep Batch \\
\hline Dry Soil Prep $\quad$ Dry Soil Prep GL-RAD-A-021 & LXM2 & $09 / 07 / 06$ & 1709 & 565882 \\
The following Analytical Methods were performed & & & \\
\hline Method & Description & & & \\
\hline 1 & DOE EML HASL-300, U-02-RC Modified
\end{tabular}

\begin{tabular}{llcc} 
Surrogate/Tracer recovery & Test & Recovery \% & Acceptable Limits \\
\hline Uranium-232 & Alphaspec U, Solid 2pCi/g & 69 & $(25 \%-125 \%)$
\end{tabular}

Notes:

The Qualifiers in this report are defined as follows :

* A quality control analyte recovery is outside of specified acceptance criteria

$<$ Result is less than value reported

$>$ Result is greater than value reported

A The TIC is a suspected aldol-condensation product

B Target analyte was detected in the associated blank

BD Results are either below the MDC or tracer recovery is low

C Analyte has been confirmed by GC/MS analysis

D Results are reported from a diluted aliquot of the sample

$\mathrm{H}$ Analytical holding time was exceeded

$\mathrm{J}$ Value is estimated

N/A Spike recovery limits do not apply. Sample concentration exceeds spike concentration by $4 \mathrm{X}$ or more

$\mathrm{R}$ Sample results are rejected

$\mathrm{U}$ Analyte was analyzed for, but not detected above the MDL, MDA, or LOD.

UI Gamma Spectroscopy-Uncertain identification

$\mathrm{X}$ Consult Case Narrative, Data Summary package, or Project Manager concerning this qualifier 


\section{GENERAL ENGINEERING LABORATORIES, LLC \\ 2040 Savage Road Charleston SC 29407 - (843) 556-8171 - www.gel.com}

\section{Certificate of Analysis}

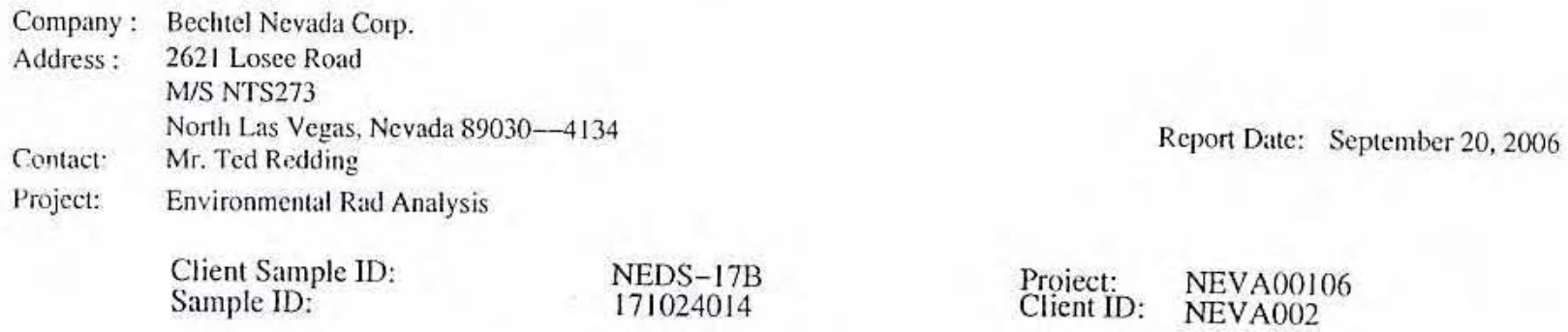

DL TPU RL

Y QC Samples were not spiked with this compound

$\hat{n}$ RPD of sample and duplicate evaluated using $+/-$ RL. Concentrations are $\angle 5 X$ the RL

$\mathrm{h}$ Preparation or preservation holding time was exceeded

The above sample is reported on a dry weight basis. 


\section{GENERAL ENGINEERING LABORATORIES, LLC \\ 2040 Savage Road Charleston SC 29407 - (843) 556-8171 - www.gel.com}

\section{Certificate of Analysis}

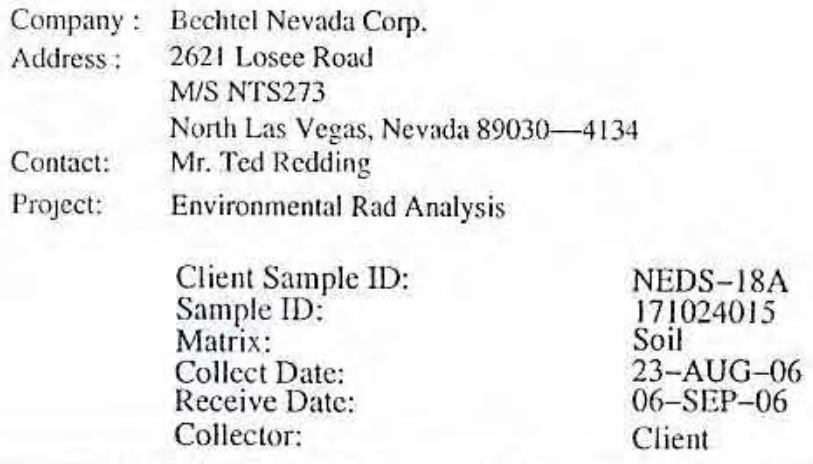

\begin{tabular}{|c|c|c|c|c|c|c|c|c|c|c|}
\hline Parameter & Qualifier & Result & Uncertainty & DL & TPU & RL & Units & DF & Analyst Date & Time Batch Mt: \\
\hline \multicolumn{11}{|c|}{ Rad Alpha Spec Analysis } \\
\hline \multicolumn{11}{|c|}{ Alphaspec U. Solid $2 p \mathrm{Ci}_{\mathrm{g}}$} \\
\hline $\begin{array}{l}\text { Uránium-233/234 } \\
\text { Uranium-235/236 } \\
\text { Uranium-238 }\end{array}$ & $\mathrm{U}$ & $\begin{array}{r}1.53 \\
-0.0334 \\
1.41\end{array}$ & $\begin{array}{r}+l-0.656 \\
+l-0.0463 \\
+l-0.626\end{array}$ & $\begin{array}{l}0.458 \\
0.386 \\
0.424\end{array}$ & $\begin{array}{r}+1-0.701 \\
+1-0.0467 \\
+1-0.666\end{array}$ & $\begin{array}{l}2.00 \\
2.00 \\
2.00\end{array}$ & $\begin{array}{l}\mathrm{pCi} / \mathrm{g} \\
\mathrm{pCi} / \mathrm{g} \\
\mathrm{pCi} / \mathrm{g}\end{array}$ & & BXLI $09 / 17 / 0$ & 60914566392 \\
\hline
\end{tabular}

The following Prep Methods were performed

\begin{tabular}{|c|c|c|c|c|c|}
\hline Method & Description & Analyst & Date & Time & Prep Batch \\
\hline Dry Soil Prep & Dry Soil Prep GL-RAD-A-021 & LXM2 & $09 / 07 / 06$ & 1709 & 565882 \\
\hline \multicolumn{6}{|c|}{ The following Analytical Methods were perlormed } \\
\hline Method & Description & & & & \\
\hline 1 & DOE EML HASL-300, U-02-RC Modified & & & & \\
\hline Surrogate/Tr & recovery & Recovery\% & \multicolumn{2}{|c|}{ Acceptable Limits } & \\
\hline Uranium-232 & Alphaspec U, Solid $2 \mathrm{pCi} / \mathrm{g}$ & 86 & \multicolumn{2}{|c|}{$(25 \%-125 \%)$} & \\
\hline
\end{tabular}

Notes:

The Qualifiers in this report are defined as follows :

* A quality control analyte recovery is outside of specified acceptance criteria

$<$ Result is less than value reported

$>$ Result is greater than value reported

A The TIC is a suspected aldol-condensation product

B Target analyte was detected in the associated blank

BD Results are either below the MDC or tracer recovery is low

C Analyte has been confirmed by GC/MS analysis

D Results are reported from a diluted aliquot of the sample

$\mathrm{H}$ Analytical holding time was exceeded

$\mathrm{J}$ Value is estimated

N/A Spike recovery limits do not apply. Sample concentration exceeds spike concentration by $4 X$ or more

R Sample results are rejected

U Analyte was analyzed for, but not detected above the MDL, MDA, or LOD.

UI Gamma Spectroscopy-Uncertain identification

X Consult Case Narrative, Data Summary package, or Project Manager concerning this qualifier 


\section{GENERAL ENGINEERING LABORATORIES, LLC \\ 2040 Savage Road Charleston SC 20407 - (843) 556-8171 - www.gel.com}

\section{Certificate of Analysis}

Company: Bechtel Nevada Corp.

Address: 2621 Losee Road

M/S NTS273

North Las Vegas, Nevada 89030--4134

Contact: $\quad$ Mr. Ted Redding

Project: Environmental Rad Analysis

Client Sample ID: $\quad$ NEDS-18A $\quad$ Project: NEVA00106

Sample ID: 171024015 Client ID: NEVA002

$\begin{array}{lllllll}\text { Parameter Qualifier Result Uncertainty } & \text { DL } & \text { TPU } & \text { RL } & \text { Units DF Analyst Date Time Batch Mt }\end{array}$

Y QC Samples were not spiked with this compound

$\wedge$ RPD of sample and duplicate evaluated using $+1-$ RL. Concentrations are $<5 X$ the $R L$

$\mathrm{h}$ Preparation or preservation holding time was exceeded

The above sample is reported on a dry weight basis. 


\section{GENERAL ENGINEERING LABORATORIES, LLC \\ 2040 Savage Road Charleston SC 29407 (843) 556-8171 - www.gel.com}

\section{Certificate of Analysis}

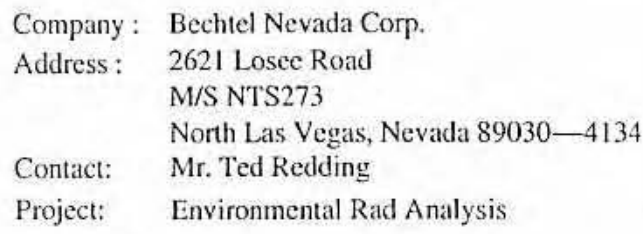

$\begin{array}{llll}\text { Client Sample ID: } & \text { NEDS-18B } & \text { Proiect: } & \text { NEVA00106 } \\ \text { Sample ID: } & 171024016 & \text { Client ID: } & \text { NEVA002 } \\ \text { Matrix: } & \text { Soil } & \\ \text { Collect Date: } & 23-A U G-06 & \\ \text { Receive Date: } & 06-S E P-06 & \\ \text { Collector: } & \text { Client } & \end{array}$

\begin{tabular}{|c|c|c|c|c|c|c|c|c|c|c|}
\hline Parameter & Qualifier & Result & Uncertainty & $\mathrm{DL}$ & TPU & RL & Units & DF & Analyst Date & Time Batch Mt \\
\hline \multicolumn{11}{|c|}{ Rad Alpha Spec Analysis } \\
\hline \multicolumn{11}{|c|}{ Alphaspec U. Solid $2 p C i / g$} \\
\hline Uranium-233/234 & & 1.46 & $+1-0.625$ & 0.436 & $+1-0.667$ & 2.00 & $\mathrm{pCi} / \mathrm{g}$ & & BXLI $09 / 17 /$ & 060914566392 \\
\hline Uranium-235/236 & $\mathrm{U}$ & 0.154 & $+1-0.273$ & 0.514 & $+1-0.274$ & 2.00 & $\mathrm{pCi} / \mathrm{g}$ & & & \\
\hline Uranium-238 & & 2.02 & $+1-0.737$ & 0.489 & $+1-0.805$ & 2.00 & $\mathrm{pCi} / \mathrm{g}$ & & & \\
\hline
\end{tabular}

The following Prep Methods were performed

\begin{tabular}{llllll}
\hline Method & Description & Analyst & Date & Time & Prep Batch \\
\hline Dry Soil Prep & Dry Soil Prep GL-RAD-A-021 & LXM2 & $09 / 07 / 06$ & 1709 & 565882
\end{tabular}

The following Analytical Methods were performed

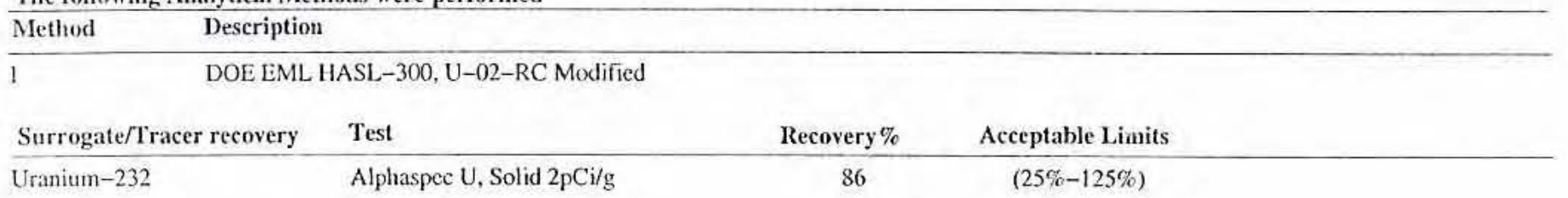

Notes:

The Qualifiers in this report are defined as follows :

* A quality control analyte recovery is outside of specified acceptance criteria

$<$ Result is less than value reported

$>$ Result is greater than value reported

A The TIC is a suspected aldol-condensation product

$B$ Target analyte was detected in the associated blank

BD Results are either below the MDC or tracer recovery is low

C Analyte has been confirmed by GC/MS analysis

D Results are reported from a diluted aliquot of the sample

$\mathrm{H}$ Analytical holding time was exceeded

$\mathrm{J}$ Value is estimated

N/A Spike recovery limits do not apply. Sample concentration exceeds spike concentration by $4 X$ or more

$\mathrm{R} \quad$ Sample results are rejected

$\mathrm{U}$ Analyte was analyzed for, but not detected above the MDL, MDA, or LOD.

UI Gamma Spectroscopy--Uncertain identification

$\mathrm{X}$ Consult Case Narrative, Data Summary package, or Project Manager concerning this qualifier 


\section{GENERAL ENGINEERING LABORATORIES, LLC}

2040 Savage Road Charleston SC 29407 - (843) 556-8171 .. www.gel.com

\section{Certificate of Analysis}

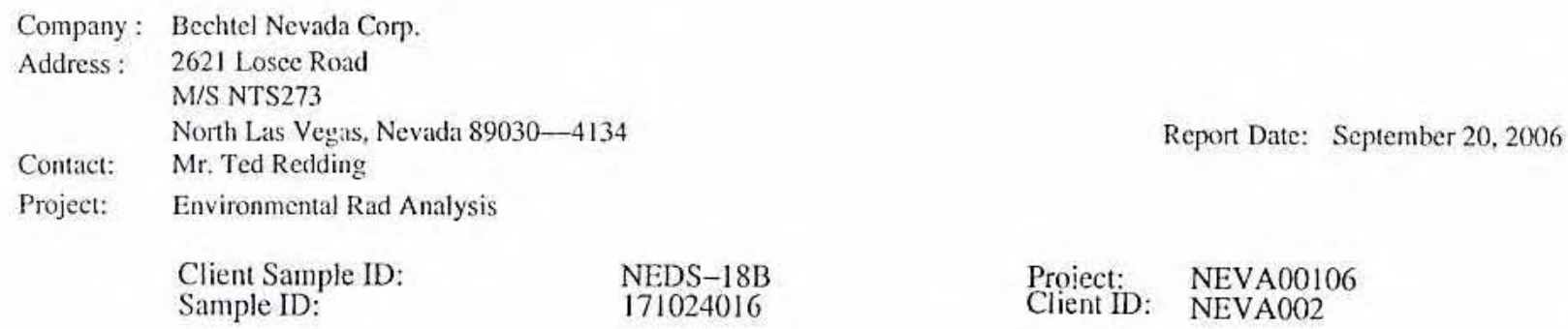

RL.

Units

Y QC Samples were not spiked with this compound

$\wedge$ RPD of sample and duplicate evaluated using $+/-$ RL. Concentrations are $<5 X$ the RL

h Preparation or preservation holding time was exceeded

The above sample is reported on a dry weight basis. 


\section{GENERAL ENGINEERING LABORATORIES, LLC}

2040 Savage Road Charleston SC 29407 - (843) 556-8171 - www.gel.com

\section{Certificate of Analysis}

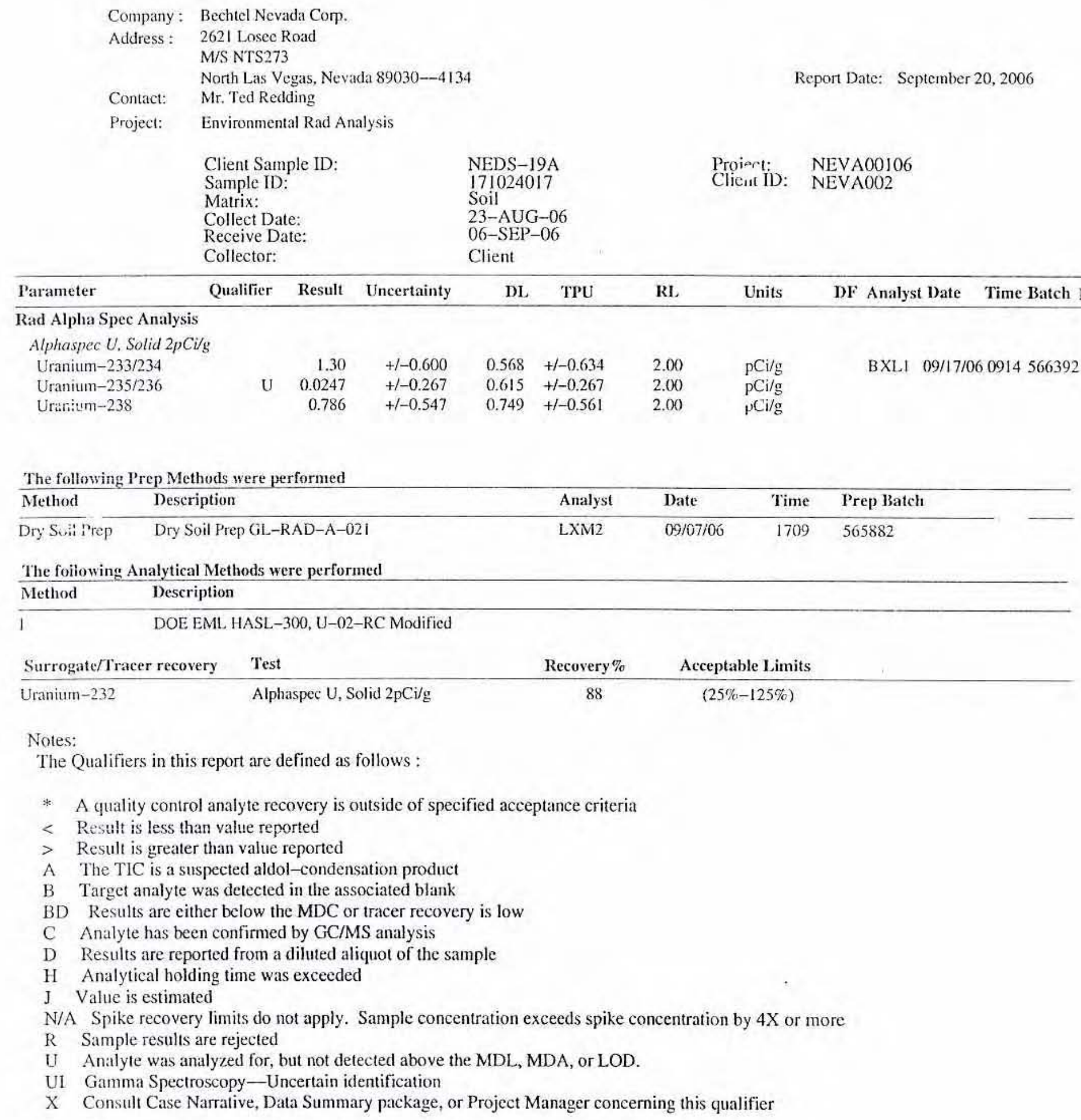




\section{GENERAL ENGINEERING LABORATORIES, LLC}

2040 Savage Road Charleston SC 29407 - (843) 556-8171 - www.gei.cum

\section{Certificate of Analysis}

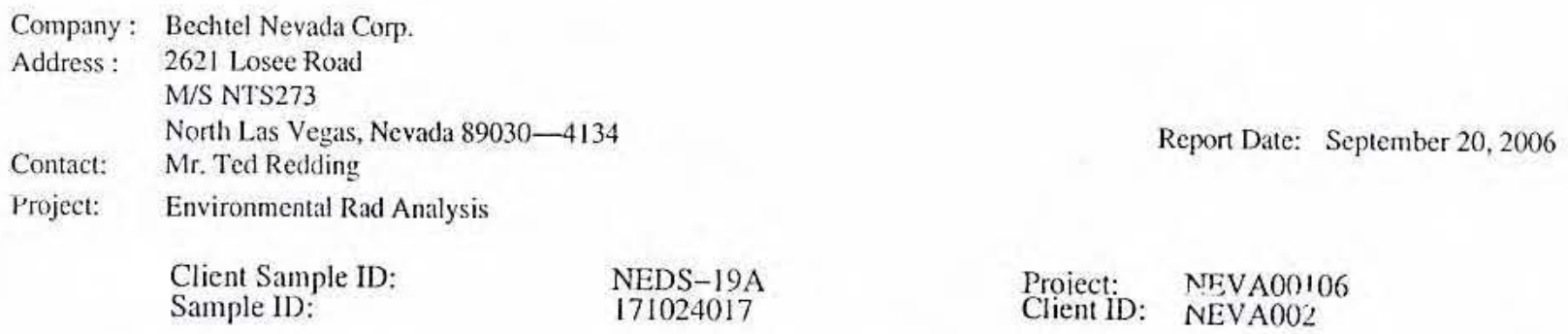

Y QC Samples were not spiked with this compound

$\wedge$ RPD of sample and duplicate evaluated using $+/-R L$. Concentrations are $<5 X$ the $R L$

$\mathrm{h}$ Preparation or preservation holding time was exceeded

The above sample is reported on a dry weight basis. 


\section{GENERAL ENGINEERING LABORATORIES, LIC}

2040 Savage Road Charleston SC 29407 - (843) 556-8171 - www.gel.com

\section{Certificate of Analysis}

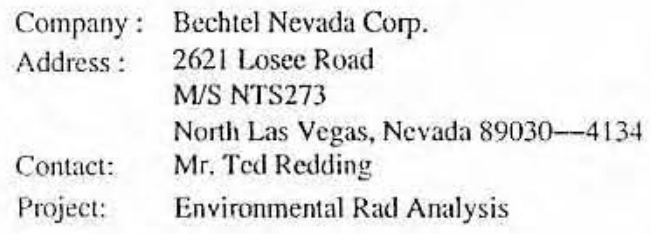

Client Sample ID:

Sample ID:

NEDS $-19 B$

171024018

Soil $23-A U G-06$

Matrix:

Receive Date: $\quad 06-S E P-06$

Collector:

Client

\begin{tabular}{|c|c|c|c|c|c|c|c|c|c|c|}
\hline Parameter & Qualifier & Result & Uncertainty & DL & TPU & RL & Units & DF & Analyst Date & Time Batch Mt \\
\hline \multicolumn{11}{|c|}{ Rad Alpha Spec Analysis } \\
\hline \multicolumn{11}{|c|}{ Alphaspec U, Solid $2 p \mathrm{Ci} g$} \\
\hline Uraniuft1-233/234 & & 1.99 & $+1-0.843$ & 0.612 & $+1-0.909$ & 2.00 & $\mathrm{pCi} / \mathrm{g}$ & \multirow{3}{*}{\multicolumn{3}{|c|}{ BXLI $09 / 17 / 060914566392$}} \\
\hline Uranium-235/236 & $\mathrm{U}$ & 0.112 & $+1-0.253$ & 0.535 & $+1-0.254$ & 2.00 & $\mathrm{pCi} / \mathrm{g}$ & & & \\
\hline Uranium-238 & & 1.96 & $+1-0.822$ & 0.483 & $+1-0.889$ & 2.00 & $\mathrm{pCi} / \mathrm{g}$ & & & \\
\hline
\end{tabular}

The following Prep Methods were performed

\begin{tabular}{llllll}
\hline Method & Description & Analyst & Date & Time & Prep Batch \\
\hline Dry Soil Prep & Dry Soil Prep GL-RAD-A-021 & LXM2 & $09 / 07 / 06$ & 1709 & 565882
\end{tabular}

The following Analytical Methods were performed

\begin{tabular}{ll} 
Method & Description \\
\hline & DOE EML HASL-300, U-02-RC Modified
\end{tabular}

\begin{tabular}{llcc} 
Surrogate/Tracer recovery & Test & Recovery\% & Acceptable Limits \\
\hline Uranium-232 & Alphaspec U, Solid 2pCi/g & 69 & $(25 \%-125 \%)$
\end{tabular}

Notes:

The Qualifiers in this report are defined as follows :

* A quality control analyte recovery is outside of specified acceptance criteria

< Result is less than value reported

$>$ Result is greater than value reported

A The TIC is a suspected aldol-condensation product

B Target analyte was detected in the associated blank

BD Results are either below the MDC or tracer recovery is low

C Analyte has been confirmed by GC/MS analysis

D Results are reported from a diluted aliquot of the sample

H Analytical holding time was exceeded

$\mathrm{J}$ Value is estimated

N/A Spike recovery limits do not apply. Sample concentration exceeds spike concentration by $4 \mathrm{X}$ or more

$\mathrm{R}$ Sample results are rejected

U Analyte was analyzed for, but not detected above the MDL, MDA, or LOD.

UI Gamma Spectroscopy-Uncertain identification

X Consult Case Narrative, Data Summary package, or Project Manager concerning this qualifier 


\section{GENERAL ENGINEERING LABORATORIES, LLC}

2040 Savage Road Charleston SC 29407 - (843) 556-8171 - www.gel.com

\section{Certificate of Analysis}

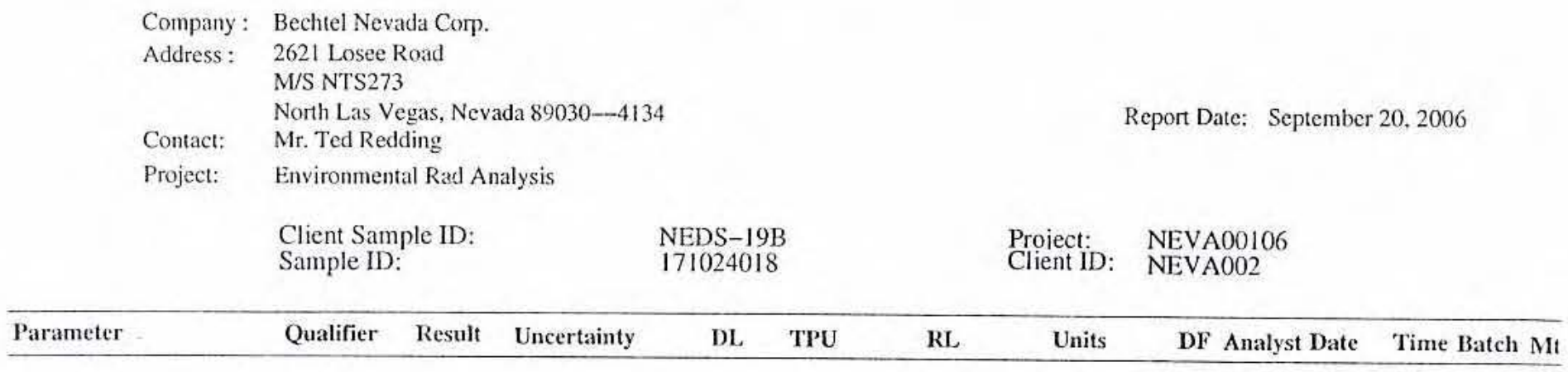

Y QC Samples were not spiked with this compound

$\wedge$ RPD of sampie and duplicate evaluated using $+/-\mathrm{RL}$. Concentrations are $\angle 5 \mathrm{X}$ the $\mathrm{RL}$

h Preparation or preservation holding time was exceeded

The above sample is reported on a dry weight basis. 


\section{GENERAL ENGINEERING LABORATORIES, LLC \\ 2040 Savage Road Charleston SC 29407 - (843) 556-8171 - www.gel.com}

\section{Certificate of Analysis}

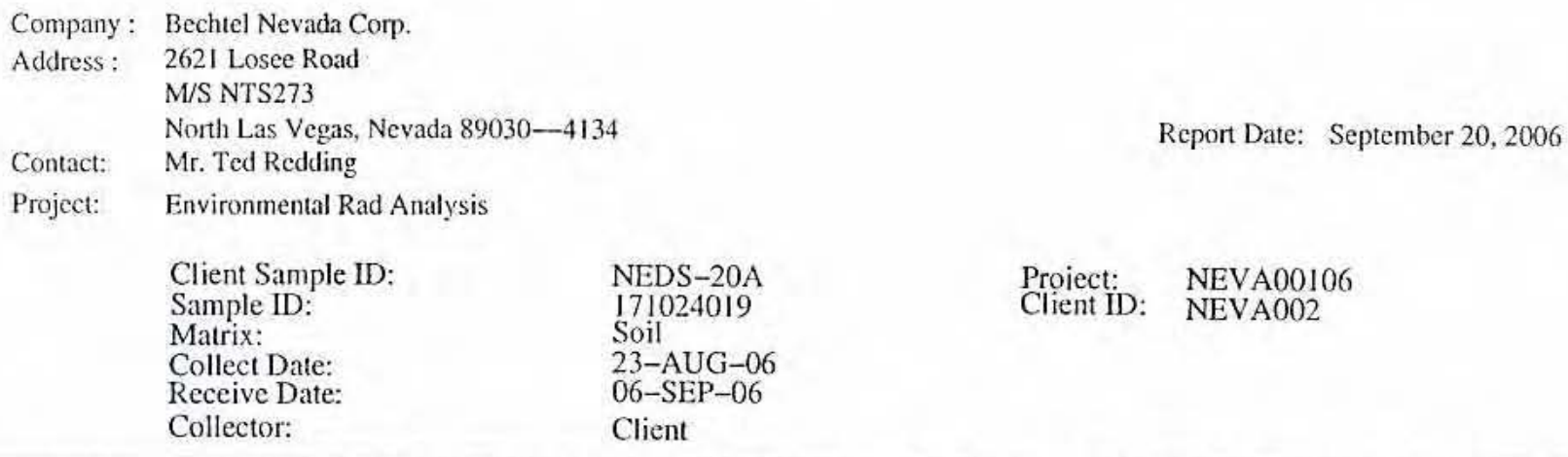

\begin{tabular}{|c|c|c|c|c|c|c|c|c|c|c|}
\hline Parameter & Qualifier & Result & Uncertainty & $\mathrm{DL}$ & TPU & RL & Units & DF & Analyst Date & Time Batch Mt \\
\hline \multicolumn{11}{|c|}{ Rad Alpha Spec Analysis } \\
\hline \multicolumn{11}{|c|}{ Alphaspec U. Solid $2 \mathrm{pCi} / \mathrm{g}$} \\
\hline Uranium-233/234 & & 1.65 & $+1-0.623$ & 0.427 & $+1-0.674$ & 2.00 & $\mathrm{pCi} / \mathrm{g}$ & & BXLI $09 / 17 / C$ & 60914566392 \\
\hline Uranium-235/236 & U & 0.250 & $+1-0.287$ & 0.448 & $+1-0.290$ & 2.00 & $\mathrm{pCi} / \mathrm{g}$ & & & \\
\hline Uranium-238 & & 1.33 & $+1-0.557$ & 0.380 & $+1-0.594$ & 2.00 & $\mathrm{pCi} / \mathrm{g}$ & & & \\
\hline
\end{tabular}

The following Prep Methods were performed

\begin{tabular}{llllll}
\hline Method & Description & Analyst & Date & Time & Prep Batch \\
\hline Dry Soil Prep & Dry Soil Prep GL-RAD- - -02i & LXM2 & $099 / 07 / 06$ & 1709 & 565882
\end{tabular}

The following Analytical Methods were performed

\begin{tabular}{ll} 
Method & Description \\
\hline & DOE EML HASL-300, U-02-RC Modified
\end{tabular}

\begin{tabular}{llcc} 
Surrogate/Tracer recovery & Test & Recovery \% & Acceptable Limits \\
\hline Uranium-232 & Alphaspec U, Solid 2pCi/g & 92 & $(25 \%-125 \%)$
\end{tabular}

Notes:

The Qualifiers in this report are defined as follows :

* A quality control analyte recovery is outside of specified acceptance criteria

$<$ Result is less than value reported

$>$ Result is greater than value reported

A The TIC is a suspected aldol-condensation product

B Target analyte was detected in the associated blank

$\mathrm{BD}$ Results are either below the MDC or tracer recovery is low

C Analyte has been confirmed by GC/MS analysis

D Results are reported from a diluted aliquot of the sample

$\mathrm{H}$ Analytical holding time was exceeded

$\mathrm{J}$ Value is estimated

N/A Spike recovery limits do not apply. Sample concentration exceeds spike concentration by $4 X$ or more

R Sample results are rejected

U Analyte was analyzed for, but not detected above the MDL, MDA, or LOD.

UI Gamma Spectroscopy--Uncertain identification

X Consult Case Narrative, Data Summary package, or Project Manager concerning this qualifier 


\section{GENERAL ENGINEERING LABORATORIES, LLC}

2040 Savage Road Charleston SC 29407 - (843) 556-8171 - www.gel.com

\section{Certificate of Analysis}

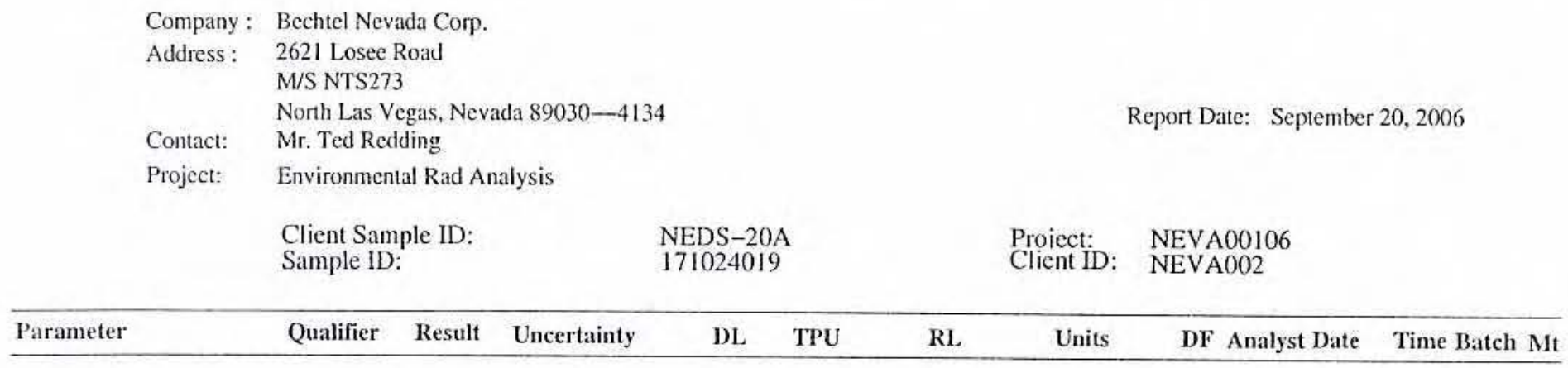

Y QC Samples were not spiked with this compound

$\wedge$ RPD of sample and duplicate evaluated using $+/-$ RL. Concentrations are $<5 X$ the RL

$h$ Preparation or preservation holding time was exceeded

The above sample is reported on a dry weight hasis. 


\section{GENERAL ENGINEERING LABORATORIES, LLC \\ 2040 Savage Road Charleston SC 29407 - (843) 556-8171 - www.gel.com}

\section{Certificate of Analysis}

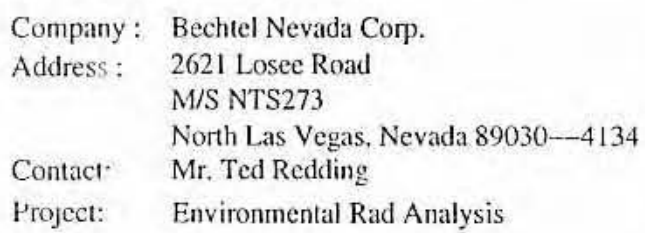

Report Date: September 20, 2006

NEDS-20B
171024020
Soil
$23-A U G-06$
06-SEP-06
Client

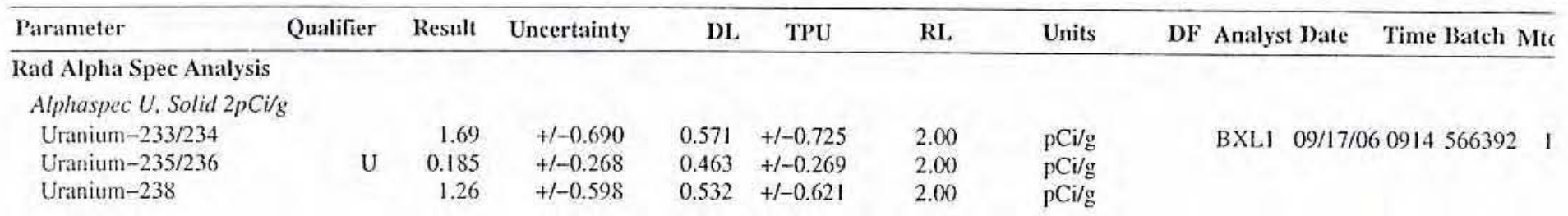

The following Prep Methods were performed

\begin{tabular}{llllll}
\hline Method & Description & Analyst & Date & Time & Prep Batch \\
\hline Dry Soil Prep & Dry Soil Prep GL-RAD-A-02i & LXM2 & $09 / 07 / 06$ & 1709 & 565882
\end{tabular}

The following Analytical Methods sere performed

\begin{tabular}{llcc}
\hline Method Description & & \\
\hline 1 & DOE EML HASL-300, U-02-RC Modified & \\
Surrogate/Tracer recovery & Test & Recovery \% & Acceptable Limits \\
\hline Uranium-232 & Alphaspec U, Solid $2 \mathrm{pCi} / \mathrm{g}$ & 80 & $(25 \%-125 \%)$
\end{tabular}

Notes:

The Qualifiers in this report are defined as follows :

* A quality control analyte recovery is outside of specified acceptance criteria

$<$ Result is less than value reported

$>$ Result is greater than value reported

A The TIC is a suspected aldol-condensation product

B Target analyte was detected in the associated blank

$\mathrm{BD}$ Results are either below the MDC or tracer recovery is low

C Analyte has been confirmed by GC/MS analysis

D Results are reported from a diluted aliquot of the sample

$\mathrm{H}$ Analytical holding time was exceeded

$\mathrm{J}$ Value is estimated

N/A Spike recovery limits do not apply. Sample concentration exceeds spike concentration by $4 X$ or more

$\mathrm{R}$ Sample results are rejected

U Analyte was analyzed for, but not detected above the MDL, MDA, or LOD.

UI Gamma Spectroscopy--Uncertain identification

X Consult Case Narrative, Data Summary package, or Project Manager concerning this qualifier 


\section{GENERAL ENGINEERING LABORATORIES, LLC \\ 2040 Savagge Road Charleston SC 29407 - (843) 556-8171 - www.gel.com}

\section{Certificate of Analysis}

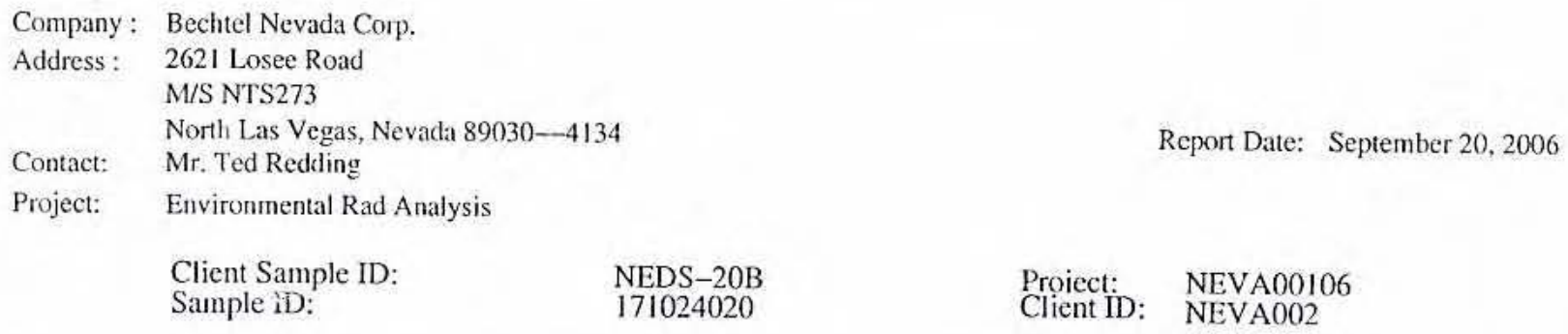

DL TPU RL

RL. Units

DF Analyst Date Time Batch Mtc

Y QC Samples were not spiked with this compound

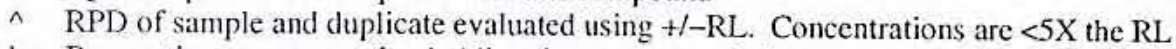

$\mathrm{h}$ Preparation or preservation holding time was exceeded

The above sample is reported on a dry weight basis. 


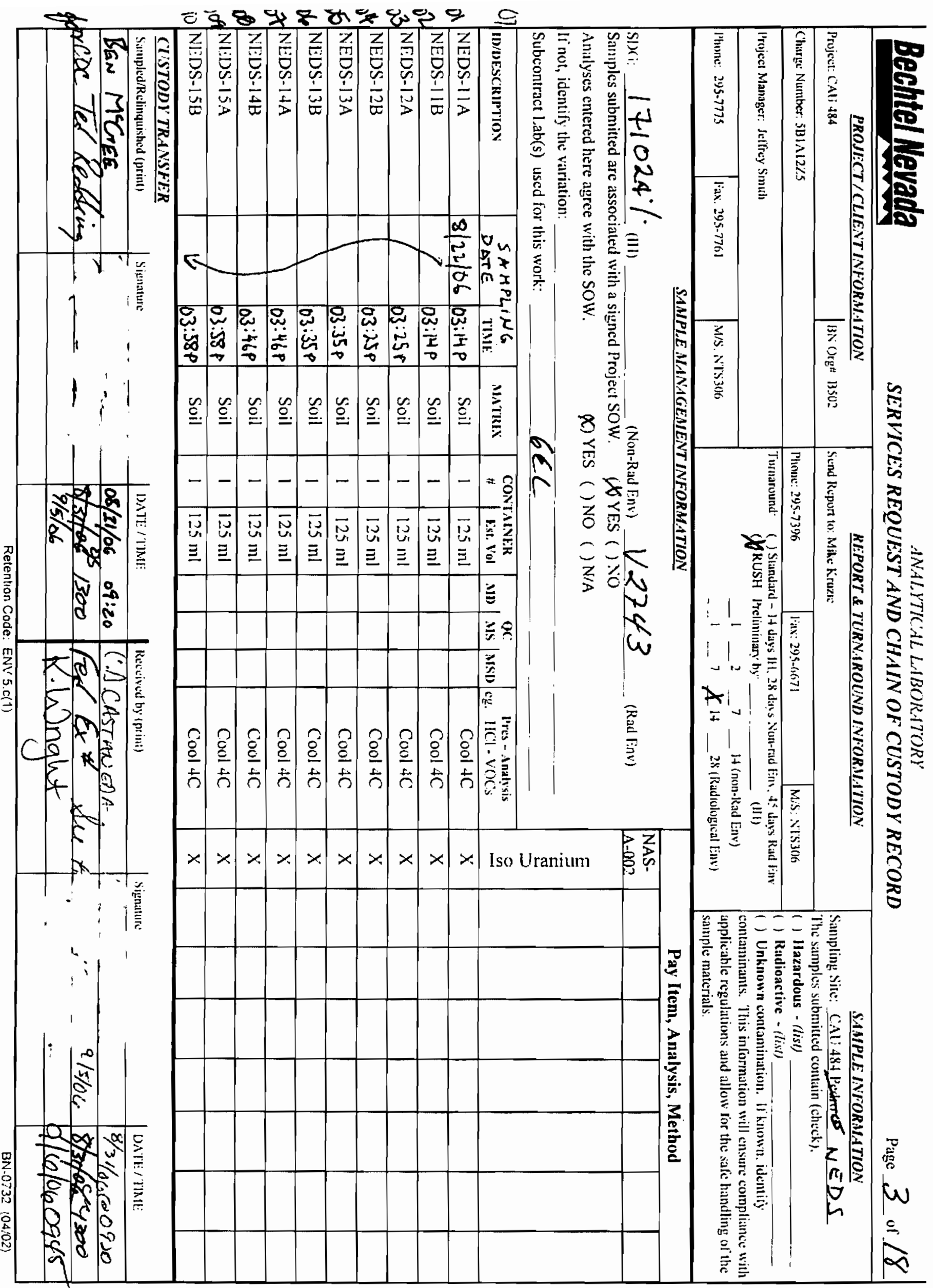




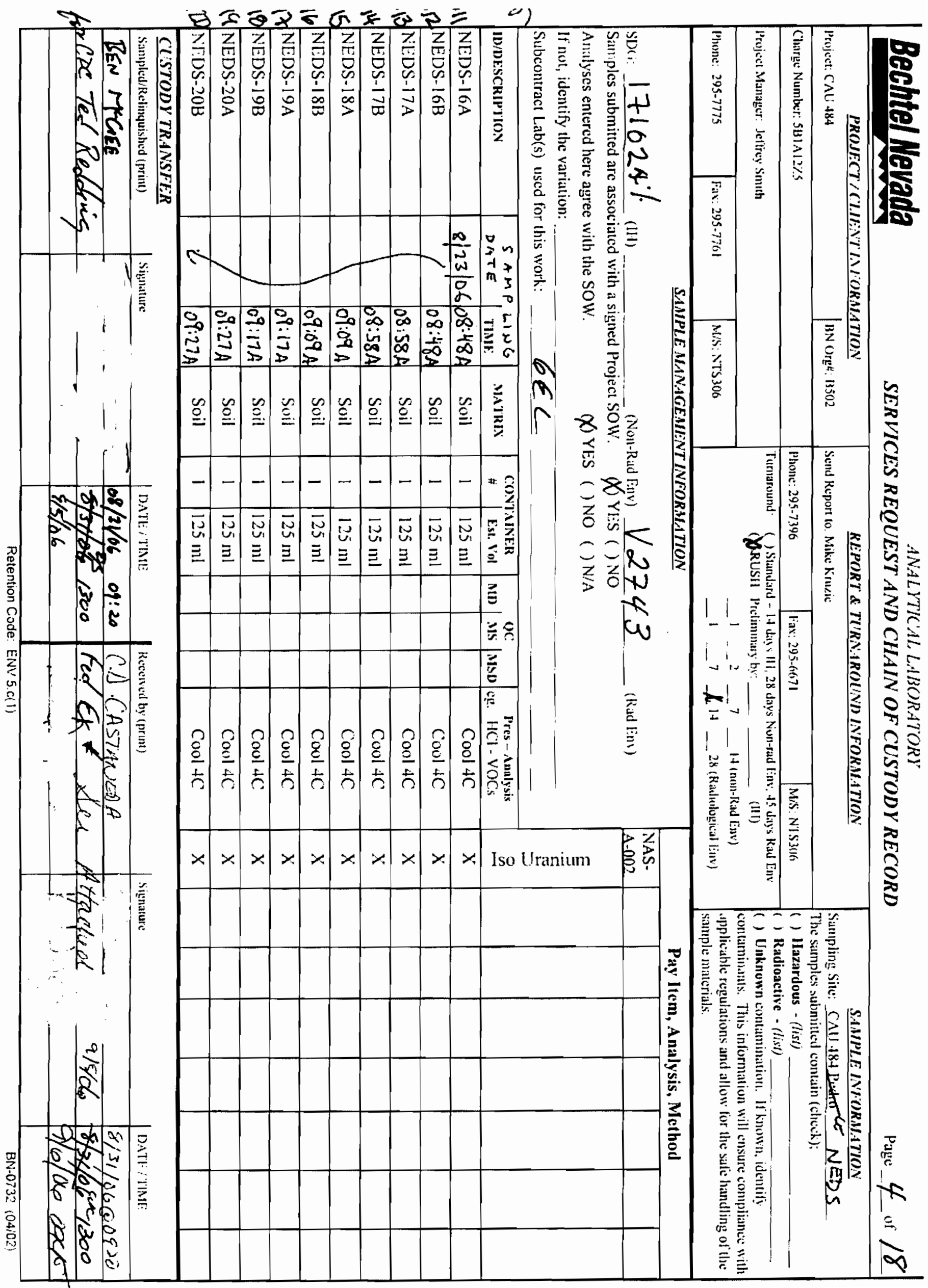




\section{GENERAL ENGINEERING LABORATORIES, LLC}

2040 Savage Road Charleston SC 29407 - (843) 556-8171 - www.gel.com

\section{Certificate of Analysis}

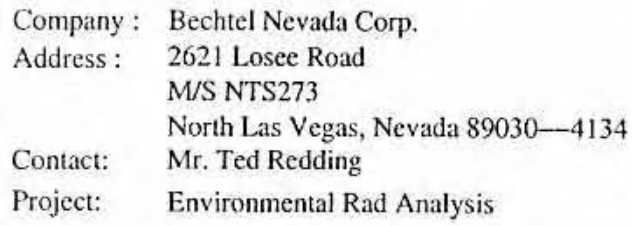

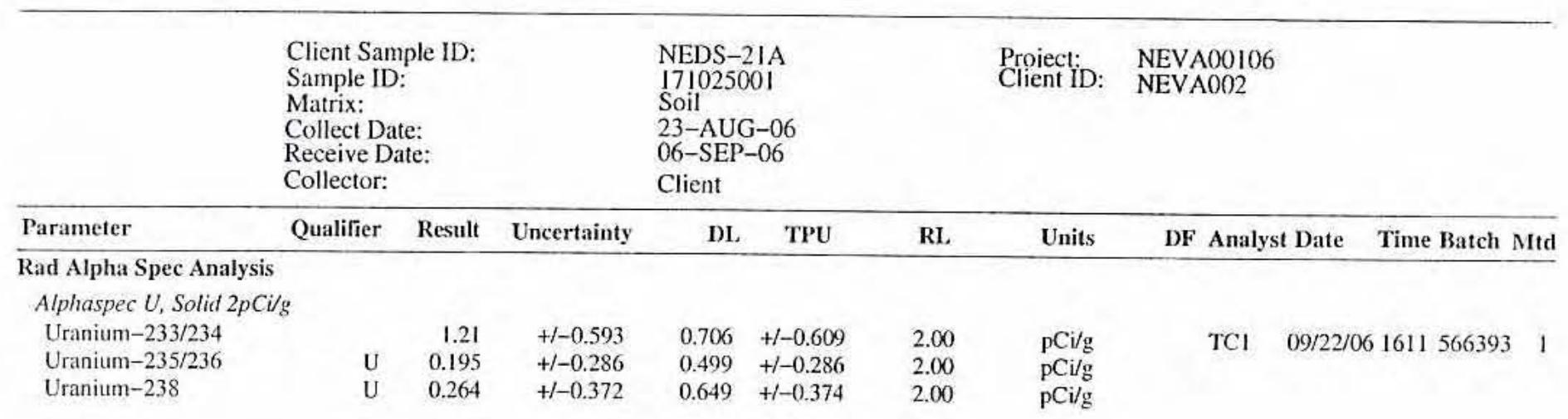

The following Prep Methods were performed

\begin{tabular}{llllll}
\hline Method & Description & Analyst & Date & Time & Prep Batch \\
\hline Dry Soil Prep & Dry Soil Prep GL-RAD-A-021 & AXP2 & $09 / 07 / 06$ & 1306 & 565885
\end{tabular}

The following Analytical Methods were performed

\begin{tabular}{ll}
\hline Method & Description \\
\hline 1 & DOE EML HASL-300, U-02-RC Modified \\
2 & DOE EML HASL-300, U-02-RC Modified
\end{tabular}

\begin{tabular}{llcc} 
Surrogate/Tracer recovery & Test & Recovery\% & Acceptable Limits \\
\hline Uranium-232 & Alphaspec U, Solid 2pCi/g & 89 & $(25 \%-125 \%)$
\end{tabular}

Notes:

The Qualifiers in this report are defined as follows :

* A quality control analyte recovery is outside of specified acceptance criteria

$<$ Result is less than value reported

$>$ Result is greater than value reported

A The TIC is a suspected aldol-condensation product

B Target analyte was detected in the associated blank

BD Results are either below the MDC or tracer recovery is low

C Analyte has been confirmed by GC/MS analysis

D Results are reported from a diluted aliquot of the sample

$\mathrm{H}$ Analytical holding time was exceeded

$\mathrm{J}$ Value is estimated

N/A Spike recovery limits do not apply. Sample concentration exceeds spike concentration by $4 \mathrm{X}$ or more

$\mathrm{R}$ Sample results are rejected

U Analyte was analyzed for, but not detected above the MDL, MDA, or LOD. 


\section{GENERAL ENGINEERING LABORATORIES, LLC \\ 2040 Savage Road Charleston SC 29407 - (843) 556-8171 - www.gel.com}

\section{Certificate of Analysis}

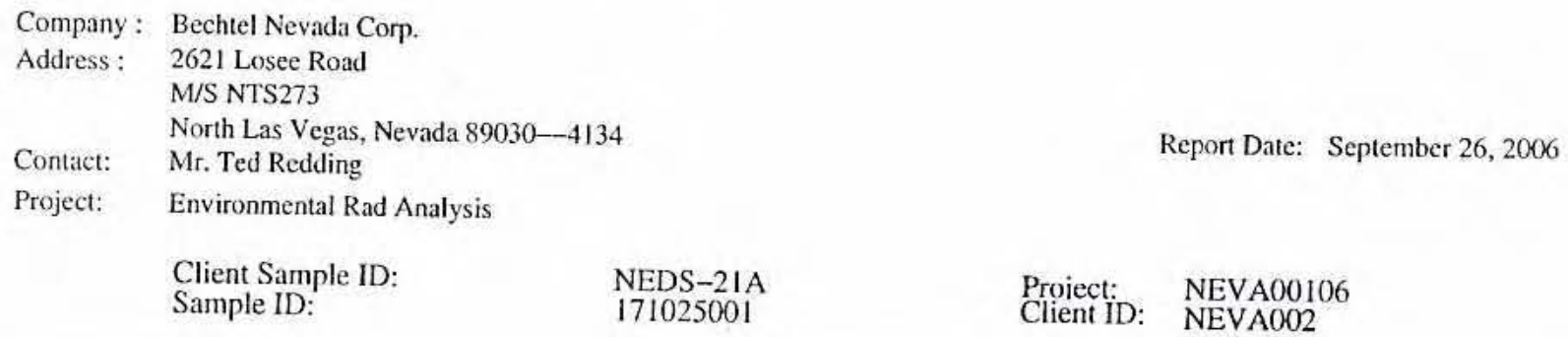

UI Gamma Spectroscopy--Uncertain identification

$\mathrm{X}$ Consult Case Narrative, Data Summary package, or Project Manager concerning this qualifier

Y QC Samples were not spiked with this compound

$\hat{A}$ RPD of sample and duplicate evaluated using $+/-R L$. Concentrations are $<5 X$ the RL

h Preparation or preservation holding time was exceeded

The above sample is reported on a dry weight basis. 


\section{GENERAL ENGINEERING LABORATORIES, LLC}

2040 Savage Road Charleston SC 29407 - (843) 556-8171 - www.gel.com

\section{Certificate of Analysis}

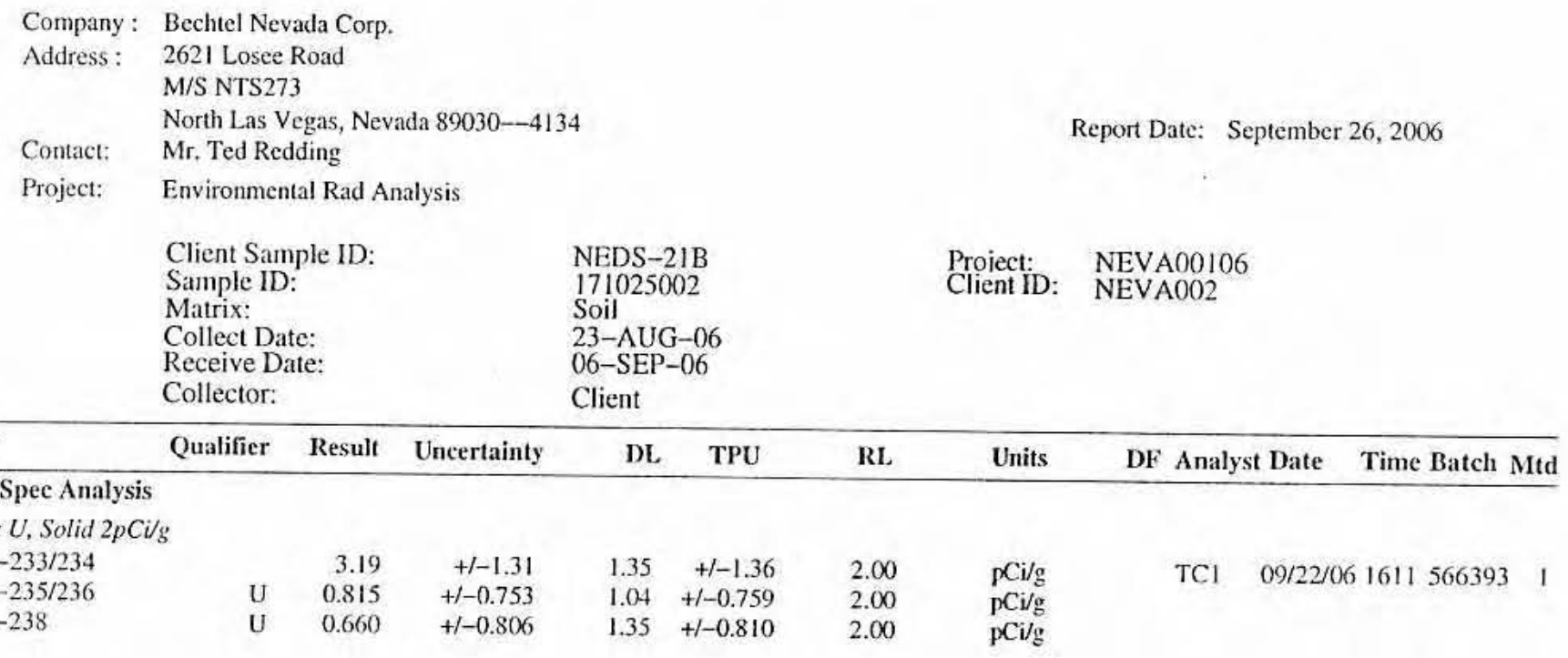

The following Prep Methods were performed

\begin{tabular}{llllll}
\hline Method & Description & Analyst & Date & Time & Prep Batch \\
\hline Dry Soil Prep & Dry Soil Prep GL-RAD-A-021 & AXP2 & $09 / 07 / 06$ & 1306 & 565885
\end{tabular}

The following Analytical Methods were performed

\begin{tabular}{ll} 
Method & Description \\
\hline 1 & DOE EML HASL-300, U-02-RC Modified \\
2 & DOE EML HASL-300, U-02-RC Modified
\end{tabular}

\begin{tabular}{llcc} 
Surrogate/Tracer recovery & Test & Recovery\% & Acceptable Limits \\
\hline Uranium-232 & Alphaspec U, Solid 2pCi/g & 79 & $(25 \%-125 \%)$
\end{tabular}

Notes:

The Qualifiers in this report are defined as follows :

* A quality control analyte recovery is outside of specified acceptance criteria

$<$ Result is less than value reported

$>$ Result is greater than value reported

A The TIC is a suspected aldol-condensation product

B Target analyte was detected in the associated blank

$\mathrm{BD}$ Results are either below the MDC or tracer recovery is low

C Analyte has been confirmed by GC/MS analysis

D Results are reported from a diluted aliquot of the sample

$\mathrm{H}$ Analytical holding time was exceeded

$\mathrm{J}$ Value is estimated

N/A Spike recovery limits do not apply. Sample concentration exceeds spike concentration by $4 X$ or more

$\mathrm{R}$ Sample results are rejected

U Analyte was analyzed for, but not detected above the MDL, MDA, or LOD.

UI Gamma Spectroscopy-Uncertain identification 


\section{GENERAL ENGINEERING LABORATORIES, LLC}

2040 Savage Road Charleston SC 29407 - (843) 556-8171 - www.gel.com

\section{Certificate of Analysis}

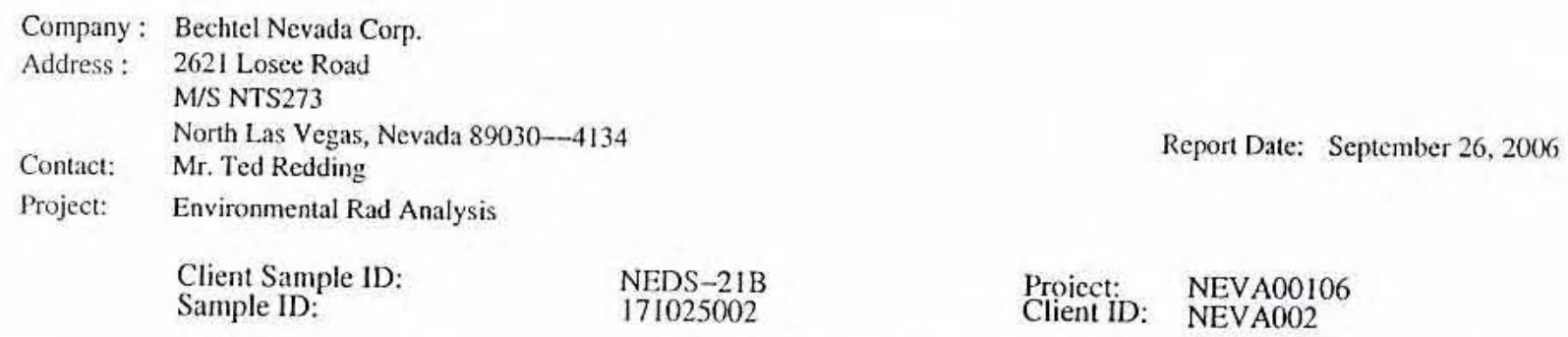

Parameter

Qualifier Result Uncertainty

DL TPU

RL

Units

DF Analyst Date

Time Batch Mtd

$\mathrm{X}$ Consult Case Narrative, Data Summary package, or Project Manager concerning this qualifier

Y QC Samples were not spiked with this compound

$\wedge \quad \mathrm{RPD}$ of sample and duplicate evaluated using $+/-\mathrm{RL}$. Concentrations are $<5 \mathrm{X}$ the RL

h Preparation or preservation holding time was exceeded

The above sample is reported on a dry weight basis. 


\title{
GENERAL ENGINEERING LABORATORIES, LLC
}

2040 Savage Road Charleston SC 29407 - (843) 556-8171 - www.gel.com

\section{Certificate of Analysis}

\author{
Company: Bechtel Nevada Corp. \\ Address : 2621 Losee Road \\ M/S NTS273 \\ North Las Vegas, Nevada 89030-4134 Report Date: September 26, 2006 \\ Contact: $\quad$ Mr. Ted Redding \\ Project: Environmental Rad Analysis \\ Client Sample ID: \\ Sample ID: \\ Matrix: \\ Collect Date: \\ Receive Date: \\ Collector:

NEDS-22A
171025003
Soil
23-AUG-06
$06-S E P-06$
Client \\ NEDS-22A \\ Soil \\ 06-SEP-06 \\ Clien

\begin{tabular}{|c|c|c|c|c|c|c|c|c|c|c|c|c|}
\hline Parameter & Qualifier & Result & Uncertainty & DL & TPU & RL & Units & DF & Analys & Date & Time Batch N & $\overline{\text { Mid }}$ \\
\hline \multicolumn{13}{|c|}{ Rad Alpha Spec Analysis } \\
\hline \multicolumn{13}{|c|}{ Alphaspec U, Solid $2 p C i / g$} \\
\hline $\begin{array}{l}\text { Uranium-233/234 } \\
\text { Uranium-235/236 } \\
\text { Uranium-238 }\end{array}$ & $\mathrm{U}$ & $\begin{array}{r}2.14 \\
0.241 \\
1.36\end{array}$ & $\begin{array}{l}+1-0.752 \\
+l-0.521 \\
+I-0.661\end{array}$ & $\begin{array}{l}0.496 \\
0.987 \\
0.717\end{array}$ & $\begin{array}{l}+1-0.781 \\
+1-0.522 \\
+l-0.674\end{array}$ & $\begin{array}{l}2.00 \\
2.00 \\
2.00\end{array}$ & $\begin{array}{l}\mathrm{pCi} / \mathrm{g} \\
\mathrm{pCi} / \mathrm{g} \\
\mathrm{pCi} / \mathrm{g}\end{array}$ & & $\mathrm{TCl}$ & $09 / 22 / 0$ & 61612566393 & 1 \\
\hline
\end{tabular} \\ The following Prep Methods were performed

\begin{tabular}{llllll}
\hline Method & Description & Analyst & Date & Time & Prep Batch \\
\hline Dry Soil Prep & Dry Soil Prep GL-RAD-A-021 & AXP2 & $09 / 07 / 06$ & 1306 & 565885
\end{tabular} \\ The following Analytical Methods were performed

\begin{tabular}{ll} 
Method & Description \\
\hline 1 & DOE EML HASL-300, U-02-RC Modified \\
2 & DOE EML HASL-300, U-02-RC Modified
\end{tabular}

\begin{tabular}{llcc} 
Surrogate/Tracer recovery & Test & Recovery\% & Acceptable Limits \\
\hline Uranium-232 & Alphaspec U, Solid 2pCi/g & 88 & $(25 \%-125 \%)$
\end{tabular}

\section{Notes:} \\ The Qualifiers in this report are defined as follows : \\ * A quality control analyte recovery is outside of specified acceptance criteria \\ $<$ Result is less than value reported \\ $>$ Result is greater than value reported \\ A The TIC is a suspected aldol-condensation product \\ B Target analyte was detected in the associated blank \\ BD Results are either below the MDC or tracer recovery is low \\ C Analyte has been confirmed by GC/MS analysis \\ D Results are reported from a diluted aliquot of the sample \\ $\mathrm{H}$ Analytical holding time was exceeded \\ $\mathrm{J}$ Value is estimated \\ N/A Spike recovery limits do not apply. Sample concentration exceeds spike concentration by $4 \mathrm{X}$ or more \\ $\mathrm{R}$ Sample results are rejected \\ U Analyte was analyzed for, but not detected above the MDL, MDA, or LOD. \\ UI Gamma Spectroscopy--Uncertain identification
}




\section{GENERAL ENGINEERING LABORATORIES, LLC \\ 2040 Savage Road Charleston SC 29407 - (843) 556-8171 - www.gel.com}

\section{Certificate of Analysis}

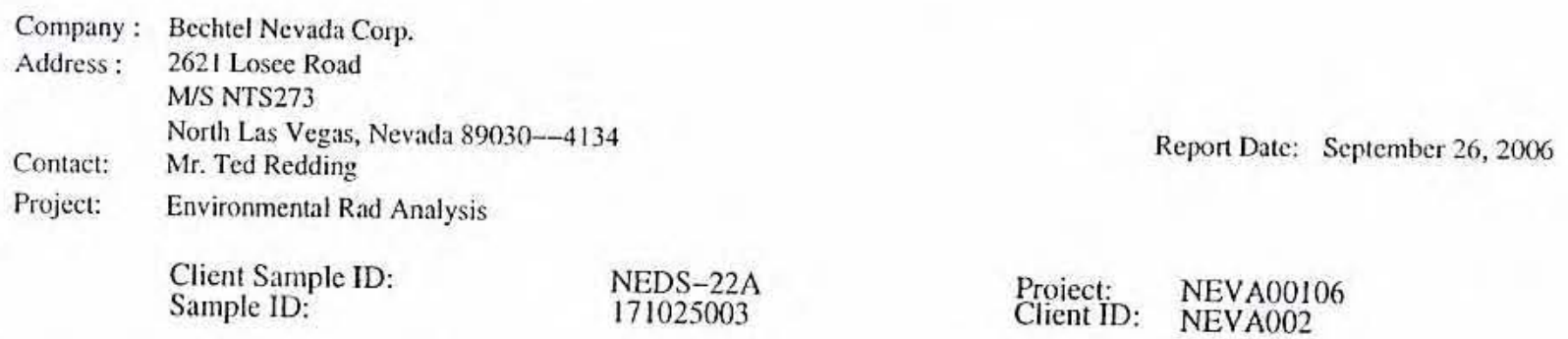

Y QC Samples were not spiked with this compound

$\hat{R}$ RPD of sample and duplicate evaluated using $+1-\mathrm{RL}$. Concentrations are $<5 \mathrm{X}$ the RL

$\mathrm{h}$ Preparation or preservation holding time was exceeded

The above sample is reported on a dry weight basis. 


\section{GENERAL ENGINEERING LABORATORIES, LLC}

2040 Savage Road Charleston SC 29407 - (843) 556-8171 - www.gel.com

\section{Certificate of Analysis}

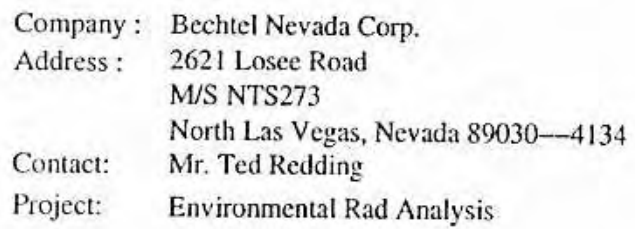

NEDS-22B

171025004

Soil

23-AUG-06

Collect Date:

Receive Date:

Collector:

Client

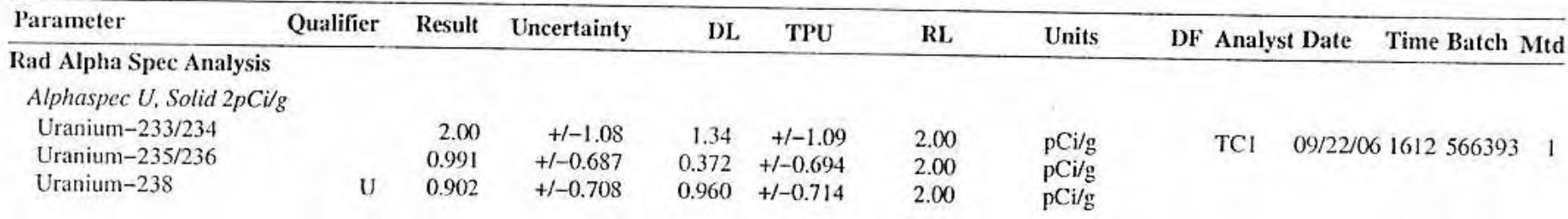

The following Prep Methods were performed

\begin{tabular}{llllll}
\hline Method & Description & Analyst & Date & Time & Prep Batch \\
\hline Dry Soil Prep & Dry Soil Prep GL-RAD-A-021 & AXP2 & $09 / 07 / 06$ & 1306 & 565885
\end{tabular}

The following Analytical Methods were performed

\begin{tabular}{ll}
\hline Method & Description \\
\hline 1 & DOE EML HASL-300, U-02-RC Modified \\
2 & DOE EML HASL-300, U-02-RC Modified
\end{tabular}

\begin{tabular}{llcc} 
Surrogate/Tracer recovery & Test & Recovery $\%$ & Acceptable Limits \\
\hline Uranium-232 & Alphaspec U, Solid 2pCi/g & 85 & $(25 \%-125 \%)$
\end{tabular}

Notes:

The Qualifiers in this report are defined as follows:

* A quality control analyte recovery is outside of specified acceptance criteria

$<$ Result is less than value reported

$>$ Result is greater than value reported

A The TIC is a suspected aldol-condensation product

$B$ Target analyte was detected in the associated blank

$\mathrm{BD}$ Results are either below the MDC or tracer recovery is low

C Analyte has been confirmed by GC/MS analysis

D Results are reported from a diluted aliquot of the sample

$\mathrm{H}$ Analytical holding time was exceeded

$\mathrm{J}$ Value is estimated

N/A Spike recovery limits do not apply. Sample concentration exceeds spike concentration by $4 X$ or more

$\mathrm{R}$ Sample results are rejected

U Analyte was analyzed for, but not detected above the MDL, MDA, or LOD.

UI Gamma Spectroscopy--Uncertain identification 


\section{GENERAL ENGINEERING LABORATORIES, LLC \\ 2040 Savage Road Charleston SC 29407 - (843) 556-8171 - www.gel.com}

\section{Certificate of Analysis}

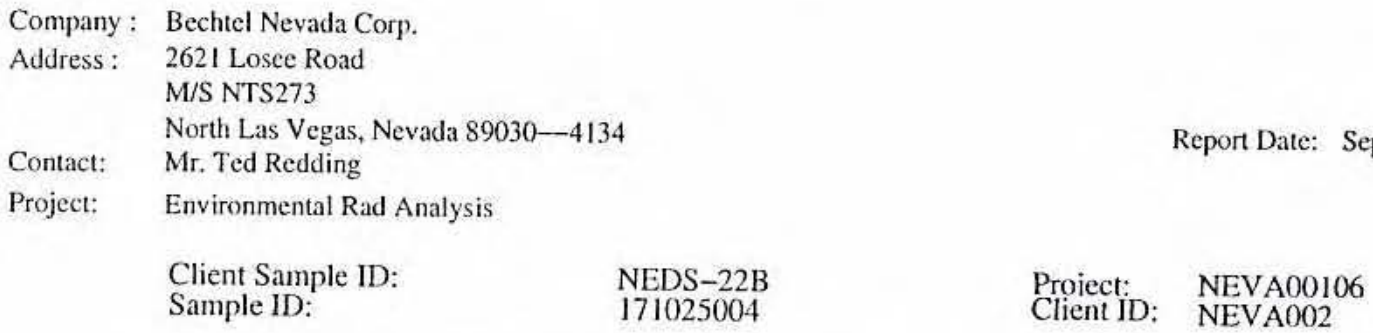

$X$ Consult Case Narrative, Data Summary package, or Project Manager concerning this qualifier

Y QC Samples were not spiked with this compound

$\wedge$ RPD of sample and duplicate evaluated using $+1-R L$. Concentrations are $<5 X$ the $R L$

$\mathrm{h}$ Preparation or preservation holding time was exceeded

The above sample is reported on a dry weight basis. 


\section{GENERAL ENGINEERING LABORATORIES, LLC \\ 2040 Savage Road Charleston SC 29407 - (843) 556-8171 - www.gel.com}

\section{Certificate of Analysis}

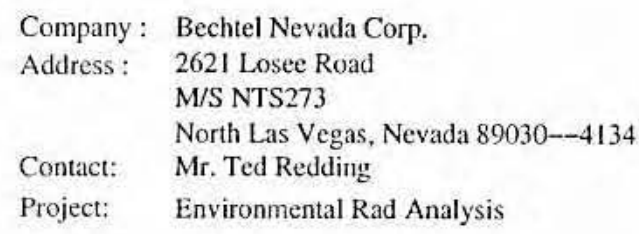

Client Sample ID:

Sample ID:

Matrix:

Collect Date:

Receive Date:

Collector:

\begin{tabular}{|c|c|c|c|c|c|c|c|c|c|c|c|c|}
\hline Parameter & Qualifier & Result & Uncertainty & $\mathrm{DL}$ & TPU & RL & Units & DF & Analyst & t Date & Time Batch N & Mtd \\
\hline \multicolumn{13}{|c|}{ Rad Alpha Spec Analysis } \\
\hline \multicolumn{13}{|c|}{ Alphaspec U. Solid $2 \mathrm{pCi} / \mathrm{g}$} \\
\hline Uranium-233/234 & $\mathrm{U}$ & 1.15 & $+1-1.08$ & 1.53 & $+1-1.09$ & 2.00 & $\mathrm{pCi} / \mathrm{g}$ & & $\mathrm{TCl}$ & $09 / 20 / 06$ & 61820566393 & 1 \\
\hline Uranium-235/236 & $\mathrm{U}$ & 0.356 & $+1-0.668$ & 1.30 & $+/-0.670$ & 2.00 & $\mathrm{pCi} / \mathrm{g}$ & & & & & \\
\hline Uranium-238 & $\mathrm{U}$ & 0.440 & $+1-0.780$ & 1.47 & $+1-0.783$ & 2.00 & $\mathrm{pCi} / \mathrm{g}$ & & & & & \\
\hline
\end{tabular}

The following Prep Methods were performed

\begin{tabular}{lllll}
\hline Method $\quad$ Description & Analyst & Date & Time & Prep Batch \\
\hline Dry Soil Prep Dry Soil Prep GL-RAD-A-021 & AXP2 & $09 / 07 / 06$ & 1306 & 565885 \\
The following Analytical Methods were performed & & & \\
\hline Dethod DOE EML HASL-300, U-02-RC Modified & & & \\
\hline $1 \quad$ Test & Recovery\% & Acceptable Limits \\
Surrogate/Tracer recovery & Alphaspec U, Solid 2pCi/g & 77 & $(25 \%-125 \%)$
\end{tabular}

Notes:

The Qualifiers in this report are defined as follows :

* A quality control analyte recovery is outside of specified acceptance criteria

$<$ Result is less than value reported

$>$ Result is greater than value reported

A The TIC is a suspected aldol-condensation product

B Target analyte was detected in the associated blank

BD Results are either below the MDC or tracer recovery is low

C Analyte has been confirmed by GC/MS analysis

D Results are reported from a diluted aliquot of the sample

$\mathrm{H}$ Analytical holding time was exceeded

$\mathrm{J}$ Value is estimated

N/A Spike recovery limits do not apply. Sample concentration exceeds spike concentration by $4 X$ or more

$\mathrm{R}$ Sample results are rejected

U Analyte was analyzed for, but not detected above the MDL, MDA, or LOD.

UI Gamma Spectroscopy-Uncertain identification

$\mathrm{X}$ Consult Case Narrative, Data Summary package, or Project Manager concerning this qualifier 


\section{GENERAL ENGINEERING LABORATORIES, LLC \\ 2040 Savage Road Charleston SC 29407 - (843) 556-8171 - www.gel.com}

\section{Certificate of Analysis}

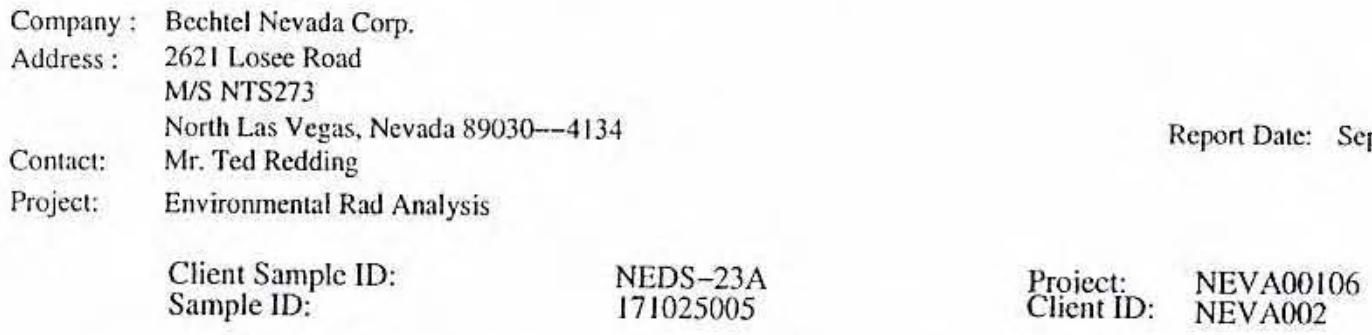

Y QC Samples were not spiked with this compound

$\wedge$ RPD of sample and duplicate evaluated using $+/-$ RL. Concentrations are $\angle 5 X$ the RL

$\mathrm{h}$ Preparation or preservation holding time was exceeded

The above sample is reported on a dry weight basis. 


\section{GENERAL ENGINEERING LABORATORIES, LLC \\ 2040 Savage Road Charleston SC 29407 - (843) 556-8171 - www.gel.com}

\section{Certificate of Analysis}

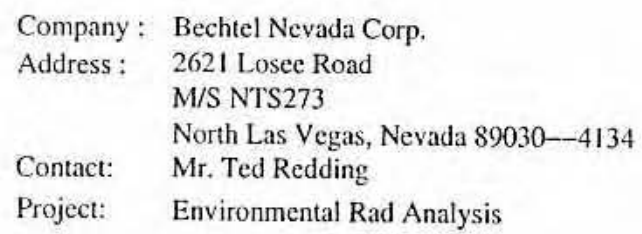

Report Date: September 26, 2006

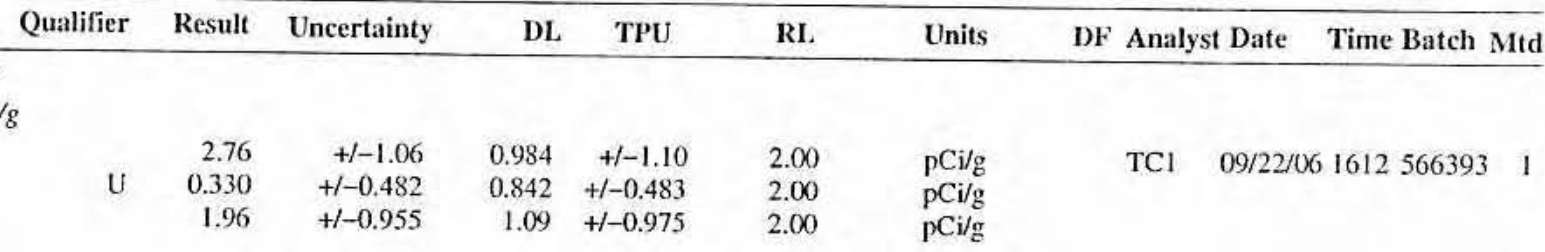

The following Prep Methods were performed

\begin{tabular}{llllll}
\hline Method & Description & Analyst & Date & Time & Prep Batch \\
\hline Dry Soil Prep & Dry Soil Prep GL-RAD-A-021 & AXP2 & $09 / 07 / 06$ & 1306 & 565885
\end{tabular}

The following Analytical Methods were performed

\begin{tabular}{ll}
\hline Method & Description \\
\hline 1 & DOE EML HASL-300, U-02-RC Modified \\
2 & DOE EML HASL-300, U-02-RC Modified
\end{tabular}

\begin{tabular}{llcc} 
Surrogate/Tracer recovery & Test & Recovery $\%$ & Acceptable Limits \\
\hline Uranium-232 & Alphaspec U, Solid 2pCi $/ g$ & 70 & $(25 \%-125 \%)$
\end{tabular}

Notes:

The Qualifiers in this report are defined as follows :

* A quality control analyte recovery is outside of specified acceptance criteria

$<$ Result is less than value reported

$>$ Result is greater than value reported

A The TIC is a suspected aldol-condensation product

B Target analyte was detected in the associated blank

$\mathrm{BD}$ Results are either below the MDC or tracer recovery is low

C Analyte has been confirmed by GC/MS analysis

D Results are reported from a diluted aliquot of the sample

$\mathrm{H}$ Analytical holding time was exceeded

$\mathrm{J}$ Value is estimated

N/A Spike recovery limits do not apply. Sample concentration exceeds spike concentration by $4 \mathrm{X}$ or more

$\mathrm{R}$ Sample results are rejected

U Analyte was analyzed for, but not detected above the MDL, MDA, or LOD.

UI Gamma Spectroscopy--Uncertain identification 


\section{GENERAL ENGINEERING LABORATORIES, LLC \\ 2040 Savage Road Charleston SC 29407 - (843) 556-8171 - www. gel.com}

\section{Certificate of Analysis}

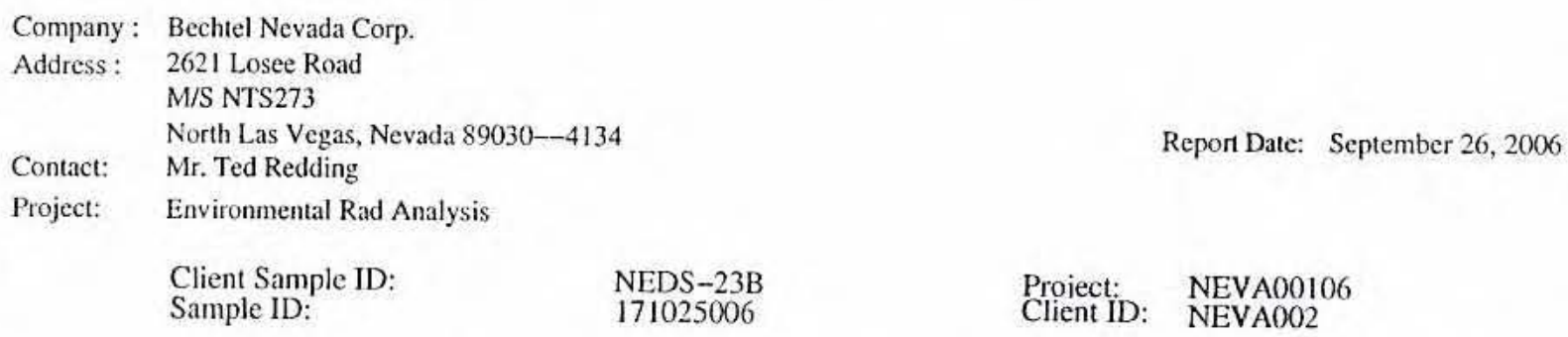

X Consult Case Narrative, Data Summary package, or Project Manager concerning this qualifier

Y QC Samples were not spiked with this compound

$\wedge$ RPD of sample and duplicate evaluated using $+/-R L$. Concentrations are $<5$ the RL.

h Preparation or preservation holding time was exceeded

The above sample is reported on a dry weight basis. 


\section{GENERAL ENGINEERING LABORATORIES, LLC}

2040 Savage Road Charleston SC 29407 - (843) 556-8171 - www.gel.com

\section{Certificate of Analysis}

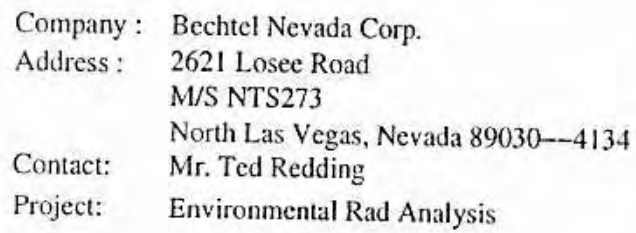

Client Sample ID:

Sample ID:

Matrix:

Collect Date:

Receive Date:

Collector:
Report Date: September 26, 2006

NEDS-24A
171025007
Soil
23-AUG-06
$06-S E P-06$
Client

Proiect: NEVA00106

Client ID: NEVA002

\begin{tabular}{|c|c|c|c|c|c|c|c|c|c|c|c|c|}
\hline Parameter & Qualifier & Result & Uncertainty & DL & TPU & RL. & Units & $\mathrm{DF}$ & Analyst & t Date & Time Batch $M$ & Mtd \\
\hline $\begin{array}{l}\text { Rad Alpha Spec Ana } \\
\text { Alphaspec U, Solid }\end{array}$ & & & & & & & & & & & & \\
\hline $\begin{array}{l}\text { Uranium-233/234 } \\
\text { Uranium-235/236 } \\
\text { Uranium-238 }\end{array}$ & $\begin{array}{l}U \\
U\end{array}$ & $\begin{array}{r}2.18 \\
0.328 \\
0.887\end{array}$ & $\begin{array}{r}+1-1.35 \\
+/-0.740 \\
+/-0.924\end{array}$ & $\begin{array}{l}1.15 \\
1.57 \\
1.27\end{array}$ & $\begin{array}{r}+/-1.40 \\
+/-0.742 \\
+/-0.934\end{array}$ & $\begin{array}{l}2.00 \\
2.00 \\
2.00\end{array}$ & $\begin{array}{l}\mathrm{pCi} / \mathrm{g} \\
\mathrm{pCi} / \mathrm{g} \\
\mathrm{pCi} / \mathrm{g}\end{array}$ & & $\mathrm{TCl}$ & $09 / 20 / 06$ & 1820566393 & 1 \\
\hline
\end{tabular}

The following Prep Methods were performed

\begin{tabular}{llllll}
\hline Method & Description & Analyst & Date & Time & Prep Batch \\
\hline Dry Soil Prep & Dry Soil Prep GL-RAD-A-021 & AXP2 & $09 / 07 / 06$ & 1306 & 565885
\end{tabular}

The following Analytical Methods were performed

\begin{tabular}{ll} 
Method & Description \\
\hline 1 & DOE EML HASL-300, U-02-RC Modified
\end{tabular}

\begin{tabular}{llcc} 
Surrogate/Tracer recovery & Test & Recovery $\%$ & Acceptable Limits \\
\hline Uranium-232 & Alphaspec U, Solid 2pCi/g & 61 & $(25 \%-125 \%)$
\end{tabular}

Notes:

The Qualifiers in this report are defined as follows :

* A quality control analyte recovery is outside of specified acceptance criteria

$<$ Result is less than value reported

$>$ Result is greater than value reported

A The TIC is a suspected aldol-condensation product

B Target analyte was detected in the associated blank

$\mathrm{BD}$ Results are either below the MDC or tracer recovery is low

C Analyte has been confirmed by GC/MS analysis

D Results are reported from a diluted aliquot of the sample

$\mathrm{H}$ Analytical holding time was exceeded

$\mathrm{J}$ Value is estimated

N/A Spike recovery limits do not apply. Sample concentration exceeds spike concentration by 4 X or more

$\mathrm{R}$ Sample results are rejected

$U$ Analyte was analyzed for, but not detected above the MDL, MDA, or LOD.

UI Gamma Spectroscopy--Uncertain identification

X Consult Case Narrative, Data Summary package, or Project Manager concerning this qualifier 


\section{GENERAL ENGINEERING LABORATORIES, LLC \\ 2040 Savage Road Charleston SC 29407 - (843) 556-8171 - www. gel.com}

\section{Certificate of Analysis}

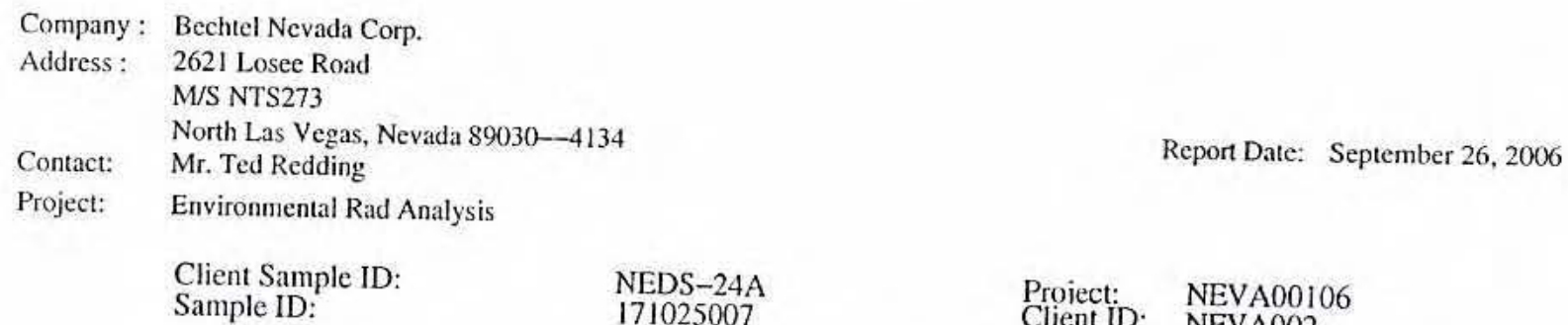

Y QC Samples were not spiked with this compound

$\hat{R}$ RPD of sample and duplicate evaluated using $+/-\mathrm{RL}$. Concentrations are $<5 \mathrm{X}$ the RL

$h$ Preparation or preservation holding time was exceeded

The above sample is reported on a dry weight basis. 


\section{GENERAL ENGINEERING LABORATORIES, LLC \\ 2040 Savage Road Charleston SC 29407 - (843) 556-8171 - www.gel.com}

\section{Certificate of Analysis}

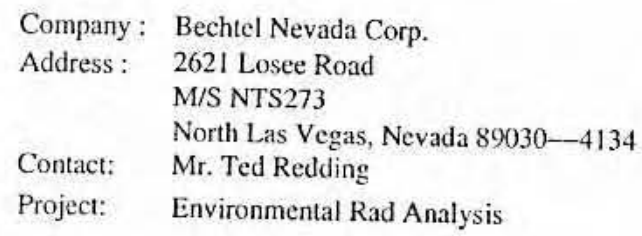

Report Date: September 26, 2006

Client Sample ID:

Sample ID:

NEDS-24B

171025008

Soil

Matrix:

Collect Date:

Receive Date:

Collector:
23-AUG-06

06-SEP-06

Client

\begin{tabular}{|c|c|c|c|c|c|c|c|c|c|c|}
\hline Parameter & Qualilier & Result & Uncertainty & DL & TPU & RI & Units & DF & Analyst Date & Time Batch Mitd \\
\hline $\begin{array}{l}\text { Rad Alpha Spec Ana } \\
\text { Alphaspec } U \text {, Solid }\end{array}$ & & & & & & & & & & \\
\hline $\begin{array}{l}\text { Uranium-233/234 } \\
\text { Uranium-235/236 } \\
\text { Uranium-238 }\end{array}$ & $\begin{array}{l}\mathrm{U} \\
\mathrm{U}\end{array}$ & $\begin{array}{r}2.28 \\
0.704 \\
0.997\end{array}$ & $\begin{array}{r}+1-1.25 \\
+1-0.846 \\
+1-0.926\end{array}$ & $\begin{array}{l}1.36 \\
1.35 \\
1.36\end{array}$ & $\begin{array}{r}+/-1.27 \\
+/-0.849 \\
+/-0.932\end{array}$ & $\begin{array}{l}2.00 \\
2.00 \\
2.00\end{array}$ & $\begin{array}{l}\mathrm{pCi} / \mathrm{g} \\
\mathrm{pCi} / \mathrm{g} \\
\mathrm{pCi} / \mathrm{g}\end{array}$ & & $09 / 22 / 0$ & $61612566393 \quad 1$ \\
\hline
\end{tabular}

The following Prep Methods were performed

\begin{tabular}{llllll}
\hline Method & Description & Analyst & Date & Time & Prep Batch \\
\hline Dry Soil Prep & Dry Soil Prep GL-RAD-A-021 & AXP2 & $09 / 07 / 06$ & 1306 & 565885
\end{tabular}

The following Analytical Methods were performed

\begin{tabular}{ll} 
Method & Description \\
\hline 1 & DOE EML HASL-300, U-02-RC Modified \\
2 & DOE EML HASL-300, U-02-RC Modificd
\end{tabular}

\begin{tabular}{llcc} 
Surrogate/Tracer recovery & Test & Recovery\% & Acceptable Limits \\
\hline Uranium-232 & Alphaspec U, Solid 2pCi/g & 69 & $(25 \%-125 \%)$
\end{tabular}

Notes:

The Qualifiers in this report are defined as follows :

* A quality control analyte recovery is outside of specified acceptance criteria

$<$ Result is less than value reported

$>$ Result is greater than value reported

A The TIC is a suspected aldol-condensation product

B Target analyte was detected in the associated blank

$\mathrm{BD}$ Results are either below the MDC or tracer recovery is low

C Analyte has been confirmed by GC/MS analysis

D Results are reported from a diluted aliquot of the sample

H Analytical holding time was exceeded

$\mathrm{J}$ Value is estimated

N/A Spike recovery limits do not apply. Sample concentration exceeds spike concentration by $4 X$ or more
R Sample results are rejected

$\mathrm{U}$ Analyte was analyzed for, but not detected above the MDL, MDA, or LOD.

U1 Gamma Spectroscopy-Uncertain identification 


\section{GENERAL ENGINEERING LABORATORIES, LLC \\ 2040 Savage Road Charleston SC 29407 - (843) 556-8171 - www.gel.com}

\section{Certificate of Analysis}

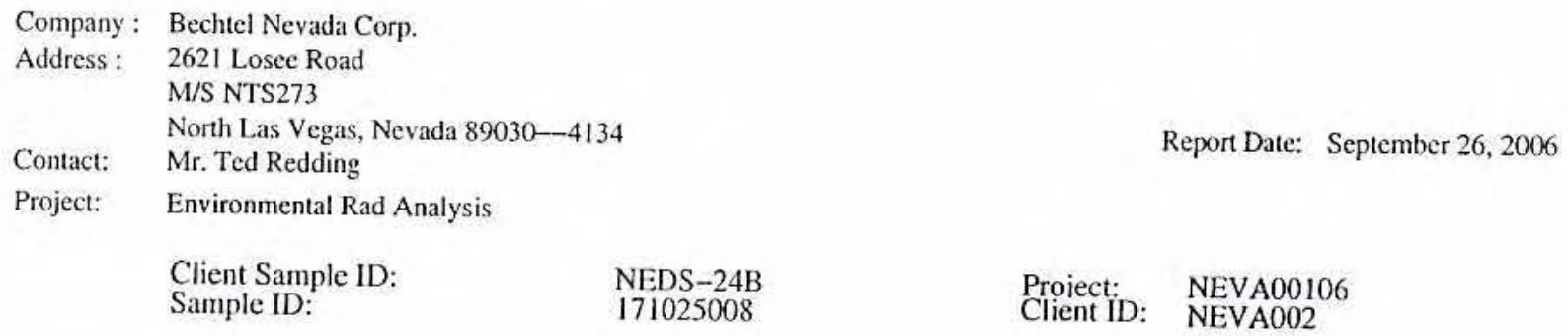

$\mathrm{X}$ Consult Case Narrative, Data Summary package, or Project Manager concerning this qualifier

Y QC Samples were not spiked with this compound

$\hat{R}$ RD of sample and duplicate evaluated using $+/-\mathrm{RL}$. Concentrations are $<5 \mathrm{X}$ the $\mathrm{RL}$

h Preparation or preservation holding time was exceeded

The above sample is reported on a dry weight basis. 


\section{GENERAL ENGINEERING LABORATORIES, LLC \\ 2040 Savage Road Charleston SC 29407 - (843) 556-8171 - www.gel.com}

\section{Certificate of Analysis}

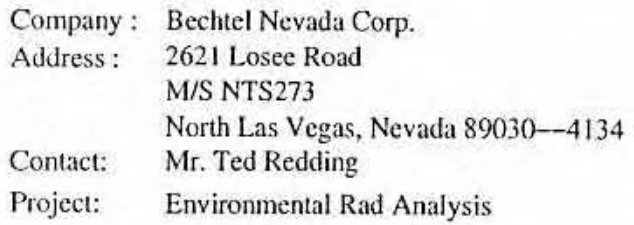

NEDS-25A
171025009
Soil
$23-A U G-06$
$06-S E P-06$
Client

\begin{tabular}{|c|c|c|c|c|c|c|c|c|c|c|c|c|}
\hline Parameter & Qualifier & Result & Uncertainty & DI. & TPU & RL & Units & DF & Analyst & Date & Time Batch M & Mtd \\
\hline \multicolumn{13}{|c|}{ Rad Alpha Spec Analysis } \\
\hline \multicolumn{13}{|c|}{ Alphaspec U. Solid $2 p C i / g$} \\
\hline $\begin{array}{l}\text { Uranium-233/234 } \\
\text { Uranium-235/236 } \\
\text { Uranium-238 }\end{array}$ & $\mathrm{U}$ & $\begin{array}{r}1.63 \\
0.459 \\
1.11\end{array}$ & $\begin{array}{l}+/-0.797 \\
+/-0.540 \\
+/-0.600\end{array}$ & $\begin{array}{l}0.914 \\
0.879 \\
0.568\end{array}$ & $\begin{array}{l}+/-0.814 \\
+/-0.542 \\
+/-0.610\end{array}$ & $\begin{array}{l}2.00 \\
2.00 \\
2.00\end{array}$ & $\begin{array}{l}\mathrm{pCi} / \mathrm{g} \\
\mathrm{pCi} / \mathrm{g} \\
\mathrm{pCi} / \mathrm{g}\end{array}$ & & $\mathrm{TCl}$ & $09 / 22 / 06$ & 1612566393 & 1 \\
\hline
\end{tabular}

The following Prep Methods were performed

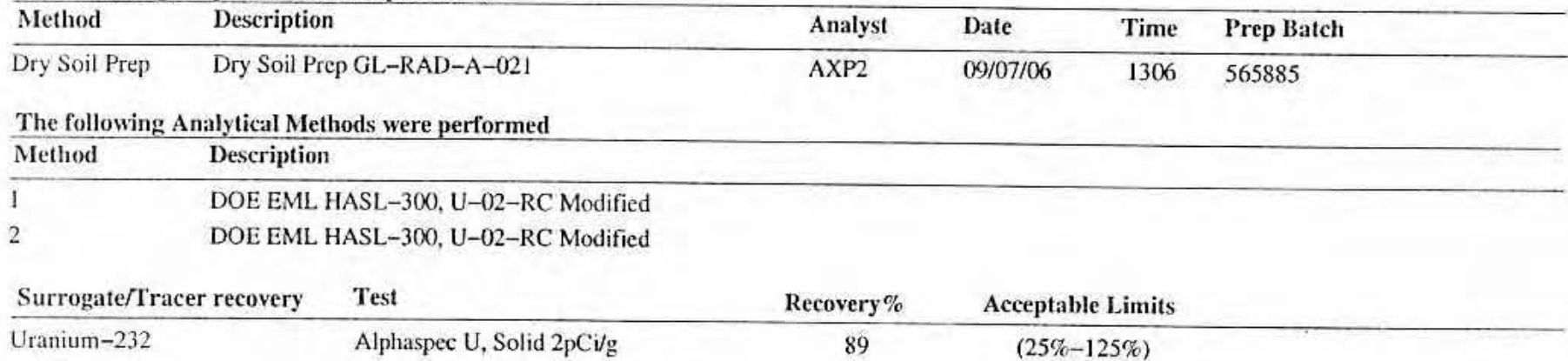

Notes:

The Qualifiers in this report are defined as follows :

* A quality control analyte recovery is outside of specified acceptance criteria

$<$ Result is less than value reported

$>$ Result is greater than value reported

A The TIC is a suspected aldol-condensation product

B Target analyte was detected in the associated blank

$\mathrm{BD}$ Results are either below the MDC or tracer recovery is low

C Analyte has been confirmed by GC/MS analysis

D Results are reported from a diluted aliquot of the sample

$\mathrm{H}$ Analytical holding time was exceeded

$\mathrm{J}$ Value is estimated

N/A Spike recovery limits do not apply. Sample concentration exceeds spike concentration by $4 X$ or more

$\mathrm{R}$ Sample results are rejected

U Analyte was analyzed for, but not detected above the MDL, MDA, or LOD.

UI Gamma Spectroscopy--Uncertain identification 


\section{GENERAL ENGINEERING LABORATORIES, LLC \\ 2040 Savage Road Charleston SC 29407 - (843) 556-8171 - www.gel.com}

\section{Certificate of Analysis}

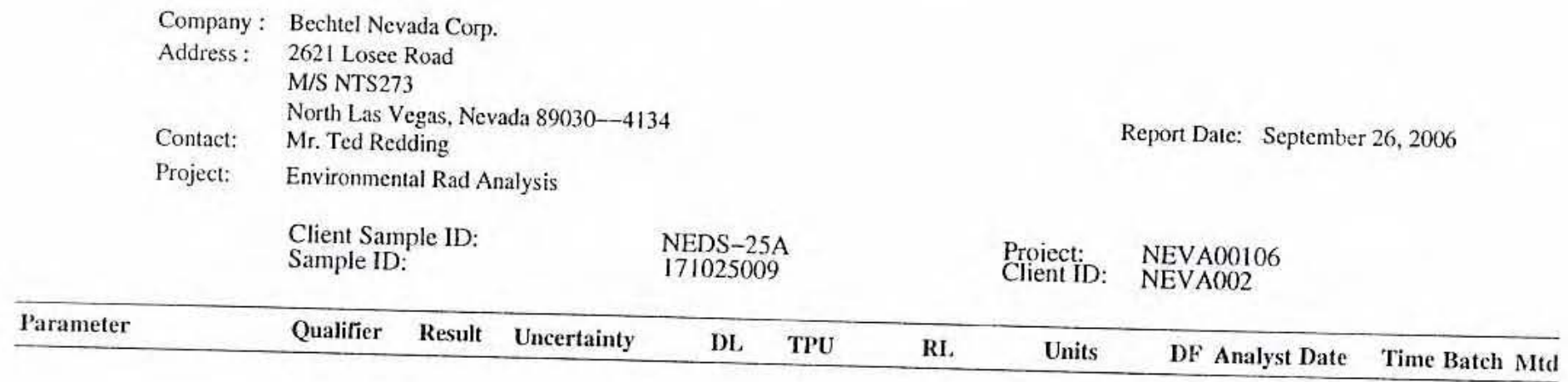

$\mathrm{X}$ Consult Case Narrative, Data Summary package, or Project Manager concerning this qualifier

Y QC Samples were not spiked with this compound

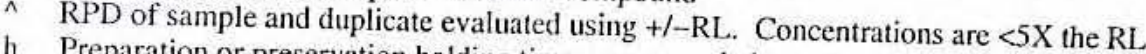

$\mathrm{h}$ Preparation or preservation holding time was exceeded

The above sample is reported on a dry weight basis. 


\section{GENERAL ENGINEERING LABORATORIES, LLC \\ 2040 Savage Road Charleston SC 29407 - (843) 556-8171 - www.gel.com}

\section{Certificate of Analysis}

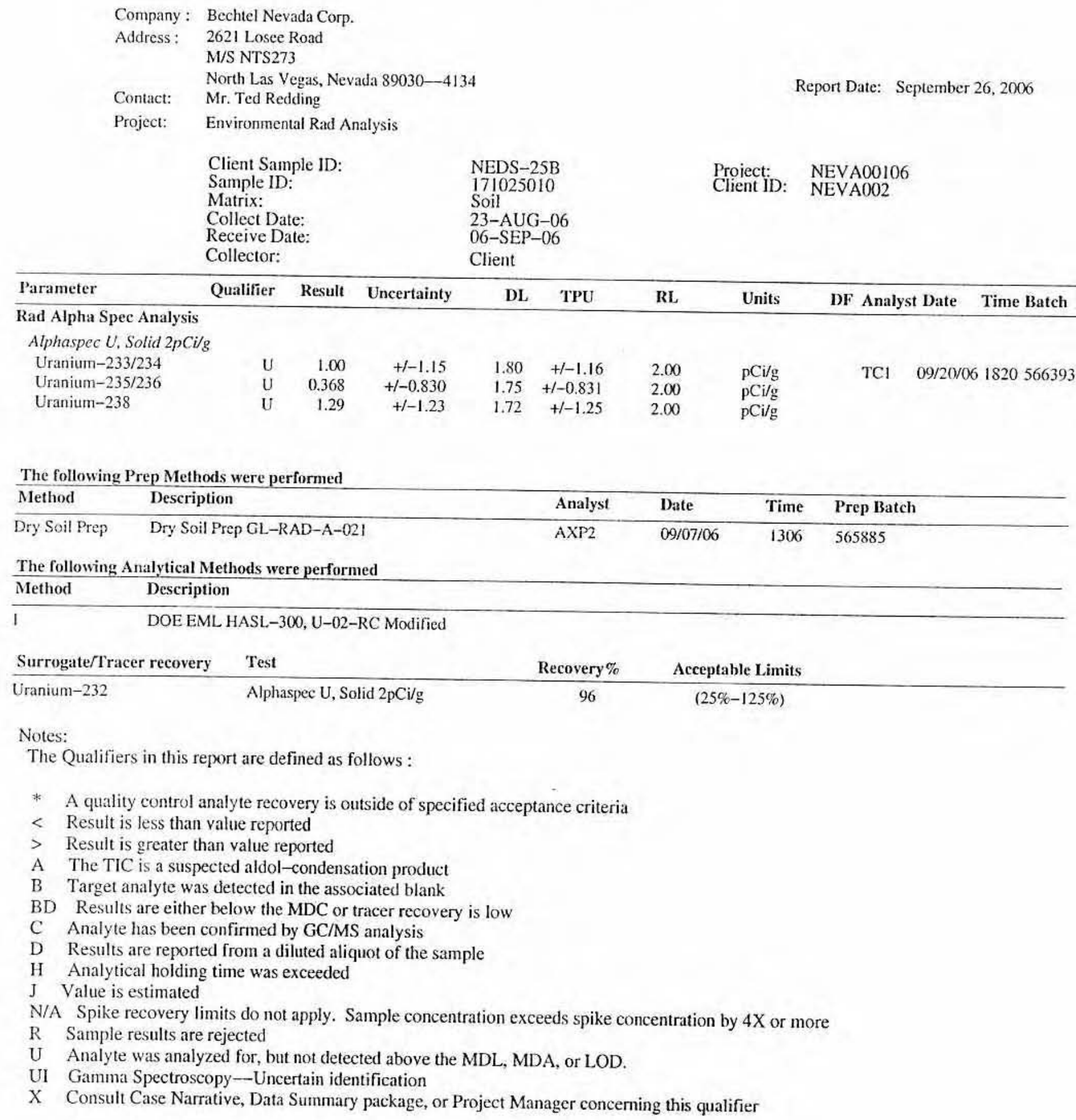




\section{GENERAL ENGINEERING LABORATORIES, LLC \\ 2040 Savage Road Charleston SC 29407 - (843) 556-8171 - www.gel.com}

\section{Certificate of Analysis}

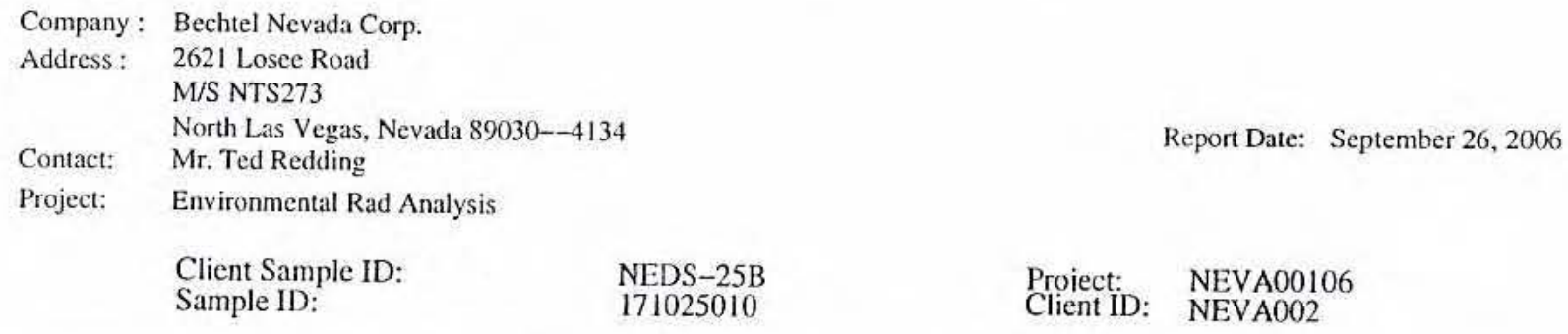

DL TPU RL

Units

Y QC Samples were not spiked with this compound

$\hat{R}$ RD of sample and duplicate evaluated using $+/-$ RL. Concentrations are $<5 X$ the RL

$\mathrm{h}$ Preparation or preservation holding time was exceeded

The above sample is reported on a dry weight basis. 


\section{GENERAL ENGINEERING LABORATORIES, LLC \\ 2040 Savage Road Charleston SC 29407 - (843) 556-8171 - www.gel.com}

\section{Certificate of Analysis}

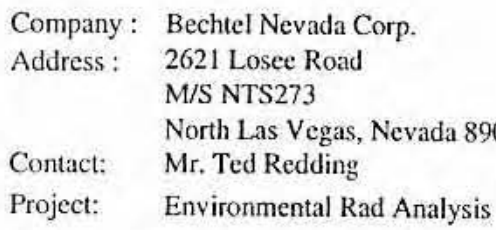

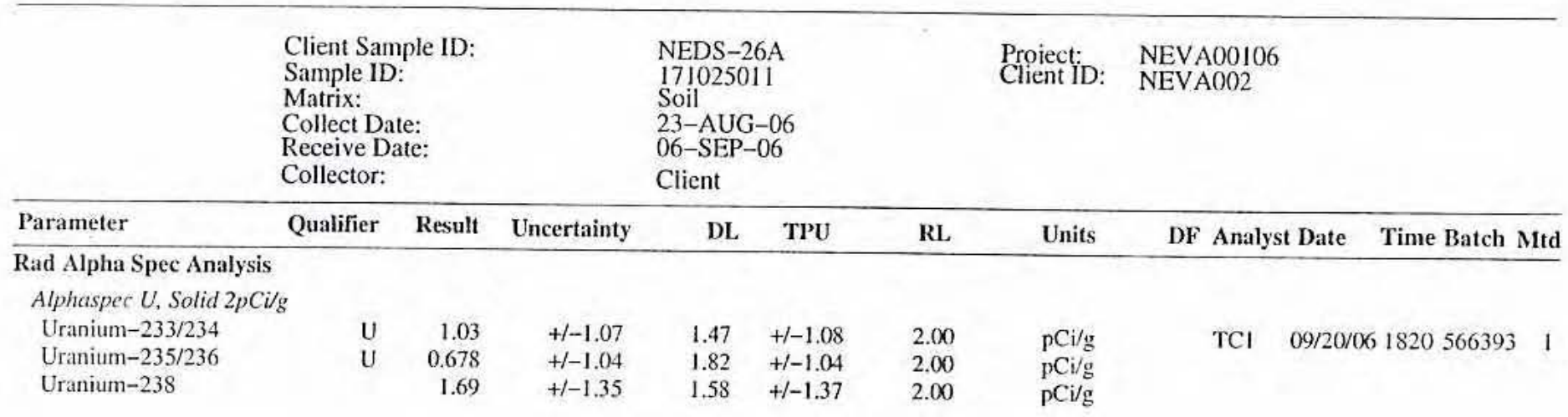

The following Prep Methods were performed

\begin{tabular}{llllll}
\hline Method & Description & Analyst & Date & Time & Prep Batch \\
\hline Dry Soil Prep & Dry Soil Prep GL-RAD-A-021 & AXP2 & $09 / 07 / 06$ & 1306 & 565885
\end{tabular}

The following Analytical Methods were performed

\begin{tabular}{ll} 
Method & Description \\
\hline 1 & DOE EML HASL-300, U-02-RC Modified
\end{tabular}

\begin{tabular}{llcc} 
Surrogate/Tracer recovery & Test & Recovery\% & Acceptable Limits \\
\hline Uranium-232 & Alphaspec U, Solid 2pCi/g & 93 & $(25 \%-125 \%)$
\end{tabular}

Notes:

The Qualifiers in this report are defined as follows:

* A quality control analyte recovery is outside of specified acceptance criteria

$<$ Resuit is less than value reported

$>$ Result is greater than value reported

A The TIC is a suspected aldol-condensation product

B Target analyte was detected in the associated blank

$\mathrm{BD}$ Results are either below the MDC or tracer recovery is low

C Analyte has been confirmed by GC/MS analysis

D Results are reported from a diluted aliquot of the sample

$\mathrm{H}$ Analytical holding time was exceeded

$\mathrm{J}$ Value is estimated

N/A Spike recovery limits do not apply. Sample concentration exceeds spike concentration by $4 \mathrm{X}$ or more

$\mathrm{R}$ Sample results are rejected

$\mathrm{U}$ Analyte was analyzed for, but not detected above the MDL, MDA, or LOD.

UI Gamma Spectroscopy-Uncertain identification 


\section{GENERAL ENGINEERING LABORATORIES, LLC \\ 2040 Savage Road Charleston SC 29407 - (843) 556-8171 - www.gel.com}

\section{Certificate of Analysis}

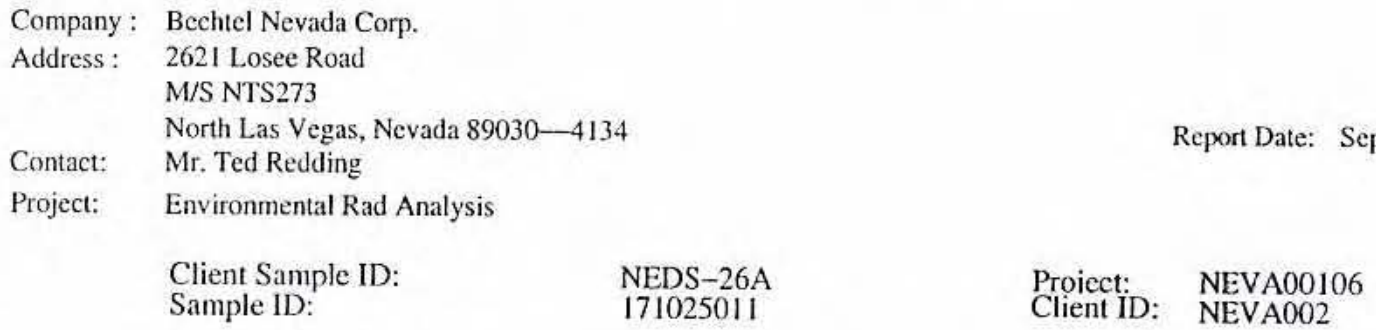

$X$ Consult Case Narrative, Data Summary package, or Project Manager concerning this qualifier

Y QC Samples were not spiked with this compound

$\wedge$ RPD of sample and duplicate evaluated using $+/-\mathrm{RL}$. Concentrations are $<5 \mathrm{X}$ the RL

h Preparation or preservation holding time was exceeded

The above sample is reported on a dry weight basis. 


\section{GENERAL ENGINEERING LABORATORIES, LLC \\ 2040 Savage Road Charleston SC 29407 - (843) 556-8171 - www.gel.com}

\section{Certificate of Analysis}

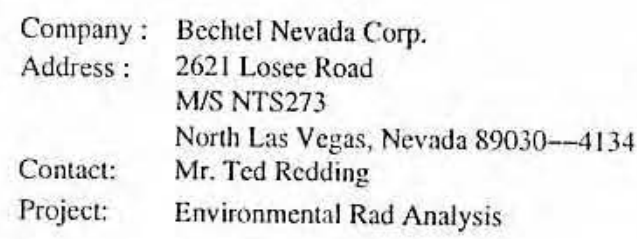

Client Sample ID:

Sample ID:

NEDS-26B

171025012

Matrix:

Collect Date:

Receive Date:

Collector:

Client

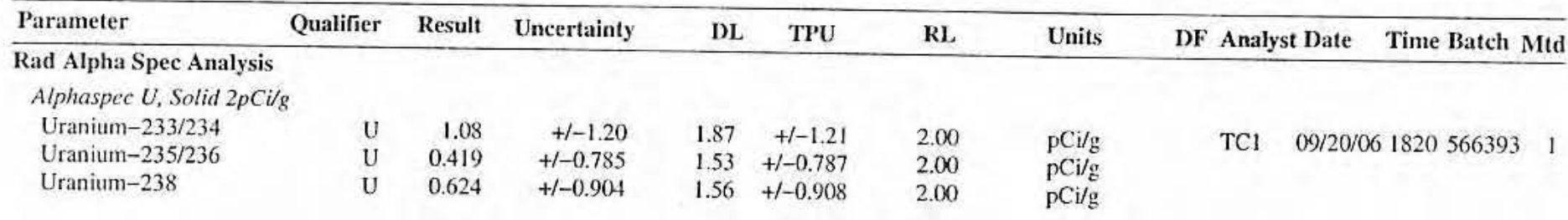

The following Prep Methods were performed

\begin{tabular}{llllll}
\hline Method & Description & Analyst & Date & Time & Prep Batch \\
\hline Dry Soil Prep & Dry Soil Prep GL-RAD-A-021 & AXP2 & $09 / 07 / 06$ & 1306 & 565885
\end{tabular}

The following Analytical Methods were performed

\begin{tabular}{ll} 
Method & Description \\
\hline 1 & DOE EML HASL-300, U-02-RC Modificd
\end{tabular}

\begin{tabular}{llcc} 
Surrogate/Tracer recovery & Test & Recovery\% & Acceptable Limits \\
\hline Uranium-232 & Alphaspec U, Solid 2pCi/g & 86 & $(25 \%-125 \%)$
\end{tabular}

Notes:

The Qualifiers in this report are defined as follows :

* A quality control analyte recovery is outside of specified acceptance criteria

$<$ Result is less than value reported

$>$ Result is greater than value reported

A The TIC is a suspected aldol-condensation product

B Target analyte was detected in the associated blank

$\mathrm{BD}$ Results are either below the MDC or tracer recovery is low

C. Analyte has been confirmed by GC/MS analysis

D Results are reported from a diluted aliquot of the sample

$\mathrm{H}$ Analytical holding time was exceeded

$\mathrm{J}$ Value is estimated

N/A Spike recovery limits do not apply. Sample concentration exceeds spike concentration by $4 \mathrm{X}$ or more

$\mathrm{R}$ Sample results are rejected

U Analyte was analyzed for, but not detected above the MDL, MDA, or LOD.

UI Gamma Spectroscopy--Uncertain identification

X Consult Case Narrative, Data Summary package, or Project Manager concerning this qualifier 


\section{GENERAL ENGINEERING LABORATORIES, LLC \\ 2040 Savage Road Charleston SC 29407 - (843) 556-8171 - www.gel.com}

\section{Certificate of Analysis}

Company : Bechtel Nevada Corp.

Address : 2621 Losec Road

M/S NTS273

North Las Vegas, Nevada $89030-4134$

Contact: Mr. Ted Redding

Report Date: September 26, 2006

Project: Environmental Rad Analysis

Client Sample ID: $\quad$ NEDS-26B

Sample ID: $\quad 171025012$

Proiect: NEVA00106

Client ID: NEVAO02

Y QC Samples were not spiked with this compound

RPD of sample and duplicate evaluated using $+/-\mathrm{RL}$. Concentrations are $<5 X$ the RL

h Preparation or preservation holding time was exceeded

The above sample is reported on a dry weight basis. 


\section{GENERAL ENGINEERING LABORATORIES, LLC \\ 2040 Savage Road Charleston SC 29407 - (843) 556-8171 - www.gel.com}

\section{Certificate of Analysis}

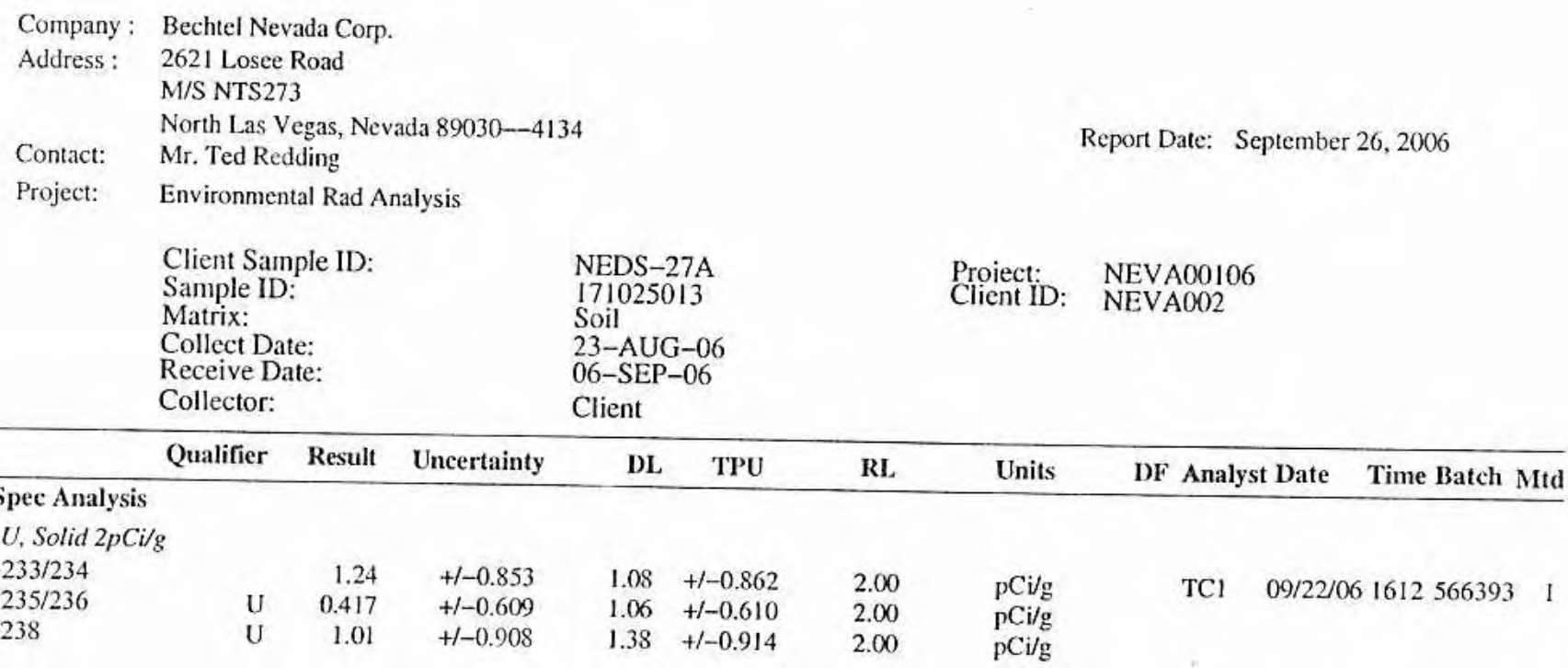

The following Prep Methods were performed

\begin{tabular}{llllll}
\hline Method & Description & Analyst & Date & Time & Prep Batch \\
\hline Dry Soil Prep & Dry Soil Prep GL-RAD-A-021 & AXP2 & $09 / 07 / 06$ & 1306 & 565885
\end{tabular}

The following Analytical Methods were performed

\begin{tabular}{ll} 
Method & Description \\
\hline 1 & DOE EML HASL-300, U-02-RC Modified \\
2 & DOE EML HASL-300, U-02-RC Modified
\end{tabular}

\begin{tabular}{llcc} 
Surrogate/Tracer recovery & Test & Recovery\% & Acceptable Limits \\
\hline Uranium-232 & Alphaspec U, Solid 2pCi/g & 91 & $(25 \%-125 \%)$
\end{tabular}

Notes:

The Qualifiers in this report are defined as follows :

* A quality control analyte recovery is outside of specified acceptance criteria

$<$ Result is less than value reported

$>$ Result is greater than value reported

A The TIC is a suspected aldol-condensation product

B Target analyte was detected in the associated blank

$\mathrm{BD}$ Results are either below the MDC or tracer recovery is low

C Analy te has been confirmed by GC/MS analysis

D Results are reported from a diluted aliquot of the sample

$\mathrm{H}$ Analytical holding time was exceeded

$\mathrm{J}$ Value is estimated

N/A Spike recovery limits do not apply. Sample concentration exceeds spike concentration by $4 \mathrm{X}$ or more

$\mathrm{R}$ Sample results are rejected

U Analyte was analyzed for, but not detected above the MDL, MDA, or LOD.

UI Gamma Spectroscopy--Uncertain identification 


\section{GENERAL ENGINEERING LABORATORIES, LLC \\ 2040 Savage Road Charleston SC 29407 - (843) 556-8171 - www.gel.com}

\section{Certificate of Analysis}

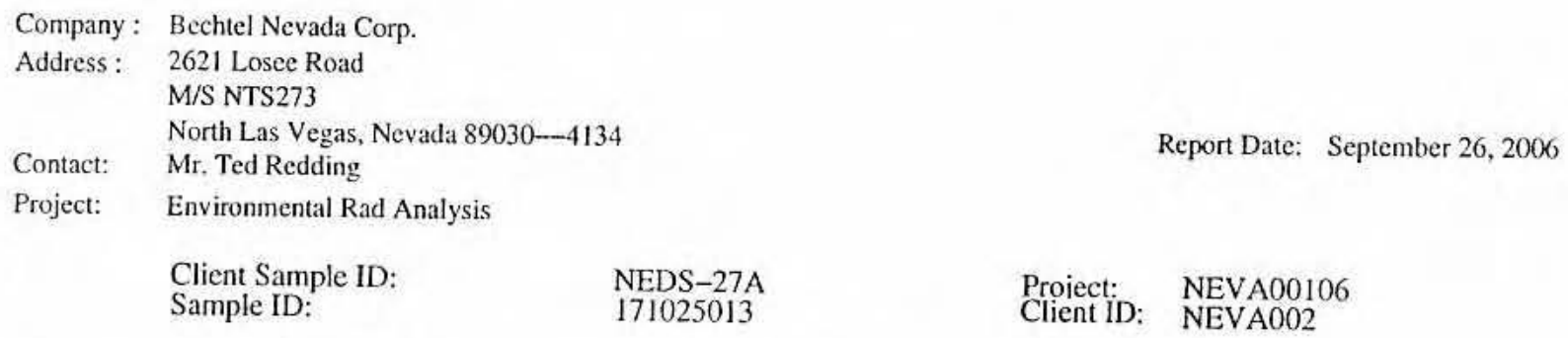

\footnotetext{
$X$ Consult Case Narrative, Data Summary package, or Project Manager concerning this qualifier

Y QC Samples were not spiked with this compound

$\wedge \quad$ RPD of sample and duplicate evaluated using $+/-$ RL. Concentrations are $<5 X$ the RL .

$\mathrm{h}$ Preparation or preservation holding time was exceeded

The above sample is reported on a dry weight basis.
} 


\section{GENERAL ENGINEERING LABORATORIES, LLC \\ 2040 Savage Road Charleston SC 29407 - (843) 556-8171 - www.gel.com}

\section{Certificate of Analysis}

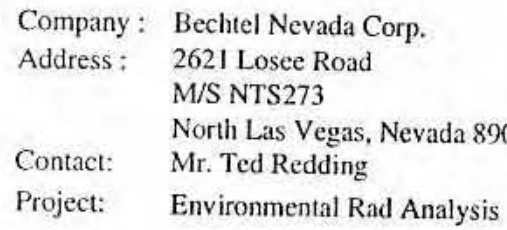

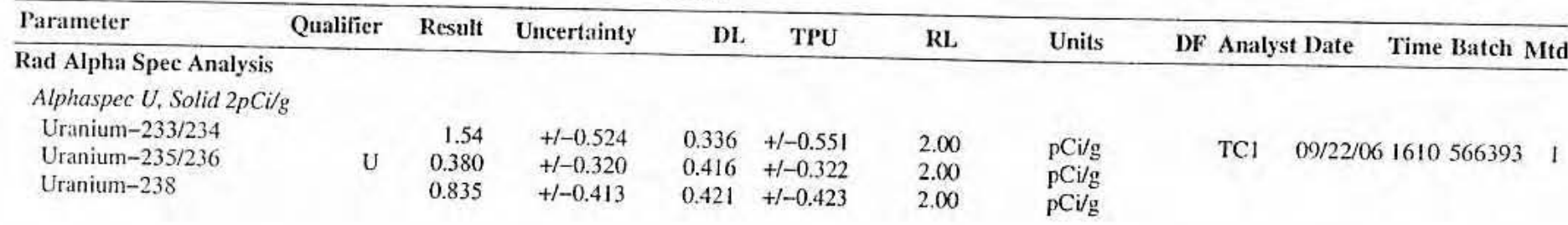

The following Prep Methods were performed

\begin{tabular}{llllll}
\hline Method & Description & Analyst & Date & Time & Prep Batch \\
\hline Dry Soil Prep & Dry Soil Prep GL-RAD-A-021 & AXP2 & $09 / 07 / 06$ & 1306 & 565885
\end{tabular}

The following Analytical Methods were performed

\begin{tabular}{ll} 
Method & Description \\
\hline 1 & DOE EML HASL-300, U-02-RC Modified \\
2 & DOE EML HASL-300, U-02-RC Modified
\end{tabular}

\begin{tabular}{llcc} 
Surrogate/Tracer recovery & Test & Recovery\% & Acceptable Limits \\
\hline Uranium-232 & Alphaspec U, Solid 2pCi/g & 91 & $(25 \%-125 \%)$
\end{tabular}

Notes:

The Qualifiers in this report are defined as follows :

* A quality control analyte recovery is outside of specified acceptance criteria

$<$ Result is less than value reported

$>$ Result is greater than value reported

A The TIC is a suspected aldol-condensation product

B Target analyte was detected in the associated blank

BD Results are either below the MDC or tracer recovery is low

C Analyte has been confirmed by GC/MS analysis

D Results are reported from a diluted aliquot of the sample

$\mathrm{H}$ Analytical holding time was exceeded

$\mathrm{J}$ Value is estimated

N/A Spike recovery limits do not apply. Sample concentration exceeds spike concentration by $4 X$ or more

R Sample results are rejected

U Analyte was analyzed for, but not detected above the MDL, MDA, or LOD.

UI Gamma Spectroscopy--Uncertain identification 


\section{GENERAL ENGINEERING LABORATORIES, LLC \\ 2040 Savage Road Charleston SC 29407 - (843) 556-8171 - www.gel.com}

\section{Certificate of Analysis}

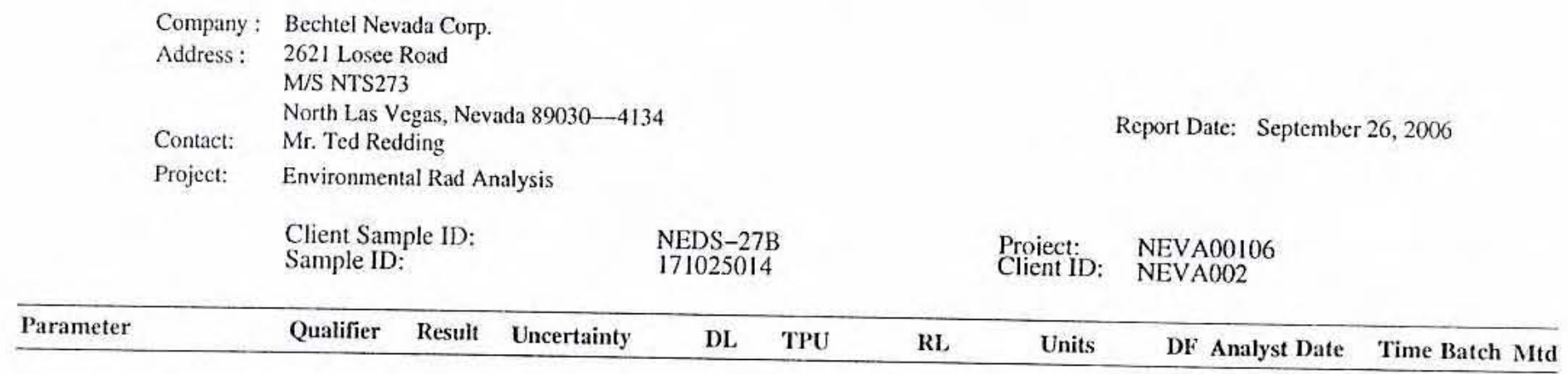

$X$ Consult Case Narrative, Data Summary package, or Project Manager concerning this qualifier

Y QC Samples were not spiked with this compound

$\hat{R}$ RPD of sample and duplicate evaluated using $+/-\mathrm{RL}$. Concentrations are $<5 \mathrm{X}$ the RL

$\mathrm{h}$ Preparation or preservation holding time was exceeded

The above sample is reported on a dry weight basis. 


\section{GENERAL ENGINEERING LABORATORIES, LLC \\ 2040 Savage Road Charleston SC 29407 - (843) 556-8171 - www. gel.com}

\section{Certificate of Analysis}

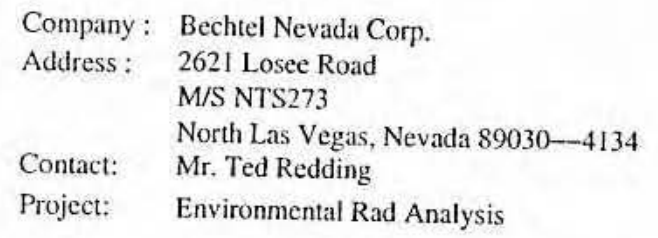

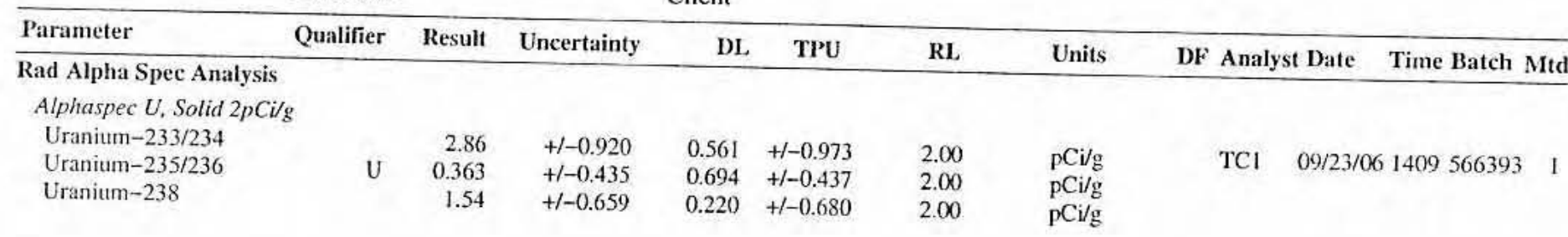

The following Prep Methods were performed

\begin{tabular}{|c|c|}
\hline Method & Description \\
\hline Dry Soil Prep & Dry Soil Prep GL-RAD-A-021 \\
\hline \multicolumn{2}{|c|}{ The following Analytical Methods were performed } \\
\hline Method & Description \\
\hline 1 & DOE EML HASL-300, U-02-RC Modified \\
\hline 2 & DOE EML HASL-300, U-02-RC Modified \\
\hline
\end{tabular}

\begin{tabular}{llcc} 
Surrogate/Tracer recovery & Test & Recovery\% & Acceptable Limits \\
\hline Uranium-232 & Alphaspec U, Solid $2 \mathrm{pCi} / \mathrm{g}$ & 88 & $(25 \%-125 \%)$
\end{tabular}

Notes:

The Qualifiers in this report are defined as follows :

* A quality control analyte recovery is outside of specified acceptance criteria

$<$ Result is less than value reported

$>$ Result is greater than value reported

A The TIC is a suspected aldol-condensation product

B Target analyte was detected in the associated blank

BD Results are either below the MDC or tracer recovery is low

C Analyte has been confirmed by GC/MS analysis

D Results are reported from a diluted aliquot of the sample

H Analytical holding time was exceeded

$\mathrm{J}$ Value is estimated

N/A Spike recovery limits do not apply. Sample concentration exceeds spike concentration by $4 X$ or more
$R$ Sample results are rejected

$U$ Analyte was analyzed for, but not detected above the MDL, MDA, or LOD.

UI Gamma Spectroscopy-Uncertain identification 


\section{GENERAL ENGINEERING LABORATORIES, LLC \\ 2040 Savage Road Charleston SC 29407 - (843) 556-8171 - www.gel.com}

\section{Certificate of Analysis}

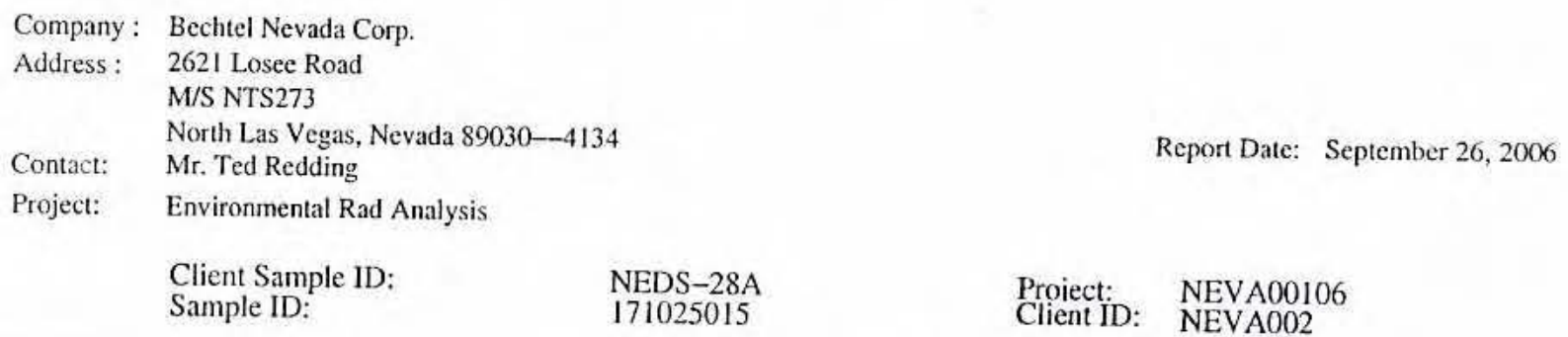

$\mathrm{X}$ Consult Case Narrative, Data Summary package, or Project Manager concerning this qualifier

Y QC Samples were not spiked with this compound

$\hat{R}$ RD of sample and duplicate evaluated using $+/-\mathrm{RL}$. Concentrations are $<5 \mathrm{X}$ the RL

$\mathrm{h}$ Preparation or preservation holding time was exceeded

The above sample is reported on a dry weight basis. 


\section{GENERAL ENGINEERING LABORATORIES, LLC \\ 2040 Savage Road Charleston SC 29407 - (843) 556-8171 - www.gel.com}

\section{Certificate of Analysis}

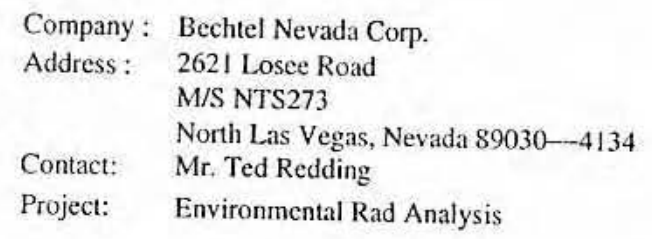

Client Sample ID:

Sample ID:

Matrix:

Collect Date:

Receive Date:

Collector:
Report Date: September 26, 2006

NEDS-28B
171025016
Soil
23-AUG-06
06-SEP-06
Client

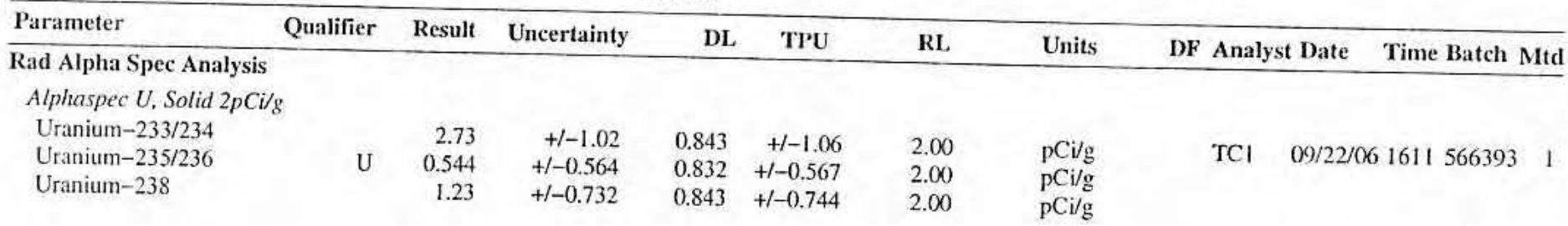

The following Prep Methods were performed

\begin{tabular}{llllll}
\hline Method & Description & Analyst & Date & Time & Prep Batch \\
\hline Dry Soil Prep & Dry Soil Prep GL-RAD-A-021 & AXP2 & $09 / 07 / 06$ & 1306 & 565885
\end{tabular}

The following Analytical Methods were performed

\begin{tabular}{ll} 
Method & Description \\
\hline 1 & DOE EML HASL-300, U-02-RC Modified \\
2 & DOE EML HASL-300, U-02-RC Modified
\end{tabular}

\begin{tabular}{llcc} 
Surrogate/Tracer recovery & Test & Recovery\% & Acceptable Limits \\
\hline Uranium-232 & Alphaspec U, Solid 2pCi/g & 87 & $(25 \%-125 \%)$
\end{tabular}

Notes:

The Qualifiers in this report are defined as follows :

* A quality control analyte recovery is outside of specified acceptance criteria

$<$ Result is less than value reported

$>$ Result is greater than value reported

A The TIC is a suspected aldol-condensation product

B Target analyte was detected in the associated blank

$\mathrm{BD}$ Results are either below the MDC or tracer recovery is low

C Analyte has been confirmed by GC/MS analysis

D Results are reported from a diluted aliquot of the sample

$\mathrm{H}$ Analytical holding time was exceeded

$\mathrm{J}$ Value is estimated

N/A Spike recovery limits do not apply. Sample concentration exceeds spike concentration by $4 \mathrm{X}$ or more
$\mathrm{R}$ Sample results are rejected

U Analyte was analyzed for, but not detected above the MDL, MDA, or LOD.

UI Gamma Spectroscopy-Uncertain identification 


\section{GENERAL ENGINEERING LABORATORIES, LLC \\ 2040 Savage Road Charleston SC 29407 - (843) 556-8171 - www. gel.com}

\section{Certificate of Analysis}

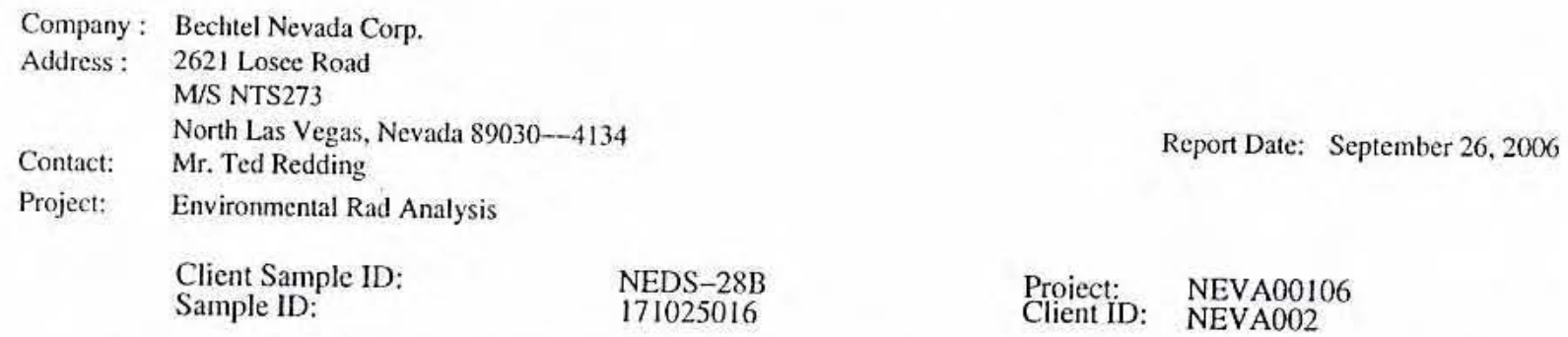

X Consult Case Narrative, Data Summary package, or Project Manager concerning this qualifier

Y QC Samples were not spiked with this compound

$\hat{A}$ RPD of sample and duplicate evaluated using $+/-\mathrm{RL}$. Concentrations are $<5 \mathrm{X}$ the RL

$\mathrm{h}$ Preparation or preservation holding time was exceeded

The above sample is reported on a dry weight basis. 


\section{GENERAL ENGINEERING LABORATORIES, LLC \\ 2040 Savage Road Charleston SC 29407 - (843) 556-8171 - www.gel.com}

\section{Certificate of Analysis}

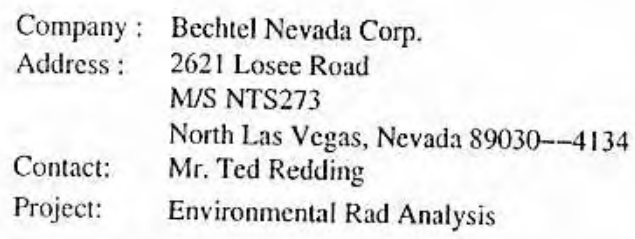

Client Sample ID:

Sample ID:

NEDS-29A

171025017

Soil

Matrix:

23-AUG-06

Receive Date:

06-SEP-06

Collector:

Client

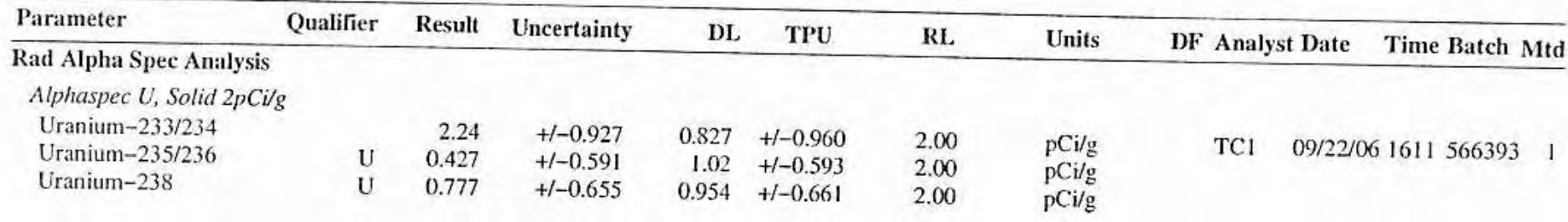

The following Prep Methods were performed

\begin{tabular}{llllll}
\hline Method & Description & Analyst & Date & Time & Prep Batch \\
\hline Dry Soil Prep & Dry Soil Prep GL-RAD-A-02i & AXP2 & $09 / 07 / 06$ & 1306 & 565885
\end{tabular}

The following Analytical Methods were performed

\begin{tabular}{ll} 
Method & Description \\
\hline I & DOE EML HASL-300, U-02-RC Modified \\
2 & DOE EML HASL-300, U-02-RC Modified
\end{tabular}

\begin{tabular}{llcc} 
Surrogate/Tracer recovery & Test & Recovery $\%$ & Acceptable Limits \\
\hline Uranium-232 & Alphaspec U, Solid 2pCi/g & 76 & $(25 \%-125 \%)$
\end{tabular}

Notes:

The Qualifiers in this report are defined as follows:

* A quality control analyte recovery is outside of specified acceptance criteria

$<$ Result is less than value reported

$>$ Result is greater than value reported

A The TIC is a suspected aldol-condensation product

$\mathrm{B}$ Target analyte was detected in the associated blank

$\mathrm{BD}$ Results are either below the MDC or tracer recovery is low

C Analyte has been confirmed by GC/MS analysis

D Results are reported from a diluted aliquot of the sample

$\mathrm{H}$ Analytical holding time was exceeded

$\mathrm{J}$ Value is estimated

N/A Spike recovery limits do not apply. Sample concentration exceeds spike concentration by $4 X$ or more
$R$ Sample results are rejected

$\mathrm{U}$ Analyte was analyzed for, but not detected above the MDL, MDA, or LOD.

UI Gamma Spectroscopy-Uncertain identification 


\section{GENERAL ENGINEERING LABORATORIES, LLC \\ 2040 Savage Road Charleston SC 29407 - (843) 556-8171 - www.gel.com}

\section{Certificate of Analysis}

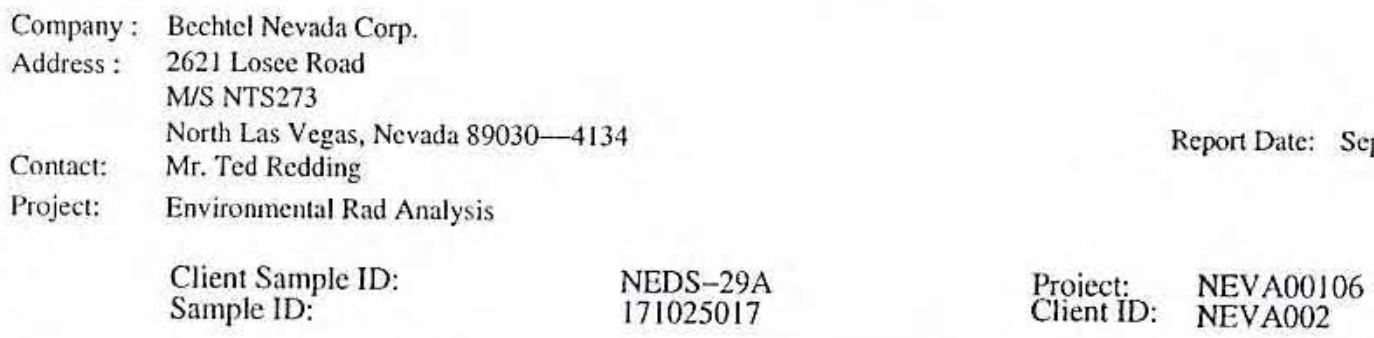

\begin{tabular}{llllllll}
\hline Parameter & Qualifier & Result Uncertainty & DL & TPU & RL & Units & DF Analyst Date Time Batch Mtd
\end{tabular}

$\mathrm{X}$ Consult Case Narrative, Data Summary package, or Project Manager concerning this qualifier

Y QC Samples were not spiked with this compound

$\wedge \quad$ RPD of sample and duplicate evaluated using $+/-\mathrm{RL}$. Concentrations are $<5 \mathrm{X}$ the $\mathrm{RL}$

h Preparation or preservation holding time was exceeded

The above sample is reported on a dry weight basis. 


\section{GENERAL ENGINEERING LABORATORIES, LLC \\ 2040 Savage Road Charleston SC 29407 - (843) 556-8171 - www.gel.com}

\section{Certificate of Analysis}

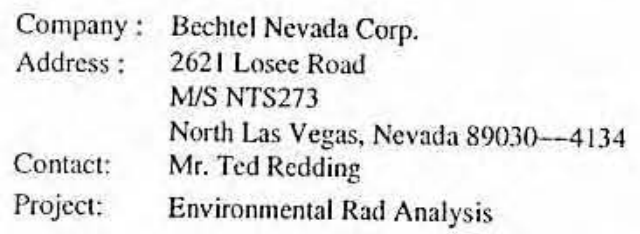

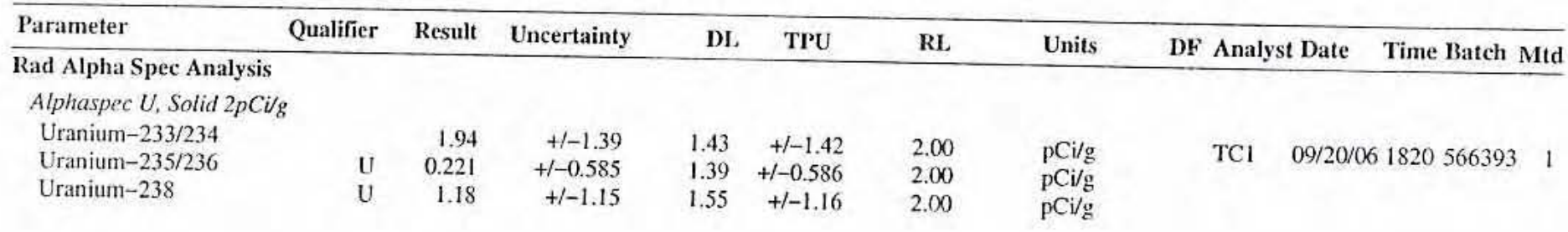

The following Prep Methods were performed

\begin{tabular}{llllll}
\hline Method & Description & Analyst & Date & Time & Prep Batch \\
\hline Dry Soil Prep & Dry Soil Prep GL-RAD-A-021 & AXP2 & $09 / 07 / 06$ & 1306 & 565885
\end{tabular}

The following Analytical Methods were performed

Method Deseription

\begin{tabular}{llcc} 
Surrogate/Tracer recovery & Test & Recovery\% & Acceptable Limits \\
\hline Uranium-232 & Alphaspec U, Solid $2 \mathrm{pCi} / \mathrm{g}$ & 91 & $(25 \%-125 \%)$
\end{tabular}

Notes:

The Qualifiers in this report are defined as follows :

* A quality control analyte recovery is outside of specified acceptance criteria

$<$ Result is less than value reported

$>$ Result is greater than value reported

A The TIC is a suspected aldol-condensation product

B Target analyte was detected in the associated blank

BD Results are either below the MDC or tracer recovery is low

C Analyte has been confirmed by GC/MS analysis

D Results are reported from a diluted aliquot of the sample

$\mathrm{H}$ Analytical holding time was exceeded

$\mathrm{J}$ Value is estimated

N/A Spike recovery limits do not apply. Sample concentration exceeds spike concentration by $4 \mathrm{X}$ or more

R Sample results are rejected

U Analyte was analyzed for, but not detected above the MDL, MDA, or LOD.

UI Gamma Spectroscopy-Uncertain identification

X Consult Case Narrative, Data Summary package, or Project Manager concerning this qualifier 


\section{GENERAL ENGINEERING LABORATORIES, LLC \\ 2040 Savage Road Charleston SC 29407 - (843) 556-8171 - www.gel.com}

\section{Certificate of Analysis}

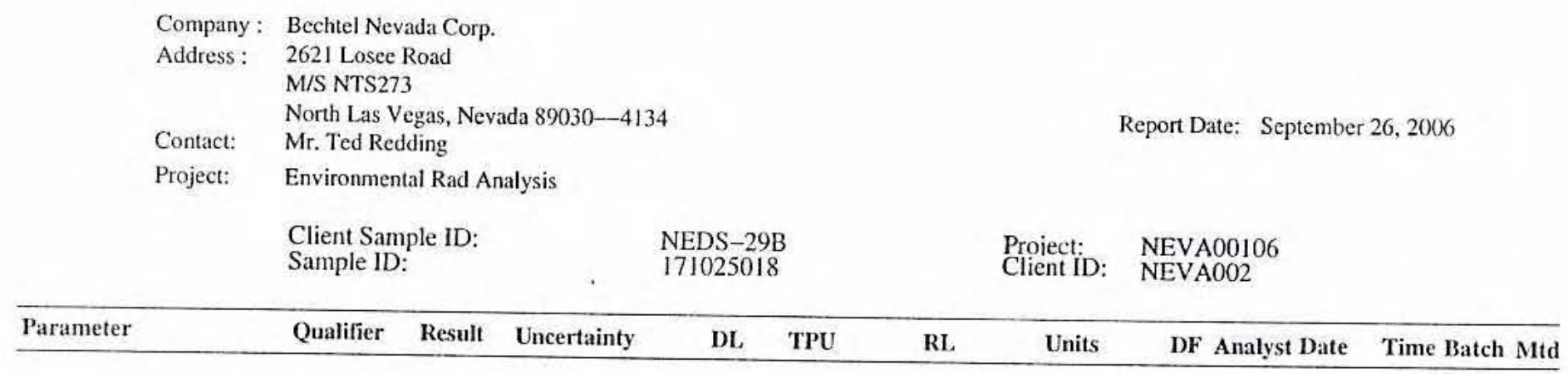

Y QC Samples were not spiked with this compound

$\hat{R}$ RPD of sample and duplicate evaluated using $+/-\mathrm{RL}$. Concentrations are $<5 \mathrm{X}$ the $\mathrm{RL}$

h Preparation or preservation holding time was exceeded

The above sample is reported on a dry weight basis. 


\section{GENERAL ENGINEERING LABORATORIES, LLC \\ 2040 Savage Road Charleston SC 29407 - (843) 556-8171 - www.gel.com}

\section{Certificate of Analysis}

Company: Bechtel Nevada Corp.

Address: 2621 Losee Road

M/S NTS273

$\begin{array}{ll} & \text { North Las Vegas, Nevada } 89030--4134 \\ \text { Contact: } & \text { Mr. Ted Redding }\end{array}$

Report Date: September 26, 2006

Project: Environmental Rad Analysis

\begin{tabular}{|c|c|c|c|c|c|c|c|c|c|c|c|c|}
\hline \multirow[b]{2}{*}{ Parameter } & \multicolumn{2}{|c|}{$\begin{array}{l}\text { Client Sample ID: } \\
\text { Sample ID: } \\
\text { Matrix: } \\
\text { Collect Date: } \\
\text { Receive Date: } \\
\text { Collector: }\end{array}$} & & \multicolumn{2}{|c|}{$\begin{array}{l}\text { NEDS-30A } \\
171025019 \\
\text { Soil } \\
23-A U G-06 \\
06-\text { SEP-06 } \\
\text { Client }\end{array}$} & \multirow[b]{2}{*}{$\mathrm{RL}$} & \multirow{2}{*}{$\begin{array}{l}\text { Proiect: } \\
\text { Client ID: } \\
\text { Units }\end{array}$} & \multicolumn{5}{|c|}{$\begin{array}{l}\text { NEVA00106 } \\
\text { NEVA002 }\end{array}$} \\
\hline & Qualifier & Result & Uncertainty & DL & TPU & & & DF & Analyst & t Date & Time Batch N & Mtd \\
\hline \multicolumn{10}{|c|}{ Rad Alpha Spec Analysis } & \multicolumn{3}{|c|}{ Alphaspec $U$, Solid $2 p C i / g$} \\
\hline $\begin{array}{l}\text { Uranium-233/234 } \\
\text { Uranium-235/236 } \\
\text { Uranium-238 }\end{array}$ & U & $\begin{array}{r}2.04 \\
0.473 \\
1.21\end{array}$ & $\begin{array}{l}+/-0.750 \\
+/-0.535 \\
+/-0.573\end{array}$ & $\begin{array}{l}0.611 \\
0.872 \\
0.488\end{array}$ & $\begin{array}{l}+1-0.783 \\
+1-0.538 \\
+1-0.588\end{array}$ & $\begin{array}{l}2.00 \\
2.00 \\
2.00\end{array}$ & $\begin{array}{l}\mathrm{pCi} / \mathrm{g} \\
\mathrm{pCi} / \mathrm{g} \\
\mathrm{pCi} / \mathrm{g}\end{array}$ & & $\mathrm{TCl}$ & $09 / 22 / 0$ & 61611566393 & 1 \\
\hline
\end{tabular}

The following Prep Methods were performed

\begin{tabular}{llllll}
\hline Method & Description & Analyst & Date & Time & Prep Batch \\
\hline Dry Soil Prep & Dry Soil Prep GL-RAD-A-021 & AXP2 & $09 / 07 / 06$ & 1307 & 565885
\end{tabular}

The following Analytical Methods were performed

\begin{tabular}{ll} 
Method & Description \\
\hline 1 & DOE EML HASL-300, U-02-RC Modified \\
2 & DOE EML HASL-300, U-02-RC Modified
\end{tabular}

\begin{tabular}{llcc} 
Surrogate/Tracer recovery & Test & Recovery\% & Acceptable Limits \\
\hline Uranium-232 & Alphaspec U, Solid 2pCi/g & 90 & $(25 \%-125 \%)$
\end{tabular}

Notes:

The Qualifiers in this report are defined as follows :

* A quality control analyte recovery is outside of specified acceptance criteria

$<$ Result is less than value reported

$>$ Result is greater than value reported

A The TIC is a suspected aldol-condensation product

B Target analyte was detected in the associated blank

$\mathrm{BD}$ Results are either below the MDC or tracer recovery is low

C Analyte has been confirmed by GC/MS analysis

D Results are reported from a diluted aliquot of the sample

$\mathrm{H}$ Analytical holding time was exceeded

J Value is estimated

N/A Spike recovery limits do not apply. Sample concentration exceeds spike concentration by $4 \mathrm{X}$ or more

$\mathrm{R}$ Sample results are rejected

U Analyte was analyzed for, but not detected above the MDL, MDA, or LOD.

UI Gamma Spectroscopy-Uncertain identification 


\section{GENERAL ENGINEERING LABORATORIES, LLC \\ 2040 Savage Road Charleston SC 29407 - (843) 556-8171 - www.gel.com}

\section{Certificate of Analysis}

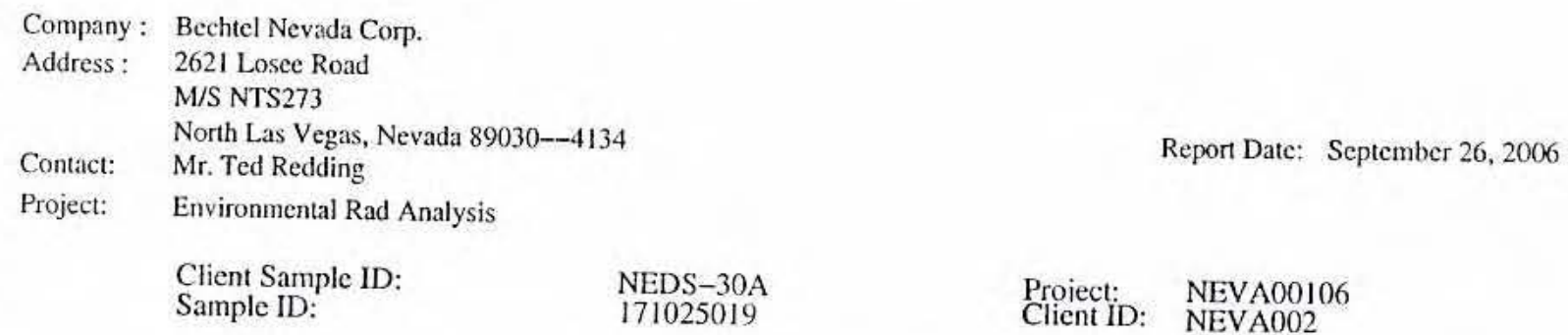

$\mathrm{X}$ Consult Case Narrative, Data Summary package, or Project Manager concerning this qualifier

Y QC Samples were not spiked with this compound

$\hat{R P D}$ of sample and duplicate evaluated using $+/-\mathrm{RL}$. Concentrations are $<5 \mathrm{X}$ the RL

$\mathrm{h}$ Preparation or preservation holding time was exceeded

The above sample is reported on a dry weight basis. 


\section{GENERAL ENGINEERING LABORATORIES, LLC \\ 2040 Savage Road Charleston SC 29407 - (843) 556-8171 - www.gel.com}

\section{Certificate of Analysis}

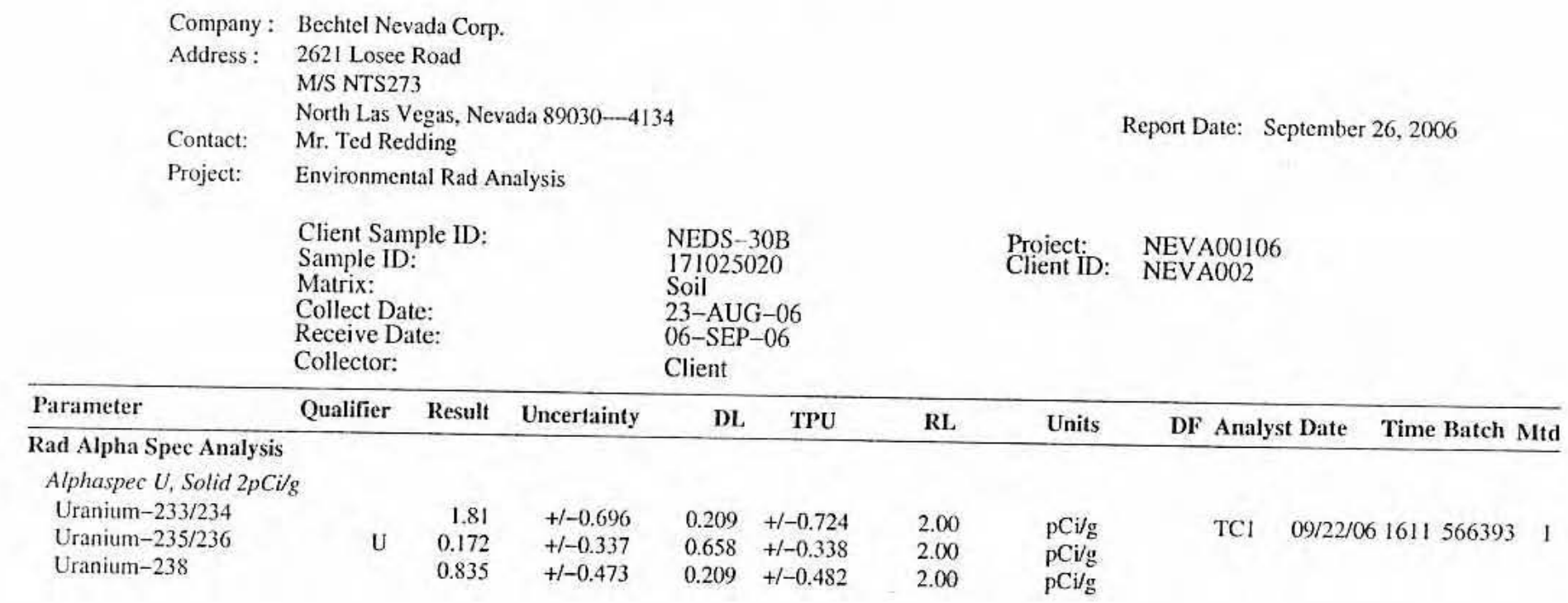

The following Prep Methods were performed

\begin{tabular}{llllll}
\hline Method & Description & Analyst & Date & Time & Prep Batch \\
\hline Dry Soil Prep & Dry Soil Prep GL-RAD-A-021 & AXP2 & $09 / 07 / 06$ & 1307 & 565885
\end{tabular}

The following Analytical Methods were performed

\begin{tabular}{ll} 
Method & Description \\
\hline & DOE EML HASL-300, U-02-RC Modified \\
2 & DOE EML HASL-300, U-02-RC Modified
\end{tabular}

\begin{tabular}{llcc} 
Surrogate/Tracer recovery & Test & Recovery\% & Acceptable Limits \\
\hline Uranium-232 & Alphaspec U, Solid 2pCi/g & 84 & $(25 \%-125 \%)$
\end{tabular}

Notes:

The Qualifiers in this report are cefined as follows :

* A quality control analyte recovery is outside of specified acceptance criteria

$<$ Result is less than value reported

$>$ Result is greater than value reported

A The TIC is a suspected aldol-condensation product

B Target analyte was detected in the associated blank

$\mathrm{BD}$ Results are either below the MDC or tracer recovery is low

C Analyte has been confirmed by GC/MS analysis

D Results are reported from a diluted aliquot of the sample

$\mathrm{H}$ Analytical holding time was exceeded

I Value is estimated

N/A Spike recovery limits do not apply. Sample concentration exceeds spike concentration by $4 \mathrm{X}$ or more

$R$ Sample results are rejected

$U$ Analyte was analyzed for, but not detected above the MDL, MDA, or LOD.

UI Gamma Spectroscopy--Uncertain identification 


\section{GENERAL ENGINEERING LABORATORIES, LLC \\ 2040 Savage Road Charleston SC 29407 - (843) 556-8171 - www.gel.com}

\section{Certificate of Analysis}

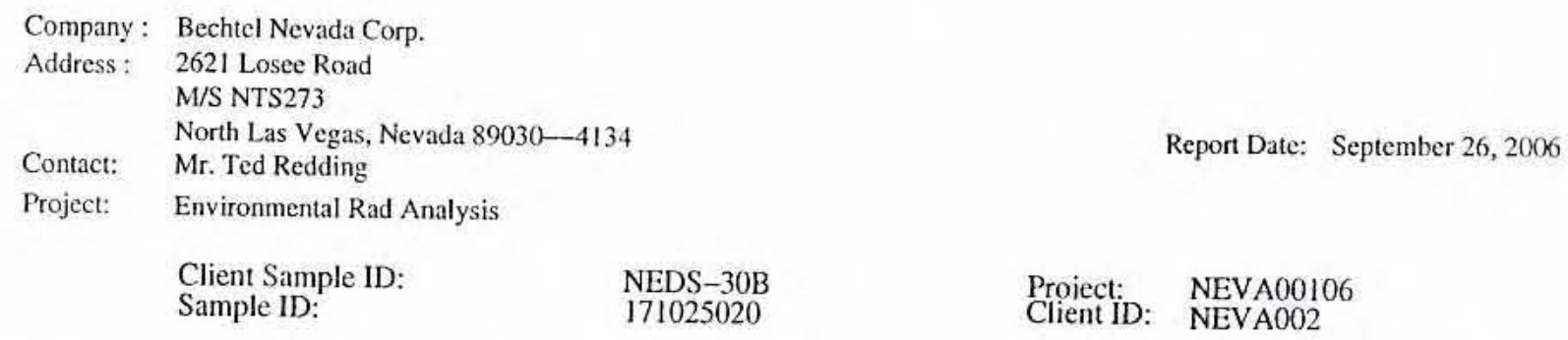

Y QC Samples were not spiked with this compound

$\wedge$ RPD of sample and duplicate evaluated using $+/-$ RL. Concentrations are $<5 X$ the RL

h Preparation or preservation holding time was exceeded

The above sample is reported on a dry weight basis. 


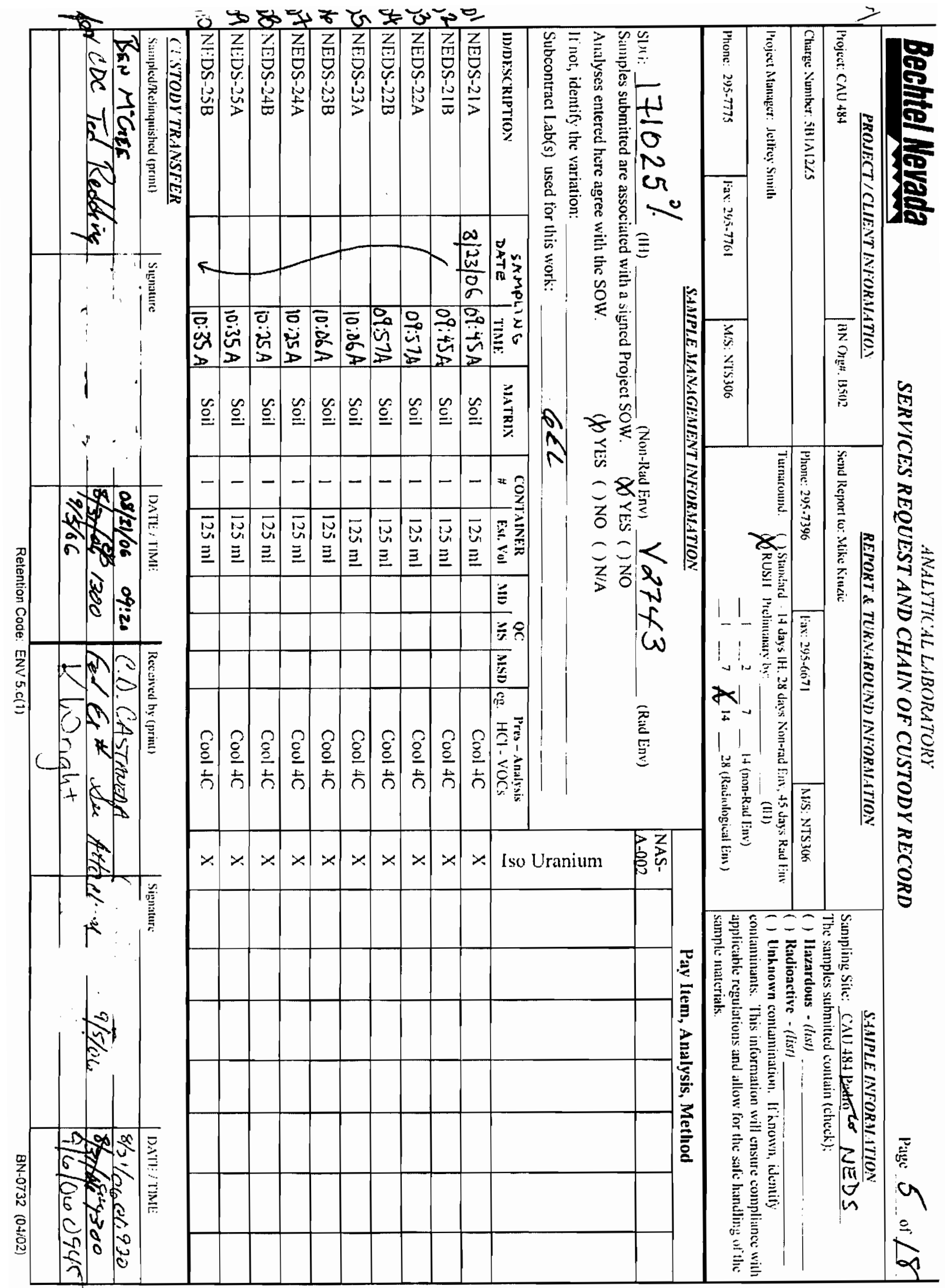




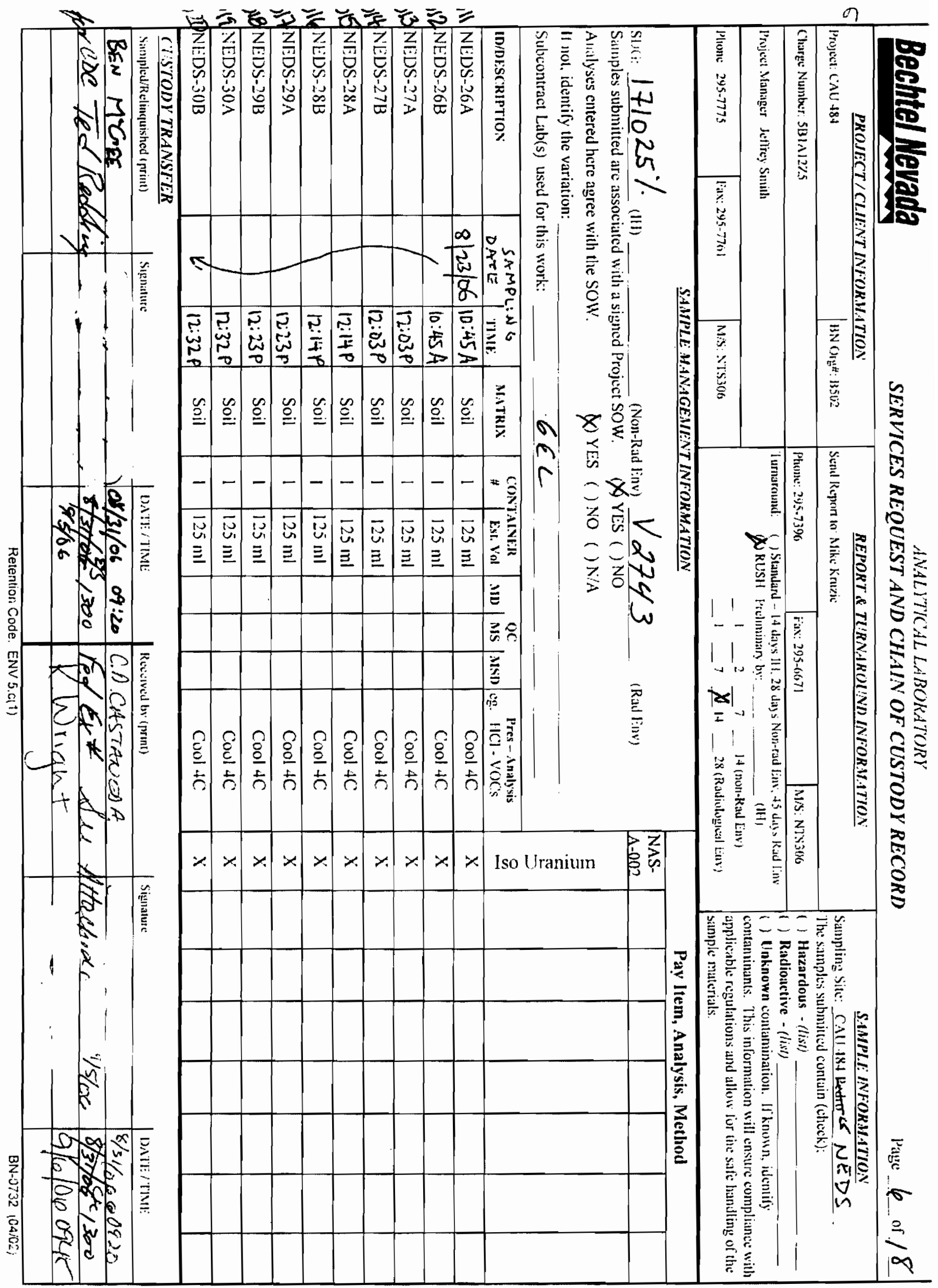




\section{GENERAL ENGINEERING LABORATORIES, LLC
2040 Savage Road Charleston SC \\ 2040 Savage Road Charleston SC 29407 - (843) 556-8171 - www.gel.com}

\section{Certificate of Analysis}

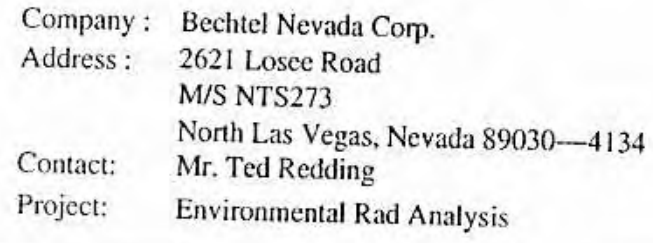

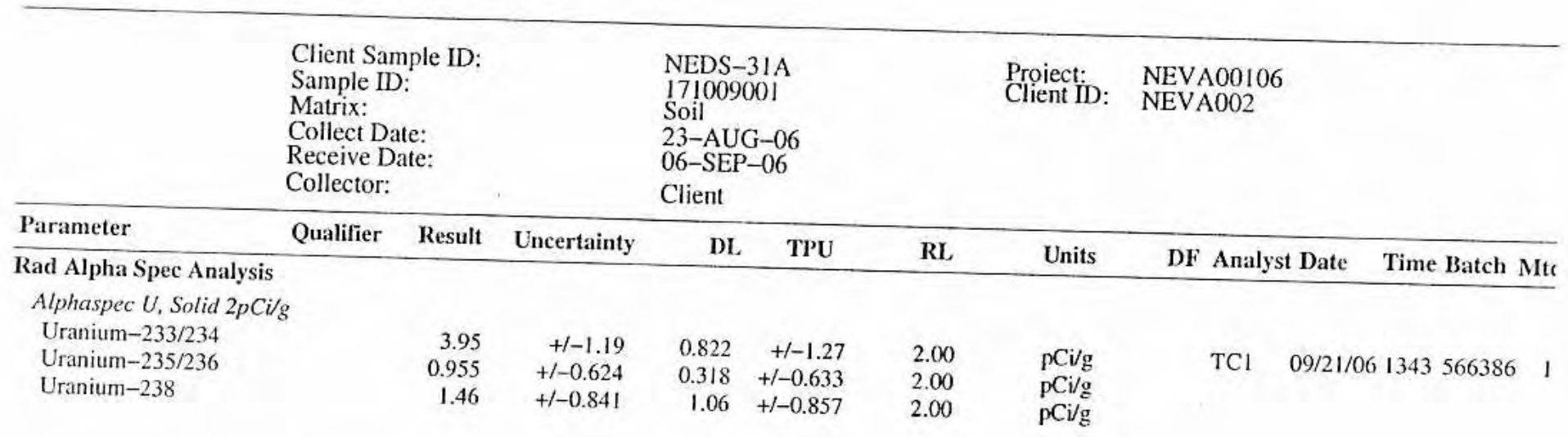

The following Prep Methods were performed

\begin{tabular}{llllll}
\hline Method & Description & Analyst & Date & Time & Prep Batch \\
\hline Dry Soil Prep & Dry Soil Prep GL-RAD-A-021 & JMB1 & $09 / 07 / 06$ & 0901 & 565866
\end{tabular}

The following Analytical Methods were performed

\begin{tabular}{ll}
\hline Method & Description \\
\hline I & DOE EML HASL-300, U-02-RC Modified \\
& DOE EML HASL-300, U-02-RC Modified
\end{tabular}

\begin{tabular}{llcc} 
Surrogate/Tracer recovery & Test & Recovery $\%$ & Acceptable Limits \\
\hline Uranium-232 & Alphaspec U, Solid 2pCi/g & 84 & $(25 \%-125 \%)$
\end{tabular}

Notes:

The Qualifiers in this report are defined as follows :

* A quality control analyte recovery is outside of specified acceptance criteria
$<\quad$ Result is less than value reported

$>$ Result is greater than value reported

A The TIC is a suspected aldol-condensation product

B Target analyte was detected in the associated blank

$\mathrm{BD}$ Results are either below the MDC or tracer recovery is low

C Analyte has been confirmed by GC/MS analysis

$\mathrm{H}$ Analytical holding trom a diluted aliquot of the sample

Analytical holding time was exceeded

I Value is estimated

N/A Spike recovery limits do not apply. Sample concentration exceeds spike concentration by $4 X$ or more
$R$ Sample results are rejected

U Analyte was analyzed for, but not detected above the MDL, MDA, or LOD. 


\section{GENERAL ENGINEERING LABORATORIES, LLC \\ 2040 Savage Road Charleston SC 29407 - (843) 556-8171 - www.gel.com}

\section{Certificate of Analysis}

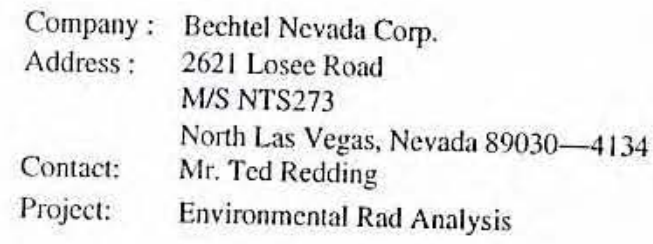

UI Gamma Spectroscopy--Uncertain identification

X Consult Case Narrative, Data Summary package, or Project Manager concerning this qualifier
Y QC Samples were not spiked with this compound

$\wedge \quad$ RPD of sample and duplicate evaluated using $+/-\mathrm{RL}$. Concentrations are $<5 \mathrm{X}$ the $\mathrm{RL}$

h Preparation or preservation holding time was exceeded

The above sample is reported on a dry weight basis. 


\section{GENERAL ENGINEERING LABORATORIES, LLC \\ 2040 Savage Foad Charleston SC 29407 - (843) 556-8171 - www.gel.com}

\section{Certificate of Analysis}

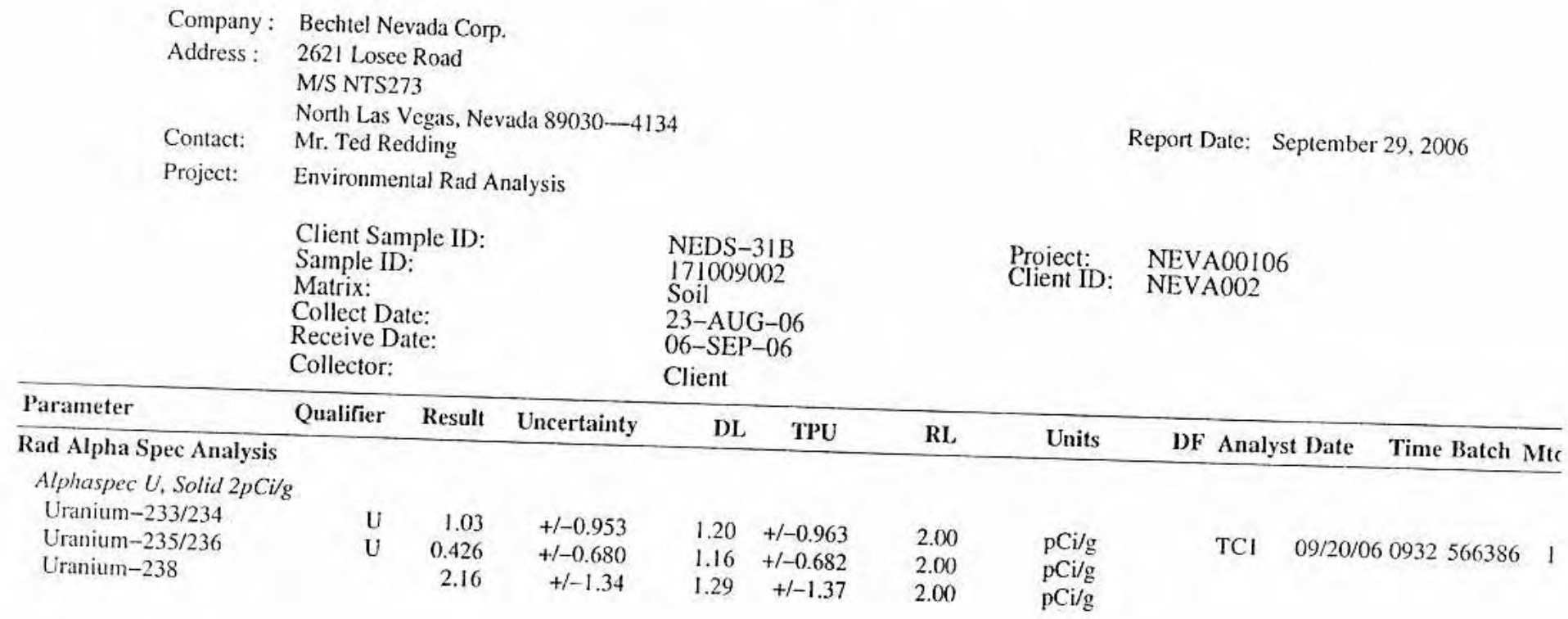

The following Prep Methods were performed

\begin{tabular}{llllll}
\hline Method & Description & Analyst & Date & Time & Prep Batch \\
\hline Dry Soil Prep & Dry Soil Prep GL-RAD-A-021 & JMB1 & $09 / 07 / 06$ & 0901 & 565866
\end{tabular}

The following Analytical Methods were performed

\begin{tabular}{ll} 
Method & Description \\
\hline & DOE EML HASL-300, U-02-RC Modified
\end{tabular}

Surrogate/Tracer recovery Test

$\begin{array}{cccc}\text { Uranium-232 } & \text { Alphaspec U, Solid 2pCi/g } & \text { Recovery \% } & \text { Acceptable Limits }\end{array}$

Notes:

The Qualifiers in this report are defined as follows :

* A quality control analyte recovery is outside of specified acceptance criteria

$>$ Result is greater than value reported

A The TIC is a suspected aldol-condensation product

$B$ Target analyte was detected in the associated blank

BD Results are either below the MDC or tracer recovery is low

C Analyte has been confirmed by GC/MS analysis

$\mathrm{H}$ Analytical holding time a diluted aliquot of the sample

J A a exceeded

$\mathrm{J}$ Value is estimated

N/A Spike recovery limits do not apply. Sample concentration exceeds spike concentration by $4 \mathrm{X}$ or more
$\mathrm{R}$ Sample results are rejected

$U$ Analyte was analyzed for

UI Gamma Spectroscopy--Uncertain detected above the MDL, MDA, or LOD.

$\mathrm{X}$ Consult Case Narrative, Data Summary package, or Project Manager concerning this qualifier 


\section{GENERAL ENGINEERING LABORATORIES, LLC \\ 2040 Savage Road Charleston SC 29407 - (843) 556-8171 - www.gel.com}

\section{Certificate of Analysis}

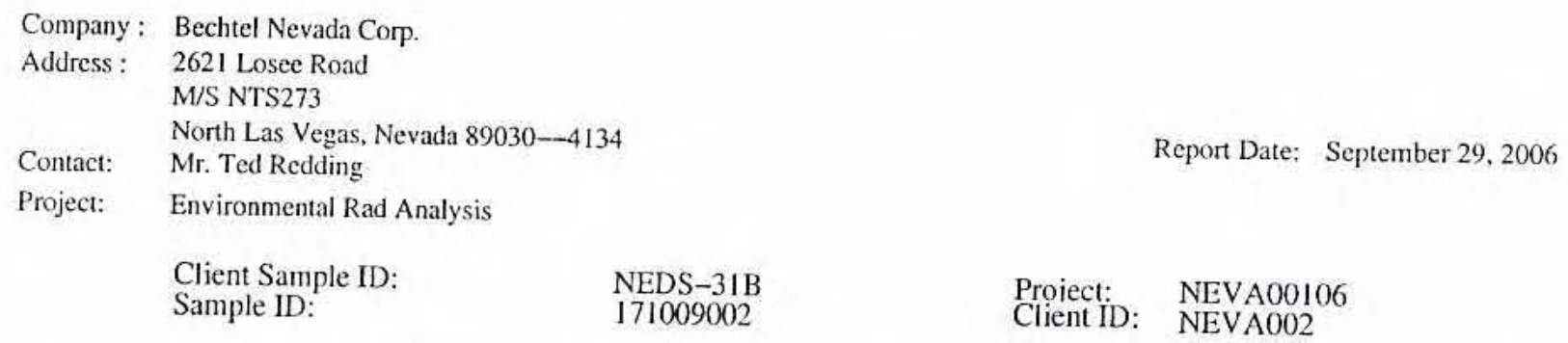

Y QC Samples were not spiked with this compound

$\hat{R}$ RPD of sample and duplicate evaluated using $+/-\mathrm{RL}$. Concentrations are $<5 \mathrm{X}$ the RL

$\mathrm{h}$ Preparation or preservation holding time was exceeded

The above sample is reported on a dry weight basis. 


\section{GENERAL ENGINEERING LABORATORIES, LLC \\ 2040 Savage Road Charleston SC 29407 - (843) 556-8171 - www.gel.com}

\section{Certificate of Analysis}

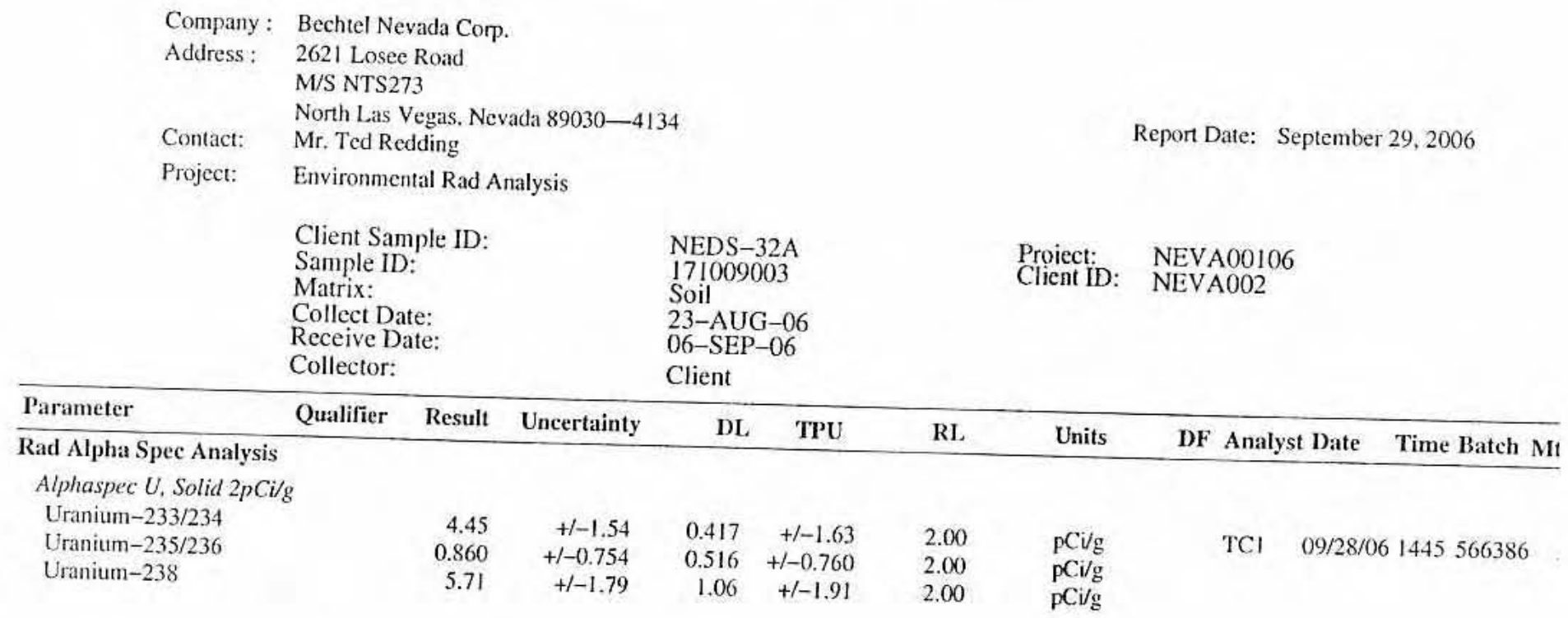

The following Prep Methods were performed

\begin{tabular}{llllll}
\hline Method & Description & Analyst & Date & Time & Prep Batch \\
\hline Dry Soil Prep & Dry Soil Prep GL-RAD-A-021 & JMBI & $09 / 07 / 06$ & 0901 & 565866
\end{tabular}

The following Analytical Methods were performed

\begin{tabular}{ll}
\hline Method & Description \\
\hline 1 & DOE EML HASL-300, U-02-RC Modified \\
2 & DOE EML HASL-300, U-02-RC Modified \\
3 & DOE EML HASL-300, U-02-RC Modified \\
4 & DOE EML HASL-300, U-02-RC Modified \\
5 & DOE EML HASL-300, U-02-RC Modified
\end{tabular}

\begin{tabular}{llcc} 
Surrogate/Tracer recovery & Test & Recovery $\%$ & Acceptable Limits \\
\hline Uranium-232 & Alphaspec U, Solid 2pCi/g & 53 & $(25 \%-125 \%)$
\end{tabular}

Notes:

The Qualifiers in this report are defined as follows :

* A quality control analyte recovery is outside of specified acceptance criteria

$<$ Result is less than value reported

$>$ Result is greater than value reported

A The TIC is a suspected aldol-condensation product

B Target analyte was detected in the associated blank

$\mathrm{BD}$ Results are either below the MDC or tracer recovery is low

C Analyte has been confirmed by GC/MS analysis

D Results are reported from a diluted aliquot of the sample

$\mathrm{H}$ Analytical holding time was exceeded

$\mathrm{J}$ Value is estimated 


\section{GENERAL ENGINEERING LABORATORIES, LLC \\ 2040 Savage Road Charleston SC 29407 - (843) 556-8171 - www.gel.com}

\section{Certificate of Analysis}

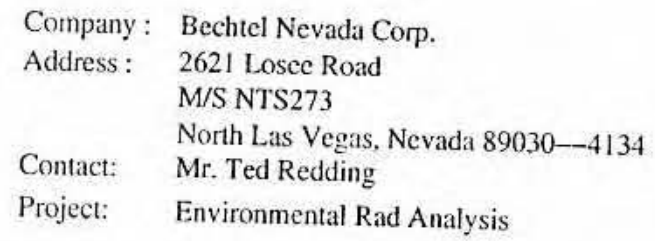

$\begin{array}{ll}\text { Client Sample ID: } & \text { NEDS-32A } \\ \text { Sample ID: } & 171009003\end{array}$

171009003

Project:

Client: NEVA00106

Parameter

Qualifier

Result Uncertainty

DL

TPU

RL

Units

DF Analyst Date

Time Batch Mtı

N/A Spike recovery limits do not apply. Sample concentration exceeds spike concentration by $4 X$ or more
$R$ Sample results are rejected

U Analyte was analyzed for, but not detected above the MDL, MDA, or LOD.

U1 Gamma Spectroscopy--Uncertain identification

$X$ Consult Case Narrative, Data Summary package, or Project Manager concerning this qualifier
Y QC Samples were not spiked with this compound

$\mathrm{h}$ Preparation or preservation hovaluated using $+/-\mathrm{RL}$. Concentrations are $<5 \mathrm{X}$ the RL

The abovesation or preservation holding time was exceeded

The above sample is reported on a dry weight basis. 


\section{GENERAL ENGINEERING LABORATORIES, LLC \\ 2040 Savage Road Charleston SC 29407 - (843) 556-8171 - www.gel.com}

\section{Certificate of Analysis}

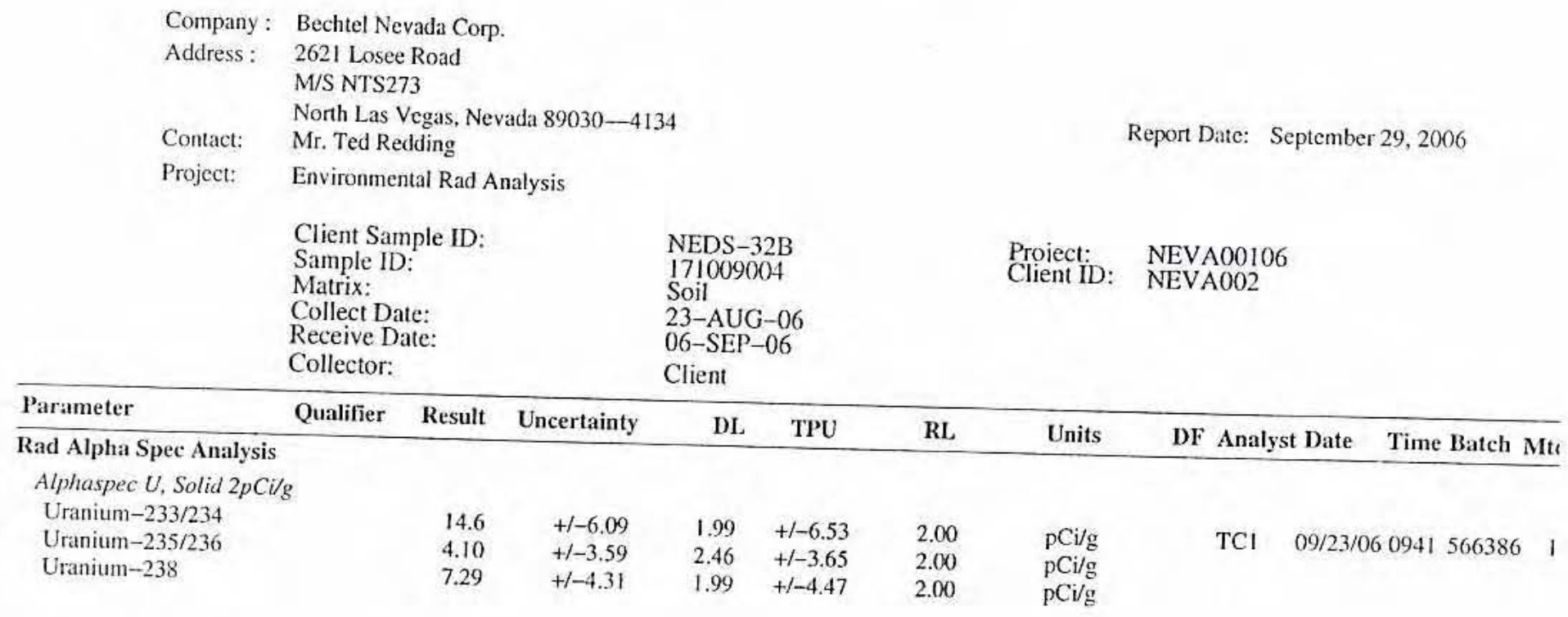

The following Prep Methods were performed

\begin{tabular}{llllll}
\hline Method & Description & Analyst & Date & Time & Prep Batch \\
\hline Dry Soil Prep & Dry Soil Prep GL-RAD-A-021 & JMBI & $09 / 07 / 06$ & 0901 & 565866
\end{tabular}

The following Analytical Methods were performed

\begin{tabular}{ll}
\hline Method & Description \\
\hline 1 & DOE EML HASL-300, U-02-RC Modified \\
3 & DOE EML HASL-300, U-02-RC Modified \\
3 & DOE EML HASL-300, U-02-RC Modified
\end{tabular}

\begin{tabular}{llcc} 
Surrogate/Tracer recovery & Test & Recovery\% & Acceptable Limits \\
\hline Uranium-232 & Alphaspec U, Solid 2pCi/g & 49 & $(25 \%-125 \%)$
\end{tabular}

Notes:

The Qualifiers in this report are defined as follows :

* A quality control analyte recovery is outside of specified acceptance criteria

$<$ Result is less than value reported

$>$ Result is greater than value reported

A The TIC is a suspected aldol-condensation product

B Target analyte was detected in the associated blank

$\mathrm{BD}$ Results are either below the MDC or tracer recovery is low

C Analyte has been confirmed by GC/MS analysis

D Results are reported from a diluted aliquot of the sample

$\mathrm{H}$ Analytical holding time was exceeded

$\mathrm{J}$ Value is estimated

N/A Spike recovery limits do not apply. Sample concentration exceeds spike concentration by $4 \mathrm{X}$ or more
$\mathrm{R}$ Sample results are rejected

U Analyte was analyzed for, but not detected above the MDL, MDA, or LOD. 


\section{GENERAL ENGINEERING LABORATORIES, LLC \\ 2040 Savage Road Charleston SC 29407 - (843) 556-8171 - www.gel.com}

\section{Certificate of Analysis}

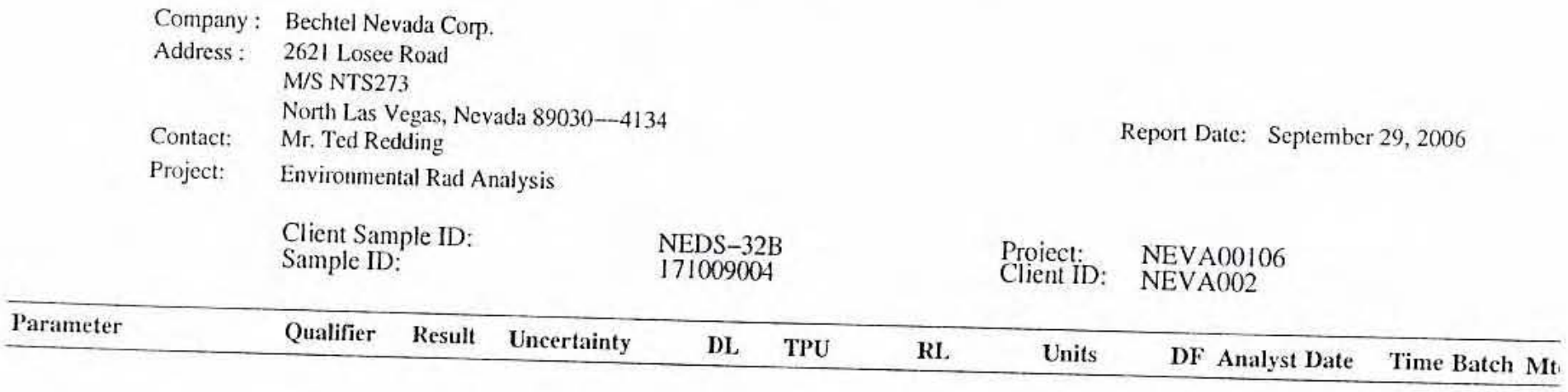

UI Gamma Spectroscopy-Uncertain identification

X Consult Case Narrative, Data Summary package, or Project Manager concerning this qualifier
Y QC Samples were not spiked with this compound

$\mathrm{h}$ Preparationple and duplicate evaluated using $+/-$ RL. Concentrations are $<5 X$ the RL

The abe saption preservation holding time was exceeded

The above sample is reported on a dry weight basis. 


\section{GENERAL ENGINEERING LABORATORIES, LLC
2040 Savage Road Charleston SC 29407 - (843) 556-8171 - www.gel.com}

\section{Certificate of Analysis}

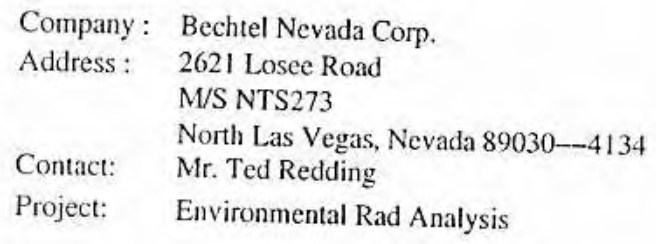

Client Sample ID:

Sample ID:

Matrix:

Collect Date:

Receive Date:

Collector:

NEDS-33A
171009005
Soil
$23-A U G-06$
$06-S E P-06$
Client

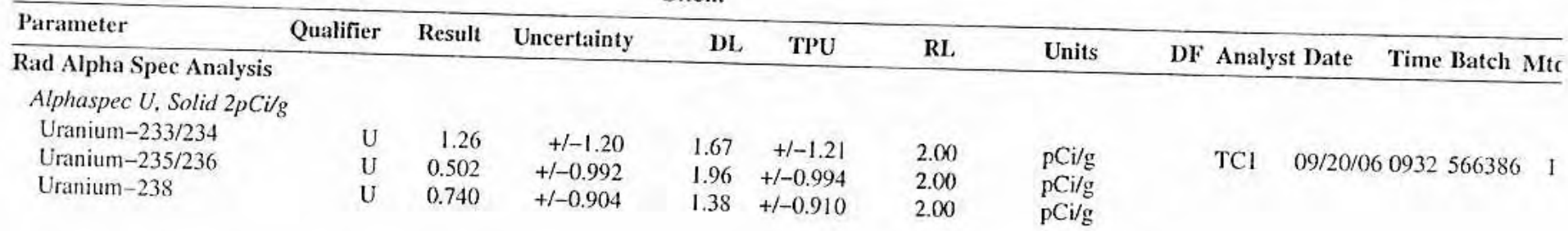

The following Prep Methods were performed

\begin{tabular}{llllll}
\hline Method & Description & Analyst & Date & Time & Prep Batch \\
\hline Dry Soil Prep & Dry Soil Prep GL-RAD-A-021 & JMB1 & $09 / 07 / 06$ & 0901 & 565866
\end{tabular}

The following Analytical Methods were performed

$\begin{array}{ll}\text { Method } & \text { Description } \\ 1 & \text { DOE EML HASL-300, U-02-RC Modified }\end{array}$

\begin{tabular}{llcc} 
Surrogate/Tracer recovery & Test & Recovery $\%$ & Acceptable Limits \\
\hline Uranium-232 & Alphaspec U, Solid 2pCi/g & 81 & $(25 \%-125 \%)$
\end{tabular}

Notes:

The Qualifiers in this report are defined as follows :

* A quality control analyte recovery is outside of specified acceptance criteria

$<$ Result is less than valuc roported

$>$ Result is greater than value reported

A The TIC is a suspected aldol-condensation product

B Target analyte was detected in the associated blank

$\mathrm{BD}$ Results are either below the MDC or tracer recovery is low

C Analyte has been confirmed by GC/MS analysis

D Results are reported from a diluted aliquot of the sample

$\mathrm{H}$ Analytical holding time was exceeded

$\mathrm{J}$ Value is estimated

N/A Spike recovery limits do not apply. Sample concentration exceeds spike concentration by $4 \mathrm{X}$ or more
$\mathrm{R}$ Sample results are rejected

Analyte was analyzed for, but not detected above the MDL, MDA, or LOD.

UI Gamma Spectroscopy--Uncertain identification

X Consult Case Narrative, Data Summary package, or Project Manager concerning this qualifier 


\section{GENERAL ENGINEERING LABORATORIES, LLC \\ 2040 Savage Road Charleston SC 29407 - (843) 556-8171 - www.gel.com}

\section{Certificate of Analysis}

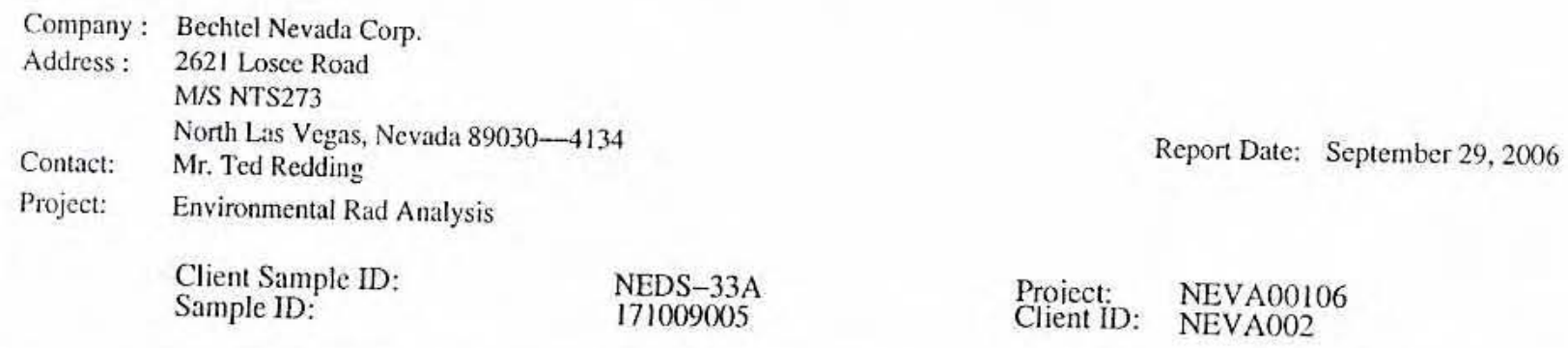

DL TPU RL

Y QC Samples were not spiked with this compound

$\hat{h}$ RPD of sample and duplicate evaluated using $+/-$ RL. Concentrations are $<5 X$ the $R L$

$h$ Preparation or preservation holding time was exceeded

The above sample is reported on a dry weight basis. 


\section{GENERAL ENGINEERING LABORATORIES, LLC \\ 2040 Savage Road Charleston SC 29407 - (843) 556-8171 - www.gel.com}

\section{$\underline{\text { Certificate of Analysis }}$}

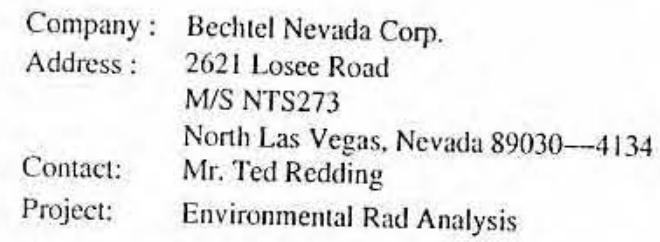

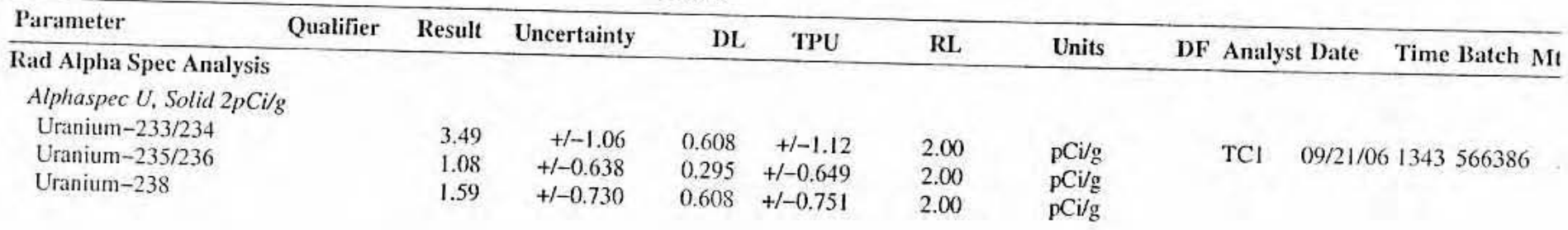

The following Prep Methods were performed

\begin{tabular}{llllll}
\hline Method & Description & Analyst & Date & Time & Prep Batch \\
\hline Dry Soil Prep & Dry Soil Prep GL-RAD-A-021 & JMB1 & $09 / 07 / 06$ & 0901 & 565866
\end{tabular}

The following Analytical Methods were performed

\begin{tabular}{ll} 
Method & Description \\
\hline 1 & DOE EML HASL-300, U-02-RC Modified \\
2 & DOE EML HASL-300, U-02-RC Modified
\end{tabular}

\begin{tabular}{llcc} 
Surrogate/Tracer recovery & Test & Recovery \% & Acceptable Limits \\
\hline Uranium-232 & Alphaspec U, Solid 2pCi/g & 92 & $(25 \%-125 \%)$
\end{tabular}

Notes:

The Qualifiers in this report are defined as follows :

* A quality control analyte recovery is outside of specified acceptance criteria

$<$ Result is less than value reported

$>$ Result is greater than value reported

A The TIC is a suspected aldol-condensation product

B Target analyte was detected in the associated blank

$\mathrm{BD}$ Results are either below the MDC or tracer recovery is low

C Analyte has been confirmed by GC/MS analysis

D Results are reported from a diluted aliquot of the sample

$\mathrm{H}$ Analytical holding time was exceeded

$\mathrm{J}$ Value is estimated

N/A Spike recovery limits do not apply. Sample concentration exceeds spike concentration by $4 X$ or more
$R$ Sample results are rejected

U Analyte was analyzed for, but not detected above the MDL, MDA, or LOD.

UI Gamma Spectroscopy--Uncertain identification 


\section{GENERAL ENGINEERING LABORATORIES, LLC \\ 2040 Savage Road Charleston SC 29407 - (843) 556-8171 - www.gel.com}

\section{Certificate of Analysis}

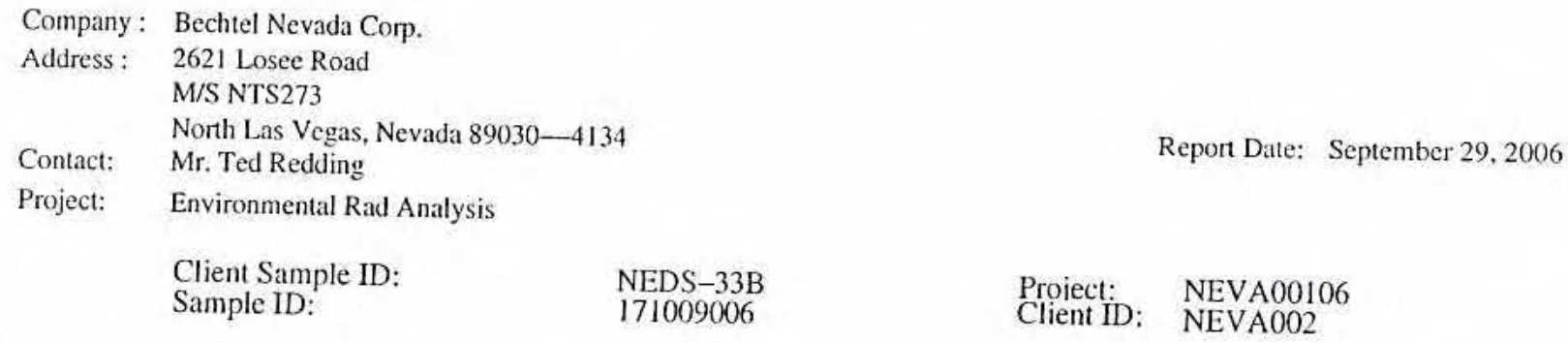

X Consult Case Narrative, Data Summary package, or Project Manager concerning this qualifier

Y QC Samples were not spiked with this compound

$\hat{h} \quad$ RPD of sample and duplicate evaluated using $+/-R L$. Concentrations are $<5 X$ the $R L$

$\mathrm{h}$ Preparation or preservation holding time was exceeded

The above sample is reported on a dry weight basis. 


\section{GENERAL ENGINEERING LABORATORIES, LLC \\ 2040 Savage Road Charleston SC 29407 - (843) 556-8171 - www.gel.com}

\section{Certificate of Analysis}

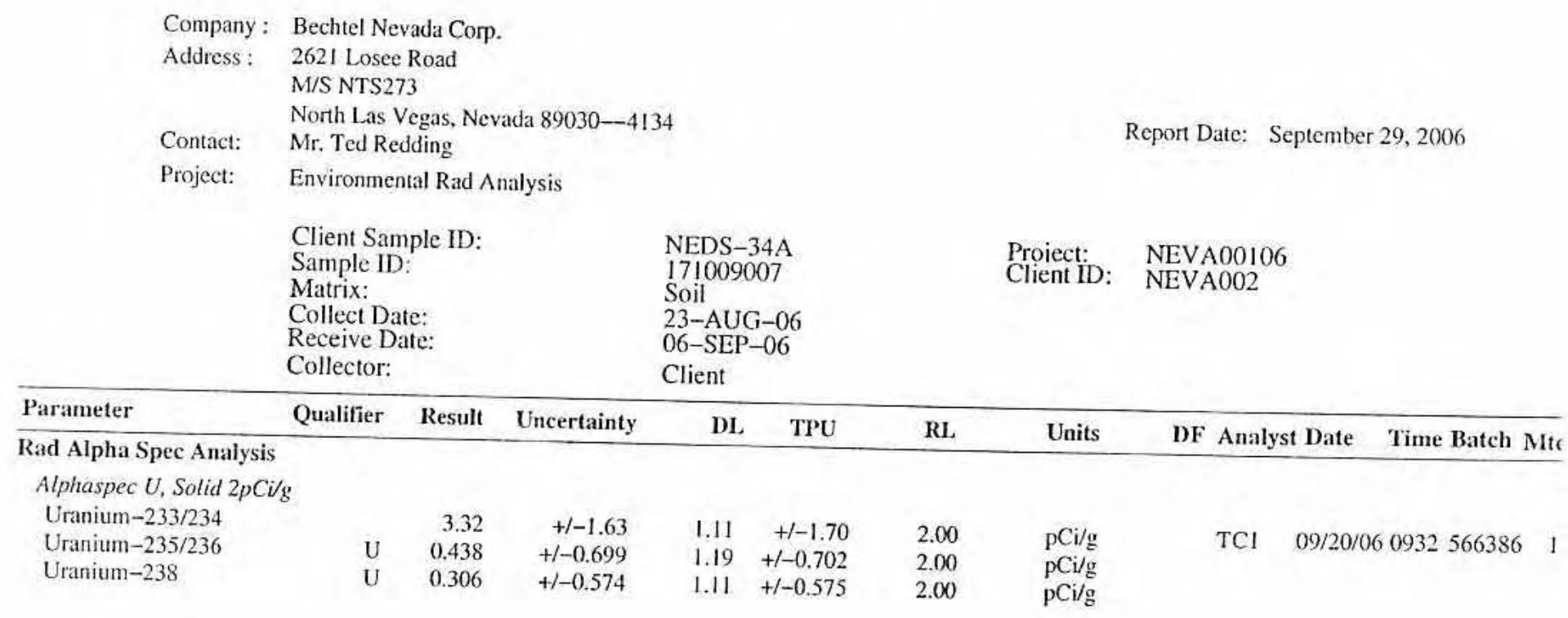

The following P'rep Methods were performed

\begin{tabular}{llllll}
\hline Method & Description & Analyst & Date & Time & Prep Batch \\
\hline Dry Soil Prep & Dry Soil Prep GL-RAD-A-021 & JMBI & $09 / 07 / 06$ & 0901 & 565866
\end{tabular}

The following Analytical Methods were performed

\begin{tabular}{ll} 
Method & Description \\
\hline DOE EML HASL-300, U-02-RC Modified
\end{tabular}

\begin{tabular}{llcc} 
Surrogate/Tracer recovery & Test & Recovery \% & Acceptable Limits \\
\hline Uranium-232 & Alphaspec U, Solid 2pCi/g & 89 & $(25 \%-125 \%)$
\end{tabular}

Notes:

The Qualifiers in this report are defined as follows :

* A quality control analyte recovery is outside of specified acceptance criteria

$<$ Result is less than value reported

$>$ Result is greater than value reported

A The TIC is a suspected aldol-condensation product

B Target analyte was detected in the associated blank

BD Results are either below the MDC or tracer recovery is low

C Analyte has been confirmed by GC/MS analysis

D Results are reported from a diluted aliquot of the sample

H Analytical holding time was exceeded

$\mathrm{J}$ Value is estimated

N/A Spike recovery limits do not apply. Sample concentration exceeds spike concentration by $4 X$ or more
$R$ Sample results are rejected

U Analyte was analyzed for, but not detected above the MDL, MDA, or LOD.

UI Gamma Spectroscopy--Uncertain identification

X Consult Case Narrative, Data Summary package, or Project Manager concerning this qualifier 


\section{GENERAL ENGINEERING LABORATORIES, LLC \\ 2040 Savage Road Charleston SC 29407 - (843) 556-8171 - www.gel.com}

\section{Certificate of Analysis}

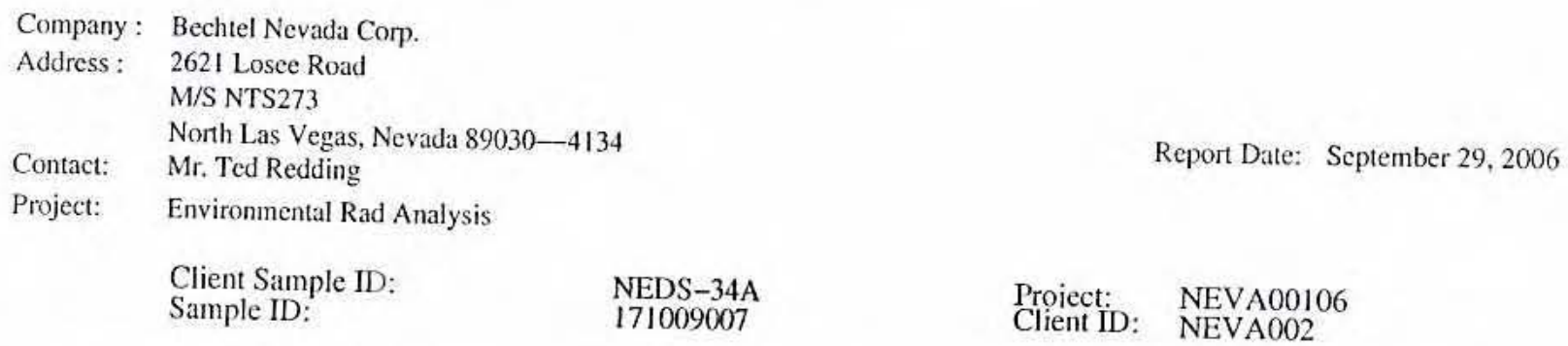

Y QC Samples were not spiked with this compound

$\hat{h}$ RPD of sample and duplicate evaluated using $+/-R L$. Concentrations are $<5 X$ the RL

$h$ Preparation or preservation holding time was exceeded

The above sample is reported on a dry weight basis. 


\section{GENERAL ENGINEERING LABORATORIES, LLC \\ 2040 Savage Road Charleston SC 29407 - (843) 556-8171 - www.gel.com}

\section{Certificate of Analysis}

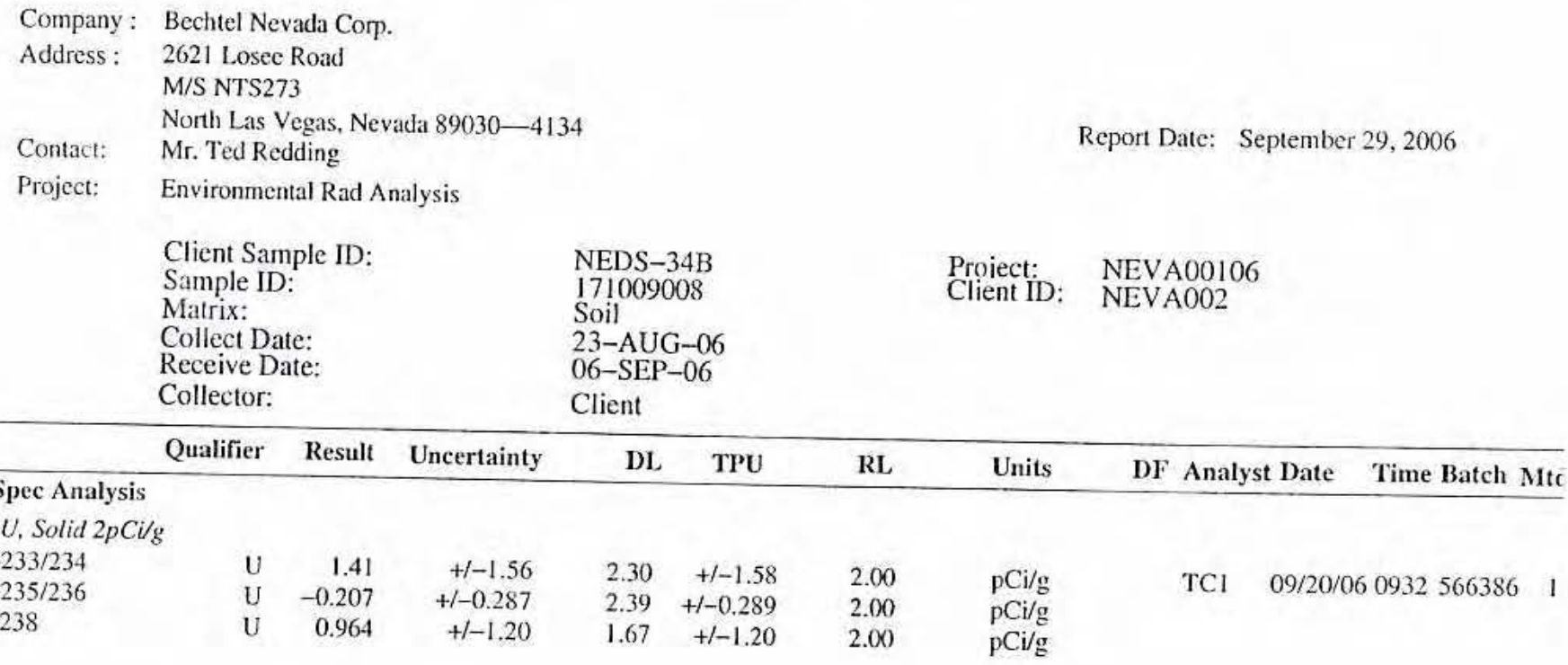

The following Prep Methods were performed

\begin{tabular}{llllll}
\hline Method & Description & Analyst & Date & Time & Prep Batch \\
\hline Dry Soil Prep & Dry Soil Prep GL-RAD-A-021 & JMBI & $09 / 07 / 06$ & 0901 & 565866
\end{tabular}

The following Analytical Methods were performed

\begin{tabular}{ll} 
Method & Description \\
\hline DOE EML HASL-300, U-02-RC Modified
\end{tabular}

\begin{tabular}{llcc} 
Surrogate/Tracer recovery & Test & Recovery\% & Acceptable Limits \\
\hline Uranium-232 & Alphaspec U, Solid 2pCi/g & 77 & $(25 \%-125 \%)$
\end{tabular}

Notes:

The Qualifiers in this report are defined as follows :

* A quality control analyte recovery is outside of specified acceptance criteria

- Result is less than value reported

$>$ Result is greater than value reported

A The TIC is a suspected aldol-condensation product

B Target analyte was detected in the associated blank

BD Results are either below the MDC or tracer recovery is low

C Analyte has been confirmed by GC/MS analysis

D Results are reported from a diluted aliquot of the sample

$\mathrm{H}$ Analytical holding time was exceeded

$\mathrm{J}$ Value is estimated

N/A Spike recovery limits do not apply. Sample concentration exceeds spike concentration by $4 X$ or more

$\mathrm{R}$ Sample results are rejected

U Analyte was analyzed for, but not detected above the MDL, MDA, or LOD.

UI Gamma Spectroscopy-Uncertain identification

$\mathrm{X}$ Consult Case Narrative, Data Summary package, or Project Manager concerning this qualifier 


\section{GENERAL ENGINEERING LABORATORIES, LLC \\ 2040 Savage Road Charleston SC 29407 - (843) 556-8171 - www.gel.com}

\section{Certificate of Analysis}

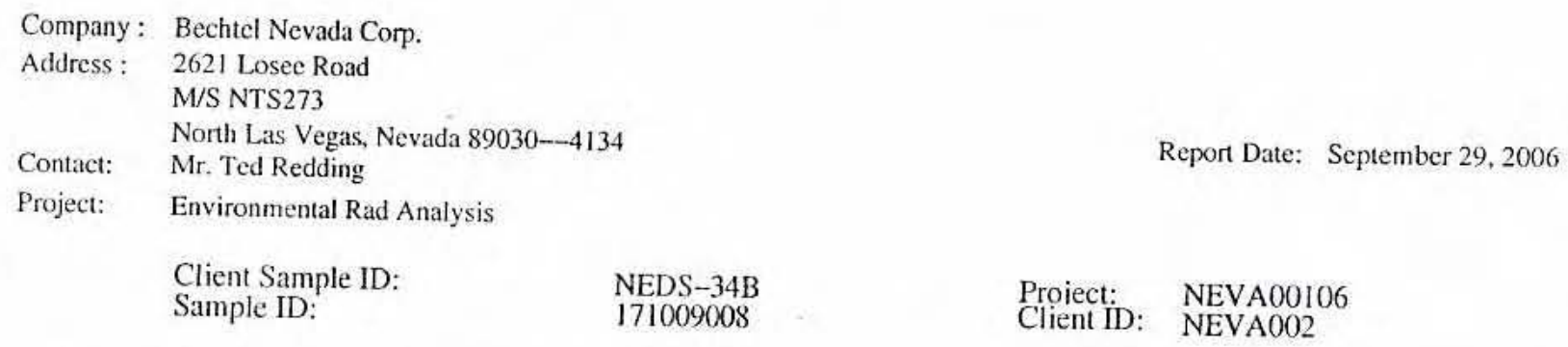

Y QC Samples were not spiked with this compound

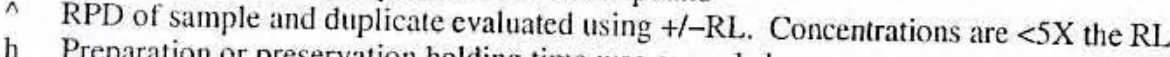

$\mathrm{h}$ Preparation or preservation holding time was exceeded

The above sample is reported on a dry weight basis. 


\section{GENERAL ENGINEERING LABORATORIES, LLC \\ 2040 Savage Road Charleston SC 29407 - (843) 556-8171 - www.gel.com}

\section{Certificate of Analysis}

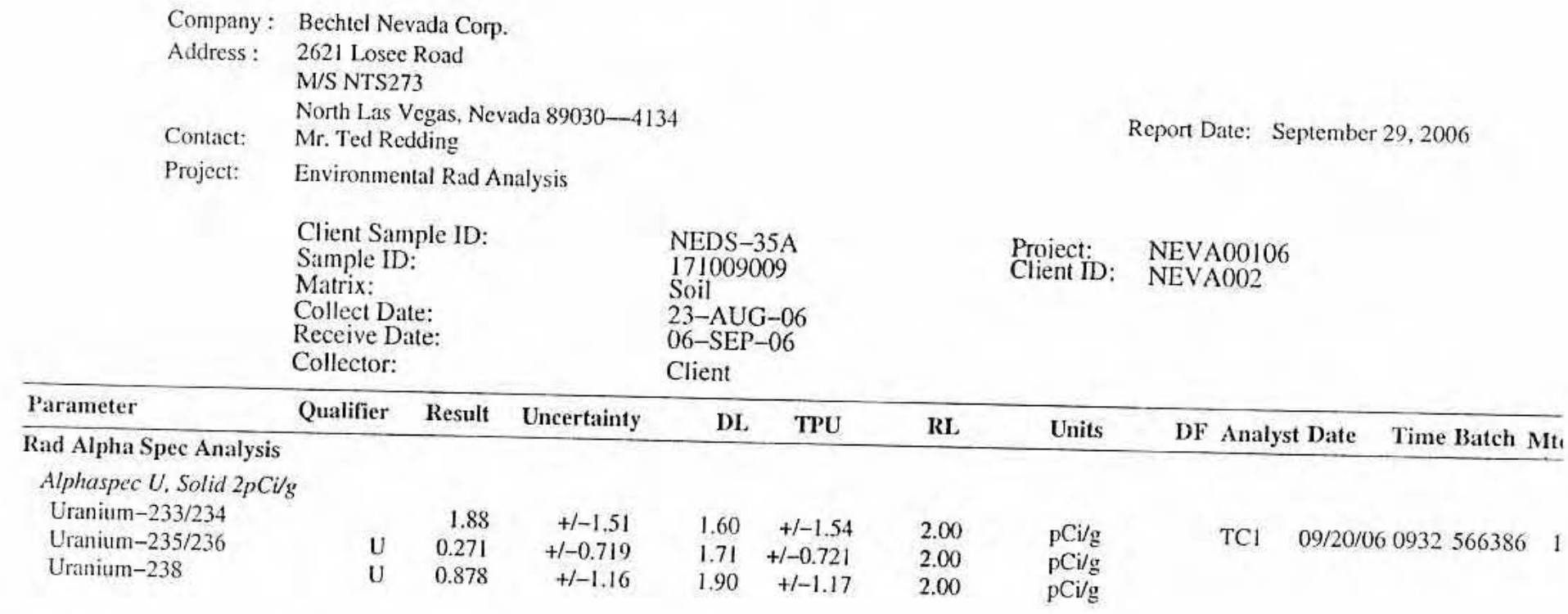

The following Prep Methods were performed

\begin{tabular}{llllll}
\hline Method & Description & Analyst & Date & Time & Prep Batch \\
\hline Dry Soil Prep & Dry Soil Prep GL-RAD-A-021 & JMBI & $09 / 07 / 06$ & 0901 & 565866
\end{tabular}

The following Analytical Methods were performed

\begin{tabular}{ll} 
Method & Description \\
\hline & DOE EML HASL-300, U-02-RC Modified
\end{tabular}

\begin{tabular}{llcc} 
Surrogate/Tracer recovery & Test & Recovery\% & Acceptable Limits \\
\hline Uranium-232 & Alphaspec U, Solid 2pCi/g & 76 & $(25 \%-125 \%)$
\end{tabular}

Notes:

The Qualifiers in this report are defined as follows :

* A quality control analyte recovery is outside of specified acceptance criteria

$<$ Result is less than value reported

$>$ Result is greater than value reported

A The TIC is a suspected aldol-condensation product

B Target analyte was detected in the associated blank

$\mathrm{BD}$ Results are either below the MDC or tracer recovery is low

C Analyte has been confirmed by GC/MS analysis

$\mathrm{D}$ Results are reported from a diluted aliquot of the sample

$\mathrm{H}$ Analytical holding time was exceeded

$\mathrm{J}$ Value is estimated

N/A Spike recovery limits do not apply. Sample concentration exceeds spike concentration by $4 X$ or more
$R$ Sample results are rejected

U Analyte was analyzed for, but not detected above the MDL, MDA, or LOD.

UI Gamma Spectroscopy--Uncertain identification

X Consult Case Narrative, Data Summary package, or Project Manager concerning this qualifier 


\section{GENERAL ENGINEERING LABORATORIES, LLC \\ 2040 Savage Road Charleston SC 29407 - (843) 556-8171 - www.gel.com}

\section{Certificate of Analysis}

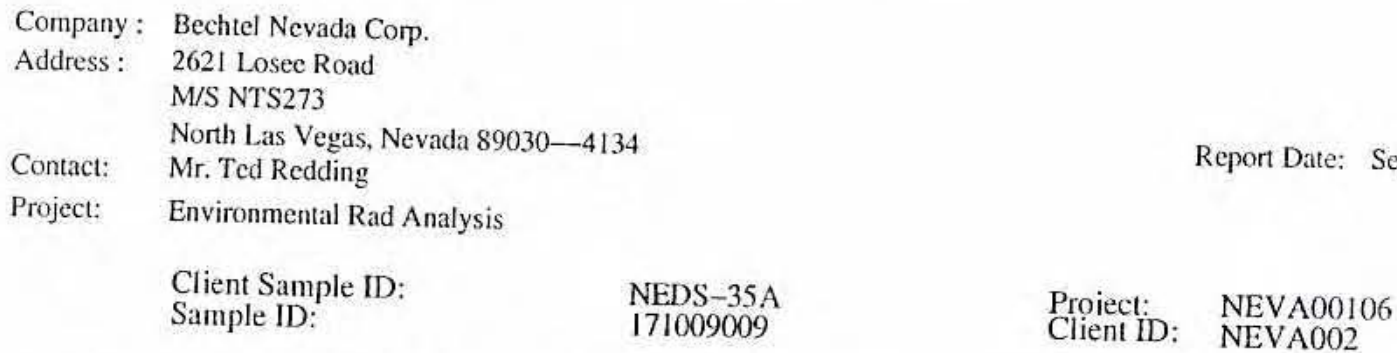

Parameter

Qualifier Result Uncertainty

DL TPU

RL

Units DF Analyst Date

Time Batch Mt,

Y QC Samples were not spiked with this compound

$\wedge$ RPD of sample and duplicate evaluated using $+/-\mathrm{RL}$. Concentrations are $<5 \mathrm{X}$ the RL

$\mathrm{h}$ Preparation or preservation holding time was exceeded

The ahove sample is reported on a dry weight basis. 


\section{GENERAL ENGINEERING LABORATORIES, LLC \\ 2040 Savage Road Charleston SC 29407 - (843) 556-8171 - www.gel.com}

\section{Certificate of Analysis}

\author{
Company: Bechtel Nevada Corp. \\ Address : 2621 Losee Road \\ M/S NTS273 \\ North Las Vegas, Nevada 89030-4134 \\ Contact: Mr. Ted Redding \\ Project: Environmental Rad Analysis
}

Report Date: September 29,2006
Proiect: NEVA00106

Client ID: NEVA002

$\begin{array}{ll}\text { Client Sample ID: } & \text { NEDS-35B } \\ \text { Sample ID: } & 171009010 \\ \text { Matrix: } & \text { Soil } \\ \text { Collect Date: } & \text { 23-AUG-06 } \\ \text { Receive Date: } & \text { 06-SEP-06 } \\ \text { Collector: } & \text { Client }\end{array}$

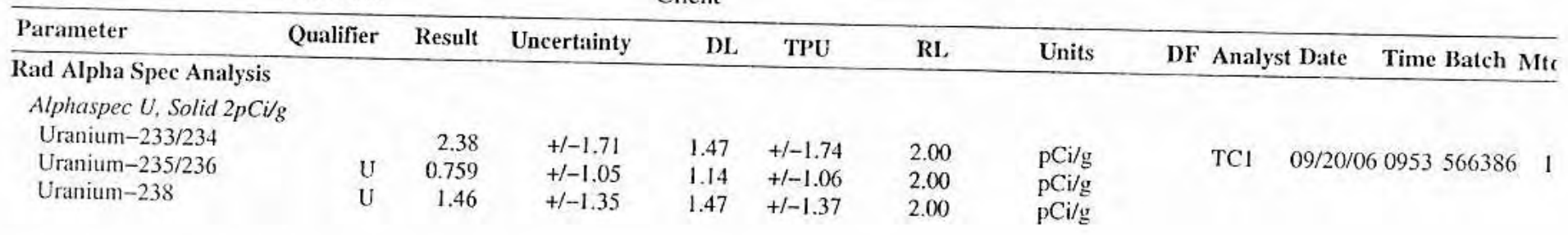

The following Prep Methods were performed

\begin{tabular}{llllll}
\hline Method & Description & Analyst & Date & Time & Prep Batch \\
\hline Dry Soil Prep & Dry Soil Prep GL-RAD-A-021 & JMBI & $09 / 07 / 06$ & 0901 & 565866
\end{tabular}

The following Analytical Methods were performed

$\begin{array}{ll}\text { Method Description } \\ 1 & \text { DOE EML HASL-300, U-02-RC Modified }\end{array}$

\begin{tabular}{llcc} 
Surrogate/Tracer recovery & Test & Recovery\% & Acceptable Limits \\
\hline Uranium-232 & Alphaspec U, Solid 2pCi/g & 96 & $(25 \%-125 \%)$
\end{tabular}

Notes:

The Qualifiers in this report are defined as follows :

* A quality control analyte recovery is outside of specified acceptance criteria

$<$ Result is less than value reported

$>$ Result is greater than value reported

A The TIC is a suspected aldol-condensation product

$\mathrm{B}$ Target analyte was detected in the associated blank

BD Results are either below the MDC or tracer recovery is low

C Analyte has been confirmed by GC/MS analysis

D Results are reported from a diluted aliquot of the sample

$\mathrm{H}$ Analytical holding time was exceeded

$\mathrm{J}$ Value is estimated

N/A Spike recovery limits do not apply. Sample concentration exceeds spike concentration by $4 X$ or more
$\mathrm{R}$ Sample results are rejected

$\mathrm{U}$ Analyte was analyzed for, but not detected above the MDL, MDA, or LOD.

UI Gamma Spectroscopy-Uncertain identification

X Consult Case Narrative, Data Summary package, or Project Manager concerning this qualifier 


\section{GENERAL ENGINEERING LABORATORIES, LLC \\ 2040 Savage Road Charleston SC 29407 - (843) 556-8171 - www.gel.com}

\section{Certificate of Analysis}

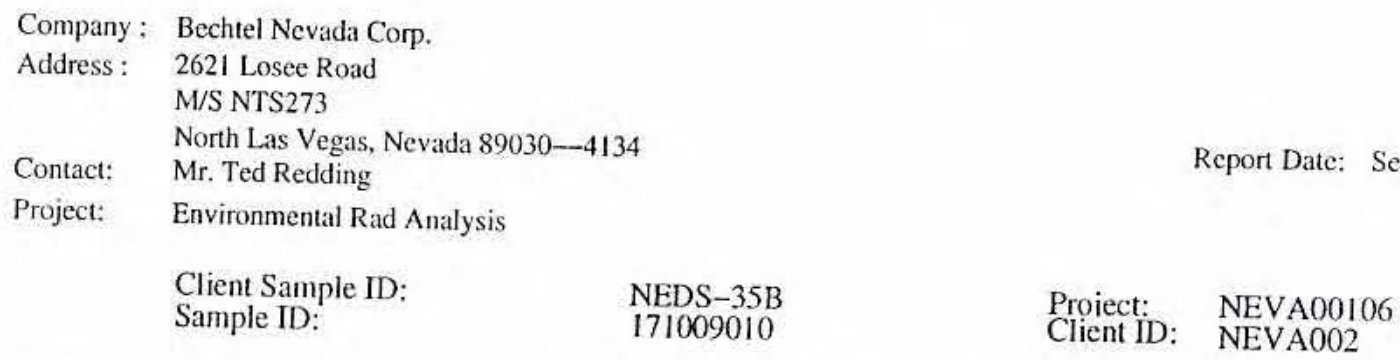

Y QC Samples were not spiked with this compound

$\hat{A}$ RPD of sample and duplicate evaluated using $+1-\mathrm{RL}$. Concentrations are $<5 \mathrm{X}$ the RL

$h$ Preparation or preservation holding time was exceeded

The above sample is reported on a dry weight basis. 


\section{GENERAL ENGINEERING LABORATORIES, LLC \\ 2040 Savage Road Charleston SC 29407 - (843) 556-8171 - www.gel.com}

\section{$\underline{\text { Certificate of Analysis }}$}

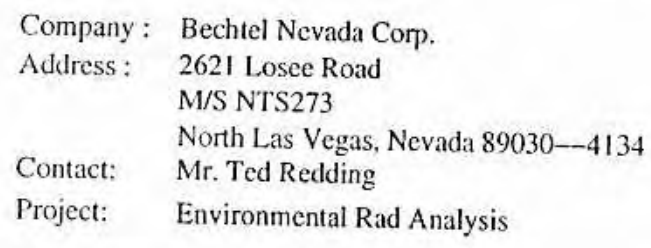

\begin{tabular}{|c|c|c|c|c|c|c|c|c|c|c|}
\hline \multirow[b]{2}{*}{ Parameter } & \multicolumn{2}{|c|}{$\begin{array}{l}\text { Client Sample ID: } \\
\text { Sample ID: } \\
\text { Matrix: } \\
\text { Collect Date: } \\
\text { Receive Date: } \\
\text { Collector: }\end{array}$} & & \multicolumn{3}{|c|}{$\begin{array}{l}\text { NEDS-36A } \\
171009011 \\
\text { Soil } \\
\text { 23-AUG-06 } \\
\text { 06-SEP-06 } \\
\text { Client }\end{array}$} & \multirow{2}{*}{$\begin{array}{l}\text { Proiect: } \\
\text { Client ID: } \\
\text { Units } \\
\end{array}$} & \multicolumn{3}{|l|}{$\begin{array}{l}\text { NEVA00106 } \\
\text { NEVA002 }\end{array}$} \\
\hline & Qualifier & Result & Uncertainty & DL & TPU & $\mathrm{RL}$ & & DF Analyst & $t$ Date & Time Batch Mtt \\
\hline \multicolumn{11}{|c|}{$\begin{array}{l}\text { Rad Alpha Spec Analysis } \\
\text { Alphaspec U. Solid } 2 p C i / \delta \\
\text { Uranium-233/234 }\end{array}$} \\
\hline $\begin{array}{l}\text { Uranium-233/234 } \\
\text { Uranium-235/236 } \\
\text { Uranium-238 }\end{array}$ & U & $\begin{array}{r}0.804 \\
0.298 \\
1.45\end{array}$ & $\begin{array}{l}+/-0.590 \\
+/-0.585 \\
+/-0.705\end{array}$ & $\begin{array}{r}0.770 \\
1.10 \\
0.615\end{array}$ & $\begin{array}{l}+/-0.597 \\
+/-0.585 \\
+/-0.724\end{array}$ & $\begin{array}{l}2.00 \\
2.00 \\
2.00\end{array}$ & $\begin{array}{l}\text { pCi/g } \\
\text { pCi/g } \\
\text { pCi/g }\end{array}$ & $\mathrm{TCl}$ & $09 / 21 / 06$ & $61343566386 \quad$ । \\
\hline
\end{tabular}

The following Prep Methods were performed

\begin{tabular}{llllll}
\hline Method & Description & Analysi & Date & Time & Prep Batch \\
\hline Dry Soil Prep & Dry Soil Prep GL-RAD-A-021 & JMBI & $09 / 07 / 06$ & 0901 & 565866
\end{tabular}

The following Analytical Methods were performed

\begin{tabular}{ll} 
Method & Description \\
\hline 1 & DOE EML HASL-300, U-02-RC Modified \\
2 & DOE EML HASL-300, U-02-RC Modified
\end{tabular}

\begin{tabular}{llcc} 
Surrogate/Tracer recovery & Test & Recovery\% & Acceptable Limits \\
\hline Uranium-232 & Alphaspec U, Solid 2pCi/g & 71 & $(25 \%-125 \%)$
\end{tabular}

Notes:

The Qualifiers in this report are defined as follows :

* A quality control analyte recovery is outside of specified acceptance criteria

$<$ Result is less than value reported

$>$ Result is greater than value reported

A The TIC is a suspected aldol-condensation product

B Target analyte was detected in the associated blank

BD Results are either below the MDC or tracer recovery is low

C Analyte has been confirmed by GC/MS analysis

D Results are reported from a diluted aliquot of the sample

H Analytical holding time was exceeded

$\mathrm{J}$ Value is estimated

N/A Spike recovery limits do not apply. Sample concentration exceeds spike concentration by $4 X$ or more
$R$ Sample results are rejected

U Analyte was analyzed for, but not detected above the MDL, MDA, or LOD. 


\section{GENERAL ENGINEERING LABORATORIES, LLC \\ 2040 Savage Road Charleston SC 29407 - (843) 556-8171 - www.gel.com}

\section{Certificate of Analysis}

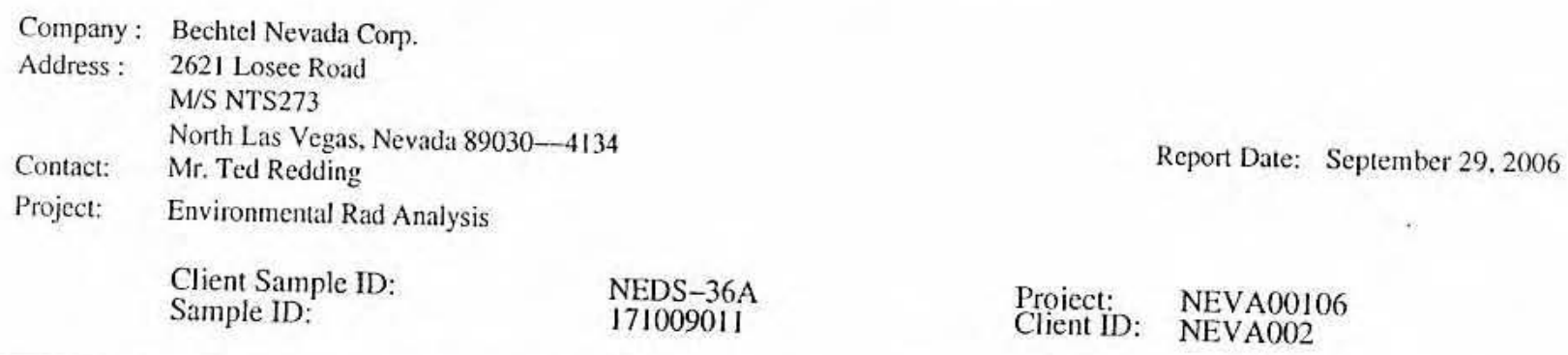

DL TPU RL

Units DF Analyst Date Time Batch Mt

UI Gamma Spectroscopy--Uncertain identification

X Consult Case Narrative, Data Summary package, or Project Manager concerning this qualifier

Y QC Samples were not spiked with this compound

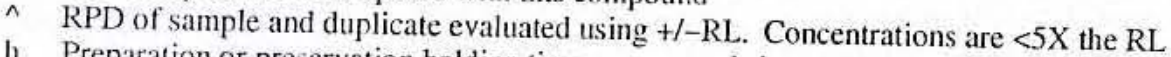

h Preparation or preservation holding time was exceeded

The above sample is reported on a dry weight basis. 


\section{GENERAL ENGINEERING LABORATORIES, LLC \\ 2040 Savage Road Charleston SC 29407 - (843) 556-8171 - www.gel.com}

\section{Certificate of Analysis}

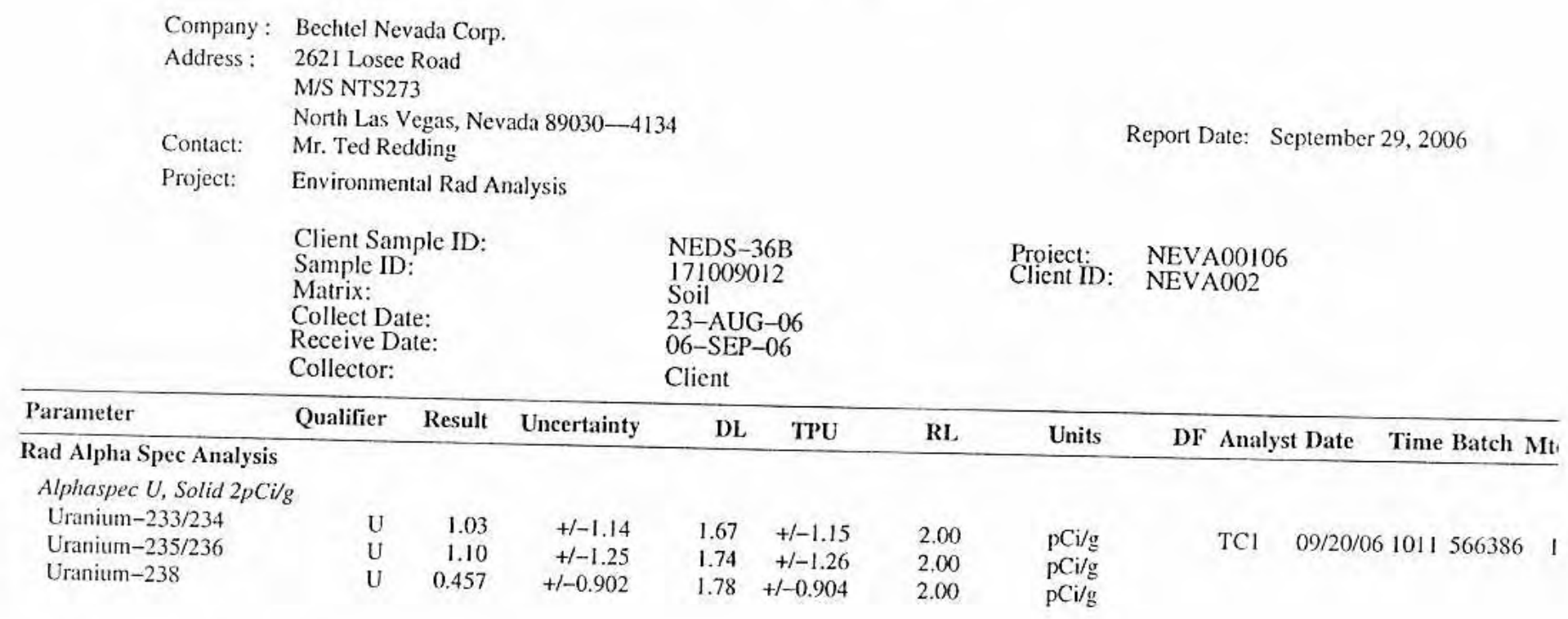

The following Prep Methods were performed

\begin{tabular}{llllll}
\hline Method & Description & Analyst & Date & Time & Prep Batch \\
\hline Dry Soil Prep & Dry Soil Prep GL-RAD-A-021 & JMB1 & $09 / 07 / 06$ & 0901 & 565866
\end{tabular}

The following Analytical Methods were performed

Method Description
1 DOE EML HASL-300, U-02-RC Modified

\begin{tabular}{llcc} 
Surrogate/Tracer recovery & Test & Recovery \% & Acceptable Limits \\
\hline Uranium-232 & Alphaspec U, Solid 2pCi/g & 82 & $(25 \%-125 \%)$
\end{tabular}

Notes:

The Qualifiers in this report are defined as follows :

* A quality control analyte recovery is outside of specified acceptance criteria

$<$ Result is less than value reported

$>$ Result is greater than value reported

A The TIC is a suspected aldol-condensation product

B Target analyte was detected in the associated blank

BD Results are either below the MDC or tracer recovery is low

C Analyte has been confirmed by GC/MS analysis

D Results are reported from a diluted aliquot of the sample

H Analytical holding time was exceeded

$\mathrm{J}$ Value is estimated

N/A Spike recovery limits do not apply. Sample concentration exceeds spike concentration by $4 \mathrm{X}$ or more
$\mathrm{R}$ Sample results are rejected

$\mathrm{U}$ Analyte was analyzed for, but not detected above the MDL, MDA, or LOD.

UI Gamma Spectroscopy-Uncertain identification

$\mathrm{X}$ Consult Case Narrative, Data Summary package, or Project Manager concerning this qualifier 


\section{GENERAL ENGINEERING LABORATORIES, LLC \\ 2040 Savage Road Charleston SC 29407 - (843) 556-8171 - www.gel.com}

\section{Certificate of Analysis}

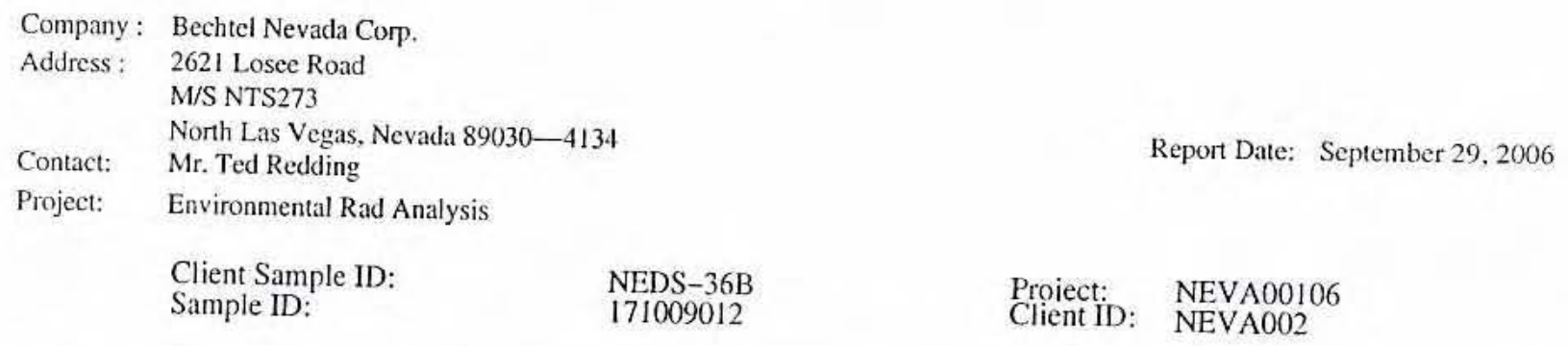

DL TPU

RL

Units DF Analyst Date

Time Batch Mtc

Y QC Samples were not spiked with this compound

$\hat{h}$ RPD of sample and duplicate evaluated using $+1-R L$. Concentrations are $<5 X$ the $R L$

$\mathrm{h}$ Preparation or preservation holding time was exceeded

The above sample is reported on a dry weight basis. 


\section{GENERAL ENGINEERING LABORATORIES, LLC \\ 2040 Savage Road Charleston SC 29407 - (843) 556-8171 - www.gel.com}

\section{Certificate of Analysis}

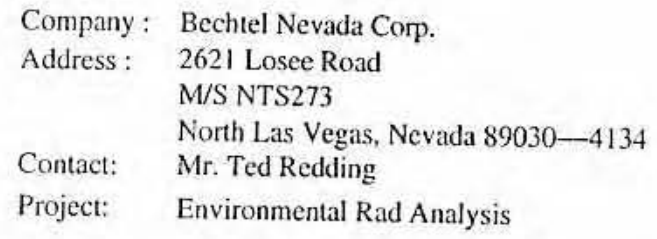

Client Sample ID:

Sample ID:

NEDS $-37 \mathrm{~A}$

171009013

Soil

Matrix:

Collect Date:

Receive Date:

Collector:
23-AUG-06

06-SEP-06

Client

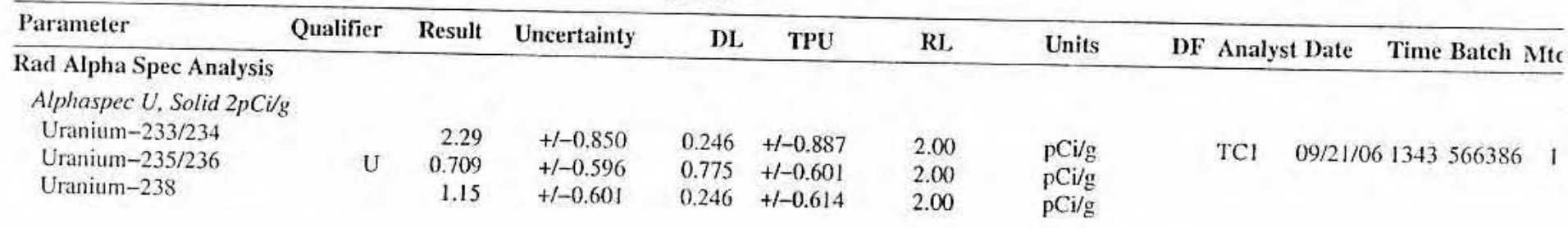

The following Prep Methods were performed

\begin{tabular}{llllll}
\hline Method & Description & Analyst & Date & Time & Prep Batch \\
\hline Dry Soil Prep & Dry Soil Prep GL-RAD-A-021 & JMBI & $09 / 07 / 06$ & 0901 & 565866
\end{tabular}

The following Analytical Methods were performed

\begin{tabular}{ll} 
Method & Description \\
\hline 1 & DOE EML HASL-300, U-02-RC Modified \\
2 & DOE EML HASL-300, U-02-RC Modified
\end{tabular}

Surrogate/Tracer recovery Test

Uranium-232 Alphaspec U, Solid 2pCi/g

Recovery\% Acceptable I.imits

Notes:

The Qualifiers in this report are defined as follows :

* A quality control analyte recovery is outside of specified acceptance criteria

$<$ Result is less than value reported

$>$ Result is greater than value reported

A The TIC is a suspected aldol-condensation product

B Target analyte was detected in the associated blank

$\mathrm{BD}$ Results are either below the MDC or tracer recovery is low

C Analyte has been confirmed by GC/MS analysis

D Results are reported from a diluted aliquot of the sample

$\mathrm{H}$ Analytical holding time was exceeded

J Value is estimated

N/A Spike recovery limits do not apply. Sample concentration exceeds spike concentration by $4 X$ or more

$\mathrm{R}$ Sample results are rejected

U Analyte was analyzed for, but not detected above the MDL, MDA, or LOD.

UI Gamma Spectroscopy-Uncertain identification 


\section{GENERAL ENGINEERING LABORATORIES, LLC \\ 2040 Savage Road Charleston SC 29407 - (843) 556-8171 - www.gel.com}

\section{Certificate of Analysis}

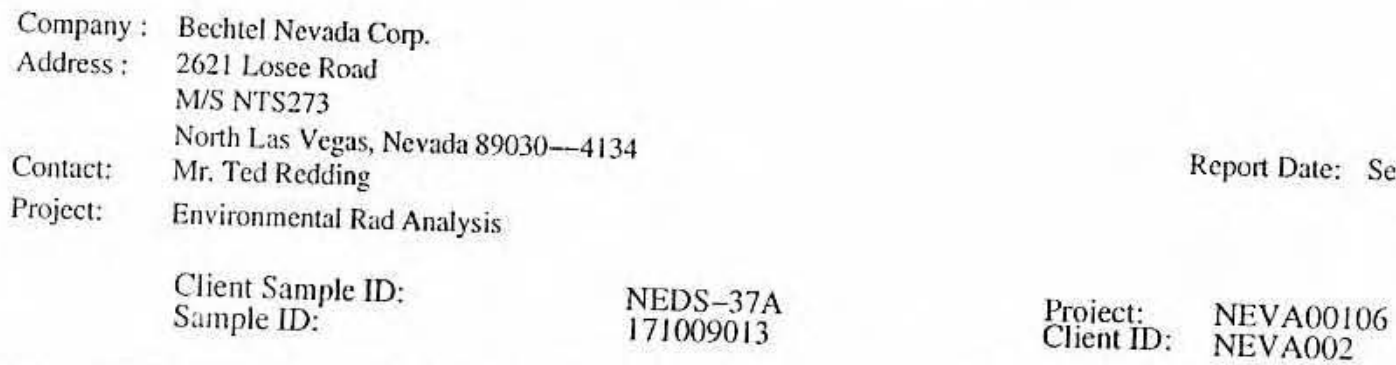

X Consult Case Narrative, Data Summary package, or Project Manager concerning this qualifier
Y QC Samples were not spiked with this compound

$\hat{h}$ Preparation or preservation holding timed using $+/-\mathrm{RL}$. Concentrations are $<5 \mathrm{X}$ the RL

The above ation or preservation holding time was exceeded

The above sample is reported on a dry weight basis. 


\section{GENERAL ENGINEERING LABORATORIES, LLC \\ 2040 Savage Road Charleston SC 29407 - (843) 556-8171 - www.gel.com}

\section{Certificate of Analysis}

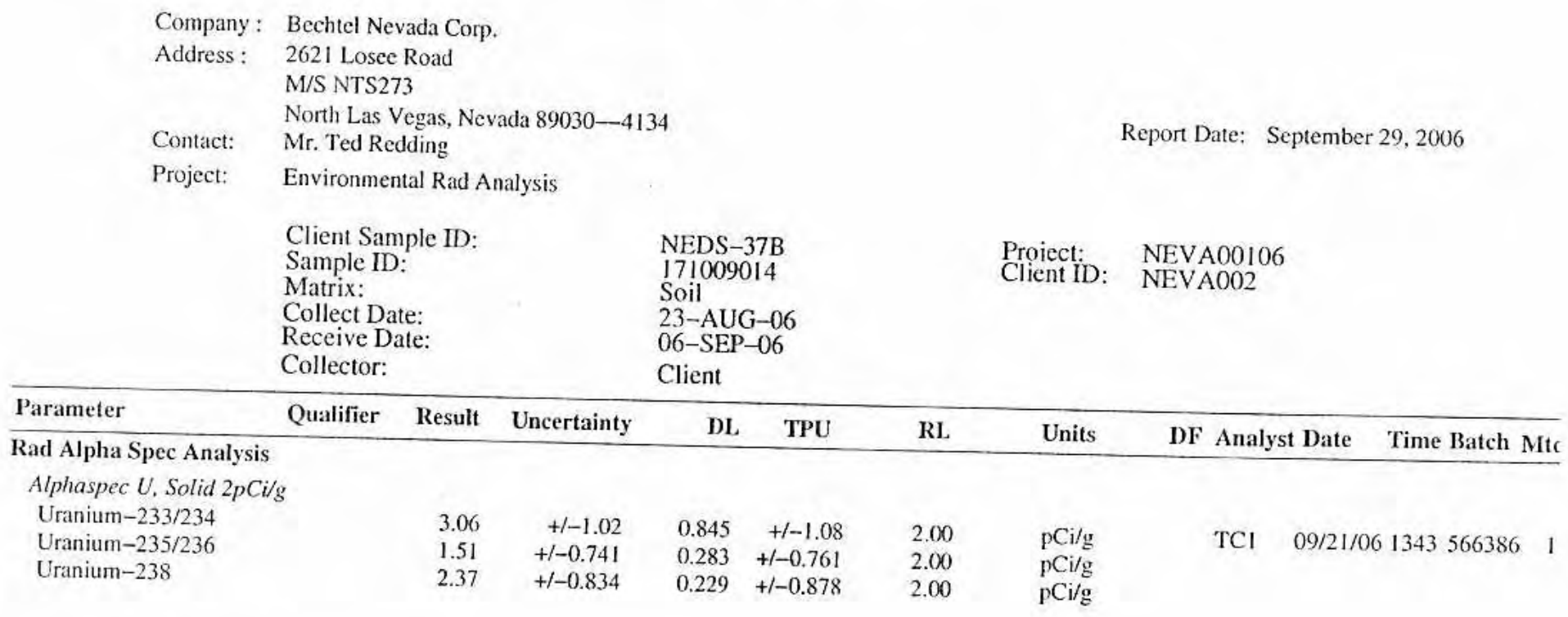

The following Prep Methods were performed

\begin{tabular}{llllll}
\hline Method & Description & Analyst & Date & Time & Prep Batch \\
\hline Dry Soil Prep & Dry Soil Prep GL-RAD-A-021 & JMB1 & $09 / 07 / 06$ & 0901 & 565866
\end{tabular}

The following Analytical Methods were performed

\begin{tabular}{ll} 
Method & Description \\
\hline 1 & DOE EML HASL-300, U-02-RC Modified \\
2 & DOE EML HASL-300, U-02-RC Modified
\end{tabular}

Surrogate/Tracer recovery Test

$\begin{array}{cccc}\text { Uranium-232 } & \text { Recovery } \% & \text { Acceptable Limi }\end{array}$

Notes:

The Qualifiers in this report are defined as follows :

* A quality control analyte recovery is outside of specified acceptance criteria

$<$ Result is less than value reported

$>$ Result is greater than value reported

A The TIC is a suspected aldol-condensation product

B Target analyte was detected in the associated blank

$\mathrm{BD}$ Results are either below the MDC or tracer recovery is low

C Analyte has been confirmed by GC/MS analysis

D Results are reported from a diluted aliquot of the sample

$\mathrm{H}$ Analytical holding time was exceeded

J Value is estimated

N/A Spike recovery limits do not apply. Sample concentration exceeds spike concentration by $4 \mathrm{X}$ or more
$\mathrm{R}$ Sample results are rejected

U Analyte was analyzed for, but not detected above the MDL, MDA, or LOD.

UI Gamma Spectroscopy--Uncertain identification 


\section{GENERAL ENGINEERING LABORATORIES, LLC \\ 2040 Savage Road Charleston SC 29407 - (843) 556-8171 - www.gel.com}

\section{Certificate of Analysis}

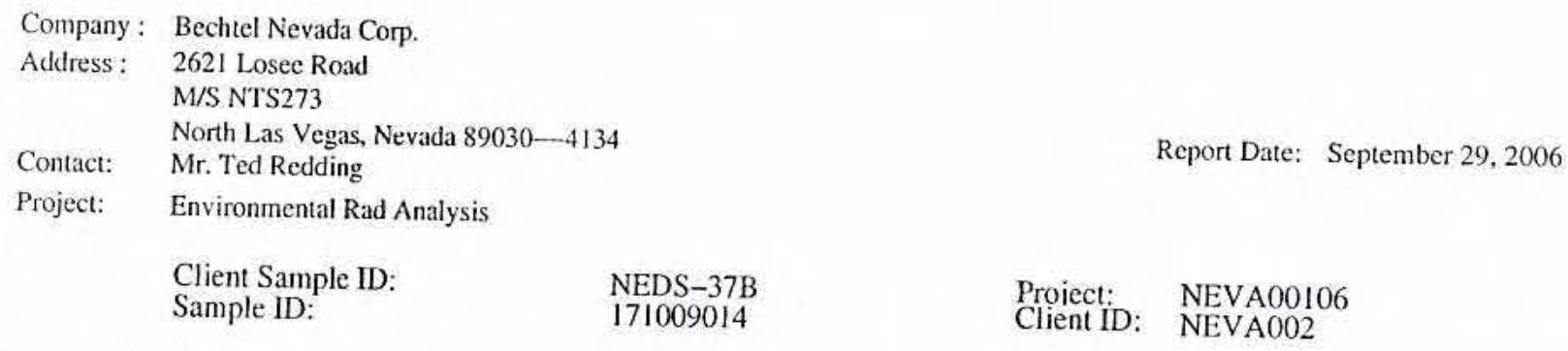

DL TPU

RL

Units DF Analyst Date

Time Batch Mts

\footnotetext{
$X$ Consult Case Narrative, Data Summary package, or Project Manager concerning this qualifier

Y QC Samples were not spiked with this compound

$\hat{R}$ RPD of sample and duplicate evaluated using $+/-\mathrm{RL}$. Concentrations are $<5 \mathrm{X}$ the $\mathrm{RL}$

h Preparation or preservation holding time was exceeded

The above sample is reported on a dry weight basis.
} 


\section{GENERAL ENGINEERING LABORATORIES, LLC \\ 2040 Savage Road Charleston SC 29407 - (843) 556-8171 - www.gel.com}

\section{Certificate of Analysis}

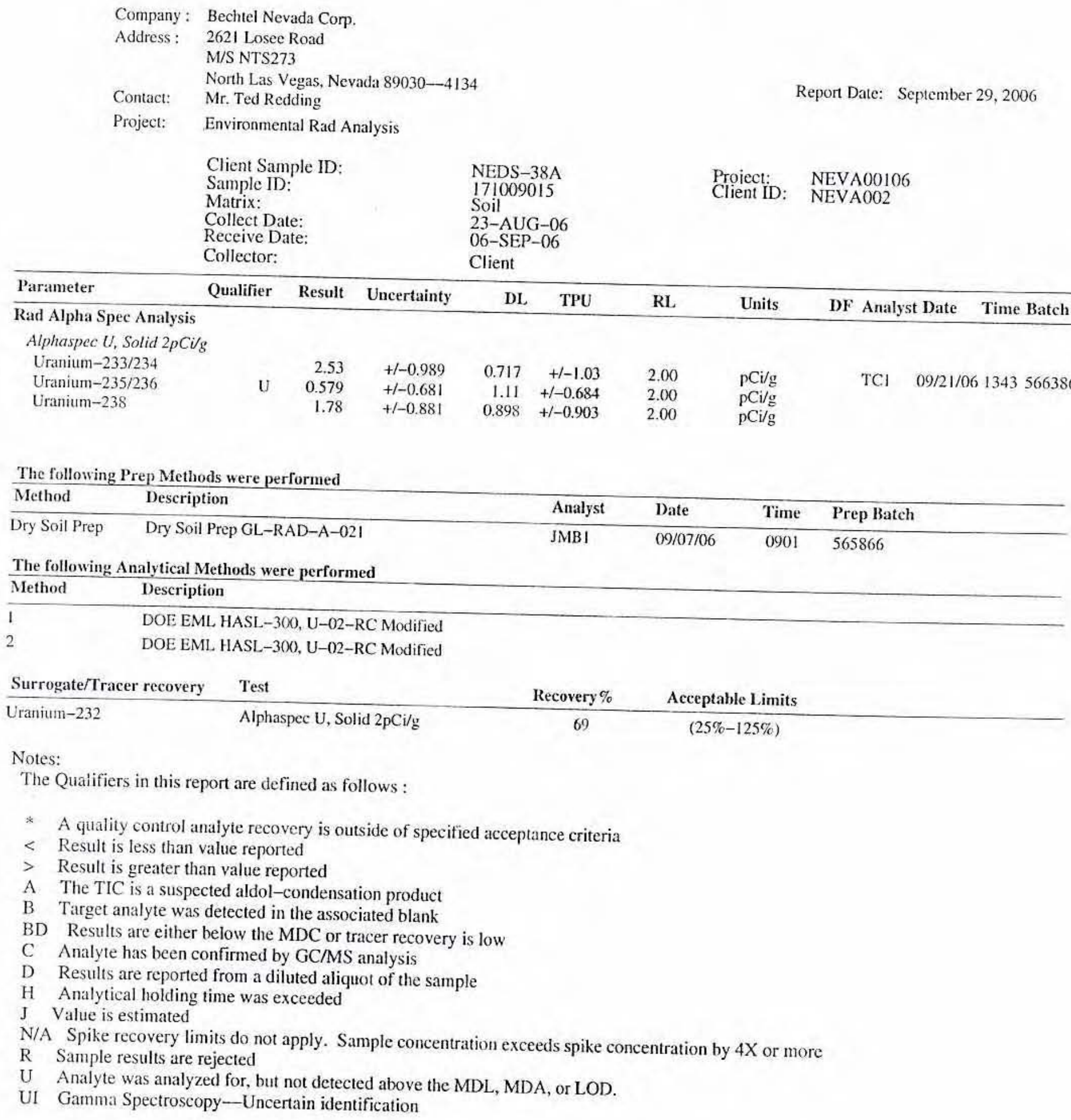




\section{GENERAL ENGINEERING LABORATORIES, LLC \\ 2040 Savage Road Charleston SC 29407 - (843) 556-8171 - www.gel.com}

\section{Certificate of Analysis}

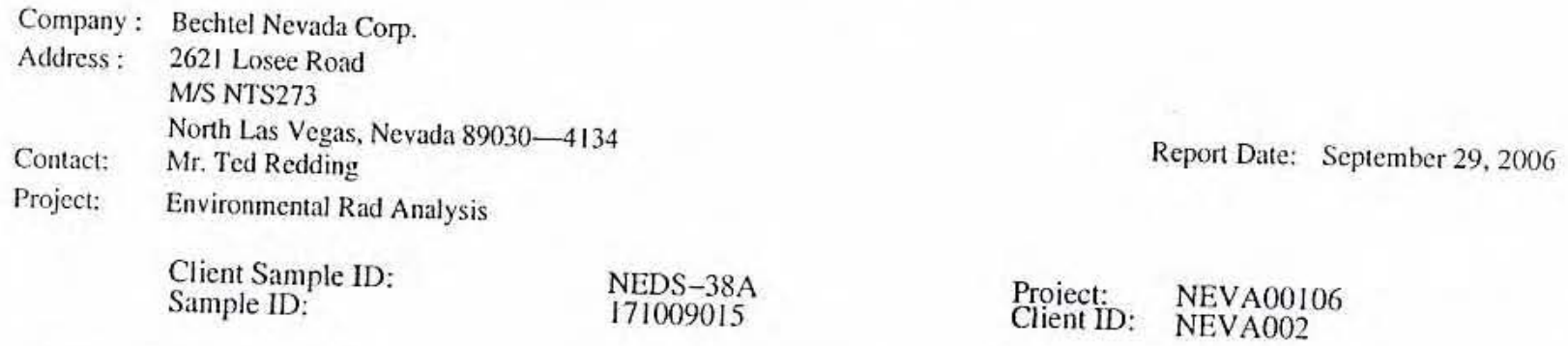

DL TPU

RL

Units

$X$ Consult Case Narrative, Data Summary package, or Project Manager concerning this qualifier

Y QC Samples were not spiked with this compound

$\hat{h}$ Preparation or preservation holding tion asing $+/-R L$. Concentrations are $<5 X$ the RL

The abaration or preservation holding time was exceeded

The above sample is reported on a dry weight basis. 


\title{
GENERAL ENGINEERING LABORATORIES, LLC \\ 2040 Savage Road Charleston SC 29407 - (843) 556-8171 - www.gel.com
}

\section{Certificate of Analysis}

\author{
Company: Bechtel Nevada Corp. \\ Address: 2621 Losee Road \\ M/S NTS273 \\ Contact: North Las Vegas, Nevada 89030-4134 \\ Project: Environmental Rad Analysis
}

Client Sample ID:

Sample ID:

Matrix:

Collect Date:

Receive Date:

Collector:

NEDS-38B
171009016
Soil
23-AUG-06
06-SEP-06
Client

Proiect: NEVA00106

Client ID: NEVA002

Client

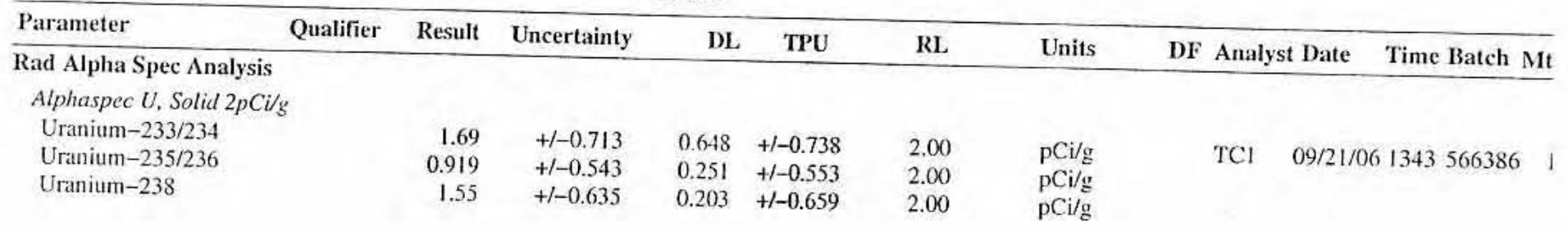

The following Prep Methods were performed

\begin{tabular}{llllll}
\hline Method & Description & Analyst & Date & Time & Prep Batch \\
\hline Dry Soil Prep & Dry Soil Prep GL-RAD-A-021 & JMB1 & $09 / 07 / 06$ & 0901 & 565866
\end{tabular}

The following Analytical Methods were performed

\begin{tabular}{ll}
\hline Method & Description \\
\hline 1 & DOE EML HASL-300, U-02-RC Modified \\
2 & DOE EML HASL-300, U-02-RC Modified
\end{tabular}

\begin{tabular}{llcc} 
Surrogate/Tracer recovery & Test & Recovery \% & Acceptable Limits \\
\hline Uranium-232 & Alphaspec U, Solid 2pCi/g & 77 & $(25 \%-125 \%)$
\end{tabular}

Notes:

The Qualifiers in this report are defined as follows :

* A quality control analyte recovery is outside of specified acceptance criteria

$<$ Result is less than value reported

$>$ Result is greater than value reported

A The TIC is a suspected aldol-condensation product

B Target analyte was detected in the associated blank

$\mathrm{BD}$ Results are either below the MDC or tracer recovery is low

C Analyte has been confirmed by GC/MS analysis

D Results are reported from a diluted aliquot of the sample

$\mathrm{H}$ Analytical holding time was exceeded

$\mathrm{J}$ Value is estimated

N/A Spike recovery limits do not apply. Sample concentration exceeds spike concentration by $4 X$ or more
$R$ Sample results are rejected

U Analyte was analyzed for, but not detected above the MDL, MDA, or LOD.

UT Gamma Spectroscopy-Uncertain identification 


\section{GENERAL ENGINEERING LABORATORIES, LLC \\ 2040 Savage Road Charleston SC 29407 - (843) 556-8171 - www.gel.com}

\section{Certificate of Analysis}

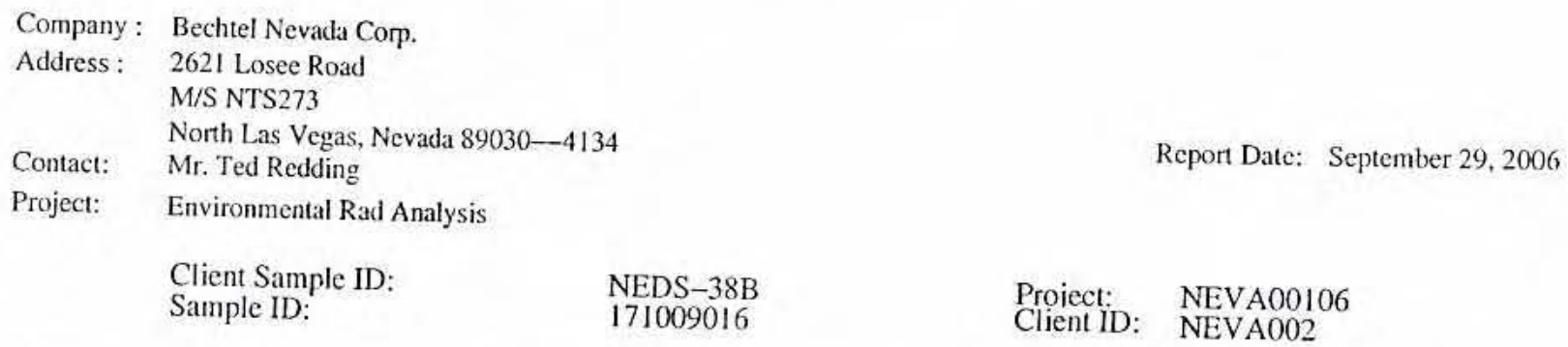

$\mathrm{X}$ Consult Case Narrative, Data Summary package, or Project Manager concerning this qualifier

Y QC Samples were not spiked with this compound

$\hat{A}$ RPD of sample and duplicate evaluated using $+/-R L$. Concentrations are $<5 X$ the RL

h Preparation or preservation holding time was exceeded

The above sample is reported on a dry weight basis. 


\section{GENERAL ENGINEERING LABORATORIES, LLC \\ 2040 Savage Road Charleston SC 29407 - (843) 556-8171 - www.gel.com}

\section{Certificate of Analysis}

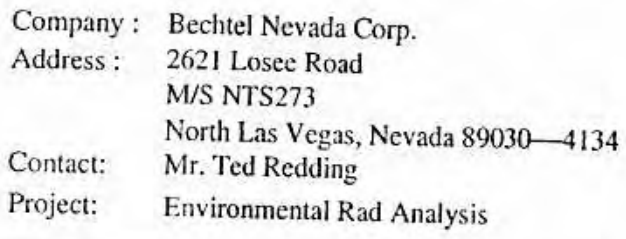

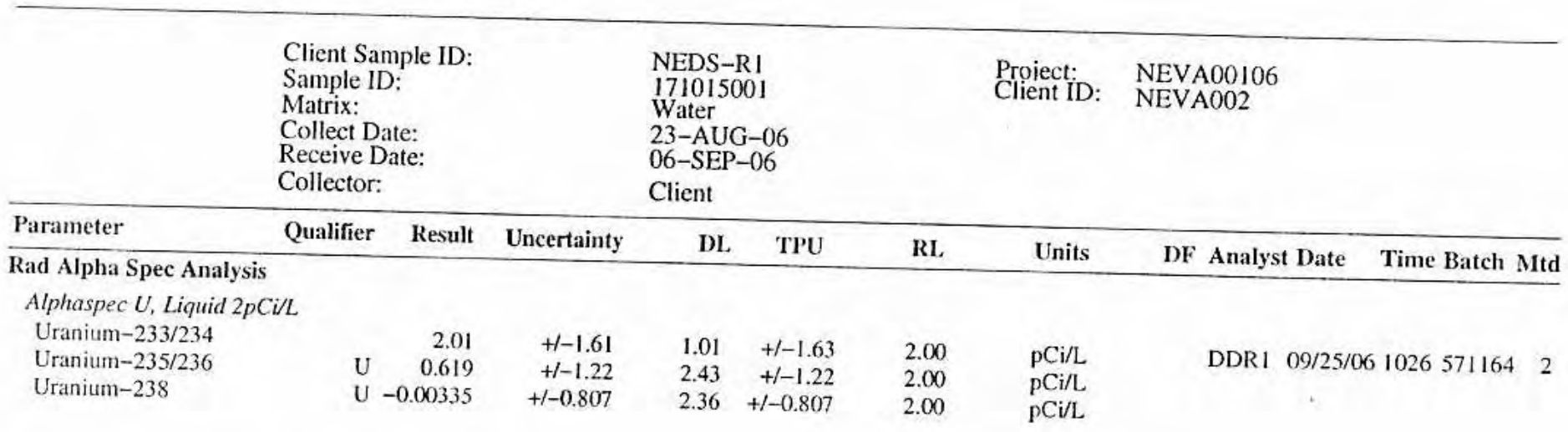

The following Analytical Methods were performed

\begin{tabular}{ll}
\hline Method & Description \\
\hline 1 & DOE EML HASL-300, U-02-RC Modified \\
2 & DOE EML HASL-300, U-02-RC Modified \\
3 & DOE EML HASL-300, U-02-RC Modified
\end{tabular}

\begin{tabular}{llcc} 
Surrogate/Tracer recovery & Test & Recovery $\%$ & Acceptable Limits \\
\hline Uranium-232 & Alphaspec U, Liquid 2pCilL & 35 & $(25 \%-125 \%)$
\end{tabular}

Notes:

The Qualifiers in this report are defined as follows :

* A quality control analyte recovery is outside of specified acceptance criteria

$<$ Result is less than value reported

$>$ Result is greater than value reported

A The TIC is a suspected aldol-condensation product

B Target analyte was detected in the associated blank

BD Results are either below the MDC or tracer recovery is low

C Analyte has been confirmed by GC/MS analysis

D Results are reported from a diluted aliquot of the sample

$\mathrm{H}$ Analytical holding time was exceeded

$\mathrm{J}$ Value is estimated

N/A Spike recovery limits do not apply. Sample concentration exceeds spike concentration by $4 X$ or more
$\mathrm{R}$ Sample results are rejected

$\mathrm{U}$ Analyte was a

UI Gamma Spectrosed the MDL, MDA, or LOD.

-Uncertain identification

Y QC Samples were not spiked with this compound 


\section{GENERAL ENGINEERING LABORATORIES, LLC}

2040 Savage Road Charleston SC 29407 - (843) 556-8171 - www.gel.com

\section{Certificate of Analysis}

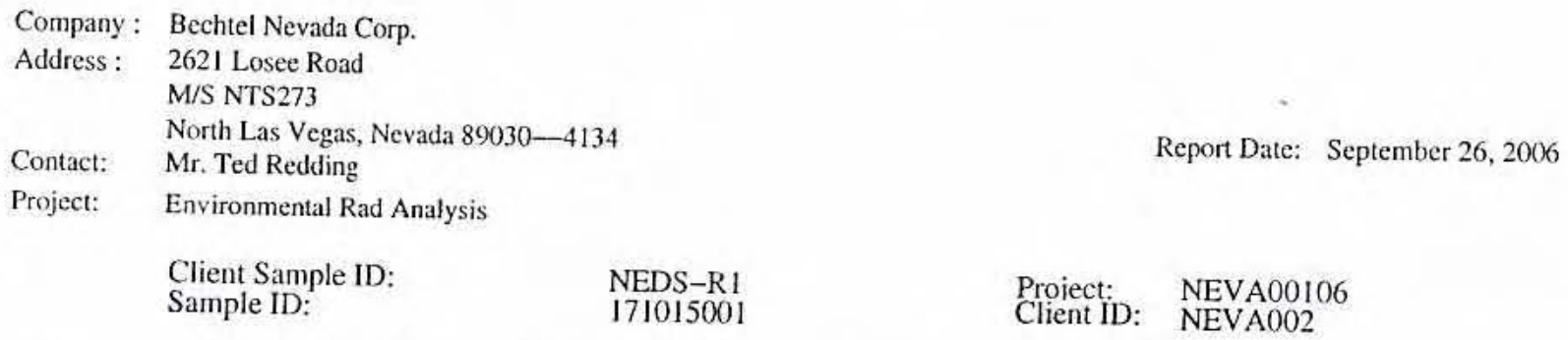

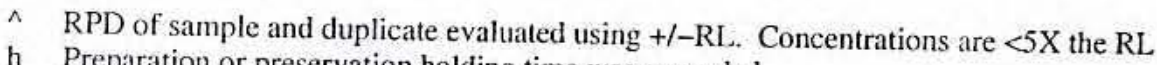

$\mathrm{h}$ Preparation or preservation holding time was exceeded

The above sample is reported on an "as received" basis. 


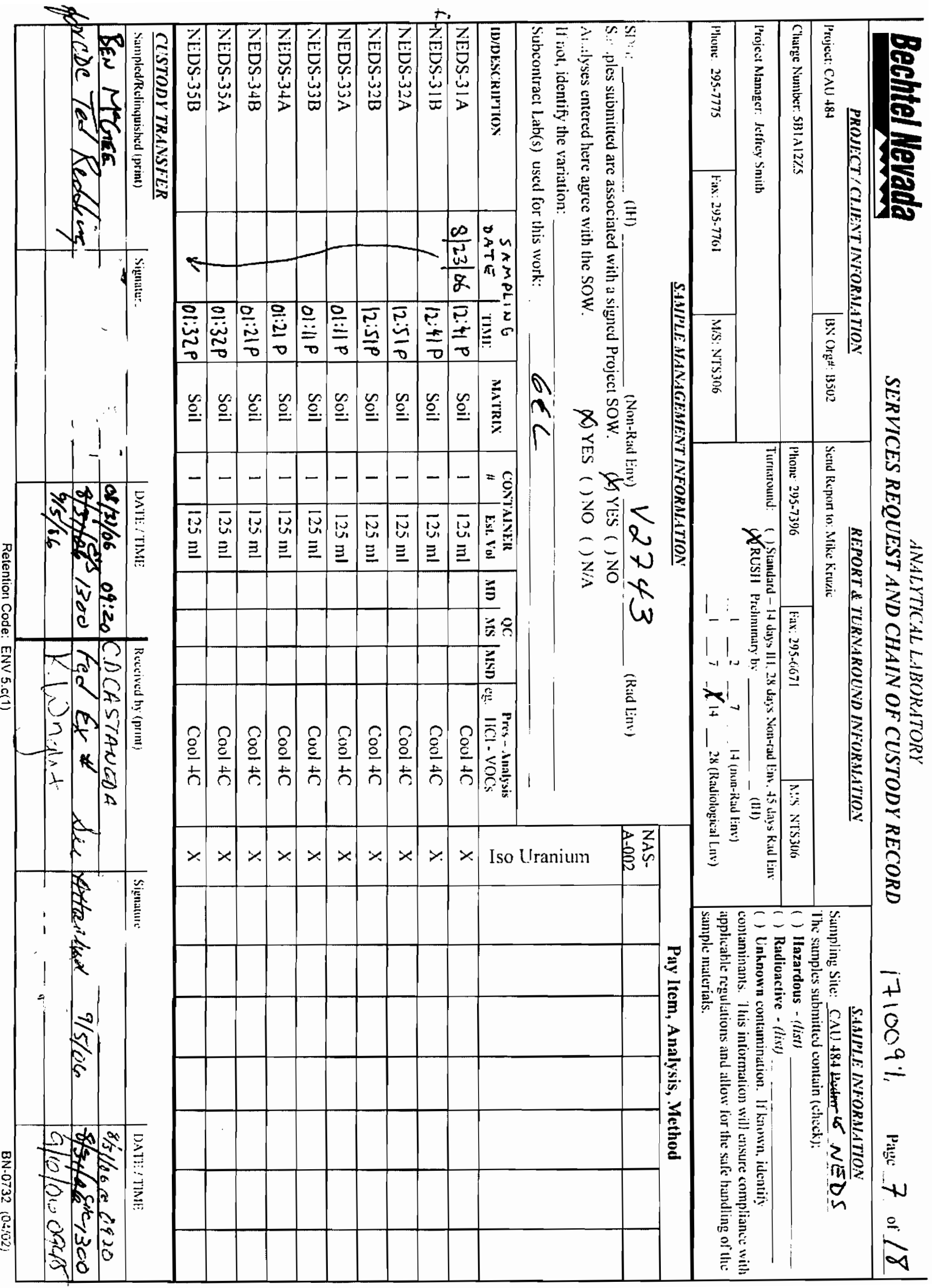




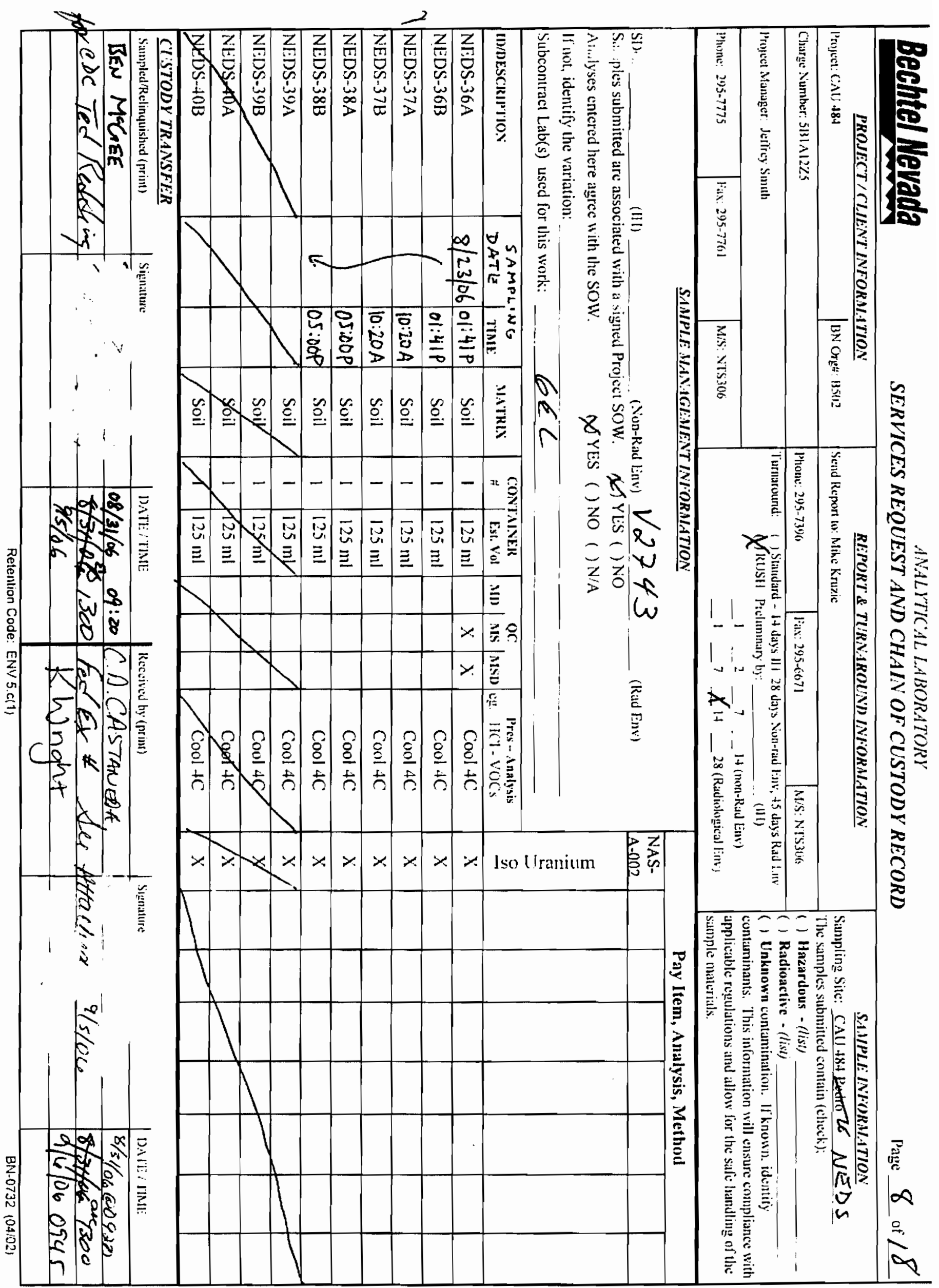




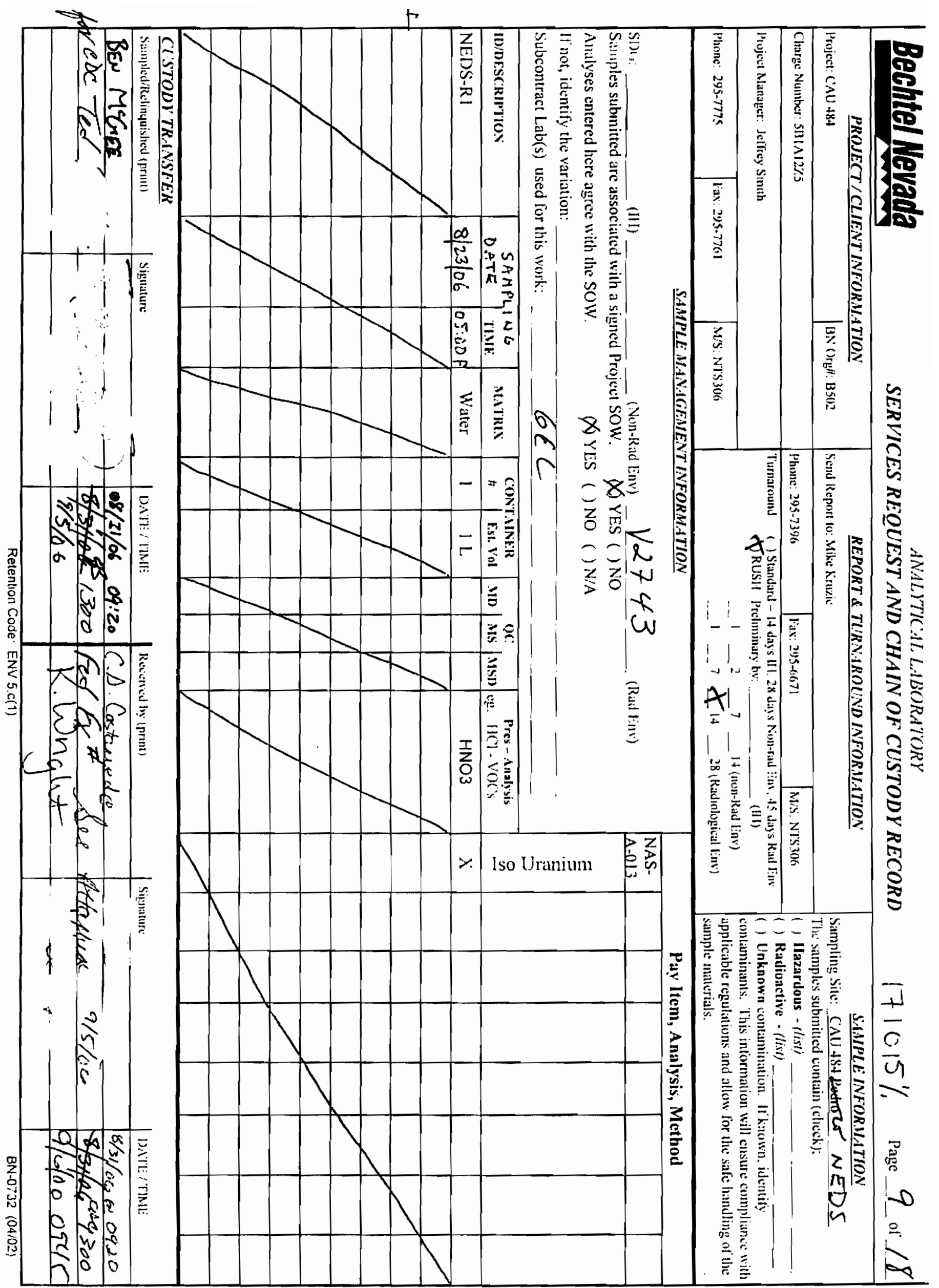




\section{INVESTIGATION (GEOPROBE ${ }^{\circledR}$ ) SOIL SAMPLE ANALYTICAL RESULTS \\ CAS TA-52-006-TAPL DEPLETED URANIUM SITE}

\section{Chemical Data}




\section{U.S. EPA}

INORGANIC ANALYSES DATA SHEET

EPA SAMPLE NO.

$$
01 \mathrm{~A}
$$

Lab Name: LIONVILLE_LABORATORY

Lab Code: LVLI

Matrix (soil/water): SOIL

Level (low/med):

은 Solids:
Contract: 60052

SAS NO.:

Date Received: 09/01/06

Concentration Units (ug/L or $\mathrm{mg} / \mathrm{kg}$ dry weight) : MG/KG

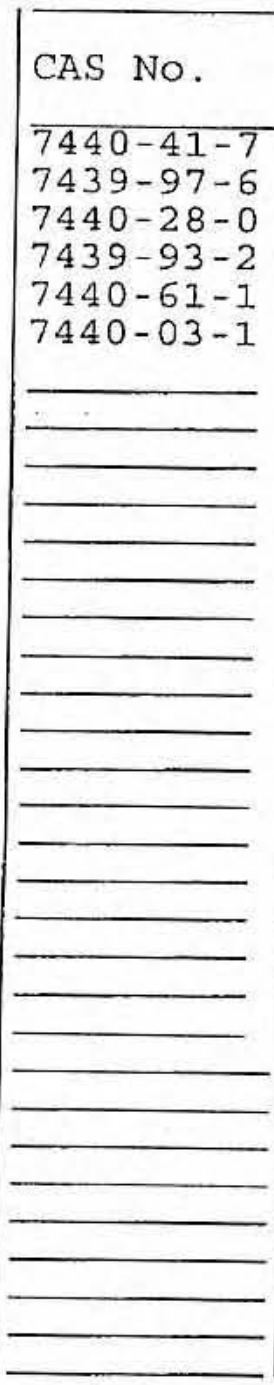

Jolor Before: Color After:

Comments :

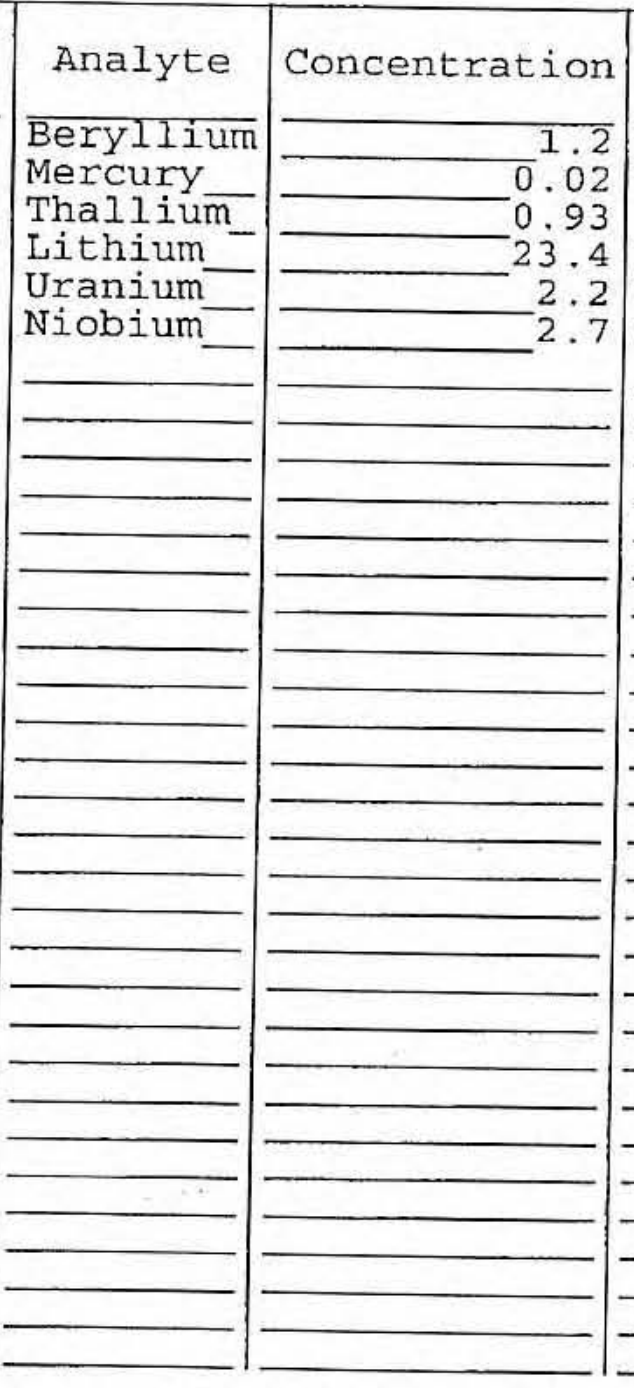

Clarity Before: Clarity After:

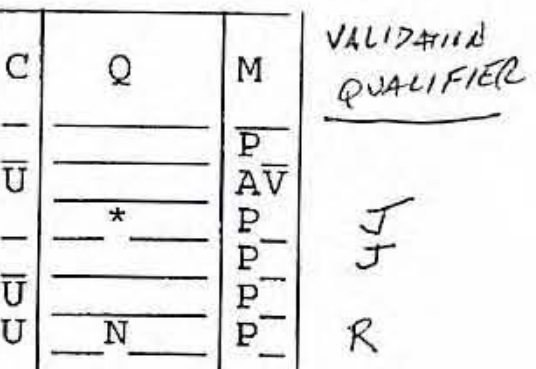

$R$

DUS - 01A 
EPA SAMPLE NO.

Lab Name: LIONVILLE_LABORATORY

Lab Code: LVLI _ Case No.: DUS

Matrix (soil/water): SOIL

Level (low/med): LOW

Solids:
Contract: 60052

SAS NO.:

Lab Sample ID: 0609L861-002

Date Received: 09/01/06

Concentration Units (ug/L or $\mathrm{mg} / \mathrm{kg}$ dry weight): MG/KG

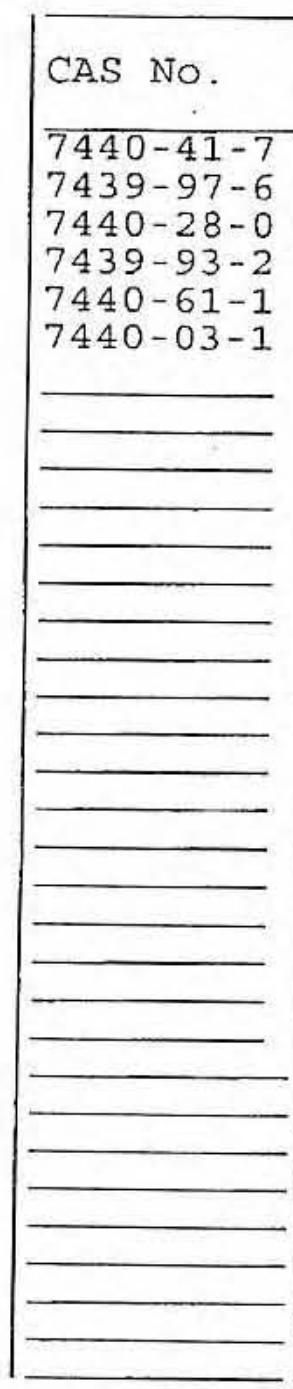

Color Before: Color After:

Comments :

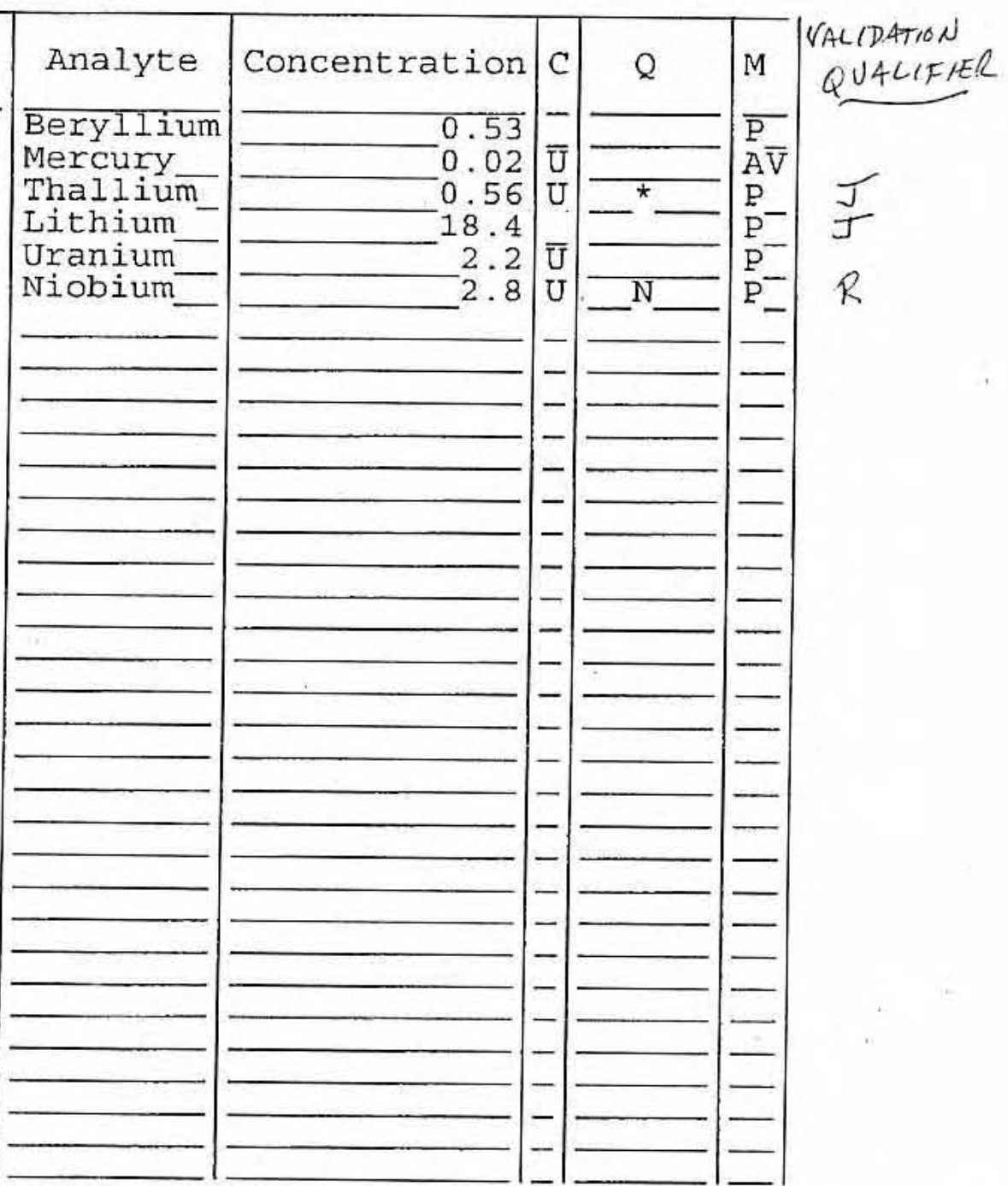

Clarity Before: Clarity After:
Texture:

Artifacts: 
Lab Name: LIONVILLE_LABORATORY

Lab Code: LVLI Matrix (soil/water): SOIL Level (low/med): \% Solids:

LOW

$-92 \overline{.8}$
Contract: 60052

SAS No.:

Lab Sample ID: 0609L861-003

Date Received: 09/01/06

Concentration Units (ug/L or $\mathrm{mg} / \mathrm{kg}$ dry weight): $\mathrm{MG} / \mathrm{KG}$

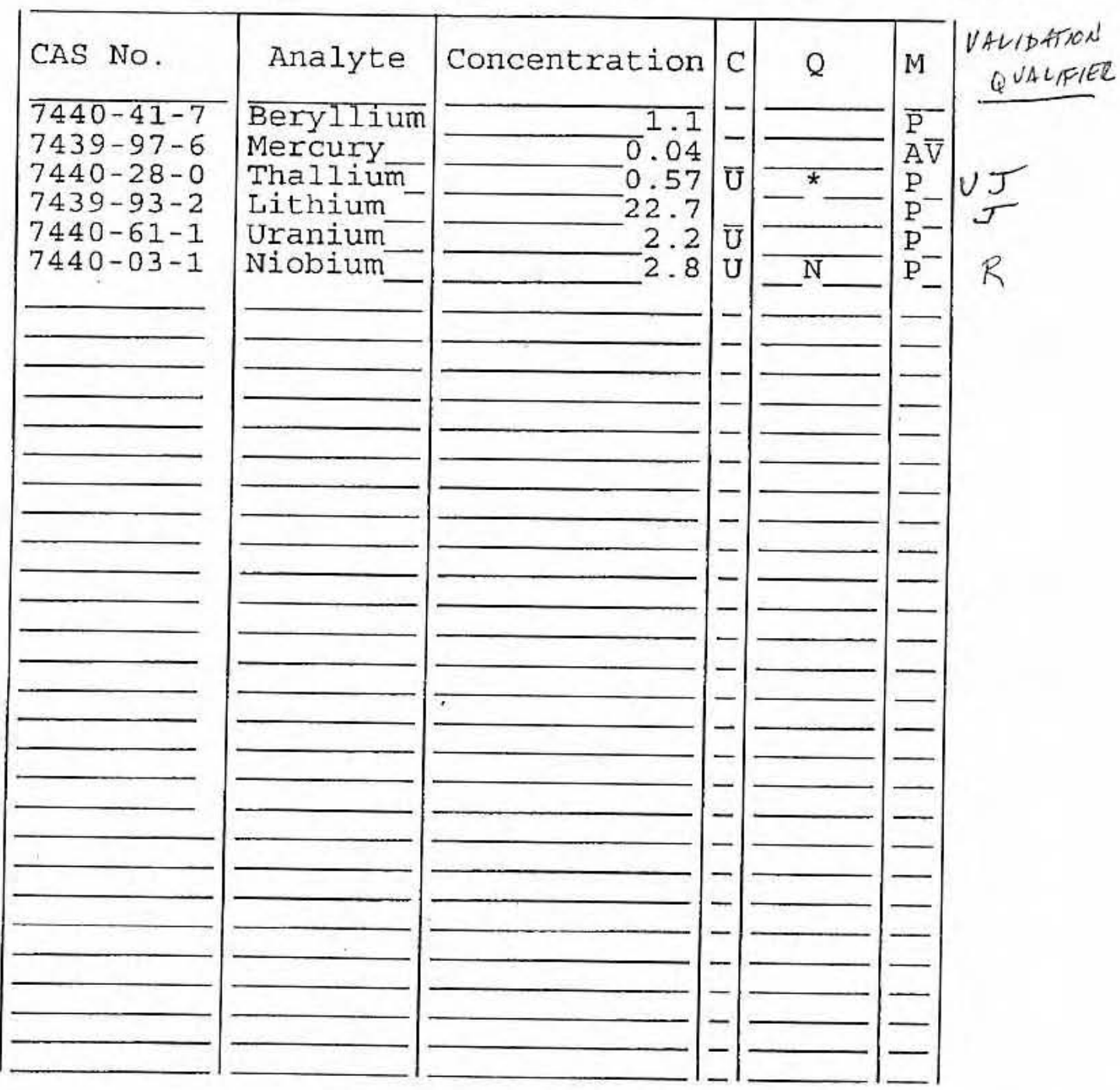

Color Before: Color After:

Comments :
Clarity Before: Clarity After:
Texture: Artifacts: 
U.S. EPA

INORGANIC ANALYYSES DATA SHEET

EPA SAMPLE NO.

Lab Name: LIONVILLE_LABORATORY

Lab Code: LVLI

Matrix (soil/water):

Level (low/med) :

: Solids:

Contract: 60052

SAS No.:

$02 B$

SOIL
LOW -
-90.2

Lab Sample

Date Received: 09/01/06

Concentration Units (ug/L or $\mathrm{mg} / \mathrm{kg}$ dry weight): MG/KG

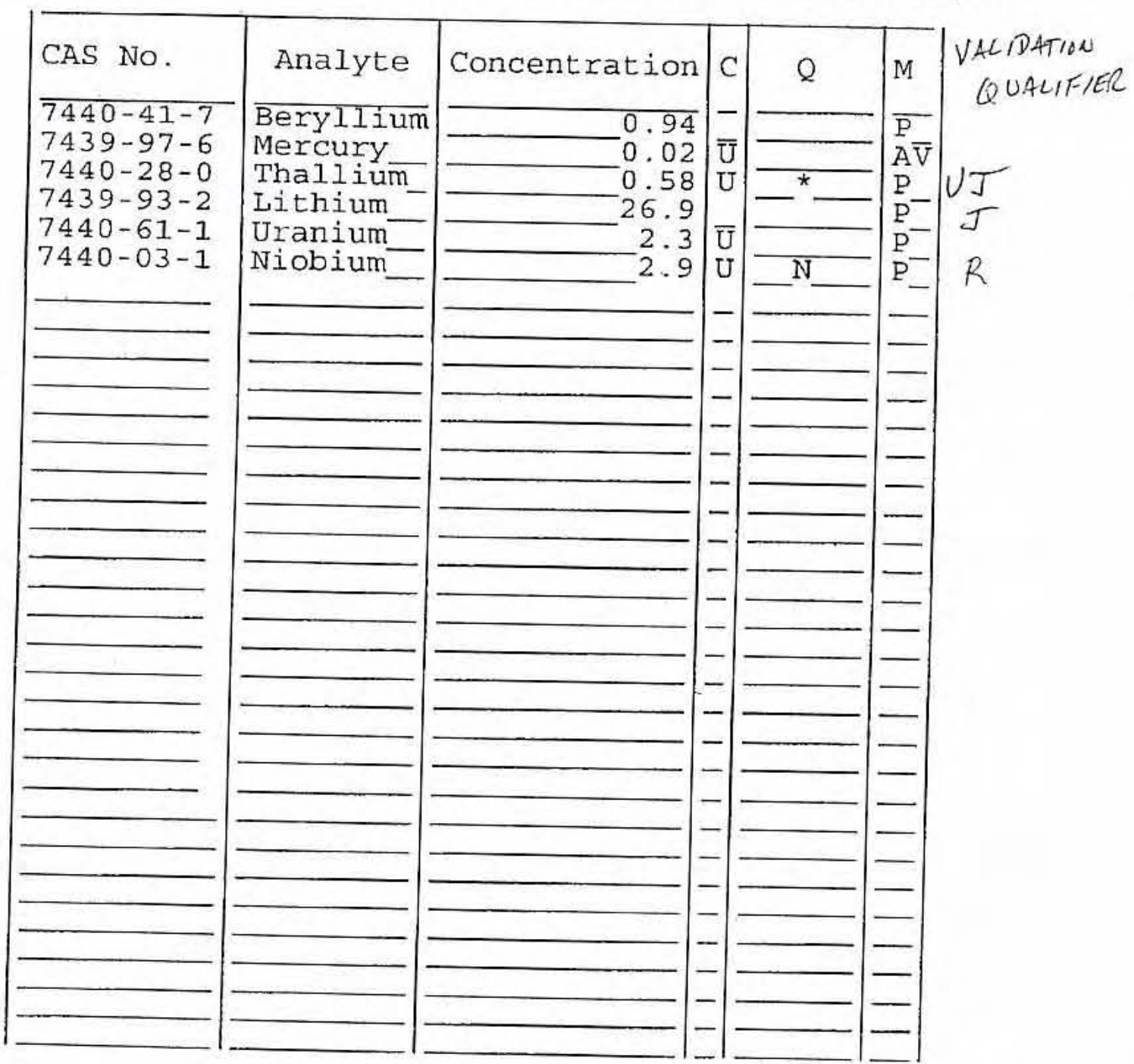

Color Before:

Color After:

Clarity Before:

Texture:

Clarity After:

Artifacts :

Comments :

DUS-02B 
U.S. EPA

1

INORGANIC ANALYSES DATA SHEET
Lab Name: LIONVILLE LABORATORY

Matrix (soil/water): SoIL

Level (low/med): LOW

\% Solids:
Contract: 60052

SAS No.:
EPA SAMPLE NO.

03A

SDG NO.: 01A

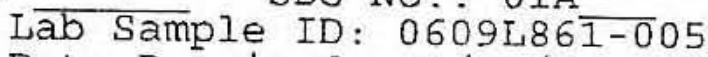

Date Received: 09/01/06

Concentration Units (ug/L or $\mathrm{mg} / \mathrm{kg}$ dry weight): MG/KG

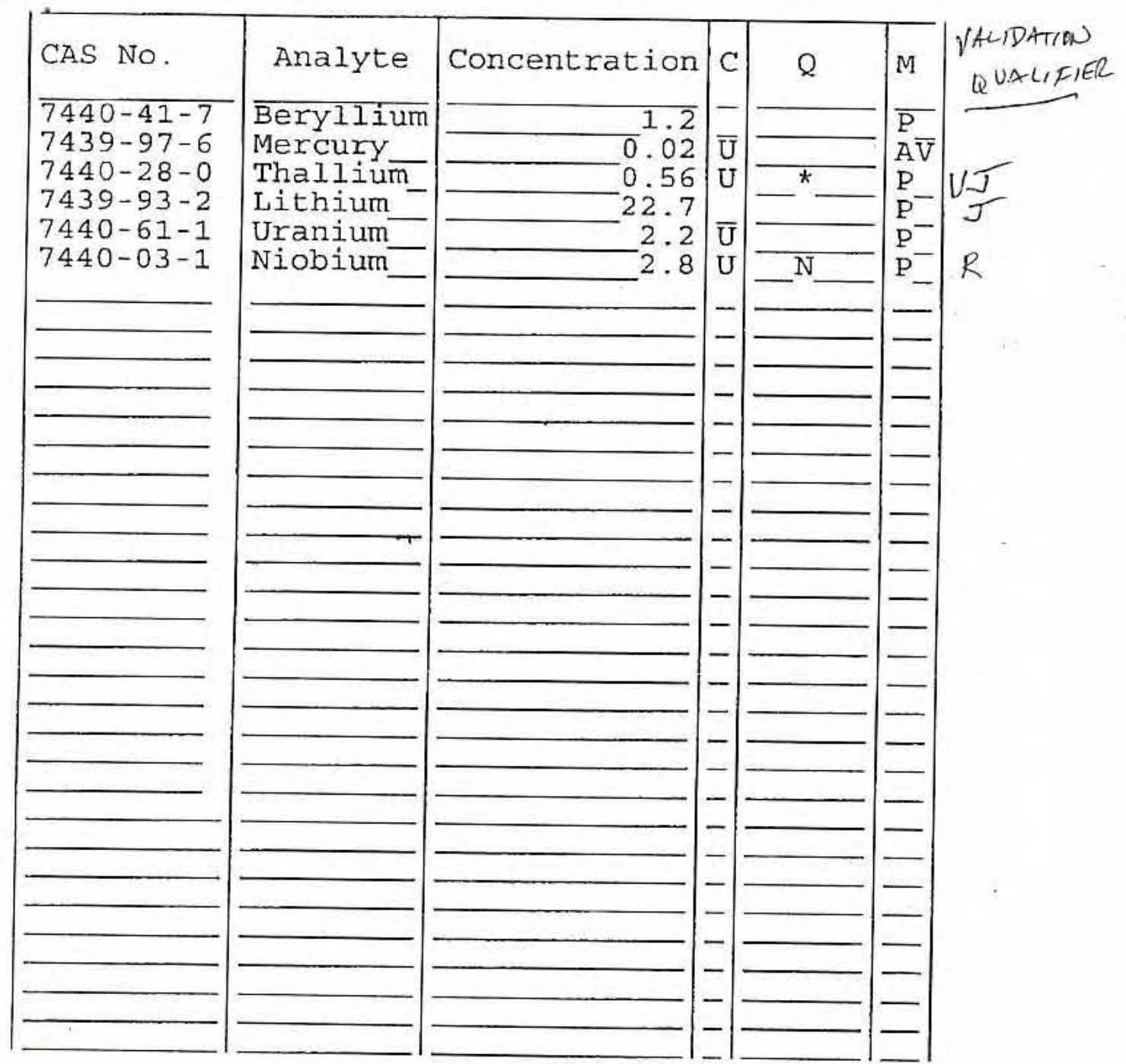

Color Before: Color After:

Clarity Before: Clarity After:
Texture:

Artifacts:

Comments :

DUS-03A 
U.S. EPA

INORGANIC ANALY $\stackrel{1}{\text { YSES DATA SHEET }}$

EPA SAMPLE NO.

Iab Name: LIONVILLE_LABORATORY

Lab Code: LVLI

Matrix (soil/water): SOIL

Level (low/med):

$\div$ Solids : LOW
Contract: 60052

SAS NO. :

O3B

SDG NO.: 01A

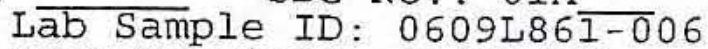

Date Received: 09/01/06

Concentration Units (ug/L or $\mathrm{mg} / \mathrm{kg}$ dry weight): MG/KG

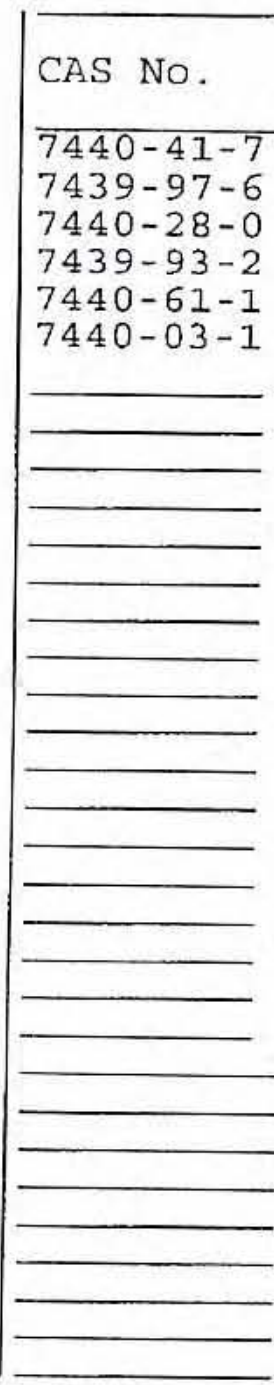

Color Before: Color After:

\section{Comments :}

DUS-03B

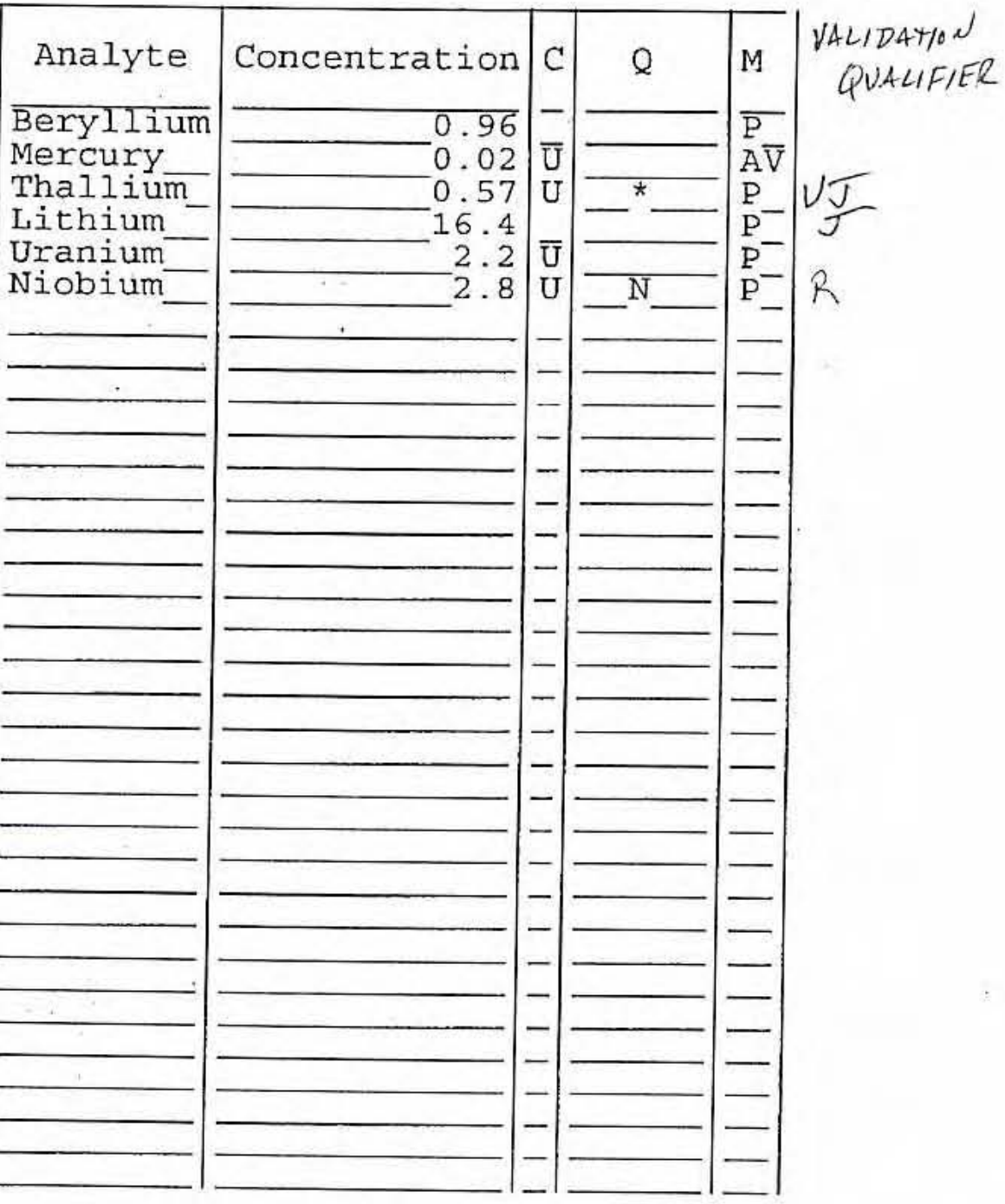

Clarity Before:

Clarity After:
Texture:

Artifacts : 


\section{U.S. EPA}

1

INORGANIC ANALYSSE DATA SHEET
EPA SAMPLE NO.

\section{O4A}

Contract: 60052

SAS NO.:

Lab Sample ID: 0609L861-

Date Received: 09/01/06
Lab Name: LIONVILLE_LABORATORY

Matrix (soil/water): SOIL

Level (low/med): LOW

: Solids:

Concentration Units (ug/L or $\mathrm{mg} / \mathrm{kg}$ dry weight): MG/KG

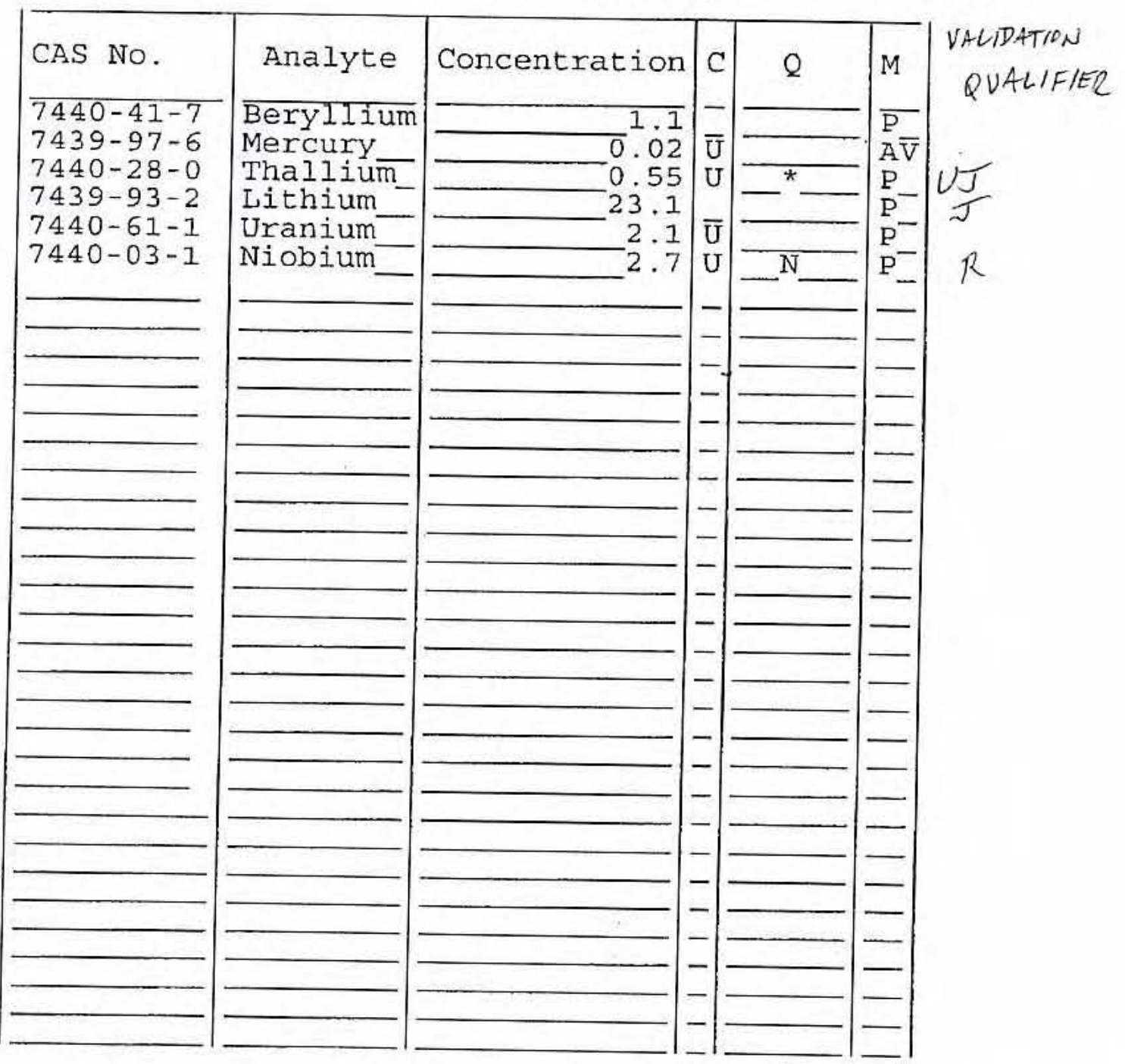

Color Before: Color After:

Comments :
Clarity Before: Clarity After:
Texture:

Artifacts: 
1

INORGANIC ANALYSES DATA SHEET
EPA SAMPLE NO.

Contract: 60052

SAS NO.:

SDG No.: 01A

04B

Lab Code: LVII_LABORATORY

Matrix (soil/water): SoIL

Level (low/med): LOW

: Solids:
Lab Sample ID: 0609L86 $\overline{1-0} 08$

Date Received: 09/01/06

\section{Concentration Units (ug/L or $\mathrm{mg} / \mathrm{kg}$ dry weight): MG/KG}

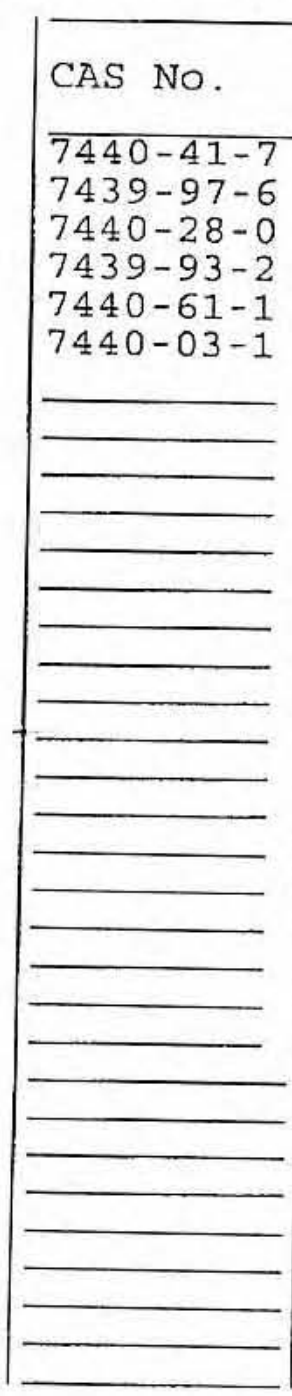

Jolor Before:

Jolor After:

\section{Jomments :}

DUS-0 $4 B$

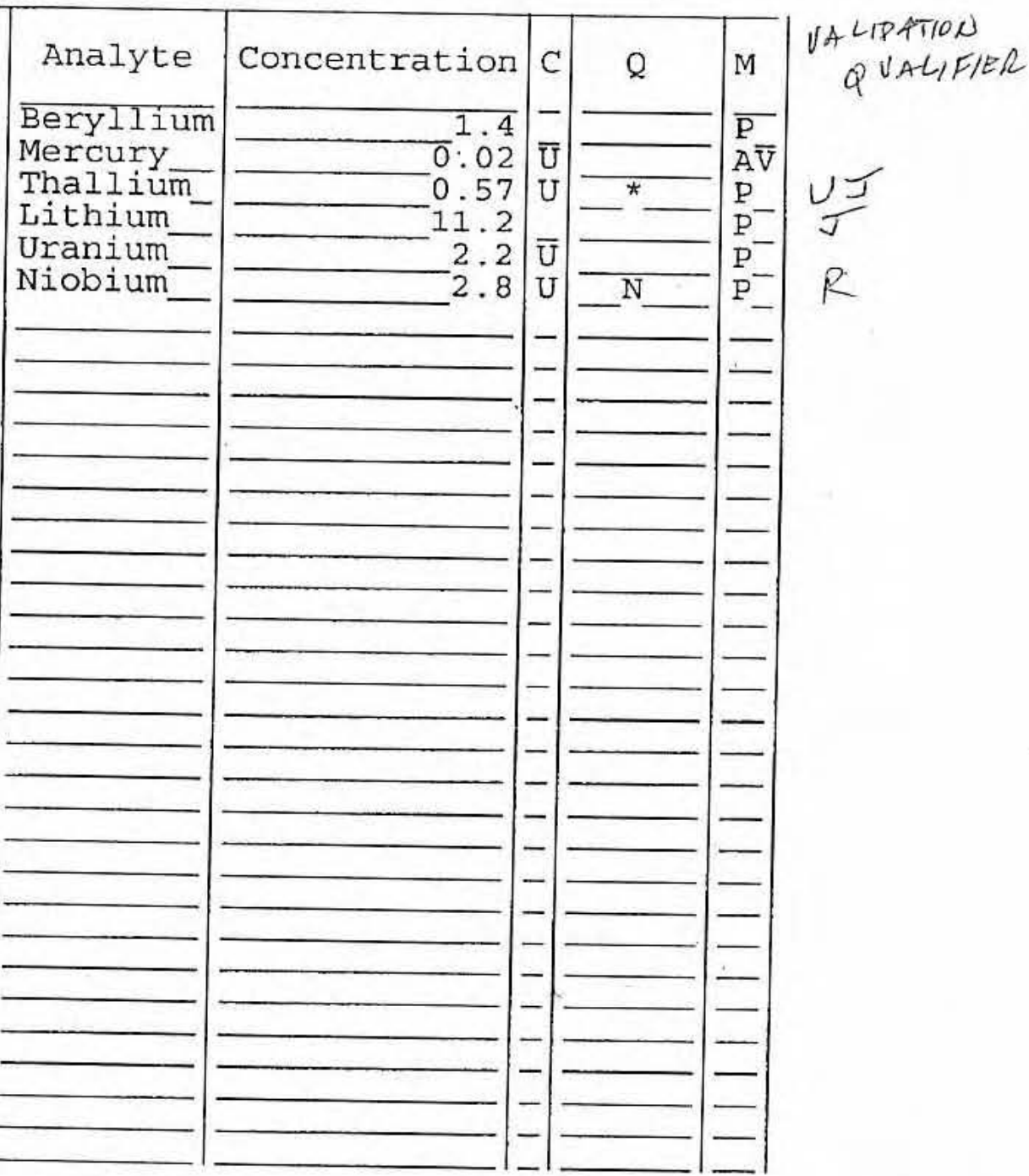

Clarity Before: Clarity After:
Texture:

Artifacts : 


\section{INORGANIC ANALYSES DATA SHEET}

Lab Name: LIONVILLE_LABORATORY

Lab Code: LVLI Matrix (soil/water): SOIL Level (low/med) : : Solids :
Contract: 60052

SAS NO.:

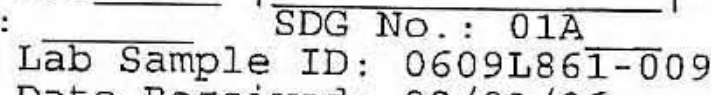

Date Received: 09/01/06
EPA SAMPLE NO.

$$
\text { 05A }
$$

\section{Concentration Units (ug/L or $\mathrm{mg} / \mathrm{kg}$ dry weight): MG/KG}

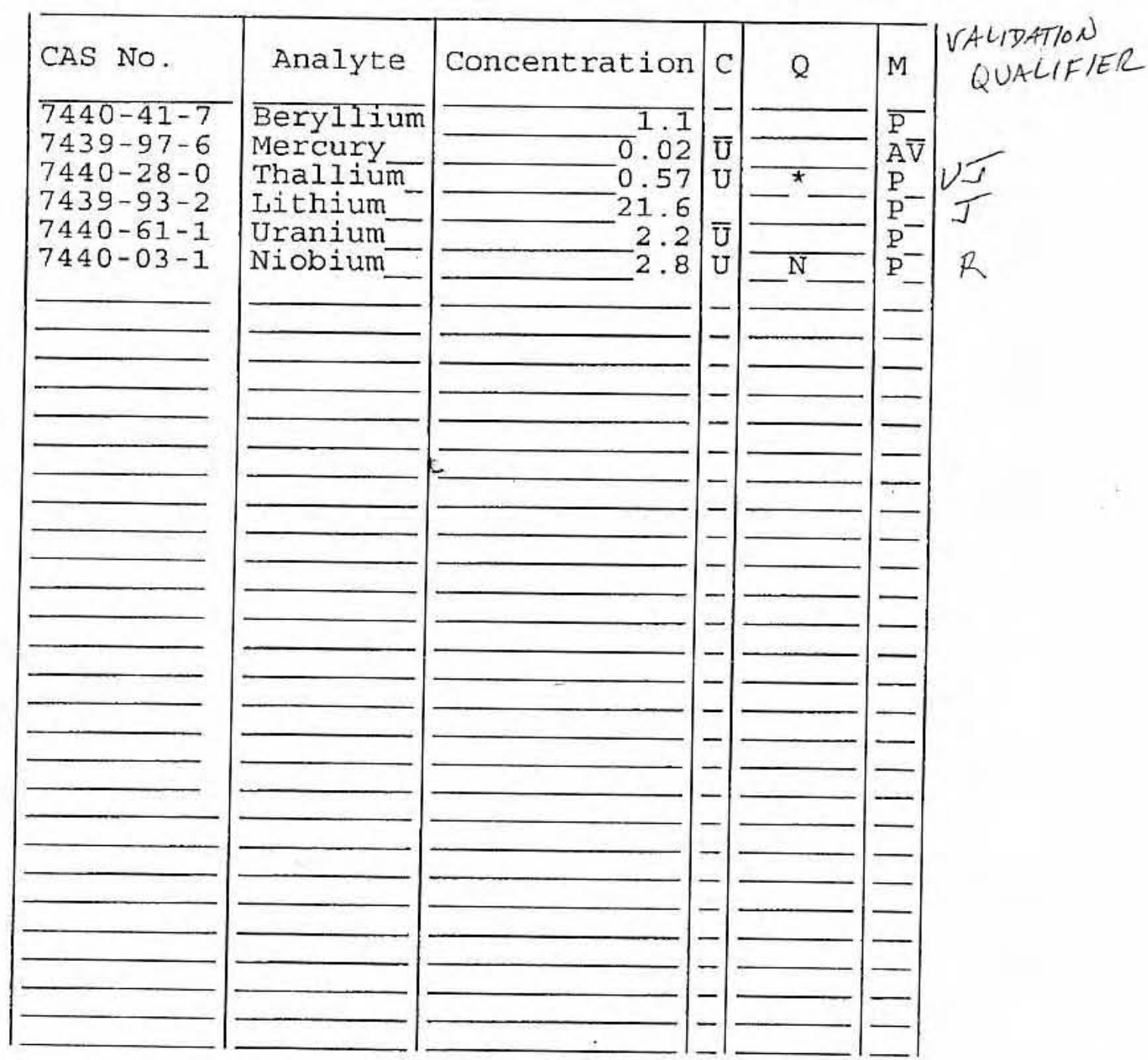

Color Before: Color After:

\section{Comments:}

DUS - 05A
Clarity Before:

Clarity After:
Texture: Artifacts: 


\section{U.S. EPA}

1

INORGANIC ANALYSES DATA SHEET
EPA SAMPLE NO.

$$
\text { 05B }
$$

Contract : 60052

SAS NO. :

SDG NO : 01A

Case No: : DUS

Lab Code: LVLI

Matrix (soil/water): SoIL

Level. (low/med):

Solids:

$89 \overline{.0}$
-8

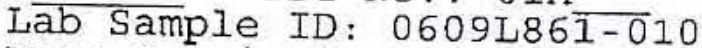

Date Received: 09/01/06

Concentration Units (ug/L or $\mathrm{mg} / \mathrm{kg}$ dry weight): MG/KG

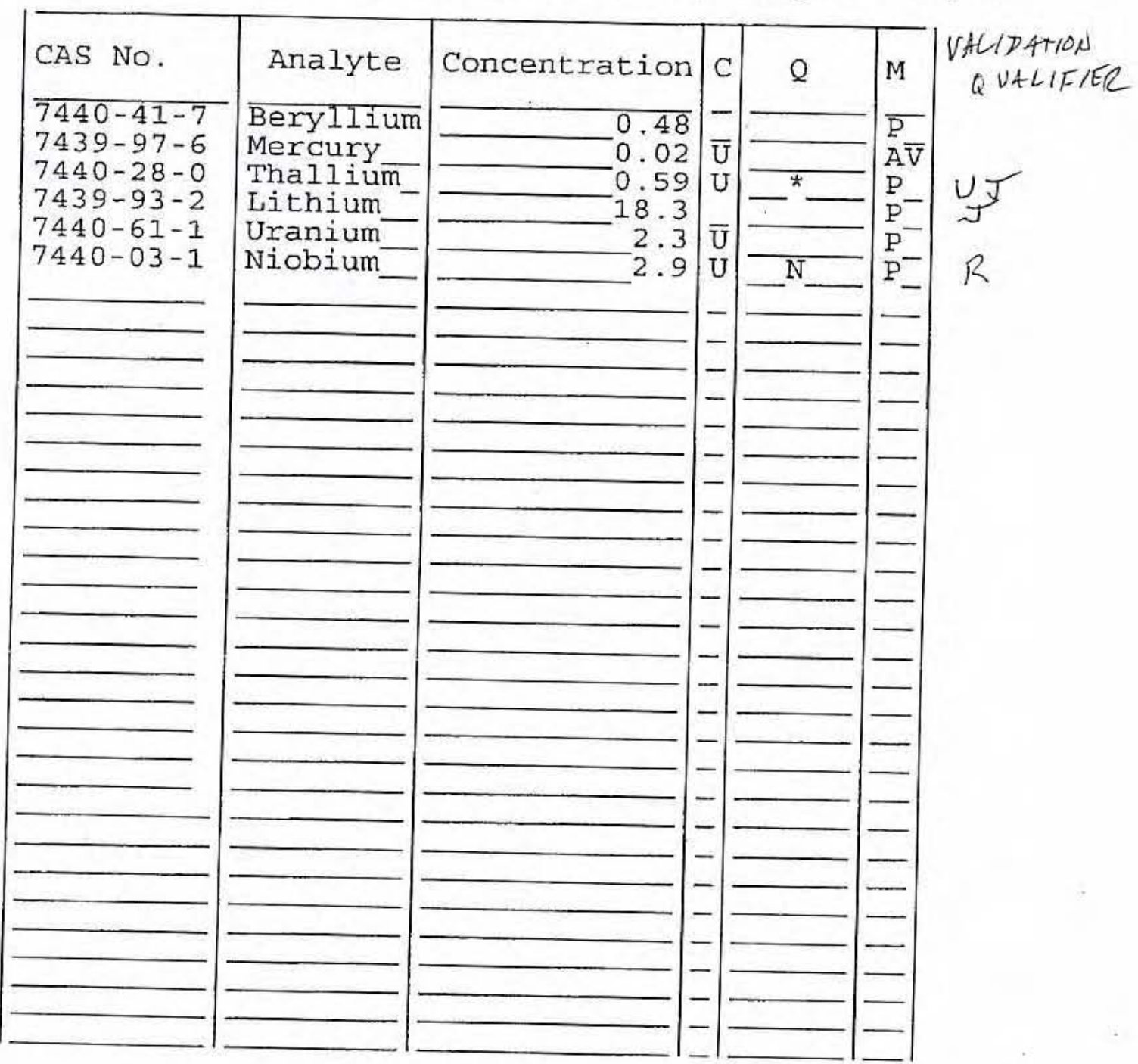

Color Before:

Color After:

\section{Comments :}

Clarity Before:

Clarity After:
Texture:

Artifacts: DUS-05B 
Lab Name: LIONVILLE_LABORATORY

Lab Code: LVLI

Matrix (soil/water): SOIL

Level (low/med): LOW

\% Solids:
Contract: 60052

SAS NO.:

$-93 . \overline{8}$
SDG NO.: 01A

$06 \mathrm{~A}$

Lab Sample ID: 0609L86/1-011

Date Received: 09/01/06

Concentration Units (ug/L or $\mathrm{mg} / \mathrm{kg}$ dry weight): MG/KG

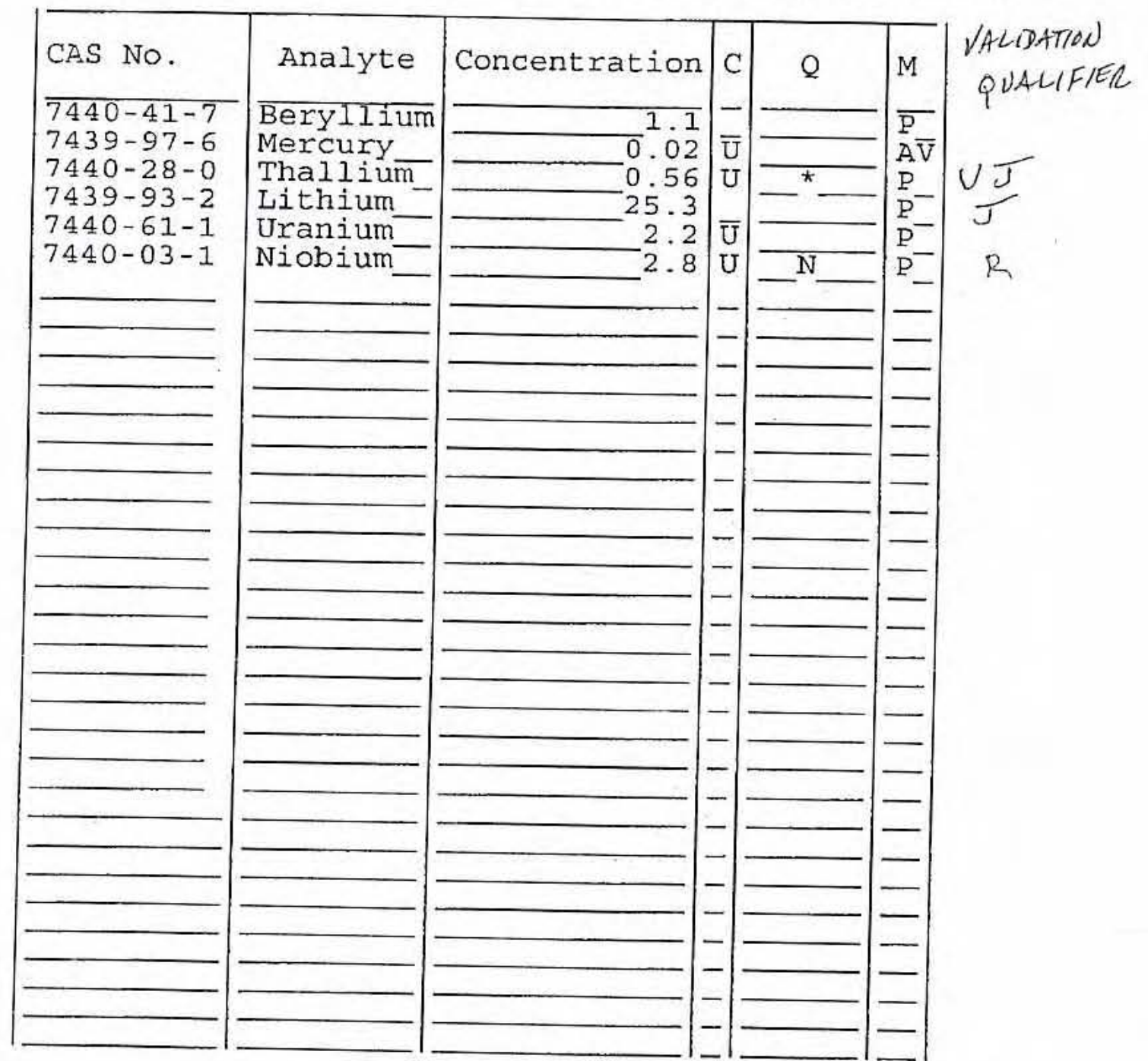

Color Before: Color After:

Comments :
Clarity Before:

Clarity After:
Texture:

Artifacts: DUS-06A 
U.S. EPA

1

INORGANIC ANALYSES DATA SHEET
EPA SAMPLE NO.

$$
\text { 06B }
$$

Lab Name: LIONVILLE_LABORATORY

Lab Code: LVLI

Matrix (soil/water): SOIL

Level (low/med)

\% Solids :
Contract: 60052

SAS NO. :

SDG NO.: 01A

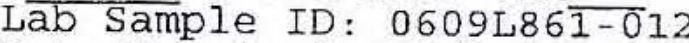

Date Received: 09/01/06

Concentration Units (ug/L or $\mathrm{mg} / \mathrm{kg}$ dry weight): MG/KG

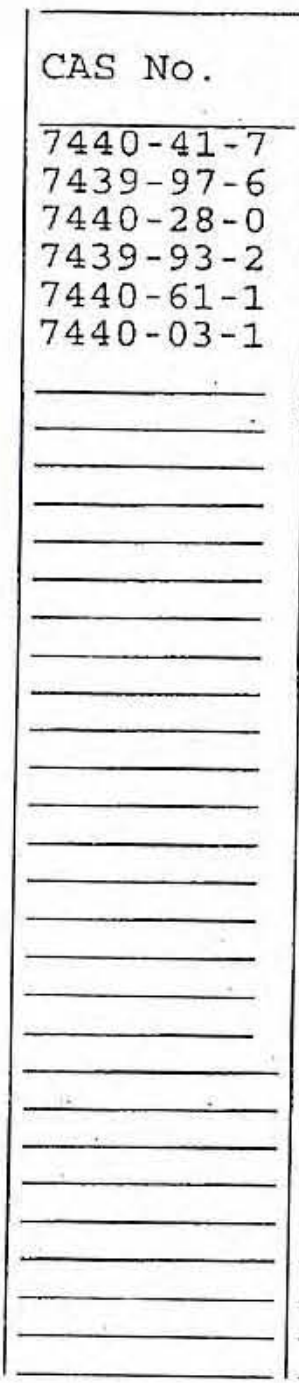

Color Before: Color After:

Comments :

DUS - $06 \mathrm{~B}$

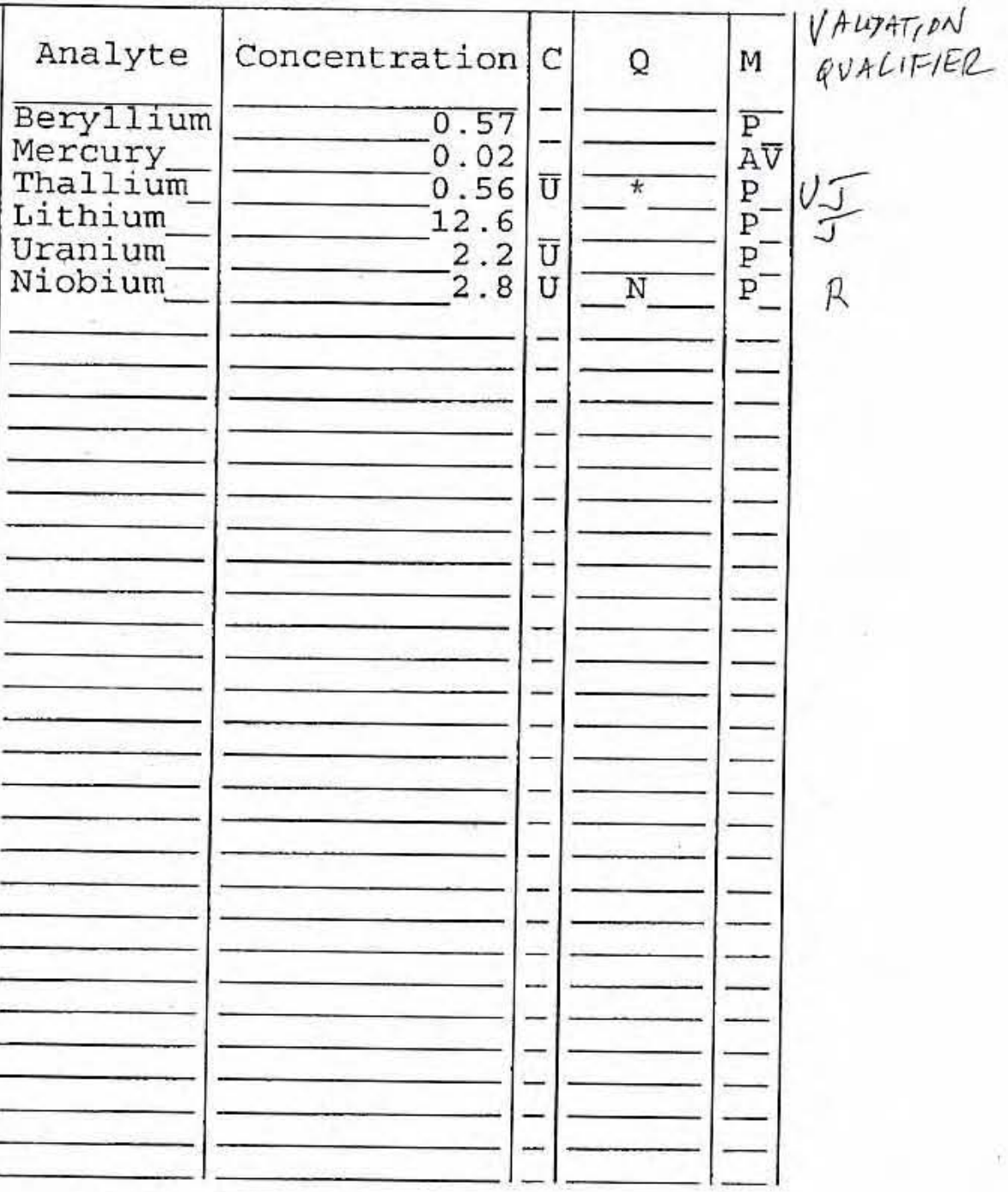

Clarity Before:

Clarity After:
Texture: Artifacts: 


\section{U.S. EPA}

\section{INORGANIC ANALYSES DATA SHEET}

EPA SAMPLE NO.

Lab Name: LIONVILLE_LABORATORY

Lab code: LVLI Matrix (soil/water): SOIL Level (low/med): LOW $\div$ Solids:
Contract: 60052

SAS NO.:

Lab Sample ID: 0609L861-013

Date Received: 09/01/06

Concentration Units (ug/L or $\mathrm{mg} / \mathrm{kg}$ dry weight): MG/KG

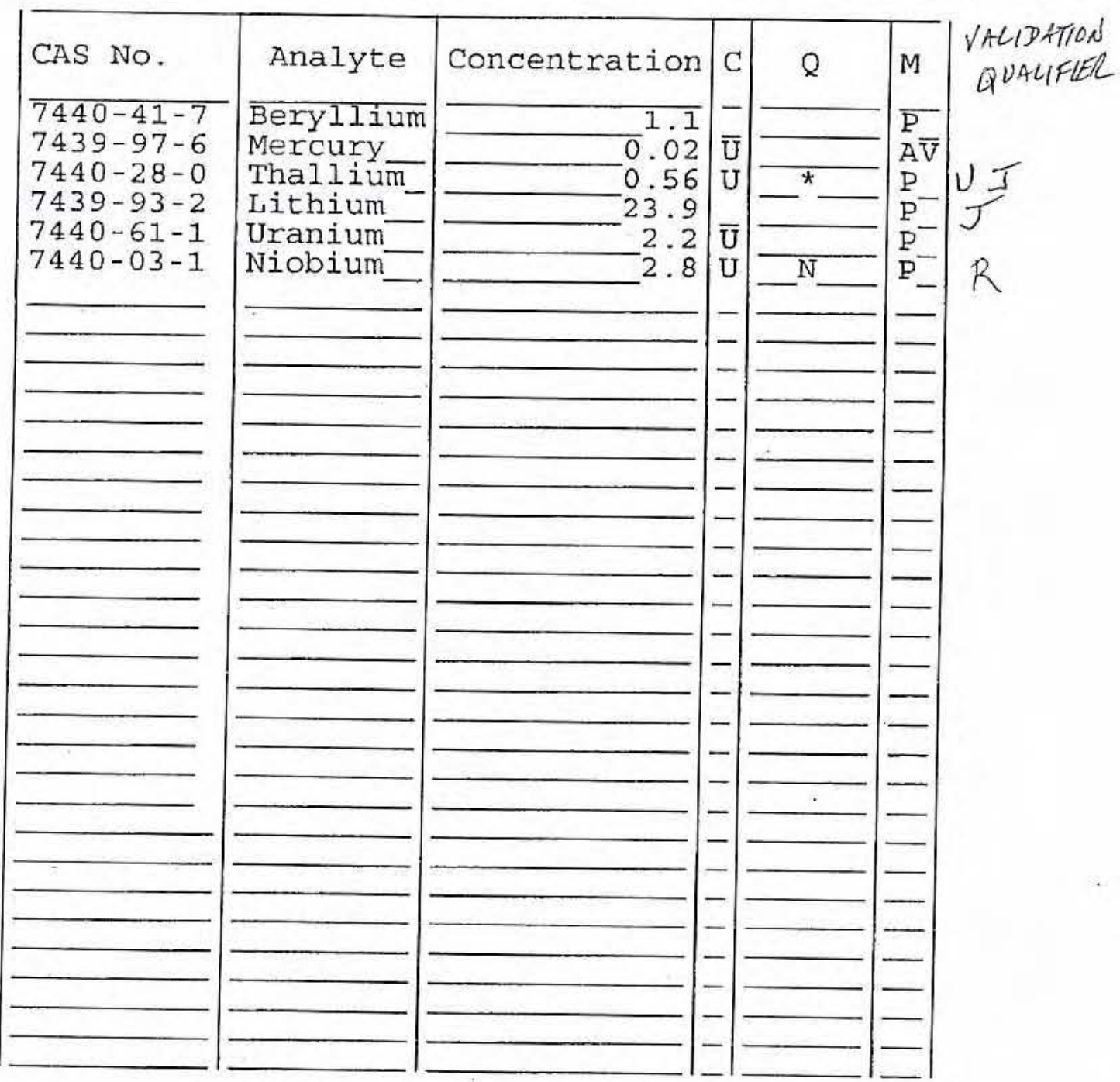

Color Before: Color After: Comments:
Clarity Before:

Clarity After:
Texture:

Artifacts: DUS - 07A 


\section{INORGANIC ANALYSES DATA SHEET}

Lab Name: LIONVILLE LABORATORY

Lab Code: LVLI - Cas
Matrix (soil/water): SoIL

Level. (low/med):

\% Solids :
Contract : 60052

SAS No. :

Lab Sample ID: 0609L86/-014

$91 . \overline{6}$
EPA SAMPLE NO.

$$
07 \mathrm{~B}
$$

Date Received: 09/01/06

Concentration Units (ug/L or $\mathrm{mg} / \mathrm{kg}$ dry weight) : MG/KG

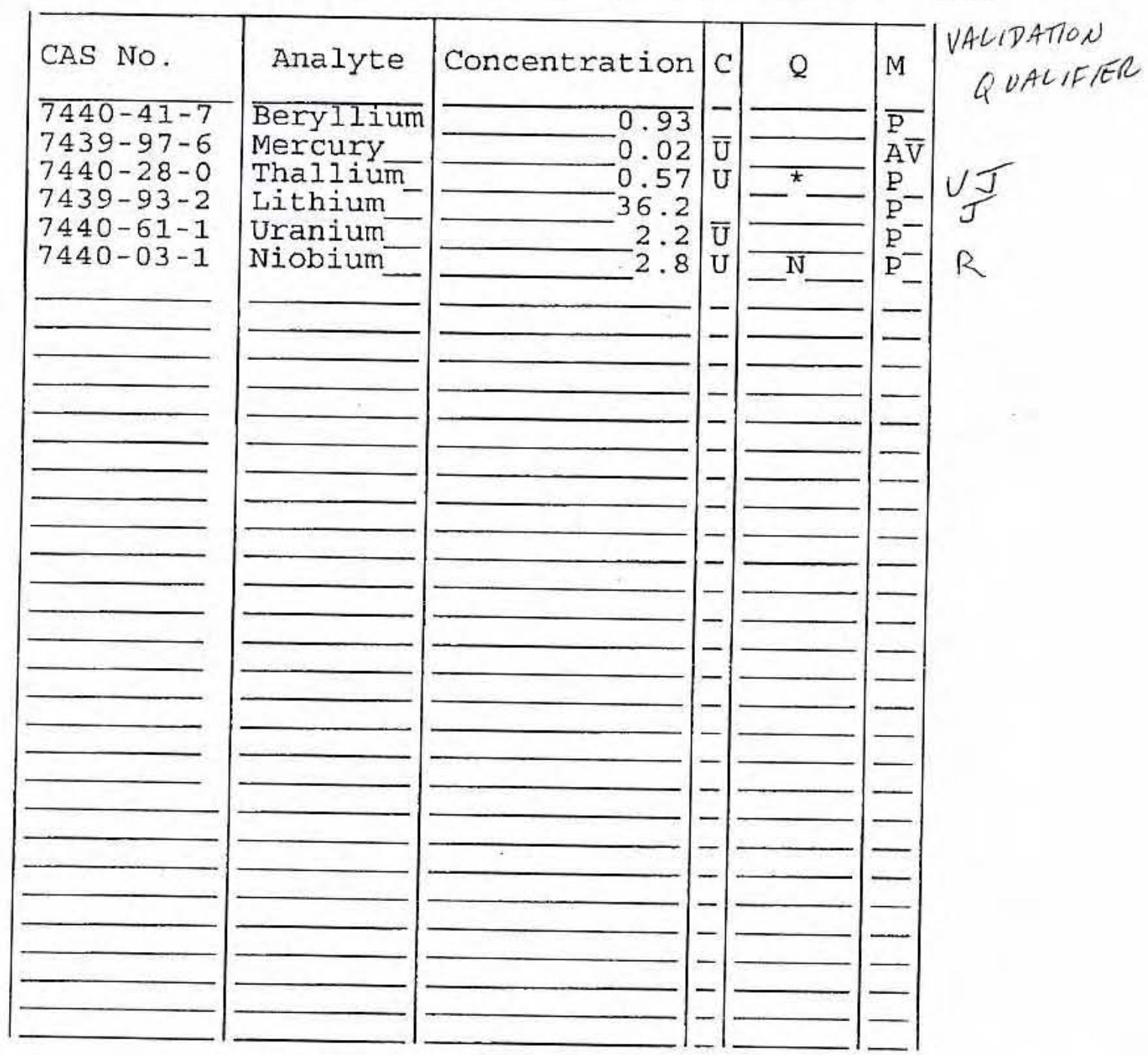

Color Before: Color After:

\section{Comments :}

Clarity Before: Clarity After:
Texture:

Artifacts: DUS-07B 


\section{U.S. EPA}

\section{INORGANIC ANALYSES DATA SHEET}

EPA SAMPLE NO.

Lab Name: LIONVILIE_LABORATORY

Lab Code: LVLI

Matrix (soil/water): Level (low/med):

\% Solids:

$$
\begin{aligned}
& \text { SOIL } \\
& \text { LOW - } \\
& -92.5
\end{aligned}
$$

Contract: 60052

Case No.: DUS

SAS NO.:

\section{A}

Lab Sample ID: 0609L86⿸广1-015

Date Received: 09/01/06

Concentration Units (ug/L or $\mathrm{mg} / \mathrm{kg}$ dry weight): MG/KG

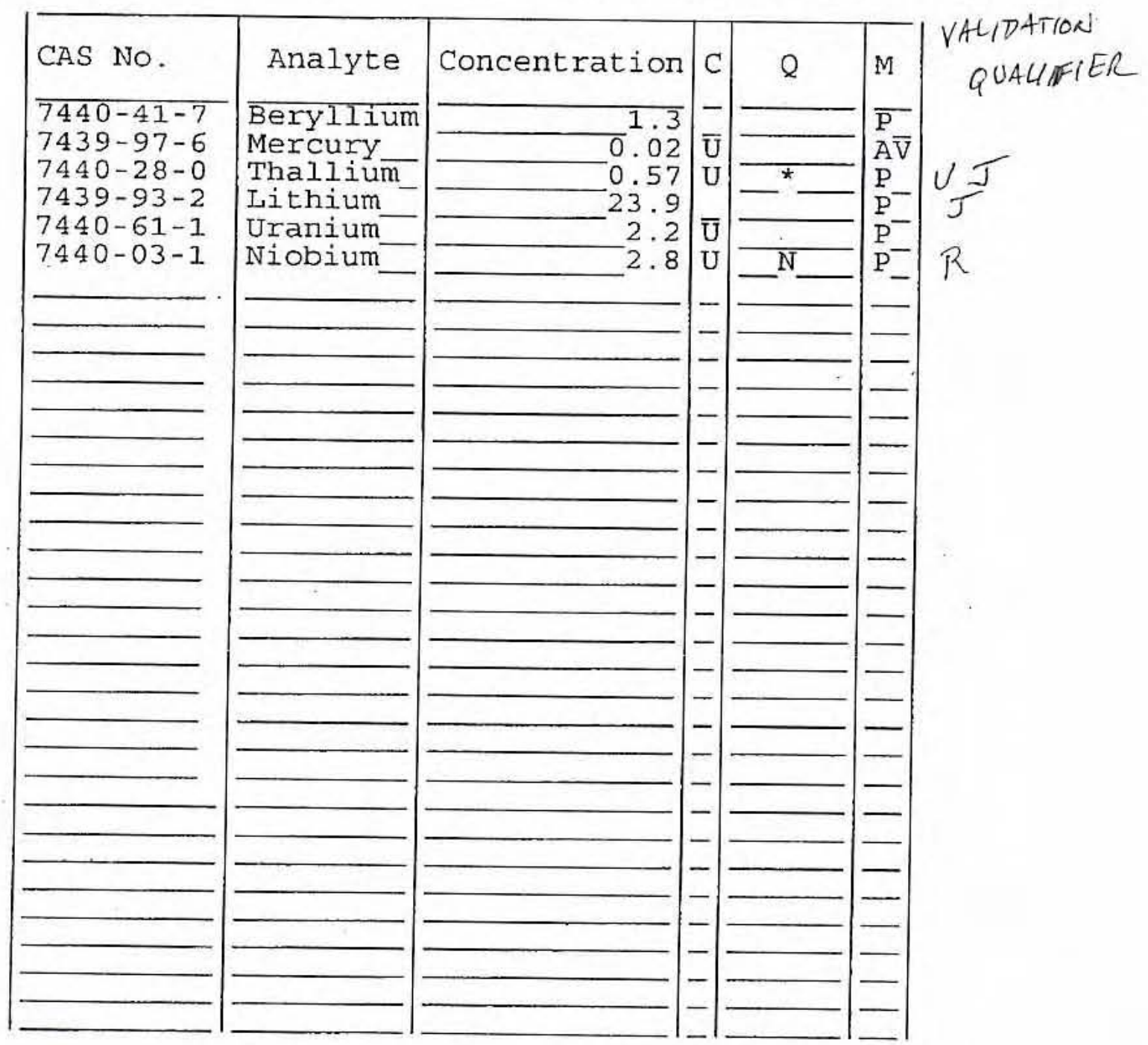

Folor Before: Jolor After:

\section{Jomments:}

DUS-08A
Clarity Before:

Clarity After:
Texture:

Artifacts: 


\section{U.S. EPA}

\section{INORGANIC ANALYSES DATA SHEET}

EPA SAMPLE NO.

Lab Name: LIONVILLE_LABORATORY

Lab Code: LVLI

Matrix (soil/water): SOIL

Level (low/med): LOW

: Solids :
Contract: 60052

SAS No.: 08B

SDG NO.: 01A

Lab Sample ID: 0609L86/-016

Date Received: 09/01/06

Concentration Units (ug/L or $\mathrm{mg} / \mathrm{kg}$ dry weight): MG/KG

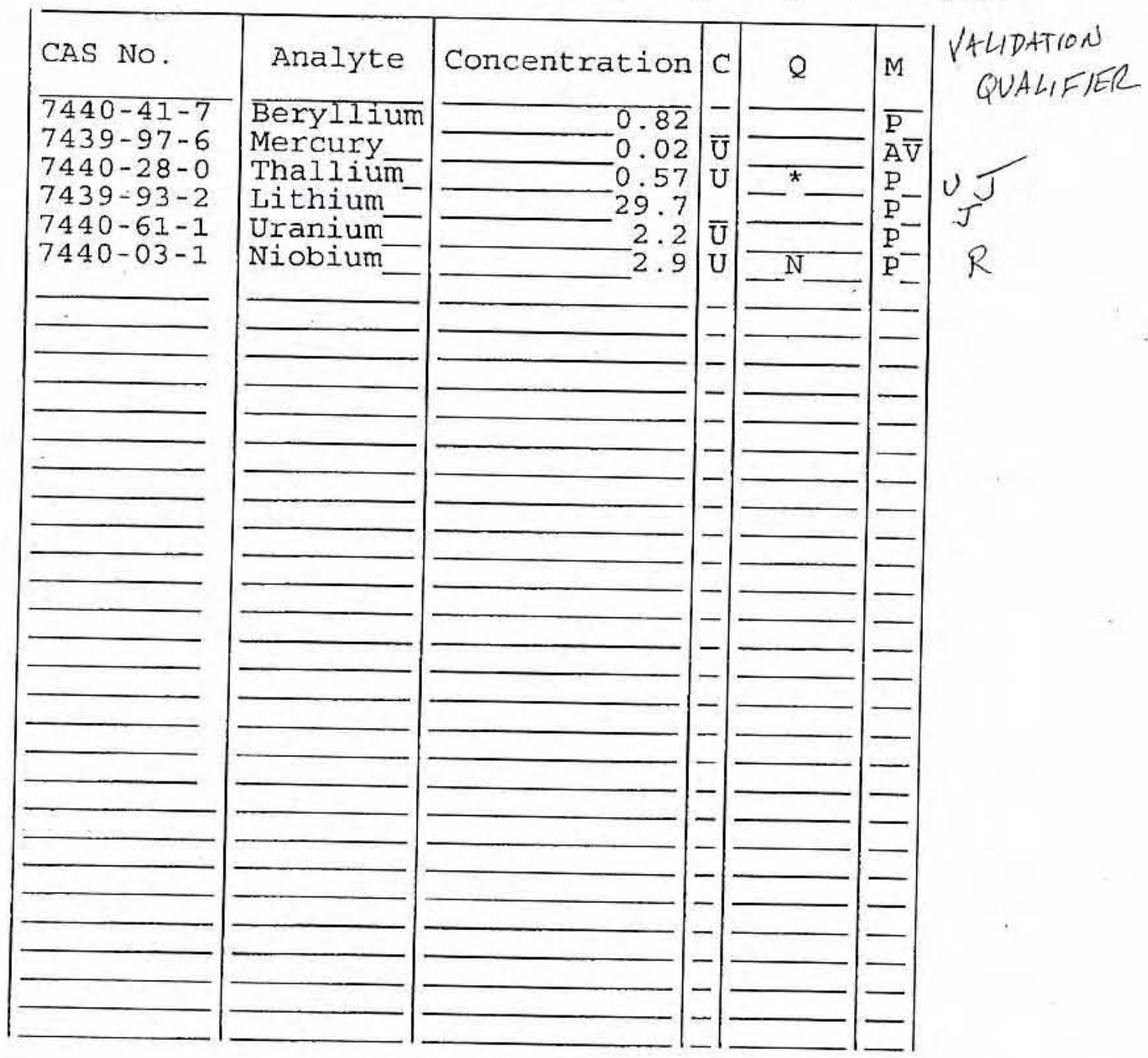

Zolor Before: Jolor After:

\section{Jomments :} DUS - $08 B$
Clarity Before:

Clarity After:
Texture:

Artifacts: 
EPA SAMPLE NO.

Lab Name: LIONVILLE_LABORATORY

Lab Code: LVLI Matrix (soil/water): SoIL Level. (low/med) \% Solids :
Contract: 60052 SAS NO.:

SDG No.: 01A Lab Sample ID: 0609L86̄-017

Date Received: 09/01/06 LOW

09A

Concentration Units (ug/L or $\mathrm{mg} / \mathrm{kg}$ ary weight): MG/KG

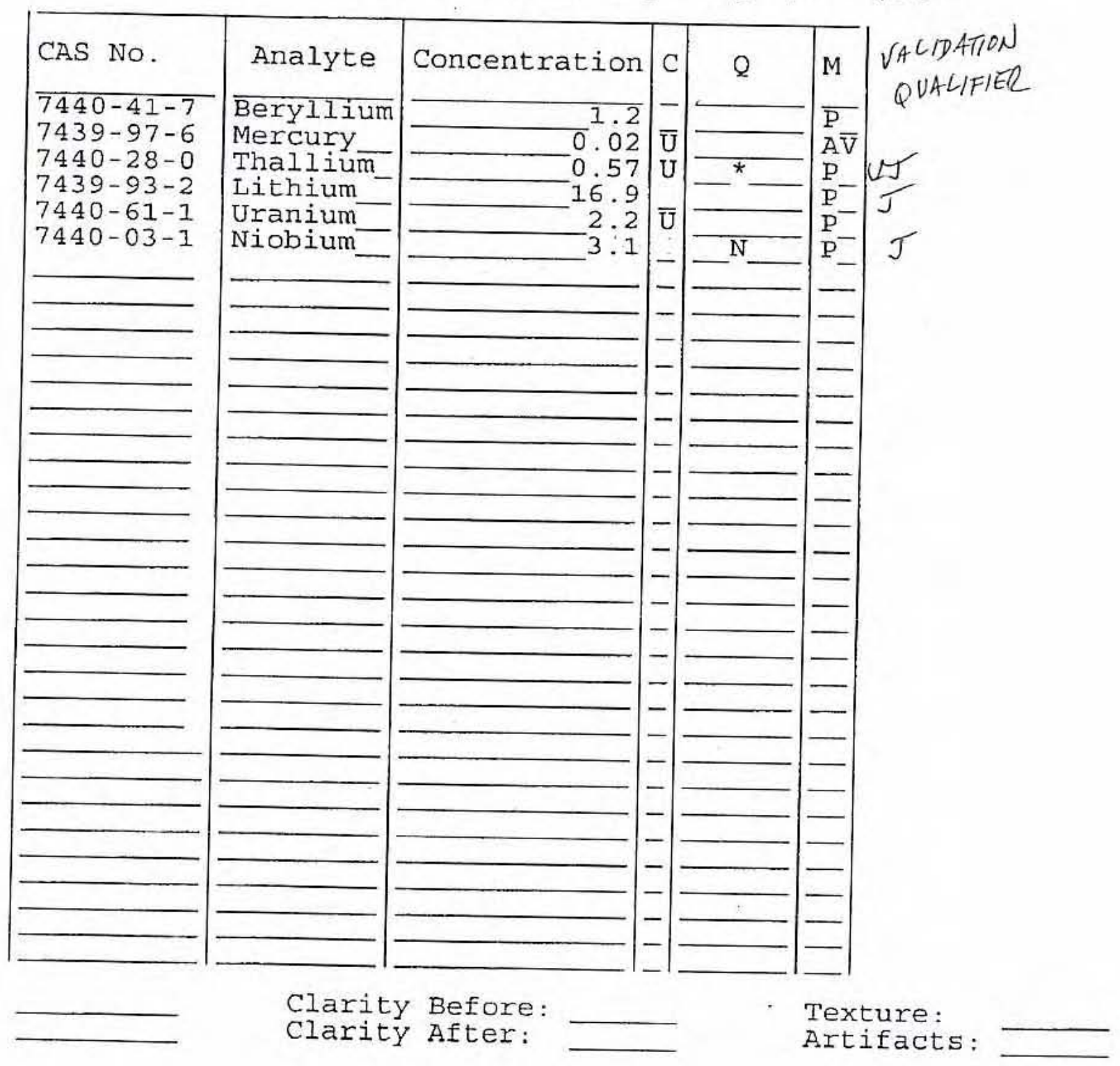

Color Before: Color After:

\section{Comments :} DUS-09A 


\section{U.S. EPA}

\section{INORGANIC ANALYSES DATA SHEET}

EPA SAMPLE NO.

Lab Name: LIONVILLE_LABORATORY

Lab Code: LVLI

$\begin{array}{ll}\text { Matrix (soil/water): SOIL } & \text { LOW } \\ \text { Level (low/med): } & \text { LOW }\end{array}$

\% Solids:

91.6
Contract: 60052

SAS NO.:
09B

SDG No.: 01A

Lab Sample ID: $0609486 \overline{1-0} 18$

Date Received: 09/01/06

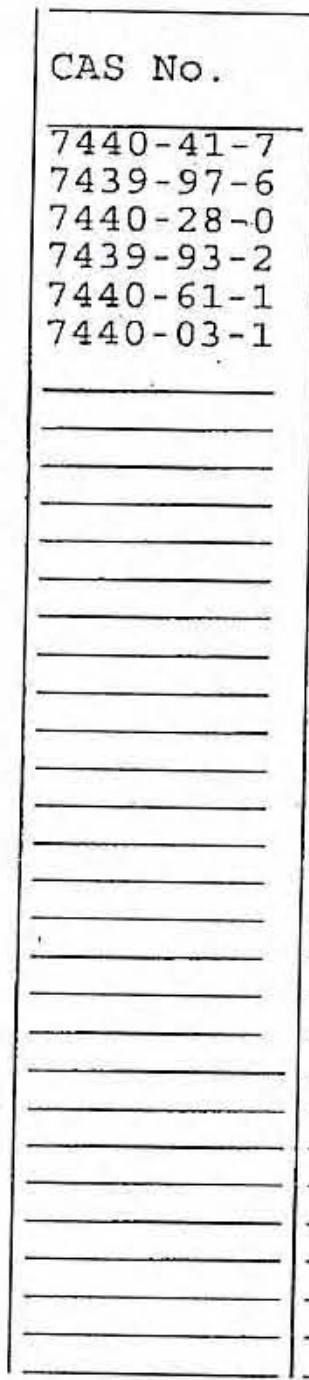

Color Before: Color After:

\section{Somments:}

DUS-09B
Concentration Units (ug/L or $\mathrm{mg} / \mathrm{kg}$ dry weight): MG/KG
Clarity Before:

Clarity After:
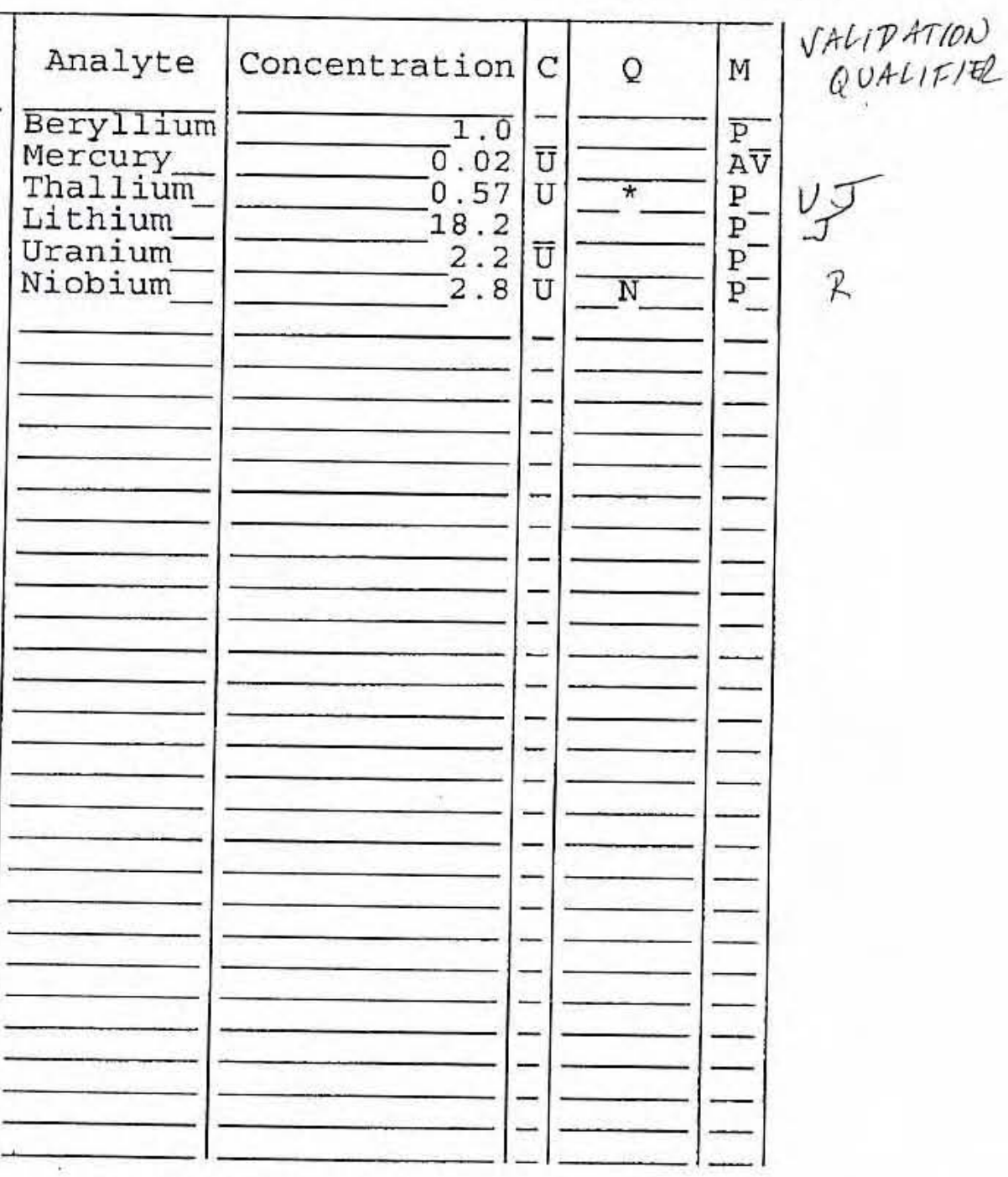

Texture:

Artifacts: 


\section{INORGANIC ANALYSES DATA SHEET}

Lab Name: LIONVILLE LABORATORY

Lab Code: LVLI

Matrix (soil/water): SOIL

Level (low/med) :

$\div$ Solids:
Contract: 60052

SAS No.:

SDG NO.: 01A

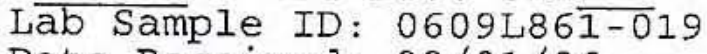

LOW

$-94 \overline{.9}$
EPA SAMPLE NO.

$10 \mathrm{~A}$

Date Received: 09/01/06

Concentration Units (ug/L or $\mathrm{mg} / \mathrm{kg}$ dry weight): MG/KG

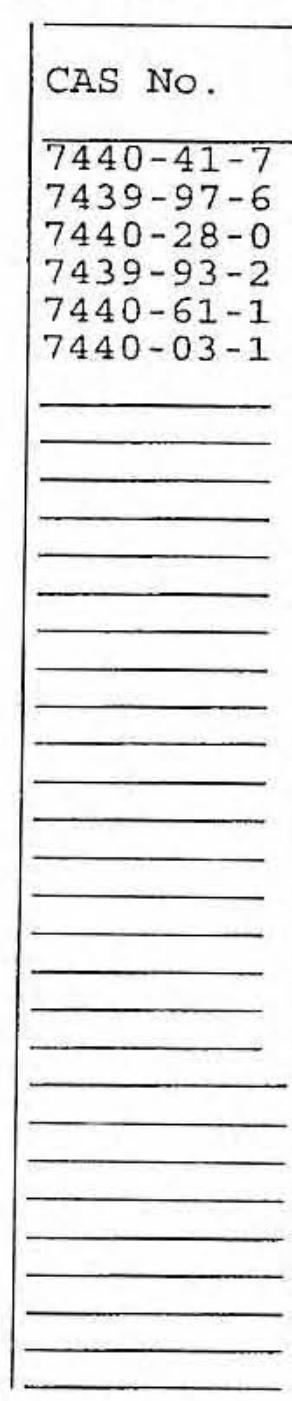

Color Before: Color After: Comments :

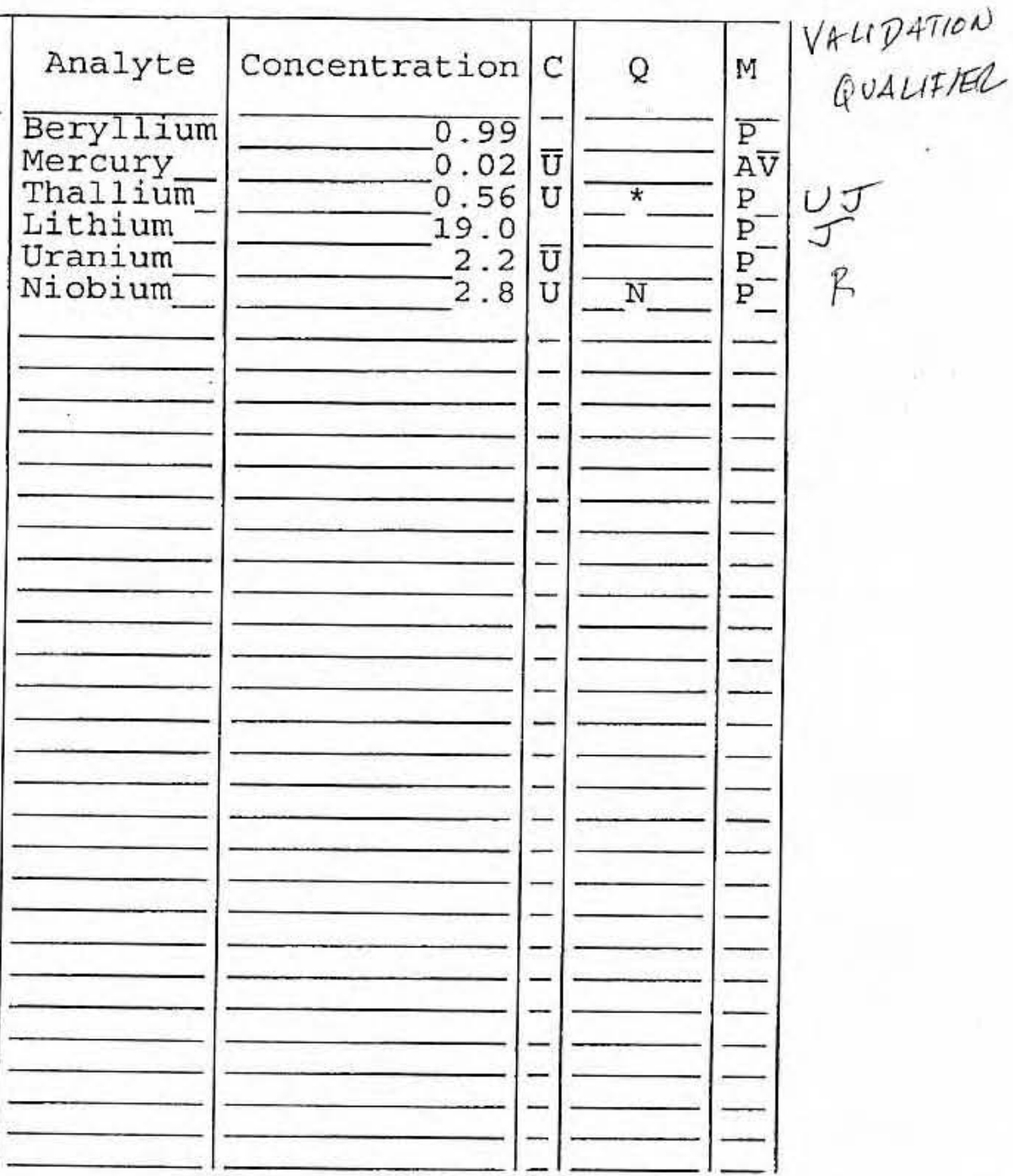

Clarity Before: Clarity After:
Texture:

Artifacts: DUS-10A 


\section{U.S. EPA}

\section{INORGANIC ANALYSES DATA SHEET}

Lab Name: LIONVILLE_LABORATORY

Lab Code: LVLI

Matrix (soil/water). SOLS

Level. (low/med):

: Solids:
Contract: 60052

SAS NO.:

LOW -
-89.8
SDG No.: 01A

EPA SAMPLE NO.

$10 \mathrm{~B}$

Lab Sample ID: 0609L86/-020

Date Received: 09/01/06

Concentration Units (ug/L or $\mathrm{mg} / \mathrm{kg}$ dry weight): MG/KG

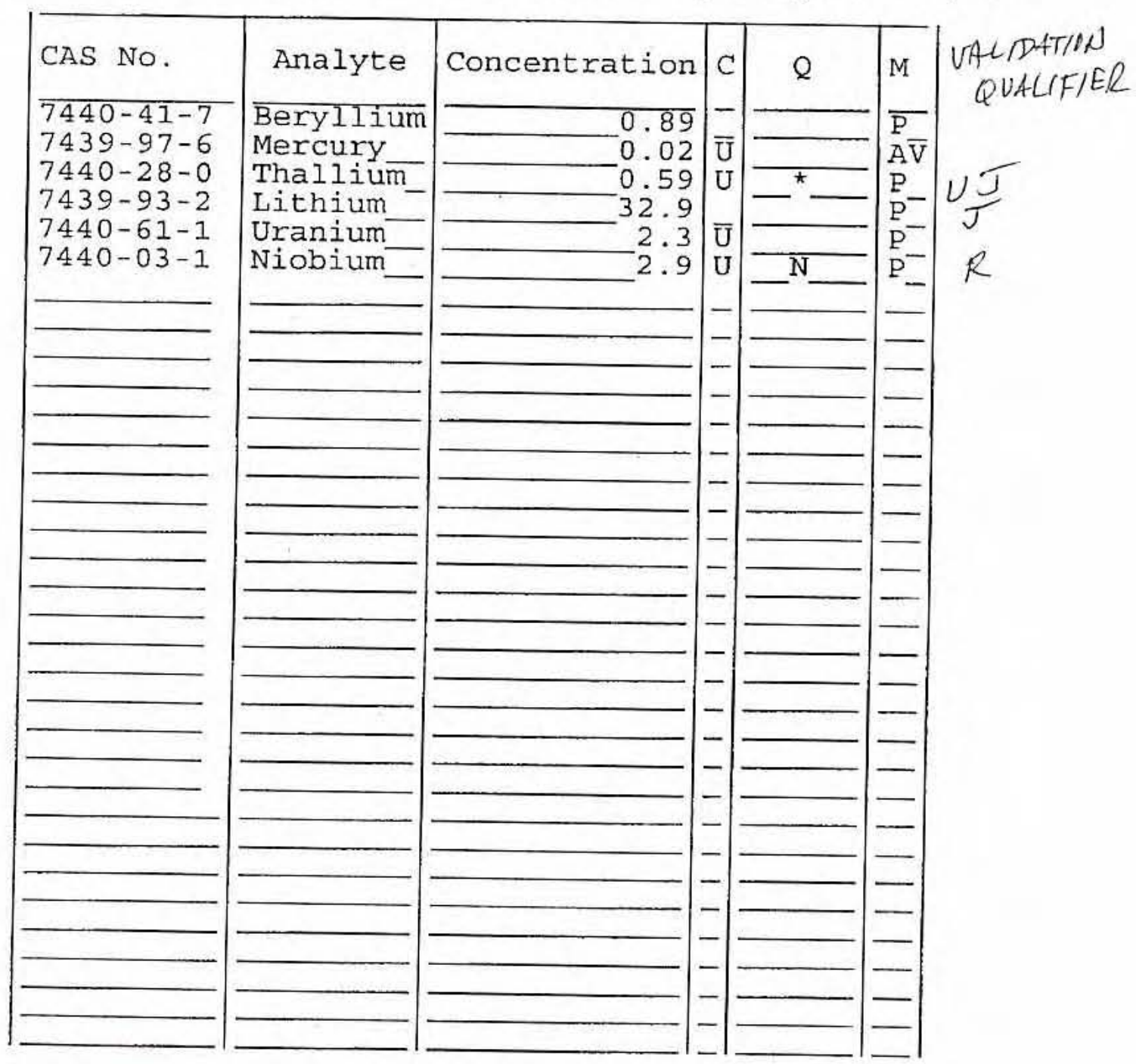

Color Before: Color After:

Comments :
Clarity Before:

Clarity After:
Texture:

Artifacts : 


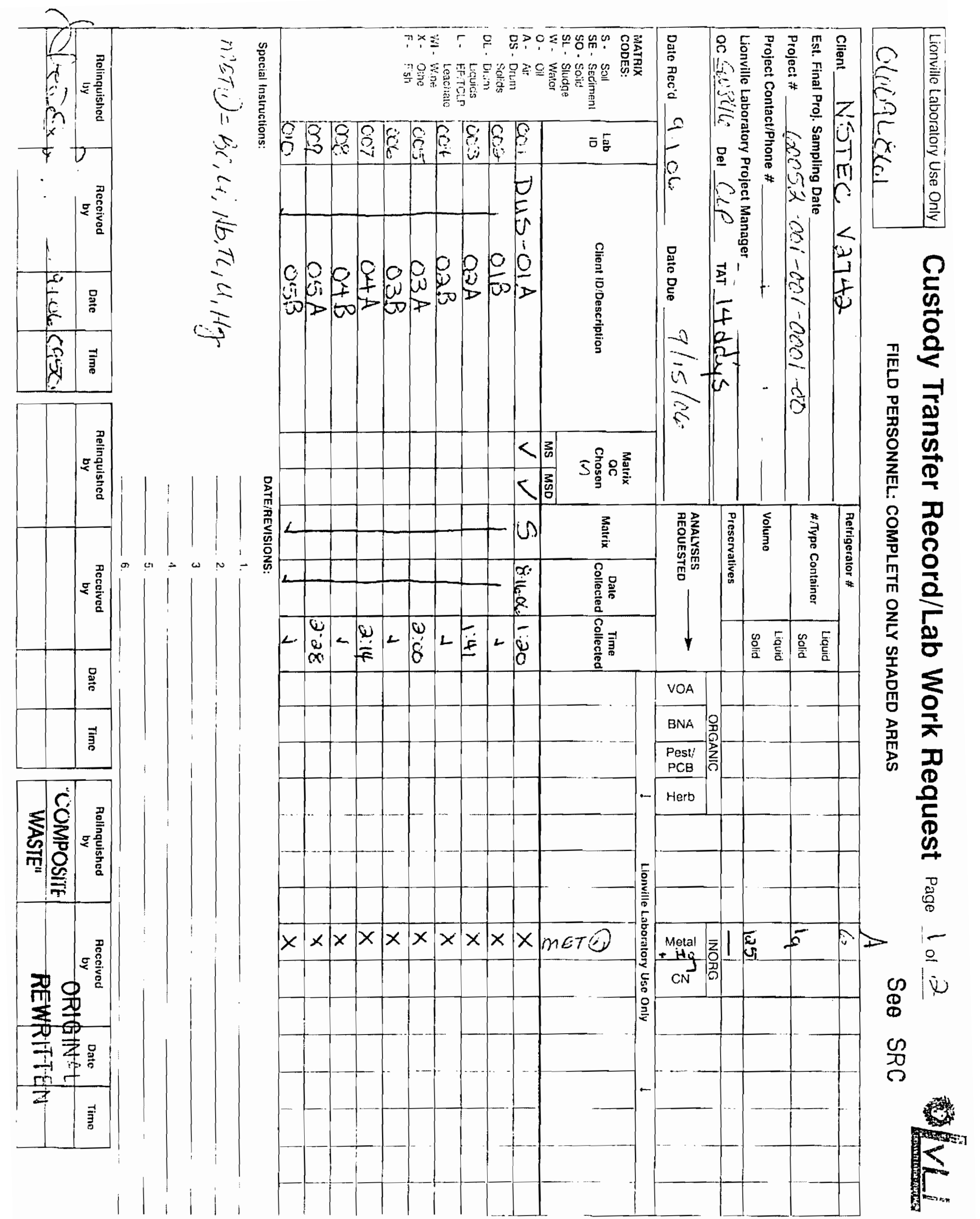




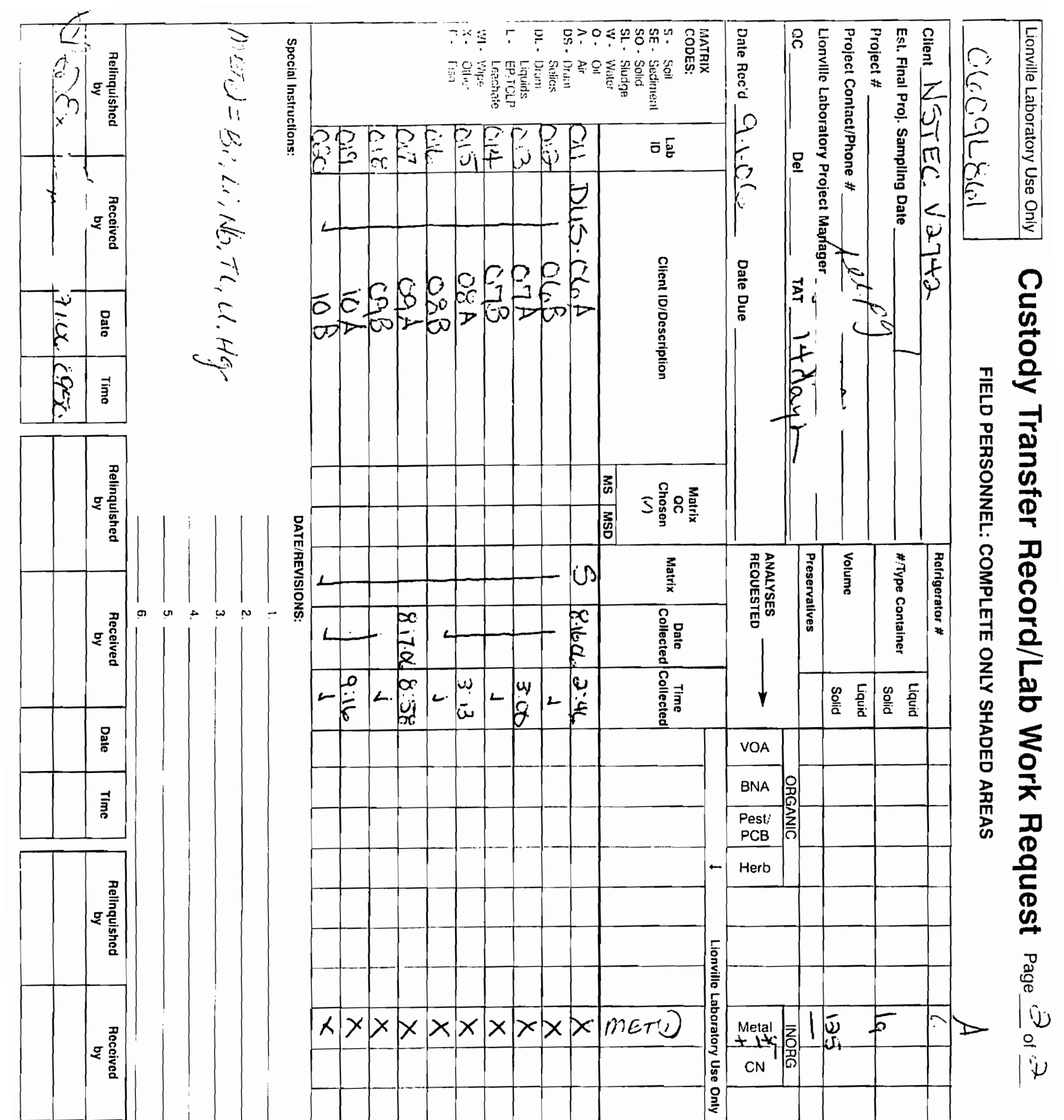




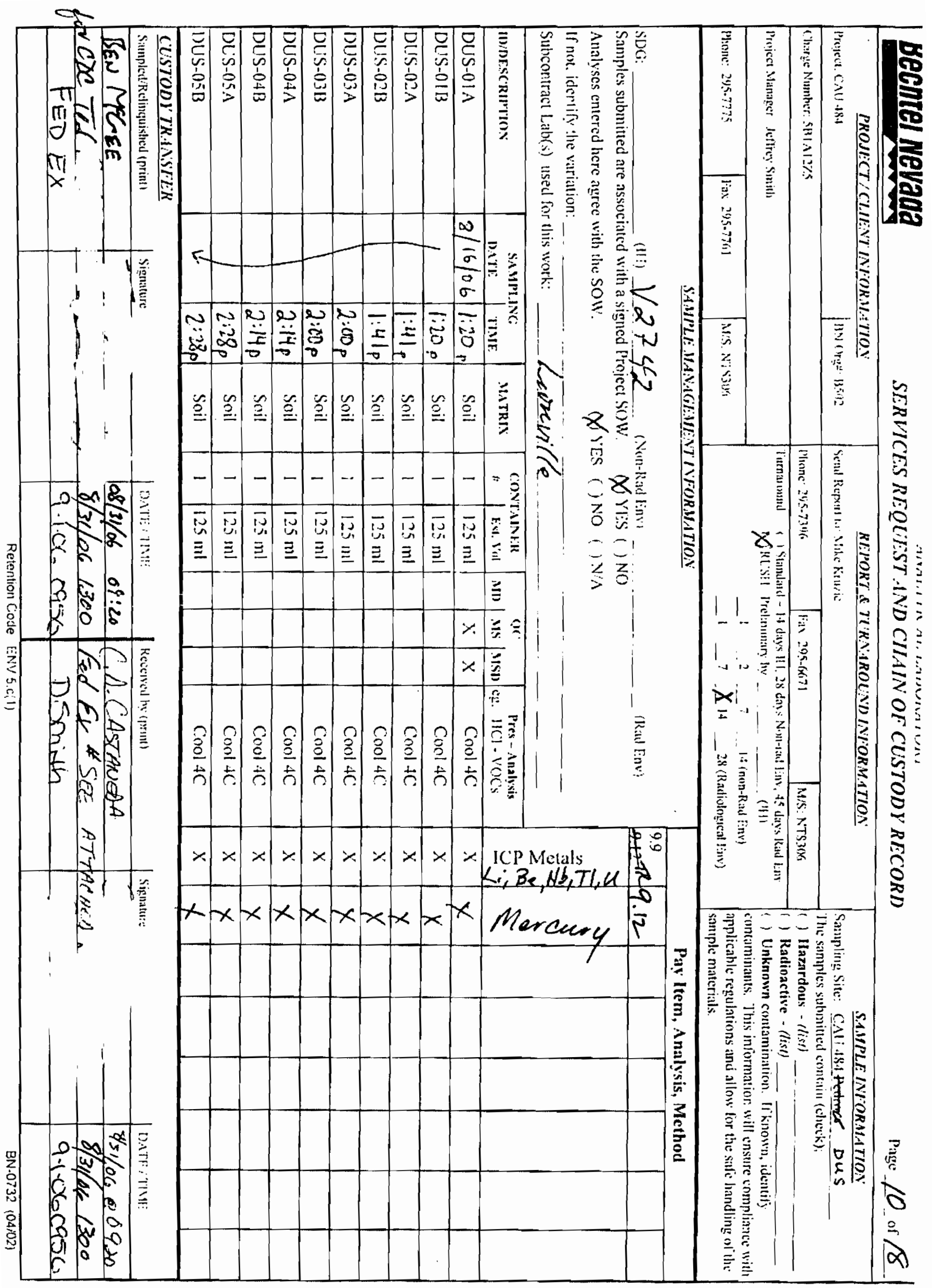




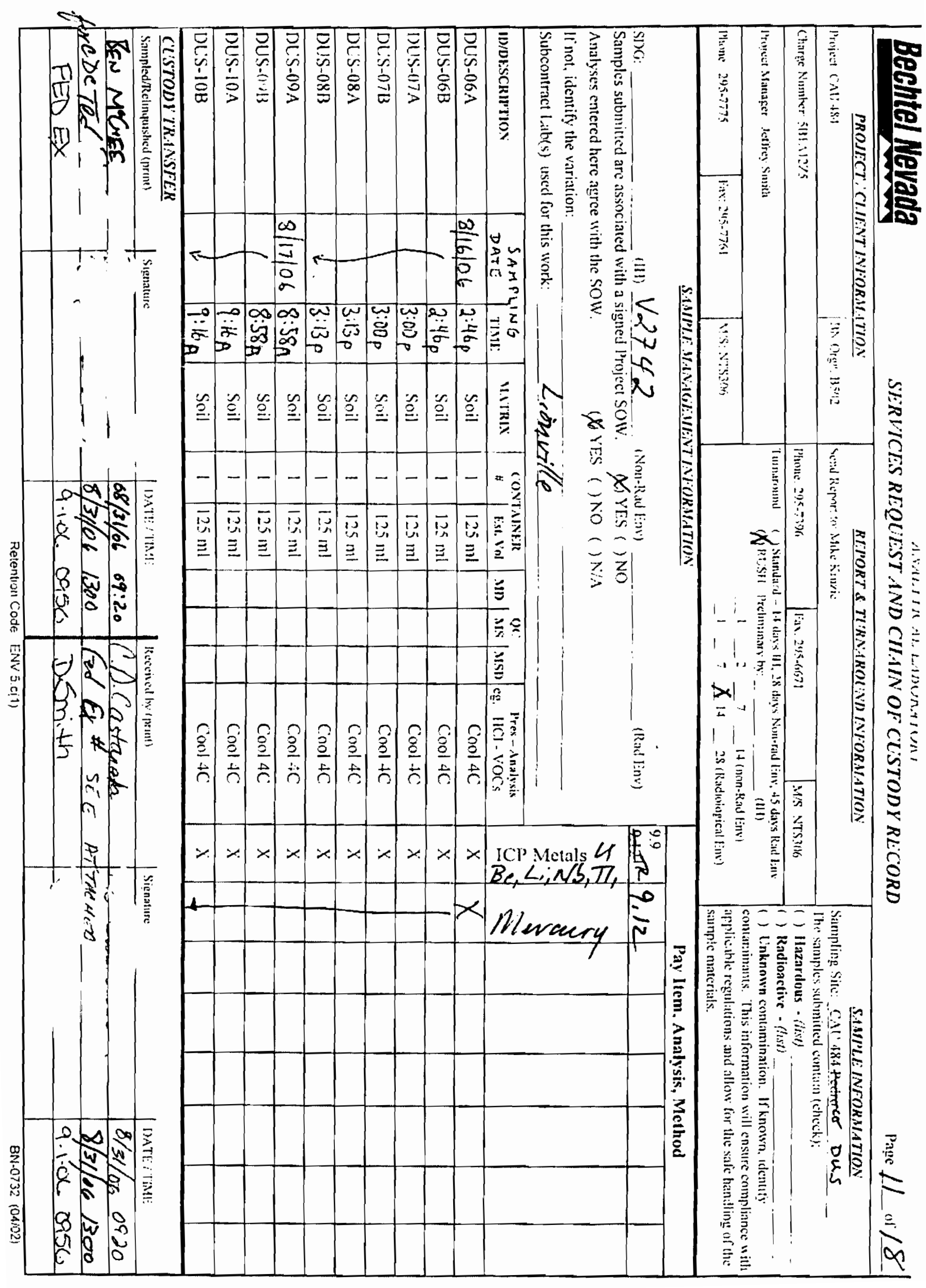


Lab Name: LIONVILLE_IABBORATORY

Lab code: IVLI Matrix (soil/water): SOII

Level (low/med):

$\because$ Solids :
Contract: 60052

SAS NO.
$11 \mathrm{~A}$

SDG NO.: 11A

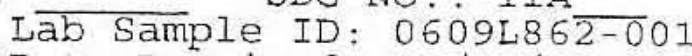
LOW
-95.2

Date Received: 09/01/06

Concentration Units (ug/L or $\mathrm{mg} / \mathrm{kg}$ dry weight): MG/KG

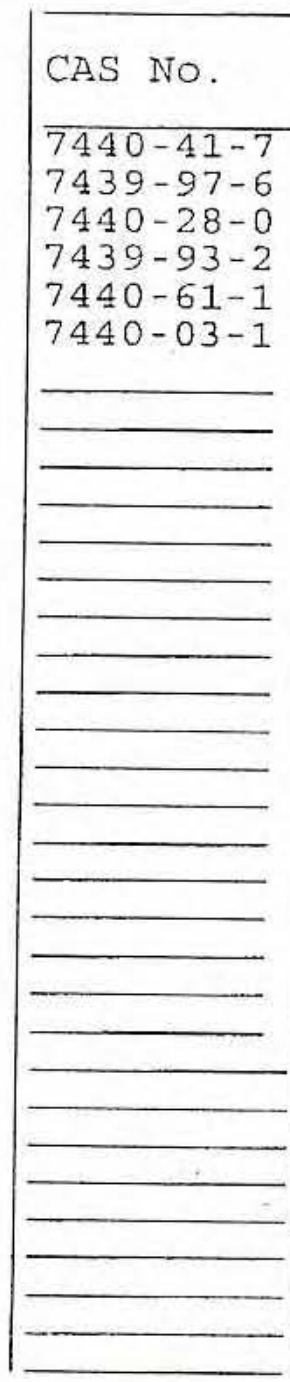

Color Before: Color After:

\section{Comments :}

DUS - 11A

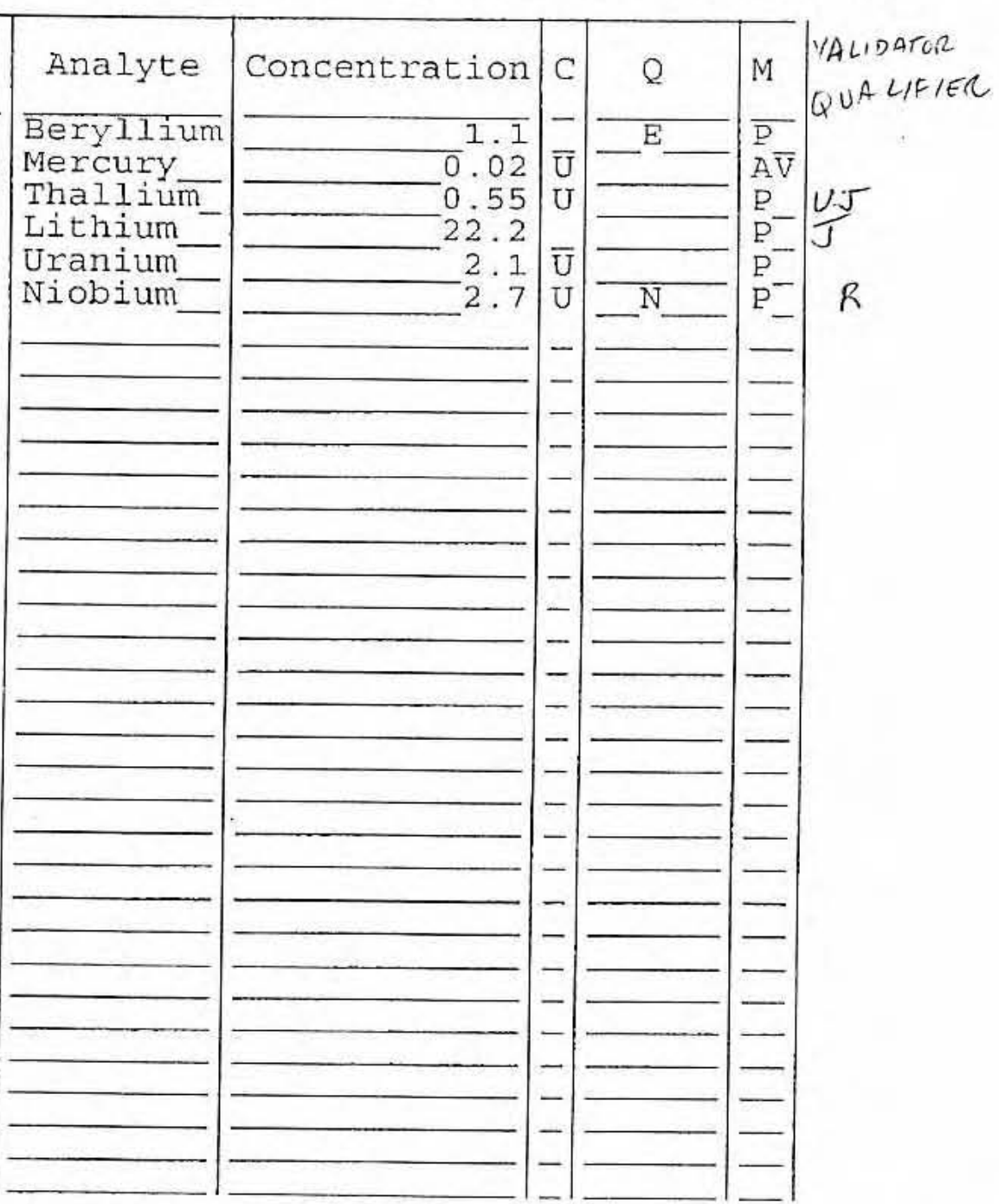

Clarity Before: Clarity After:
Texture:

Artifacts: 


\section{U.S. EPA}

1

INORGANIC ANALYSES DATA SHEET
Lab Name: LIONVILLE_LABORATORY

Lab Code: LVLI Case No.: DUS

Matrix (soil/water): SOIL

Level, (low/med):

$\%$ Solids:

$-90 \overline{.4}$
Contract: 60052

SAS NO.:

LOW -
SDG No.: 11A

EPA SAMPLE NO.

\section{$11 \mathrm{~B}$}

Date Received: 09/01/06

Concentration Units (ug/L or $\mathrm{mg} / \mathrm{kg}$ dry weight): $\mathrm{MG} / \mathrm{KG}$

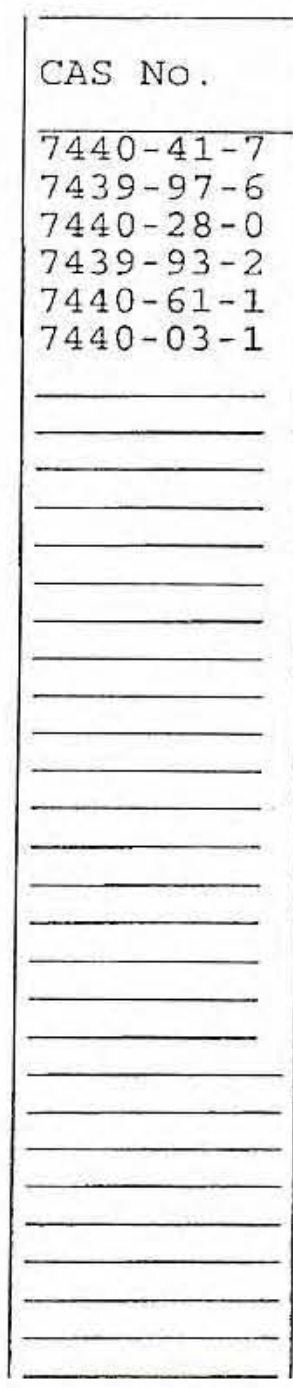

Color Before: Color After:

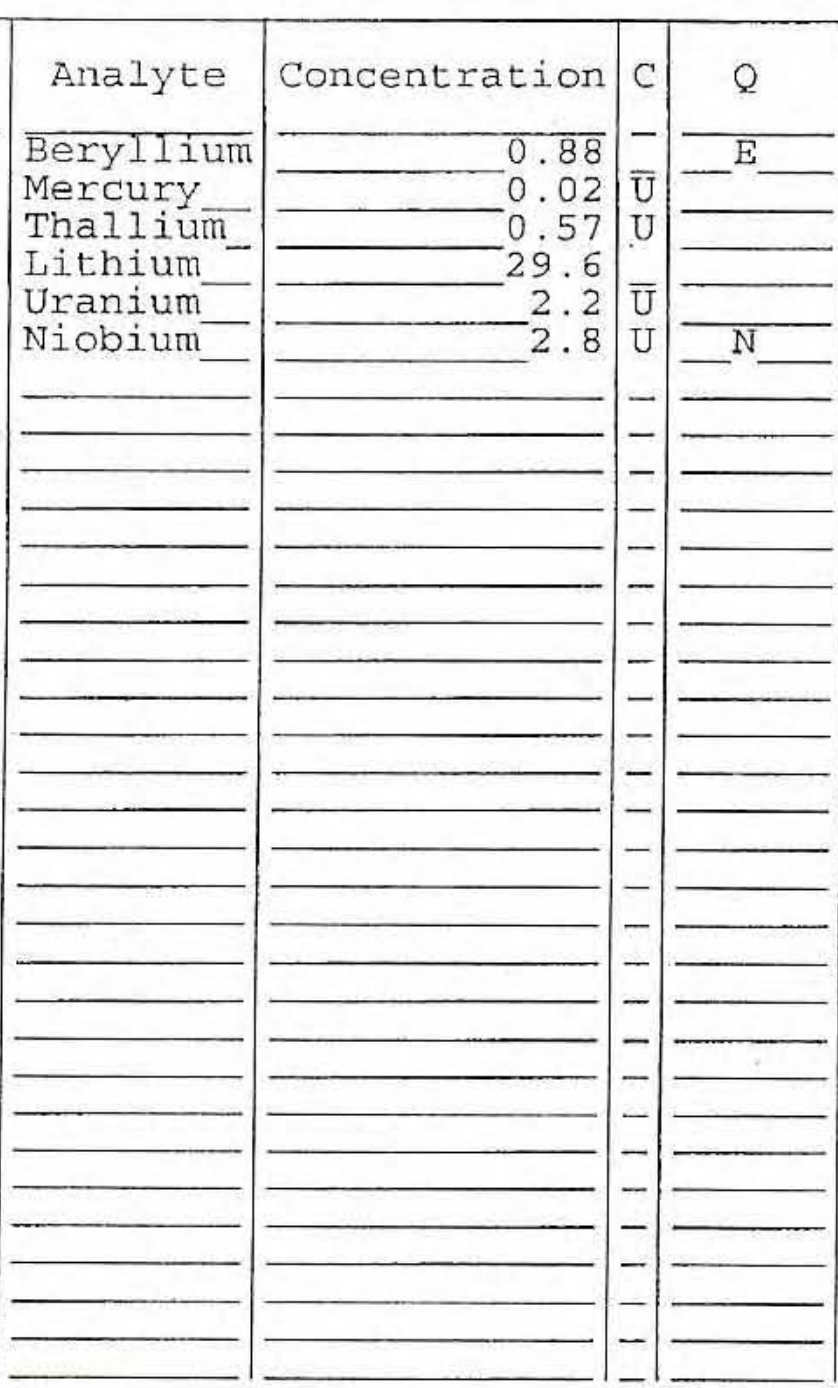

Clarity Before: Clarity After:

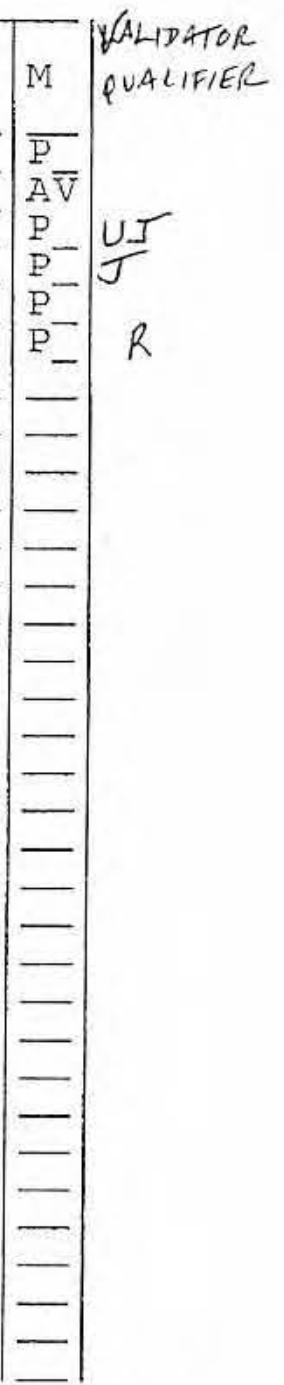

Texture: Artifacts:

Comments :

DUS - IIB 
EPA SAMPLE NO.

\section{$12 \mathrm{~A}$}

Lab Name: LIONVILLE LABORATORY Lab Code: LVLI Matrix (soil/water): SOIL Level. (low/med): LOW $\div$ Solids:
Contract: 60052

SAS NO.:

SDG NO.: $11 \mathrm{~A}$

Lab Sample ID: $0609186 \overline{2-0} 03$ $-92 \overline{9}$

Date Received: 09/01/06

Concentration Units (ug/L or $\mathrm{mg} / \mathrm{kg}$ dry weight): MG/KG

Color Before: Color After:

\section{Comments :}

DUS - 12A

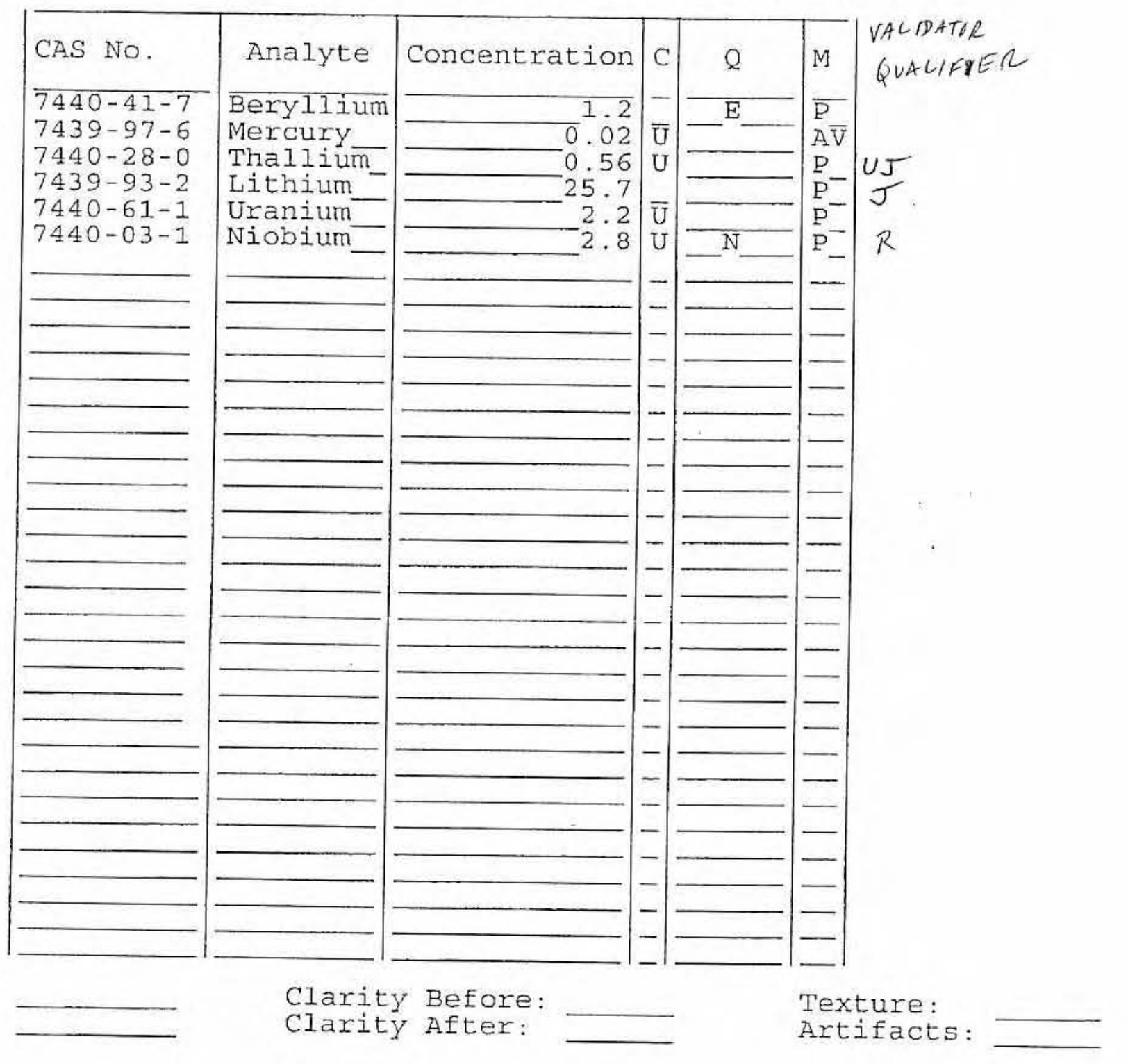




\section{U.S. EPA}

Lab Name: LIONVILLE_LABORATORY

Lab Code: LVII

Matrix (soil/water): SOIL
Level (low/med):

: Solids:
Contract: 60052

SAS NO.:

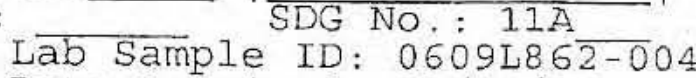

Date Received: 09/01/06

Concentration Units (ug/L or $\mathrm{mg} / \mathrm{kg}$ dry weight): MG/KG

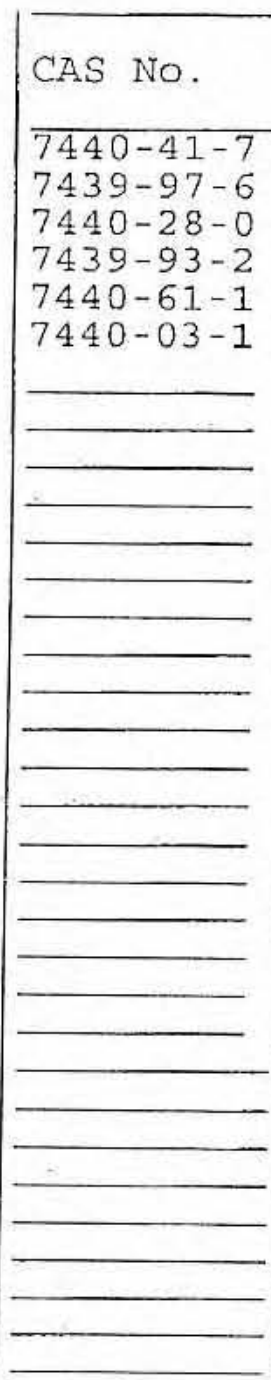

Color Before: Color After:

Comments :

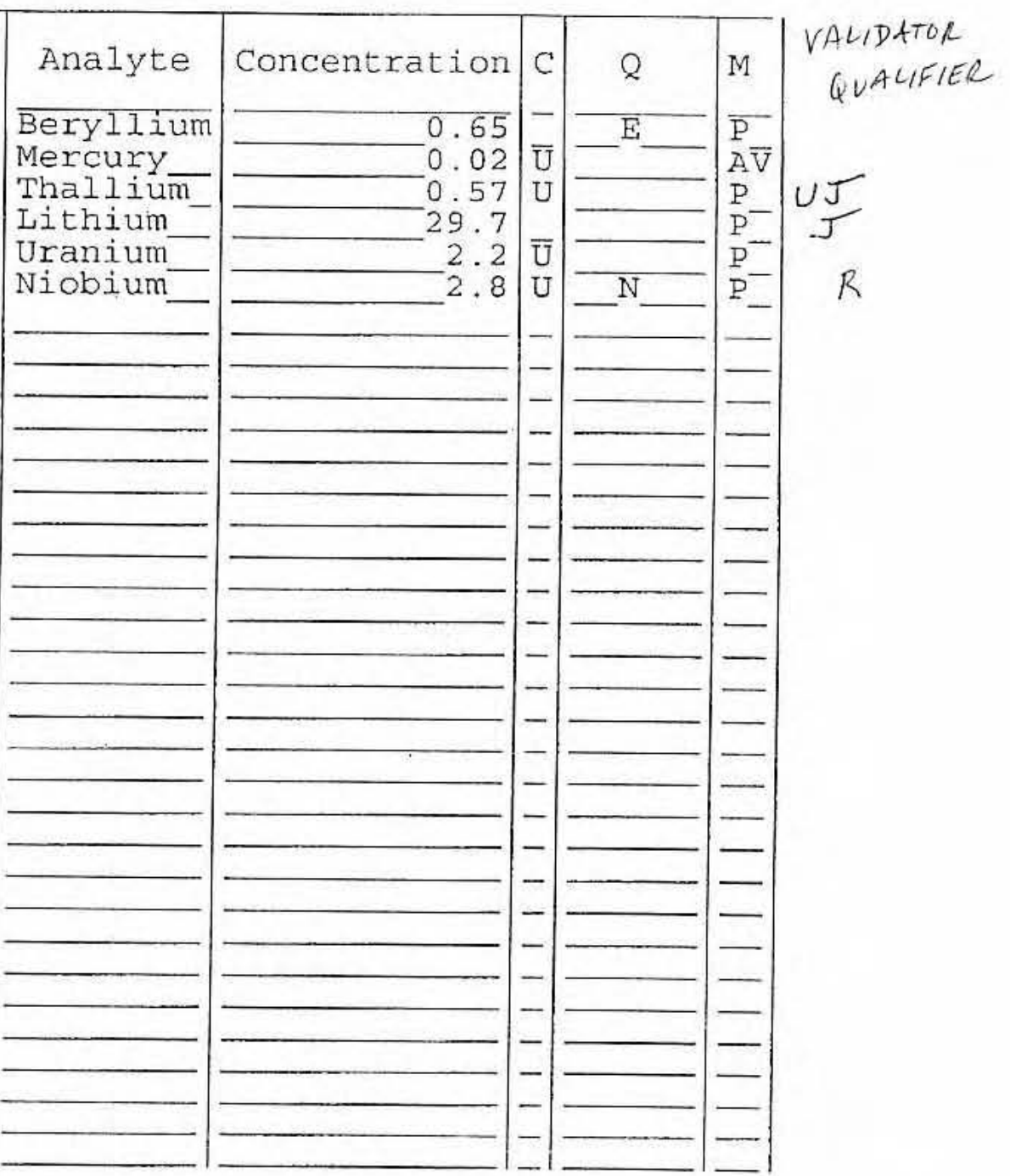

Clarity Before: Clarity After:
Texture:

Artifacts: 


\section{INORGANIC ANALY $\stackrel{1}{\text { YSES DATA SHEET }}$}

Lab Name: LIONVILLE LABORATORY

Lab Code: LVLI

Matrix (soil/water): SOIL

Level (low/med): LOW

Solids:
EPA SAMPLE NO.

\section{$13 \mathrm{~A}$}

Contract: 60052

SAS NO. :

Lab Sample ID: 06091862-005

Date Received: 09/01/06

Concentration Units (ug/L or mg/kg dry weight): MG/KG

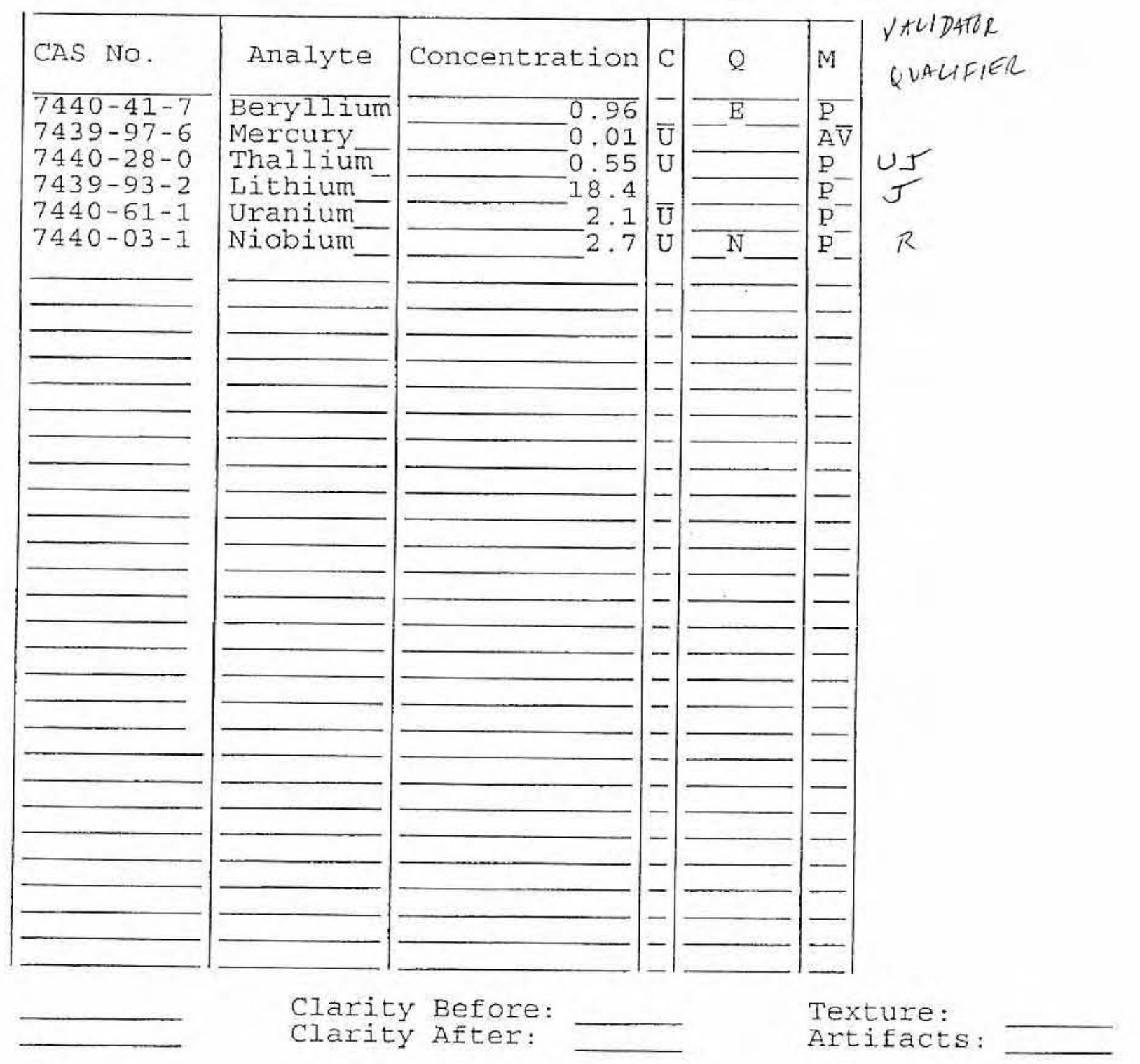

Color Before:

Color After:
Clarity Before:

Clarity After:

\section{Comments:}

DUS - $13 \mathrm{~A}$ 
U.S. EPA

1

INORGANIC ANALYSES DATA SHEET
EPA SAMPLE NO.

\section{$13 \mathrm{~B}$}

Lab Name: LIONVILIE__LABORATORY

Lab Code: IVII

Matrix (soil/water): SoIL

Level. (low/med): LOW

$\div$ Solids:
Contract: 60052

SAS NO.: Lab Sample ID: $0609186 \overline{2-006}$

Date Received: 09/01/06

Concentration Units (ug/L or mg/kg dry weight): MG/KG

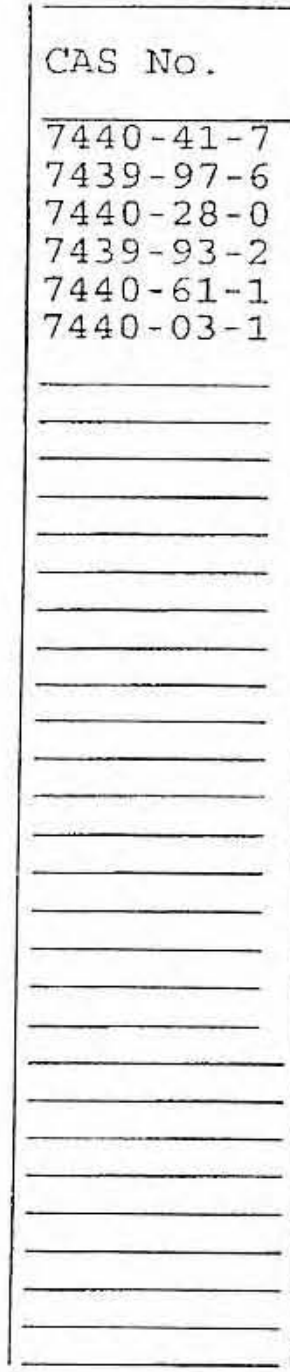

Color Before:

Color After:

\section{Comments:}

DUS $-13 B$

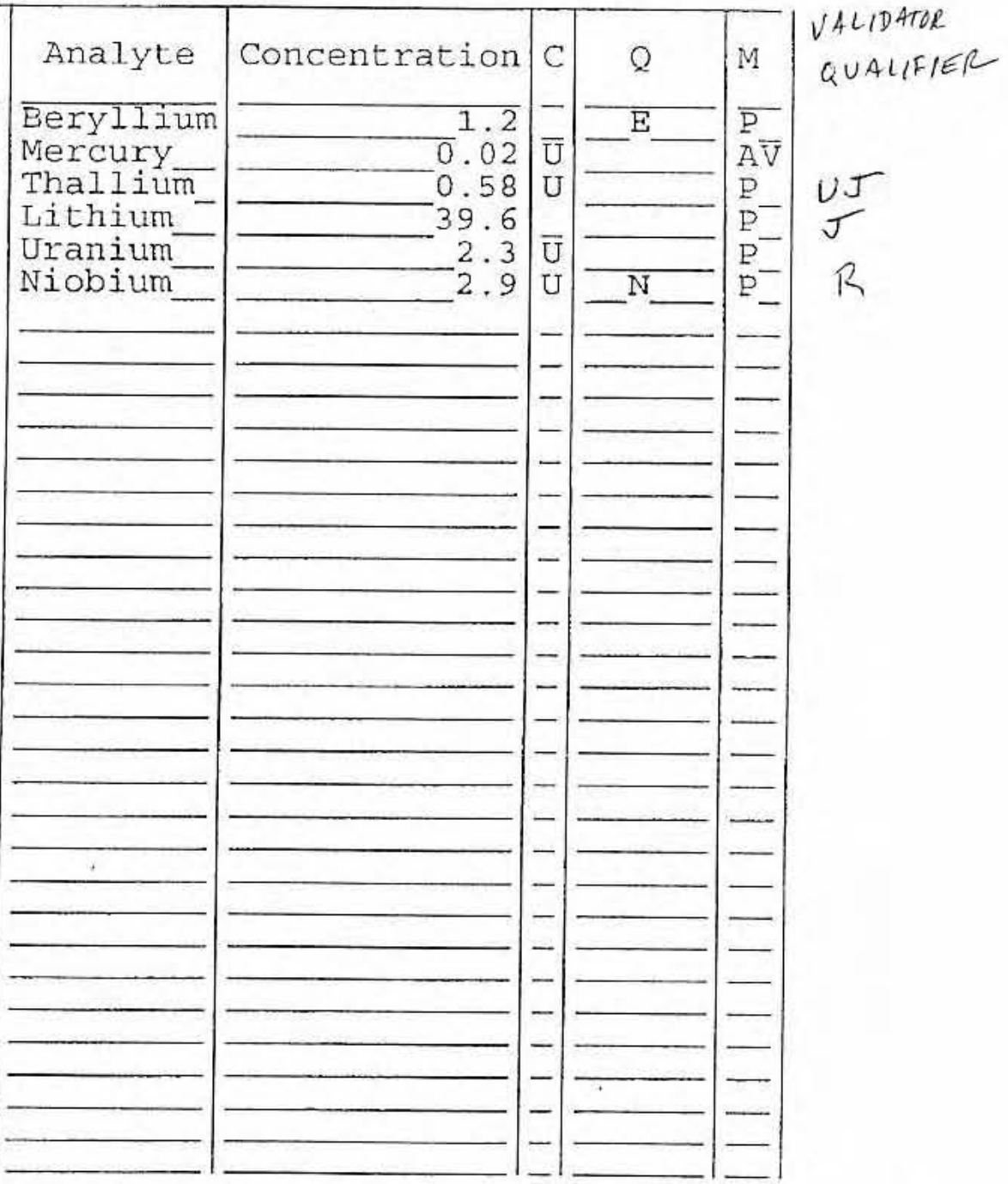

Clarity Before:

Clarity After:

Texture:

Artifacts: 
U.S. EPA

1

INORGANIC ANALYSES DATA SHEET
EPA SAMPLE NO.

\section{$14 \mathrm{~A}$}

Contract: 60052

SAS NO.:

SDG NO : 11A

Matrix (soil/water): SOIL
Level (low/med):

Solids:
Lab Sample ID: 0609L865-007

Date Received: 09/01/06

Concentration Units (ug/L or $\mathrm{mg} / \mathrm{kg}$ dry weight): MG/KG

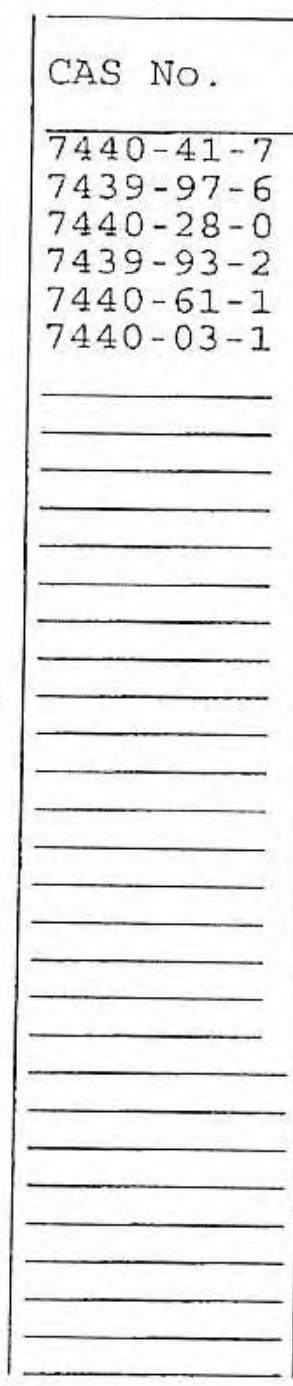

Color Before:

Color After:

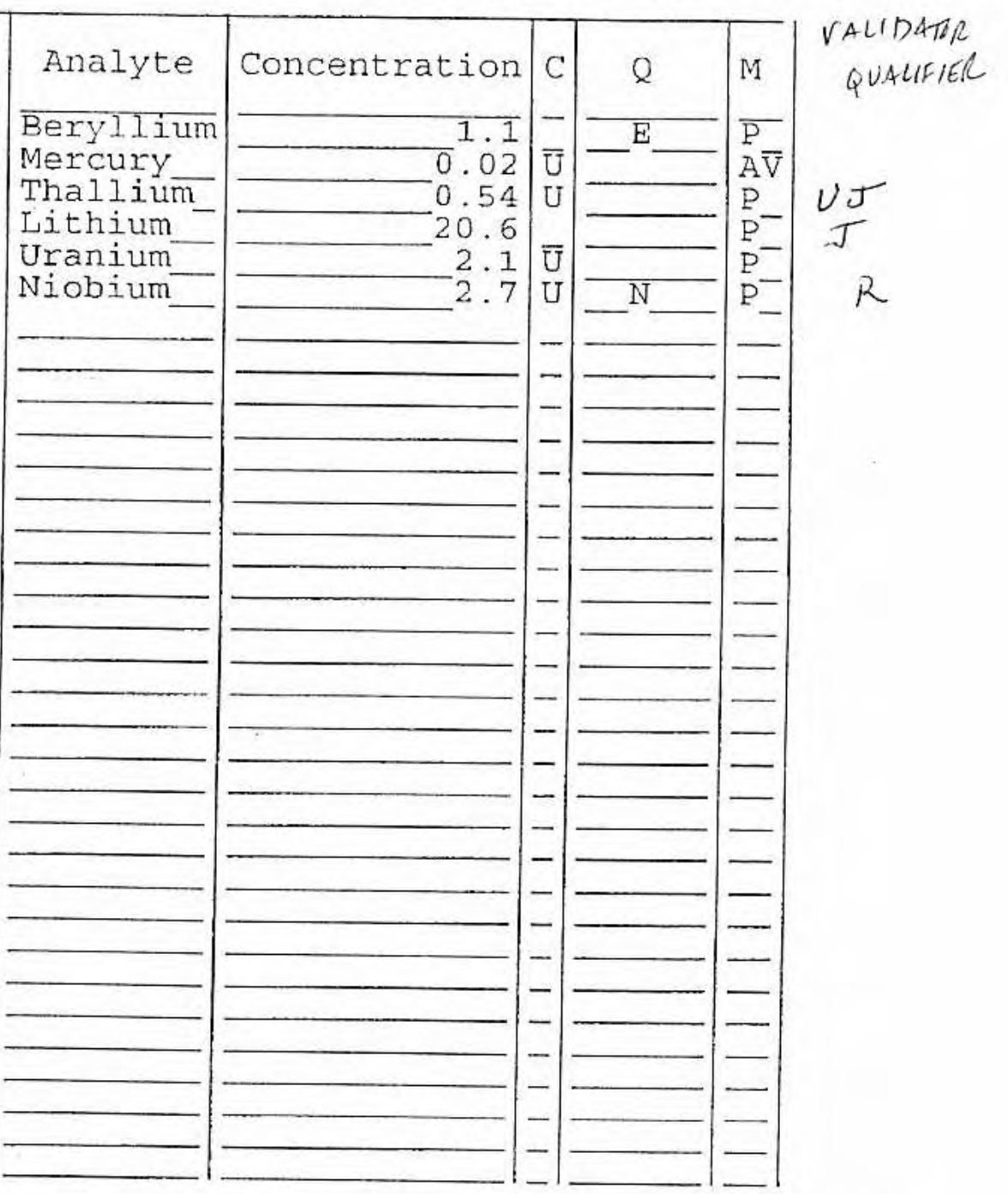

Clarity Before:

Clarity After:

Texture:

Artifacts:

\section{Comments :}

DUS - 14A 
1

\section{INORGANIC ANALYSES DATA SHEET}

Lab Name: LIONVILLE LABORATORY

Lab Code: IVLI

Matrix (soil/water): SOIL

Level (low/med): LOW

$\div$ solids:
Contract: 60052 SAS NO.:

SDG NO.: 11A

$14 B$

Lab Sample ID: 0609L86 $\overline{2-0} 08$

Date Received: 09/01/06

Concentration Units (ug/L or $\mathrm{mg} / \mathrm{kg}$ dry weight): MG/KG

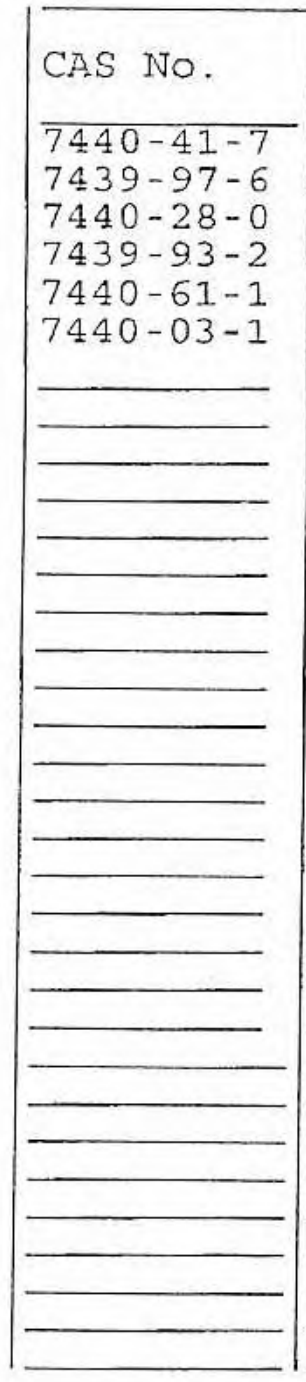

Color Before: Color After:

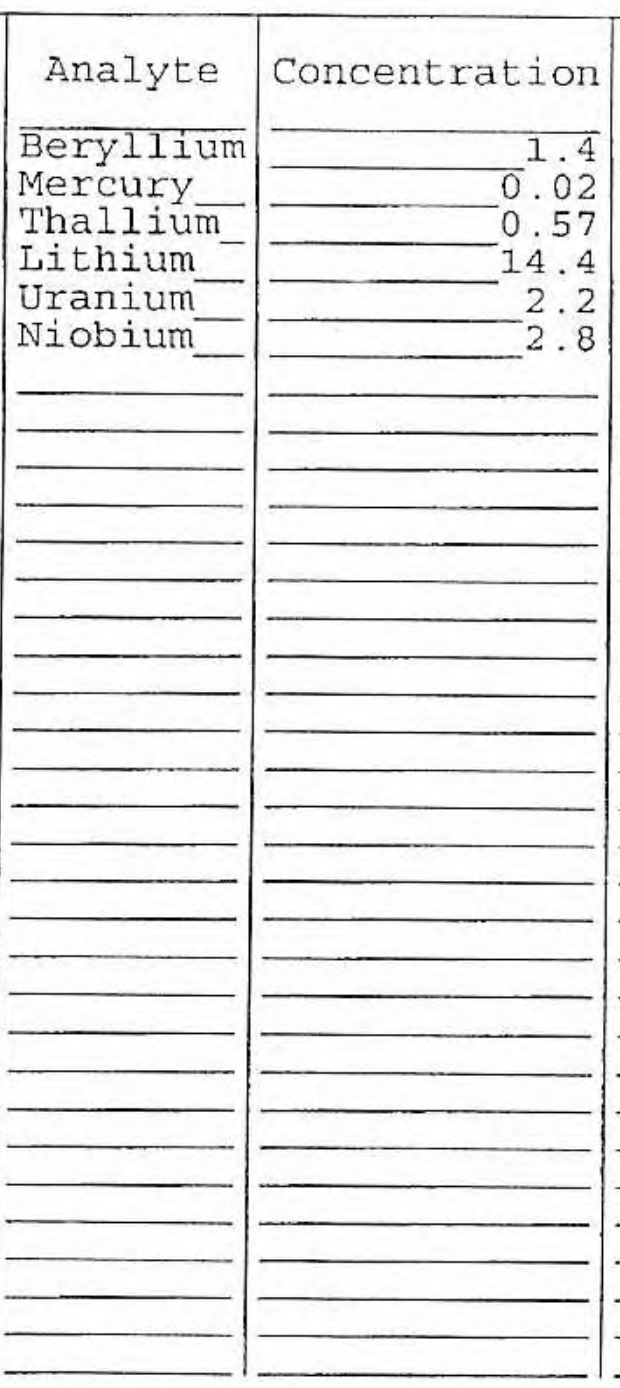

Clarity Before: Clarity After:

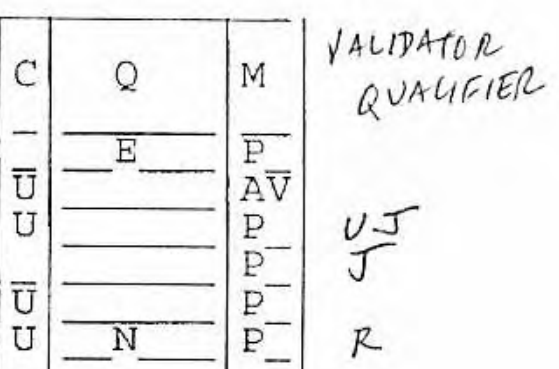

Comments:

DUS - 14B 


\section{U.S. EPA}

1

INORGANIC ANALYSES DATA SHEET
EPA SAMPLE NO.

$15 \mathrm{~A}$

Contract: 60052

SAS NO, :

Lab Sample ID: $0609486 \overline{2-0} 09$

Date Received: 09/01/06

\section{(1)}

Lab Nane: LIONVILLE LABORATORY

Lab Code: LVLI Case No.: DUS

Level (low/med) :

\% Solids: $-93 . \overline{7}$

\section{Concentration Units (ug/L or $\mathrm{mg} / \mathrm{kg}$ dry weight): MG/KG}

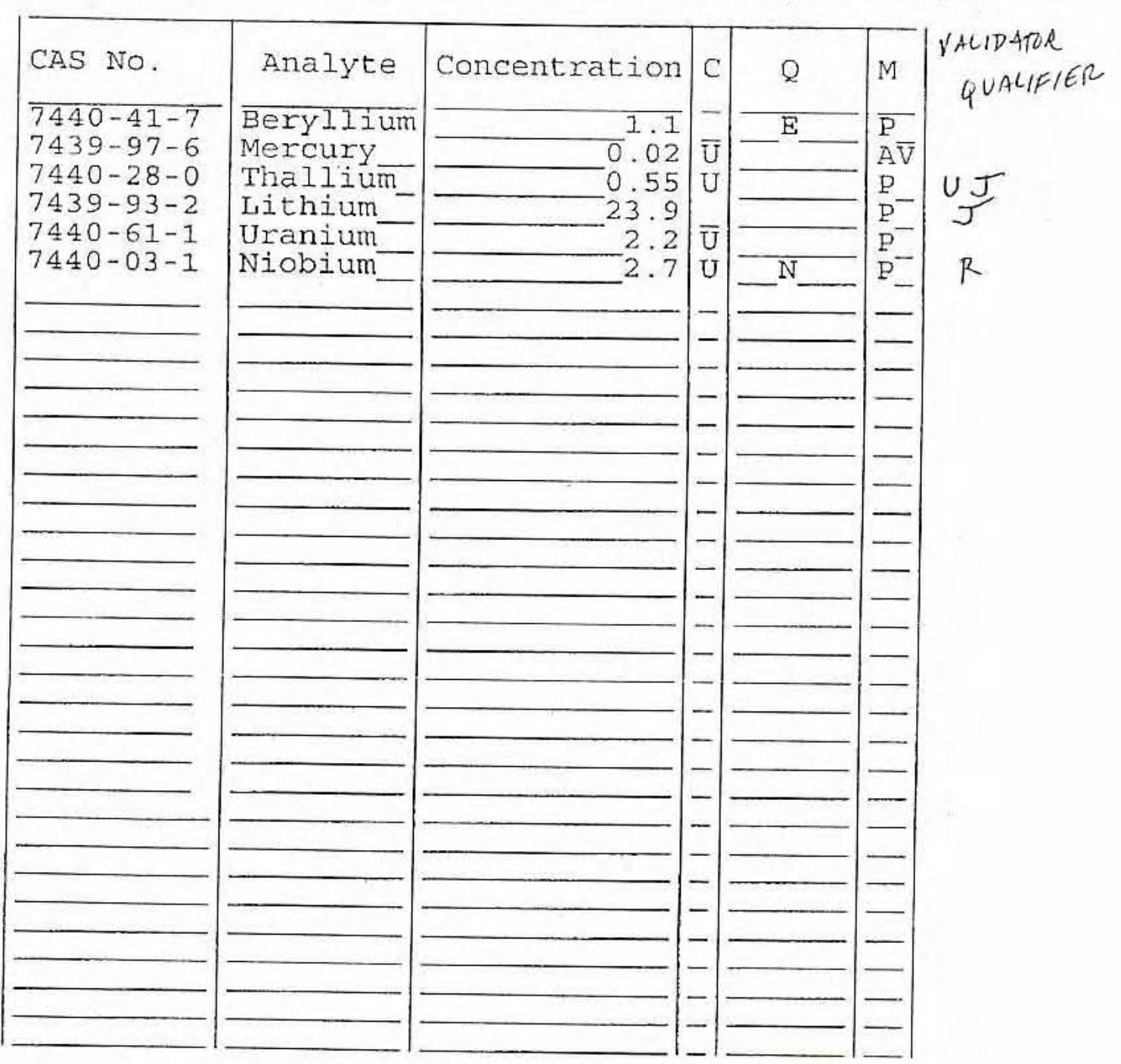

Color Before:

Color After:
Clarity Before:

Clarity After:
Texture: Artifacts:

\section{Comments :}

\section{DUS-15A}


1

INORGANIC ANALYSES DATA SHEET
EPA SAMPLE NO.

\section{$15 \mathrm{~B}$}

Contract: 60052

SAS NO. : Lab Sample ID: 06091862-010 Date Received: 09/01/06
Lab Name: LIONVILLE_LABORATORY

Lab Code: LVLI

Matrix (soil/water):
Level (low/med):

: Solids:

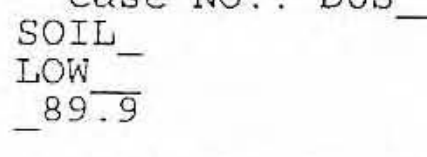

Concentration Units (ug/L or $\mathrm{mg} / \mathrm{kg}$ dry weight): $\mathrm{MG} / \mathrm{KG}$

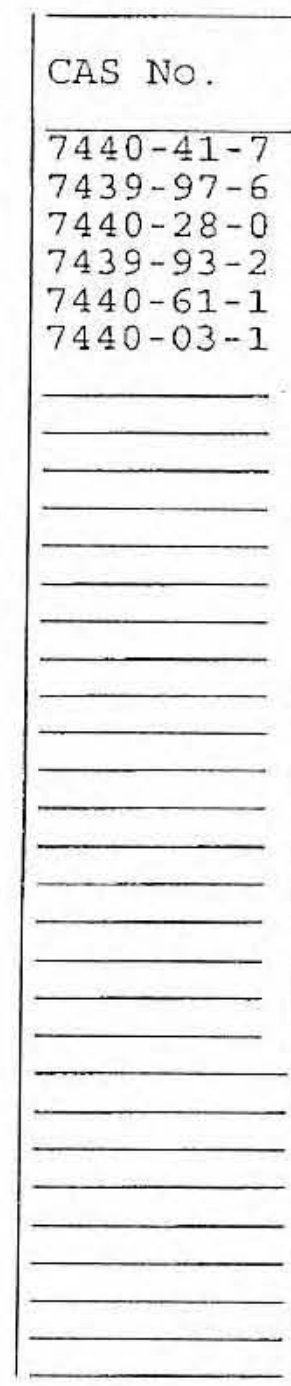

Color Before:

Color After:

\section{Comments :}

DUS - 15B

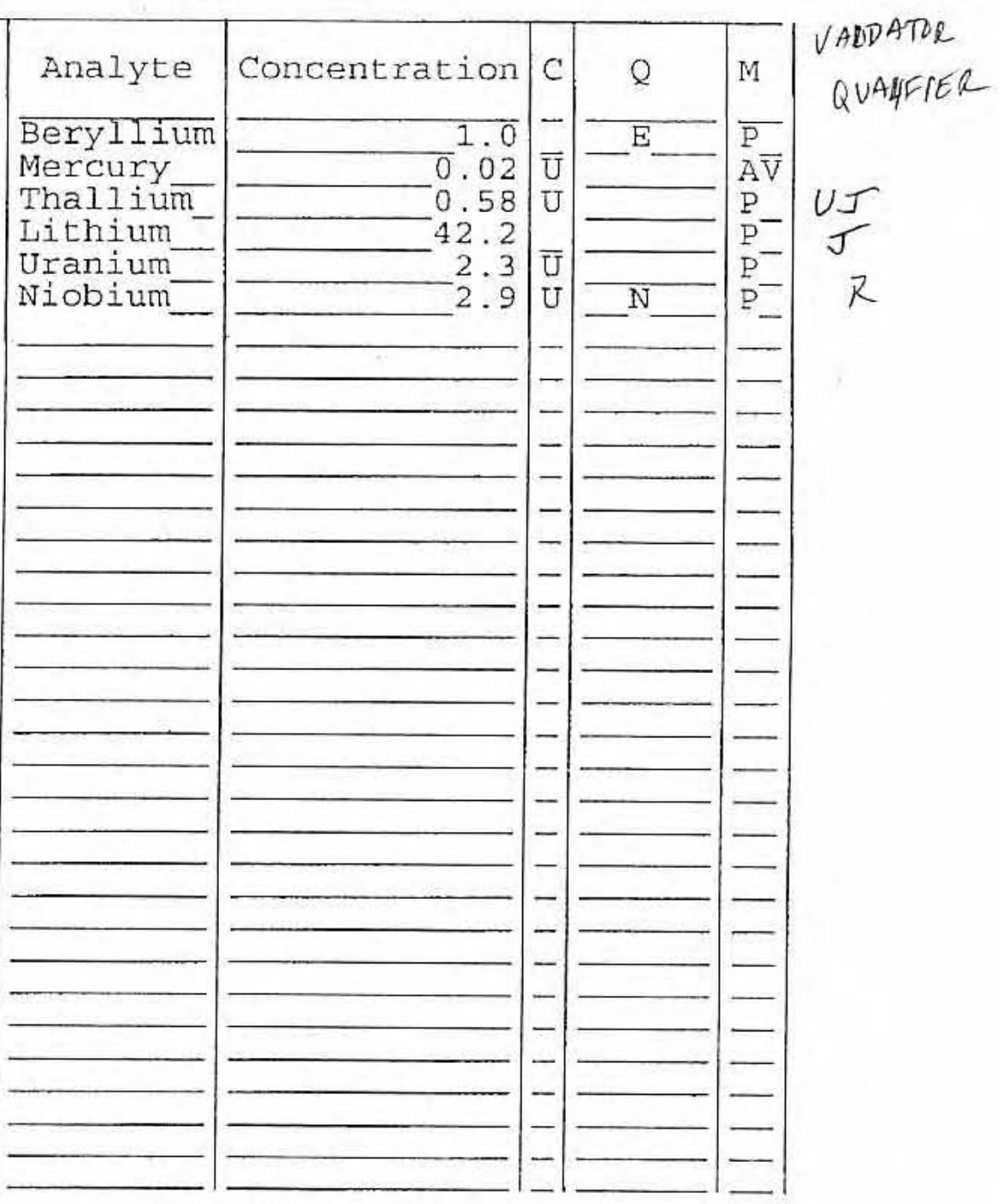

Clarity Before: Clarity After:
Texture:

Artifacts: 


\section{U.S. EPA}

\section{1}

INORGANIC ANALYSES DATA SHEET
EPA SAMPLE NO.

$16 \mathrm{~A}$

Lab Name: LIONVILLE_LABORATORY

Contract: 60052

SAS NO.:

Lab sample ID: 0609118

Date Received: 09/01/06
Matrix (soil/water): SOIL

$\begin{array}{ll}\text { o Solids: } & \text { LOW } \\ & \end{array}$

Concentration Units (ug/L or $\mathrm{mg} / \mathrm{kg}$ dry weight): MG/KG

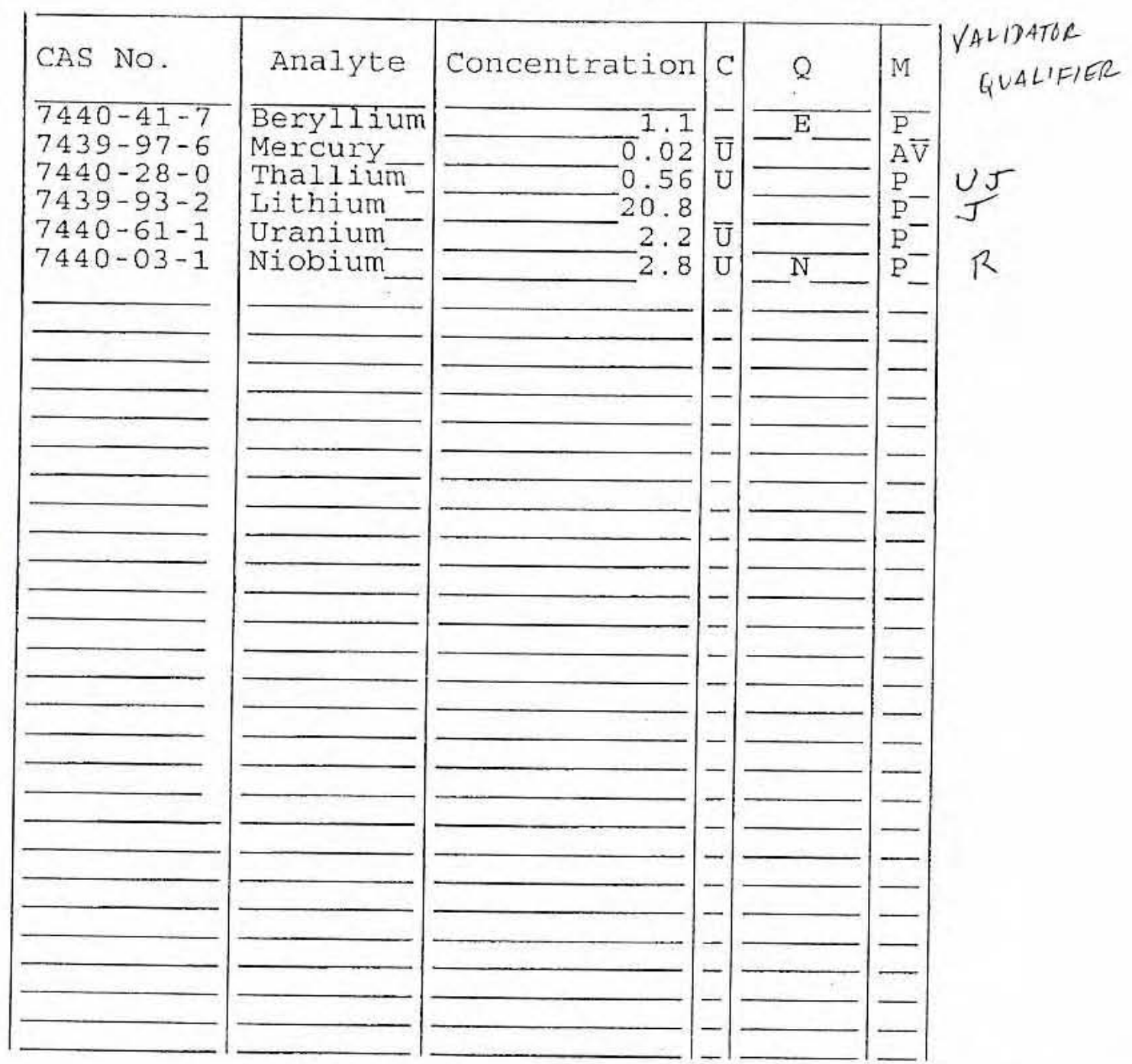

Color Before:

Color After:
Clarity Before: Clarity After:
Texture:

Artifacts:

Comments:

DUS $-16 \mathrm{~A}$ 
1

INORGANIC ANALYSES DATA SHEET
EPA SAMPLE NO.

$$
16 \mathrm{~B}
$$

Lab Name: LIONVILLE LABORATORY

Lab Code: LVLI Matrix (soil/water): SOIL

Level (low/med):

Solids:
Contract: 60052

SAS NO.:

Lab Sample ID: 0609L862-012

Date Received: 09/01/06

Concentration Units (ug/L or $\mathrm{mg} / \mathrm{kg}$ dry weight): $\mathrm{MG} / \mathrm{KG}$

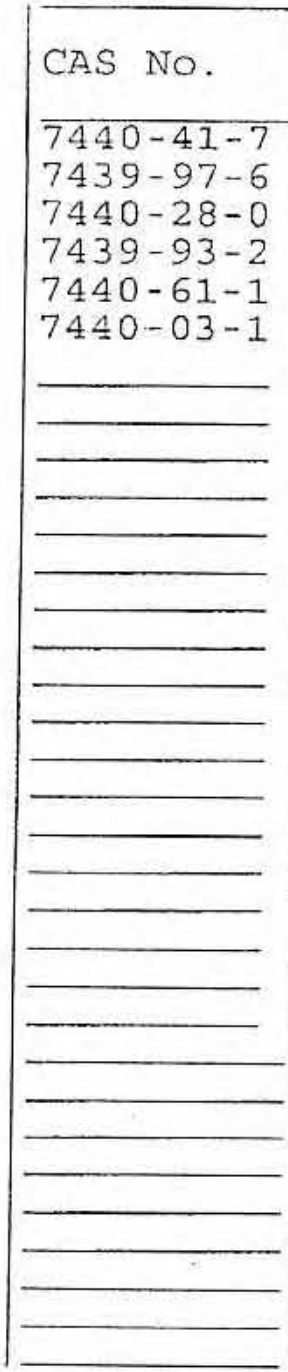

Color Before:

Color After:

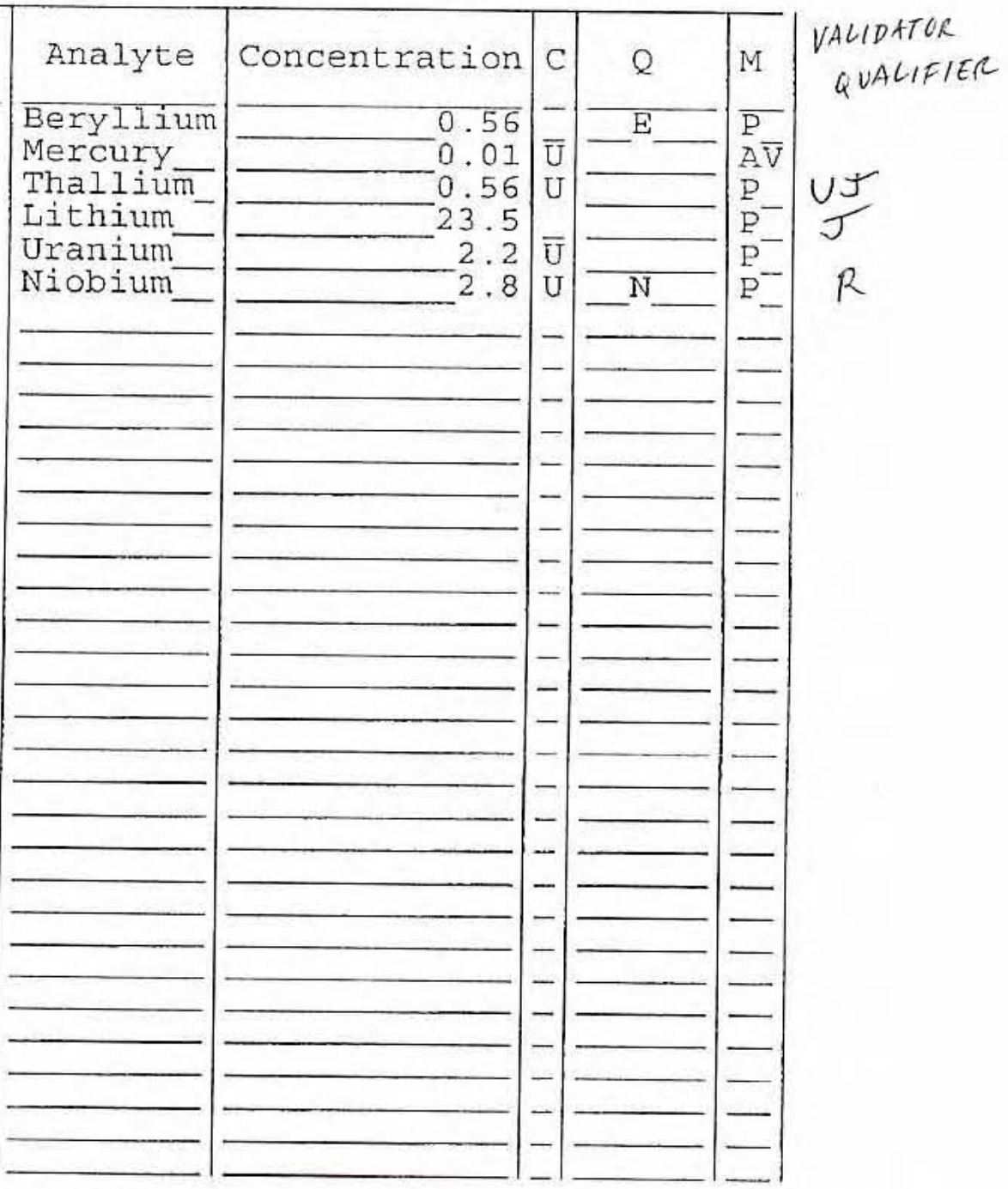

Clarity Before:

Clarity After:
Texture:

Artifacts:

\section{Comments:} DUS - I6B 


\section{U.S. EPA.}

1 INORGANIC ANALYSES DATA SHEET
EPA. SAMPLE NNO.

$17 \mathrm{~A}$

Lab Name: LIONVILLE LABORATORY

Lab Code: LVLI

Matrix (soil/water): SoIl

Level (low/med): LOW

5olids:
Contract: 60052

SAS NO.:

SDG NO.: 11A

Lab Sample ID: 0609L86 $\overline{2-013}$ $-95 \overline{.1}$

Date Received: 09/01/06

Concentration Units (ug/I or mg/kg dry weight): $\mathrm{MG} / \mathrm{KG}$

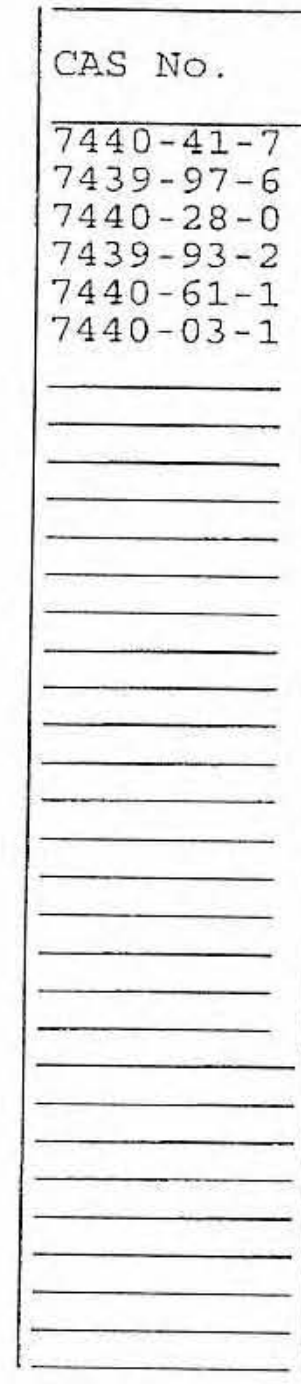

Color Before:

Color After:

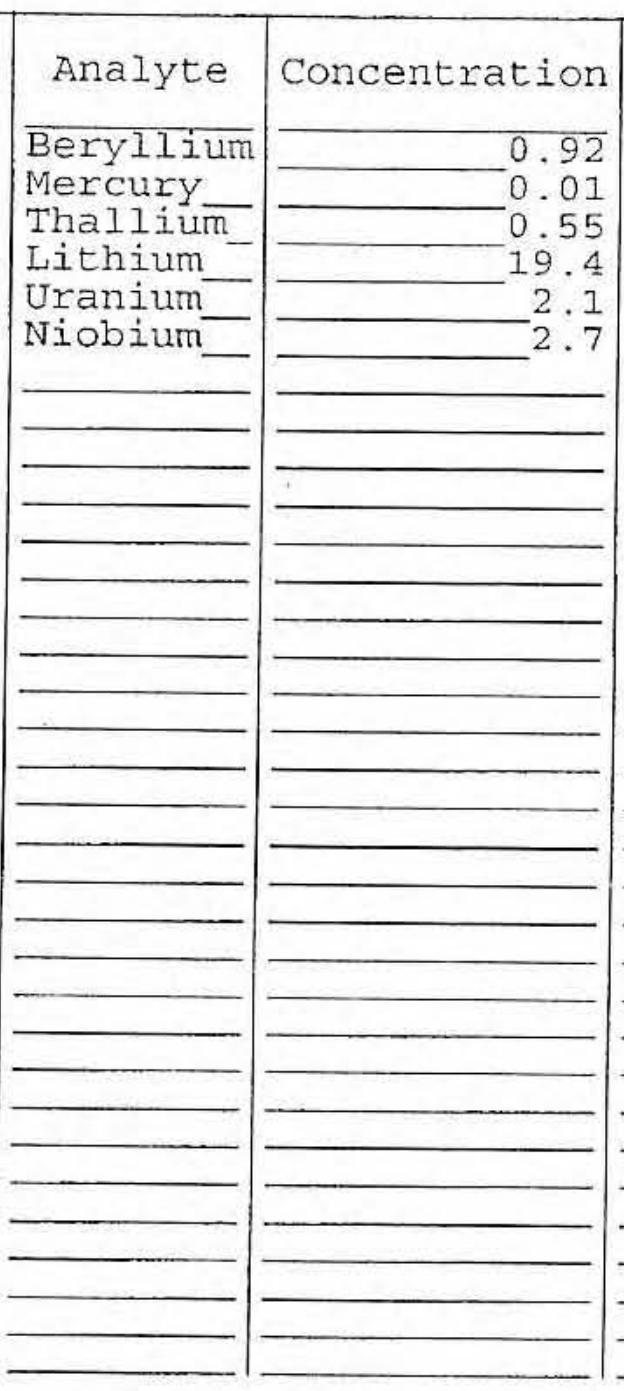

Clarity Before: Clarity After:
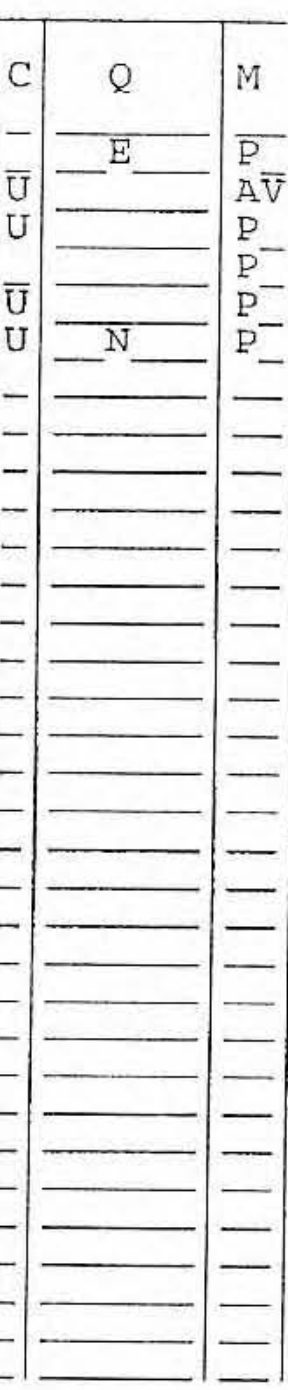

Texture: Artifacts: $\checkmark A L D A T R$ QUALFIER

UJ

$R$

\section{Comments:}

DUS - 17A 
U.S. EPA

1

INORGANIC ANALYSES DATA SHEET
EPA SAMPLE NO.

\section{$17 \mathrm{~B}$}

Lab Name: LIONVILLE_LABORATORY

Lab code: LVLI

Matrix (soil/water): SoIL

Level, (low/med):

: Solids:
Contract: 60052

SAS NO. :

SDG NO.: 11A

LOW
$-90 . \overline{7}$

Lab Sample ID: 0609L86 $\overline{2-0} 14$

Date Received: 09/01/06

Concentration Units (ug/L or $\mathrm{mg} / \mathrm{kg}$ dry weight): $\mathrm{MG} / \mathrm{KG}$

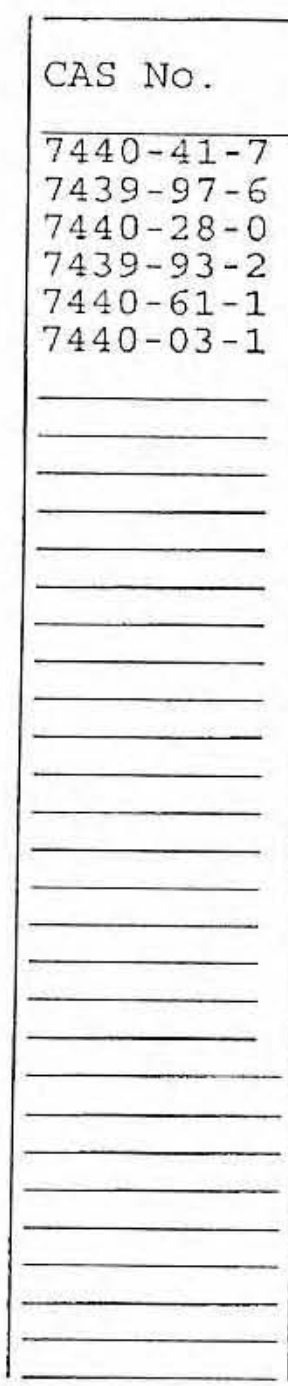

Color Before:

Color After:

\section{Comments :}

DUS $-17 \mathrm{~B}$

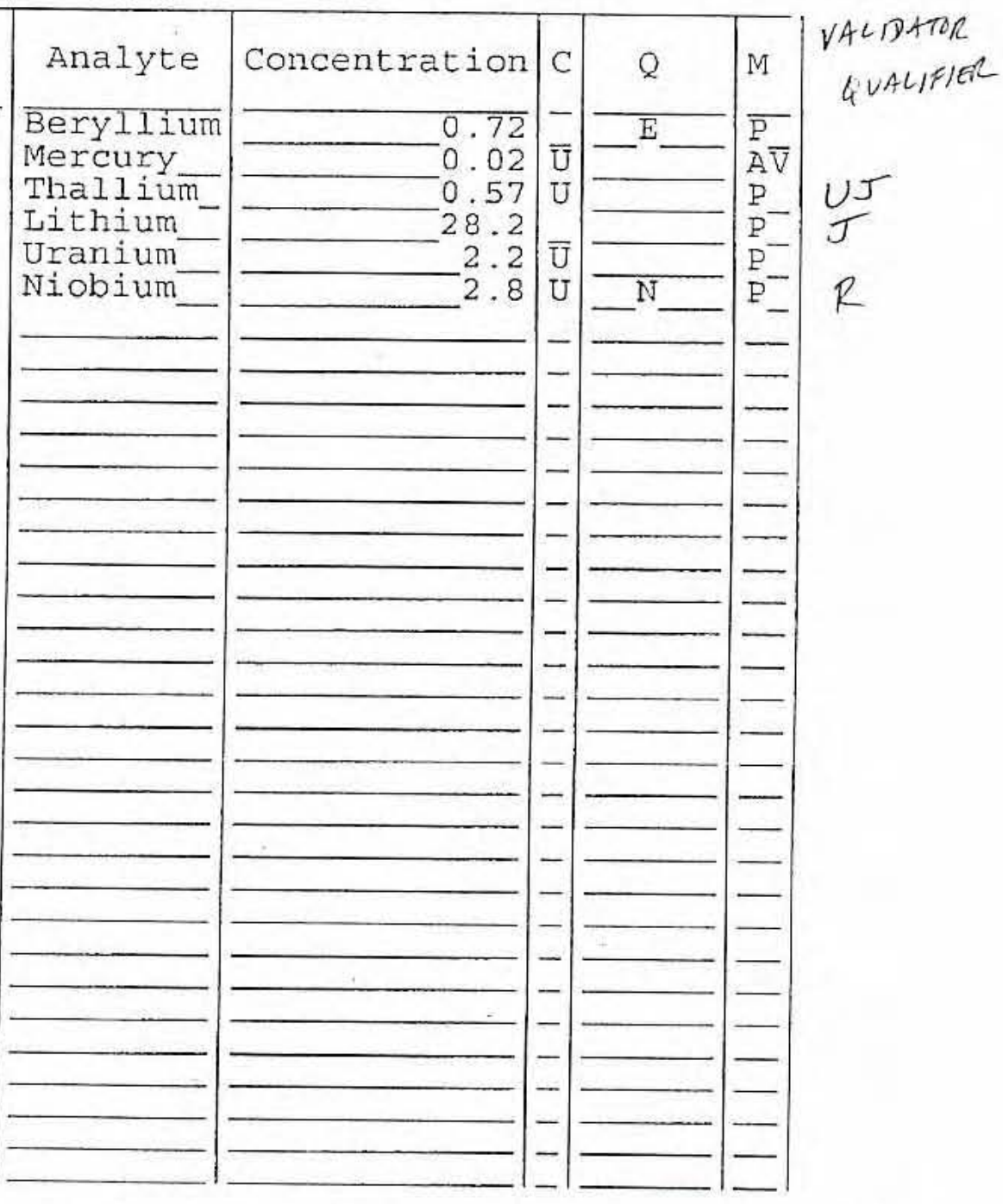

Clarity Before:

Clarity After:

Texture:

Artifacts: 
1 INORGANIC ANALYSES DATA SHEET
Lab Name: LIONVILIE LABORATORY

Lab Code: LVLI

Matrix (soil/water)

Level (low/med):

$\because$ Solids: SOIL

LOW
Contract: 60052

SAS NO.:

SDG NO.: $11 \mathrm{~A}$

$-93 \overline{.8}$
EPA SAMPLE NO.

$18 \mathrm{~A}$

Date Received: 09/01/06

Concentration Units (ug/L or $\mathrm{mg} / \mathrm{kg}$ dry weight): MG/KG

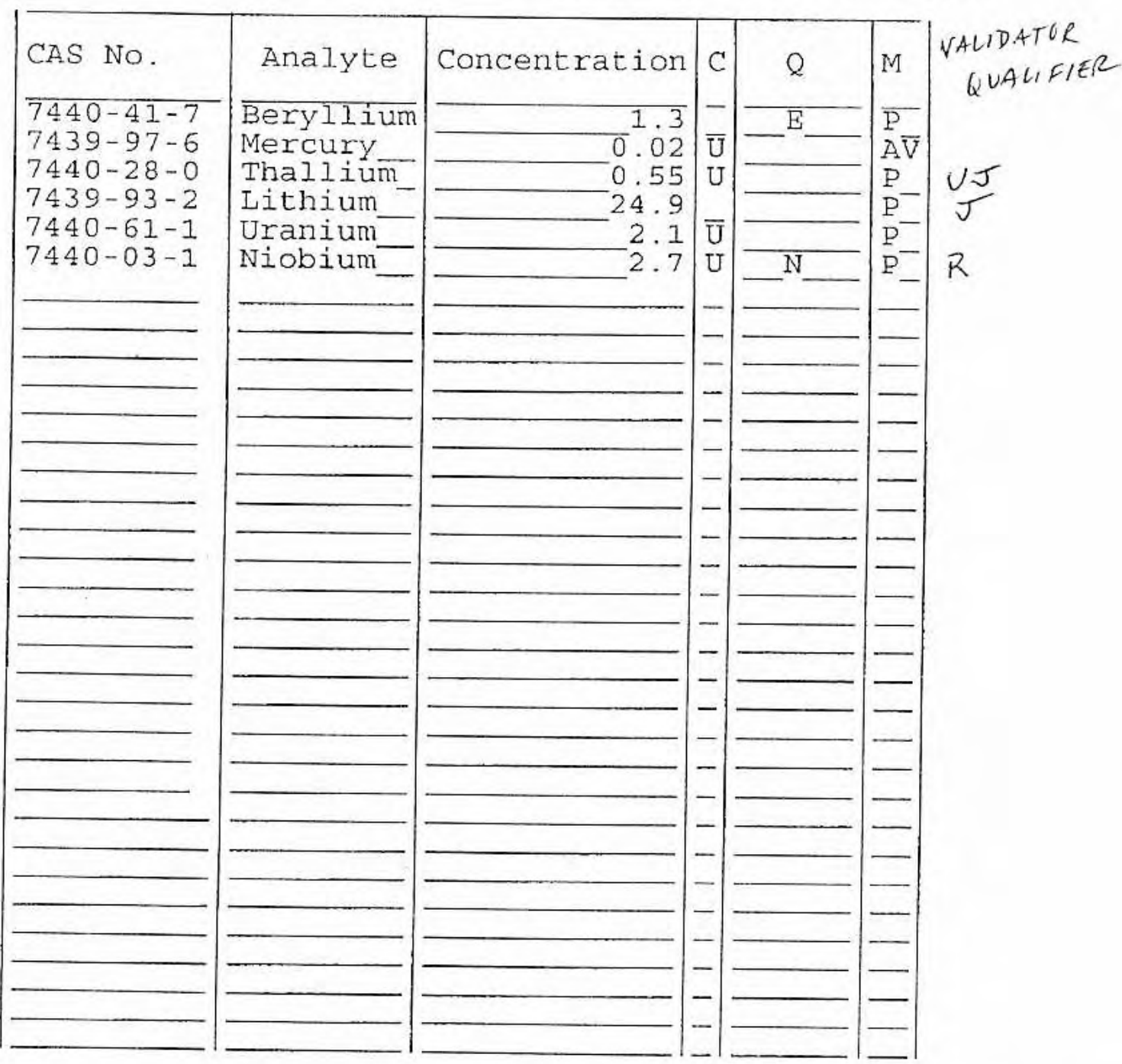

Color Before:

Color After:
Clarity Before:

Clarity After:
Texture:

Artifacts:

\section{Comments:}

DUS-18A 
U.S. EPA

1

INORGANIC ANALYSES DATA SHEET
EPA SAMPLE NO.

$18 \mathrm{~B}$

Lab Name: LIONVILLE LABORATORY

Lab Code: LVLI Matrix (soil/water): SoIL Level (low/med): LOW : Solids:
Contract: 60052 SAS NO. :

SDG No.: 11A

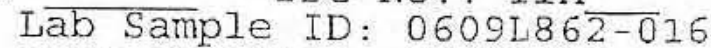

Date Received: 09/01/06

Concentration Units (ug/L or $\mathrm{mg} / \mathrm{kg}$ dry weight): MG/KG

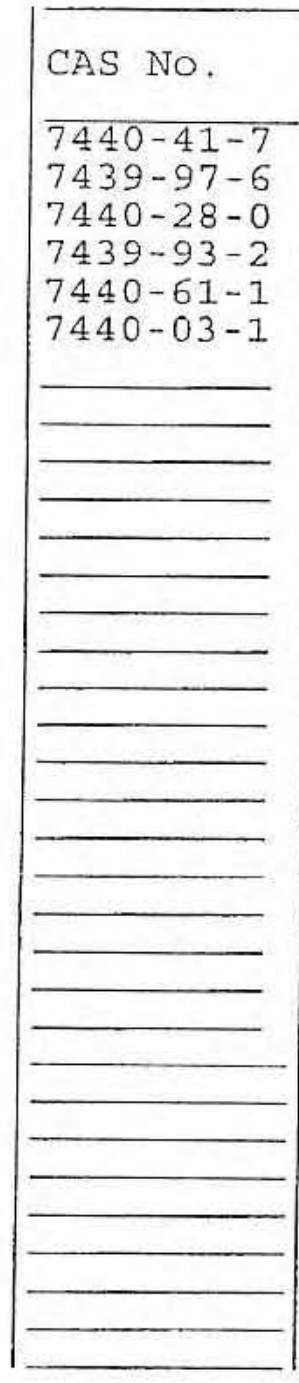

Color Before:

Color After:

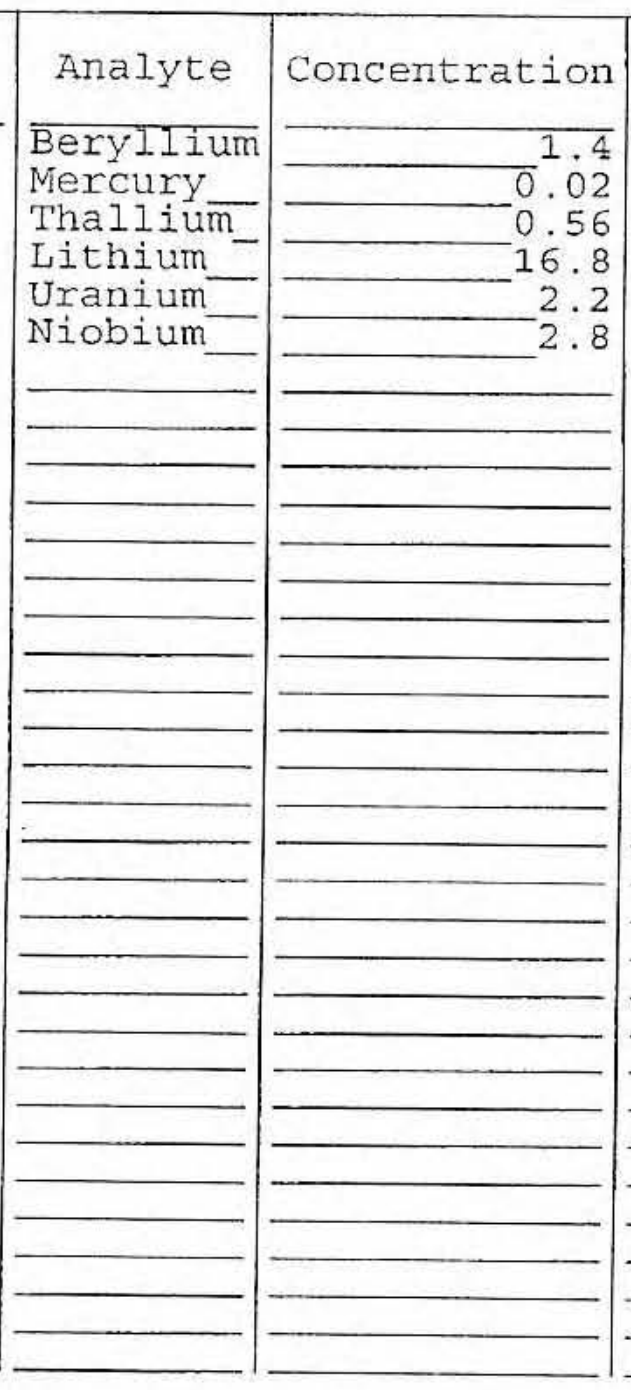

Clarity Before: Clarity After:

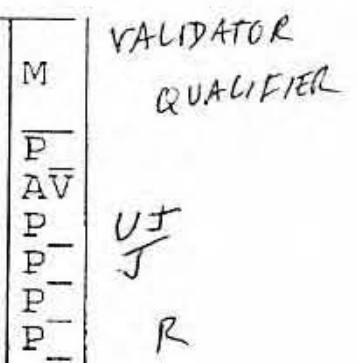

R

\section{Comments:}

\section{DUS $-18 B$}


Lab Name: LIONVILLE LABORATORY Lab Code: LVLI
Matrix (soil/water): SoIL Level (low/med): LOW $\therefore$ Solids:
Contract: 60052 SAS NO.:
$19 \mathrm{~A}$

SDG NO.: 11A

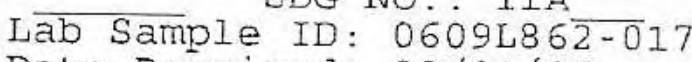

Date Received: 09/01/06

Concentration Units (ug/L or mg/kg dry weight): MG/KG

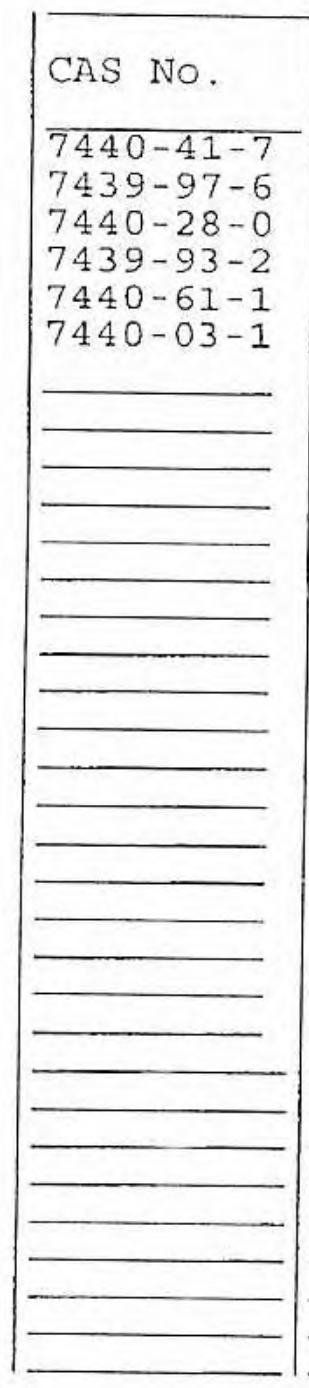

Color Before:

Color After:

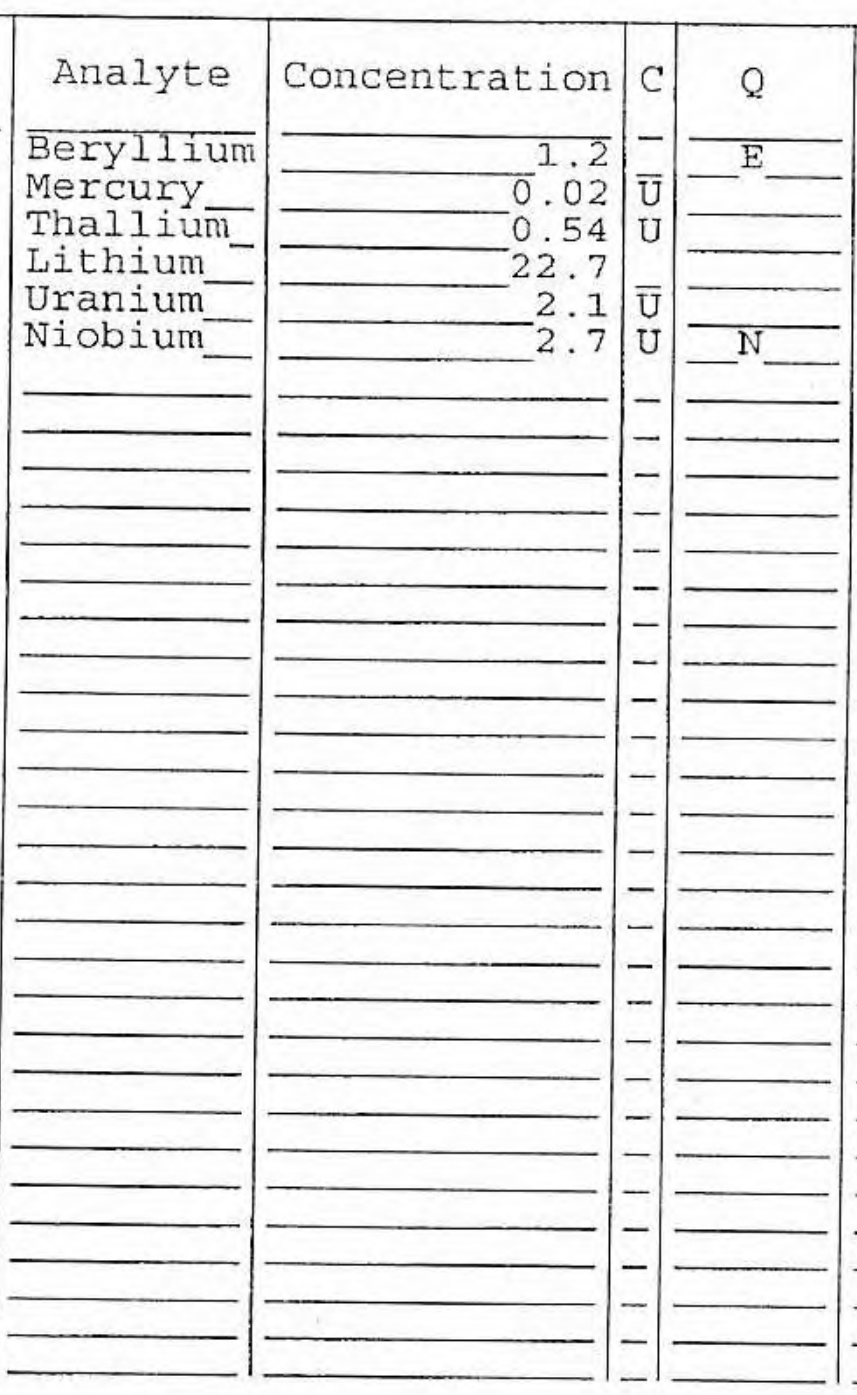

Clarity Before: Clarity After:

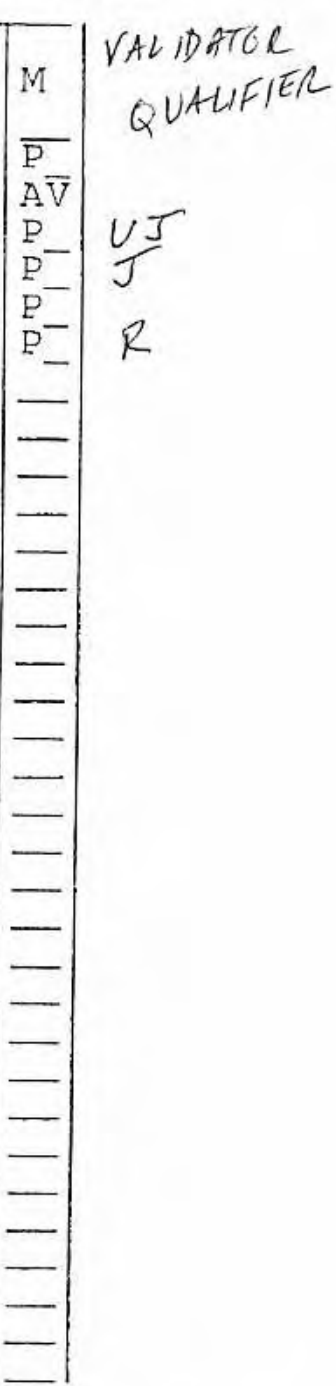

Texture:

Artifacts:

Comments:

DUS - 19A 
1 INORGANIC ANALYSES DATA SHEET
Lab Name: LIONVILLE LABORATORY

Lab Code: LVLI Matrix (soil/water): SOIL Level (low/med): LOW \% Solids:
Contract: 60052

$$
\text { SAS NO.: }
$$

Lab Sample ID: 0609L86 $\overline{2-0} 18$

$-90 \overline{.1}$
SDG NO.: 11A

EPA SAMPLE NO.

I.9B

Date Received: 09/01/06

Concentration Units (ug/L or $\mathrm{mg} / \mathrm{kg}$ dry weight): MG/KG

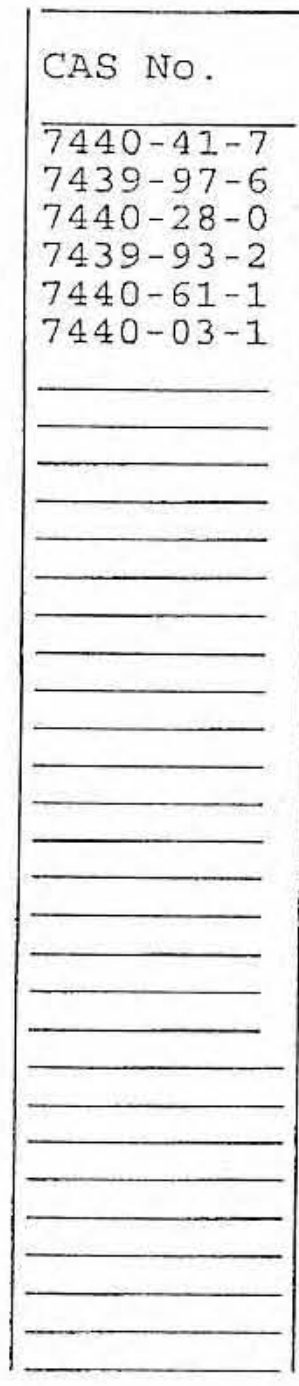

Color Before: Color After:

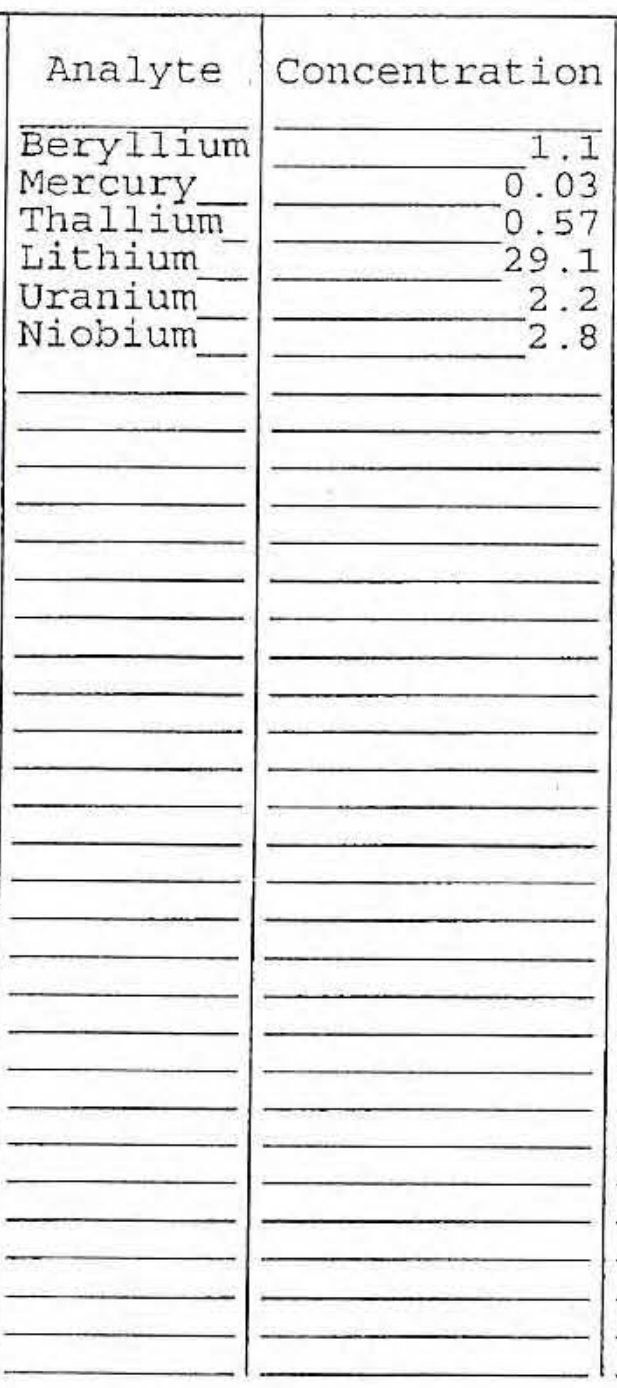

Clarity Before: Clarity After:

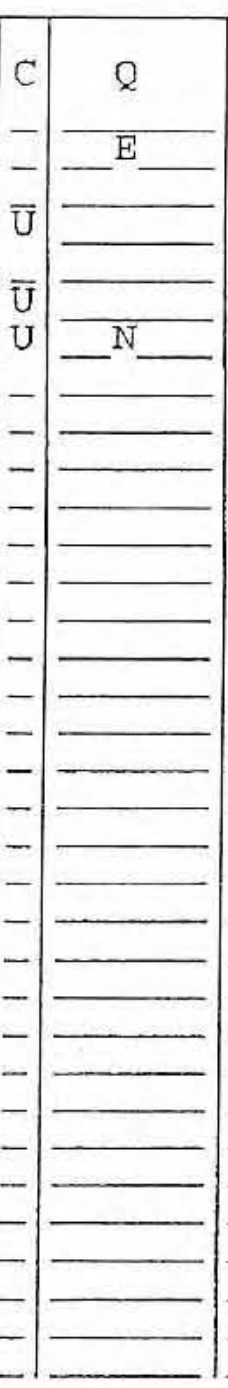

TIVACIDATOR QUALIFIER UJ $R$

Texture: Artifacts:

Comments :

DUS - 19B 
1 INORGANIC ANALYSES DATA SHEET
EPA SAMPLE NO.

$20 \mathrm{~A}$

Date Received: 09/01/06
SDG NO.: 11A

SA.S NO.:

Contract: 60052

Lab Sample ID: $0609486 \overline{2-019}$
Matrix (soil/water): SOIL

\% Solids:

Concentration Units (ug/L or $\mathrm{mg} / \mathrm{kg}$ dry weight): $\mathrm{MG} / \mathrm{KG}$

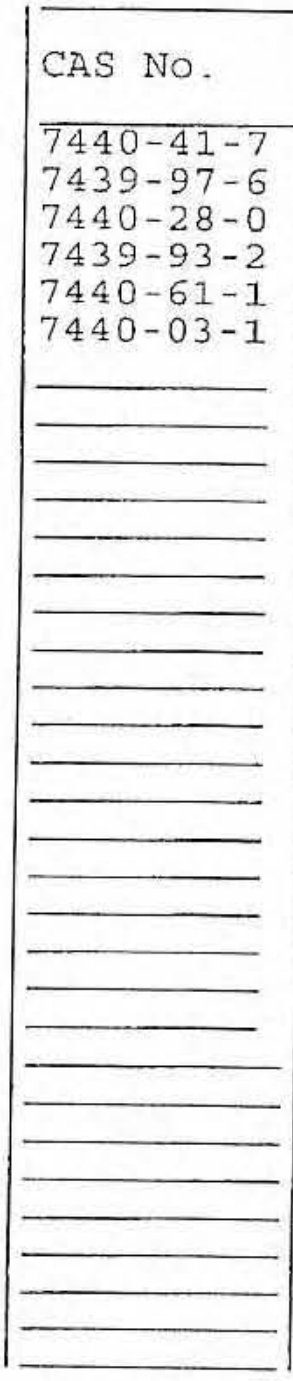

Color Before:

Color After:

Comments :

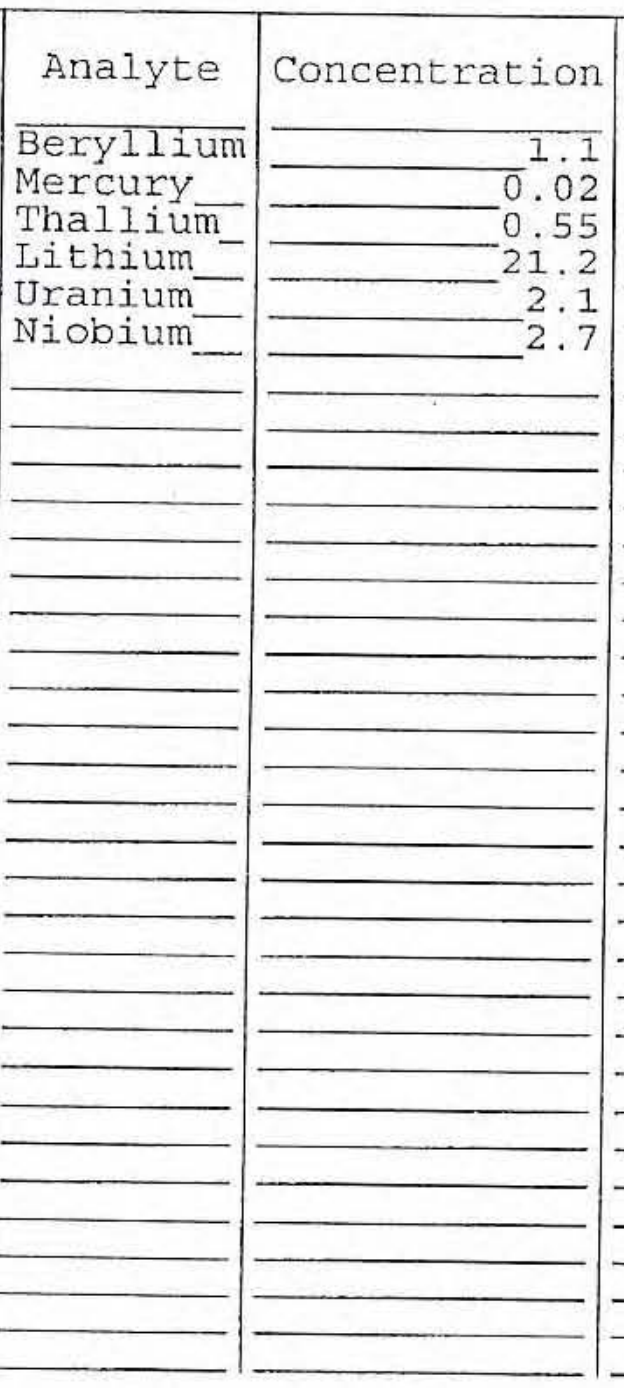

Clarity Before:

Clarity After:

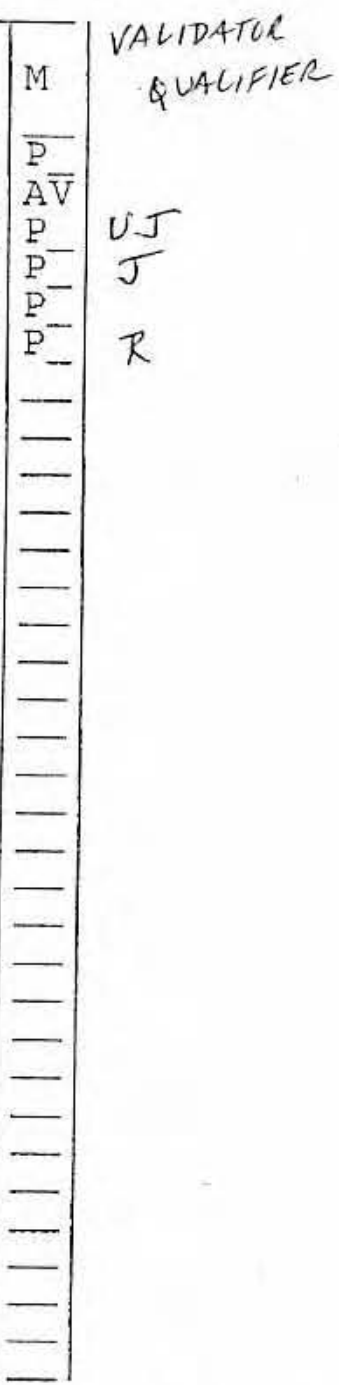

Texture: Artifacts:

DUS-20A 


\section{INORGANIC ANALYSES DATA SHEET}

EPA SAMPLE NO.

$$
20 B
$$

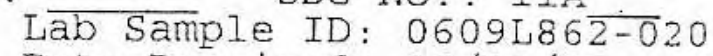

SDG NO.: $11 \mathrm{~A}$ SAS No.:

Date Received: 09/01/06

Contract: 60052
Matrix (soil/water): SOIL

Level (low/med): LOW

$\div$ Solids:

Concentration Units (ug/L or mg/kg dry weight): MG/KG

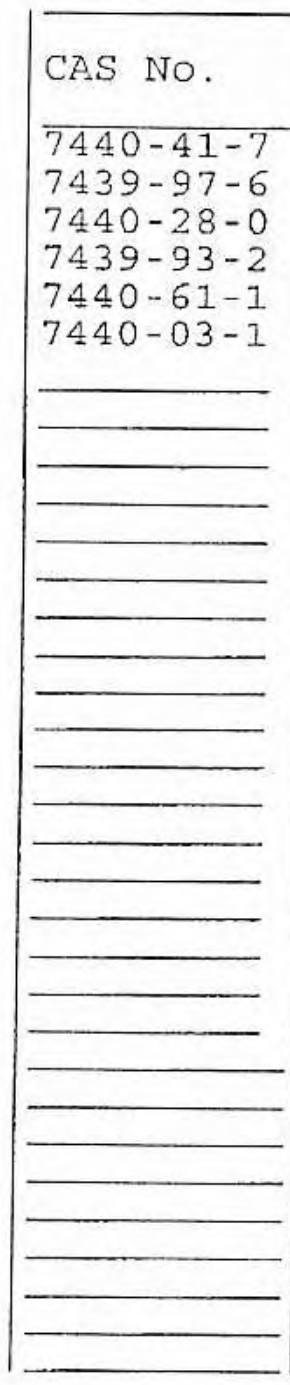

Color Before:

Color After:

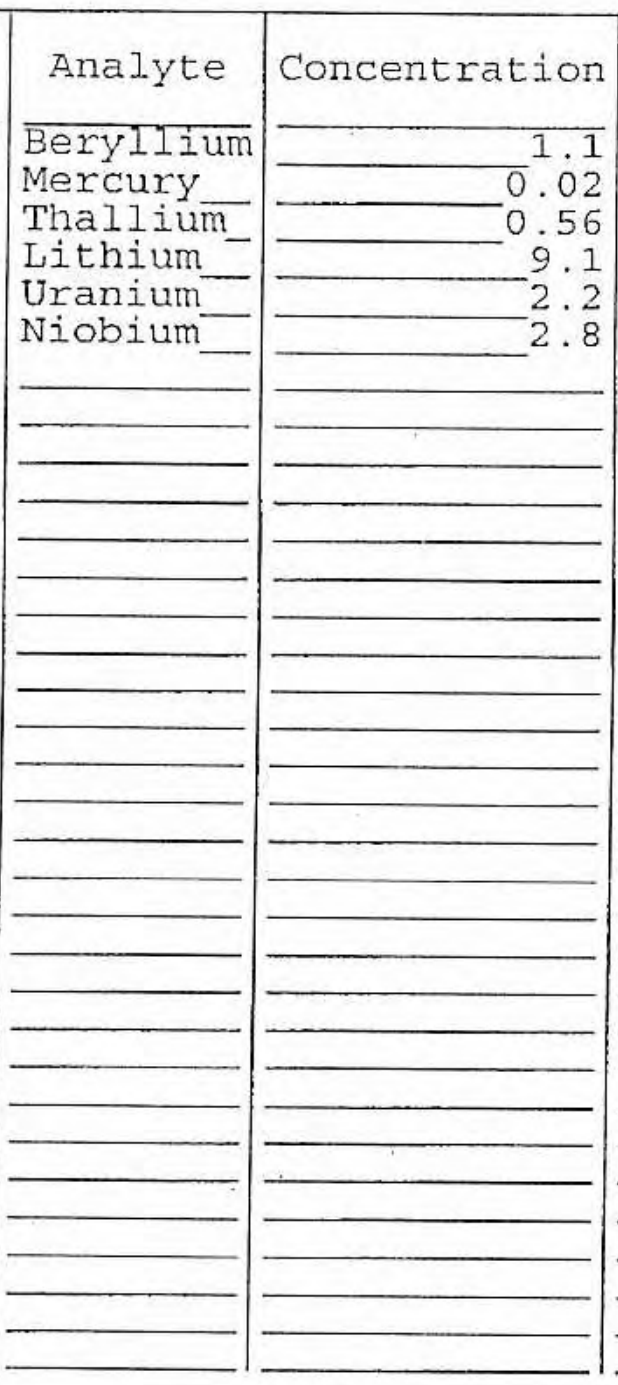

Clarity Before: Clarity After:

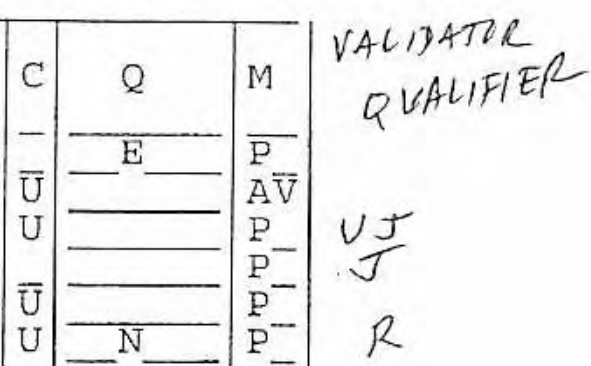

QULIJATIR
QVALIFIER

$\frac{\pi}{R T}$

Comments :

DUS - 2OB 


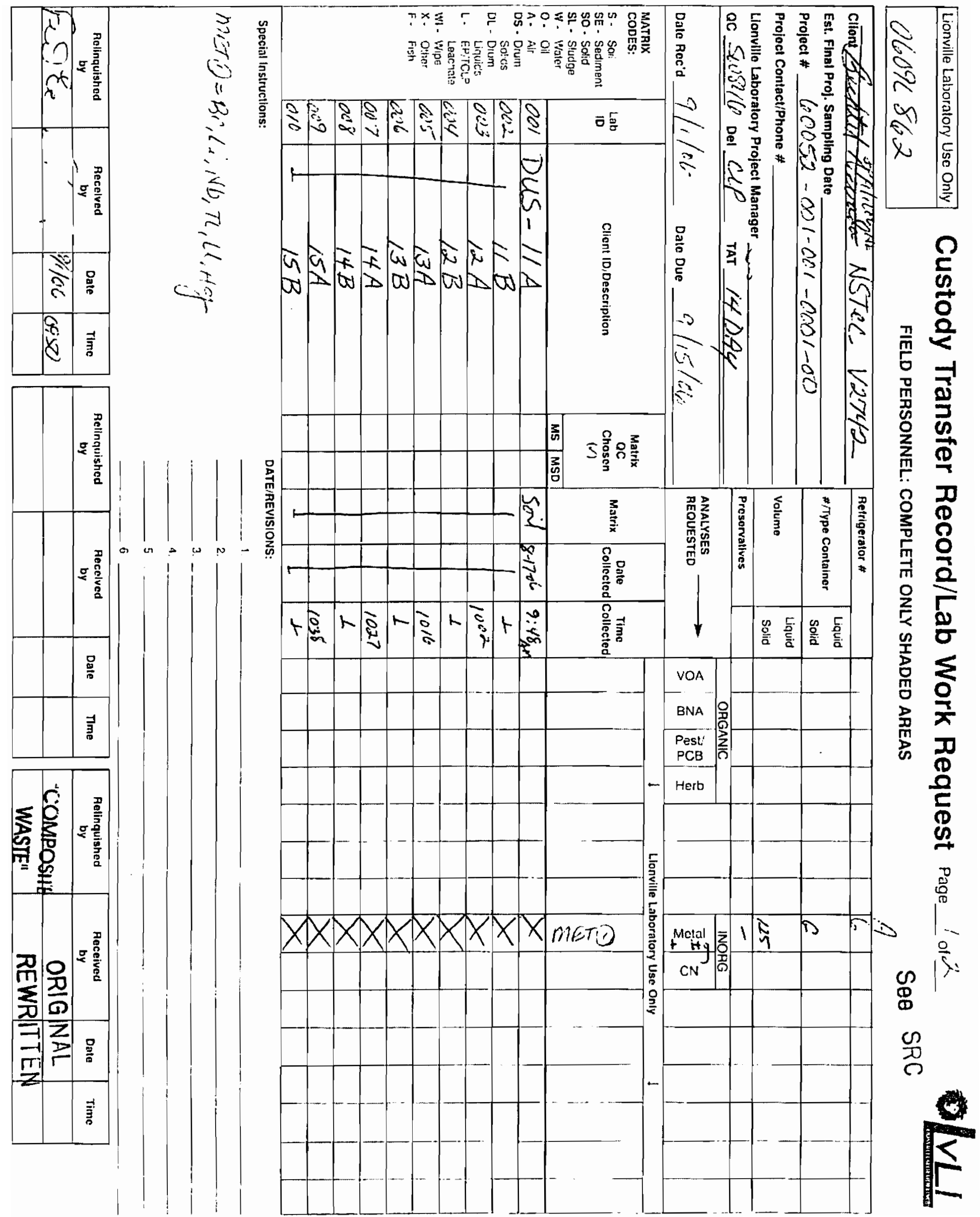




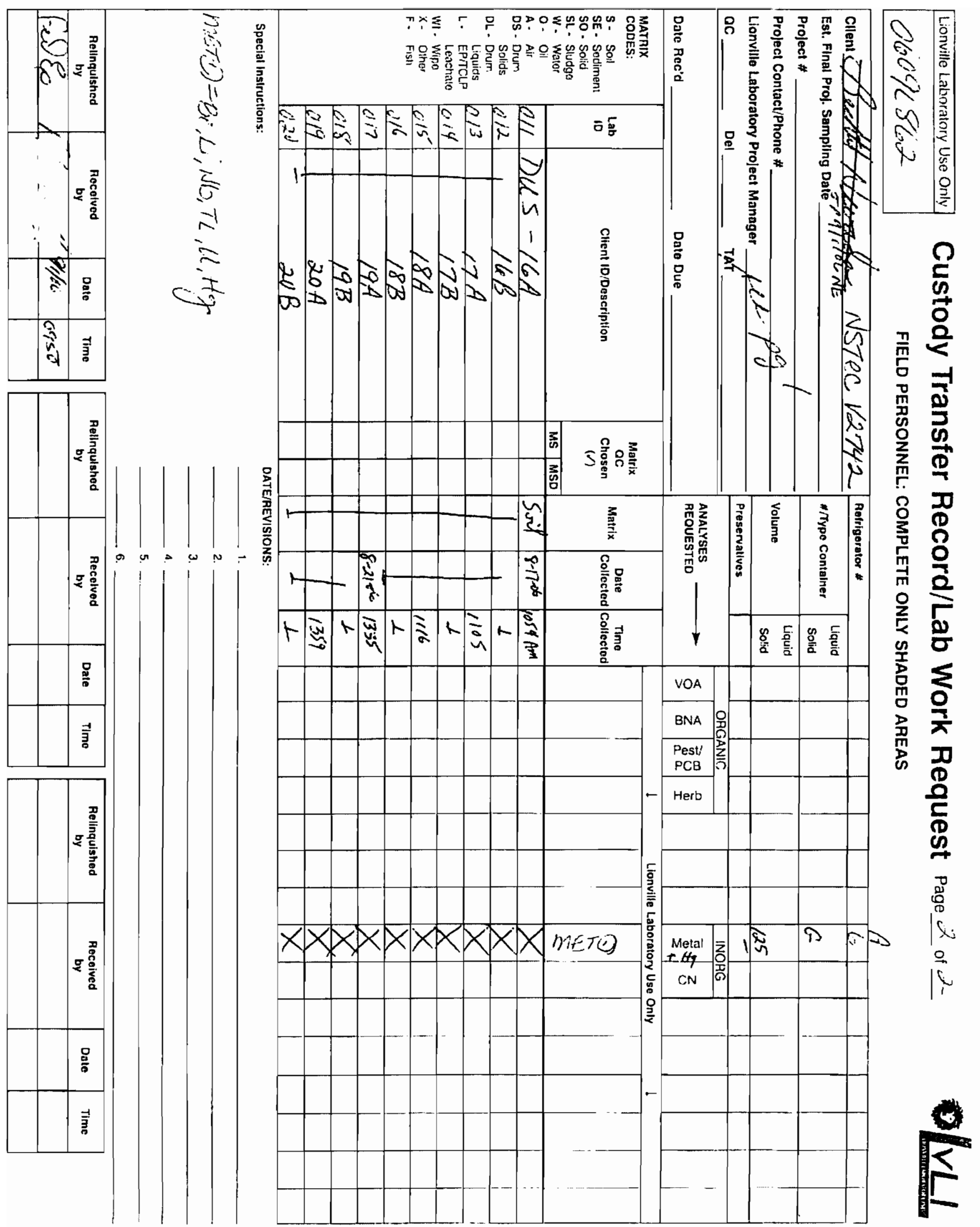




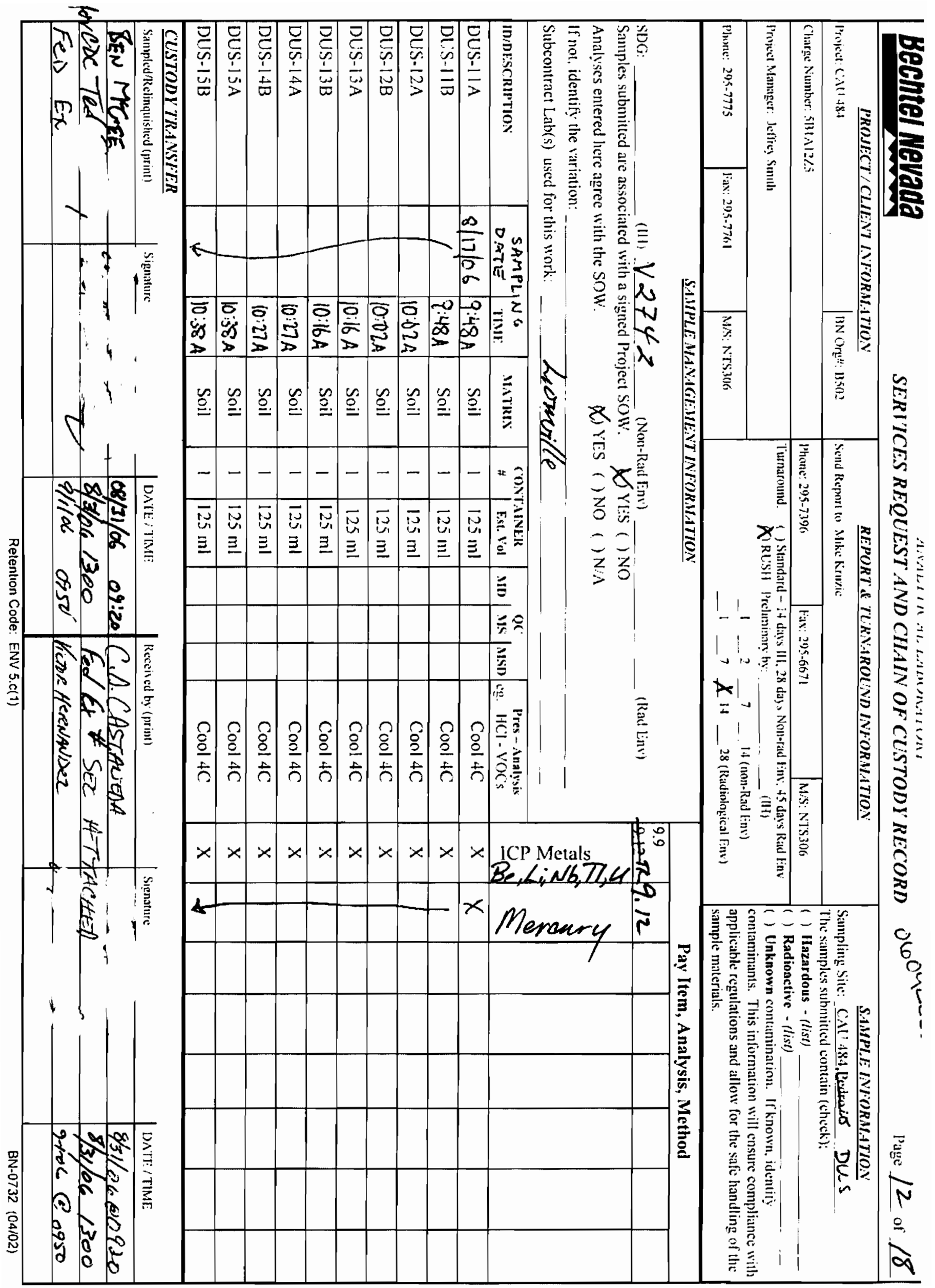




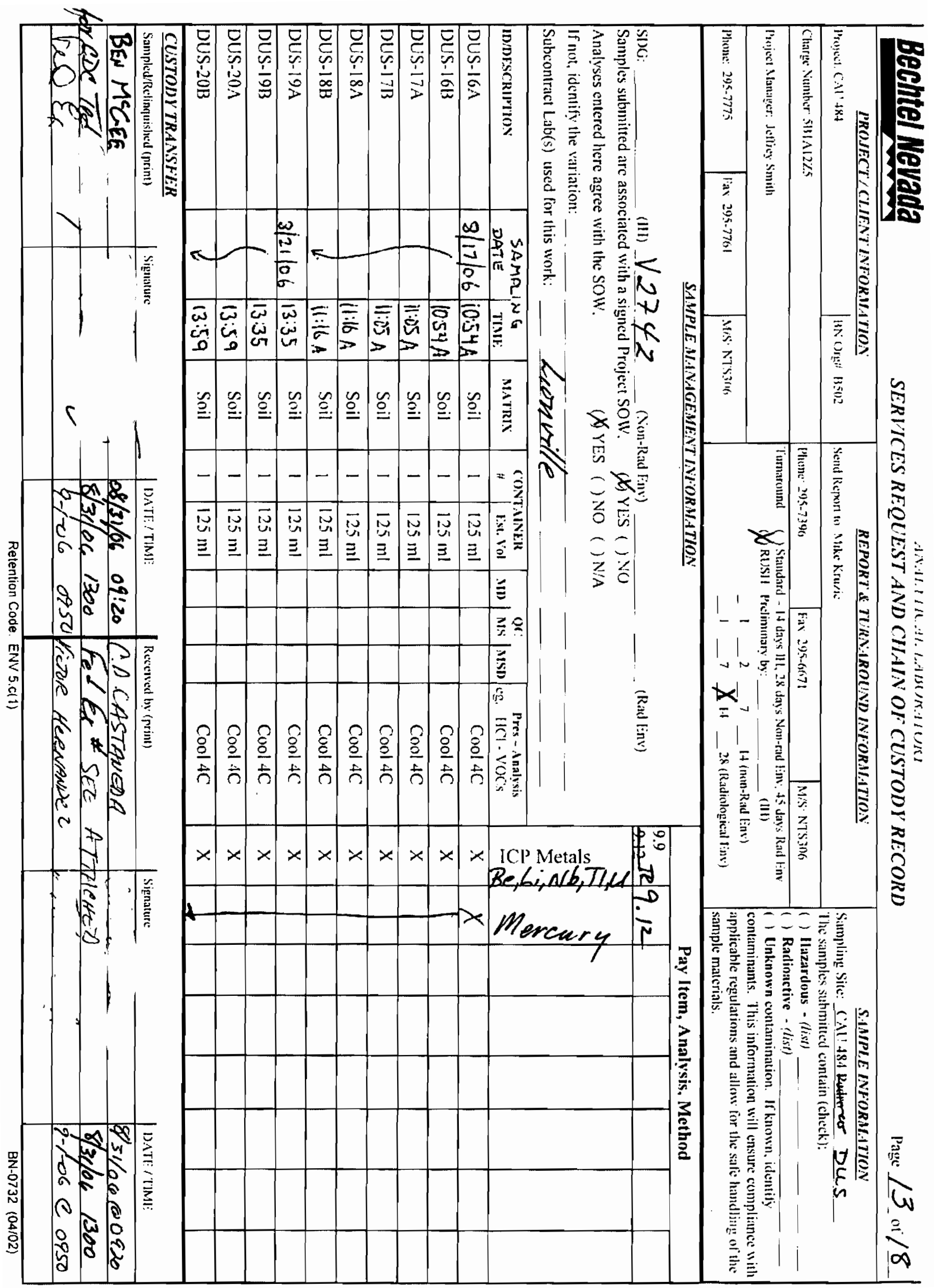


1

\section{INORGANIC ANALYSES DATA SHEET}

Lab Name: LIONVILLE LABORATORY Lab Code: LVII Matrix (soil/water): SoIL Level. (low/med): LOW \% Solids:
Contract: 60052 SAS NO.:

SDG NO.: V2742

Lab Sample ID: 0609L863-001 $-96 . \overline{5}$
EPA SAMPLE NO.

$21 \mathrm{~A}$

Date Received: 09/01/06

Concentration Units (ug/L or $\mathrm{mg} / \mathrm{kg}$ dry weight): $\mathrm{MG} / \mathrm{KG}$

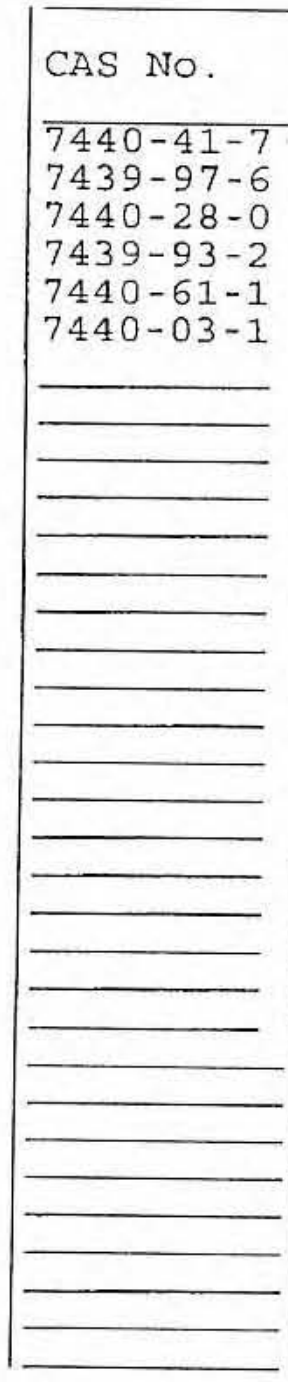

Color Before:

Color After:

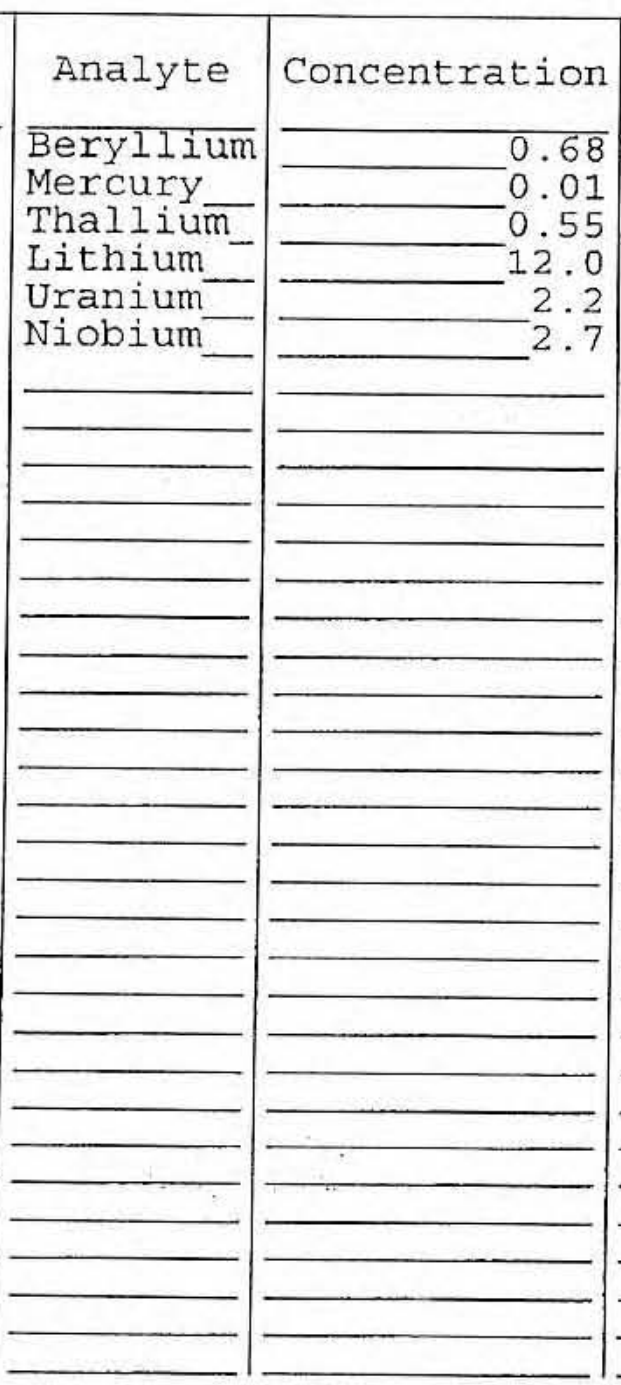

Clarity Before: Clarity After:

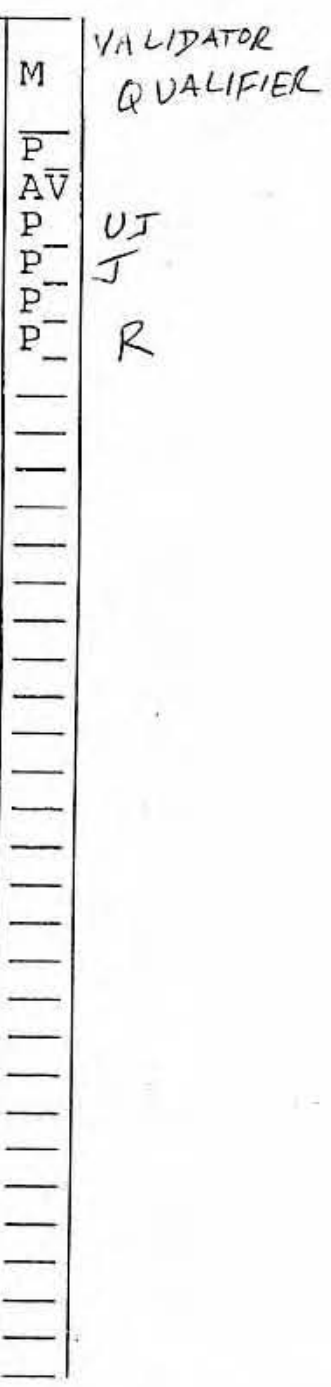

Texture: Artifacts:

Comments:

DUS - 21A 


\section{INORGANIC ANALYSES DATA SHEET}

Lab Name: LIONVILLE LABORATORY

Lab Code: LVLI Matrix (soil/water): SOIL

Level (low/med):

\% Solids:

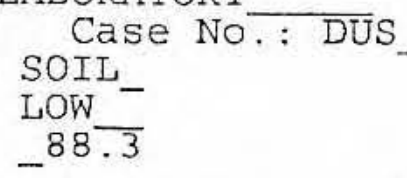

EPA SAMPLE NO.

$21 B$

SDG NO.: V2742

Date Received: 09/01/06

Concentration Units (ug/L or $\mathrm{mg} / \mathrm{kg}$ dry weight): MG/KG

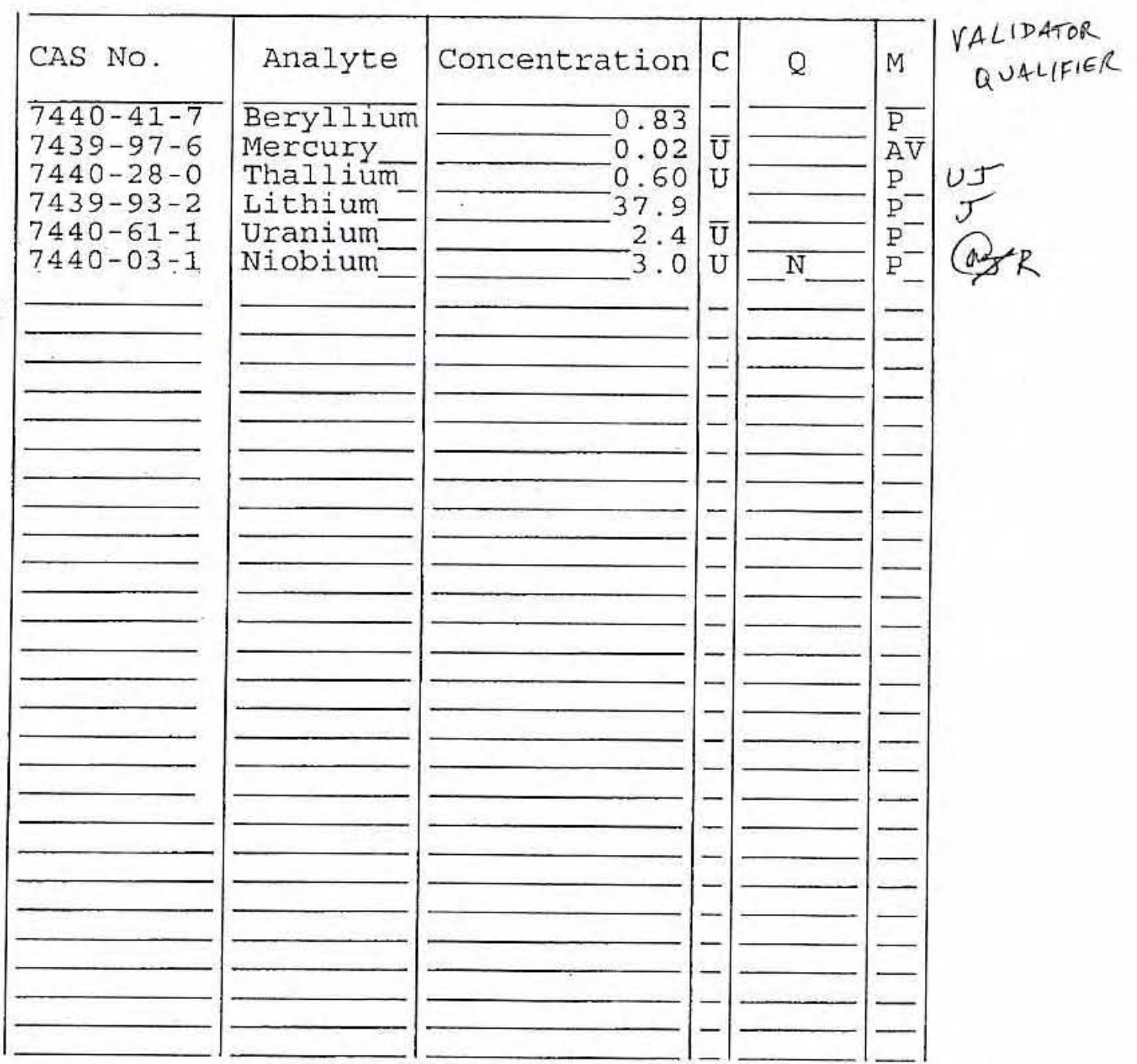

Color Before:

Color After:
Clarity Before:

Clarity After:
Texture: Artifacts:

Comments :

DUS - $21 B$ 
1

INORGANIC ANALYSES DATA SHEET
EPA SAMPLE NO.

$22 \mathrm{~A}$

Contract: 60052 SAS NO.:

SDG No.: V2742

Date Received: 09/01/06
Lab Name: LIONVILLE LABORATORY

Lab Code: LVLI Matrix (soil/water): SOIL

Level (low/med): LOW

\% Solids: $\quad-95 \overline{.0}$
Case No.: DUS

Concentration Units (ug/L or $\mathrm{mg} / \mathrm{kg}$ dry weight): MG/KG

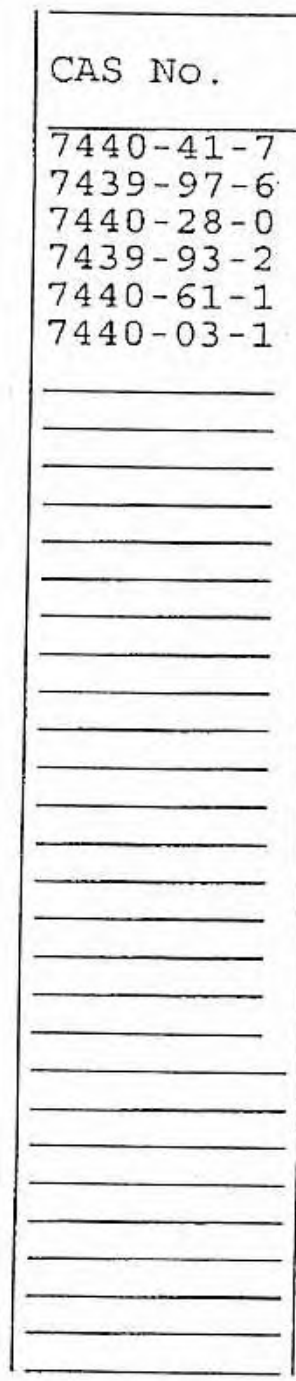

Color Before:

Color After:

Comments :

DUS $-22 \mathrm{~A}$

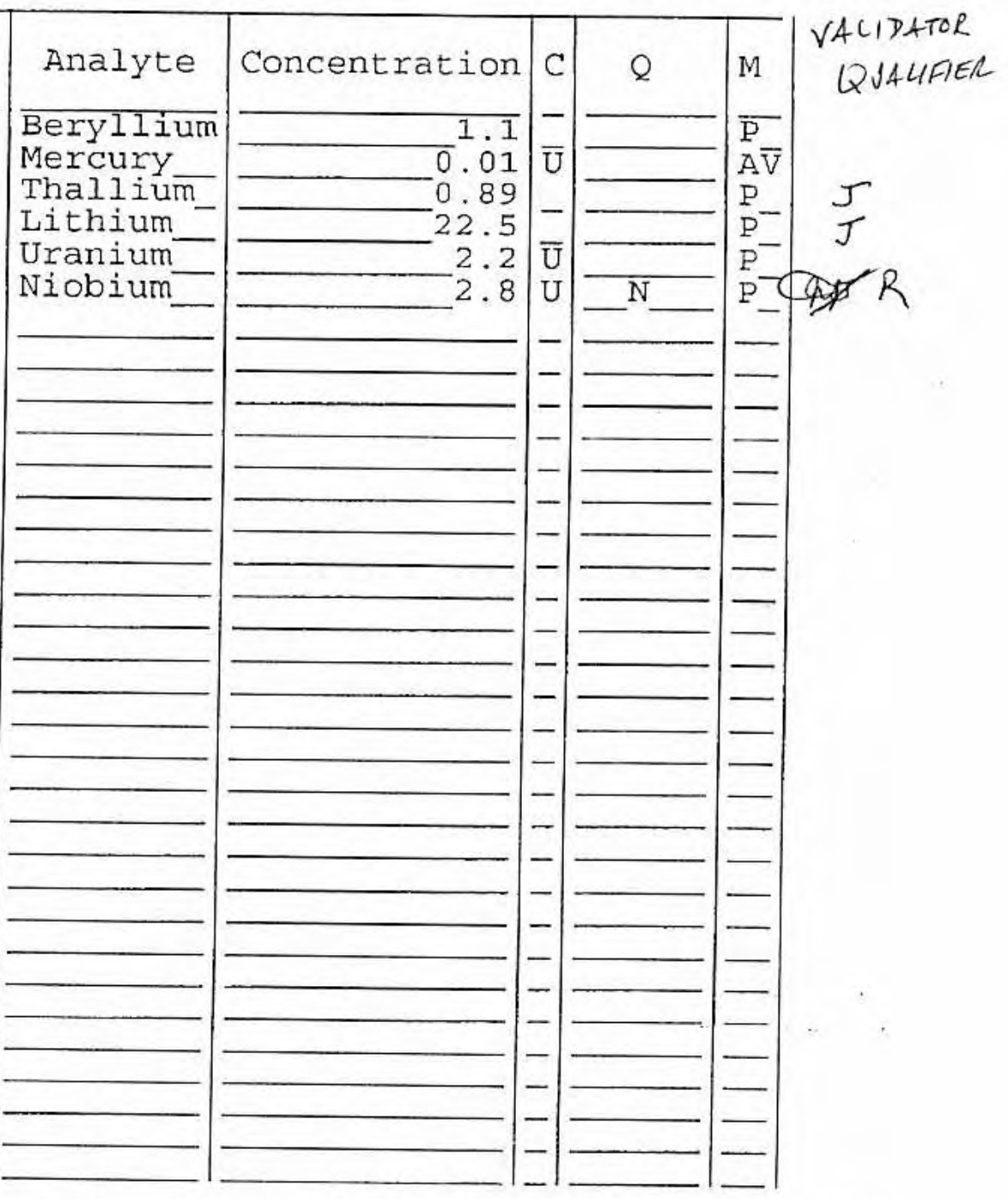

Clarity Before:

Texture: Clarity After: 
EPA SAMPLE NO.

$$
22 \mathrm{~B}
$$

Lab Name: LIONVILLE LABORATORY Lab Code: LVLI Matrix (soil/water): SoIL Level (low/med): LOW \% Solids:
Contract: 60052

SAS NO.:

SDG NO : V2742

Lab Sample ID: 0609L863-0̄04

Date Received: 09/01/06

Concentration Units (ug/L or $\mathrm{mg} / \mathrm{kg}$ ary weight): MG/KG

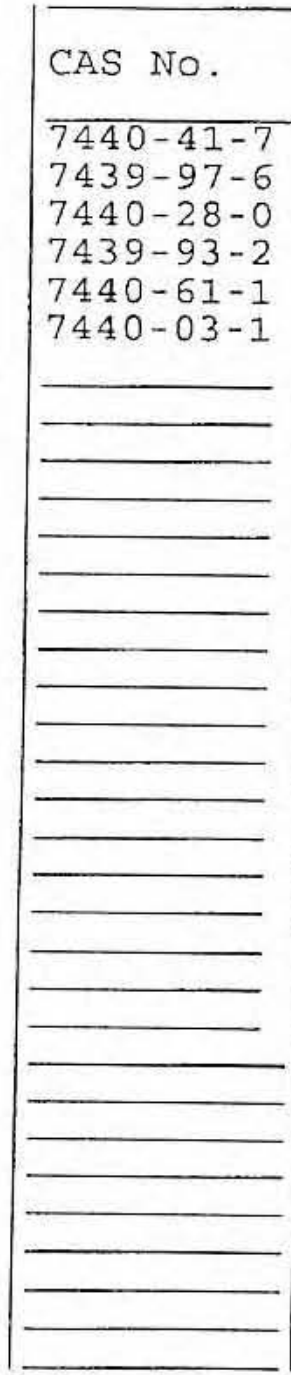

Color Before: Color After:

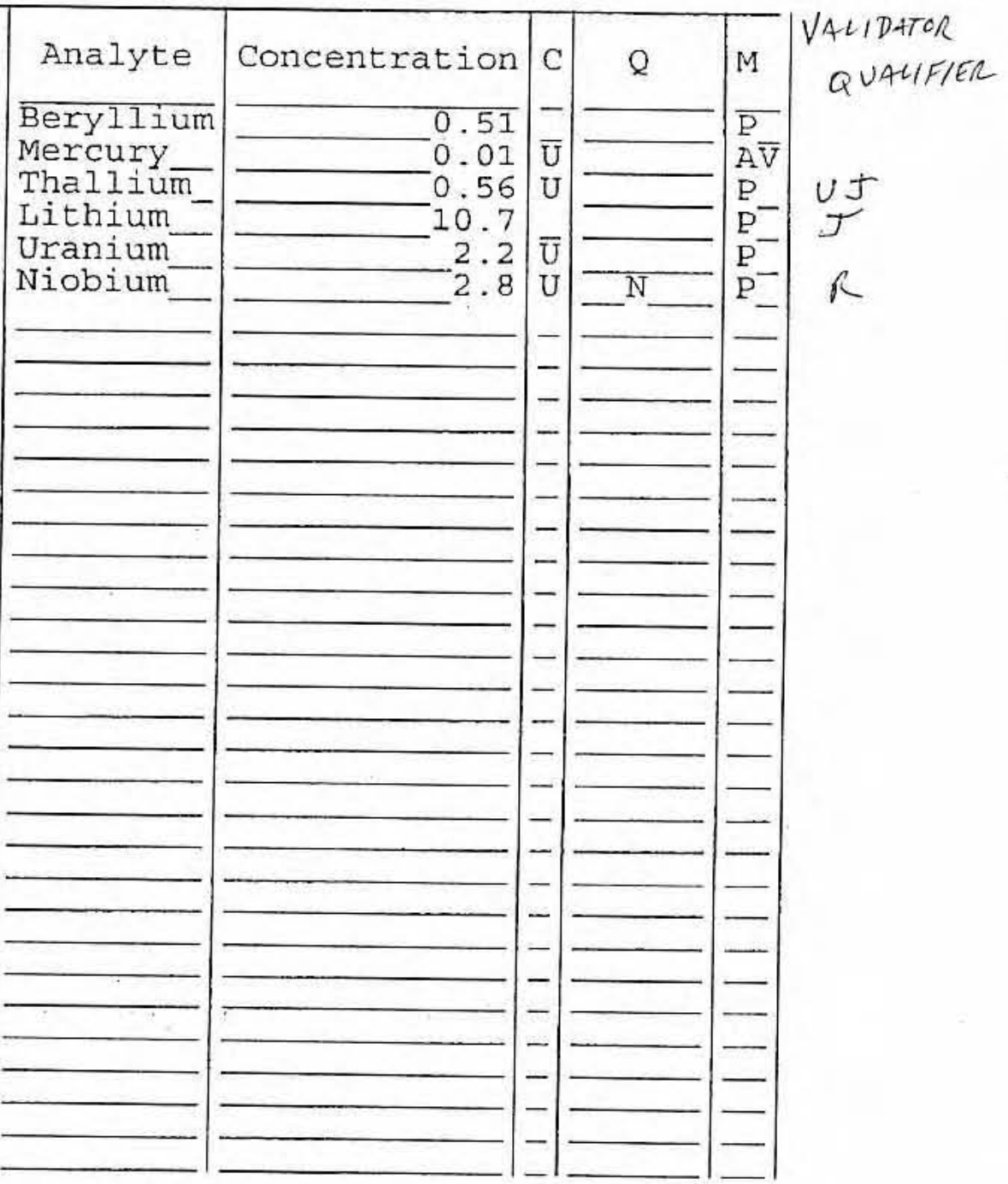

Clarity Before: Clarity After:
Texture: Artifacts :

\section{Comments :} DUS $-22 B$ 
Lab Name: LIONVILLE_LABORATORY

Lab Code: LVLI

Matrix (soil/water): SoIL

Level. (low/med): LOW

\% Solids:
$23 \mathrm{~A}$

SDG NO.: V2742

SAS NO.

Lab Sample ID: 0609L863-005

Date Received: 09/01/06

Concentration Units (ug/L or mg/kg dry weight): MG/KG

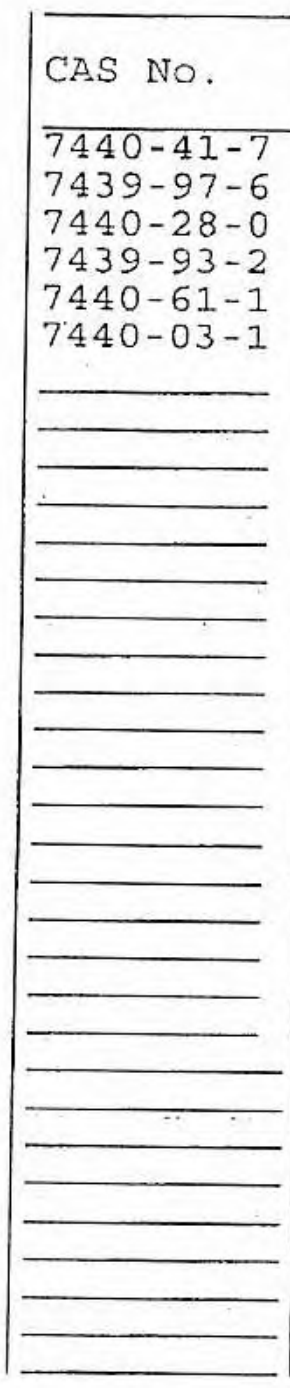

Color Before: Color After:

Comments:

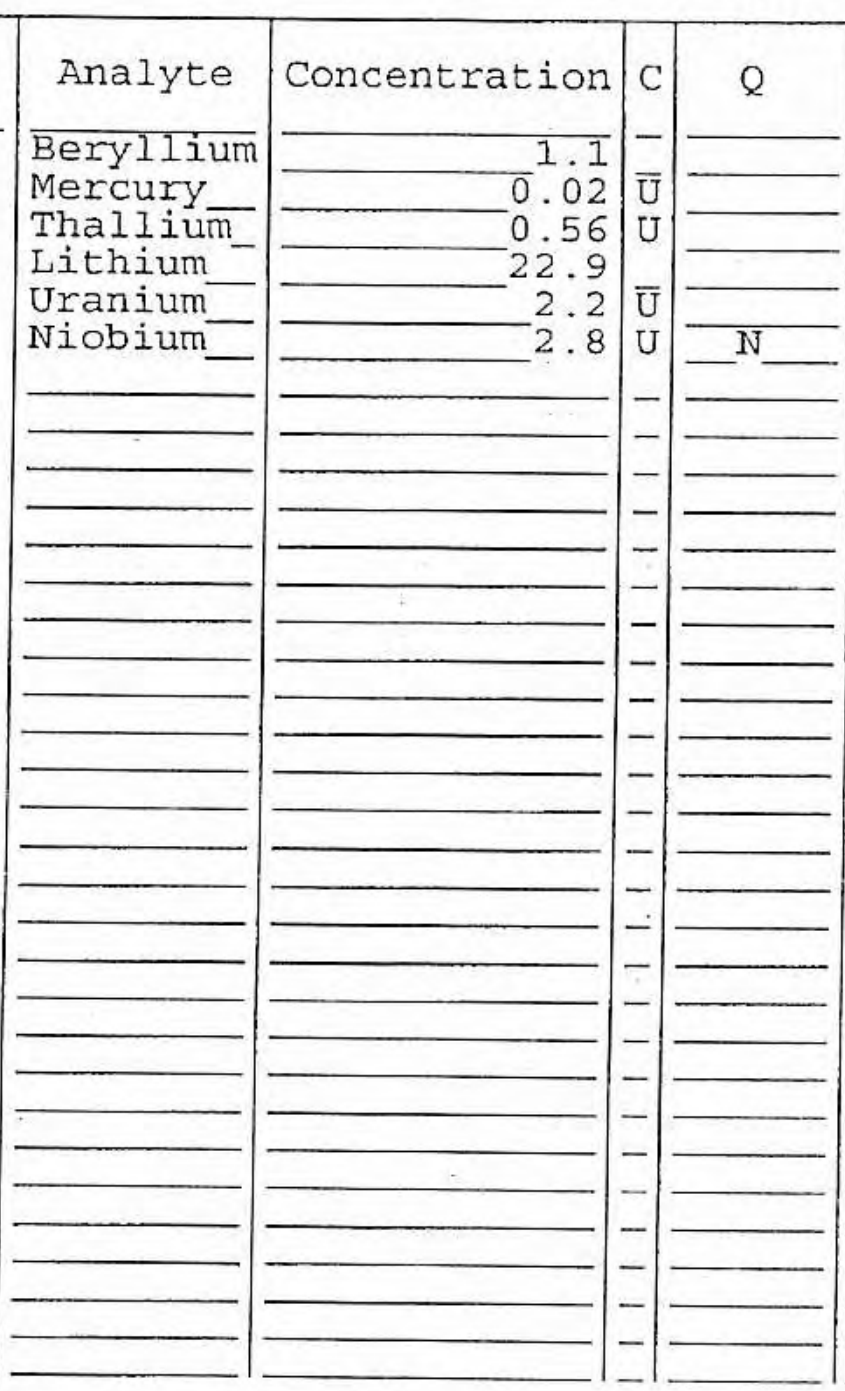

Clarity Before: Clarity After:

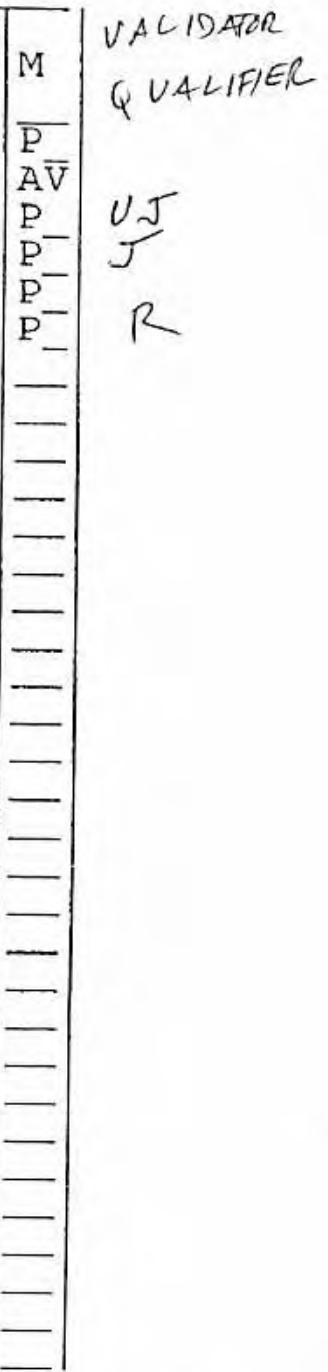

Texture: Artifacts: DUS - 23A 
EPA SAMPLE NO.

$$
23 \mathrm{~B}
$$

Lab Name: LIONVILLE LABORATORY

Lab Code: LVLI

Matrix (soil/water): SOIL_

Level. (low/med): LOW-

\% Solids: $\quad-92 \overline{1}$

Contract: 60052

SAS NO.:

SDG NO : V2742

Lab Sample ID: 0609L863-006

Date Received: 09/01/06

Concentration Units (ug/L or $\mathrm{mg} / \mathrm{kg}$ dry weight): MG/KG

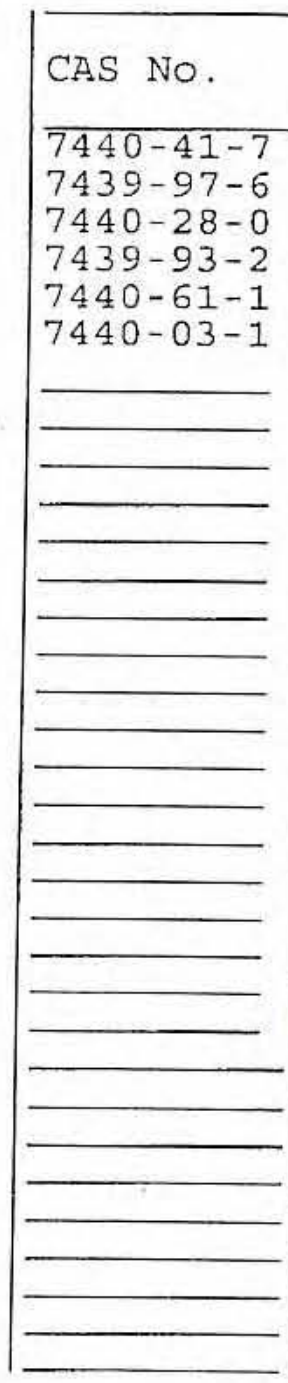

Color Before:

Color After:
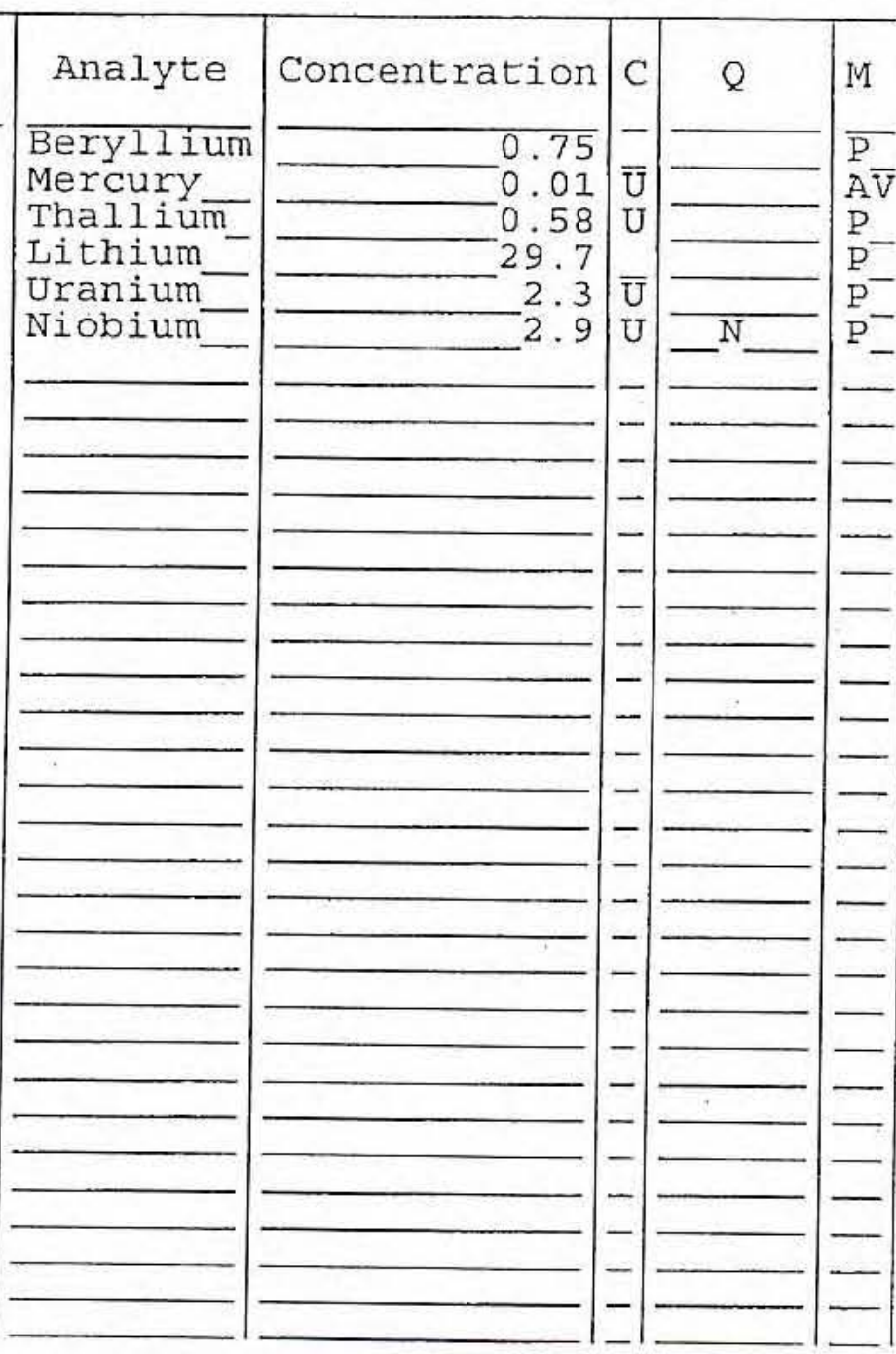

VALIDATRL QUAUFIER

A

UJ

J

$R$

Clarity Before:

Clarity After:
Texture:

Artifacts :

Comments :

DUS - $23 B$ 
U.S. EPA

1

INORGANIC ANALŸSES DATA SHEET
EPA SAMPLE NO.

$$
24 \mathrm{~A}
$$

Contract: 60052

SAS NO.:

SDG No.: V2742

Matrix (soil/water): SoIL

Level (low/med): LOW -

Lab Sample ID: 0609L863-̄007

Date Received: 09/01/06

Concentration Units (ug/L or $\mathrm{mg} / \mathrm{kg}$ dry weight): MG/KG
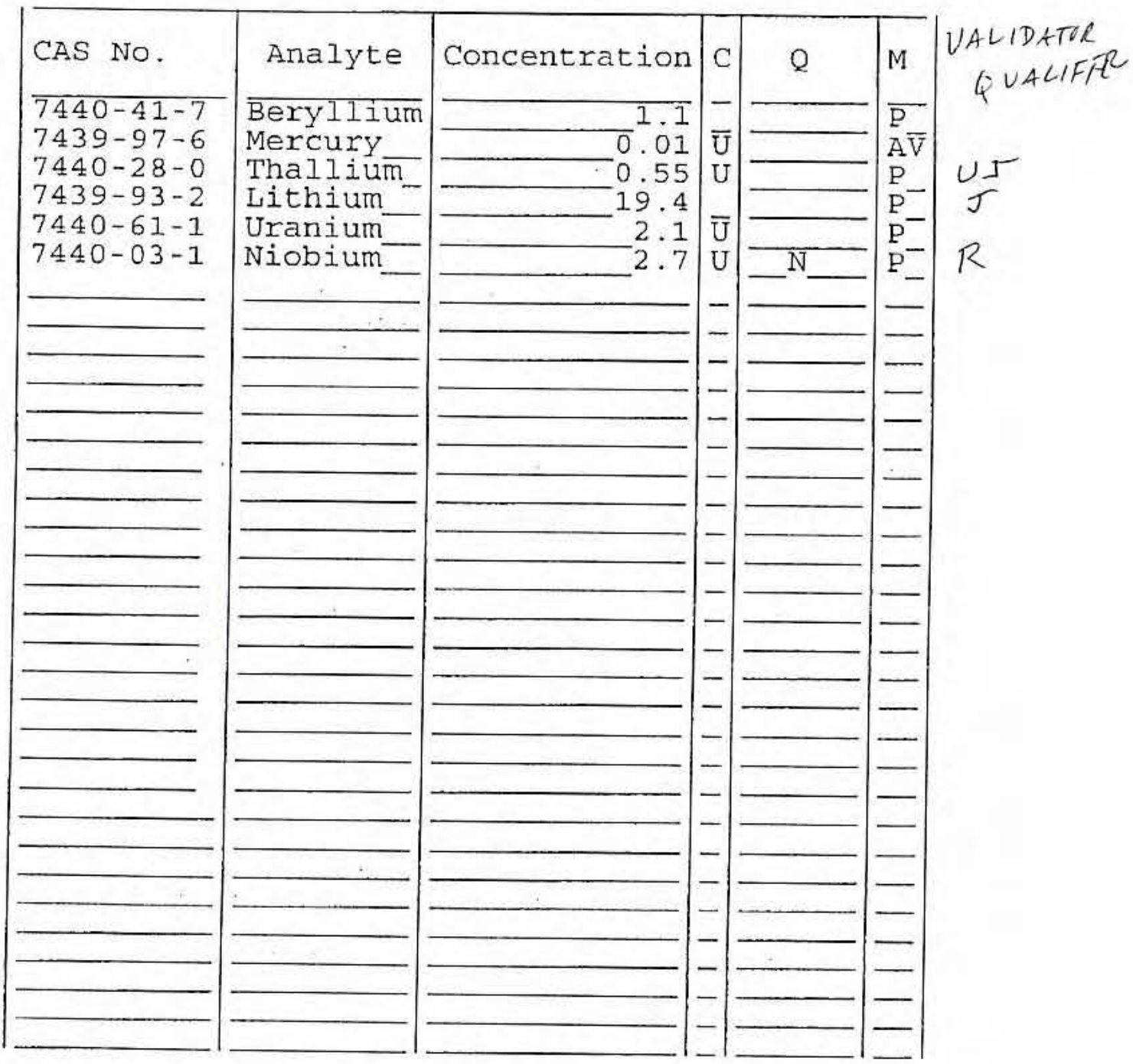

M UALIDATVR

\% Solids :

Color Before:

Color After:

Clarity Before:

Clarity After:

Texture:

Artifacts:

Comments :

DUS $-24 \mathrm{~A}$ 
Lab Name: LIONVILLE_LABORATORY

Lab Code: LVLI Matrix (soil/water): SOIL Level. (low/med): LOW Solids:
Contract: 60052 SAS NO. :
$24 B$

SDG NO.: V2742

uab Sample ID: 0609L863-008

Date Received: 09/01/06

Concentration Units (ug/L or $\mathrm{mg} / \mathrm{kg}$ dry weight): MG/KG

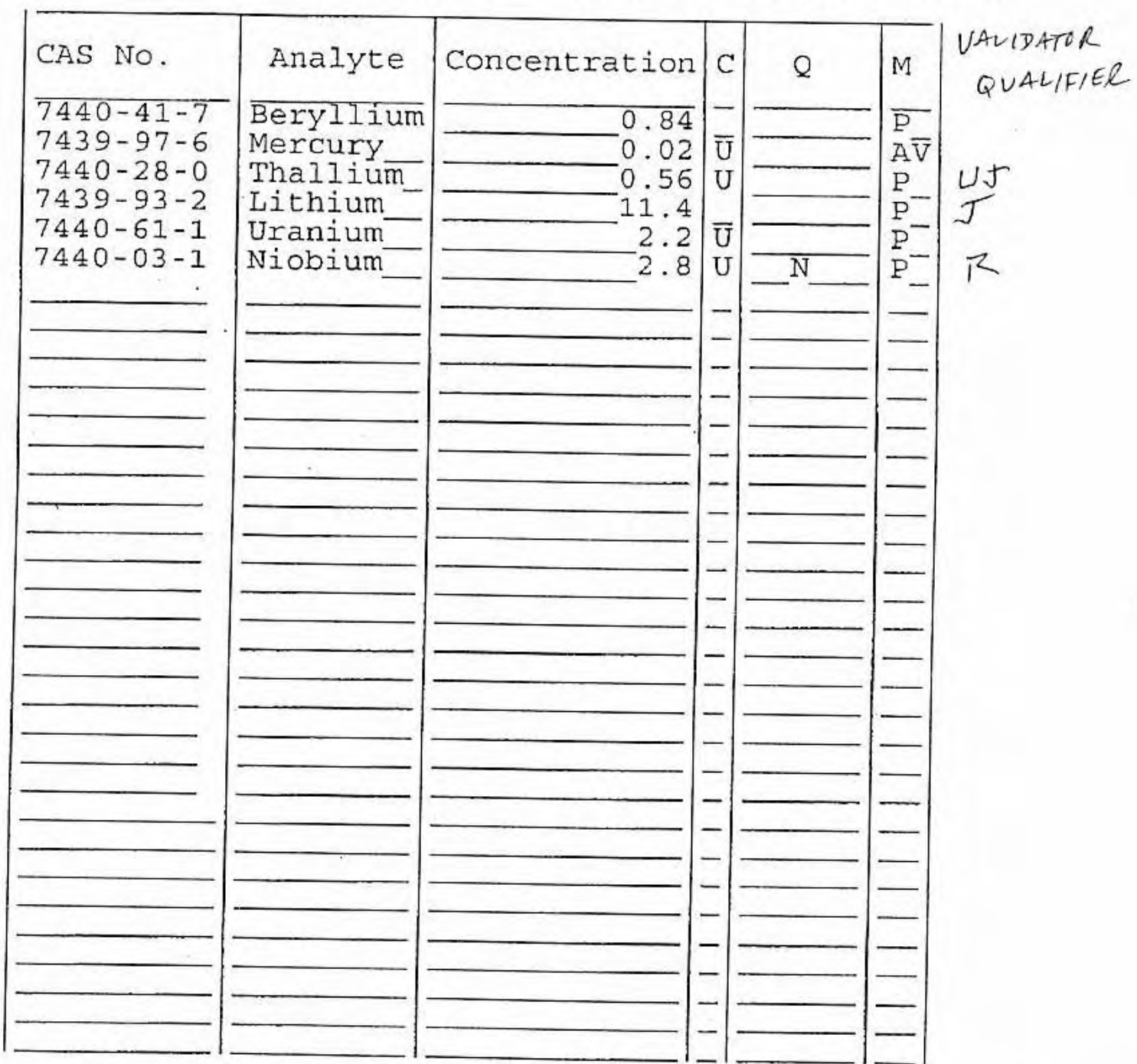

Color Before: Color After:

Comments:
Clarity Before: Clarity After:
Texture: Artifacts:

\section{DUS-24B}




\section{U.S. EPA}

1

INORGANIC ANALYSES DATA SHEET
EPA SAMPLE NO.

$$
25 \mathrm{~A}
$$

SDG NO.: V2742

SAS NO.:

Lab Sample ID: 0609L863-009

Date Received: 09/01/06

Level (low LOW
-94.8

Concentration Units (ug/L or $\mathrm{mg} / \mathrm{kg}$ dry weight): MG/KG

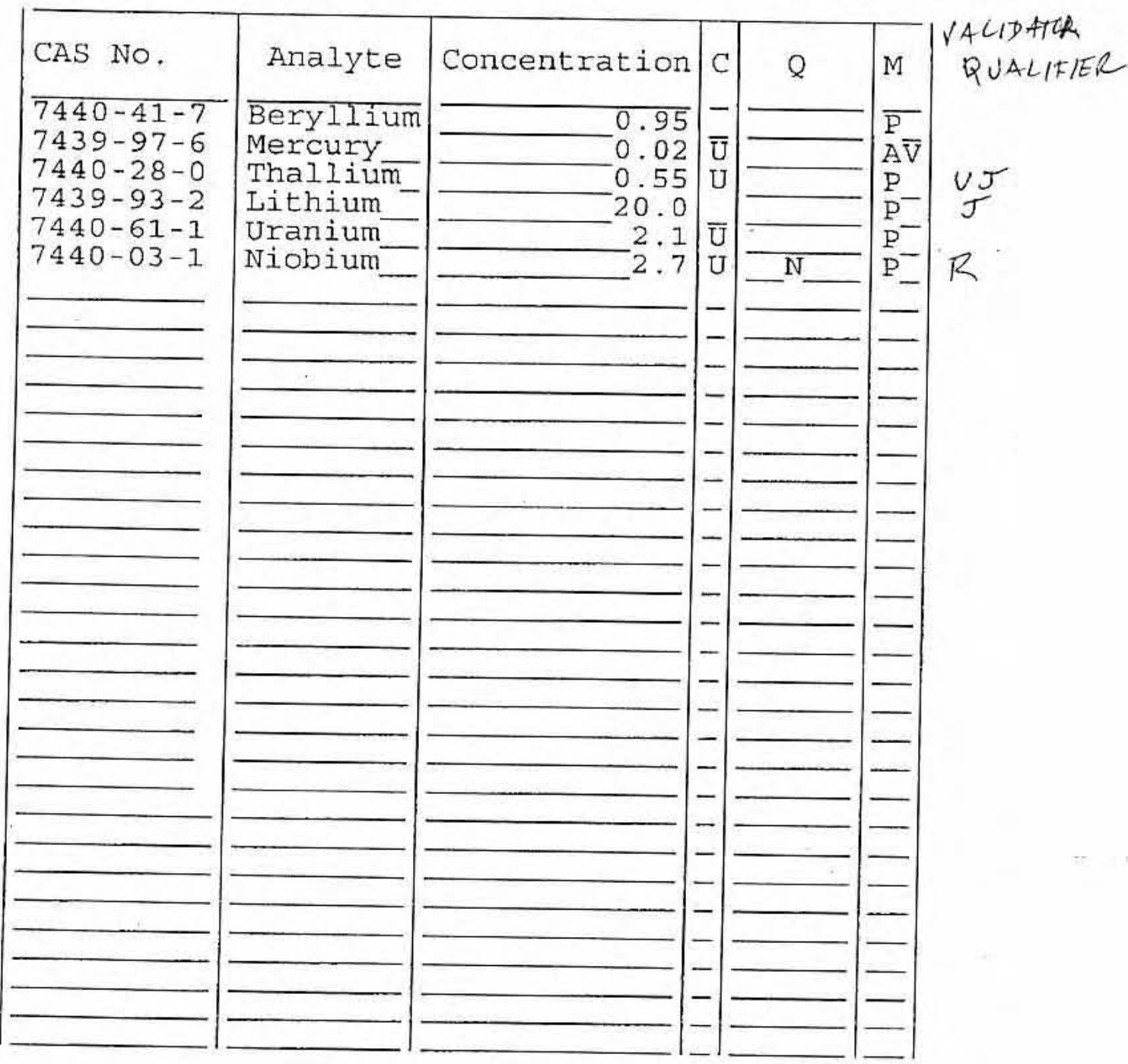

Color Before: Color After:

\section{Comments :}

DUS - 25A
Clarity Before:

Clarity After:
Texture: Artifacts: 
U.S. EPA

1

INORGANIC ANALYSES DATA SHEET
EPA SAMPLE NO.

$$
25 B
$$

Lab Name: LIONVILLE_LABORATORY

Lab Code: LVLI

Matrix (soil/water): SoIL

Level. (low/med) :

: Solids:
Contract: 60052

SAS NO.:

SDG NO.: V2742

Lab Sample ID: 0609L863-0̄10

Date Received: 09/01/06

Concentration Units (ug/L or $\mathrm{mg} / \mathrm{kg}$ dry weight): MG/KG

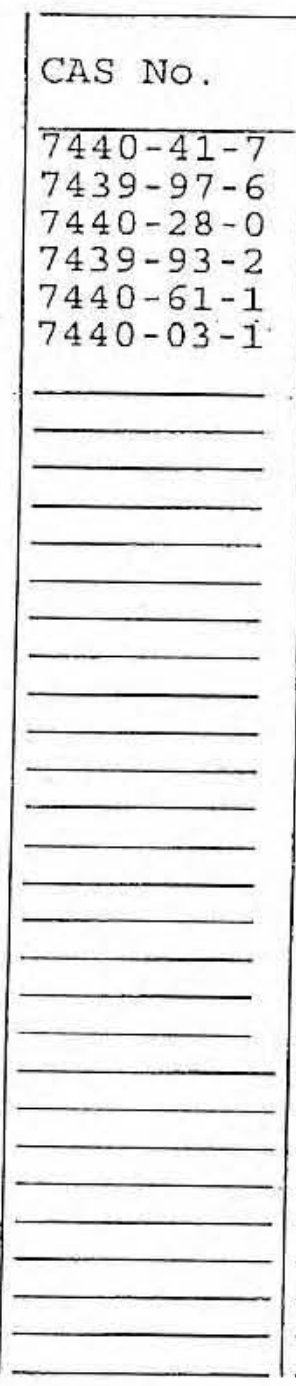

Color Before: Color After:

Comments :

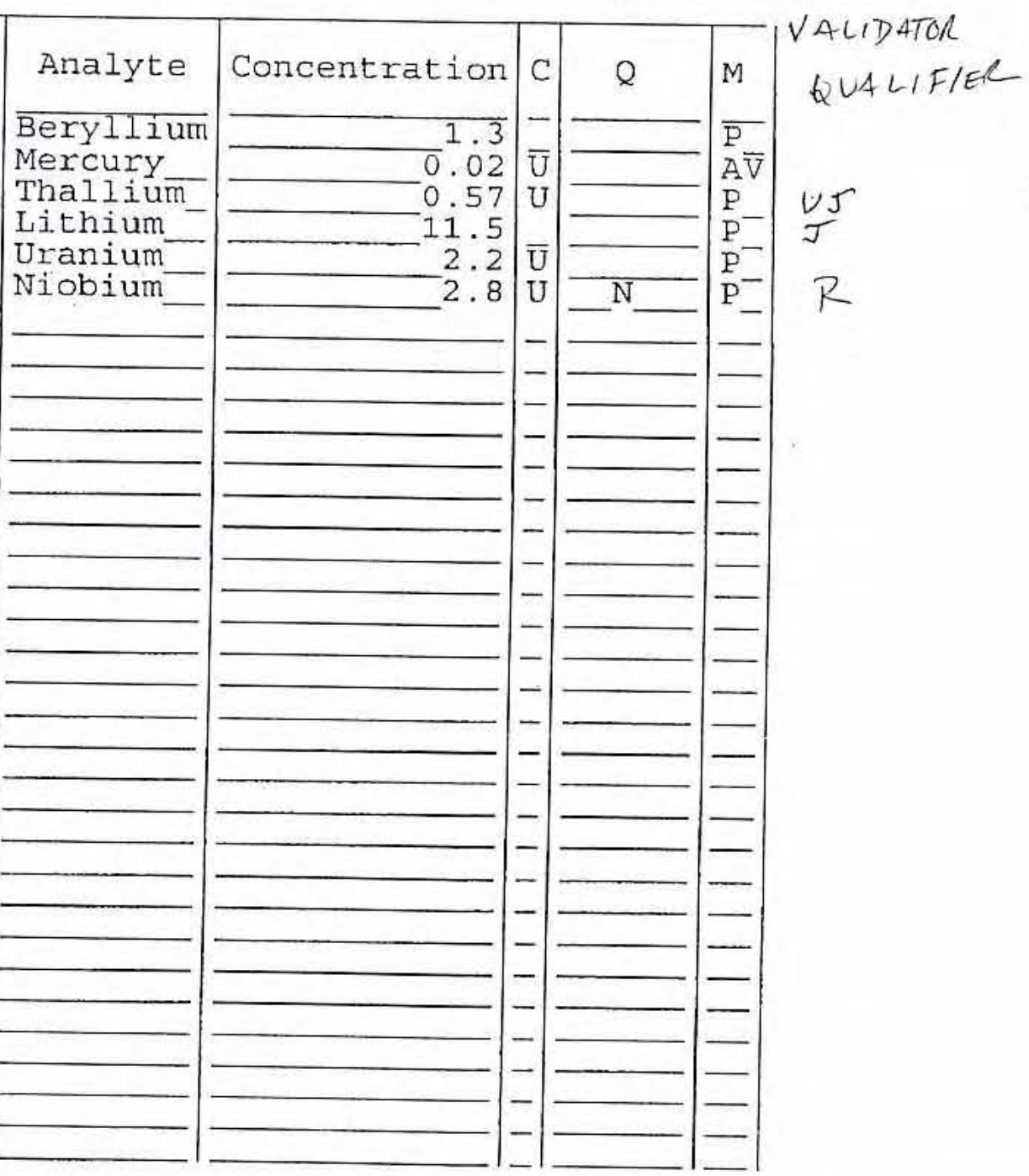

Clarity Before:

Clarity After:
Texture:

Artifacts:

DUS - 25B 


\section{U.S. EPA}

1

INORGANIC ANALYSES DATA SHEET
EPA SAMPLE NO.

$$
26 \mathrm{~A}
$$

Contract: 60052

SAS NO.:

SDG NO: V2742

Lab Sample ID: 0609L863-0̄11 Matrix (soil/water): SoIL

Level (low/med): LOW

Solids:

Date Received: 09/01/06

Concentration Units (ug/L or $\mathrm{mg} / \mathrm{kg}$ dry weight): MG/KG

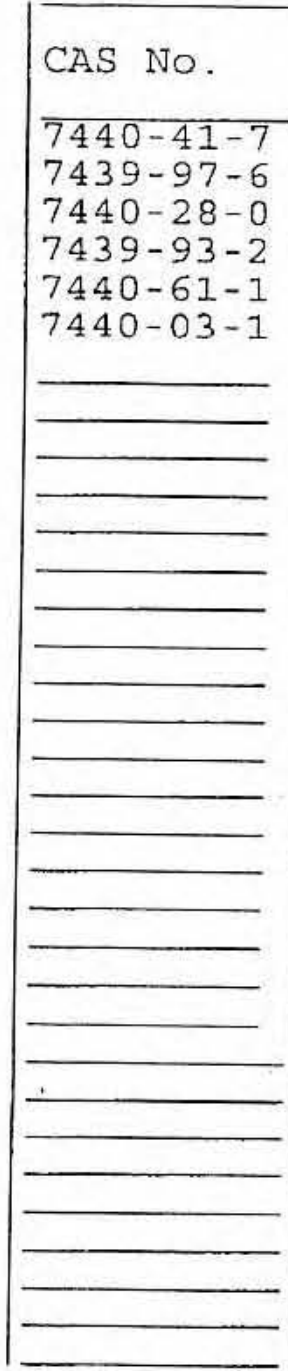

Color Before:

Color After:

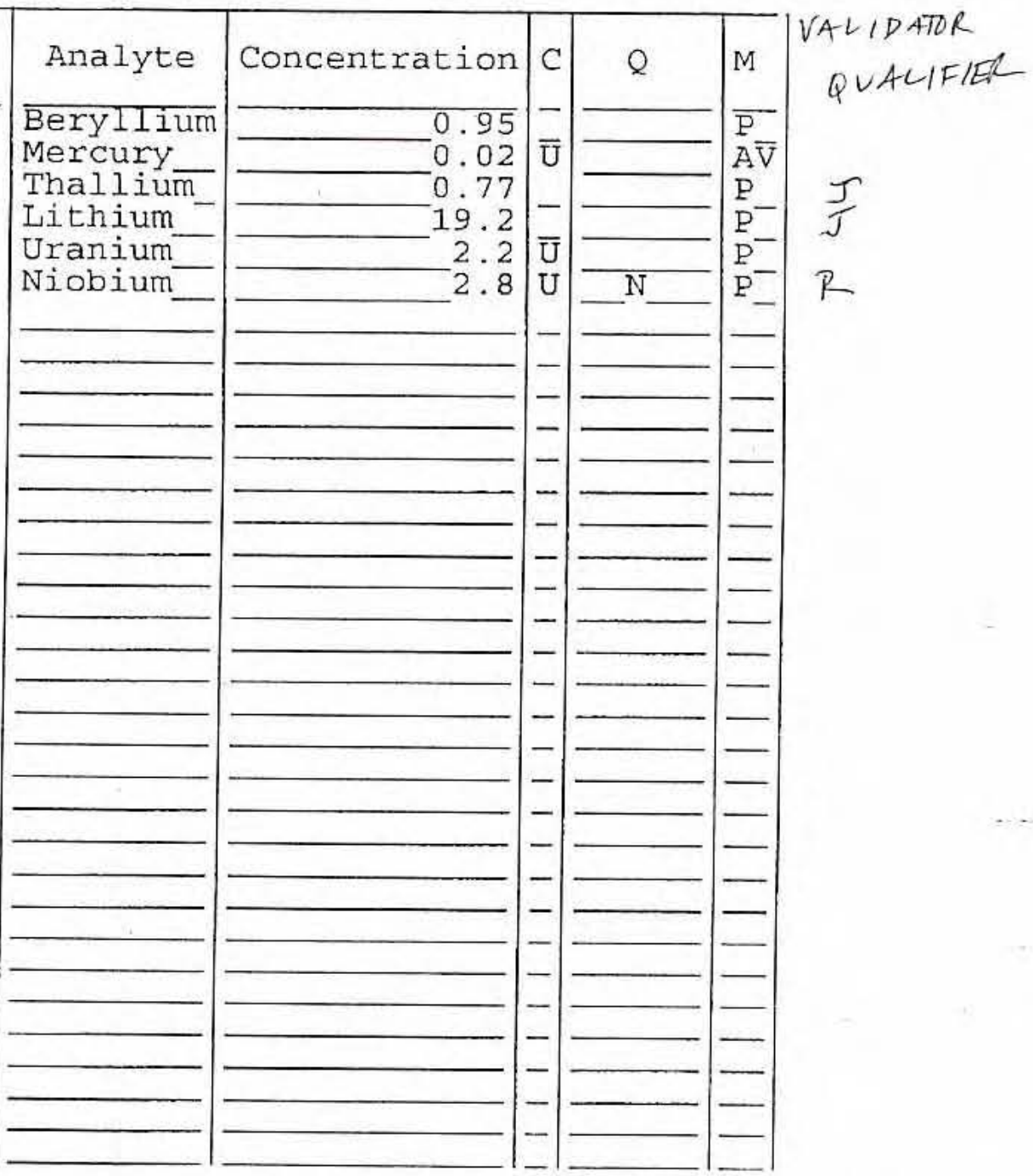

Clarity Before: Clarity After:
Texture: Artifacts:

\section{Comments :}

DUS - 26A 


\section{U.S. EPA}

Lab Name: LIONVILLE LABORATORY Lab Code: LVLI Matrix (soil/water): SoIL Level (low/med): LOW : Solids: $-93 \overline{.8}$
1

INORGANIC ANALYSES DATA SHEET
Contract: 60052 SAS No.:

SDG No.: V2742

Lab Sample ID: 0609L863-0̄12

Date Received: 09/01/06
EPA SAMPLE NO.

$26 B$

Concentration Units (ug/L or $\mathrm{mg} / \mathrm{kg}$ dry weight): MG/KG

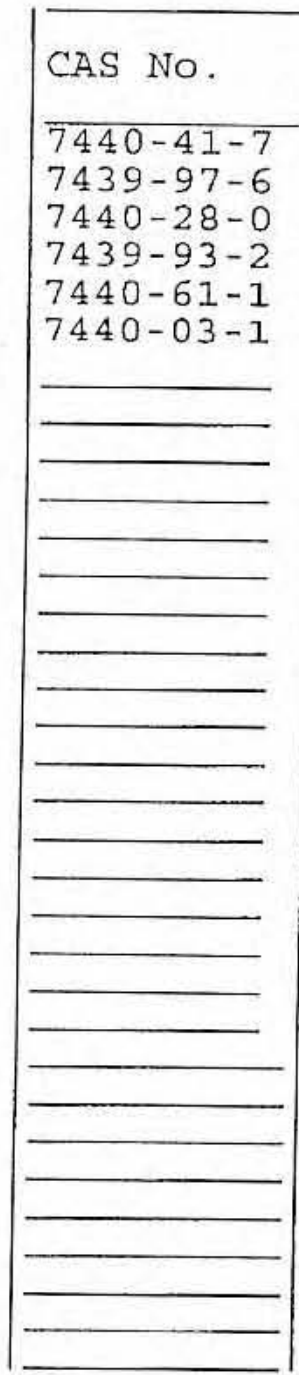

Color Before:

Color After:

\section{Comments:}

DUS $-26 \mathrm{~B}$

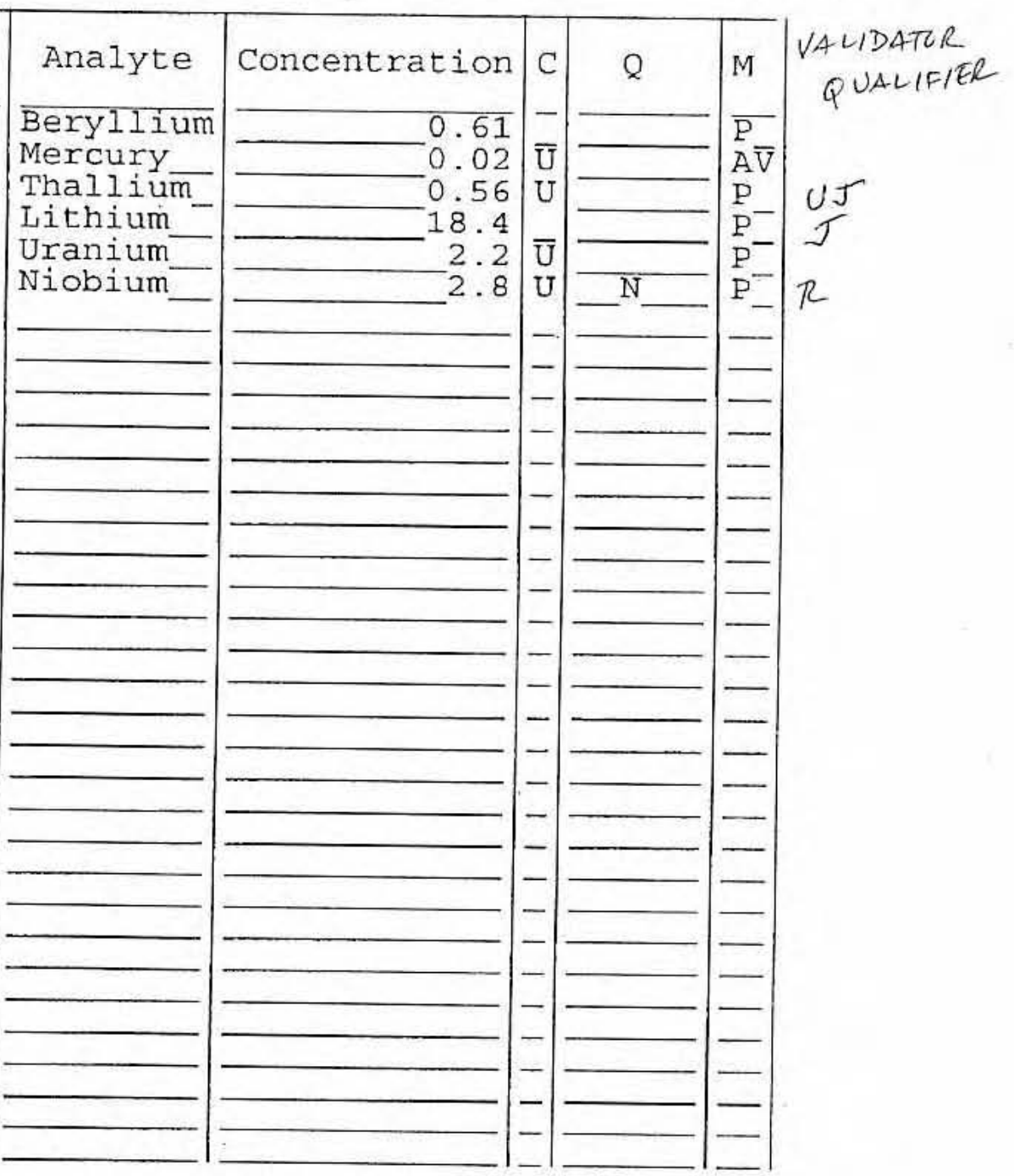

Clarity Before: Clarity After:
Texture: Artifacts: 


\section{U.S. EPA}

1
INORGANIC ANALYSES DATA SHEET

EPA SAMPLE NO.

$$
27 \mathrm{~A}
$$

Contract: 60052

SAS NO.:

SDG NO: V2742

Lab Code: LVLI Case No.: DUS

Matrix (soil/water): SOIL

Level (low/med): LOW

\% Solids: $\quad-95 \overline{.2}$

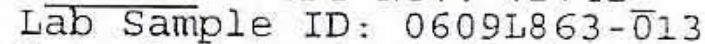

Date Received: 09/01/06

Concentration Units (ug/L or $\mathrm{mg} / \mathrm{kg}$ dry weight): MG/KG

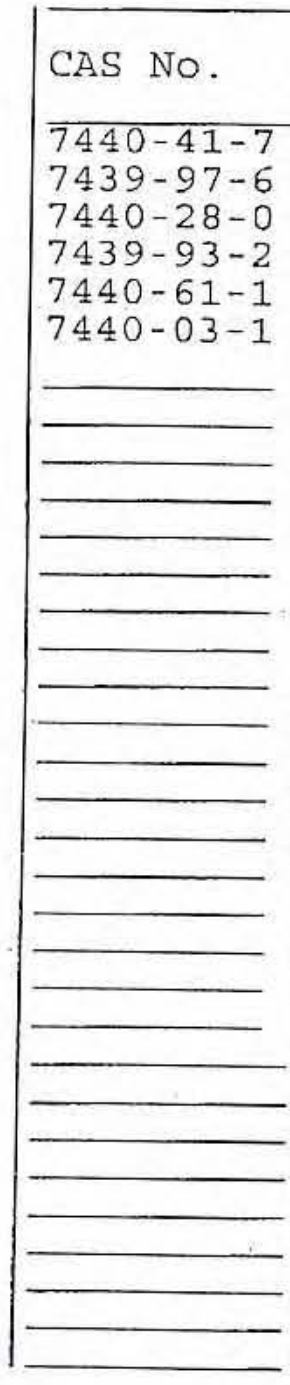

Color Before:

Color After:

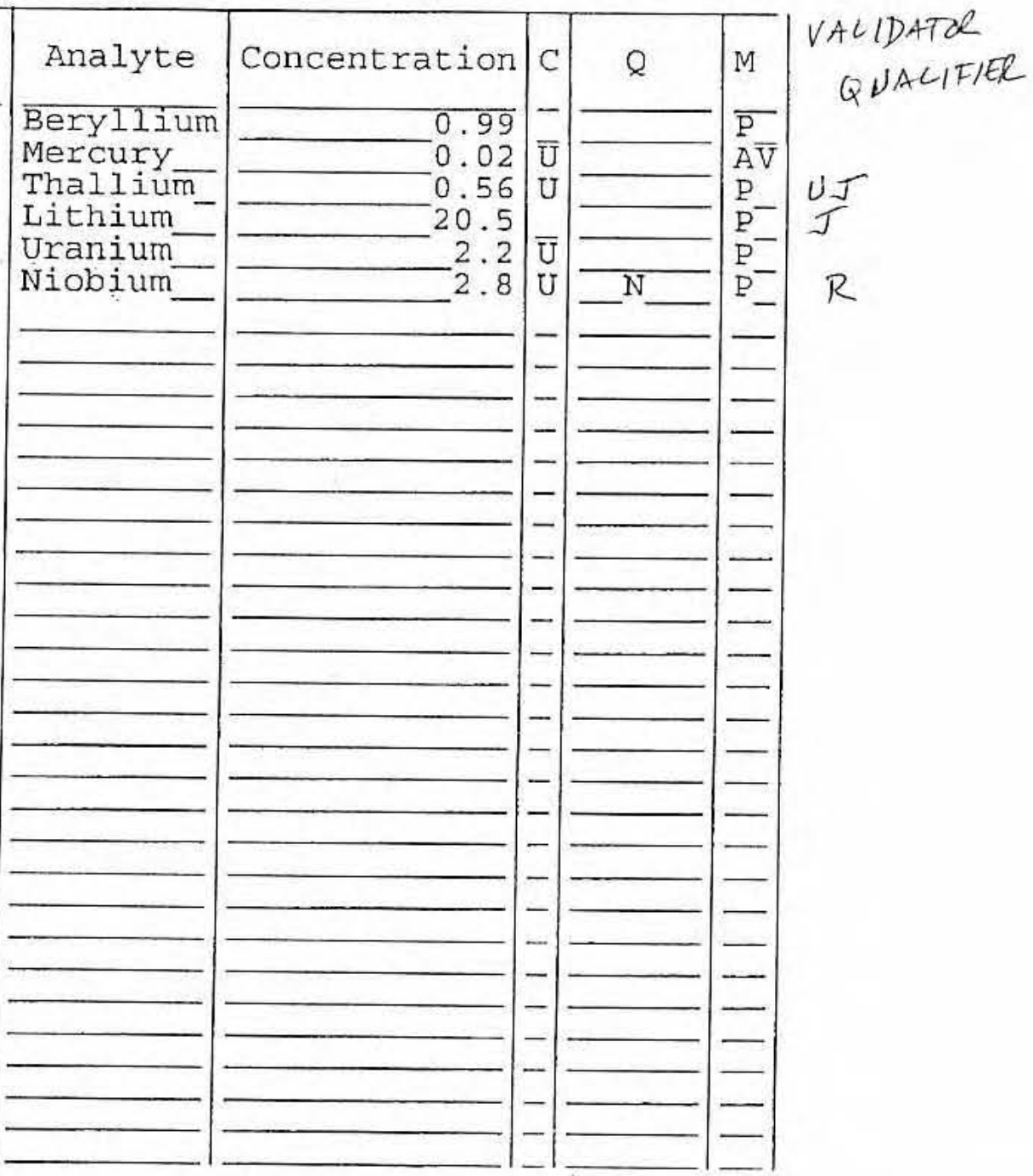

Clarity Before: Clarity After:
Texture: Artifacts:

\section{Comments:}

DUS-27A 
U.S. EPA

1

INORGANIC ANALYSES DATA SHEET
EPA SAMPLE NO.

$27 \mathrm{~B}$

Contract: 60052

SAS NO.:

SDG NO.: V2742

Lab Sample ID: 0609L863-0̄14

Date Received: 09/01/06

Level (low/med): $\quad$ LOW

$\div$ Solids :

$-92.0$

Concentration Units (ug/L or $\mathrm{mg} / \mathrm{kg}$ dry weight): MG/KG

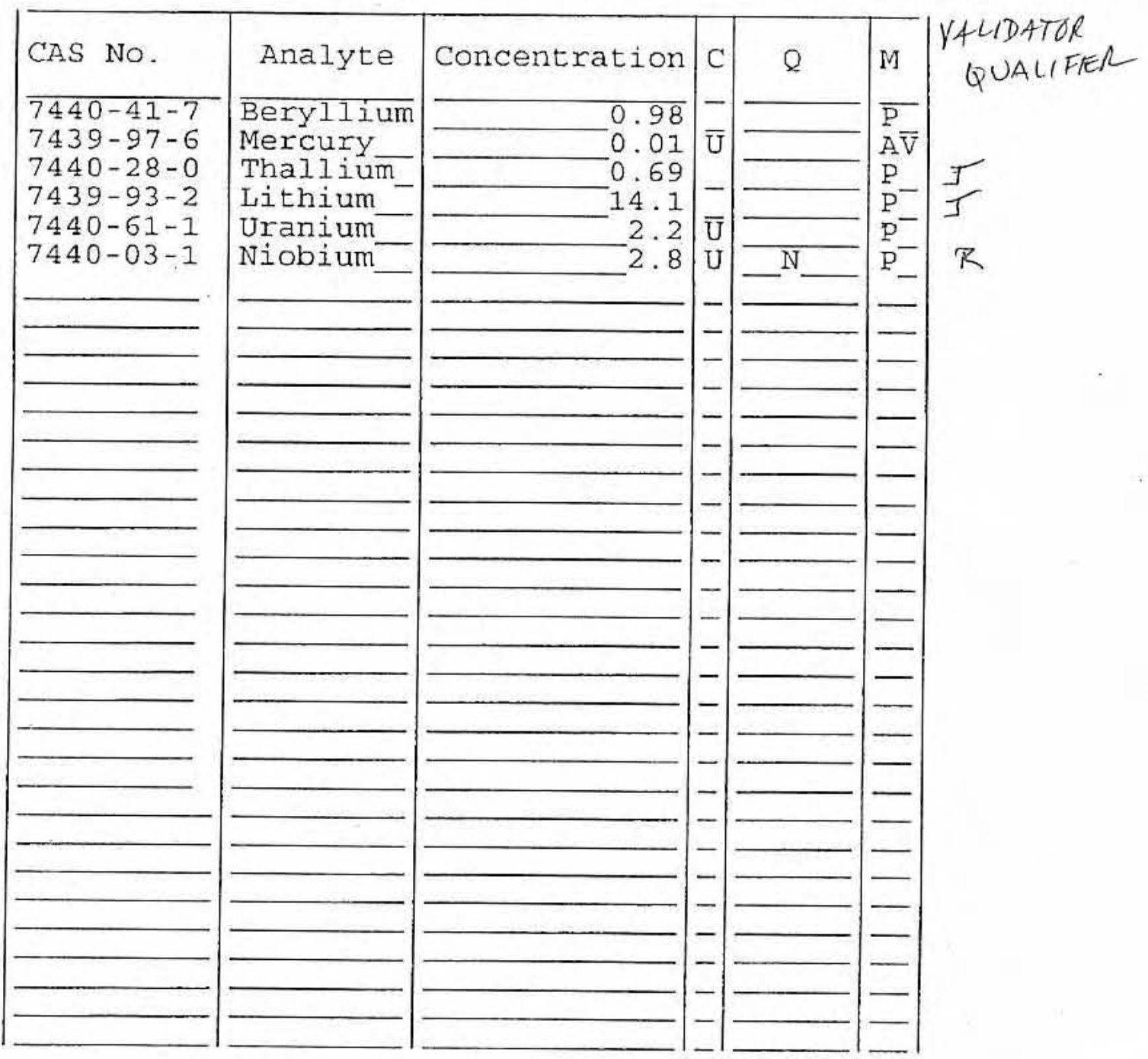

Color Before:

Color After:
Clarity Before:

Clarity After:
Texture:

Artifacts:

Comments:

DUS $-27 B$ 


\section{U.S. EPA}

1 INORGANIC ANALYSES DATA SHEET
EPA SAMPLE NO.

$$
28 \mathrm{~A}
$$

Lab Name: LIONVILLE LABORATORY

Lab Code: LVLI

Case No.: DUS

Matrix (soil/water): SOIL

Level (low/med): LOW

\% Solids:
Contract: 60052

SAS NO.:

SDG No.: V2742

Date Received: 09/01/06

Concentration Units (ug/L or $\mathrm{mg} / \mathrm{kg}$ dry weight): $\mathrm{MG} / \mathrm{KG}$

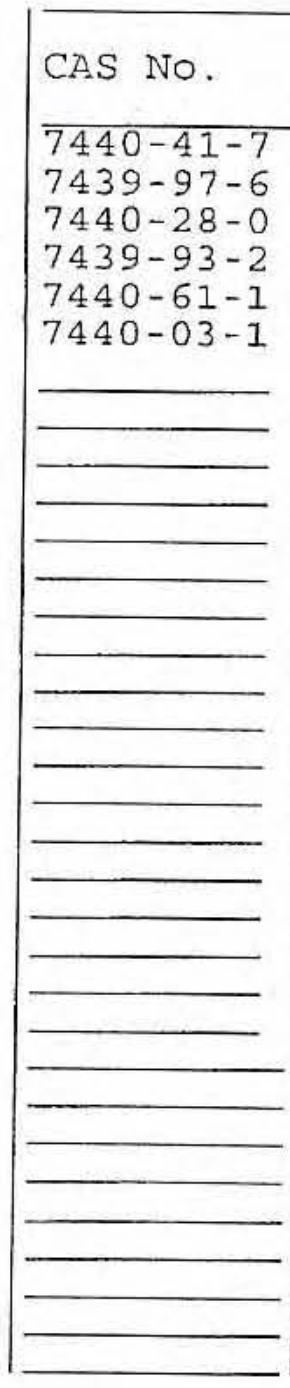

Color Before:

Color After:

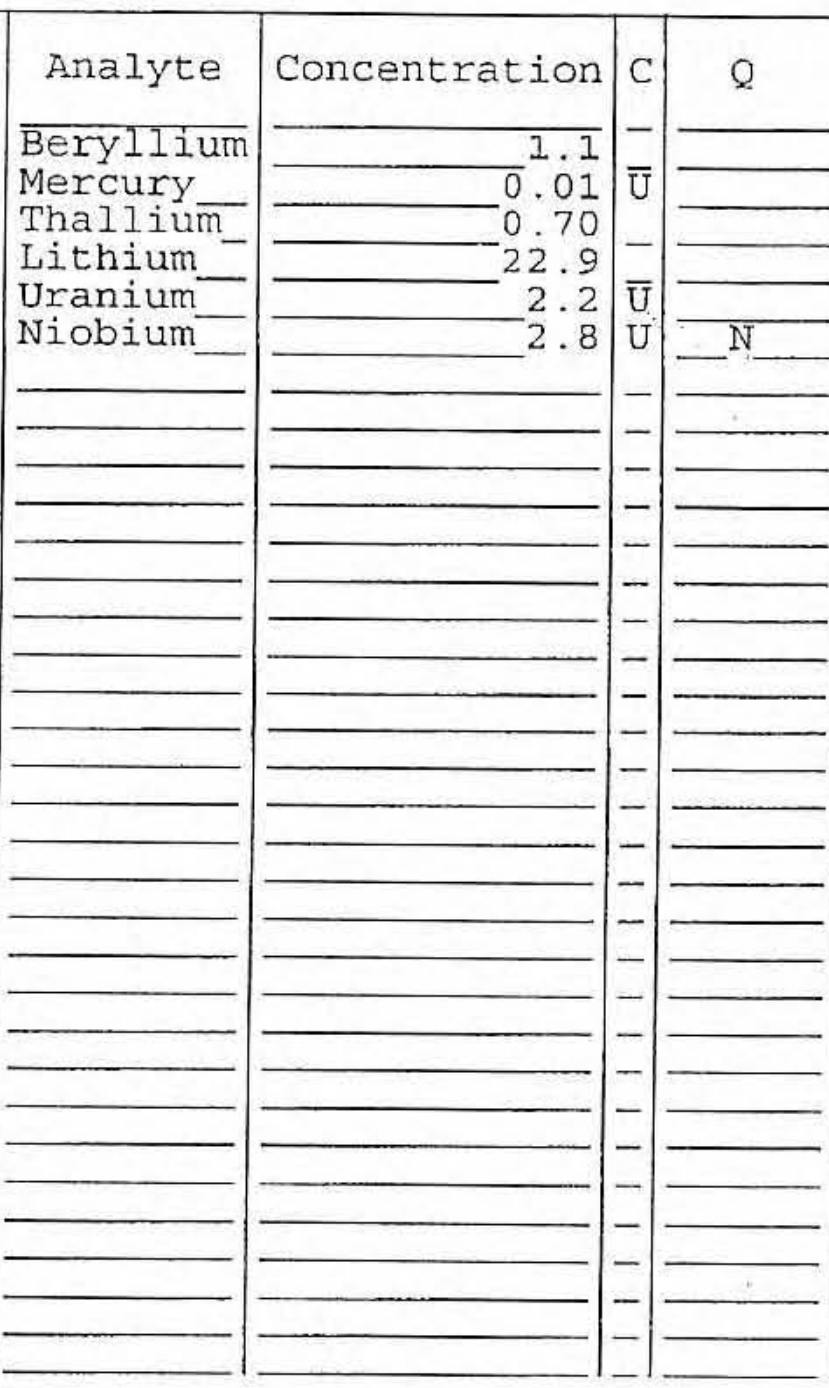

Clarity Before: Clarity After:

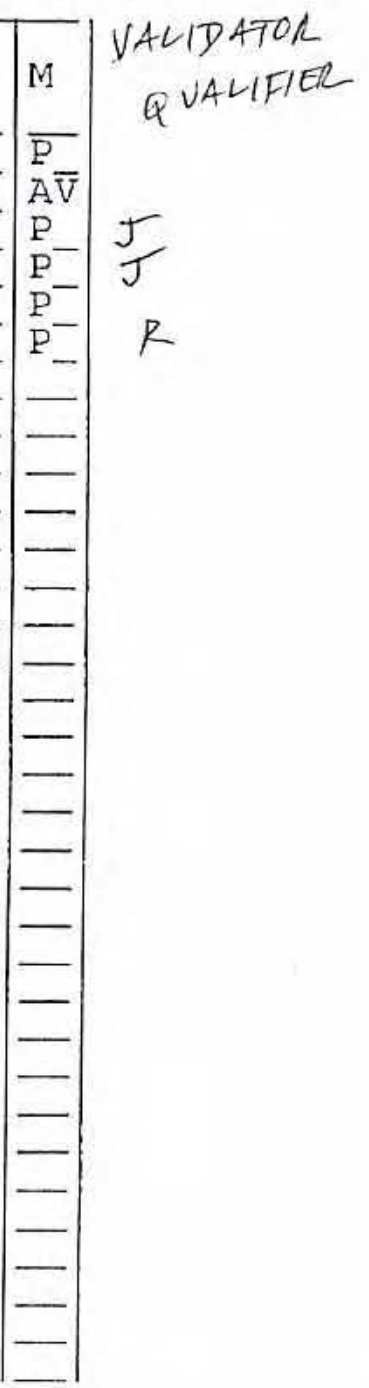

Texture: Artifacts:

Comments :

DUS - 28A 
1

INORGANIC ANALYSES DATA SHEET
EPA SAMPLE NO.

$$
28 \mathrm{~B}
$$

Contract: 60052

SAS NO.:

DDG NO : V2742

Lab Sample ID: 0609L863-0̄16

Date Received: 09/01/06
Matrix (soil/water): SoIL
Level (low/med):

\% Solids:

Concentration Units (ug/L or $\mathrm{mg} / \mathrm{kg}$ dry weight) : MG/KG

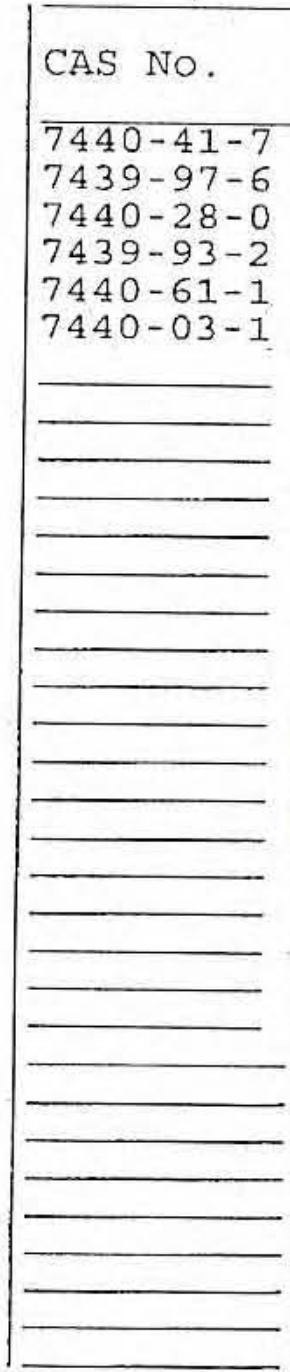

Color Before:

Color After:

\section{Comments:}

DUS $-28 B$

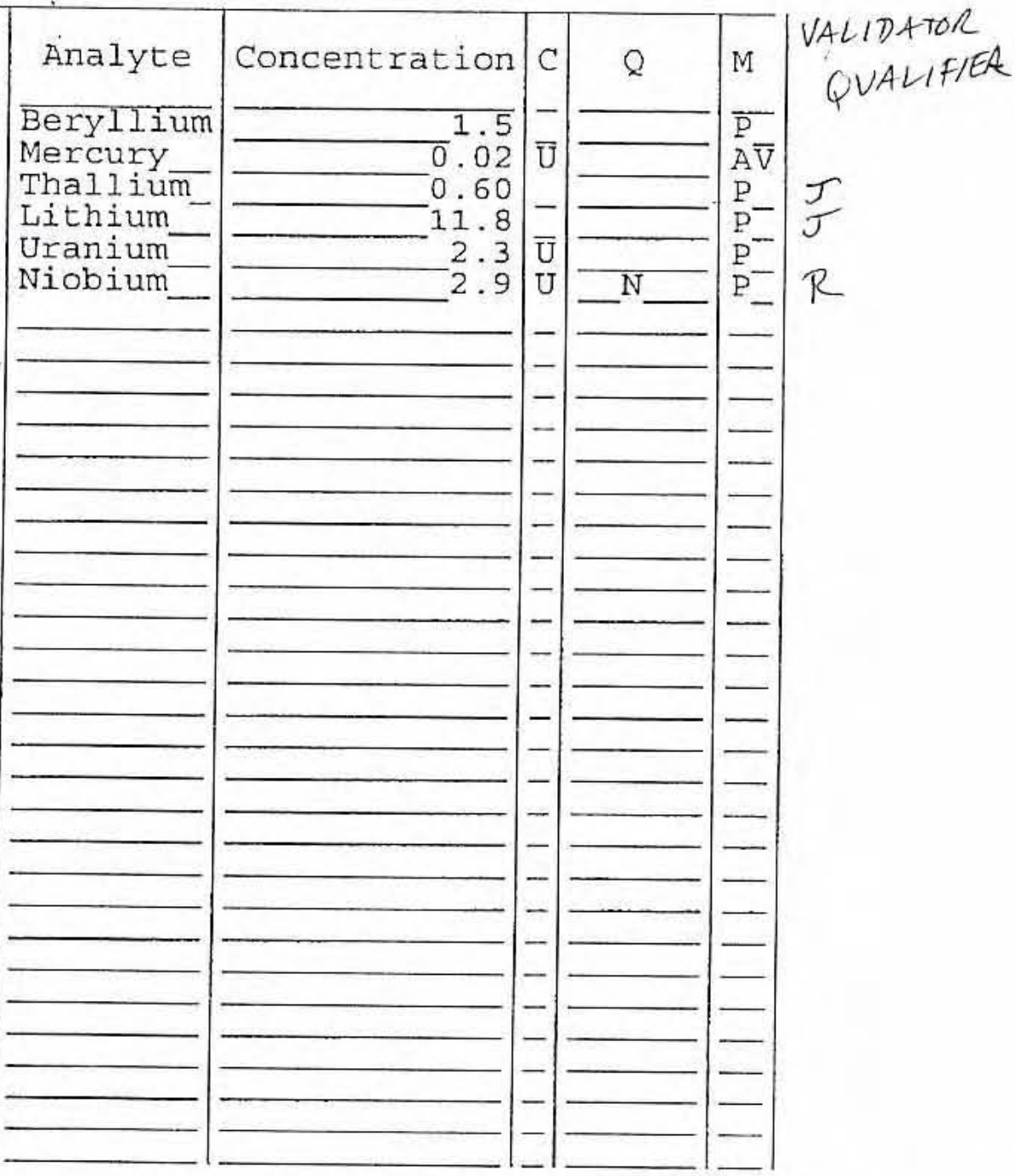

Clarity Before: Clarity After:
Texture: Artifacts: 


\section{U.S, EPA}

1

INORGANIC ANALYSES DATA SHEET
EPA SAMPLE NO.

$29 \mathrm{~A}$

Contract: 60052 SAS NO,:

SDG NO.: V2742

Lab Sample ID: 0609L863-0̄17

Date Received: 09/01/06
Matrix (soil/water): SOI
Level (low/med):

$\div$ Solids:

$$
\text { LOW - }
$$

Concentration Units (ug/L or $\mathrm{mg} / \mathrm{kg}$ dry weight): MG/KG

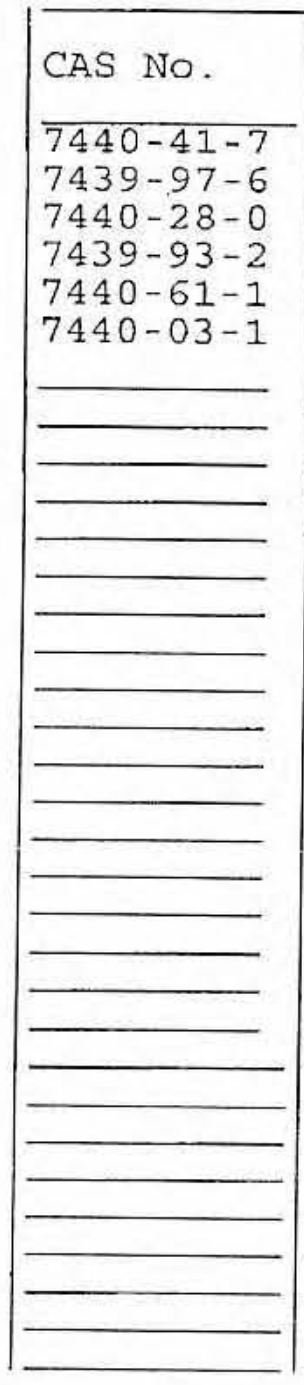

Color Before: Color After:

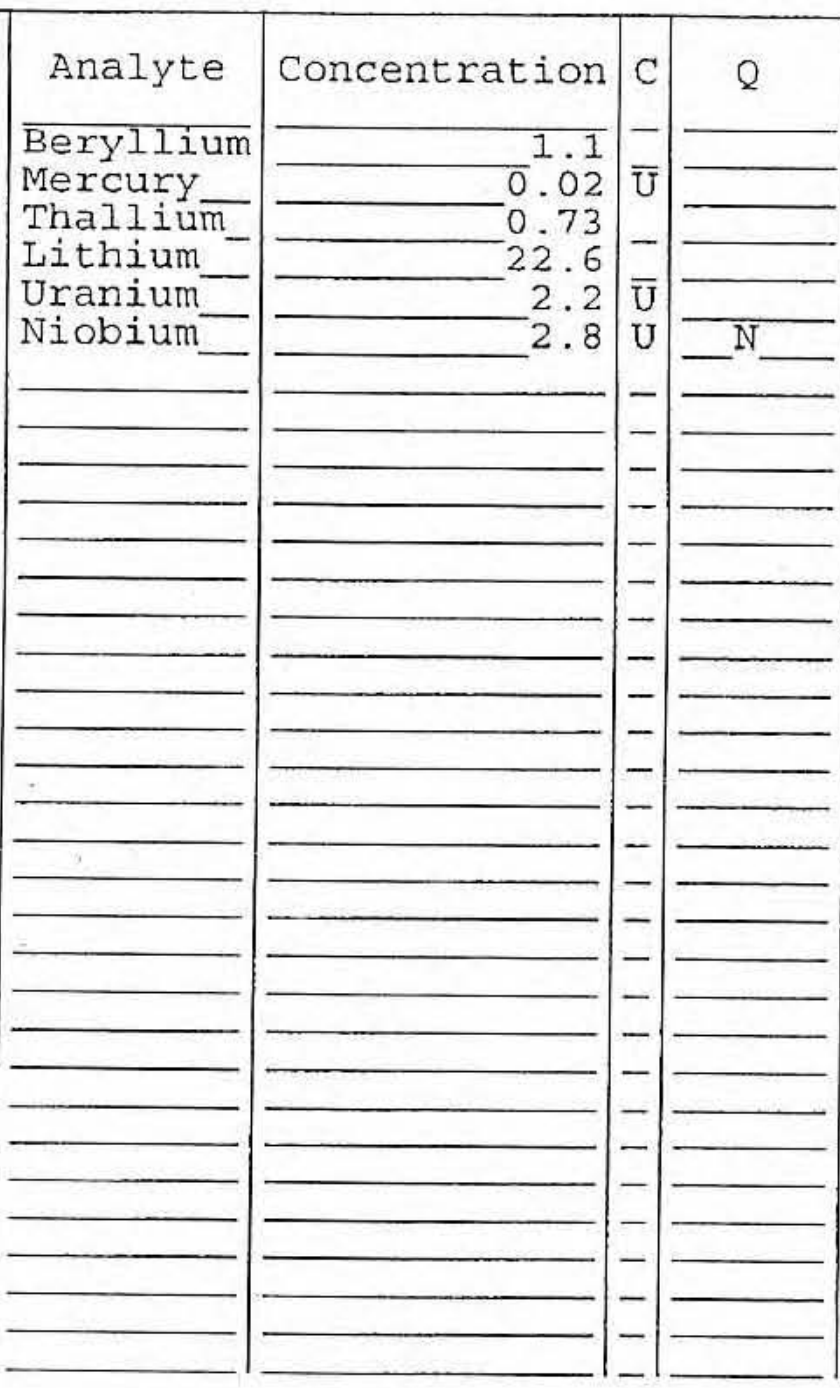

Clarity Before: Clarity After:

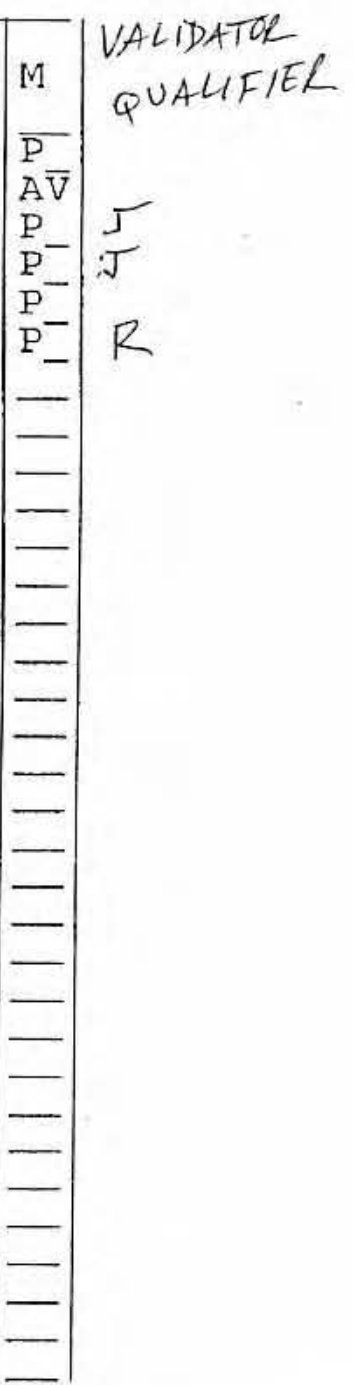

Texture: Artifacts:

Comments:

DUS - 29A 
U.S. EPA

1

INORGANIC ANALYSES DATA SHEET
EPA SAMPLE NO.

$$
29 B
$$

Lab Name: LIONVILLE_LABORATORY

Contract: 60052

Lab Code: LVLI

Matrix (soil/water): SOIL

Level (low/med):

\% Solids:
Case No.: DUS

SAS NO. :

SDG NO.: V2742

Lab Sample ID: 0609L863-0̄18 LOW

$-93 . \overline{4}$

Date Received: 09/01/06

Concentration Units. (ug/L or $\mathrm{mg} / \mathrm{kg}$ dry weight): MG/KG

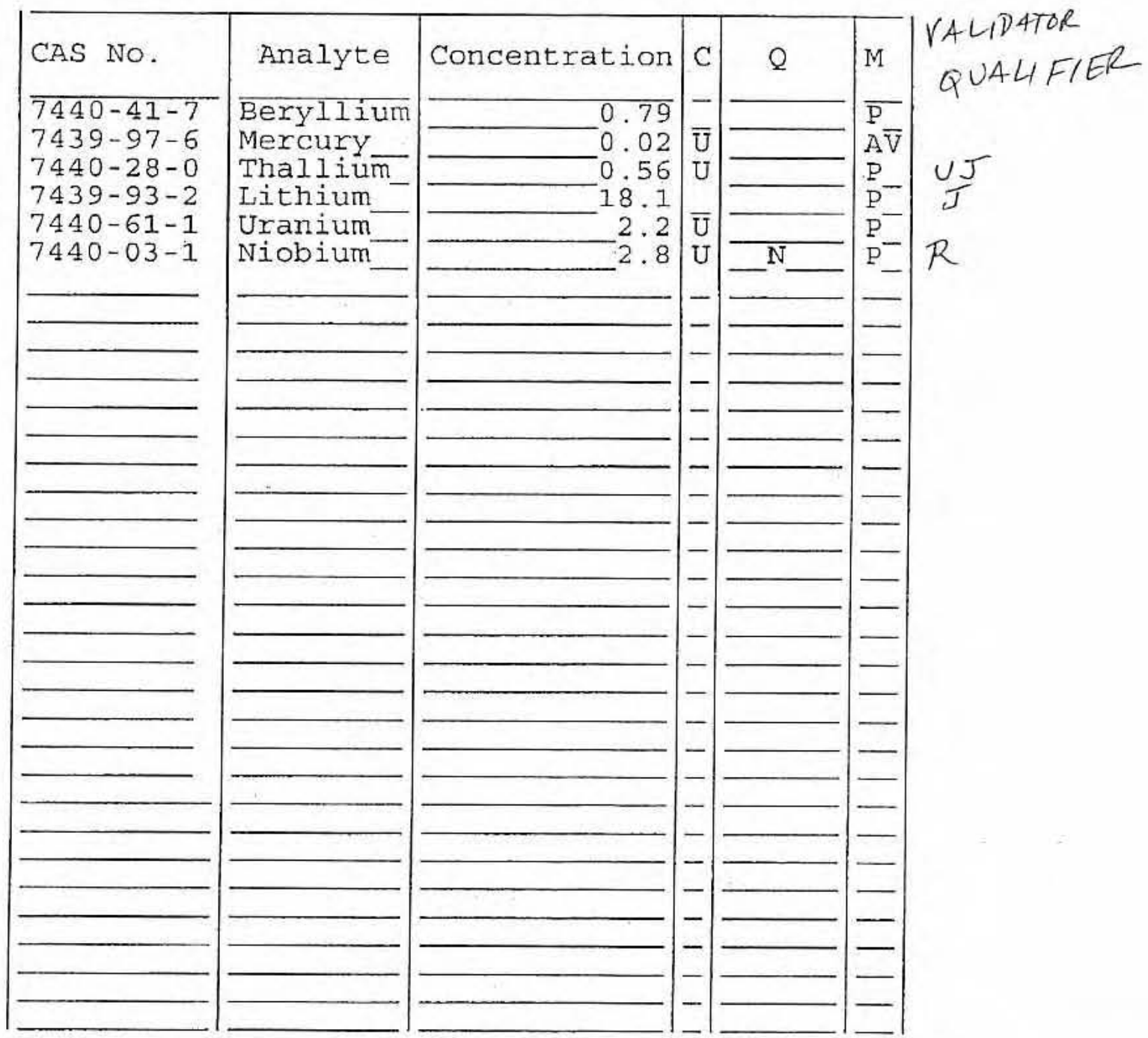

Color Before:

Color After:

Comments :
Clarity Before:

Clarity After:
Texture:

Artifacts: 
1

\section{INORGANIC ANALYSES DATA SHEET}

Lab Name: LIONVILLE LABORATORY

Lab Code: LVLI Materilsater): SOIL Level (low/med): \% Solids:
Contract: 60052 SAS No. :

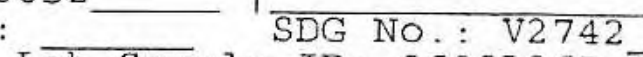

Lab Sample ID: 0609L863-0̄19 LOW
-96.0
EPA SAMPLE NO.

$30 \mathrm{~A}$

Date Received: 09/01/06

Concentration Units (ug/L or $\mathrm{mg} / \mathrm{kg}$ dry weight): MG/KG

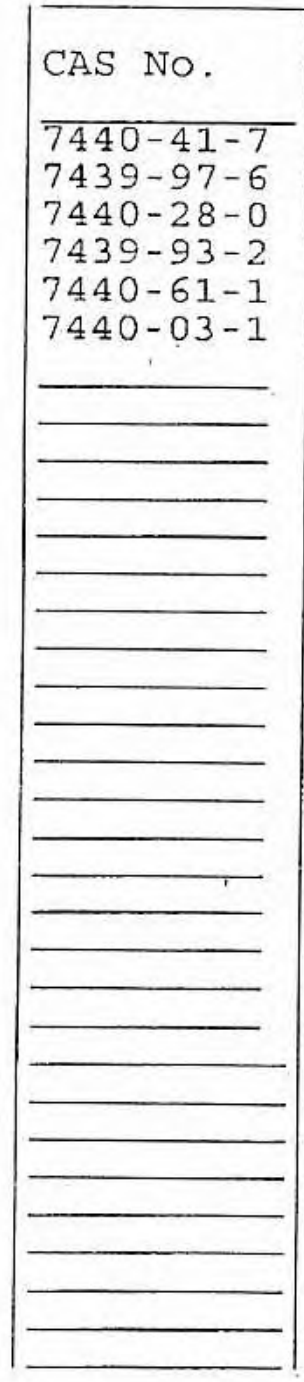

Color Before: Color After:

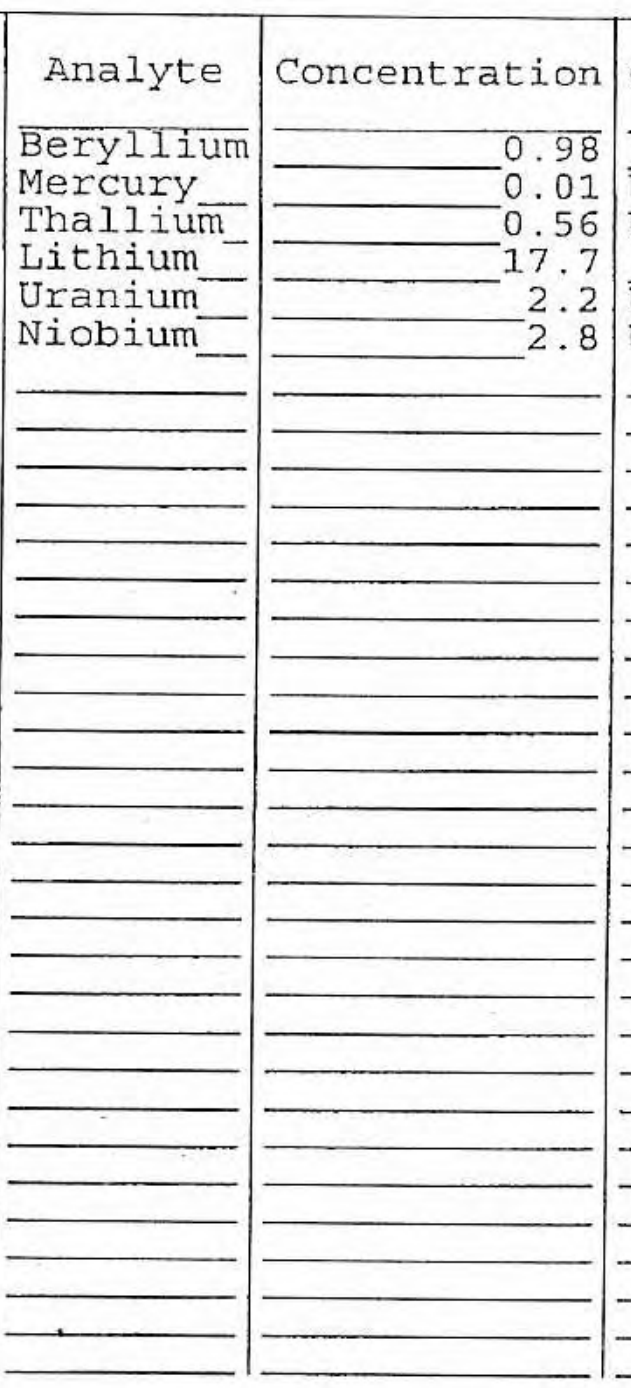

Clarity Before: Clarity After:

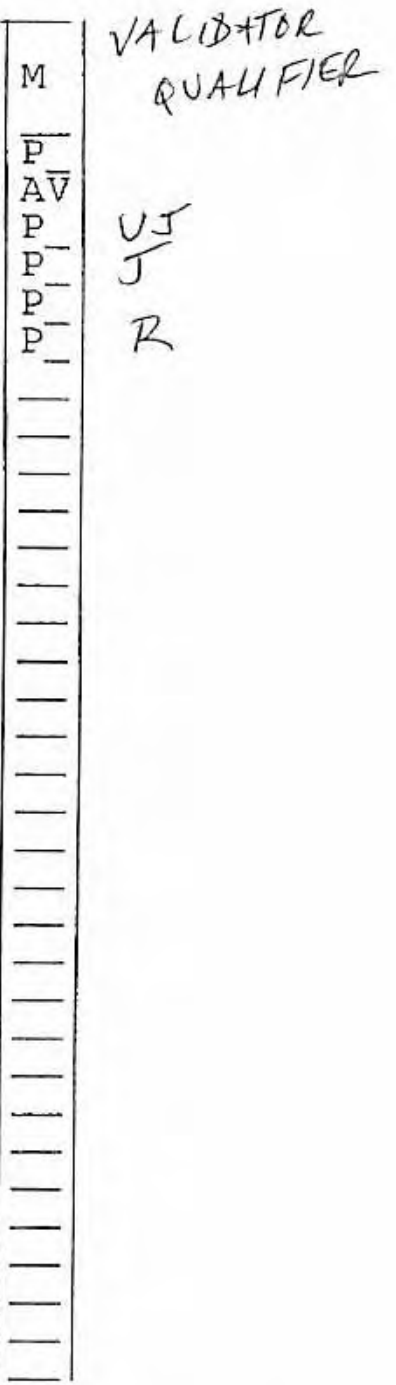

Texture: Artifacts:

Comments:

DUS - 3OA 
U.S. EPA

INORGANIC ANALYSES DATA SHEET

EPA SAIMPLE NO.

Lab Name: LIONVILLE LABORATORY $30 \mathrm{~B}$

Lab Code: LVLI

Contract: 60052

Case No.: DUS

Matrix (soil/water): SOIL

Level (low/med): LOW

Solids:

SAS NO.: - $95 \overline{.2}$

SDG NO: V2742

Lab Sample ID: 0609L863-020

Date Received: 09/01/06

Concentration Units (ug/L or $\mathrm{mg} / \mathrm{kg}$ dry weight): MG/KG

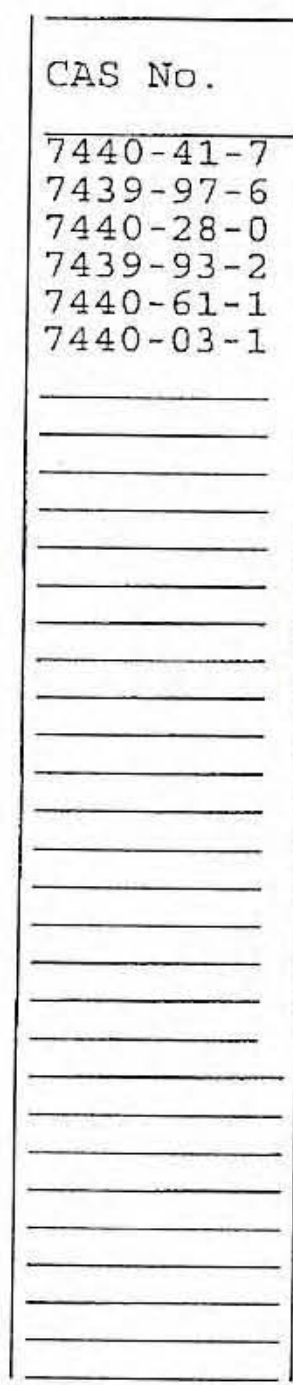

Color Before:

Color After:

\section{Comments :}

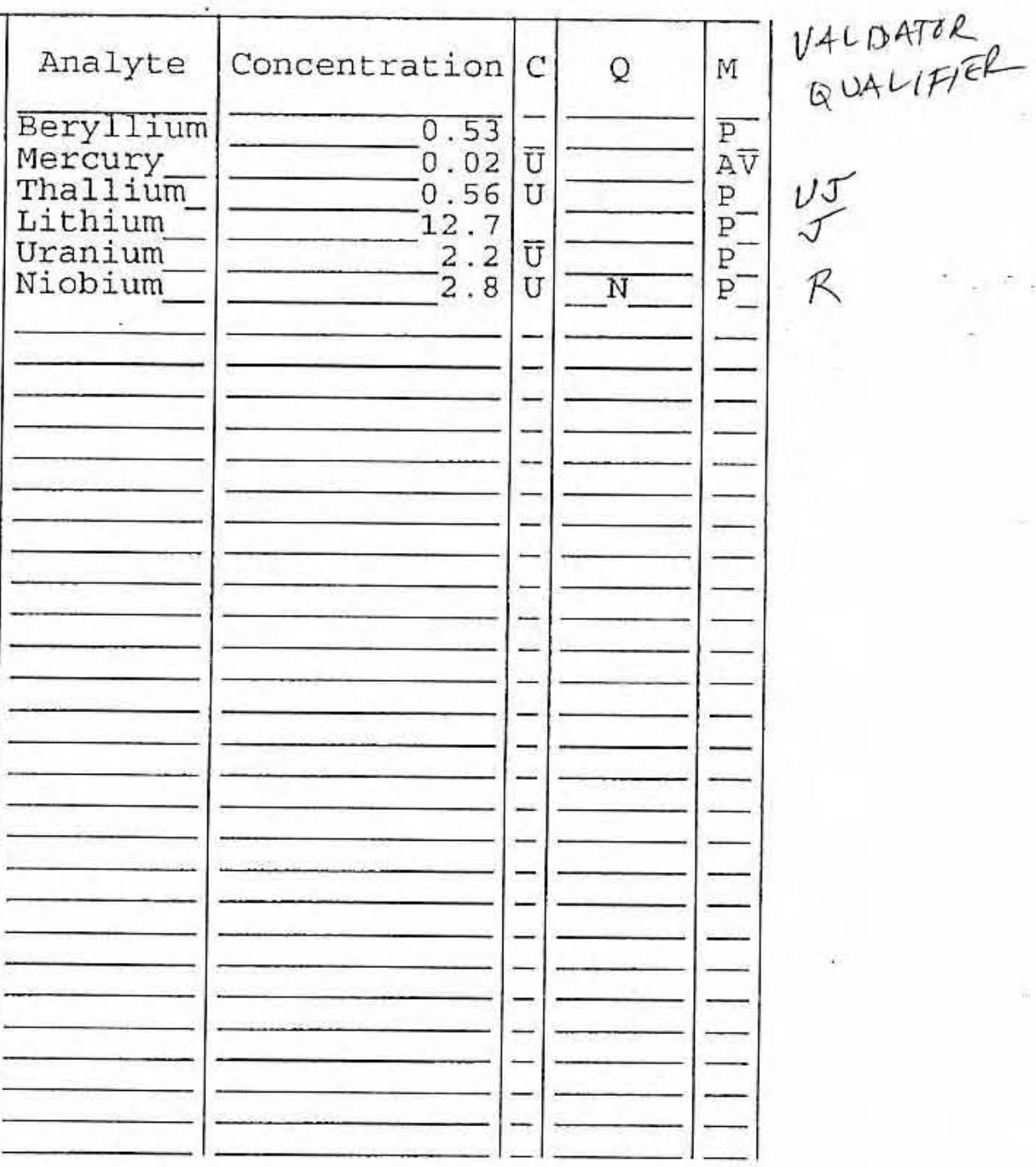

Clarity Before:

Clarity After:
Texture:

Artifacts: 


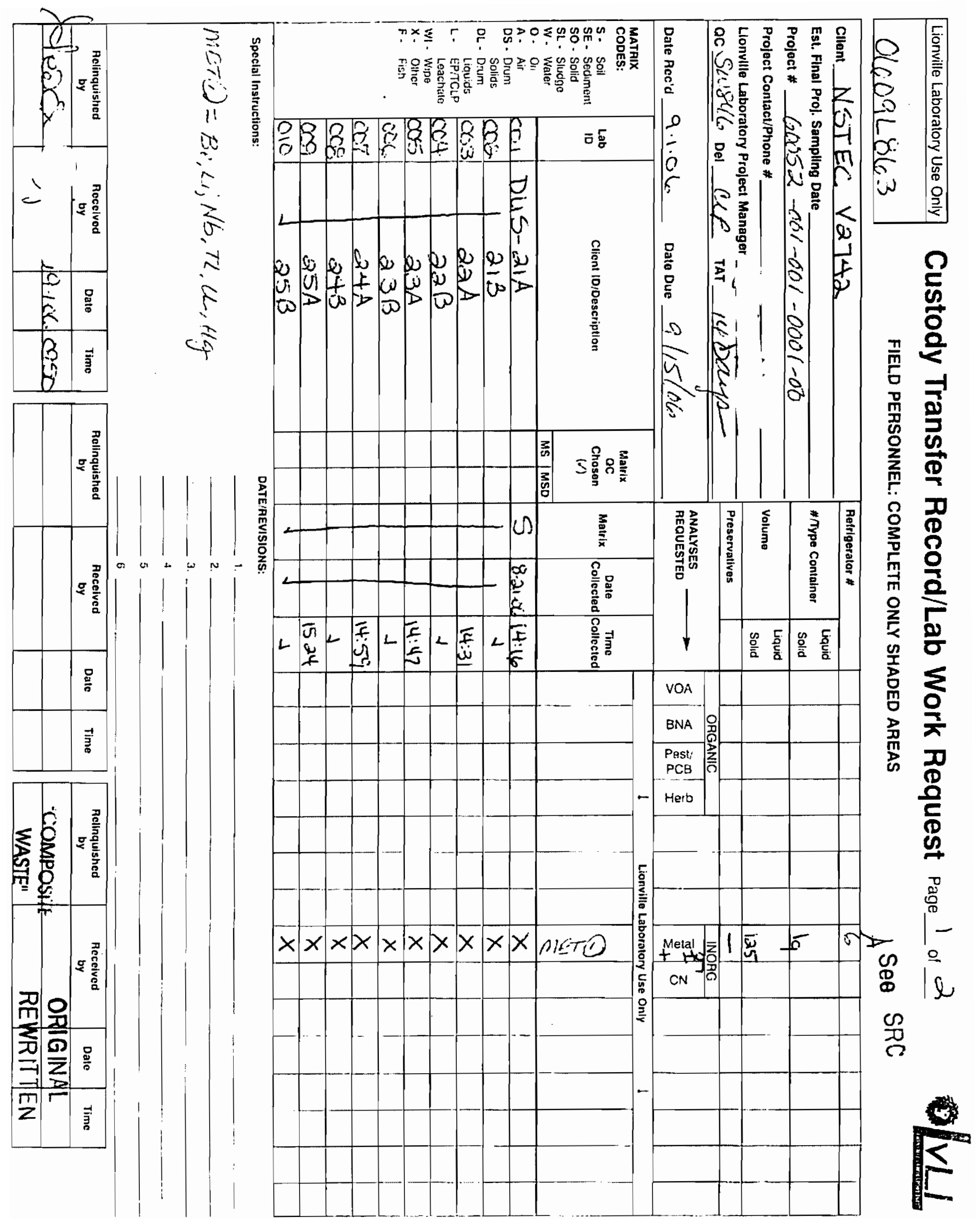




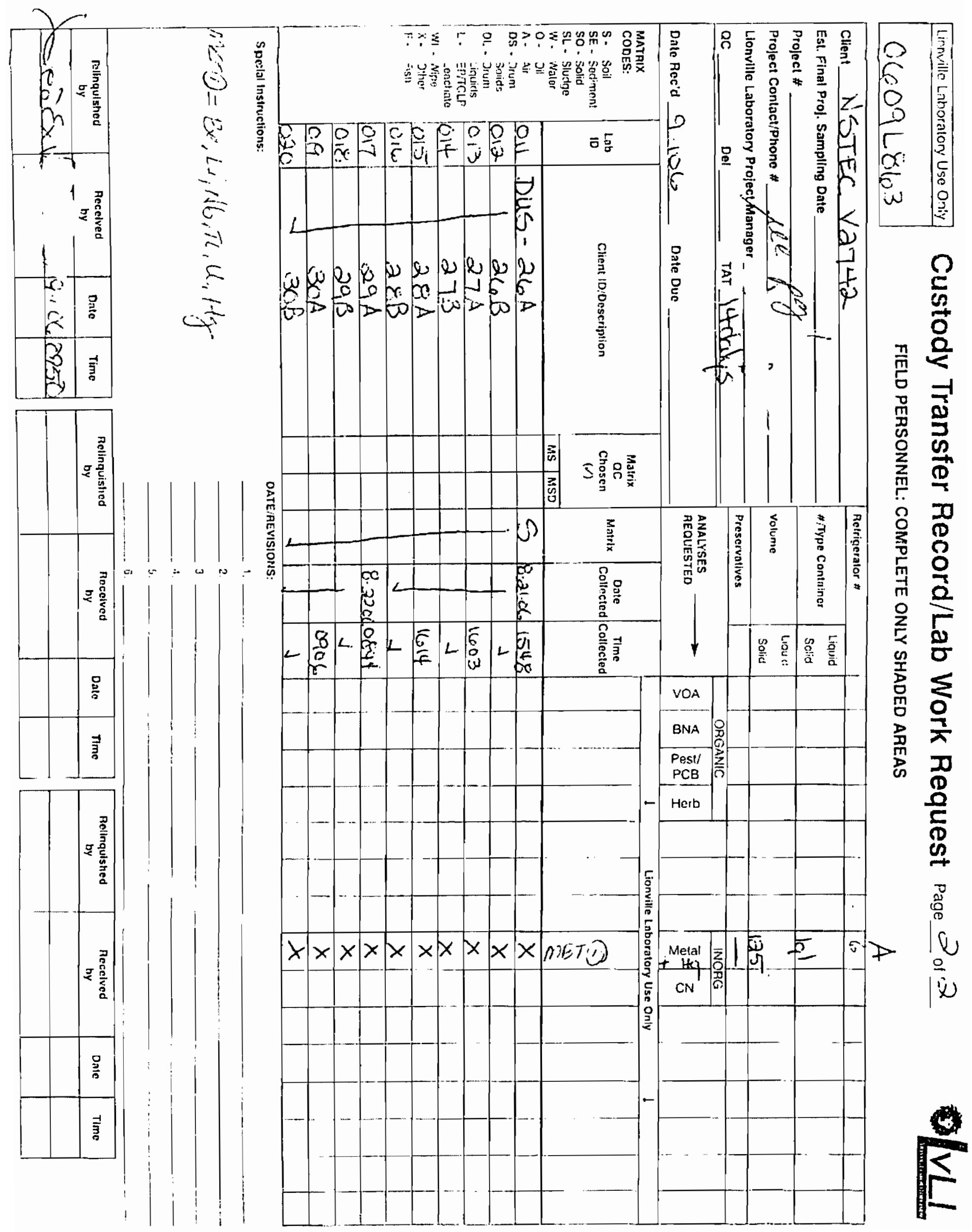




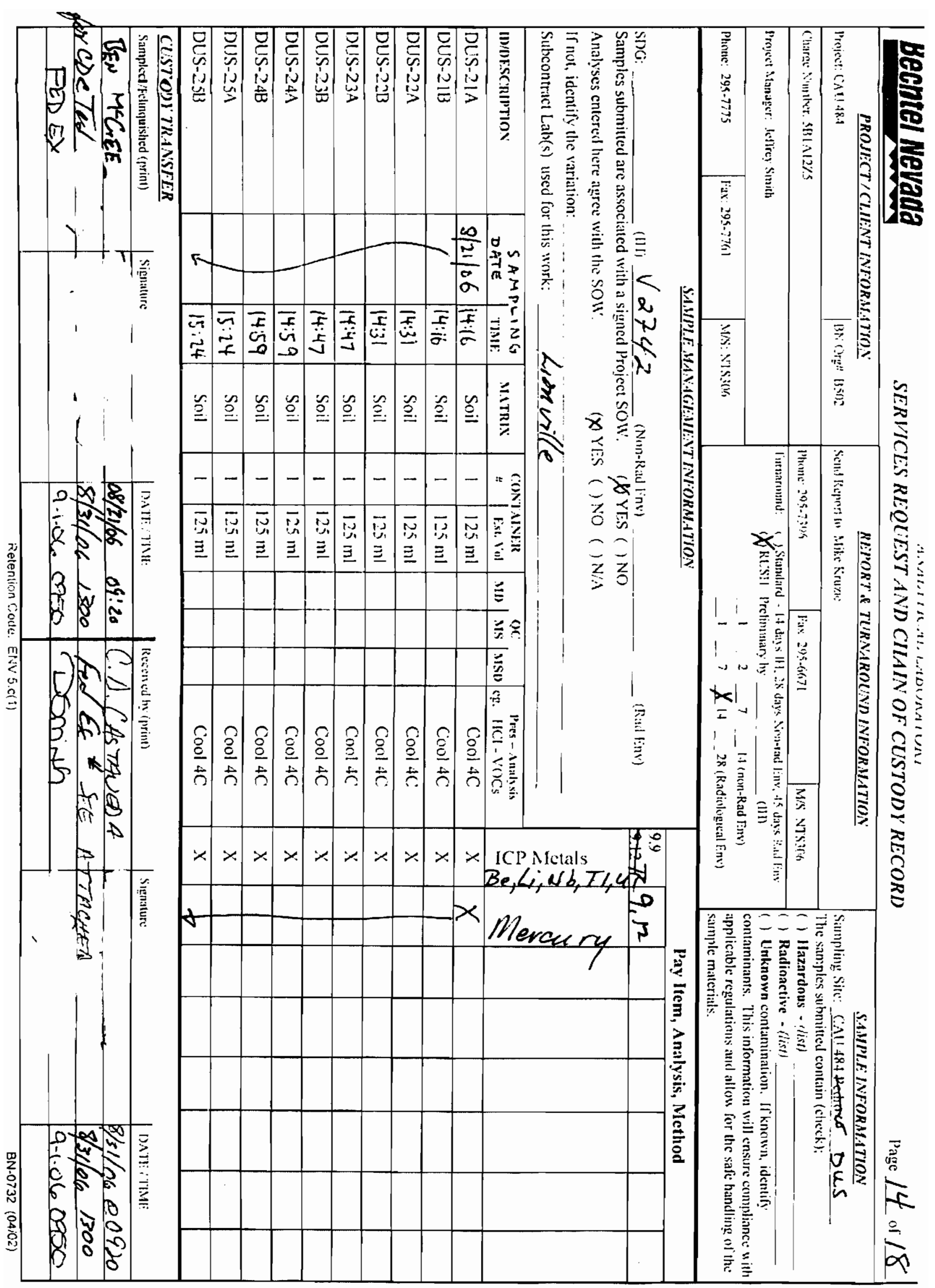




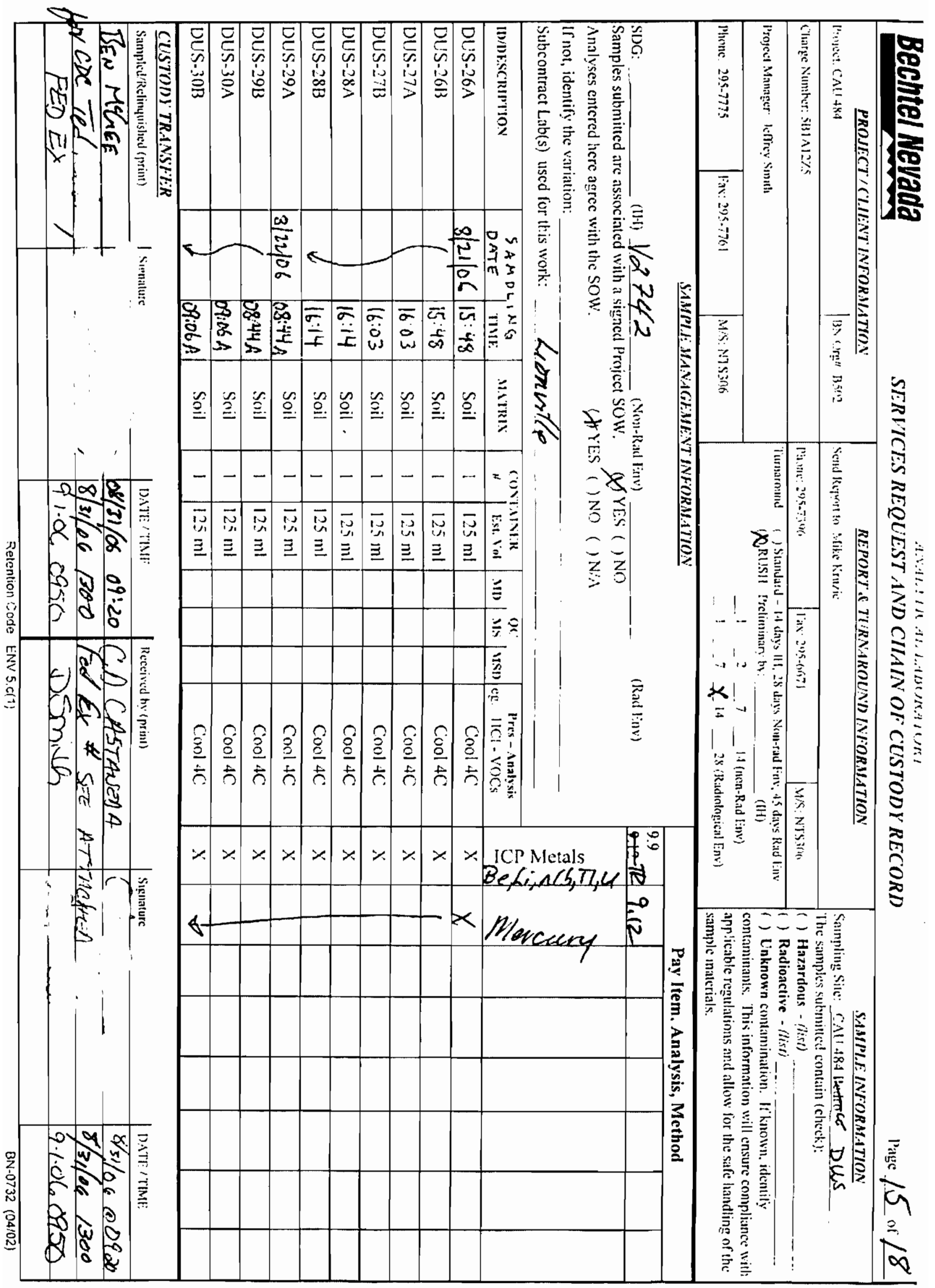




\section{U.S. EPA}

1.

INORGANIC ANALYSES DATA SHEET
EPA SAMPLE NO.

$31 \mathrm{~A}$

Contract: 60052

SAS No.:

Lab Sample ID: $060918 \overline{64-0} 01$

Date Received: 09/01/06

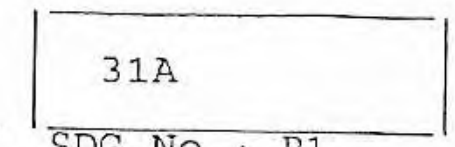

Lab Name: LIONVILLE LABORATORY

Lab Code: LVLI

Matrix (soil/water): SoIL

Level (low/med): LOW

\% Solids :
$-91 \overline{.9}$

Concentration Units (ug/L or mg/kg dry weight): MG/KG

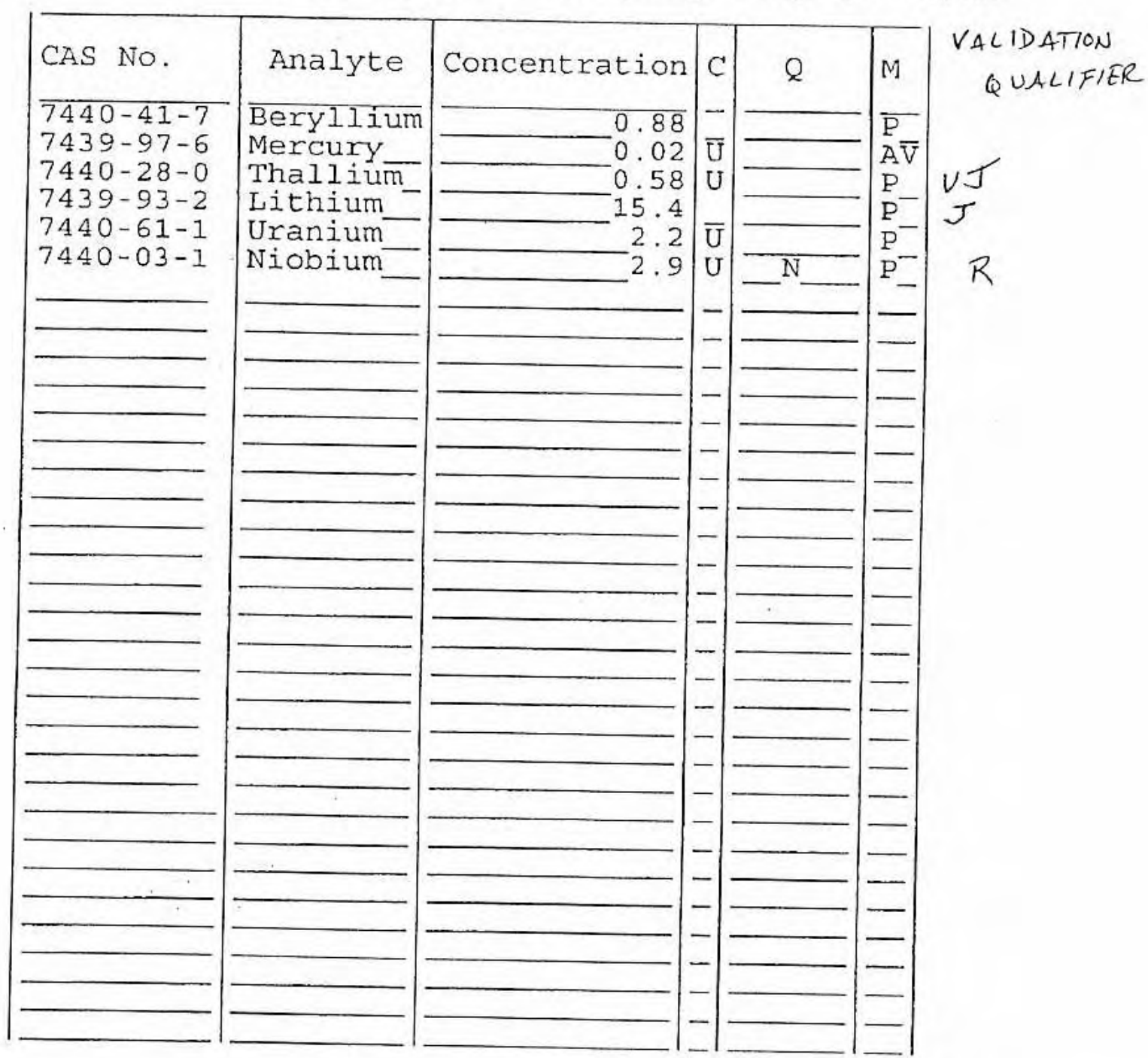

Color Before:

Color After:

Comments:

DUS - 3 IA
Clarity Before: Clarity After:
Texture:

Artifacts: 
U.S. EPA

1

INORGANIC ANALYSES DATA SHEET
EPA SAMPLE NO.

$31 B$

Lab Name: LIONVILLE LABORATORY

Lab Code: LVLI Matrix (soil/water): SoIL

Level (low/med): LOW

\% Solids: $\quad-92 . \overline{1}$
Contract: 60052

SAS NO.:

SDG NO.: R1

Lab sample ID: 0609L8 $\overline{64-002}$

Date Received: 09/01/06

\section{Concentration Units (ug/L or $\mathrm{mg} / \mathrm{kg}$ dry weight): MG/KG}

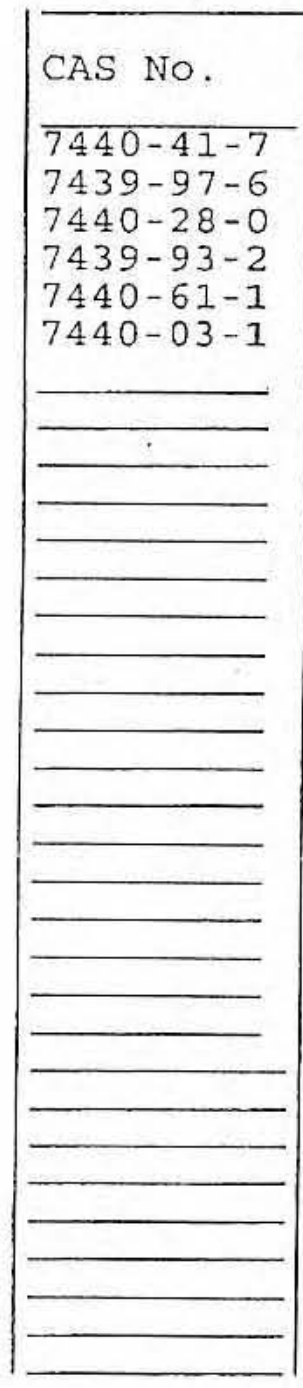

Color Before:

Color After:

Comments :

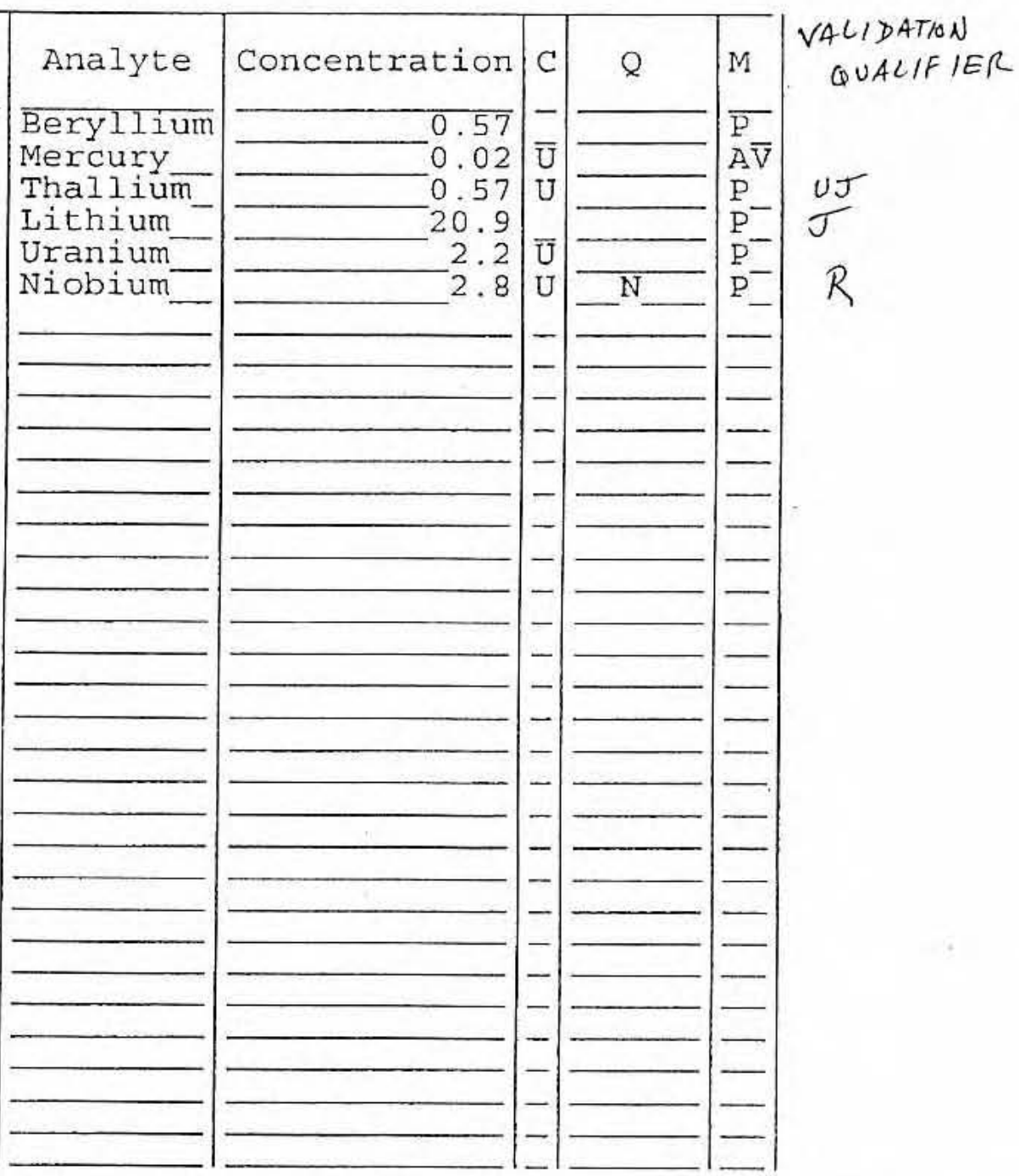

Clarity Before: Clarity After:
Texture:

Artifacts:

DUS - 31B 
U.S. EPA

1

INORGANIC ANALYSES DATA SHEET
EPA SAMPLE NO.

$32 \mathrm{~A}$

Contract: 60052

SAS NO. :

SDG NO.: R1

Lab Sample ID: 0609L8 $\overline{64-0} 03$

Date Received: 09/01/06
Case NO.: DUS

Matrix (soil/water): SOIL

Level (low/med): LOW : Solids: $\quad-94 \overline{.3}$

Concentration Units (ug/L or $\mathrm{mg} / \mathrm{kg}$ dry weight): MG/KG

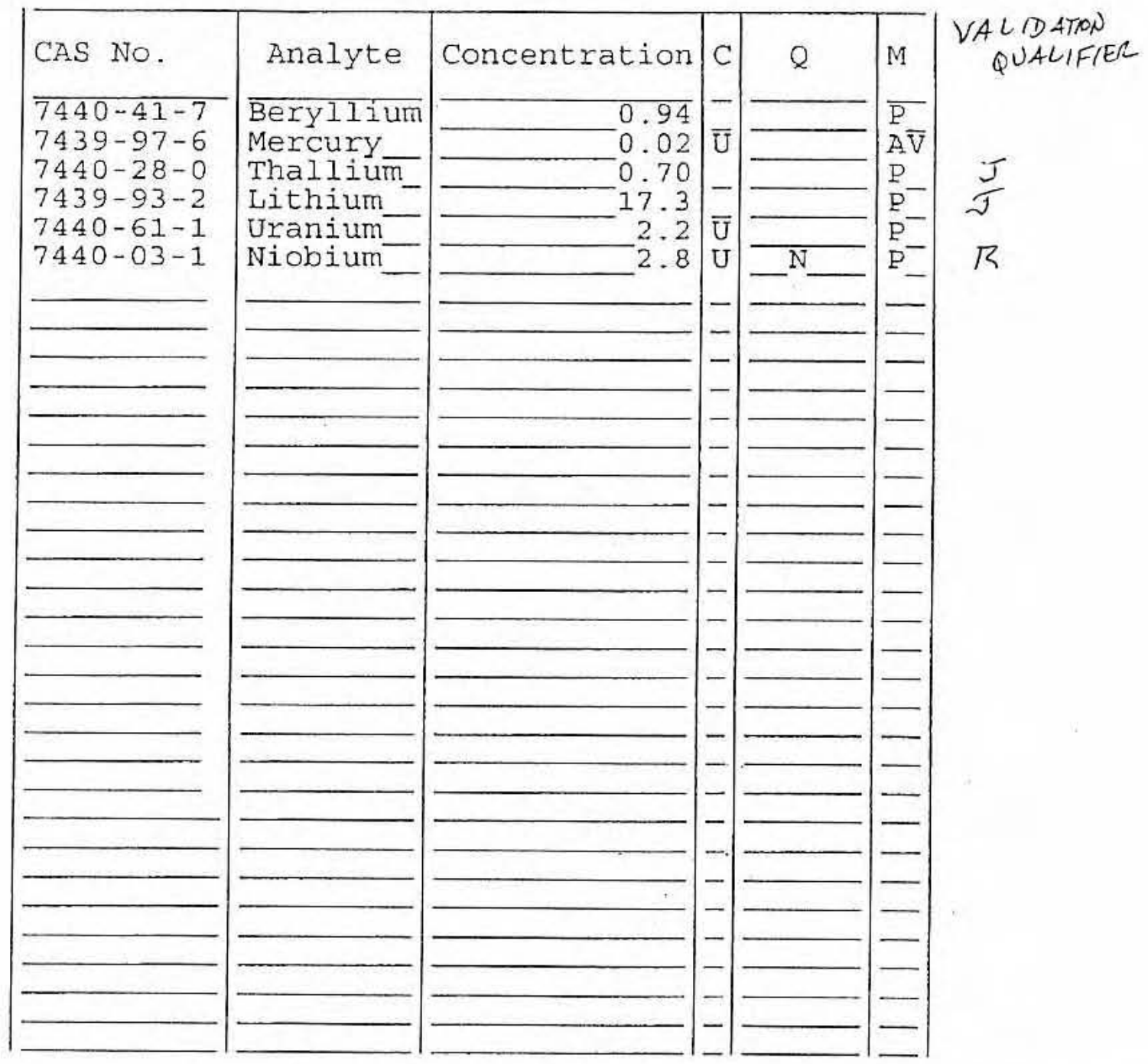

Color Before:

Color After:

Comments:

DUS $-32 \mathrm{~A}$
Clarity Before:

Clarity After:
Texture:

Artifacts: 
EPA SAMPLE NO.

\section{$32 \mathrm{~B}$}

Lab Name: LIONVILLE LABORATORY

Lab Code: LVLI

Matrix (soil/water): SOIL_

Level (low/med): LOW

: Solids:
Contract: 60052

SAS NO.:

Lab Sample ID: $060918 \overline{64-004}$

Date Received: 09/01/06

Concentration Units (ug/L or $\mathrm{mg} / \mathrm{kg}$ dry weight): MG/KG

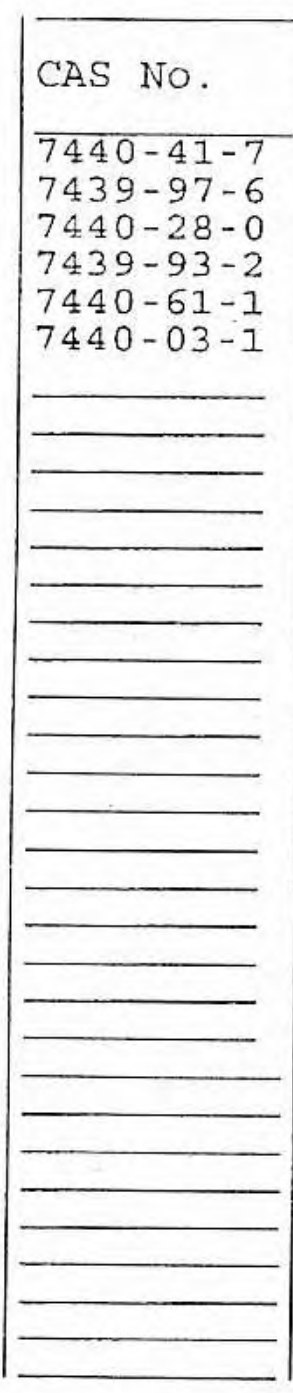

Color Before:

Color After:

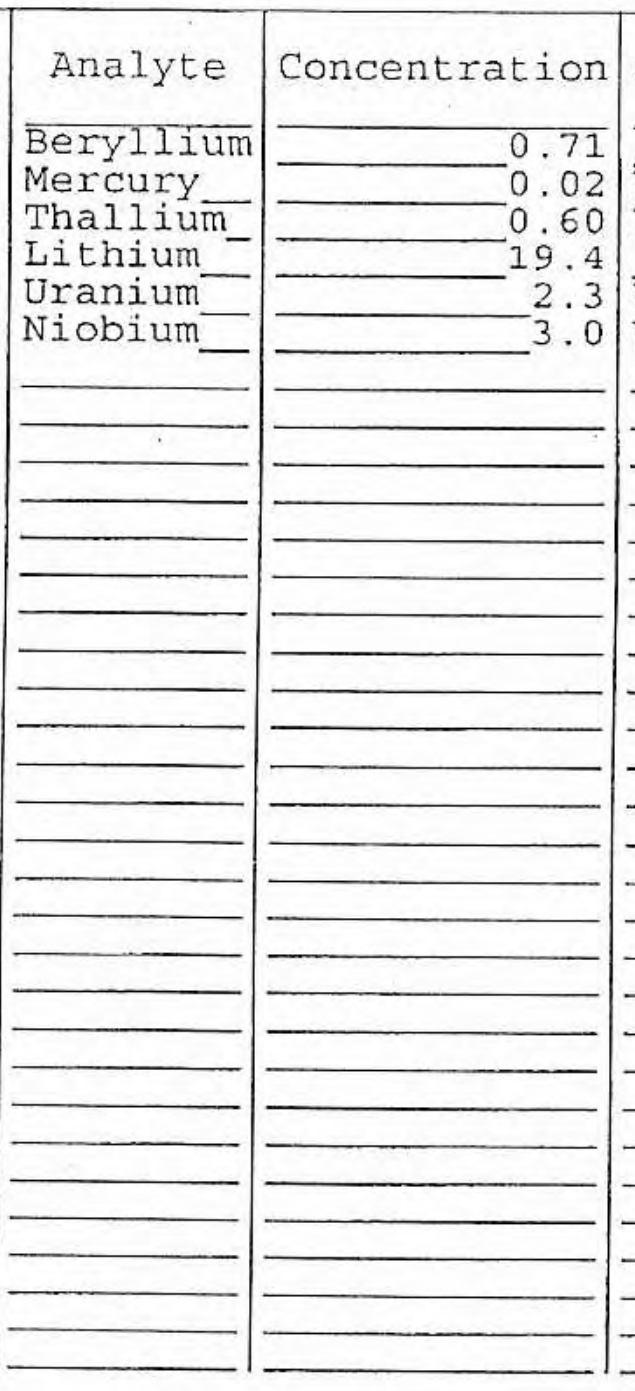

Clarity Before:

Clarity After:

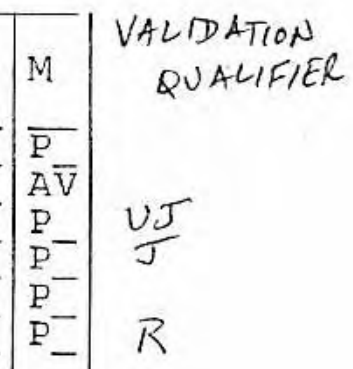

QUALIFIER

Comments:

DUS - 32B

Texture:

Artifacts: 
EPA SAMPLE NO.

$33 \mathrm{~A}$

Lab Name: LIONVILLE LABORATORY

Lab Code: LVLI Matrix (soil/water): SOIL Level. (low/med): LOW \% Solids:

Contract: 60052
SAS NO.:

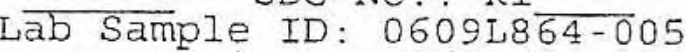
$-94 \overline{.2}$

Date Received: 09/01/06

Concentration Units (ug/L or $\mathrm{mg} / \mathrm{kg}$ dry weight): MG/KG

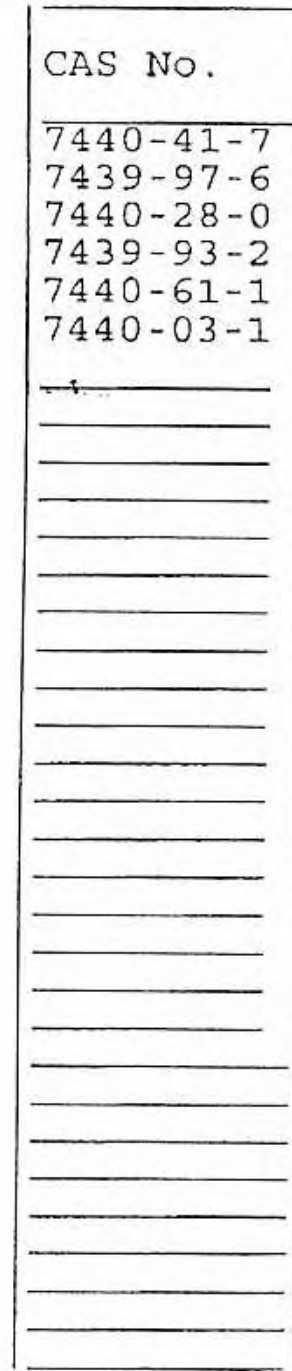

Color Before: Color After:

\section{Comments :}

DUS $-33 A$

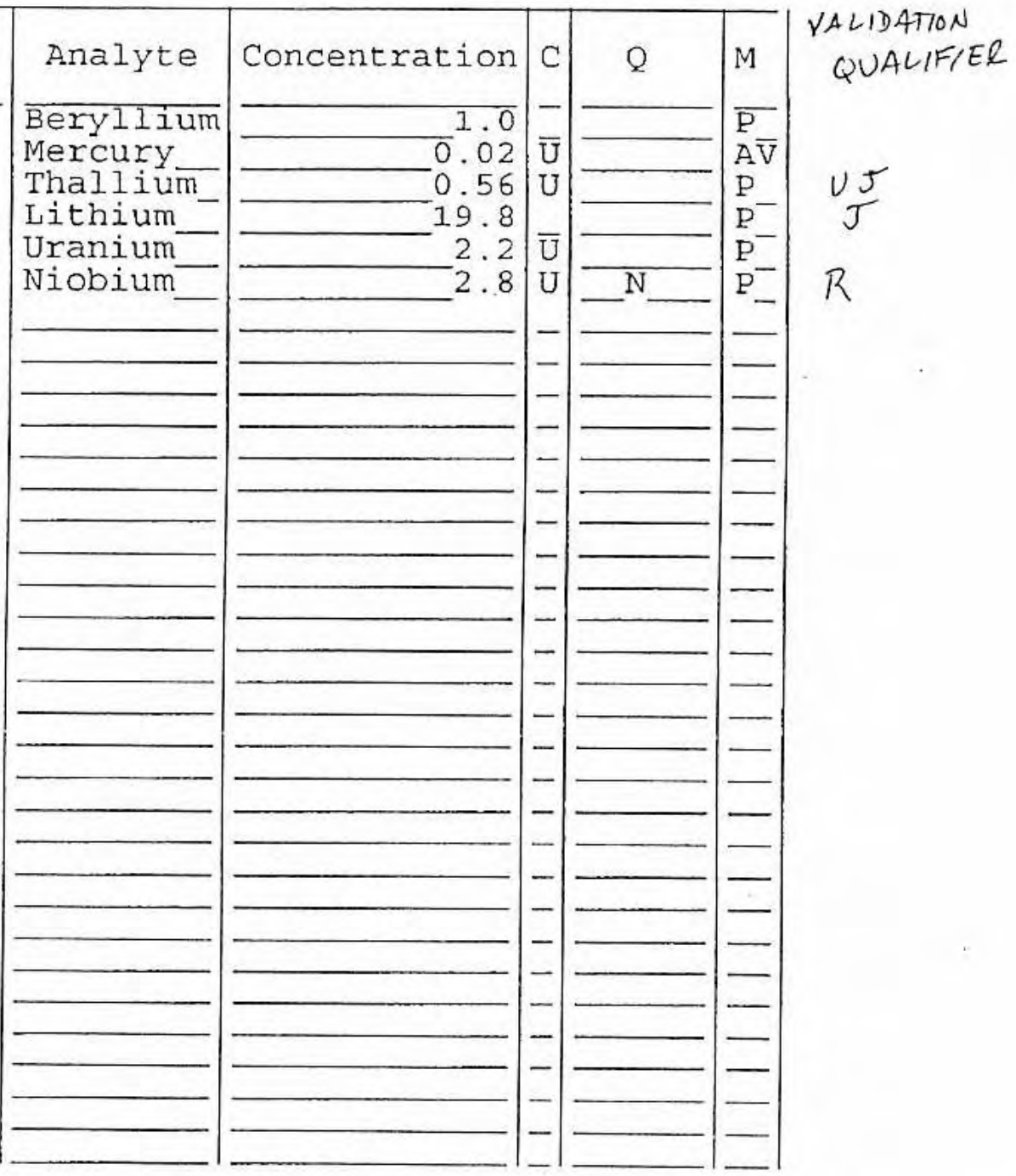

Clarity Before: Clarity After:
Texture: Artifacts: 
1

INORGANIC ANALYSES DATA SHEET
EPA SAMPLE NO.

\section{$33 \mathrm{~B}$}

Contract: 60052

SAS No. :

Lab Sample ID: $060918 \overline{64-006}$

Date Received: 09/01/06
Lab Code: LVLI_CASE NO.: DUS

Matrix (soil/water): SOIL

Level (low/med): LOW

\% Solids:
Concentration Units (ug/L or $\mathrm{mg} / \mathrm{kg}$ dry weight): MG/KG
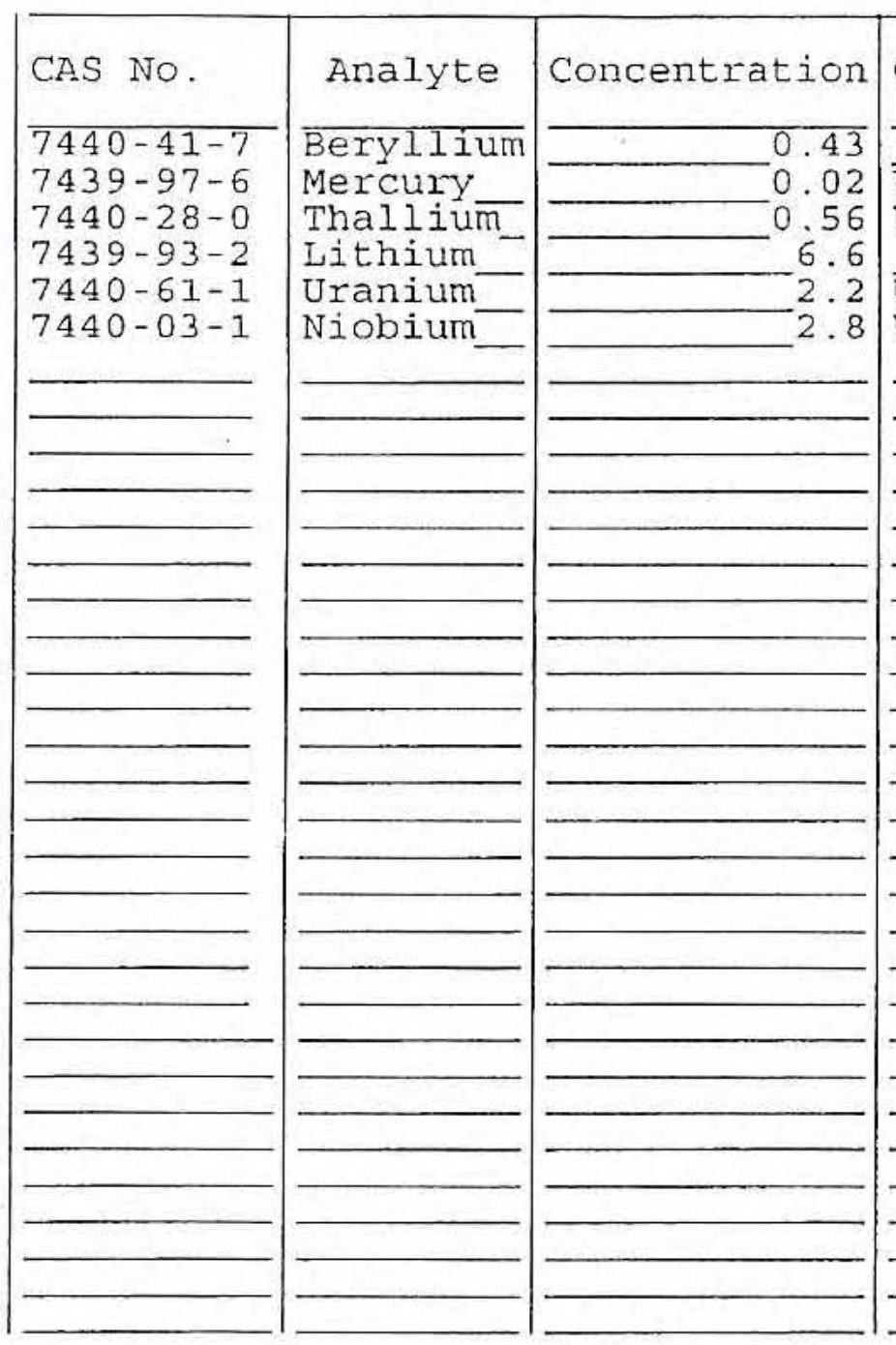

Clarity Before:

Clarity After:

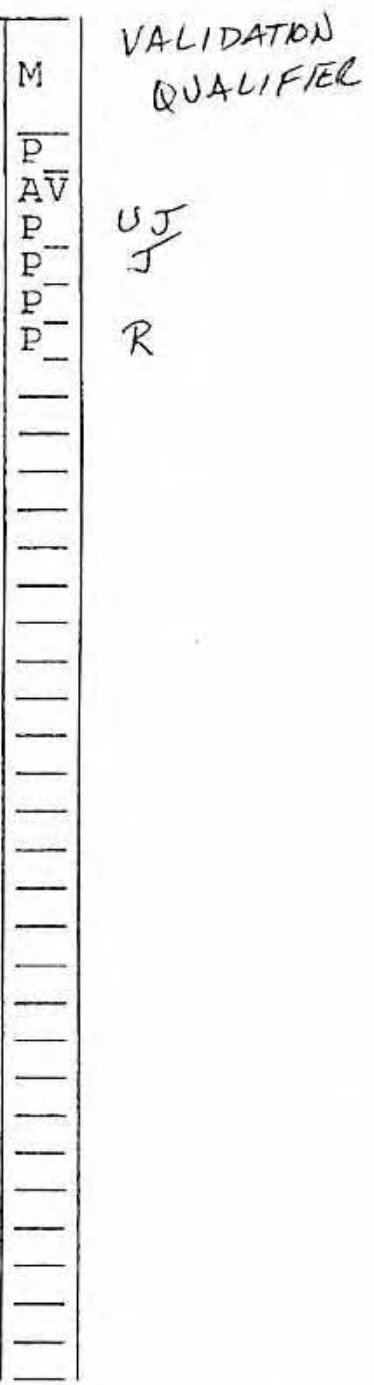

Texture:

Artifacts:

Comments:

DUS - 33B 


\section{U.S. EPA}

1

INORGANIC ANALYSES DATA SHEET
EPA SAMPLE NO.

Contract: 60052

SAS NO.:

Lab Sample ID: 0609L8 0

Date Received: 09/01/06
$34 \mathrm{~A}$

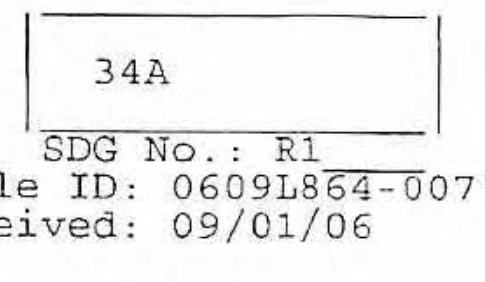

Lab Name: LIONVILLE_LABORATORY

Lab Code: LVLI

Matrix (soil/water): SOIL

Level (low/med): LOW-

\% Solids: $\quad-94 \overline{.9}$

Concentration Units (ug/L or $\mathrm{mg} / \mathrm{kg}$ dry weight) : $\mathrm{MG} / \mathrm{KG}$

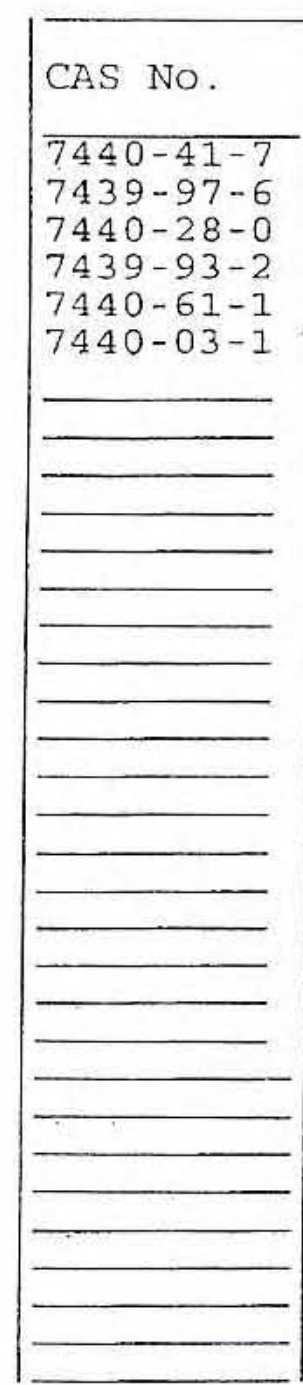

Color Before:

Color After:
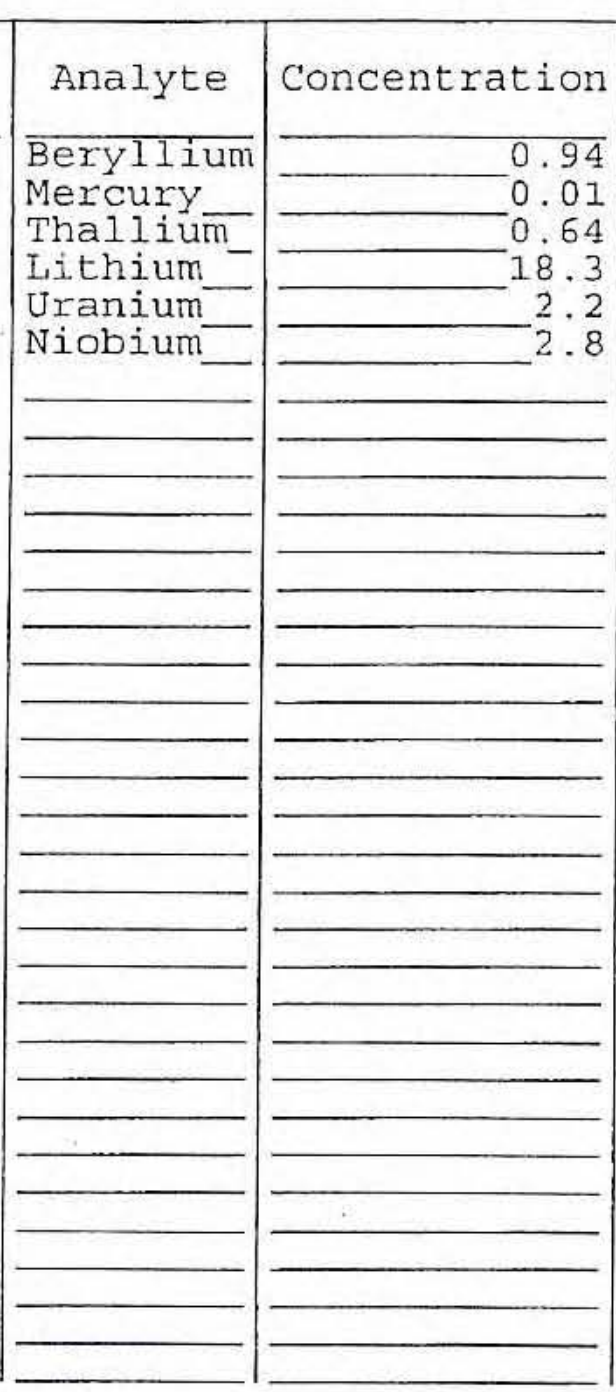

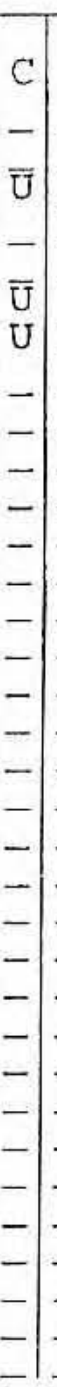

Clarity Before:

Clarity After:

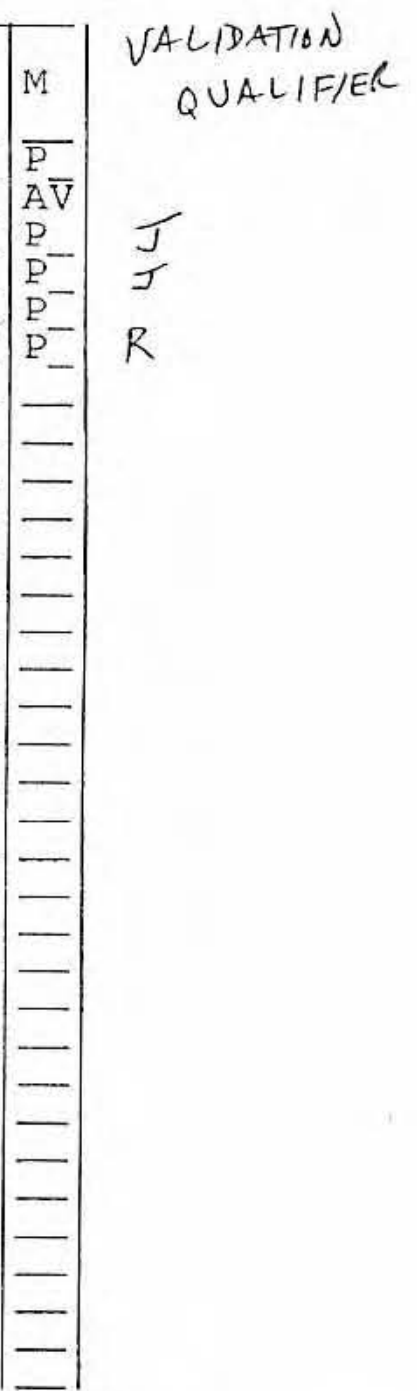

Texture: Artifacts :

Comments :

DUS $-34 \mathrm{~A}$ 
U.S. EPA

Lab Name: LIONVILLE_LABORATORY

Lab Code: LVLI

Matrix (soil/water); SOIL

Level. (low/med): LOW

Solids:

$-91 \overline{7}$

Concentration Units (ug/L or mg/kg dry weight): MG/KG

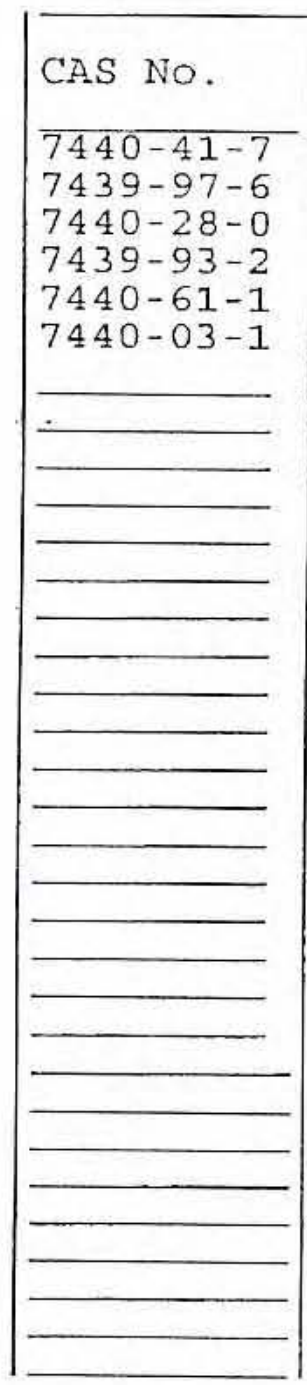

Color Before: Color After:

Comments:

DUS $-34 \mathrm{~B}$

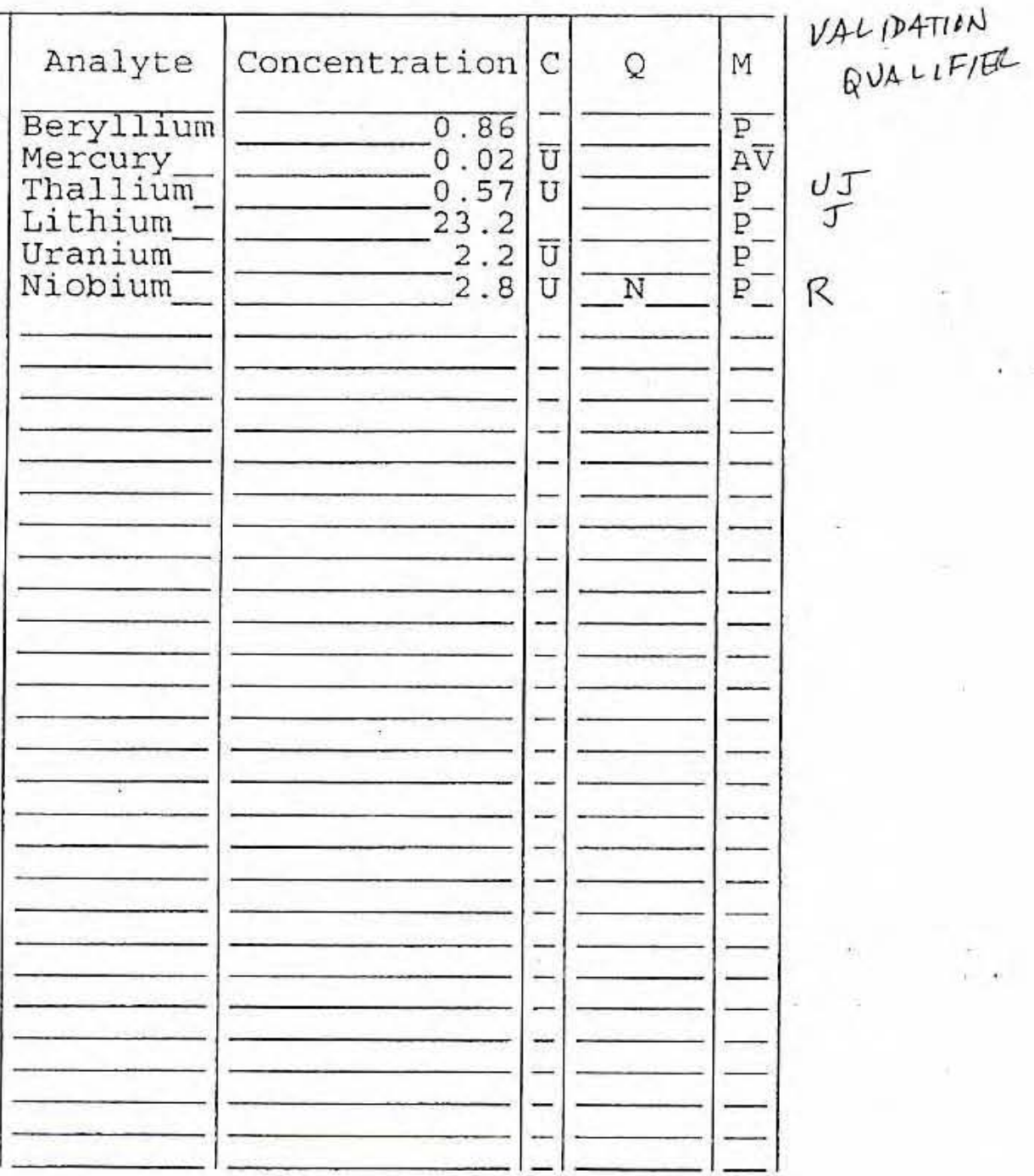

Clarity Before:

Texture: Artifacts:
1

INORGANIC ANALYSES DATA SHEET
Contract: 60052

SAS NO.:

Lab Sample ID: 0609L8 $\overline{64-0} 08$

Date Received: 09/01/06
EPA SAMPLE NO.

$$
34 \mathrm{~B}
$$


1

INORGANIC ANALYSES DATA SHEET
EPA SAMPLE NO.

\section{$35 \mathrm{~A}$}

Lab Name: LIONVILLE LABORATORY

Lab Code: LVLI

Level (low/med): LOW

\%olids: $\quad-92 \overline{8}$
Contract: 60052

SAS NO.:

Lab Sample ID: $060918 \overline{64-009}$

Date Received: 09/01/06

Concentration Units (ug/L or $\mathrm{mg} / \mathrm{kg}$ dry weight): MG/KG

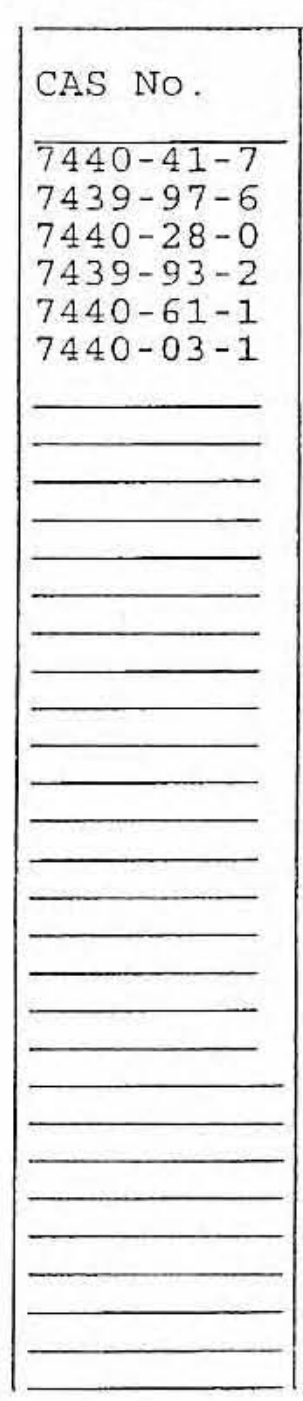

Color Before:

Color After:

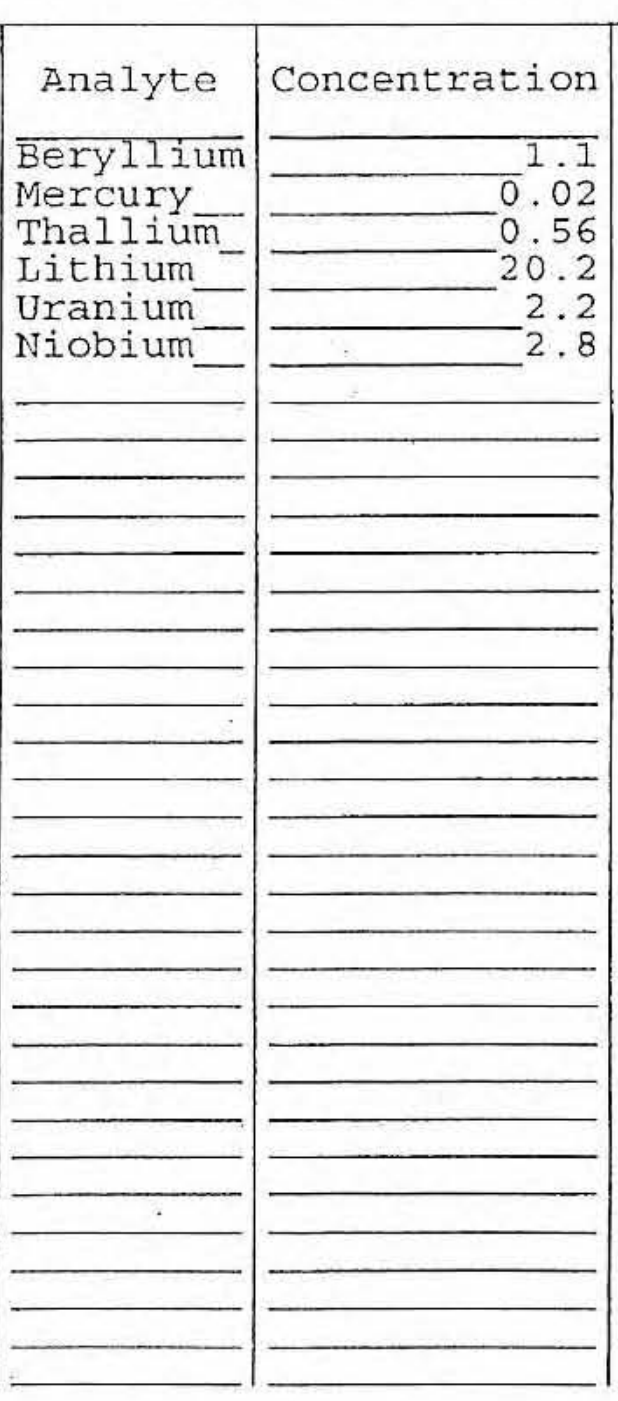

Clarity Before:

Clarity After:

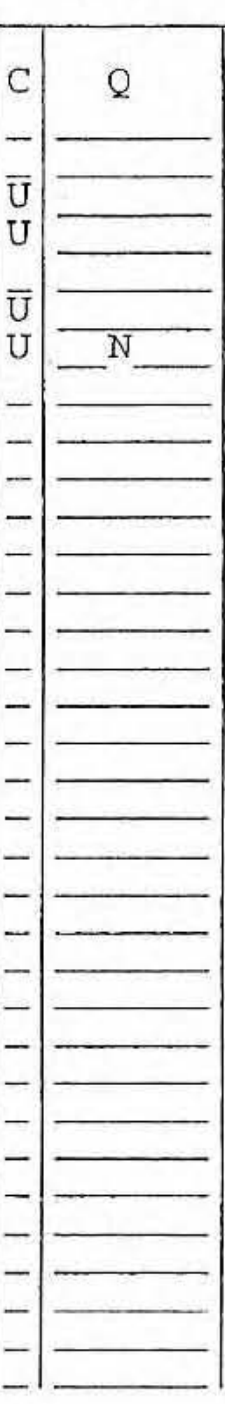

TIVALDATION M QUALIFIER $\overline{\mathrm{P}}$ AV P UJ P- J $\mathrm{P}^{-} \mathrm{R}$

Comments:

DUS - 35A 
U.S. EPA

1

INORGANIC ANALYSES DATA SHEET
EPA SAMPLE NO.

$35 B$

Contract: 60052

SAS NO. :

Lab Cocie: LVII - Case No.: DUS

Matrix (soil/water): SoIL

Level (low/med): LOW

$\div$ Solids:
Lab Sample ID: 0609L8 $\overline{64-010}$

Date Received: 09/01/06

Concentration Units (ug/L or $\mathrm{mg} / \mathrm{kg}$ dry weight): MG/KG

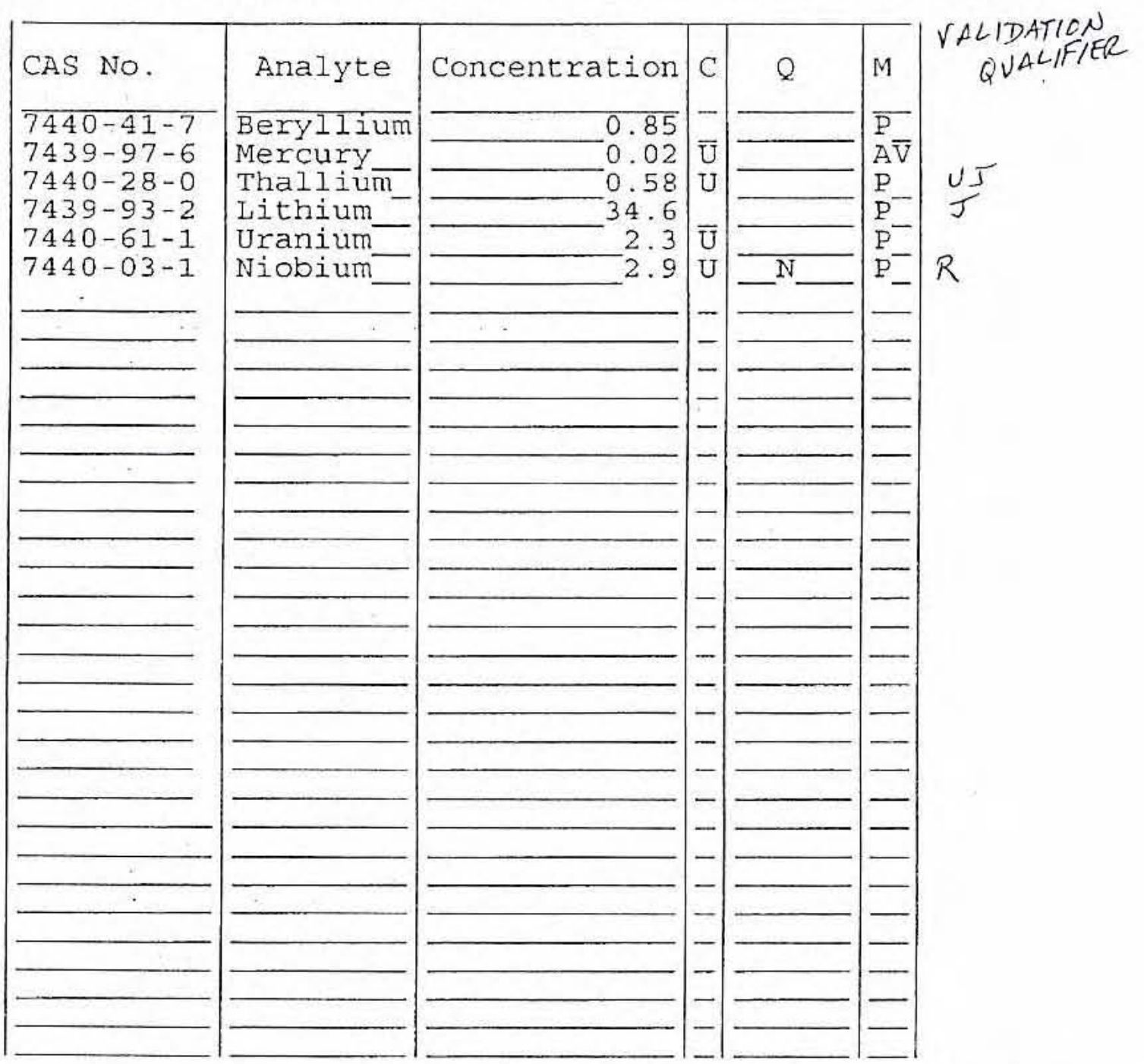

Color Before:

Clarity Before:

Color After:

Clarity After:

Texture:

Artifacts:

\section{Comments:}

DUS - 35B 
1

INORGANIC ANALYYSES DATA SHEET
EPA SAMPLE NO.

$36 \mathrm{~A}$

Contract: 60052

SAS NO. :

Lab Sample ID: 0609L8 $\overline{64-011}$

Date Received: 09/01/06
Lab Name: LIONVILLE_LABORATORY

Matrix (soil/water): SoIL

Level (low/med): LOW

\% Solids:
Concentration Units (ug/L or $\mathrm{mg} / \mathrm{kg}$ ary weight): MG/KG
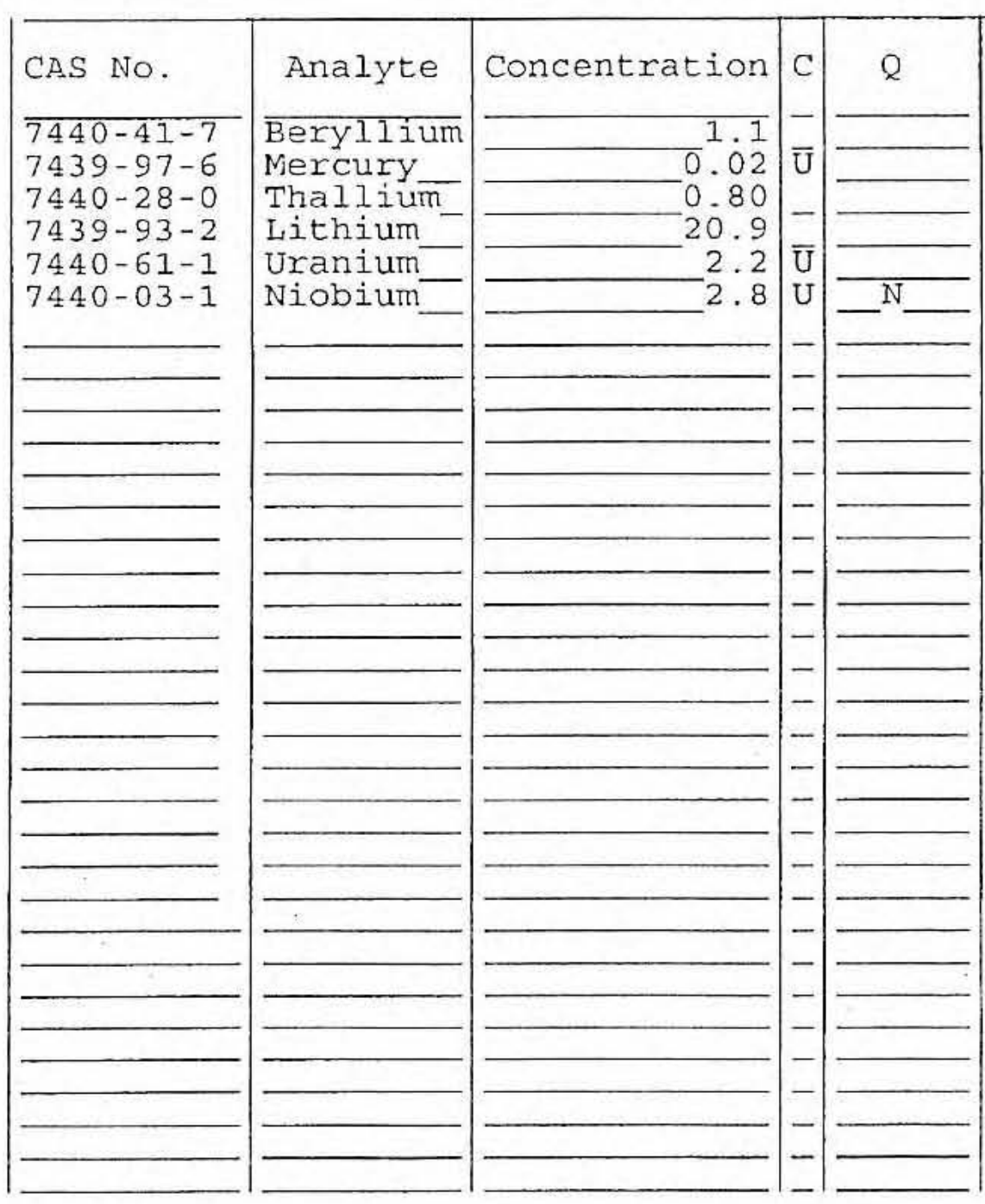

Clarity Before:

Clarity After:

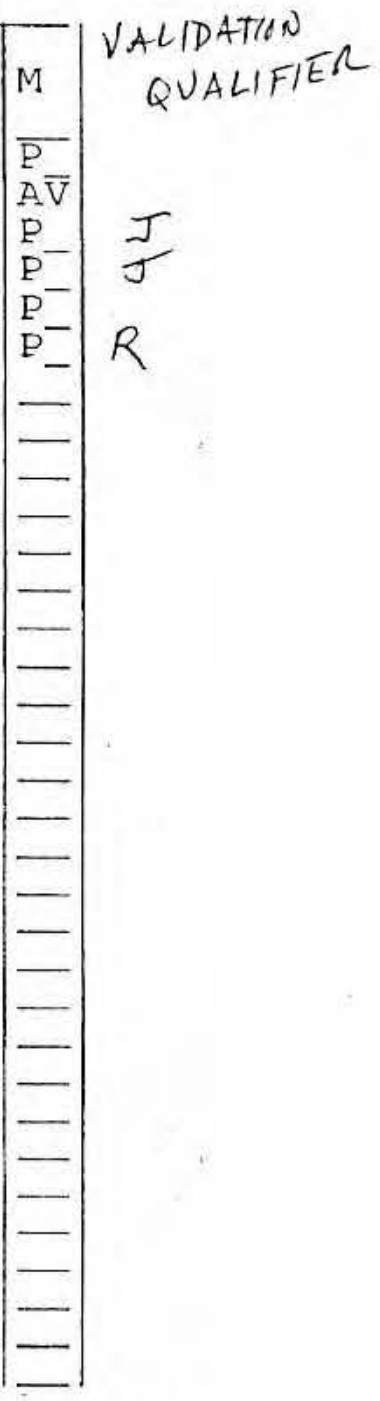

Texture: Artifacts:
Color Before:

Color After:
Comments :

DUS - $36 \mathrm{~A}$ 
1

INORGANIC ANALYSES DATA SHEET
EPA SAMPLE NO.

$36 \mathrm{~B}$

Contract: 60052

SAS NO.:

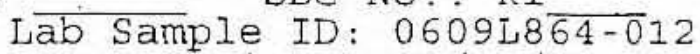

Date Received: 09/01/06
Lab Name: LIONVILLE LABORATORY

Lab code: LVLI Case No.: DUS Matrix (soil/water): SOIL

Level (low/med): LOW $\div$ Solids:
$-91 \overline{1}$

Concentration Units (ug/L or mg/kg dry weight): MG/KG

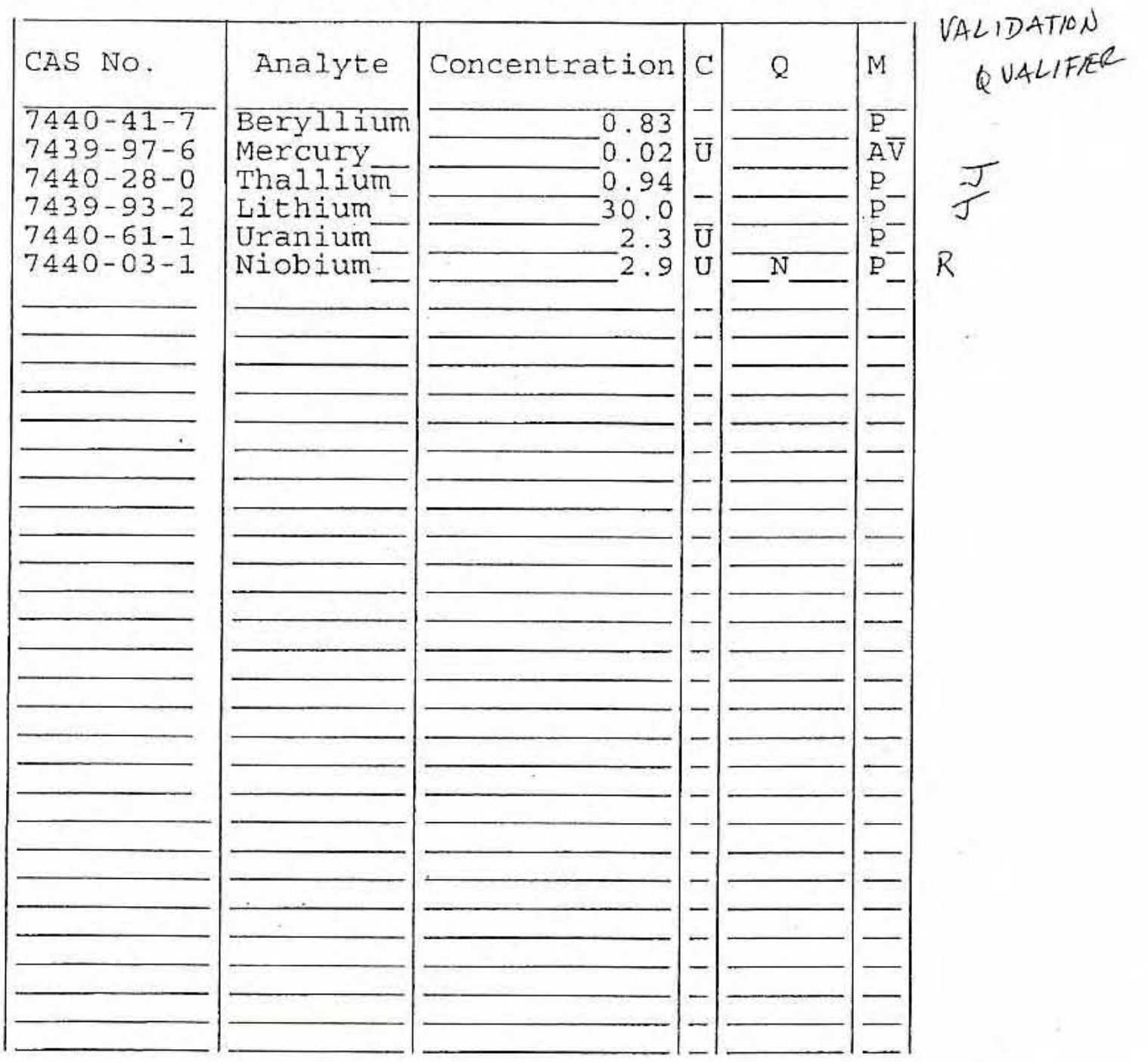

Clarity Before: Clarity After:
Texture:

Artifacts:
Color Before:

Color After:

\section{Comments:}

DUS $-36 \mathrm{~B}$ 
EPA SAMPLE NO.

Lab Name: LIONVILIE LABORATORY

Lab Code: LVLI

Matrix (soil/water)

Level (low/med):

$\%$ Solids: SOIL LOW $-94 \overline{.9}$
Contract: 60052

SAS NO.:

Case No.: DUS
$37 \mathrm{~A}$

SDG NO.: RI

Lab Sample ID: 0609L8त्64-013

Date Received: 09/01/06

Concentration Units (ug/L or $\mathrm{mg} / \mathrm{kg}$ dry weight): $\mathrm{MG} / \mathrm{KG}$

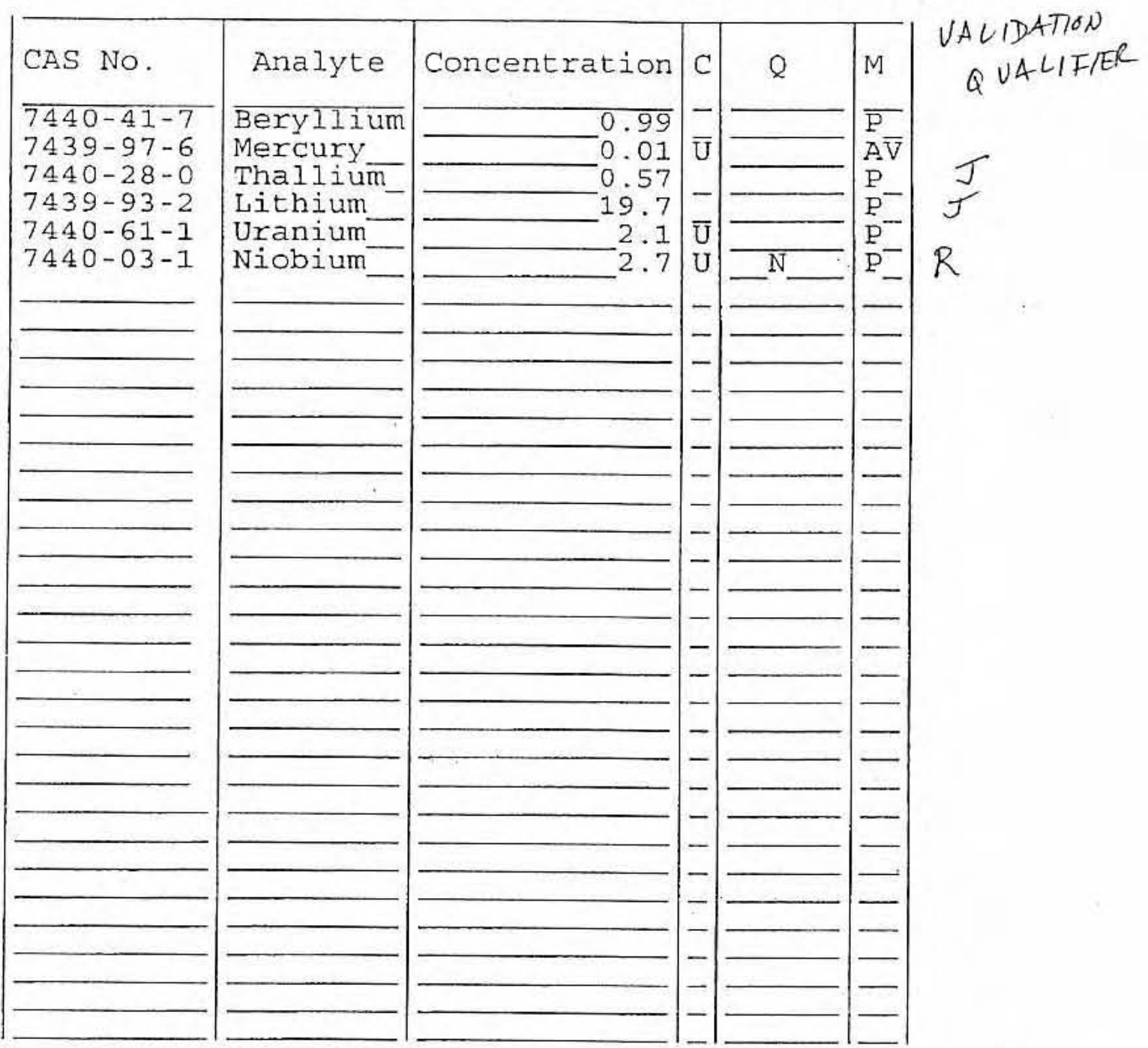

Color Before:

Clarity Before:

Clarity After:

Texture:

Artifacts:

\section{Comments:}

DUS - 37A 
U.S. EPA

1

INORGANIC ANALYSES DATA SHEET
EPA SAMPLE NO.

$$
37 \mathrm{~B}
$$

Lab Name: LIONVILLE_LABORATORY

Lab Code: LVLI

Matrix (soil/water): SoIL

Level (low/med): LOW

: Solids: $\quad-89 \overline{.7}$
Contract: 60052

SAS NO. :

Lab Sample ID: 0609L8 $\overline{64-014}$

Date Received: $09 / 01 / 06$

Concentration Units (ug/L or $\mathrm{mg} / \mathrm{kg}$ dry weight): MG/KG

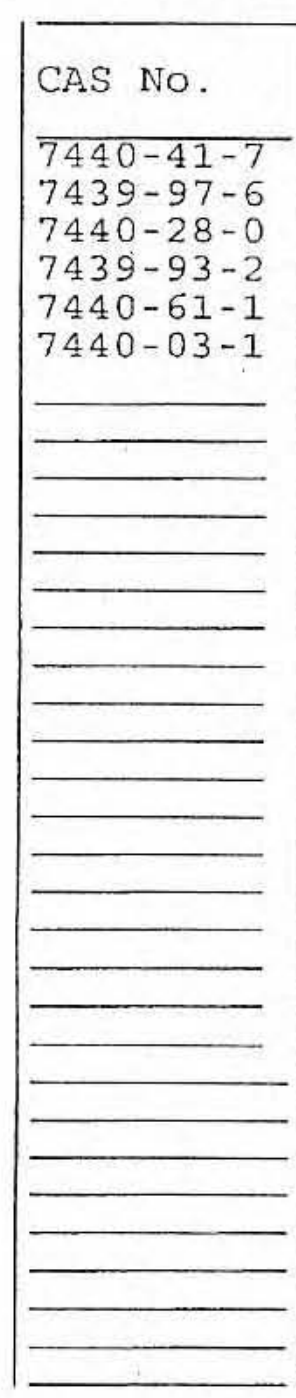

Color Before: Color After:

Comments:

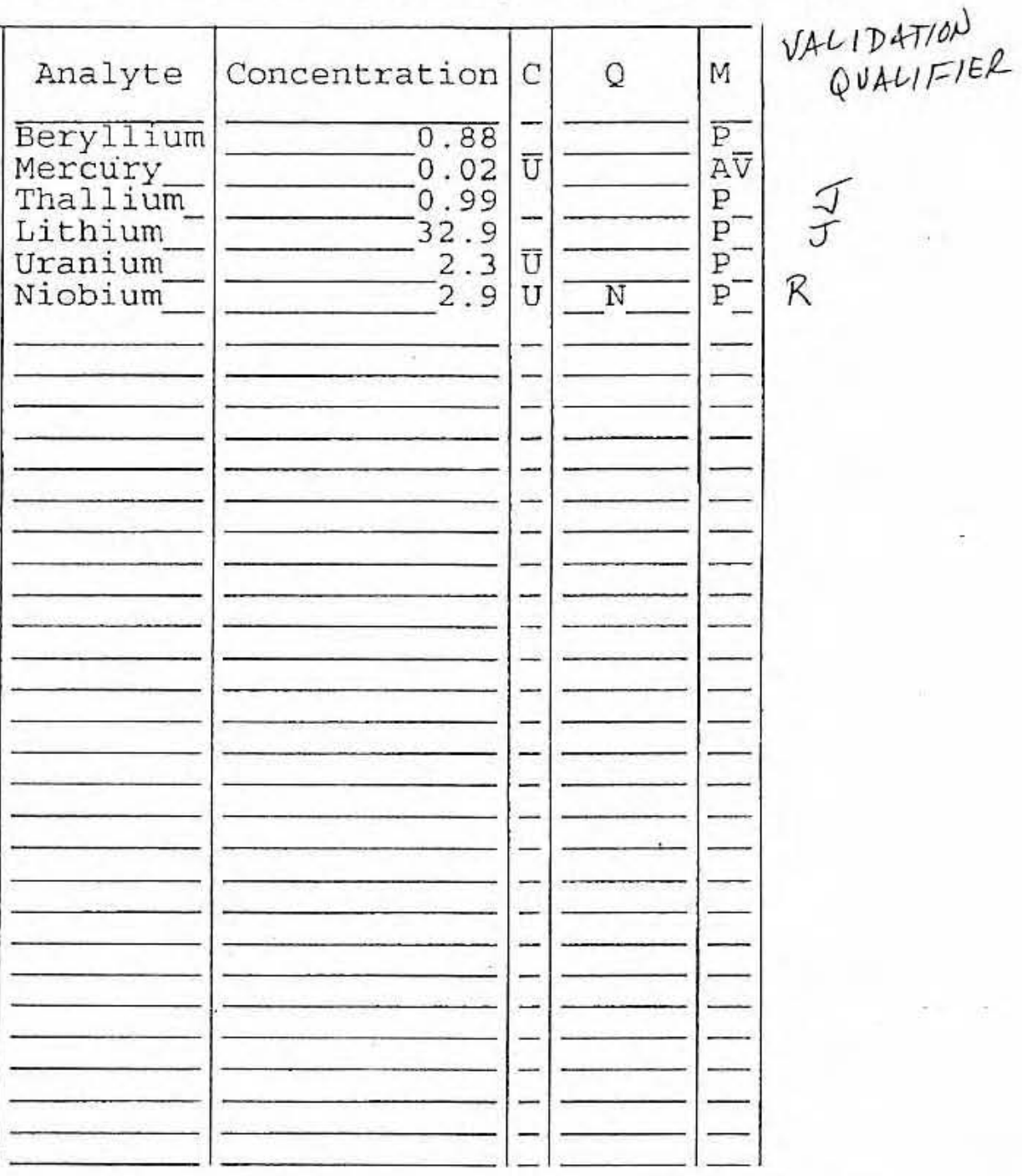

Clarity Before: Clarity After:
Texture: Artifacts:

DUS $-37 \mathrm{~B}$ 
1 INORGANIC ANALYSES DATA SHEET
Lab Name: LIONVILLE LABORATORY

Lab Code: LVLI Matrix (soil/water): SoII Level (low/med): $\div$ Solids: LOW
-94.7
Contract: 60052 SAS NO.:

Lab Sample ID: 0609L8 $\overline{64-015}$
EPA SAMPLE NO.

$38 \mathrm{~A}$

Date Received: 09/01/06

Concentration Units (ug/L or $\mathrm{mg} / \mathrm{kg}$ dry weight): MG/KG

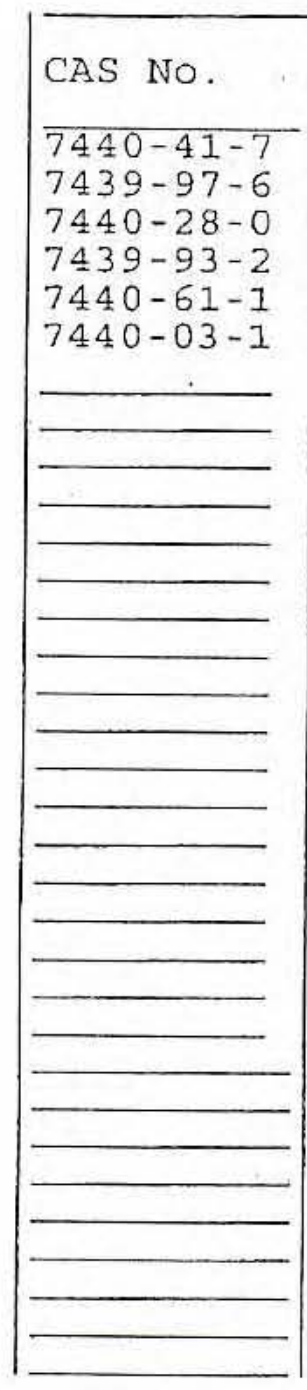

Color Before: Color After:
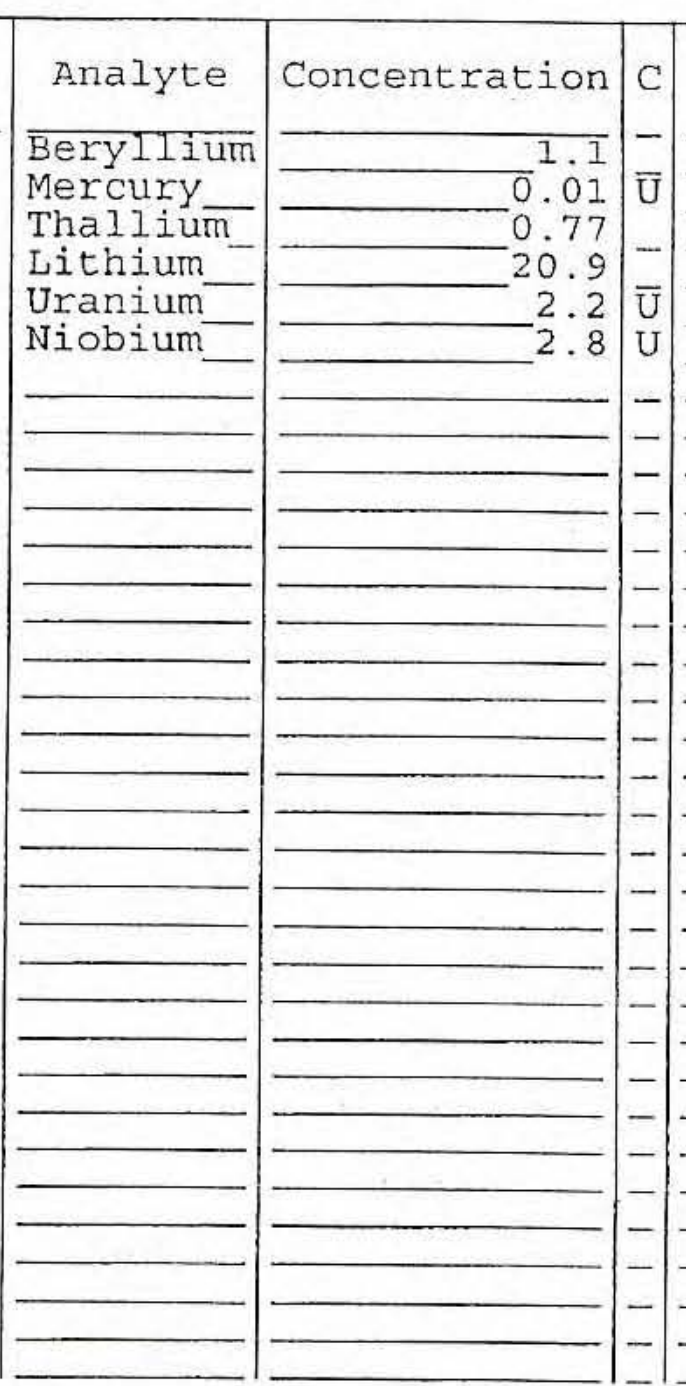

Clarity Before: Clarity After:

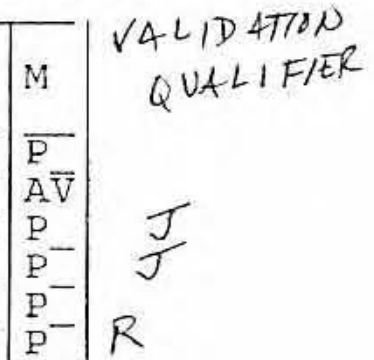

\section{Comments :}

DUS - 38A 


\section{U.S. EPA}

1

INORGANIC ANALYSES DATA SHEET
EPA SAMPLE NO.

\section{$38 \mathrm{~B}$}

Contract: 60052

SAS NO. :

Lab. Sample ID: 0609L8 $\overline{64-016}$

Date Received: 09/01/06
Lab Code: LVLI

Matrix (soil/water): SOIL
Level (low/med): LOW

solids:
$-92 \overline{1}$
ORATORY

Case No.: DUS$$
\text { - }
$$

Concentration Units (ug/L or $\mathrm{mg} / \mathrm{kg}$ dry weight) : MG/KG

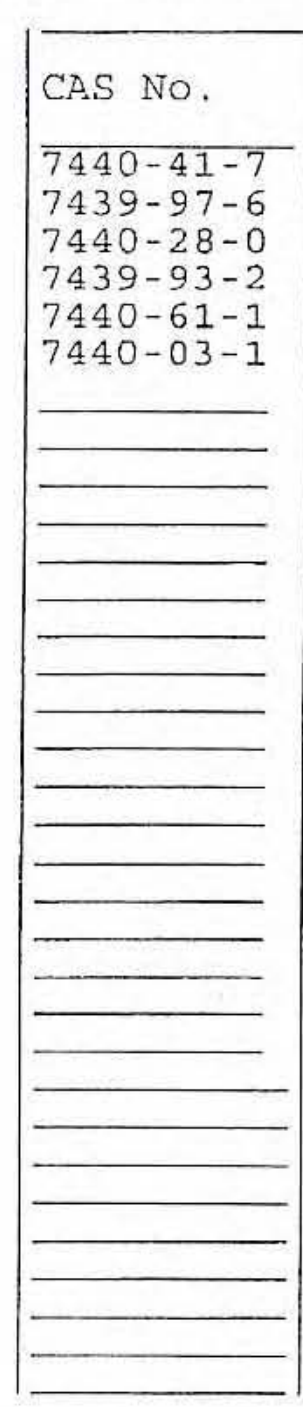

Color Before:

Color After:

Comments:

DUS - $38 \mathrm{~B}$

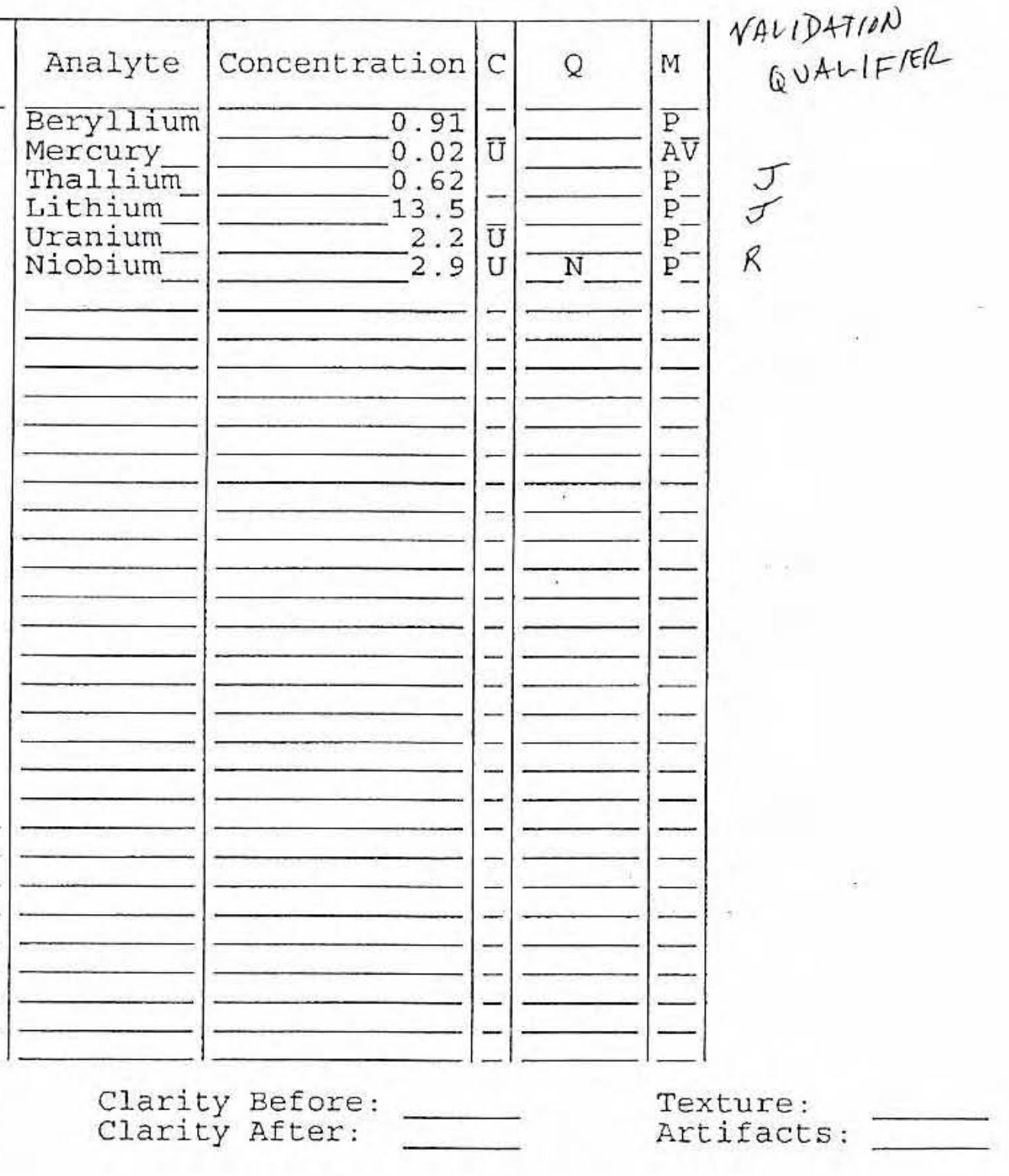


1

INORGANIC ANALȲSES DATA SHEET
EPA SAMPLE NO.

RI

Contract: 60052 SAS NO.:

SDG NO.: R1

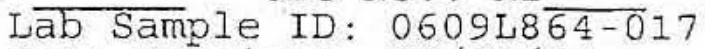

Date Received: 09/01/06
Lab Name: LIONVILLE_LABORATORY
Lab Code: LVII Case No.: DUS

Matrix (soil/water): WATER

Level (low/med): LOW

: Solids :
LOW

Concentration Units (ug/L or mg/kg dry weight): UG/L_

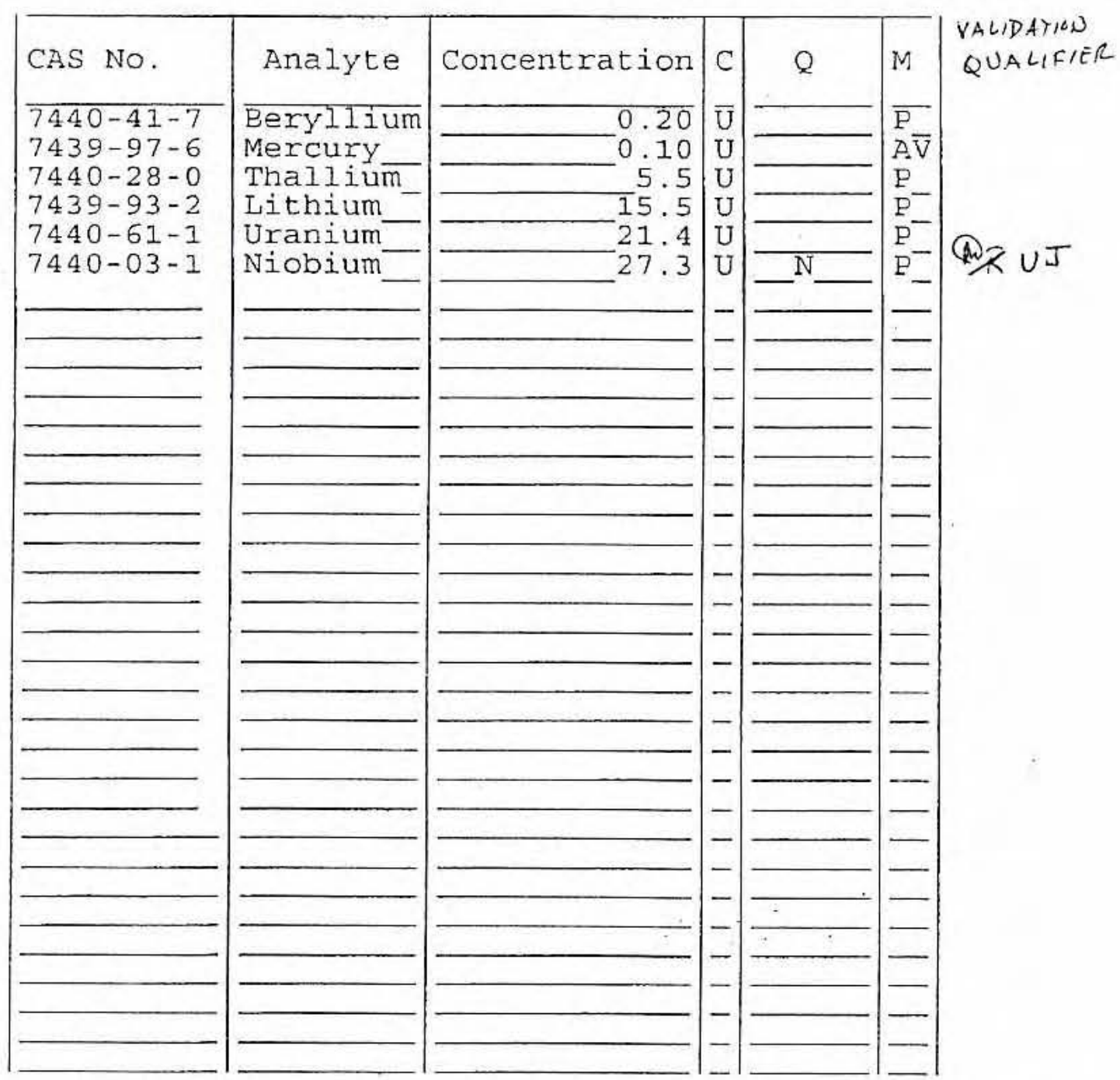

Color Before:

Color After:

Comments :
Clarity Before:

Clarity After:
Texture: Artifacts: 


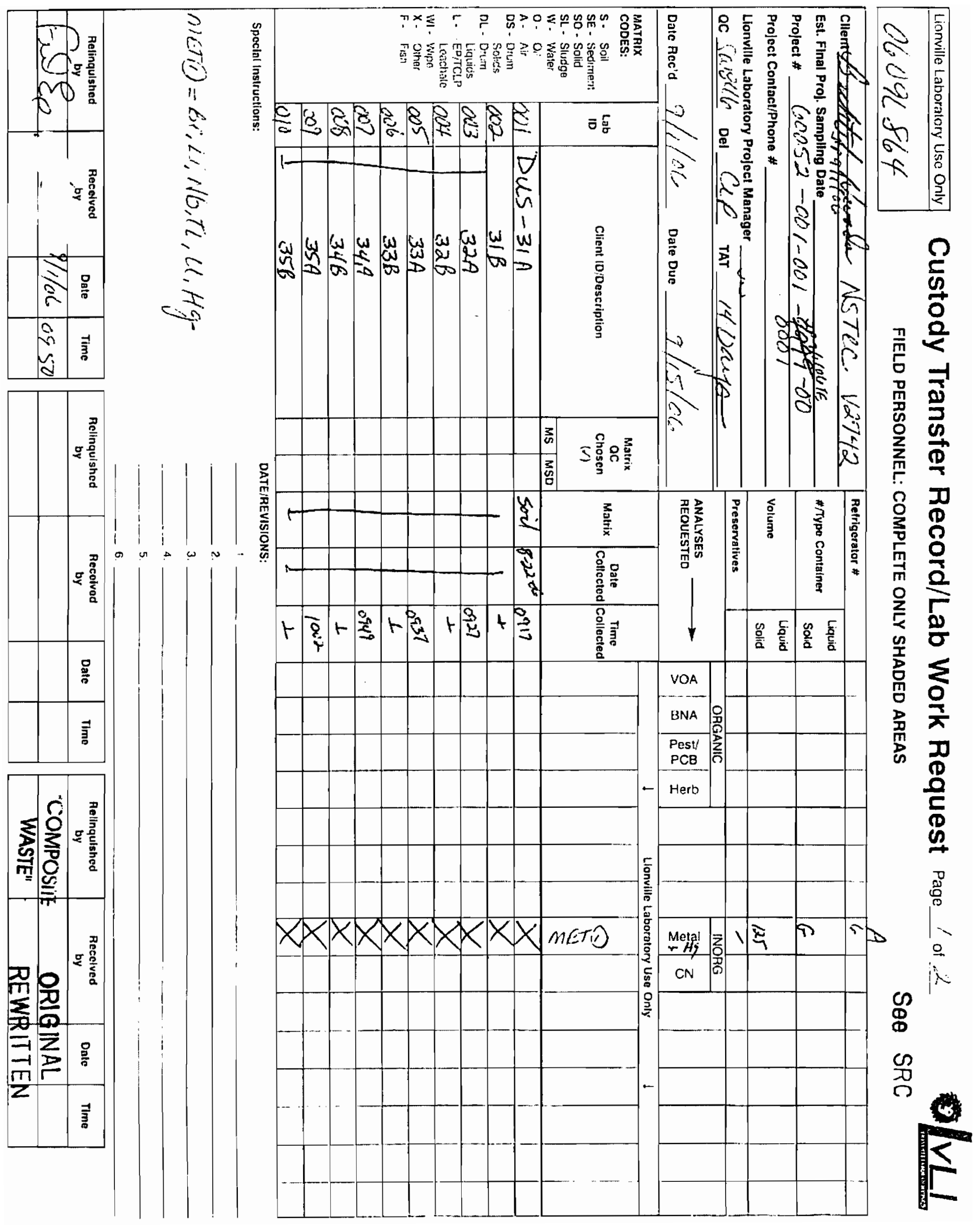




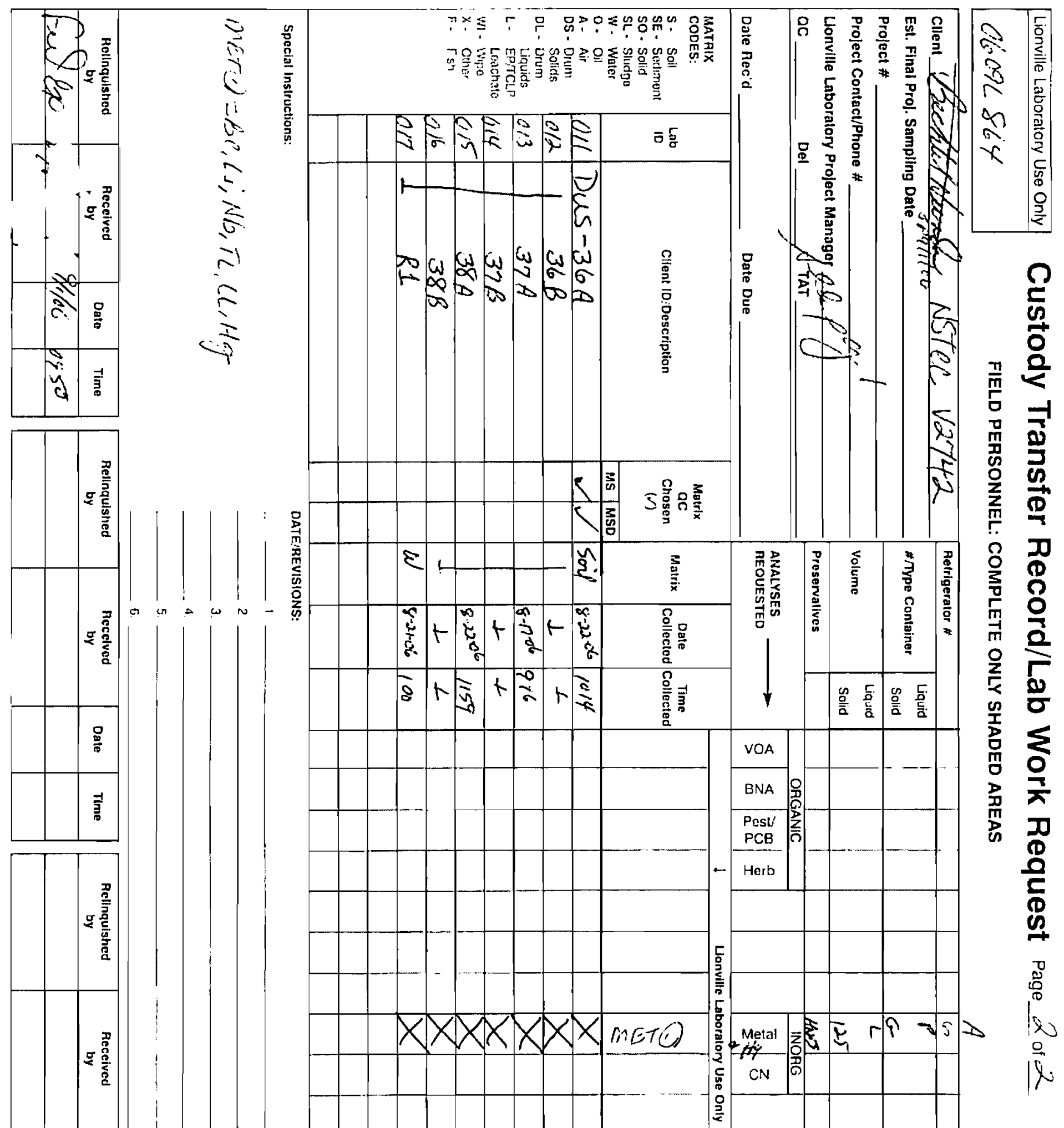




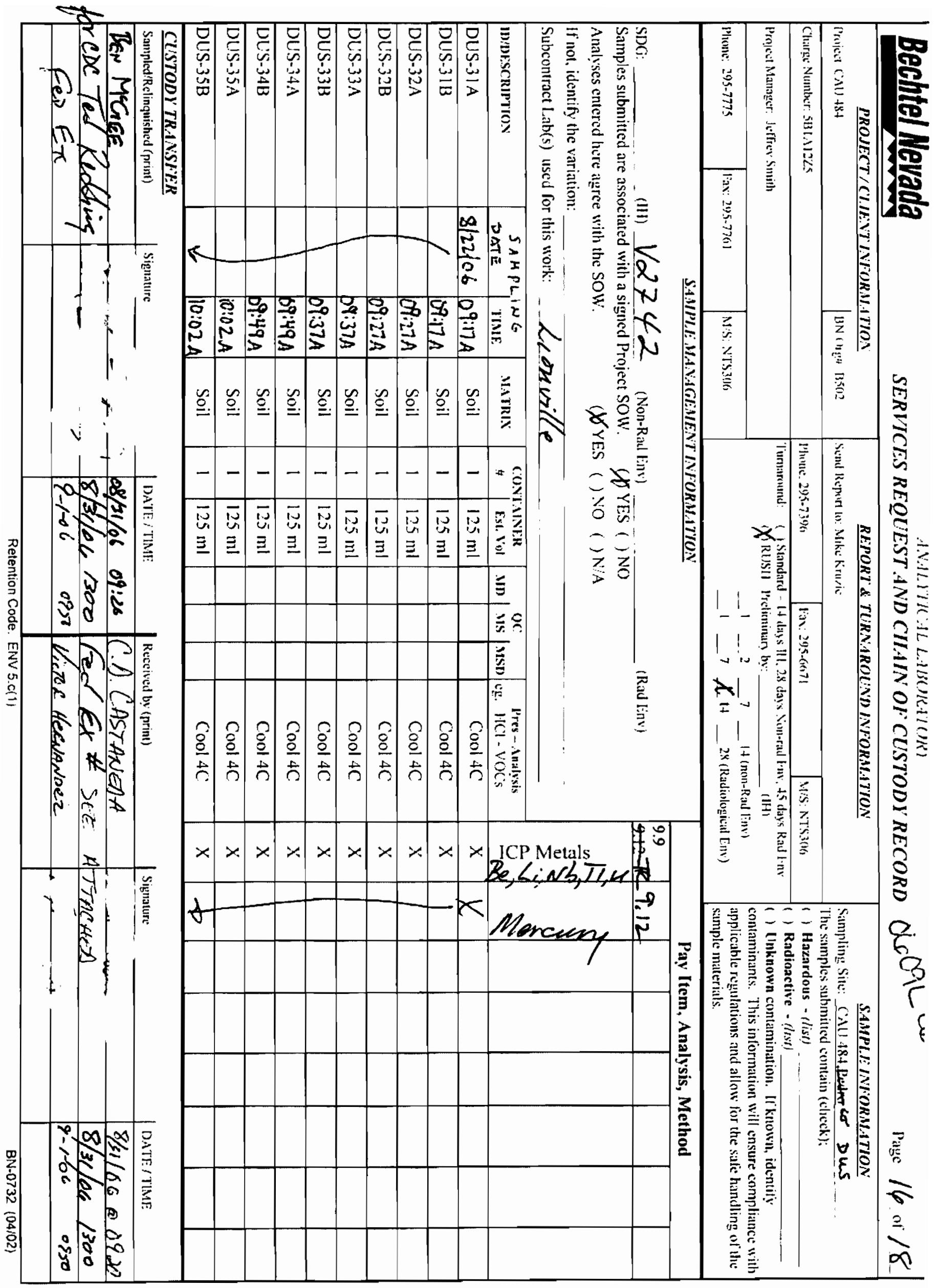




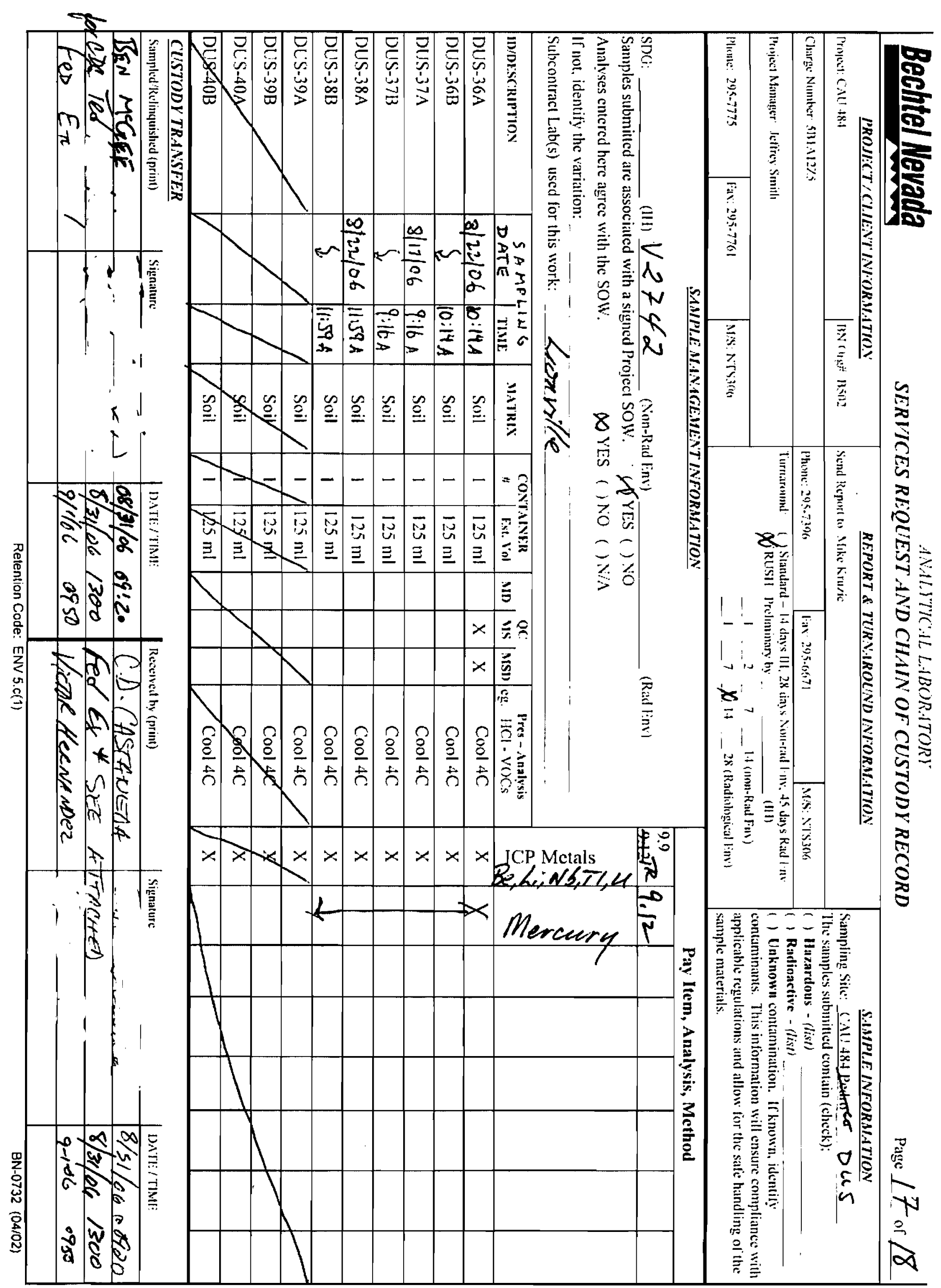




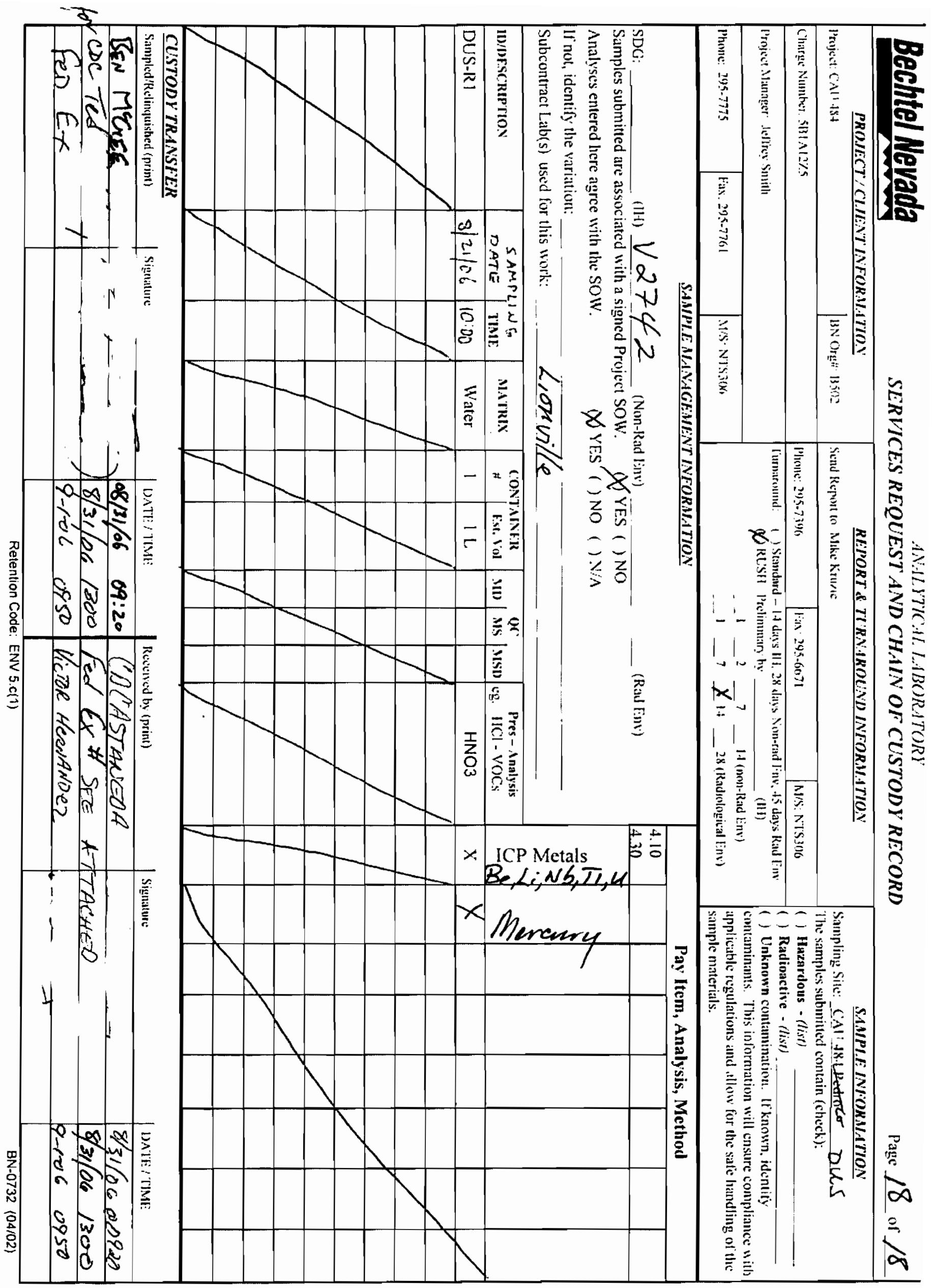




\title{
INVESTIGATION (GEOPROBE ${ }^{\circledR}$ ) SOIL SAMPLE ANALYTICAL RESULTS \\ CAS TA-52-006-TAPL DEPLETED URANIUM SITE
}

\author{
Radiological Data
}




\section{GENERAL ENGINEERING LABORATORIES, LLC \\ 2040 Savage Road Charleston SC 29407 - (843) 556-8171 - www.gel.com}

\section{Certificate of Analysis}

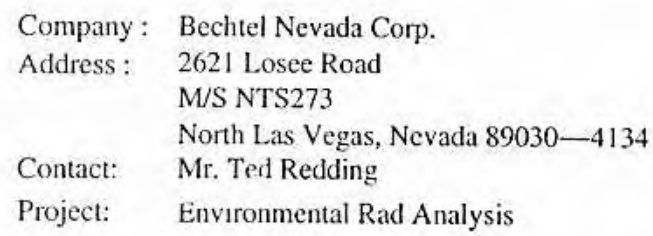

Client Sample ID:

Sample ID:

Matrix:

Collect Date:

Receive Date:

Collector:

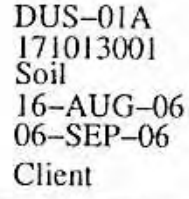

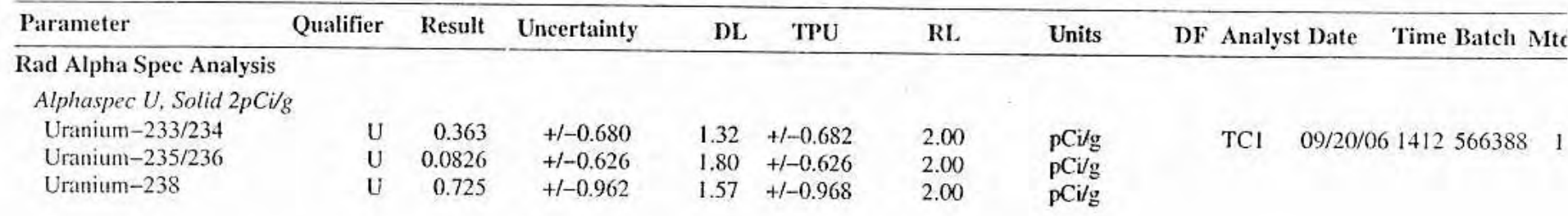

The following Prep Methods were performed

\begin{tabular}{llllll}
\hline Method & Description & Analyst & Date & Time & Sep Batch \\
\hline Dry Soil Prep & Dry Soil Prep GL-RAD-A-021 & AXP2 & $09 / 07 / 06$ & 1321 & 565874
\end{tabular}

The following Analytical Methods were performed

\begin{tabular}{ll} 
Method & Description \\
\hline DOE EML HASL-300, U-02-RC Modified
\end{tabular}

\begin{tabular}{llcc} 
Surrogate/Tracer recovery & Test & Recovery \% & Acceptable Limits \\
\hline Uranium-232 & Alphaspec U, Solid 2pCi/g & 67 & $(25 \%-125 \%)$
\end{tabular}

Notes:

The Qualifiers in this report are defined as follows :

* A quality control analyte recovery is outside of specified acceptance critcria

$<$ Result is less than value reported

$>$ Result is greater than value reported

A The TIC is a suspected aldol-condensation product

B Target analyte was detected in the associated blank

BD Results are either below the MDC or tracer recovery is low

C Analyte has been confirmed by GC/MS analysis

D Results are reported from a diluted aliquot of the sample

$\mathrm{H}$ Analytical holding time was exceeded

$\mathrm{J}$ Value is estimated

N/A Spike recovery limits do not apply. Sample concentration exceeds spike concentration by $4 X$ or more

$\mathrm{R}$ Sample results are rejected

$\mathrm{U}$ Analyte was analyzed for, but not detected above the MDL, MDA, or LOD.

UI Gamma Spectroscopy-Uncertain identification 


\section{GENERAL ENGINEERING LABORATORIES, LLC \\ 2040 Savage Road Charleston SC 29407 - (843) 556-8171 - www.gel.com}

\section{Certificate of Analysis}

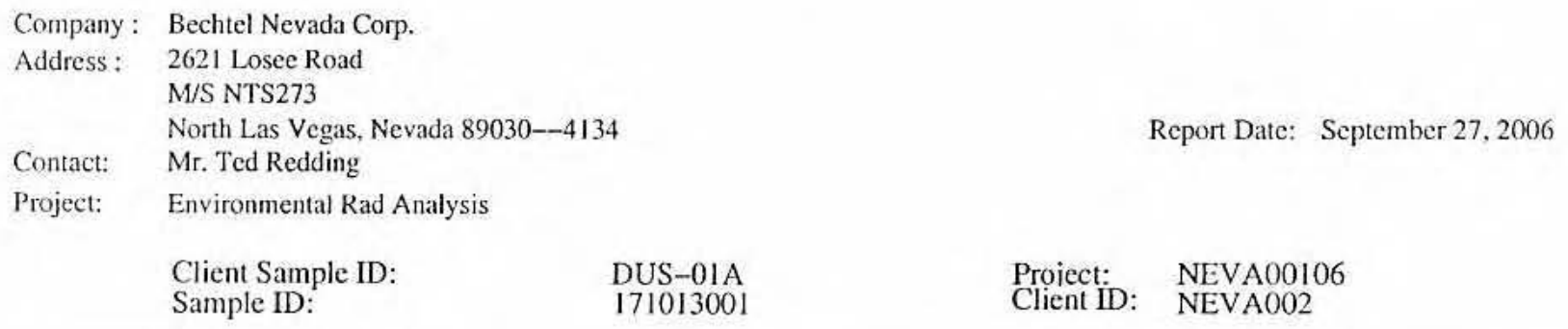

\begin{tabular}{lllllllll}
\hline Parameter & Qualifier & Result & Uncertainty & DL & TPU & RI. & Units & DF Analyst Date Time Batch Mti
\end{tabular}

X Consult Case Narrative, Data Summary package, or Project Manager concerning this qualifier

Y QC Samples were not spiked with this compound

$\wedge$ RPD of sample and duplicate evaluated using $+/-$ RL. Concentrations are $<5 \mathrm{X}$ the RL

h Preparation or preservation holding time was exceeded

The above sample is reported on a dry weight basis. 


\section{GENERAL ENGINEERING LABORATORIES, LLC \\ 2040 Savage Road Charleston SC 29407 - (843) 556-8171 - wrw..gel.com}

\section{Certificate of Analysis}

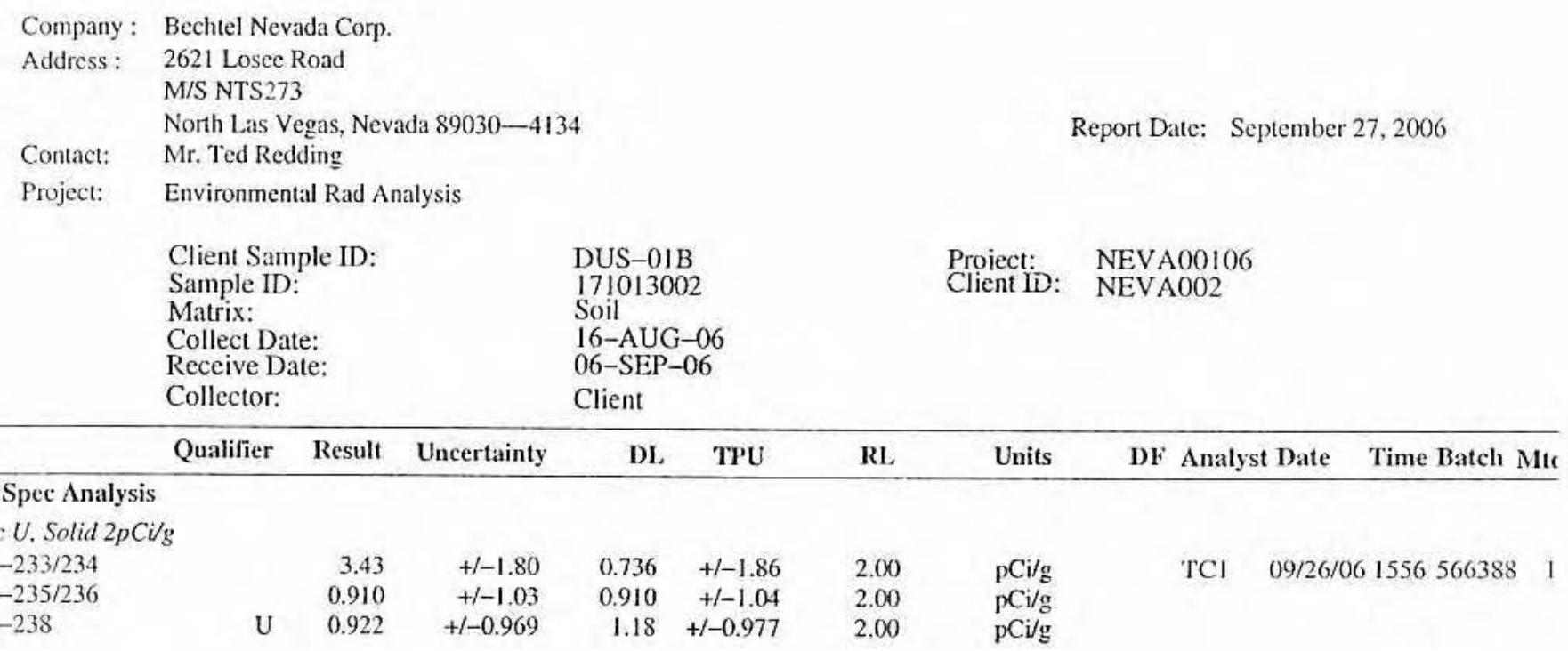

The following l'rep Methods were performed

\begin{tabular}{llllll}
\hline Method & Description & Analyst & Date & Time & Prep Batch \\
\hline Di; Soil Prep & Dry Soil Prep GL-RAD-A-021 & AXP2 & $09 / 07 / 06$ & 1321 & 565874
\end{tabular}

The following Analytical Methods were performed

\begin{tabular}{ll} 
Method & Description \\
\hline 1 & DOE EML HASL-300, U-02-RC Modified \\
2 & DOE EML HASL-300, U-02-RC Modificd
\end{tabular}

\begin{tabular}{llcc} 
Surrogate/Tracer recovery & Test & Recovery $\%$ & Acceptable Limits \\
\hline Uranium-232 & Alphaspec U, Solid $2 \mathrm{pCi} / \mathrm{g}$ & 90 & $(25 \%-125 \%)$
\end{tabular}

Notes:

The Qualifiers in this report are defined as follows:

* A quality control analyte recovery is outside of specified acceptance criteria

$<$ Result is less than value reported

$>$ Result is greater than value reported

A The TIC is a suspected aldol-condensation product

B Target analyte was detected in the associated blank

$\mathrm{BD}$ Results are either below the MDC or tracer recovery is low

C Analyte has been confirmed by GC/MS analysis

D Results are reported from a diluted aliquot of the sample

$\mathrm{H}$ Analytical holding time was exceeded

$\mathrm{J}$ Value is estimated

N/A Spike recovery limits do not apply. Sample concentration exceeds spike concentration by $4 \mathrm{X}$ or more

R Sample results are rejected

$\mathrm{U}$ Analyte was analyzed for, but not detected above the MDL, MDA, or LOD.

UI Gamma Spectroscopy--Uncertain identification 


\section{GENERAL ENGINEERING LABORATORIES, LLC}

2040 Savage Road Charleston SC 29407 - (843) 556-8171 - ww' gel com

\section{Certificate of Analysis}

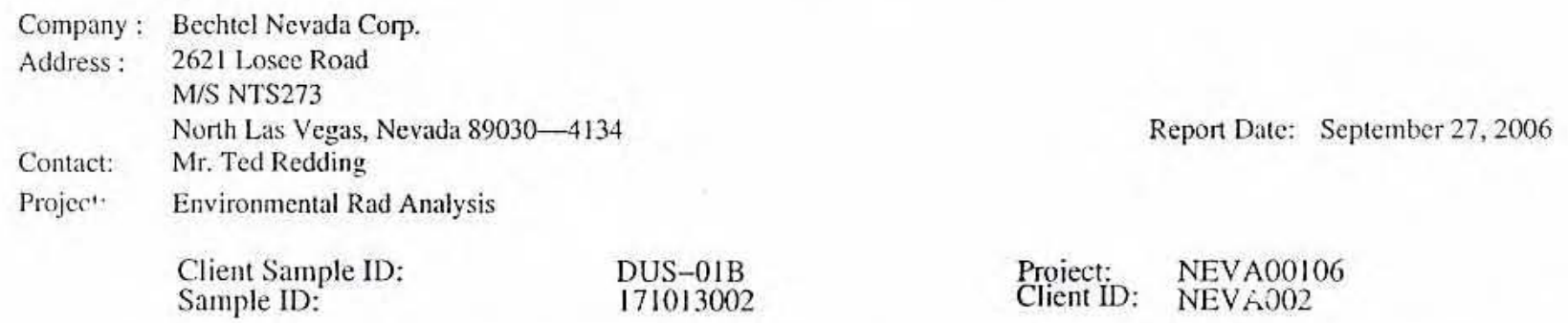

\begin{tabular}{llllllll}
\hline Parameter & Qualifier & Result & Uncertainty & DL & TPU & RL & Units
\end{tabular}

$\mathrm{X}$ Consult Case Narrative, Data Summary package, or Project Manager concerning this qualifier

Y QC Samples were not spiked with this compound

$\wedge \quad \mathrm{RPD}$ of sample and duplicate evaluated using $+/-\mathrm{RL}$. Concentrations are $<5 \mathrm{X}$ the RL

h Preparation or preservation holding time was exceeded

The above sample is reported on a dry weight basis. 


\section{GENERAL ENGINEERING LABORATORIES, LLC}

2040 Savage Road Charleston SC 29407 - (843) 556-8171 - www.ge!.com

\section{Certificate of Analysis}

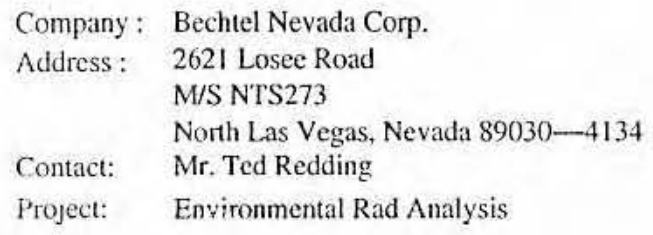

Soil

Client
Report Date: September 27, 2006

16-AUG-06

06-SEP-06

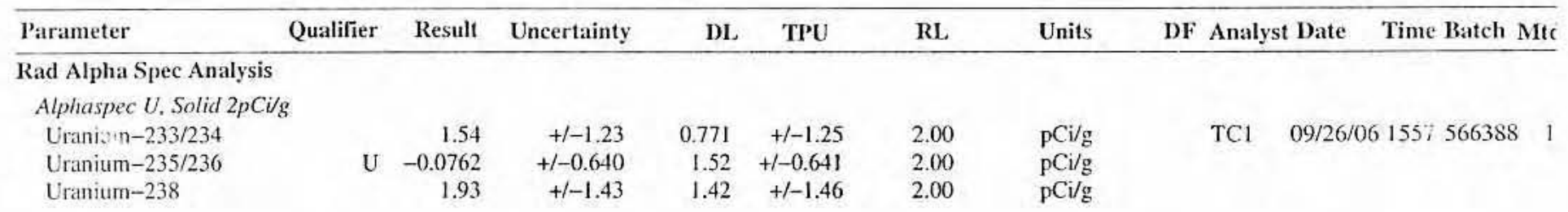

The following P'rep Methods were performed

\begin{tabular}{llllll}
\hline Method & Description & Analyst & Date & Time & Prep Batch \\
\hline Dry Soil Prep & Diy Goil Prep GL-RAD-A-021 & AXP2 & $09 / 07 / 06$ & 1321 & 565874
\end{tabular}

The following Analytical Methods were performed

\begin{tabular}{ll} 
Method & Description \\
\hline 1 & DOE EML HASL-300, U-02-RC Modified \\
2 & DOE EML HASL-300, U-02-RC Modified
\end{tabular}

$\begin{array}{llll}\text { Surrogate/Tracer recovery } & \text { Test } & \text { Recovery } \% & \text { Acceptable Limits }\end{array}$

\begin{tabular}{llll}
\hline Uranium-232 Alphaspec U, Solid 2pCi/g & 93 & $(25 \%-125 \%)$
\end{tabular}

Notes:

The Qualifiers in this report are defined as follows :

* A quality control analyte recovery is outside of specified acceptance criteria

$<$ Result is less than value reported

$>$ Result is greater than value reported

A The TIC is a suspected aldol-condensation product

B Target analyte was detected in the associated blank

$\mathrm{BD}$ Results are either below the MDC or tracer recovery is low

C Analyte has been confirmed by GC/MS analysis

D Results are reported from a diluted aliquot of the sample

$\mathrm{H}$ Analytical holding time was exceeded

$\mathrm{J}$ Value is estimated

N/A Spike recovery limits do not apply. Sample concentration exceeds spike concentration by $4 \mathrm{X}$ or more

$R$ Sample results are rejected

U Analyte was analyzed for, but not detected above the MDL, MDA, or LOD.

U1 Gamma Spectroscopy-Uncertain identification 


\section{GENERAL ENGINEERING LABORATORIES, LLC \\ 2040 Savage Road Charleston SC 29407 - (843) 556-8171 - www.gel.com}

\section{Certificate of Analysis}

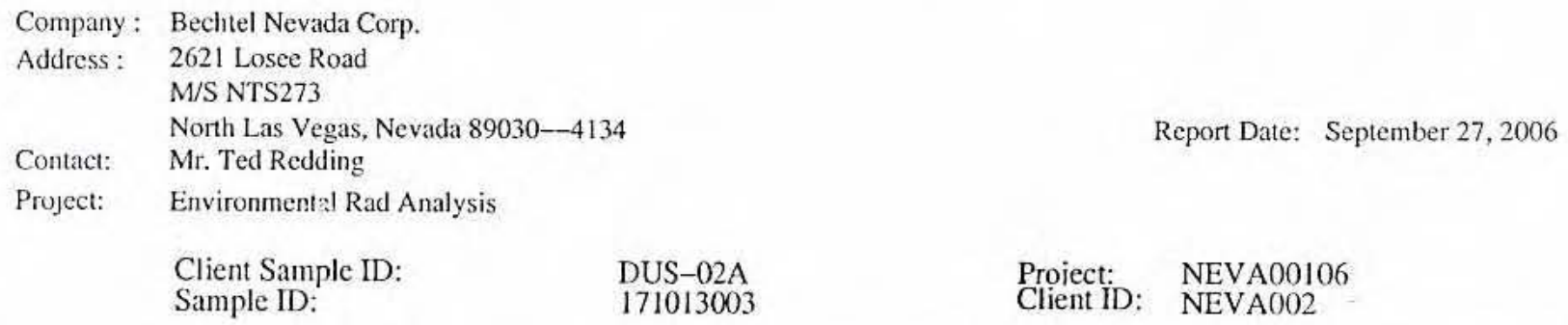

$\mathrm{X}$ Consult Case Narrative, Data Summary package, or Project Manager concerning this qualifier

$\mathrm{Y}$ QC Sami 'es were not spiked with this compound

$\wedge$ RPD of sample and duplicate evaluated using $+/-$ RL. Concentrations are $<5 \mathrm{X}$ the $\mathrm{RL}$

$\mathrm{h}$ Preparation or preservation holding time was exceeded

The above sa. le is reported on a dry weight basis. 


\section{GENERAL ENGINEERING LABORATORIES, LLC \\ 2040 Savage Road Charleston SC 29407 - (843) 556-8171 - www.gel.com}

\section{Certificate of Analysis}

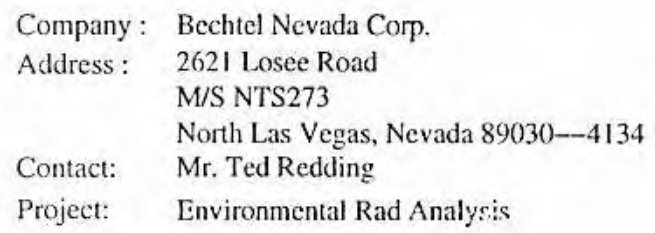

Report Date: September 27,2006

Proiect: NEVA00106

Client ID: NEVA002

\begin{tabular}{|c|c|c|c|c|c|c|c|c|c|c|c|}
\hline Parameter & Qualifier & Result & Uncertainty & DL & TPU & RL & Units & DF & Analyst & Date & Time Batch Mtc \\
\hline \multicolumn{12}{|c|}{ Rad Alpha Spec Analysis } \\
\hline \multicolumn{12}{|c|}{ Alphaspec U. Solid $2 p C \ddot{g} g$} \\
\hline Uranium-233/234 & & 1.55 & $+1-0.968$ & 0.864 & $+/-0.986$ & 2.00 & $\mathrm{pCi} / \mathrm{g}$ & & $\mathrm{TCl}$ & $09 / 23 / 0$ & $61411566388 \quad$ । \\
\hline Uranium-235/236 & $\mathrm{U}$ & -0.184 & $+1-0.438$ & 1.28 & $+1-0.438$ & 2.00 & $\mathrm{pCi} / \mathrm{g}$ & & & & \\
\hline Uranium-238 & $\mathrm{U}$ & 0.146 & $+1-0.457$ & 1.04 & $+1-0.458$ & 2.00 & $\mathrm{pCi} / \mathrm{g}$ & & & & \\
\hline
\end{tabular}

The following Prep Methods were performed

\begin{tabular}{llllll}
\hline Method & Description & Analyst & Date & Time & Prep Batch \\
\hline Dry Soil Prep & Dry Soil Prep GL-RAD-A-v21 & AXP2 & $09 / 07 / 06$ & 1321 & 565874
\end{tabular}

The following An-lytical Methods were periormed

\begin{tabular}{ll} 
Method & Description \\
\hline 1 & DOE EML HASL-300, U-02-RC Modified
\end{tabular}

\begin{tabular}{llcc} 
Surrogate/Tracer recovery & Test & Recovery \% & Acceptable Limits \\
\hline Uranium-232 & Alphaspec U, Solid 2pCi/g & 87 & $(25 \%-125 \%)$
\end{tabular}

Notes:

The Qualifiers in this report are defined as follows :

* A quality control analyte recovery is outside of specified acceptance criteria

$<$ Result is less than value reported

$>$ Result is greater than value reported

A The TIC is a suspected aldol-condensation product

B Target analyte was detected in the associated blank

$\mathrm{BD}$ Results are either below the MDC or tracer recovery is low

C Analyte has been confirmed by GC/MS analysis

D Results are reported from a diluted aliquot of the sample

$\mathrm{H}$ Analytical holding time was exceeded

$\mathrm{J}$ Value is estimated

N/A Spike recovery limits do not apply. Sample concentration exceeds spike concentration by $4 X$ or more

$\mathrm{R}$ Sample results are rejected

U Analyte was analyzed for, but not detected above the MDL, MDA, or LOD.

UI Gamma Spectroscopy-Uncertain identification 


\section{GENERAL ENGINEERING LABORATORIES, LLC \\ 2040 Savage Road Charleston SC 29407 - (843) 556-8171 - www.gel.com}

\section{Certificate of Analysis}

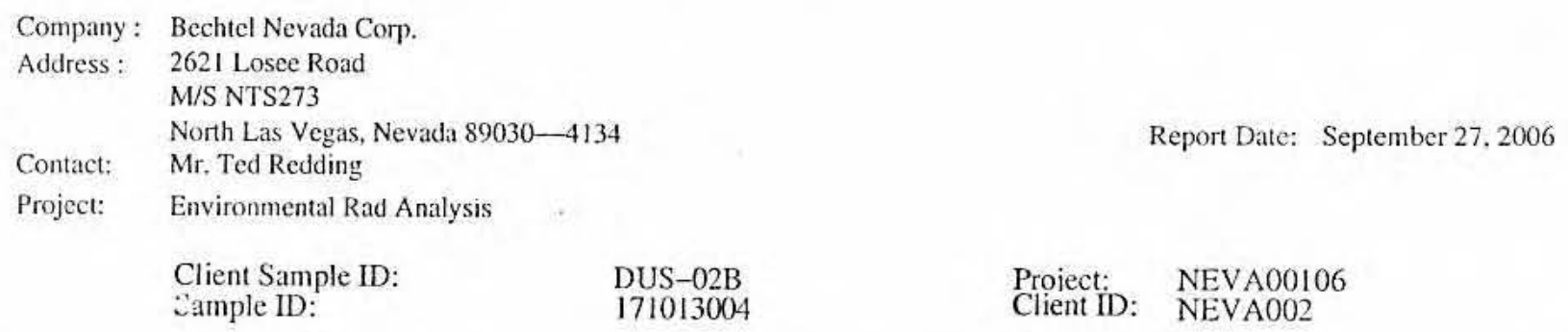

$\wedge \quad$ RPD of sample and duplicate evaluated using $+/-\mathrm{RL}$. Concentrations are $<5 X$ the $R L$

h Preparation or preservation holding time was exceeded

The above sample is reported on a dry weight basis. 


\section{GENERAL ENGINEERING LABORATORIES, LLC}

2040 Savage Road Charleston SC 29407 - (843) 556-8171 - www.gel.com

\section{Certificate of Analysis}

\author{
Company : Bechtel Nevada Corp. \\ Address: 2621 Losee Road \\ M/S NTS273 \\ North Las Vegas, Nevada 89030-4134 \\ Contact: Mr. Ted Redding \\ Froject: Environmental Rad Analysis
}

Report Date: September 27,2006

DUS-03A
171013005
Soil
16-AUG-06
06-SEP-06
Client

Client Sample ID: DUS-03A Proiect: NEVA00106

Matrix:

Collect Date:

Receive Date:

Collector:
Client ID: NEVA002

\begin{tabular}{|c|c|c|c|c|c|c|c|c|c|c|c|c|}
\hline Parameter & Qualifier & Result & Uncertainty & DL & TPU & RL & Units & DF & Analyst & Date & Time Batch $\mathrm{M}$ & Mtc \\
\hline \multicolumn{13}{|c|}{ Rad Alpha Spec Analysis } \\
\hline \multicolumn{13}{|c|}{ Alphaspec U, Solid $2 p C i / g$} \\
\hline Uranium-233/234 & $\mathrm{U}$ & 1.31 & $+1-1.41$ & 2.19 & $+1-1.42$ & 2.00 & $\mathrm{pCi} / \mathrm{g}$ & & $\mathrm{TCl}$ & $09 / 20 / 0$ & 61412566388 & 1 \\
\hline Uranium-235/236 & $\mathrm{U}$ & 0.544 & $+1-1.07$ & 2.12 & $+1-1.08$ & 2.00 & $\mathrm{pCi} / \mathrm{g}$ & & & & & \\
\hline Uranium-238 & $\mathrm{U}$ & -0.108 & $+1-0.556$ & 1.81 & $+1-0.556$ & 2.00 & $\mathrm{pCi} / \mathrm{g}$ & & & & & \\
\hline
\end{tabular}

The following Prep Methods were performed

\begin{tabular}{llllll}
\hline Method & Description & Analyst & Date & Time & Prep Batch \\
\hline Dry Soil Prep & Dry Soil Prep GL-RAD-A-021 & AXP2 & $09 / 07 / 06$ & 1321 & 565874
\end{tabular}

The following Analytical Methods were performed

\begin{tabular}{ll} 
Method & Description \\
\hline DOE EML HASL-300, U-02-RC Modified
\end{tabular}

\begin{tabular}{llcc} 
Surrogate/Tracer recovery & Test & Recovery $\%$ & Acceptable Linits \\
\hline Uranium-232 & Alphaspec U, Solid 2pCi/g & 84 & $(25 \%-125 \%)$
\end{tabular}

Notes:

The Qualifiers in this report are defined as follows:

* A quality control analyte recovery is outside of specified acceptance criteria

$<$ Result is less than value reported

$>$ Result is greater than value reported

A The TIC is a suspected aldol-condensation product

B Target analyte was detected in the associated blank

$\mathrm{BD}$ Results are either below the MLC or tracer recovery is low

C Analyte has been confirmed by GC/MS analysis

D Results are reported from a diluted aliquot of the sample

$\mathrm{H}$ Analytical holding time was exceeded

$\mathrm{J}$ Value is estimated

N/A Spike recovery limits do not apply. Sample concentration exceeds spike concentration by $4 \mathrm{X}$ or more

$\mathrm{R} \quad$ Sample results are rejected

U Analyte was analyzed for, but not detected above the MDL, MDA, or LOD.

UI Gamma Spectroscopy--Unccrtain identification

X Consult Case Narrative, Data Summary package, or Project Manager conceming this qualifier 


\section{GENERAL ENGINEERING LABORATORIES, LLC}

2040 Savage Road Charleston SC 29407 - (843) 556-8171 - www.gel.com

\section{Certificate of Analysis}

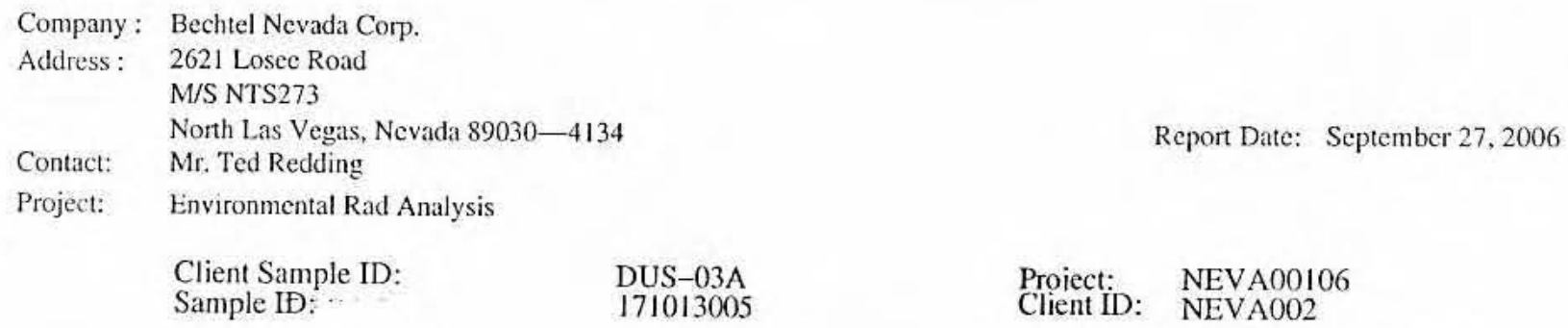

RL.

Units

DF Analyst Date

Time Batch Mtc

Y QC Samples were not spiked with this compound

$\wedge$ RPD of sample and duplicate evaluated using $+/-\mathrm{Ri}$. Concentrations are $<5 \mathrm{X}$ the RL

h Preparation or preservation holding time was exceeded

The above sample is reported on a dry weight basis. 


\section{GENERAL ENGINEERING LABORATORIES, LLC \\ 2040 Savage Road Charlesion SC 29407 - (843) 556-8171 - www.gel.com}

\section{Certificate of Analysis}

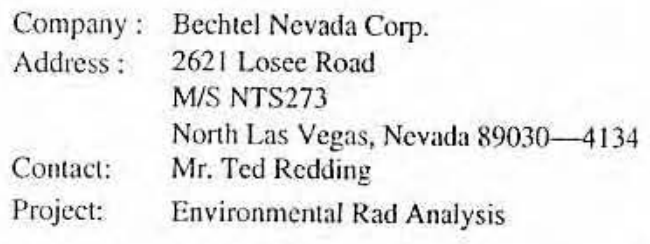

Client
Report Date: September 27, 2006

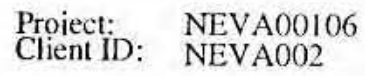

\begin{tabular}{|c|c|c|c|c|c|c|c|c|c|c|c|c|}
\hline Parameter & Qualifier & Result & Uncertainty & DL & TPU & RL & Units & DF & Analys & t Date & Time Batch Mt & Mtc \\
\hline Rad Alpha Spec Ana & & & & & & & & & & & & \\
\hline Alphaspec U, Solid & & & & & & & & & & & & \\
\hline Uranium-233/234 & & 2.25 & $+/-1.20$ & 1.06 & $+1-1.23$ & 2.00 & $\mathrm{pCi} / \mathrm{g}$ & & $\mathrm{TCl}$ & $09 / 23 / 06$ & 61411566388 & \\
\hline Uranium-235/236 & & 0.744 & $+/-0.729$ & 0.558 & $+/-0.734$ & 2.00 & $\mathrm{pCi} / \mathrm{g}$ & & & & & \\
\hline Uranium-238 & & 2.11 & $+1-1.10$ & 0.451 & $+1-1,13$ & 2.00 & $\mathrm{pCi} / \mathrm{g}$ & & & & & \\
\hline
\end{tabular}

The following Prep Methods were performed

\begin{tabular}{|c|c|c|c|c|c|}
\hline Method & Description & Analyst & Date & Time & Prep Batch \\
\hline Dry Soil Prep & Dry Soil Prep GL-RAD-A-021 & $\mathrm{AXP2}$ & $09 / 07 / 06$ & 1321 & 565874 \\
\hline \multicolumn{6}{|c|}{ The following Analytical Methods were performed } \\
\hline Method & Description & & & & \\
\hline 1 & DOE EML HASL-3(X), U-02-RC Modified & & & & \\
\hline 2 & DOE EML HASL-300, 11-02-RC Modified & & & & \\
\hline Surrogate/Tr & recovery & Recovery\% & \multicolumn{3}{|c|}{ Acceptable Limits } \\
\hline Uranium-232 & Alphaspec U, Solid 2pCi/g & 85 & \multicolumn{3}{|c|}{$(25 \%-125 \%)$} \\
\hline
\end{tabular}

Notes:

The Qualifiers in this report are defined as follows :

* A quality control analyte recovery is outside of specified acceptance criteria

$<$ Result is less than value reported

$>$ Result is greater than value reported

A The TIC is a suspected aldol-condensation product

B Target analyte was detected in the associated blank

BD Results are either below the MDC or tracer recovery is low

C Analyte has been confirmed by GC/MS analysis

D Results are reported from a diluted aliquot of the sample

$\mathrm{H}$ Analytical holding time was exceeded

$\mathrm{J}$ Value is estimated

N/A Spike recovery limits do not apply. Sample concentration exceeds spike concentration by $4 X$ or more

$R$ Sample results are rejected

U Analyte was analyzed for, but not detected above the MDL, MDA, or LOD.

UI Gamma Spectroscopy--Uncertain identification 


\section{GENERAL ENGINEERING LABORATORIES, LLC \\ 2040 Savage Road Charleston SC 29407 - (843) 556-8171 - www.gel.com}

\section{Certificate of Analysis}

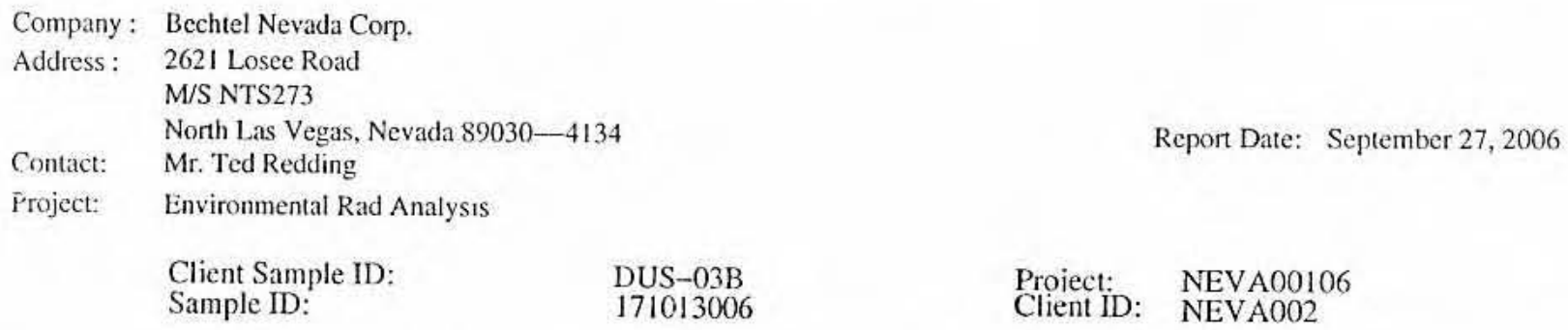

DL TPU

RL

Units

DF Analyst Date

Time Batch Mtc

X Consult Case Narrative, Data Summary package, or Project Manager concerning this qualifier

Y QC Samples were not spiked with this compound

$\wedge$ RPD of sample and duplicate evaluated using $+/-$ RL. Concentrations are $\angle 5 X$ the RL

h Preparation or preservation holding time was exceeded

The above sample is reported on a dry weight basis. 


\section{GENERAL ENGINEERING LABORATORIES, LLC \\ 2040 Savage Road Charleston SC 29407 - (843) 556-8171 - www.gel.com}

\section{Certificate of Analysis}

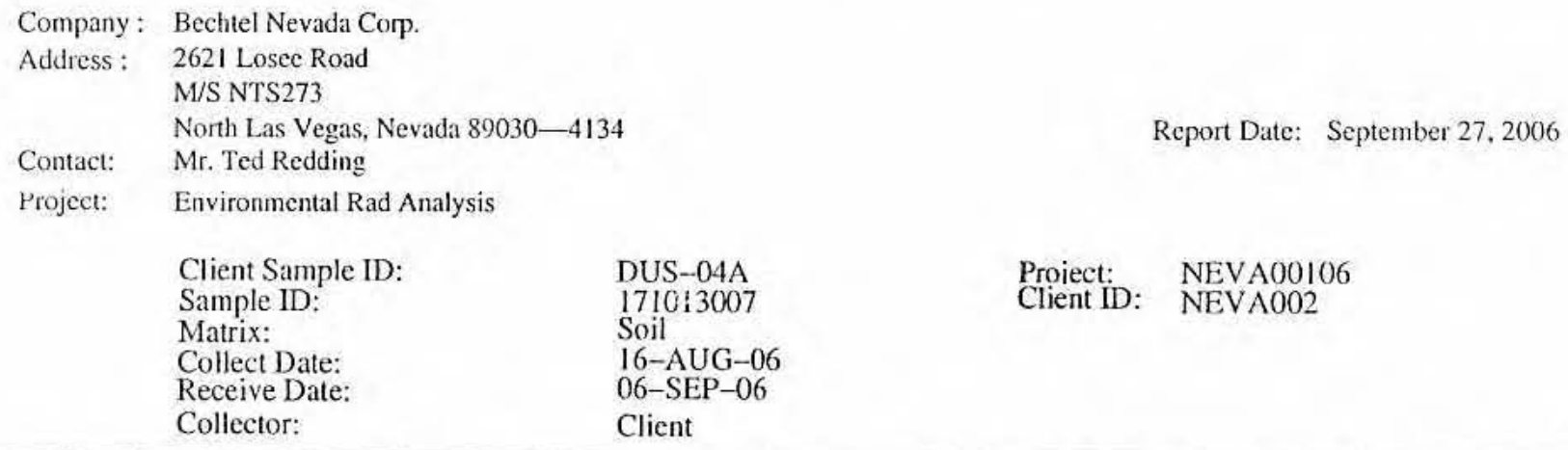

\begin{tabular}{|c|c|c|c|c|c|c|c|c|c|c|c|}
\hline Parameter & Qualifier & Result & Uncertainty & DL & TPU & RL & Units & DF & \multicolumn{3}{|c|}{ Analyst Date } \\
\hline \multicolumn{12}{|c|}{ Rad Alpha Spec Analysis } \\
\hline \multicolumn{12}{|c|}{ Alphaspec U, Solid $2 p C i / g$} \\
\hline Uranium-233/234 & $\mathrm{U}$ & 1.18 & $+1-1.11$ & 1.57 & $+/-1.12$ & 2.00 & $\mathrm{pCV} / \mathrm{g}$ & & $\mathrm{TCl}$ & $09 / 20 / 0$ & 61412566388 \\
\hline Uranium-235/236 & $\mathrm{U}$ & 0.364 & $+1-0.683$ & 1.33 & $+1-0.685$ & 2.00 & $\mathrm{pCi} / \mathrm{g}$ & & & & \\
\hline Uranium-238 & & 1.56 & $+/-1.15$ & 128 & $+1-1.18$ & 2.00 & pCig & & & & \\
\hline
\end{tabular}

The following Prep Methods were performed

\begin{tabular}{|c|c|c|c|c|c|}
\hline Method & Description & Analyst & Date & Time & Prep Batch \\
\hline Dry Soil Prep & Dry Soil Prep GL-RAD-A-021 & AXP2 & $09 / 07 / 06$ & 1.321 & 565874 \\
\hline \multicolumn{6}{|c|}{ The following Analytical Methods were performed } \\
\hline Method & Description & & & & \\
\hline
\end{tabular}

$\begin{array}{llll}\text { Surrogate/Tracer recovery } & \text { Test Recovery\% Acceptable Limits }\end{array}$

$\begin{array}{llll}\text { Uranium-232 } & \text { Alphaspec U, Solid 2pCi/g } & 78 & (25 \%-125 \%)\end{array}$

Notes:

The Qualifiers in this report are defined as follows:

* A quality control analyte recovery is outside of specified acceptance criteria

$<$ Result is less than value reported

$>$ Result is greater than value reported

A The TIC is a suspected aldol-condensation product

B Target analyte was detected in the associated blank

$\mathrm{BD}$ Results are either below the MDC or tracer recovery is low

C Analyte has been confirmed by GC/MS analysis

D Results are reported from a diluted aliquot of the sample

$\mathrm{H}$ Analytical holding time was exceeded

$\mathrm{J}$ Value is estimated

N/A Spike recovery limits do not apply. Sample concentration exceeds spike concentration by $4 X$ or more

$\mathrm{R}$ Sample results are rejected

U Analyte was analyzed for, but not detected above the MDL, MDA, or LOD.

UI Gamma Spectroscopy-Uncertain identification

X Consult Case Narrative, Data Summary package, or Project Manager concerning this qualifier 


\section{GENERAL ENGINEERING LABORATORIES, LLC \\ 2040 Savage Road Charleston SC 29407 - (843) 556-8171 - www.gel.com}

\section{Certificate of Analysis}

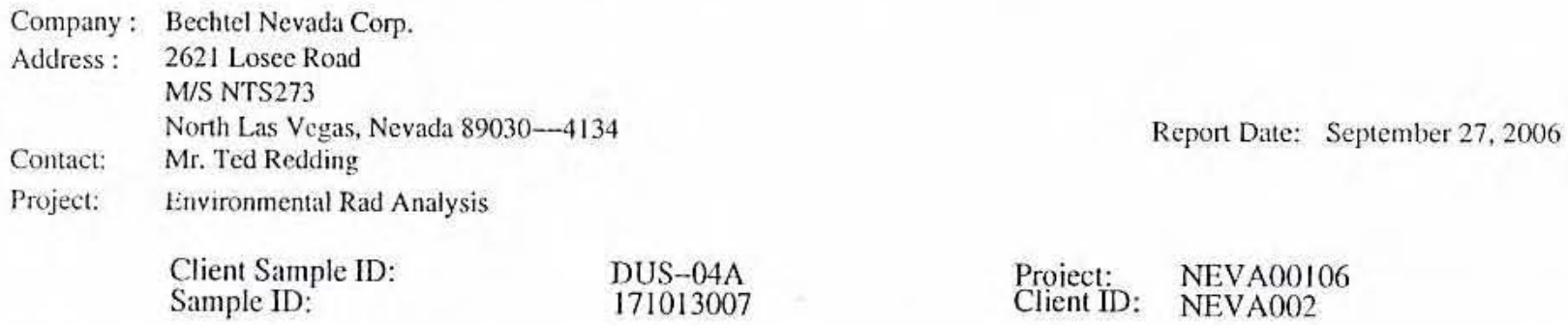

\begin{tabular}{lllllll}
\hline Parameter & Qualinier Result Uncertainty & DL & TPU & RL & Units & DF Analyst Date Time Batch Mti
\end{tabular}

Y QC Samples were not spiked with this compound

$\wedge \quad \mathrm{RPD}$ of sample and duplicate evaluated using $+/-\mathrm{RL}$. Concentrations are $<5 \mathrm{X}$ the RL

$\mathrm{h}$ Preparation or preservation holding time was exceeded

The above sample is reported on a dry weight basis. 


\section{GENERAL ENGINEERING LABORATORIES, LLC \\ 2040 Savage Road Charleston SC 29407 - (843) 556-8171 - www.gel.com}

\section{Certificate of Analysis}

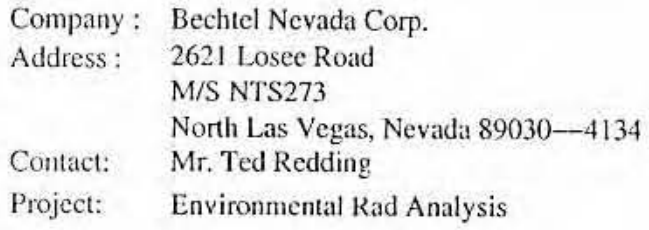

Client

\begin{tabular}{|c|c|c|c|c|c|c|c|c|c|c|c|}
\hline Parameter & Qualifier & Result & Uncertainty & DL & TPU & RL & Units & DF & Analys & Date & Time Batch Mti \\
\hline \multicolumn{12}{|c|}{ Rad Alpha Spec Analysis } \\
\hline \multicolumn{12}{|c|}{ Alphaspec $U$, Solid $2 p C i / g$} \\
\hline Uranium-233/234 & & 3.83 & $+1-1.63$ & 1.40 & $+1-1.69$ & 2.00 & $\mathrm{pCi} / \mathrm{g}$ & & $\mathrm{TCl}$ & $09 / 25 / 0$ & $61025566388 \quad 1$ \\
\hline Uranium-235/236 & U & 0.690 & $+1-0.798$ & 1.16 & $+/-0.803$ & 2.00 & $\mathrm{pCi} / \mathrm{g}$ & & & & \\
\hline Uranium-238 & & 2.63 & $+/-1.30$ & 0.937 & $+1-1.34$ & 2.00 & $\mathrm{pCi} / \mathrm{g}$ & & & & \\
\hline
\end{tabular}

The following Prep Methods were performed

\begin{tabular}{llllll}
\hline Method & Description & Analyst & Date & Time & Prep Batch \\
\hline Dry Soil Prep & Dry Soil Prep GL-RAD-A-021 & AXP2 & $09 / 07 / 06$ & 1321 & 565874
\end{tabular}

The following Analytical Methods were performed

\begin{tabular}{ll}
\hline Method & Description \\
\hline 1 & DOE EML HASL-300, U-02-RC Modified \\
2 & DOE EML HASL-300, U-02-RC Modified
\end{tabular}

$\begin{array}{llll}\text { Surrogate/Tracer recovery } & \text { Test Recovery\% Acceptable Limits }\end{array}$

Uranium-232 Alphaspec U, Solid 2pCi/g $\quad 83 \quad(25 \%-125 \%)$

Notes:

The Qualifiers in this report are defined as follows:

* A quality control analyte recovery is outside of specified acceptance criteria

$<$ Result is less than value reported

$>$ Result is greater than value reported

A The TIC is a suspected aldol-condensation product

B Target analyte was detected in the associated blank

BD Results are either below the MDC or tracer recovery is low

C Analyte has been confinned by GC/MS analysis

D Results are reported from a diluted aliquot of the sample

$\mathrm{H}$ Analytical holding time was exceeded

J Value is estimated

N/A Spike recovery limits do not apply. Sample concentration exceeds spike concentration by $4 \mathrm{X}$ or more

R Sample results are rejected

U Analyte was analyzed for, but not detected above the MDL, MDA, or LOD.

UI Gamma Spectroscopy--Uncertain identification 


\section{GENERAL ENGINEERING LABORATORIES, LLC \\ 2040 Savage Road Charleston SC 29407 - (843) 556-8171 - www.gel.com}

\section{Certificate of Analysis}

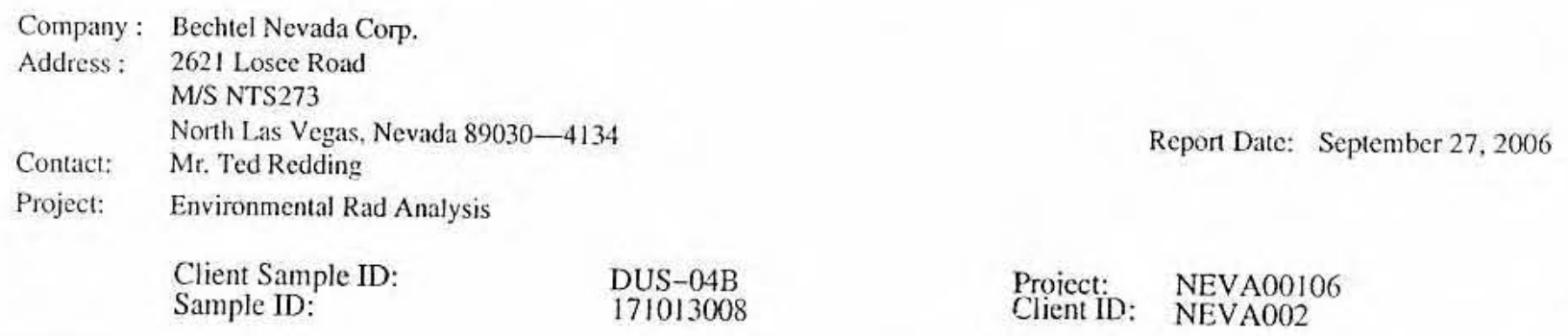

\begin{tabular}{lllllll}
\hline Parameter & Qualifier Result Uncertainty & DL & TPU & RL & Units & DF Analyst Date Time Batch Mtc
\end{tabular}

X Consult Case Narrative, Data Summary package, or Project Manager concerning this qualifier

Y QC Samples were not spiked with this compound

$\hat{R}$ RPD of sample and duplicate evaluated using $+/-\mathrm{RL}$. Concentrations are $<5 \mathrm{X}$ the RL

h Preparation or preservation holding time was exceeded

The above sample is reported on a dry weight basis. 


\section{GENERAL ENGINEERING LABORATORIES, LLC \\ 2040 Savage Road Charleston SC 29407 - (843) 556-8171 - wsw.gel.com}

\section{Certificate of Analysis}

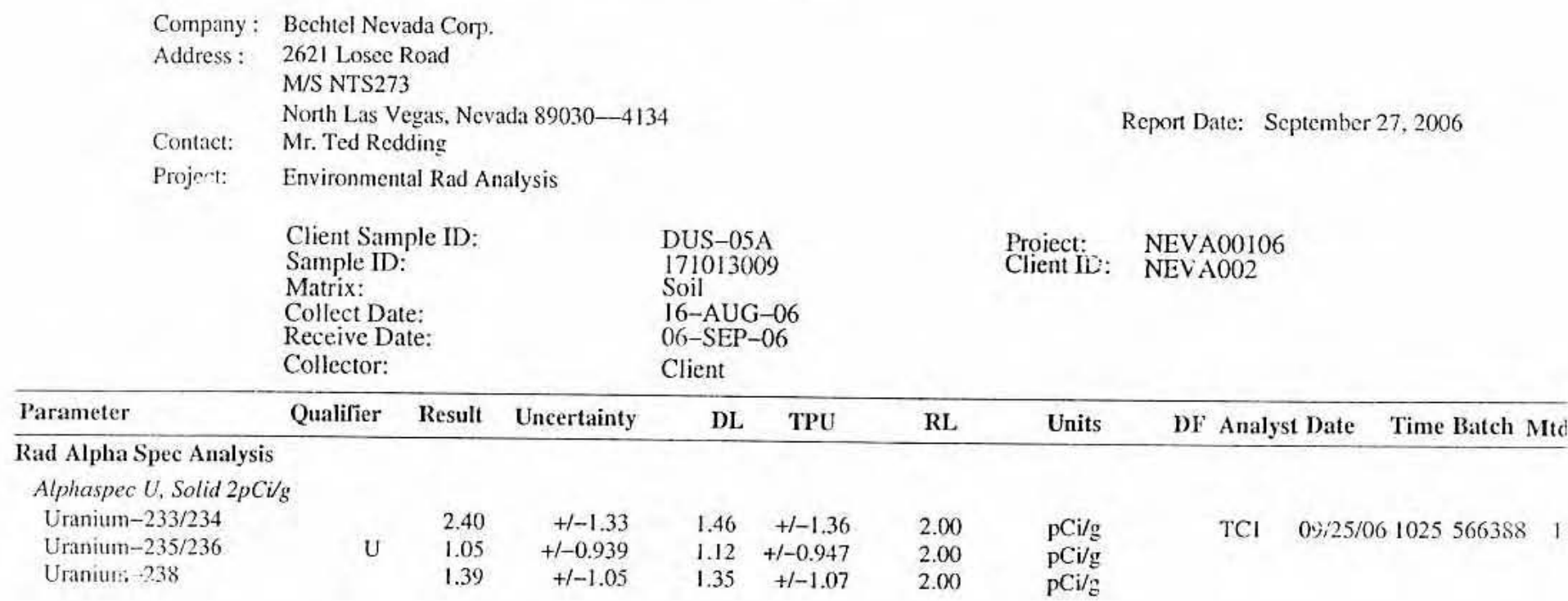

The following Prep Methods were performed

\begin{tabular}{llllll}
\hline Method & Description & Analyst & Date & Time & Prep Batch \\
\cline { 1 - 5 } & Dry Soi! 1 $\mathrm{N}_{\mathrm{p}}$ & AXP2 & $09 / 07 / 06$ & 1321 & 565874
\end{tabular}

The following Analytical Methods were performed

\begin{tabular}{ll} 
Method & Description \\
\hline 1 & DOE EML HASL-300, U-02-RC Modified \\
2 & DOE EML HASL-300, U-02-RC Modified
\end{tabular}

$\begin{array}{llll}\text { Surrogate/Tracer recovery } & \text { Test } & \text { Recovery } \% & \text { Acceptable Limits }\end{array}$

Notes:

The Qualifiers in this repert are defined as follows :

* A quality control analyte recovery is outside of specified acceptance criteria

$<$ Result is less than value reported

$>$ Result is greater than value reported

A The TIC is a suspected aldol-condensation product

B Target analyte was detected in the associated blank

BD Results are either below the MDC or tracer recovery is low

C Analyte has been confirmed by GC/MS analysis

D Results are reported from a diluted aliquot of the sample

$\mathrm{H}$ Analytical holding time was exceeded

$\mathrm{J}$ Value is estimated

N/A Spike recovery limits do not apply. Sample concentration exceeds spike concentration by $4 X$ or more

$\mathrm{R}$ Sample results are rejected

U Analyte was analyzed for, but not detected above the MDL, MDA, or LOD.

UI Gamma Spectroscopy-Uncertain identification 


\section{GENERAL ENGINEERING LABORATORIES, LLC \\ 2040 Savage Road Charleston SC 29407 - (843) 556-8171 - www.gel.com}

\section{Certificate of Analysis}

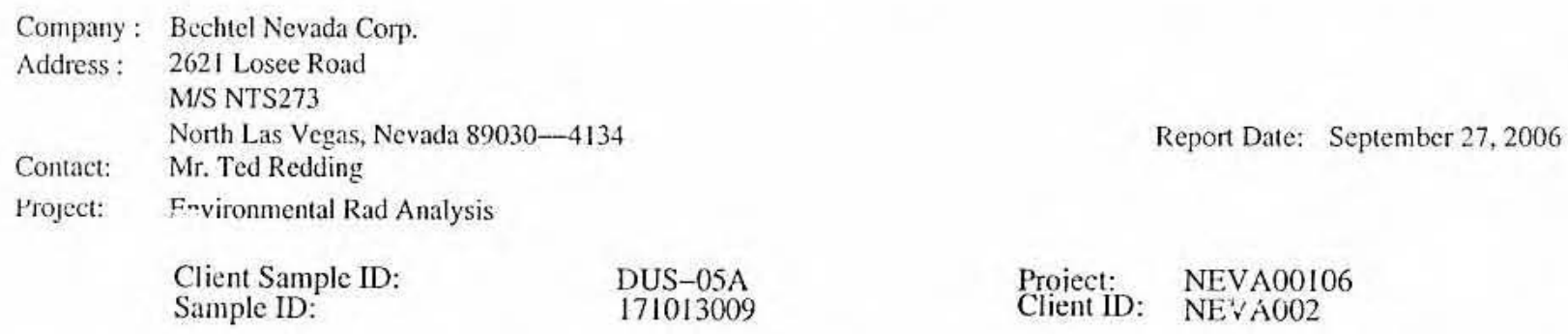

\begin{tabular}{lllllllll}
\hline Parameter & Qualifier & Result & Uncertainty & DL & TPU & RL & Units & DF Analyst Date Time Batch Mtc
\end{tabular}

$X$ Consult Case Narrative, Data Summary package, or Project Manager concerning this qualifier

Y QC Samples were not spiked with this compound

$\wedge$ RPD of sample and duplicate evaluated using $+/-$ RL. Concentrations are $<5 X$ the $R L$

h Preparation or preservation holding time was exceeded

Tht above sample is reported on a dry weight basis. 


\section{GENERAL ENGINEERING LABORATORIES, LLC \\ 2040 Savage Road Charleston SC 29407 - (843) 556-8171 - www.gel.com}

\section{Certificate of Analysis}

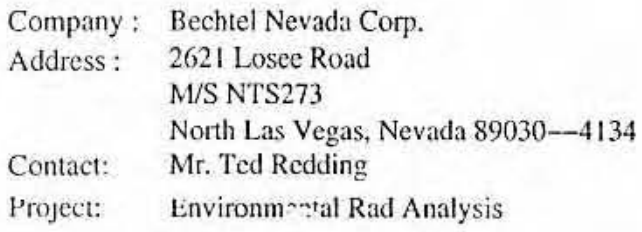

Report Date: September 27, 2006

Soil

16-AUG-06

06-SEP-06

Client

\begin{tabular}{|c|c|c|c|c|c|c|c|c|c|c|c|}
\hline Parameter & Qualifier & Result & Uncertainty & DL & TPU & RL & Units & DF & Analyst & st Date & Time Batch Mtc \\
\hline \multicolumn{12}{|c|}{ Rad Alpha Spec Analysis } \\
\hline \multicolumn{12}{|c|}{ Alphaspec $U$, Solid $2 p C i / g$} \\
\hline Uranium-232 34 & $\mathrm{U}$ & 0.475 & $+/-0.945$ & 1.84 & $+/-0.948$ & 2.00 & $\mathrm{pCi} / \mathrm{g}$ & & $\mathrm{TCl}$ & $09 / 20 / 0$ & $61547566380 \quad 1$ \\
\hline Uranium-235/236 & $\mathrm{U}$ & -0.0677 & $+1-0.133$ & 1.35 & $+1-0.133$ & 2.00 & $\mathrm{pCi} / \mathrm{g}$ & & & & \\
\hline Uranium-238 & $\mathrm{U}$ & 0.247 & $+/-0.833$ & 1.84 & $+1-0.833$ & 2.00 & $\mathrm{pCi} / \mathrm{g}$ & & & & \\
\hline
\end{tabular}

The following Prep Methods were performed

\begin{tabular}{llllll}
\hline Method & Description & Analyst & Date & Time & Prep Batch \\
\hline Dry Soil Prep & Dry So:1 1'icy GL-RAD-A-021 & AXP2 & $09 / 07 / 06$ & 1321 & 565874
\end{tabular}

The following Analytical Metiods were performed

\begin{tabular}{ll} 
Method & Description \\
\hline & DOE EML HASL-300, U-02-RC Modified
\end{tabular}

\begin{tabular}{llcc} 
Surrogate/Tracer recovery & Test & Recovery\% & Acceptable Limits \\
\hline Uranium-232 & Alphaspec U, Solid 2pCi/g & 78 & $(25 \%-125 \%)$
\end{tabular}

Notes:

The Qualifiers in this report are defined as follows:

* A quality control analyte recovery is outside of specified acceptance criteria

$<$ Result is less than value reported

$>$ Result is greater than value reported

A The TIC is a suspected aldol-condensation product

B Target analyte was detected in the associated blank

$\mathrm{BD}$ Results are either below the MDC or tracer recovery is low

C Analyte has been confirmed by GC/MS analysis

D Results are reported from a diluted aliquot of the sample

$\mathrm{H}$ Analytical holding time was exceeded

$\mathrm{J}$ Value is estimated

N/A Spike recovery limits do not apply. Sample concentration exceeds spike concentration by $4 \mathrm{X}$ or more

$\mathrm{R}$ Sample results are rejected

U Analyte was analyzed for, but not detected above the MDL, MDA, or LOD.

UJ Gamma Spectroscopy-Uncertain identification

X Consult Case Narrative, Data Summary package, or Project Manager concerning this qualifier 


\section{GENERAL ENGINEERING LABORATORIES, LLC}

2040 Savage Road Charleston SC 29407 - (843) 556-8171 - www.gel.com

\section{Certificate of Analysis}

Company: Bechtel Nevada Corp.

Address : 2621 Losee Road

M/S NTS273

North Las Vegas, Nevada 89030-4134 Report Date: September 27, 2006

Contact: Mr. Ted Redding

Project: Environmental Rad Analysis

Client Sample ID: $\quad$ DUS-05B

Sample ID: $\quad 171013010$

Proiect: NEVA00106

Client ID: NEVA002

\begin{tabular}{llllllll}
\hline Parameter & Qualifier Result Uncertainty & DL & TPU & RL & Units & DF Analyst Date Time Batch Mte
\end{tabular}

Y QC Samples were not spiked with this compound

$\wedge \quad \mathrm{RPD}$ of sample anc duplicate evaluated using $+/-\mathrm{RL}$. Concentrations are $<5 \mathrm{X}$ the $\mathrm{RL}$

h Preparation or preservation holding time was exceeded

The above sample is reported on a dry weight basis. 


\section{GENERAL ENGINEERING LABORATORIES, LLC}

2040 Savage Road Charleston SC 29407 - (843) 556-8171 - www.gel.com

\section{Certificate of Analysis}

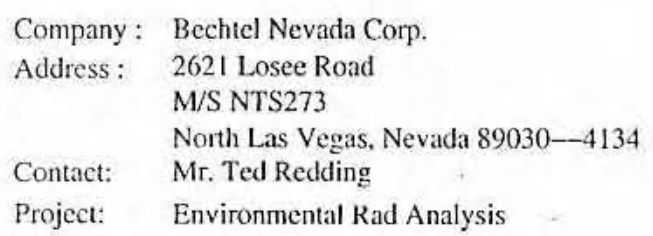

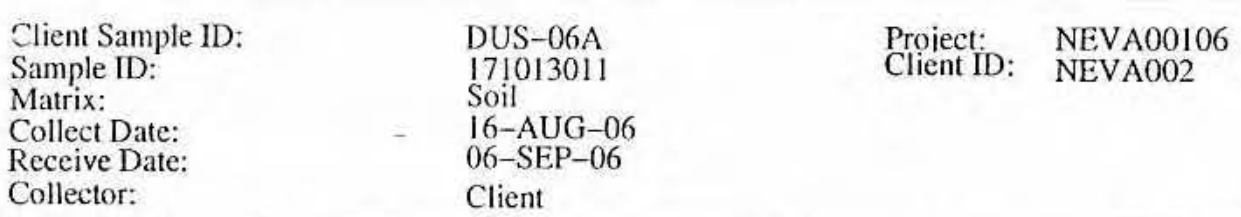

\begin{tabular}{|c|c|c|c|c|c|c|c|c|c|c|c|}
\hline Parameter & Qualifier & Result & Uncertainty & $\mathrm{DL}$ & TPU & RL & Units & DF & Analyst & Date & Time Batch Mtc \\
\hline \multicolumn{12}{|l|}{ Rad Alpha Spec Analysis } \\
\hline Alphaspec U, Solid $2 p \mathrm{Ci} / \mathrm{g}$ & $\therefore$ & & & & & & & & & & \\
\hline Uranium-233/234 & $\mathrm{U}$ & 1.07 & $+1-1.09$ & 1.71 & $+\mid-1.11$ & 2.00 & $\mathrm{pCi} / \mathrm{g}$ & & $\mathrm{TCl}$ & $09 / 20 / 0$ & $61547566388 \quad 1$ \\
\hline Uranium-235/236 & U & 1.11 & $-+1-1.14$ & 1.72 & $+/-1.15$ & 2.00 & $\mathrm{pCi} / \mathrm{g}$ & & & & \\
\hline Uranium-238 & $\mathrm{U}$ & 0.193 & $+1-0.843$ & 1.77 & $+1-0.844$ & 2.00 & $\mathrm{pCi} / \mathrm{g}$ & & & & \\
\hline
\end{tabular}

The following Prep Methods were performed

\begin{tabular}{llllll}
\hline Method & Description & Analyst & Date & Time & Prep Batch \\
\hline Dry Soil Prep & Dry Soil Prep GL-RAD-A-021 & AXP2 & $09 / 07 / 06$ & 1321 & 565874
\end{tabular}

The following Analytical Methods were performed

\begin{tabular}{ll} 
Method & Description \\
\hline DOE EML HASL-300, U-02-RC Modificd
\end{tabular}

$\begin{array}{llll}\text { Surrogate/Tracer recovery } & \text { Test } & \text { Recovery\% } & \text { Acceptable Limits }\end{array}$

$\begin{array}{llll}\text { Uranium-232 } & \text { Alphaspec U, Solid 2pCi/g } & 84 & (25 \%-125 \%)\end{array}$

Notes:

The Qualifiers in this report are defined as follows :

* A quality control analyte recovery is outside of specified acceptance criteria

$<$ Result is less than value reported

$>$ Result is greater than value reported

A The TIC is a suspected aldol-condensation product

B Target analyte was detected in the associated blank

$\mathrm{BD}$ Results are either below the MDC or tracer recovery is low

C Analyte has been confirmed by GC/MiS analysis

D Results are reported from a diluted aliquot of the sample

$\mathrm{H}$ Analytical holding time was exceeded

$\mathrm{J}$ Value is estimated

N/A Spike recovery limits do not apply. Sample concentration exceeds spike concentration by $4 \mathrm{X}$ or more

R Sample results are rejected

U Analyte was analyzed for, but not detected above the MDL, MDA, or LOD.

UI Gamma Spectroscopy-Uncertain identification 


\section{GENERAL ENGINEERING LABORATORIES, LLC \\ 2040 Savage Road Charleston SC 29407 - (843) 556-8171 - www.gel.com}

\section{Certificate of Analysis}

Company: Bechtel Nevada Corp.

Address : 2621 Losee Road

M/S NTS273

North Las Vegas, Nevada 89030-4134 Report Date: September 27, 2006

Contact: $\quad$ Mr. Ted Redding

Project: Environmental Rad Analysis

Client Sample ID: $\quad$ DUS-06A Proiect: NEVA00106

vainple D: $171013011 \quad$ Client ID: NEVA002

DL TPU

RL

Units

DF Analyst Date

Time Batch Mtc

$X$ Consult Case Narrative, Data Summary package, or Project Manager concerning this qualifier

Y QC Samples were noi spiked with this compound

$\wedge$ RPD of sample and duplicate evaluated using $+/-$ RL. Concentrations are $<5 X$ the RL

$\mathrm{h}$ Preparation or preservation holding time was exceeded

The above sample is reported on a dry weik', 1 t basis. 


\section{GENERAL ENGINEERING LABORATORIES, LLC \\ 2040 Savagge Foad Charleston SC 29407 - (843) 556-8171 - www.gel.com}

\section{Certificate of Analysis}

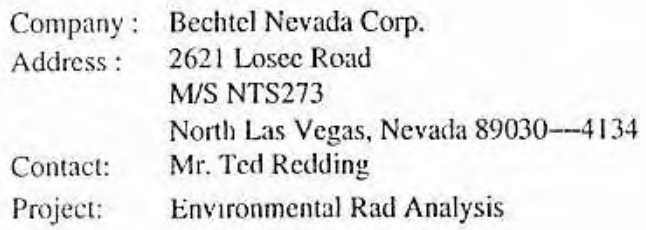

\begin{tabular}{|c|c|c|c|c|c|c|c|c|c|c|c|}
\hline Parameter & Qualifier & Result & Uncertainty & DL & TPU & RL & Units & DF & Analyst & Date & Time Batch Mtc \\
\hline \multicolumn{12}{|c|}{ Rad Alpha Spec Analysis } \\
\hline \multicolumn{12}{|c|}{ Alphaspec U, Solid $2 p$ Ci/g } \\
\hline Uranium-233/234 & & 1.89 & $+1-0.974$ & 0.914 & $+1-1.00$ & 2.00 & $\mathrm{pCi} / \mathrm{g}$ & & TCI & $09 / 25 / 0$ & 61025566388 \\
\hline Uranium-235/236 & & 0.426 & $+/-0.482$ & 0.426 & $+1-0.484$ & 2.00 & $\mathrm{pCi} / \mathrm{g}$ & & & & \\
\hline Uranium-238 & & 1.03 & $+/-0.675$ & 0.344 & $i \prime-0.686$ & 2.00 & $\mathrm{pCi} / \mathrm{g}$ & & & & \\
\hline
\end{tabular}

The following Prep Methods were performed

\begin{tabular}{|c|c|c|c|c|c|}
\hline Method & Description & Analyst & Date & Time & Prep Batch \\
\hline Dry Soil Prep & Dry Soil Prep GL-RAD-A-021 & $\mathrm{AXP} 2$ & $09 / 07 / 06$ & 1321 & 565874 \\
\hline \multicolumn{6}{|c|}{ The following Analytical Methods were performed } \\
\hline Method & Description & & & & \\
\hline 1 & DOE EML HASL-300, U-02-RC Modified & & & & \\
\hline 2 & DOE EML HASL-300, U-02-RC Modified & & & & \\
\hline \multicolumn{2}{|c|}{ Surrogate/Tracer recovery } & Recovery \% & \multicolumn{2}{|c|}{ Acceptable Limits } & \\
\hline Uranium-232 & Alphaspec U, Solid $2 \mathrm{pCi} / \mathrm{g}$ & 87 & $(25 \%$ & $25 \%)$ & \\
\hline
\end{tabular}

Notes:

The Qualifiers in this report are defined as follows :

* A quality control analyte recovery is outside of specified acceptance criteria

$<$ Result is less than value reported

$>$ Result is greater than value reported

A The TIC is a suspected aldol-condensation product

B Target analyte was detected in the associated blank

$\mathrm{BD}$ Results are either below the MDC or tracer recovery is low

C Analyte has been confirmed by GC/MS analysis

D Results are reported from a diluted aliquot of the sample

$\mathrm{H}$ Analytical holding time was exceeded

$\mathrm{J}$ Value is estimated

N/A Spike recovery limits do not apply. Sample concentration exceeds spike concentration by $4 \mathrm{X}$ or more

$\mathrm{R}$ Sample results are rejected

U Analyte was analyzed for, but not detecisd above the MDL, MDA, or LOD.

UI Gamma Spectroscopy-Uncertain identification 


\section{GENERAL ENGINEERING LABORATORIES, LLC}

2040 Savage Road Charleston SC 29407 - (843) 556-8171 - www.gel.com

\section{Certificate of Analysis}

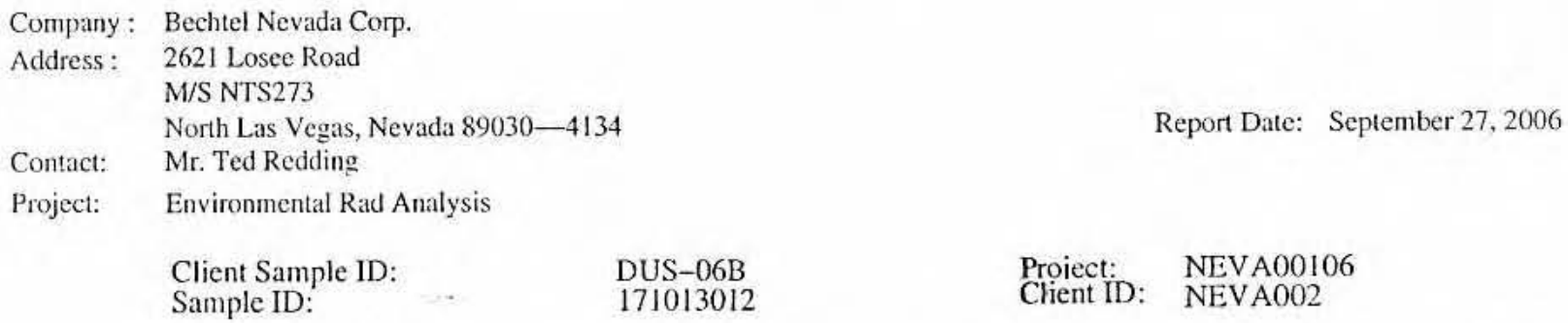

\begin{tabular}{lllllllll}
\hline Parameter & Qualifier & Result & Uncertainty & DL & TPU & RL & Units & DF Analyst Date Time Batch Mtd
\end{tabular}

X Consult Case Narrative, Data Summary package, or Project Manager concerning this qualifier

Y QC Samples were not spiked with this compuund

$\wedge \quad$ RPD of sample and duplicate evaluated using $+/-$ RL. Concentrations are $<5 X$ the RL

h Preparation or preservation holding time was exceeded

The above sample is reported on a dry weight basis. 


\section{GENERAL ENGINEERING LABORATORIES, LLC \\ 2040 Savage Road Charleston SC 29407 - (843) 556-8171 - www.gel.com}

\section{Certificate of Analysis}

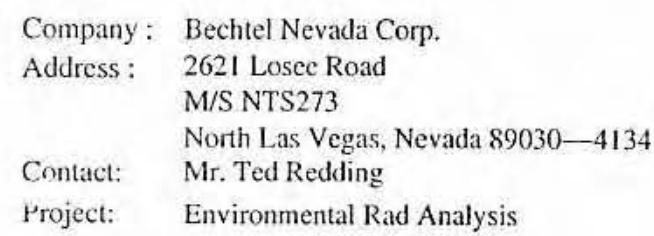

\begin{tabular}{|c|c|c|c|c|c|c|c|c|c|c|c|}
\hline Parameter & Qualifier & Result & Uncertainty & DL & TPU & RL & Units & DF & Analyst & Date & Time Batch Mtc \\
\hline \multicolumn{12}{|c|}{ Rad Alpha Spec Analysis } \\
\hline \multicolumn{12}{|c|}{ Alphaspec U, Solid $2 p \mathrm{Ci} / g$} \\
\hline Uranium-233/234 & $\mathrm{U}$ & 0.621 & $+1-1.18$ & 2.21 & $+1-1.18$ & 2.00 & $\mathrm{pCi} / \mathrm{g}$ & & $\mathrm{TCl}$ & $09 / 20 / 06$ & $1547566388 \quad 1$ \\
\hline $\begin{array}{l}\text { Uranium-235/236 } \\
\text { Uranium-238 }\end{array}$ & U & $\begin{array}{r}0.158 \\
1.19\end{array}$ & $\begin{array}{r}+1-0.974 \\
+1-122\end{array}$ & 2.28 & $+1-0.974$ & 2.00 & $\mathrm{pCi} / \mathrm{g}$ & & & & \\
\hline Uranium-238 & U & 1.19 & $+1-1.22$ & 1.84 & $+/-1.23$ & 2.00 & $\mathrm{pCi} / \mathrm{g}$ & & & & \\
\hline
\end{tabular}

The following Prep Methods were performed

\begin{tabular}{llllll}
\hline Method & Description & Analyst & Date & Time & Prep Batch \\
\hline Dry Soil Prep & Dry Soil Prep GL-RAD-A-021 & AXP2 & $09 / 07 / 06$ & 1321 & 565874
\end{tabular}

The following Analytical Methods were performed

$\begin{array}{ll}\text { Method } & \text { Description } \\ 1 & \text { DOE EML HASL-300, U-02-RC Modified }\end{array}$

\begin{tabular}{llcc} 
Surrogate/Tracer recovery & Test & Recovery $\%$ & Acceptable Limits \\
\hline Uranium-232 & Alphaspec U, Solid 2, Ci/g & 89 & $(25 \%-125 \%)$
\end{tabular}

Notes:

The Qualifiers in this report are defined as follows:

* A quality control analyte recovery is outside of specified acceptance criteria

$<$ Result is less than value reported

$>$ Result is greater than value reported

A The TIC is a suspected aldol-condensation product

B Target analyte was detected in the associated blank

BD Results are either below the MDC or tracer recovery is low

C Analyte has been confirmed by GC/MS analysis

D Results are reported from a diluted aliquot of the sample

$\mathrm{H}$ Analytical holding time was exceeded

$\mathrm{J}$ Value is estimated

N/A Spike recovery limits do not apply. Sample concentration exceeds spike concentration by $4 X$ or more

$R$ Sample results are rejected

U Analyte was analyzed for, but not detected above the MDL, MDA, or LOD.

UI Gamma Spectroscopy-Uncertain identification

X Consult Case Narrative, Data Summary package, or Project Manager concerning this qualifier 


\section{GENERAL ENGINEERING LABORATORIES, LLC \\ 2040 Savage Road Charleston SC 29407-- (843) 556-8171 - www.gel.com}

\section{Certificate of Analysis}

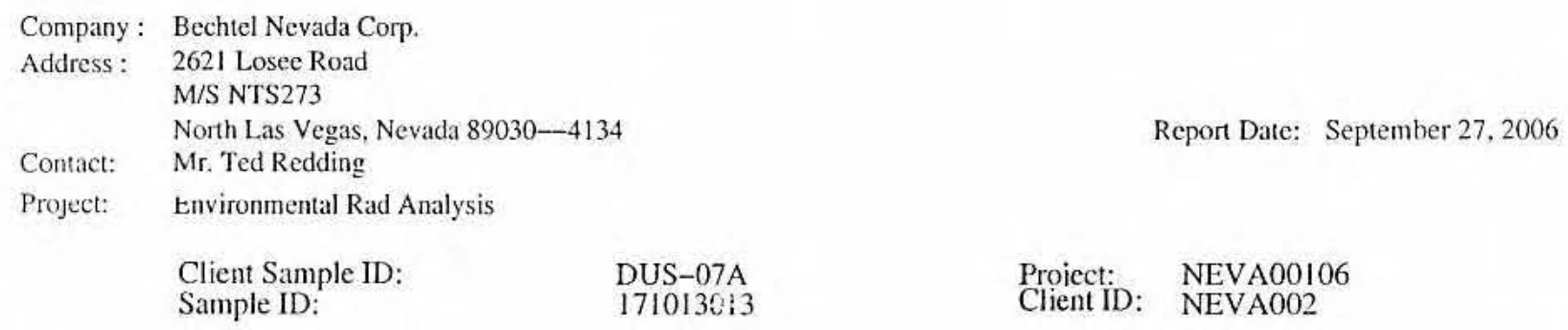

RL

Units

Y QC Samples were not spiked with this compound

$\wedge$ RPD of sample and duplicate evaluated using $+/-R L$. Concentrations are $<5 X$ the $R L$

h Preparation or preservation holding time was exceeded

The above sample is reported on a dry weight basis. 


\title{
GENERAL ENGINEERING LABORATORIES, LLC \\ 2040 Savage Road Charleston SC 29407 - (843) 556-8171 - www.gel.com
}

\section{Certificate of Analysis}

\author{
Company: Bechtel Nevada Corp. \\ Address : 2621 Losee Road \\ M/S NTS273 \\ North Las Vegas, Nevada $89030-4134$ \\ Contact: $\quad$ Mr. Ted Redding \\ Project: Environmental Rad Analysis

$\begin{array}{ll}\text { Client Sample ID: } & \text { DUS-07B } \\ \text { Sample ID: } & 171013014 \\ \text { Matrix: } & \text { Soil } \\ \text { Collect Date: } & 16 \text {-AUG-06 } \\ \text { Receive Date: } & 06-\text { SEP-06 } \\ \text { Collector: } & \text { Client }\end{array}$

\begin{tabular}{|c|c|c|c|c|c|c|c|c|c|c|c|c|}
\hline Parameter & Qualifier & Result & Uncertainty & DL & TPU & RL & Units & DF & Analyst & Date & Time Batch $\mathrm{M}$ & Mtc \\
\hline \multicolumn{13}{|c|}{ Rad Alpha Spec Analysis } \\
\hline \multicolumn{13}{|c|}{ Alphaspec U. Solid $2 p C i / g$} \\
\hline Uranium-233/234 & & 3.24 & $+1-1.16$ & 0.324 & $+1-1.22$ & 2.00 & $\mathrm{pCi} / \mathrm{g}$ & & $\mathrm{TCl}$ & $09 / 25 / 0$ & 61026566388 & 1 \\
\hline Uranium-235/236 & & 1.20 & $+/-0.784$ & 0.400 & $+/-0.797$ & 2.00 & $\mathrm{pCi} / \mathrm{g}$ & & & & & \\
\hline Uranium-238 & & 2.10 & $+/-0.952$ & 0.633 & $+/-0.984$ & 2.00 & $\mathrm{pCi} / \mathrm{g}$ & & & & & \\
\hline
\end{tabular}

The following Prep Methods were performed

\begin{tabular}{llllll}
\hline Method & Description & Analyst & Date & Time & Prep Batch \\
\hline Dry Soil Prep & Dry Soil Prep GL-RAD-A-021 & AXP2 & $09 / 07 / 06$ & 1321 & 565874
\end{tabular}

The following Analytical Methods were performed

\begin{tabular}{ll} 
Method & Description \\
\hline 1 & DOE EML HASL-300, U-02-RC Modified \\
2 & DOE EML HASL-300, U-02-RC Modified
\end{tabular}

\begin{tabular}{llcc} 
Surrogate/Tracer recovery & Test & Recovery \% & Acceptable Limits \\
\hline Uranium-232 & Alphaspec U, Solid 2pCi/g & 87 & $(25 \%-125 \%)$
\end{tabular}

Notes:

The Qualifiers in this report are defined as follows:

* A quality control analyte recovery is outside of specified acceptance criteria

$<$ Result is less than value reported

$>$ Result is greater than value reported

A The TIC is a suspected aldol-condensation product

B Target analyte was detected in the associated blank

BD Results are either below the MDC or tracer recovery is low

C Analyte has been confirmed by GC/MS analysis

D Results are reported from a diluted aliquot of the sample

$\mathrm{H}$ Analytical holding time was exceeded

$\mathrm{J}$ Value is estimated

N/A Spike recovery limits do not apply. Sample concentration exceeds spike concentration by $4 \mathrm{X}$ or more

$\mathrm{R}$ Sample results are rejected

U Analyte was analyzed for, but not detected above the MDL, MDA, or LOD.

UI Gamma Spectroscopy--Uncertain identification 


\section{GENERAL ENGINEERING LABORATORIES, LLC \\ 2040 Savage Road Charleston SC 29407 - (843) 556-8171 - www gel.com}

\section{Certificate of Analysis}

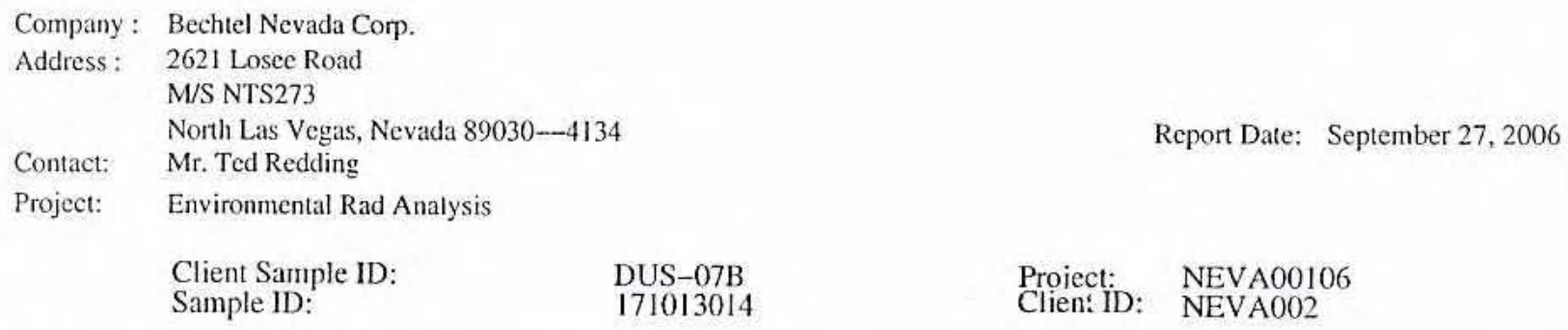

\begin{tabular}{llllllll}
\hline Parameter & Qualifier Result Uncertainty & DL & TPU & RL & Units & DF Analyst Date Time Batch MItd
\end{tabular}

$\mathrm{X}$ Consult Case Narrative, Data Summary package, or Project Manager concerning this qualifier

Y QC Samples were not spiked with this compound

$\wedge \quad$ RPD of sample and duplicate evaluated using $+/-$ RL. Concentrations are $<5 X$ the RL

$\mathrm{h}$ Preparation or preservation holding time was exceeded

The above sample is reported on a dry weight basis. 


\section{GENERAL ENGINEERING LABORATORIES, LLC}

2040 Savage Road Charleston SC 29407 - (843) 556-8171 - www.gel com.

\section{Certificate of Analysis}

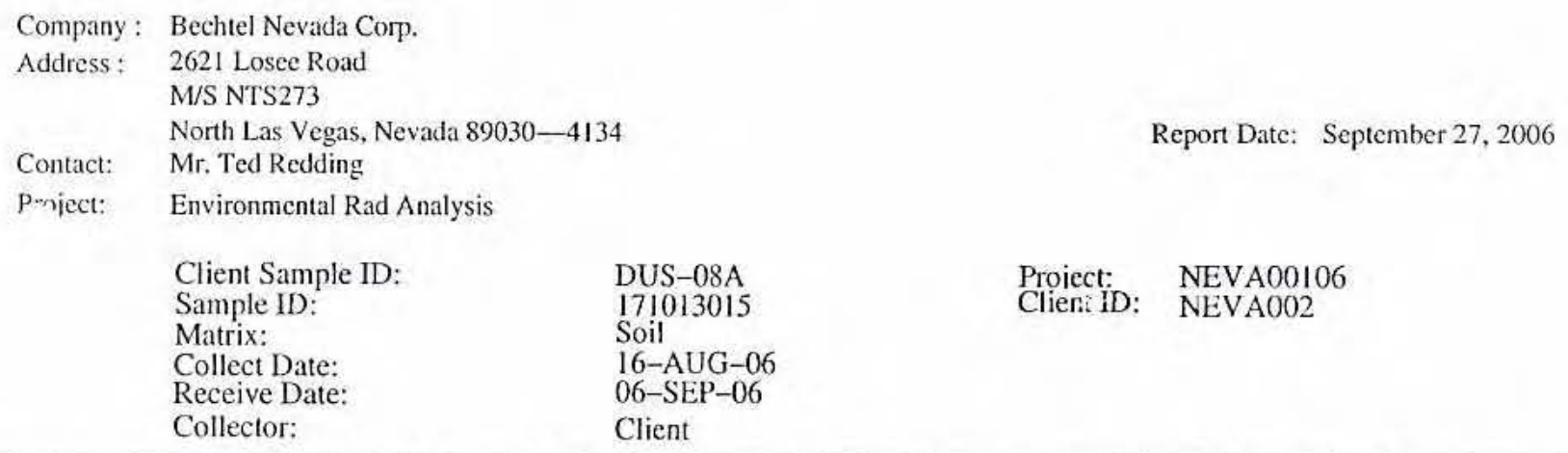

\begin{tabular}{|c|c|c|c|c|c|c|c|c|c|c|c|c|}
\hline Parameter & Qualifier & Result & Uncertainty & DL & TPU & $\mathrm{RL}$ & Units & DF & Analyst & Date & Time Batch N & Mtd \\
\hline \multicolumn{13}{|c|}{ Rad Alpha Spec Analysis } \\
\hline \multicolumn{13}{|c|}{ Alphaspec U. Solid $2 p \mathrm{Ci} / g$} \\
\hline Uranium-233/234 & $\mathrm{U}$ & 0.852 & $+1-1.01$ & 1.57 & $+1-1.02$ & 2.00 & $\mathrm{pCi} / \mathrm{g}$ & & $\mathrm{TCl}$ & $09 / 20 / 0$ & 61547566388 & 1 \\
\hline Uranium-235/236 & $\mathrm{U}$ & -0.0665 & $+1-0.130$ & 1.33 & $+|-0.13|$ & 2.00 & $\mathrm{pCi} / \mathrm{g}$ & & & & & \\
\hline Urai:ium-238 & $\mathrm{U}$ & 0.906 & $+1-1.01$ & 1.48 & $+/-1.01$ & 2.00 & $\mathrm{pCi} / \mathrm{g}$ & & & & & \\
\hline
\end{tabular}

The following Prep Methods were performed

\begin{tabular}{llllll}
\hline Method & Description & Analyst & Date & Time & Prep Batch \\
\hline Diy Soil Prep & Dry Soil Prep GL-RAD-A-021 & AXP2 & $09 / 07 / 06$ & 1321 & 565874
\end{tabular}

The following Analytical Methods were performed

\begin{tabular}{ll} 
Method & Description \\
\hline & DOE EML HASL-300, U-02-RC Modified
\end{tabular}

\begin{tabular}{llcc} 
Surrogate/Tracer recovery & Test & Recovery $\%$ & Acceptable Limits \\
\hline Uranium-232 & Alphaspec U, Solid 2pCi/g & 91 & $(25 \%-125 \%)$
\end{tabular}

Notes:

The Qualifiers in this report are defined as follows:

* A quality control analyte recovery is outside of specified acceptance criteria

$<$ Result is less than value reported

$>$ Result is greater than value reported

A The TIC is a suspected aldol-condensation product

B Target analyte was detected in the associated blank

$\mathrm{BD}$ Results are either below the MDC or tracer recovery is low

C Analyte has been confirmed by GC/MS analysis

D Results are reported from a diluted aliquot of the sample

$\mathrm{H}$ Analytical holding time was exceeded

$\mathrm{J}$ Value is estimated

N/A Spike recovery limits do not apply. Sample concentration exceeds spike concentration by $4 \mathrm{X}$ or more

$\mathrm{R}$ Sample results are rejected

U Analyte was analyzed for, but not detected above the MDL, MDA, or LOD.

UI Gamma Spectroscopy--Uncertain identification

$\mathrm{X}$ Consult Case Narrative, Data Summary package, or Project Manager concerning this qualifier 


\section{GENERAL ENGINEERING LABORATORIES, LLC \\ 2040 Savage Road Charleston SC 29407 - (843) 556-8171 - www.gel.com}

\section{Certificate of Analysis}

Company: Bechtel Nevada Corp.

Address: 2621 Losee Road

M/S NTS273

North Las Vegas, Nevada 89030-4134

Contact: Mr. Ted Redding

Report Date: September 27,2006

Project: Fnvironmental Rad Analysis

Client Sample ID: $\quad$ DUS-08A

Sample ID: $\quad 171013015$

Proiect: NEVA00106

Client ID: NEVAG02

DL TPU

RI.

Units

DF Analyst Date

Time Batch Mts

Y QC Samples were not spiked with this compound

$\wedge$ RPD of sample and duplicate evaluated using $+/-$ RL. Concentrations are $<5 X$ the RL

h Preparation or preservation holding time was exceeded

The above sample is reported on a dry weight basis. 


\section{GENERAL ENGINEERING LABORATORIES, LLC \\ 2040 Savage Road Charleston SC 29407 - (843) 556-8171 - www.gel.ccm}

\section{Certificate of Analysis}

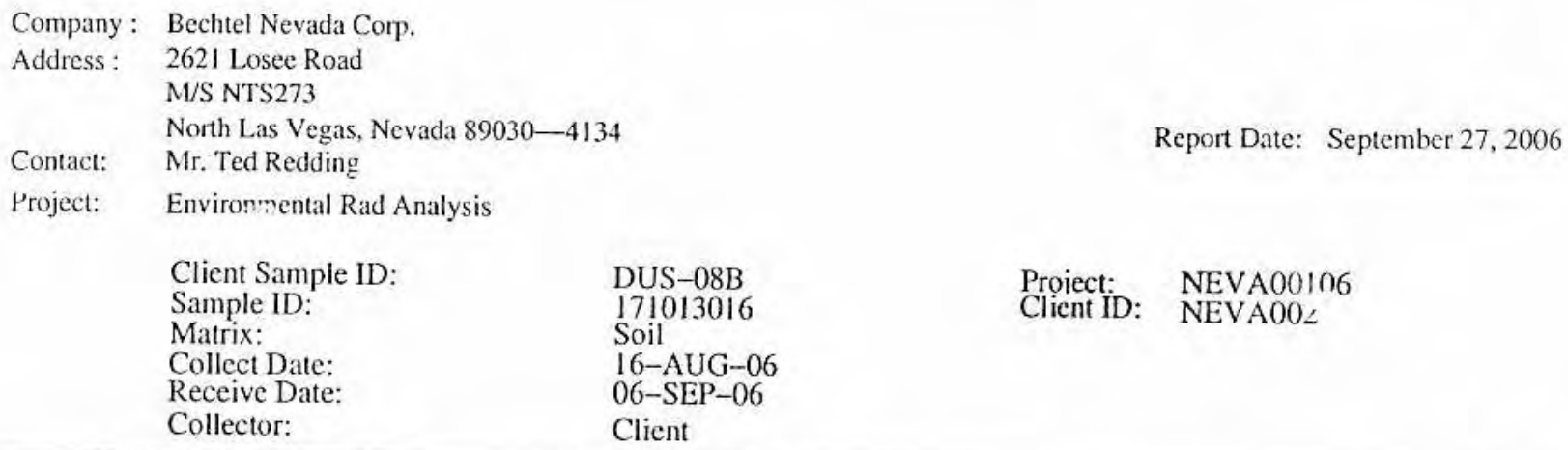

\begin{tabular}{|c|c|c|c|c|c|c|c|c|c|c|c|}
\hline Parameter & Qualifier & Result & Uncertainty & DL & TPU & RL & Units & DF & Analyst & Date & Time Batch Mts \\
\hline \multicolumn{12}{|c|}{ Rad Alpha Spec Analysis } \\
\hline \multicolumn{12}{|c|}{ Alphaspec U, Solid $2 p C i / g$} \\
\hline Uranium-23/234 & $\mathrm{U}$ & 1.51 & $+/-1.47$ & 1.98 & $+/-1.48$ & 2.00 & $\mathrm{pCi} / \mathrm{g}$ & & $\mathrm{TCl}$ & $09 / 20 / 06$ & 1547566388 \\
\hline Uranium-235/236 & U & 0.653 & $+1-1.04$ & 1.78 & $+1-1.05$ & 2.00 & $\mathrm{pCi} / \mathrm{g}$ & & & & \\
\hline Uranium-238 & $\mathrm{U}$ & 1.21 & $+1-1.35$ & 1.98 & $+1-1.36$ & 2.00 & $\mathrm{pCi} / \mathrm{g}$ & & & & \\
\hline
\end{tabular}

The following Prep Methods were performed

\begin{tabular}{|c|c|c|c|c|c|}
\hline Method & Description & Analyst & Date & Time & Prep Batch \\
\hline Dry Soil Prep & Dry Sont Irep GL-RAD-A-021 & AXP2 & $09 / 07 / 06$ & 1321 & 565874 \\
\hline \multicolumn{6}{|c|}{ The following Analytical Methods were performed } \\
\hline Method & Description & & & & \\
\hline
\end{tabular}

\begin{tabular}{llcc} 
Surrogate/Tracer recovery & Test & Recovery \% & Acceptable Limits \\
\hline Uranium-232 & Alphaspec U, Solid 2pCi/g & 96 & $(25 \%-125 \%)$
\end{tabular}

Notes:

The Qualifiers in this report are defined as follows:

* A quality control analyte recovery is outside of specified acceptance criteria

$<$ Result is iess than vaiue reported

$>$ Result is greater than value reported

A The TIC is a suspected aldol-condensation product

B Target analyte was detected in the associated blank

$\mathrm{BD}$ Results are either below the MDC or tracer recovery is low

C Analyte has been confirmed by GC/MS analysis

D Results are reported from a diluted aliquot of the sample

$\mathrm{H}$ Analytical holding time was exceeded

$\mathrm{J}$ Value is estimated

N/A Spike recovery limits do not apply. Sample concentration exceeds spike concentration by $4 \mathrm{X}$ or more

$\mathrm{R}$ Sample results are rejected

$\mathrm{U}$ Analyte was analyzed for, but not detected above the MDL, MDA, or LOD.

UI Gamma Spectroscopy-Uncertain identification

X Consult Case Narrative, Data Summary package, or Project Manager concerning this qualifier 


\section{Certificate of Analysis}

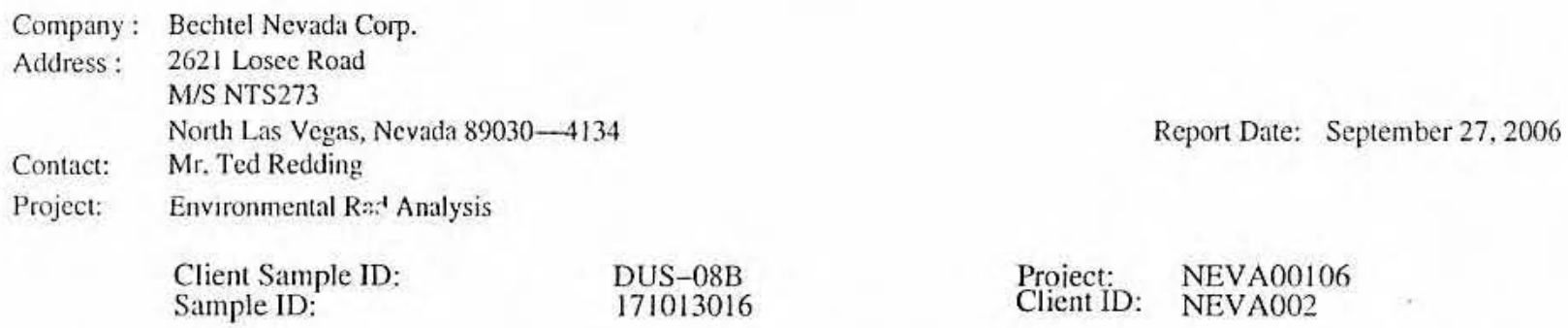

Parameter Qualifier Result Uncertainty DL TPU RL Units DF Analyst Date Time Batch MIt

Y QC Samples were not spiked with this compound

$\wedge \quad$ RPD of sample and duplicate evaluated using $+1-$ RL. Concentrations are $<5 \mathrm{X}$ the $\mathrm{RL}$

h Preparation or preservation holding time was exceeded

The above sample is reported on a dry weight basis. 


\section{GENERAL ENGINEERING LABORATORIES, LLC}

2040 Savage Road Charleston SC 29407 - (843) 556-8171 - www.gel.com

\section{Certificate of Analysis}

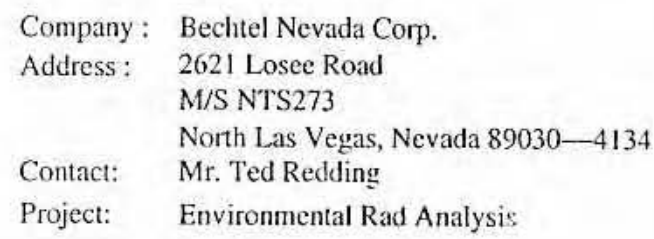

Report Date: September 27, 2006 $\begin{array}{ll}\text { Proiect: } & \text { NEVA00106 } \\ \text { Client ID: } & \text { NEVA002 }\end{array}$

DUS-09A
171013017
Soil
17-AUG-06
06-SEP-06
Client

DUS-09A

$\begin{array}{ll}\text { Proiect: } & \text { NEVA00106 } \\ \text { Client ID: } & \text { NEVA002 }\end{array}$

\begin{tabular}{|c|c|c|c|c|c|c|c|c|c|c|c|c|}
\hline Parameter & Qualifier & Result & Uncertainty & DL & TPU & RL & Units & DF & Analyst & Date & Time Batch Mt & It \\
\hline \multicolumn{13}{|c|}{ Rad AIpha Spec Analysis } \\
\hline \multicolumn{13}{|c|}{ Alphaspec U, Solid $2 p C i / g$} \\
\hline Uranium-233/234 & & 1.80 & $+1-1.21$ & 0.985 & $+/-1.24$ & 2.00 & $\mathrm{pCi} / \mathrm{g}$ & & $\mathrm{TCl}$ & $09 / 20 / 0$ & 61547566388 & 1 \\
\hline Uranium-235/236 & U & -0.244 & $+1-0.239$ & 1.67 & $+1-0.242$ & 2.00 & $\mathrm{pCi} / \mathrm{g}$ & & & & & \\
\hline Uranium-238 & $\mathrm{U}$ & 1.04 & $+1-1.01$ & 1.35 & $+1-1.02$ & 2.00 & $\mathrm{pCi} / \mathrm{g}$ & & & & & \\
\hline
\end{tabular}

The following Prep Methods were performed

\begin{tabular}{lllllll}
\hline Method & Description & Analyst & Date & Time & Prep Batch \\
\hline Dry Soil Prep & Dry Soil Prep GL-RAD A-v21 & AXP2 & $09 / 07 / 06$ & 1321 & 565874
\end{tabular}

The following Analytical Methods were performed

\begin{tabular}{|c|c|c|c|}
\hline Descripti & & & \\
\hline DOE EMI & ASL -300, U-02-RC Modified & & \\
\hline Surrogate/Tracer recovery & Test & Recovery \% & Acceptable Limits \\
\hline Uranium-232 & Alphaspec U, Solid 2pCi/g & 78 & $(25 \%-125 \%)$ \\
\hline
\end{tabular}

Notes:

The Qualificrs in this report are defined as follows :

* A quality control analyte recovery is outside of specified acceptance criteria

$<$ Result is less than value reporied

$>$ Result is greater than value reported

A The TIC is a suspected aldol-condensation product

B Target analyte was detected in the associated blank

$\mathrm{BD}$ Results are either below the MDC or tracer recovery is low

C Analyte has been confirmed by GC/MS analysis

D Results are reported from a diluted aliquot of the sample

$\mathrm{H}$ Analytical holding time was exceeded

$\mathrm{J}$ Value is estimated

N/A Spike recovery limits do not apply. Sample concentration exceeds spike concentration by $4 \mathrm{X}$ or more

$\mathrm{R}$ Sample results are rejected

U Analyte was analyzed for, but not detected above the MDL, MDA, or LOD.

UI Gamma Spectroscopy--Uncertain identification

$\mathrm{X}$ Consult Case Narrative, Data Summary package, or Project Manager concerning this qualifier 


\section{GENERAL ENGINEERING LABORATORIES, LLC \\ 2040 Savage Road Charleston SC 29407 - (843) 556-8171 - www.gel.com}

\section{Certificate of Analysis}

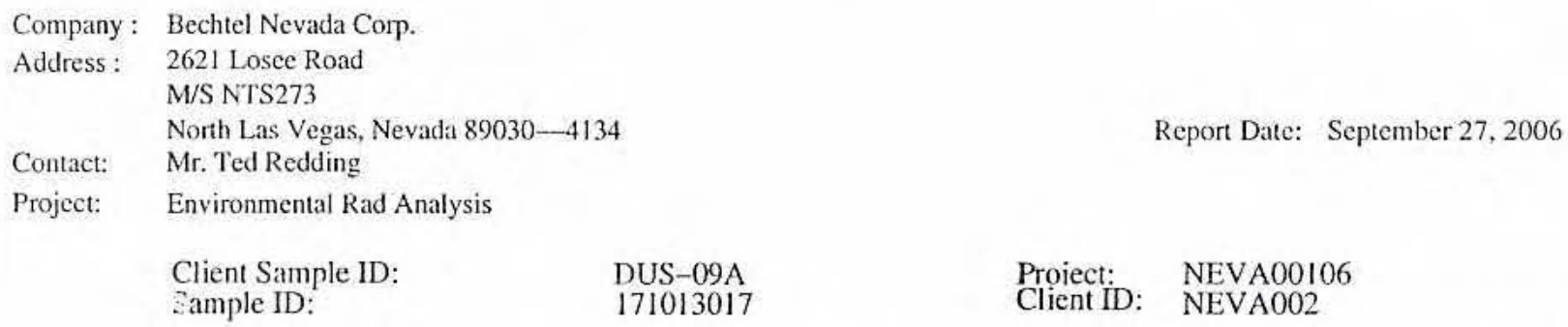

\begin{tabular}{llllllll}
\hline Parameter Qualifier Result Uncertainty & DL & TPU & RI & Units & DF Analyst Date Time Batch Mt
\end{tabular}

\section{Y QC Samples were not spiked with this compound}

$\wedge$ RPD of sample and duplicatc evaluated using $+/-\mathrm{RL}$. Concentrations are $<5 \mathrm{X}$ the RL

h Preparation or preservation holding time was exceeded

The above sample is reported on a dry weight hasis. 


\section{GENERAL ENGINEERING LABORATORIES, LLC}

2040 Savage Road Charleston SC 29407 - (843) 556-8171 - www.gel.com

\section{Certificate of Analysis}

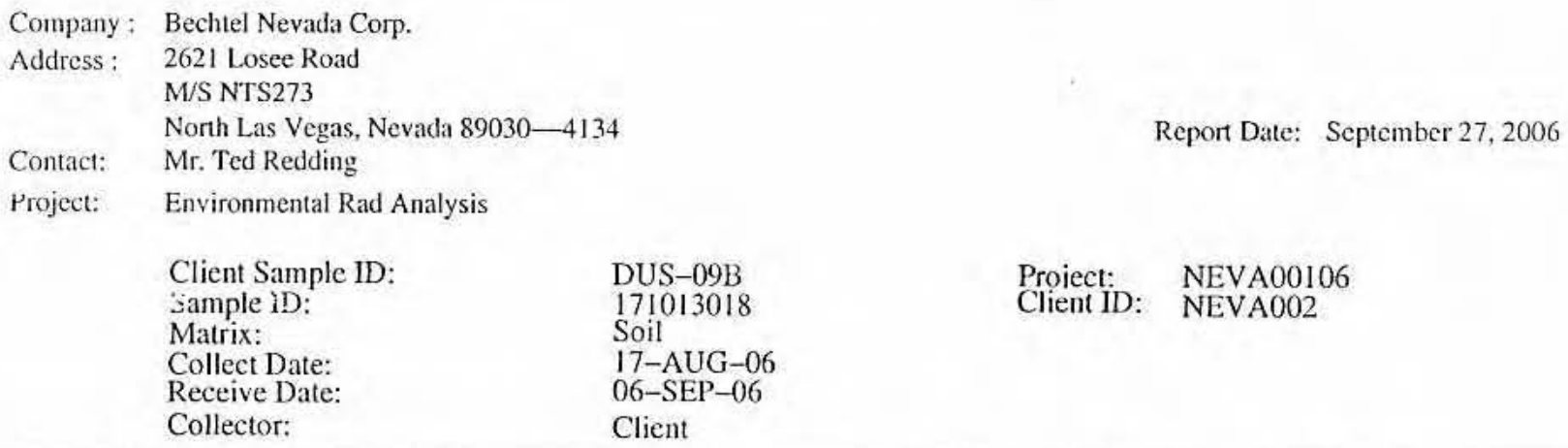

\begin{tabular}{|c|c|c|c|c|c|c|c|c|c|c|c|}
\hline Parameter & Qualifier & Result & Uncertainty & DL & TPU & RL & Units & DF & Analys & t Date & Time Batch Mti \\
\hline \multicolumn{12}{|c|}{ Rad Alpha Spec Analysis } \\
\hline \multicolumn{12}{|c|}{ Alphaspec U. Solid $2 p C i / g$} \\
\hline Uranium-233/234 & $\mathrm{U}$ & 0.865 & $+/-0.961$ & 1.50 & $+1-0.969$ & 2.00 & $\mathrm{pCi} / \mathrm{g}$ & & $\mathrm{TCl}$ & $09 / 20 / 0$ & $61547566388 \quad$ I \\
\hline Uranium-235/236 & U & 0.451 & $+1-0.779$ & 1.46 & $+1-0.781$ & 2.00 & $\mathrm{pCi} / \mathrm{g}$ & & & & \\
\hline Uranium-238 & $\mathrm{U}$ & -0.0787 & $+1-0.407$ & 1.32 & $+1-0.407$ & 2.00 & $\mathrm{pCi} / \mathrm{g}$ & & & & \\
\hline
\end{tabular}

The following Prep Methods were performed

\begin{tabular}{llllll}
\hline Method & Description & Analyst & Date & Time & Prep Batch \\
\hline Dry Soil Prep & Dry Soil Prep GL-RAD-A-021 & AXP2 & $09 / 07 / 06$ & 1321 & 565874
\end{tabular}

The following Analytical Methods were performed

\begin{tabular}{|c|c|c|c|}
\hline Method Descriptio & & & \\
\hline DOE EML & ASL-300, U-02-RC Modified & & \\
\hline Surrogate/Tracer recovery & Test & Recovery\% & Acceptable Limits \\
\hline Uranium-232 & Alphaspec U, Solid 2pCi/g & 82 & $(25 \%-125 \%)$ \\
\hline
\end{tabular}

Notes:

The Qualifiers in this report are defined as follows :

* A quality control analyte recovery is outside of specified acceptance criteria

$<$ Result is less than value reported

$>$ Result is greater than value reported

A The TIC is a suspected aldol-condensation product

B Target analyte was detected in the associated blank

$\mathrm{BD}$ Results are either below the MDC or tracer recovery is low

C Analyte has been confirmed by GC/MS analysis

D Results are reported from a diluted aliquot of the sample

$\mathrm{H}$ Analytical holding time was exceeded

$\mathrm{J}$ Value is estimated

N/A Spike recovery limits do not apply. Sample concentration exceeds spike concentration by $4 \mathrm{X}$ or more

$\mathrm{R}$ Sample results are rejected

U Analyte was analyzed for, but not detected above the MDL, MDA, or LOD.

UI Gamma Spectroscopy-Uncertain identification

$X$ Consult Case Narrative, Data Summary package, or Project Manager concerning this qualifier 


\section{GENERAL ENGINEERING LABORATORIES, LLC \\ 2040 Savage Road Charleston SC 29407 - (843) 556-8171 - www.gel.com}

\section{Certificate of Analysis}

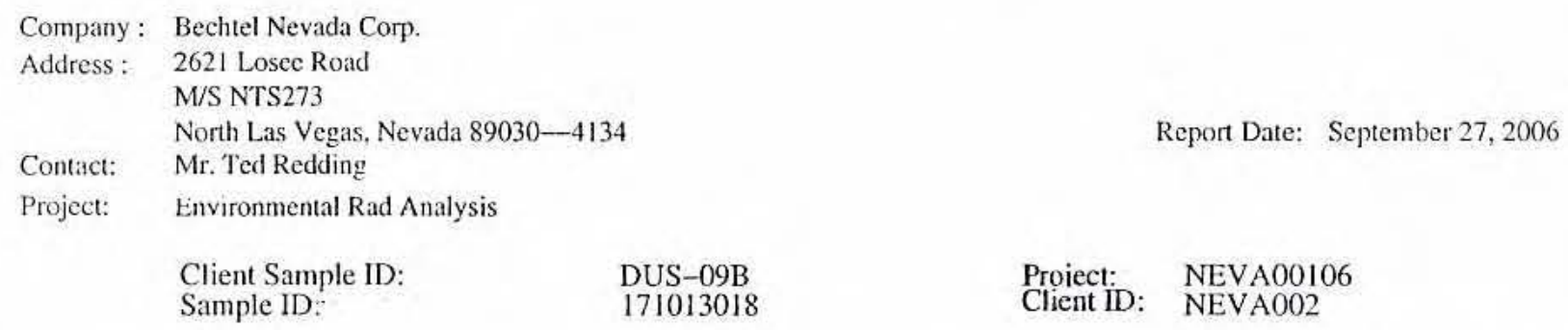

\begin{tabular}{lllllllll}
\hline Parameter & Qualifier & Result & Uncertainty & DI & TPU & RL & Units & DF Analyst Date Time Batch Mits
\end{tabular}

Y QC Samples were not spiked with this compound

$\wedge$ RPD of sample and duplicate evaluated using $+/-\mathrm{KL}$. Concentrations are $<5 \mathrm{X}$ the $\mathrm{RL}$

$h$ Preparation or preservation holding time was exceeded

The above sample is reported on a dry weight basis. 


\section{GENERAL ENGINEERING LABORATORIES, LLC}

2040 Savage Foad Charleston SC 29407 - (843) 556-8171 - www.gel.com

\section{Certificate of Analysis}

\author{
Company: Bechtel Nevada Corp. \\ Address: 2621 Losee Road \\ M/S NTS273 \\ North Las Vegas, Nevada 89030-4134 \\ Contact: Mr. Ted Redding \\ Project: Environmental Rad Analysis
}

Report Date: September 27, 2006

\author{
DUS-10A Proiect: NEVA00106 \\ $171013019 \quad$ Client ID: NEVA002 \\ 17-AUG-06 \\ 06-SEP-06 \\ Client
}

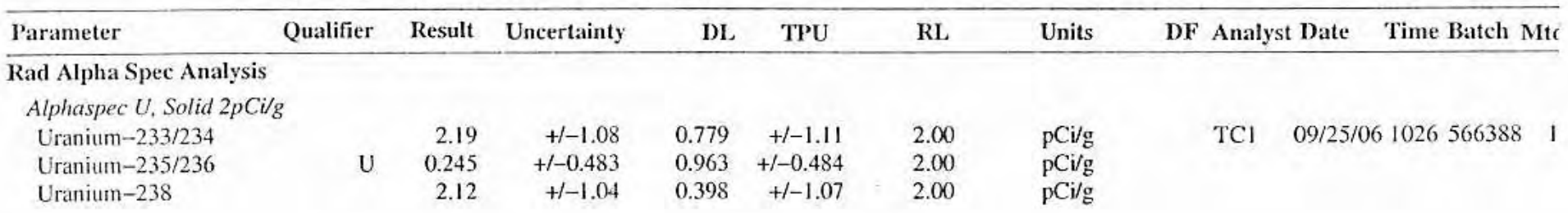

The following Prep Methods were performed

\begin{tabular}{llllll}
\hline Method & Description & Analyst & Date & Time & Prep Batch \\
\hline Dry Soil Prep & Dry Soil Prep GL-RAD-A-021 & AXP2 & $09 / 07 / 06$ & 1321 & 565874
\end{tabular}

The following Analytical Methods were performed

\begin{tabular}{ll} 
Method & Description \\
\hline 1 & DOE EML HASL-300, U-02-RC Modified \\
2 & DOE EML HASL-300, U-02-RC Modified
\end{tabular}

\begin{tabular}{llcc} 
Surrogate/Tracer recovery & Test & Recovery $\%$ & Acceptable Limits \\
\hline Uranium-232 & Alphaspec U, Solid 2pCi/g & 86 & $(25 \%-125 \%)$
\end{tabular}

Notes:

The Qualifiers in this report are defined as follows :

* A quality control analyte recovery is outside of specified acceptance criteria

$<$ Result is less than value reported

$>$ Result is greater than value reported

A The TIC is a suspected aldol-condensation product

B Target analyte was detected in the associated blank

$\mathrm{BD}$ Results are either below the MDC or tracer recovery is low

C Analyte has been confirmed by GC/MS analysis

D Results are reported from a diluted aliquot of the sample

H Analytical holding time was exceeded

$\mathrm{J}$ Value is estimated

N/A Spike recovery limits do not apply. Sample concentration exceeds spike concentration by $4 \mathrm{X}$ or more

R Sample results are rejected

$\mathrm{U}$ Analyte was analyzed for, but not detected above the MDL, MDA, or LOD.

UI Gamma Spectroscopy--Uncertain identification 


\section{GENERAL ENGINEERING LABORATORIES, LLC}

2040 Savage Road Charleston SC: 29407 - (843) 556-8171 - www.gel.com

\section{Certificate of Analysis}

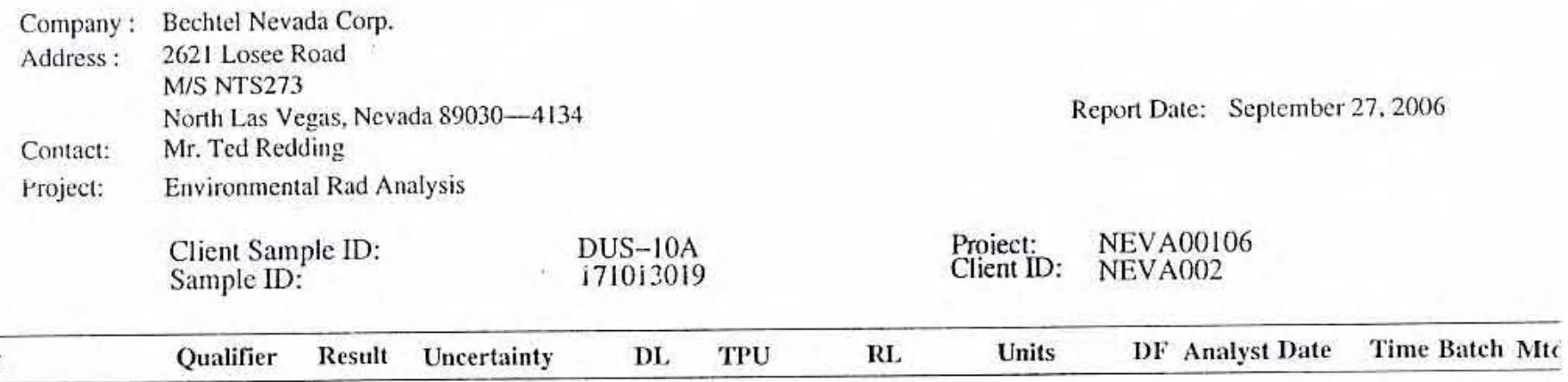

X Consult Case Narrative, Data Summary package, or Project Manager concerning this qualifier

Y QC Samples were not spiked with this compound

$\wedge$ RPD of sample and duplicate evaluated using $+/-\mathrm{RL}$. Concentrations are $<5 \mathrm{X}$ the RL

$h$ Preparation or preservation holding time was exceeded

The above sample is reported on a dry weight basis. 


\section{GENERAL ENGINEERING LABORATORIES, LLC \\ 2040 Savage Road Charleston SC. 29407 - (843) 556-8171 - www.gel.com}

\section{Certificate of Analysis}

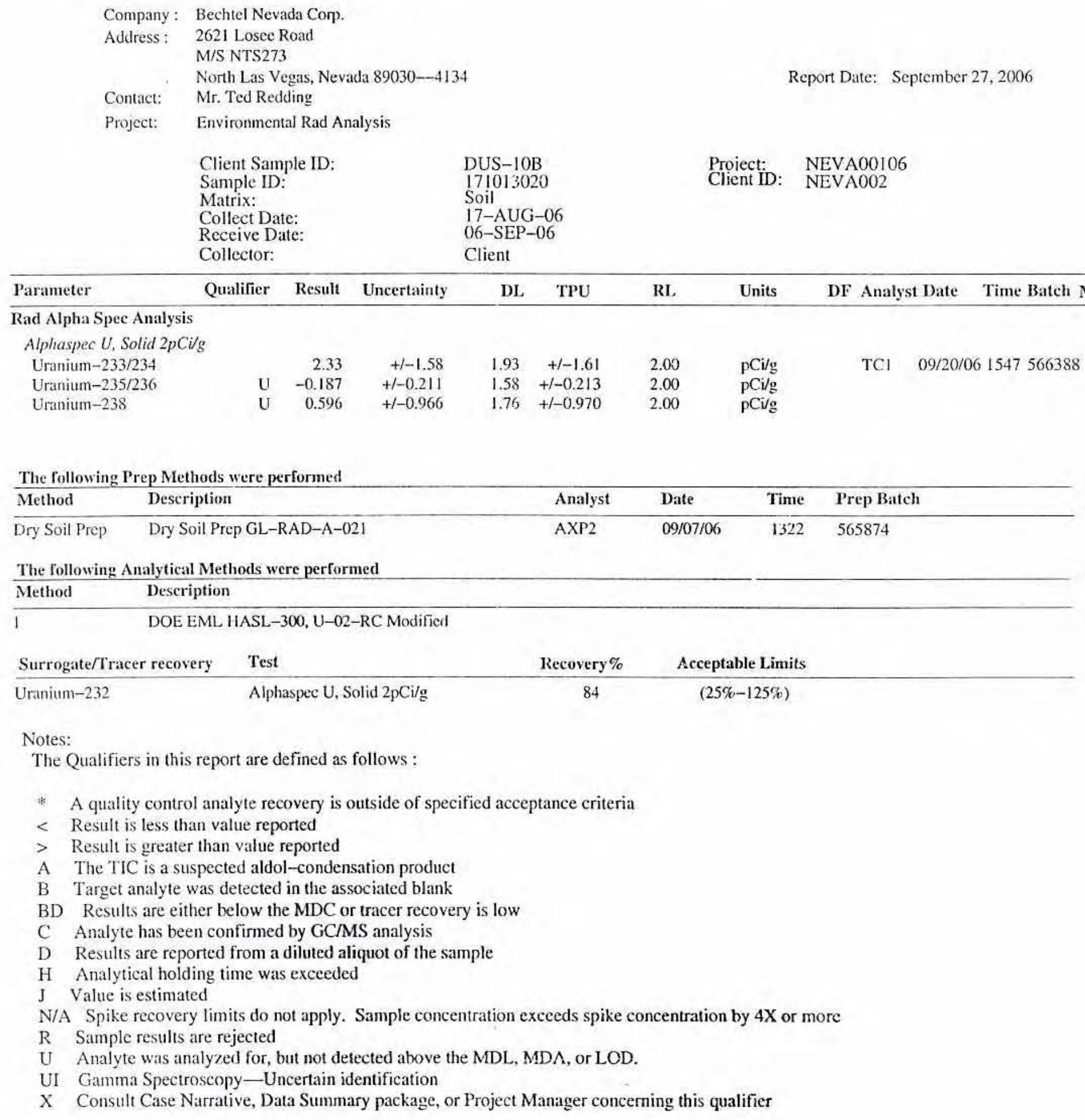




\section{GENERAL ENGINEERING LABORATORIES, LLC \\ 2040 Savage Road Charleston SC 29407 - (843) 556-8171 - www.gel.com}

\section{Certificate of Analysis}

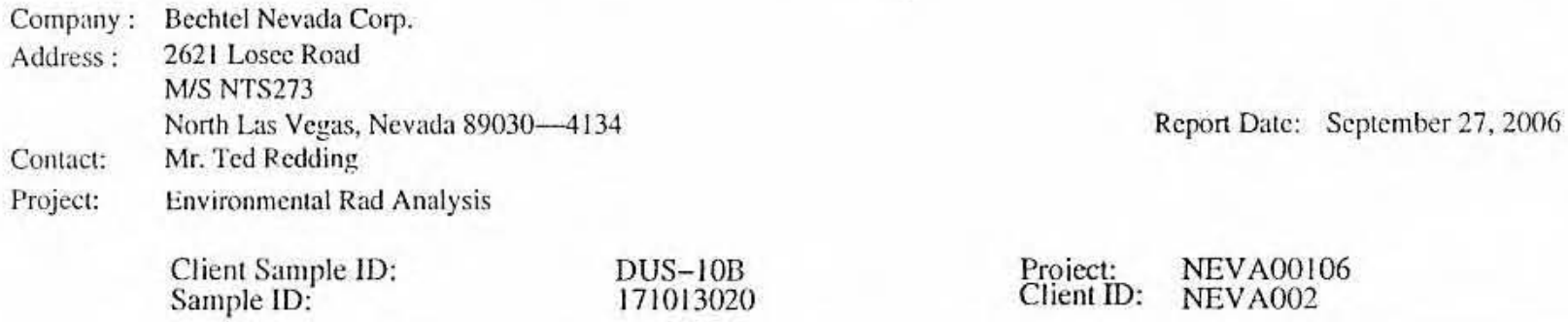

\begin{tabular}{llllllll}
\hline Parameter Qualifier Result Uncertainty & DL & TPU & RL & Units & DF Analyst Date Time Batch Mitc
\end{tabular}

Y QC Samples were not spiked with this compound

$\wedge$ RPD of sample and duplicate evaluated using $+/-$ RL. Concentrations are $<5 X$ the RL

$\mathrm{h}$ Preparation or preservation holding time was exceeded

The above sample is reported on a dry weight basis. 


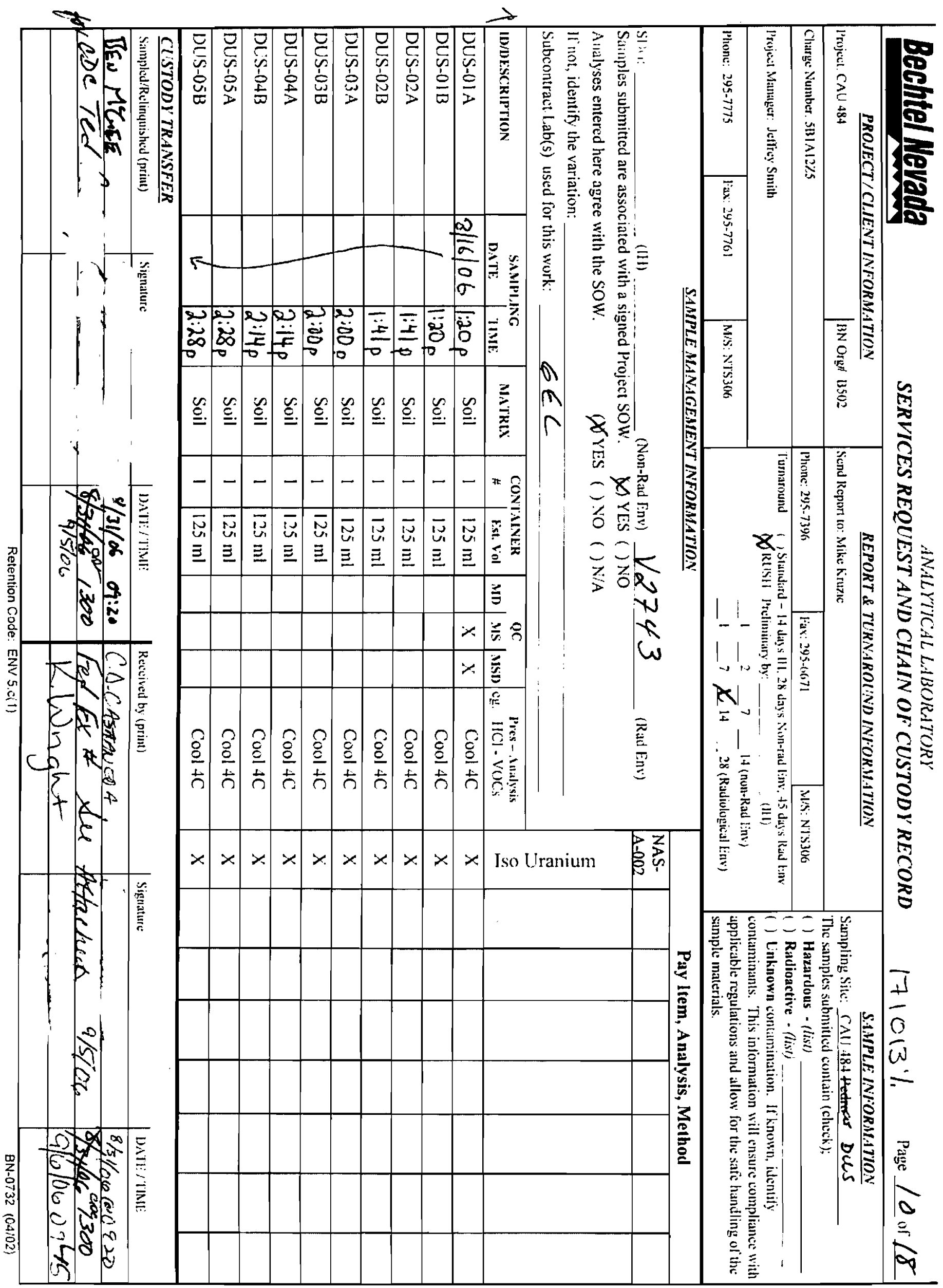




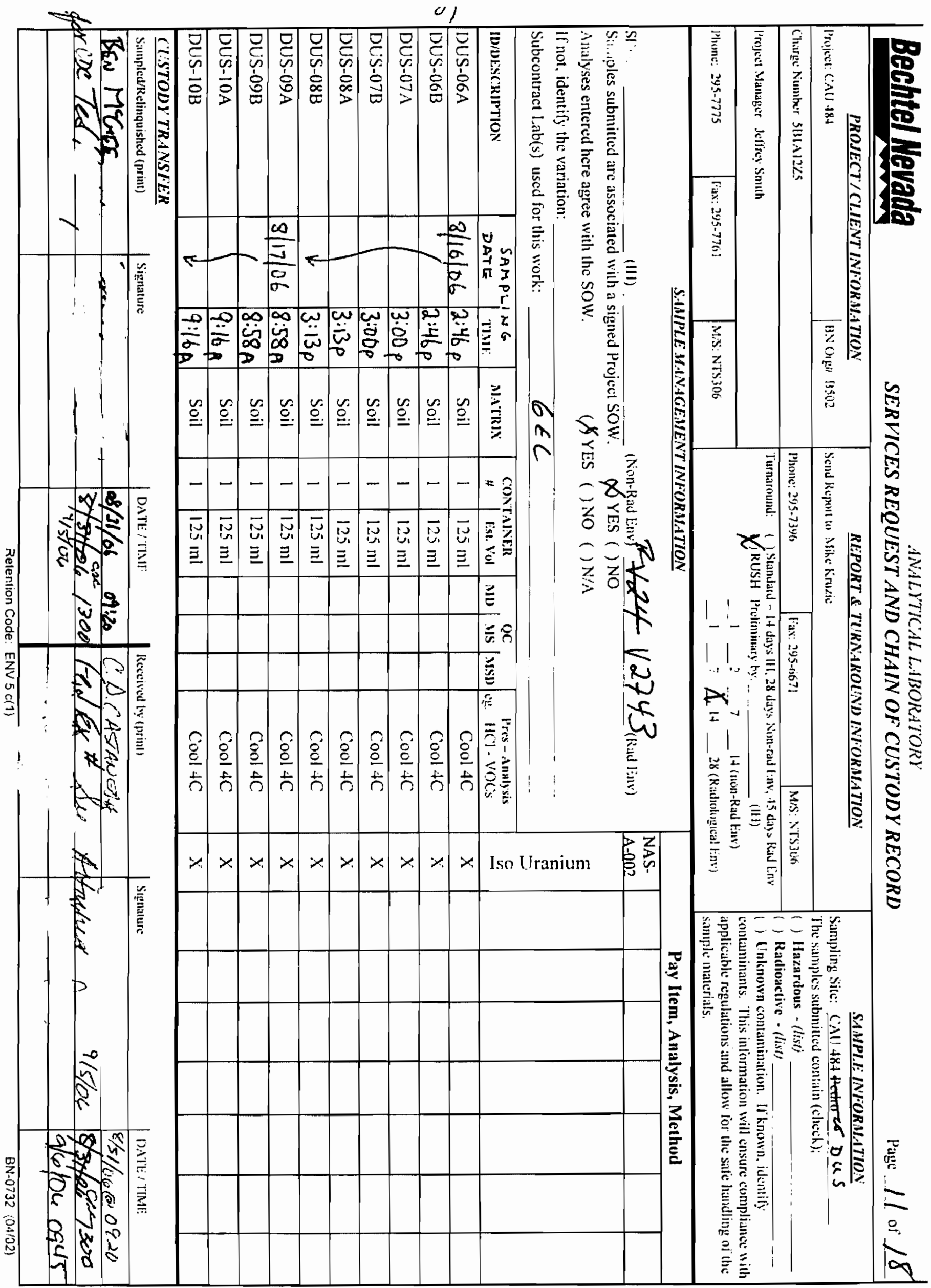




\section{GENERAL ENGINEERING LABORATORIES, LLC}

2040 Savage Road Charleston SC 29407 - (843) 556-8171 - www.gel.com

\section{Certificate of Analysis}

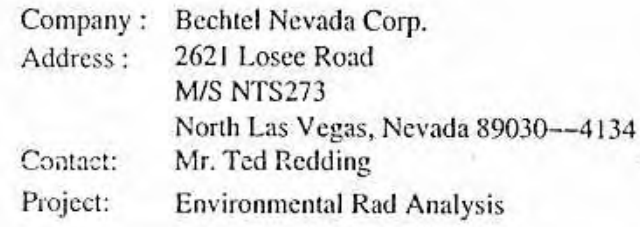

Client Sample ID:

Sample ID:

Matrix:

Collect Date:

Receive Date:

Collector:

$\begin{array}{lll}\text { DUS-11A } & \text { Proiect: } & \text { NEVA00106 } \\ \text { 171011001 } & \text { Client ID: } & \text { NEVA002 } \\ \text { Soil } & & \\ \text { 17-AUG-06 } & \\ \text { 06-SEP-06 } & \\ \text { Client } & & \end{array}$

RL Units

DF Analyst Date

Time Batch Mtd

Rad Alpha Spec Analysis

Alphaspec U. Solid $2 p C i / g$

Uranium-233/234

Uranium-235/236

Uranium-238

Qualifier Result Uncertainty

$\begin{array}{llrlrl}\mathrm{U} & 0.861 & +/-1.28 & 2.26 & +/-1.29 & 2.00 \\ \mathrm{U} & 0.525 & +/-0.985 & 1.91 & +/-0.988 & 2.00 \\ \mathrm{U} & 0.649 & +/-1.15 & 2.17 & +/-1.15 & 2.00\end{array}$

2.00
$\mathrm{pCi} / \mathrm{g}$

$\mathrm{pCi} / \mathrm{g}$

The following Prep Methods were performed

\begin{tabular}{llllll}
\hline Method & Description & Analyst & Date & Time & Prep Batch \\
\hline Dry Soil Prep & Dry Soil Prep GL-RAD-A-02I & JMBI & $09 / 07 / 06$ & 0913 & 565870
\end{tabular}

The following Analytical Methods were performed

$\begin{array}{ll}\text { Method } & \text { Description } \\ 1 & \text { DOE EML HASL-300, U-02-RC Modified }\end{array}$

Surrogate/Tracer recovery

Test

Recovery\%

Acceptable Limits

Uranium-232

Alphaspec U, Solid 2pCi'g

79

$(25 \%-125 \%)$

Notes:

The Qualifiers in this report are defined as follows :

* A quality control analyte recovery is outside of specified acceptance criteria

$<$ Result is less than value reported

$>$ Result is greater than value reported

A The TIC is a suspected aldol-condensation product

B Target analyte was detected in the associated blank

$\mathrm{BD}$ Results are either below the MDC or tracer recovery is low

C Analyte has been confirmed by GC/MS analysis

D Results are reported from a diluted aliquot of the sample

$\mathrm{H}$ Analytical holding time was exceeded

$\mathrm{J}$ Value is estimated

N/A Spike recovery limits do not apply. Sample concentration exceeds spike concentration by $4 X$ or more

$\mathrm{R}$ Sample results are rejected

U Analyte was analyzed for, but not detected above the MDL, MDA, or LOD.

UI Gamma Spectroscopy--Uncertain identification 


\section{GENERAL ENGINEERING LABORATORIES, LLC}

2040 Savage Road Charleston SC 29407 - (843) 556-8171 - www.gel.com

\section{Certificate of Analysis}

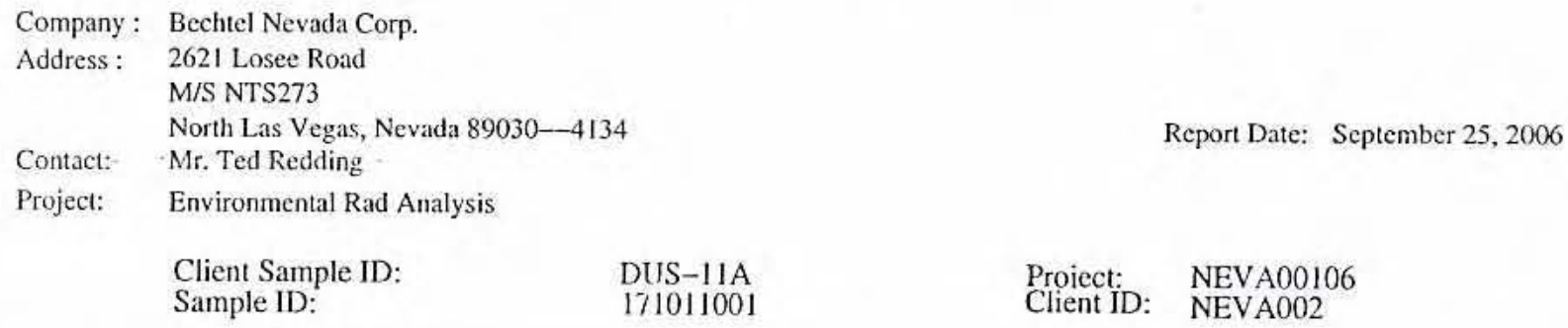

$\wedge$ RPD of sample and duplicate evaluated using $+/-$ RL. Concentrations are $<5 X$ the RL

$\mathrm{h}$ Preparation or preservation holding time was exceeded

The above sample is reported on a dry weight basis. 


\section{GENERAL ENGINEERING LABORATORIES, LLC}

2040 Savage Road Charleston SC 29407 - (843) 556-8171 - www.gel.com

\section{Certificate of Analysis}

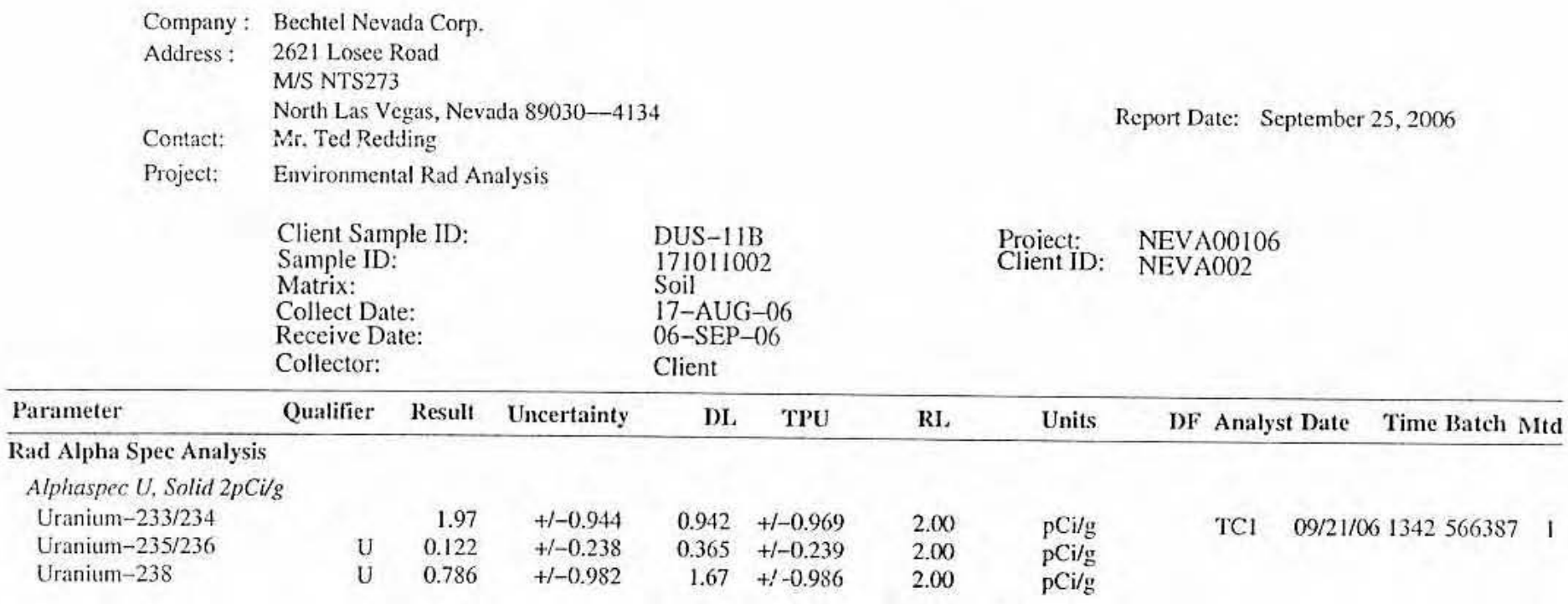

The following Prep Methods were performed

\begin{tabular}{llllll} 
Method & Description & Analyst & Date & Time & Prep Batch \\
\hline Dry Soil Prep & Dry Soil Prep GL-RAD-A-021 & JMB1 & $09 / 07 / 06$ & $09 \mathrm{i} 3$ & 565870
\end{tabular}

The following Analytical Methods were performed

\begin{tabular}{ll} 
Method & Description \\
\hline 1 & DOE EML HASL-300, U-02-RC Modified \\
2 & DOE EML HASL-300, U-02-RC Modified
\end{tabular}

\begin{tabular}{llcc} 
Surrogate/Tracer recovery & Test & Recovery\% & Acceptable Limits \\
\hline Uranium-232 & Alphaspec U, Solid $2 \mathrm{pCi} / \mathrm{g}$ & 87 & $(25 \%-125 \%)$
\end{tabular}

Notes:

The Qualifiers in this report are defined as follows :

* A quality control analyte recovery is outside of specified acceptance criteria

$<$ Result is less than value reported

$>$ Result is greater than value reported

A The TIC is a suspected aldol-condensation product

B Target analyte was detected in the associated blank

$\mathrm{BD}$ Results are either below the MDC or tracer recovery is low

C Analyte has been confirmed by GC/MS analysis

D Results are reported from a diluted aliquot of the sample

$\mathrm{H}$ Analytical holding time was exceeded

$\mathrm{J}$ Value is estimated

N/A Spike recovery limits do not apply. Sample concentration exceeds spike concentration by $4 X$ or more

$\mathrm{R}$ Sample results are rejected

U Analyte was analyzed for, but not detected above the MDL, MDA, or LOD.

U1 Gamma Spectroscopy--Uncertain identification 


\section{GENERAL ENGINEERING L.ABORATORIES, LLC}

2040 Savage Road Charleston SC 29407 - (843) 556-8171 - www.gel.com

\section{Certificate of Analysis}

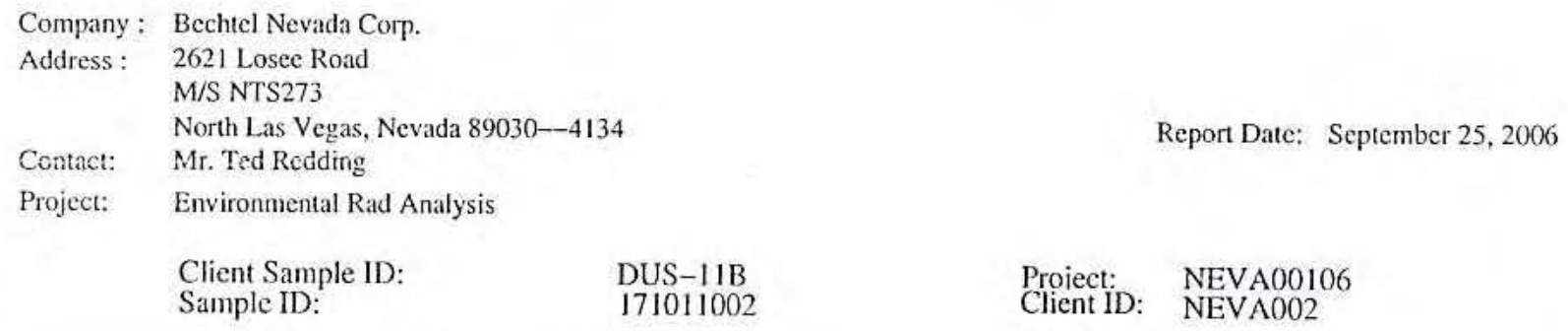

X Consult Case Narrative, Data Summary package, or Project Manager concerning this qualifier

Y QC Samples were not spiked with this compound

$\wedge$ RPD of sample and duplicate evaluated using $+/-$ RL. Concentrations are $<5 X$ the RL

h Preparation or preservation holding time was exceeded

The above sample is reported on a dry weight basis. 


\section{GENERAL ENGINEERING LABORATORIES, LLC}

2040 Savage Road Charleston SC 29407 - (843) 556-8171 - www.gel.com

\section{Certificate of Analysis}

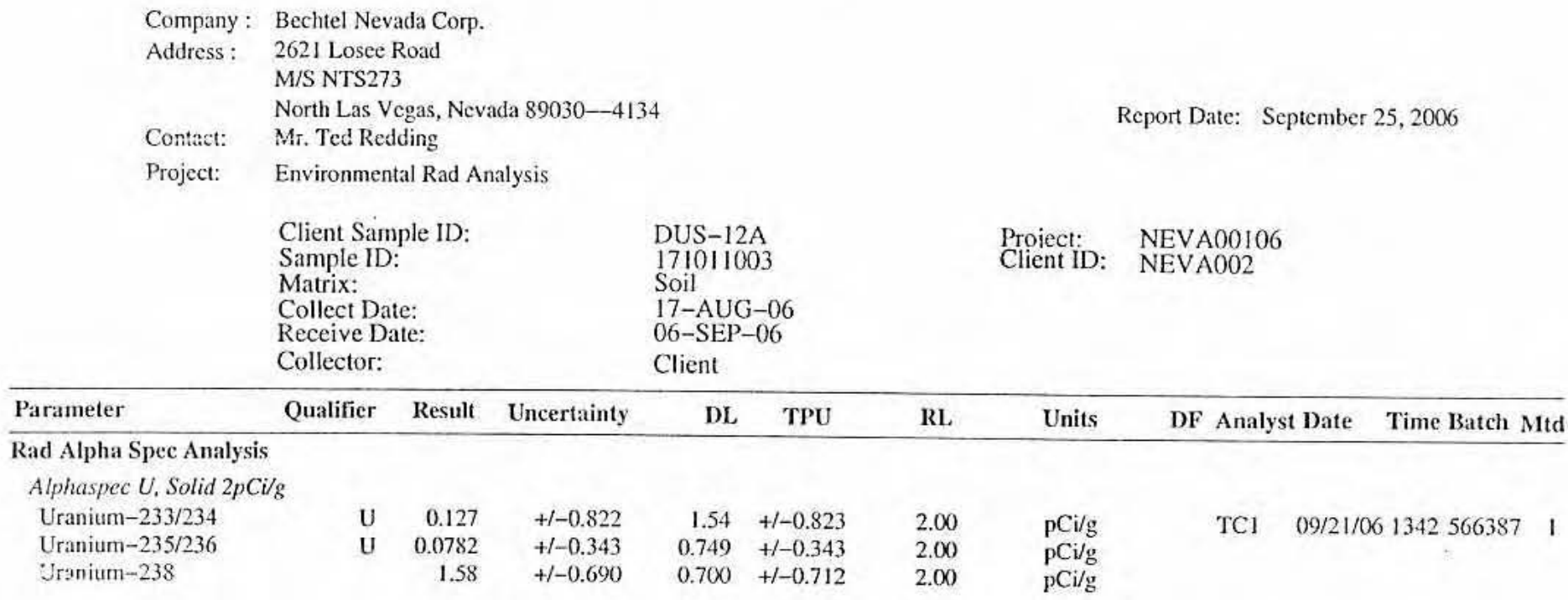

The following Prep Methods were performed

\begin{tabular}{llllll}
\hline Method & Description & Analyst & Date & Time & Prep Batch \\
\hline D:; Soil Prep & Dry Soil Prep GL-RAD-A-021 & JMB1 & $09 / 07 / 06$ & 0913 & 565870
\end{tabular}

Thc following Analytical Methods were performed

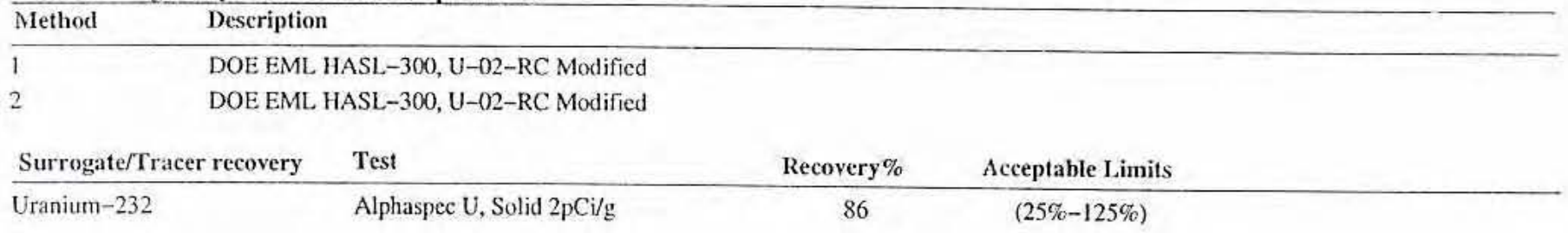

Notes:

The Qualifiers in this report are defined as follows :

* A quality control analyte recovery is outside of specified acceptance criteria

$<$ Result is less than value reported

$>$ Result is greater than value reported

A The TIC is a suspected aldol-condensation product

B Target analyte was detected in the associated blank

$\mathrm{BD}$ Results are either below the MDC or tracer recovery is low

C Analyte has been confirmed by GC/MS analysis

D Results are reported from a diluted aliquot of the sample

$\mathrm{H}$ Analytical holding time was exceeded

$\mathrm{J}$ Value is estimated

N/A Spike recovery limits do not apply. Sample concentration exceeds spike concentration by $4 \mathrm{X}$ or more

$R$ Sample results are rejected

U Analyte was analyzed for, but not detected above the MDL, MDA, or LOD.

UI Gamma Spectroscopy--Uncertain identification 


\section{GENERAL ENGINEERING LABORATORIES, LLC .}

2040 Savage Road Charleston SC 29407 - (843) 556-8171 - www.gel.com

\section{Certificate of Analysis}

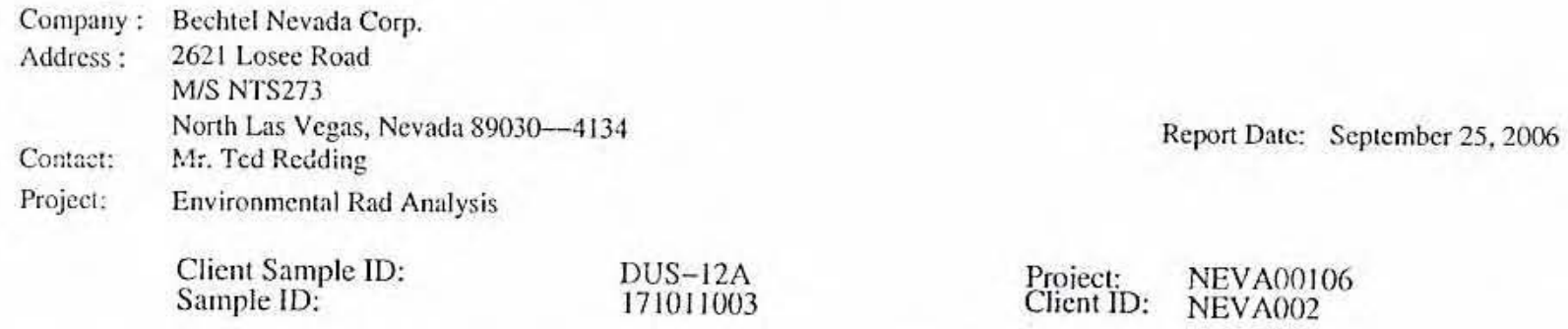

X Consult Case Narrative, Data Summary package, or Project Manager concerning this qualifier

Y QC Samples were not spiked with this compound

$\wedge$ RPD of sample and duplicate evaluated using $+1-\mathrm{RL}$. Concentrations are $<5 \mathrm{X}$ the RL

h Preparation or preservation holding time was exceeded

The above sample is reported on a dry weight basis. 


\section{GENERAL ENGINEERING LABORATORIES, LLC}

2040 Savage Road Charleston SC 29407 - (843) 556-8171 - www.gel.com

\section{Certificate of Analysis}

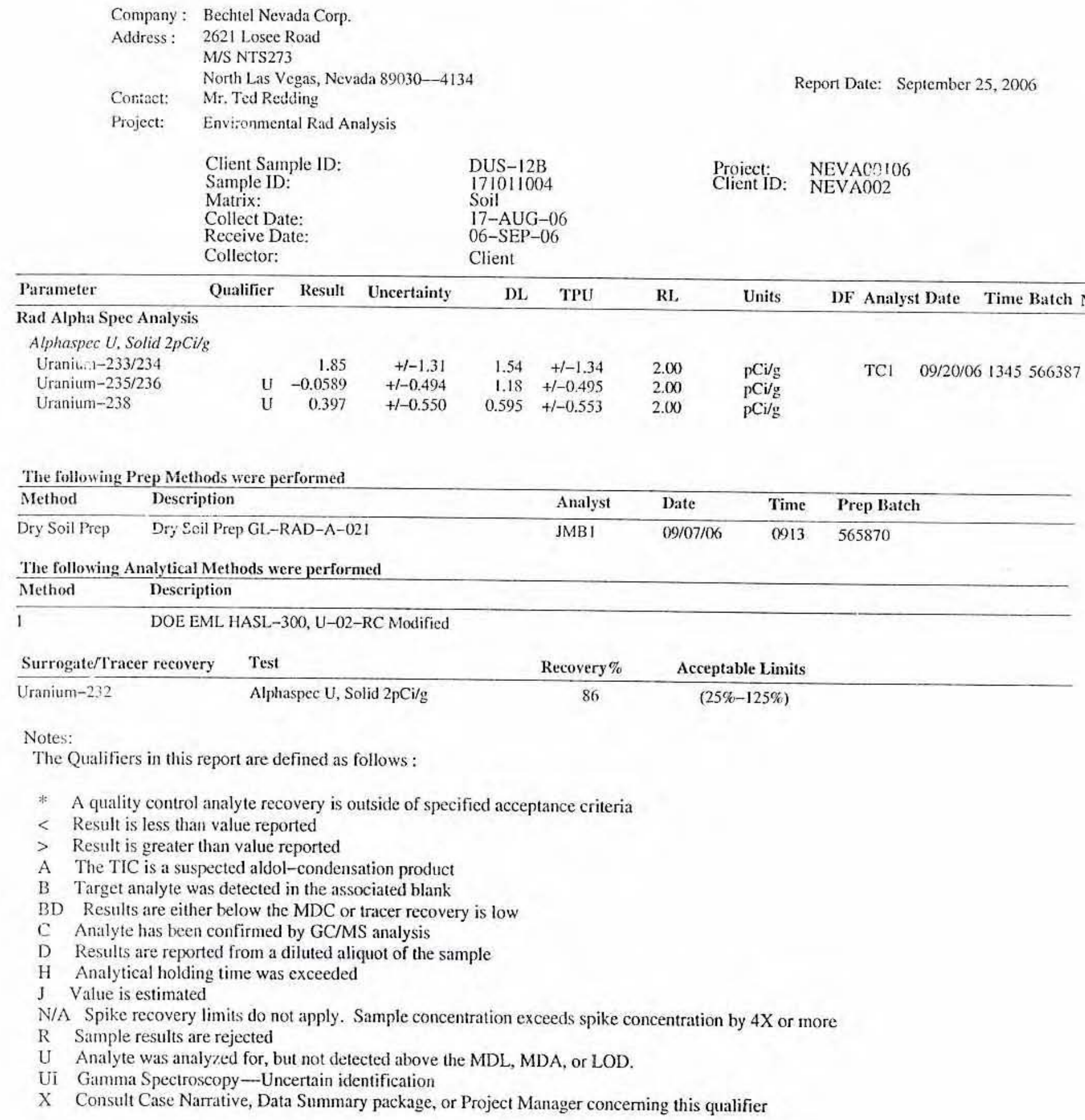




\section{GENERAL ENGINEERING LABORATORIES, LLC}

2040 Savage Road Charleston SC 29407 - (843) 556-8171 - www.gel.com

\section{Certificate of Analysis}

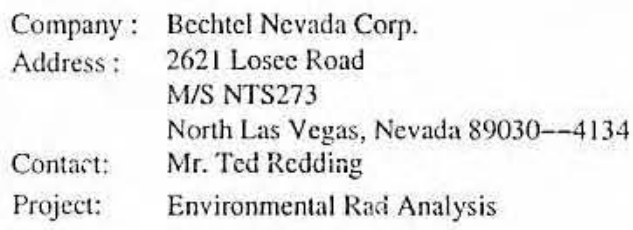

Y QC Samples were not spiked with this compound

$\wedge \quad \mathrm{RPD}$ of sample and duplicate evaluated using $+/-\mathrm{RL}$. Concentrations are $<5 \mathrm{X}$ the $\mathrm{RL}$

$h$ Preparation or preservation holding time was exceeded

The above sample is reported on a dry weight basis. 


\section{GENERAL ENGINEERING LABORATORIES, LLC \\ 2040 Savage Road Charleston SC 29407 - (843) 556-8171 - www.gel.com}

\section{Certificate of Analysis}

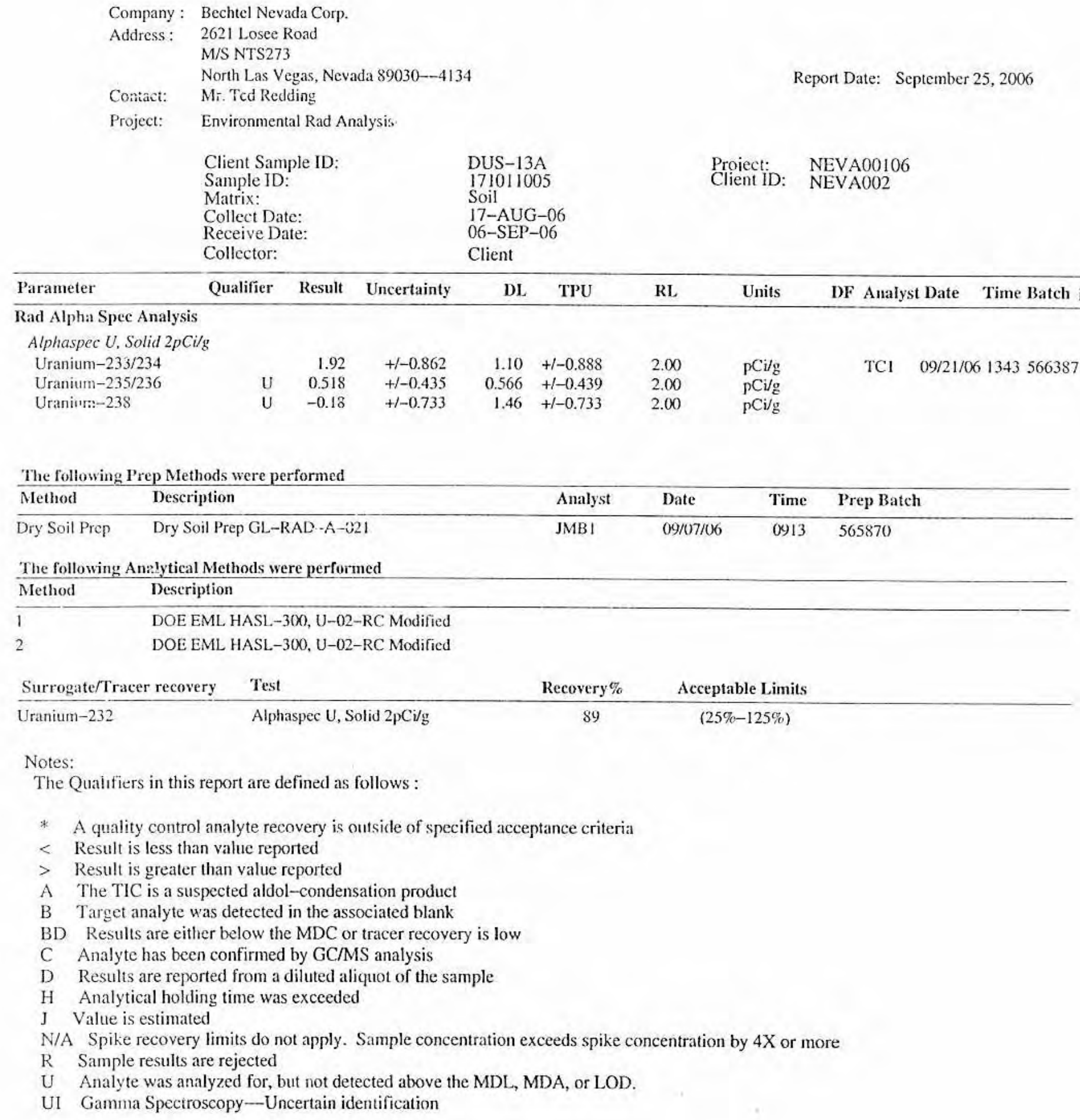




\section{GENERAL ENGINEERING LABORATORIES, LLC \\ 2040 Savage Road Charleston SC 29407 - (843) 556-8171 - www.gel.com}

\section{Certificate of Analysis}

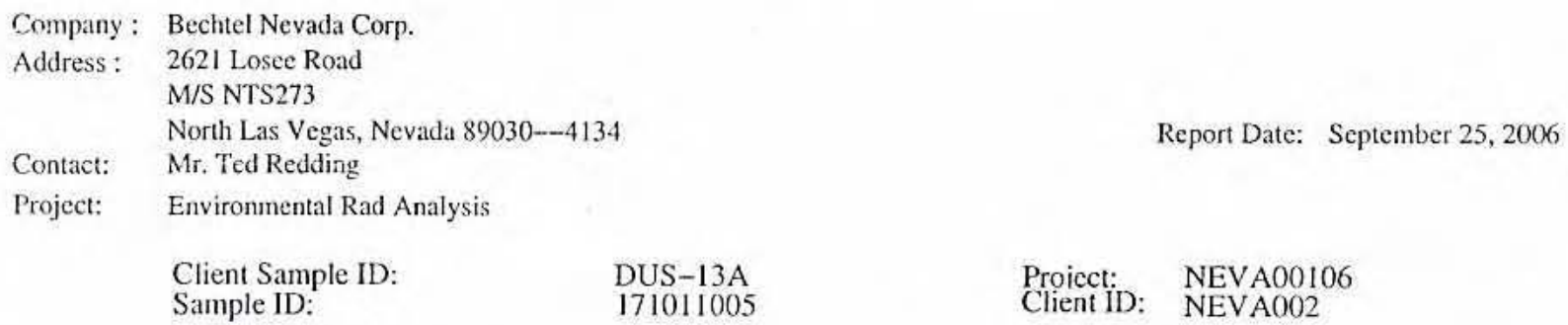

\begin{tabular}{lllllll}
\hline Parameter Qualifier Result Uncertainty & DL TPU RL & Units DF Analyst Date Time Batch MItd
\end{tabular}

X Consult Case Narrative, Data Summary package, or Project Manager conceming this qualifier

Y QC Samples were not spiked with this compound

$\wedge$ RPD of sample and duplicate evaluated using $+1-$ RL. Concentrations are $<5 X$ the RL

$h$ Preparation or preservation holding time was exceeded

The above sample is reported on a diry weight basis. 


\section{GENERAL ENGINEERING LABORATORIES, LLC}

2040 Savage Road Charleston SC 29407 - (843) 556-8171 - www.gel.com

\section{Certificate of Analysis}

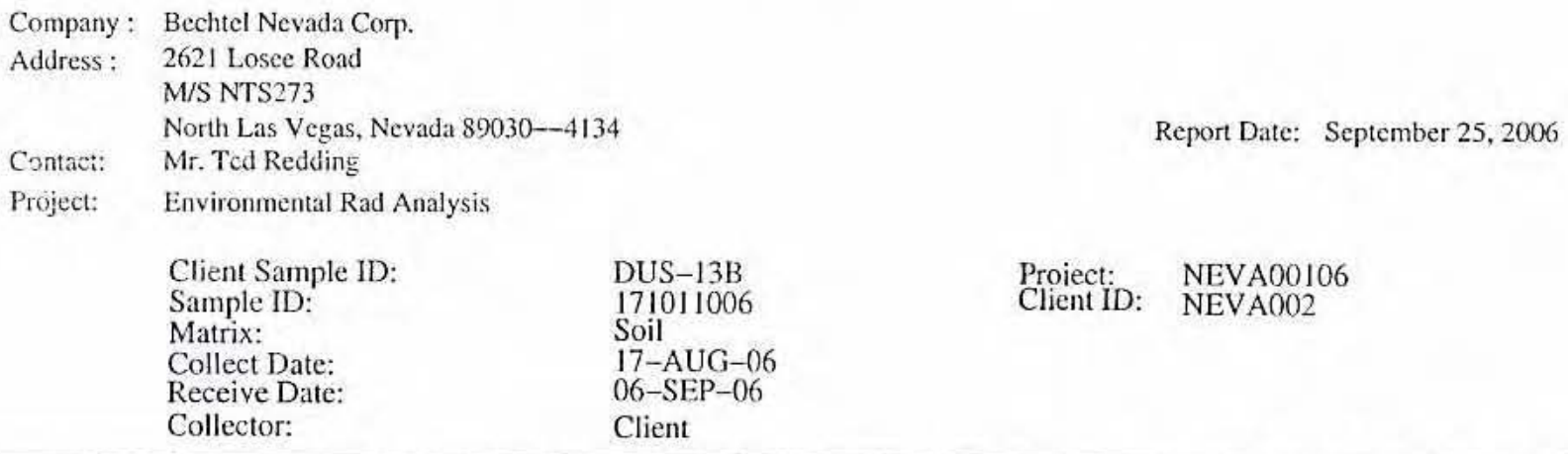

\begin{tabular}{|c|c|c|c|c|c|c|c|c|c|c|c|}
\hline Parameter & Qualifier & Result & Uncertainty & DL & TPU & RL & Units & DF & \multicolumn{3}{|c|}{ Analyst } \\
\hline \multicolumn{12}{|c|}{ Rad Alpha Spec Analysis } \\
\hline \multicolumn{12}{|c|}{ Alphaspec U. Solid 2pCi/g } \\
\hline Uranium-233/234 & $\mathrm{U}$ & 1.48 & $+1-1.06$ & 1.57 & $+1-1.07$ & 2.00 & $\mathrm{pCi} / \mathrm{g}$ & & $\mathrm{TCl}$ & $09 / 21 / 06$ & 1342566387 \\
\hline Uranium-235/236 & & 0.344 & $+1-0.389$ & 0.344 & $+1-0.391$ & 2.00 & $\mathrm{pCi} / \mathrm{g}$ & & & & \\
\hline Uranium-238 & & 1.58 & $+1-0.944$ & 1.24 & $+1-0.960$ & 2.00 & $\mathrm{pCi} / \mathrm{g}$ & & & & \\
\hline
\end{tabular}

The following Prep Methods were performed

\begin{tabular}{llllll}
\hline Method & Description & Analyst & Date & Time & Prep Batch \\
\hline Dry Soil Prep & Dry Soil Prep GL-RAD-A-021 & JMB1 & $09 / 07 / 06$ & 0913 & 565870
\end{tabular}

The following Analytical Methods were performed

\begin{tabular}{ll} 
Method & Description \\
\hline 1 & DOE EML HASL-300, U-02-RC Modified \\
2 & DOE EML HASL-300, U-02-RC Modified
\end{tabular}

$\begin{array}{llll}\text { Surrogate/Tracer recovery Test } & \text { Recovery\% } & \text { Acceptable Limits }\end{array}$

\begin{tabular}{llll}
\hline Uranium-232 Alphaspec U, Solid 2pCi/g & 89 & $(25 \%-125 \%)$
\end{tabular}

Notes:

The Qualifiers in this report are defined as follows :

* A quality control analyte recovery is outside of specified acceptance criteria

$<$ Result is less than value reported

$>$ Result is greater than value reported

A The TIC is a suspected aldol-condensation product

B Target analyte was detected in the associated blank

BD Results are either below the MDC or tracer recovery is low

C Analyte has been confirmed by GC/MS analysis

D Results are reported from a diluted aliquot of the sample

$\mathrm{H}$ Analytical holding time was exceeded

$\mathrm{J}$ Value is estimated

N/A Spike recovery limits do not apply. Sample concentration exceeds spike concentration by $4 X$ or more

$R$ Sample results are rejected

U Analyte was analyzed for, but not detected above the MDL, MDA, or LOD.

UI Gamma Spectroscopy--Uncertain identification 


\section{GENERAL ENGINEERING LABORATORIES, LLC}

2040 Savage Road Charleston SC 29407 - (843) 556-8171 - www.gel.com

\section{Certificate of Analysis}

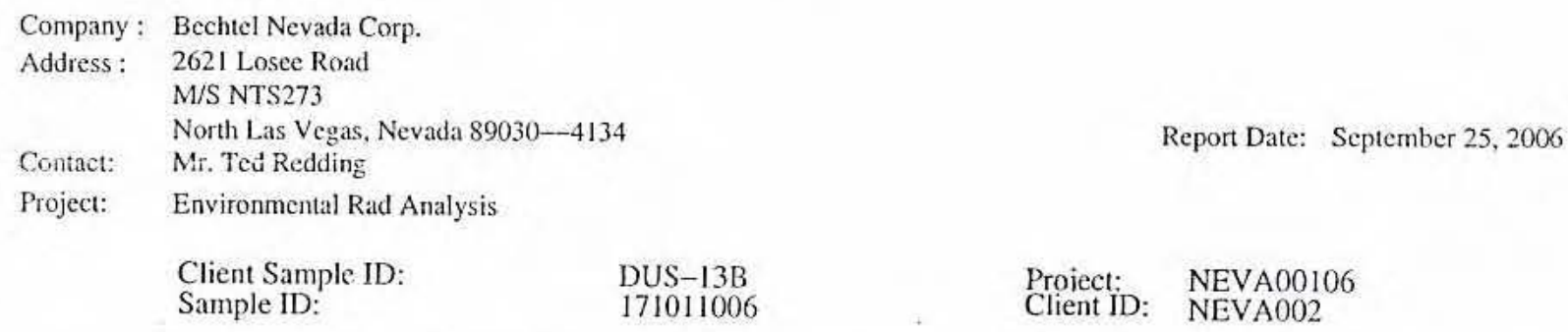

$X$ Consult Case Narrative, Data Summary package, or Project Manager concerning this qualifier

Y QC Samples were not spiked with this compound

$\wedge$ RPD of sample and duplicate evaluated using $+/-$ RL. Concentrations are $<5 X$ the RL

h Preparation or preservation holding time was excceded

The above sample is reported on a dry weight basis. 


\section{GENERAL ENGINEERING LABORATORIES, LLC \\ 2040 Savage Road Charleston SC 29407 - (843) 556-8171 - www.gel.com}

\section{Certificate of Analysis}

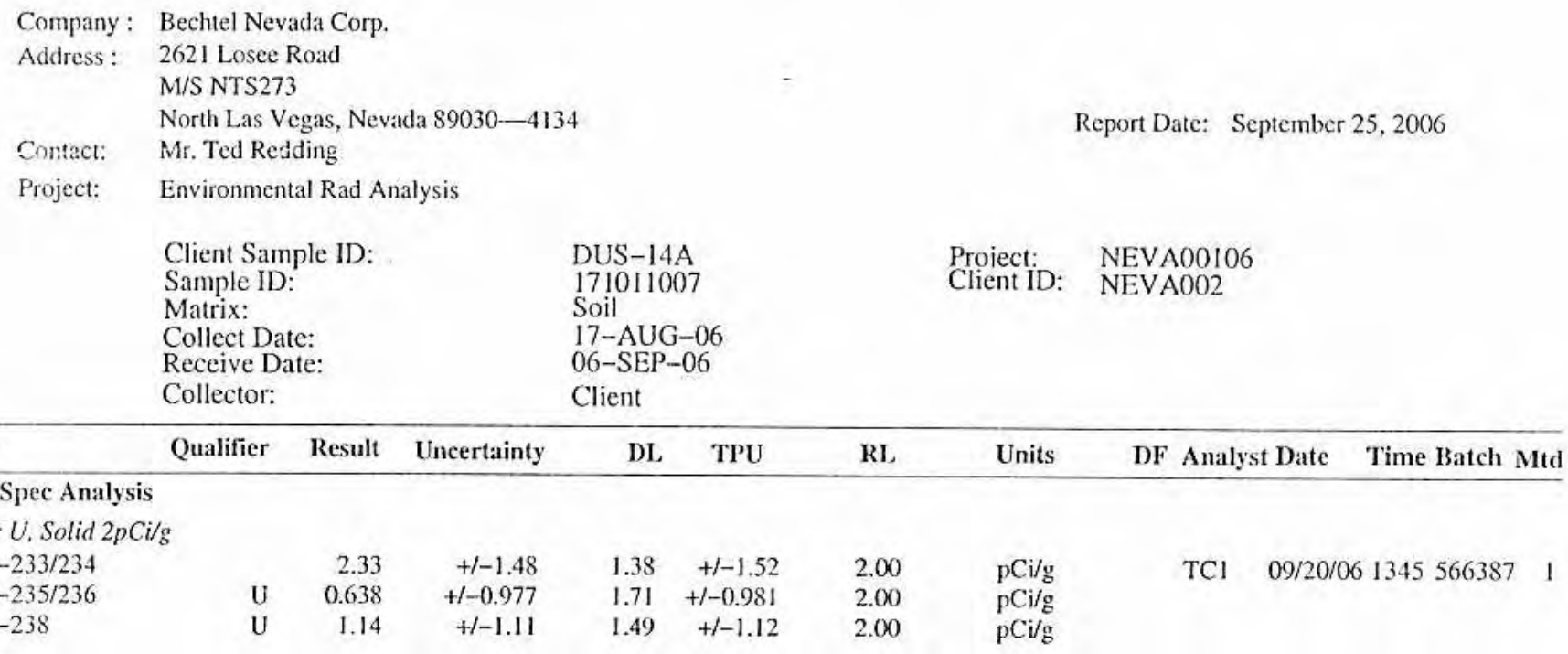

The following Prep Methods were performed

\begin{tabular}{llllll}
\hline Method & Description & Analyst & Date & Time & Prep Batch \\
\hline Dry Soil Prep & Dry Soil Prep GL-RAD-A-021 & JMBI & $09 / 07 / 06$ & 0913 & 565870
\end{tabular}

The following Analytical Methods were performed

\begin{tabular}{ll} 
Method & Description \\
\hline & DOE EML HASL-300, U-02-RC Modified
\end{tabular}

Surrogate/Tracer recovery Test Recovery\% Acceptable Limits

$\begin{array}{llll}\text { Uranium-232 Alphaspec U, Solid 2pCi/g } & 77 & (25 \%-125 \%)\end{array}$

Notes:

The Qualifiers in this report are defined as follows :

* A quality control analyte recovery is outside of specified acceptance criteria

$<$ Result is less than value reported

$>$ Result is greater than value reported

A The TIC is a suspected aldol-condensation product

B Target analyte was detected in the associated blank

$\mathrm{BD}$ Results are either below the MDC or tracer recovery is low

C Analyte has been confirmed by GC/MS analysis

D Results are reported from a diluted aliquot of the sample

$\mathrm{H}$ Analytical holding time was exceeded

$\mathrm{J}$ Value is estimated

N/A Spike recovery limits do not apply. Sample concentration exceeds spike concentration by $4 \mathrm{X}$ or more

$\mathrm{R}$ Sample results are rejected

U Analyte was analyzed for, but not detected above the MDL, MDA, or LOD.

UI Gamma Spectroscopy-Uncertain identification

$\mathrm{X}$ Consult Case Narrative, Data Summary package, or Project Manager concerning this qualifier 


\section{GENERAL-ENGINEERING LABORATORIES, LLC}

2040 Savage Road Charleston SC 29407 - (843) 556-8171 - www. gel.com

\section{Certificate of Analysis}

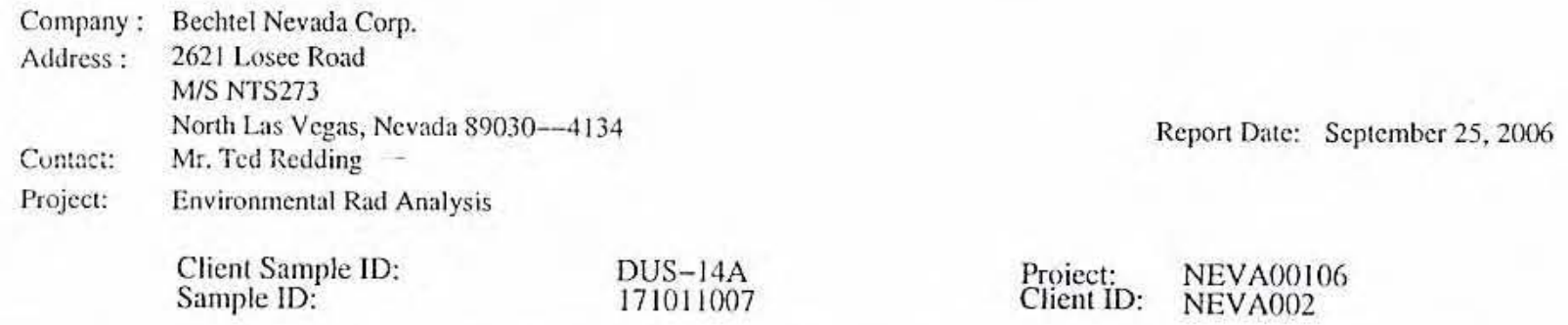

$\begin{array}{lllllll}\text { Parameter } & \text { Qualifier Result Uncertainty } & \text { DL } & \text { TPU } & \text { RL } & \text { Units } & \text { DF Analyst Date Time Batch Mtd }\end{array}$

Y QC Samples were not spiked with this compound

A RP of sample and duplicate evaluated using $+/-\mathrm{RL}$. Concentrations are $<5 \mathrm{X}$ the $\mathrm{RL}$

$h$ Preparation or preservation holding time was exceeded

The above sample is reported on a dry weight basis. 


\section{GENERAL ENGINFERING LABORATORIES, LLC \\ 2040 Savage Road Charleston SC 29407 - (843) 556-8171 - www.gel.com}

\section{Certificate of Analysis}

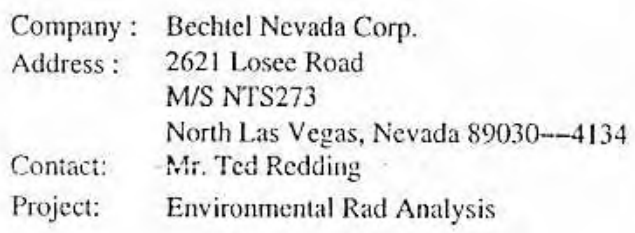

$\begin{array}{llll}\text { Client Sample ID: } & \text { DUS-14B } & \text { Proiect: } & \text { NEVA00106 } \\ \text { Sample ID: } & 171011008 & \text { Client ID: } & \text { NEVA002 } \\ \text { Matrix: } & \text { Soil } & & \\ \text { Collect Date: } & 17-\text { AUG-06 } & \\ \text { Receive Date: } & \text { 06-SEP-06 } & \\ \text { Collector: } & \text { Client } & & \end{array}$

\begin{tabular}{|c|c|c|c|c|c|c|c|c|c|c|}
\hline Parameter & Qualifier & Result & Uncertainty & $\mathrm{DL}$ & TPU & RL & Units & $\mathrm{DF}$ & Analyst Date & Time Batch Mtd \\
\hline \multicolumn{11}{|c|}{ Rad Alpha Spec Analysis } \\
\hline \multicolumn{11}{|c|}{ Alphaspec U. Solid $2 p C i / g$} \\
\hline Uranium-233/234 & & 2.67 & $+1-0.837$ & 0.715 & $+1-0.892$ & 2.00 & $\mathrm{pCi} / \mathrm{g}$ & & $09 / 23 / 0$ & $60941566387 \quad 1$ \\
\hline Uranium-235/236 & $\mathrm{U}$ & 0.0719 & $+1-0.141$ & 0.216 & $+1-0.141$ & 2.00 & $\mathrm{pCi} / \mathrm{g}$ & & & \\
\hline Uranium-238 & & 1.57 & $+1-0.634$ & 0.557 & $+1-0.659$ & 2.00 & $\mathrm{pCi} / \mathrm{g}$ & & & \\
\hline
\end{tabular}

The following Prep Methods were performed

\begin{tabular}{llllll}
\hline Method & Description & Analyst & Date & Time & Prep Batch \\
\hline Dry Soil Prep & Dry Soil Prep GL-RAD-A-021 & JMB1 & $09 / 07 / 06$ & U913 & 565870
\end{tabular}

The following Analytical Methods were performed

\begin{tabular}{ll} 
Method & Description \\
\hline 1 & DOE EML HASL-300, U-02-RC Modified \\
2 & DOE EML HASL-300, U-02-RC Modified
\end{tabular}

\begin{tabular}{llcc} 
Surrogate/Tracer recovery & Test & Recovery\% & Acceptable Limits \\
\hline Uranium-232 & Alphaspec U, Solid 2pCi/g & 63 & $(25 \%-125 \%)$
\end{tabular}

Notes:

The Qualifiers in this report are defined as follows :

* A quality control analyte recovery is outside of specified acceptance criteria

$<$ Result is less than value reported

$>$ Result is greater than value reported

A The TIC is a suspected aldol-condensation product

B Target analyte was detected in the associated blank

$\mathrm{BD}$ Results are either below the MDC or tracer recovery is low

C Analyte has been confirmed by GC/MS analysis

D Results are reported from a diluted aliquot of the sample

$\mathrm{H}$ Analytical holding time was exceeded

$\mathrm{J}$ Value is estimated

N/A Spike recovery limits do not apply. Sample concentration exceeds spike concentration by $4 \mathrm{X}$ or more

$\mathrm{R}$ Sample results are rejected

U Analyte was analyzed for, but not detected above the MDL, MDA, or LOD.

UI Gamma Spectroscopy-Uncertain identification 


\section{GENERAL ENGINEERING LABORATORIES, LLC}

2040 Savage Road Charleston SC 29407 - (843) 556-8171 - www.gel.com

\section{Certificate of Analysis}

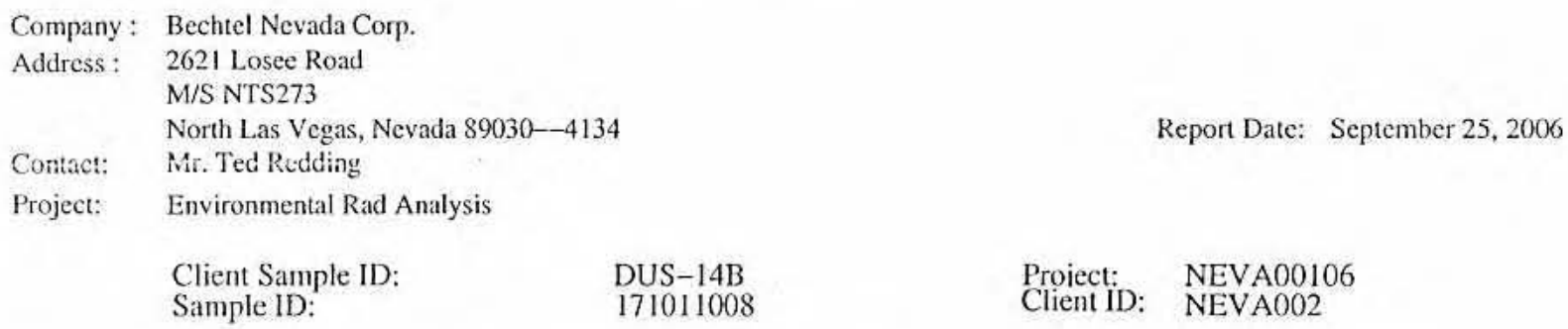

\begin{tabular}{llllllll}
\hline Parameter Qualifier Result Uncertainty & DL & TPU & RL & Units & DF Analyst Date Time Batch MItd
\end{tabular}

X Consult Case Narrative, Data Summary package, or Project Manager concerning this qualifier

Y QC Samples were not spiked with this compound

$\wedge$ RPD of sample and duplicate evaluated using $+/-$ RL. Concentrations are $<5 X$ the $R L$

h Preparation or preservation holding time was exceeded

The above sample is reported on a dry weight basis. 


\section{GENERAL ENGINEERING LABORATORIES, LLC \\ 2040 Savage Road Charleston SC 29407 - (843) 556-8171 - www.gel.com}

\section{Certificate of Analysis}

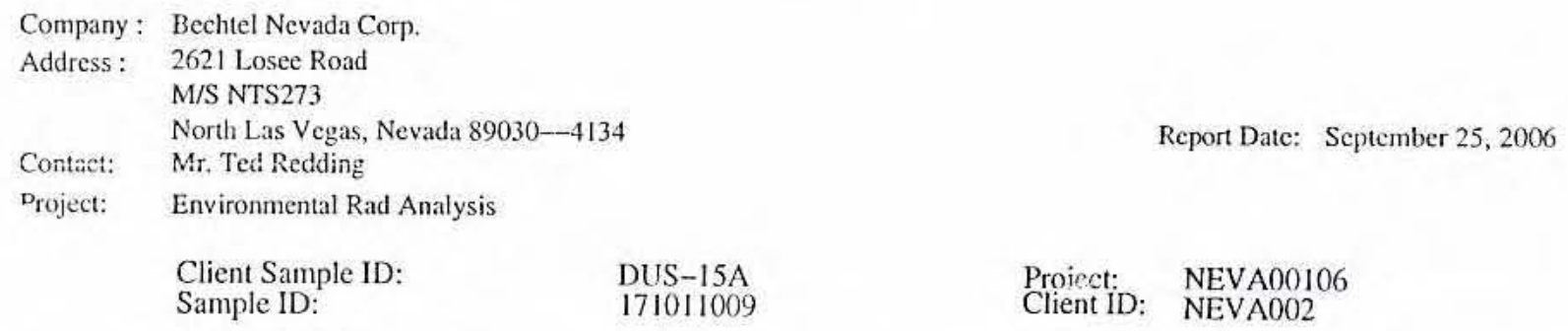

Y QC Samples were not spiked with this compound

$\wedge$ RPD of sample and duplicate evaluated using $+/-$ RL. Concentrations are $<5 X$ the RL

$\mathrm{h}$ Preparation or preservation holding time was exceeded

The above sample is reported on a dry weight basis. 


\section{GENERAL ENGINEERING LABORATORIES, LLC}

2040 Savage Road Charleston SC 29407 - (843) 556-8171 - www.gel.com

\section{Certificate of Analysis}

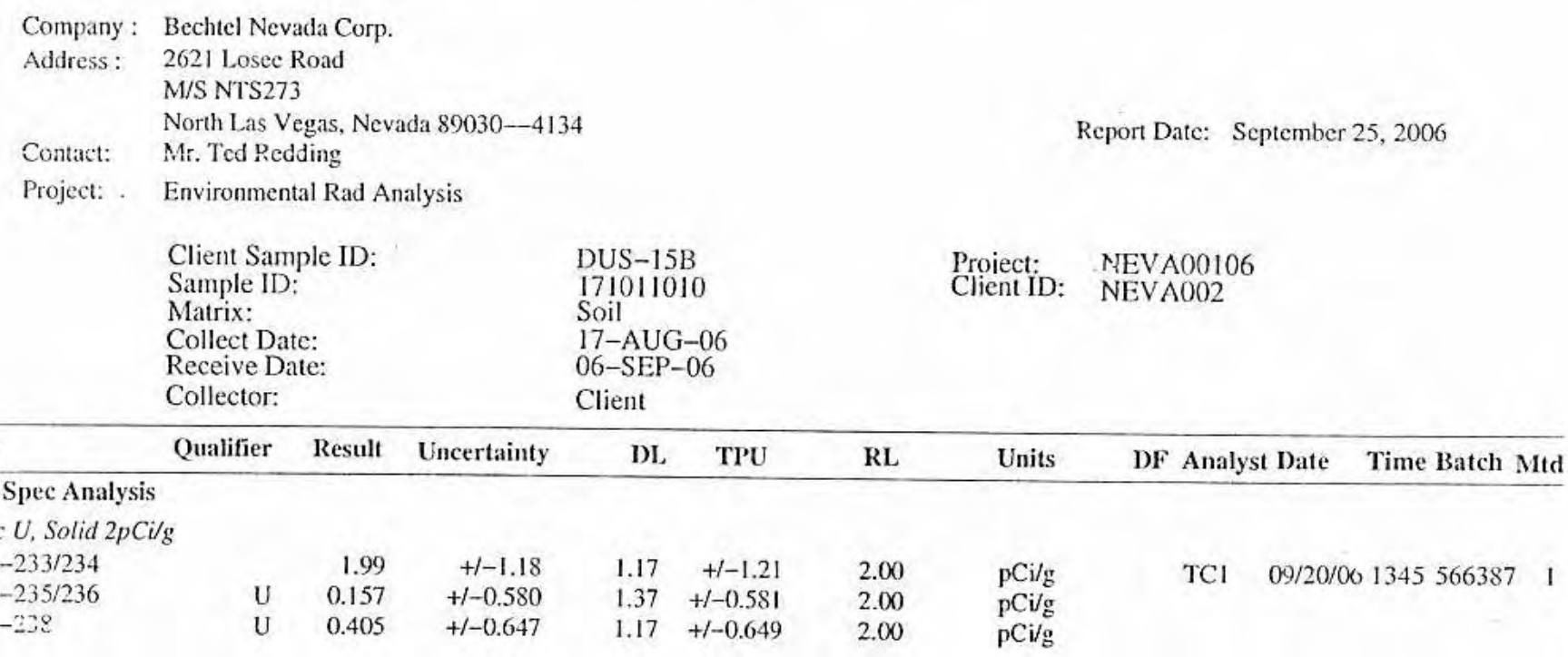

The following Prep Methods were performed

\begin{tabular}{llllll}
\hline Method & Description & Analyst & Date & Time & Prep Batch \\
\hline Dry Soil Prep & Dry Soil Prep GL-RAD-A-021 & JMBI & $09 / 07 / 06$ & 0913 & 565870
\end{tabular}

The following Analytical Methods were performed

\begin{tabular}{ll} 
Method Description \\
\hline DOE EML HASL-300, U-02-RC Modified
\end{tabular}

\begin{tabular}{llcc} 
Surrogate/Tracer recovery & Test & Recovery\% & Acceptable Limits \\
\hline Uranium-232 & Alphaspec U, Solid 2pCi/g & 86 & $(25 \%-125 \%)$
\end{tabular}

Notes:

The Qualifiers in this report are defined as follows :

* A quality control analyte recovery is outside of specified acceptance criteria

$<$ Result is less than value reported

$>$ Result is greater than value reported

A The TIC is a suspected aldol-condensation product

B Target analyte was detected in the associated blank

$\mathrm{BD}$ Results are either below the MDC or tracer recovery is low

C Analyte has been confirmed by GC/MS analysis

D Results are reported from a diluted aliquot of the sample

$\mathrm{H}$ Analytical holding time was exceeded

$\mathrm{J}$ Value is estimated

N/A Spike recovery limits do not apply. Sample concentration exceeds spike concentration by $4 X$ or more

$\mathrm{R}$ Sample results are rejected

U Analyte was analyzed for, but not detected above the MDL, MDA, or LOD.

UI Gamma Spectroscopy--Uncertain identification

X Consult Case Narrative, Data Summary package, or Project Manager concerning this qualifier 


\section{GENERAL ENGINEERING LABORATORIES, LLC}

2040 Savage Road Charleston SC 29407 - (843) 556-8171 - www.gel.com

\section{Certificate of Analysis}

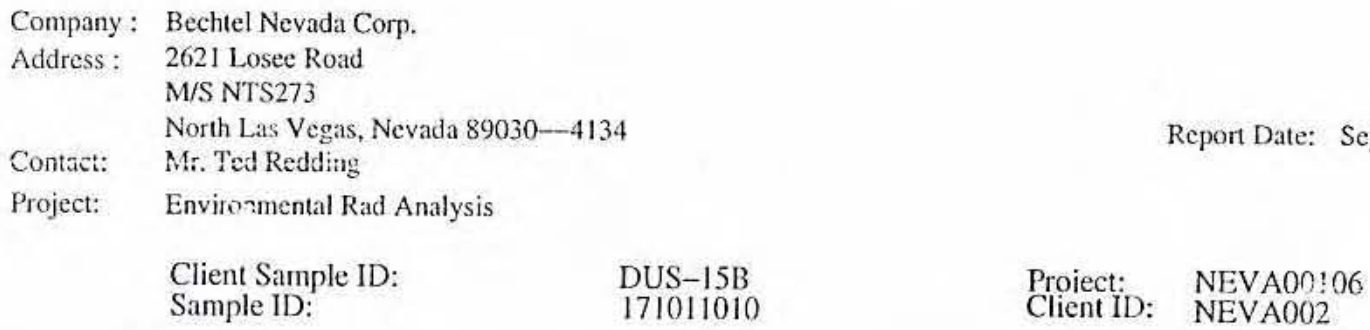

Y QC Samples were not spiked with this compound

$\hat{R}$ RPD of sample and duplicate evaluated using $+/-\mathrm{RL}$. Concentrations are $<5 \mathrm{X}$ the RL

h Preparation or preservation holding time was exceeded

The above sample is reported on a dry weight basis. 


\section{GENERAL ENGINEERING LABORATORIES, LLC \\ 2040 Savage Road Charleston SC 29407 - (843) 556-8171 - www.gel.com}

\section{Certificate of Analysis}

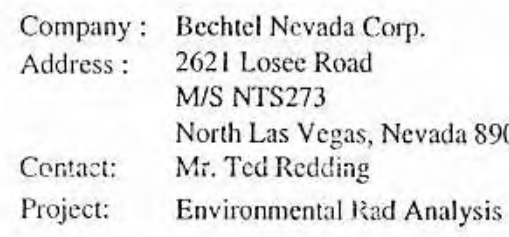

\begin{tabular}{|c|c|c|c|c|c|c|c|c|c|c|c|c|}
\hline \multirow[b]{2}{*}{ Parameter } & \multicolumn{2}{|c|}{$\begin{array}{l}\text { Client Sample ID: } \\
\text { Sample ID: } \\
\text { Matrix: } \\
\text { Collect Date: } \\
\text { Receive Date: } \\
\text { Collector: }\end{array}$} & \multicolumn{3}{|c|}{$\begin{array}{l}\text { DUS-16A } \\
171011011 \\
\text { Soil } \\
17-A U G-06 \\
06-S E P-06 \\
\text { Client }\end{array}$} & \multirow[b]{2}{*}{$\mathrm{RL}$} & \multirow{2}{*}{$\begin{array}{l}\text { Proiect: } \\
\text { Client ID: } \\
\text { Units }\end{array}$} & \multicolumn{5}{|c|}{$\begin{array}{l}\text { NEVA00106 } \\
\text { NEVA002 }\end{array}$} \\
\hline & Qualifier & Result & Uncertainty & $\mathrm{DL}$ & TPU & & & DF & Anal & st Date & Time Batch $\mathrm{N}$ & Mtd \\
\hline \multicolumn{13}{|c|}{ Rad Alpha Spec Analysis } \\
\hline \multicolumn{13}{|c|}{ Aiphaspec U, Solvi $2 p C i / g$} \\
\hline Uranium-233/234 & $\mathrm{U}$ & 1.68 & $+/-1.54$ & 2.21 & $+/-1.56$ & 2.00 & $\mathrm{pCi} / \mathrm{g}$ & & $\mathrm{TCl}$ & $09 / 20 / 6$ & 661345566387 & \\
\hline Uranium-235/236 & $-U$ & 0.792 & $+1-1.09$ & 1.74 & $+1-1.09$ & 2.00 & $\mathrm{pCi} / \mathrm{g}$ & & & & & \\
\hline Uranium-238 & $\mathrm{U}$ & 0.519 & $+1-0.896$ & 1.68 & $+1-0.899$ & 2.00 & $\mathrm{pCi} / \mathrm{g}$ & & & & & \\
\hline
\end{tabular}

The following Prep Methods were performed

\begin{tabular}{llllll}
\hline Method & Description & Analyst & Date & Time & Prep Batch \\
\hline Dry Soil Prep & Dry Soil Prep GL-RAD-A-021 & JMBI & $09 / 07 / 06$ & 0913 & 565870
\end{tabular}

The following Analytical Metiods were performed

\begin{tabular}{llcc}
\hline Method Description & & \\
\hline DOE EML HASL-300, U-02-RC Modified & & \\
Surrogate/Tracer recovery & Test & Recovery\% & Acceptable Limits \\
\hline Uranium-232 & Alphaspec U, Solid 2pCi/g & 81 & $(25 \%-125 \%)$
\end{tabular}

Notes:

The Qualifiers in this report are defined as follows :

* A quality control analyte recovery is outside of specified acceptance criteria

$<$ Result is less than value reported

$>$ Result is greater than value reported

A The TIC is a suspected aldol-condensation product

B Target analyte was detected in the associated blank

$\mathrm{BD}$ Results are either below the MDC or tracer recovery is low

C Analyte has been confirmed by GC/MS analysis

D Results are reported from a diluted aliquot of the sample

$\mathrm{H}$ Analytical holding time was exceeded

J Value is estimated

N/A Spike recovery limits do not apply. Sample concentration exceeds spike concentration by $4 \mathrm{X}$ or more

$\mathrm{R}$ Sample results are rejected

$\mathrm{U}$ Analyte was analyzed for, but not detected above the MDL, MDA, or LOD.

UI Ganma Spectroscopy--Uncertain identification 


\section{GENERAL ENGINEERING LABORATORIES, LLC \\ 2040 Savage Road Charleston SC 29407 - (843) 556-8171 - www.gel.com}

\section{Certificate of Analysis}

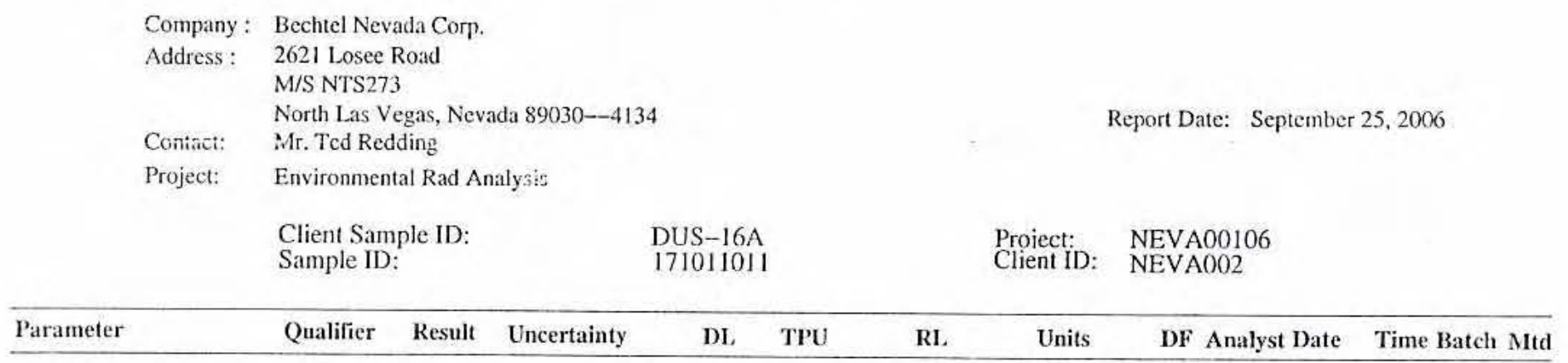

$\mathrm{X}$ Consult Case Narrative, Data Summary package, or Project Manager concerning this qualifier

Y QC Samples were not spiked with this compound

A RPD of sample and duplicate evaluated using $+/-\mathrm{RL}$. Concentrations are $\angle 5 X$ the RL

$h$ Preparation or preservation holding time was exceeded

The above sample is reposted on a dry weight basis. 


\section{GENERAL ENGINEERING LABORATORIES, LLC \\ 2040 Savage Road Charleston SC 29407 - (843) 556-8171 - www.gel.com}

\section{Certificate of Analysis}

\author{
Company: Bechtel Nevada Corp. \\ Address : 2621 Losee Road \\ M/S NTS273 \\ North Las Vegas, Nevada 89030--4134 \\ Contact: Mr. Ted Redding \\ Project: Environmental Rad Analysis
}

Report Date: September 25, 2006

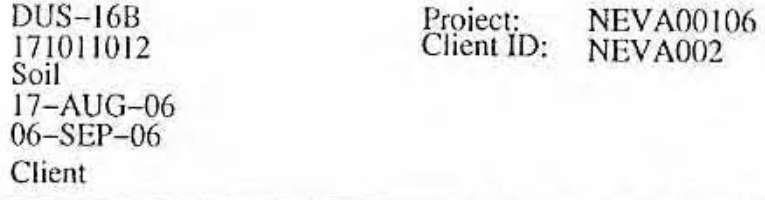

\begin{tabular}{|c|c|c|c|c|c|c|c|c|c|c|c|c|}
\hline Parameter & Qualifier & Result & Uncertainty & $\mathrm{DL}$ & TPU & RL & Units & DF & Analyst & Date & Time Batch $\mathrm{N}$ & Mtd \\
\hline \multicolumn{13}{|c|}{ Rad Alpha Spec Analysis } \\
\hline \multicolumn{13}{|c|}{ Alphaspec $U$, Solid $2 p C i / g$} \\
\hline Uranium-233/234 & $\mathrm{U}$ & 1.19 & $+1-1.40$ & 2.25 & $+/-1.41$ & 2.00 & $\mathrm{pCi} / \mathrm{g}$ & & $\mathrm{TCl}$ & $09 / 20 / 06$ & 1345566387 & 1 \\
\hline Uranium-235/236 & U & -0.538 & $+/-0.398$ & 2.48 & $+1-0.405$ & 2.00 & $\mathrm{pCi} / \mathrm{g}$ & & & & & \\
\hline Uranium-238 & $U$ & 0.404 & +i. 0.928 & 1.91 & $+/-0.930$ & 2.00 & $\mathrm{pCi} / \mathrm{g}$ & & & & & \\
\hline
\end{tabular}

The following Prep Methods were performed

\begin{tabular}{llllll}
\hline Method & Description & Analyst & Date & Time & Prep Batch \\
\hline Dry Soil Prep & Dry Soil Prep GL-RAD-A-02! & JMBi & $09 / 07 / 06$ & 0913 & 565870
\end{tabular}

The following Analytical Methods were performed

$\frac{\text { Description }}{1}$ DOE EML HASL-300, U-02-RC Modified

\begin{tabular}{llcc} 
Surrogate/Tracer recovery & Test & Recovery\% & Acceptable Limits \\
\hline Uranium-232 & Alphaspec U, Solid 2pCi/g & 86 & $(25 \%-125 \%)$
\end{tabular}

\section{Notes:}

The Qualifiers in this report are defined as follows :

* A quality control analyte recovery is outside of specified acceptance criteria

$<$ Result is less than value reported

$>$ Result is greater than value reported

A The TIC is a suspected aldol-condensation product

B Target analyte was detected in the associated blank

BD Results are either below the MDC or tracer recovery is low

C Analyte has been confirmed by GC/MS analysis

D Results are reported from a diluted aliquot of the sample

H Analytical holding time was exceeded

$\mathrm{J}$ Value is estimated

N/A Spike recovery limits do not apply. Sample concentration exceeds spike concentration by $4 \mathrm{X}$ or more

$\mathrm{R}$ Sample results are rejected

$\mathrm{U}$ Analyte was analyzed for, but not detected above the MDL, MDA, or LOD.

UI Gamma Spectroscopy-Uncertain identification

$\mathrm{X}$ Consult Case Narrative, Data Summary package, or Project Manager concerning this qualifier 


\section{GENERAL ENGINEERING LABORATORIES, LLC \\ 2040 Savage Road Charleston SC 29407 - (843) 556-8171 - www.gel.com}

\section{Certificate of Analysis}

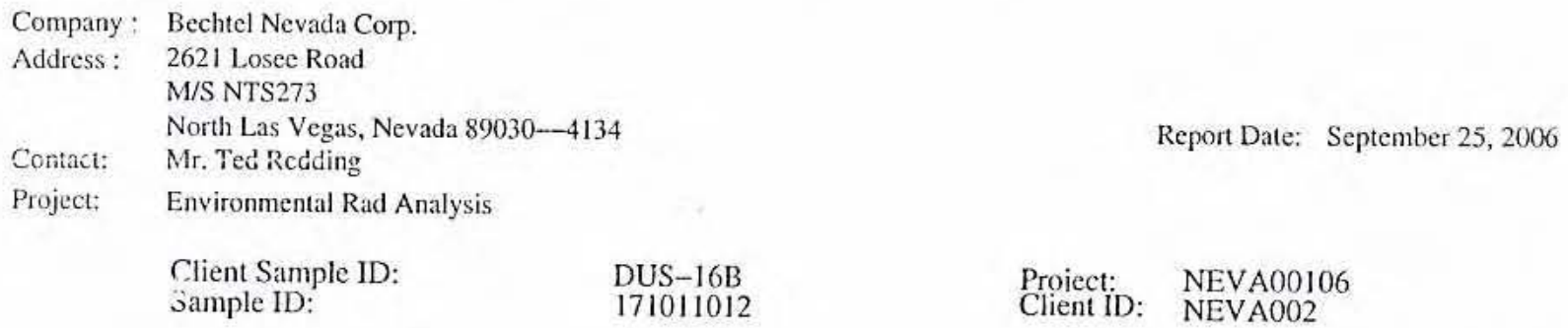

Y QC Samples were not spiked with this compound

$\wedge$ RPD of sample and duplicate evaluatect using $+/-$ RL. Concentrations are $\angle 5 X$ the $R L$

h Preparation or preservation holding time was exceeded

The above sample is reported on a dry weight basis. 


\section{Certificate of Analysis}

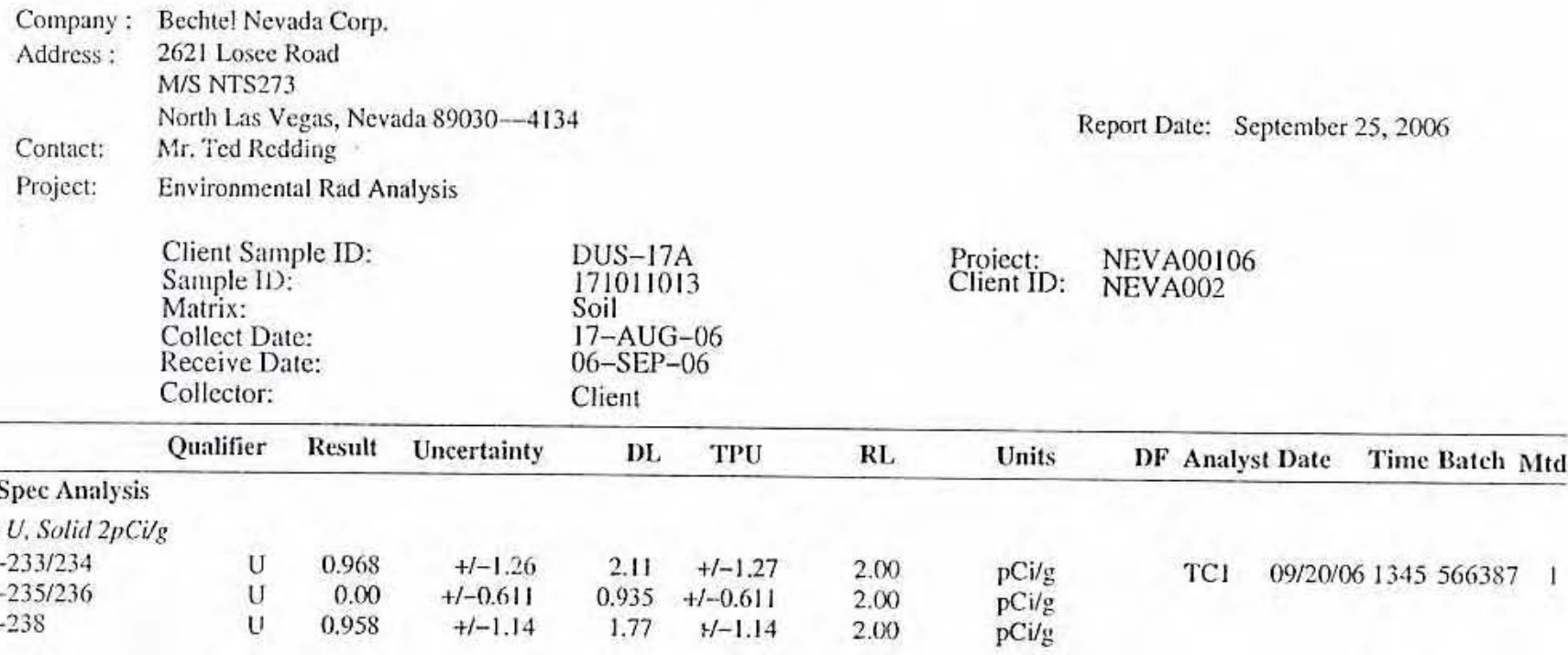

The following Prep Methods were performed

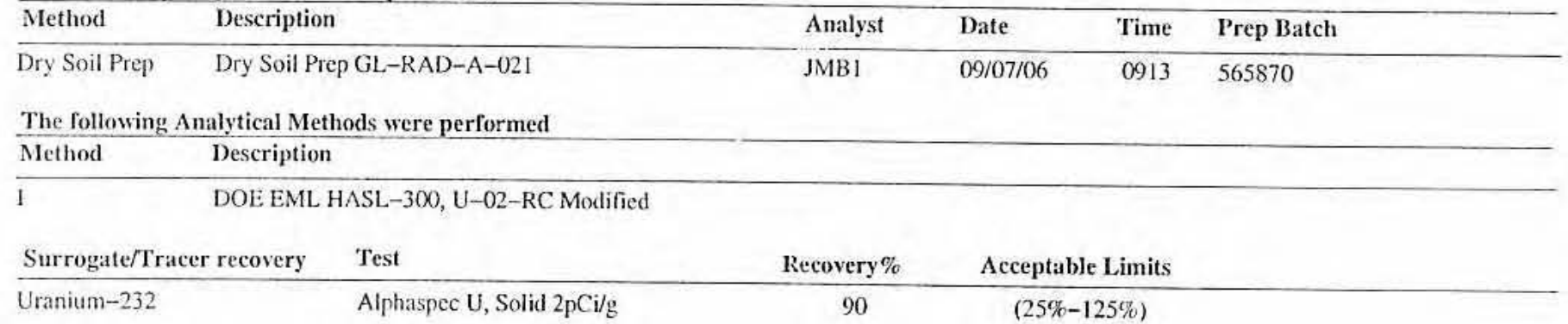

Notes:

The Qualifiers in this report are defined as follows :

* A quality control analyte recovery is outside of specified acceptance criteria

$<$ Result is less than value reported

$>$ Result is greater than value reported

A The TIC is a suspected aldol-condensation product

B Target analyte was detected in the associated blank

$\mathrm{BD}$ Results are either below the MDC or tracer recovery is low

C Analyte has been confirmed by GC/MS analysis

D Results are reported from a diluted aliquot of the sample

$\mathrm{H}$ Analytical holding time was exceeded

$\mathrm{J}$ Value is estimated

N/A Spike recovery limits do not apply. Sample concentration exceeds spike concentration by $4 \mathrm{X}$ or more

$\mathrm{R}$ Sample results are rejected

U Analyte was analyzed for, but not detected above the MDL, MDA, or LOD.

UI Gamma Spectroscopy--Uncertain identification

$\mathrm{X}$ Consult Case Narrative, Dita Summary package, or Project Manager concerning this qualifier 


\section{GENERAL ENGINEERING LABORATORIES, LLC \\ 2040 Savage Road Charleston SC 29407 - (843) 556-8171 - www.gel.com}

\section{Certificate of Analysis}

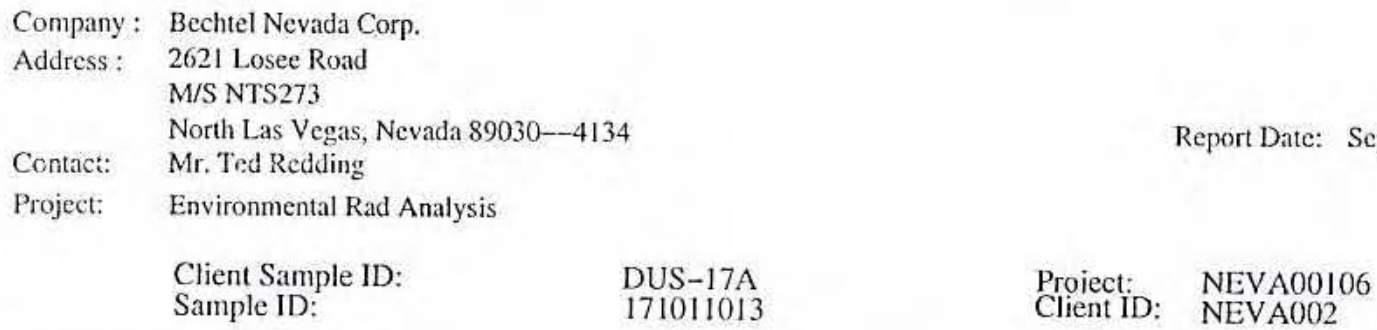

\begin{tabular}{llllllll}
\hline Parameter & Qualifier Result Uncertainty & DL & TPU & RL & Units & DF Analyst Date Time Batch Mtd
\end{tabular}

Y QC Samples were not spiked with this compound

A RPD of sample and duplicate evaluated using $+/-\mathrm{RL}$. Concentrations are $<5 \mathrm{X}$ the $\mathrm{RL}$

$\mathrm{h}$ Preparation or preservation holding time was exceeded

The above sample is reported on a dry weight basis. 


\section{GENERAL ENGINEERING LABORATORIES, LLC}

2040 Savage Hoad Charieston SC 29407 - (843) 556-8171 - www.gel.com

\section{Certificate of Analysis}

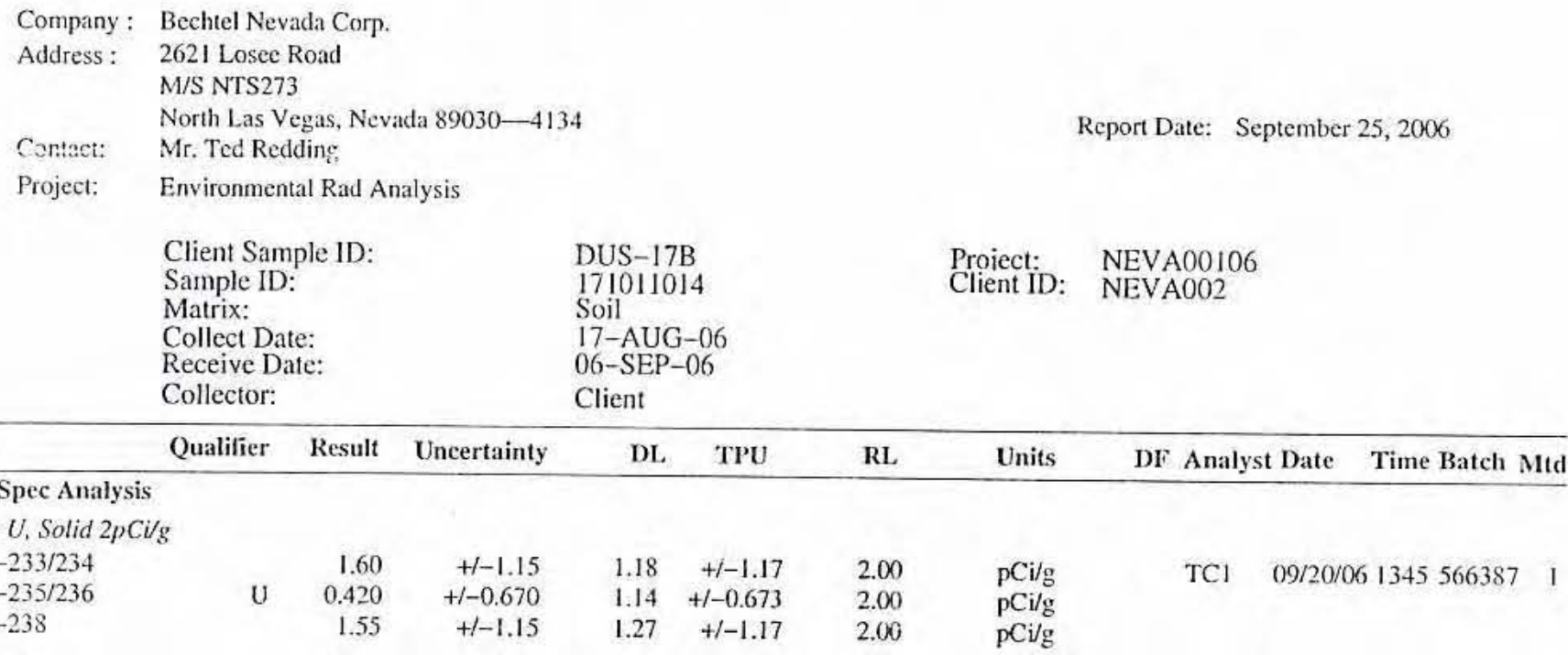

The following l'rep Methods were perfornied

\begin{tabular}{llllll}
\hline Method & Description & Analyst & Date & Time & Prep Batch \\
\hline Dry Soil Prep & Dry Soil Prep GL-RAD-A-021 & JMBI & $09 / 07 / 06$ & 0913 & 565870
\end{tabular}

The following Analytical Methods were performed

\begin{tabular}{ll} 
Method & Description \\
\hline & DOE EML HASL-300, U-02-RC Modified
\end{tabular}

\begin{tabular}{llcc} 
Surrogate/Tracer recovery & Test & Recovery\% & Acceptable Limits \\
\hline Uranium-232 & Alphaspec U, Sclid 2pCi/g & 70 & $(25 \%-125 \%)$
\end{tabular}

Notes:

The Qualifiers in this report are defined as follows :

* A quality control analyte recovery is outside of specified acceptance criteria

$<$ Result is less than value reported

$>$ Result is greater than value reported

A The TIC is a suspected aldol-condensation product

B Target analyte was detected in the associated blank

BD Results are either below the MDC or tracer recovery is low

C Analyte has been confirmed by GC/MS analysis

D Results are reported from a diluted aliquot of the sample

$\mathrm{H}$ Analytical holding time was exceeded

J Value is estimated

N/A Spike recovery limits do not apply. Sample concentration exceeds spike concentration by $4 \mathrm{X}$ or more

$R$ Sample results are rejected

U Analyte was analyzed for, but not detected above the MDL, MDA, or LOD.

UI Gamma Spectroscopy-Uncertain identification

X Consult Case Narrative, Data Summary package, or Projec: Manager concerning this qualifier 


\section{GENERAL ENGINEERING LABORATORIES, LLC \\ 2040 Savage Road Charleston SC 29407 - (843) 556-8171 - www.gel.com}

\section{Certificate of Analysis}

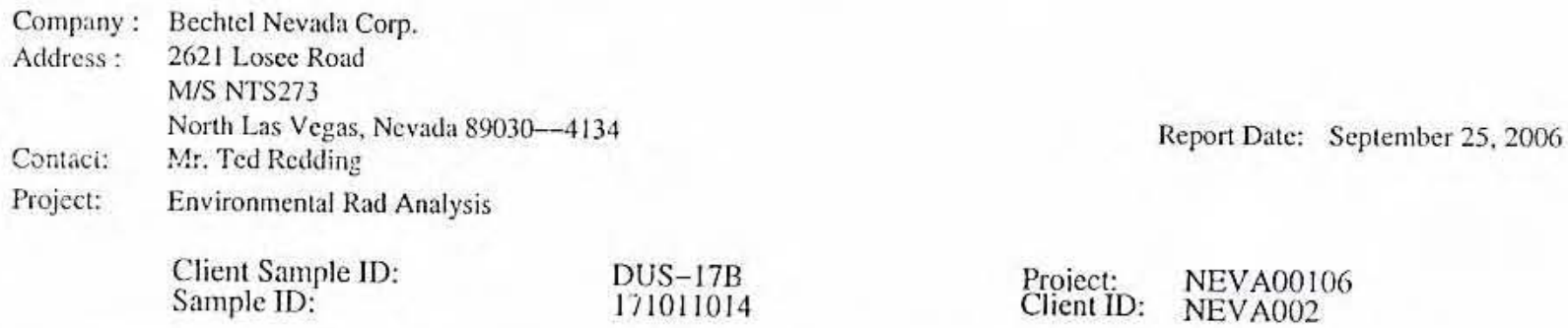

Y QC Samples were not spiked with this compound

$A$ RPD of sample and duplicate evaluated using $+/-R L$. Concentrations are $<5 X$ the RL

h Preparation or preservation holding time was exceeded

The above sample is reported on a dry weight basis. 


\section{GENERAL ENGINEERING LABORATORIES, LLC \\ 2040 Savage Road Charleston SC 29407 - (843) 556-8171 - www.gel.com}

\section{Certificate of Analysis}

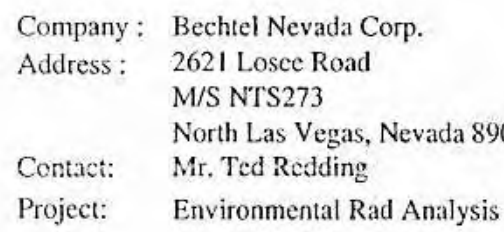

$\begin{array}{ll}\text { Client Sample ID: } & \text { DUS-18A } \\ \text { Sample ID: } & 171011015 \\ \text { Matrix: } & \text { Soil } \\ \text { Collect Date: } & 17-\text { AUG-06 } \\ \text { Receive Date: } & \text { 06-SEP-06 } \\ \text { Collector: } & \text { Client }\end{array}$

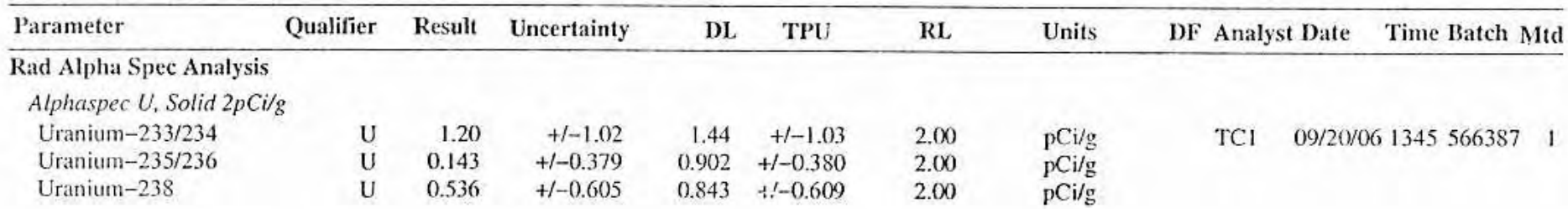

The following Prep Methols were performed

\begin{tabular}{llllll}
\hline Method & Description & Analyst & Date & Time & Prep Batch \\
\hline Dry Soil Prep & Dry Soil Prep GL-RAD-A-021 & JMB1 & $09 / 07 / 06$ & 0913 & 565870
\end{tabular}

The following Analytical Methods were performed

\begin{tabular}{ll} 
Method & Description \\
\hline 1 & DOE EML HASL-300, U-02-RC Modified
\end{tabular}

\begin{tabular}{llcc} 
Surrogate/Tracer recovery & Test & Recovery\% & Acceptable Limits \\
\hline Uranium-232 & Alphaspec U, Solid 2pCi/g & 82 & $(25 \%-125 \%)$
\end{tabular}

Notes:

The Qualifiers in this report are defined as follows:

* A quality control analyte recovery is outside of specified acceptance criteria

$<\quad$ Result is less than value reported

$>$ Result is greater than value reported

A The TIC is a suspected aldol-condensation product

B Target analyte was detected in the associated blank

BD Results are either below the MDC or tracer recovery is low

C Analyte has been confirmed by GC/MS analysis

D Results are reported from a diluted aliquot of the sample

$\mathrm{H}$ Analytical holding time was exceeded

$\mathrm{J}$ Value is estimated

N/A Spike recovery limits do not apply. Sample concentration exceeds spike concentration by $4 \mathrm{X}$ or more

$R$ Sample results are rejected

U Analyte was analyzed for, but not detected above the MDL, MDA, or LOD.

UI Gamma Spectroscopy--Uncertain identification

X Consult Case Narrative, Data Summary package, or Project Manager concerning this qualifier 


\section{GENERAL ENGINEERING I.ABORATORIES, LLC \\ 2040 Savage Road Charleston SC 29407 - (843) 556-8171 - www.gel.com}

\section{Certificate of Analysis}

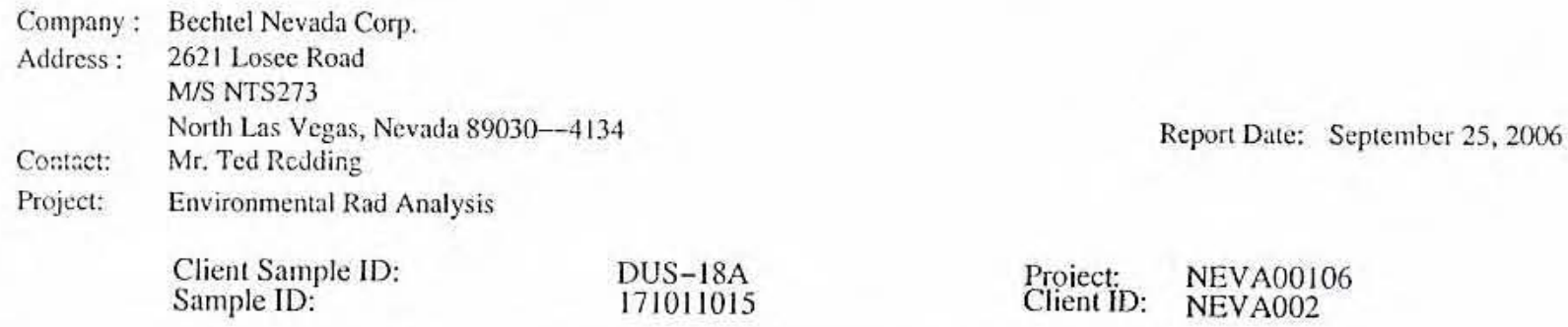

Y QC Samples were not spiked with this compound

$\wedge$ RPD of sample and duplicate evaluated using $+/-\mathrm{RL}$. Concentrations are $<5 X$ the RL

$\mathrm{h}$ Preparation or preservation holding time was exceeded

The above sample is reported on a dry weight basis. 


\section{GENERAL ENGINEERING LABORATORIES, LLC}

2040 Savage Road Charleston SC 29407 - (843) 556-8171 - www.gel.com

\section{Certificate of Analysis}

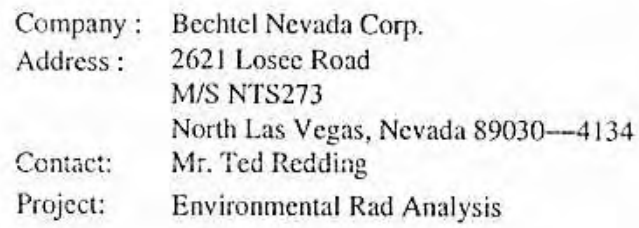

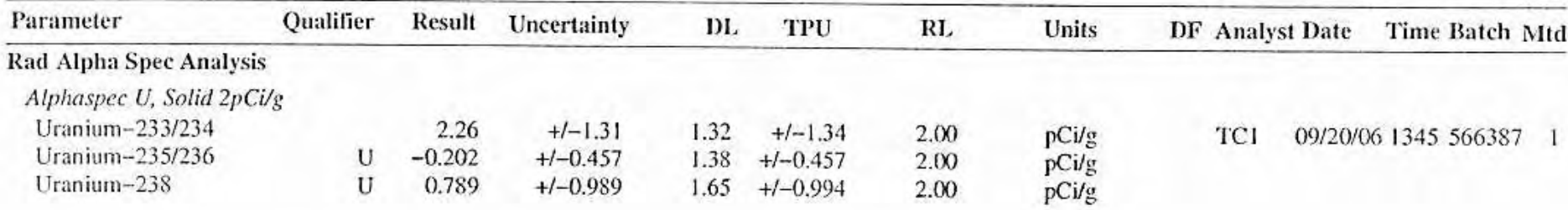

The following Prep Methods were performed

\begin{tabular}{llllll}
\hline Method & Description & Analyst & Date & Time & Prep Batch \\
\hline D.y Soil Prep & Dry Soil Prep GL-RAD-A-021 & JMBI & $09 / 07 / 06$ & 0913 & 565870
\end{tabular}

The following Analytical Methods were performed

\begin{tabular}{ll} 
Method & Description \\
\hline 1 & DOE EML HASL-300, U-02-RC Modified
\end{tabular}

\begin{tabular}{llcc} 
Surrogate/Tracer recovery & Test & Recovery \% & Acceptable Limits \\
\hline Uranium-232 & Alphaspec U, Solid 2pCi/g & 92 & $(25 \%-125 \%)$
\end{tabular}

Notes:

The Qualifiers in this report are defined as follows :

* A quality control analyte recovery is outside of specified acceptance criteria

$<$ Result is less than value reported

$>$ Result is greater than value reported

A The TIC is a suspected aldol-condensation product

B Target analyte was detected in the associated blank

BD Results are either below the MDC or tracer recovery is low

C Analyte has been confirmed by GC/MS analysis

D Results are reported from a diluted aliquot of the sample

$\mathrm{H}$ Analytical holding time was exceeded

$\mathrm{J}$ Value is estimated

N/A Spike recovery limits do not apply. Sample concentration exceeds spike concentration by $4 \mathrm{X}$ or more

$\mathrm{R}$ Sample results are rejected

U Analyte was analyzed for, but not detected above the MDL, MDA, or LOD.

UI Gamma Spectroscopy--Uncertain identification

X Consult Case Narrative, Data Summary package, or Project Manager concerning this qualifier 


\section{GENERAL ENGINEERING LABORATORIES, LLC}

2040 Savage Road Charleston SC 29407 - (843) 556-8171 - www.gel.com

\section{Certificate of Analysis}

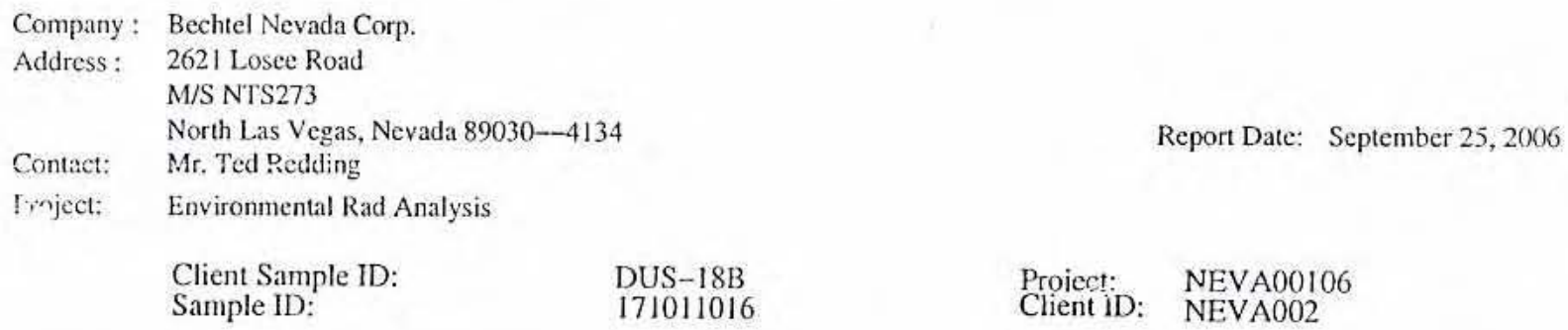

\begin{tabular}{lllllll}
\hline Parameter & Qualilier Result Uncertainty DL TPU & DL & Units & DF Analyst Date Time Batch Mtd
\end{tabular}

Y QC Samples were not spiked with this compound

$\wedge$ RPD of sample and duplicate evaluated using $+/-$ RL. Concentrations are $<5 X$ the RL

$\mathrm{h}$ Preparation or preservation holding time was exceeded

The above sample is reported on a dry weight basis. 


\section{GENERAL ENGINEERING LABORATORIES, LLC}

2040 Savage Road Charleston SC 29407 - (843) 556-8171 - www.gel.com

\section{Certificate of Analysis}

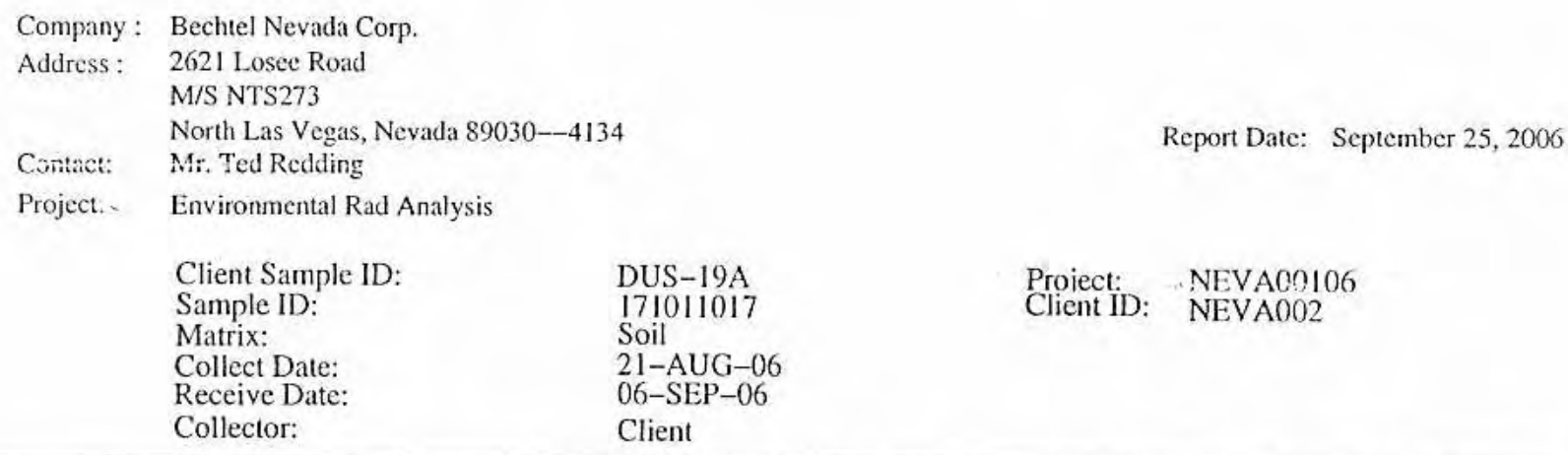

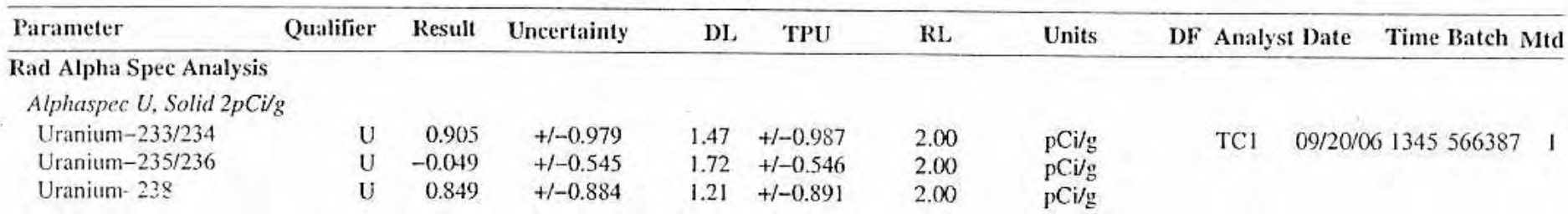

The following Prep Methods were performed

\begin{tabular}{llllll}
\hline Method & Description & Analyst & Date & Time & Prep Batch \\
\hline Dry Soil Prep & Dry Soil Prep GL-RAD-A-021 & JMB1 & $09 / 07 / 06$ & 0913 & 565870
\end{tabular}

The following Analytical Methods were performed

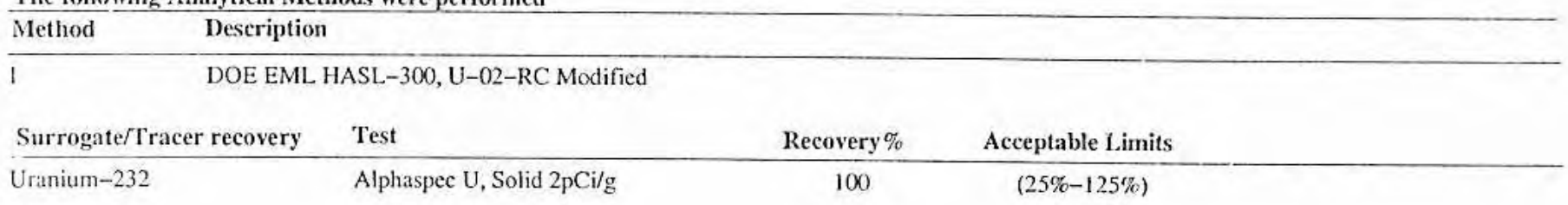

Notes:

The Qualifiers in this report are defined as follows :

* A quality control analyte recovery is outside of specified acceptance criteria

$<$ Result is less than value reported

$>$ Result is greater than value reported

A The TIC is a suspected aldol-condensation product

B Target analyte was detected in the associated blank

$\mathrm{BD}$ Results are either below the MDC or tracer recovery is low

C Analyte has been confirmed by GC/MS analysis

D Results are reported from a diluted aliquot of the sample

$\mathrm{H}$ Analytical holding time was exceeded

$\mathrm{J}$ Value is estimated

N/A Spike recovery limits do not apply. Sample concentration exceeds spike concentration by $4 \mathrm{X}$ or more

R Sample results are rejected

U Analyte was analyzed for, but not detected above the MDL, MDA, or LOD.

UI Gamma Spectroscopy-Uncertain identification

X Consult Case Narrative, Data Summary package, or Project Manager conceming this qualifier 


\section{GENERAL ENGINEERING LABORATORIES, LLC \\ 2040 Savage Road Charleston SC 29407 - (843) 556-8171 - www.gel.com}

\section{Certificate of Analysis}

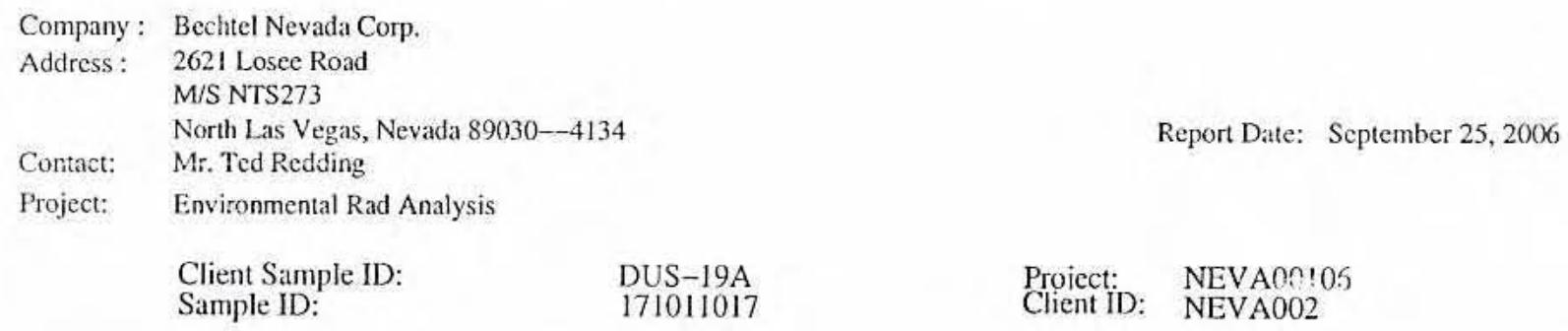

RL.

Units

DF Analyst Date

Time Batch Mtd

Y QC Samples were not spiked with this compound

$\wedge$ KPD of sample and duplicate evaluated using $+/-$ RL. Concentrations are $<5 \mathrm{X}$ the RL

h Preparation or preservation holding time was exceeded

The above sample is reported on a dry weight basis. 


\section{GENERAL ENGINEERING LABORATORIES, LLC \\ 2040 Savage Road Charleston SC 29407 - (843) 556-8171 - www.gel.com}

\section{Certificate of Analysis}

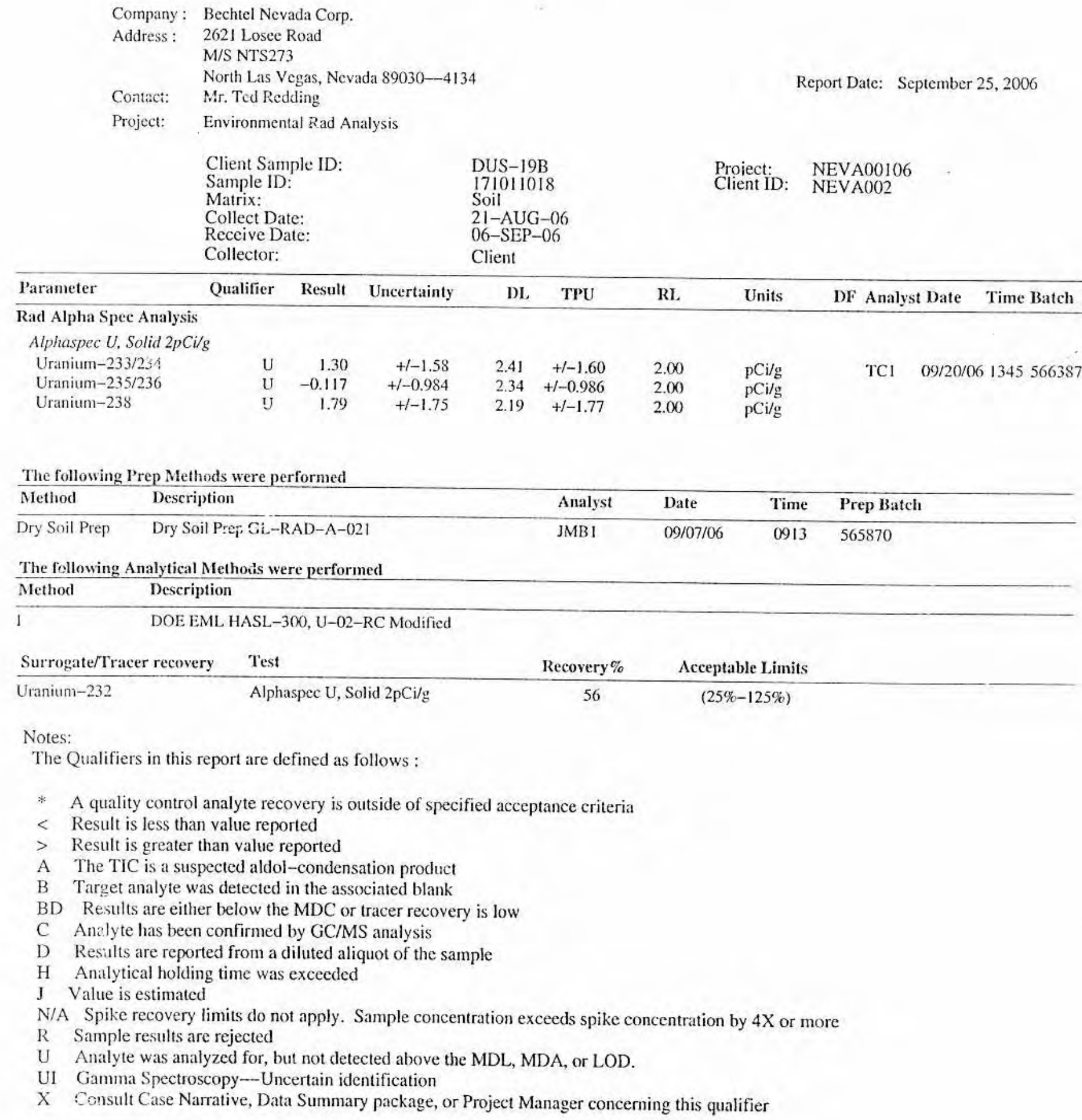




\section{GENERAL ENGINEERING LABORATORIES, LLC}

2040 Savage Road Charleston SC 29407 - (843) 556-8171 - www.gel.com

\section{Certificate of Analysis}

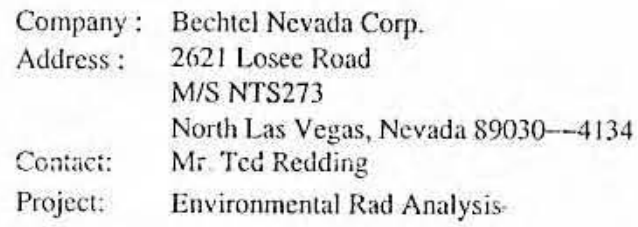

Client Sample ID: $\quad$ DUS-19B

Sample ID: $\quad 171011018$

Proiect: NEVA00106

Client ID: NEVA002

Y QC Samples were not spiked with this compound

$\wedge$ RPD of sample and diplicate evaluated using $+1-$ RL. Concentrations are $<5 X$ the RL

$\mathrm{h}$ Preparation or preservation holding time was exceeded

The above sample is reported on a dry weight basis. 


\section{GENERAL ENGINEERING LABORATORIES, LLC}

2040 Savage Road Charleston SC 29407 - (843) 556-8171 - www.gel.com

\section{Certificate of Analysis}

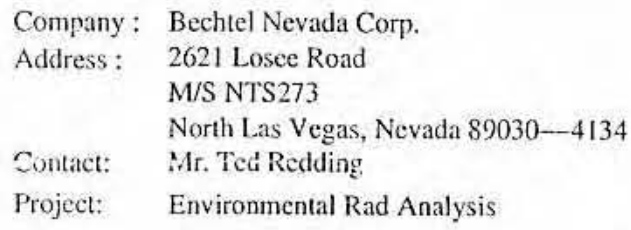

Report Date: September 25, 2006

$\begin{array}{ll}\text { Client Sample ID: } & \text { DUS-20A } \\ \text { Sample ID: } & 171011019 \\ \text { Matrix: } & \text { Soil } \\ \text { Collect Date: } & 21-A U G-06 \\ \text { Receive Date: } & 06-\text { SEP-06 } \\ \text { Collector: } & \text { Client }\end{array}$

Proiect: NEVA00106

Client ID: NEVA002

\begin{tabular}{|c|c|c|c|c|c|c|c|c|c|c|c|c|}
\hline Parameter & Qualifier & Result & Uncertainty & DL & TPU & RL & Units & DF & Analyst & t Date & Time Batch M & Mtd \\
\hline \multicolumn{13}{|c|}{ Rad Alpha Spec Analysis } \\
\hline \multicolumn{13}{|c|}{ Alphaspec U. Solid $2 p C i / g$} \\
\hline $\begin{array}{l}\text { Uranium-233/234 } \\
\text { Uranium-235/236 } \\
\text { Uranium-238 }\end{array}$ & $\mathrm{U}$ & $\begin{array}{r}1.86 \\
-0.0525 \\
1.51\end{array}$ & $\begin{array}{r}+/-1.16 \\
+1-0.441 \\
+/-1.05\end{array}$ & $\begin{array}{r}0.981 \\
1.05 \\
0.981\end{array}$ & $\begin{array}{r}+/-1.19 \\
+/-0.442 \\
+1-1.07\end{array}$ & $\begin{array}{l}2.00 \\
2.00 \\
2.00\end{array}$ & $\begin{array}{l}\mathrm{pCi} / \mathrm{g} \\
\mathrm{pCi} / \mathrm{g} \\
\mathrm{pCi} / \mathrm{g}\end{array}$ & & $\mathrm{TCl}$ & $09 / 20 / 0$ & 61345566387 & 1 \\
\hline
\end{tabular}

The following Prep Methods were performed

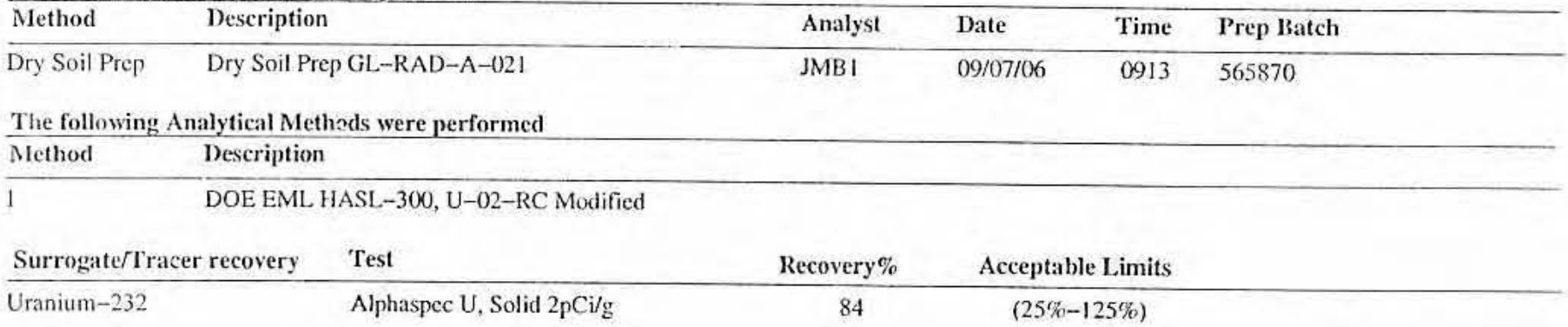

Notes:

The Qualifiers in this report are defined as follows :

* A quality control analyte recovery is outside of specified acceptance criteria

$<$ Result is less than value reported

$>$ Result is greater than value reported

A The TIC is a suspected aldol-condensation product

B Target analyte was detected in the associated blank

$\mathrm{BD}$ Results are either below the MDC or tracer recovery is low

C Analyte has been confirmed by GC/MS analysis

D Results are reported from a diluted aliquot of the sample

$\mathrm{H}$ Analytical holding time was exceeded

$\mathrm{J}$ Value is estimated

N/A Spike recovery limits do not apply. Sample concentration exceeds spike concentration by $4 X$ or more

R Sample results are rejected

$\mathrm{U}$ Analyte was analyzed for, but not detected above the MDL, MDA, or LOD.

UI Gamma Spectroscopy-Uncertain identification

X Consult Case Narrative, Data Summary package, or Project Manager concerning this qualifier 


\section{GENERAL ENGINEERING LABORATORIES, LLC \\ 2040 Savage Road Charleston SC 29407 - (843) 556-8171 - www.gel.com}

\section{Certificate of Analysis}

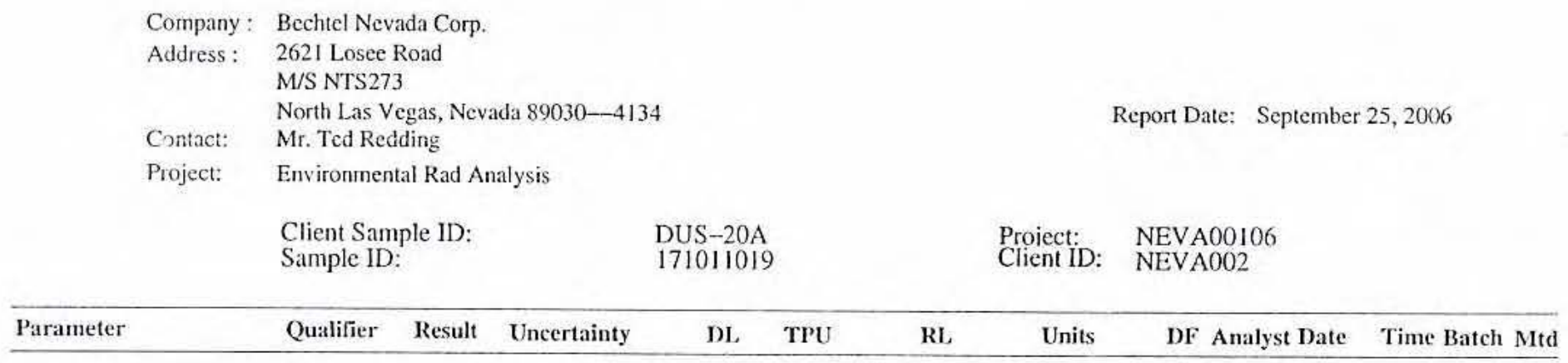

Y QC Samples were not spiked with this compound

$\wedge$ RPD of sample and duplicate evaluated osing $+1-\mathrm{RL}$. Concentrations are $<5 X$ the RL

$h$ Preparation or preservation holding time was exceeded

The above sample is reported on a dry weight basis. 


\section{GENERAL. ENGINEERING LABORATORIES, LLC \\ 2040 Savage Road Charleston SC 29407 - (843) 556-8171 - www.gel.com}

\section{Certificate of Analysis}

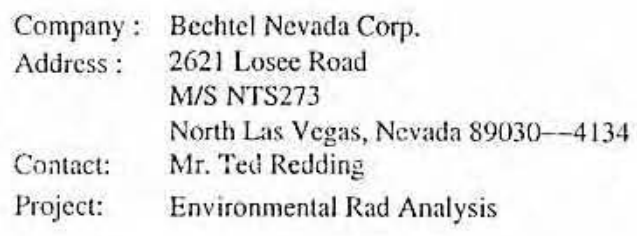

Report Date: September 25, 2006

Client Sample ID:

Sample ID:

Matrix:

Collect Date:

Receive Date:

Collector:

\author{
DUS-20B \\ 171011020 \\ Proiect: NEVA00106 \\ Client ID: NEVA002
}

\begin{tabular}{|c|c|c|c|c|c|c|c|c|c|c|c|c|}
\hline Parameter & Qualifier & Result & Uncertainty & $\mathrm{DL}$ & TPU & RL & Units & DF & Analys & t Date & Time Batch N & Mtd \\
\hline \multicolumn{13}{|c|}{ Rad Alpha Spec Analysis } \\
\hline \multicolumn{13}{|c|}{ Alphaspec $U$, Solid $2 p C i / g$} \\
\hline Uranium-233/234 & & 2.40 & $+l-2.0 \mathrm{i}$ & 2.34 & $+/-2.04$ & 2.00 & $\mathrm{pCi} / \mathrm{g}$ & & $\mathrm{TCl}$ & $09 / 20 / 0$ & 61412566387 & 1 \\
\hline Uranium-235/236 & $\mathrm{U}$ & 0.473 & $+1-0.926$ & 1.42 & $+1-0.929$ & 2.00 & $\mathrm{pCi} / \mathrm{g}$ & & & & & \\
\hline Uranium-238 & U & 1.35 & $+/-1.52$ & 2.12 & $t_{i}^{t}-1.53$ & 2.00 & $\mathrm{pCi} / \mathrm{g}$ & & & & & \\
\hline
\end{tabular}

The following Prep Methods were performed

\begin{tabular}{llllll}
\hline Method & Description & Analyst & Date & Time & Prep Batch \\
\hline Dry Soil Prep & Dry Soil Prep GL-RAD-A-021 & jMBI & $09 / 07 / 06$ & 0913 & 565870
\end{tabular}

The following Analytical Methods were performed

\begin{tabular}{ll} 
Method & Description \\
\hline & DOE EML HASL-300, U-02-RC Modified
\end{tabular}

\begin{tabular}{llcc} 
Surrogate/Tracer recovery & Test & Recovery\% & Acceptable Limits \\
\hline Uranium-232 & Alphaspec U, Solid 2pCi/g & 86 & $(25 \%-125 \%)$
\end{tabular}

Notes:

The Qualifiers in this report are defined as follows :

* A quality control analyte recovery is outside of specified acceptance criteria

$<$ Result is less than value reported

$>$ Result is greater than value reported

A The TIC is a suspected aldol-condensation product

B Target analyte was detected in the associated blank

$\mathrm{BD}$ Results are either below the MDC or tracer recovery is low

C Analyte has been confirmed by GC/MS analysis

D Results are repotted from a diluted aliquol of the sample

$\mathrm{H}$ Analytical holding time was exceeded

$\mathrm{J}$ Value is estimated

N/A Spike recovery limits do not apply. Sample concentration exceeds spike concentration by $4 X$ or more

R Sample results are rejected

U Analyte was analyzed for, but not detected above the MDL, MDA, or LOD.

UI Gamma Spectroscopy-Uncertain identification

X Consult Case Narrative, Data Summary package, or Project Manager concerning this qualifier 


\section{GENERAL ENGINEERING LABORATORIES, LLC}

2040 Savage Hoad Charleston SC 29407 - (843) 556-8171 - www.gel.com

\section{Certificate of Analysis}

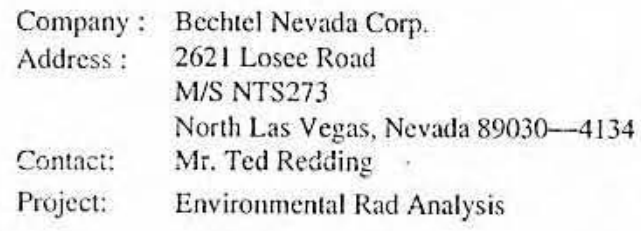

\begin{tabular}{llllllll}
\hline Parameter & Qualifier & Result Uncertainty & DL. TPU & RL & Units & DF Analyst Date Time Batch Mitd
\end{tabular}

Y QC Samples were not spiked with this compound

$\wedge \mathrm{RPD}$ of sample and duplicate evaluated using $+/-\mathrm{RL}$. Concentrations are $<5 \mathrm{X}$ the $\mathrm{RL}$.

h Preparation or preservation holding time was exceeded

The above sample is reported on a dry weight basis. 


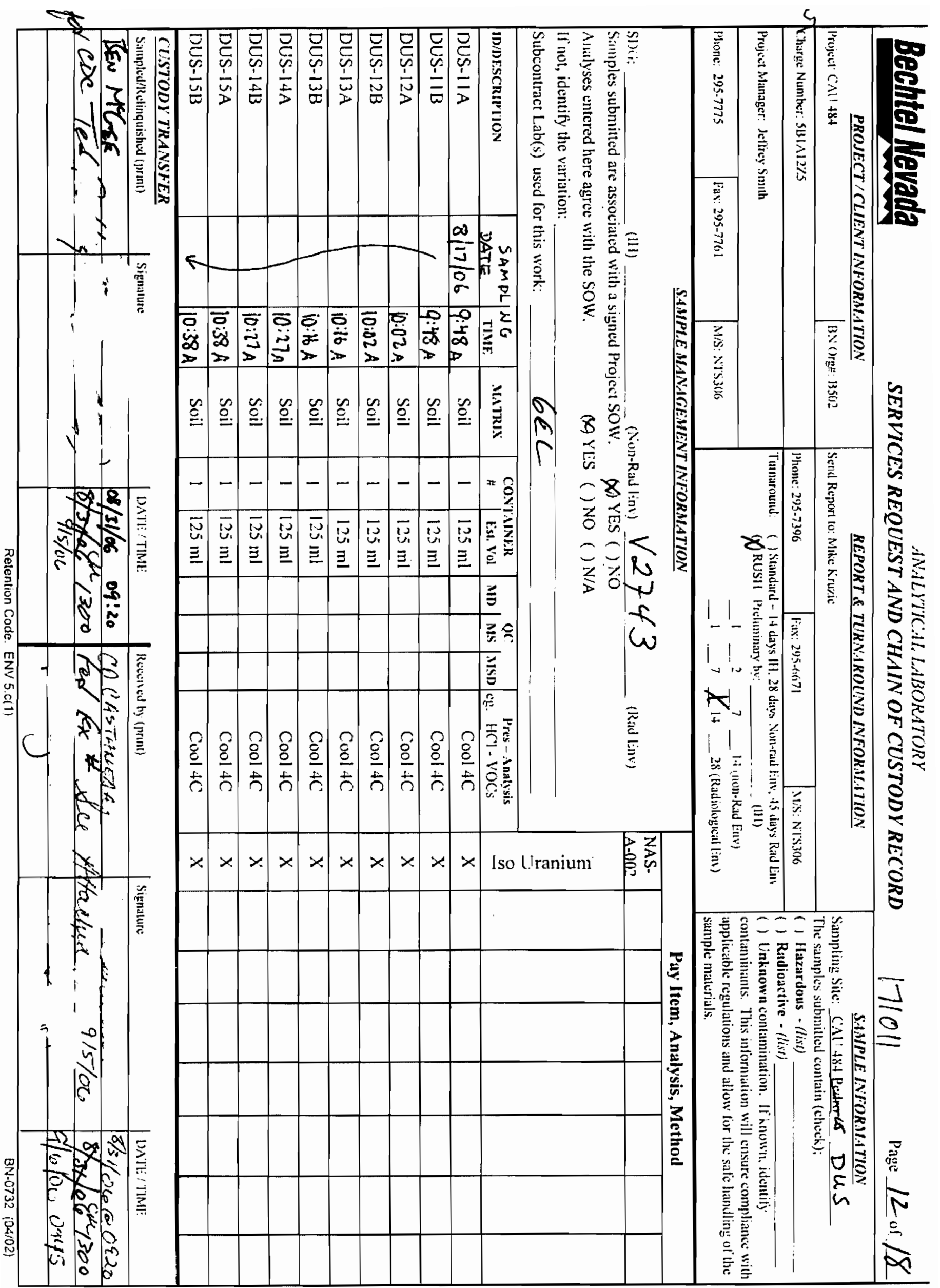




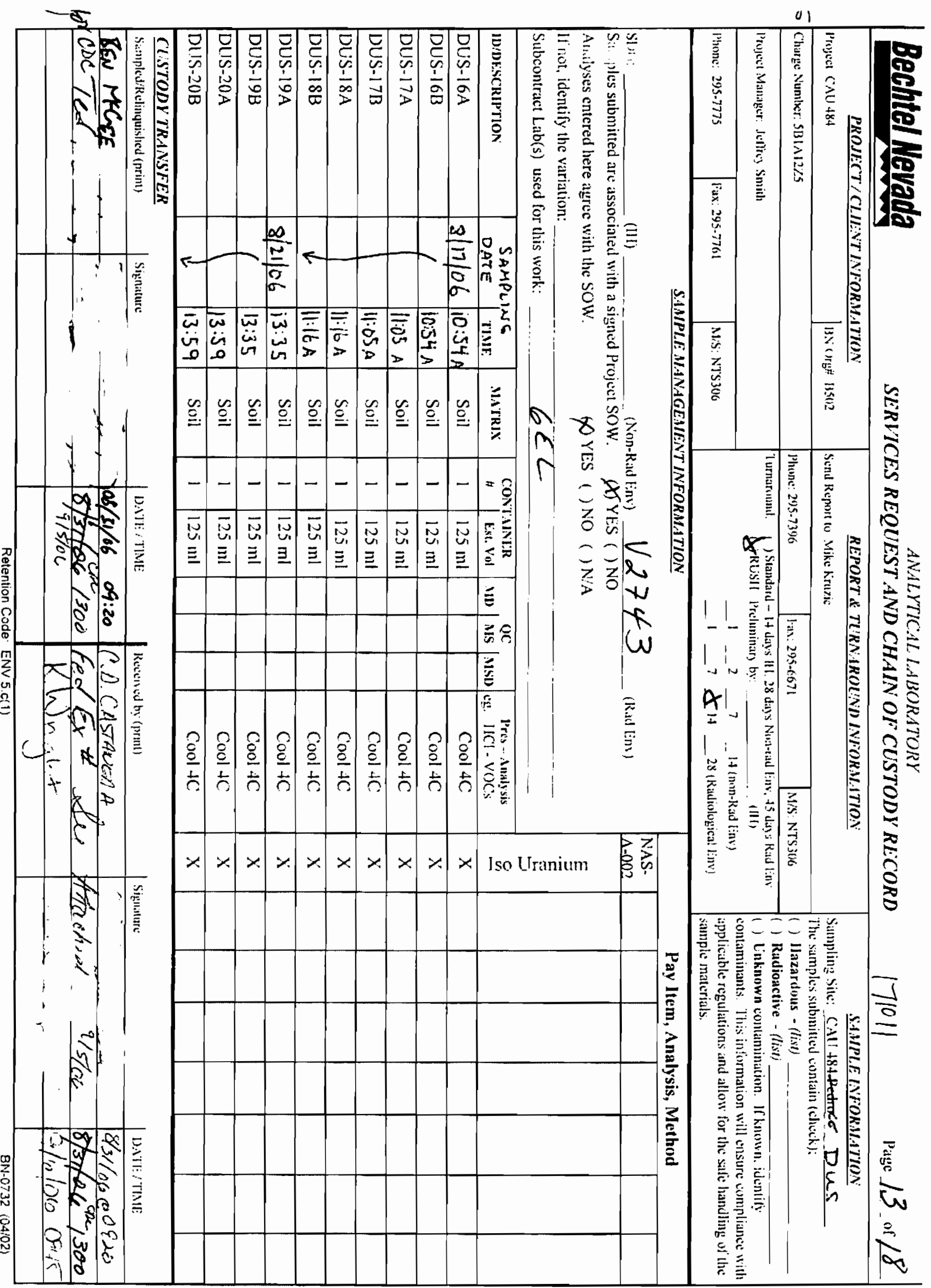




\section{Isotopic Uranium By Alpha Spectroscopy Sample Results Summary}

Client Name: National Sercurity Technologies, LLC Client Project Name: CAU 484 Client Project Number: V2760

\author{
Laboratory Name: Paragon Analytics \\ PAI Work Order: 0610009
}

Page: 1 of 1

Reported on: Tuesday, October 17, 2006

12:36:05 PM

\begin{tabular}{|c|c|c|c|c|c|c|c|c|c|c|}
\hline $\begin{array}{c}\text { Lab } \\
\text { Sample ID }\end{array}$ & Client Sample ID & $\begin{array}{l}\text { Sample } \\
\text { Type }\end{array}$ & Nuclide & Result +/- 2 s TPU & MDC & Units & Matrix & Prep Batch & $\begin{array}{c}\text { Date } \\
\text { Analyzed }\end{array}$ & Flags \\
\hline $0610009-1$ & DUS-21A & Sample & $U-233 / 234$ & $1.60 \mathrm{E}+00+1-4.79 \mathrm{E}-01$ & $2.94 E-01$ & $\mathrm{pCi} / \mathrm{g}$ & SOIL & AS061010-5 & $10 / 13 / 2006$ & LT \\
\hline $0610009-1$ & DUS-21A & Sample & U-235 & $1.40 \mathrm{E}-01+l-1.38 \mathrm{E}-01$ & $1.96 \mathrm{E}-01$ & $\mathrm{pCi} / \mathrm{g}$ & SOIL & AS061010-5 & $10 / 13 / 2006$ & $u$ \\
\hline 0610009-1 & DUS-21A & Sample & U-238 & $2.42 \mathrm{E}+00+/-6.22 \mathrm{E}-01$ & $2.02 E-01$ & $\mathrm{pCi} / \mathrm{g}$ & SOIL & AS061010-5 & $10 / 13 / 2006$ & \\
\hline
\end{tabular}

\section{Comments:}

Data Package ID: U0610009-1
Qualifiers/Flags:
$U$ - Result is less than the sample specific MDC.
LT - Result is less than Requested MDC, greater than sample specific MDC.
Y1. Chemical Yield is in control at $100-110 \%$, Quantitative Yield is assumed.
Y2 - Chemical Yield outside default limits.
$M$ - The requested MDC was not met.
M3. The requested MDC was not met, but the reported activity is greater than the reported MDC.

\section{Abbreviations:}

TPU - Total Propagated Uncertainty (see PAI SOP 743)

MDC - Minimum Detectable Concentration (see PAI SOP 709)

BDL - Below Detection Limit 


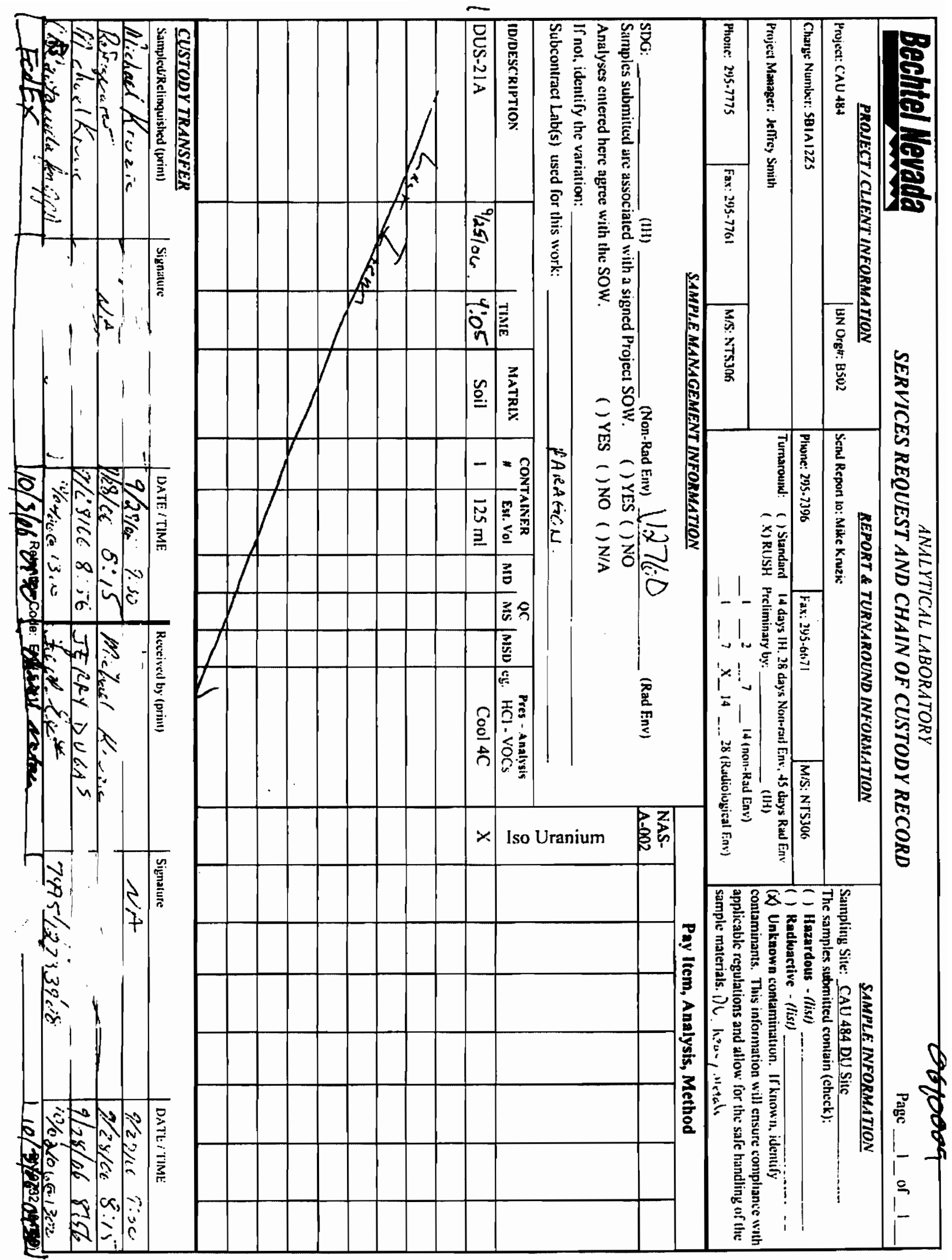




\section{GENERAL ENGINEERING LABORATORIES, LLC \\ 2040 Savage Road Charleston SC 29407 - (843) 556-8171 - www.gel.com}

\section{Certificate of Analysis}

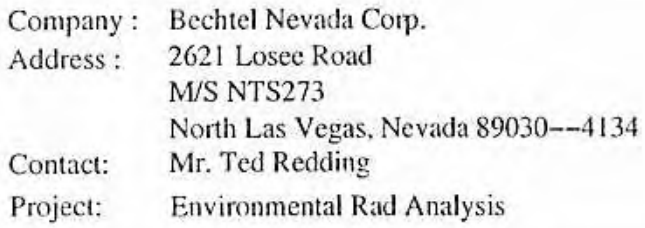

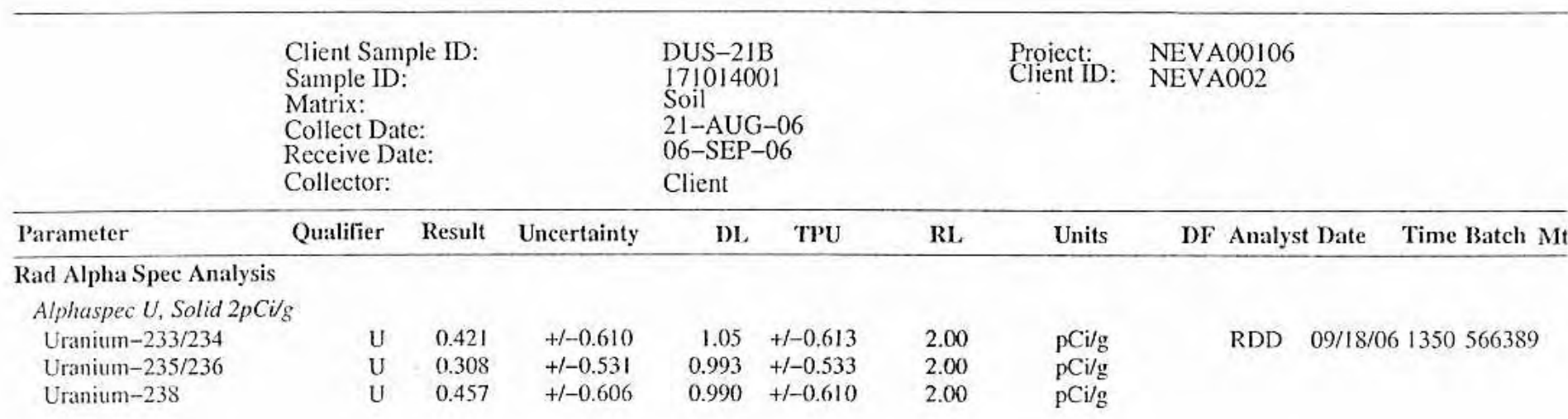

The following Prep Methods were performed

\begin{tabular}{llllll}
\hline Method & Description & Analyst & Date & Time & Prep Batch \\
\hline Dry Soil Prep & Dry Soil Prep GL-RAD-A-021 & LXM2 & $09 / 07 / 06$ & 1002 & 565877
\end{tabular}

The following Analytical Methods were performed

$\begin{array}{ll}\text { Miethod Description } \\ 1 & \text { DOE EML HASL-300, U-02-RC Modified }\end{array}$

\begin{tabular}{llcc} 
Surrogate/Tracer recovery & Test & Recovery\% & Acceptable Limits \\
\hline Uranium-232 & Alphaspec U, Solid 2pCi/g & 80 & $(25 \%-125 \%)$
\end{tabular}

Notes:

The Qualifiers in this report are defined as follows :

* A quality control analyte recovery is outside of specified acceptance criteria

$<$ Result is less than value reported

$>$ Result is greater than value reported

A The TIC is a suspected aldol-condensation product

B Target analyte was detected in the associated blank

$\mathrm{BD}$ Results are either below the MDC or tracer recovery is low

C Analyte has been confirmed by GC/MS analysis

D Results are reported from a diluted aliquot of the sample

$\mathrm{H}$ Analytical holding time was exceeded

$\mathrm{J}$ Value is estimated

N/A Spike recovery limits do not apply. Sample concentration exceeds spike concentration by $4 X$ or more

$\mathrm{R}$ Sample results are rejected

$\mathrm{U}$ Analyte was analyzed for, but not detected above the MDL, MDA, or LOD.

UI Gamma Spectroscopy--Uncertain identification 


\section{GENERAL. ENGINEERING LABORATORIES, LLC \\ 2040 Savage Road Charleston SC 29407 - (843) 556-8171 - www.gel.com}

\section{Certificate of Analysis}

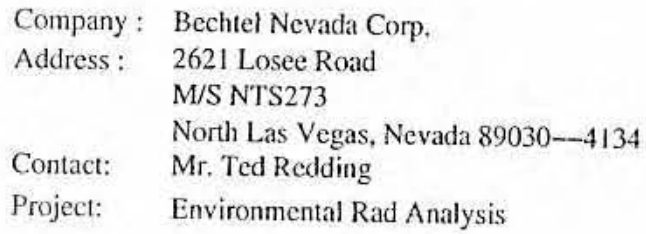

DL TPU

RL

Units

DF Analyst Date

Time Batch Mt

$\mathrm{X}$ Consult Case Narrative, Data Summary package, or Project Manager concerning this qualifier

Y QC Samples were not spiked with this compound

$\wedge$ RPD of sample and duplicate evaluated using $+/-$ RL. Concentrations are $<5 X$ the RL

$\mathrm{h}$ Preparation or preservation holding time was exceeded

The above sample is reported on a dry weight basis. 


\section{GENERAL ENGINEERING LABORATORIES, LLC}

2040 Savage Road Charleston SC 29407 - (843) 556-8171 - www.gel.com

\section{Certificate of Analysis}

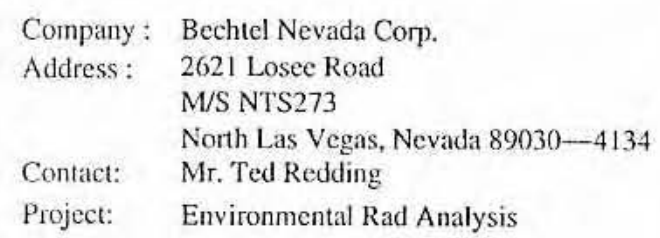

\begin{tabular}{|c|c|c|c|c|c|c|c|c|c|c|}
\hline Parameter & Qualifier & Result & Uncertainty & $\mathrm{DL}$ & TPU & $\mathrm{RL}$ & Units & $\mathrm{DF}$ & Analyst Date & Time Batch Mt \\
\hline \multicolumn{11}{|c|}{ Rad Alpha Spec Analysis } \\
\hline \multicolumn{11}{|c|}{ Alphaspec U, Solid $2 p \mathrm{C} / \mathrm{g}$} \\
\hline Uranium-233/234 & & 1.17 & $+1-0.764$ & 0.766 & $+1-0.782$ & 2.00 & $\mathrm{pCi} / \mathrm{g}$ & & RDD $\quad 09 / 18 / 1$ & 6.1350566389 \\
\hline Uranium-235/236 & U & 0.471 & $+1-0.523$ & 0.769 & $+1-0.527$ & 2.00 & $\mathrm{pCi} / \mathrm{g}$ & & & \\
\hline Uranium-238 & $\mathrm{U}$ & 0.586 & $+1-0.569$ & 0.766 & $+/-0.575$ & 2.00 & $\mathrm{pCi} / \mathrm{g}$ & & & \\
\hline
\end{tabular}

The following Prep Methods were performed

\begin{tabular}{llllll}
\hline Method & Description & Analyst & Date & Time & Prep Batch \\
\hline Dry Soil Prep & Dry Soil Prep GL-RAD-A-021 & LXM2 & $09 / 07 / 06$ & 1002 & 565877
\end{tabular}

The following Analytical Methods were performed

Method $\frac{\text { Description }}{1}$ DOE EML HASL-300, U-02-RC Modified

\begin{tabular}{llcc} 
Surrogate/1racer recovery & Test & Recovery $\%$ & Acceptable Limits \\
\hline Uranium-232 & Alphaspec U, Solid 2pCi/g & 89 & $(25 \%-125 \%)$
\end{tabular}

Notes:

The Qualifiers in this report are defined as follows :

* A quality control analyte recovery is outside of specified acceptance criteria

$<$ Result is less than value reported

$>$ Result is greater than value reported

A The TIC is a suspected aldot-condensation product

B Target analyte was detected in the associated blank

BD Results are either below the MDC or tracer recovery is low

C Analyte has been confirmed by GC/MS analysis

D Results are reported from a diluted aliquot of the sample

$\mathrm{H}$ Analytical holding time was exceeded

$\mathrm{J}$ Value is estimated

N/A Spike recovery limits do not apply. Sample concentration excceds spike concentration by $4 X$ or more

R Sample results are rejected

U Analyte was analyzed for, but not detected above the MDL, MDA, or LOD.

UI Gamma Spectroscopy-Uncertain identification

$X$ Consult Case Narrative, Data Summary package, or Project Manager concerning this qualifier 


\section{GENERAL ENGINEERING LABORATORIES, LLC \\ 2040 Savage Road Charleston SC 29407 - (843) 556-8171 - www.gel.com}

\section{Certificate of Analysis}

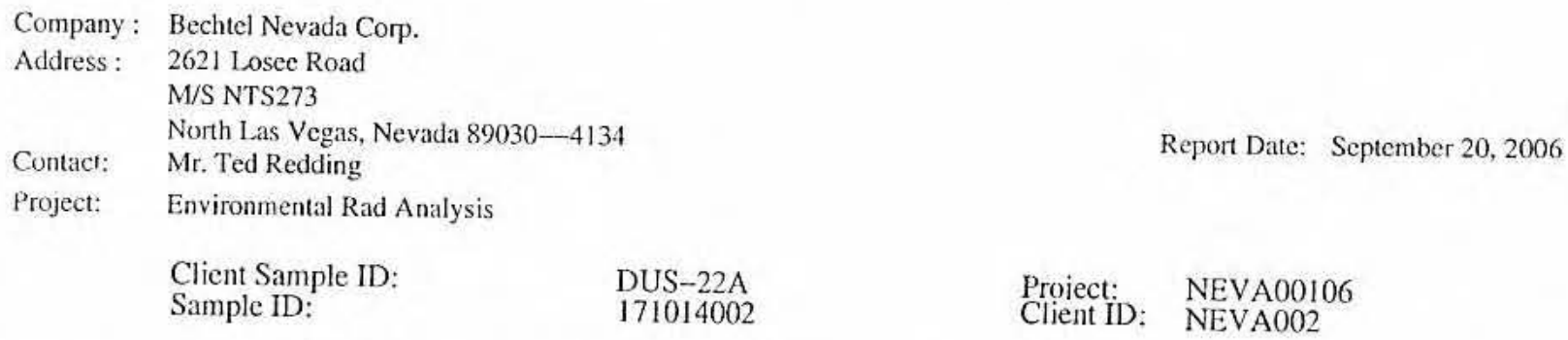

RL

Units

Y QC Samples were not spiked with this compound

$\wedge \quad$ RPD of sample and duplicate evaluated using $+/-$ RL. Concentrations are $\angle 5 X$ the RL

h Preparation or preservation holding time was exceeded

The above sample is reported on a dry weight basis. 


\section{GENERAL ENGINEERING LABORATORIES, LLC \\ 2040 Savage Road Charleston SC 29407 - (843) 556-8171 - www.gel.com}

\section{Certificate of Analysis}

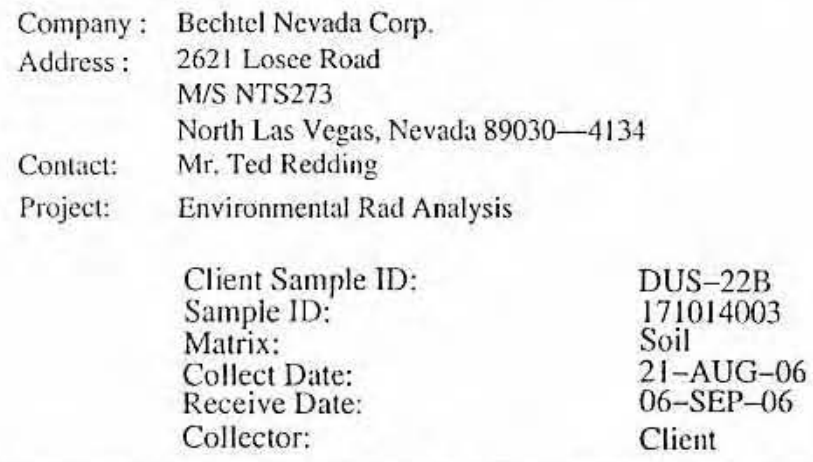

\begin{tabular}{|c|c|c|c|c|c|c|c|c|c|c|}
\hline Parameter & Qualifier & Result & Uncertainty & DL. & TPU & $\mathrm{RL}$ & Units & DF & Analyst Date & Time Batch MI \\
\hline \multicolumn{11}{|c|}{ Rad Alpha Spec Analysis } \\
\hline \multicolumn{11}{|c|}{ Alphaspec U. Solid $2 p C i / g$} \\
\hline Uranium-233/234 & $\mathrm{U}$ & 0.790 & +0.829 & 1.01 & $+1-0.840$ & 2.00 & $\mathrm{pCi} / \mathrm{g}$ & & RDD $\quad 09 / 18 / 0$ & 61350566389 \\
\hline Uranium-235/236 & $\mathrm{U}$ & -0.354 & $+/-0.262$ & 1.63 & $+1-0.269$ & 2.00 & $\mathrm{pCi} / \mathrm{g}$ & & & \\
\hline Uranium-238 & $\mathrm{U}$ & 0.218 & $+1-0.615$ & 1.38 & $+1-0.616$ & 2.00 & $\mathrm{pCi} / \mathrm{g}$ & & & \\
\hline
\end{tabular}

The following Prep Methods were performed

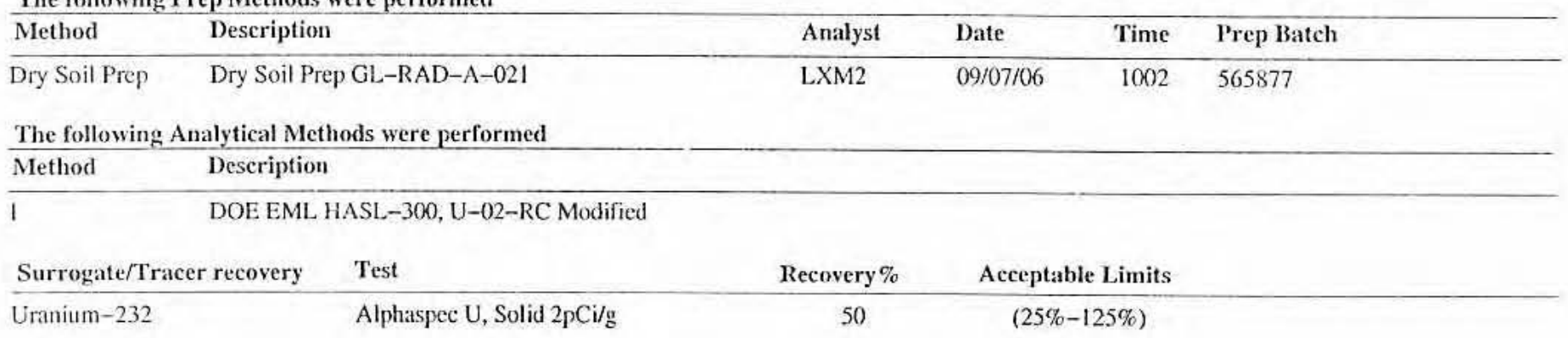

Notes:

The Qualifiers in this report are defined as follows :

* A quality control analyte recovery is outside of specified acceptance criteria

$<$ Result is less than value reported

$>$ Result is greater than value reported

A The TIC is a suspected aldol-condensation product

B Target analyte was detected in the associated blank

$\mathrm{BD}$ Results are either below the MDC or tracer recovery is low

C Analyte has been confirmed by GC/MS analysis

D Results are reported from a diluted aliquot of the sample

$\mathrm{H}$ Analytical holding time was exceeded

$\mathrm{J}$ Value is estimated

N/A Spike recovery limits do not apply. Sample concentration exceeds spike concentration by $4 X$ or more

$\mathrm{R}$ Sample results are rejected

U Analyte was analyzed for, but not detected above the MDL, MDA, or LOD.

UI Gamma Spectroscopy--Uncertain identification

$X$ Consult Case Narrative, Data Summary package, or Project Manager concerning this qualifier 


\section{GENERAL ENGINEERING LABORATORIES, LLC}

2040 Savage Road Charleston SC 29407 - (843) 556-8171 - www.gel.com

\section{Certificate of Analysis}

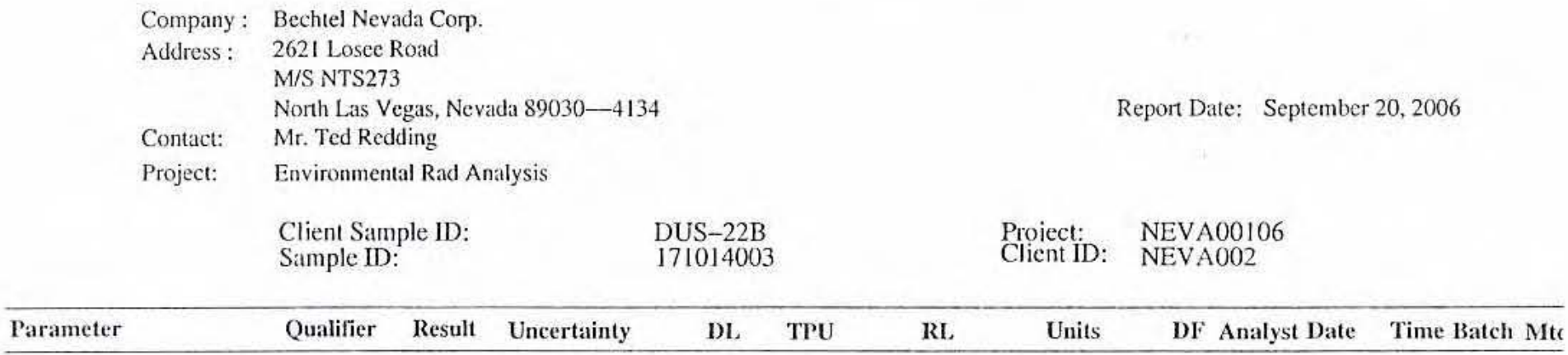

Y QC Samples were not spiked with this compound

$\wedge$ RPD of sample and duplicate evaluated using $+/-$ RL. Concentrations are $\angle 5 X$ the RL

h Preparation or preservation holding time was exceeded

The above sample is reported on a dry weight basis. 


\section{GENERAL ENGINEERING LABORATORIES, LLC \\ 2040 Savage Hoad Charleston SC 29407 - (843) 556-8171 - www.gel.com}

\section{Certificate of Analysis}

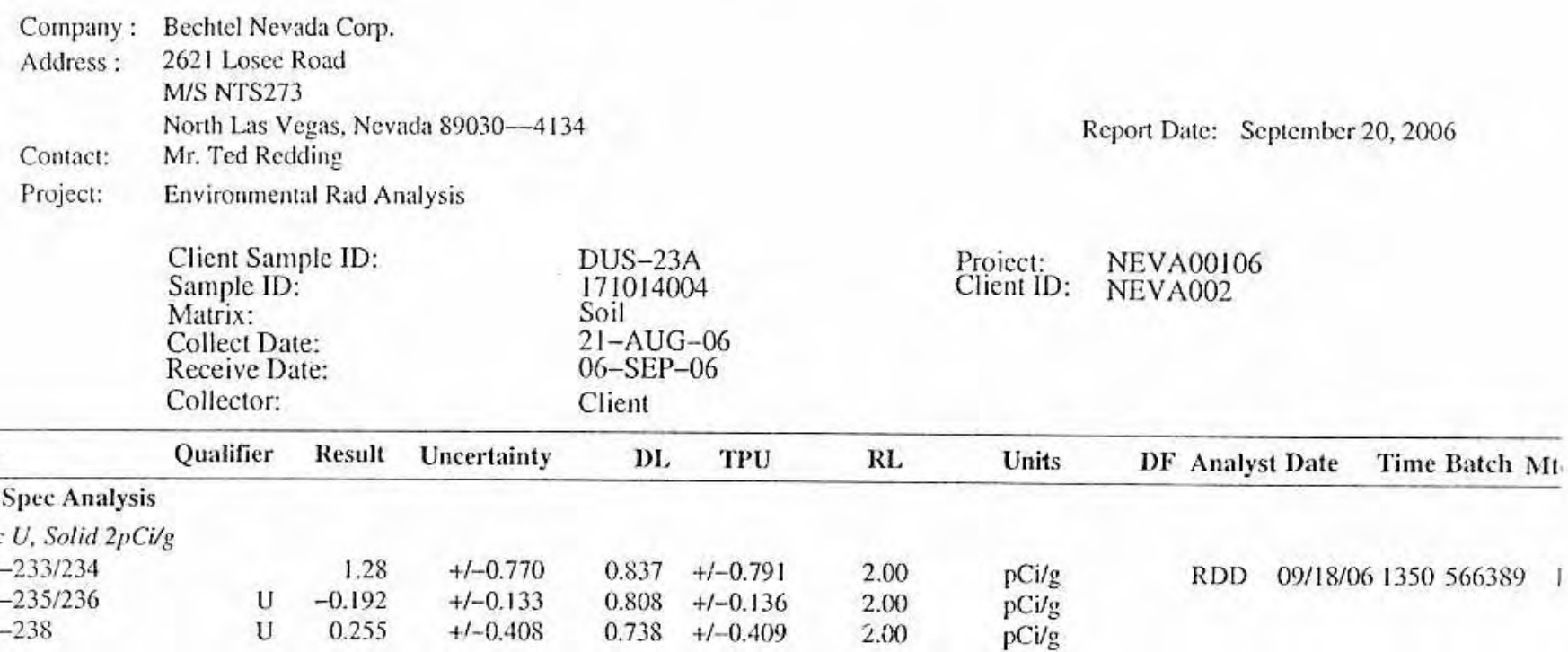

The following Prep Methods were performed

\begin{tabular}{llllll}
\hline Method & Description & Analyst & Date & Time & Prep Batch \\
\hline Dry Soil Prep $\quad$ Dry Soil Prep GL-RAD-A-02I & LXM2 & $09 / 07 / 06$ & 1002 & 565877 \\
The following Analytical Methods were performed & & & \\
\hline Method & Description & & & \\
\hline 1 & DOE EML HASL-300, U-02-RC Modified & &
\end{tabular}

$\begin{array}{llll}\text { Surrogate/Tracer recovery } & \text { Test } & \text { Recovery\% } & \text { Acceptable Limits }\end{array}$

$\begin{array}{llll}\text { Uranium-232 Alphaspec U, Solid 2pCi/g } & 90 & (25 \%-125 \%)\end{array}$

Notes:

The Qualifiers in this report are defined as follows :

* A quality control analyte recovery is outside of specified acceptance criteria

$<$ Resuit is less than vaiue reported

$>$ Result is greater than value reported

A The TIC is a suspected aldol-condensation product

B Target analyte was detected in the associated blank

BD Results are either below the MDC or tracer recovery is low

C Analyte has been confirmed by GC/MS analysis

D Results are reported from a diluted aliquot of the sample

$\mathrm{H}$ Analytical holding time was exceeded

$\mathrm{J}$ Value is estimated

N/A Spike recovery limits do not apply. Sample concentration exceeds spike concentration by $4 \mathrm{X}$ or more

R Sample results are rejected

U Analyte was analyzed for, but not detected above the MDL, MDA, or LOD.

UI Gamma Spectroscopy-Uncertain identification

X Consult Case Narrative, Data Summary package, or Project Manager concerning this qualifier 


\section{GENERAL ENGINEERING LABORATORIES, LLC \\ 2040 Savage Road Charleston SC 29407 - (843) 556-8171 - www.gel.com}

\section{$\underline{\text { Certificate of Analysis }}$}

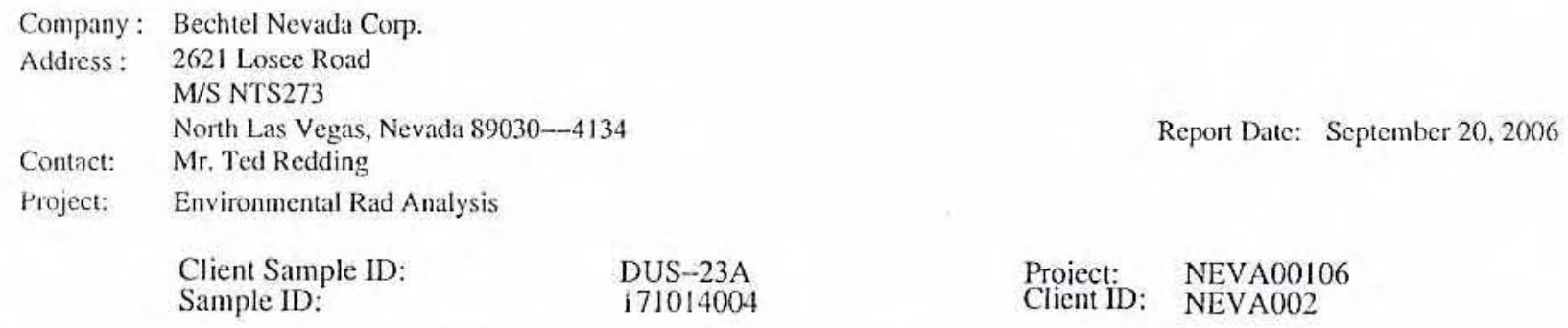

Parameter Qualifier Result Uncertainty DL TPU RL Units DF Analyst Date Time Batch Mit

Y QC Samples were not spiked with this compound

$\wedge$ RPD of sample and duplicate evaluated using $+/-R L$. Concentrations are $<5 \mathrm{X}$ the RL

$\mathrm{h}$ Preparation or preservation holding time was exceeded

The above sample is reported on a dry weight basis. 


\section{GENERAL ENGINEERING LABORATORIES, LLC \\ 2040 Savage Road Charleston SC 29407 (343) 556-8171 - www.gel.com}

\section{Certificate of Analysis}

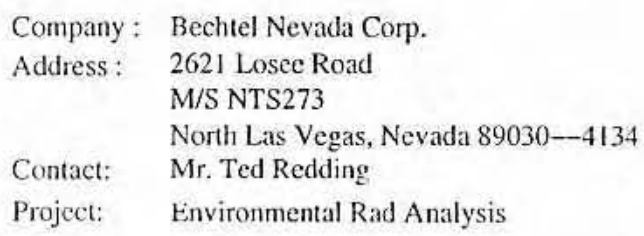

Client Sample ID: $\quad$ DUS-23B

Sample ID:

Matrix:

Collect Date:

Receive Date:

Collector:

171014005
Report Date: September 20,2006

Proiect: NEVA00106

Client ID: NEVA002

\begin{tabular}{|c|c|c|c|c|c|c|c|c|c|c|}
\hline Parameter & Qualifier & Result & Uncertainty & DL & TPU & RI & Units & $\mathrm{DF}$ & Analyst Date & Time Batch M \\
\hline \multicolumn{11}{|c|}{ Rad Alpha Spec Analysis } \\
\hline \multicolumn{11}{|c|}{ Alphaspec U. Solid $2 p C i / g$} \\
\hline Uranium-233/234 & $\mathrm{U}$ & 0.885 & $+/-0.708$ & 0.948 & $+1-0.718$ & 2.00 & pCi/g & & RDD $\quad 09 / 18 / 0$ & 61350566389 \\
\hline Uranium-235/236 & $\mathrm{U}$ & 0.378 & $+1-0.481$ & 0.785 & $+1-0.484$ & 2.00 & $\mathrm{pCi} / \mathrm{g}$ & & & \\
\hline Uranium-238 & $\mathrm{U}$ & 0.673 & $+1-0.644$ & 0.948 & $+1-0.651$ & 2.00 & $\mathrm{pCi} / \mathrm{g}$ & & & \\
\hline
\end{tabular}

The following Prep Methods were performed

\begin{tabular}{llllll}
\hline Method & Description & Analyst & Date & Time & Prep Batch \\
\hline Dry Soil Prep & Dry Soil Prep GL-RAD-A-021 & LXM2 & $09 / 07 / 06$ & 1002 & 565877
\end{tabular}

The following Analytical Methods were performed

\begin{tabular}{ll}
\hline Method & Description \\
\hline 1 & DOE EML HASL-300, U-02-RC Modified
\end{tabular}

\begin{tabular}{llcc} 
Surrogate/Tracer recovery & Test & Recovery \% & Acceptable Limits \\
\hline Uranium-232 & Alphaspec U, Solid 2pCi/g & 96 & $(25 \%-125 \%)$
\end{tabular}

Notes:

The Qualifiers in this report are defined as follows :

* A quality control analyte recovery is outside of specified acceptance criteria

$<$ Result is less than value reported

$>$ Result is greater than value reported

A The TIC is a suspected aldol-condensation product

B Target analyte was detected in the associated blank

BD Results are either below the MDC or tracer recovery is low

C Analyte has been confirmed by GC/MS analysis

D Results are reported from a diluted aliquot of the sample

$\mathrm{H}$ Analytical holding time was exceeded

$\mathrm{J}$ Value is estimated

N/A Spike recovery limits do not apply. Sample concentration exceeds spike concentration by $4 \mathrm{X}$ or more

$\mathrm{R}$ Sample results are rejected

U Analyte was analyzed for, but not detected above the MDL, MDA, or LOD.

UI Gamma Spectroscopy-Uncertain identification

X Consult Case Narrative, Data Summary package, or Project Manager concerning this qualifier 


\section{GENERAL ENGINEERING LABORATORIES, LLC \\ 2040 Savage Road Charleston SC 29407 - (843) 5568171 - www.gel.com}

\section{Certificate of Analysis}

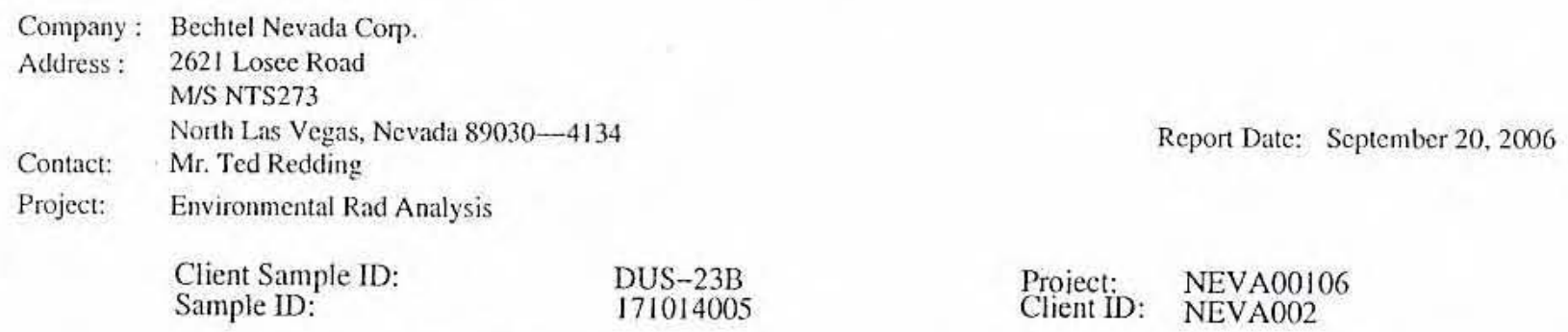

Y QC Samples were not spiked with this compound

$\wedge$ RPD of sample and duplicate evaluated using $+/-$ RL. Concentrations are $<5 X$ the RL

h Preparation or preservation holding time was exceeded

The above sample is reported on a dry weight basis. 


\section{GENERAL ENGINEERING LABORATORIES, LLC \\ 2040 Savage Road Charleston SC 29407 - (843) 556-8171 - www.gel.com}

\section{Certificate of Analysis}

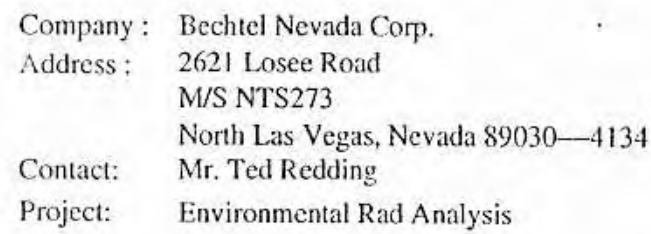

Report Date: September 20,2006

DUS-24A
171014006
Soil
21-AUG-06
06-SEP-06
Client

Proiect: NEVA00106

Client ID: NEVA002

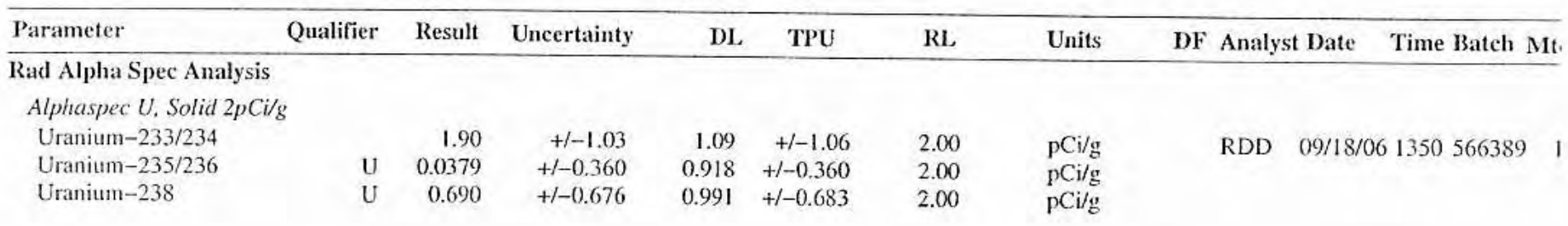

The following Prep Methods were performed

\begin{tabular}{llllll}
\hline Method & Description & Analyst & Date & Time & Prep Batch \\
\hline Dry Soil Prep & Dry Soil Prep GL-RAD-A-021 & LXM2 & $09 / 07 / 06$ & 1002 & 565877
\end{tabular}

The following Analytical Methods were performed

$\begin{array}{ll}\text { Method } & \text { Description } \\ 1 & \text { DOE EML HASL-300, U-02-RC Modified }\end{array}$

\begin{tabular}{llcc} 
Surrogate/Tracer recovery & Test & Recovery\% & Acceptable Limits \\
\hline Uranium-232 & Alphaspec U, Solid 2pCi/g & 90 & $(25 \%-125 \%)$
\end{tabular}

Notes:

The Qualifiers in this report are defined as follows :

* A quality control analyte recovery is outside of specified acceptance criteria

$<$ Result is less than value reported

$>$ Result is greater than value reported

A The TIC is a suspected aldol-condensation product

B Target analyte was detected in the associated blank

$\mathrm{BD}$ Results are either below the MDC or tracer recovery is low

C Analyte has been confirmed by GC/MS analysis

D Results are reported from a diluted aliquot of the sample

$\mathrm{H}$ Analytical holding time was exceeded

J Value is estimated

N/A Spike recovery limits do not apply. Sample concentration exceeds spike concentration by $4 \mathrm{X}$ or more

$\mathrm{R}$ Sample results are rejected

U Analyte was analyzed for, but not detected above the MDL, MDA, or LOD.

UI Gamma Spectroscopy--Uncertain identification

X Consult Case Narrative, Data Summary package, or Project Manager concerning this qualifier 


\section{GENERAL ENGINEERING LABORATORIES, LLC \\ 2040 Savage Road Charleston SC 29407 - (843) 556-8171 www.gei.com}

\section{Certificate of Analysis}

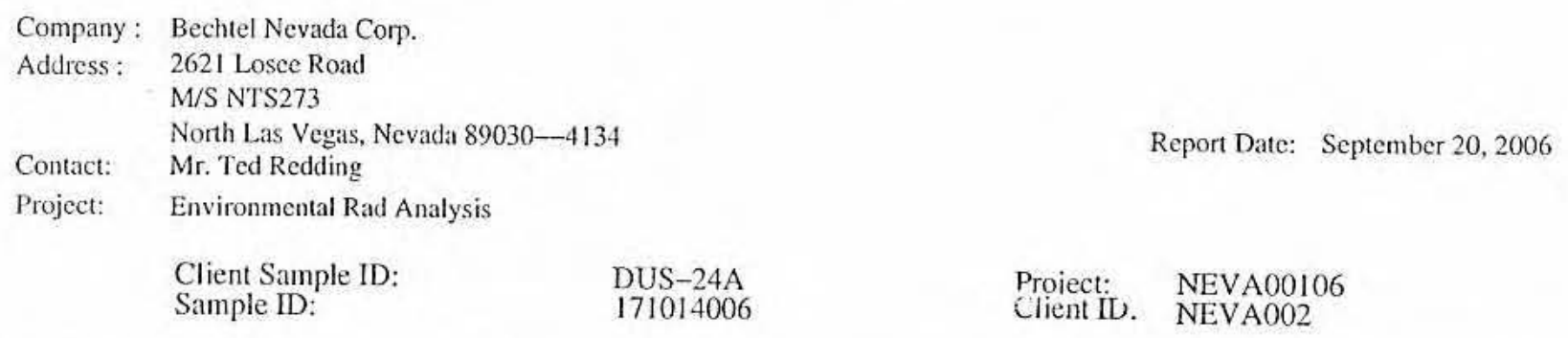

Y QC Samples were not spiked with this compound

$\wedge$ RPD of sample and duplicate evaluated using $+/-$ RL. Concentrations are $<5 X$ the RL

$\mathrm{h}$ Preparation or preservation holding time was exceeded

The above sample is reported on a dry weight basis. 


\section{GENERAL ENGINEERING LABORATORIES, LLC \\ 2040 Savage Road Charleston SC 29407 - (843) 556-81ii - wrww.gei.com}

\section{Certificate of Analysis}

Company: Bechtel Nevada Corp.

Address : 2621 Losee Road

M/S NTS273

North Las Vegas, Nevada 89030-4134 Report Date: September 20, 2006

Contact: $\quad$ Mr. Ted Redding

Project: Environmental Rad Analysis

Client Sample ID: $\quad$ DUS-24B Proiect: NEVA00106

Sample ID: $\quad 171014007 \quad$ Client ii): NEVA002

Matrix: Soil

Collect Date: $\quad$ 21-AUG-06

Receive Date: $\quad$ 06-SEP-06

Collector: Client

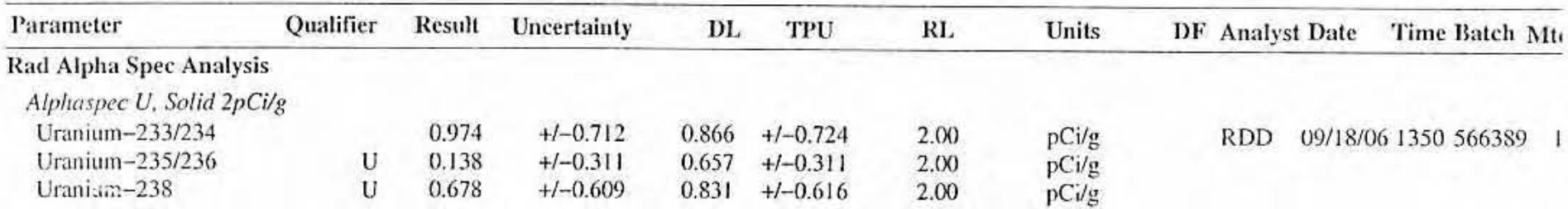

The following Prep Methods were performed

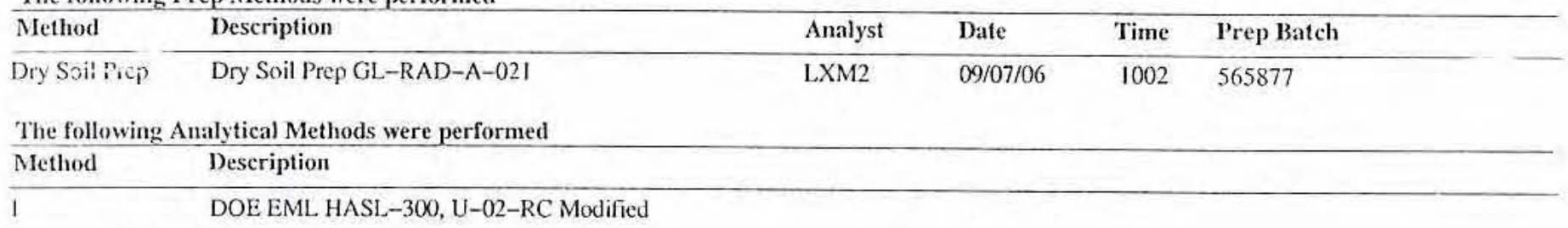

$\begin{array}{llll}\text { Surrogate/Tracer recovery } & \text { Test } & \text { Recovery\% } & \text { Acceptable Limits }\end{array}$

$\begin{array}{llll}\text { Uranium-232 Alphaspec U, Solid 2pCi/g } & 92 & (25 \%-125 \%)\end{array}$

Notes:

The Qualifiers in this report are defined as follows :

* A quality control analyte recovery is outside of specified acceptance criteria

$<$ Result is less than value reported

$>$ Result is greater than value reported

A The TIC is a suspected aldol-condensation product

B Target analyte was detected in the associated blank

BD Results are either below the MDC or tracer recovery is low

C Analyte has been confirmed by GC/MS analysis

D Results are reported from a diluted aliquot of the sample

$\mathrm{H}$ Analytical holding time was exceeded

$\mathrm{J}$ Value is estimated

N/A Spike recovery limits do not apply. Sample concentration exceeds spike concentration by $4 X$ or more

R Sample results are rejected

U Analyte was analyzed for, but not detected above the MDL, MDA, or LOD.

UI Gamma Spectroscopy--Uncertain identification

$\mathrm{X}$ Consult Case Narrative, Data Summary package, or Project Manager concerning this qualifier 


\section{GENERAL ENGINEERING LABORATORIES, LI.C}

2040 Savage Road Charleston SC 29407 - (843) 556-8171 - www.yei.cuin

\section{$\underline{\text { Certificate of Analysis }}$}

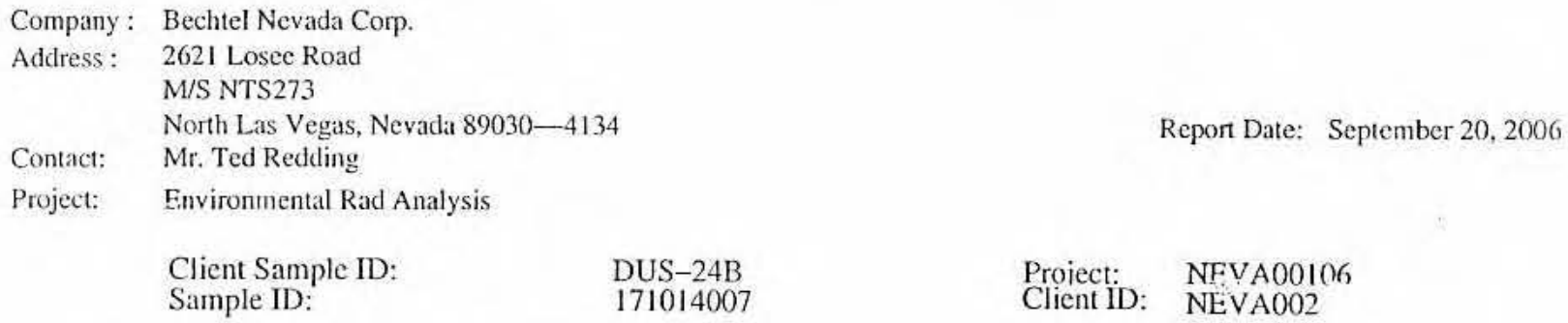

Parameter Qualifier Result Uncertainty DL TPU RL Units DF Analyst Date Time Bateh Mtd

Y QC Samples were not spiked with this compound

$\wedge \quad$ RPD of sample and duplicate evaluated using $+/-$ RL. Concentrations are $<5 X$ the RL

h Preparation or preservation holding time was exceeded

The above sample is reported on a dry weight basis. 


\section{GENERAL ENGINEERING LABORATORIES, LLC}

2040 Savage Road Charleston SC 29407 - (843) 556-8171 - www.gel.com

\section{Certificate of Analysis}

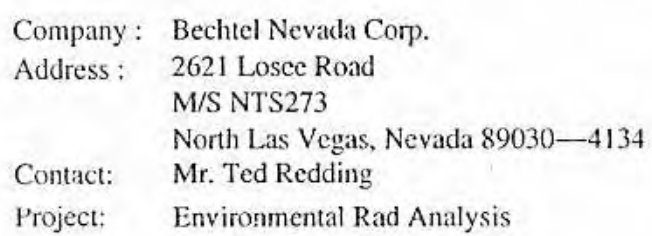

Client Sample ID: $\quad$ DUS-25A

Sample ID: $\quad 171014008$

Matrix:

Collect Date:

Receive Date:

Collector:
Report Date: September 20,2006

Soil

21-AUG-06

06-SEP-06

Client

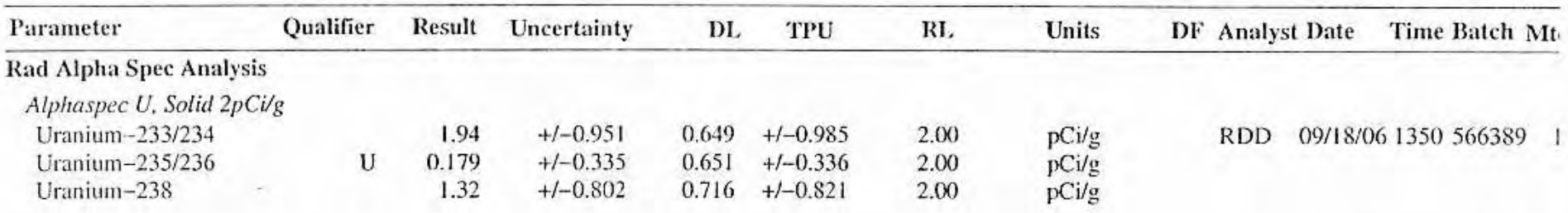

The following Prep Methods were performed

\begin{tabular}{|c|c|c|c|c|c|}
\hline Method & Description & Analyst & Date & Time & Prep Batch \\
\hline Dry Soil Prep & Dry Soil Prep GL-RAD-A-021 & LXM2 & $09 / 07 / 06$ & 1002 & 565877 \\
\hline \multicolumn{6}{|c|}{ The following Analytical Methods were performed } \\
\hline Method & Description & & & & \\
\hline
\end{tabular}

$\begin{array}{llll}\text { Surrogate/Tracer recovery } & \text { Test Recovery\% } & \text { Acceptable Limits }\end{array}$

$\begin{array}{llll}\text { Uranium-232 Alphaspec U.Solid 2pCi/g } & 94 & (25 \%-125 \%)\end{array}$

Notes:

The Qualifiers in this report are defined as follows:

* A quality control analyte recovery is outside of specified acceptance criteria

$<$ Result is less than value reported

$>$ Result is greater than value reported

A The TIC is a suspected aldol-condensation product

B Target analyte was detected in the associated blank

BD Results are either below the MDC or tracer recovery is low

C Analyte has been confirmed by GC/MS analysis

D Results are reported from a diluted aliquot of the sample

$\mathrm{H}$ Analytical holding time was exceeded

$\mathrm{J}$ Value is estimated

N/A Spike recovery limits do not apply. Sample concentration exceeds spike concentration by $4 X$ or more

$R$ Sample results are rejected

U Analyte was analyzed for, but not detected above the MDL, MDA, or LOD.

UI Gamma Spectroscopy--Uncertain identification

$\mathrm{X}$ Consult Case Narrative, Data Summary package, or Project Manager concerning this qualifier 


\section{GENERAL ENGINEERING LABORATORIES, LLC \\ 2040 Savage Road Charleston SC 29407 - (843) 556-8171 - www.gel.com}

\section{Certificate of Analysis}

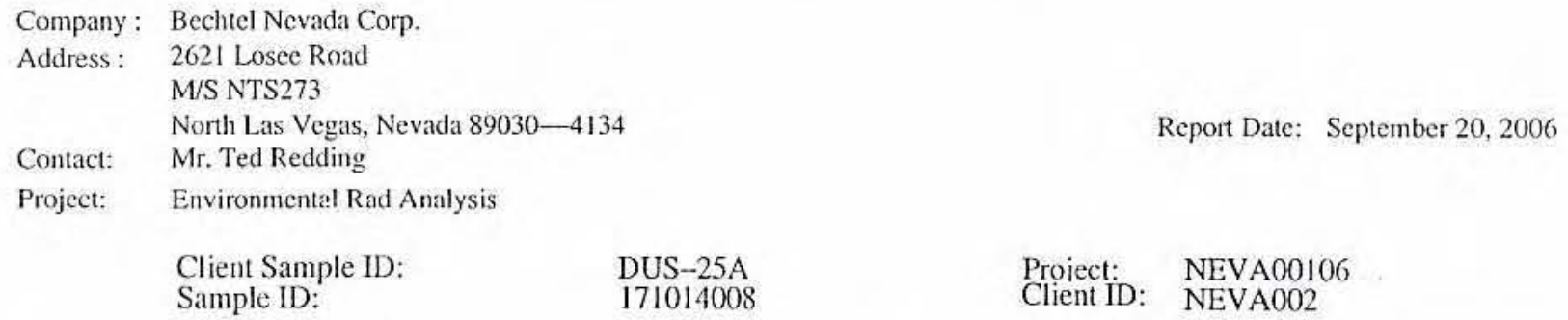

Parameter Qualifier Result Uncertainty DL TPU RL Units DF Analyst Date Time Batch Mt.

Y QC Samples were not spiked with this compound

$\wedge$ RPD of sample and duplicate evaluated using $+/-$ RL. Concentrations are $<5 X$ the RL

h Preparation or preservation holding time was exceeded

The above sample is reported on a dry weight basis. 


\section{GENERAL ENGINEERING LABORATORIES, LLC \\ 2040 Savage Road Charleston SC 29407 - (843) 556-8171 - www.gel.com}

\section{Certificate of Analysis}

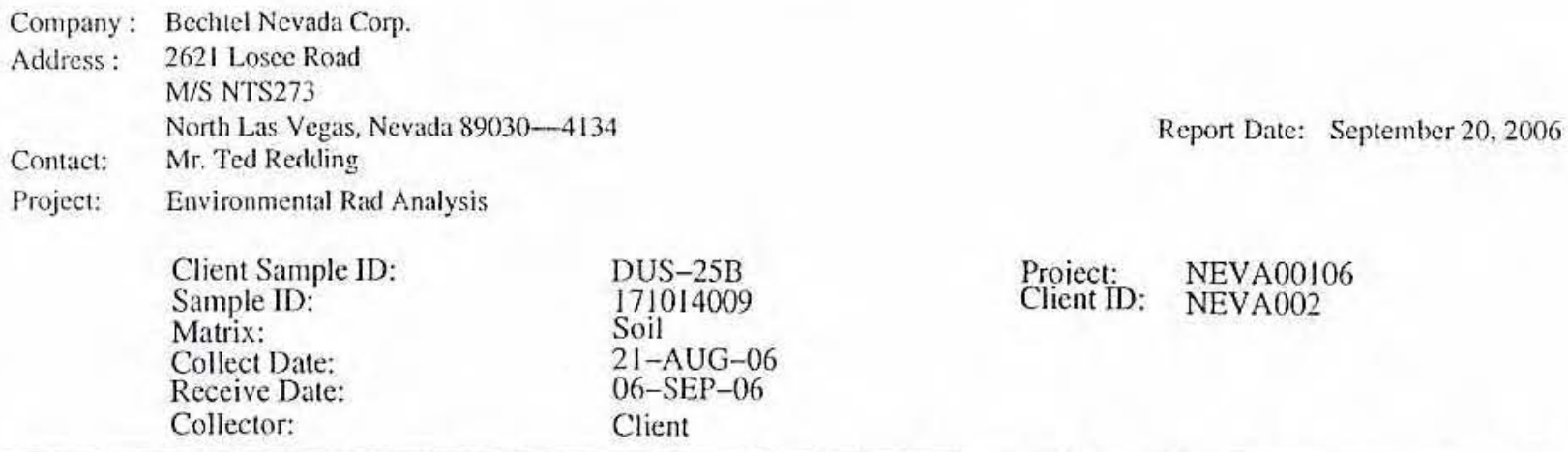

\begin{tabular}{|c|c|c|c|c|c|c|c|c|c|c|c|c|}
\hline Parameter & Qualifier & Result & Uncertainty & $\mathrm{DL}$ & TPU & RL & Units & DF & Analyst & Date & Time Batch M & Mt \\
\hline Rad Alpha Spec Analysis & & & & & & & & $=$ & & & & \\
\hline Alphaspec U, Solid $2 p C i / g$ & & & & & & & & & & & & \\
\hline Uranium-233/234 & & 2.09 & $+1-1,09$ & 0.795 & $+/-1.13$ & 2.00 & $\mathrm{pCi} / \mathrm{g}$ & & RDD & $09 / 18 / 0$ & 61350566389 & 1 \\
\hline Uranium-235/236 & $\mathrm{U}$ & -0.104 & $+1-0.117$ & 0.880 & $+1-0.118$ & 2.00 & $\mathrm{pCi} / \mathrm{g}$ & & & & & \\
\hline Tranium-238 & $U$ & 0.615 & $+1-0.640$ & 0.877 & $+1-0.646$ & 2.00 & $\mathrm{pCi} / \mathrm{g}$ & & & & & \\
\hline
\end{tabular}

The following Prep Methods were performed

\begin{tabular}{llllll}
\hline Method & Description & Analyst & Date & Time & Prep Batch \\
\hline Dry Soil Prep & Dry Soil Prep GL-RAD-A-021 & LXM2 & $09 / 07 / 06$ & 1002 & 565877
\end{tabular}

The following Analytical Methods were performed

Method Description

\begin{tabular}{llcc} 
Surrogate/Tracer recovery & Test & Recovery\% & Acceptable Limits \\
\hline Uranium-232 & Alphaspec U, Solid 2pCi/g & 83 & $(25 \%-125 \%)$
\end{tabular}

Notes:

The Qualifiers in this report are defined as follows :

* A quality control analyte recovery is outside of specified acceptance criteria

$<$ Result is less than value reported

$>$ Result is greater than value reported

A The TIC is a suspected aldol-condensation product

B Target analyte was detected in the associated blank

$\mathrm{BD}$ Restilts are either below the MDC or tracer recovery is low

C Analyte has been confirmed by GC/MS analysis

D Results are reported from a diluted aliquot of the sample

$\mathrm{H}$ Analytical holding time was exceeded

$\mathrm{J}$ Value is estimated

N/A Spike recovery limits do not apply. Sample concentration exceeds spike concentration by $4 X$ or more

$\mathrm{R}$ Sample results are rejected

$\mathrm{U}$ Analyte was analyzed for, but not detected above the MDL, MDA, or LOD.

UI Gamma Spectroscopy--Uncertain identification

$\mathrm{X}$ Consult Case Narrative, Data Summary package, or Project Manager concerning this qualifier 


\section{GENERAL ENGINEERING LABORATORIES, LLC \\ 2040 Savage Road Charleston SC 29407 - (843) 556-8171 - www.gel.com}

\section{Certificate of Analysis}

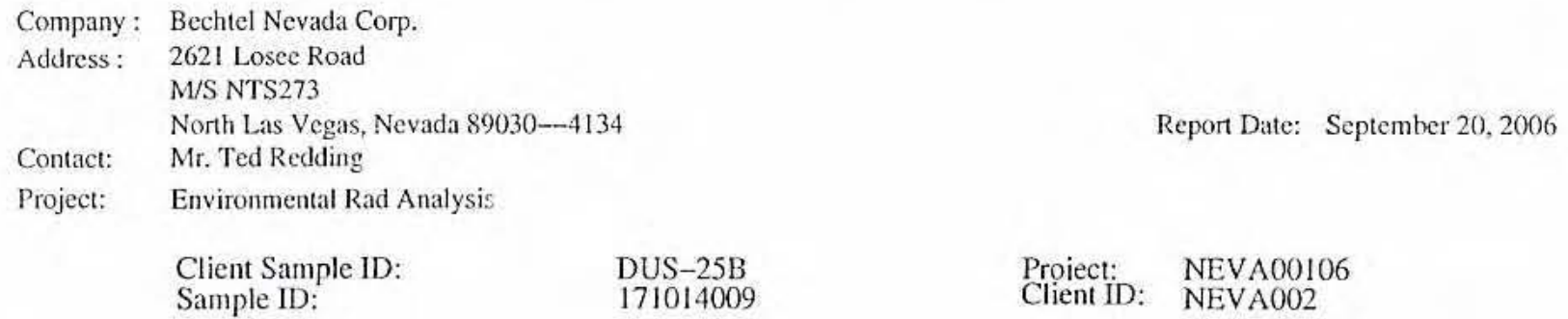

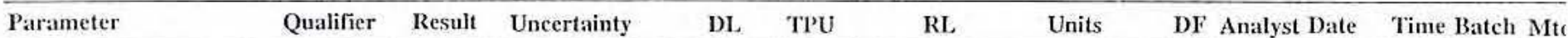

Y QC Samples were not spiked with this compound

$\wedge$ RPD of sample and duplicate evaluated using $+/-$ RL. Concentrations are $<5 X$ the $R L$

$\mathrm{h}$ Preparation or preservation holding time was exceeded

The above sample is reported on a dry weight basis. 


\section{GENERAL ENGINEERING LABORATORIES, LLC \\ 2040 Savage Road Charleston SC 29407 - (843) 556-8171 - www.gel.com}

\section{Certificate of Analysis}

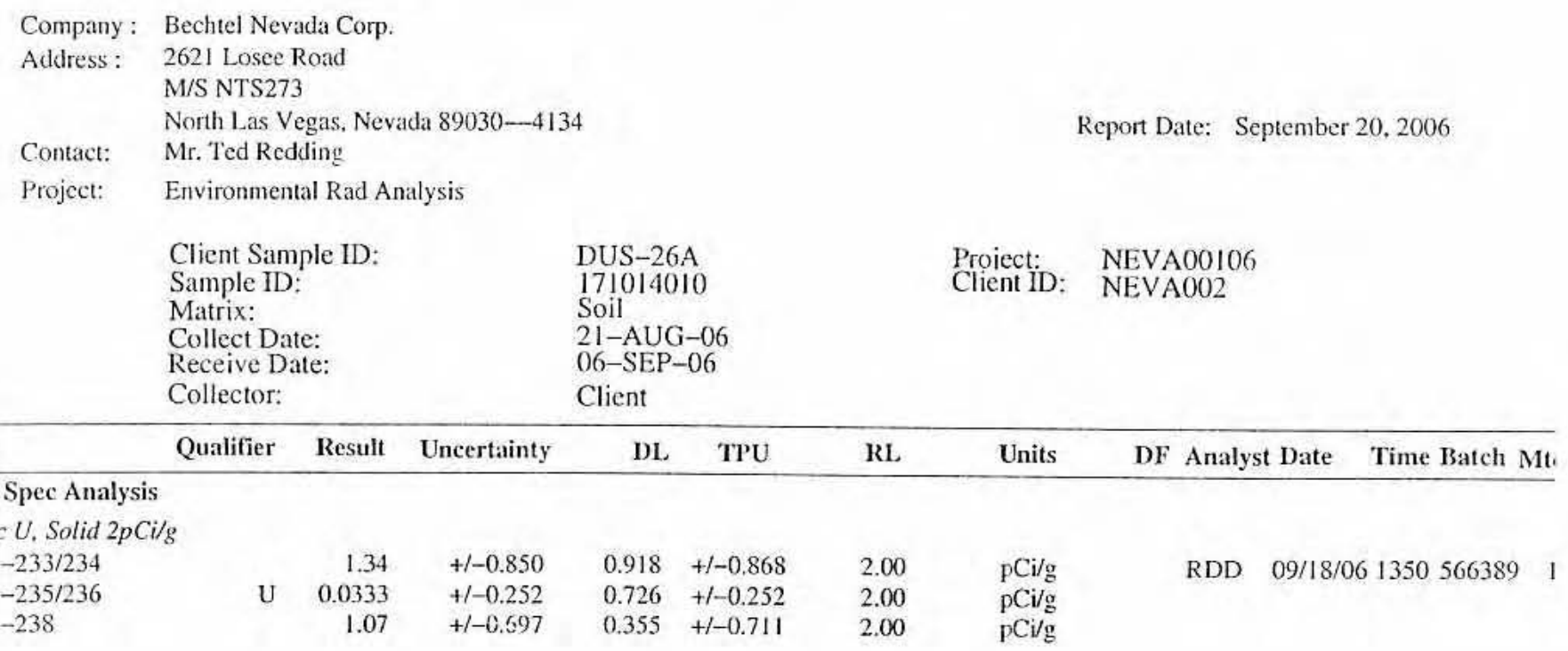

The following Prep Methods were performed

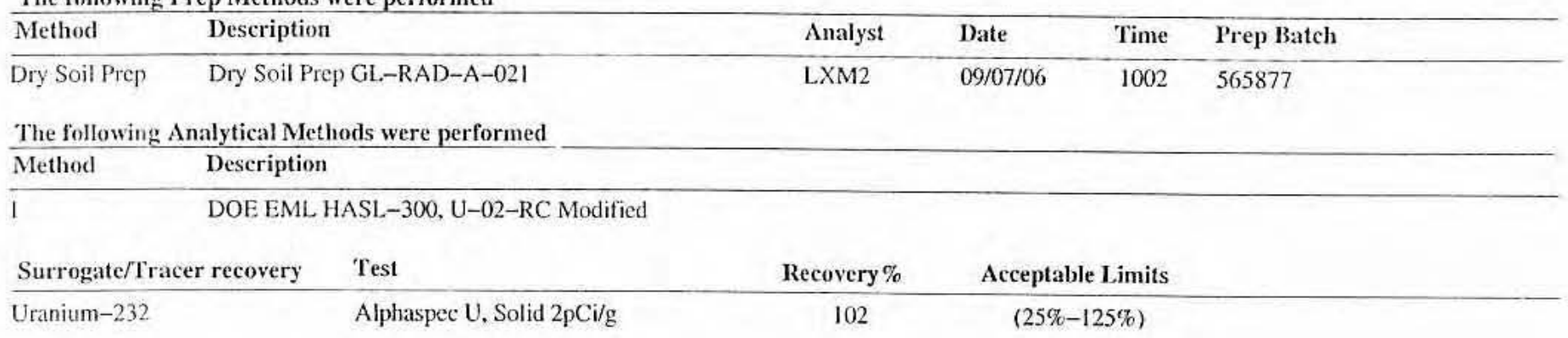

Notes:

The Qualifiers in this report are defined as follows :

* A quality control analyte recovery is outside of specified acceptance criteria

$<$ Result is less than value reported

$>$ Result is greater than value reported

A The TIC is a suspected aldol-condensation product

B Target analyte was detected in the associated blank

$\mathrm{BD}$ Results are either below the MDC or tracer recovery is low

C Analyte has been confirmed by GC/MS analysis

D Results are reported from a diluted aliquot of the sample

$\mathrm{H}$ Analytical holding time was exceeded

$\mathrm{J}$ Value is estimated

N/A Spike recovery limits do not apply. Sample concentration exceeds spike concentration by $4 X$ or more

R Sample results are rejected

U Analyte was analyzed for, but not detected above the MDL, MDA, or LOD.

UI Gamma Spectroscopy-Uncertain identification

X Consult Case Narrative, Data Summary package, or Project Manager concerning this qualifier 


\section{GENERAL ENGINEERING LABORATORIES, LLC \\ 2040 Savage Road Charleston SC 29407 - (843) 556-8171 - www.gel.com}

\section{Certificate of Analysis}

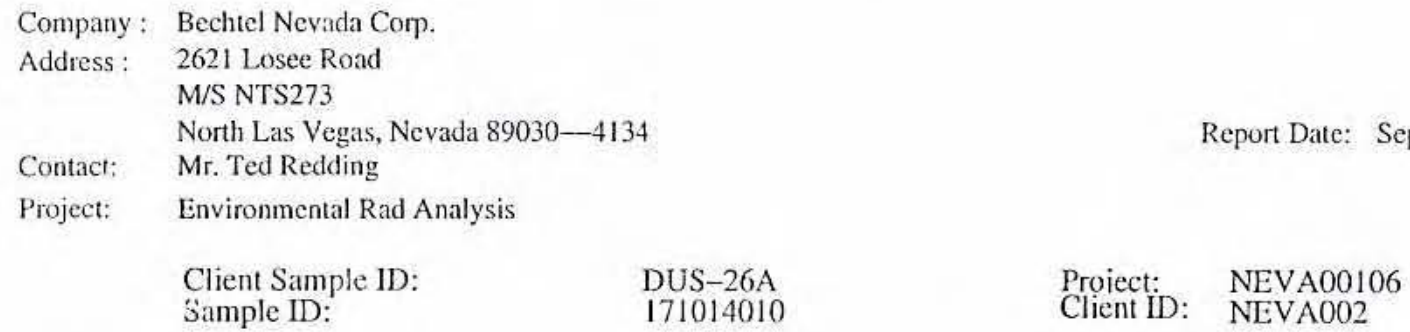

Y QC Samples were not spiked with this compound

$\wedge$ RPD of sample and duplicate evaluated using $+/-$ RL. Concentrations are $<5 X$ the RL

$\mathrm{h}$ Preparation or preservation holding time was exceeded

The above sample is reported on a dry weight basis. 


\section{Certificate of Analysis}

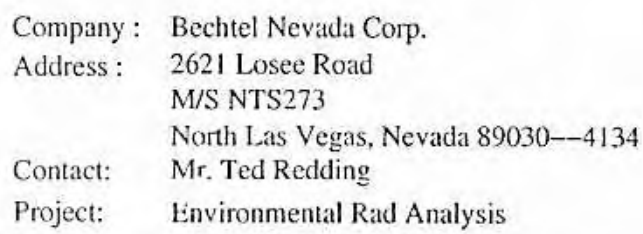

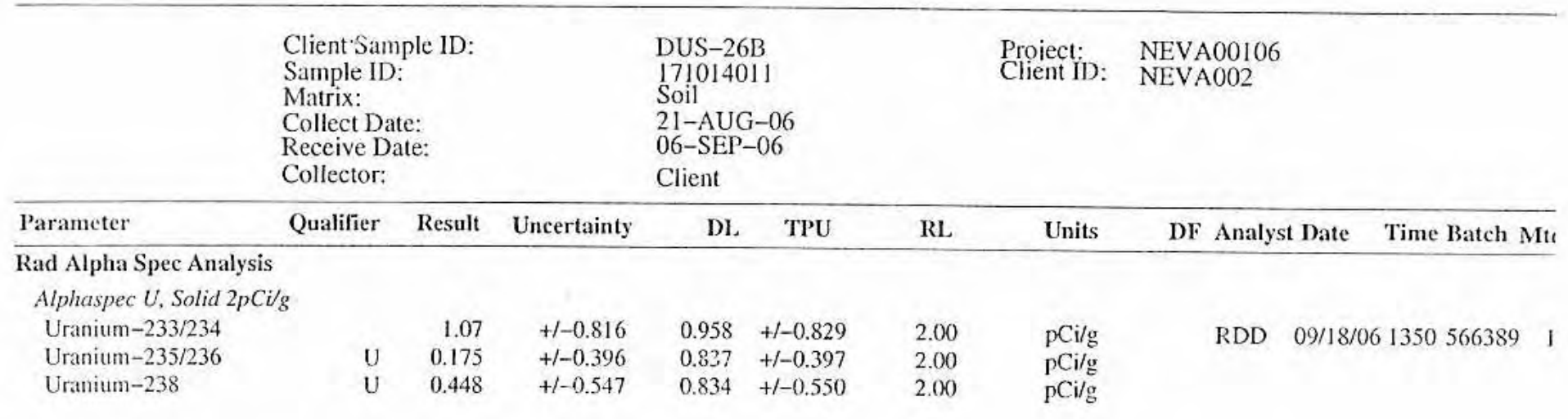

The following Prep Methods were performed

\begin{tabular}{llllll}
\hline Method & Description & Analyst & Date & Time & Prep Bateh \\
\hline Dry Soil Prep & Dry Soil Prep GL-RA.D-A-021 & LXM2 & $09 / 07 / 06$ & 1002 & 565877
\end{tabular}

The following Analytical Methods were performed

\begin{tabular}{ll} 
Method & Description \\
\hline DOE EML HASL-300, U-02-RC Modified
\end{tabular}

\begin{tabular}{llcc} 
Surrogate/Tracer recovery & Test & Recovery\% & Acceptable Limits \\
\hline Uranium-232 & Alphaspec U, Solid 2pCi/g & 90 & $(25 \%-125 \%)$
\end{tabular}

Notes:

The Qualifiers in this report are defined as follows :

* A quality control analyte recovery is outside of specified acceptance criteria

$<$ Result is less than value reported

$>$ Result is greater than value reported

A The TIC is a suspected aldol-condensation product

B Target analyte was detected in the associated blank

$\mathrm{BD}$ Results are either below the MDC or tracer recovery is low

C Analyte has been confirmed by GC/MS analysis

D Results are reported from a diluted aliquot of the sample

$\mathrm{H}$ Analytical holding time was exceeded

$\mathrm{J}$ Value is estimated

N/A Spike recovery limits do not apply. Sample concentration exceeds spike concentration by $4 X$ or more

$\mathrm{R}$ Sample results are rejected

U Analyte was analyzed for, but not detected above the MDL, MDA, or LOD.

UI Gamma Spectroscopy--Uncertain identification 


\section{GENERAL ENGINEERING LABORATORIES, LLC \\ 2040 Savage Foad Charleston SC 29407 - (843) 556-817i - www.gel.com}

\section{Certificate of Analysis}

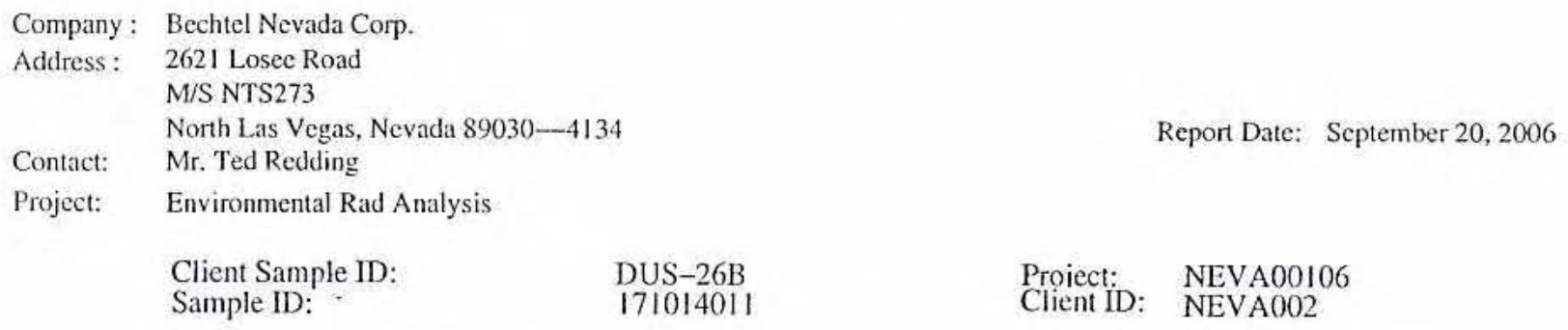

\begin{tabular}{lllllllll}
\hline Parameter & Qualifier & Result & Uncertainty & DL & TPU & RL & Units & DF Analyst Date Time Batch Mtt
\end{tabular}

$\mathrm{X}$ Consult Case Narrative, Data Summary package, or Project Manager concerning this qualifier

Y QC Samples were not spiked with this compound

$\wedge \quad$ RPD of sample and duplicate evaluated using $+/-$ RL. Concentrations are $<5 X$ the RL

h Preparation or preservation holding time was exceeded

The above sample is reported on a dry weight basis. 


\section{GENERAL ENGINEERING LABORATORIES, LLC}

2040 Savage Road Charleston SC 29407 - (843) 556-8171 - www.gel.com

\section{Certificate of Analysis}

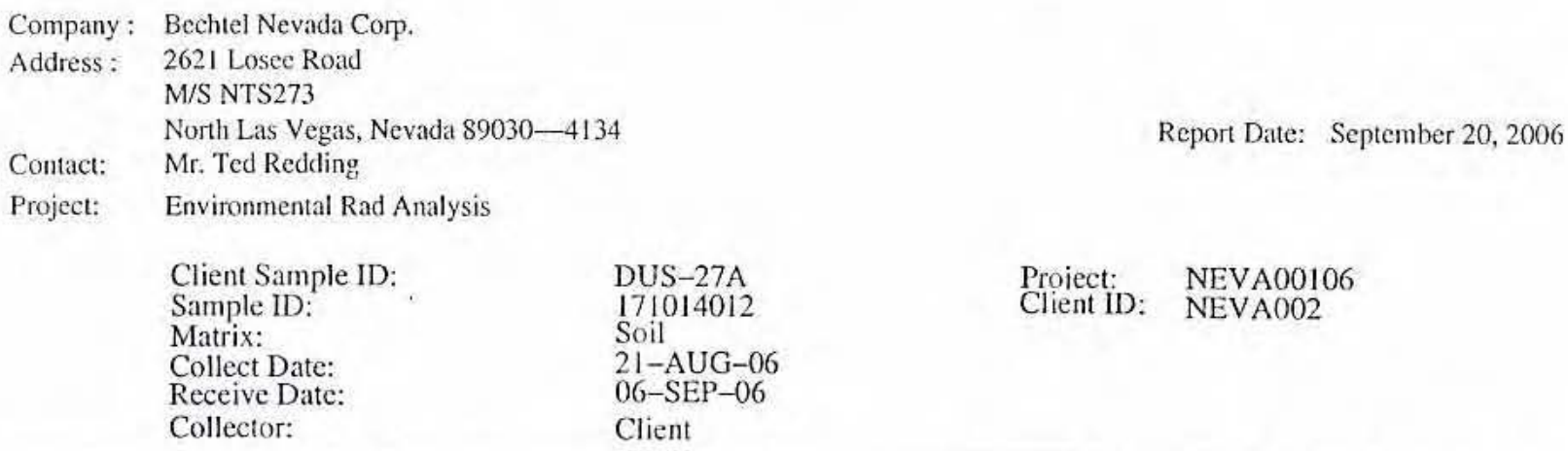

\begin{tabular}{|c|c|c|c|c|c|c|c|c|c|c|c|}
\hline Parameter & Qualifier & Result & Uncertainty & DI, & TPU & $\mathrm{RL}$ & Units & DF & Analyst & t Date & Time Batch Mt \\
\hline \multicolumn{12}{|c|}{ Rad Alpha Spec Analysis } \\
\hline \multicolumn{12}{|c|}{ Alphaspec U, Solid $2 p C i / g$} \\
\hline Uranium-233/234 & & 1.59 & $+/-0.880$ & 0.859 & $+1-0.903$ & 2.00 & $\mathrm{pCi} / \mathrm{g}$ & & RDD & $09 / 18 / 0$ & $61350566389 \quad 1$ \\
\hline Uranium-235/236 & $\mathrm{U}$ & -0.0178 & $+1-0.346$ & 0.932 & $+1-0.346$ & 2.00 & $\mathrm{pCi} / \mathrm{g}$ & & & & \\
\hline Uranium-238 & $\mathrm{U}$ & 0.590 & $+1-0.591$ & 0.859 & $+1-0.590$ & 2.00 & $\mathrm{pCi} / \mathrm{g}$ & & & & \\
\hline
\end{tabular}

The following Prep Methods were performed

\begin{tabular}{|c|c|c|c|c|c|}
\hline Method & Description & Analyst & Date & Time & Prep Batch \\
\hline Dry Soil Prep & Dry Soil Prep GL-RAD-A-02I & LXML & $09 / 07 / 06$ & 1002 & 565877 \\
\hline \multicolumn{6}{|c|}{ The following Analytical Methods were performed } \\
\hline Method & Description & & & & \\
\hline 1 & DOE EML HASL-300, U-02-RC Modified & & & & \\
\hline Surrogate/Tr: & r recovery & Recovery $\%$ & \multicolumn{2}{|c|}{ Acceptable Limits } & \\
\hline Uranium-232 & Alphaspec U, Solid 2pCi/g & 97 & \multicolumn{2}{|c|}{$(25 \%-125 \%)$} & \\
\hline
\end{tabular}

Nutes:

The Qualifiers in this report are defined as follows :

* A quality control analyte recovery is outside of specified acceptance criteria

$<$ Result is less than value reported

$>$ Result is greater than value reported

A The TIC is a suspected aldol-condensation product

B Target analyte was detected in the associated blank

$\mathrm{BD}$ Results are either below the MDC or tracer recovery is low

C Analyte has been confirmed by GC/MS analysis

D Results are reported from a diluted aliquot of the sample

$\mathrm{H}$ Analytical holding time was exceeded

$\mathrm{J}$ Value is estimated

N/A Spike recovery limits do not apply. Sample concentration exceeds spike concentration by $4 \mathrm{X}$ or more

R Sample results are rejected

U Analyte was analyzed for, but not detected above the MDL, MDA, or LOD.

UI Gamma Spectroscopy-Uncertain identification

X Consult Case Narrative, Data Summary package, or Project Manager concerning this qualifier 


\section{GENERAL ENGINEERING LABORATORIES, LLC}

2040 Savage Hoad Charleston SC 29407 - (843) 556-8171 - www.gel.com

\section{Certificate of Analysis}

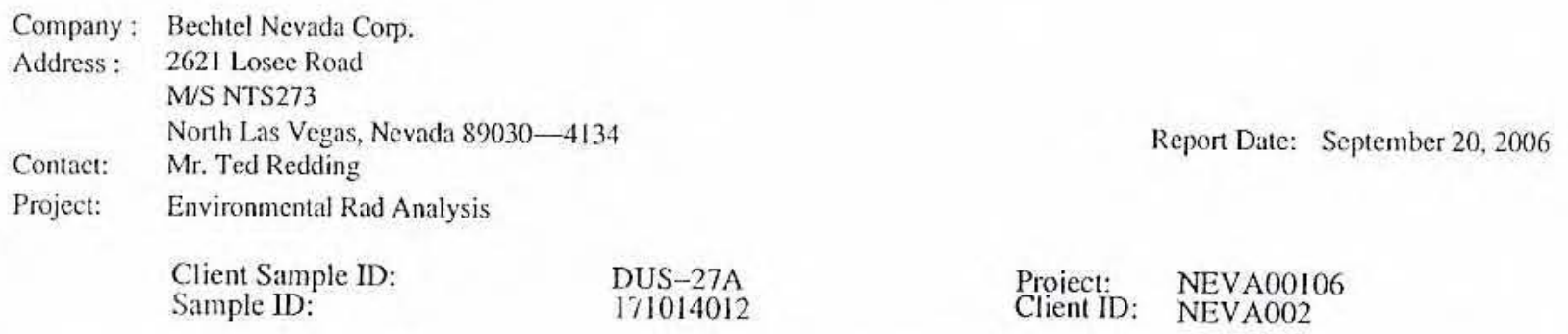

Y QC Samples were not spiked with this compound

$\wedge \quad$ RPD of sample and duplicate evaluated using $+/-R L$. Concentrations are $<5 X$ the RL

h Preparation or preservation holding time was exceeded

The above sample is reported on a dry weight basis. 


\section{GENERAL ENGINEERING LABORATORIES, LLC \\ 2040 Savage Road Charleston SC 29407 - (843) 556-8111 - www.gel.com}

\section{Certificate of Analysis}

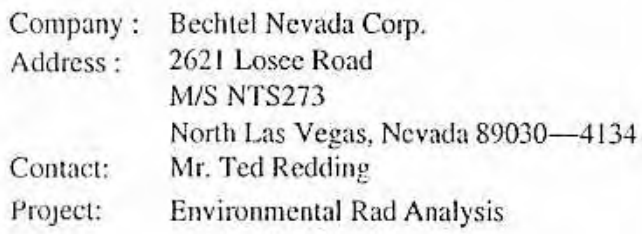

Report Date: September 20, 2006

Proiect: NEVA00106

Client ID: NEVA002

\begin{tabular}{|c|c|c|c|c|c|c|c|c|c|c|}
\hline Parameter & Qualifier & Result & Uncertainty & DL & TPU & RI & Units & DF & Analyst Date & Time Batch M \\
\hline \multicolumn{11}{|c|}{ Rad Alpha Spec Analysis } \\
\hline \multicolumn{11}{|c|}{ Alphaspec U, Solid $2 \mathrm{pCi} / g$} \\
\hline Uranium-233/234 & & 1.18 & $+1-0.846$ & 0.871 & $+1-0.861$ & 2.00 & $\mathrm{pCi} / \mathrm{g}$ & & RDD $\quad 09 / 18 / 0$ & 61350566389 \\
\hline Uranium-235/236 & $\mathrm{U}$ & 0.0457 & $+/-0.434$ & 1.11 & $+1-0.434$ & 2.00 & $\mathrm{pCi} / \mathrm{g}$ & & & \\
\hline Uranium-238 & U & 0.861 & $+1-0.751$ & 0.939 & $+1-0.760$ & 2.00 & $\mathrm{pCi} / \mathrm{g}$ & & & \\
\hline
\end{tabular}

The following Prep Methods were performed

\begin{tabular}{llllll}
\hline Method & Description & Analyst & Date & Time & Prep Batch \\
\hline Dry Soil Prep & Dry Soil Prep GL-RAD-A-021 & LXM2 & $09 / 07 / 06$ & 1002 & 565877
\end{tabular}

The following Analytical Methods were performed

Method Description
1

\begin{tabular}{llcc} 
Surrogate/Tracer recovery & Test & Recovery \% & Acceptable Limits \\
\hline Uranium-232 & Alphaspec U, Solid 2pCi/g & 81 & $(25 \%-125 \%)$
\end{tabular}

Notes:

The Qualifiers in this report are defined as follows :

* A quality control analyte recovery is outside of specified acceptance criteria

$<$ Resuit is less than value reported

$>$ Result is greater than value reported

A The TIC is a suspected aldol-condensation product

B Target analyte was detected in the associated blank

$\mathrm{BD}$ Results are either below the MDC or tracer recovery is low

C Analyte has been confirmed by GC/MS analysis

D Results are reported from a diluted aliquot of the sample

$\mathrm{H}$ Analytical holding time was exceeded

$\mathrm{J}$ Value is estimated

N/A Spike recovery limits do not apply. Sample concentration exceeds spike concentration by $4 X$ or more

$\mathrm{R}$ Sample results are rejected

U Analyte was analyzed for, but not detected above the MDL, MDA, or LOD.

UI Gamma Spectroscopy-Uncertain identification

X Consult Case Narrative, Data Summary package, or Project Manager concerning this qualifier 


\section{GENERAL ENGINEERING LABORATORIES, LLC \\ 2040 Savage Road Charleston SC 29407 - (343) 556-8171 - www.gel.com}

\section{Certificate of Analysis}

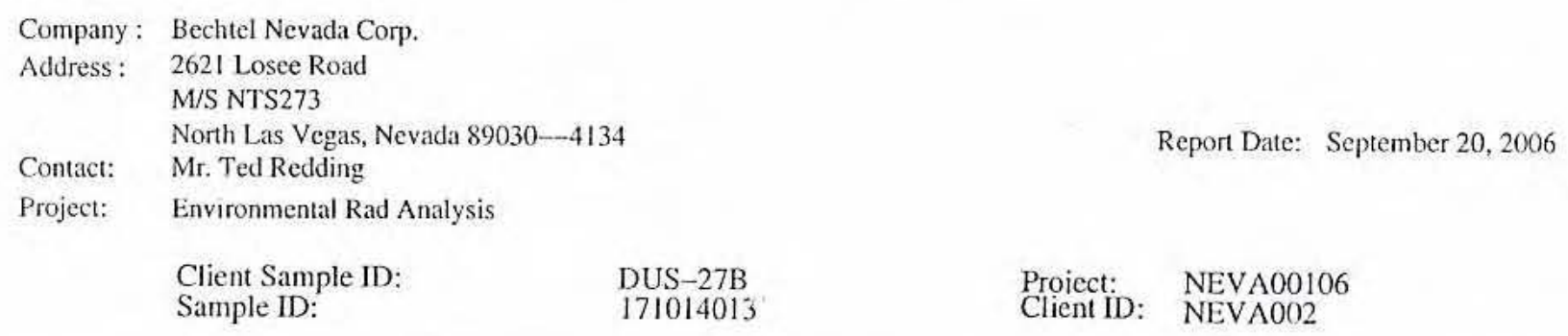

\begin{tabular}{llllllll}
\hline Parameter Qualifier Result Uncertainty & DL & TPU & RL & Units & DF Analyst Date Time Batch Mt
\end{tabular}

Y QC Samples were not spiked with this compound

$\wedge$ RPD of sample and duplicate evaluated using $+/-R L$. Concentrations are $<5 X$ the Ri

h Preparation or preservation holding time was exceeded

The above sample is reported on a dry weight basis. 


\section{GENERAL ENGINEERING LABORATORIES, LLC}

2040 Savage Road Charleston SC 29407 - \{343\} 556-8i71 - www.gel.com

\section{Certificate of Analysis}

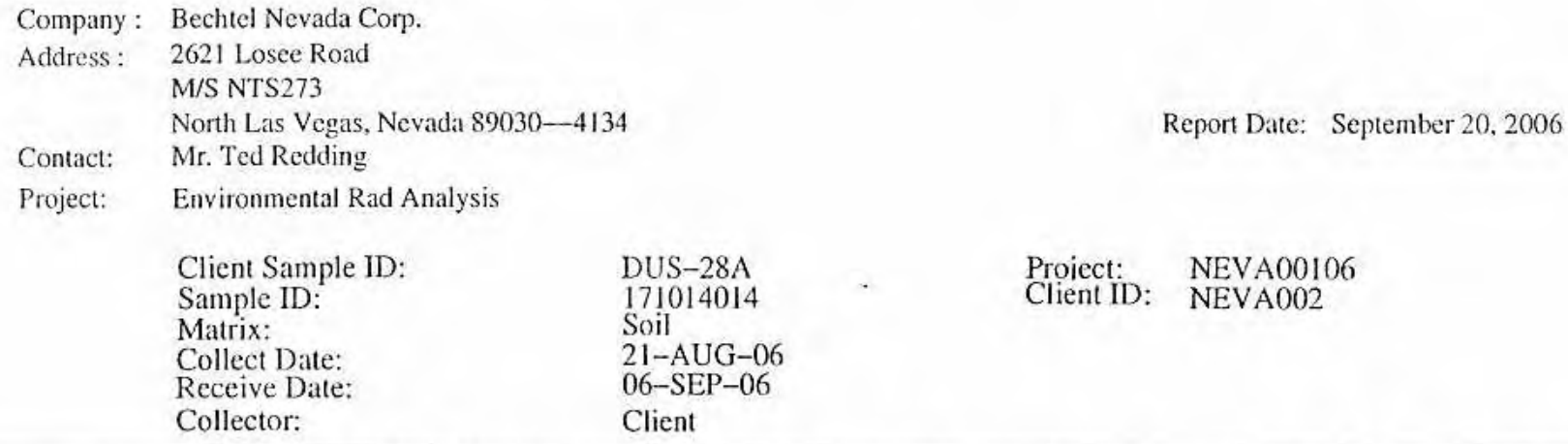

\begin{tabular}{|c|c|c|c|c|c|c|c|c|c|c|}
\hline Parameter & Qualifier & Result & Uncertainty & DL & TPU & RL & Units & $\mathrm{DF}$ & Analyst Date & Time Batch Mt \\
\hline \multicolumn{11}{|c|}{ Rad Alpha Spec Analysis } \\
\hline \multicolumn{11}{|c|}{ Alphaspec U. Solid $2 p C i / g$} \\
\hline Uranium-233/234 & & 1.05 & $+/-0.792$ & 0.998 & $+/-0.804$ & 2.00 & $\mathrm{pCi} / \mathrm{g}$ & & RDD $\quad 09 / 18 /($ & 61350566389 \\
\hline Uranium-235/236 & $\mathrm{U}$ & 0.392 & $+1-0.478$ & 0.729 & $+1-0.481$ & 2.00 & $\mathrm{pCi} / \mathrm{g}$ & & & \\
\hline Uranium-238 & $\mathrm{U}$ & 0.809 & $+1-0.672$ & 0.835 & $+1-0.680$ & 2.00 & $\mathrm{pCi} / \mathrm{g}$ & & & \\
\hline
\end{tabular}

The following Prep Methods were performed

\begin{tabular}{|c|c|c|c|c|c|}
\hline Method & Description & Analyst & Date & Time & nrep Bateh \\
\hline Dry Soil Prep & Dry Soil Prep GL-RAD-A-021 & LXM2 & $09 / 07 / 06$ & 1002 & 565877 \\
\hline \multicolumn{6}{|c|}{ The following Analytical Methods were performed } \\
\hline Method & Description & & & & \\
\hline
\end{tabular}

\begin{tabular}{llcc} 
Surrogate/Tracer recovery & Test & Recovery\% & Acceptable Limits \\
\hline Uranium-232 & Alphaspec U, Solid 2pCi/g & 92 & $(25 \%-125 \%)$
\end{tabular}

Notes:

The Qualifiers in this report are defined as follows :

* A quality control analyte recovery is outside of specified acceptance criteria

$<$ Result is less than value reported

$>$ Result is greater than value reported

A The TIC is a suspected aldol-condensation product

B Target analyte was detected in the associated blank

$\mathrm{BD}$ Results are either below the MDC or tracer recovery is low

C Analyte has been confirmed by GC/MS analysis

D Results are reported from a diluted aliquot of the sample

$\mathrm{H}$ Analytical holding time was exceeded

$\mathrm{J}$ Value is estimated

N/A Spike recovery limits do not apply. Sample concentration exceeds spike concentration by $4 \mathrm{X}$ or more

$\mathrm{R}$ Sample results are rejected

$\mathrm{U}$ Analyte was analyzed for, but not detected above the MDL, MDA, or LOD.

UI Gamma Spectroscopy-Uncertain identification

X Consult Case Narrative, Data Summary package, or Project Manager concerning this qualifier 


\section{GENERAL ENGINEERING LABORATORIES, LLC \\ 2040 Savage Road Charleston SC 29407 - (843) 55C-817i - www.gel.com}

\section{Certificate of Analysis}

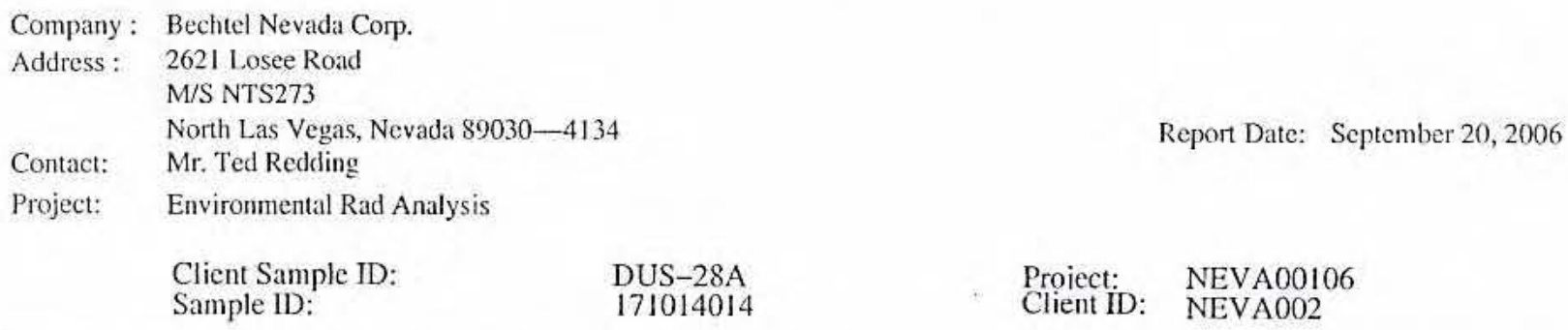

Parameter Qualifier Result Uncertainty DL TPU RL Units DF Analyst Date Time Batch Mt

Y QC Samples were not spiked with this compound

$\wedge \quad$ RPD of sample and duplicate evaluated using $+/-$ RL. Concentrations are $<5 X$ the RL

$\mathrm{h}$ Preparation or preservation holding time was exceeded

The above sample is reported on a dry weight basis. 


\section{GENERAL ENGINEERING LABORATORIES, LLC \\ 2040 Savage Road Charleston SC 29407 - (843) 556-817i - www.gel.com}

\section{Certificate of Analysis}

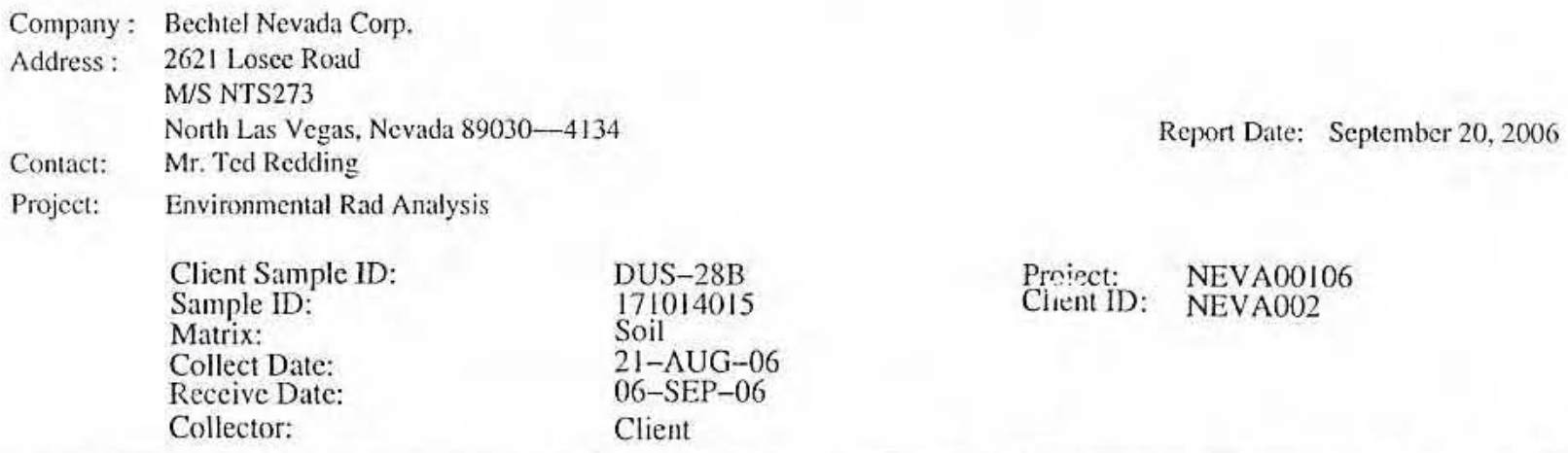

\begin{tabular}{|c|c|c|c|c|c|c|c|c|c|c|c|c|}
\hline Parameter & Qualifier & Result & Uncertainty & DL. & TPU & RL & Units & DF & Analyst & Date & Time Batch M & Ite \\
\hline \multicolumn{13}{|c|}{ Rad Alpha Spec Analysis } \\
\hline \multicolumn{13}{|c|}{ Alphaspec $U$, Solid $2 p C i / g$} \\
\hline Uranium-233/234 & & 3.75 & $+1-1.41$ & 0.992 & $+/-1.49$ & 2.00 & $\mathrm{pCi} / \mathrm{g}$ & & RDD & $09 / 18 / 06$ & 61350566389 & 1 \\
\hline Uranium-235/236 & $\mathrm{U}$ & 0.354 & $+1-0.440$ & 0.616 & $+1-0.443$ & 2.00 & $\mathrm{pCi} / \mathrm{g}$ & & & & & \\
\hline Uranium-238 & & 3.11 & $+1-1.26$ & 0.782 & $+1-1.33$ & 2.00 & $\mathrm{pCi} / \mathrm{g}$ & & & & & \\
\hline
\end{tabular}

The following Prep Methods were performed

\begin{tabular}{|c|c|c|c|c|c|}
\hline Method & Description & Analyst & Date & Time & Prep Batch \\
\hline Dry Soil Prep & Dry Soil Prep GL-RAD-A-021 & LXM2 & $09 / 07 / 06$ & 1002 & 565877 \\
\hline \multicolumn{6}{|c|}{ The following Analytical Methods were performed } \\
\hline \multicolumn{6}{|c|}{ DOE EML HASI } \\
\hline
\end{tabular}

Notes:

The Qualifiers in this report are defined as follows :

* A quality control analyte recovery is outside of specified acceptance criteria

$<$ Result is less than value reported

$>$ Result is greater than value reported

A The TIC is a suspected aldol-condensation product

B Target analyte was detected in the associated blank

BD Results are either below the MDC or tracer recovery is low

C Analyte has been confirmed by GC/MS analysis

D Results are reported from a diluted aliquot of the sample

$\mathrm{H}$ Analytical holding time was exceeded

$\mathrm{J}$ Value is estimated

N/A Spike recovery limits do not apply. Sample concentration exceeds spike concentration by $4 \mathrm{X}$ or more

$\mathrm{R}$ Sample results are rejected

U Analyte was analyzed for, but not detected above the MDL, MDA, or LOD.

Ui Gamma Spectroscopy - Uncertain identification

X Consult Case Narrative, Data Summary package, or Project Manager concerning this qualifier 


\section{GENERAL ENGINEERING LABORATORIES, LLC \\ 2040 Savage Road Charleston SC 29407 - (843) 556-8171 - www.yel.com}

\section{Certificate of Analysis}

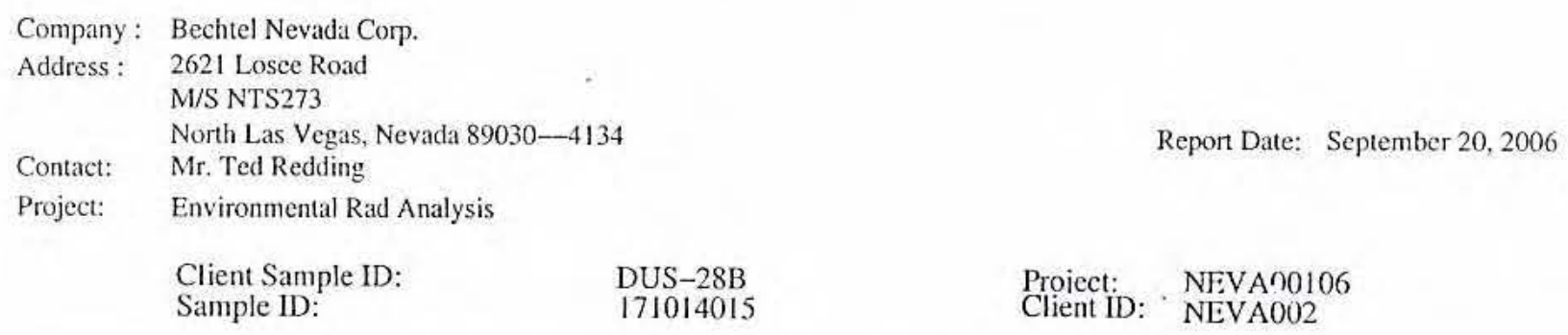

\begin{tabular}{lllllll}
\hline Parameter Qualifier Result Uncertainty & DL & TPU & RL & Units DF Analyst Date Time Batch Mt
\end{tabular}

Y QC Samples were not spiked with this compound

$\wedge$ RPD of sample and duplicate evaluated using $+/-$ RL. Concentrations are $<5 X$ the RL

$\mathrm{h}$ Preparation or preservation holding time was exceeded

The above sample is reported on a dry weight basis. 


\section{GENERAL ENGINEERING LABORATORIES, LLC \\ 2040 Savage Road Charleston SC 29407 - (843) 556-8171 - www.gel.com}

\section{Certificate of Analysis}

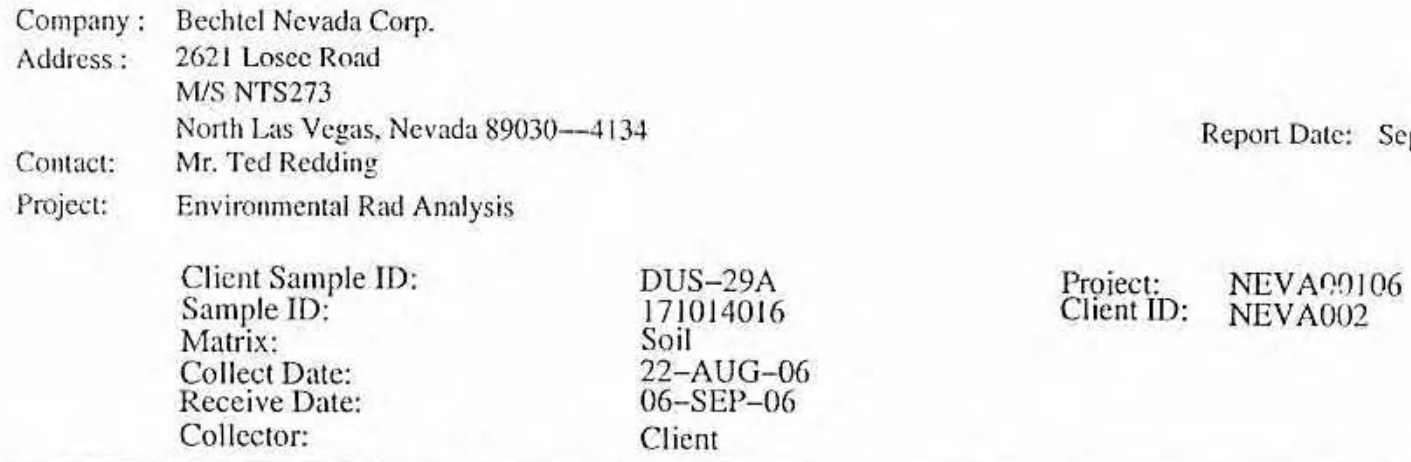

\begin{tabular}{|c|c|c|c|c|c|c|c|c|c|c|c|}
\hline Parameter & Qualifier & Result & Uncertainty & DL & TPU & RL & Units & DF & Analyst & Date & Time Batch Mt \\
\hline \multicolumn{12}{|c|}{ Rad Alpha Spec Analysis } \\
\hline \multicolumn{12}{|c|}{ Alphaspec U, Solid $2 p \mathrm{Ci} / g$} \\
\hline Uranium $-233 / 234$ & $\mathrm{U}$ & 0.516 & $+1-0.592$ & 0.925 & $+1-0.596$ & 2.00 & $\mathrm{pCi} / \mathrm{g}$ & & RDD & $09 / 18 / 0$ & 61350566389 \\
\hline Uranium-235/236 & $\mathrm{U}$ & 0.0671 & $+1-0.359$ & 0.886 & $+1-0.360$ & 2.00 & $\mathrm{pCi} / \mathrm{g}$ & & & & \\
\hline Uranium-238 & U & 0.664 & $+1-0.634$ & 0.883 & $+1-0.640$ & 2.00 & $\mathrm{pCi} / \mathrm{g}$ & & & & \\
\hline
\end{tabular}

The following Prep Methods were performed

\begin{tabular}{llllll}
\hline Method & Description & Analyst & Date & Time & Prep Batch \\
\hline Dry Soil Prep & Dry Soil Prep GL-RAD-A-021 & LXM2 & $09 / 07 / 06$ & 1002 & 565877
\end{tabular}

The following Analyticai Methods were performed

\begin{tabular}{llcc}
\hline Method Description & & \\
\hline DOE EML HASL-300, U-02-RC Modified & & \\
Surrogate/Tracer recovery & Test & Recovery \% & Acceptable Limits \\
\hline Uranium-2.32 & Alphaspec U, Solid 2pCi/g & 90 & $(25 \%-125 \%)$
\end{tabular}

Notes:

The Qualifiers in this report are defined as follows:

* A quality control analyte recovery is outside of specified acceptance criteria

$<$ Result is less than value reported

$>$ Result is greater than value reported

A The TIC is a suspected aldol-condensation product

B Target analyte was detected in the associated blank

$\mathrm{BD}$ Results are either below the MDC or tracer recovery is low

C. Analyte has been confirmed by GC/MS analysis

D Results are reported from a diluted aliquot of the sample

$\mathrm{H}$ Analytical holding time was exceeded

$\mathrm{J}$ Value is estimated

N/A Spike recovery limits do not apply. Sample concentration exceeds spike concentration by $4 X$ or more

$\mathrm{R}$ Sample results are rejected

U Analyte was analyzed for, but not detected above the MDL, MDA, or LOD.

UI Gamma Spectroscopy--Uncertain identification

X Consult Case Narrative, Data Summary package, or Project Manager concerning this qualifier 


\section{GENERAL ENGINEERING LABORATORIES, LLC \\ 2040 Savage Road Charleston SC 29407 - (843) 556-8171 - www.gel.com}

\section{Certificate of Analysis}

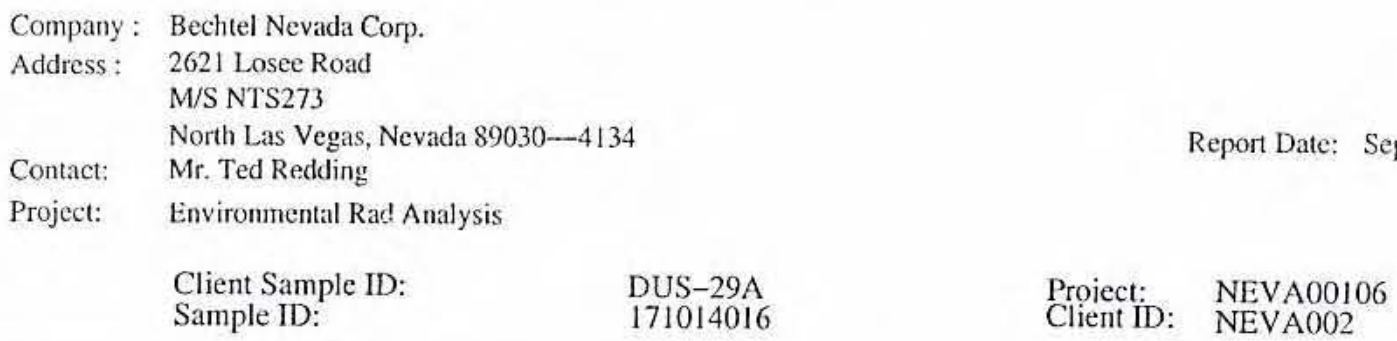

Y QC Samples were not spiked with this compound

$\wedge$ RPD of sample and duplicate evaluated using $+1-$ RL. Concentrations are $<5 X$ the RL

h Preparation or preservation holding time was exceeded

The above sample is reported on a dry weight basis. 


\section{GENERAL ENGINEERING LABORATORIES, LLC \\ 2040 Savage Road Charleston SC 29407 - (843) 556-8171 - www.gel.com}

\section{Certificate of Analysis}

\author{
Company: Bechtel Nevada Corp. \\ Address : 2621 Losee Road \\ M/S NTS273 \\ North Las Vegas, Nevada $89030-4134$ \\ Contact: Mr. Ted Redding \\ Project: Environmental Rad Analysis
}

Report Date: September 20,2006

DUS-29B
171014017
Soil
22-AUG-06
$06-S E P-06$
Client

Proiect: NEVA00106

Client ID: NEVA002

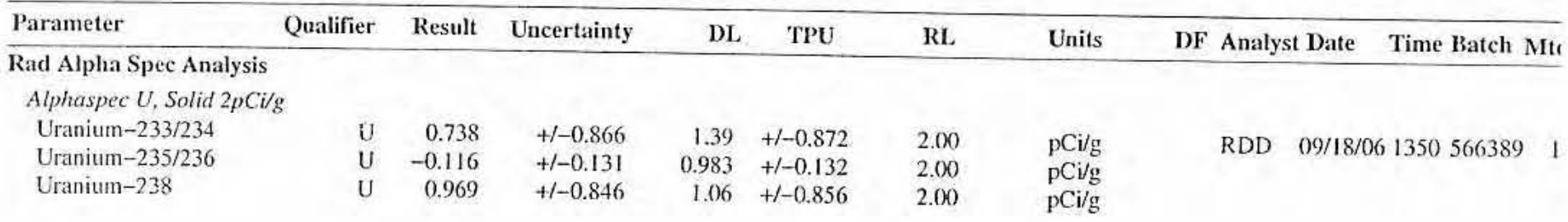

The following Prep Methodis were performed

\begin{tabular}{lllllll}
\hline Method & Deseription & Analyst & Date & Time & Prep Batch \\
\hline Dry Soil Prep & Dry Soil Prep GL-RAD-A-02i & LXM2 & $09 / 07 / 06$ & 1002 & 565877
\end{tabular}

The following Analytical Methods were performed

$\begin{array}{ll}\text { Method } & \text { Description } \\ 1 & \text { DOE EML HASL-300, U-02-RC Modified }\end{array}$

\begin{tabular}{llcc} 
Surrogate/Tracer recovery & Test & Recovery \% & Acceptable Limits \\
\hline Uranium-232 & Alphaspec U, Solid 2pCi/g & 79 & $(25 \%-125 \%)$
\end{tabular}

Notes:

The Qualifiers in this report are defined as follows :

* A quality control analyte recovery is outside of specified acceptance criteria

$<$ Result is less than value reported

$>$ Result is greater than value reported

A The TIC is a suspected aldol-condensation product

B Target analyte was detected in the associated blank

$\mathrm{BD}$ Results are either below the MDC or tracer recovery is low

C Analyte has been confirmed by GC/MS analysis

D Results are reported from a diluted aliquot of the sample

$\mathrm{H}$ Analytical holding time was exceeded

$\mathrm{J}$ Value is estimated

N/A Spike recovery limits do not apply. Sample concentration exceeds spike concentration by $4 X$ or more

$\mathrm{R}$ Sample results are rejected

U Analyte was analyzed for, but not detected above the MDL, MDA, or LOD.

UI Gamma Spectroscopy-Uncertain identification

X Consult Case Narrative, Data Summary package, or Project Manager concerning this qualifier 


\section{GENERAL ENGINEERING LABORATORIES, LLC \\ 2040 Savage Road Charleston SC 29407 - (843) 556-8171 - www.gel.com}

\section{Certificate of Analysis}

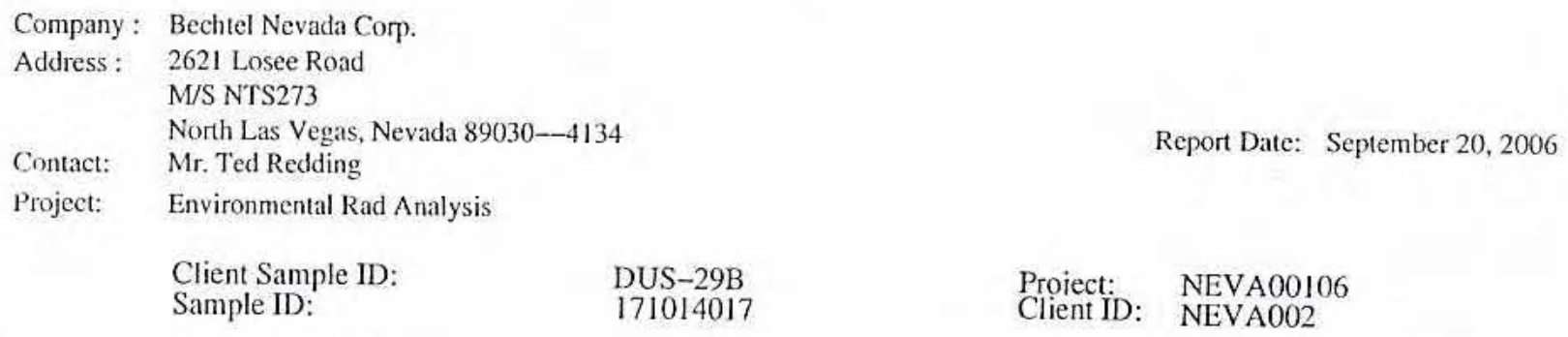

DL TPU RL

Y QC Samples were not spiked with this compound

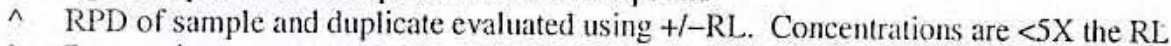

$\mathrm{h}$ Preparation or preservation holding time was exceeded

The above sample is reported on a dry weight basis. 


\section{GENERAL ENGINEERING LABORATORIES, LLC \\ 2040 Savage Road Charleston SC 29407 - (843) 556-8171 - www.gel.com}

\section{Certificate of Analysis}

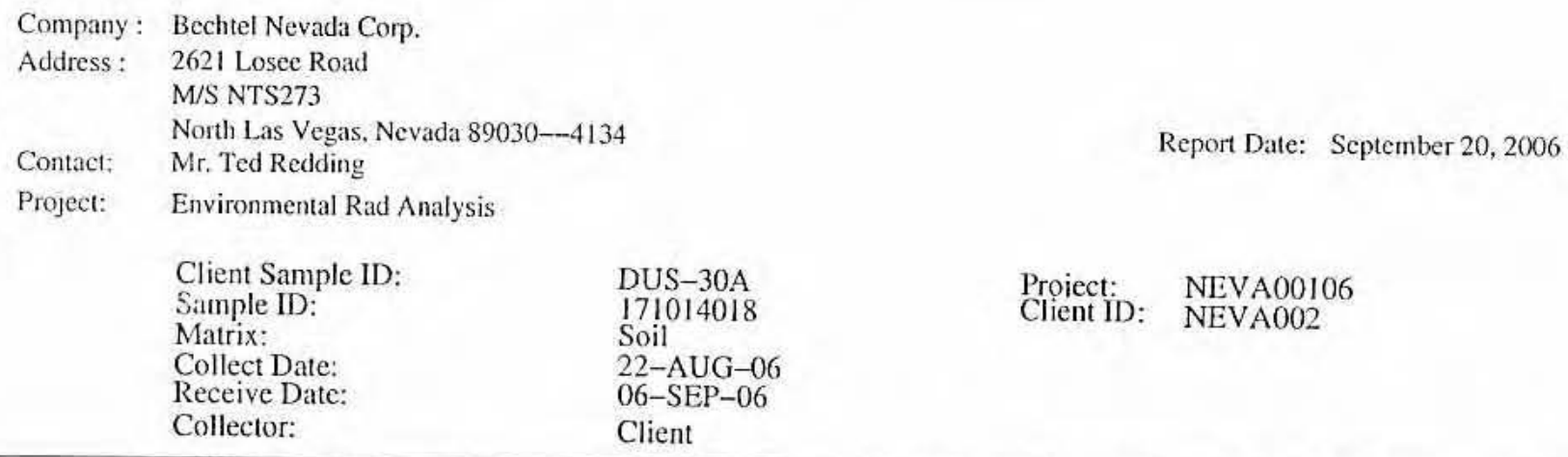

\begin{tabular}{|c|c|c|c|c|c|c|c|c|c|c|}
\hline Parameter & Qualifier & Result & Uncertainty & DL & TPU & $\mathrm{RL}$ & Units & DF & Analyst Date & Time Batch M \\
\hline \multicolumn{11}{|c|}{ Rad Alpha Spec Analysis } \\
\hline \multicolumn{11}{|c|}{$\begin{array}{l}\text { Alphaspec U, Solid } 2 p C i / g \\
\text { Uranium-233/234 }\end{array}$} \\
\hline Uranium-233/234 & & 1.22 & $+1-0.883$ & 1.10 & $+1-0.897$ & 2.00 & $\mathrm{pCi} / \mathrm{g}$ & & RDD $\quad 09 / 18 / 0$ & 61350566389 \\
\hline $\begin{array}{l}\text { Uranium-235/236 } \\
\text { Uranium-238 }\end{array}$ & $U$ & $\begin{array}{r}-0.209 \\
0.706\end{array}$ & $+1-0.319$ & 1.14 & $+1-0.320$ & 2.00 & $\mathrm{pCi} / \mathrm{g}$ & & & \\
\hline Uranium-238 & $\mathrm{U}$ & 0.706 & $+1-0.675$ & 0.940 & $+1-0.681$ & 2.00 & $\mathrm{pCi} / \mathrm{g}$ & & & \\
\hline
\end{tabular}

The following Prep Methods were performed

\begin{tabular}{llllll}
\hline Method & Description & Analyst & Date & Time & Prep Batch \\
\hline Dry Soil Prep & Dry Soil Prep GL-RAD-A-021 & LXM2 & $09 / 07 / 06$ & 1002 & 565877
\end{tabular}

The following Analytical Methods were perlormed

\begin{tabular}{ll} 
Method & Description \\
\hline DOE EML HASL-300, U-02-RC Modified
\end{tabular}

\begin{tabular}{llcc} 
Surrogate/Tracer recovery & Test & Recovery\% & Acceptable Limits \\
\hline Uranium-232 & Alphaspec U, Solid 2pCi/g & 93 & $(25 \%-125 \%)$
\end{tabular}

Notes:

The Qualifiers in this report are defined as follows :

* A quality control analyte recovery is outside of specified acceptance criteria

$<$ Result is less than value reported

$>$ Result is greater than value reported

A The TIC is a suspected aldol-condensation product

B Target analyte was detected in the associated blank

$\mathrm{BD}$ Results are either below the MDC or tracer recovery is low

C Analyte has been confirmed by GC/MS analysis

D Results are reported from a diluted aliquot of the sample

$\mathrm{H}$ Analytical holding time was exceeded

$\mathrm{J}$ Value is estimated

N/A Spike recovery limits do not apply. Sample concentration exceeds spike concentration by $4 \mathrm{X}$ or more
$\mathrm{R}$ Sample results are rejected

U Analyte was analyzed for, but not detected above the MDL, MDA, or LOD.

UI Gamma Spectroscopy-Uncertain identification

X Consult Case Narrative, Data Summary package, or Project Manager concerning this qualifier 


\section{GENERAL ENGINEERING LABORATORIES, LLC \\ 2040 Savage Road Charleston SC 29407 - (843) 556-8171 - www.gel.com}

\section{Certificate of Analysis}

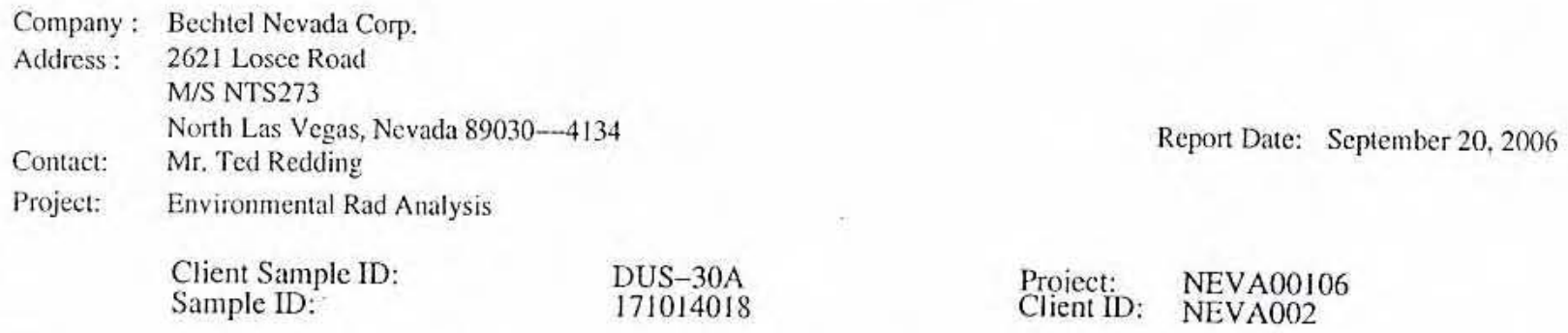

DL TPU

RI

Units

DF Analyst Date

Time Batch Mt,

Y QC Samples were not spiked with this compound

$\hat{R}$ RPD of sample and duplicate evaluated using $+/-\mathrm{KL}$. Concentrations are $<5 \mathrm{X}$ the RL

h Preparation or preservation holding time was exceeded

The above sample is reported on a dry weight basis. 


\section{GENERAL ENGINEERING LABORATORIES, LLC \\ 2040 Savage Road Charleston SC 29407 - (843) 556-8171 - www.gel.com}

\section{Certificate of Analysis}

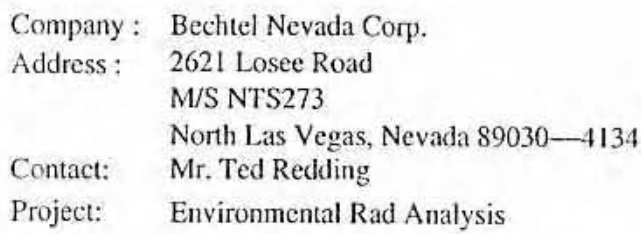

Report Date: September 20, 2006

DUS-30B
171014019
Soil
22-AUG-06
$06-$ SEP-06
Client

Proiect: NEVA00106

Client ID: NEVA002

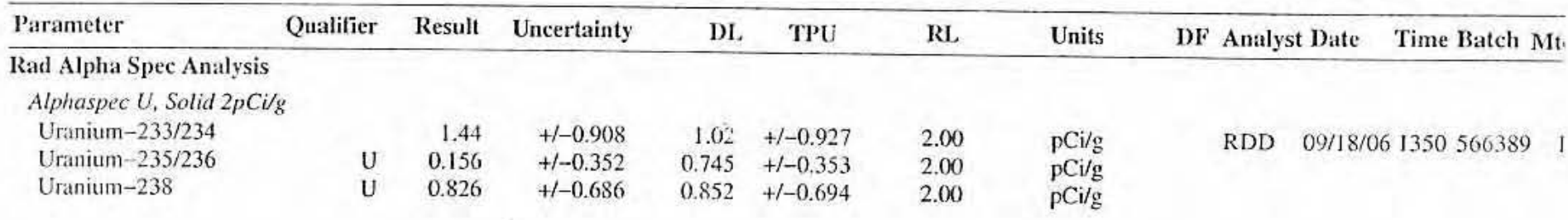

The following Prep Methods were performed

\begin{tabular}{llllll}
\hline Method & Description & Analyst & Date & Time & Prep Batch \\
\hline Dry Soil Prep & Dry Soil Prep GL-RAD-A-021 & LXM2 & $09 / 07 / 06$ & 1002 & 565877
\end{tabular}

The following Analytical Methods were performed

Method Description

I DOE EML HASL-300, U-02-RC Modified

\begin{tabular}{llcc} 
Surrogate/Tracer recovery & Test & Recovery\% & Acceptable Limits \\
\hline Uranium-232 & Alphaspec U, Solid 2pCi/g & 91 & $(25 \%-125 \%)$
\end{tabular}

Notes:

The Qualifiers in this report are defined as follows :

* A quality control analyte recovery is outside of specified acceptance criteria

$<$ Result is less than value reported

$>$ Result is greater than value reported

A The TIC is a suspected aldol-condensation product

B Target analyte was detected in the associated blank

$\mathrm{BD}$ Results are either below the MDC or tracer recovery is low

C Analyte has been confirmed by GC/MS analysis

D Results are reported from a diluted aliquot of the sample

$\mathrm{H}$ Analytical holding time was exceeded

$\mathrm{J}$ Value is estimated

N/A Spike recovery limits do not apply. Sample concentration exceeds spike concentration by $4 X$ or more

$R$ Sample results are rejected

U Analyte was analyzed for, but not detected above the MDL, MDA, or LOD.

UI Gamma Spectroscopy--Uncertain identification

X Consult Case Narrative, Data Summary package, or Project Manager concerning this qualifier 


\section{GENERAL ENGINEERING LABORATORIES, LLC \\ 2040 Savage Hoad Charleston SC 29407 - (843) 556-8171 - www.gel.com}

\section{Certificate of Analysis}

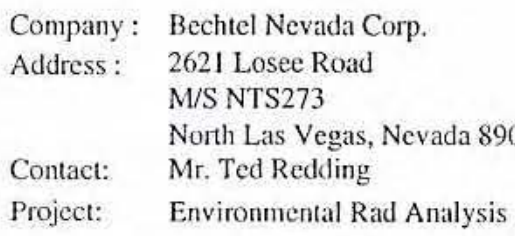

\begin{tabular}{lllllllll}
\hline Parameter & Qualifier & Result Uncertainty & DL & TPU & RL & Units & DF Analyst Date Time Batch Mt
\end{tabular}

Y QC Samples were not spiked with this compound

$\wedge$ RPD of sample and duplicate evaluated using $+/-$ RL. Concentrations are $<5 X$ the $R L$

h Preparation or preservation holding time was exceeded

The above sample is reported on a dry weight basis. 


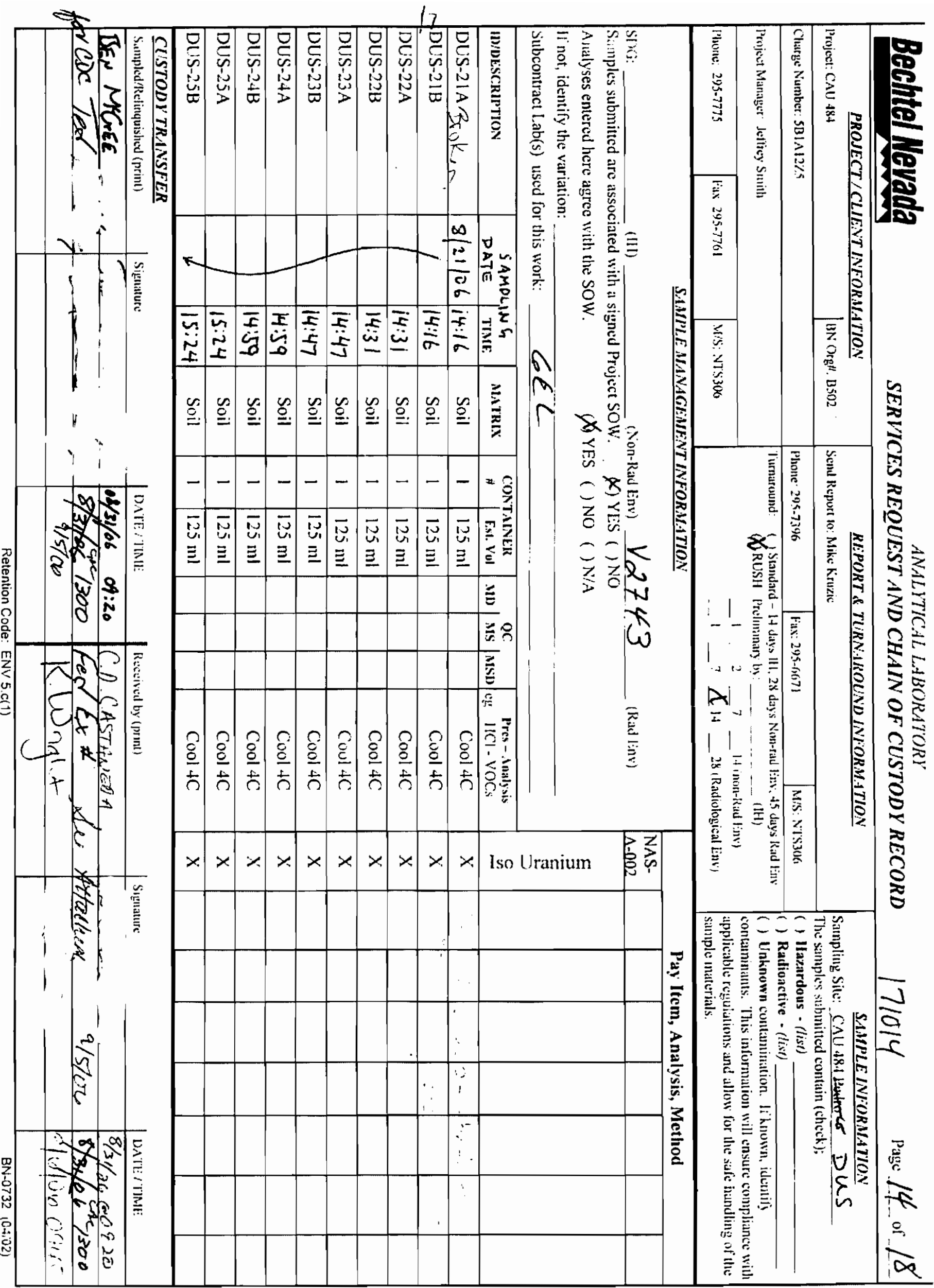




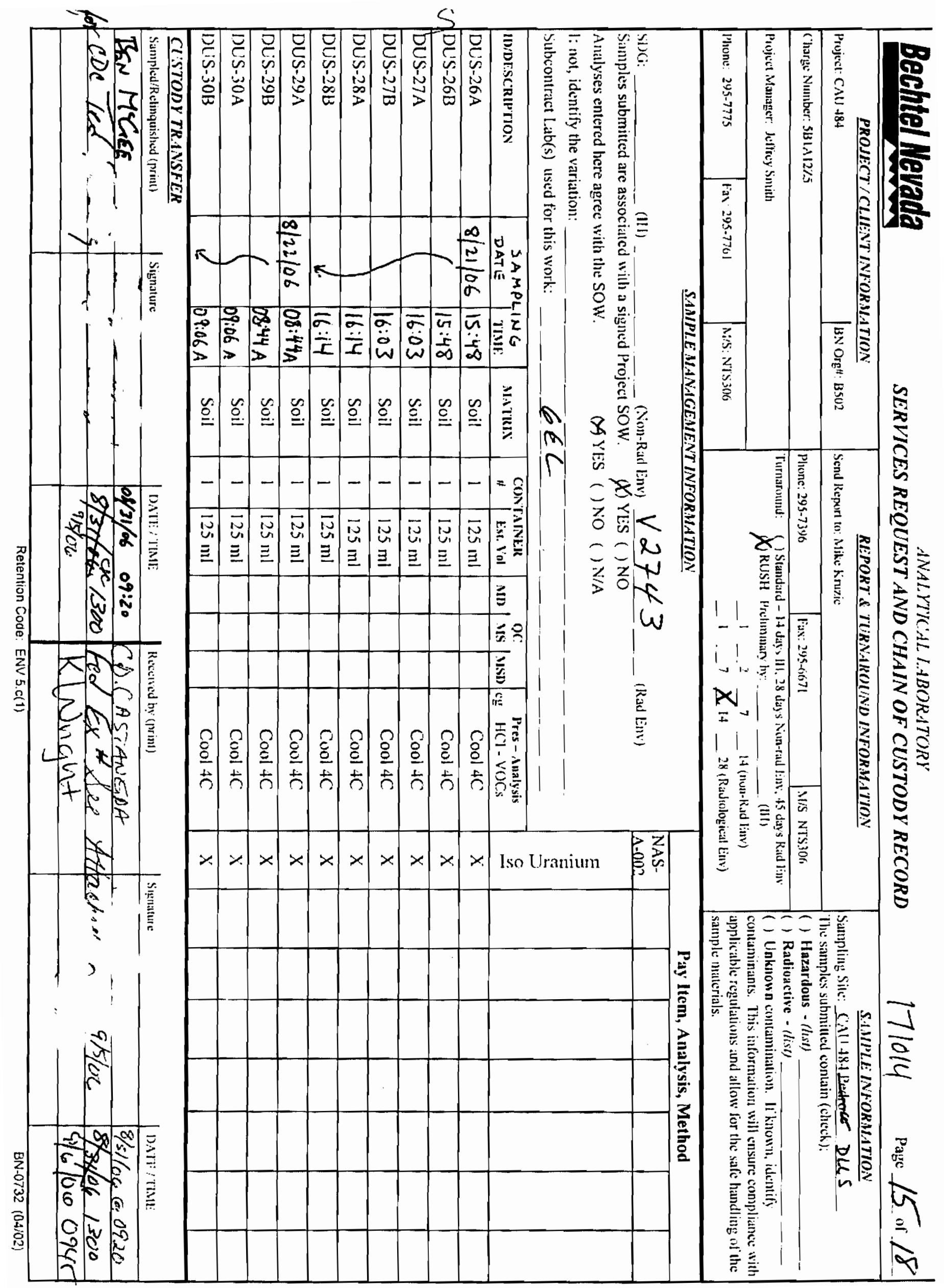




\section{GENERAL ENGINEERING LABORATORIES, LLC \\ 2040 Savage Road Charleston SC 29407 - (843) 556-8171 - www.gel.com}

\section{Certificate of Analysis}

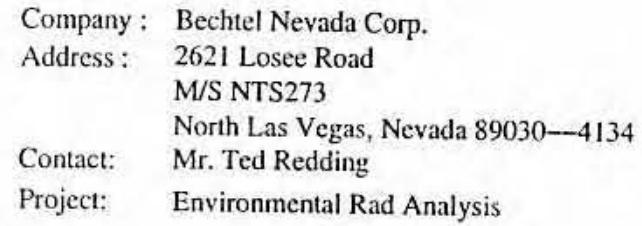

\begin{tabular}{|c|c|c|c|c|c|c|c|c|c|}
\hline \multirow[b]{2}{*}{ Parameter } & \multicolumn{2}{|c|}{$\begin{array}{l}\text { Client Sample ID: } \\
\text { Sample ID: } \\
\text { Matrix: } \\
\text { Collect Date: } \\
\text { Receive Date: } \\
\text { Collector: }\end{array}$} & \multicolumn{3}{|c|}{$\begin{array}{l}\text { DUS-31A } \\
171003001 \\
\text { Soil } \\
\text { 22-AUG-06 } \\
\text { 06-SEP-06 } \\
\text { Client }\end{array}$} & \multirow[b]{2}{*}{ RI. } & \multirow{2}{*}{$\begin{array}{l}\text { Proiect: } \\
\text { Client ID: } \\
\text { Units }\end{array}$} & \multicolumn{2}{|l|}{$\begin{array}{l}\text { NEVA00106 } \\
\text { NEVA002 }\end{array}$} \\
\hline & Qualifier & Result & Uncertainty & DL & TPU & & & DF Analyst Date & Time Batch Mtd \\
\hline $\begin{array}{l}\text { Rad Alpha Spec Anal } \\
\text { Alphaspec U, Solid } 2\end{array}$ & & & \multicolumn{7}{|c|}{ Alphaspec $U$, Solid $2 p C i / g$} \\
\hline $\begin{array}{l}\text { Uranium-233/234 } \\
\text { Uranium-235/236 } \\
\text { Uranium-238 }\end{array}$ & & $\begin{array}{r}3.84 \\
0.566 \\
2.11\end{array}$ & $\begin{array}{r}+/-1.27 \\
+/-0.496 \\
+/-0.860\end{array}$ & $\begin{array}{r}1.13 \\
0.339 \\
0.275\end{array}$ & $\begin{array}{r}+/-1.34 \\
+1-0.500 \\
+1-0.892\end{array}$ & $\begin{array}{l}2.00 \\
2.00 \\
2.00\end{array}$ & $\begin{array}{l}\mathrm{pCi} / \mathrm{g} \\
\mathrm{pCi} / \mathrm{g} \\
\mathrm{pCi} / \mathrm{g}\end{array}$ & $09 / 21 / 0$ & $61342566385 \quad$ I \\
\hline
\end{tabular}

The following Prep Methods were performed

\begin{tabular}{llllll}
\hline Method & Description & Analyst & Date & Time & Prep Batch \\
\hline Dry Soil Prep & Dry Soil Prep GL-RAD-A-021 & JMBI & $09 / 07 / 06$ & 0856 & 565864
\end{tabular}

The following Analytical Methods were performed

\begin{tabular}{ll}
\hline Method & Description \\
\hline 1 & DOE EML HASL-300, U-02-RC Modified \\
2 & DOE EML HASL-300, U-02-RC Modified
\end{tabular}

\begin{tabular}{llcc} 
Surrogate/Tracer recovery & Test & Recovery\% & Acceptable Limits \\
\hline Uranium-232 & Alphaspec U, Solid 2pCi/g & 80 & $(25 \%-125 \%)$
\end{tabular}

Notes:

The Qualifiers in this report are defined as follows :

* A quality control analyte recovery is outside of specified acceptance criteria

$<$ Result is less than value reported

$>$ Result is greater than value reported

A The TIC is a suspected aldol-condensation product

B Target analyte was detected in the associated blank

BD Results are either below the MDC or tracer recovery is low

C Analyte has been confirmed by GC/MS analysis

D Results are reported from a diluted aliquot of the sample

$\mathrm{H}$ Analytical holding time was exceeded

$\mathrm{J}$ Value is estimated

N/A Spike recovery limits do not apply. Sample concentration exceeds spike concentration by $4 X$ or more
$\mathrm{R}$ Sample results are rejected

$\mathrm{U}$ Analyte was analyzed for, but not detected above the MDL, MDA, or LOD. 


\section{GENERAL ENGINEERING LABORATORIES, LLC \\ 2040 Savage Road Charleston SC 29407 - (843) 556-8171 - www.gel.com}

\section{Certificate of Analysis}

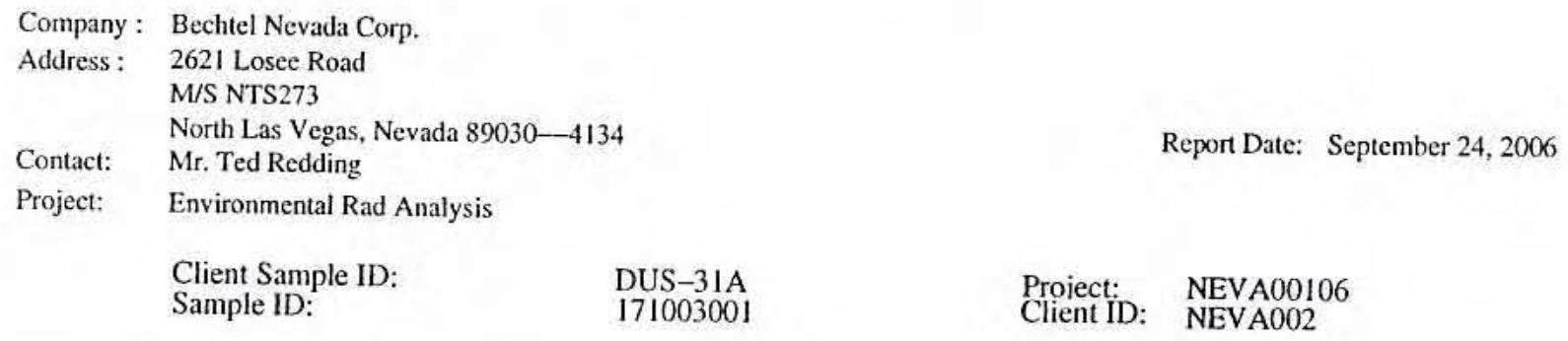

UI Gamma Spectroscopy-Uncertain identification

$\mathrm{X}$ Consult Case Narrative, Data Summary package, or Project Manager concerning this qualifier

Y QC Samples were not spiked with this compound

$\hat{h}$ RPD of sample and duplicate evaluated using $+/-\mathrm{RL}$. Concentrations are $<5 \mathrm{X}$ the $\mathrm{RL}$

$\mathrm{h}$ Preparation or preservation holding time was exceeded

The above sample is reported on a dry weight basis. 


\section{GENERAL ENGINEERING LABORATORIES, LLC \\ 2040 Savage Road Charleston SC 29407 - (843) 556-8171 - www.gel.com}

\section{Certificate of Analysis}

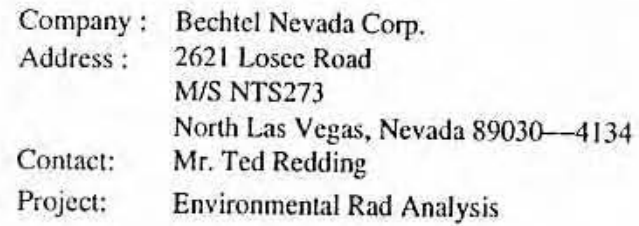

Client Sample ID: Sample ID:

Matrix:

Collect Date:

Receive Date:

Collector:
Report Date: September 24, 2006

DUS-31B
171003002
Soil
22-AUG-06
06-SEP-06
Client

Proiect:

Client ID: NEVA002

\begin{tabular}{|c|c|c|c|c|c|c|c|c|c|c|c|}
\hline Parameter & Qualifier & Result & Uncertainty & DL & TPU & RI & Units & DF Analys & st Date & Time Batch M & Mtd \\
\hline \multicolumn{12}{|c|}{ Rad Alpha Spec Analysis } \\
\hline $\begin{array}{l}\text { Uranium-233/234 } \\
\text { Uranium-235/236 } \\
\text { Uranium-238 }\end{array}$ & & $\begin{array}{l}5.51 \\
1.01 \\
1.84\end{array}$ & $\begin{array}{r}+1-1.52 \\
+/-0.782 \\
+/-0.939\end{array}$ & $\begin{array}{l}0.978 \\
0.966 \\
0.978\end{array}$ & $\begin{array}{r}+/-1.64 \\
+/-0.790 \\
+/-0.961\end{array}$ & $\begin{array}{l}2.00 \\
2.00 \\
2.00\end{array}$ & $\begin{array}{l}\mathrm{pCi} / \mathrm{g} \\
\mathrm{pCi} / \mathrm{g} \\
\mathrm{pCi} / \mathrm{g}\end{array}$ & $\mathrm{TCl}$ & $09 / 21 / 06$ & 61342566385 & 1 \\
\hline
\end{tabular}

The following Prep Methods were performed

\begin{tabular}{llllll}
\hline Method & Description & Analyst & Date & Time & Prep Batch \\
\hline Dry Soil Prep & Dry Soil Prep GL-RAD-A-021 & JMB1 & $09 / 07 / 06$ & 0856 & 565864
\end{tabular}

The following Analytical Methods were performed

\begin{tabular}{ll}
\hline Method & Description \\
\hline 1 & DOE EML HASL-300, U-02-RC Modified \\
2 & DOE EML HASL-300, U-02-RC Modified
\end{tabular}

\begin{tabular}{llcc} 
Surrogate/Tracer recovery & Test & Recovery $\%$ & Acceptable Limits \\
\hline Uranium-232 & Alphaspec U, Solid 2pCi/g & 82 & $(25 \%-125 \%)$
\end{tabular}

Notes:

The Qualifiers in this report are defined as follows :

* A quality control analyte recovery is outside of specified acceptance criteria

$<$ Result is less than value reported

$>$ Result is greater than value reported

A The TIC is a suspected aldol-condensation product

B Target analyte was detected in the associated blank

$\mathrm{BD}$ Results are either below the MDC or tracer recovery is low

C Analyte has been confirmed by GC/MS analysis

D Results are reported from a diluted aliquot of the sample

$\mathrm{H}$ Analytical holding time was exceeded

J Value is estimated

N/A Spike recovery limits do not apply. Sample concentration exceeds spike concentration by $4 \mathrm{X}$ or more

$\mathrm{R}$ Sample results are rejected

$\mathrm{U}$ Analyte was analyzed for, but not detected above the MDL, MDA, or LOD.

UI Gamma Spectroscopy-Uncertain identification 


\section{GENERAL ENGINEERING LABORATORIES, LLC \\ 2040 Savage Road Charleston SC 29407 - (843) 556-8171 - www.gel.com}

\section{Certificate of Analysis}

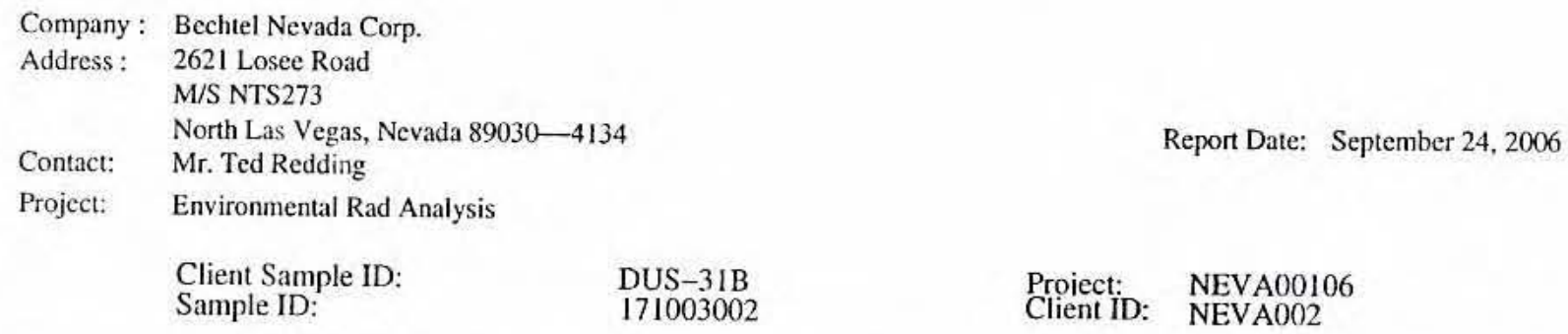

DL TPU RL

Units DF Analyst Date Time Batch Mtd

\footnotetext{
$\mathrm{X}$ Consult Case Narrative, Data Summary package, or Project Manager concerning this qualifier

Y QC Samples were not spiked with this compound

$\hat{R}$ RPD of sample and duplicate evaluated using $+/-\mathrm{RL}$. Concentrations are $<5 \mathrm{X}$ the RL

h Preparation or preservation holding time was exceeded

The above sample is reported on a dry weight basis.
} 


\section{GENERAL ENGINEERING LABORATORIES, LLC \\ 2040 Savage Road Charleston SC 29407 - (843) 556-8171 - www.gel.com}

\section{Certificate of Analysis}

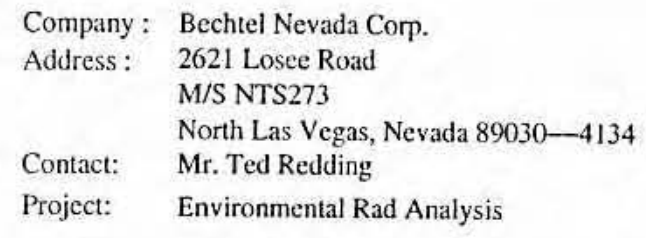

\begin{tabular}{|c|c|c|c|c|c|c|c|c|c|c|c|c|}
\hline Parameter & Qualifier & Result & Uncertainty & DI. & TPU & RL & Units & DF & Analyst & Date & Time Batch M & Mtd \\
\hline \multicolumn{13}{|c|}{ Rad Alpha Spec Analysis } \\
\hline \multicolumn{13}{|c|}{ Alphaspec U, Solid $2 \mathrm{pCi} g$} \\
\hline $\begin{array}{l}\text { Uranium-233/234 } \\
\text { Uranium-235/236 } \\
\text { Uranium-238 }\end{array}$ & $U$ & $\begin{array}{r}1.93 \\
0.435 \\
2.27\end{array}$ & $\begin{array}{r}+1-1.46 \\
+1-0.816 \\
+1-1.53\end{array}$ & $\begin{array}{l}1.80 \\
1.59 \\
1.63\end{array}$ & $\begin{array}{r}+1-1.49 \\
+/-0.819 \\
+/-1.56\end{array}$ & $\begin{array}{l}2.00 \\
2.00 \\
2.00\end{array}$ & $\begin{array}{l}\mathrm{pCi} / \mathrm{g} \\
\mathrm{pCi} / \mathrm{g} \\
\mathrm{pCi} / \mathrm{g}\end{array}$ & & $\mathrm{TCl}$ & $09 / 20 / 0$ & 60932566385 & 1 \\
\hline
\end{tabular}

The following Prep Methods were performed

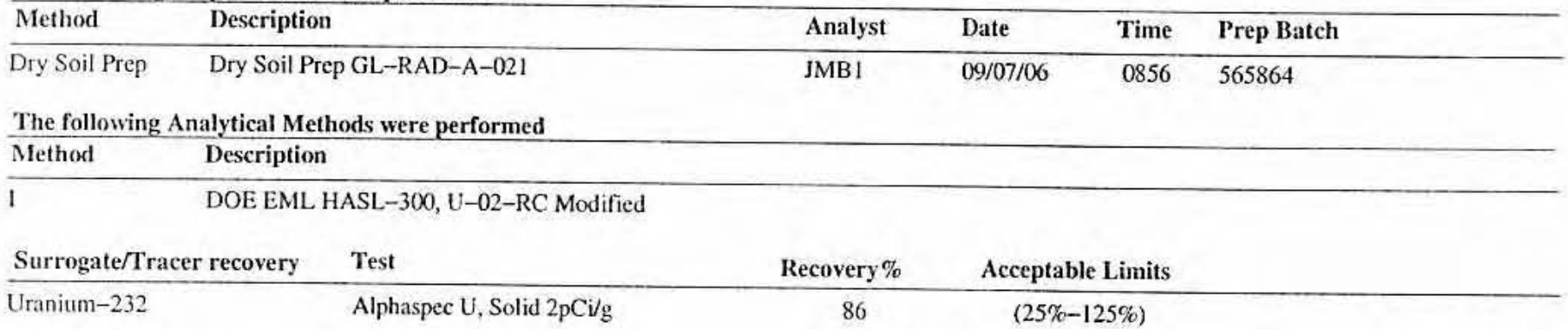

Notes:

The Qualifiers in this report are defined as follows :

* A quality control analyte recovery is outside of specified acceptance criteria

$<$ Result is less than value reported

$>$ Result is greater than value reported

A The TIC is a suspected aldol-condensation product

B Target analyte was detected in the associated blank

BD Results are either below the MDC or tracer recovery is low

C Analyte has been confirmed by GC/MS analysis

D Results are reported from a diluted aliquot of the sample

$\mathrm{H}$ Analytical holding time was exceeded

$\mathrm{J}$ Value is estimated

N/A Spike recovery limits do not apply. Sample concentration exceeds spike concentration by $4 \mathrm{X}$ or more

$\mathrm{R}$ Sample results are rejected

U. Analyte was analyzed for, but not detected above the MDL, MDA, or LOD.

UI Gamma Spectroscopy--Uncertain identification

X Consult Case Narrative, Data Summary package, or Project Manager concerning this qualifier 


\section{GENERAL ENGINEERING LABORATORIES, LLC \\ 2040 Savage Road Charleston SC 29407 - (843) 556-8171 - www.gel.com}

\section{Certificate of Analysis}

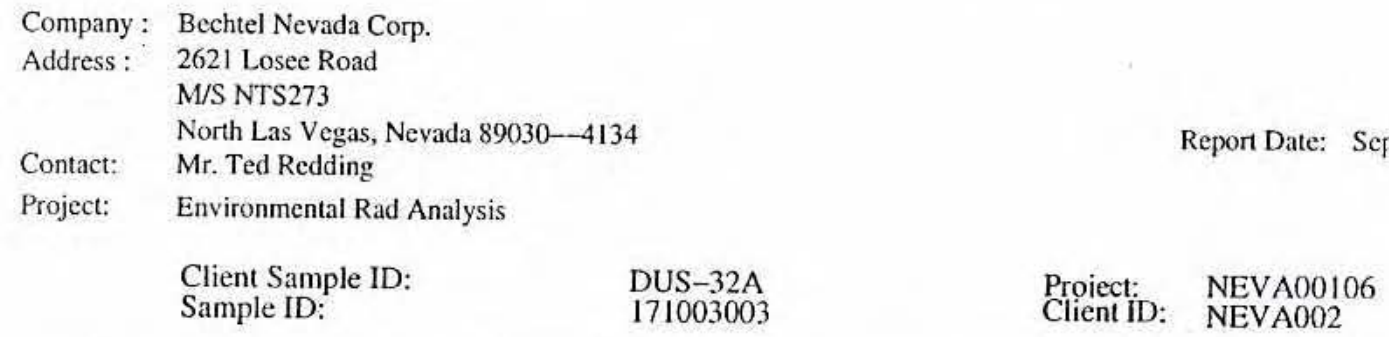

DL TPU

RL

Units

DF Analyst Date

Time Batch Mtd

Y QC Samples were not spiked with this compound

$\wedge \quad$ RPD of sample and duplicate evaluated using $+1-\mathrm{RL}$. Concentrations are $<5 X$ the RL

$\mathrm{h}$ Preparation or preservation holding time was exceeded

The above sample is reported on a dry weight basis. 


\section{GENERAL ENGINEERING LABORATORIES, LLC \\ 2040 Savage Road Charleston SC 29407 - (843) 556-8171 - www.gel.com}

\section{Certificate of Analysis}

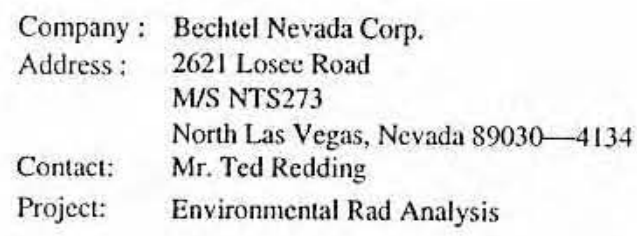

Report Date: September 24, 2006

Client Sample ID:

Sample ID:

Matrix:

Collect Date:

Receive Date:

Collector:

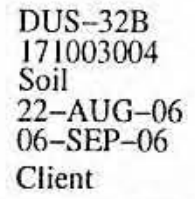

DUS-32B

171003004

Soil

22-AUG-06

06-SEP-06

Client

\begin{tabular}{|c|c|c|c|c|c|c|c|c|c|c|c|c|}
\hline Parameter & Qualifier & Result & Uncertainty & DL & TPU & RL & Units & DF & Analyst & t Date & Time Batch M & Mtd \\
\hline \multicolumn{13}{|c|}{ Rad Alpha Spec Analysis } \\
\hline \multicolumn{13}{|c|}{ Alphaspec U, Solid $2 p C i / g$} \\
\hline $\begin{array}{l}\text { Uranium-233/234 } \\
\text { Uranium-235/236 } \\
\text { Uranium-238 }\end{array}$ & $\begin{array}{l}\mathrm{U} \\
\mathrm{U}\end{array}$ & $\begin{array}{r}2.05 \\
0.487 \\
0.709\end{array}$ & $\begin{array}{l}+1-0.900 \\
+1-0.573 \\
+1-0.673\end{array}$ & $\begin{array}{r}0.970 \\
0.933 \\
1.06\end{array}$ & $\begin{array}{l}+1-0.929 \\
+1-0.575 \\
+1-0.678\end{array}$ & $\begin{array}{l}2.00 \\
2.00 \\
2.00\end{array}$ & $\begin{array}{l}\mathrm{pCi} / \mathrm{g} \\
\mathrm{pCi} / \mathrm{g} \\
\mathrm{pCi} / \mathrm{g}\end{array}$ & & $\mathrm{TCl}$ & $09 / 2 \mathrm{1} / 06$ & 1342566385 & 1 \\
\hline
\end{tabular}

The following Prep Methods were performed

\begin{tabular}{llllll}
\hline Method & Description & Analyst & Date & Time & Prep Batch \\
\hline Dry Soil Prep & Dry Soil Prep GL-RAD-A-021 & JMBI & $09 / 07 / 06$ & 0856 & 565864
\end{tabular}

The following Analytical Methods were performed

\begin{tabular}{ll} 
Method & Description \\
\hline 1 & DOE EML HASL-300, U-02-RC Modified \\
2 & DOE EML HASL-300, U-02-RC Modified
\end{tabular}

\begin{tabular}{llcc} 
Surrogate/Tracer recovery & Test & Recovery\% & Acceptable Limits \\
\hline Uranium-232 & Alphaspec U, Solid 2pCig & 82 & $(25 \%-125 \%)$
\end{tabular}

Notes:

The Qualifiers in this report are defined as follows :

* A quality control analyte recovery is outside of specified acceptance criteria

$<$ Result is less than value reported

$>$ Result is greater than value reported

A The TIC is a suspected aldol-condensation product

B Target analyte was detected in the associated blank

$\mathrm{BD}$ Results are either below the MDC or tracer recovery is low

C Analyte has been confirmed by GC/MS analysis

D Results are reported from a diluted aliquot of the sample

$\mathrm{H}$ Analytical holding time was exceeded

$\mathrm{J}$ Value is estimated

N/A Spike recovery limits do not apply. Sample concentration exceeds spike concentration by $4 \mathrm{X}$ or more

$\mathrm{R}$ Sample results are rejected

U Analyte was analyzed for, but not detected above the MDL, MDA, or LOD.

UI Gamma Spectroscopy-Uncertain identification 


\section{GENERAL ENGINEERING LABORATORIES, LLC \\ 2040 Savage Road Charleston SC 29407 - (843) 556-8171 - www.gel.com}

\section{$\underline{\text { Certificate of Analysis }}$}

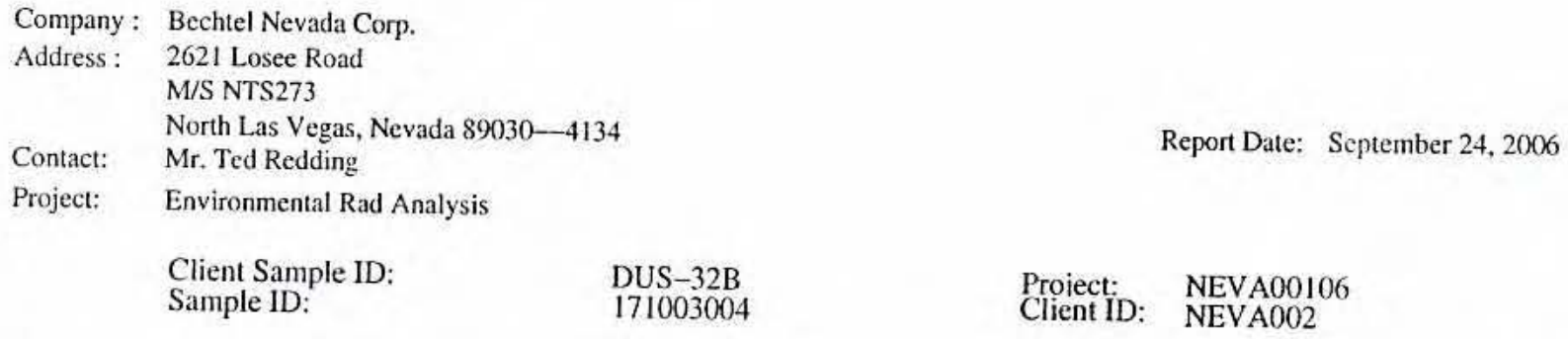

\footnotetext{
X Consult Case Narrative, Data Summary package, or Project Manager concerning this qualifier
Y QC Samples were not spiked with this compound

$\wedge$ RPD of sample and duplicate evaluated using $+/-$ RL. Concentrations are $<5 X$ the RL

h Preparation or preservation holding time was exceeded

The above sample is reported on a dry weight basis.
} 


\section{GENERAL ENGINEERING LABORATORIES, LLC \\ 2040 Savage Road Charleston SC 29407 - (843) 556-8171 - www.gel.com}

\section{Certificate of Analysis}

\author{
Company: Bechtel Nevada Corp. \\ Address: 2621 Losee Road \\ M/S NTS273 \\ North Las Vegas, Nevada 89030-4134 \\ Contact: Mr. Ted Redding \\ Project: Environmental Rad Analysis
}

Report Date: September 24,2006

$\begin{array}{llll}\text { Client Sample ID: } & \text { DUS-33A } & \text { Proiect: } & \text { NEVA00106 } \\ \text { Sample ID: } & 171003005 & \text { Client ID: } & \text { NEVA002 } \\ \text { Matrix: } & \text { Soil } & \\ \text { Collect Date: } & \text { 22-AUG-06 } & \\ \text { Receive Date: } & \text { 06-SEP-06 } & \\ \text { Collector: } & \text { Client } & & \end{array}$

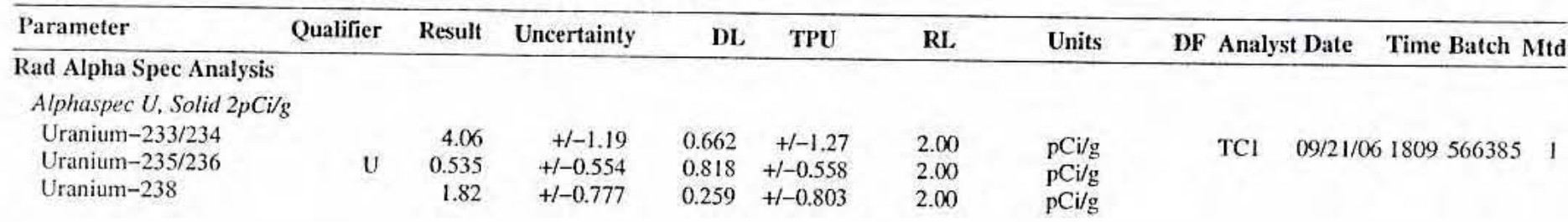

The following Prep Methods were performed

\begin{tabular}{llllll}
\hline Method & Description & Analyst & Date & Time & Prep Batch \\
\hline Dry Soil Prep & Dry Soil Prep GL-RAD-A-021 & JMBI & $09 / 07 / 06$ & 0856 & 565864
\end{tabular}

The following Analytical Methods were performed

\begin{tabular}{ll} 
Method & Description \\
\hline 1 & DOE EML HASL-300, U-02-RC Modified \\
2 & DOE EML HASL-300, U-02-RC Modified
\end{tabular}

Surrogate/Tracer recovery

\begin{tabular}{llcc} 
Surrogate/Tracer recovery & Test & Recovery\% & Acceptable Limits \\
\hline Uranium-232 & Alphaspec U, Solid 2pCi/g & 78 & $(25 \%-125 \%)$
\end{tabular}

Notes:

The Qualifiers in this report are defined as follows :

* A quality control analyte recovery is outside of specified acceptance criteria

$<$ Result is less than value reported

$>$ Result is greater than value reported

A The TIC is a suspected aldol-condensation product

B Target analyte was detected in the associated blank

$\mathrm{BD}$ Results are either below the MDC or tracer recovery is low

C Analyte has been confirmed by GC/MS analysis

D Results are reported from a diluted aliquot of the sample

$\mathrm{H}$ Analytical holding time was exceeded

$\mathrm{J}$ Value is estimated

N/A Spike recovery limits do not apply. Sample concentration exceeds spike concentration by $4 \mathrm{X}$ or more

$R$ Sample results are rejected

U Analyte was analyzed for, but not detected above the MDL, MDA, or LOD.

UI Gamma Spectroscopy-Uncertain identification 


\section{GENERAL ENGINEERING LABORATORIES, LLC \\ 2040 Savage Road Charleston SC 29407 - (843) 556-8171 - www.gel.com}

\section{Certificate of Analysis}

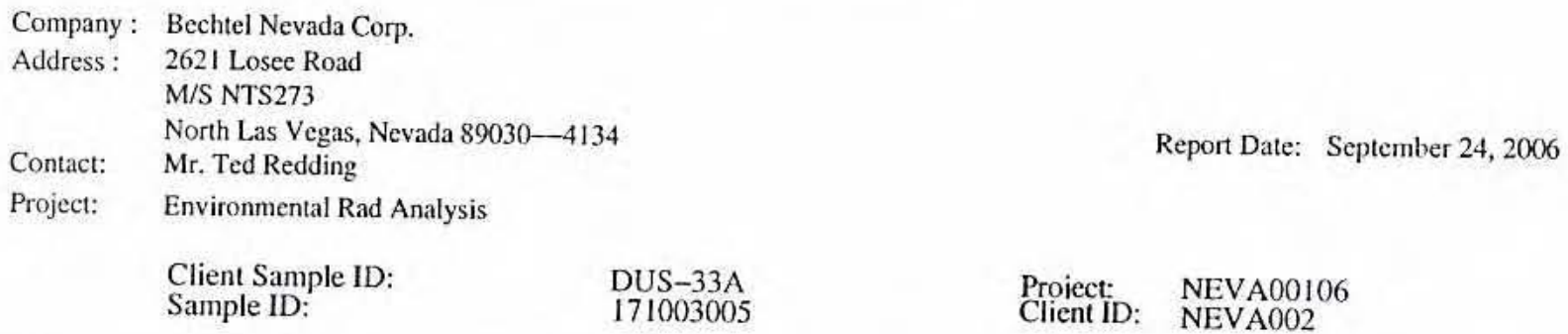

$X$ Consult Case Narrative, Data Summary package, or Project Manager concerning this qualifier

Y QC Samples were not spiked with this compound

$\wedge$ RPD of sample and duplicate evaluated using $+1-$ RL. Concentrations are $<5 X$ the RL

h Preparation or preservation holding time was exceeded

The above sample is reported on a dry weight basis. 


\section{GENERAL ENGINEERING LABORATORIES, LLC \\ 2040 Savage Road Charleston SC 29407 - (843) 556-8171 - www.gel.com}

\section{Certificate of Analysis}

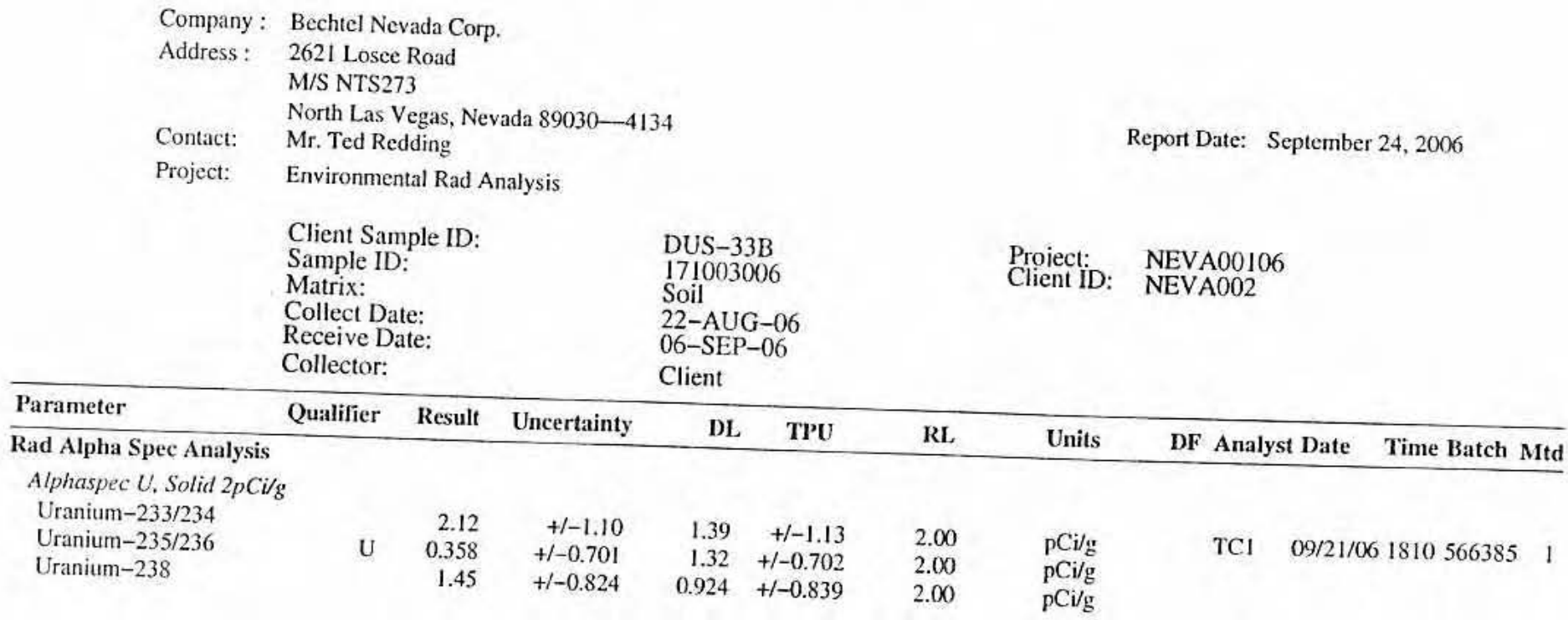

The following Prep Methods were performed

\begin{tabular}{llllll} 
Method & Description & Analyst & Date & Time & Prep Batch \\
\hline Dry Soil Prep & Dry Soil Prep GL-RAD-A-021 & JMBi & $09 / 07 / 06$ & 0856 & 565864
\end{tabular}

The following Analytical Methods were performed

\begin{tabular}{ll}
\hline Method & Description \\
\hline 1 & DOE EML HASL-300, U-02-RC Modified \\
2 & DOE EML HASL-300, U-02-RC Modified
\end{tabular}

Surrogate/Tracer recovery Test

$\begin{array}{cccc}\text { Uranium-232 } & \text { Recovery\% } & \text { Acceptable Limits }\end{array}$

Notes:

The Qualifiers in this report are defined as follows :

* A quality control analyte recovery is outside of specified acceptance criteria

$<$ Result is less than value reported

$>$ Result is greater than value reported

A The TIC is a suspected aldol-condensation product

B Target analyte was detected in the associated blank

$\mathrm{BD}$ Results are either below the MDC or tracer recovery is low

C Analyte has been confirmed by GC/MS analysis

D Results are reported from a diluted aliquot of the sample

Analytical holding time was exceeded

$\mathrm{J}$ Value is estimated

N/A Spike recovery limits do not apply. Sample concentration exceeds spike concentration by $4 \mathrm{X}$ or more
$\mathrm{R}$ Sample results are rejected

U Analyte was analyzed for, buted

U Analyte was analyzed for, but not detected above the MDL, MDA, or LOD.
UI Gamma Spectroscopy-Uncertain identification 


\section{GENERAL ENGINEERING LABORATORIES, LLC \\ 2040 Savage Road Charleston SC 29407 - (843) 556-8171 - www.gel.com}

\section{Certificate of Analysis}

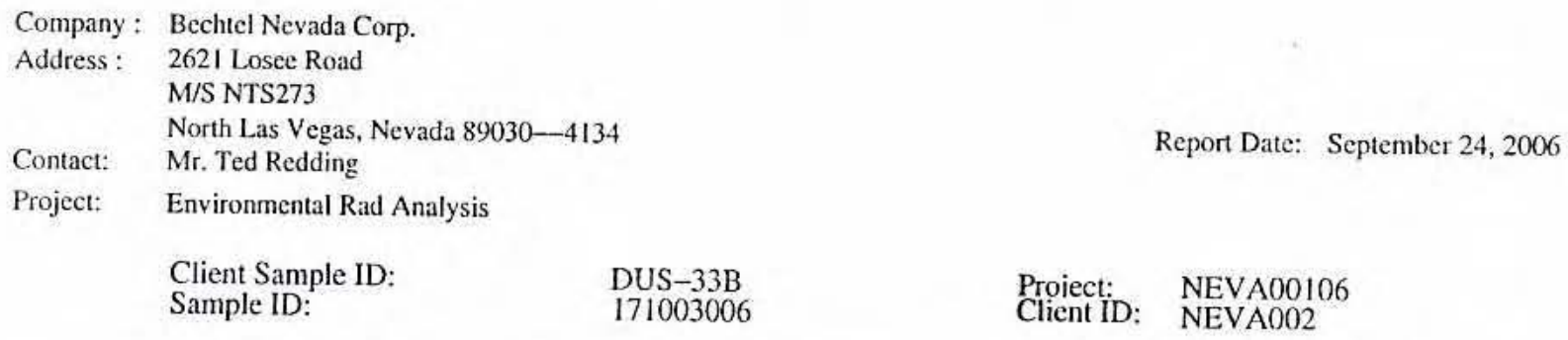

DL TPU

RL

Units

DF Analyst Date

Time Batch Mitd

$X$ Consult Case Narrative, Data Summary package, or Project Manager concerning this qualifier

Y QC Samples were not spiked with this compound

$\hat{R P D}$ of sample and duplicate evaluated using $+/-\mathrm{RL}$. Concentrations are $<5 \mathrm{X}$ the $\mathrm{RL}$

$\mathrm{h}$ Preparation or preservation holding time was exceeded

The above sample is reported on a dry weight basis. 


\section{GENERAL ENGINEERING LABORATORIES, LLC \\ 2040 Savage Road Charleston SC 29407 - (843) 556-8171 - www.gel.com}

\section{Certificate of Analysis}

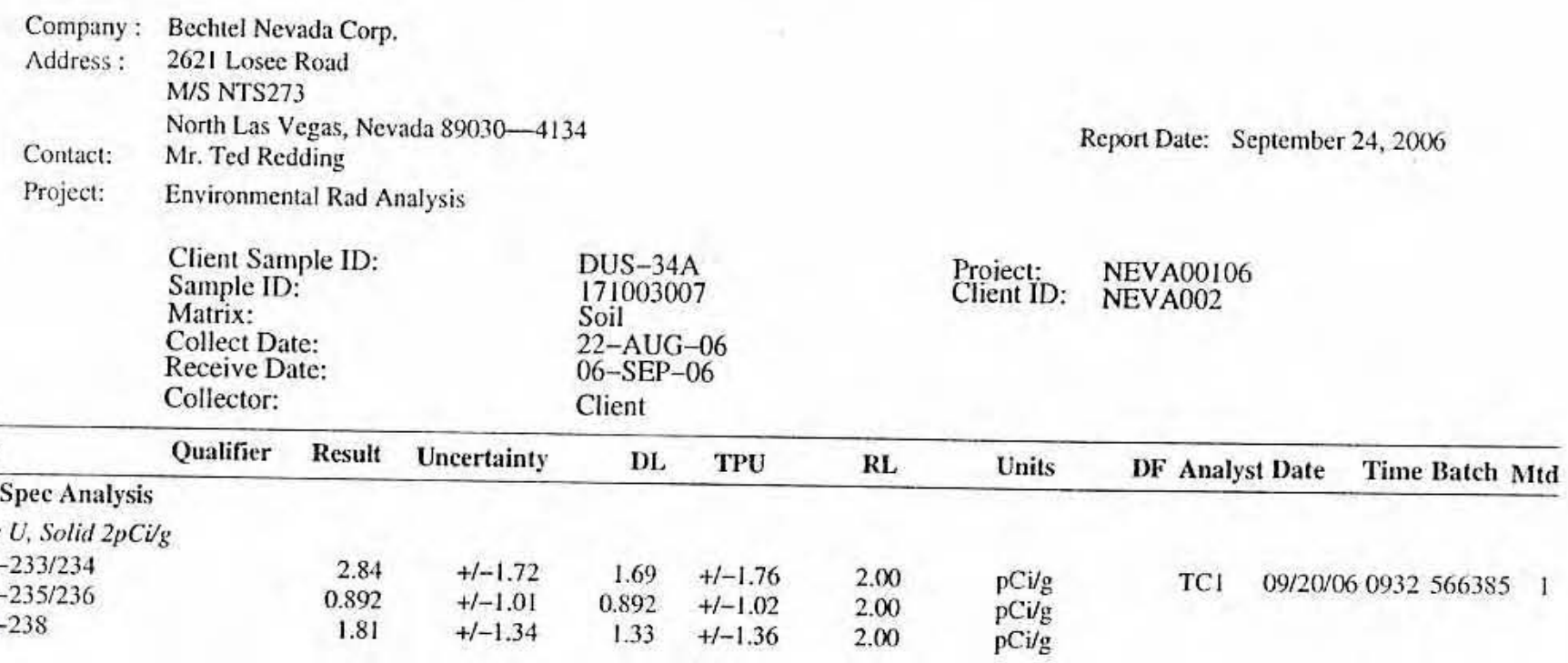

The following Prep Methods were performed

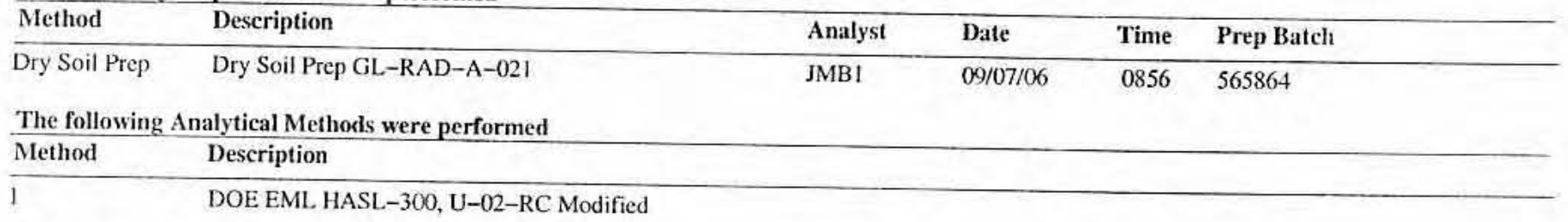

\begin{tabular}{llcc} 
Surrogate/Tracer recovery & Test & Recovery\% & Acceptable Limits \\
\hline Uranium-232 & Alphaspec U, Solid 2pCi/g & 92 & $(25 \%-125 \%)$
\end{tabular}

Notes:

The Qualifiers in this report are defined as follows :

* A quality control analyte recovery is outside of specified acceptance criteria

$<$ Result is less than value reported

$>$ Result is greater than value reported

A The TIC is a suspected aldol-condensation product

B Target analyte was detected in the associated blank

BD Results are either below the MDC or tracer recovery is low

C Analyte has been confirmed by GC/MS analysis

D Results are reported from a diluted aliquot of the sample

$\mathrm{H}$ Analytical holding time was exceeded

$\mathrm{J}$ Value is estimated

N/A Spike recovery limits do not apply. Sample concentration exceeds spike concentration by $4 \mathrm{X}$ or more

$\mathrm{R}$ Sample results are rejected

$\mathrm{U}$ Analyte was analyzed for, but not detected above the MDL, MDA, or LOD.

UI Gamma Spectroscopy--Uncertain identification

$X$ Consult Case Narrative, Data Summary package, or Project Manager concerning this qualifier 


\section{GENERAL ENGINEERING LABORATORIES, LLC \\ 2040 Savage Road Charleston SC 29407 - (843) 556-8171 - www.gel.com}

\section{Certificate of Analysis}

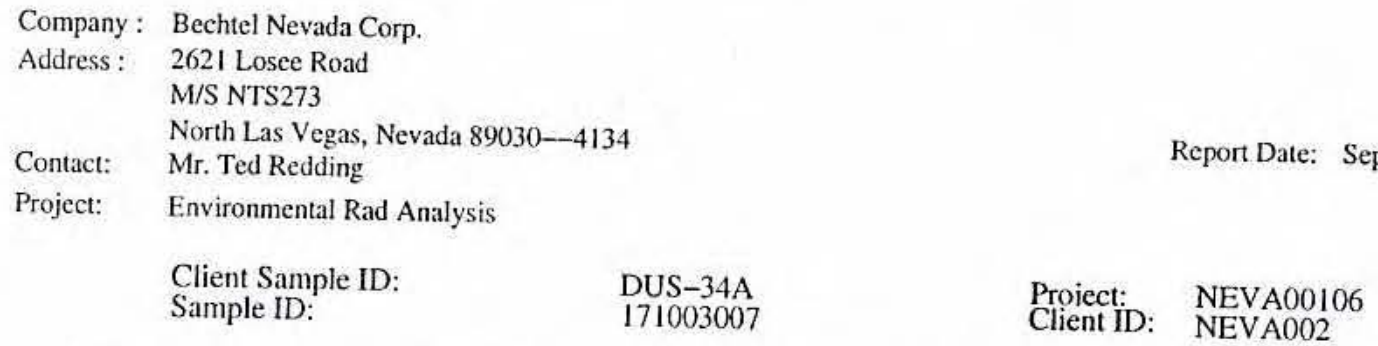

Y QC Samples were not spiked with this compound

$\wedge \quad$ RPD of sample and duplicate evaluated using $+/-\mathrm{RL}$. Concentrations are $<5 \mathrm{X}$ the $\mathrm{RL}$

$\mathrm{h}$ Preparation or preservation holding time was exceeded

The above sample is reported on a dry weight basis. 


\section{GENERAL ENGINEERING LABORATORIES, LLC \\ 2040 Savage Road Charleston SC 29407 - (843) 556-8171 - www.gel.com}

\section{Certificate of Analysis}

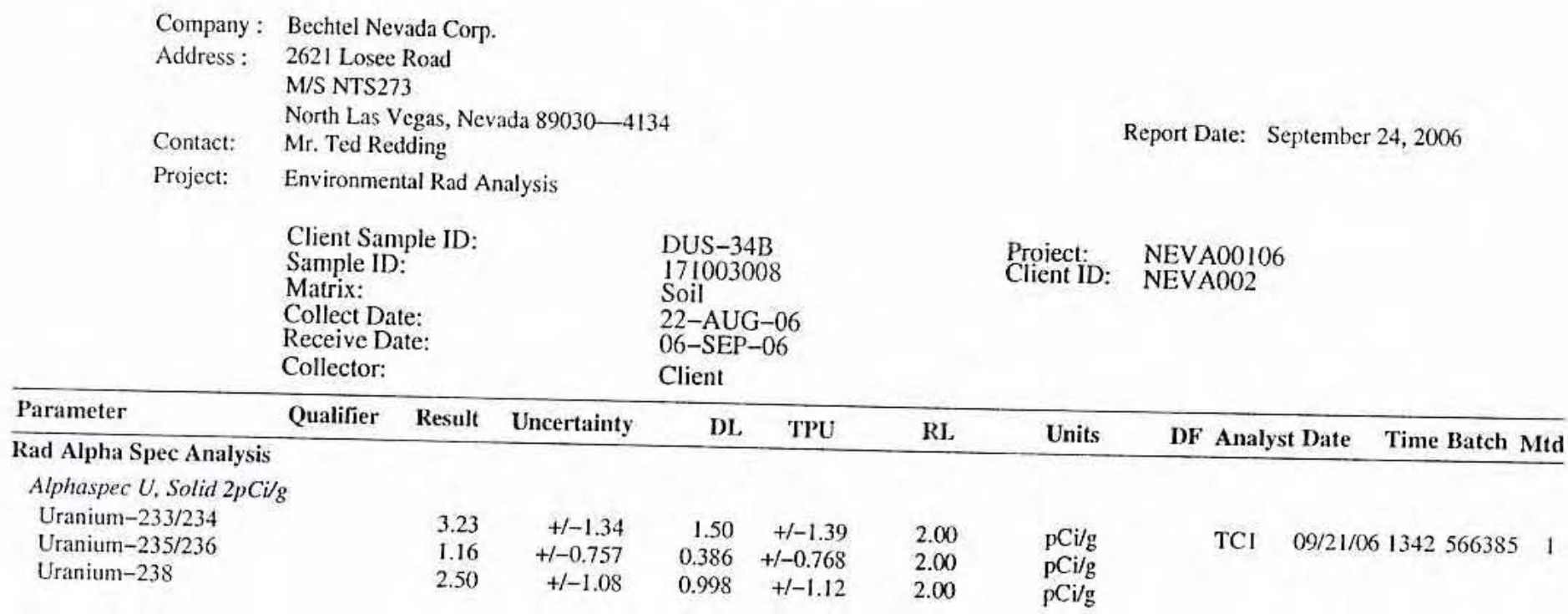

The following Prep Methods were performed

\begin{tabular}{llllll}
\hline Method & Description & Analyst & Date & Time & Prep Batch \\
\hline Dry Soil Prep & Dry Soil Prep GL-RAD-A-021 & JMBI & $09 / 07 / 06$ & 0856 & 565864
\end{tabular}

The following Analytical Methods were performed

\begin{tabular}{ll} 
Method & Description \\
\hline 1 & DOE EML HASL-300, U-02-RC Modified \\
2 & DOE EML HASL-300, U-02-RC Modified
\end{tabular}

\begin{tabular}{llcc} 
Surrogate/Tracer recovery & Test & Recovery\% & Acceptable Limits \\
\hline Uranium-232 & Alphaspec U, Solid $2 \mathrm{pCi} / \mathrm{g}$ & 72 & $(25 \%-125 \%)$
\end{tabular}

Notes:

The Qualifiers in this report are defined as follows :

* A quality control analyte recovery is outside of specified acceptance criteria

$<$ Result is less than value reported

$>$ Result is greater than value reported

A The TIC is a suspected aldol-condensation product

B Target analyte was detected in the associated blank

$\mathrm{BD}$ Results are either below the MDC or tracer recovery is low

C Analyte has been confirmed by GC/MS analysis

D Results are reported from a diluted aliquot of the sample

$\mathrm{H}$ Analytical holding time was exceeded

$\mathrm{J}$ Value is estimated

N/A Spike recovery limits do not apply. Sample concentration exceeds spike concentration by $4 X$ or more
$R$ Sample results are rejected

$\mathrm{U}$ Analyte was analyzed for, but not detected above the MDL, MDA, or LOD.

UI Gamma Spectroscopy--Uncertain identification 


\section{GENERAL ENGINEERING LABORATORIES, LLC \\ 2040 Savage Road Charleston SC 29407 - (843) 556-8171 - www.gel.com}

\section{Certificate of Analysis}

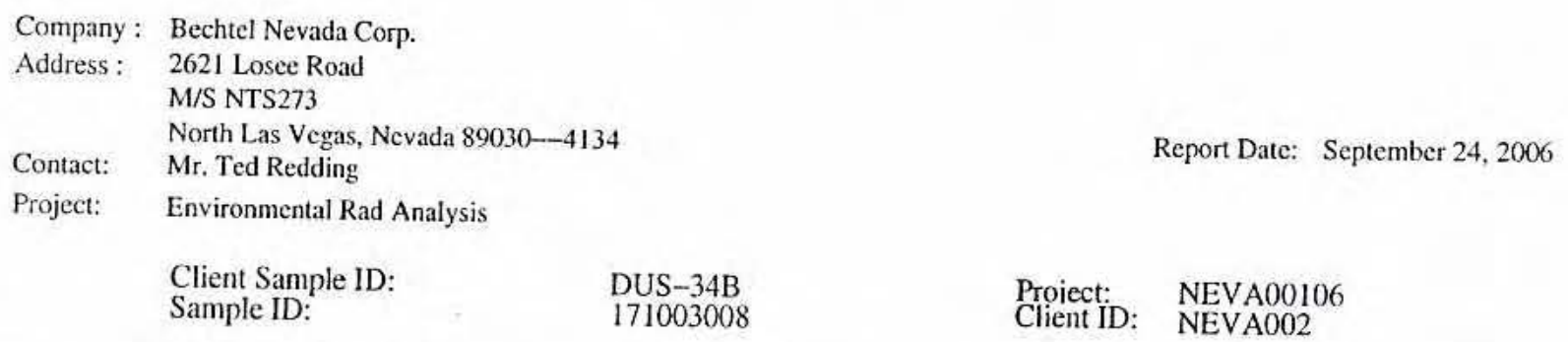

Parameter

Qualifier Result Uncertainty

DL TPU

RI

Units

DF Analyst Date

Time Batch Mtd

$X$ Consult Case Narrative, Data Summary package, or Project Manager concerning this qualifier

Y QC Samples were not spiked with this compound

$\hat{n}$ RPD of sample and duplicate evaluated using $+1-\mathrm{RL}$. Concentrations are $\angle 5 \mathrm{X}$ the $\mathrm{RL}$

$\mathrm{h}$ Preparation or preservation holding time was exceeded

The above sample is reported on a dry weight basis. 


\section{GENERAL ENGINEERING LABORATORIES, LLC \\ 2040 Savage Road Charleston SC 29407 - (843) 556-8171 - www.gel.com}

\section{Certificate of Analysis}

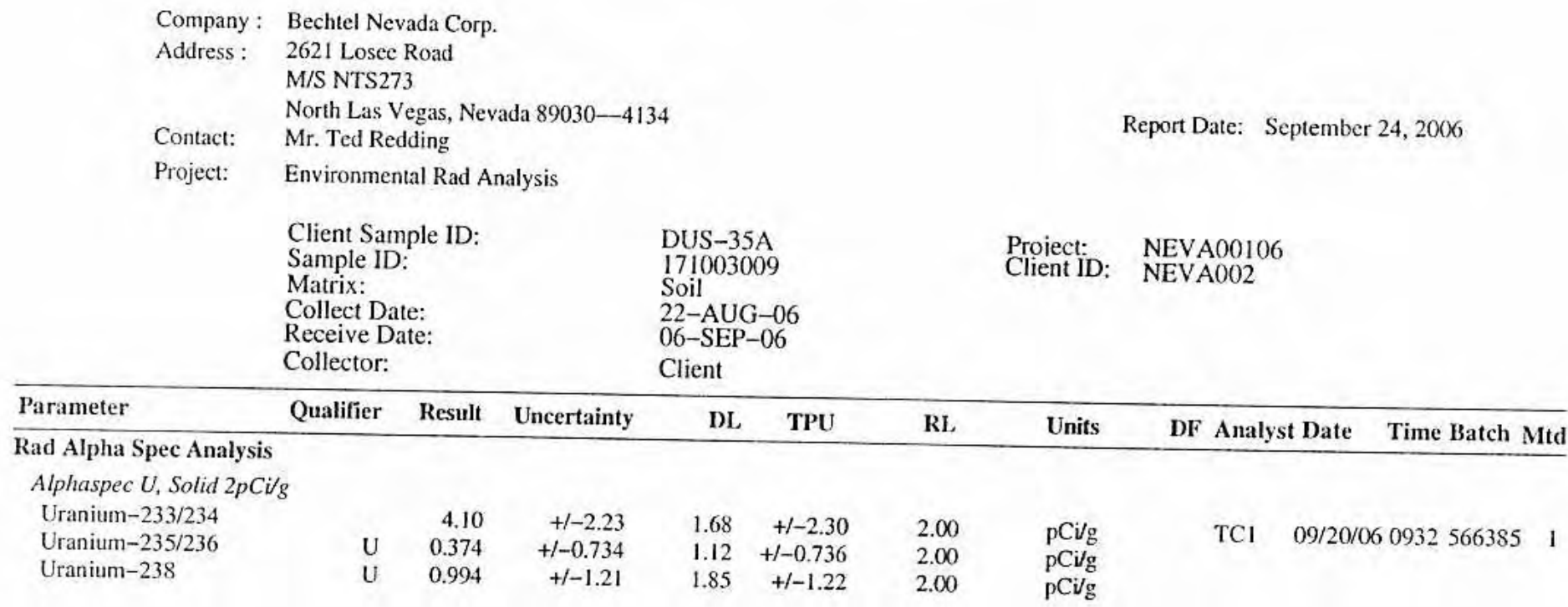

The following Prep Methods were performed

\begin{tabular}{llllll}
\hline Method & Description & Analyst & Date & Time & Prep Batch \\
\hline Dry Soil Prep & Dry Soil Prep GL-RAD-A-021 & JMB1 & $09 / 07 / 06$ & 0856 & 565864
\end{tabular}

The following Analytical Methods were performed

\begin{tabular}{ll} 
Method & Description \\
\hline & DOE EML HASL-300, U-02-RC Modified
\end{tabular}

\begin{tabular}{llcc} 
Surrogate/Tracer recovery & Test & Recovery $\%$ & Acceptable Limits \\
\hline Uranium-232 & Alphaspec U, Solid 2pCi/g & 95 & $(25 \%-125 \%)$
\end{tabular}

Notes:

The Qualifiers in this report are defined as follows :

* A quality control analyte recovery is outside of specified acceptance criteria

$<$ Result is less than value reported

$>$ Result is greater than value reported

A The TIC is a suspected aldol-condensation product

B Target analyte was detected in the associated blank

BD Results are either below the MDC or tracer recovery is low

C Analyte has been confirmed by GC/MS analysis

D Results are reported from a diluted aliquot of the sample

$\mathrm{H}$ Analytical holding time was exceeded

J Value is estimated

N/A Spike recovery limits do not apply. Sample concentration exceeds spike concentration by $4 \mathrm{X}$ or more

$\mathrm{R}$ Sample results are rejected

$\mathrm{U}$ Analyte was analyzed for, but not detected above the MDL, MDA, or LOD.

UI Gamma Spectroscopy-Uncertain identification

X Consult Case Narrative, Data Summary package, or Project Manager conceming this qualifier 


\section{GENERAL ENGINEERING LABORATORIES, LLC \\ 2040 Savage Road Charleston SC 29407 - (843) 556-8171 - www.gel.com}

\section{Certificate of Analysis}

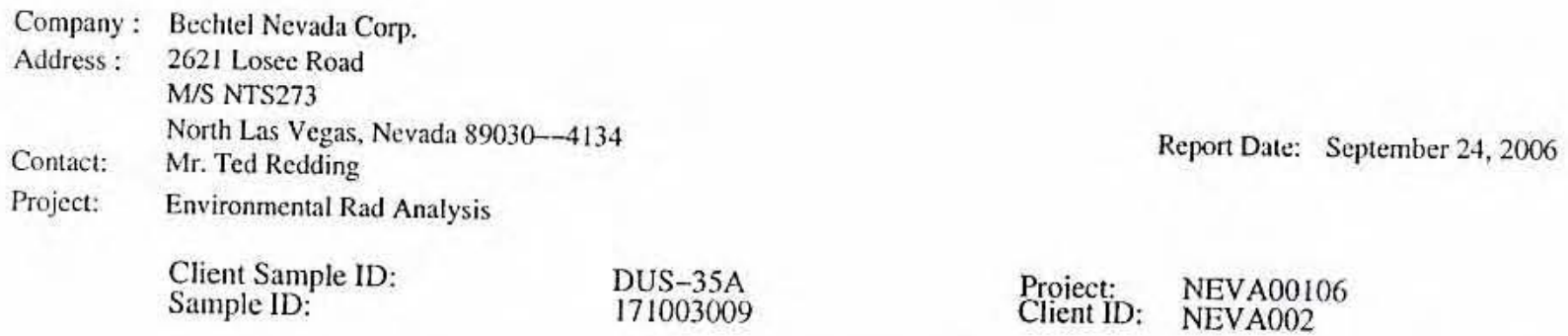

Y QC Samples were not spiked with this compound

$\hat{R}$ RPD of sample and duplicate evaluated using $+/-\mathrm{RL}$. Concentrations are $<5 \mathrm{X}$ the RL

$\mathrm{h}$ Preparation or preservation holding time was exceeded

The above sample is reported on a dry weight basis. 


\section{GENERAL ENGINEERING LABORATORIES, LLC \\ 2040 Savage Road Charleston SC 29407 - (843) 556-8171 - www.gel.com}

\section{Certificate of Analysis}

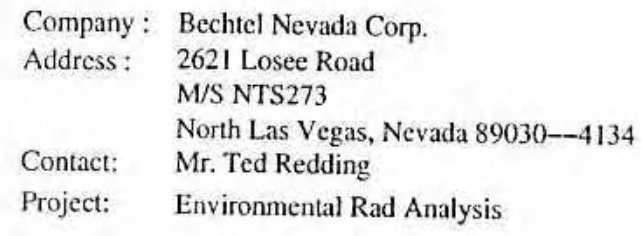

Client Sample ID:

Sample ID:

Matrix:

DUS-35B

171003010

Soil

Collect Date:

Receive Date:

22-AUG-06

Collector:

06-SEP-06

Client

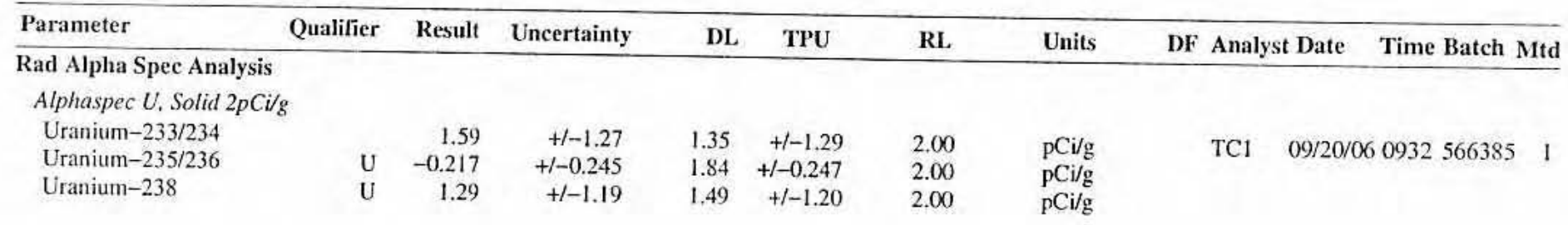

The following Prep Methods were performed

\begin{tabular}{llllll}
\hline Method & Description & Analyst & Date & Time & Prep Batch \\
\hline Dry Soil Prep & Dry Soil Prep GL-RAD-A-021 & JMBI & $09 / 07 / 06$ & 0856 & 565864
\end{tabular}

The following Analytical Methods were performed

Method Description

1 DOE EML HASL-300, U-02-RC Modified

\begin{tabular}{llcc} 
Surrogate/Tracer recovery & Test & Recovery $\%$ & Acceptable Limits \\
\hline Uranium-232 & Alphaspec U, Solid 2pCi/g & 96 & $(25 \%-125 \%)$
\end{tabular}

Notes:

The Qualifiers in this report are defined as follows :

* A quality control analyte recovery is outside of specified acceptance criteria

$<$ Result is less than value reported

$>$ Result is greater than value reported

A The TIC is a suspected aldol-condensation product

B Target analyte was detected in the associated blank

$\mathrm{BD}$ Results are either below the MDC or tracer recovery is low

C Analyte has been confirmed by GC/MS analysis

D Results are reported from a diluted aliquot of the sample

$\mathrm{H}$ Analytical holding time was exceeded

$\mathrm{J}$ Value is estimated

N/A Spike recovery limits do not apply. Sample concentration exceeds spike concentration by $4 \mathrm{X}$ or more

$\mathrm{R}$ Sample results are rejected

$\mathrm{U}$ Analyte was analyzed for, but not detected above the MDL, MDA, or LOD.

UI Gamma Spectroscopy--Uncertain identification

X Consult Case Narrative, Data Summary package, or Project Manager concerning this qualifier 


\section{GENERAL ENGINEERING LABORATORIES, LLC \\ 2040 Savage Road Charleston SC 29407 - (843) 556-8171 - www.gel.com}

\section{Certificate of Analysis}

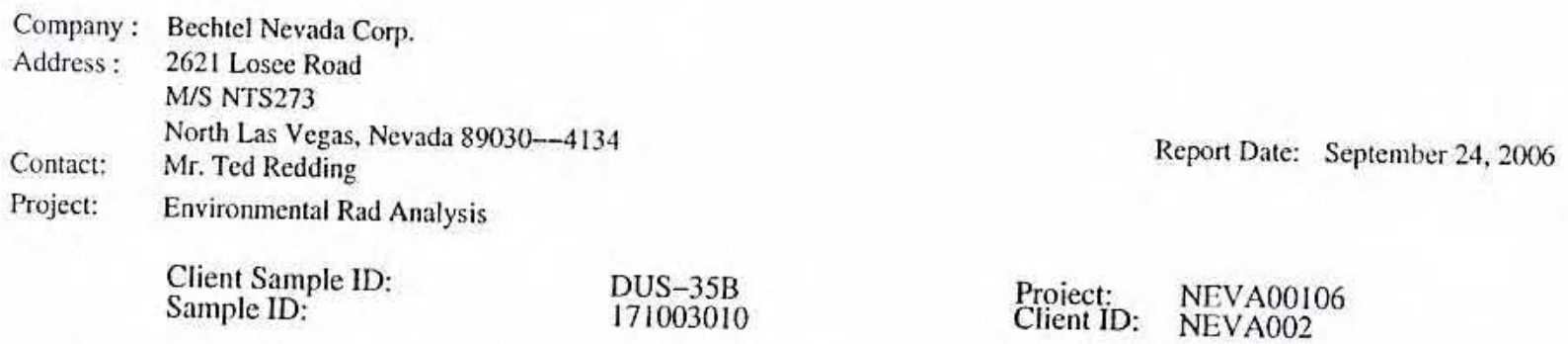

Y QC Samples were not spiked with this compound

$\hat{R}$ RP of sample and duplicate evaluated using $+/-\mathrm{RL}$. Concentrations are $<5 \mathrm{X}$ the $\mathrm{RL}$

$\mathrm{h}$ Preparation or preservation holding time was exceeded

The above sample is reported on a dry weight basis. 


\section{GENERAL ENGINEERING LABORATORIES, LLC \\ 2040 Savage Road Charleston SC 29407 - (843) 556-8171 - www.gel.com}

\section{Certificate of Analysis}

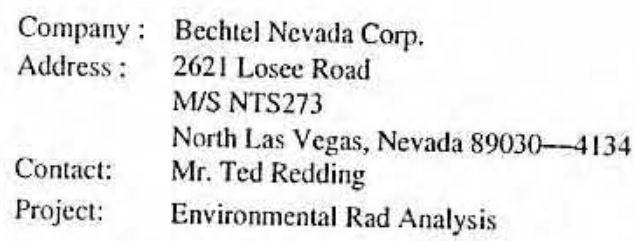

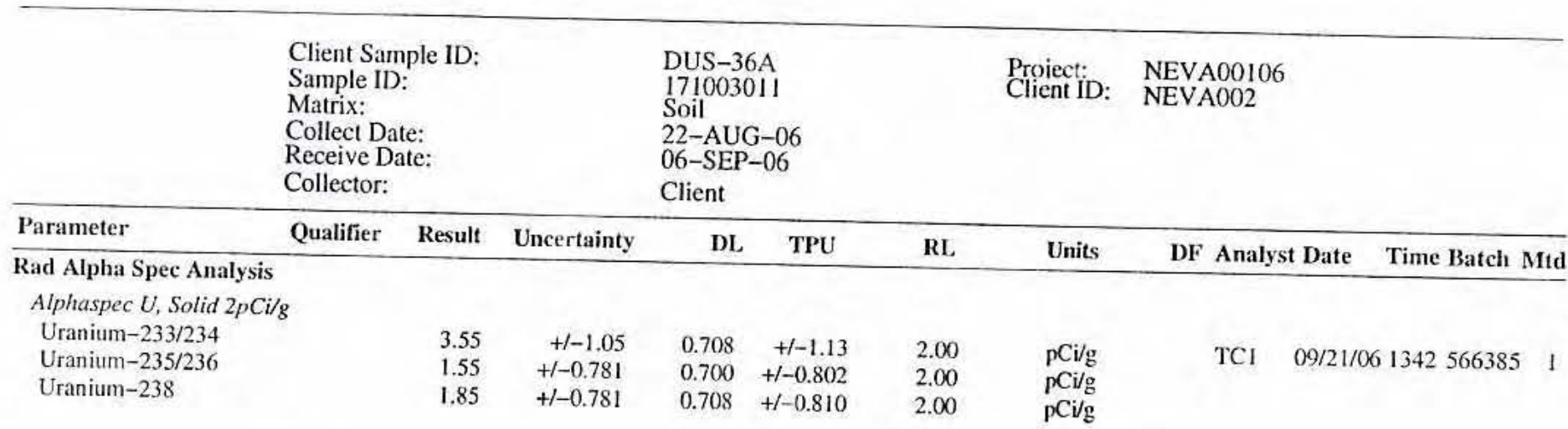

The following Prep Methods were performed

\begin{tabular}{llllll}
\hline Method & Description & Analyst & Date & Time & Prep Batch \\
\hline Dry Soil Prep & Dry Soil Prep GL-RAD-A-02I & JMBI & $09 / 07 / 06$ & 0856 & 565864
\end{tabular}

The following Analytical Methods were performed

\begin{tabular}{ll} 
Method & Description \\
\hline 1 & DOE EML HASL-300, U-02-RC Modified \\
2 & DOE EML HASL-300, U-02-RC Modified
\end{tabular}

\begin{tabular}{llcc} 
Surrogate/Tracer recovery & Test & Recovery\% & Acceptable Limits \\
\hline Uranium-232 & Alphaspec U, Solid 2pCi/g & 50 & $(25 \%-125 \%)$
\end{tabular}

\section{Notes:}

The Qualifiers in this report are defined as follows :

* A quality control analyte recovery is outside of specified acceptance criteria

$<$ Result is less than value reported

$>$ Result is greater than value reported

A The TIC is a suspected aldol-condensation product

B Target analyte was detected in the associated blank

BD Results are either below the MDC or tracer recovery is low

C Analyte has been confirmed by GC/MS analysis

D Results are reported from a diluted aliquot of the sample

$\mathrm{H}$ Analytical holding time was exceeded

$\mathrm{J}$ Value is estimated

N/A Spike recovery limits do not apply. Sample concentration exceeds spike concentration by $4 X$ or more
R Sample results are rejected

$\mathrm{U}$ Analyte was analyzed for, but not detected above the MDL, MDA, or LOD. 


\section{GENERAL ENGINEERING LABORATORIES, LLC \\ 2040 Savage Road Charleston SC 29407 - (843) 556-8171 - www.gel.com}

\section{Certificate of Analysis}

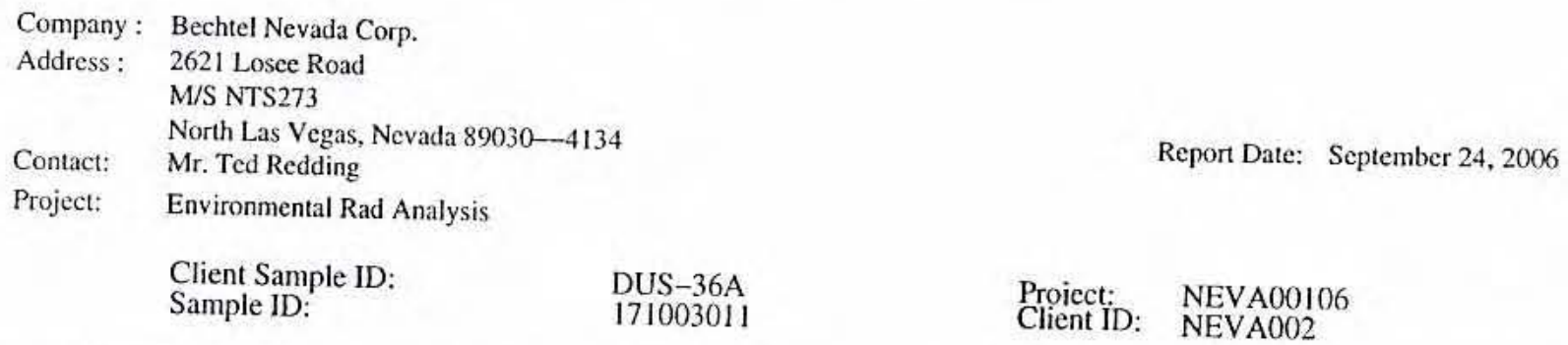

DL TPU

RL

Units

DF Analyst Date Time Batch Mtd

UI Gamma Spectroscopy--Uncertain identification

X Consult Case Narrative, Data Summary package, or Project Manager concerning this qualifier
Y QC Samples were not spiked with this compound

$\hat{h}$ RPD of sample and duplicate evaluated using $+/-$ RL. Concentrations are $<5 X$ the RL.

$\mathrm{h}$ Preparation or preservation holding time was exceeded

The above sample is reported on a dry weight basis. 


\section{GENERAL ENGINEERING LABORATORIES, LLC}

2040 Savage Road Charleston SC 29407 - (843) 556-8171 - www.gel.com

\section{Certificate of Analysis}

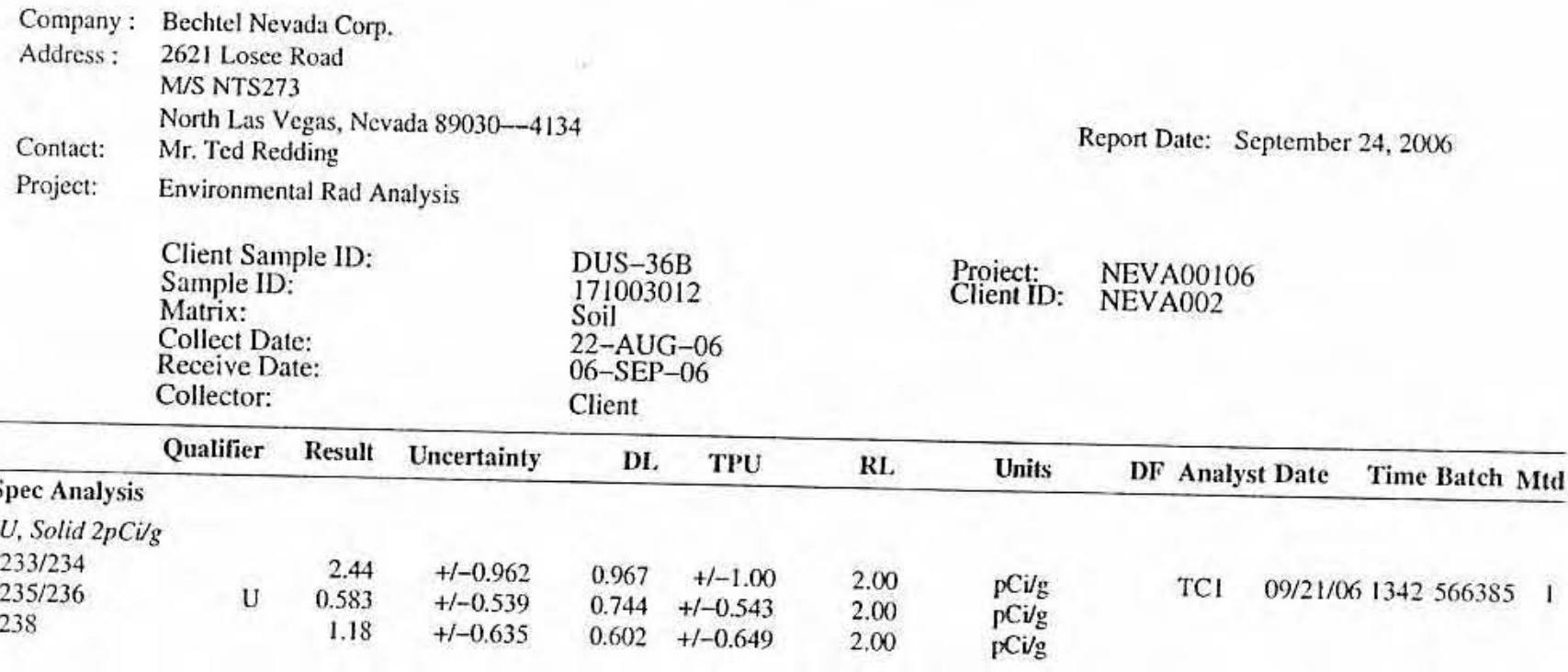

The following Prep Methods were performed

\begin{tabular}{llllll}
\hline Method & Description & Analyst & Date & Time & Prep Bateh \\
\hline Dry Soil Prep & Dry Soil Prep GL-RAD-A-021 & JMB1 & $09 / 07 / 06$ & 0856 & 565864
\end{tabular}

The following Analytical Methods were performed

\begin{tabular}{ll}
\hline Method & Description \\
\hline 1 & DOE EML HASL-300, U-02-RC Modified \\
2 & DOE EML HASL-300, U-02-RC Modified
\end{tabular}

\begin{tabular}{llcc} 
Surrogate/Tracer recovery & Test & Recovery\% & Acceptable Limits \\
\hline Uranium-232 & Alphaspec U, Solid 2pCi/g & 77 & $(25 \%-125 \%)$
\end{tabular}

Notes:

The Qualifiers in this report are defined as follows :

* A quality control analyte recovery is outside of specified acceptance criteria

$<$ Result is less than value reported

$>$ Result is greater than value reported

A The TIC is a suspected aldol-condensation product

$B$ Target analyte was detected in the associated blank

BD Results are either below the MDC or tracer recovery is low

C Analyte has been confirmed by GC/MS analysis

D Results are reported from a diluted aliquot of the sample

$H$ Analytical holding time was exceeded

$\mathrm{J}$ Value is estimated

N/A Spike recovery limits do not apply. Sample concentration exceeds spike concentration by $4 X$ or more

$\mathrm{U}$ Analyte was analyzed for, but not detected above the MDL, MDA, or LOD.

UI Gamma Spectroscopy-Uncertain identification 


\section{GENERAL ENGINEERING LABORATORIES, LLC}

2040 Savage Road Charleston SC 29407 - (843) 556-8171 - www.gel.com

\section{Certificate of Analysis}

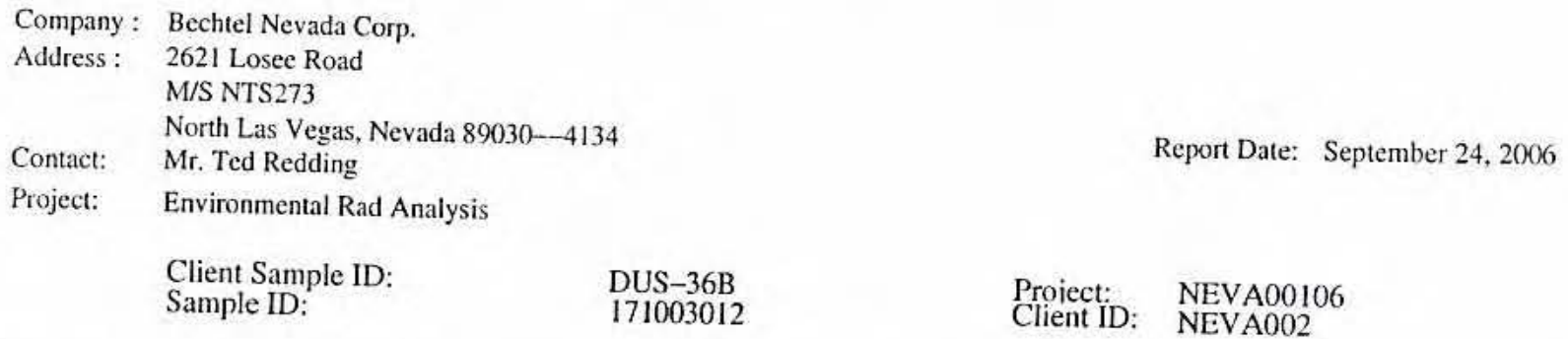

X Consult Case Narrative, Data Summary package, or Project Manager concerning this qualifier

Y QC Samples were not spiked with this compound

$\wedge \quad \mathrm{RPD}$ of sample and duplicate evaluated using $+/-\mathrm{RL}$. Concentrations are $<5 \mathrm{X}$ the RL

$\mathrm{h}$ Preparation or preservation holding time was exceeded

The above sample is reported on a dry weight basis. 


\section{GENERAL ENGINEERING LABORATORIES, LLC \\ 2040 Savage Road Charleston SC 29407 - (843) 556-8171 - www.gel.com}

\section{Certificate of Analysis}

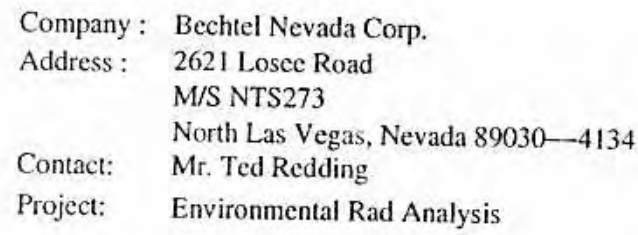

Client Sample ID: Sample ID:

Matrix:

Collect Date:

Receive Date:

Collector:
Report Date: September 24, 2006

DUS-37A
171003013
Soil
17-AUG-06
$06-S E P-06$
Client

Proiect: NEVA00106

Client ID: NEVA002

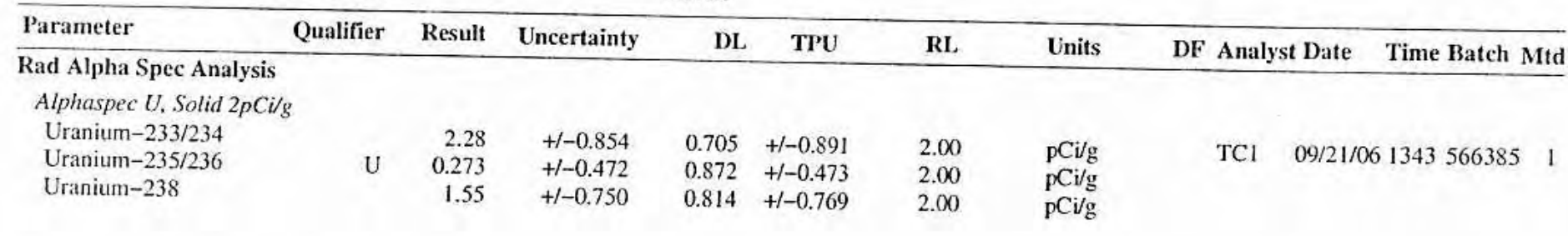

The following Prep Methods were performed

\begin{tabular}{llllll}
\hline Method & Description & Analyst & Date & Time & Prep Batch \\
\hline Dry Soil Prep & Dry Soil Prep GL-RAD-A-021 & JMB1 & $09 / 07 / 06$ & 0856 & 565864
\end{tabular}

The following Analytical Methods were performed

\begin{tabular}{ll} 
Method & Description \\
\hline 1 & DOE EML HASL-300, U-02-RC Modified \\
2 & DOE EML HASL-300, U-02-RC Modified
\end{tabular}

\begin{tabular}{llcc} 
Surrogate/Tracer recovery & Test & Recovery $\%$ & Acceptable Limits \\
\hline Uranium-232 & Alphaspec U, Solid 2pCi/g & 82 & $(25 \%-125 \%)$
\end{tabular}

Notes:

The Qualifiers in this report are defined as follows :

* A quality control analyte recovery is outside of specified acceptance criteria

$<$ Result is less than value reported

$>$ Result is greater than value reported

A The TIC is a suspected aldol-condensation product

$B$ Target analyte was detected in the associated blank

$\mathrm{BD}$ Results are either below the MDC or tracer recovery is low

C Analyte has been confirmed by GC/MS analysis

D Results are reported from a diluted aliquot of the sample

$\mathrm{H}$ Analytical holding time was exceeded

$\mathrm{J}$ Value is estimated

N/A Spike recovery limits do not apply. Sample concentration exceeds spike concentration by $4 X$ or more

$\mathrm{R}$ Sample results are rejected

U Analyte was analyzed for, but not detected above the MDL, MDA, or LOD.

UI Gamma Spectroscopy--Uncertain identification 


\section{GENERAL ENGINEERING LABORATORIES, LLC \\ 2040 Savage Road Charleston SC 29407 - (843) 556-8171 - www.gel.com}

\section{Certificate of Analysis}

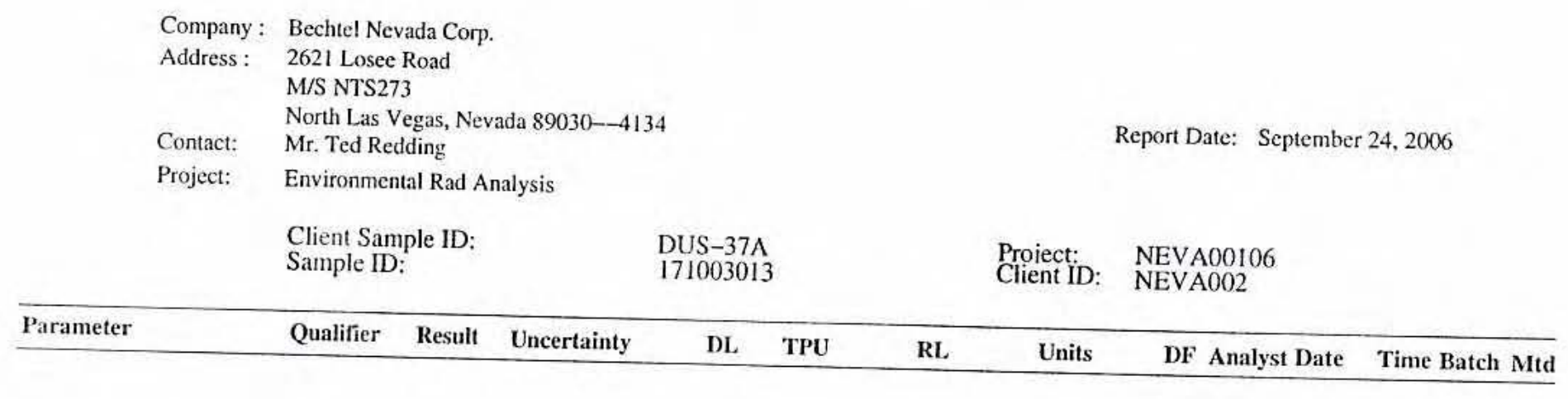

$\mathrm{X}$ Consult Case Narrative, Data Summary package, or Project Manager conceming this qualifier

Y QC Samples were not spiked with this compound

h Preparation or preservation holding time was exceeded

The preparation or preservation holding time was exceeded

The above sample is reported on a dry weight basis. 


\section{GENERAL ENGINEERING LABORATORIES, LLC \\ 2040 Savage Road Charleston SC 29407 - (843) 556-8171 - www.gel.com}

\section{Certificate of Analysis}

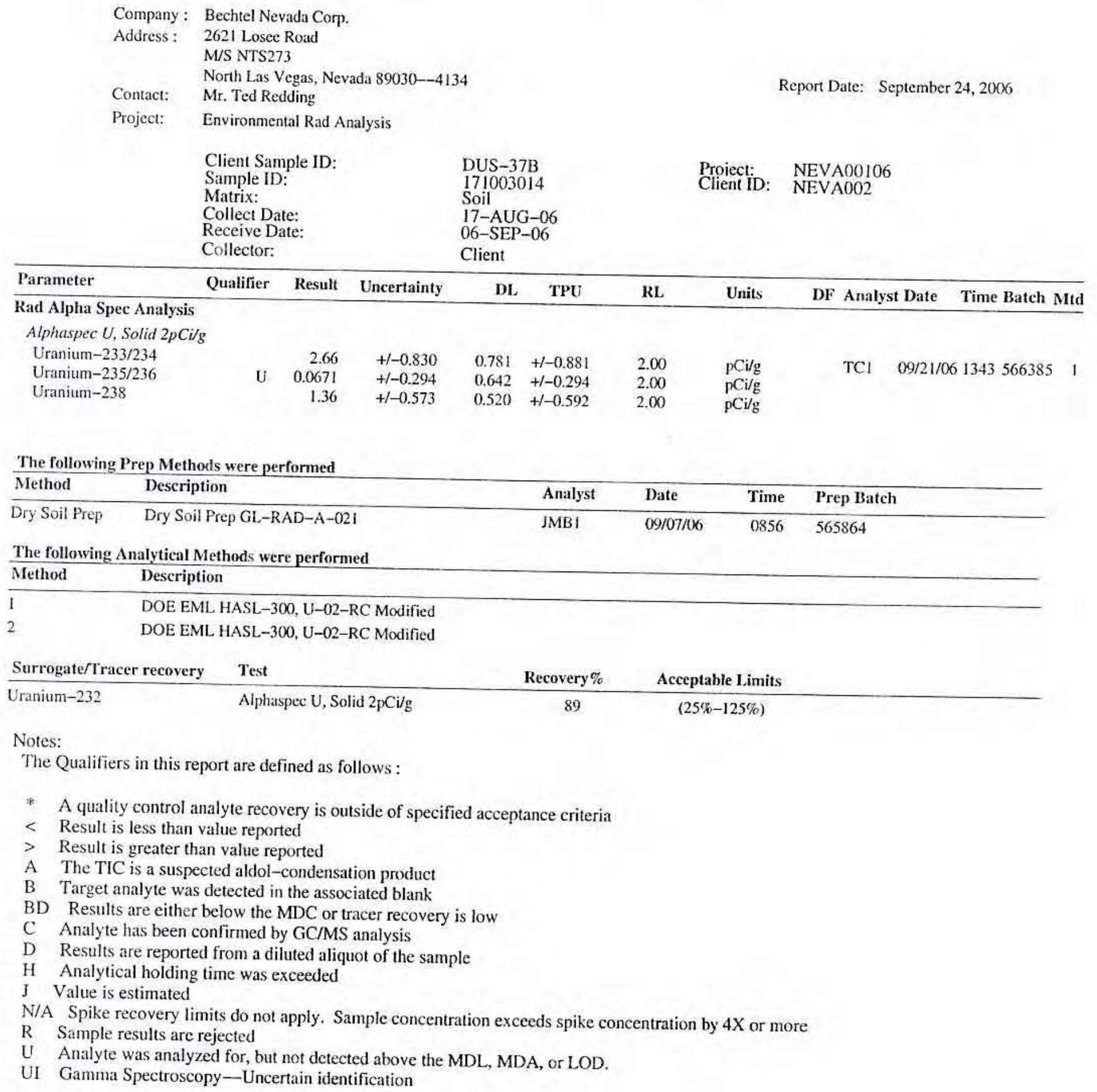




\section{GENERAL ENGINEERING LABORATORIES, LLC \\ 2040 Savage Road Charleston SC 29407 - (843) 556-8171 - www.gel.com}

\section{Certificate of Analysis}

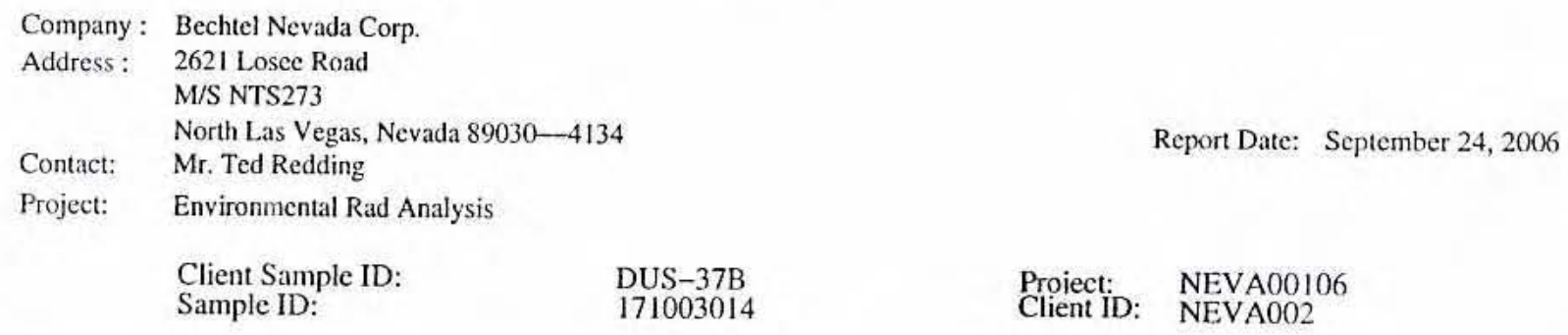

\begin{tabular}{llllllll}
\hline Parameter & Qualifier & Result & Uncertainty & DL & TPU & RL & Units
\end{tabular}

$X$ Consult Case Narrative, Data Summary package, or Project Manager concerning this qualifier

$\mathrm{Y}$ QC Samples were not spiked with this compound

$\wedge$ RPD of sample and duplicate evaluated using $+/-$ RL. Concentrations are $<5$ the RL

h Preparation or preservation holding time was exceeded

The above sample is reported on a dry weight basis. 


\section{GENERAL ENGINEERING LABORATORIES, LLC \\ 2040 Savage Road Charleston SC 29407 - (843) 556-8171 - www.gel.com}

\section{Certificate of Analysis}

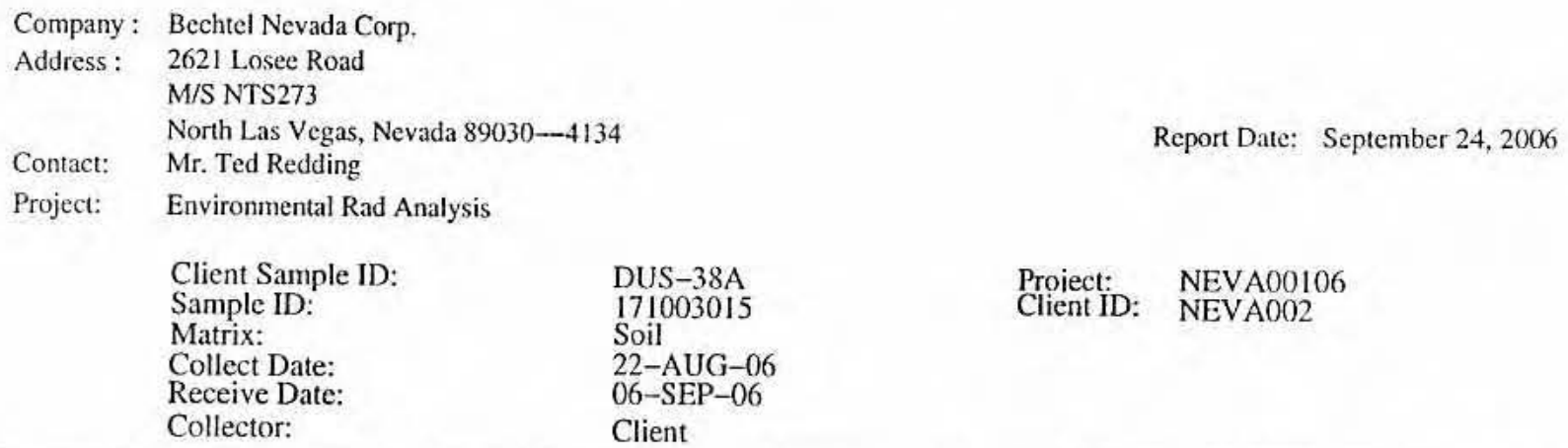

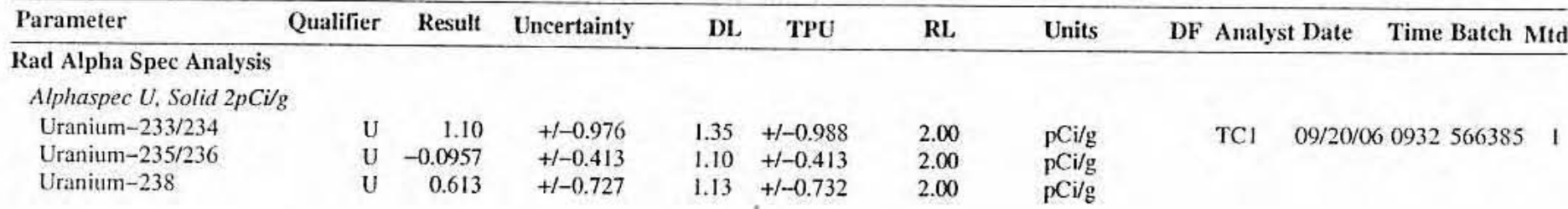

The following Prep Methods were performed

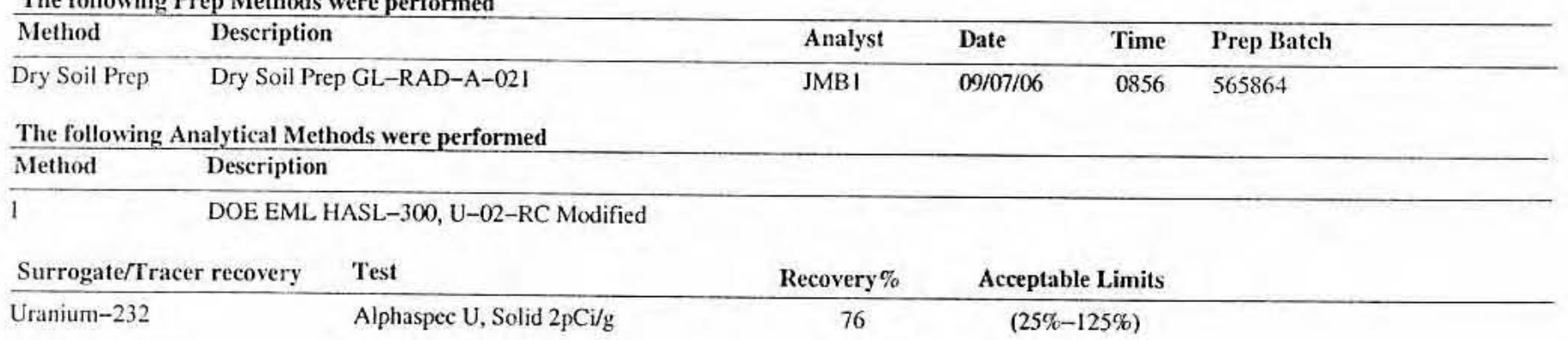

Notes:

The Qualifiers in this report are defined as follows :

* A quality control analyte recovery is outside of specified acceptance criteria

$<$ Result is less than value reported

$>$ Result is greater than value reported

A The TIC is a suspected aldol-condensation product

B Target analyte was detected in the associated blank

BD Results are either below the MDC or tracer recovery is low

C Analyte has been confirmed by GC/MS analysis

D Results are reported from a diluted aliquot of the sample

$\mathrm{H}$ Analytical holding time was exceeded

$\mathrm{J}$ Value is estimated

N/A Spike recovery limits do not apply. Sample concentration exceeds spike concentration by $4 X$ or more

$\mathrm{R}$ Sample results are rejected

U Analyte was analyzed for, but not detected above the MDL, MDA, or LOD.

UI Gamma Spectroscopy--Uncertain identification

$\mathrm{X}$ Consult Case Narrative, Data Summary package, or Project Manager concerning this qualifier 


\section{GENERAL ENGINEERING LABORATORIES, LLC \\ 2040 Savage Road Charleston SC 29407 - (843) 556-8171 - www.gel.com}

\section{Certificate of Analysis}

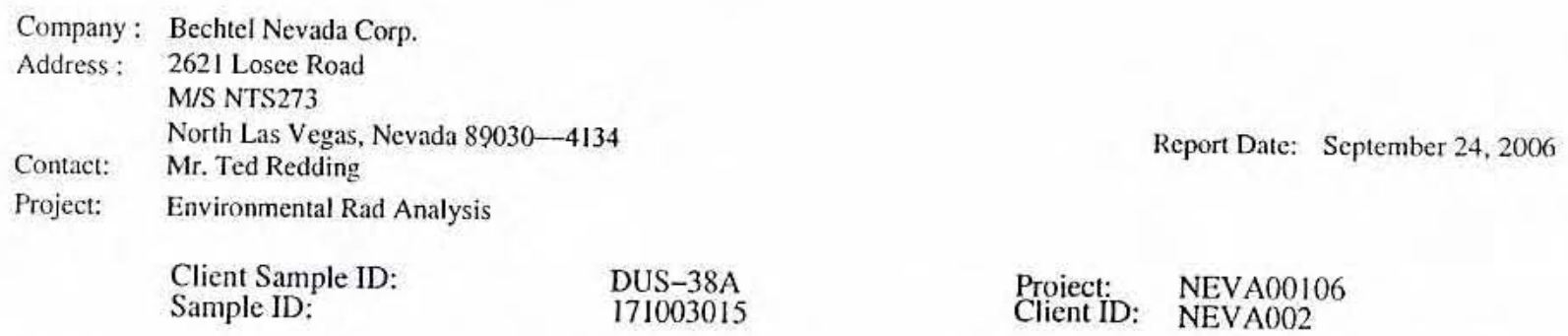

\begin{tabular}{llllllll}
\hline Parameter & Qualifier & Result & Uncertainty & DL & TPU & RL & Units
\end{tabular}

Y QC Samples were not spiked with this compound

$\hat{R P D}$ of sample and duplicate evaluated using $+/-\mathrm{RL}$. Concentrations are $<5 \mathrm{X}$ the RL

$\mathrm{h}$ Preparation or preservation holding time was exceeded

The above sample is reported on a dry weight basis. 


\section{GENERAL ENGINEERING LABORATORIES, LLC \\ 2040 Savage Road Charleston SC 29407 - (843) 556-8171 - www.gel.com}

\section{Certificate of Analysis}

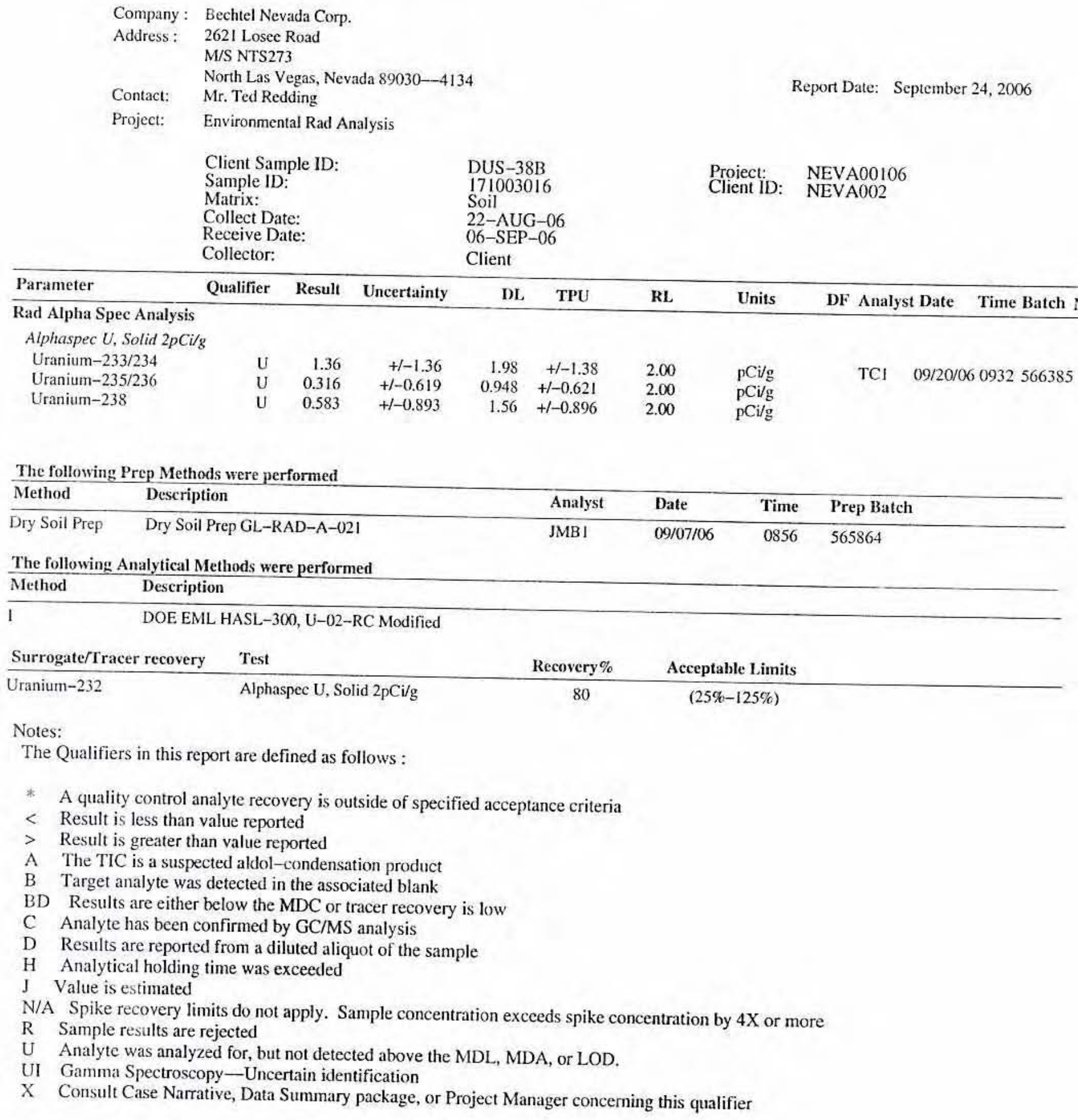




\section{GENERAL ENGINEERING LABORATORIES, LLC \\ 2040 Savage Road Charleston SC 29407 - (843) 556-8171 - www.gel.com}

\section{Certificate of Analysis}

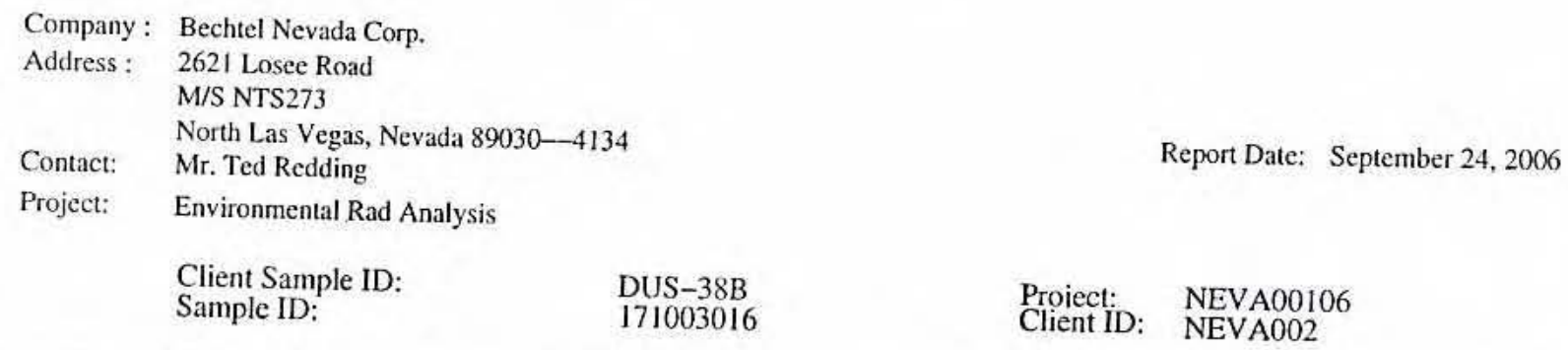

DL TPU

RL

Units

DF Analyst Date Time Batch Mtd

Y QC Samples were not spiked with this compound $\mathrm{RPD}$ of sample and duplicate evaluated using $+/-\mathrm{RL}$. Concentrations are $<5 \mathrm{X}$ the RL

$\mathrm{h}$ Preparation or preservation holding time was exceeded

The above sample is reported on a dry weight basis. 


\section{Certificate of Analysis}

\begin{tabular}{|c|c|c|c|c|c|c|c|c|c|c|c|}
\hline Parameter & Qualifier & Result & Uncertainty & $\mathrm{DL}$ & TPU & RL & Units & DF & Analyst & Date & Time Batch Mtd \\
\hline \multicolumn{12}{|c|}{ Rad Alpha Spec Analysis } \\
\hline \multicolumn{12}{|c|}{ Alphaspec U. Liquid $2 p C i / L$} \\
\hline Uranium-233/234 & $\mathrm{U}$ & 1.90 & $+1-3.04$ & 5.18 & $+1-3.06$ & 2.00 & $\mathrm{pCi} / \mathrm{L}$ & & $\mathrm{TCl}$ & $09 / 20 / 0$ & $61012566391 \quad 1$ \\
\hline Uranium-235/236 & $\mathrm{U}$ & -0.32 & $+1-2.69$ & 6.40 & $+1-2.70$ & 2.00 & $\mathrm{pCi} / \mathrm{L}$ & & & & \\
\hline Uranium-238 & $\mathrm{U}$ & 1.08 & $+1-2.12$ & 3.24 & $+1-2.13$ & 2.00 & $\mathrm{pCi} / \mathrm{L}$ & & & & \\
\hline
\end{tabular}

The following Analytical Methods were performed

Description
DOE EML HASL-300, U-02-RC Modified
DOE EML HASL-300, U-02-RC Modified
Surrogate/Tracer recovery Test $\quad$ Alphaspec U, Liquid 2pCi/L
iranium-232
Notes:
The Qualifiers in this report are defined as follows :
* A quality control analyte recovery is outside of specified acceptance criteria
< Result is less than value reported
A Result is greater than value reported
A The TIC is a suspected aldol-condensation product
B Target analyte was detected in the associated blank
BD Results are either below the MDC or tracer recovery is low
C Analyte has been confirmed by GC/MS analysis
D Results are reported from a diluted aliquot of the sample
H Analytical holding time was exceeded
J Value is estimated
N/A Spike recovery limits do not apply. Sample concentration exceeds spike concentration by 4 X or more
R Sample results are rejected
U Analyte was analyzed for, but not detected above the MDL, MDA, or LOD.
UI Gamma Spectroscopy--Uncertain identification
X Consult Case Narrative, Data Summary package, or Project Manager concerning this qualifier
Y QC Samples were not spiked with this compound
A RPD of sample and duplicate evaluated using +/-RL. Concentrations are < $5 X$ the RL
h Preparation or preservation holding time was exceeded




\section{GENERAL ENGINEERING LABORATORIES, LLC}

2040 Savage Road Charleston SC 29407 - (843) 556-8171 - www.gel.com

\section{Certificate of Analysis}

Company: Bechtel Nevada Corp.

Address: 2621 Losee Road

M/S NTS273

North Las Vegas, Nevada 89030--4134 Report Date: November 28, 2006

Contact: $\quad$ Mr. Ted Redding

Project: Environmental Rad Analysis

Client Sample ID: $\quad$ DUS-R1

Sample ID:

171015002

Proiect: NEVA00106

Client ID: NEVA002

\begin{tabular}{lllllllll}
\hline Parameter & Qualifier & Result & Uncertainty & DL & TPU & RL & Units & DF Analyst Date \\
\hline
\end{tabular}

The above sample is reported on an "as received" basis. 


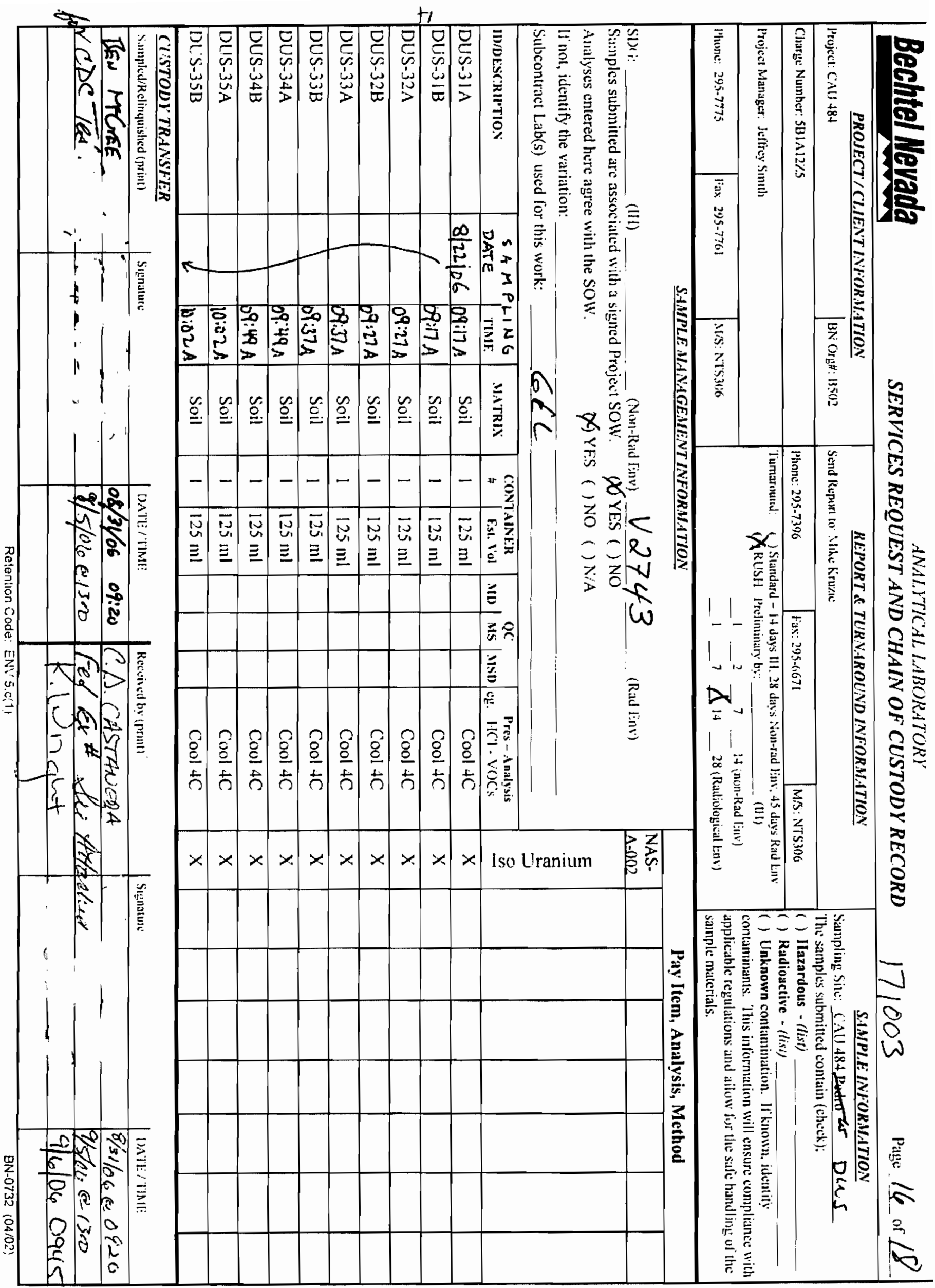




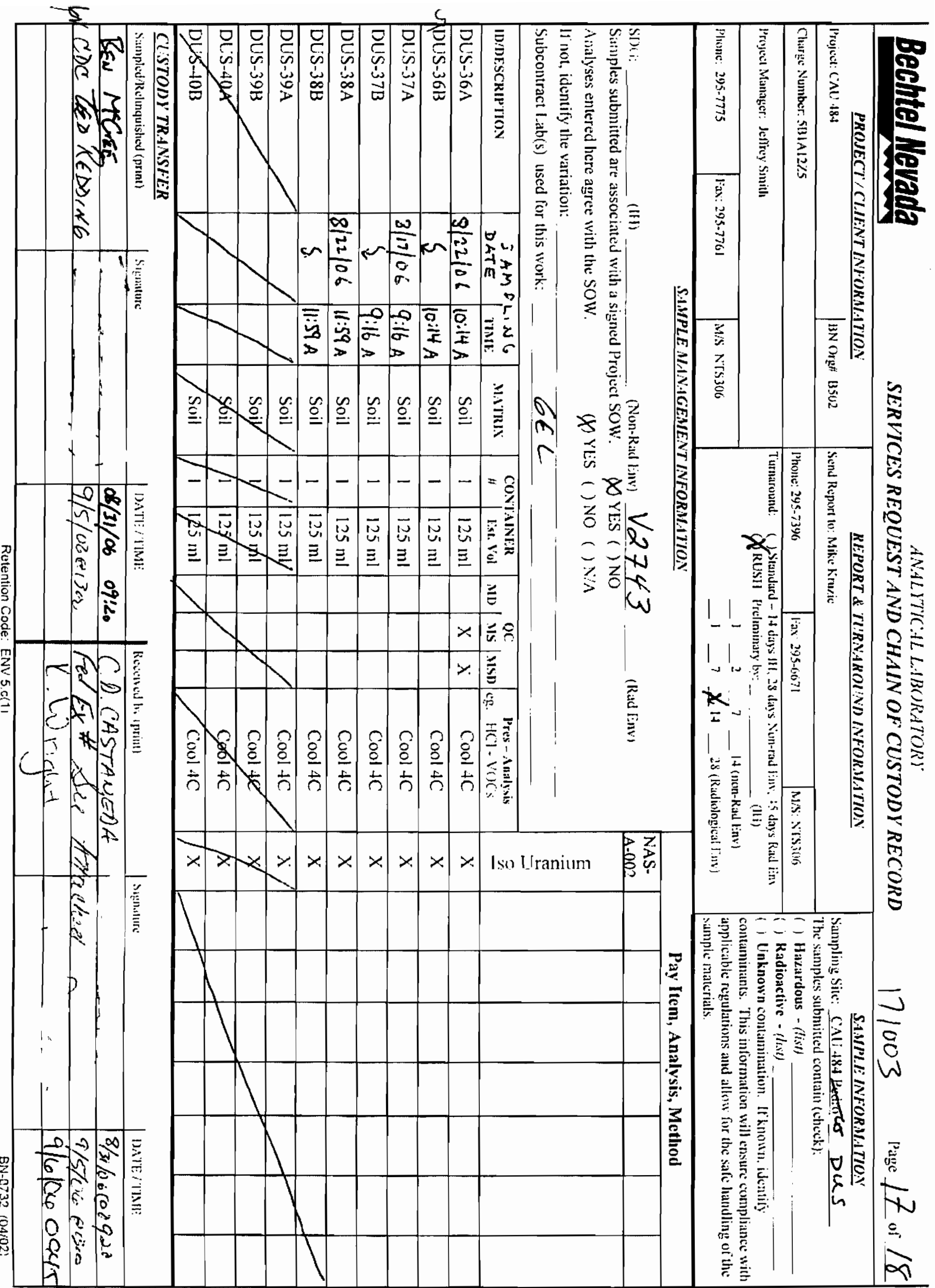




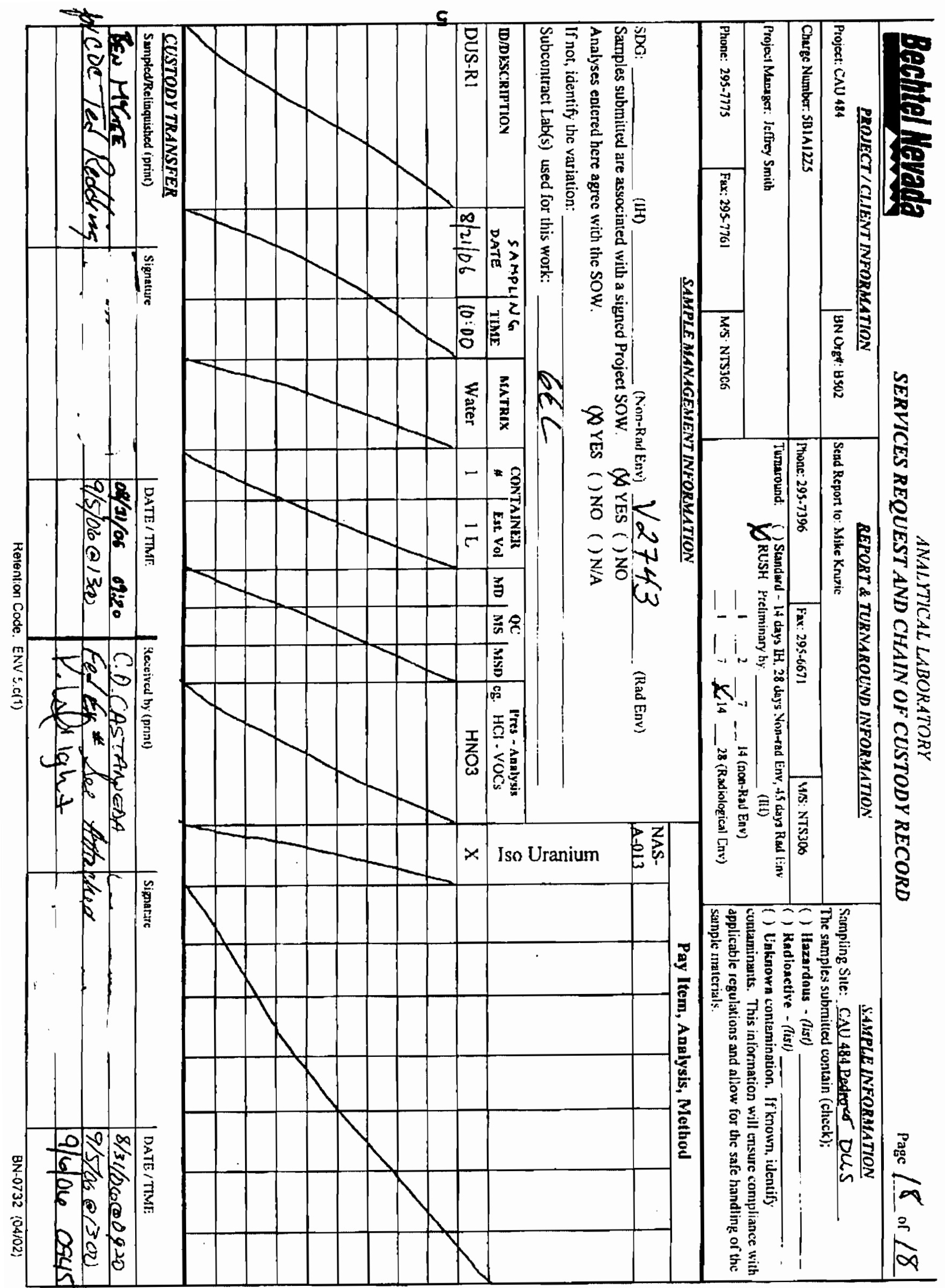




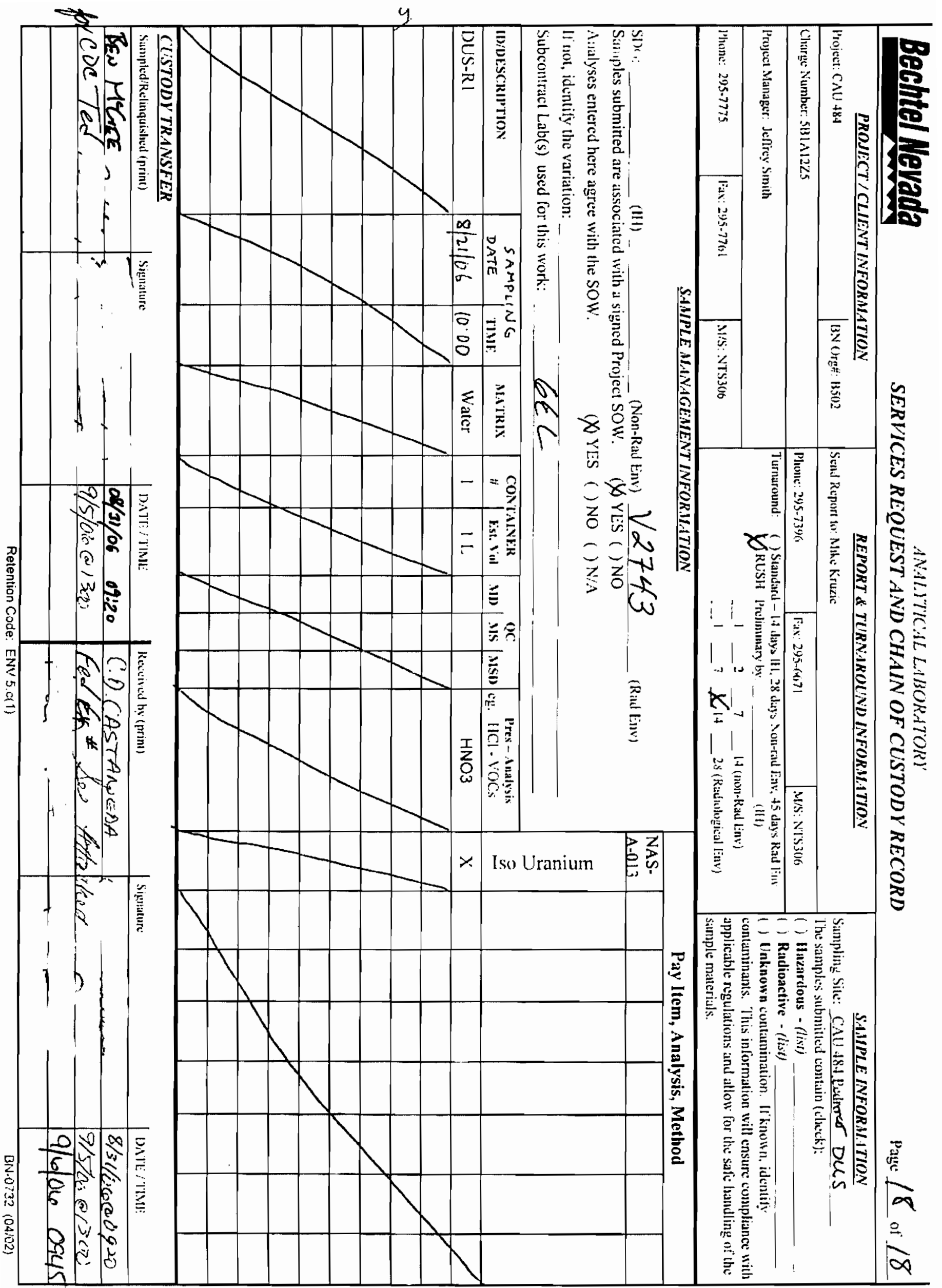




\title{
INVESTIGATION (GEOPROBE ${ }^{\circledR}$ ) SOIL SAMPLE ANALYTICAL RESULTS \\ CAS TA-52-004-TAAL METAL PARTICLE DISPERSION TEST
}

\author{
Chemical Data
}


1

\section{INORGANIC ANALYSES DATA SHEET}

EPA SAMPLE NO.

$$
01 \mathrm{~A}
$$

Contract: 60052

SAS NO. :

Lab Sample ID: 0609L894-001

Date Received: 09/07/06
Lab Name: LIONVILLE LABORATORY

Lab Code: LVLI

Matrix (soil/water): SoII_

Level (low/med): LOW

: Solids :

$-90 . \overline{7}$
Concentration Units (ug/L or $\mathrm{mg} / \mathrm{kg}$ dry weight): MG/KG

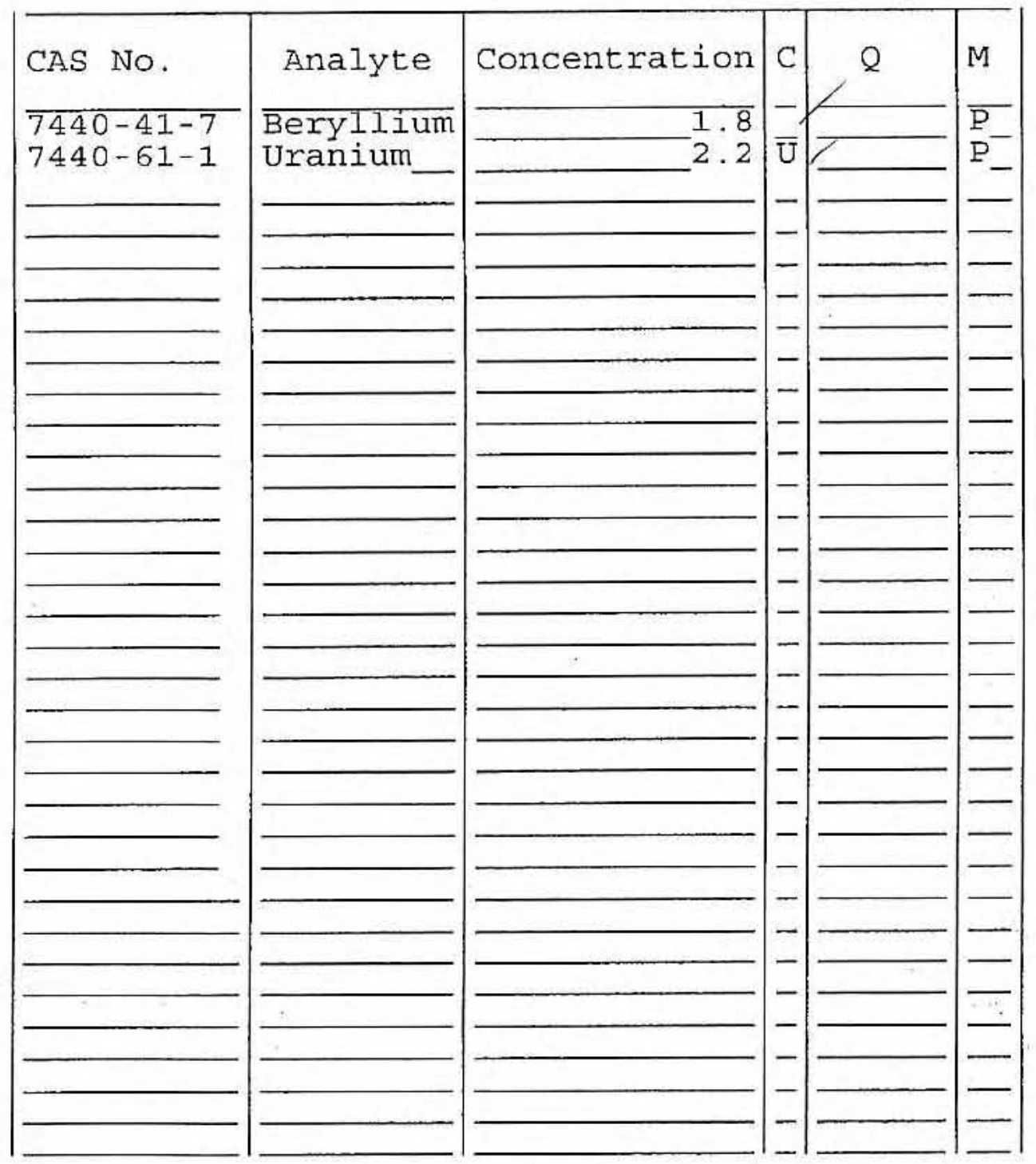

Color Before:

Clarity Before:

Clarity After:
Texture :

Artifacts :

Comments:

MPDT-01A 
1

INORGANIC ANALYSES DATA SHEET
EPA SAMPLE NO.

$$
\text { O1B }
$$

Contract: 60052 SAS NO. :

Lab Sample ID: 0609L894-0̄02 Date Received: 09/07/06
Lab Name: LIONVILLE_LABORATORY

Lab Code: LVLI Matrix (soil/water): SOIL Level (low/med): \% Solids :

LOW -
-89.0

Case NO.: MPDT

Concentration Units (ug/L or $\mathrm{mg} / \mathrm{kg}$ dry weight): MG/KG

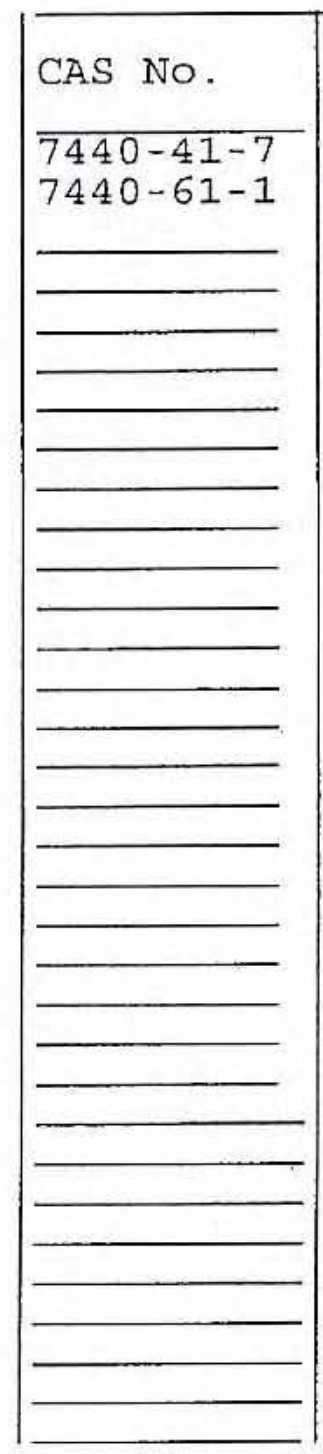

Color Before: Color After:

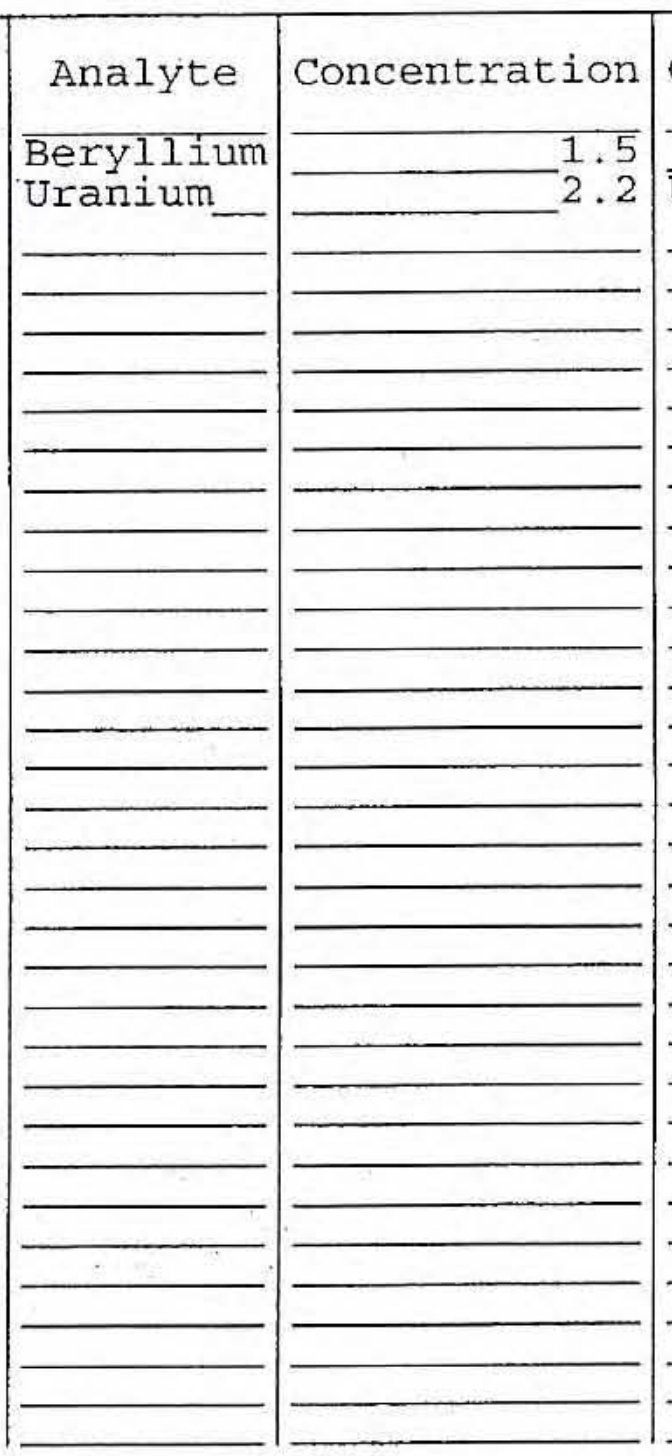

Clarity Before: Clarity After:

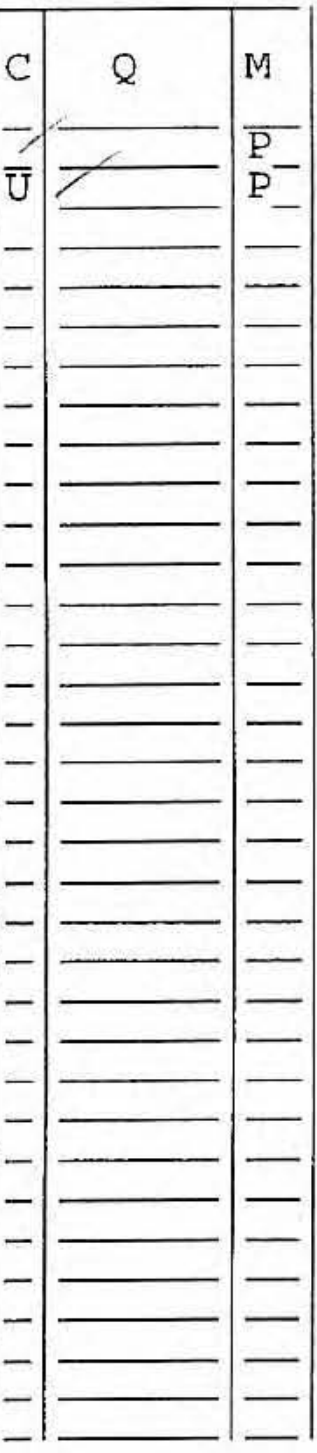

Texture: Artifacts :

Comments :

MPDT - O1B 
1
INORGANIC ANALYSES DATA SHEET

EPA SAMPLE NO.

$02 \mathrm{~A}$

Contract: 60052

SAS No.:

Lab Sample ID: 0609L894-ס̄03

Date Received: 09/07/06 $\begin{array}{ll}\text { Lab Code: LVLI } & \text { Ca } \\ \text { Matrix (soil/water): SOIL } \\ \text { Level (low/med): }\end{array}$

Lab Code: LVLI
Matrix (soil/water): SOIL
Level (low/med):

Lolids:
$-91 \overline{.4}$

Concentration Units (ug/L or $\mathrm{mg} / \mathrm{kg}$ dry weight): MG/KG

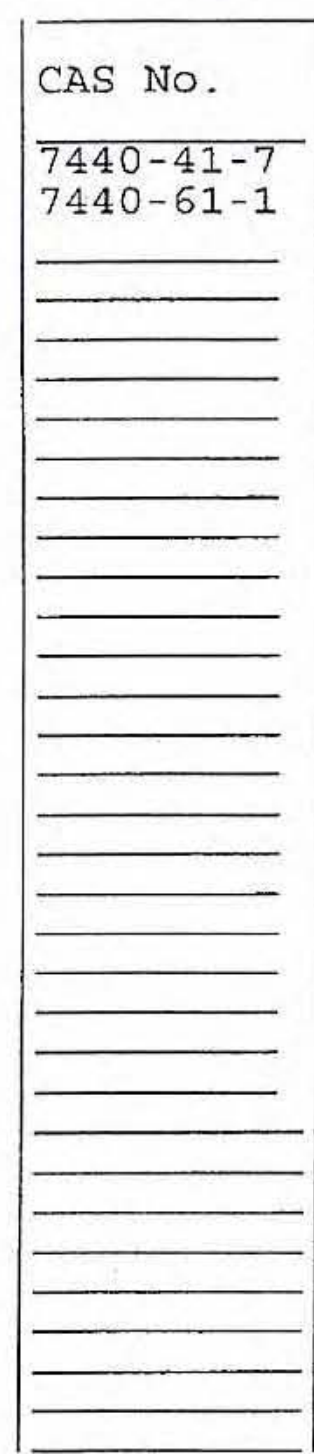

Color Before:

Color After:

Comments:

MPDT-02A

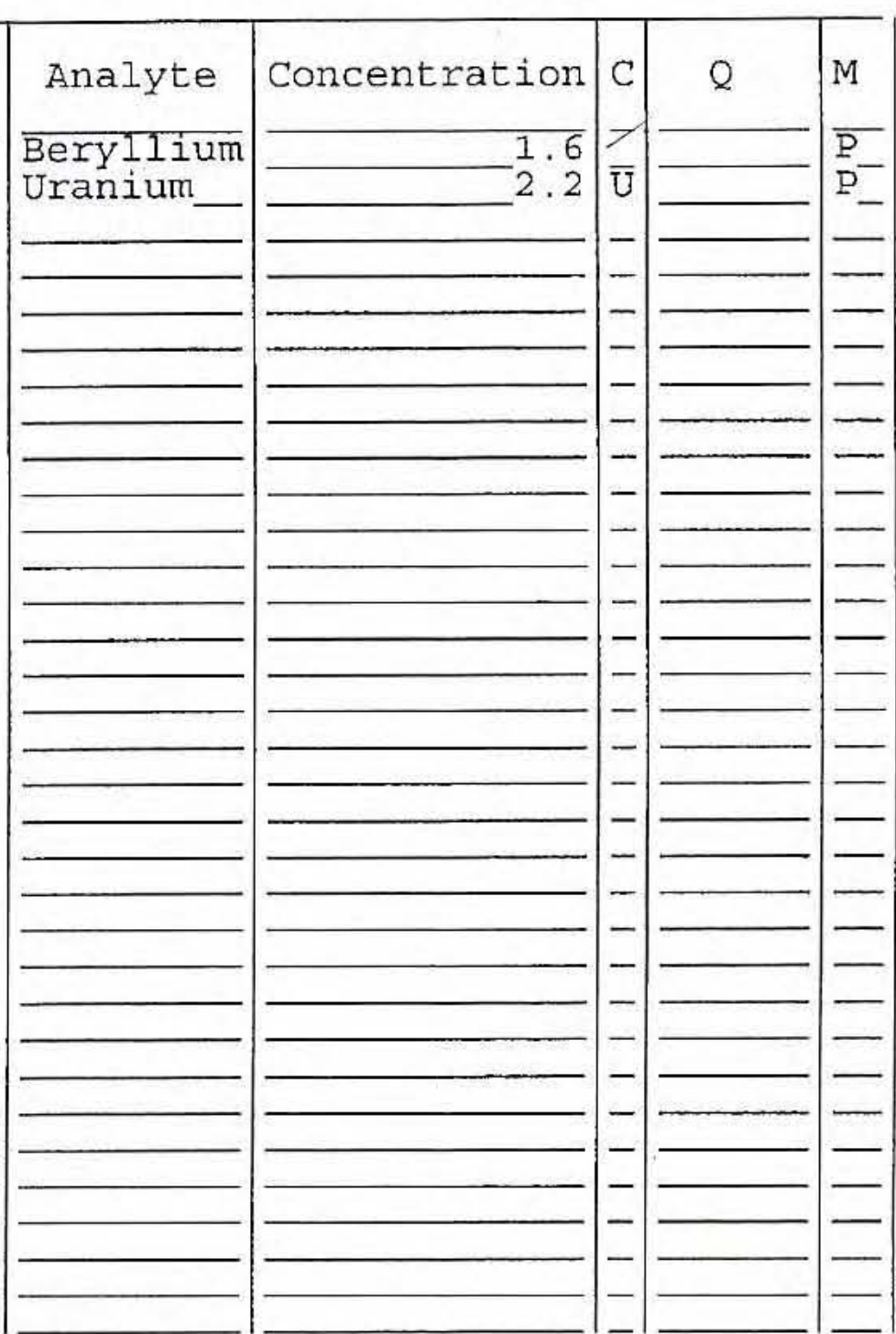

Clarity Before: Clarity After:
Texture:

Artifacts: 


\section{1 INORGANIC ANAIYSES DATA SHEET}

EPA SAMPLE NO.

Lab Name: LIONVILLE LABORATORY

Lab Code: LVLI Matrix (soil/water): SoIL Level (low/med): LOW : Solids:

$-89 \overline{.0}$
Contract : 60052

SAS NO. :

SDG NO.: V2745

$02 \mathrm{~B}$
Lab Sample ID: 0609L894-004

Date Received: 09/07/06

Concentration Units (ug/L or $\mathrm{mg} / \mathrm{kg}$ dry weight): MG/KG

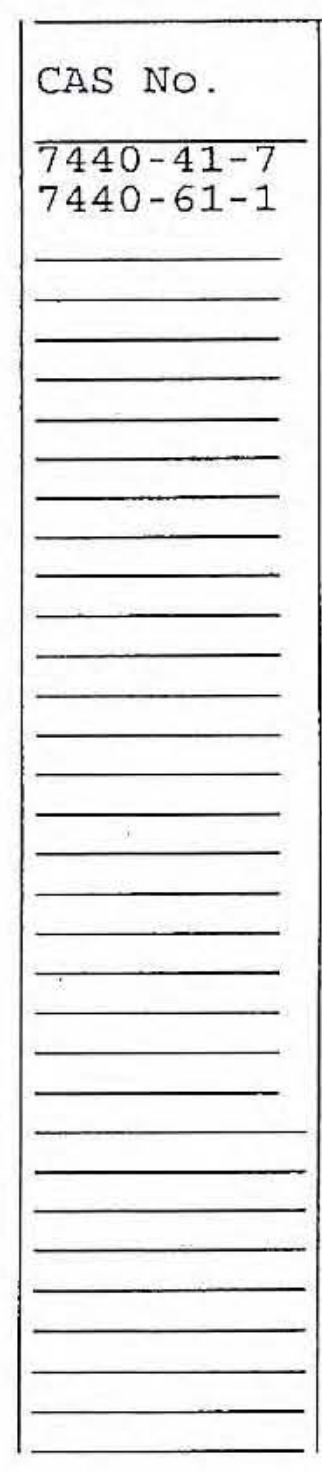

Color Before: Color After:

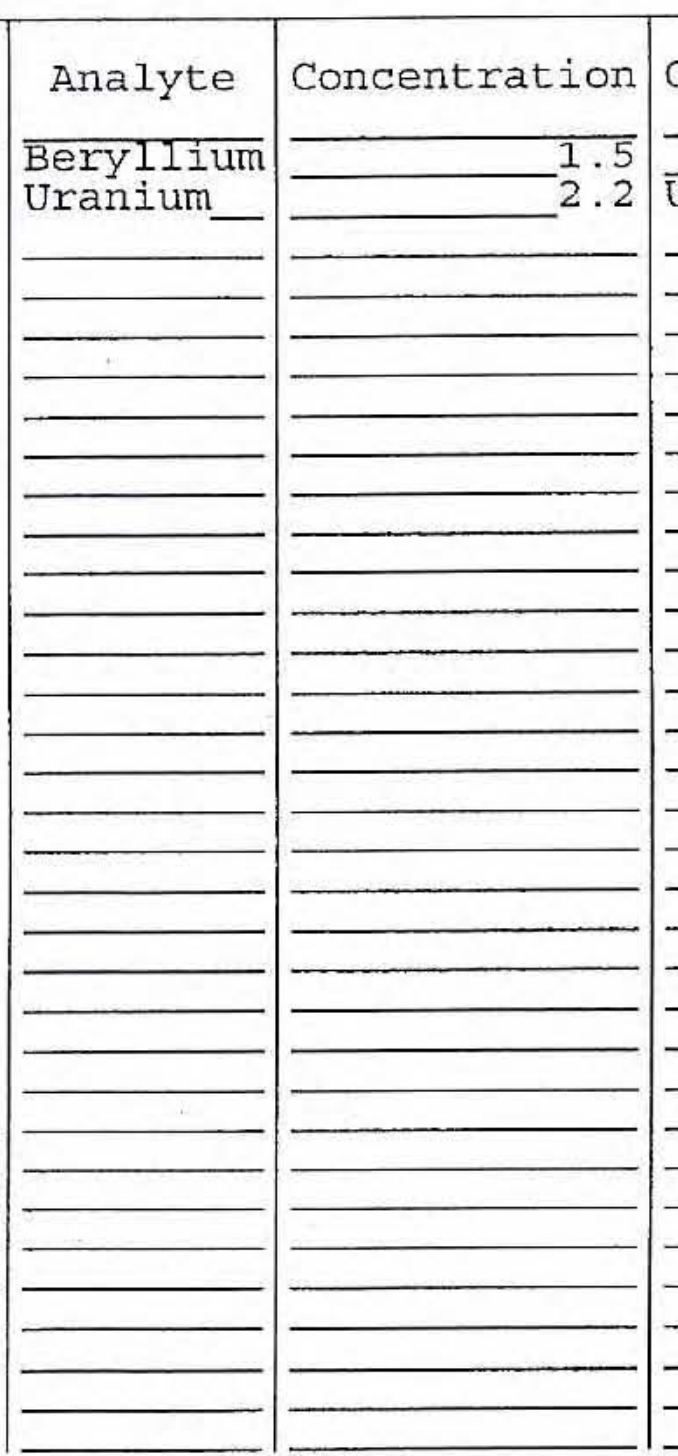

Clarity Before: Clarity After:

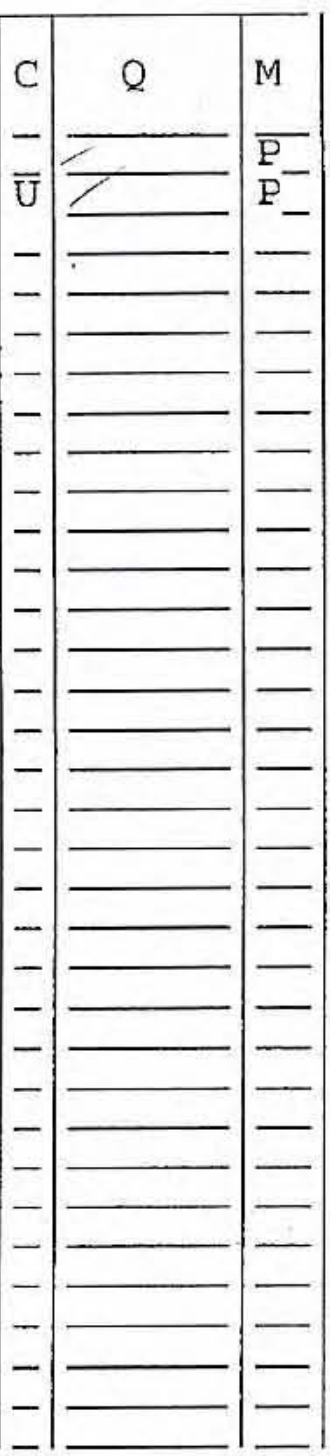

Texture: Artifacts :

Comments:

MPDT $-02 B$ 


\section{INORGANIC ANALYSES DATA SHEET}

EPA SAMPLE NO.

$03 \mathrm{~A}$

Contract: 60052

SAS No.:

Lab Sample ID: 0609L894-005

Date Received: 09/07/06
Lab Name: LION
Lab Code: LVII Matrix (soil/wat
Level (low/med): \% Solids :

\section{Case No.: MPDT}

$$
\begin{aligned}
& \text { SOIL } \\
& \text { LOW - } \\
& -91.2
\end{aligned}
$$

Concentration Units (ug/L or $\mathrm{mg} / \mathrm{kg}$ dry weight): MG/KG

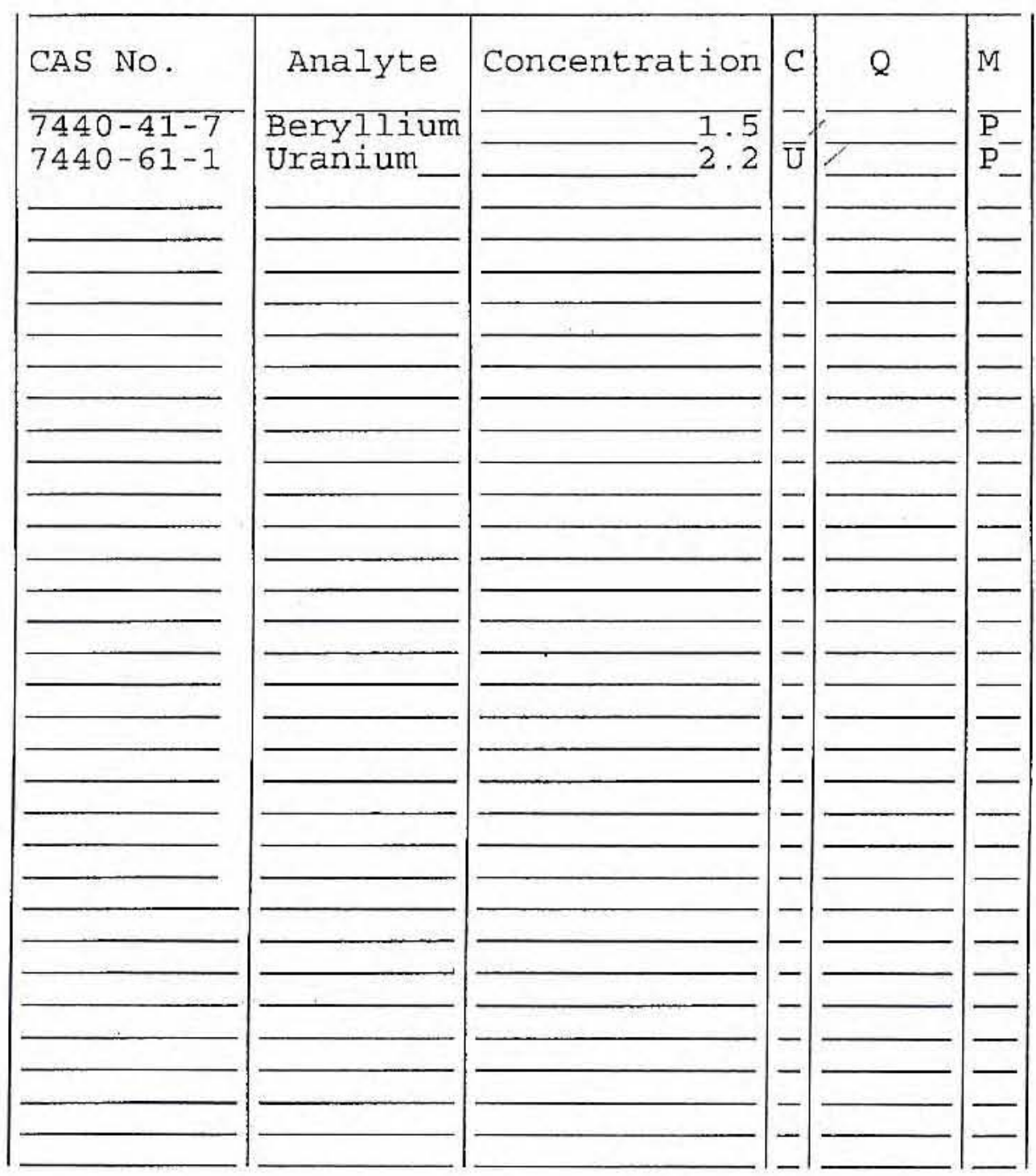

Color Before:

Color After:
Clarity Before:

Clarity After:
Texture:

Artifacts:

Comments :

MPDT-03A 


\section{1}

INORGANIC ANALYSES DATA SHEET
EPA SAMPLE NO.

$$
\text { O3B }
$$

Contract: 60052

SAS NO. :

SDG NO: V2745

Lab Sample ID: 0609L894-0006

Date Received: 09/07/06
Matrix (soil/water): SoIL

Level (low/med): LOW

$\%$ Solids :
Concentration Units (ug/L or $\mathrm{mg} / \mathrm{kg}$ dry weight): MG/KG

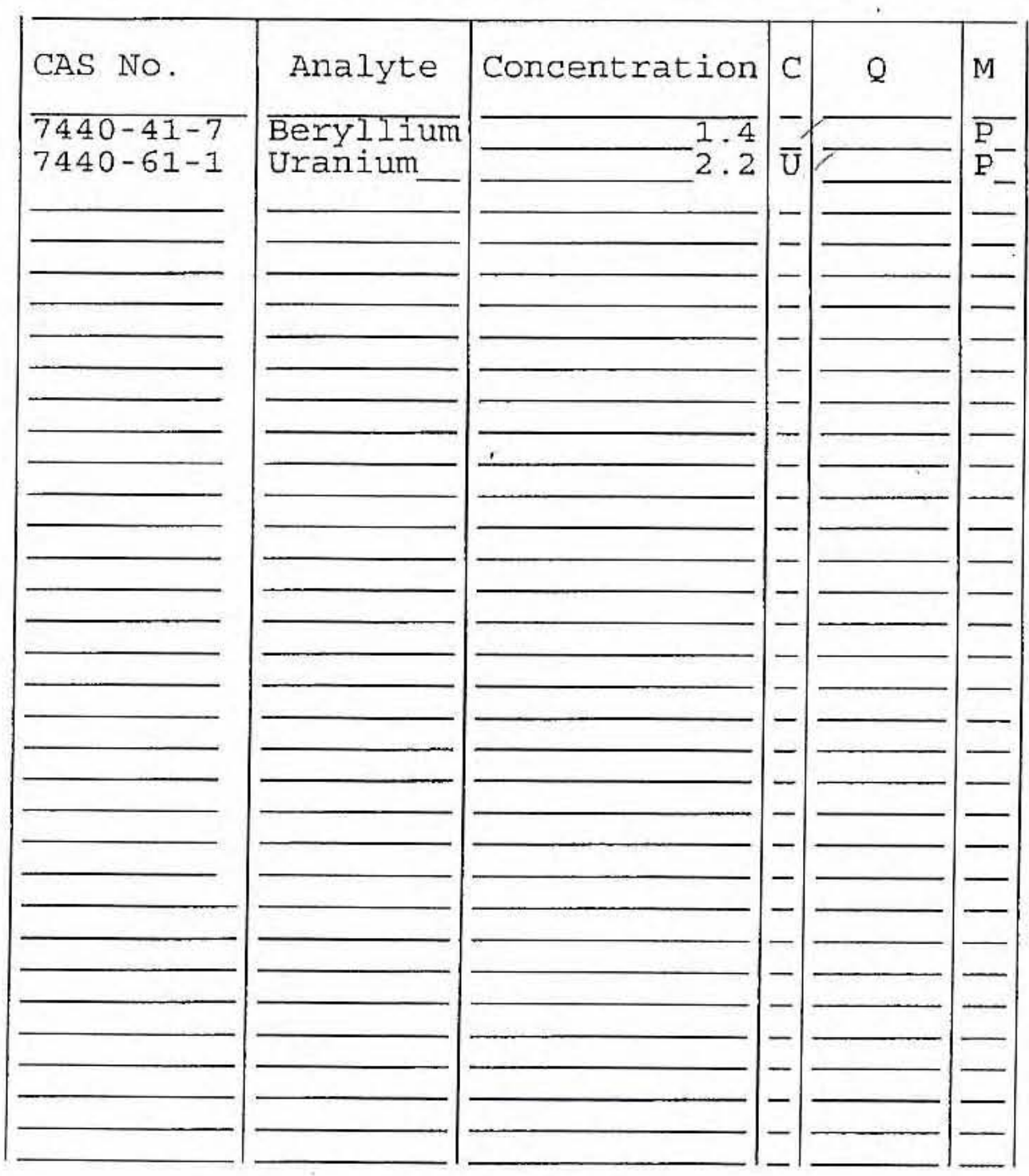

Clarity Before: Clarity After:
Texture:

Artifacts:
Color Before: Color After:

Comments :

MPDT-03B 


\section{1}

INORGANIC ANALYSES DATA SHEET
EPA SAMPLE NO.

$04 \mathrm{~A}$

Contract: 60052

SAS NO. :

SDG No.: V2745

Lab Sample ID: 06091894-0̄07

Date Received: 09/07/06
Lab Name: LION
Lab Code: LVLI

Matrix (soil/water): SoIL

Level (low/med): LOW

Solids:
$-91 . \overline{1}$

Concentration Units (ug/L or $\mathrm{mg} / \mathrm{kg}$ dry weight): MG/KG

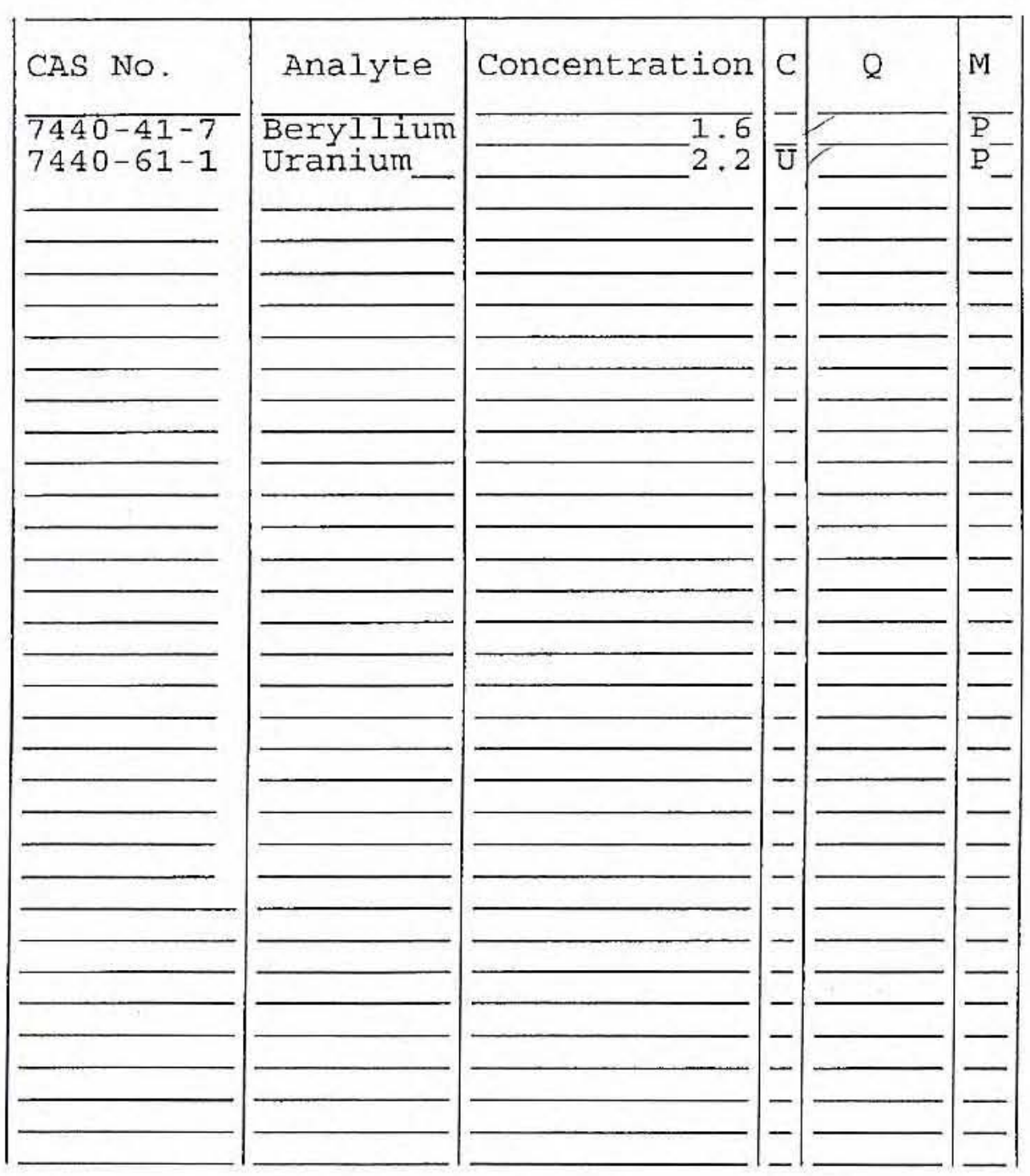

Clarity Before:

Clarity After:
Texture:

Artifacts:

Color Before
Color After:

Comments :

MPDT-04A 
1 INORGANIC ANALYSES DATA SHEET
Lab Name: LIONVILLE_LABORATORY

Lab code: LVLI

Matrix (soil/water): SOIL

Level (low/med) :

Solids:
Contract: 60052

SAS No. :
SDG No.: V2745

EPA SAMPLE NO.

\section{O4B}

Lab Sample ID: 0609L894-0008

Date Received: 09/07/06

\section{Concentration Units (ug/L or $\mathrm{mg} / \mathrm{kg}$ dry weight): MG/KG}

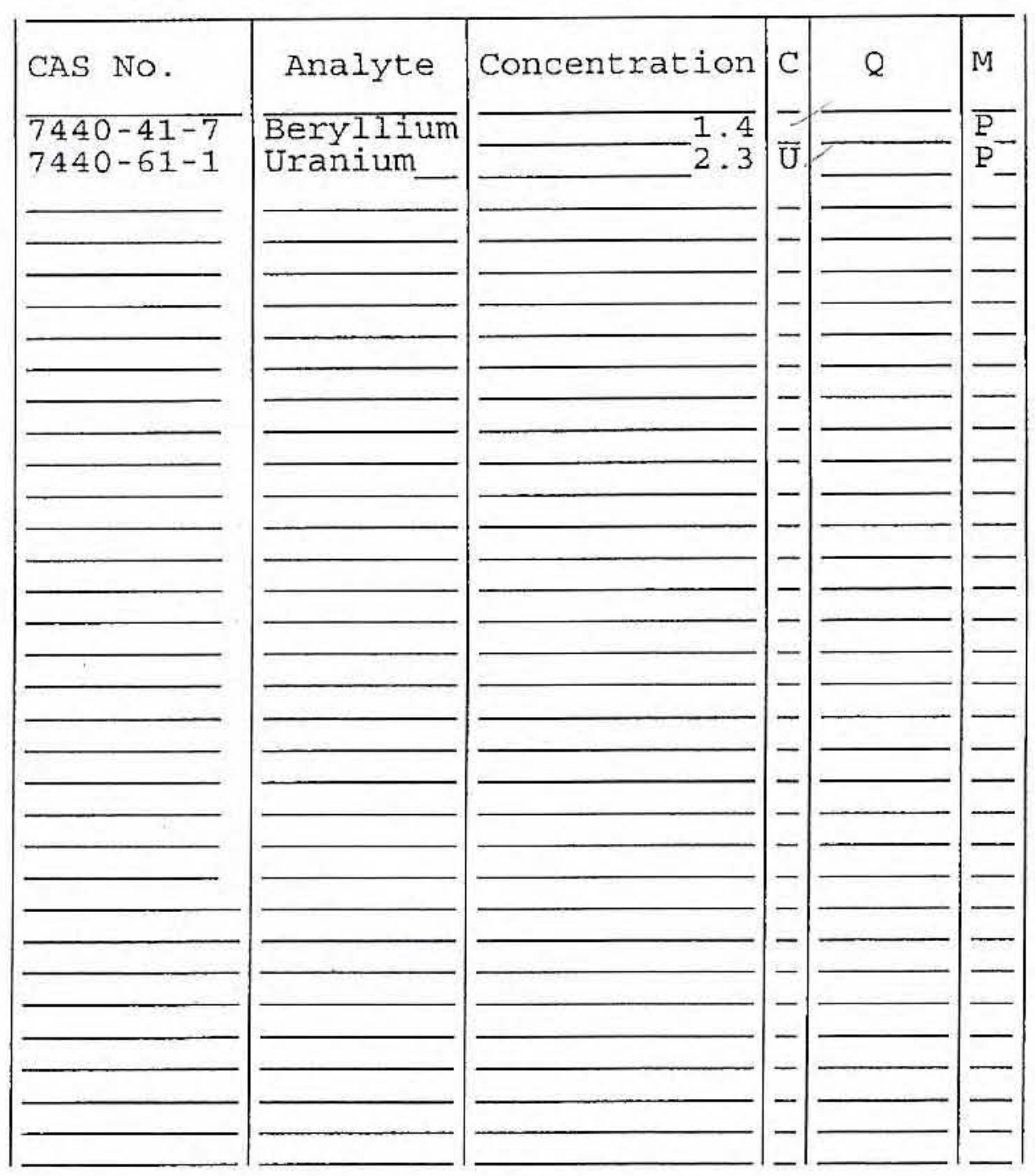

Color Before:

Color After:
Clarity Before:

Clarity After:
Texture:

Artifacts :

Comments :

MPDT $-04 B$ 
EPA SAMPLE NO.

$$
\text { 05A }
$$

Lab Name: IIONVILIE_LABORATORY

Contract: 60052

Case No.: MPDT_ SAS No.:

Lab Sample ID: 0609L894-0009

$\begin{array}{ll}\text { Matrix (soil/water): } & \text { SOIL } \\ \text { Level (low/med): } & \text { LOW } \\ \text { \% Solids: } & -90.7\end{array}$

$\begin{array}{ll}\text { Matrix (soil/water): } & \text { SOIL } \\ \text { Level (low/med): } & \text { LOW } \\ \text { \% Solids: } & -90.7\end{array}$

Date Received: 09/07/06

Concentration Units (ug/L or $\mathrm{mg} / \mathrm{kg}$ dry weight): $\mathrm{MG} / \mathrm{KG}$

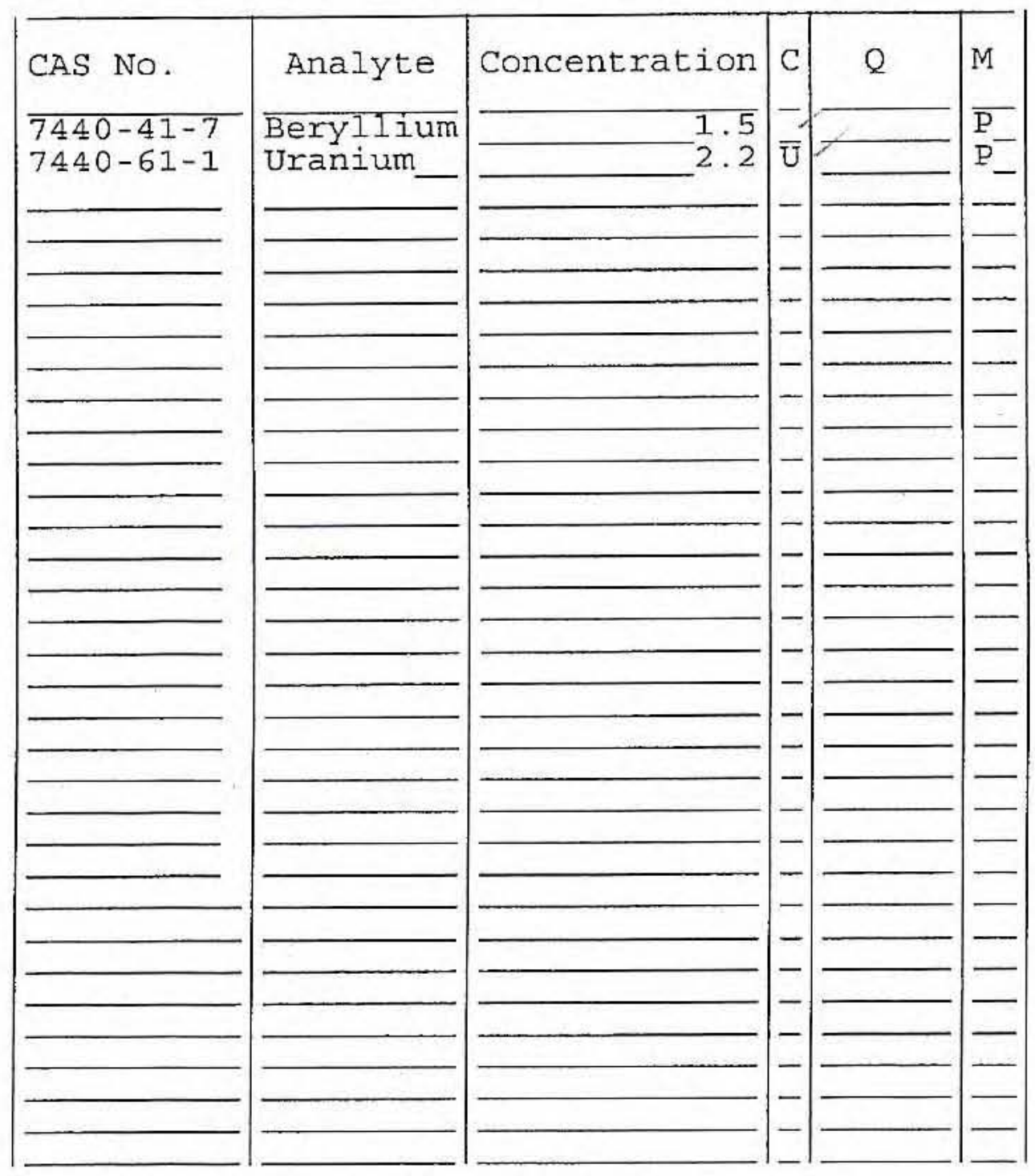

Color Before:

Color After:
Clarity Before:

Clarity After:
Texture: Artifacts:

Comments :

MPDT-05A 


\section{1 \\ INORGANIC ANALYSES DATA SHEET}

Lab Name: LIONVILLE_LABORATORY

Lab Code: IVLI.

Matrix (soil/water): SOIL Level (low/med): \% Solids :
Contract: 60052

SAS NO.:

SDG NO.: V2745

Lab Sample ID: 0609L894-0̄10

Date Received: 09/07/06

Concentration Units (ug/L or $\mathrm{mg} / \mathrm{kg}$ dry weight): MG/KG

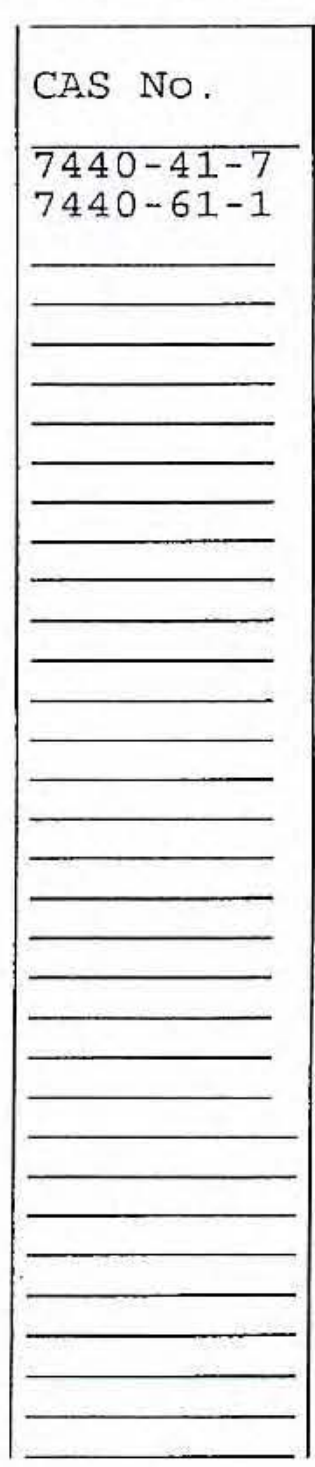

Color Before:

Color After:

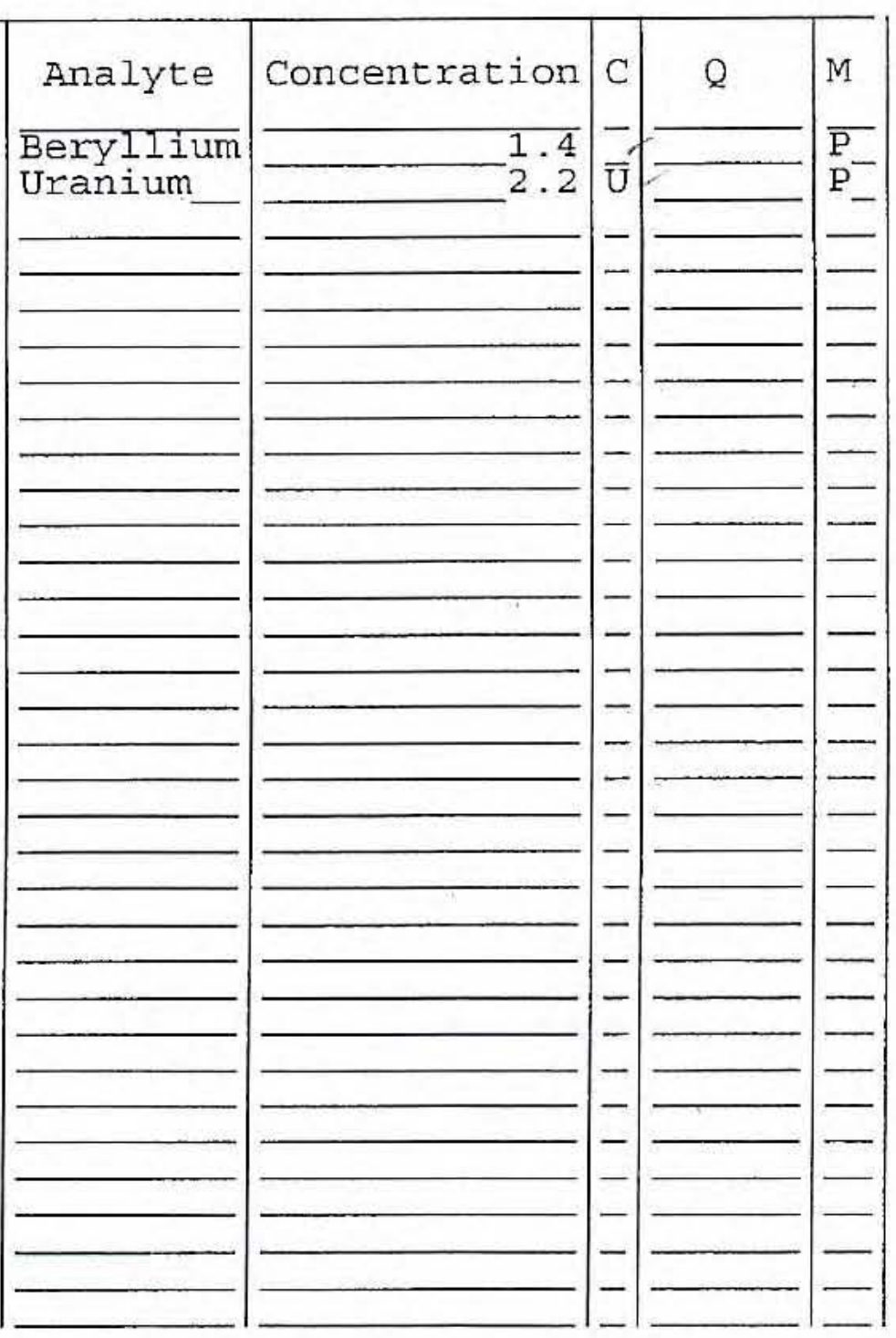

Clarity Before: Clarity After:
Texture:

Artifacts:

Comments :

MPDT $-05 B$ 
1

INORGANIC ANALYYSES DATA SHEET
EPA SAMPLE NO.

Contract: 60052

SAS NO.:

Lab Sample ID: 0609L894-0̄11 Date Received: 09/07/06
O6A
Lab Name: LION
Lab Code: LVLI Matrix (soil/water): SOIL Level (low/med): : Solids:
Case No.: MPDT

LOW
-91.6

Concentration Units (ug/L or $\mathrm{mg} / \mathrm{kg}$ dry weight): MG/KG

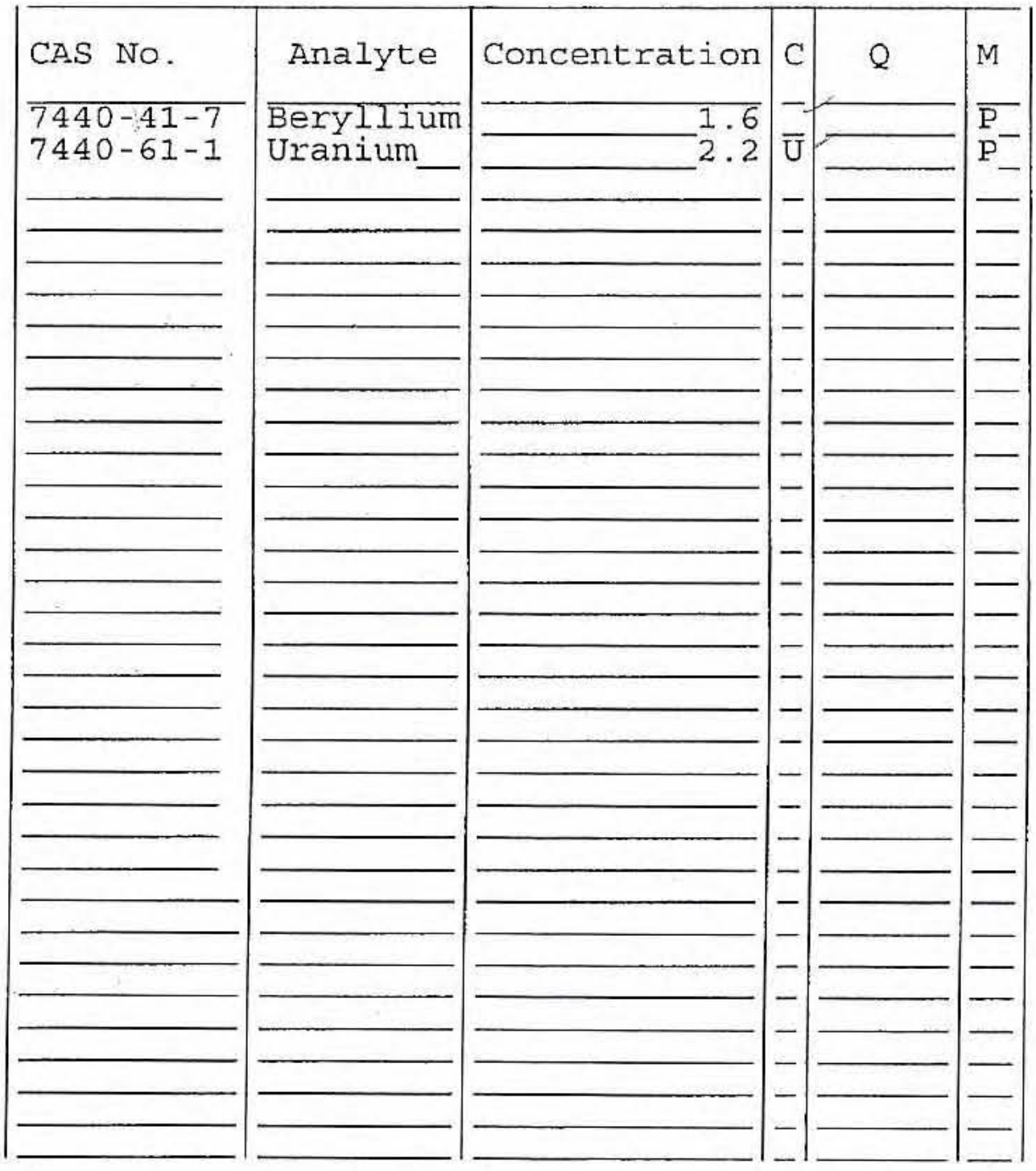

Color Before:

Color After:
Clarity Before:

Clarity After:
Texture:

Artifacts:

Comments :

MPDT-06A. 
Lab Name: LIONVILLE_LABORATORY Lab Code: LVLI Matrix (soil/water): SoIL Level (low/med): \% Solids:
LOW

$-88 \overline{.7}$
Contract: 60052 SAS NO.:

SDG NO.: V2745 Lab Sample ID: 0609L894-0̄12 Date Received: 09/07/06

Concentration Units (ug/L or $\mathrm{mg} / \mathrm{kg}$ dry weight): MG/KG
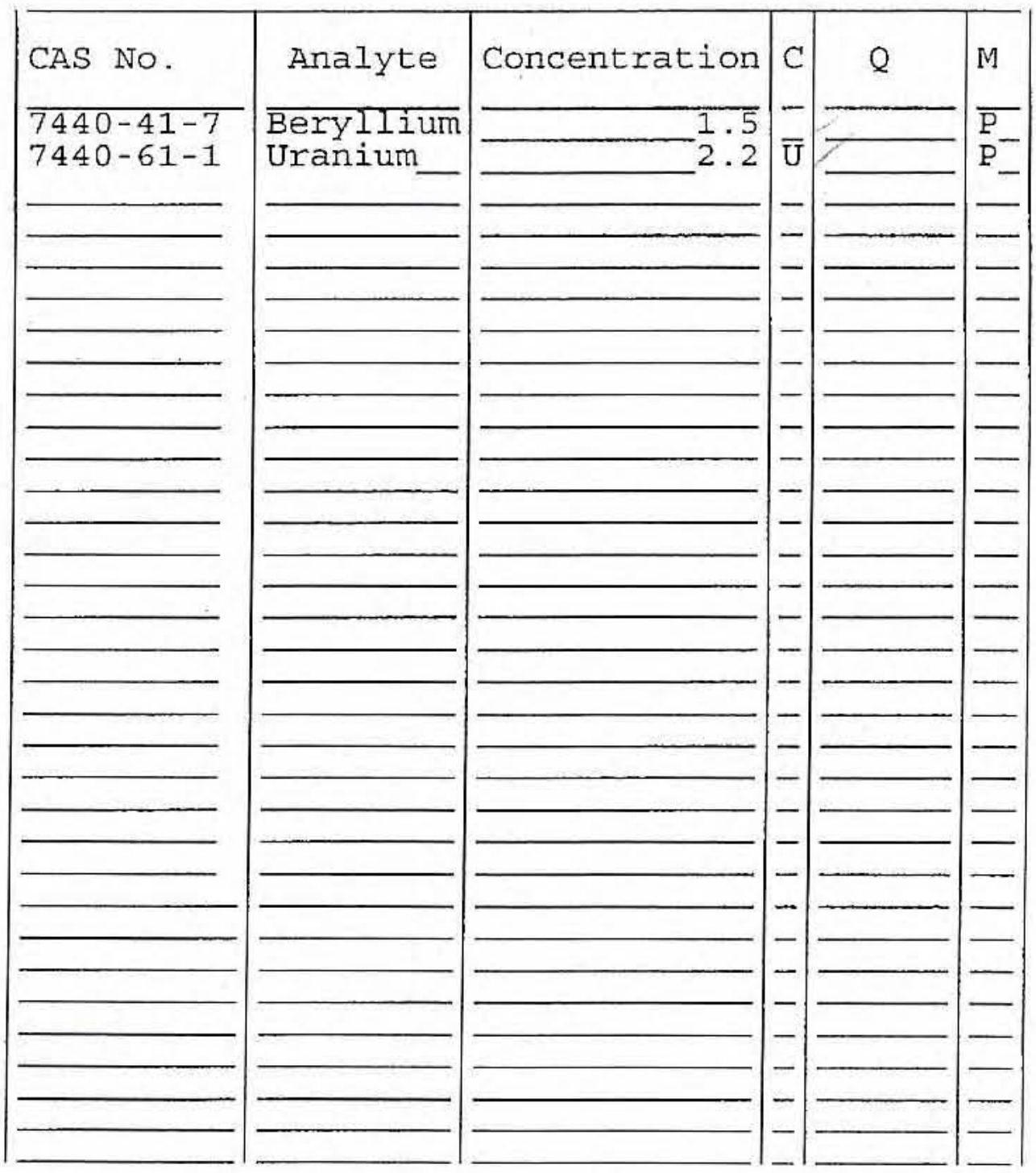

Color Before:

Color After:

\section{$06 B$}


Iab Name: IIONVILLE_LABORATORY Lab Code: LVLI Matrix (soil/water): Level (low/med): $\div$ Solids:

\section{SOIL} LOW$$
-90 \overline{7}
$$

Contract: 60052 SAS NO.:

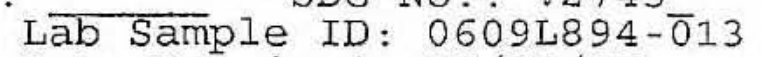

Date Received: 09/07/06

Concentration Units (ug/L or $\mathrm{mg} / \mathrm{kg}$ dry weight): MG/KG

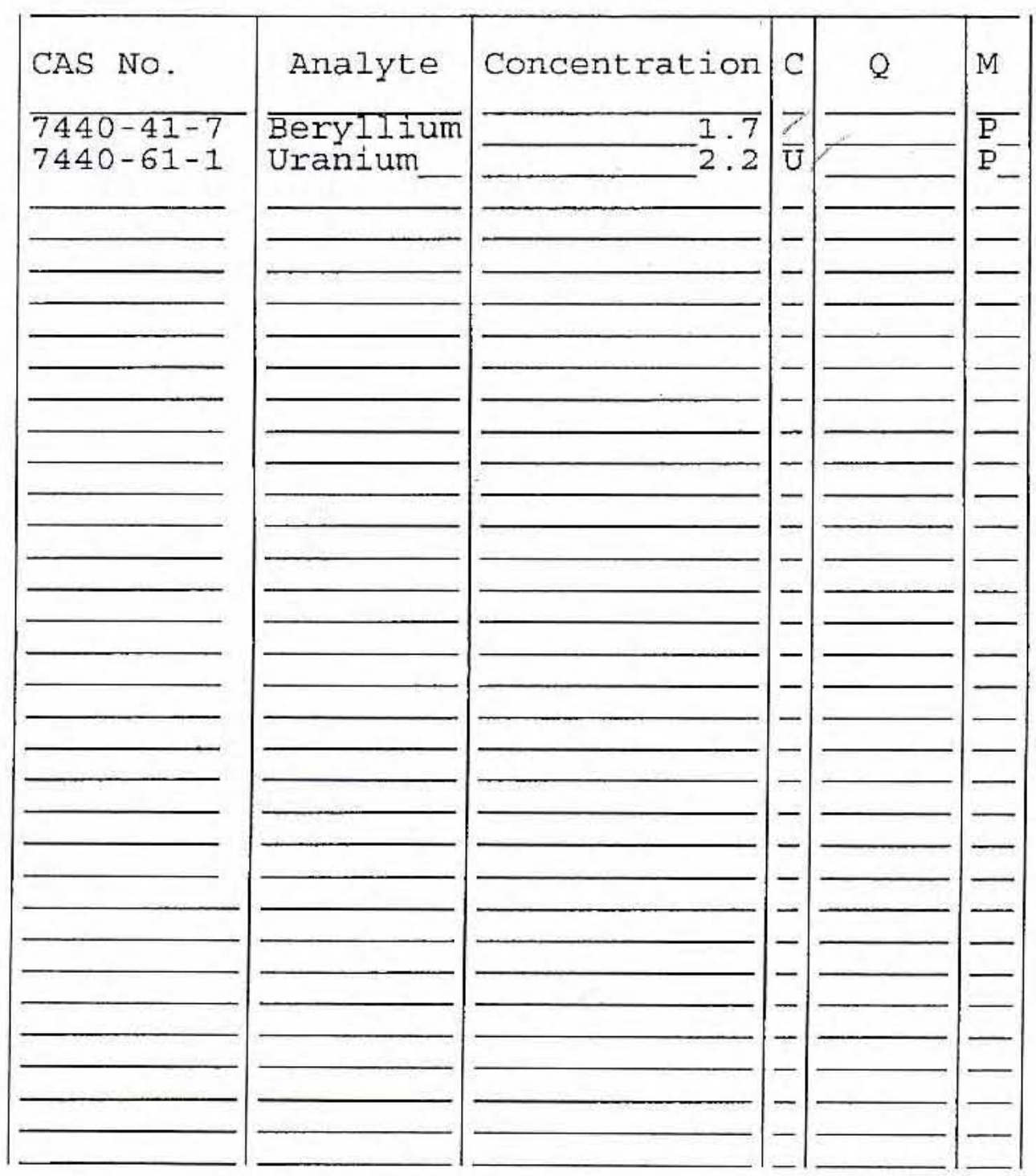

Color Before: Color After:

Comments :

MPDT-O7A

$$
07 \mathrm{~A}
$$

SDG NO.: V2745

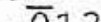




\section{INORGANIC ANAIYYSES DATA SHEET}

EPA SAMPLE NO.

Lab Name: LIONVILLE_LABORATORY

\section{Iab Code: LVLI}

Matrix (soil/water) : SOIL

Level (low/med): LOW

$\because$ Solids:
Contract: 60052

$$
\text { 07B }
$$

SDG NO.: V2745

Lab Sample ID: 06091894-0̄14 Date Received: 09/07/06

Concentration Units (ug/L or $\mathrm{mg} / \mathrm{kg}$ dry weight): MG/KG

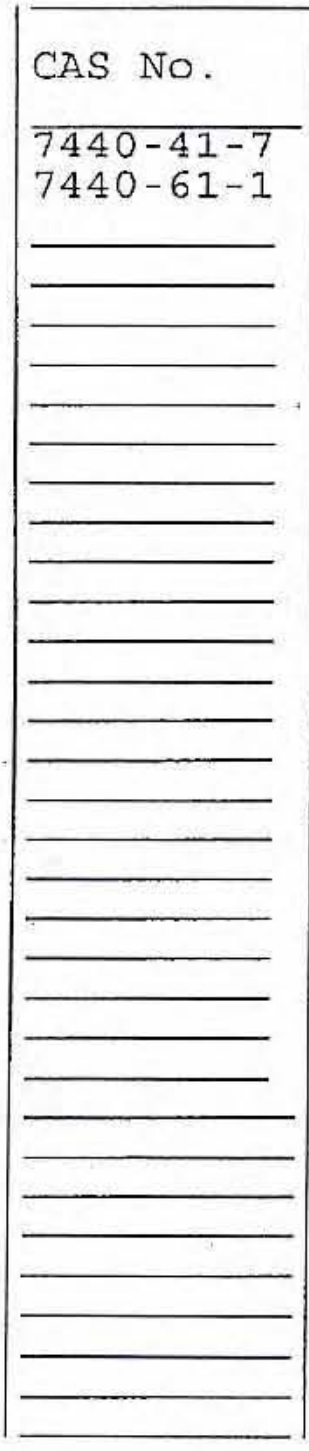

Color Before:

Color After:

Comments :

MPDT-07B

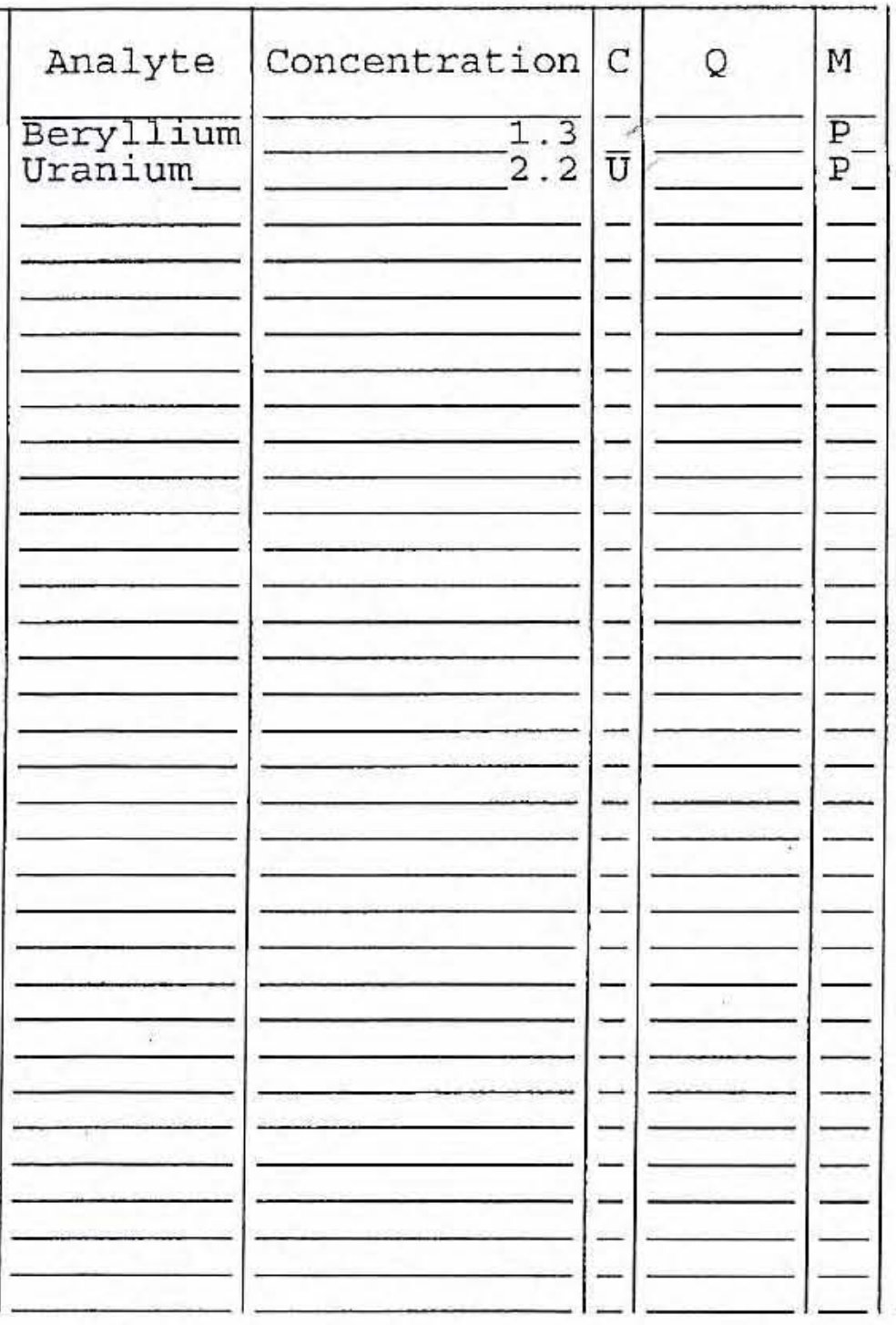

Clarity Before: Clarity After:
Texture:

Artifacts: 
1 INORGANIC ANALYSES DATA SHEET
EPA SAMPLE NO.

\section{$08 \mathrm{~A}$}

SDG NO.: V2745

Lab Sample ID: 06094894-0̄15

Date Received: 09/07/06
Case No.:MPDT_ SAS No.:

LOW-
Lab Name: LION
Lab Code: LVLI

Level (1ow/med) Solids :

Concentration Units (ug/L or $\mathrm{mg} / \mathrm{kg}$ dry weight): MG/KG

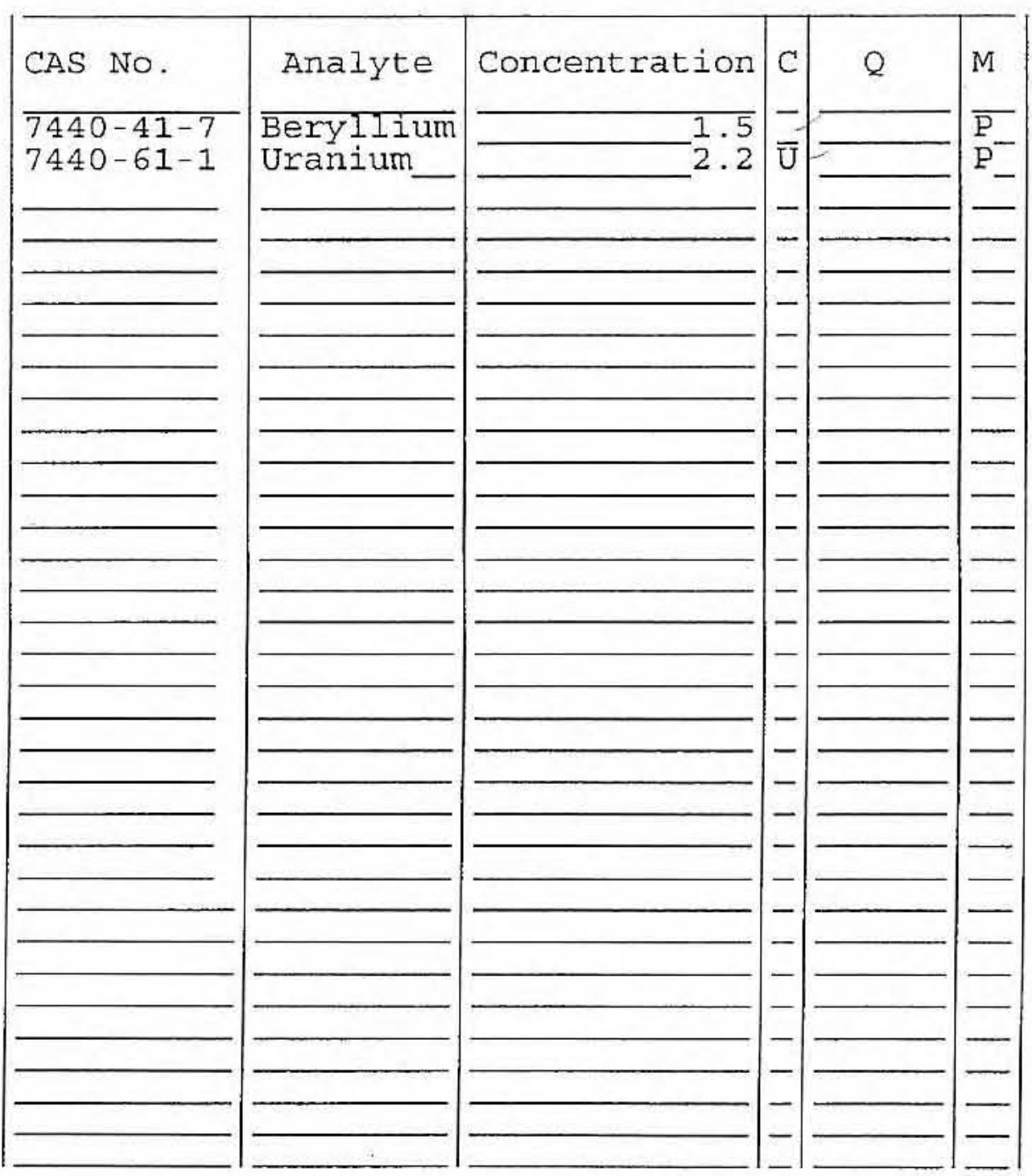

Color Before:

Color After:

Comments:

MPDT-08A
Clarity Before:

Clarity After:
Texture:

Artifacts: 
1 INORGANIC ANALYSES DATA SHEET
EPA SAMPLE NO.

\section{O8B}

Contract: 60052

SAS NO.:

Lab Sample ID: 06091894-0̄16

Date Received: 09/07/06
Lab Code: LVLI

Matrix (soil/water):

Level (low/med):

Solids:
SOIL

LOW

$-89 \overline{.4}$

Concentration Units (ug/L or $\mathrm{mg} / \mathrm{kg}$ dry weight): MG/KG

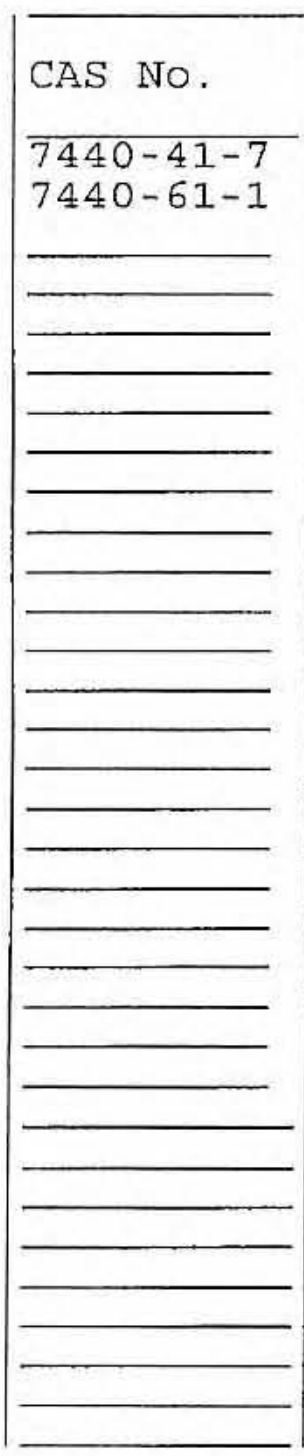

Color Before: Color After:

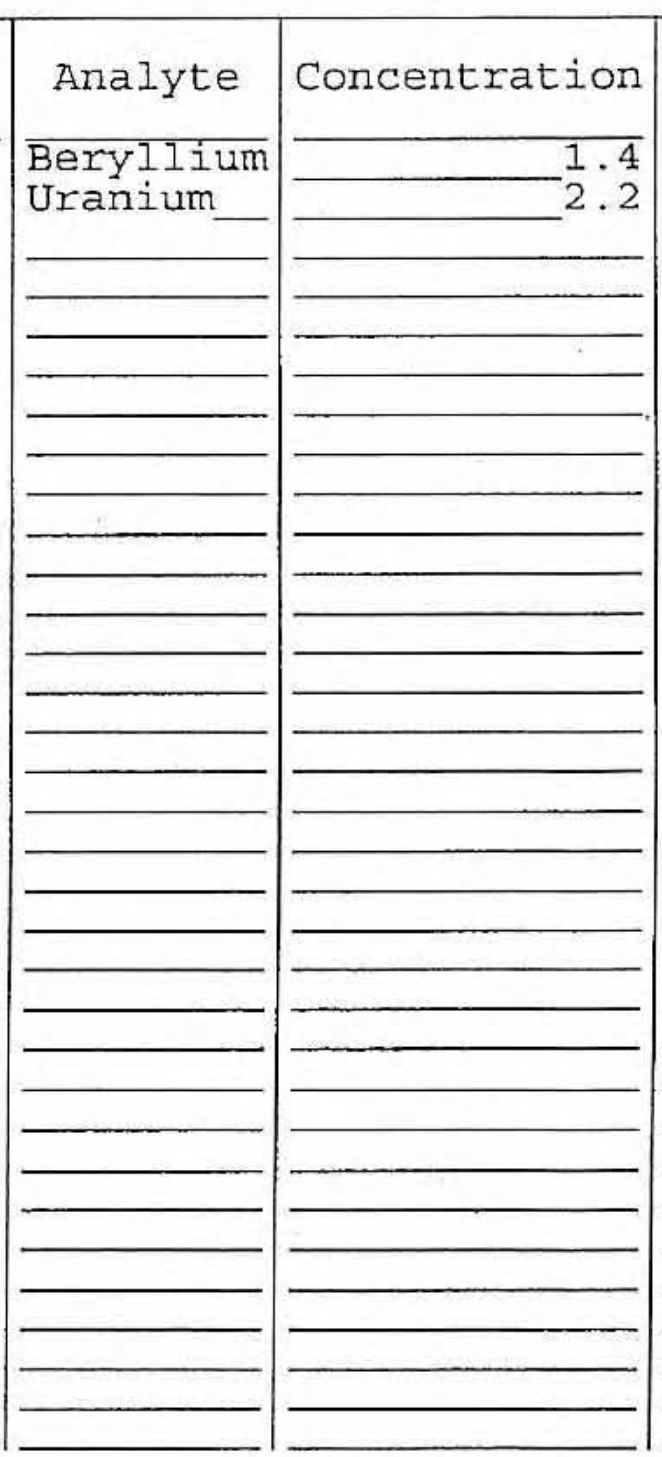

Clarity Before:

Clarity After:

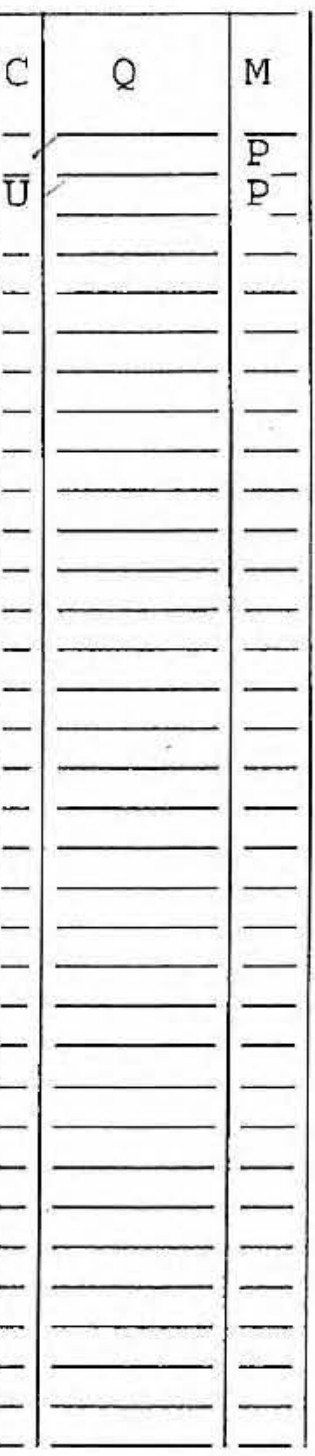

Texture : Artifacts :

Comments :

MPDT-08B 
1
INORGANIC ANALYSES DATA SHEET

EPA SAMPLE NO.

$$
\text { 09A }
$$

Contract: 60052

SAS NO. :

Case No.: MPDT

$$
\begin{aligned}
& \text { SOIL_ } \\
& \text { LOW } \\
& \text {-91.7 }
\end{aligned}
$$

Lab Sample SDG No.: V2745

Date Received: 09/07/06

Concentration Units (ug/L or $\mathrm{mg} / \mathrm{kg}$ dry weight): MG/KG

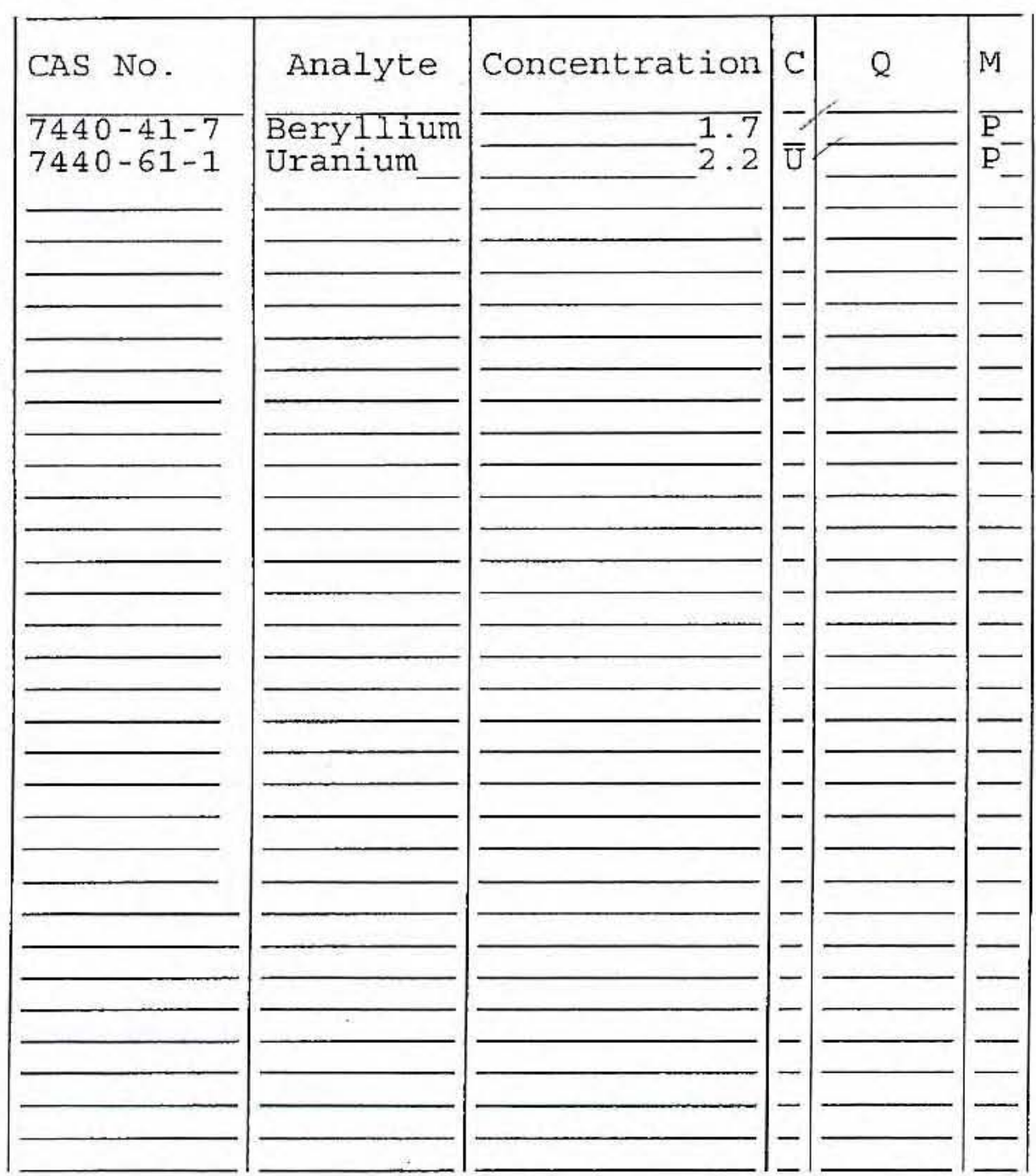

Color Before: Color After:

Comments:

MPDT-09A
Clarity Before:

Clarity After:
Texture:

Artifacts: 
1 INORGANIC ANALYSES DATA SHEET
EPA SAMPLE NO.

\section{B}

Contract: 60052

SAS NO.:

SDG No.: V2745 SOIL

LOW

Lab Sample ID: 0609L894-0̄18

$89 \overline{.4}$

Date Received: 09/07/06
Level (low/med)

응 Solids:

Concentration Units (ug/L or $\mathrm{mg} / \mathrm{kg}$ dry weight) : MG/KG

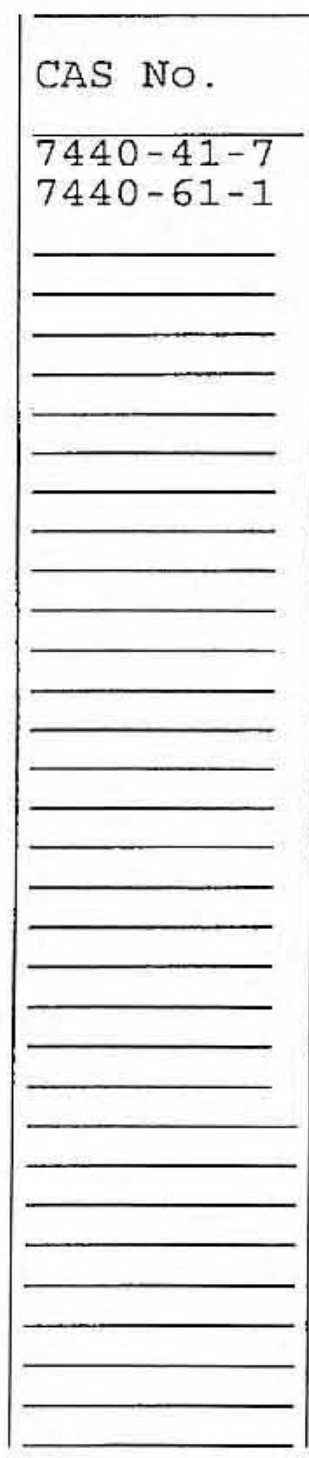

Color Before: Color After:

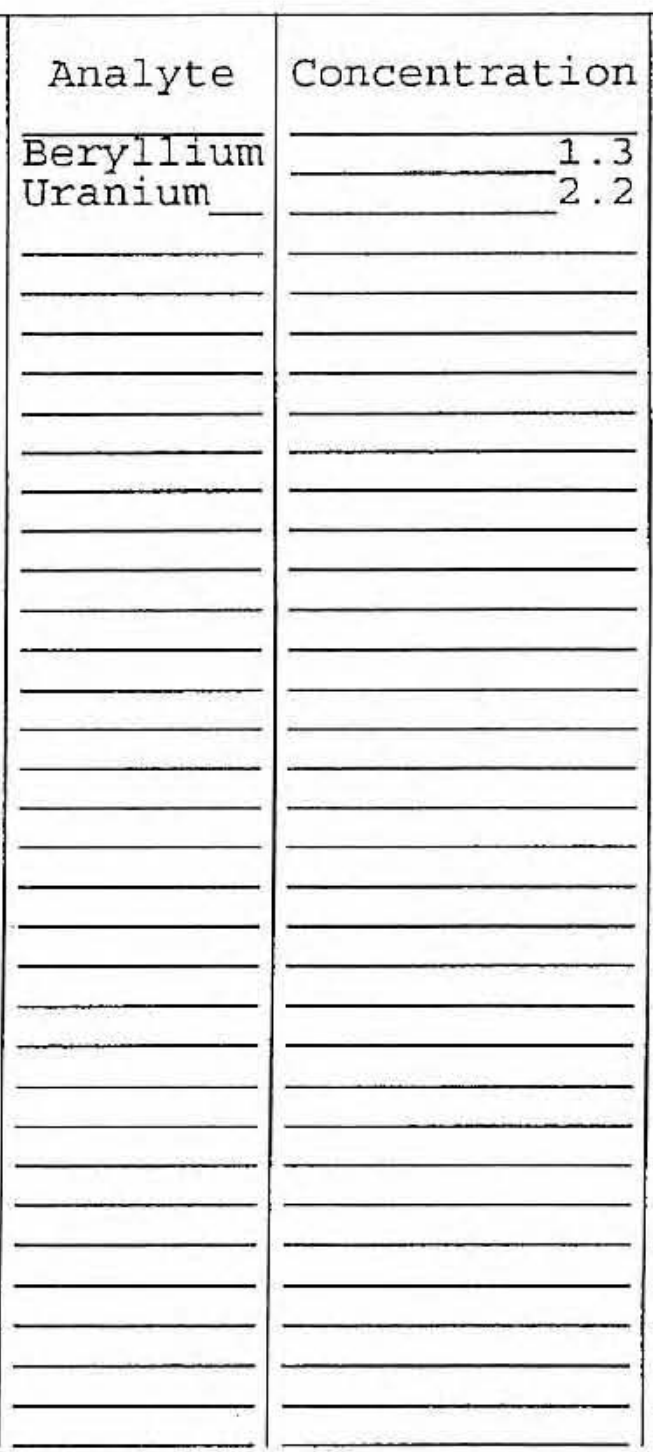

Clarity Before: Clarity After:

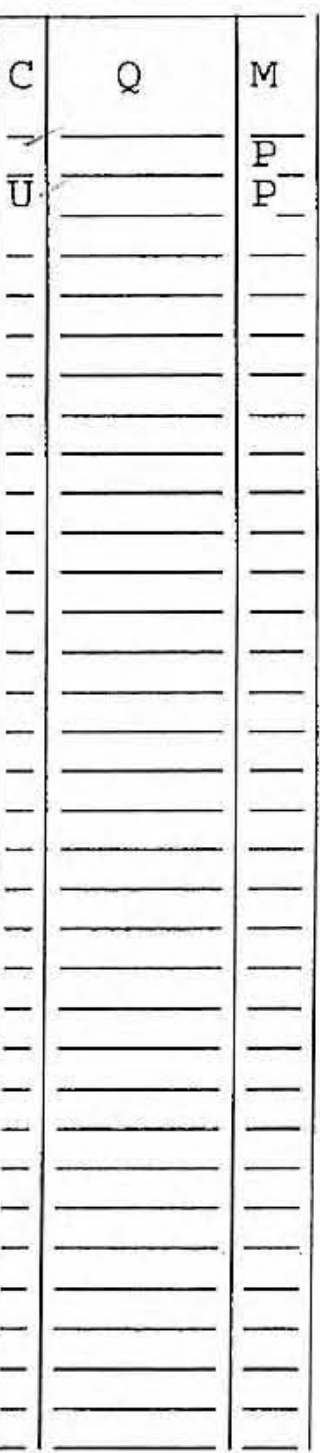

Texture: Artifacts :

Comments :

MPDT-09B 


\section{1}

INORGANIC ANALYSES DATA SHEET
EPA SAMPLE NO.

$10 \mathrm{~A}$

Contract: 60052

SAS NO.:

SDG NO.: V2745

Lab Sample ID: 0609L894-0̄19

Date Received: 09/07/06
Lab Name: LION
Lab Code: LVLI

Matrix (soil/wat
Level (low/med)

Solids:
Case No.: MPDT

$$
\begin{aligned}
& \text { SOIL } \\
& \text { LOW } \\
& -92.6
\end{aligned}
$$

Concentration Units (ug/L or $\mathrm{mg} / \mathrm{kg}$ dry weight): MG/KG

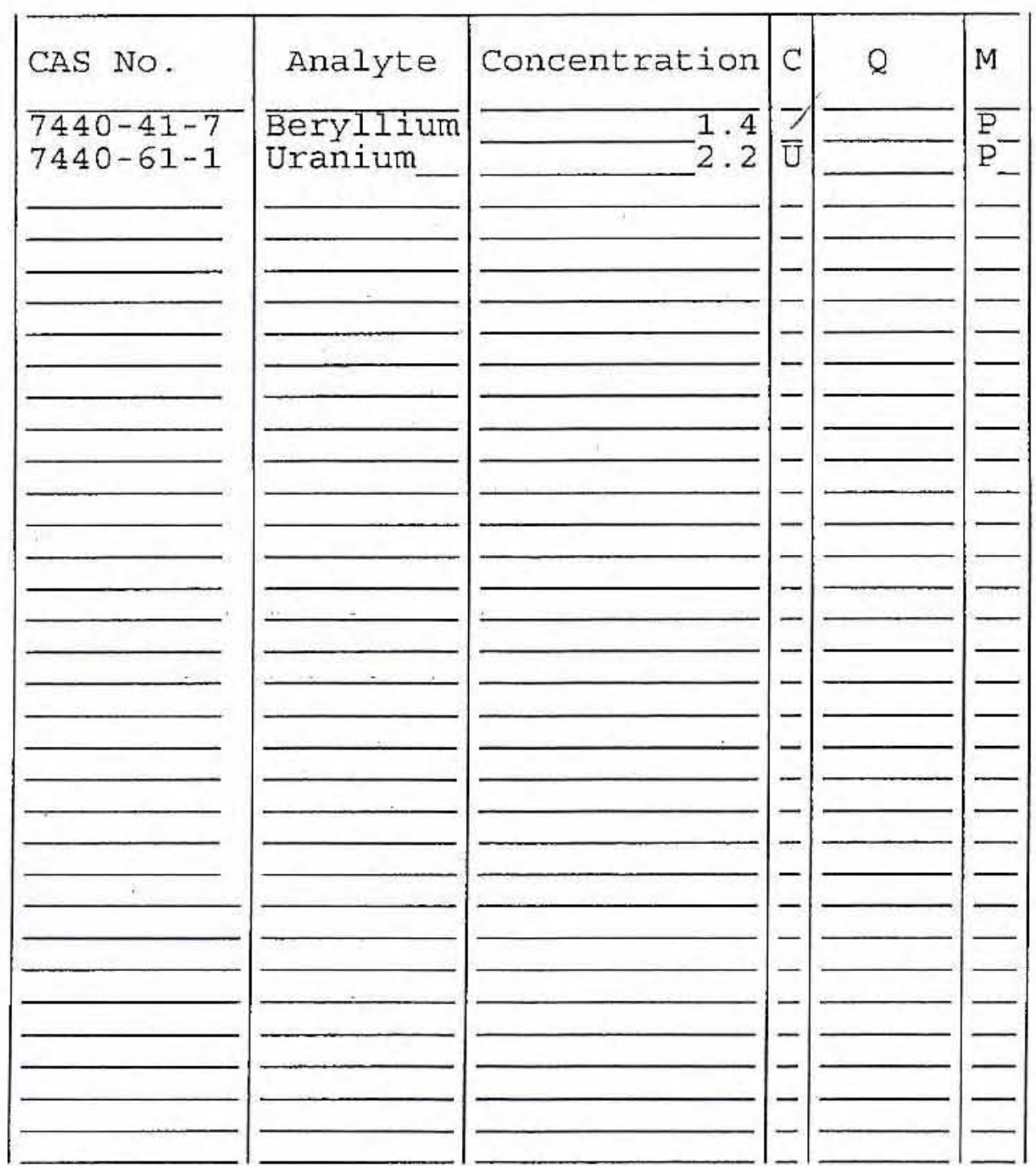

Color Before: Color After:
Clarity Before:

Clarity After:
Texture:

Artifacts:

Comments:

MPDT-IOA 
1 INORGANIC ANALYSES DATA SHEET

EPA SAMPLE NO.

$$
10 B
$$

Contract: 60052

SAS NO.:

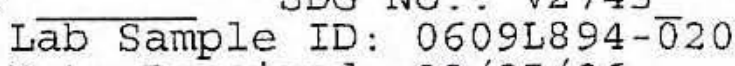
SOIL LOW $-89 \overline{.8}$

Date Received: 09/07/06
Case NO.: MPDT
Lab Code: LVLI Matrix (soil/wat $\%$ Solids:

Concentration Units (ug/L or $\mathrm{mg} / \mathrm{kg}$ dry weight): $\mathrm{MG} / \mathrm{KG}$

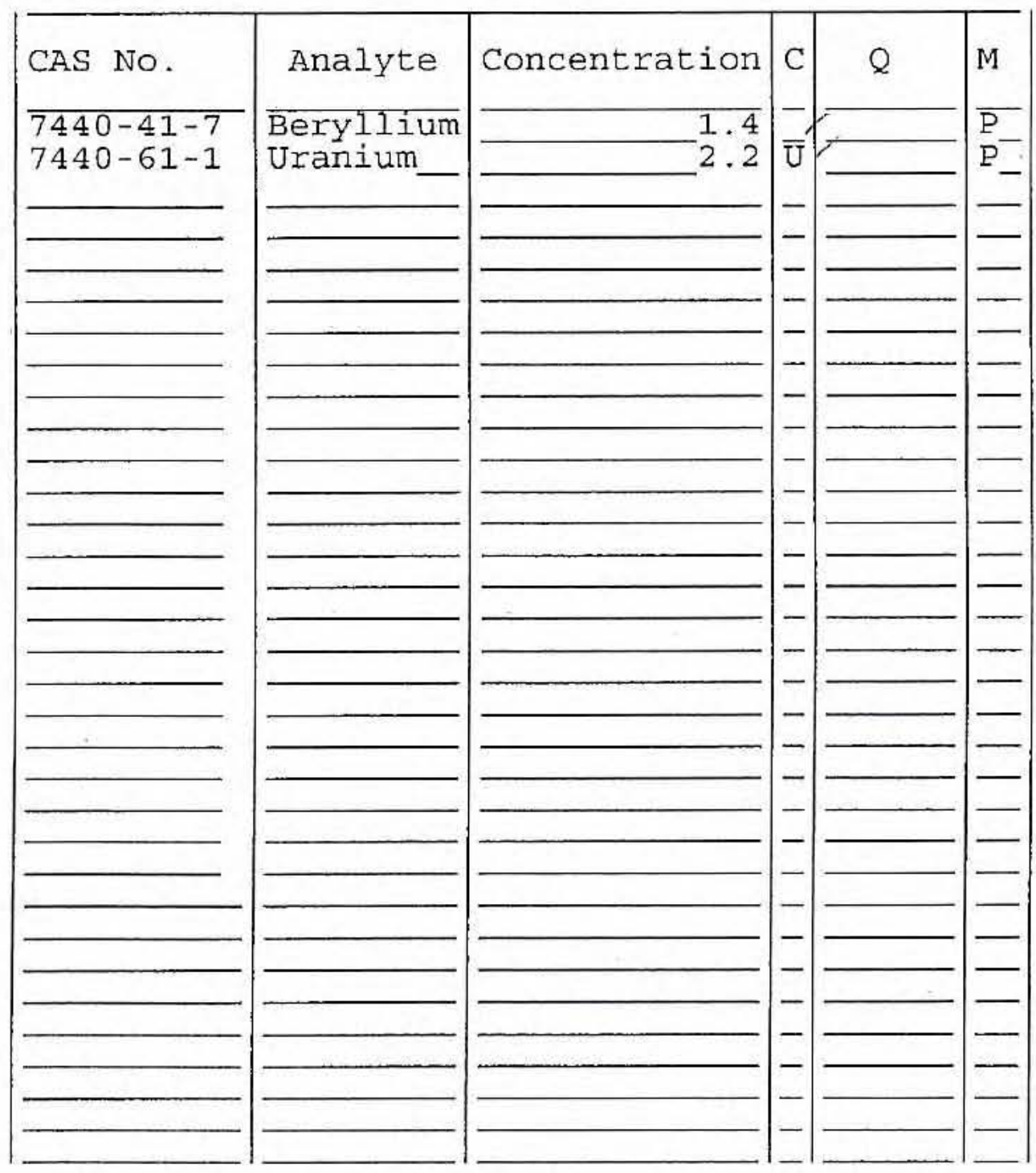

Color Before: Color After:
Clarity Before:

Clarity After:
Texture:

Artifacts:

Comments :

MPDT $-10 B$ 


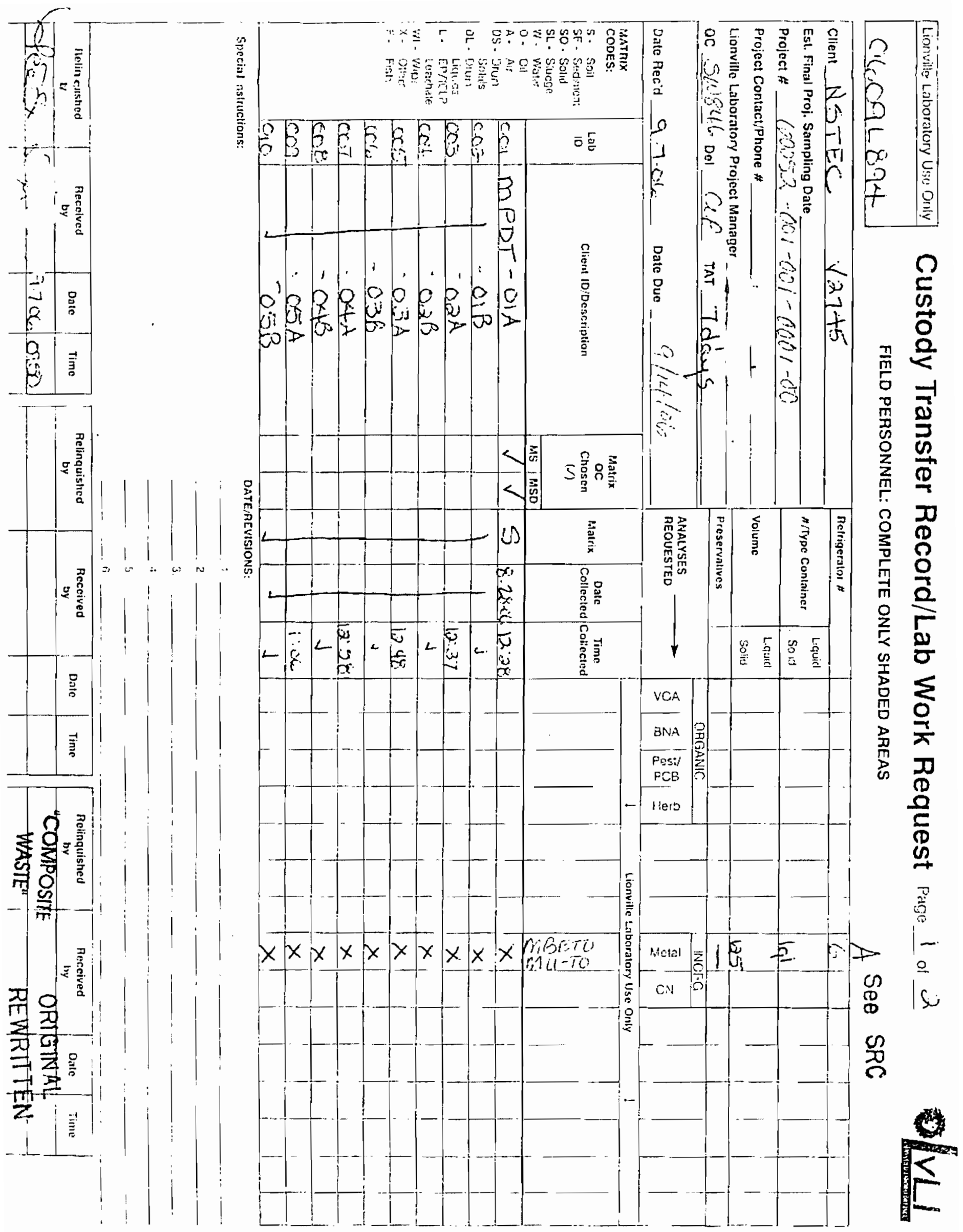




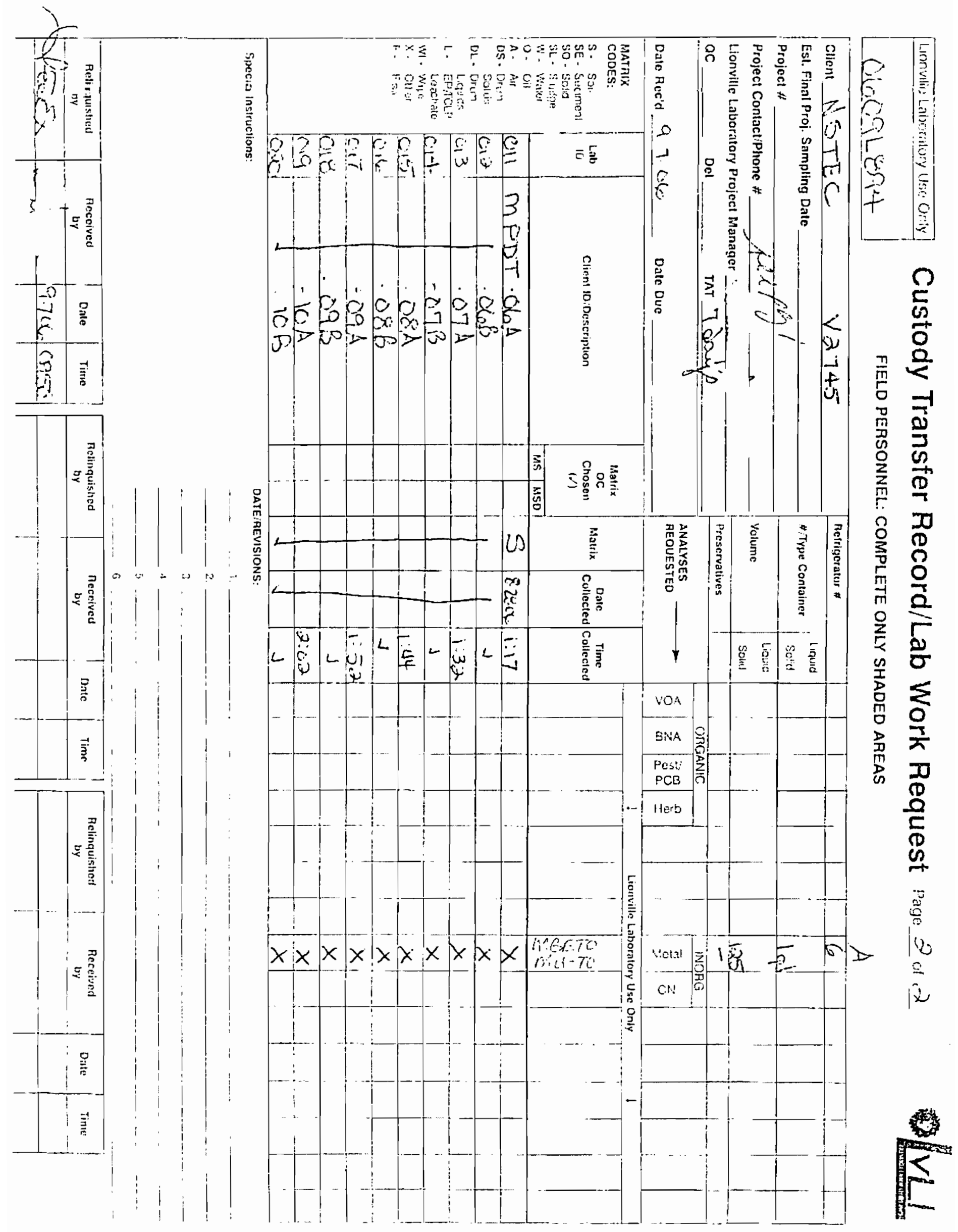




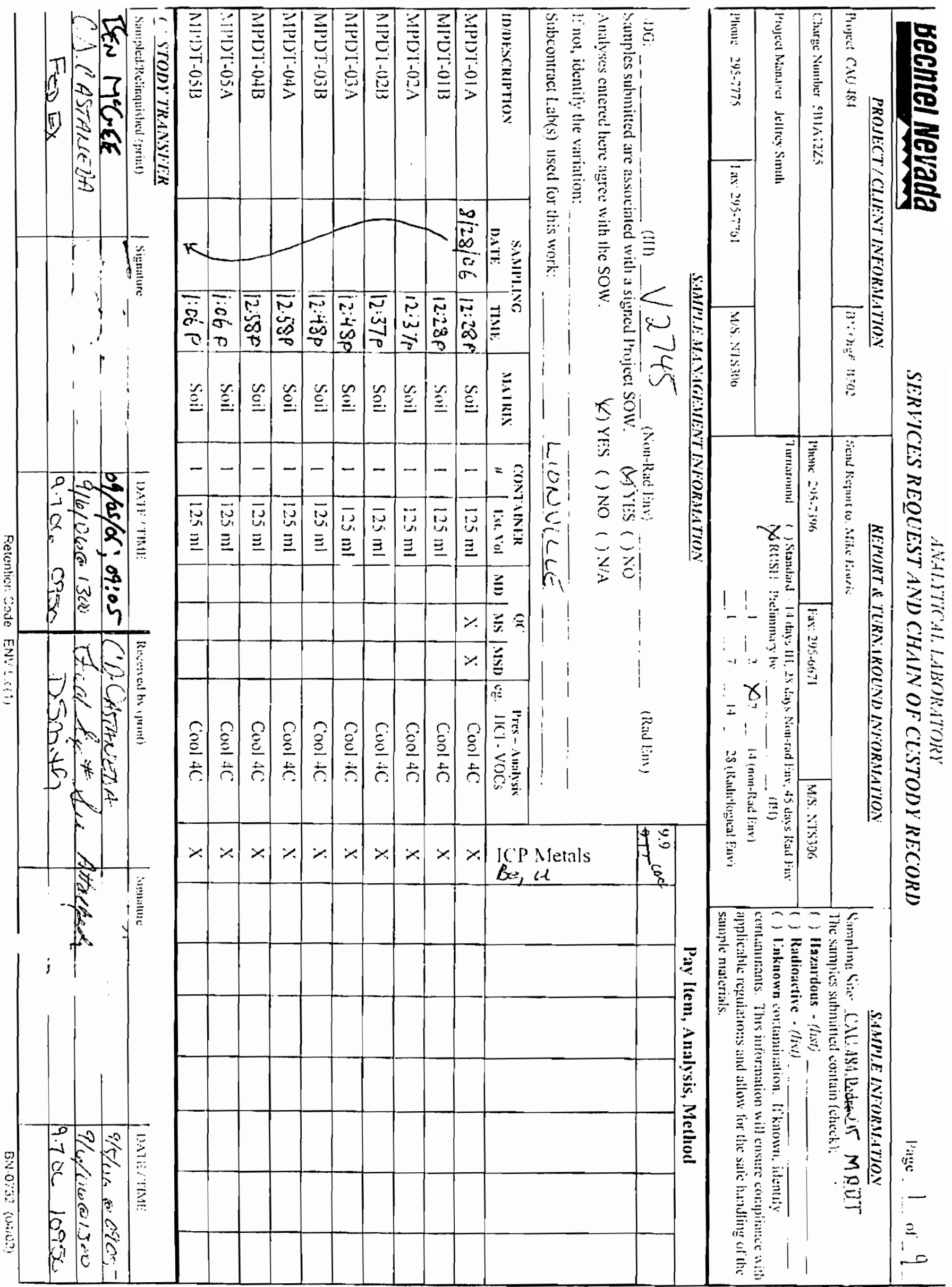




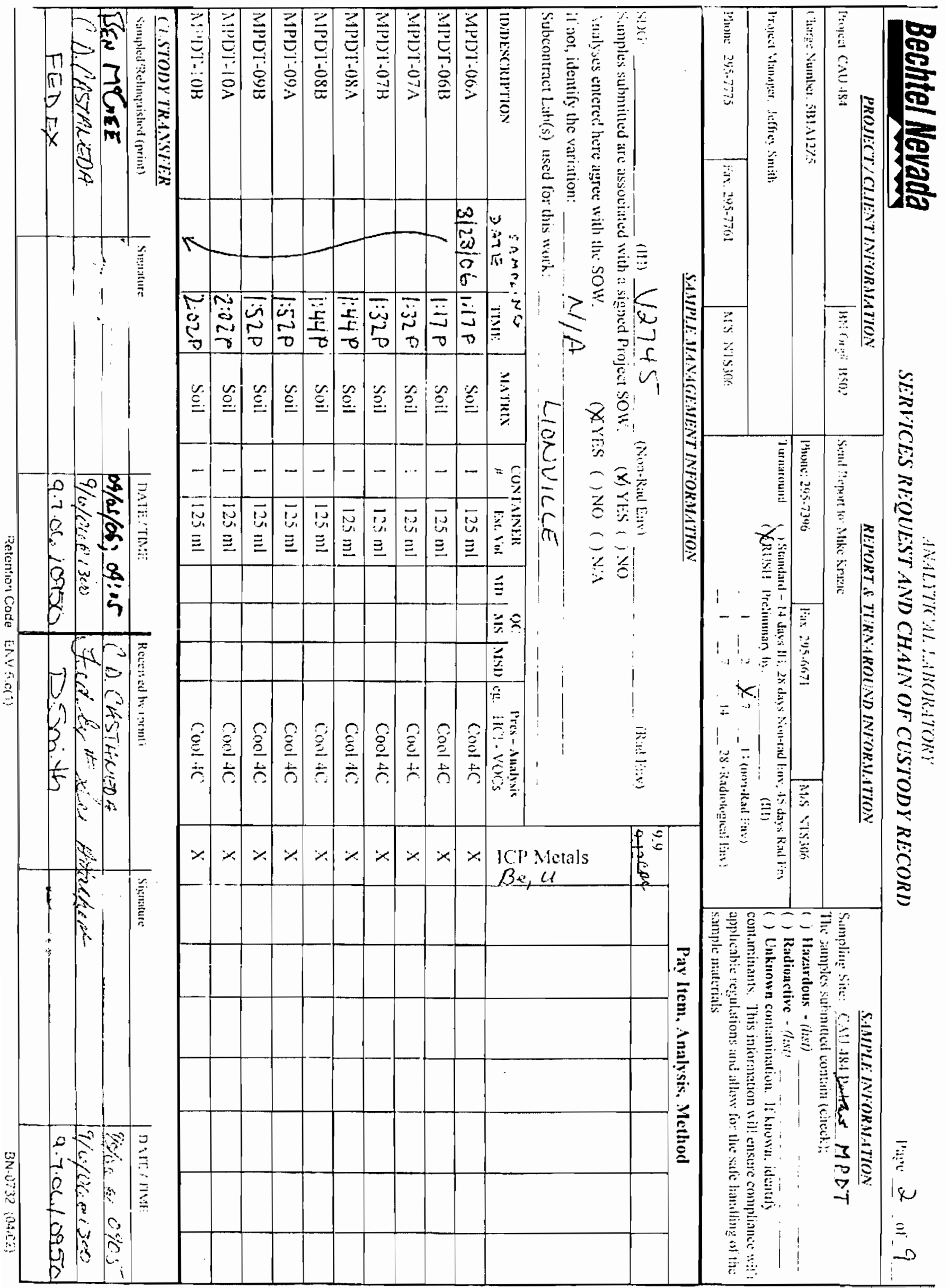


1

\section{INORGANIC ANALYSES DATA SHEET}

Lab NaLTe: LIONVILIE_LABORATORY

Lab Code: LVLI

Matrix (soil/water): SOIL Level (low/med):

$\div$ Solids:
Contract: 60052

SAS No.: LOW $-89 \overline{.9}$
SDG No.: 11A

EPA SAMPLE NO.

$11 \mathrm{~A}$

Lab Sample ID: 0609L895-001

Date Received: 09/07/06

Concentration Units (ug/L or $\mathrm{mg} / \mathrm{kg}$ dry weight): $\mathrm{MG} / \mathrm{KG}$

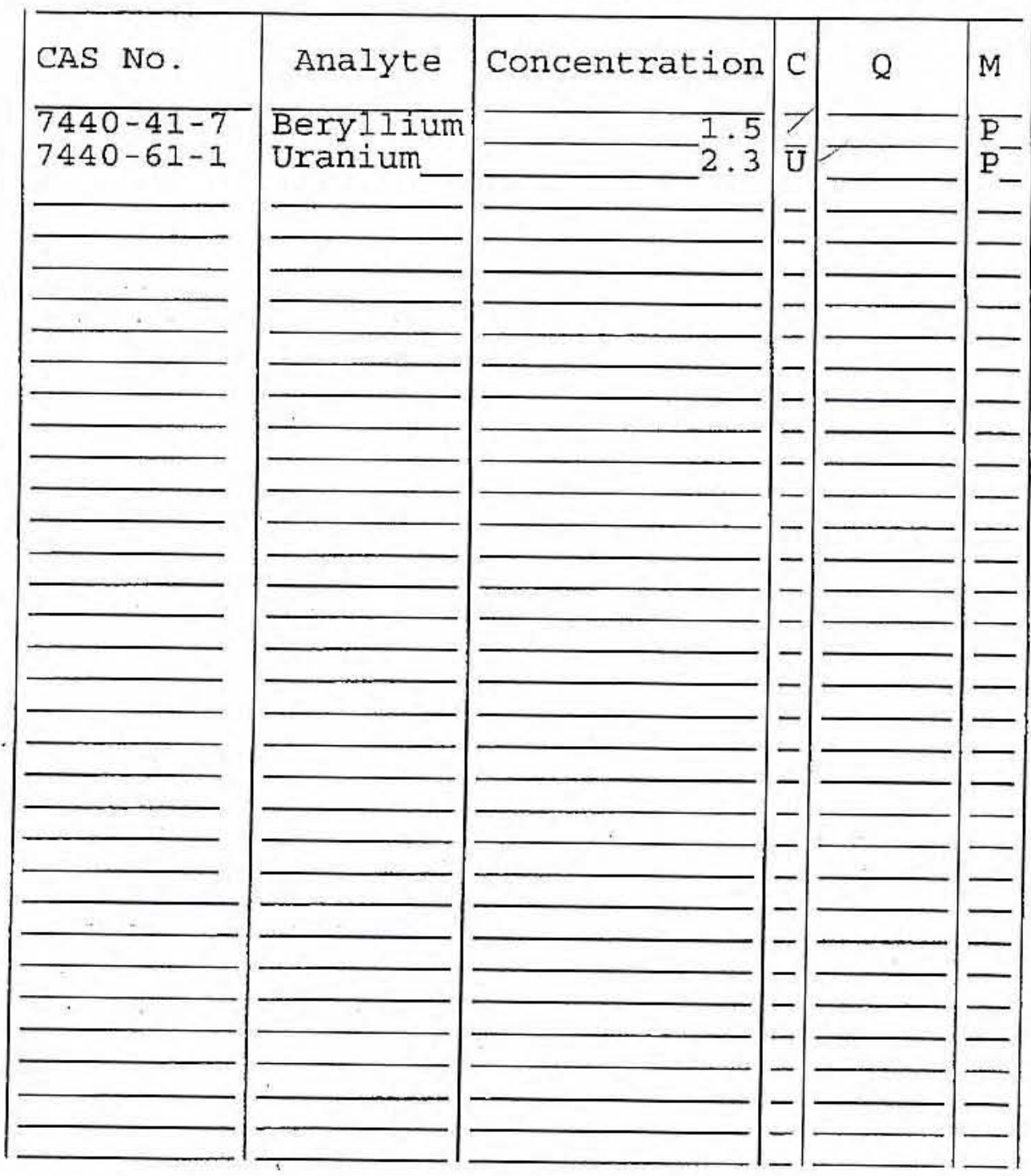

Color Before: Color After:

Comments :

MPDI - $11 \mathrm{~A}$
Clarity Before:

Clarity After:
Texture: Artifacts: 
1 INORGANIC ANALYSES DATA SHEET
EPA SAMPLE NO.

\section{$11 \mathrm{~B}$}

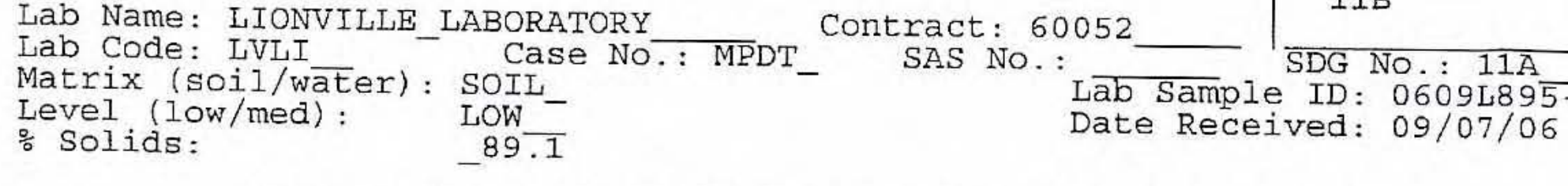

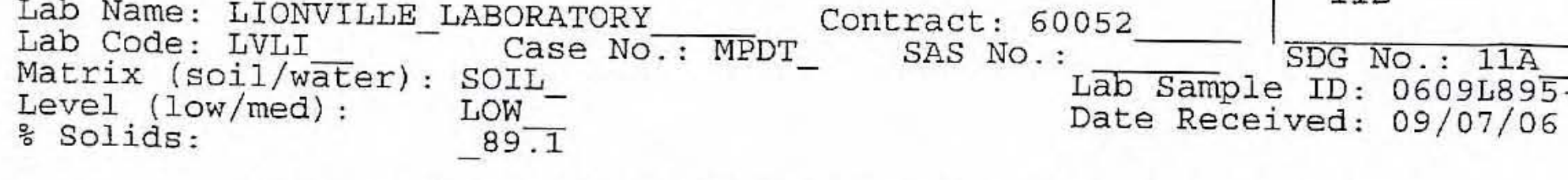

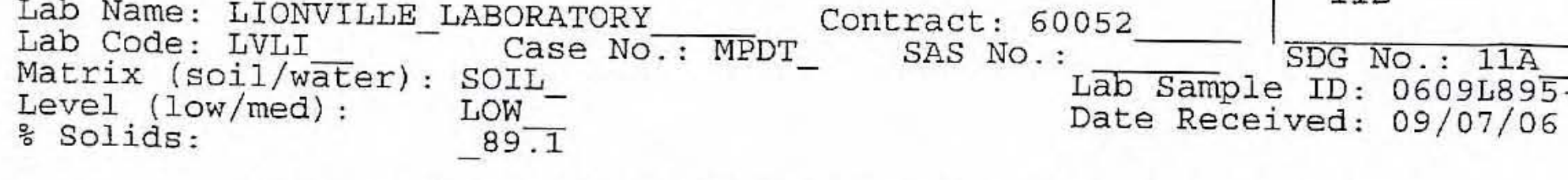

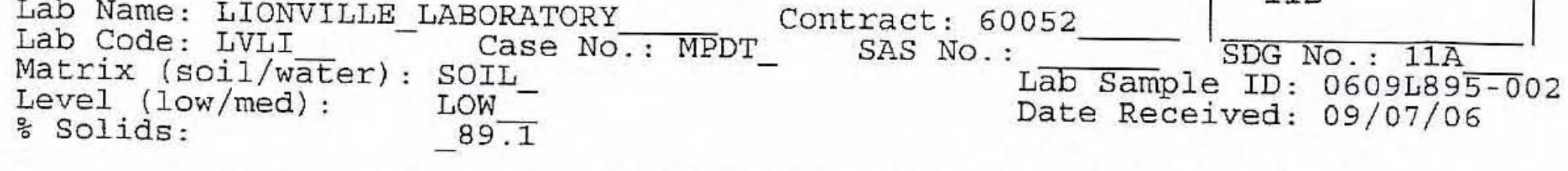

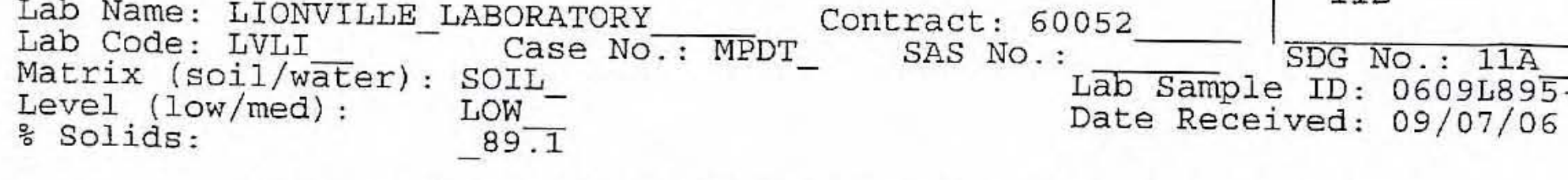

Concentration Units (ug/L or $\mathrm{mg} / \mathrm{kg}$ dry weight): MG/KG

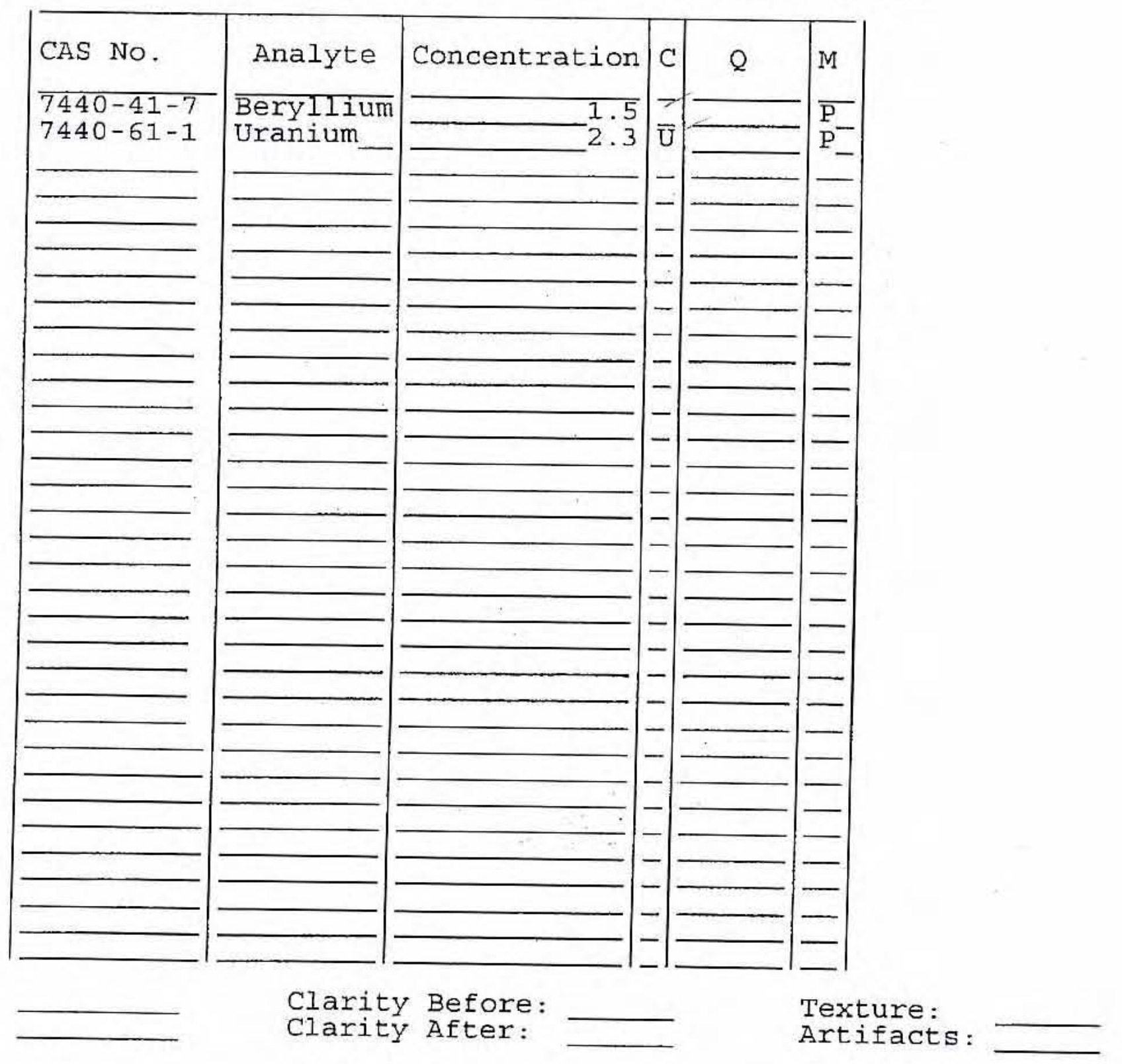

Color Before:

Color After:

Comments :

MPDT- $11 \mathrm{~B}$
Clarity Before:

Clarity After: Artifacts : 
1

INORGANIC ANALYSES DATA SHEET
EPA SAMPLE NO.

$12 \mathrm{~A}$

Lab Sample ID: 0609L895-003
SDG NO.: 11A SAS NO. :

Date Received: 09/07/06

Contract: 60052
Lab Name: LIONVILLE_IAABORATORY

Lab Code: IVLI

Matrix (soil/water): SoIL

Level (low/med):

\% Solids :
LOW

$-90 \overline{5}$

Concentration Units (ug/L or $\mathrm{mg} / \mathrm{kg}$ dry weight): MG/KG

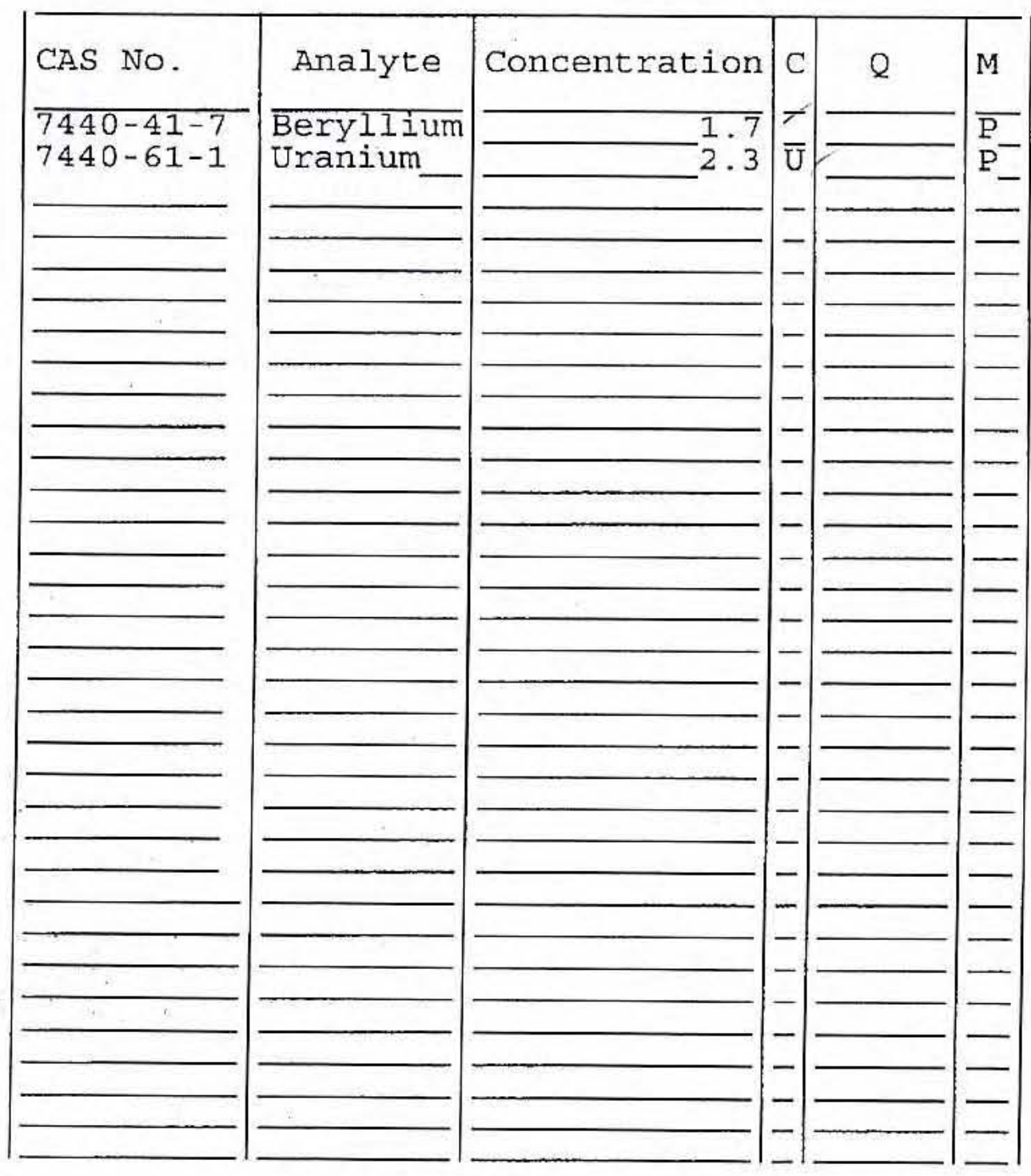

Color Before:

Color After:
Clarity Before:

Clarity After:
Texture :

Artifacts:

Comments :

MPDT - 12A 
1

INORGANIC ANALYSES DATA SHEET
EPA SAMPLE NO.

\section{$12 \mathrm{~B}$}

SDG No.: 11A

Lab Sample ID: 0609L895-004

Date Received: 09/07/06
Lab Name: LIONVILLE_LABORATORY Contract: 60052
Lab Code: LVLI Case No.: MPDT_ SAS No.:

Lab Name: LIONVILLE_LABORATORY
Lab Code: LVLI Case No.: MPDT_ Contract: 60
SAS No.:

Matrix (soil/water): SOIL

Level (low/med): LOW

$\%$ Solids:
$-89 \overline{.4}$

Concentration Units (ug/L or $\mathrm{mg} / \mathrm{kg}$ dry weight): MG/KG

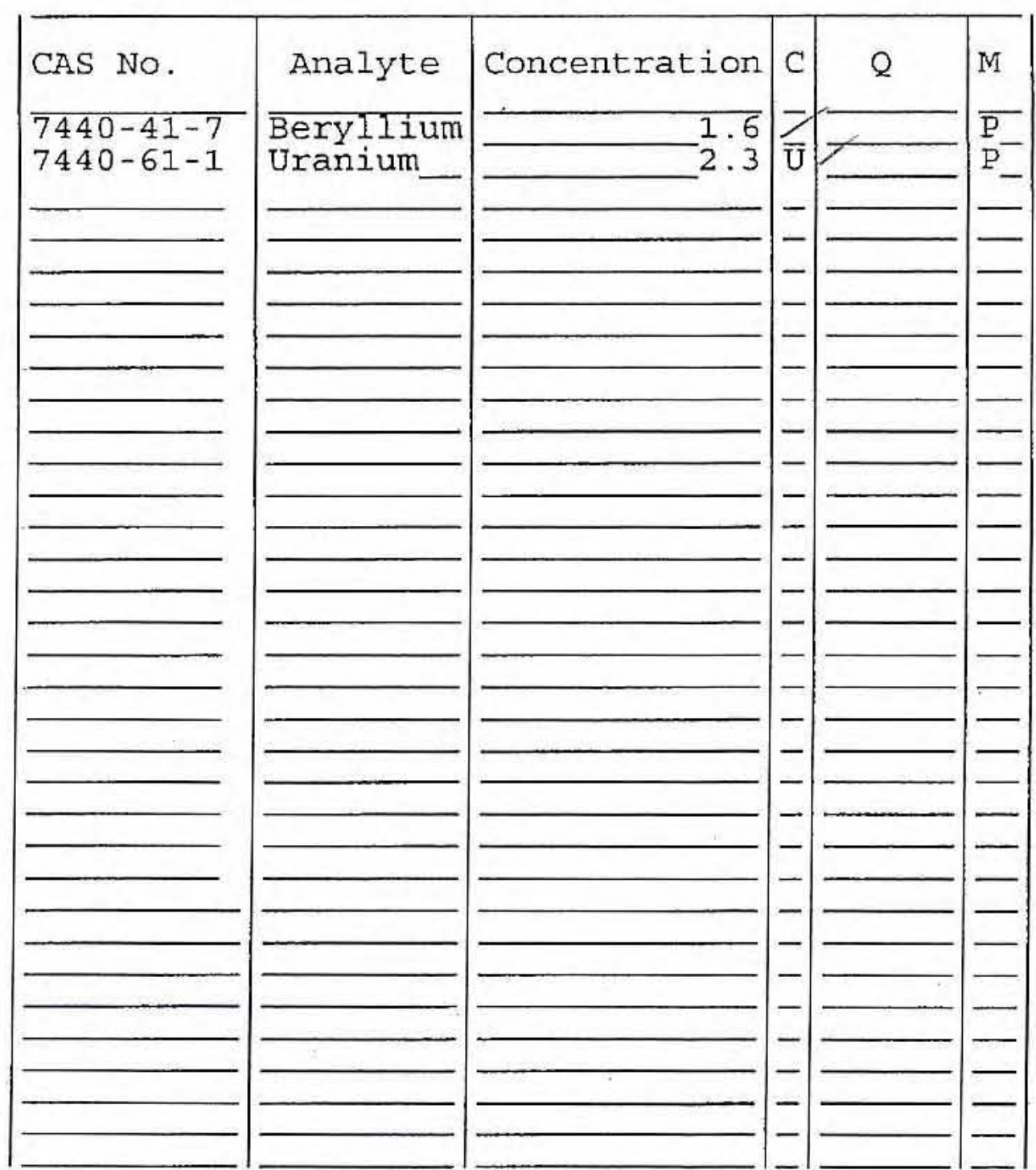

Color Before:

Color After:
Clarity Before:

Clarity After:
Texture:

Artifacts :

Comments :

MPDT - 12B 
1

INORGANIC ANALYSES DATA SHEET
EPA SAMPLE NO.

\section{$13 \mathrm{~A}$}

SDG NO.: 11A

Case No.: MPDT_ SAS No. SOIL
LOW
91.4
Lab Sample ID: 0609L895-005

Date Received: 09/07/06
Lab Code: LVLI Level (low/med): \% Solids :

Concentration Units (ug/L or $\mathrm{mg} / \mathrm{kg}$ dry weight): MG/KG

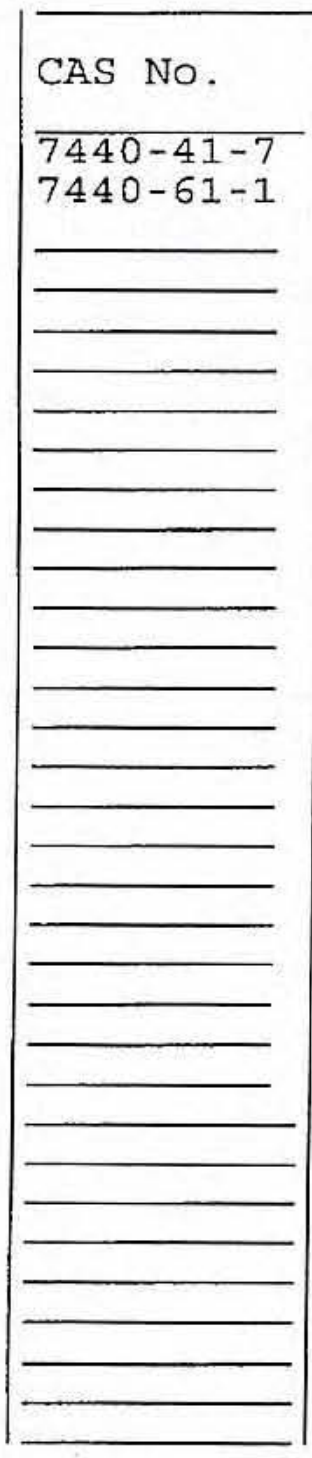

Color Before: Color After:

Comments :

MPDT-13A

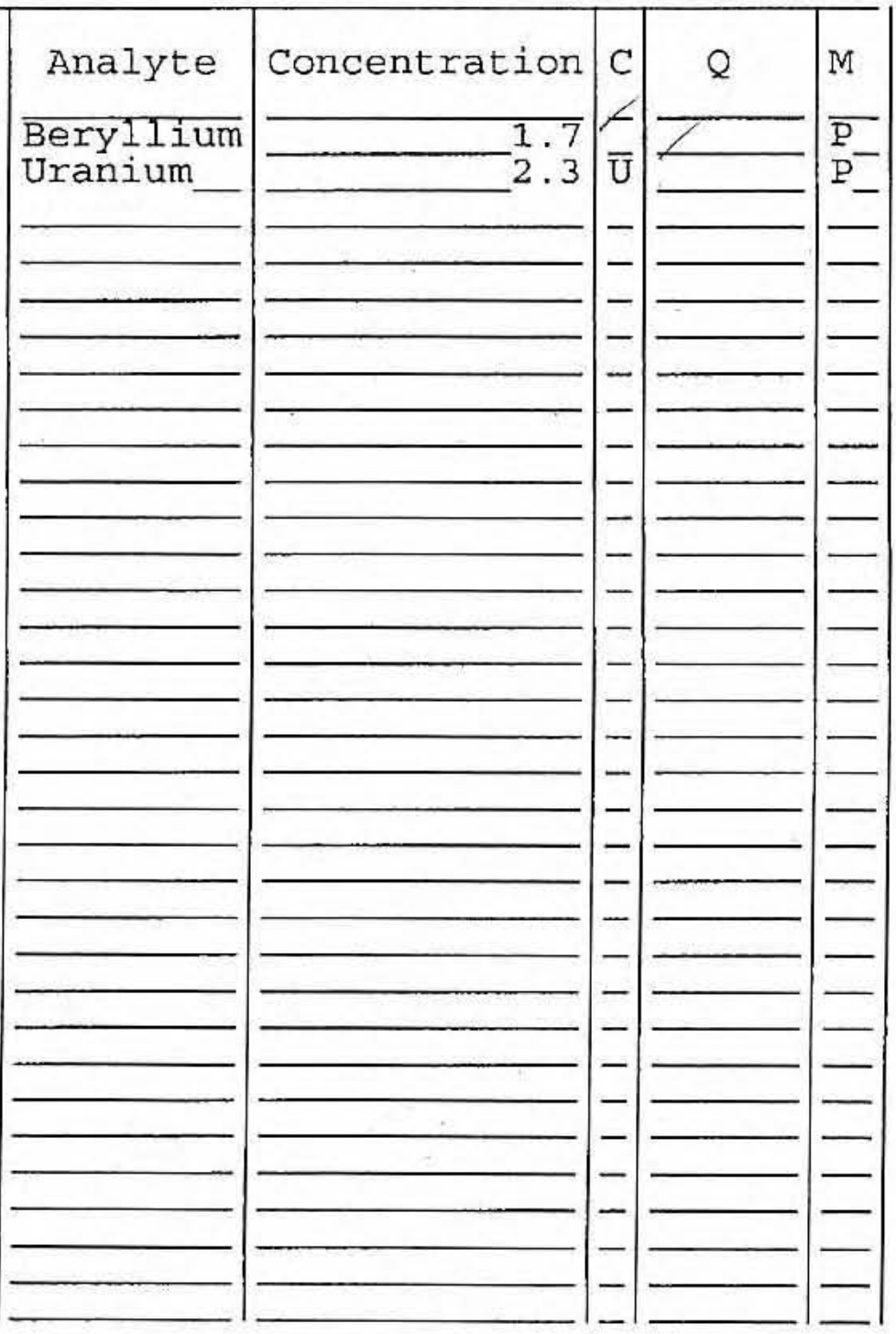

Clarity Before:

Texture:

Artifacts: 
1 INORGANIC ANALYSES DATA SHEET
EPA SAMPLE NO.

$13 B$
Lab Name: LIONVILLE_LABORATORY

Lab Code: LVLI Matrix (soil/water): SOIL Level (low/med): LOW \% Solids:
Contract: 60052

SAS NO.:

SDG No.: 11A

Lab Sample ID: 0609L895-006

Date Received: 09/07/06

Concentration Units (ug/L or $\mathrm{mg} / \mathrm{kg}$ dry weight): MG/KG

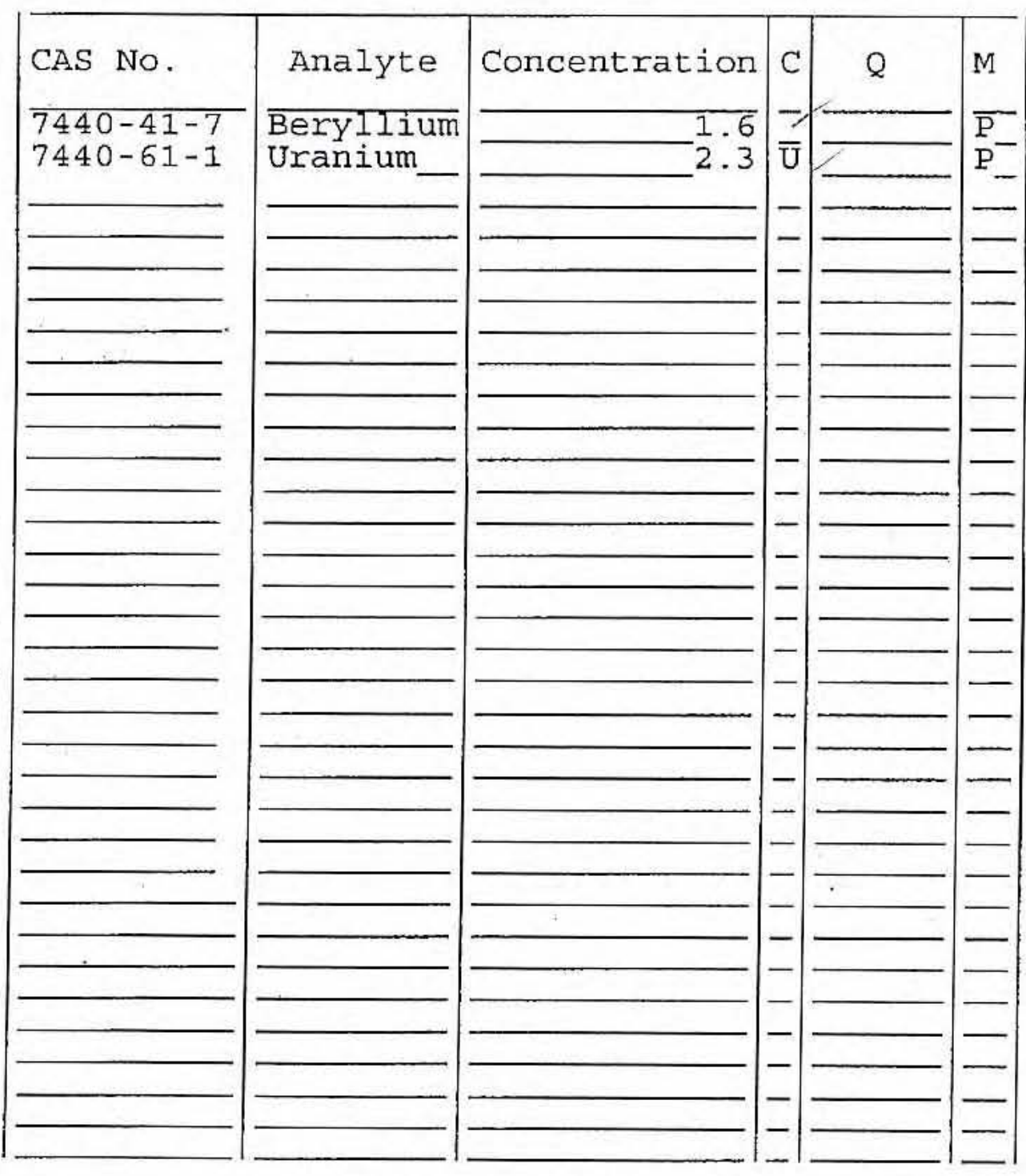

Color Before: Color After:
Clarity Before: Clarity After:
Texture: Artifacts:

Comments :

MPDT $-13 B$ 
1 INORGANIC ANALYSES DATA SHEET
EPA SAMPLE NO.

$14 \mathrm{~A}$

Contract: 60052

SAS NO.:

SDG NO.: 11A

Lab Sample ID: 06091895-007 SOIL LOW

Date Received: 09/07/06
Lab Code: LVLI

Matrix (soil/wat Solids:
$-92 \overline{4}$

Concentration Units (ug/L or $\mathrm{mg} / \mathrm{kg}$ dry weight): $\mathrm{MG} / \mathrm{KG}$

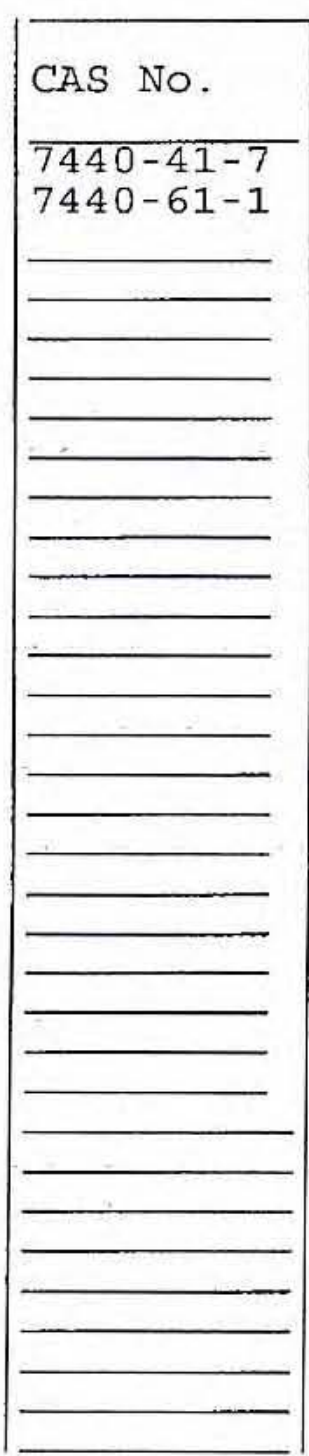

Color Before: Color After:

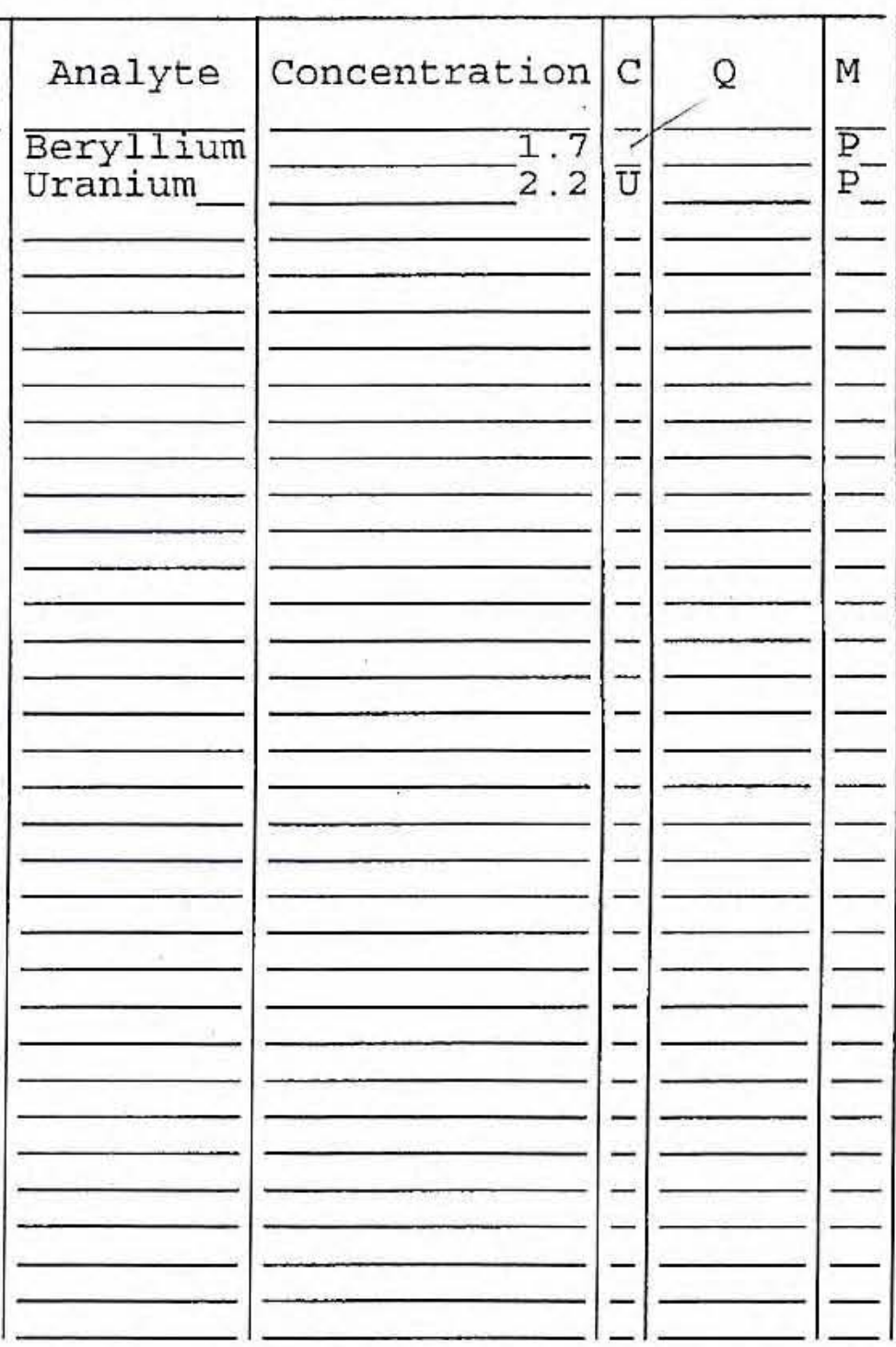

Clarity Before: Clarity After:
Texture: Artifacts:

Comments :

MPDT - $14 \mathrm{~A}$ 
1

INORGANIC ANALYSES DATA SHEET
EPA SAMPLE NO.

\section{$14 \mathrm{~B}$}

Contract: 60052

SAS NO. :

Lab Sample ID: 0609L895-008

Date Received: 09/07/06

Level (low/med): LOW

$\%$ Solids :

$-89 \overline{.5}$

Concentration Units (ug/L or $\mathrm{mg} / \mathrm{kg} \mathrm{dry}$ weight): $\mathrm{MG} / \mathrm{KG}$

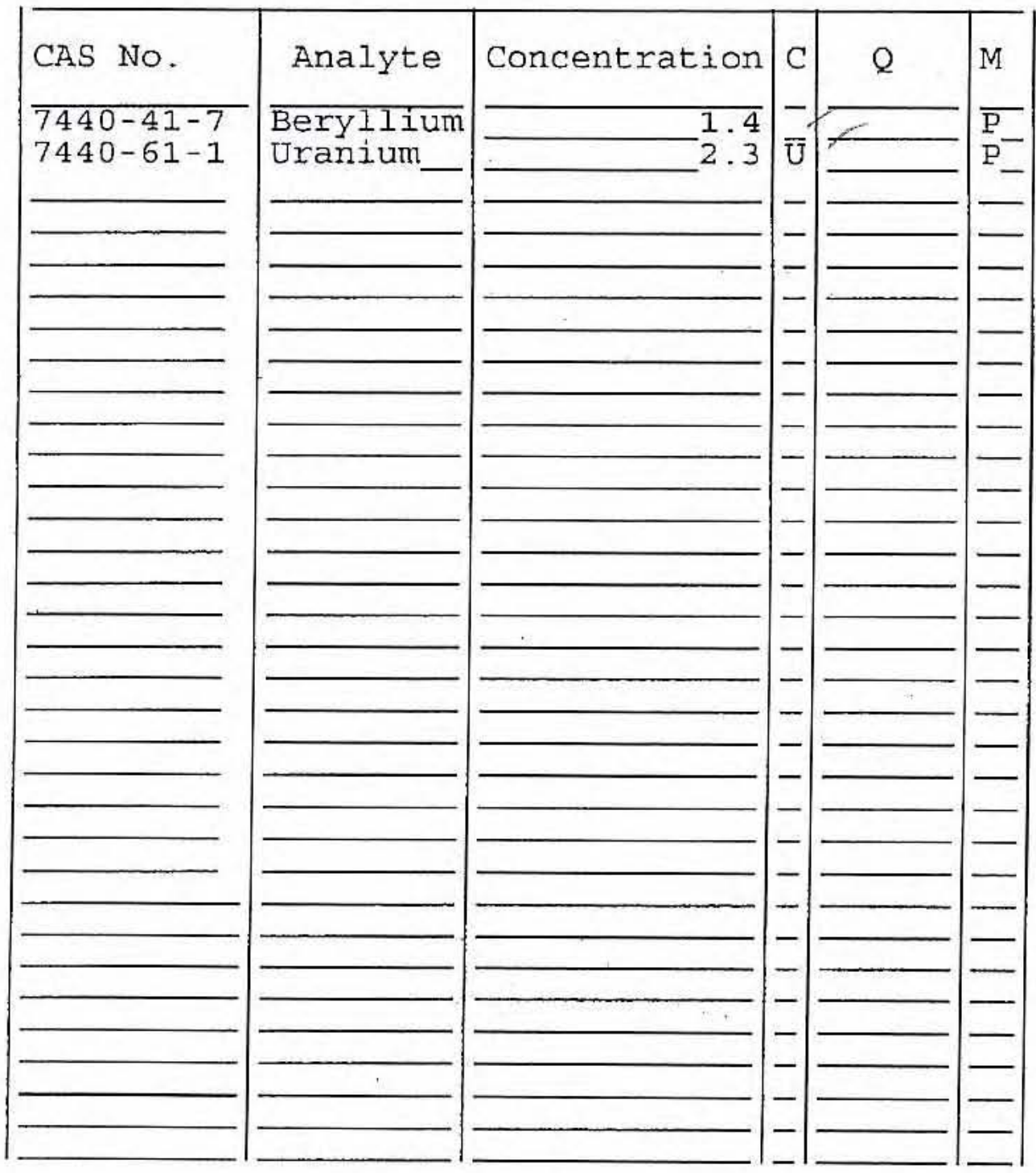

Color Before:

Color After:

Comments :

MPDT-14B
Clarity Before:

Clarity After:
Texture:

Artifacts : 
1

INORGANIC ANALYSES DATA SHEET
EPA SAMPLE NO.

$15 \mathrm{~A}$

Contract: 60052

SAS No. :

SDG No.: 11A

Case No.: MPDT

$$
\begin{aligned}
& \text { SOIL_ } \\
& \text { LOW - } \\
& -90 . \overline{9}
\end{aligned}
$$

Lab Sample ID: 0609L895-009

Date Received: 09/07/06

Concentration Units (ug/L or $\mathrm{mg} / \mathrm{kg} \mathrm{dry}$ weight): $\mathrm{MG} / \mathrm{KG}$

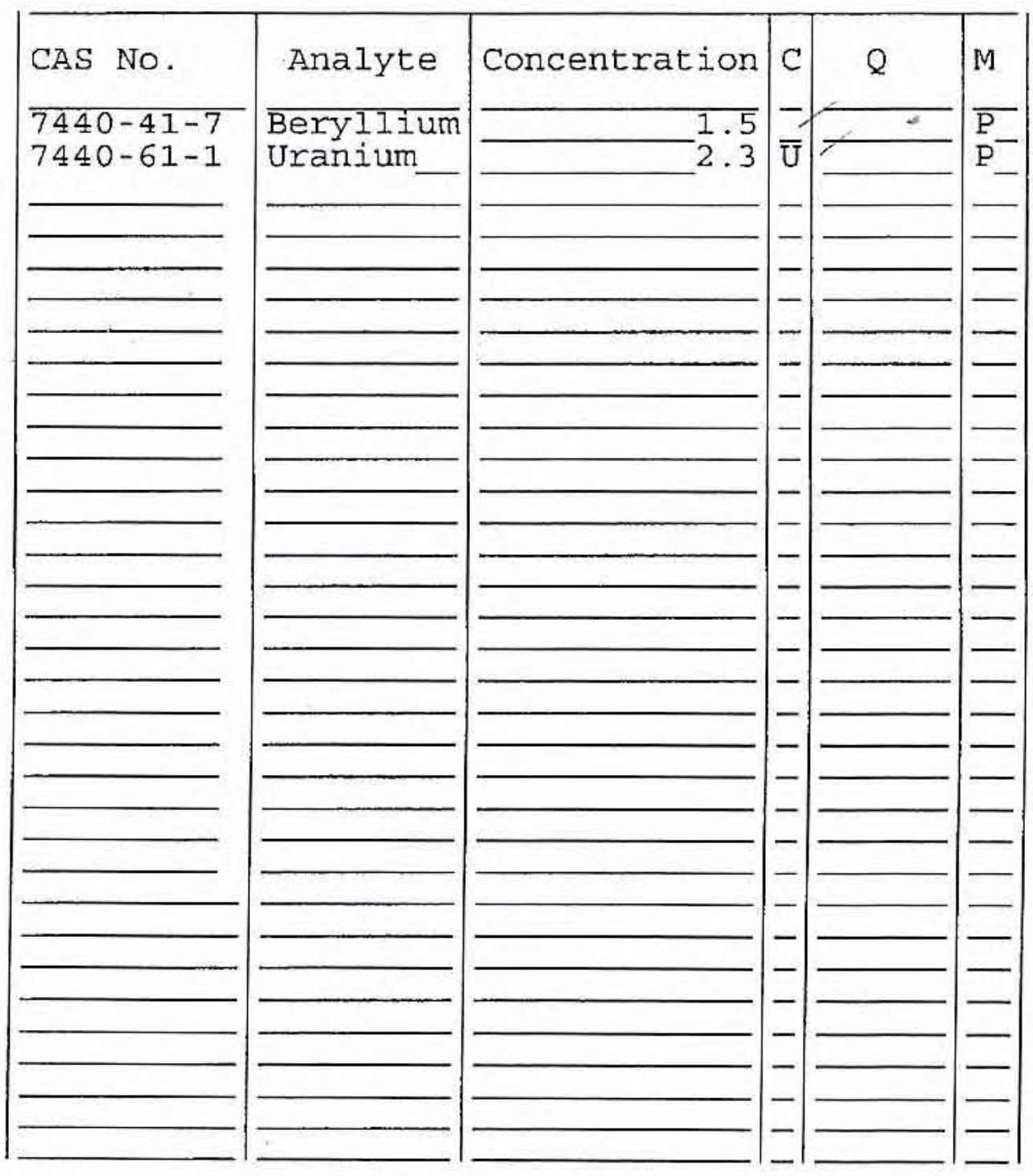

Color Before: Color After:
Clarity Before: Clarity After:
Texture:

Artifacts:

Comments :

MPDT- 15A 


\section{U.S. EPA}

1

INORGANIC ANALYSES DATA SHEET
EPA SAMPLE NO.

\section{$15 \mathrm{~B}$}

Contract: 60052

SAS NO.:

SDG NO: $11 \mathrm{~A}$

Lab Sample ID: 0609L895-010

Date Received: 09/07/06
Lab Name: LIONVILLE_LABORATORY

Lab Code: LVLI

Matrix (soil/water): SOIL

Level (low/med):

: Solids:

\section{LOW}

$-82 \overline{9}$

Concentration Units (ug/L or $\mathrm{mg} / \mathrm{kg}$ dry weight) : MG/KG

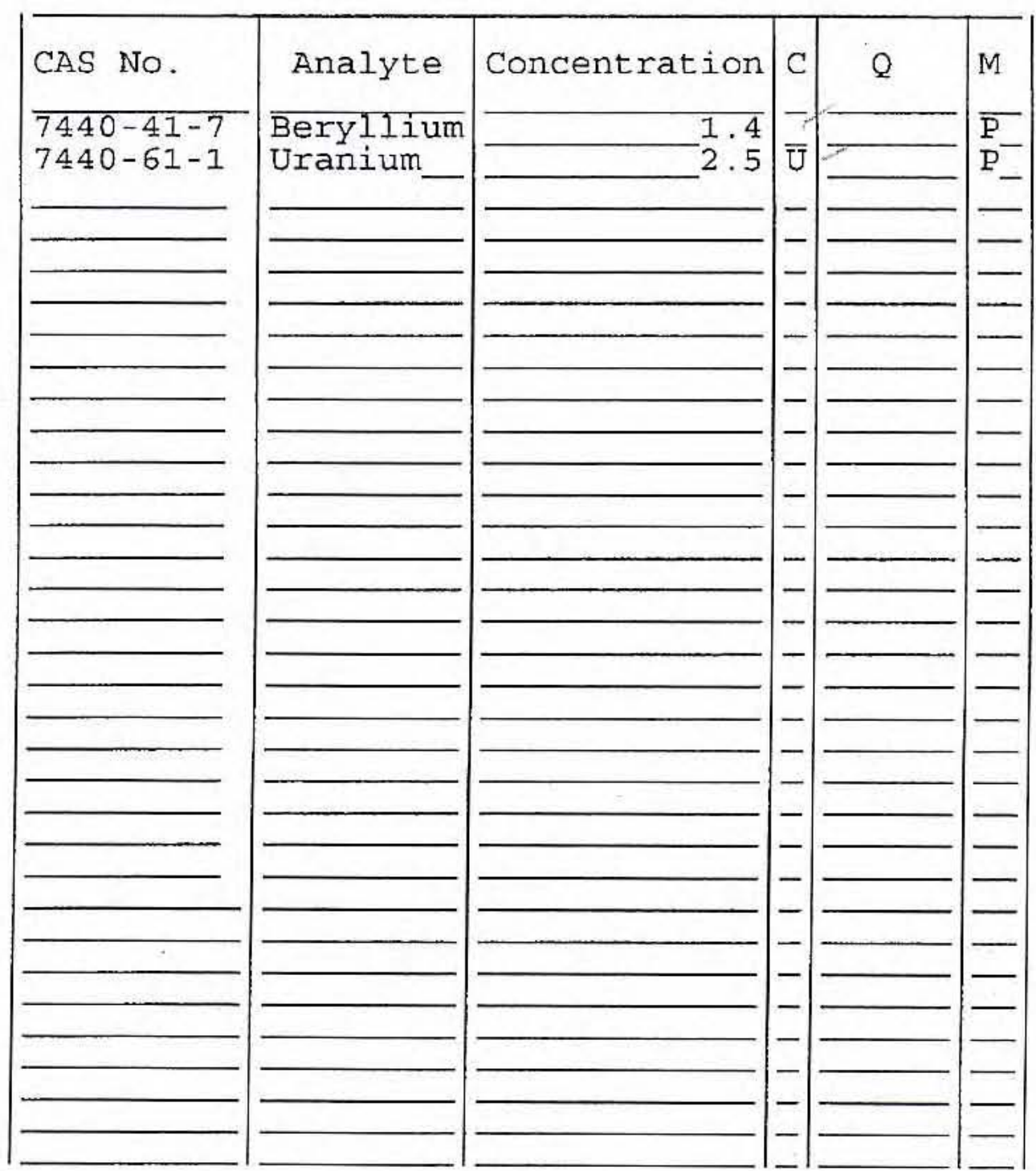

Color Before: Color After:

Comments:

MPDT-15B
Clarity Before: Clarity After:
Texture: Artifacts: 
1

INORGANIC ANALYSES DATA SHEET
EPA SAMPLE NO.

\section{$16 \mathrm{~A}$}

Contract: 60052

SAS NO.:

Lab Sample ID: 06091895-011

Date Received: 09/07/06
Lab Name: LION
Lab Code: LVLI

Matrix (soil/water): soIL

Level (low/med): LOW

$\%$ Solids:
Case No.: MPDT

$-92 \overline{.6}$

Concentration Units (ug/L or $\mathrm{mg} / \mathrm{kg}$ dry weight): MG/KG

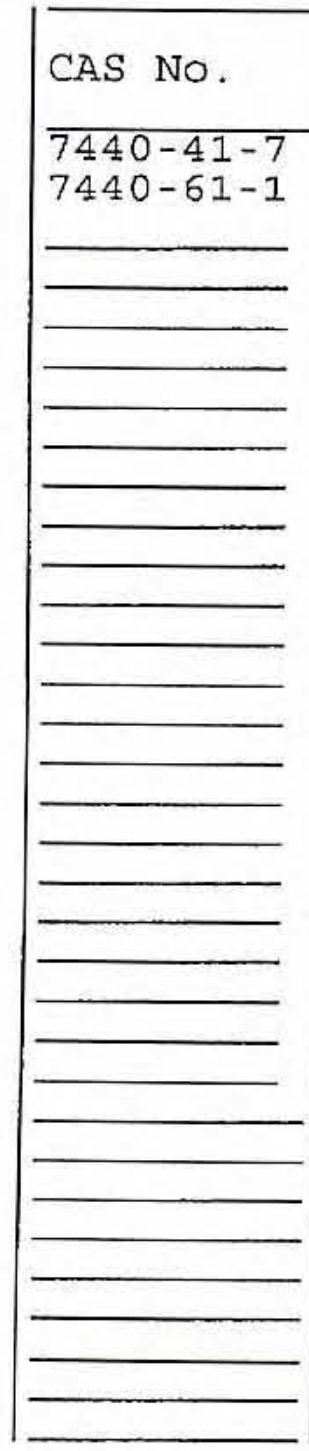

Color Before:

Color After:

Comments :

MPDT-16A

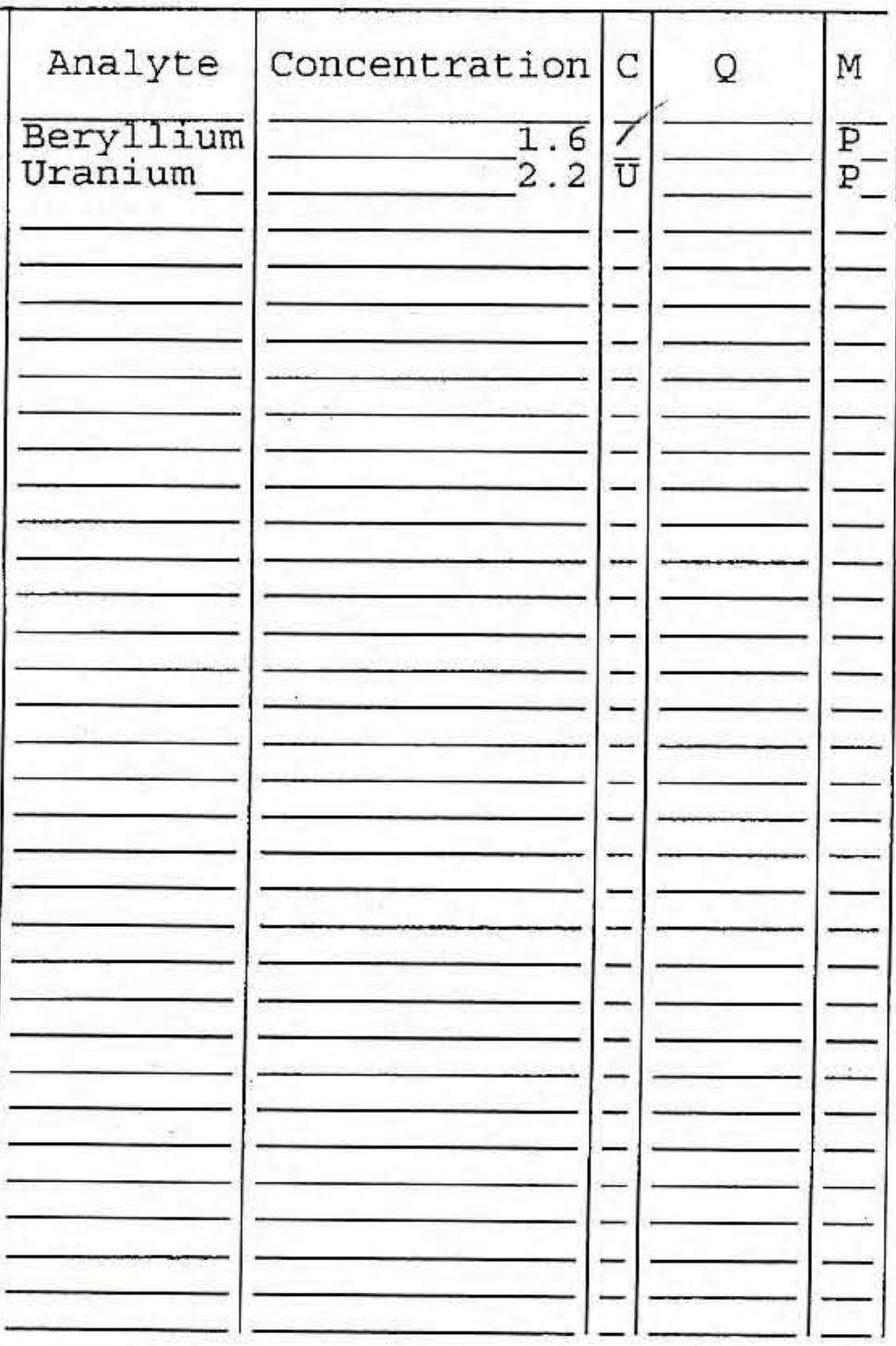

Clarity Before:

Clarity After:
Texture:

Artifacts: 
1

INORGANIC ANALYSES DATA SHEET
EPA SAMPLE NO.

$16 \mathrm{~B}$

Lab Name: LIONVILLE LABORATORY

Lab Code: LVLI Matrix (soil/water): SoIL

Level (low/med): LOW

\% Solids: $\quad-89 \overline{.8}$
Contract: 60052

SAS NO. :

Lab sample ID: 0609L895-012

Date Received: 09/07/06

Concentration Units (ug/L or $\mathrm{mg} / \mathrm{kg} \mathrm{dry}$ weight): MG/KG

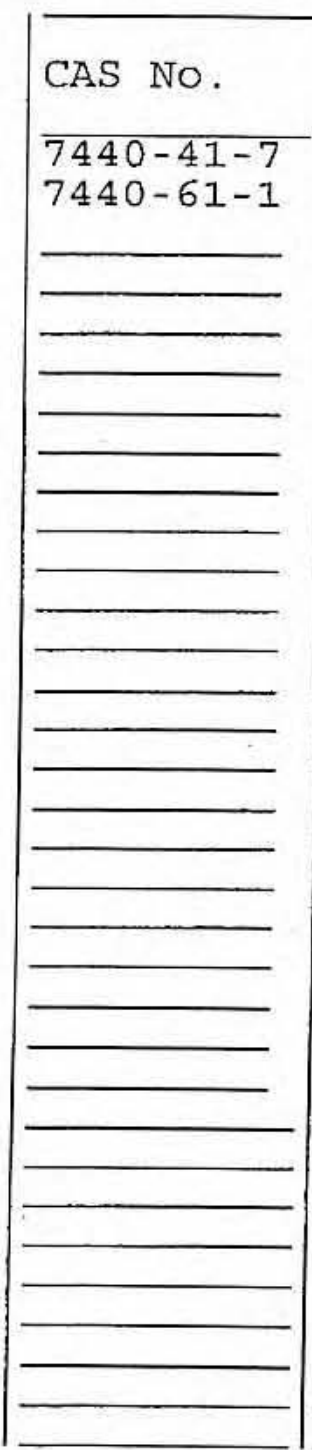

Color Before:

Color After:

Comments :

MPDT $-16 B$

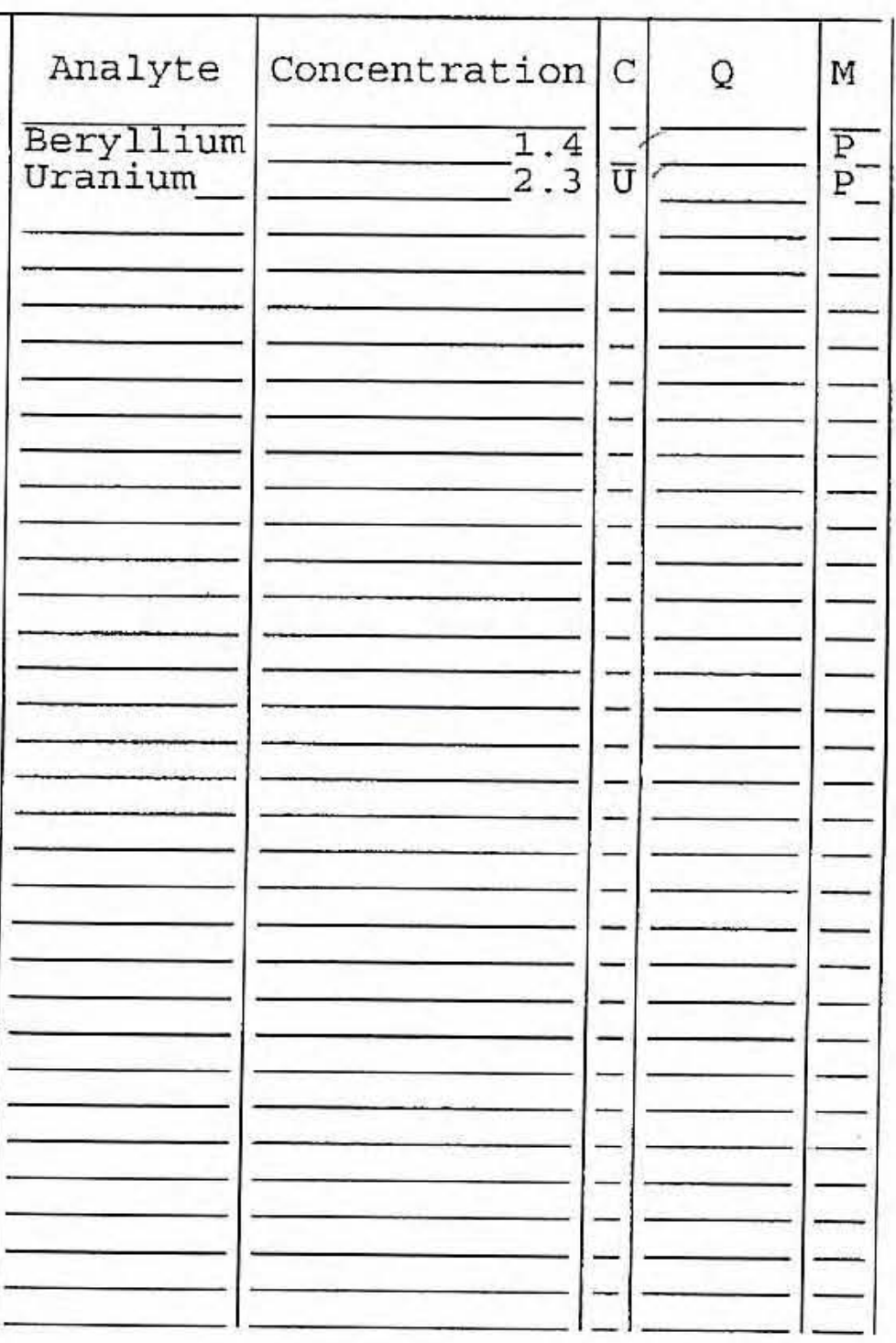

Clarity Before:

Clarity After:
Texture: Artifacts : 
1

\section{INORGANIC ANALYSES DATA SHEET}

EPA SAMPLE NO.

$17 \mathrm{~A}$

Contract: 60052

SAS No.:

SDG No. : 11A

Lab Sample ID: 0609L895-013

Date Received: 09/07/06
Lab Name: LIONVILLE_LABORATORY Matrix (soil/water): Level (low/med): 음 Solids:

LOW-
-91.1

Concentration Units ( $\mathrm{ug} / \mathrm{L}$ or $\mathrm{mg} / \mathrm{kg} \mathrm{dry}$ weight): $\mathrm{MG} / \mathrm{KG}$

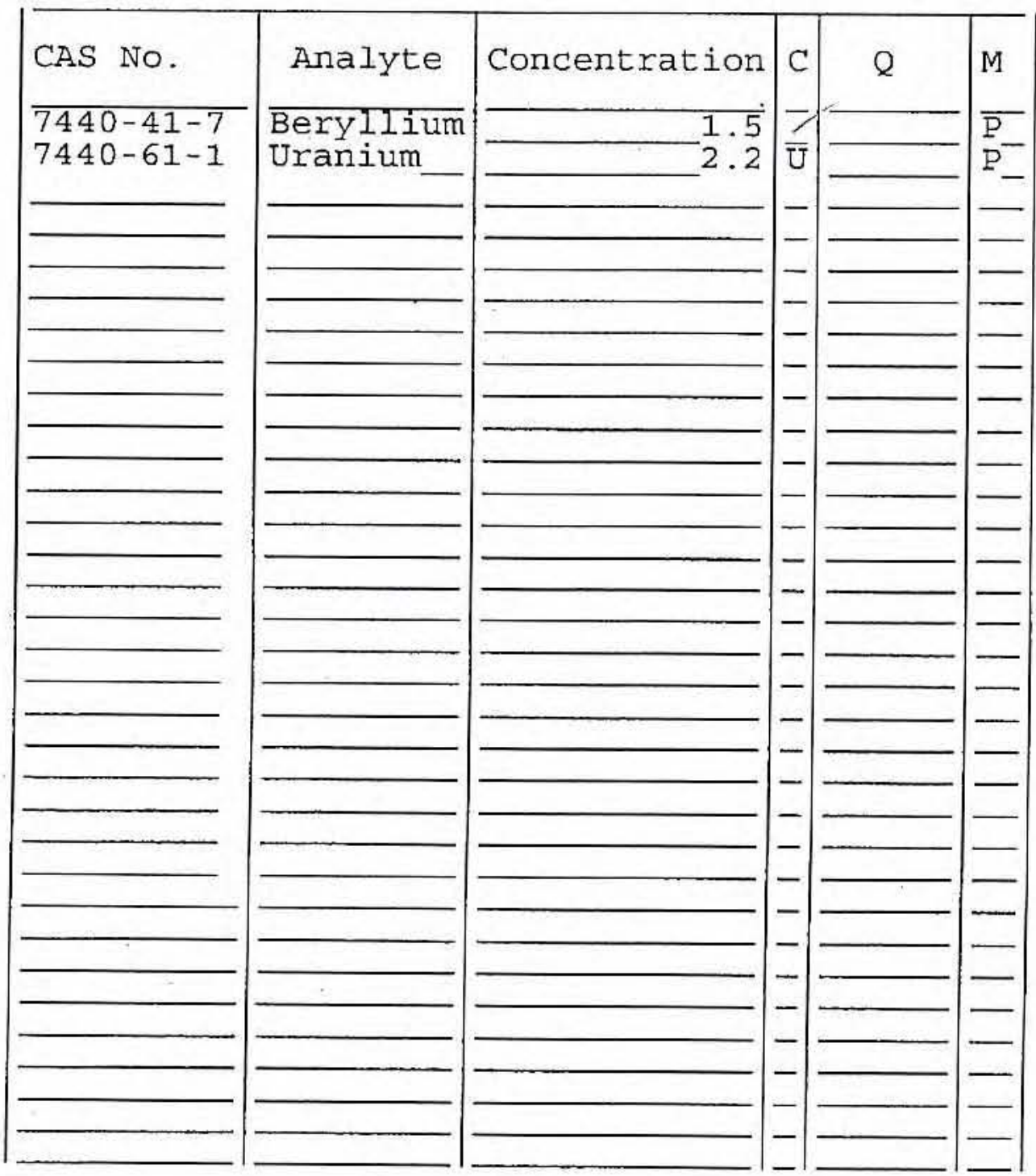

Clarity Before:

Clarity After:
Texture:

Artifacts :

Comments:

MPDT - 17A 
1

\section{INORGANIC ANALYSES DATA SHEET}

EPA SAMPLE NO.

$17 \mathrm{~B}$ Lab Sample ID: $0609189 \overline{5-0} 14$

Date Received: 09/07/06
Lab Name: LIONVILLE_LABORATORY
Case No.: MPDT_Contract: 60052
Lab

Lab Name: LIONVILLE_LABORATORY
Case No.: MPDT_Code: LVLI COntract: 60
SAS No.: Matrix (soil/water): SOIL Level (low/med): Solids:

$$
\begin{array}{r}
\text { LOW } \\
-89.7
\end{array}
$$

Concentration Units (ug/L or $\mathrm{mg} / \mathrm{kg}$ dry weight) : MG/KG

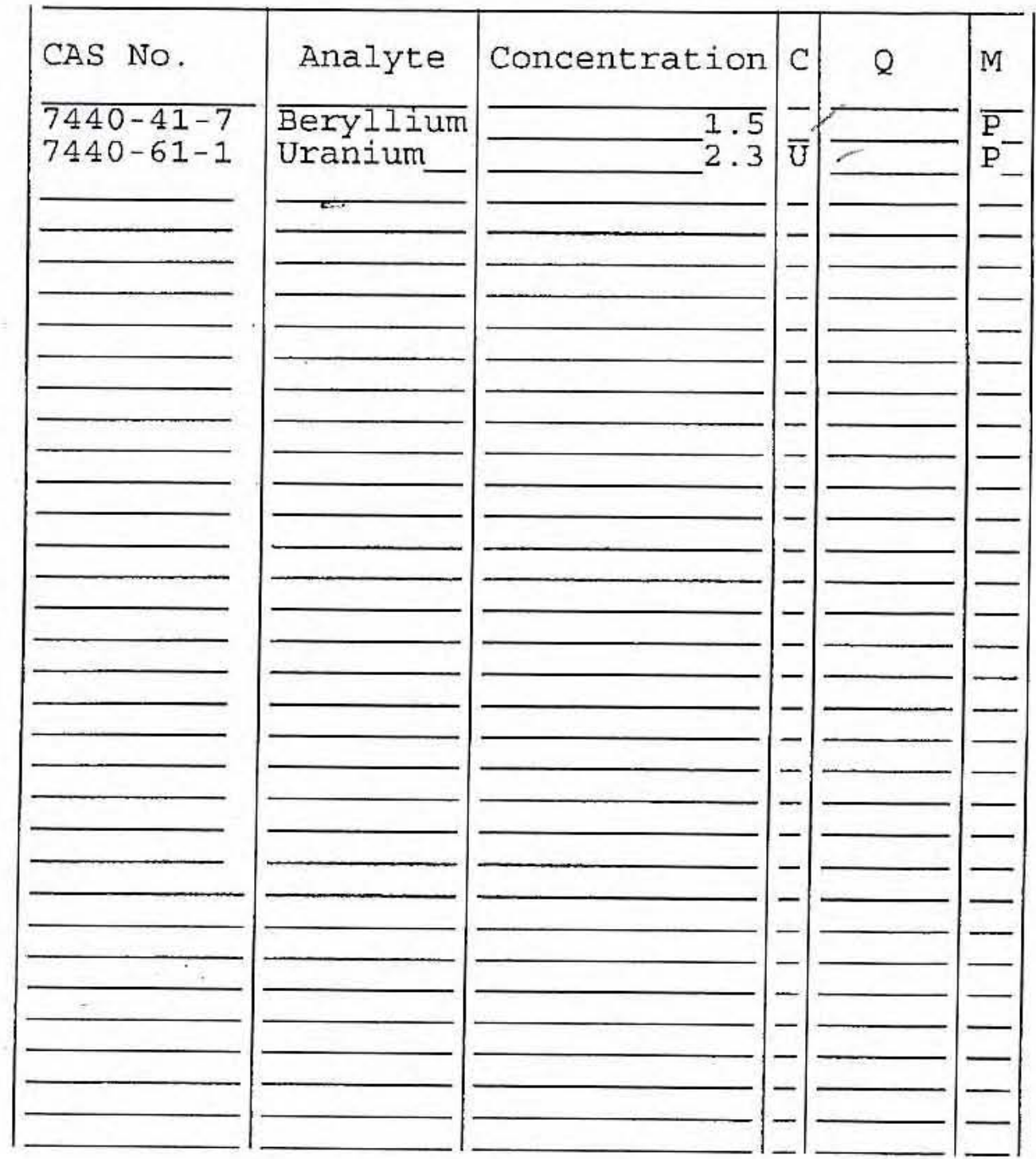

Clarity Before:

Clarity After:
Texture:

Artifacts :
Color Before:

Color After:

Comments :

MPDT-17B 
Lab Name: LIONVILLE LABORATORY

Lab Code: LVLI Matrix (soil/water): SOIL Level (low/med):

\% Solids: LOW

$-91 . \overline{4}$
Contract: 60052 SAS NO. :

SDG NO.: 11A

$18 \AA$

Lab Sample ID: 0609L895-015

Date Received: 09/07/06

Concentration Units (ug/L or $\mathrm{mg} / \mathrm{kg}$ dry weight): MG/KG
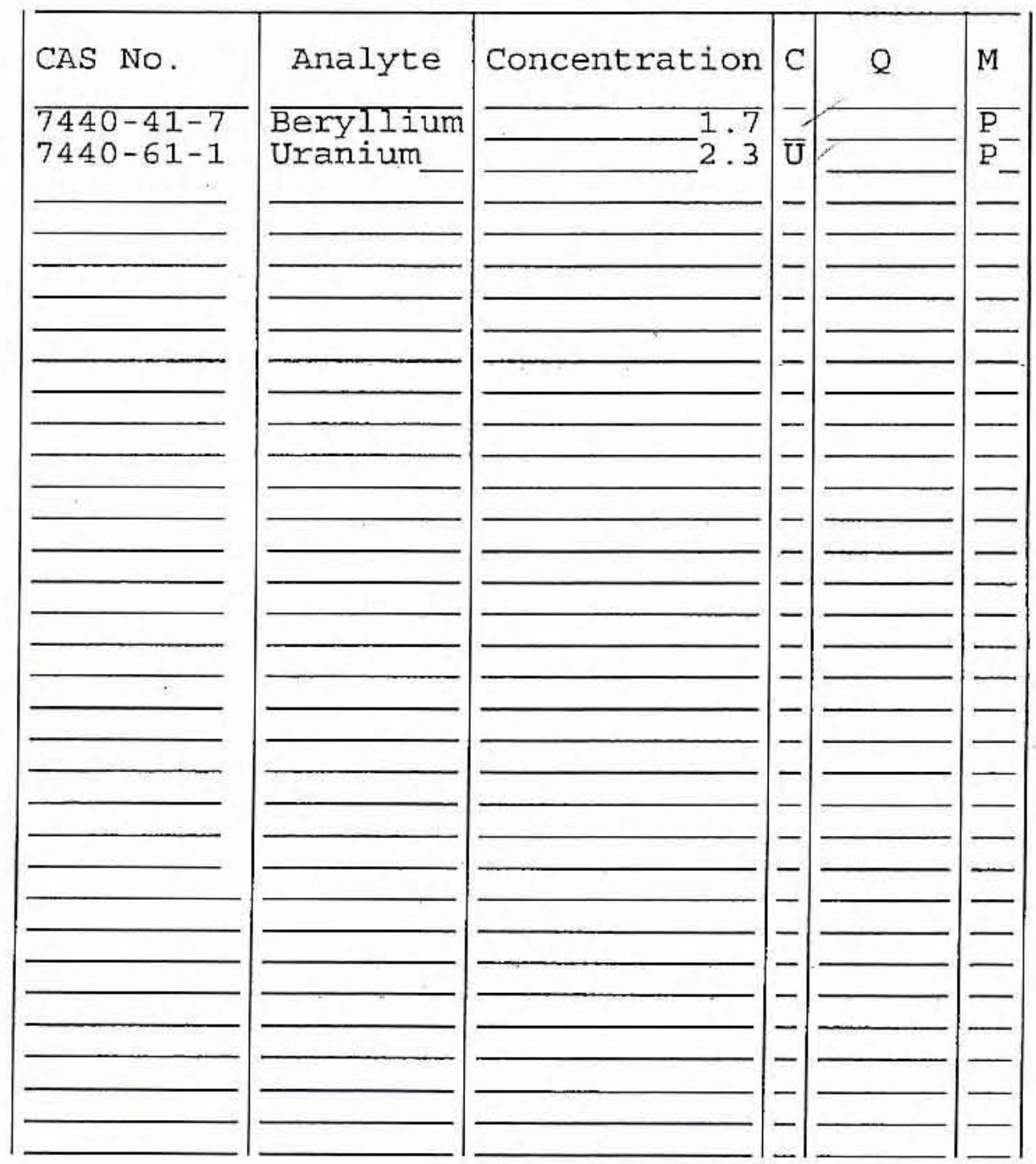

Color Before:

Color After:
Clarity Before: Clarity After:
Texture: Artifacts:

Comments :

MPDT-18A 


\section{U.S. EPA}

1

\section{INORGANIC ANALYSES DATA SHEET}

EPA SAMPLE NO.

$18 \mathrm{~B}$

Contract: 60052

SAS No.:

Lab Sample ID: 0609L895-016

Date Received: 09/07/06
Lab Name: LION
Lab Code: LVLI

Matrix (soil/water): SoIL

Level. (low/med): LOW

$\div$ Solids:

Concentration Units (ug/L or $\mathrm{mg} / \mathrm{kg}$ dry weight): MG/KG

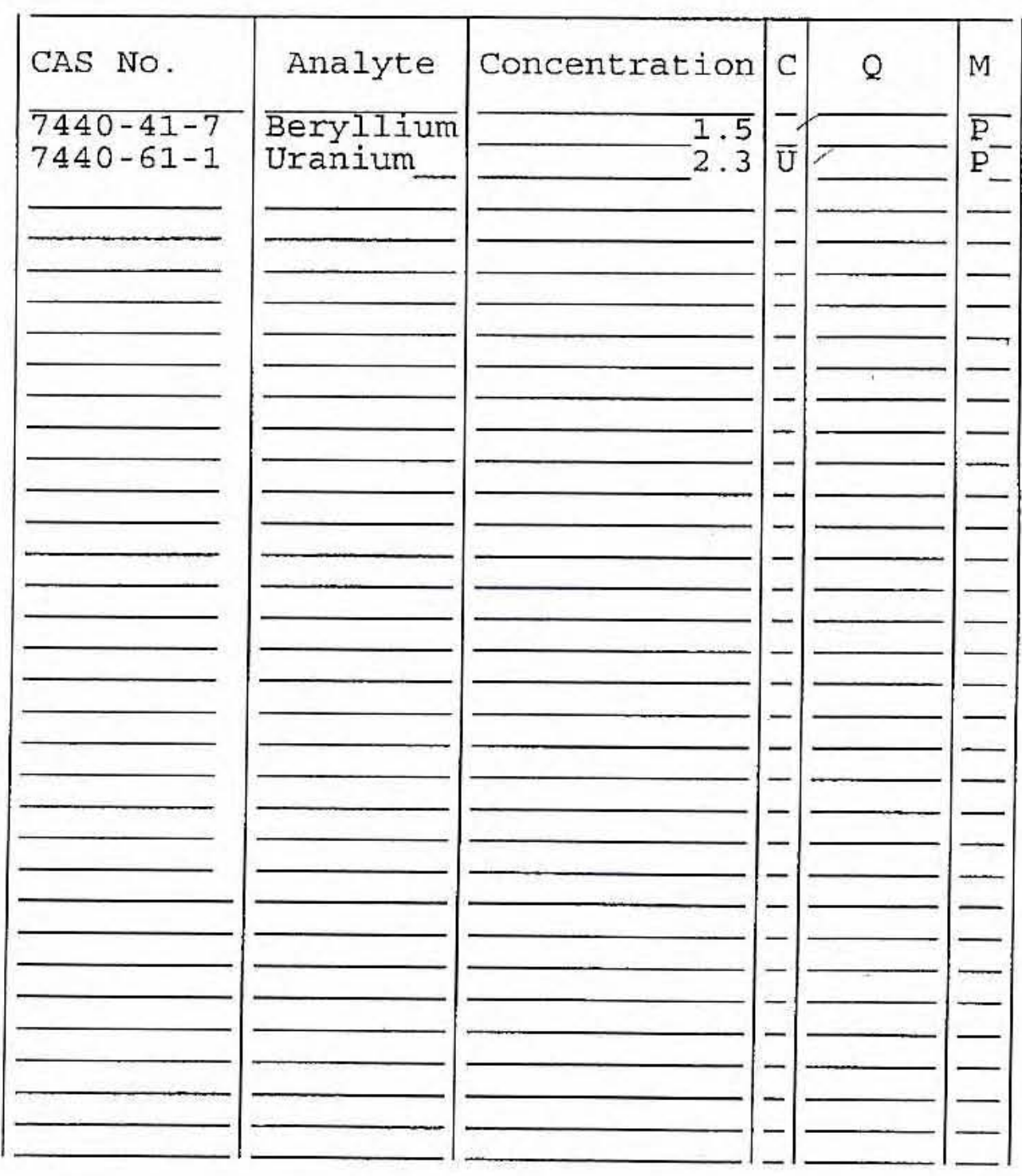

Clarity Before:

Clarity After:
Texture:

Artifacts:

Comments :

MPDT-18B 
1

INORGANIC ANALYSES DATA SHEET
EPA SAMPLE NO.

\section{$19 A$}

Contract: 60052

SAS No.:

Lab Sample ID: 0609L895-017

Date Received: 09/07/06
Lab Code: LVLI

Matrix (soil/water): SOIL

Level (low/med): LOW

Solids:
$-91.5$
Case No.: MPDT 5

injs (ug/L or $\mathrm{mg} / \mathrm{kg}$ dry weight): $M G / \mathrm{KG}$

Concentration Units (ug/L or $\mathrm{mg} / \mathrm{kg}$ dry weight): $\mathrm{MG} / \mathrm{KG}$
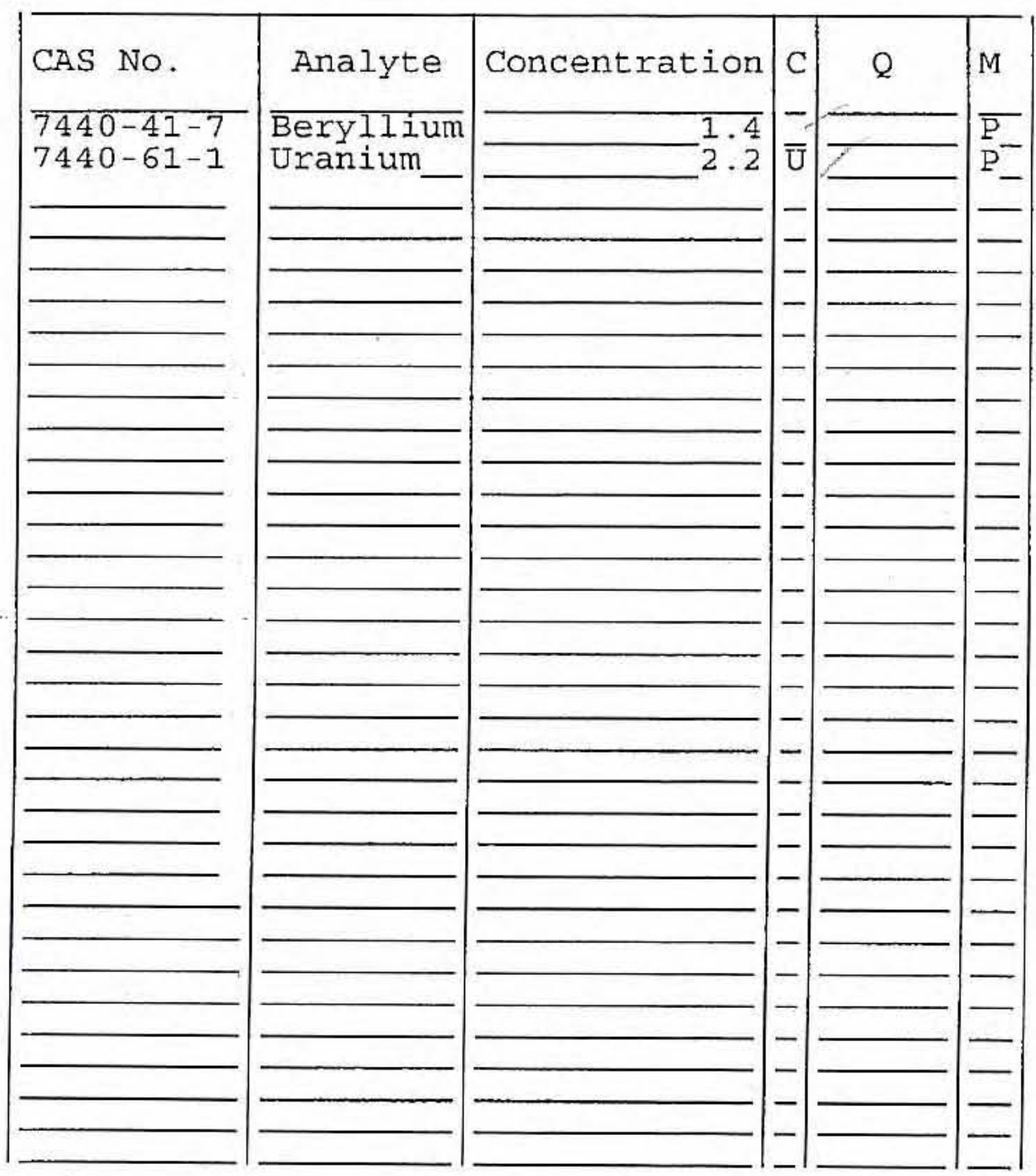

Clarity Before:

Clarity After:

Texture:

Artifacts : 
1 INORGANIC ANALYSES DATA SHEET
EPA SAMPLE NO.

$19 B$

Contract: 60052

SAS NO. :

SDG No.: 11A

Lab Sample ID: 0609L895-018

Date Received: 09/07/06

Lab Name: LIONVILLE_LABORATO
Lab Code: LVLI
Matrix (soil/water): SOIL
Level (low/med):
LOW Solids:

Lab Name: LIONVILLE_LABORATO
Lab Code: LVLI
Matrix (soil/water): SOIL
Level (low/med):
LOW Solids:

Lab Name: LIONVILLE_LABORATO
Lab Code: LVLI
Matrix (soil/water): SoIL
Level (low/med):
LOW Solids:

Concentration Units (ug/L or $\mathrm{mg} / \mathrm{kg}$ dry weight): $\mathrm{MG} / \mathrm{KG}$

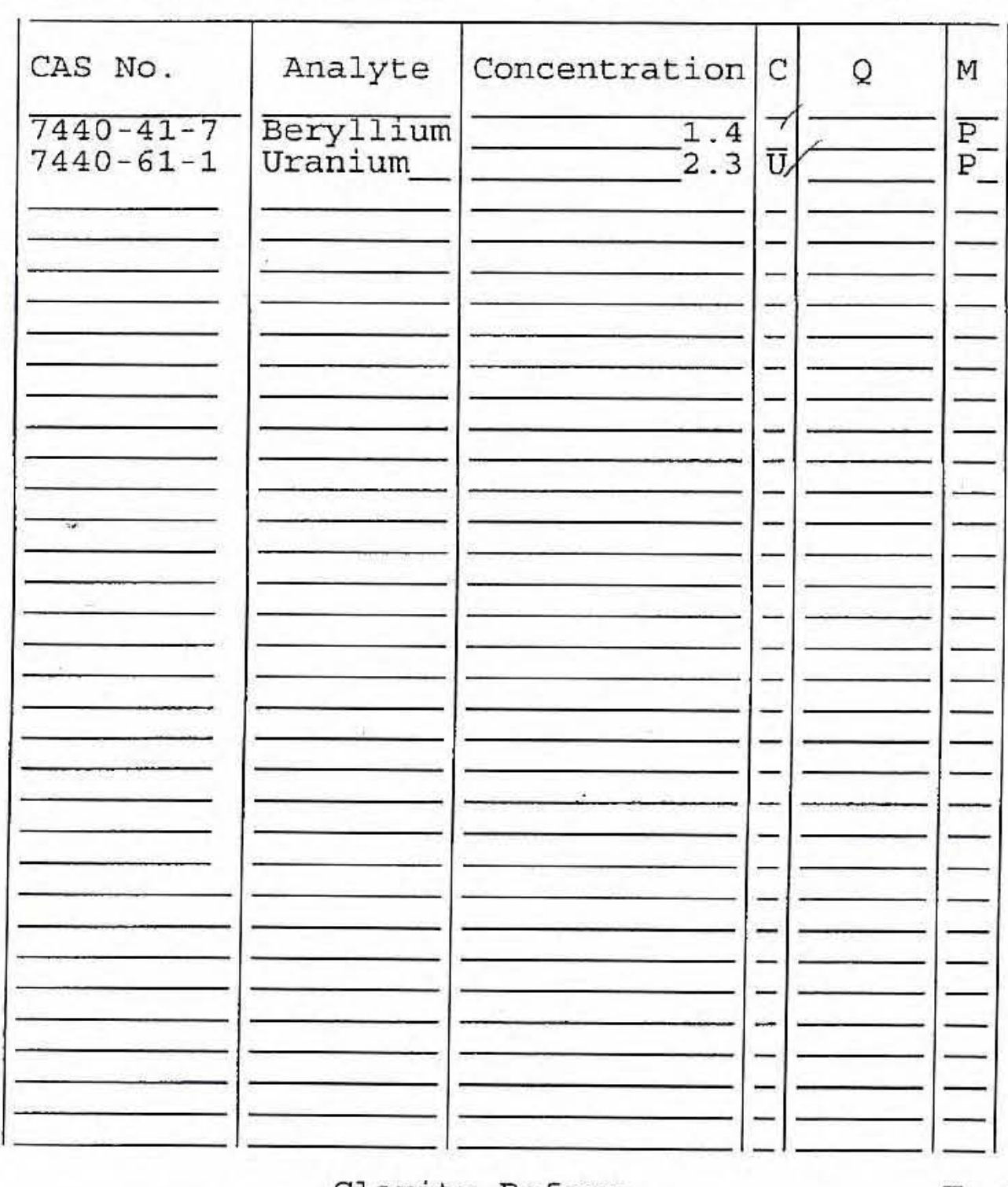

Color Before:

Color After:

Lab Name: LIONVILLE_LABORATO
Lab Code: LVLI
Matrix (soil/water): SOIL
Level (low/med):
LOW Solids:

LABORATORY

NO.: MPDT

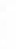

Lab Name: LIONVILLE_LABORATO
Lab Code: LVLI
Matrix (soil/water): SOIL
Level (low/med):
LOW Solids:

Lab Name: LIONVILLE_LABORATO
Lab Code: LVLI
Matrix (soil/water): SOIL
Level (low/med):
LOW Solids:

Lab Name: LIONVILLE_LABORATO
Lab Code: LVLI
Matrix (soil/water): SOIL
Level (low/med):
LOW Solids:

wejght): MG/KG

Clarity Before:

Clarity After:

Texture:

Artifacts:

Comments:

MPDT-19B 
U.S. EPA

1

INORGANIC ANALYSES DATA SHEET
EPA SAMPLE NO.

\section{$20 \mathrm{~A}$}

Contract: 60052

SAS No. :

SDG No.: 11A

Lab Sample ID: 0609L895-019

Date Received: 09/07/06
Matrix (soil/water): SOIL_

Level (low/med): LOW

\% Solids:

Concentration Units (ug/L or $\mathrm{mg} / \mathrm{kg}$ dry weight): MG/KG

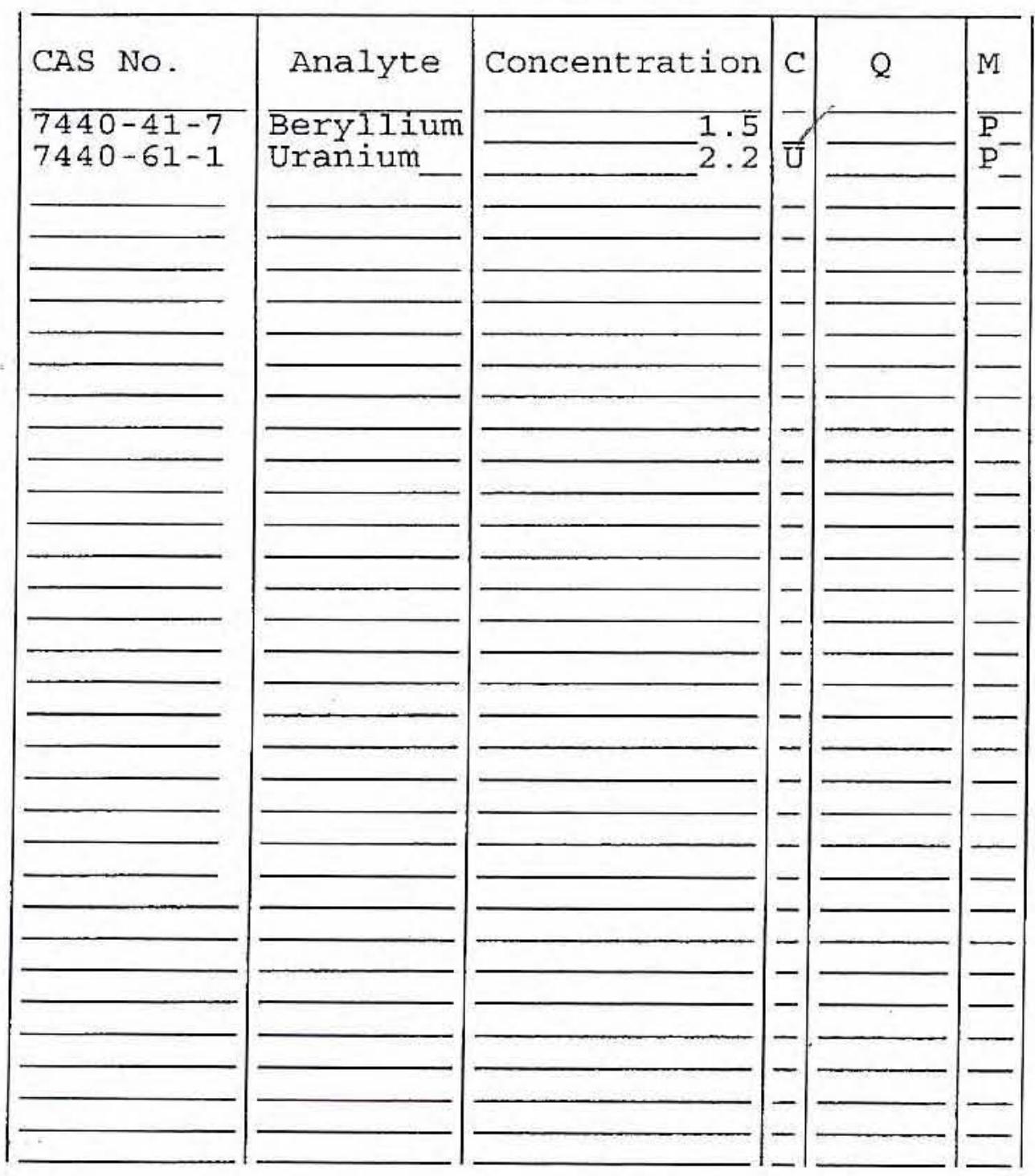

Color Before:

Color After:
Clarity Before:

Clarity After:
Texture:

Artifacts :

Comments :

MPDT-20A 
1 INORGANIC ANALYSES DATA SHEET

\section{Lab Name: LIONVILLE_LABORATORY}

Lab Code: LVLI Matrix (soil/water) : Level (low/med): \% Solids:

Contract: 60052

Case No.: MPDT_ SAS No.:

SOIL
LOW -
- 89.9

EPA SAMPLE NO.

$$
20 B
$$

SDG NO.: 11A

Lab Sample ID: 0609L895-020

Date Received: 09/07/06

Concentration Units (ug/L or $\mathrm{mg} / \mathrm{kg}$ dry weight): $\mathrm{MG} / \mathrm{KG}$

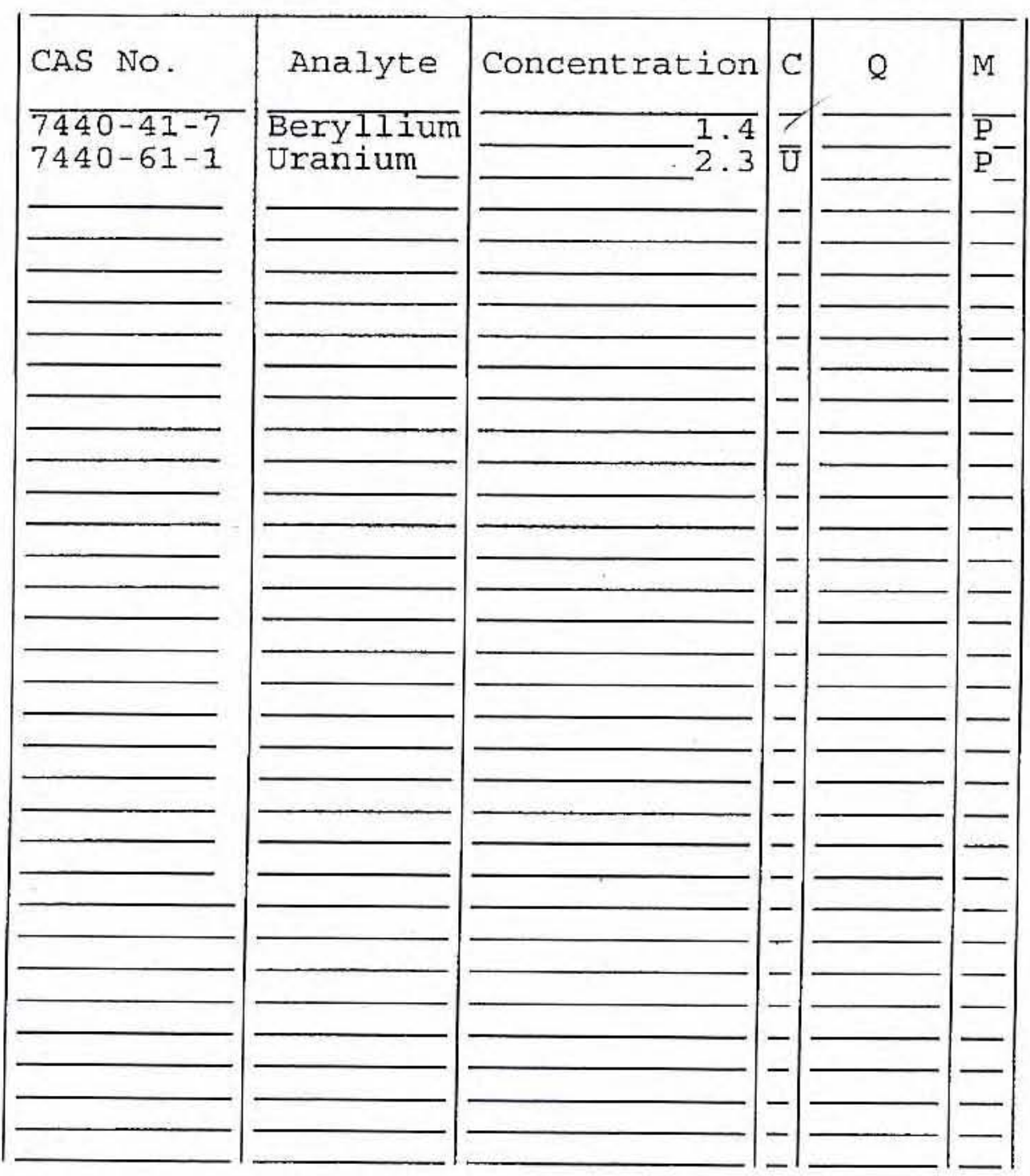

Color Before:

Color After:
Clarity Before:

Clarity After:
Texture:

Artifacts:

Comments :

MPDT $-20 B$ 


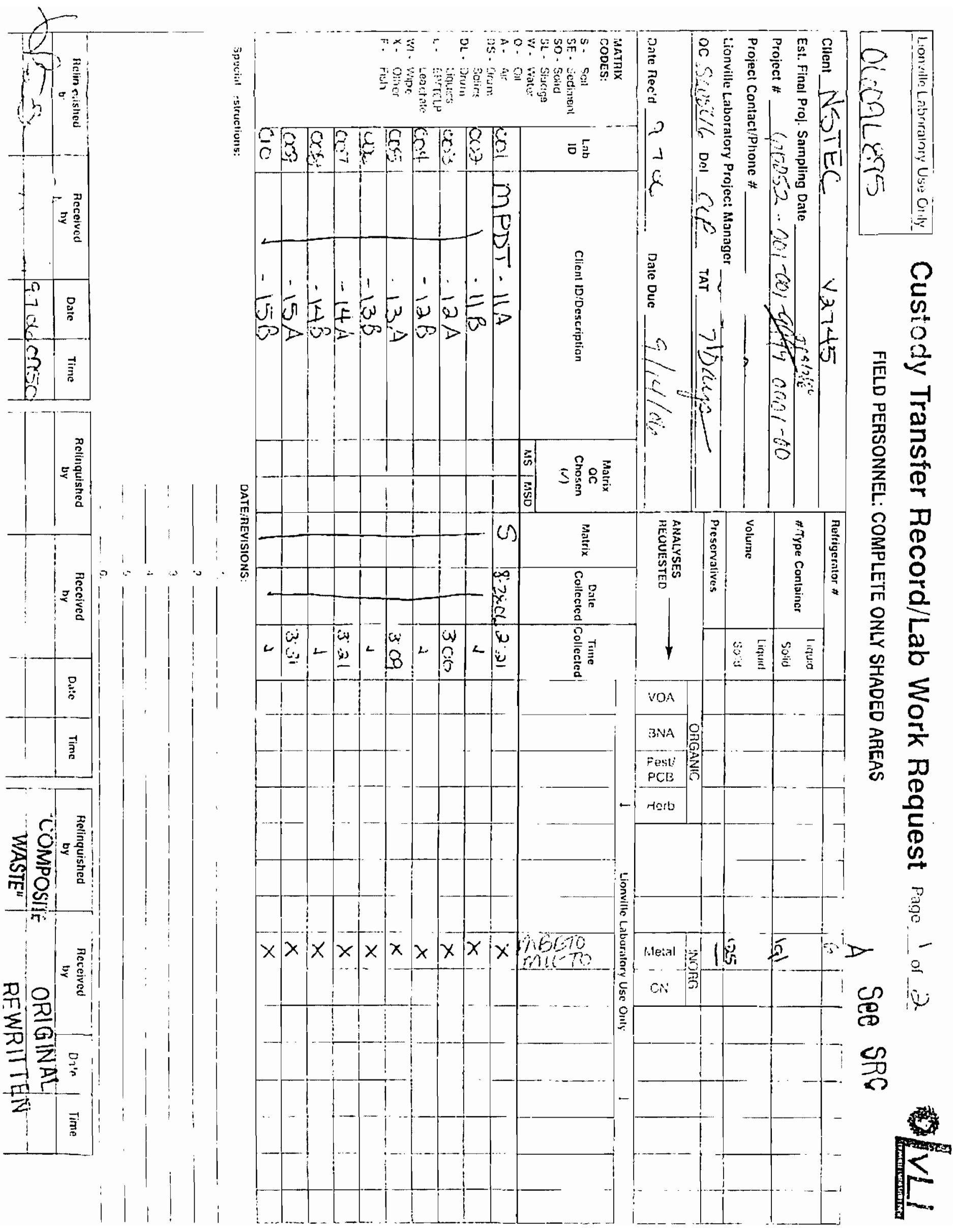




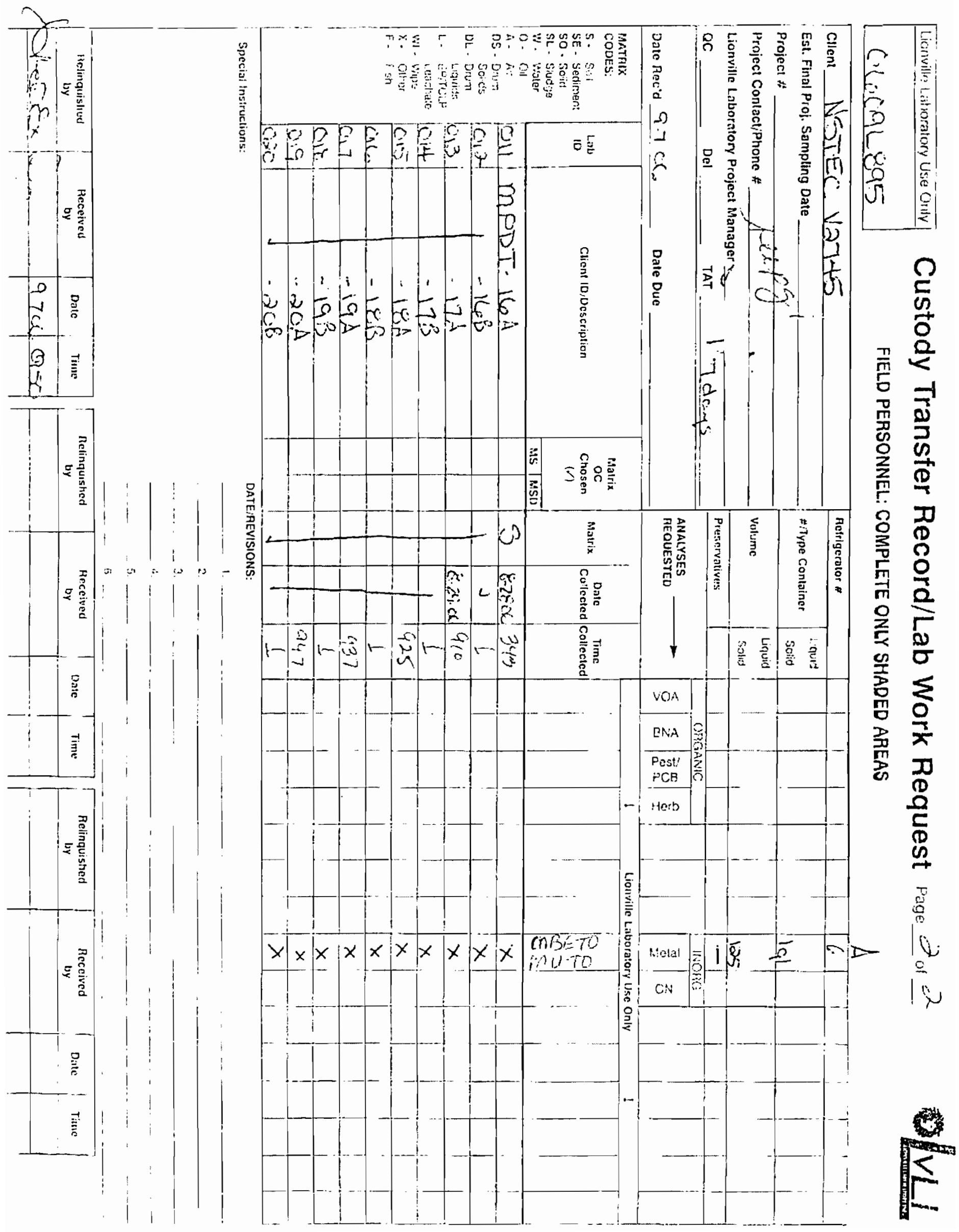




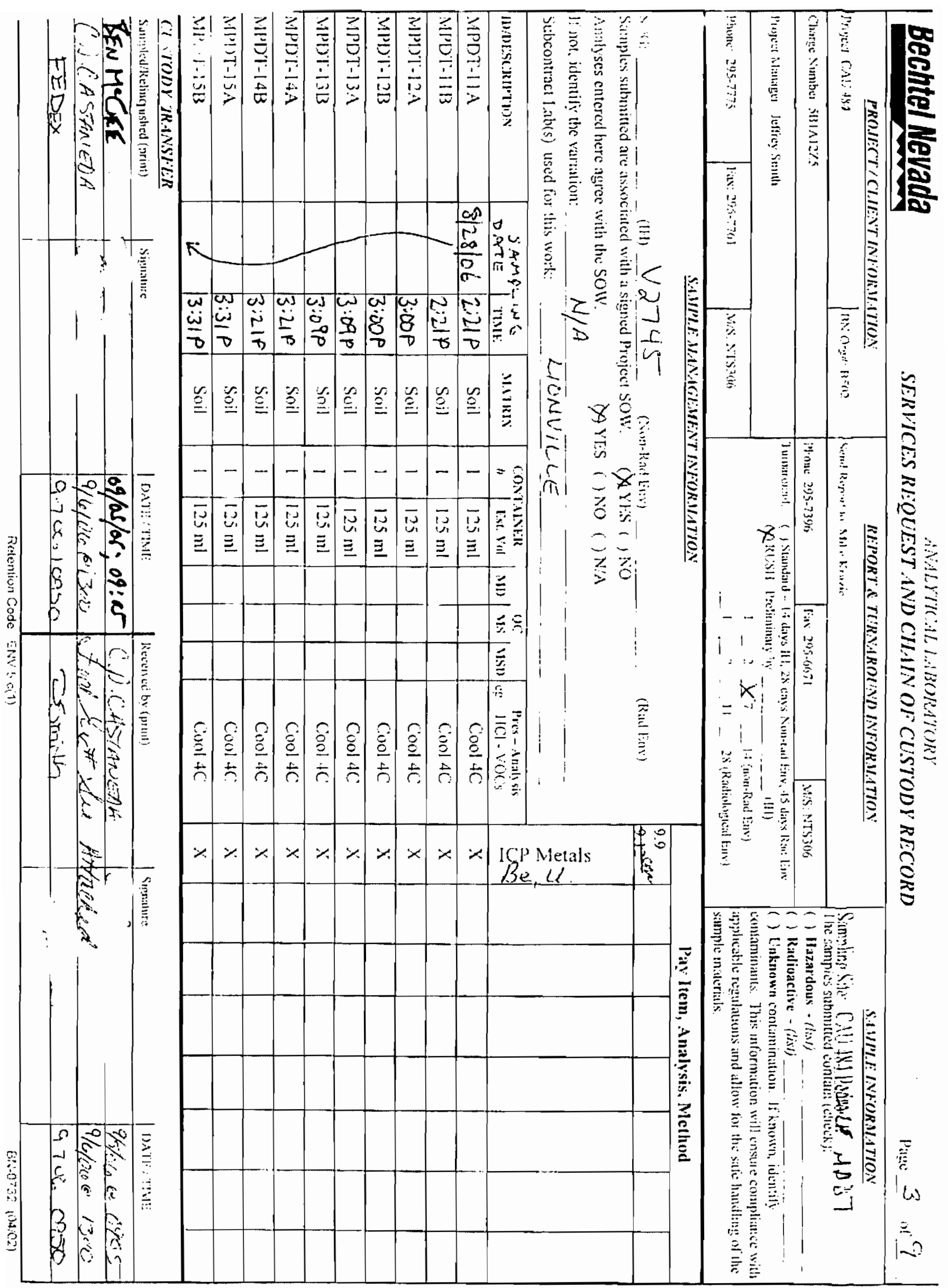




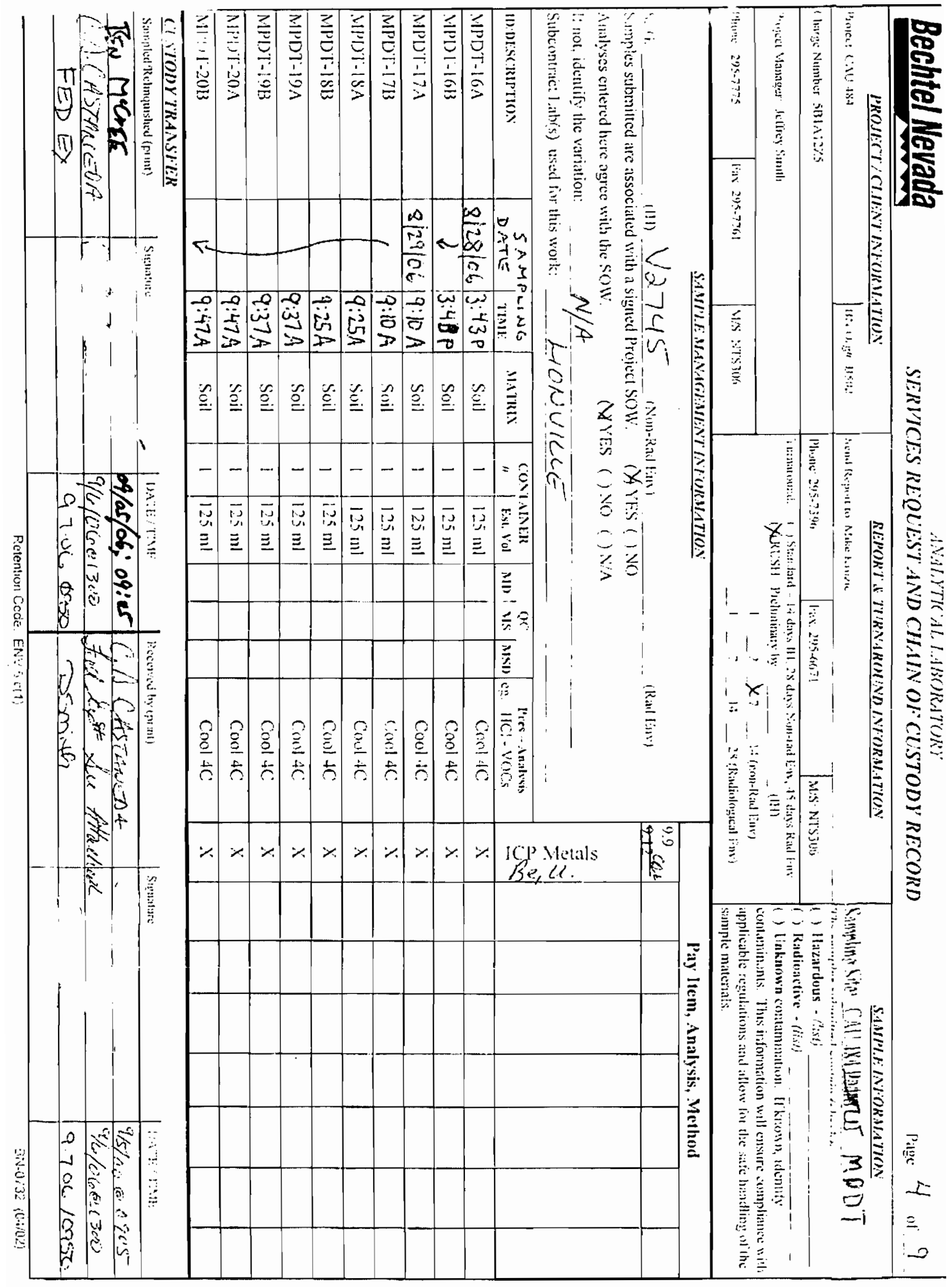




\section{U.S. EPA}

1

INORGANIC ANALYSES DATA SHEET
EPA SAMPLE NO.

\section{$21 \mathrm{~A}$}

Contract: 60052

Case No.: MPDT SAS No.: - SDG NO.: 21A

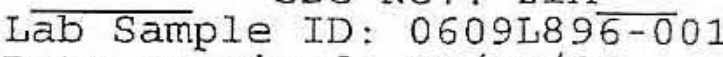

Date Received: 09/07/06
Matrix (soil/water): SOIL Level (low/med): \% Solids :

$$
\begin{array}{r}
\text { LOW } \\
-90.4
\end{array}
$$

Concentration Units (ug/L or mg/kg ary weight): $\mathrm{MG} / \mathrm{KG}$

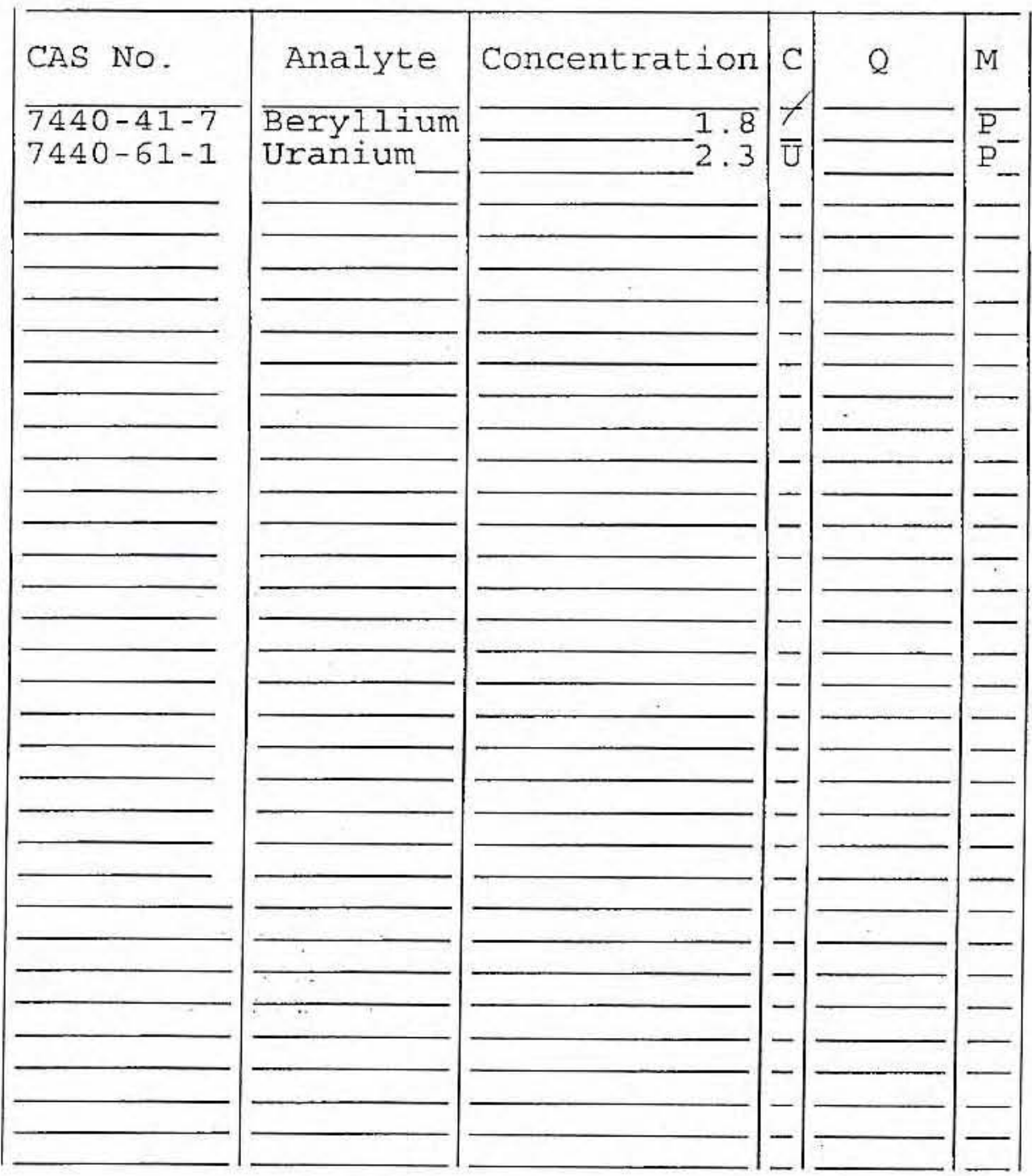

Color Before:

Color After:
Clarity Before:

Clarity After:
Texture:

Artifacts:

Comments :

MPDT - 21A. 
1

INORGANIC ANALYSES DATA SHEET
EPA SAMPLE NO.

\section{$21 B$}

Contract: 60052

Lab Sample ID: $0609489 \overline{6-0} 02$
SAS NO. :

Date Received: 09/07/06

SDG NO.: $21 \mathrm{~A}$
Lab Name: LIONVILLE_LABORATORY Matrix (soil/water): SOIL Level (low/med): Solids: LOW

Concentration Units (ug/L or $\mathrm{mg} / \mathrm{kg}$ dry weight): $\mathrm{MG} / \mathrm{KG}$

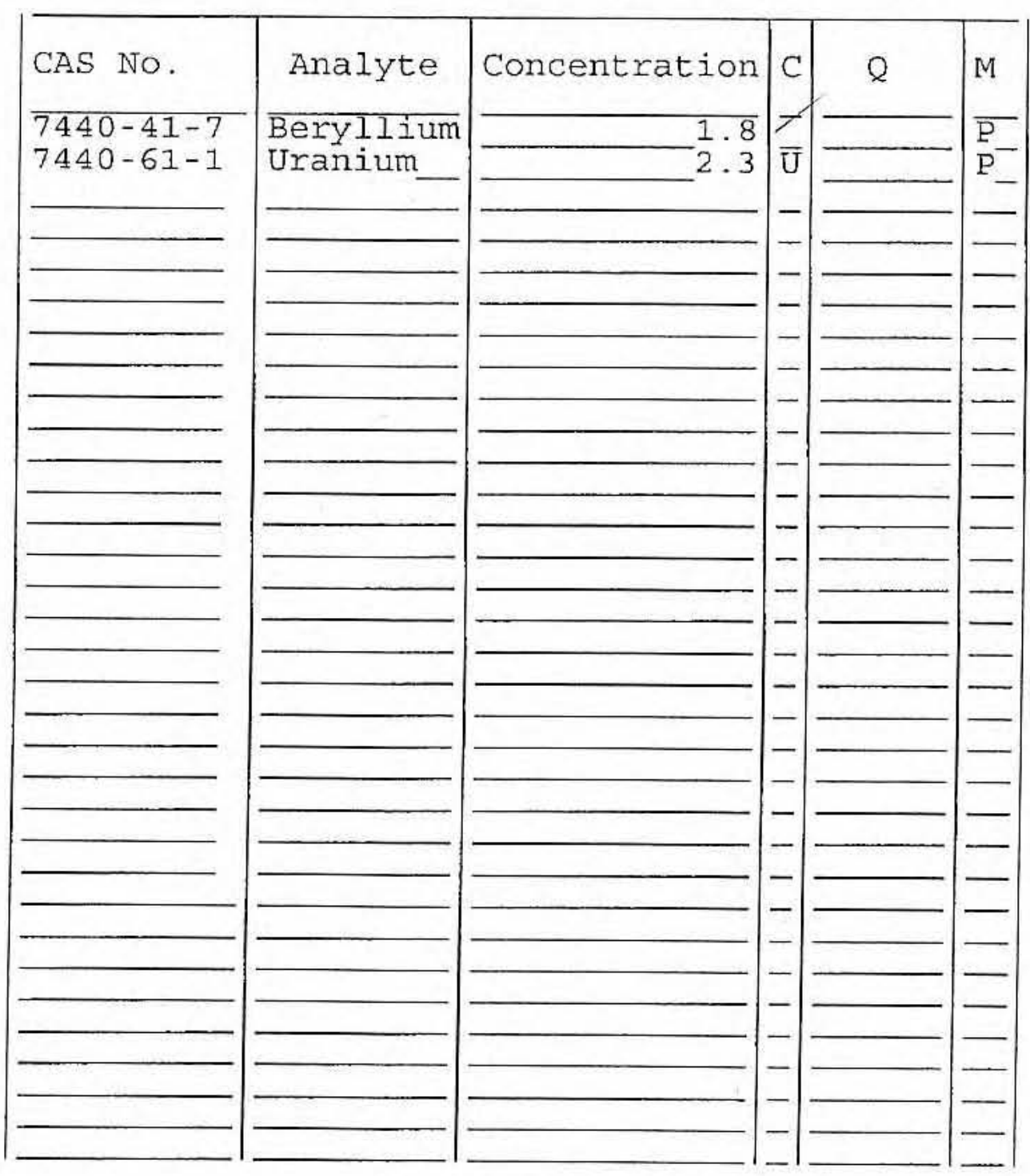

Color Before:

Color After:

\section{Comments :}

MPDT-21B
Clarity Before:

Clarity After:
Texture:

Artifacts: 


\section{U.S. EPA}

1 INORGANIC ANALYSES DATA SHEET
EPA SAMPLE NO.

\section{$22 \mathrm{~A}$}

Contract: 60052

SAS NO.:

SDG NO.: $21 \mathrm{~A}$

Lab Sample ID: 0609L896-003

Date Received: 09/07/06
Lab Name: LION Matrix (soil/water): SOIL Level (low/med): LOW \% Solids:
$-91 \overline{.2}$
Case No.: MPDT_

Concentration Units (ug/L or $\mathrm{mg} / \mathrm{kg}$ dry weight): MG/KG

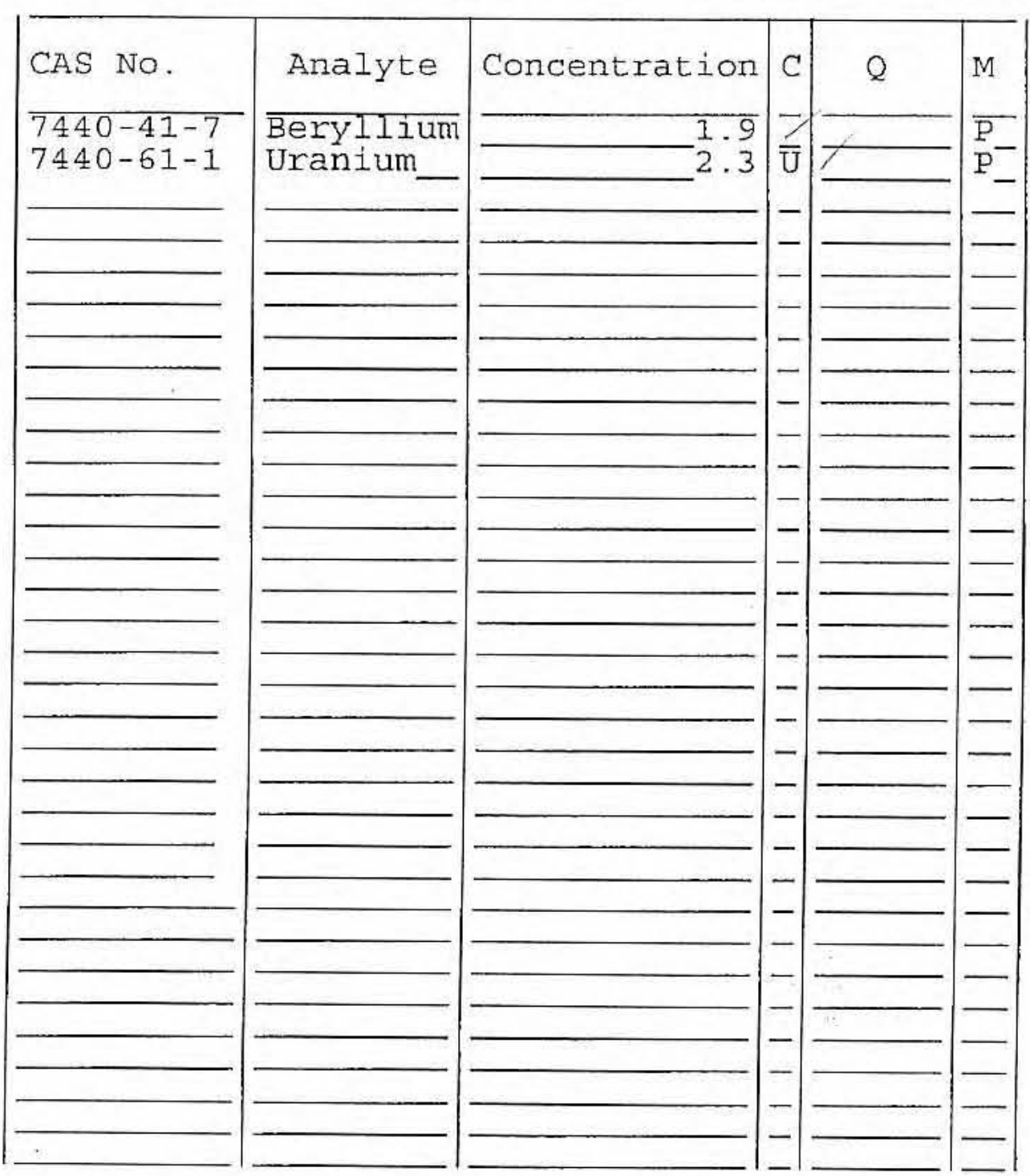

Color Before:

Color After:
Clarity Before:

Clarity After:
Texture:

Artifacts:

Comments:

MPDT $-22 \mathrm{~A}$ 
1

INORGANIC ANALYSES DATA SHEET

EPA SAMPLE NO.

$$
22 \mathrm{~B}
$$

Contract: 60052

SAS NO.:

Case No.: MPDT SOIL
LOW-
$88 \overline{.7}$
SDG NO.: 21A

Lab Sample ID: 0609L89 Date Received: 09/07/06
Lab Code: LVLI

Matrix (soil/water):

Level (low/med): Solids:
Concentration Units (ug/L or $\mathrm{mg} / \mathrm{kg}$ dry weight): MG/KG
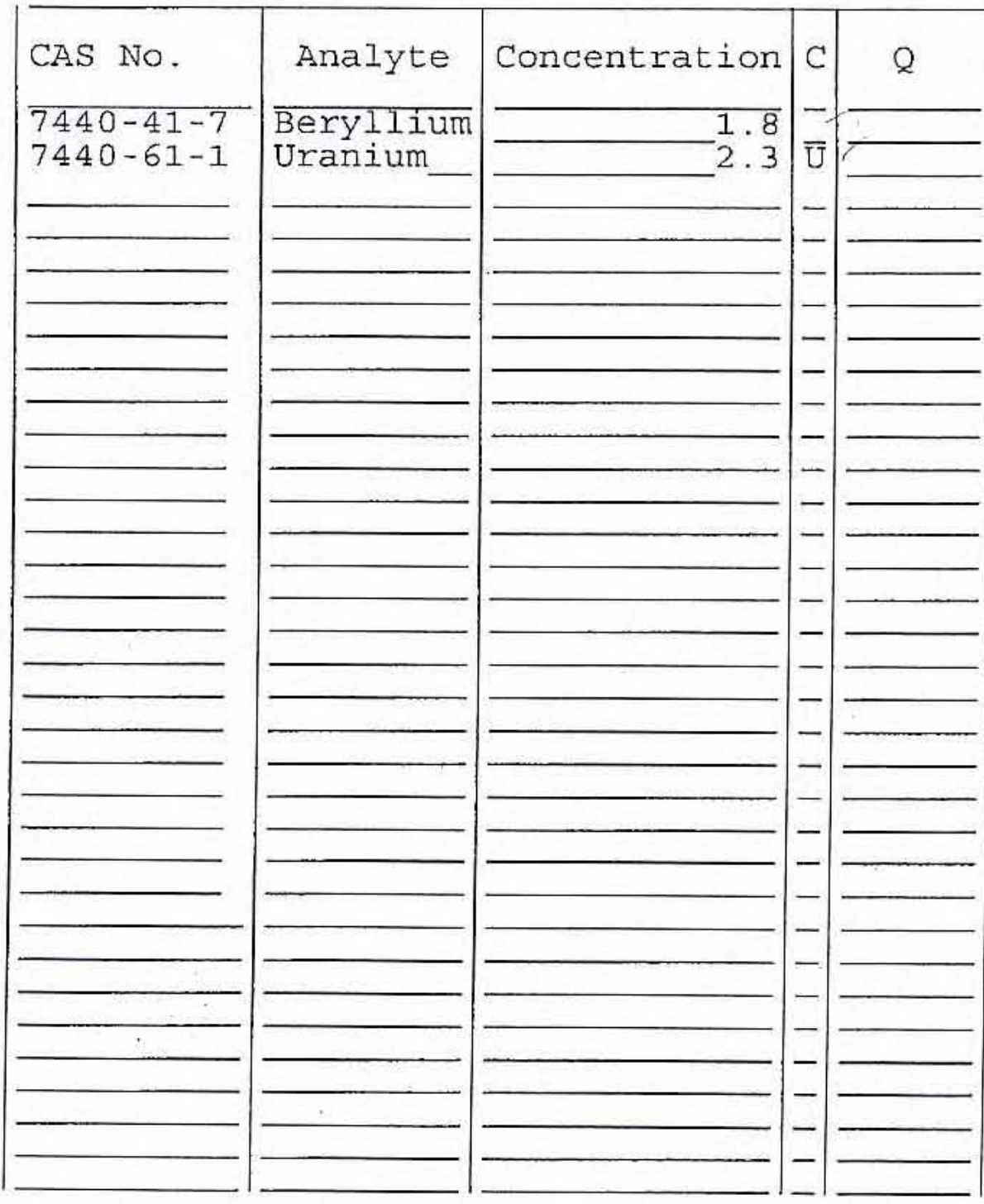

Clarity Before: Clarity After:

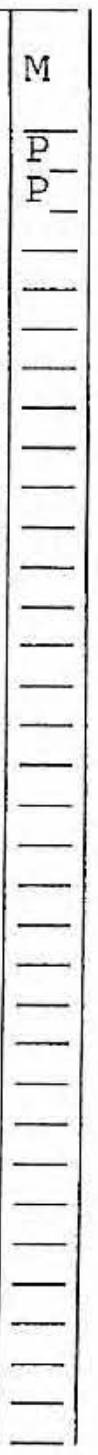

Texture: Artifacts:
Color Before

Comments:

MPDT $-22 B$ 
1

INORGANIC ANALYSES DATA SHEET
EPA SAMPLE NO.

\section{$23 \mathrm{~A}$}

Contract: 60052 SAS NO.:

Lab Sample ID: 0609L89

Date Received: 09/07/06
Lab Name: LIONVILLE_LABORATORY

Lab Code: IVLI

Matrix (soil/water): SoIL_

$\begin{array}{lr}\text { Level (low/med) : } & \text { LOW } \\ \% \text { Solids: } & 90 \overline{.6}\end{array}$

Concentration Units (ug/L or mg/kg dry weight): MG/KG

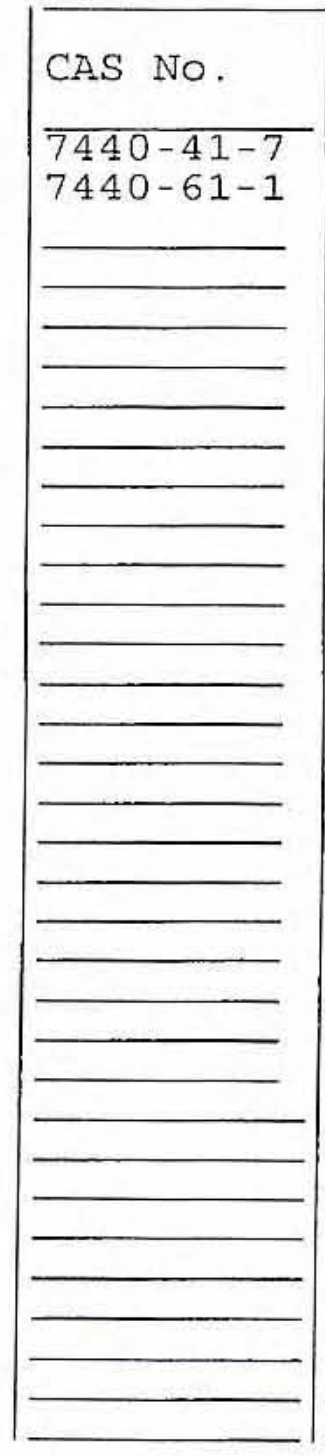

Color Before:

Color After:

Comments :

MPDT-23A

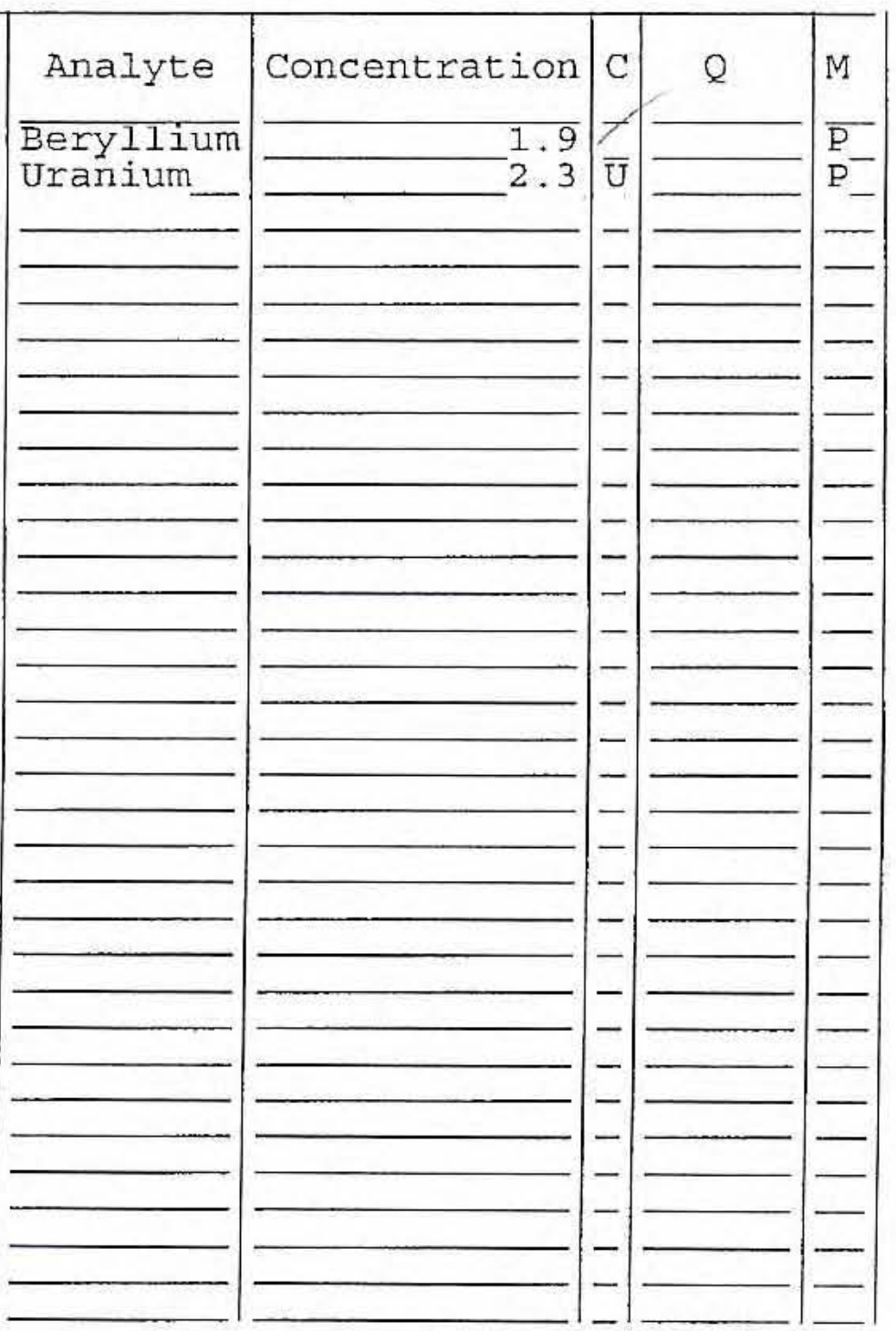

Clarity Before: Clarity After:
Texture:

Artifacts: 
EPA SAMPLE NO.

$$
23 \mathrm{~B}
$$

Lab Name: LIONVILLE_LABORATORY

Lab code: LVLI Matrix (soil/water): SOIL Level (low/med): Solids:
Contract: 60052 SAS NO.:

La: $21 \mathrm{~A}$ LOW
Lab Sample ID: 0609L896-006

Date Received: 09/07/06

Concentration Units (ug/L or $\mathrm{mg} / \mathrm{kg}$ dry weight): MG/KG

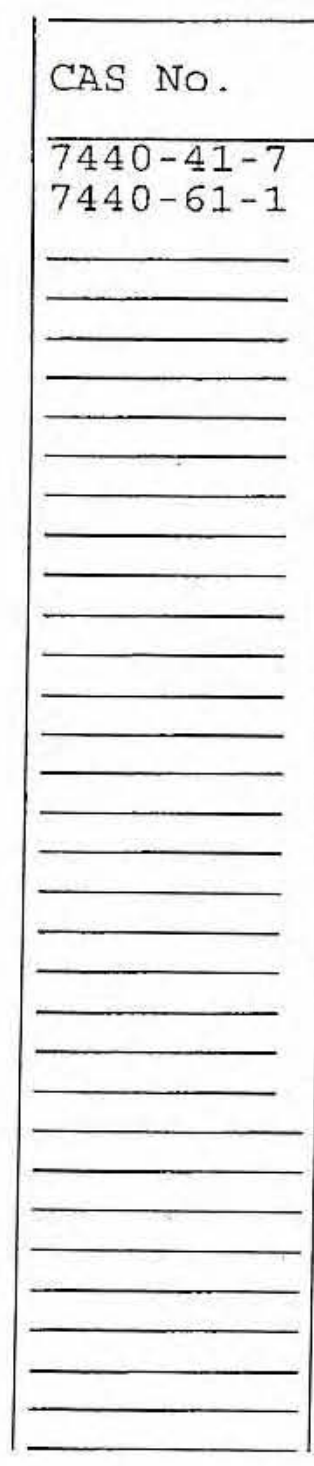

Color Before: Color After:

Comments:

MPDT $-23 B$

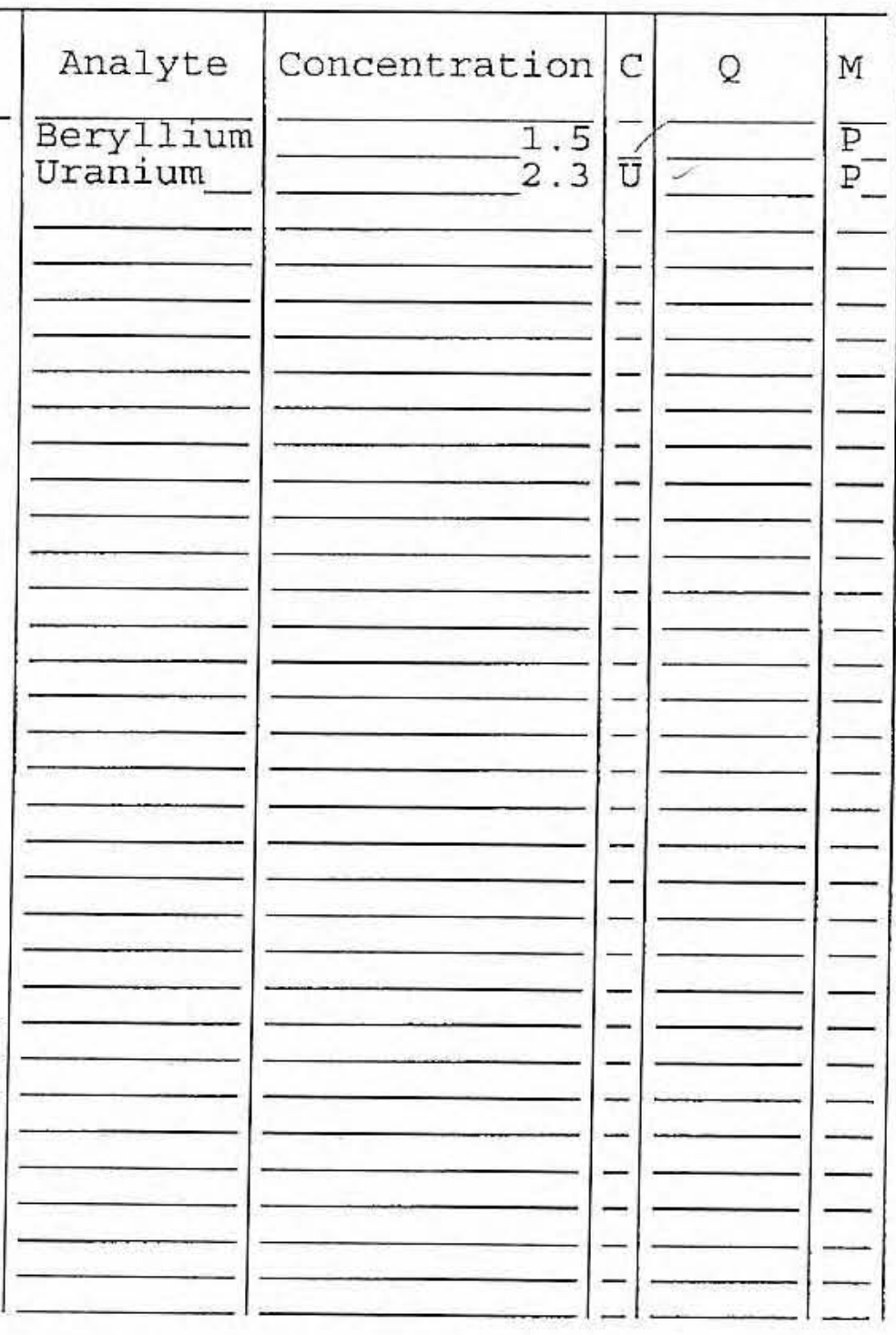

Clarity Before:

Texture:

Artifacts: 
1

\section{INORGANIC ANALYSES DATA SHEET}

EPA SAMPLE NO.

$$
24 \mathrm{~A}
$$

Contract: 60052

Case No.: MPDT_ SAS No.: _ SDG No.: $21 \mathrm{~A}$

Case No.: MPDT_ SAS No.:

Lab Sample ID: 0609L89

LOW

Concentration Units (ug/L or $\mathrm{mg} / \mathrm{kg}$ dry weight): MG/KG

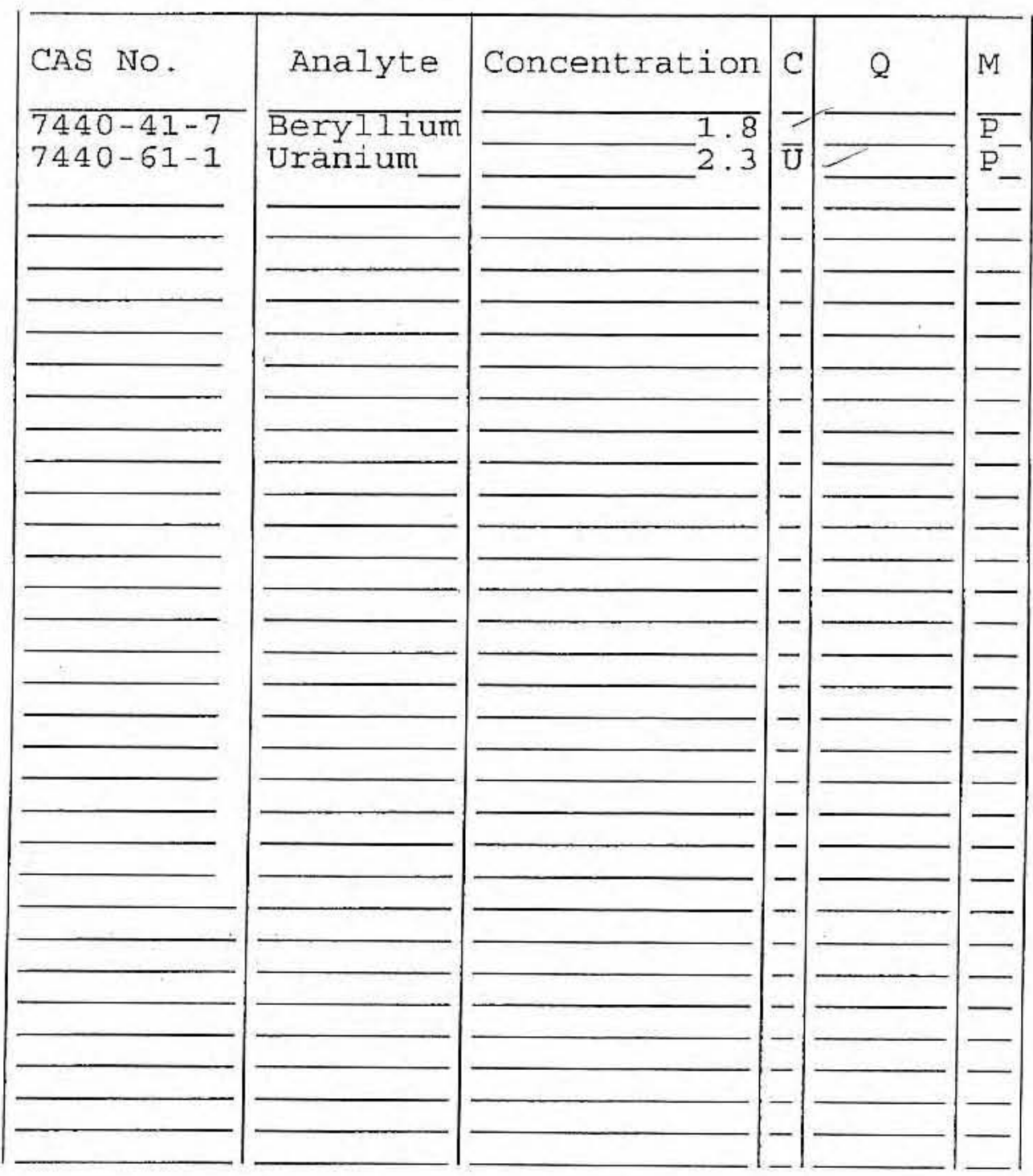

Color Before:

Color After:
Clarity Before:

Clarity After:
Texture:

Artifacts:

Comments:

$M P D T-24 \mathrm{~A}$ 


\section{U.S. EPA}

1

INORGANIC ANALYSES DATA SHEET
EPA SAMPLE NO.

$$
24 \mathrm{~B}
$$

SDG NO.: $21 \bar{A}$

Lab Sample ID: 0609L89

Date Received: 09/07/06

Lab Name: LIONVILLE_LABORATORY__Contract: 60052

Lab Code: LVLI

Matrix (soil/water): SoIL Level (low/med) :

oolids :
LOW

$-88 \overline{.6}$
SAS NO. :

Concentration Units (ug/L or $\mathrm{mg} / \mathrm{kg}$ dry weight): MG/KG

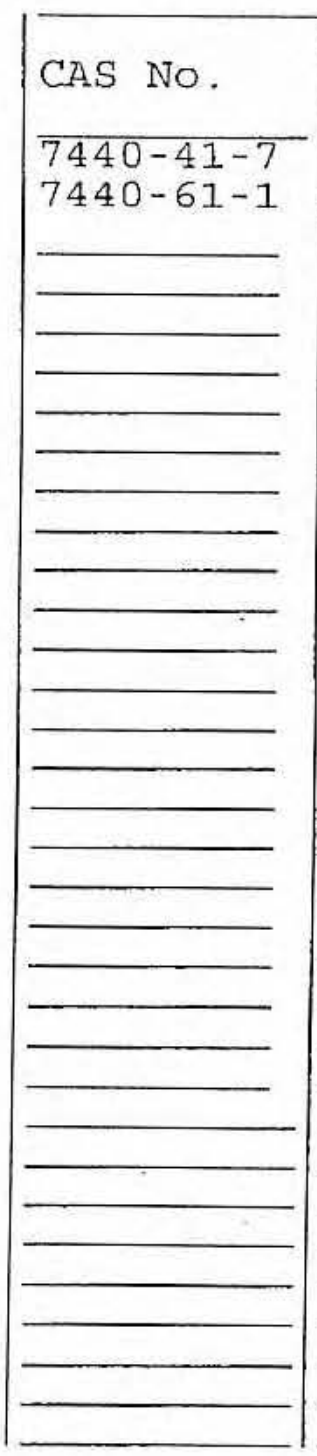

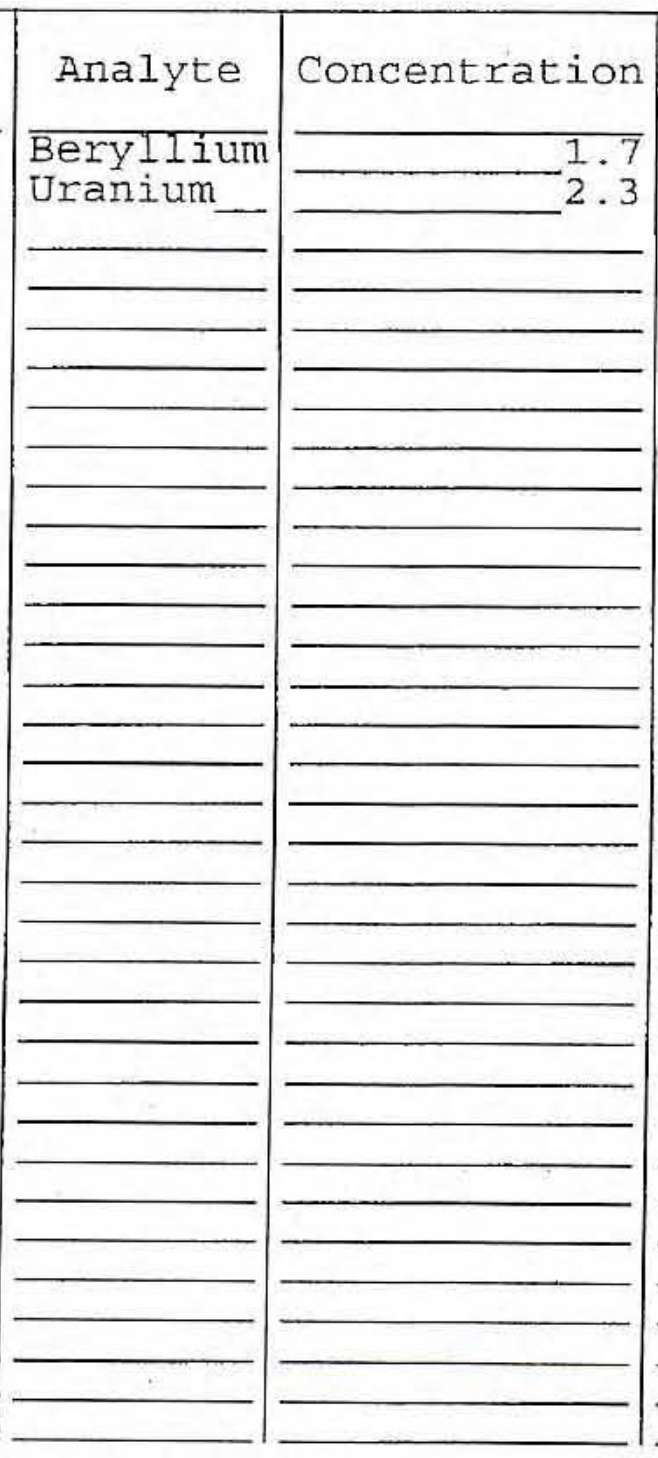

Clarity Before:

Clarity After:

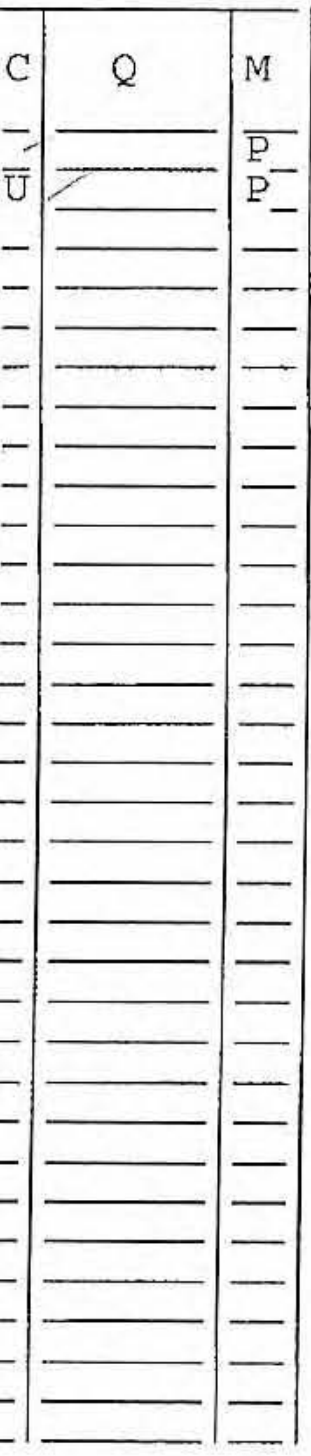

Texture: Artifacts:

Color Before
Color After:

Comments:

MPDT-24B 
U.S. EPA

1.

INORGANIC ANALYSES DATA SHEET
EPA SAMPLE NO.

$25 \mathrm{~A}$
Lab Name: LIONVILLE_LABORATORY

Lab Code: LVLI

Matrix (soil/water): SoIL_

Level (low/med):

\% Solids:
LOW
-91.8
SDG NO : : $21 \mathrm{~A}$

Lab Sample ID: $0609489 \overline{6-009}$
Date Received: $09 / 07 / 06$

Lab Sample ID: $0609489 \overline{6}-0$
Date Received: $09 / 07 / 06$

Contract: 60052

SAS NO. :

Concentration Units (ug/L or $\mathrm{mg} / \mathrm{kg}$ dry weight): MG/KG

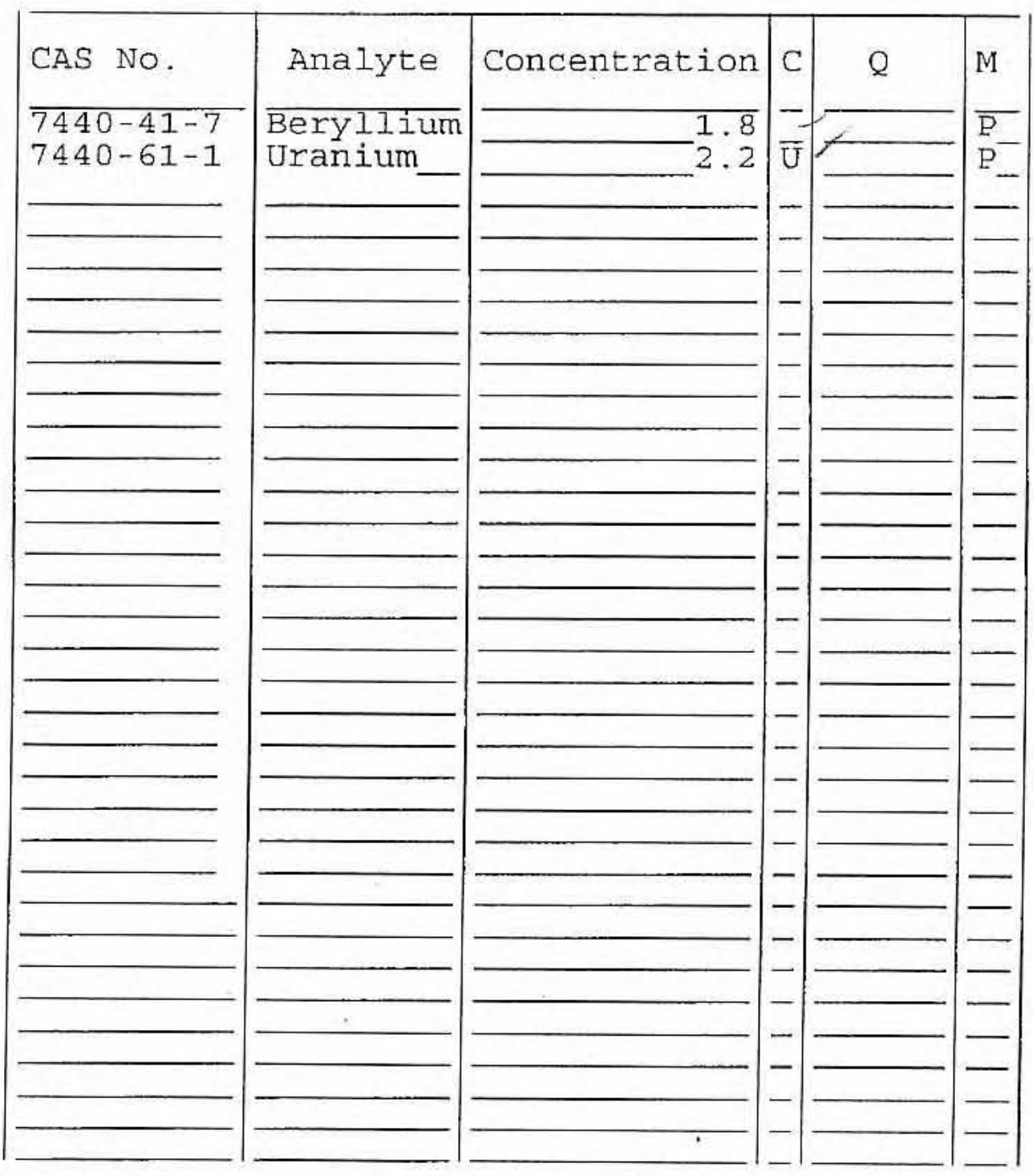

Color Before:

Color After:
Clarity Before:

Clarity After:
Texture:

Artifacts:

Comments :

MPDT - 25A 
1

INORGANIC ANALYYSES DATA SHEET
EPA SAMPLE NO.

$$
25 B
$$

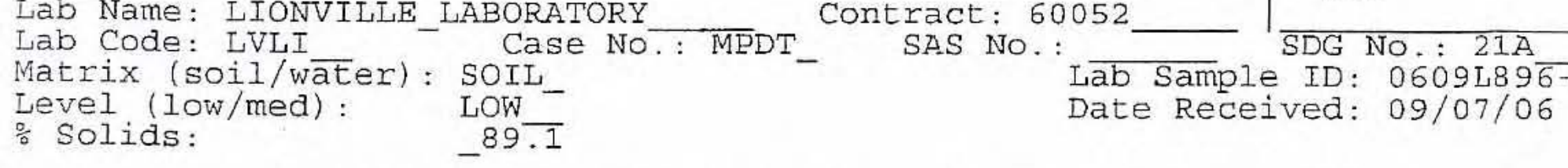

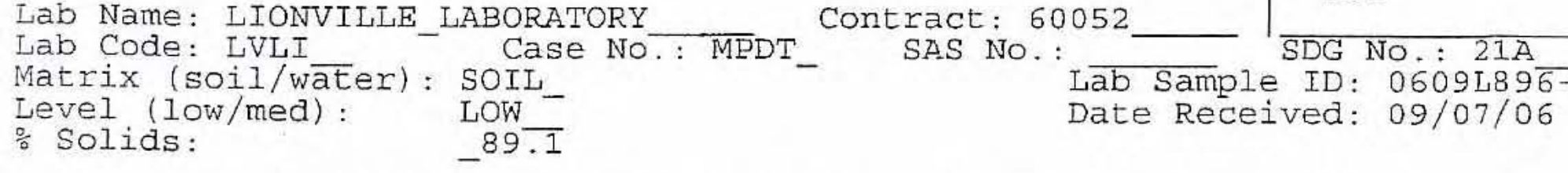

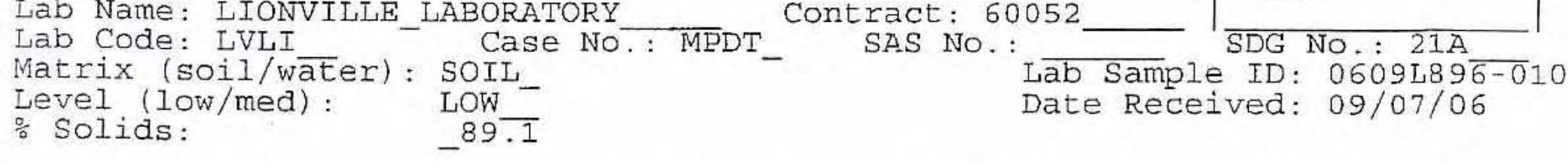

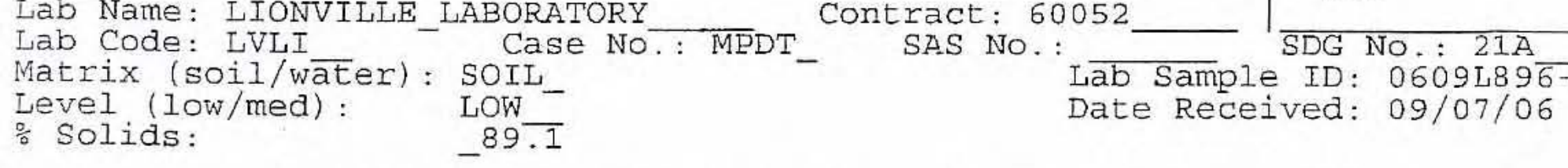

Concentration Units (ug/L or $\mathrm{mg} / \mathrm{kg}$ dry weight): MG/KG

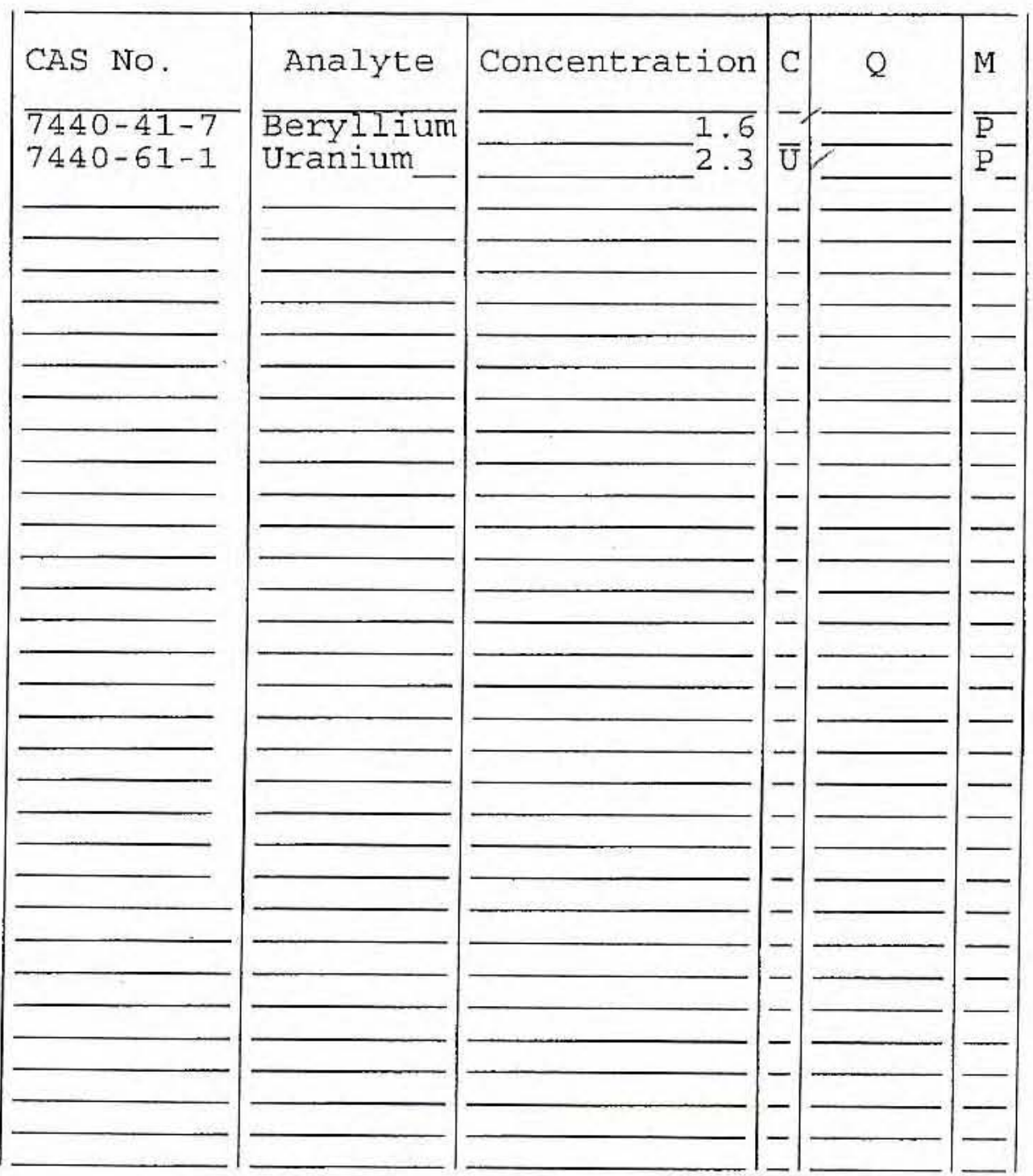

Color Before:

Color After:

Comments:

MPDT - 25B
Clarity Before:

Clarity After:
Texture:

Artifacts: 
1

INORGANIC ANALYSSES DATA SHEET
EPA SAMPLE NO.

$$
\text { 26A }
$$

SDG NO : $21 \mathrm{~A}$

SAS NO. :

Lab Sample ID: 0609L895-011

Date Received: 09/07/06

\section{wab Name: LIONVILLE_LABORATORY \\ Matrix (soil/water): SOIL \\ Level (low/med): \\ $\div$ Solids: \\ LOW \\ $- 9 2 \longdiv { . 9 }$}

Contract: 60052

Concentration Units (ug/L or $\mathrm{mg} / \mathrm{kg}$ dry weight): MG/KG

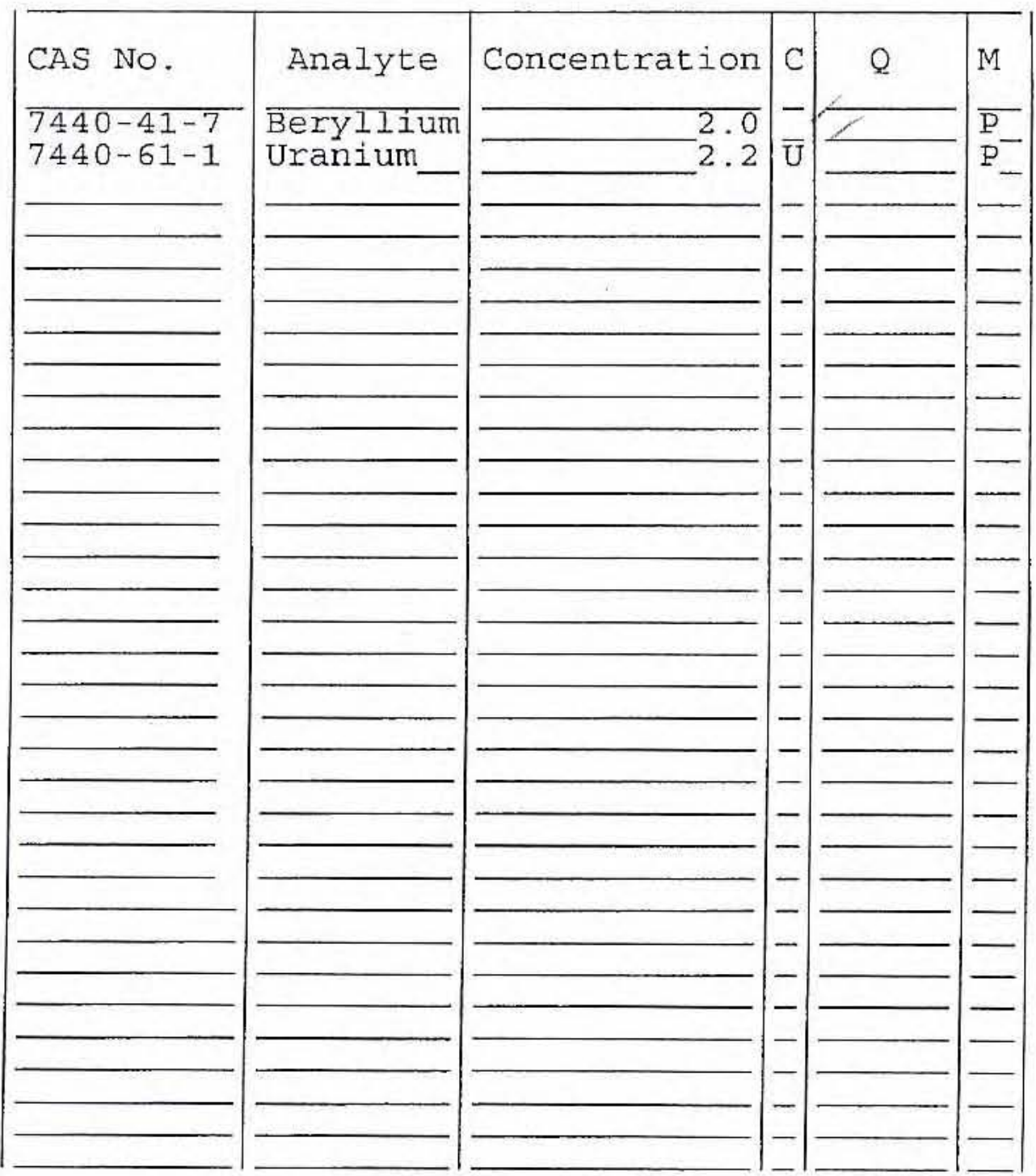

Color Before:

Color After:
Clarity Before: Clarity After:
Texture:

Artifacts:

Comments:

MPDT-26A 
1

INORGANIC ANALYSES DATA SHEET
EPA SAMPLE NO.

\section{$26 B$}

Contract : 60052

SAS NO.:

SDG NO.: $21 \mathrm{~A}$

Lab Sample ID: 0609L89 $\overline{6-012}$

Date Received: 09/07/06
Matrix (soil/water): soIL

Level. (low/med): LOW

$\div$ Solids:

Concentration Units (ug/L or $\mathrm{mg} / \mathrm{kg}$ dry weight): MG/KG

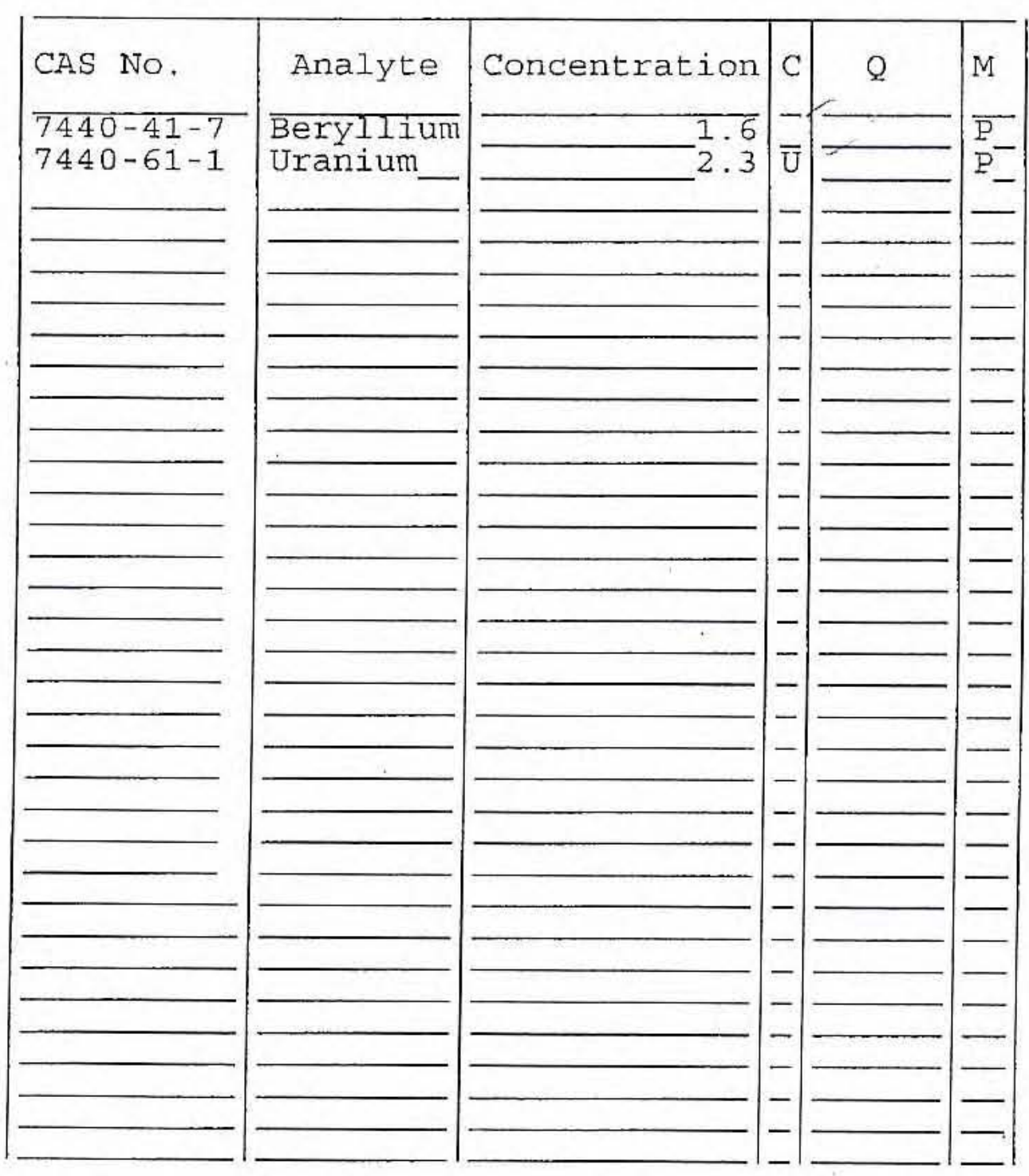

Clarity Before:

Clarity After:
Texture:

Artifacts:

Comments :

MPDT $-26 B$ 
1
INORGANIC ANALYSES DATA SHEET

EPA SAMPLE NO.

$$
27 \mathrm{~A}
$$

SDG NO , : 21A

Contract: 60052

Case No.: MPDT_ SAS No.:

Lab Sample ID: 0609L89 $\overline{6-0} 13$

Date Received: 09/07/06
Matrix (soil/water): SOIL Level (low/med): $\div$ Solids:

LOW

Concentration Units (ug/L or $\mathrm{mg} / \mathrm{kg}$ dry weight): $\mathrm{MG} / \mathrm{KG}$

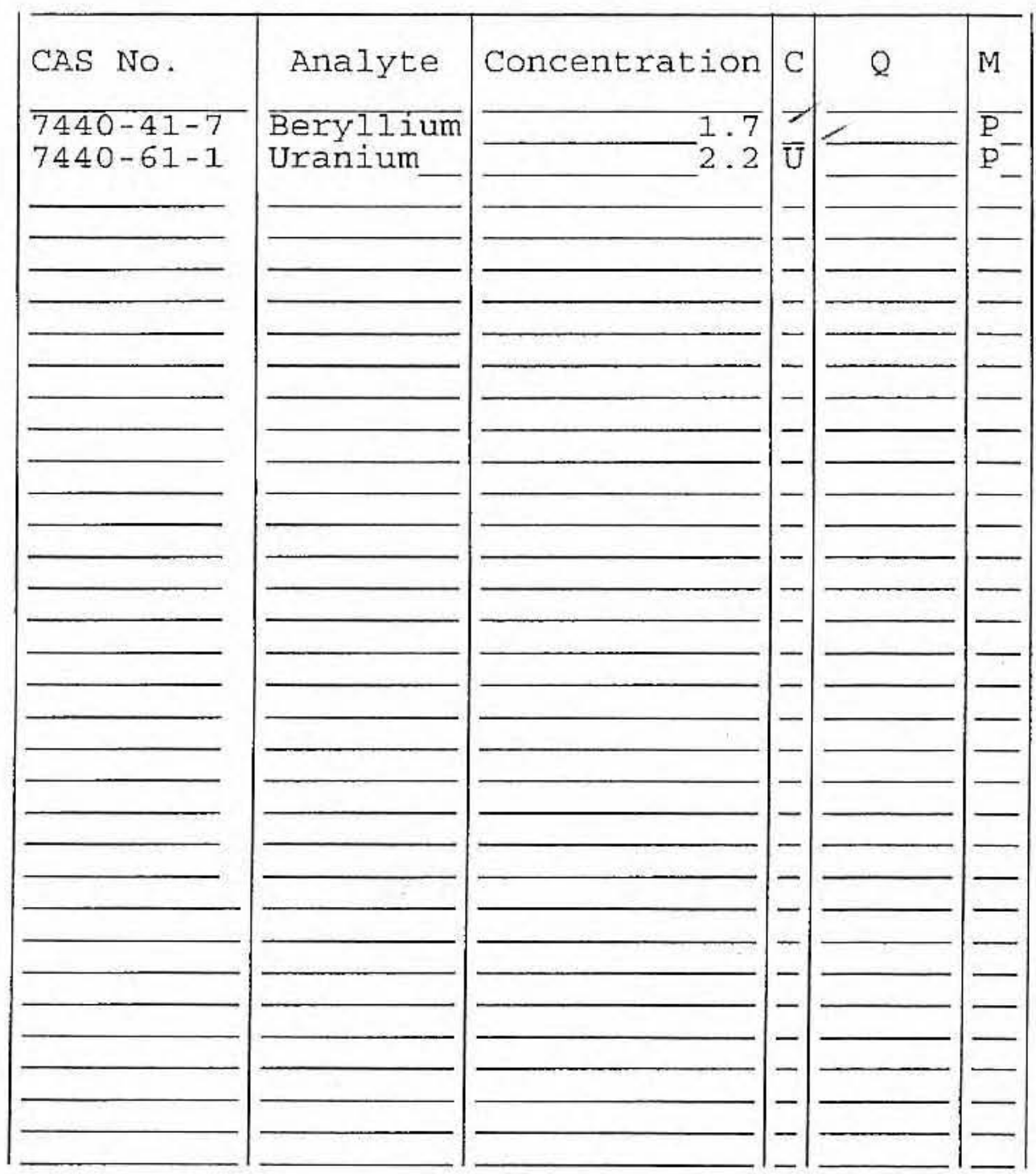

Color Before: Color After:
Clarity Before:

Clarity After:
Texture: Artifacts :

Comments:

MPDT - 27A 
1

\section{INORGANIC ANALYSES DATA SHEET}

\section{Lab Name: IIONVILLE_ILABORATORY}

Lab Code: LVLI Matrix (soil/water): SOIL_ Level (low/med): $\therefore$ Solids:

LOW
-89.4

EPA SAMPLE NO.

$27 \mathrm{~B}$

Contract: 60052

SAS NO.:

SDG No.: 21A

Lab Sample ID: 0609L89

Date Received: 09/07/06

Concentration Units (ug/L or $\mathrm{mg} / \mathrm{kg}$ dry weight): $\mathrm{MG} / \mathrm{KG}$

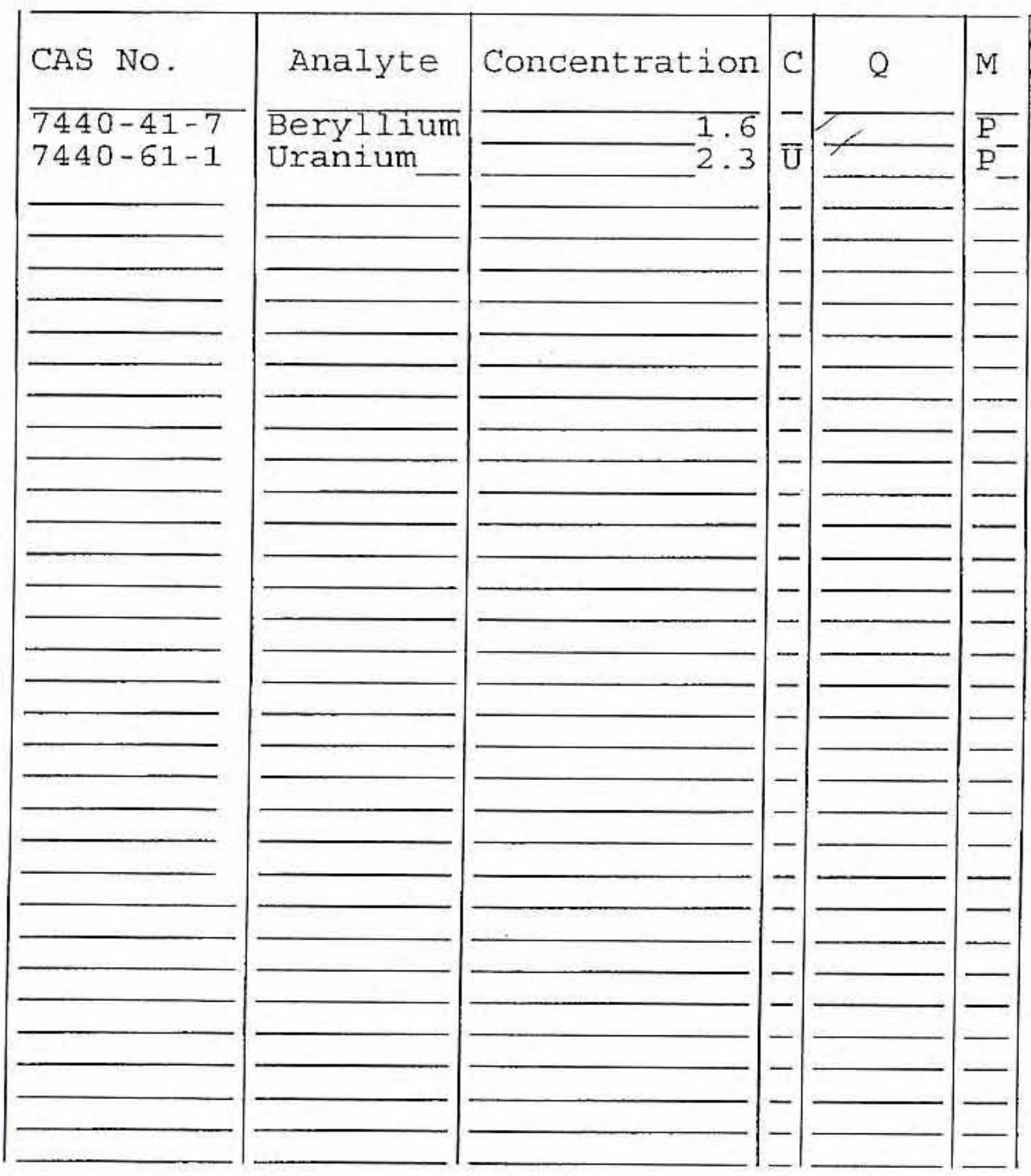

Color Before:

Color After:
Clarity Before:

Clarity After:
Texture:

Artifacts :

Comments :

MPDT $-27 B$ 
1

\section{INORGANIC ANALYSES DATA SHEET}

Iab Narme: LIONVILLE_LABORATORY

Lab Code: IVLI

Matrix (soil/water): Level (Low/med): $\div$ Solids: LOW
Contract: 60052

SAS NO. :

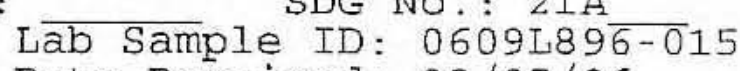

$-90 . \overline{1}$
EPA SAMPLE NO.

\section{$28 \mathrm{~A}$}

Date Received: 09/07/06

Concentration Units (ug/L or mg/kg dry weight): MG/KG

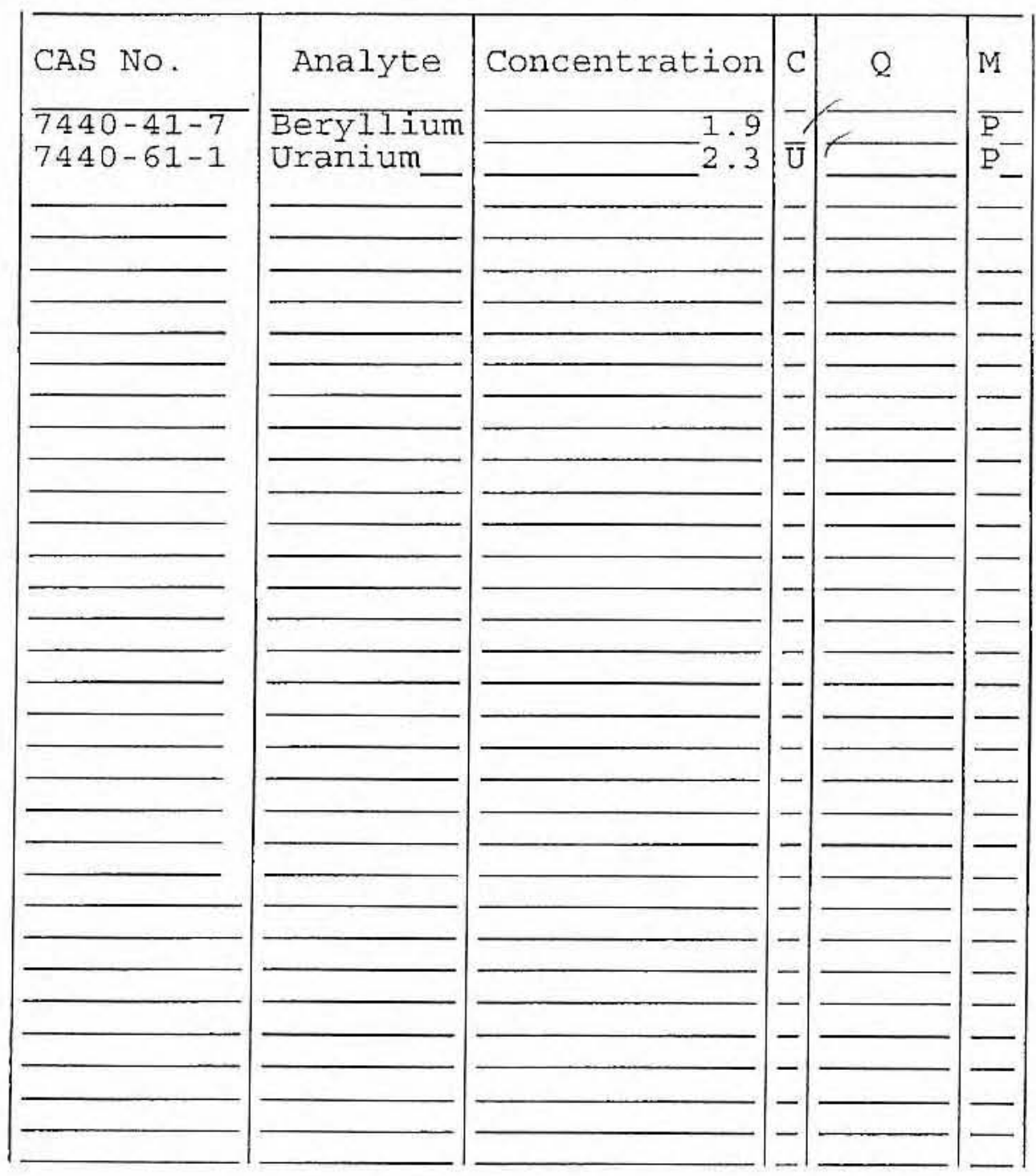

Color Before: Color After:
Clarity Before:

Clarity After:
Texture:

Artifacts:

Comments:

MPDT-28A 
1

INORGANIC ANALYSES DATA SHEET
EPA SAMPLE NO.

$28 B$

Contract: 60052

SAS NO.:

Iab Sample ID: 0609L89 $\overline{6-016}$

Date Received: 09/07/06
Lab Name: LION
Lab Code: LVII Matrix (soil/wat solids:
SOIL

LOW

$8 \overline{8.4}$

Concentration Units (ug/L or $\mathrm{mg} / \mathrm{kg}$ dry weight): $\mathrm{MG} / \mathrm{KG}$

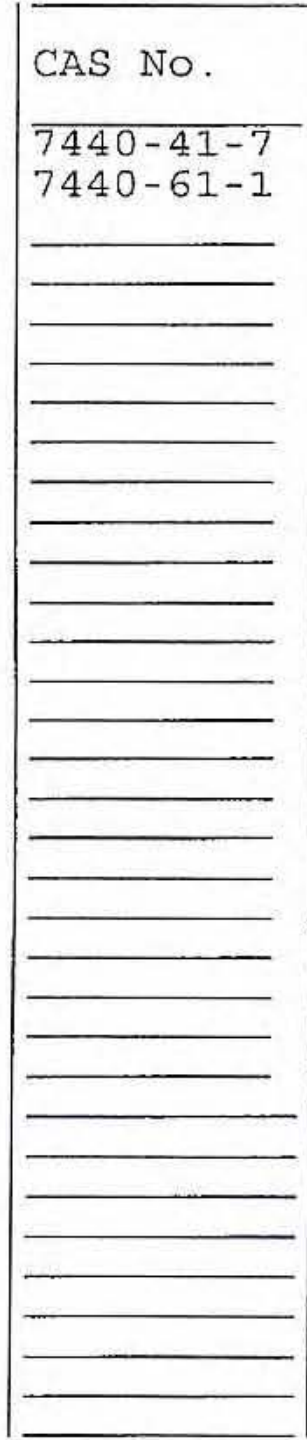

Color Before: Color After:

Comments : MPDT $-28 B$

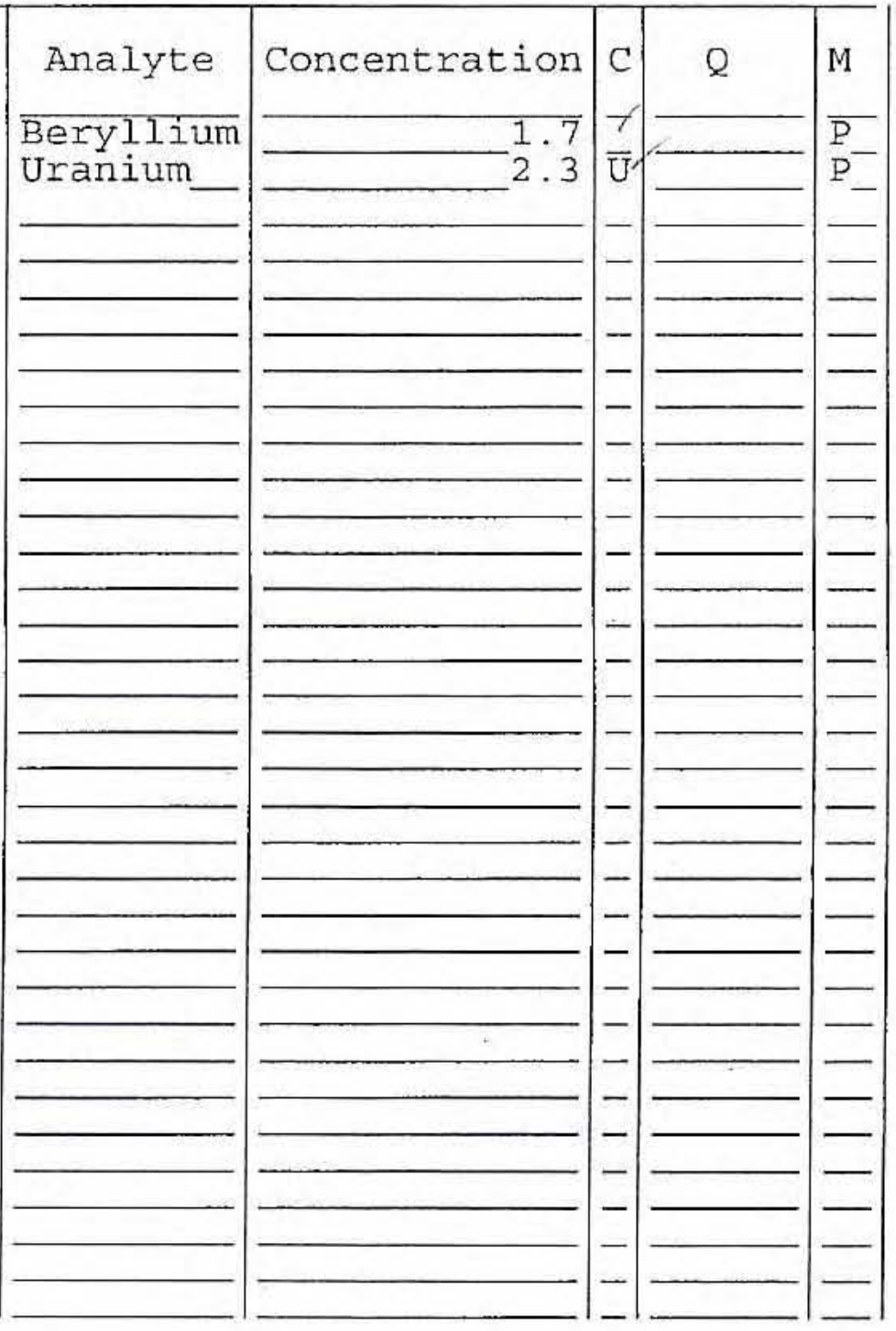

Clarity Before: Clarity After:
Texture:

Artifacts: 
1

INORGANIC ANALYSES DATA SHEET
EPA SAMPLE NO.

$$
29 \mathrm{~A}
$$

SDG NO.: $21 \mathrm{~A}$

Lab Name: LIONVILLE_LABORATORY

Contract: 60052

Case No.: MPDT_ SAS NO.:

Lab Sample ID: 06091896-017

Date Received: 09/07/06 LOW

Matrix (soil/water): SOIL

Level (low/med):

: Solids:
$91 \overline{7}$

Concentration Units (ug/L or mg/kg dry weight): MG/KG

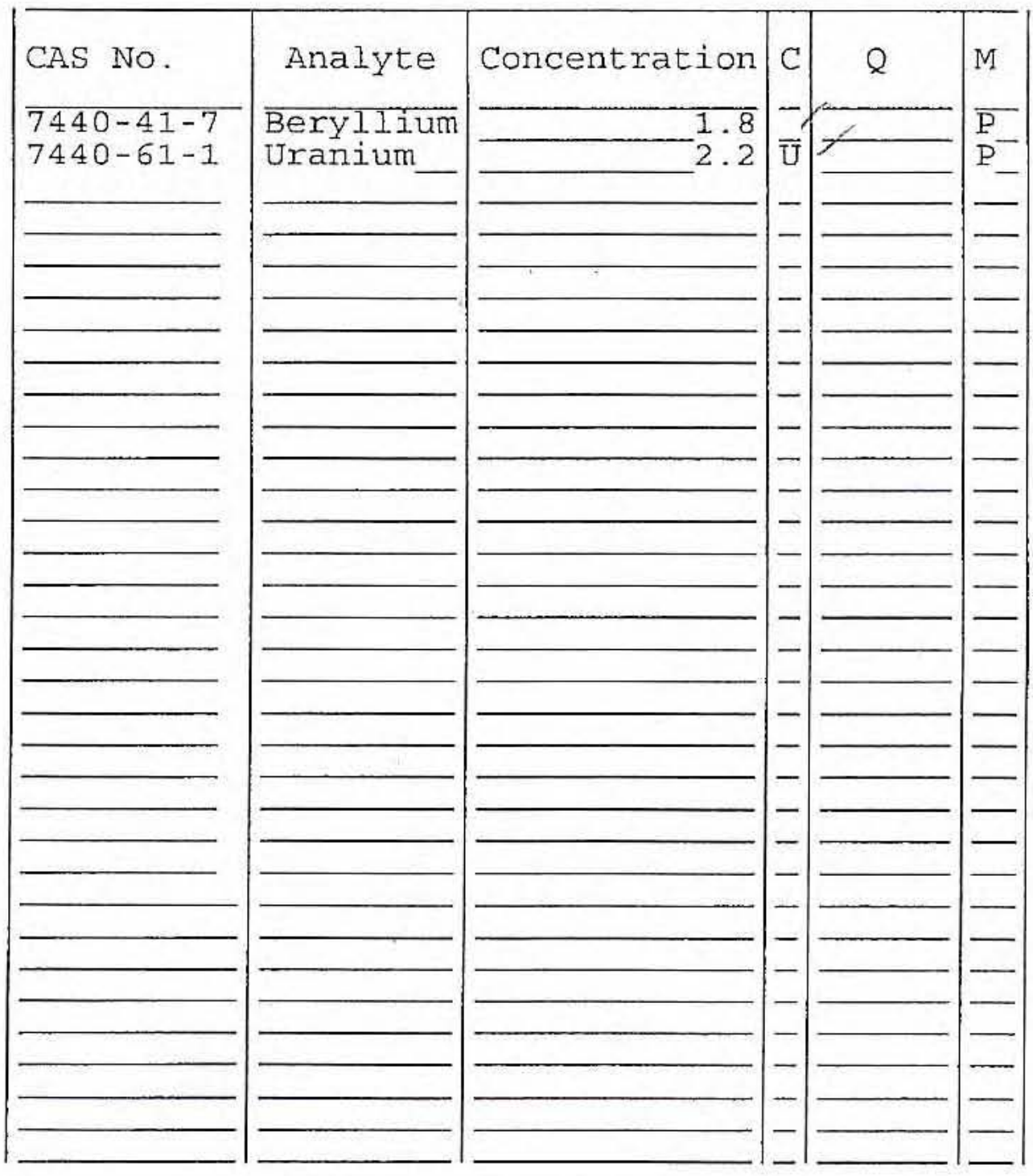

Color Before:

Color After:
Clarity Before: Clarity After:
Texture: Artifacts:

Comments:

MPDT-29A 
1

INORGANIC ANALYSES DATA SHEET
EPA SAMPLE NO.

$$
29 B
$$

SDG NO.: $21 \mathrm{~A}$

Case No.: MPDT_ SAS No.

$$
\begin{aligned}
& \text { SOIL } \\
& \text { LOW } \\
& -88 . \overline{3}
\end{aligned}
$$

Lab Sample ID: 06091896-018 Date Received: 09/07/06

Concentration Units (ug/L or $\mathrm{mg} / \mathrm{kg}$ dry weight): MG/KG

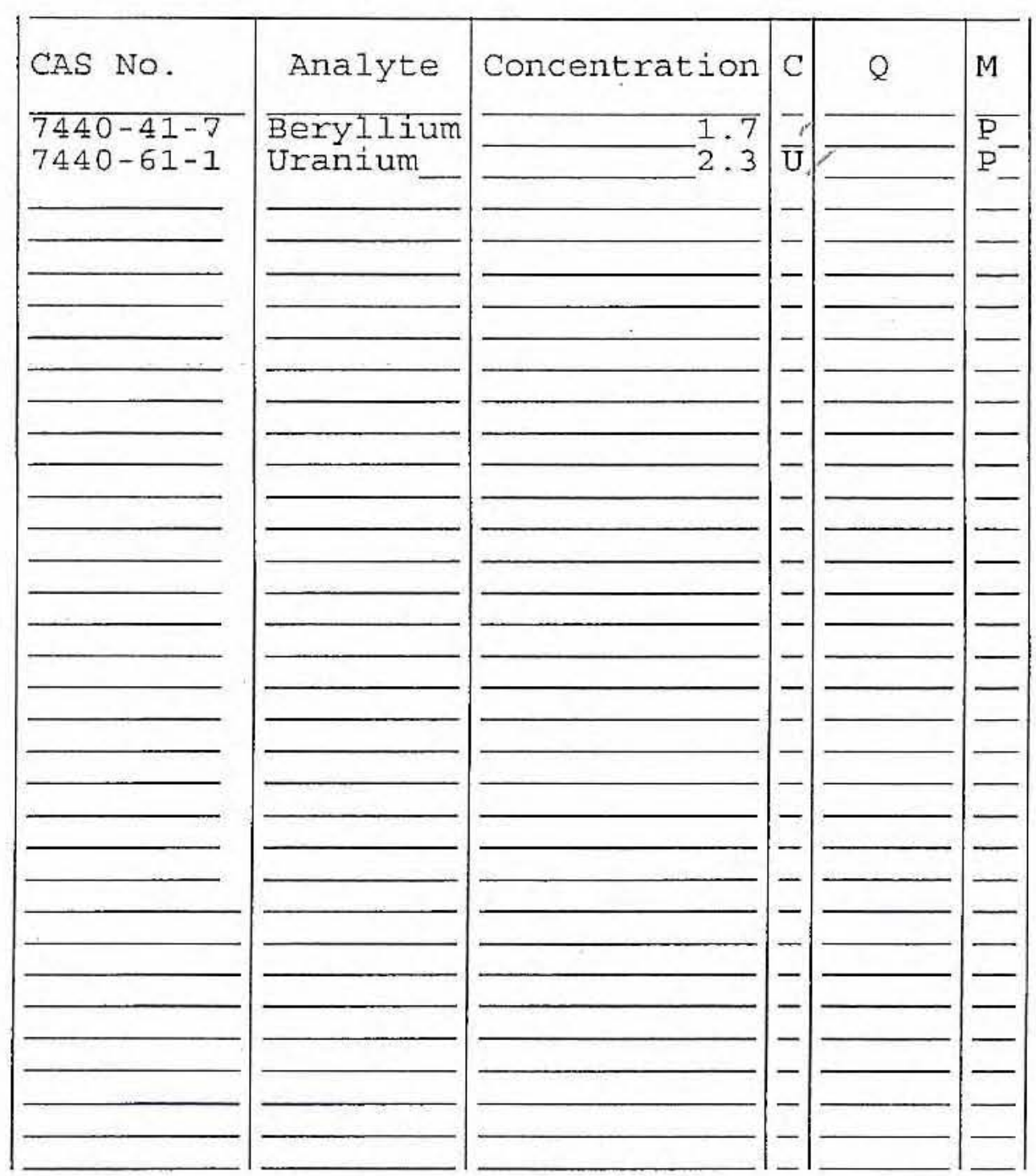

Color Before:

Color After:
Clarity Before: Clarity After:
Texture: Artifacts:

Comments:

MPDT $-29 B$ 
1 INORGANIC ANALÝSES DATA SHEET
EPA SAMPLE NO.

$$
30 \mathrm{~A}
$$

Contract: 60052

SAS NO.:

SDG No.: 21A.

Lab Sample ID: 0609L896-019

Date Received: 09/07/06
Lab Code: LVLI

Matrix (soil/wat
Level (low/med). \% Solids:

SOIL
$-91.1$
Case NO.: MPDT_

Concentration Units (ug/L or mg/kg dry weight): $\mathrm{MG} / \mathrm{KG}$

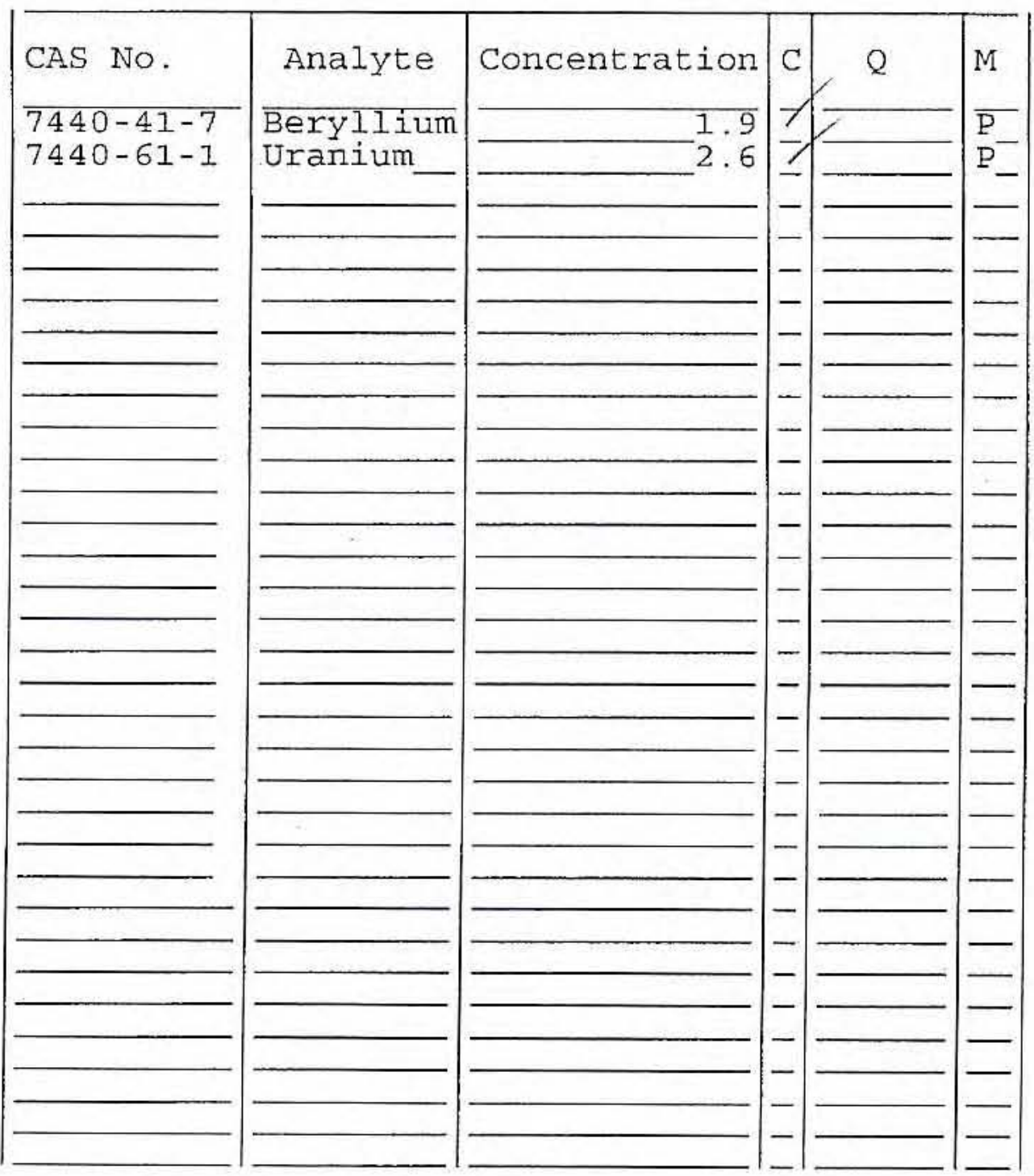

Clarity Before:

Clarity After:
Texture:

Artifacts:

Comments:

MPDT - 30A 
1

INORGANIC ANALYSES DATA SHEET
EPA SAMPLE NO.

$$
30 B
$$

SDG NO.: 21A SAS NO. :

Lab Sample ID: $0609189 \overline{6-0} 20$ Date Received: 09/07/06
Lab Name: IION
Lab Code: LVLI Matrix (soil/water): SOIL Ievel (low $/$ med) : Solids:

$$
\begin{aligned}
& \text { LOW } \\
& -88.7
\end{aligned}
$$

CORATORY

Concentration Units (ug/L or $\mathrm{mg} / \mathrm{kg}$ dry weight): MG/KG

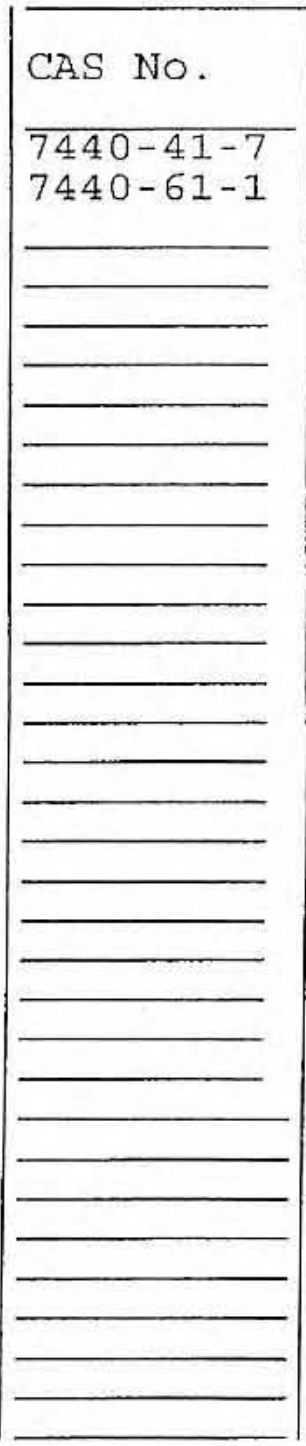

Color Before:

Color After:

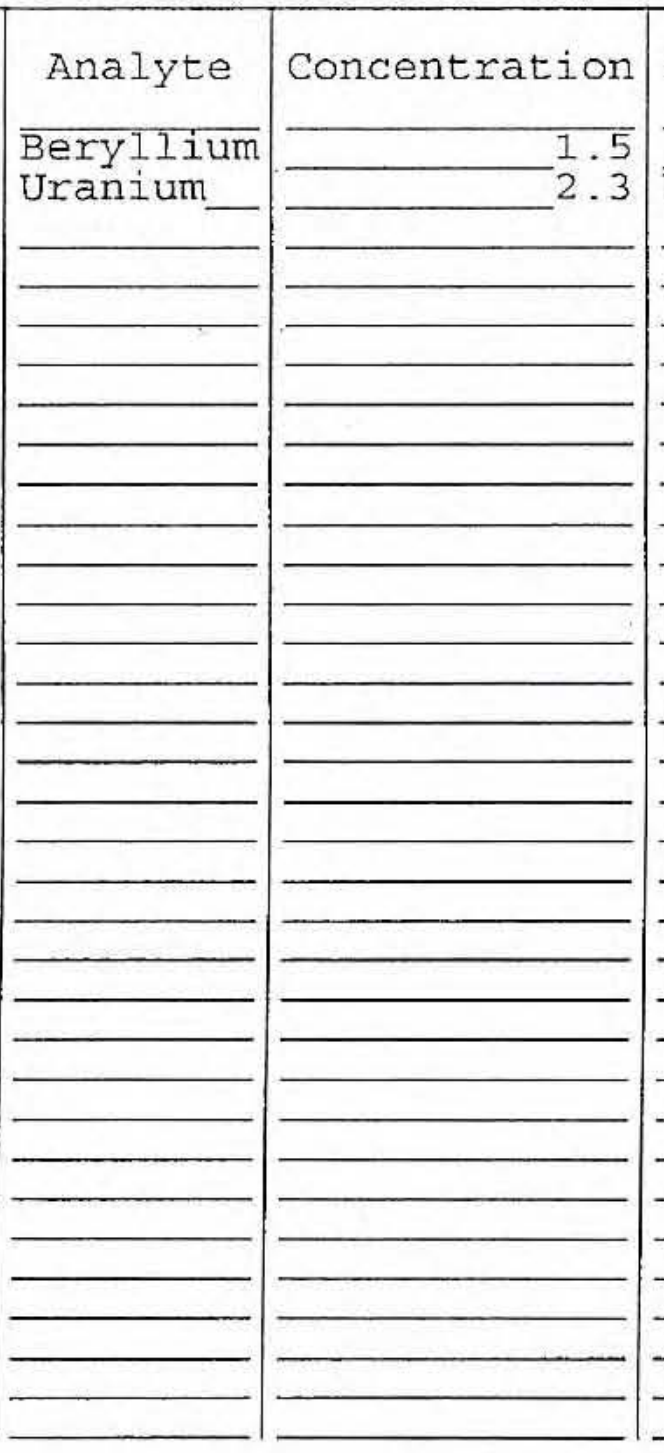

Clarity Before: Clarity After:

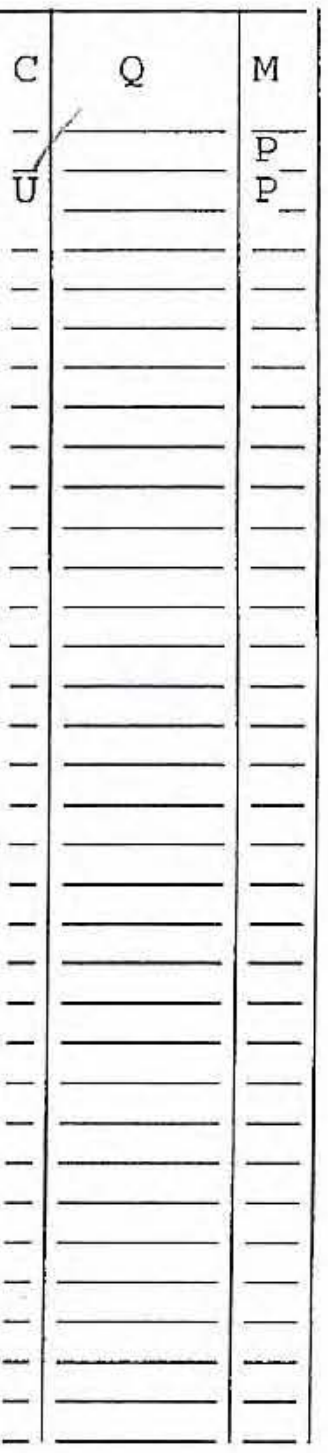

Texture: Artifacts :

Comments :

MPDT - 3OB 


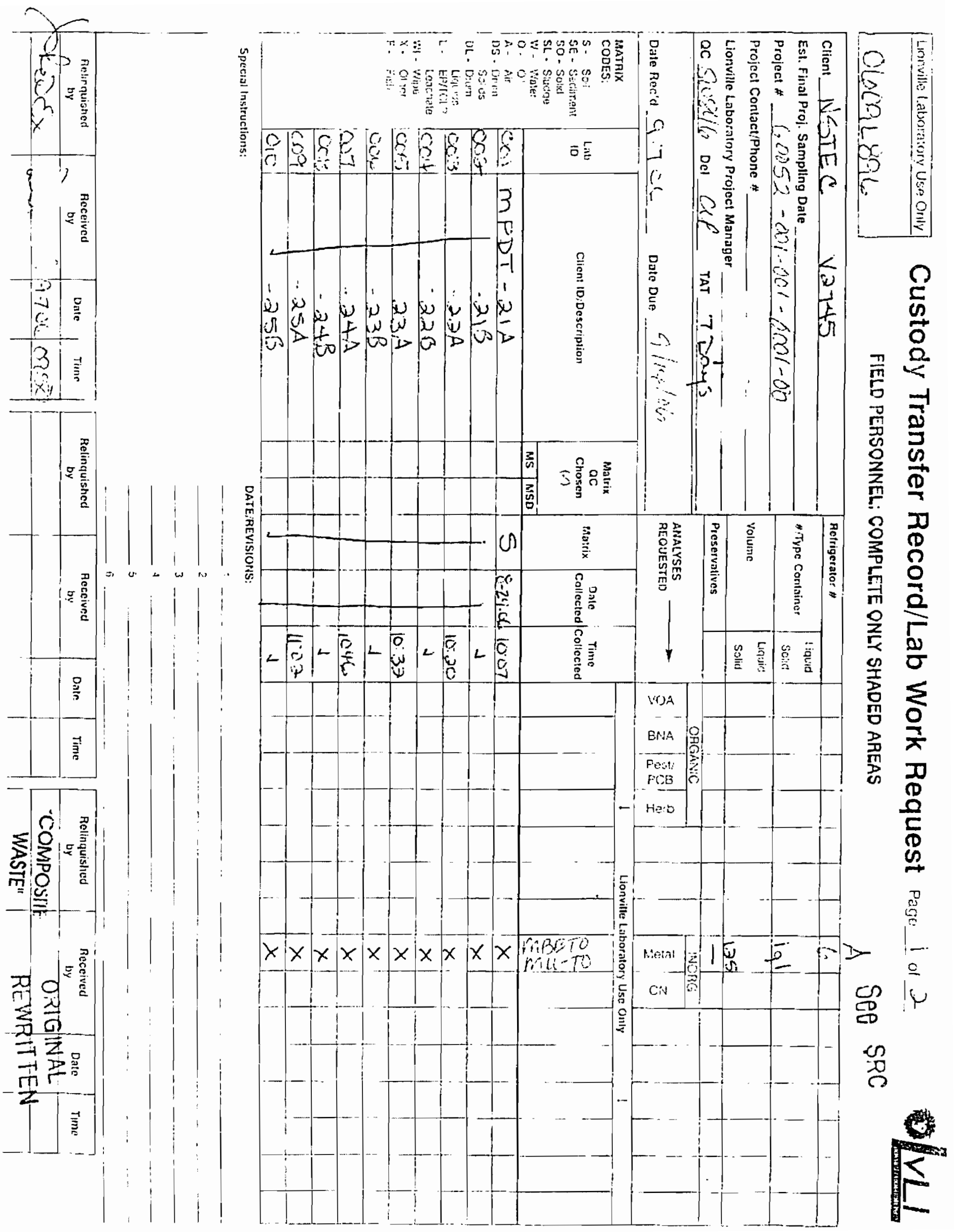




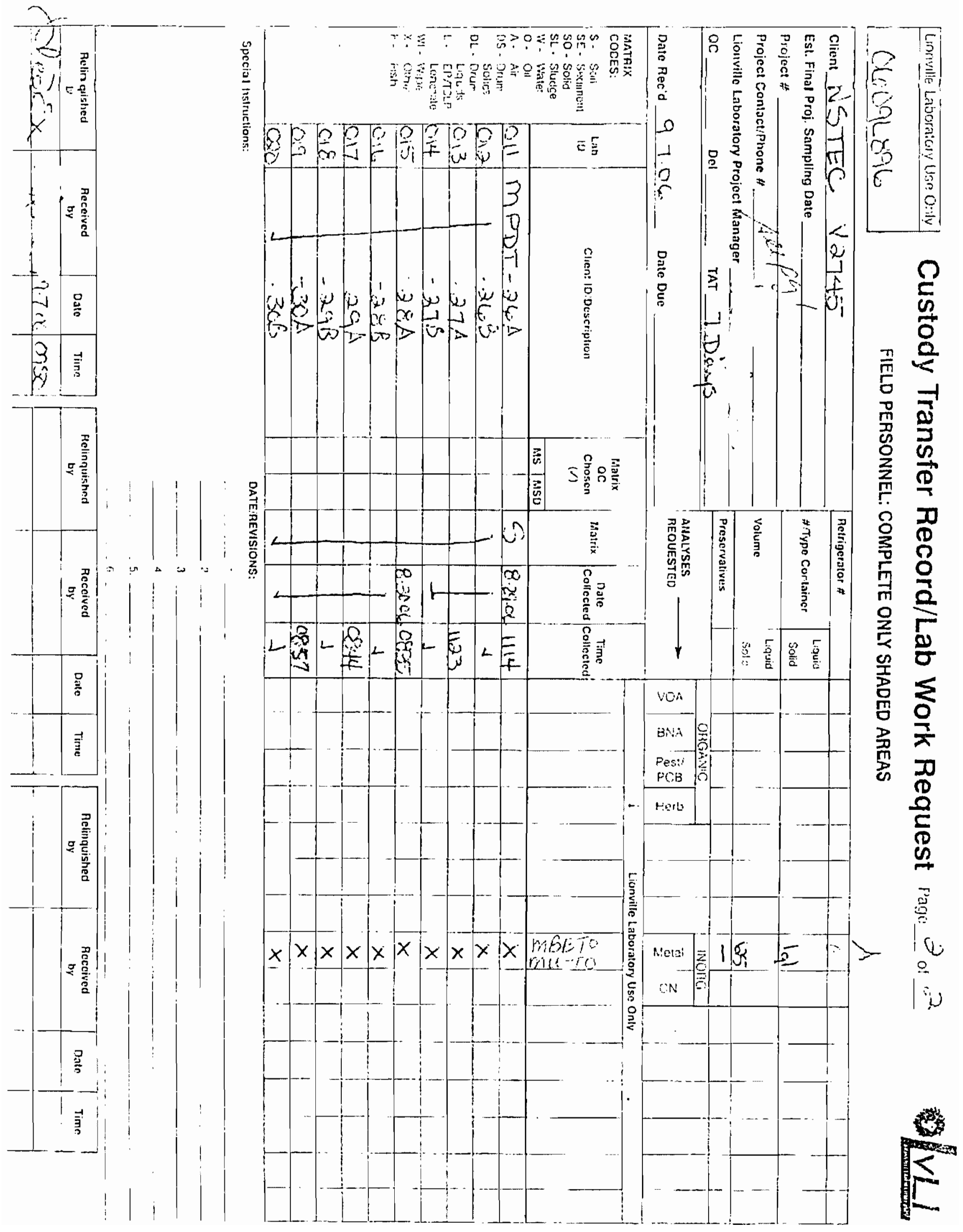




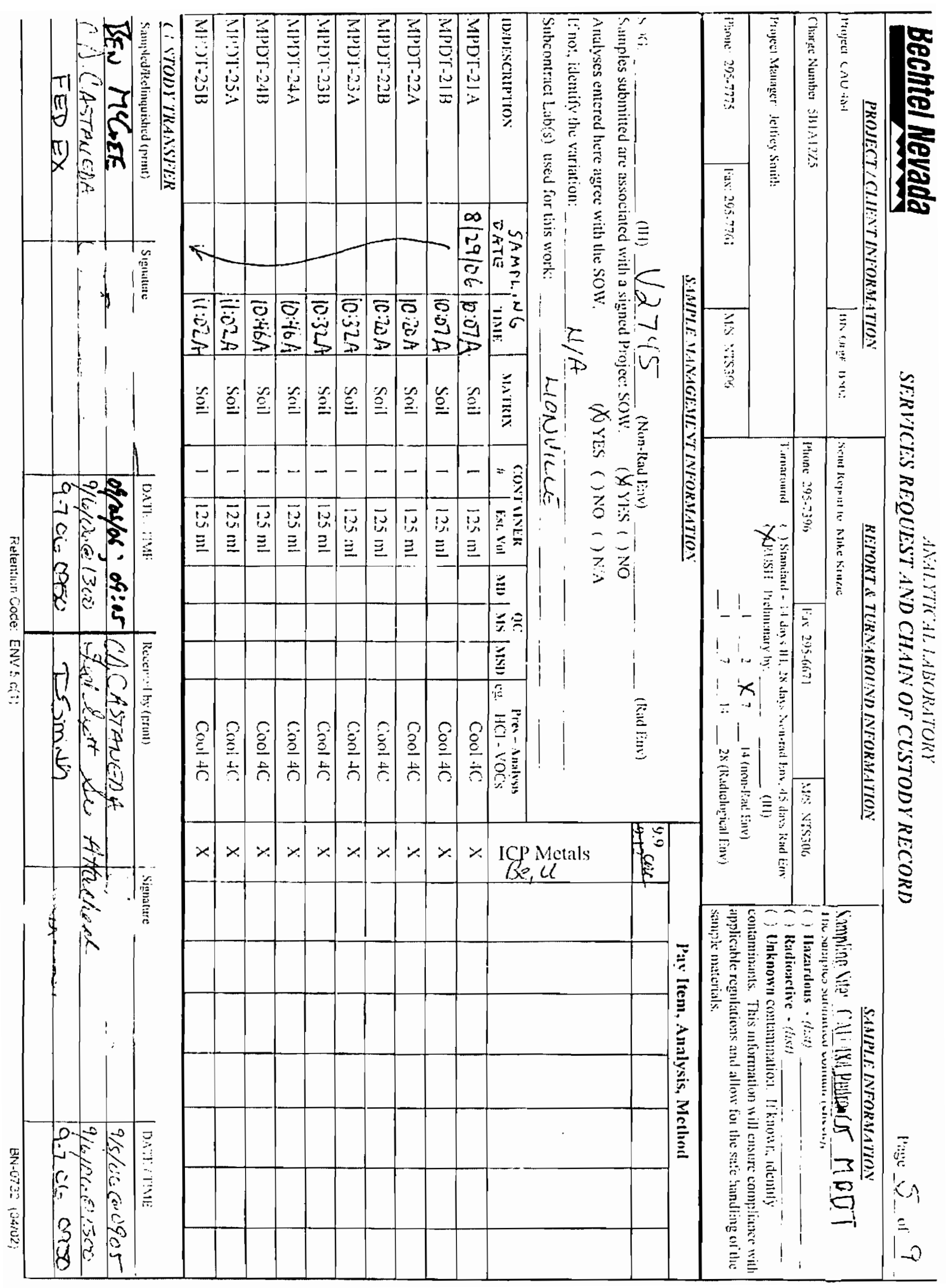




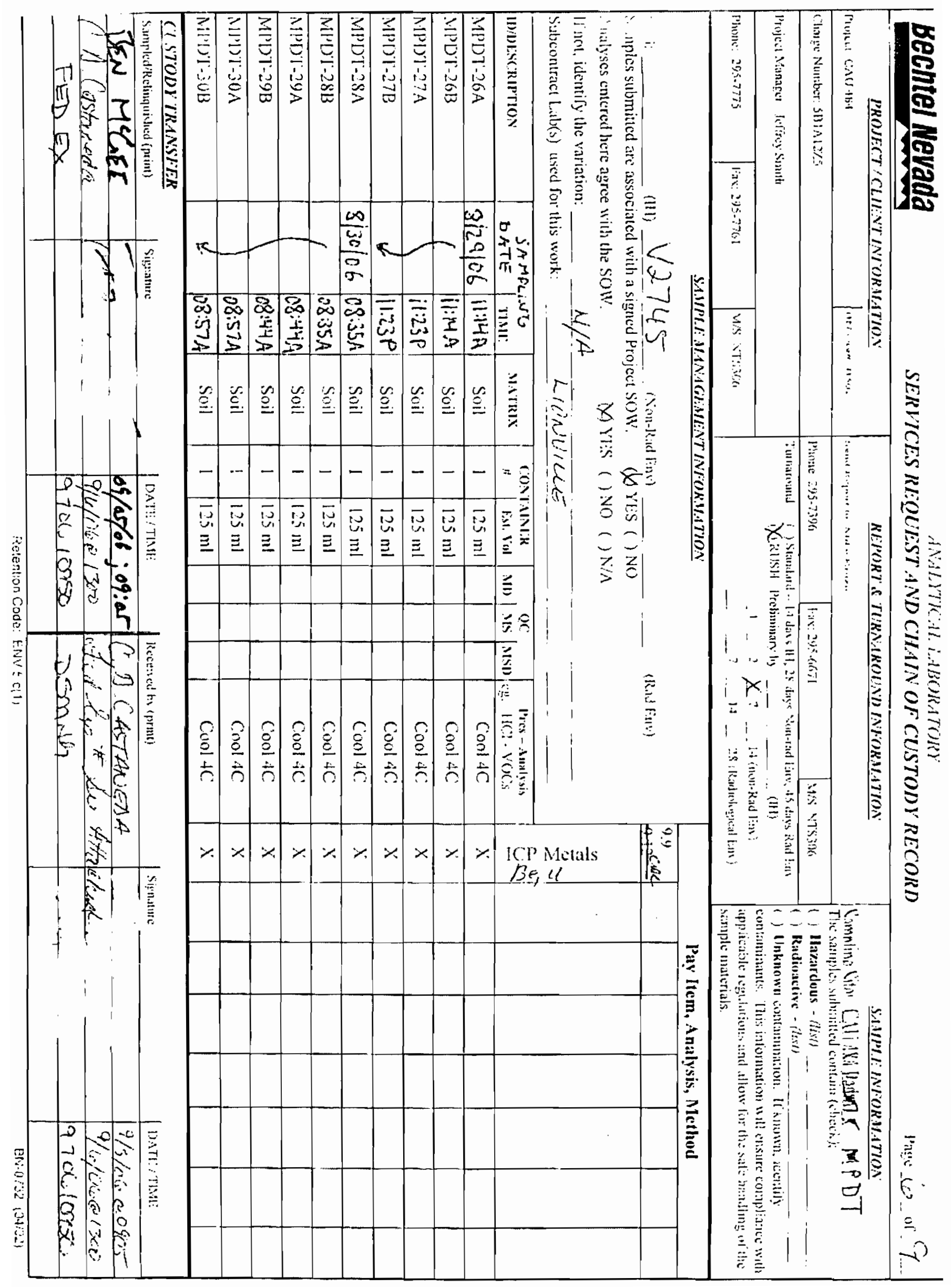


1

INORGANIC ANALYSES DATA SHEET
EPA SAMPLE NO.

$$
3 I A
$$

Lab Name: LIONVILLE_LABORATORY

Lab Code: LVLI

Matrix (soil/water): SoIL

Level (low/med):

: Solids :
LOW

$-90 \overline{.8}$
Contract: 60052

SAS No.:

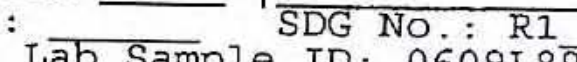

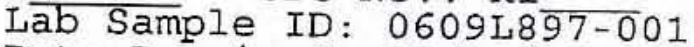

Date Received: 09/07/06

Concentration Units (ug/L or $\mathrm{mg} / \mathrm{kg}$ dry weight): MG/KG

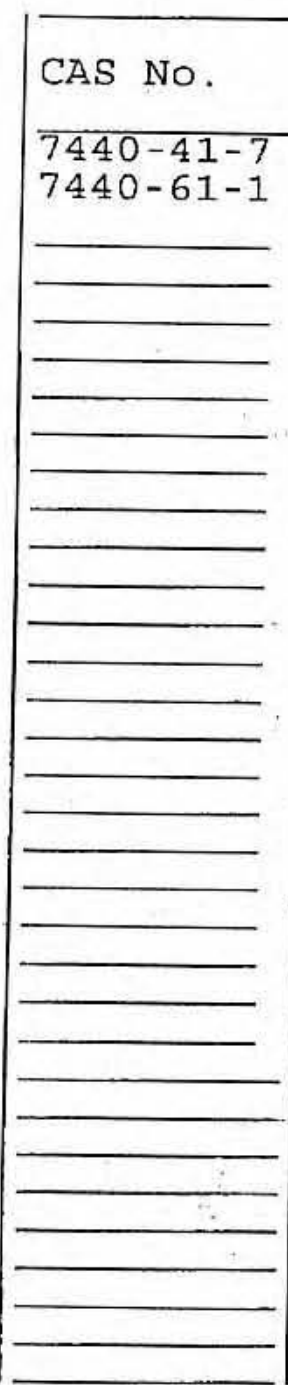

Color Before:

Color After:

Comments :

MPDT - 31A

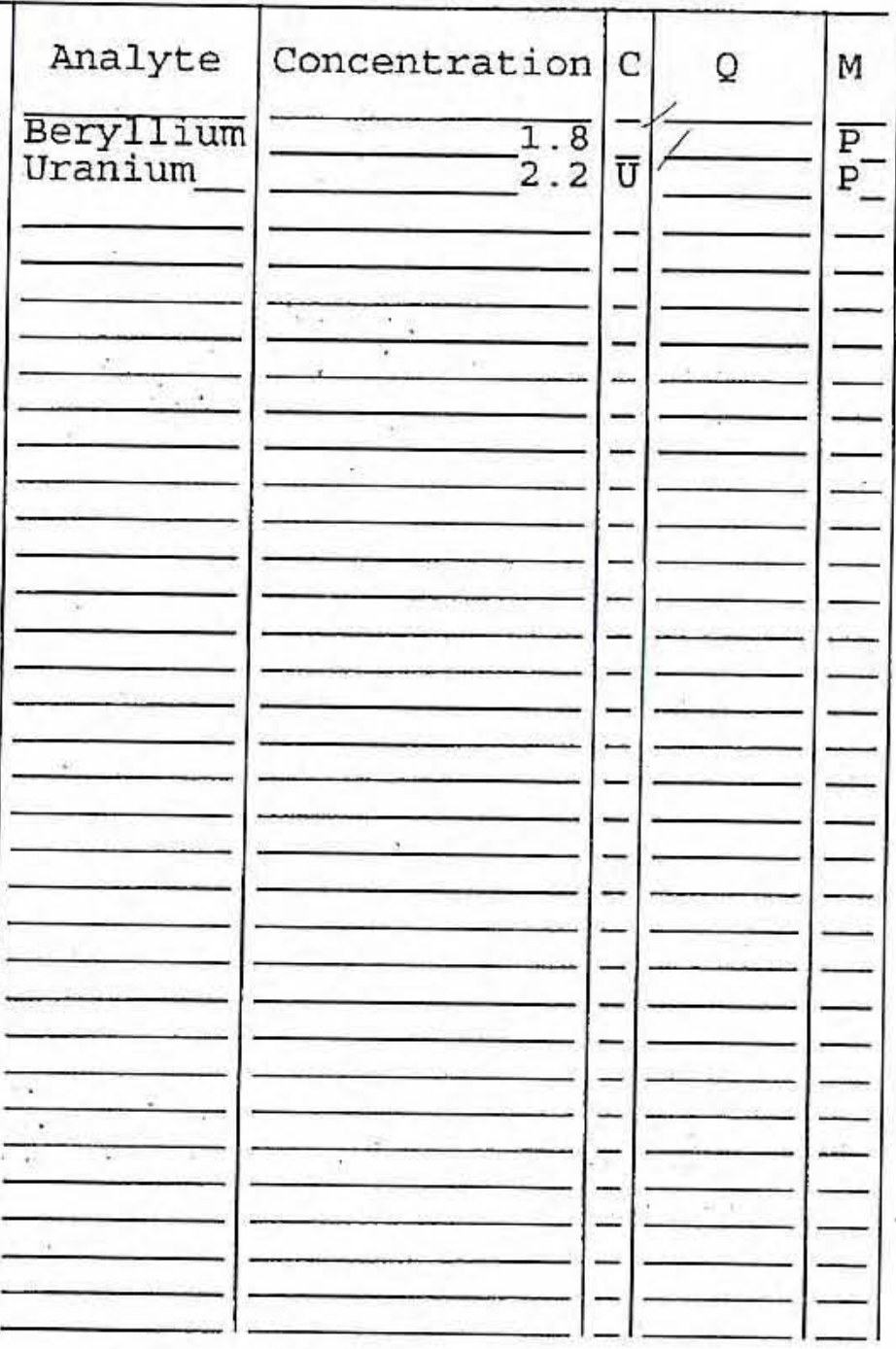

Clarity Before:

Clarity After:
Texture:

Artifacts: 
1

INORGANIC ANALYSES DATA SHEET
EPA SAMPLE NO.

\section{$31 B$}

SDG NO.: RI

SAS NO.: Lab Sample ID: 0609L8 $\overline{97-002}$ Date Received: 09/07/06
Lab Name: LION
Lab Code: LVLI

Matrix (soil/water): SOIL

Level (low/med): LOW

$\%$ Solids:
$-88 \overline{.8}$

Contract: 60052

Case No: : MPDT 8

Concentration Units (ug/L or $\mathrm{mg} / \mathrm{kg}$ dry weight): $\mathrm{MG} / \mathrm{KG}$

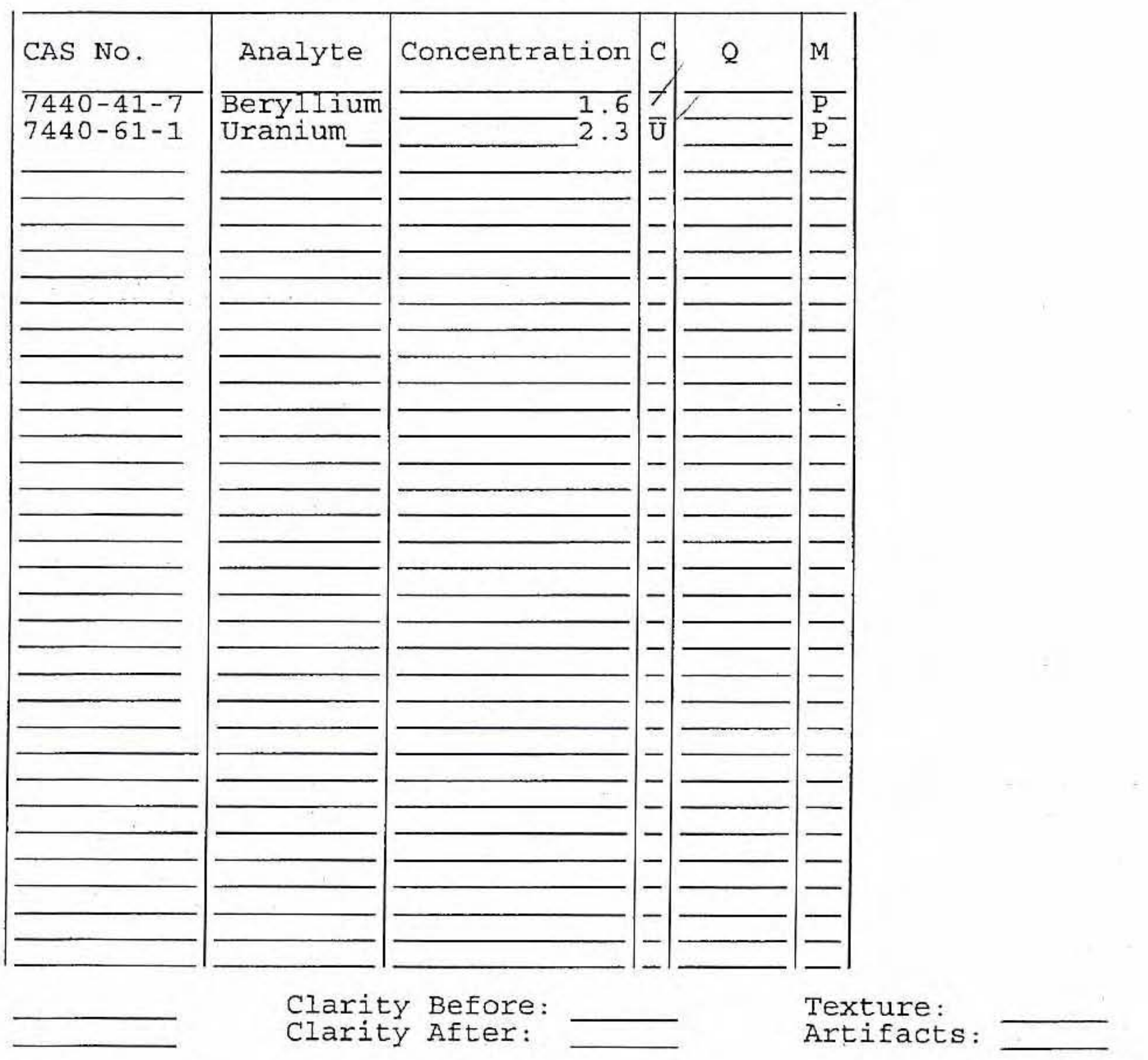

Color Before:

Color After:

Comments:

MPDT $-31 B$
Clarity Before:

Texture:

Artifacts: 
1

INORGANIC ANALYSES DATA SHEET
EPA SAMPLE NO.

$32 \mathrm{~A}$

Contract: 60052

SAS No.:

Lab Sample ID: 0609L8

Date Received: 09/07/06
Lab Name: LION
Lab Code: LVLI

Matrix (soil/water): SoIL

Level (low/med): LOW

$\div$ Solids:

$$
-90.7
$$

Concentration Units (ug/L or $\mathrm{mg} / \mathrm{kg}$ dry weight): MG/KG

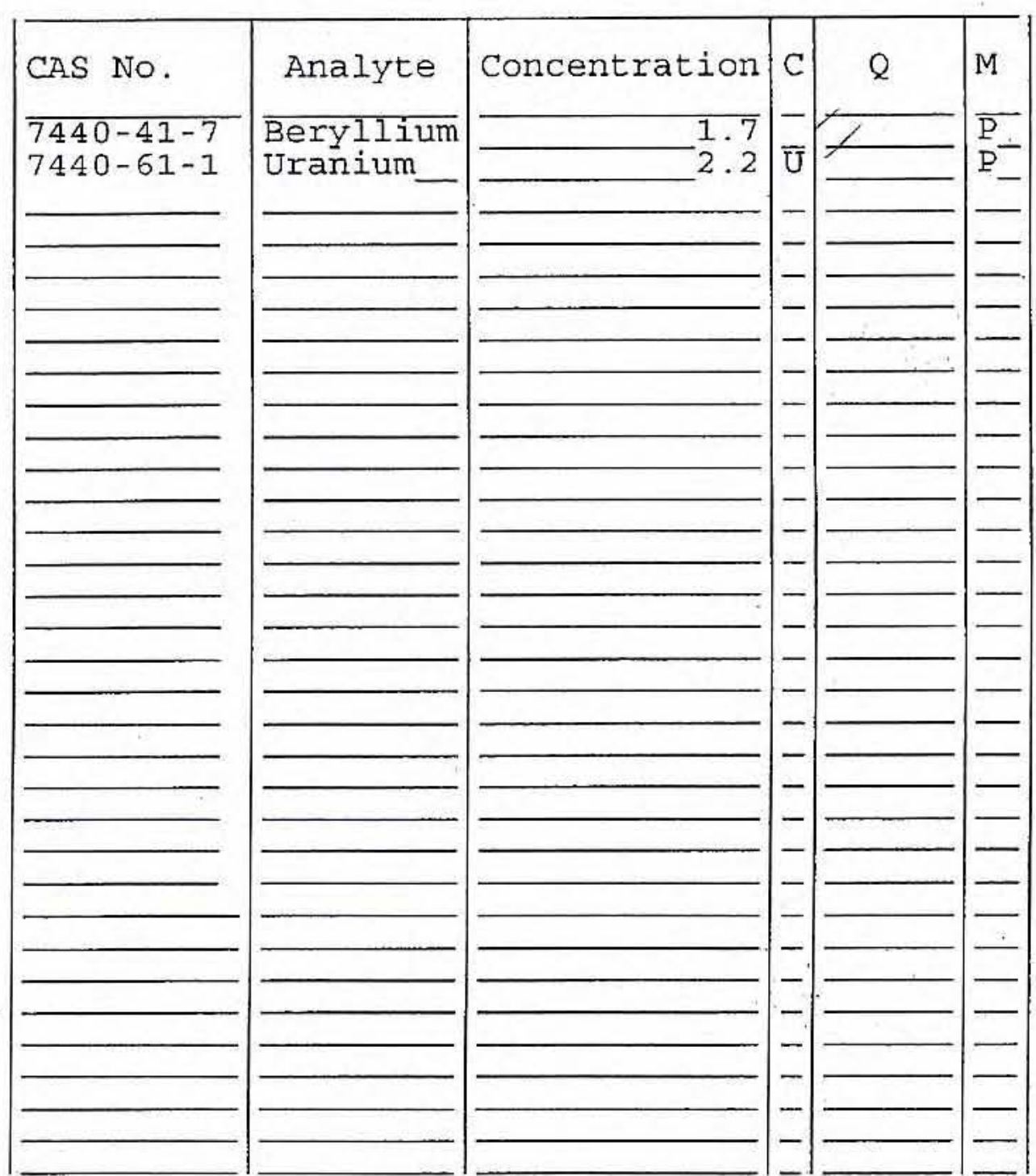

Color Before:

Color After:

Comments :

MPDT-32A
Clarity Before:

Clarity After:
Texture:

Artifacts: 
1

INORGANIC ANALYSES DATA SHEET
EPA SAMPLE NO.

\section{$32 \mathrm{~B}$}

Contract: 60052
SAS NO.:

Lab Sample ID: 0609L8 $\overline{97-004}$ SOIL
LOW-
-89.6

Date Received: 09/07/06
Lab Name: LION
Lab Code: LVLI Matrix (soil/water): Level (low/med): $\div$ Solids :

Concentration Units (ug/L or $\mathrm{mg} / \mathrm{kg}$ dry weight): MG/KG

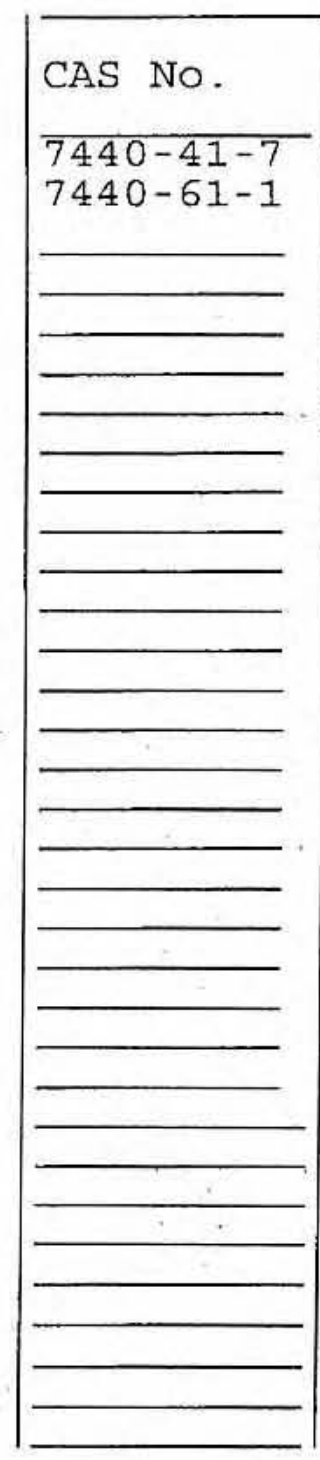

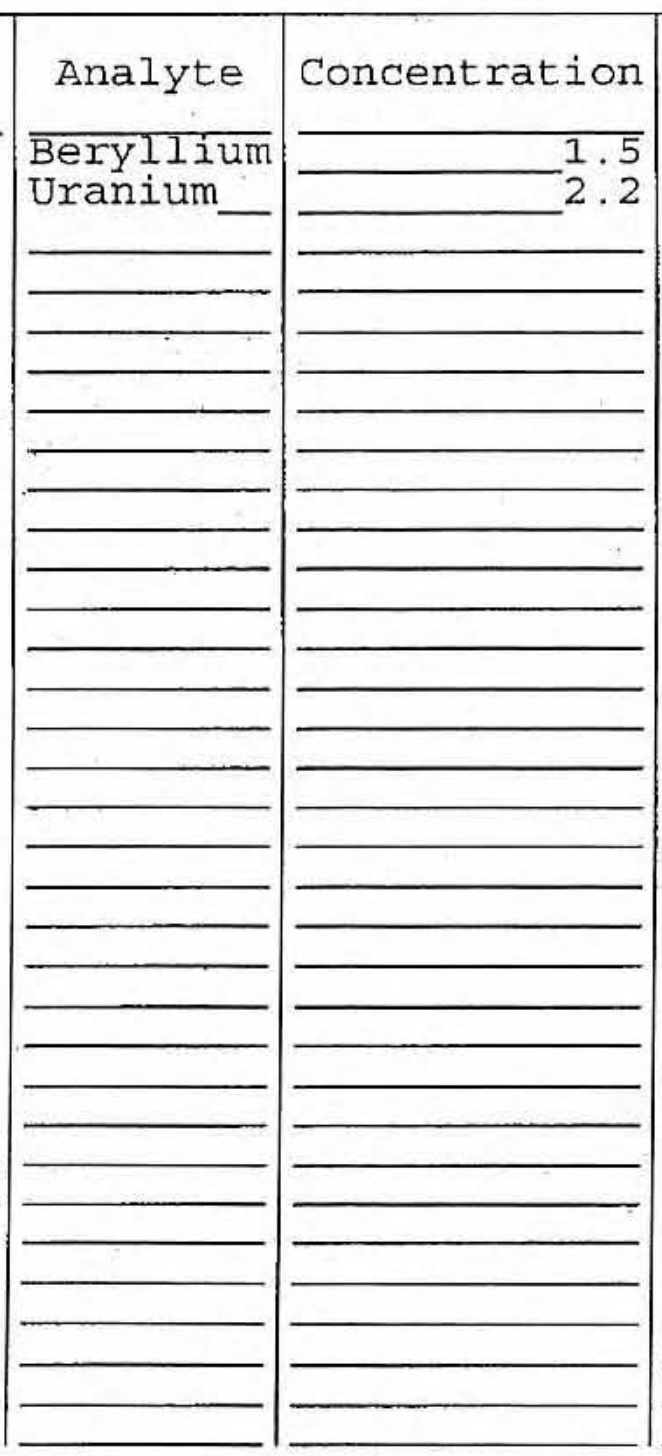

Clarity Before: Clarity After:

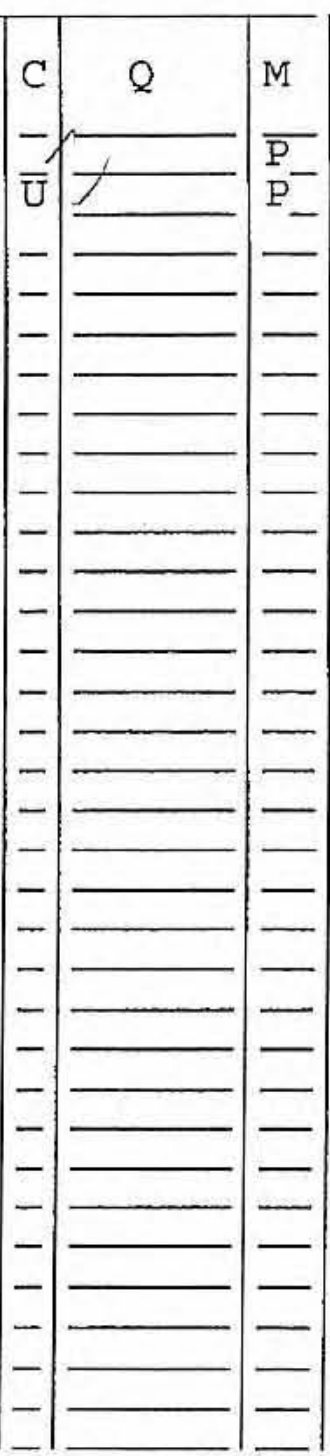

Texture:

Artifacts:

Color Before
Color After:

Comments :

MPDT $-32 B$ 
Lab Name: LIONVILLE_LABORATORY

Lab Code: LVLI Matrix (soil/water): SolL_ Level (low/med): \% Solids: LOW -91.6
1

INORGANIC ANALYSSES DATA SHEET
EPA SAMPLE NO.

\section{$33 \mathrm{~A}$}

Contract: 60052

SAS No. :

SDG NO : RI

Lab Sample ID: 06091897-005

Date Received: 09/07/06

Concentration Units (ug/L or $\mathrm{mg} / \mathrm{kg}$ dry weight): MG/KG

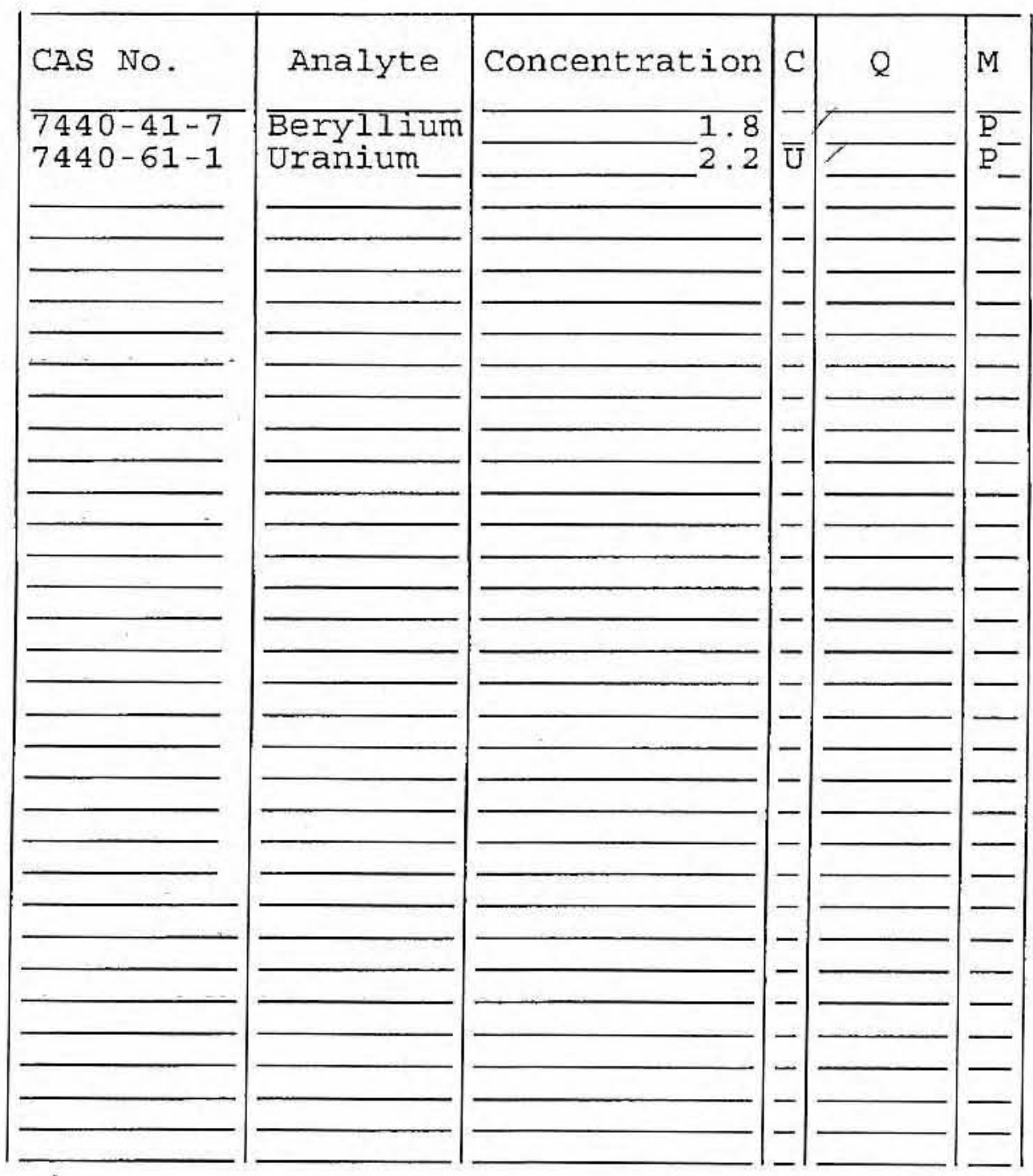

Color Before:

Color After:

Clarity Before:

Clarity After:
Texture:

Artifacts :

Comments:

MPDT-33A 


\section{INORGANIC ANALYSES DATA SHEET}

EPA SAMPLE NO.

$33 \mathrm{~B}$

Contract: 60052

SAS NO.:

Lab Sample ID: 0609L8 $\overline{97-006}$

Date Received: 09/07/06
Lab Name: LION
Lab Code: LVLI

Matrix (soil/wat

Level (lo
SOIL

LOW

$-89 \overline{.2}$
Case No.: MPDT

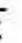

Concentration Units (ug/L or $\mathrm{mg} / \mathrm{kg}$ dry weight): MG/KG

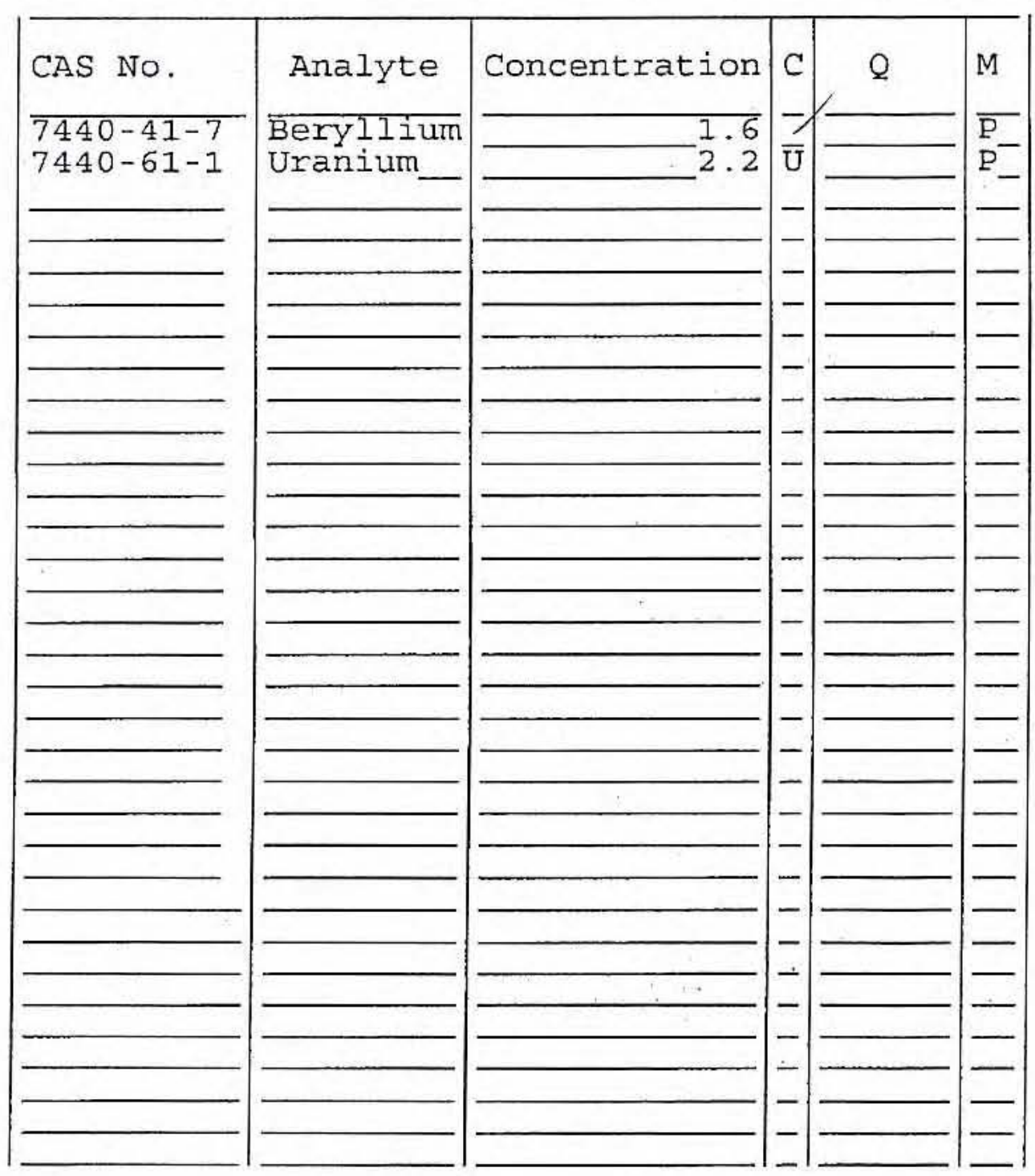

Color Before:

Color After:
Clarity Before:

Clarity After:
Texture:

Artifacts:

Comments:

MPDT-33B 
1

INORGANIC ANALYSES DATA SHEET
EPA SAMPLE NO.

\section{$34 \mathrm{~A}$}

Contract: 60052

SAS NO.:

Lab Sample ID: $060918 \overline{97-0} 07$

Case No.: MPDT SOIL LOW
$-91 \overline{1}$
Matrix (soil/w
Level (low/med) :
\% Solids :$$
\text { (- Un }
$$

Concentration Units (ug/L or $\mathrm{mg} / \mathrm{kg}$ dry weight): MG/KG

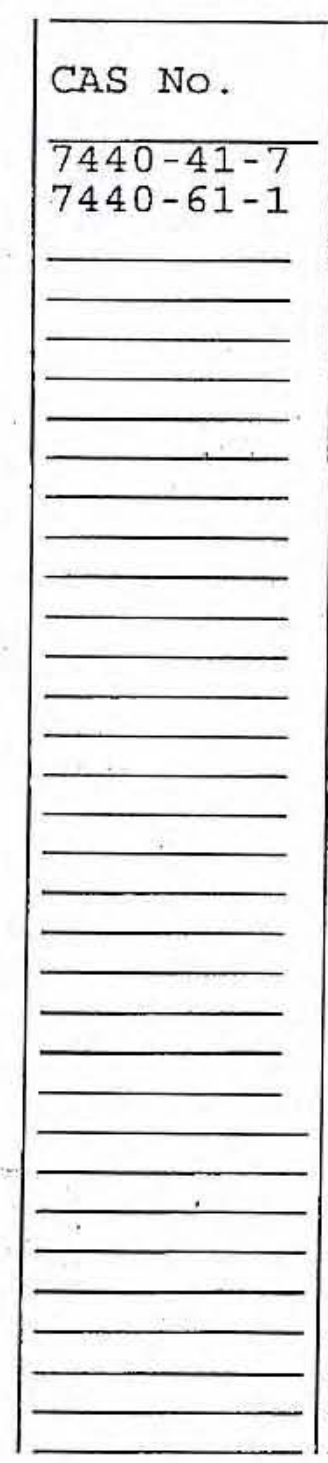

Color Before:

Color After:

Comments :

MPDT $-34 \mathrm{~A}$

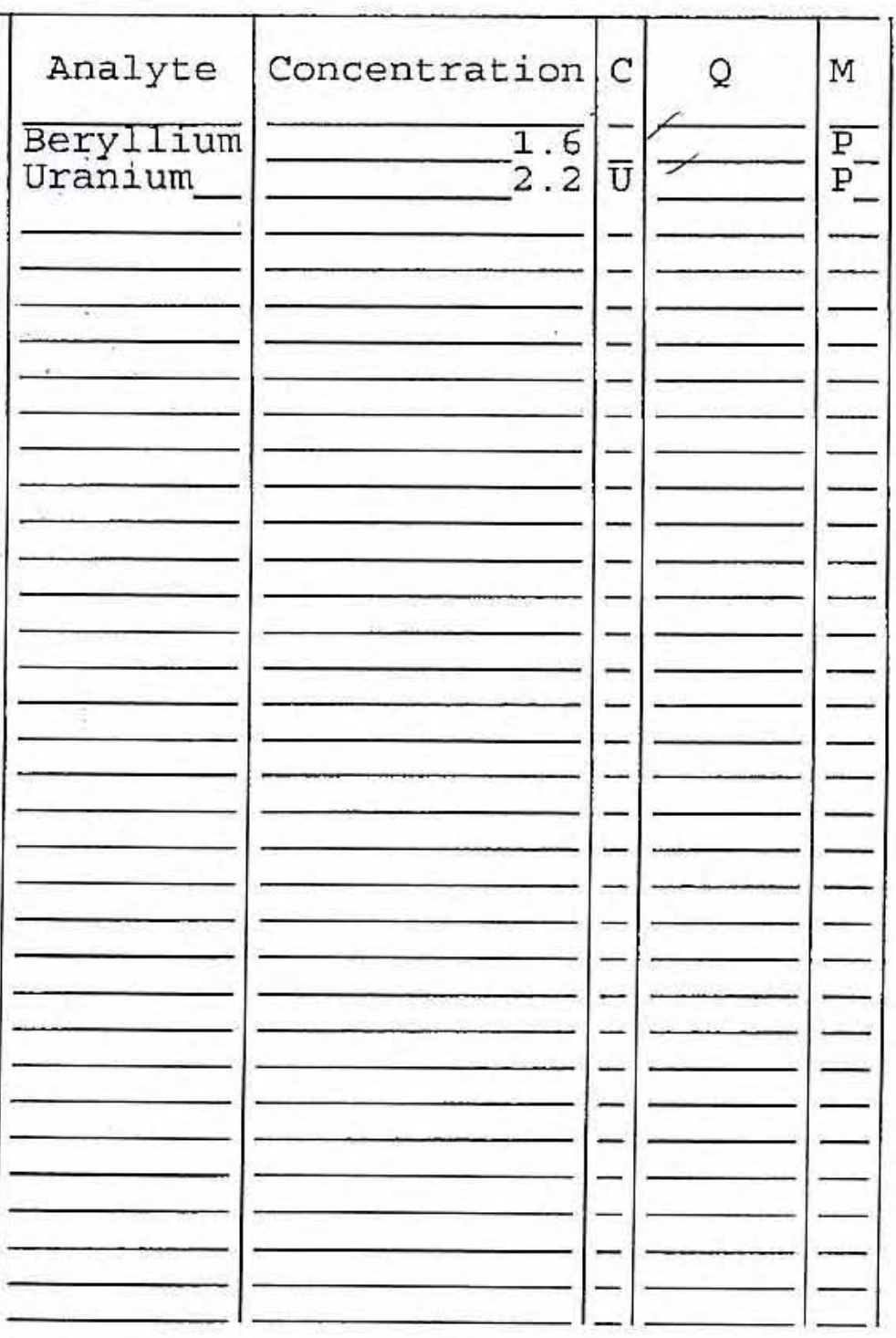

Clarity Before:

Clarity After:

Texture:

Artifacts: 
1

INORGANIC ANALYSES DATA SHEET
EPA SAMPLE NO.

\section{$34 B$}

Contract: 60052 SAS NO. :

SDG No.: R1

Case No.: MPDT_

Lab Code: LVLI Matrix (soil/water): SOIL_ Level (low/med): \% Solids:
Lab Sample ID: 0609L8 $\overline{97-0} 08$ Date Received: 09/07/06 $-89 . \overline{0}$

Concentration Units (ug/L or mg/kg dry weight): MG/KG

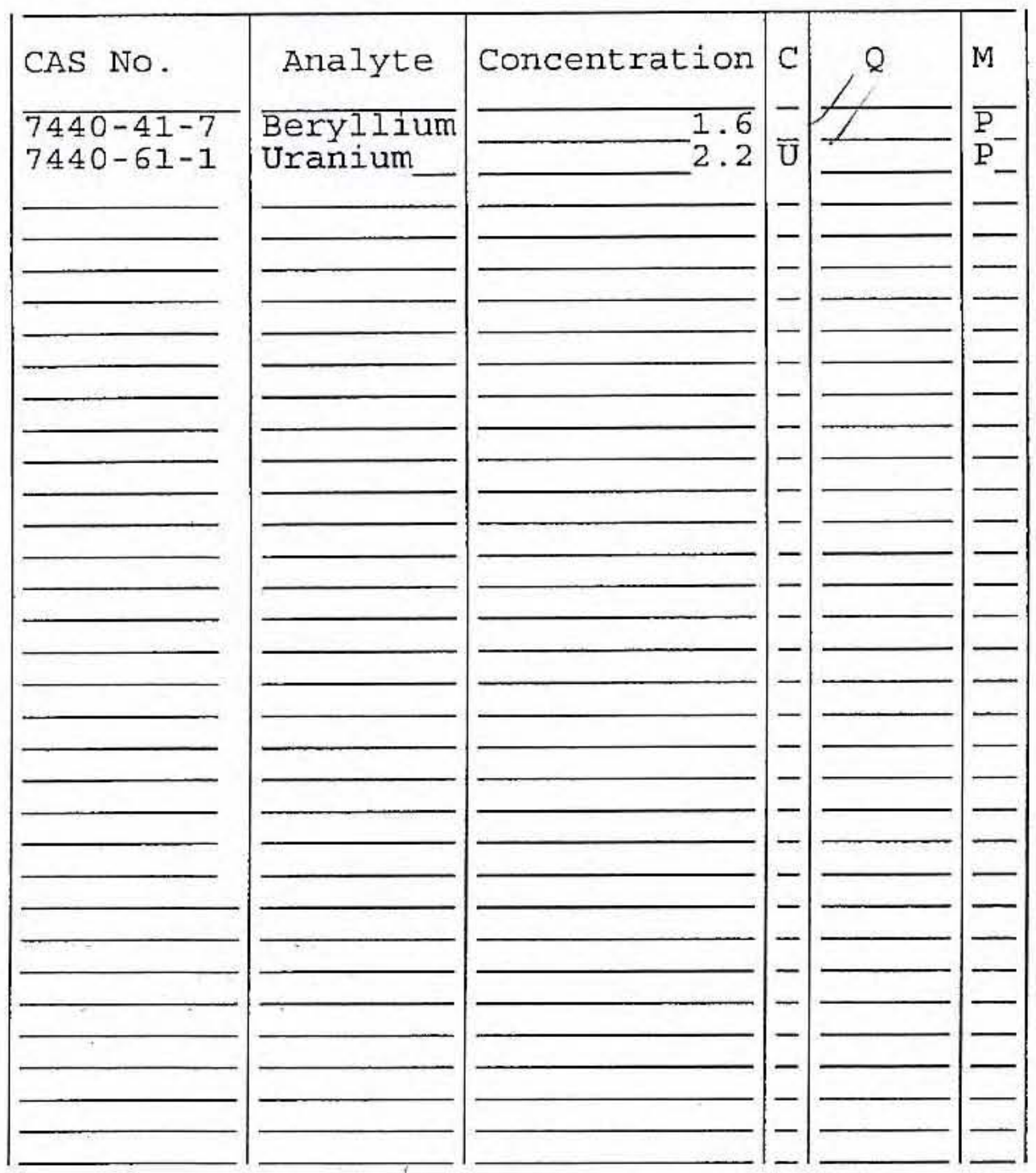

Color Before: Color After:
Clarity Before: Clarity After:
Texture: Artifacts :

Comments:

MPDT $-34 B$ 
Lab Name: LIONVILLE_LABORATORY

Lab Code: LVLI

Matrix (soil/water): SOIL

Level (low/med):

\% Solids :
Contract: 60052 SAS NO.: LOW
-91.6
SDG NO.: R1

\section{$35 \mathrm{~A}$}

Lab Sample ID: 0609L897-009

Date Received: 09/07/06

Concentration Units (ug/L or $\mathrm{mg} / \mathrm{kg}$ dry weight): $\mathrm{MG} / \mathrm{KG}$
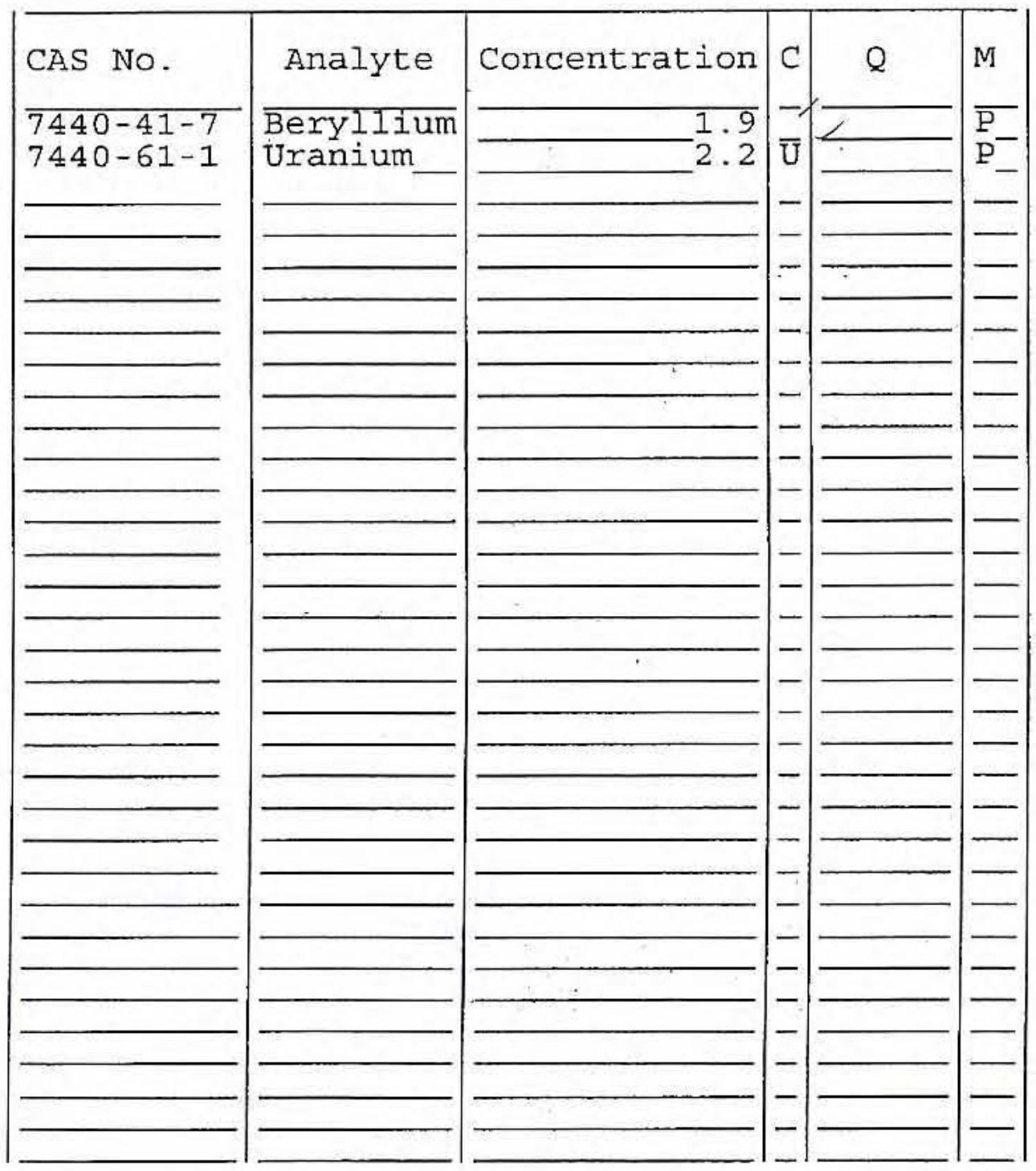

Color Before:

Color After:
Clarity Before:

Clarity After:
Texture:

Artifacts:

Comments :

MPDT $-35 \mathrm{~A}$ 
1

INORGANIC ANALYSES DATA SHEET
EPA SAMPLE NO.

$$
35 B
$$

SDG NO.: R1

Iab Sample ID: 0609L8 $\overline{97-010}$

Date Received: 09/07/06
Lab Name: LION
Lab Code: LVII

Matrix (soil/water): SoIL

Level (low/med): LOW

\% Solids :
$-89 \overline{.2}$
SAS NO.:

0052

Concentration Units (ug/I or $\mathrm{mg} / \mathrm{kg}$ dry weight): MG/KG

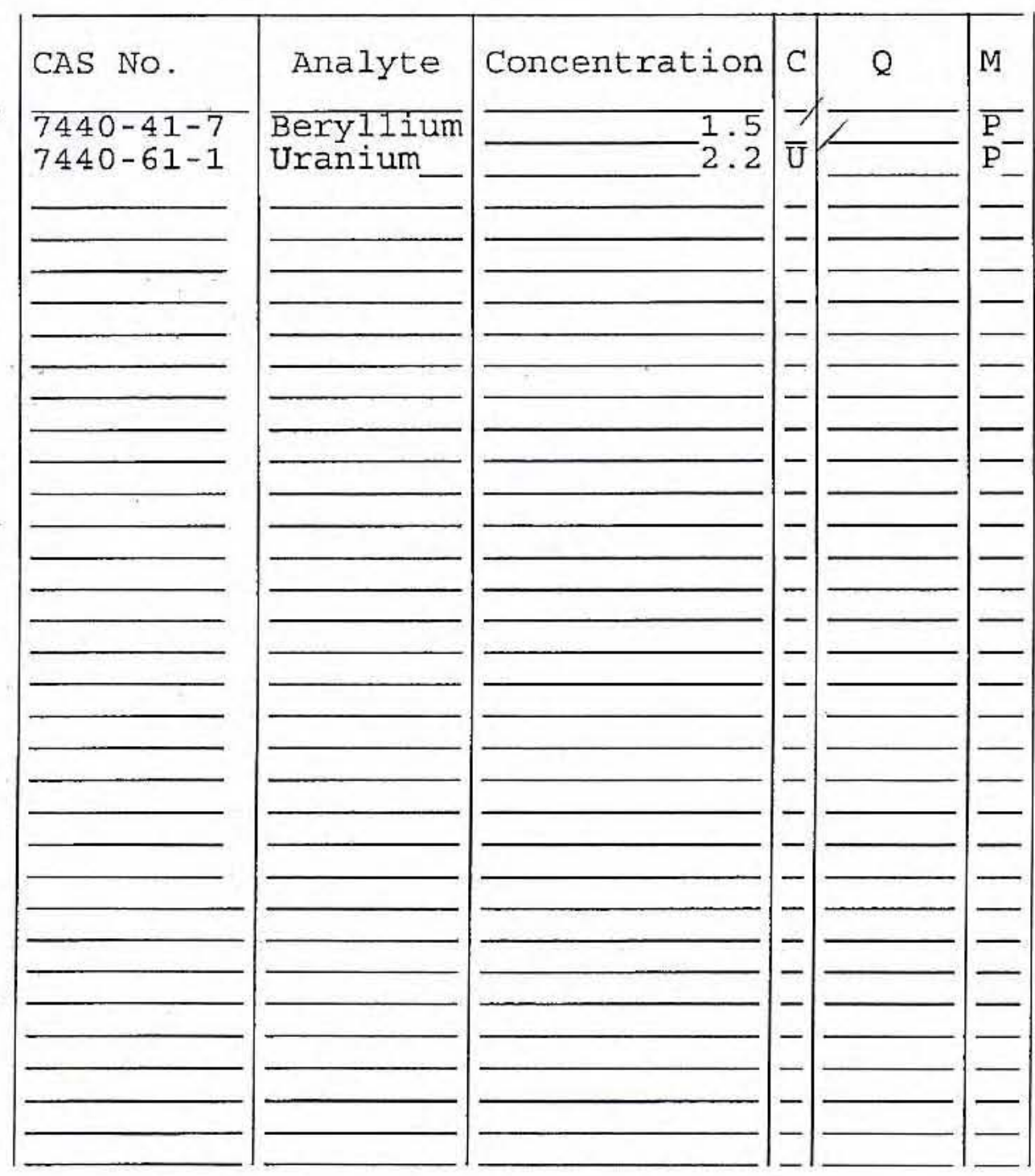

Color Before: Color After:

Comments :
Clarity Before:

Clarity After:
Texture:

Artifacts: 
1

INORGANIC ANALYSES DATA SHEET
EPA SAMPLE NO.

\section{$36 \mathrm{~A}$}

Lab Sample ID: $060918 \overline{907-011}$ SAS NO. :

Date Received: 09/07/06

Contract : 60052
Lab Name: LION
Lab Code: LVLI Matrix (soil/water): SoIL Level (low $/ \mathrm{med}$ ): \% Solids:

$$
\begin{array}{r}
\text { LOW } \\
-90.9
\end{array}
$$

Concentration Units (ug/L or $\mathrm{mg} / \mathrm{kg}$ dry weight): MG/KG

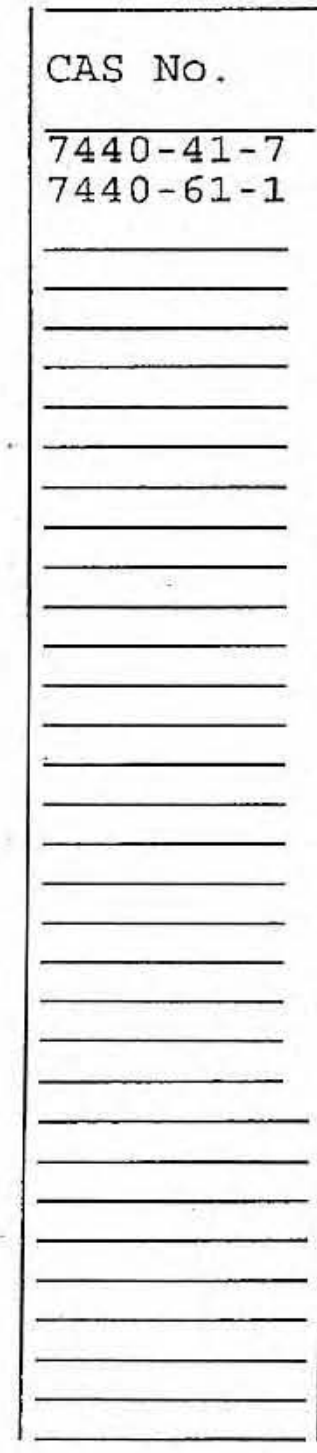

Color Before:

Color After:

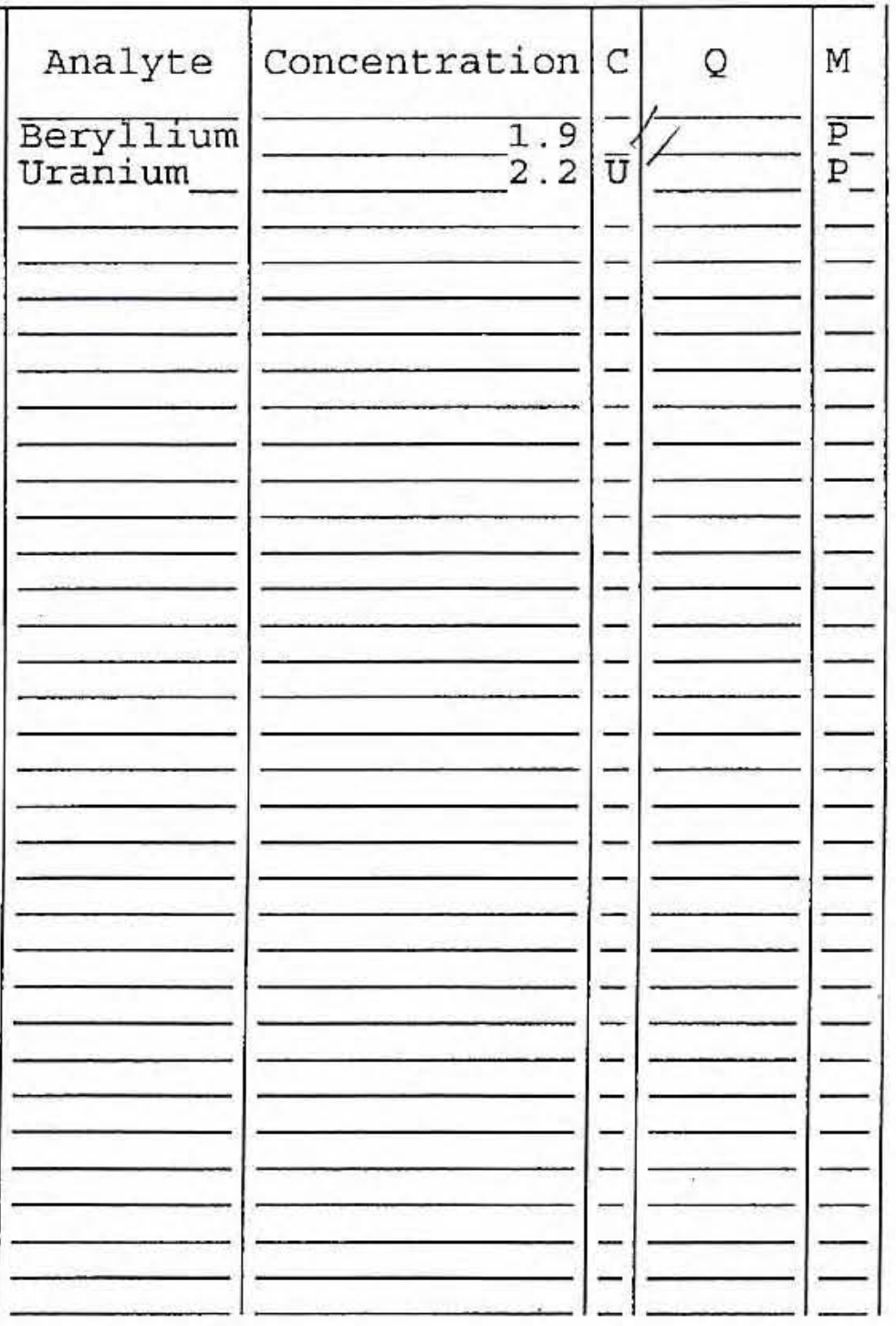

Clarity Before: Clarity After:
Texture:

Artifacts:

Comments:

MPDT $-36 \mathrm{~A}$ 


\section{EPA SAMPLE NO.}

\section{$36 \mathrm{~B}$}

Lab Name: LIONVILLE_LABORATORY Lab Code: LVLI Matrix (soil/water): SOIL Level (low/med): $\%$ Solids :
Contract: 60052 SAS NO. :

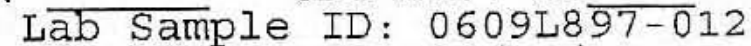
Date Received: 09/07/06

Concentration Units (ug/L or $\mathrm{mg} / \mathrm{kg}$ dry weight): MG/KG

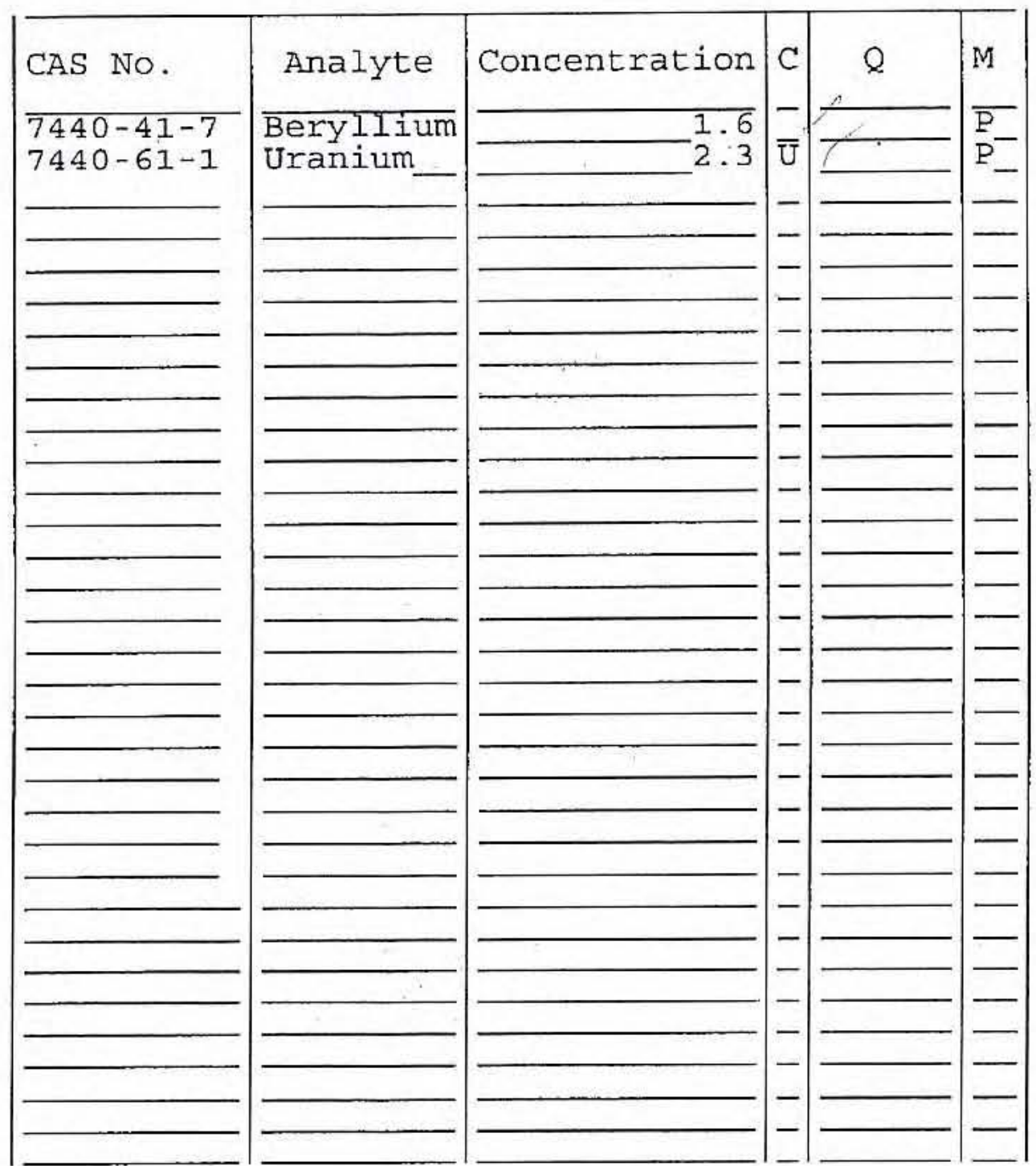

Color Before: Color After:
Clarity Before: Clarity After:
Texture: Artifacts :

Comments :

MPDT-36B 
Lab Name: LIONVILLE LABORATORY

Lab Code: LVLI Matrix (soil/water): soll Level (low/med): $\div$ Solids:

$$
\begin{aligned}
& \text { SOIL_ } \\
& \text { LOW- } \\
& -93.0
\end{aligned}
$$

Contract: 60052

SAS No.:

\section{$37 \mathrm{~A}$}

SDG NO.: R1

Lab Sample ID: 0609L8 $\overline{97-013}$

Date Received: 09/07/06

\section{Concentration Units (ug/L or $\mathrm{mg} / \mathrm{kg}$ dry weight): MG/KG}

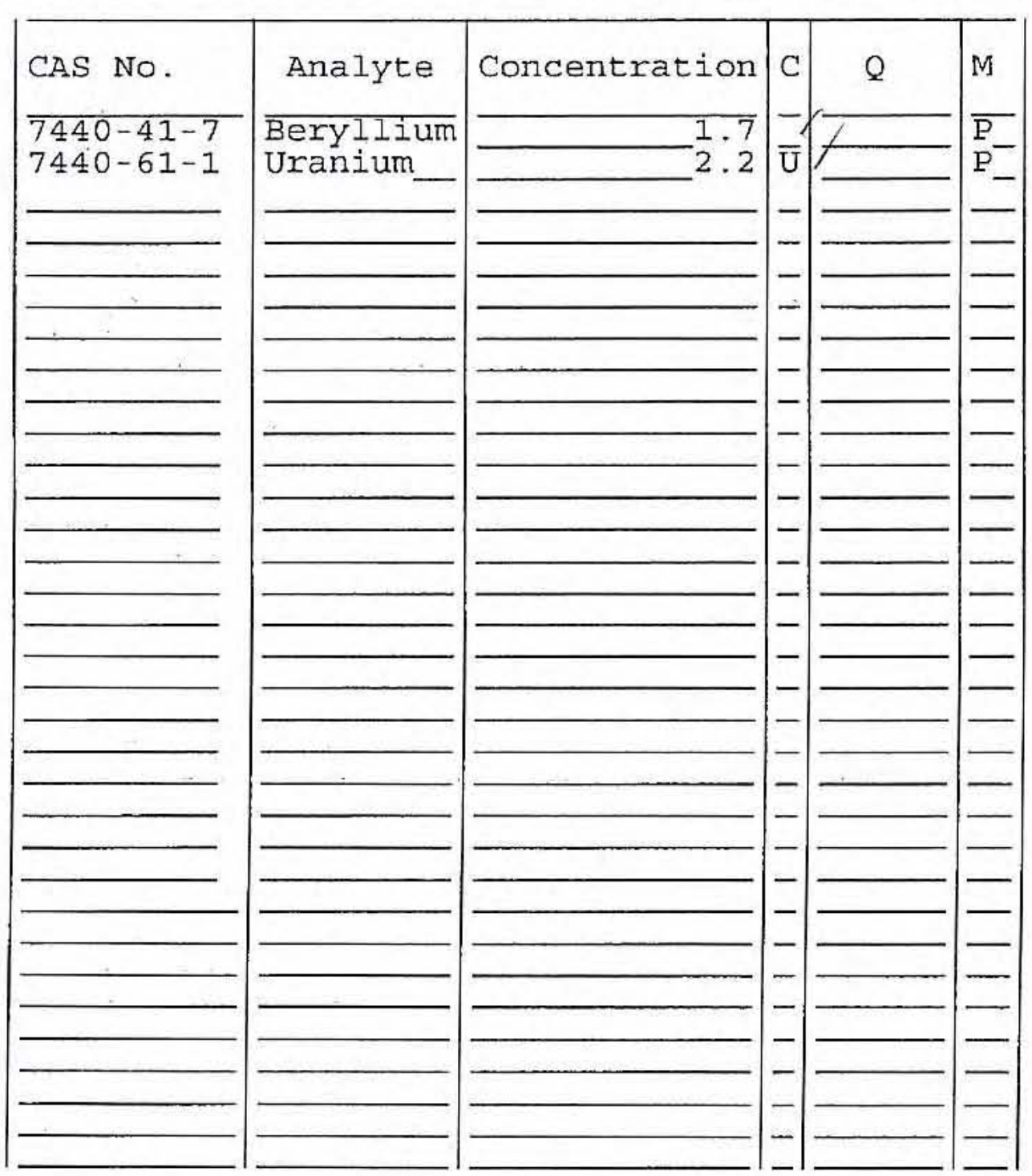

Color Before:

Color After:
Clarity Before:

Clarity After:
Texture:

Artifacts:

Comments :

MPDT $-37 \mathrm{~A}$ 
Lab Name: LIONVILIE_LABORATORY

Lab Code: LVLI Matrix (soil/water): SoIL_ Level (low/med): \% Solids: LOW
1

\section{INORGANIC ANALYSES DATA SHEET}

Contract: 60052
EPA SAMPLE NO.

$37 B$

SDG NO.: R1

Lab Sample ID: 0609L8 $\overline{97-014}$

Date Received: 09/07/06

Concentration Units (ug/L or $\mathrm{mg} / \mathrm{kg}$ dry weight): MG/KG

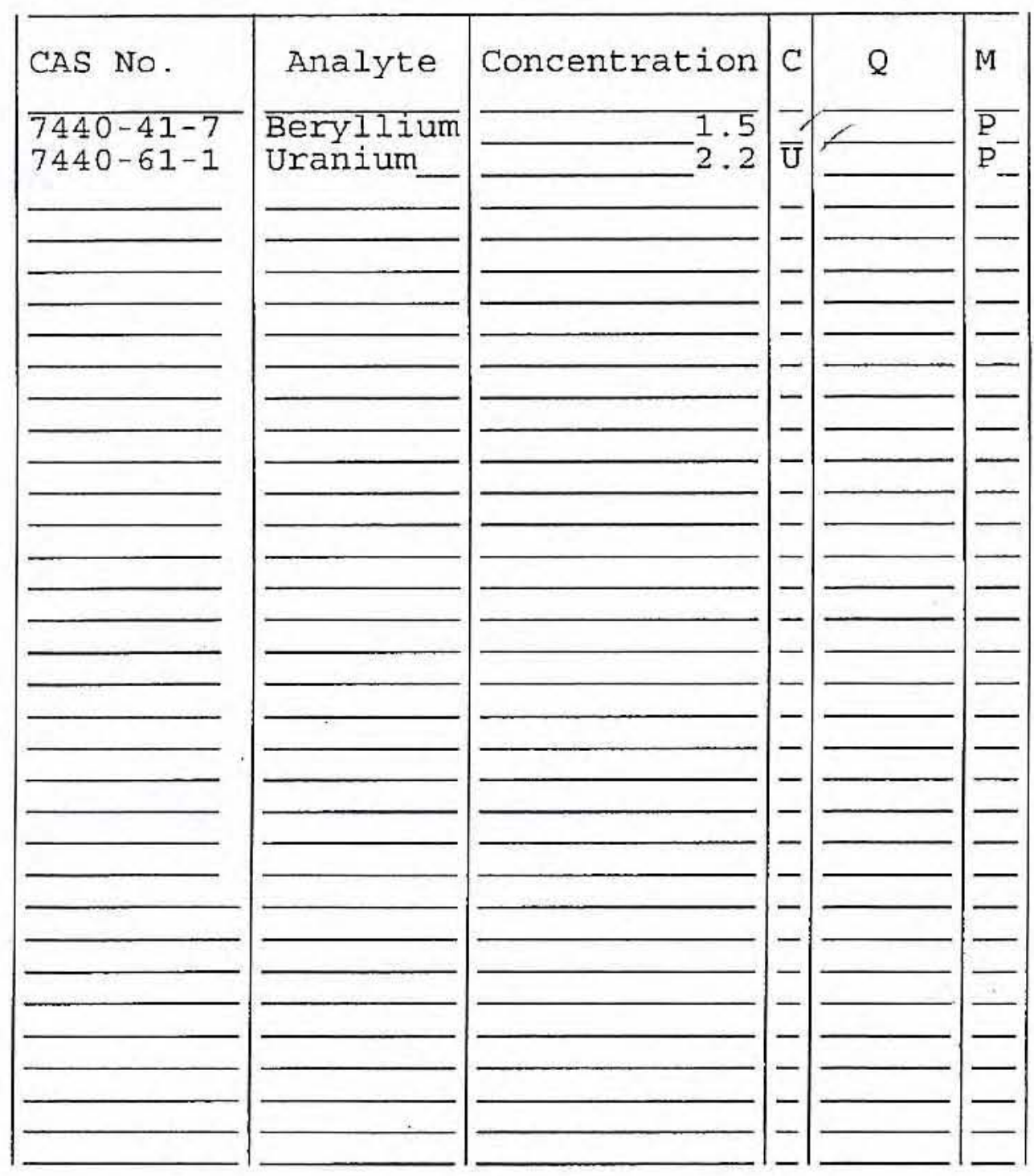

Color Before:

Color After:
Clarity Before:

Clarity After:
Texture: Artifacts:

Comments :

MPDT $-37 \mathrm{~B}$ 
1

INORGANIC ANALYSES DATA SHEET
EPA SAMPLE NO.

\section{$38 \mathrm{~A}$}

SDG No. : R1

Contract: 60052

SAS NO.:

Lab Sample ID: 0609L8̄97-015

Date Received: 09/07/06
Lab Name: LION
Lab Code: LVLI

Matrix (soil/wat
Level (low/med): \% Solids:
Case No.: MPDT_

$$
\begin{aligned}
& \text { SOIL_ } \\
& \text { LOW- } \\
& \quad 92.8
\end{aligned}
$$

Concentration Units (ug/L or $\mathrm{mg} / \mathrm{kg}$ dry weight): $\mathrm{MG} / \mathrm{KG}$

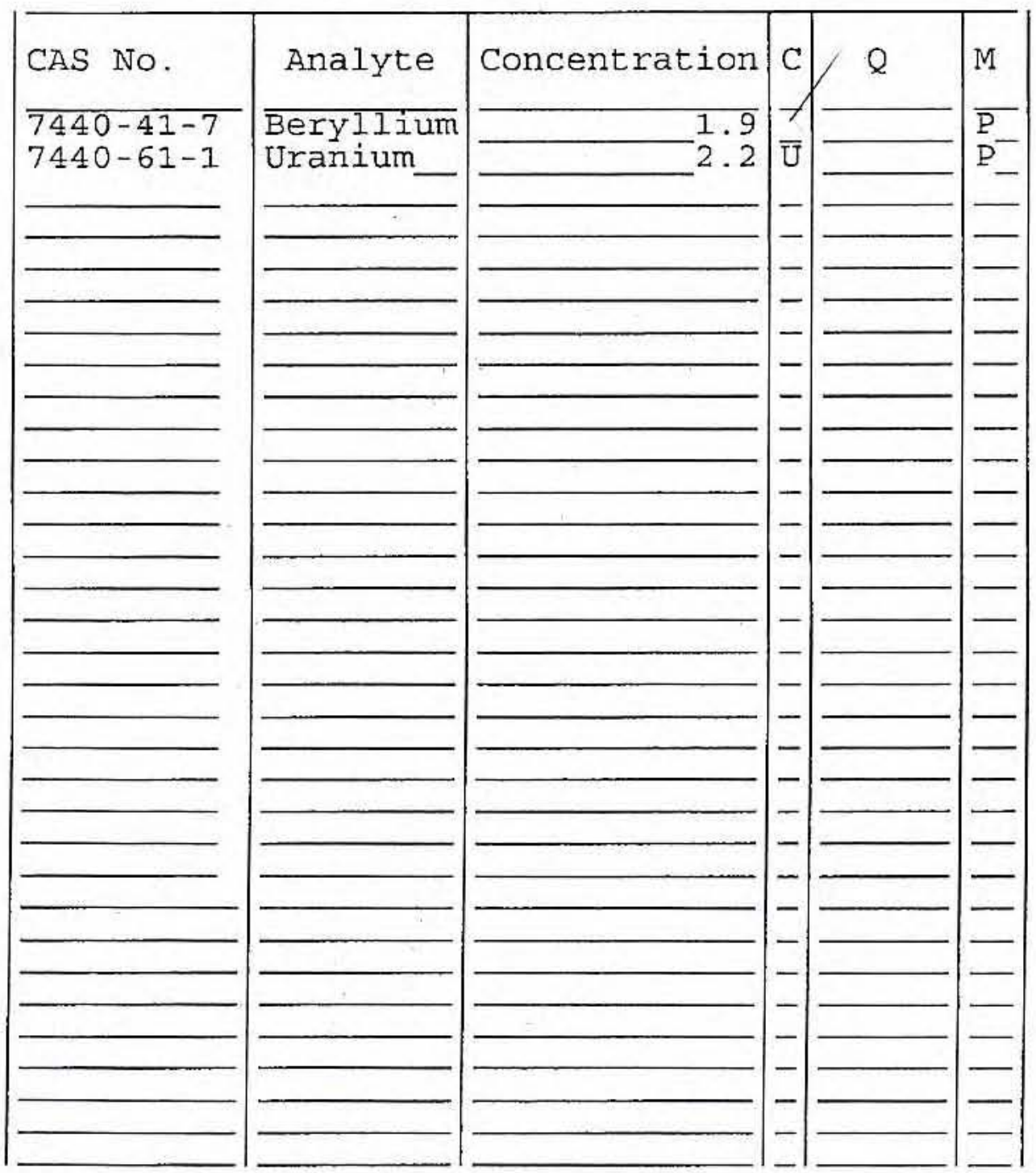

Color Before:

Color After:
Clarity Before: Clarity After:
Texture:

Artifacts :

Comments :

MPDT $-38 \mathrm{~A}$ 
1

INORGANIC ANALYSES DATA SHEET
EPA SAMPLE NO.

\section{$3-8 \mathrm{~B}$}

Lab Sample ID: 0609L8 $\overline{97-016}$
SDG NO.: RI

SAS NO. :

Date Received: 09/07/06

Contract: 60052
Lab Name: LIONVILLE_LABORATORY

Lab Code: LVLI
Matrix (soil/water): SoIL

Level (low/med) :

\% Solids :
LOW

$-90 \overline{1.1}$

Concentration Units (ug/L or $\mathrm{mg} / \mathrm{kg}$ dry weight): MG/KG

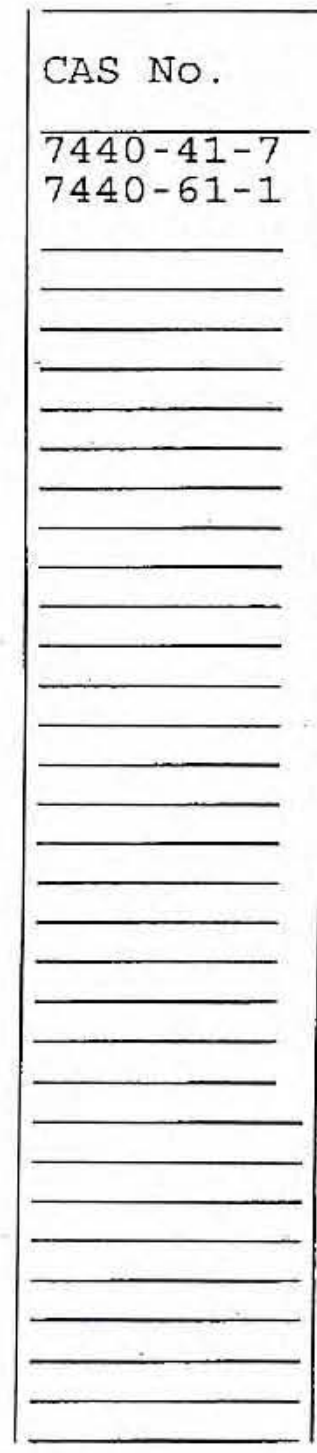

Color Before:

Color After:

Comments :

MPDT $-38 B$

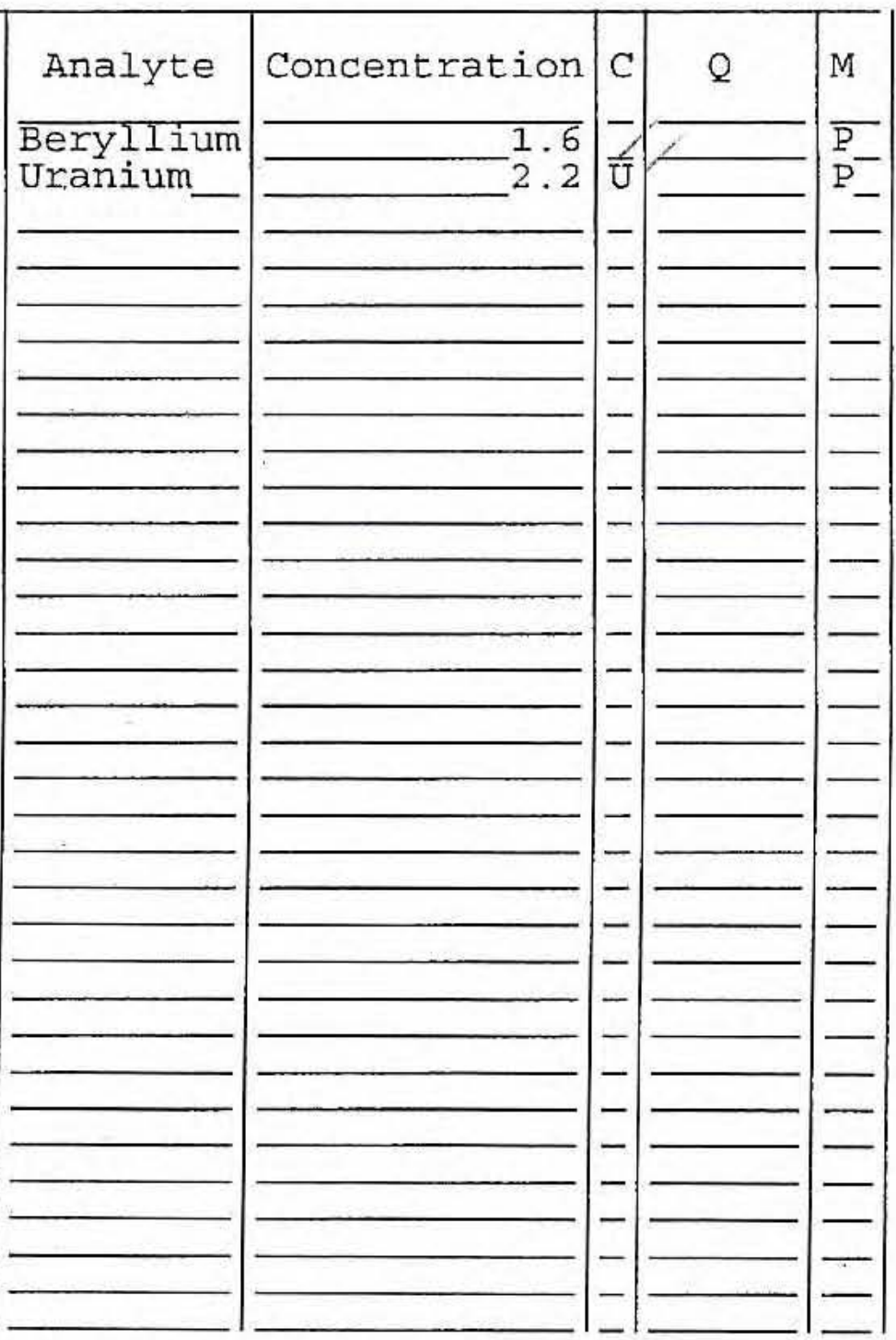

Clarity Before: Clarity After:
Texture: Artifacts : 


\section{INORGANIC ANALYSES DATA SHEET}

Lab Name: LIONVILLE LABORATORY

Lab Code: LVLI

Matrix (soil/water): WATER

Level (low $/ \mathrm{med}$ ): LOW

Solids:
EPA SAMPLE NO.

R1

Contract: 60052

SAS NO. :

Lab Sample ID: $060918 \overline{97-017}$

Date Received: 09/07/06

Concentration Units (ug/L or $\mathrm{mg} / \mathrm{kg}$ dry weight): UG/L_

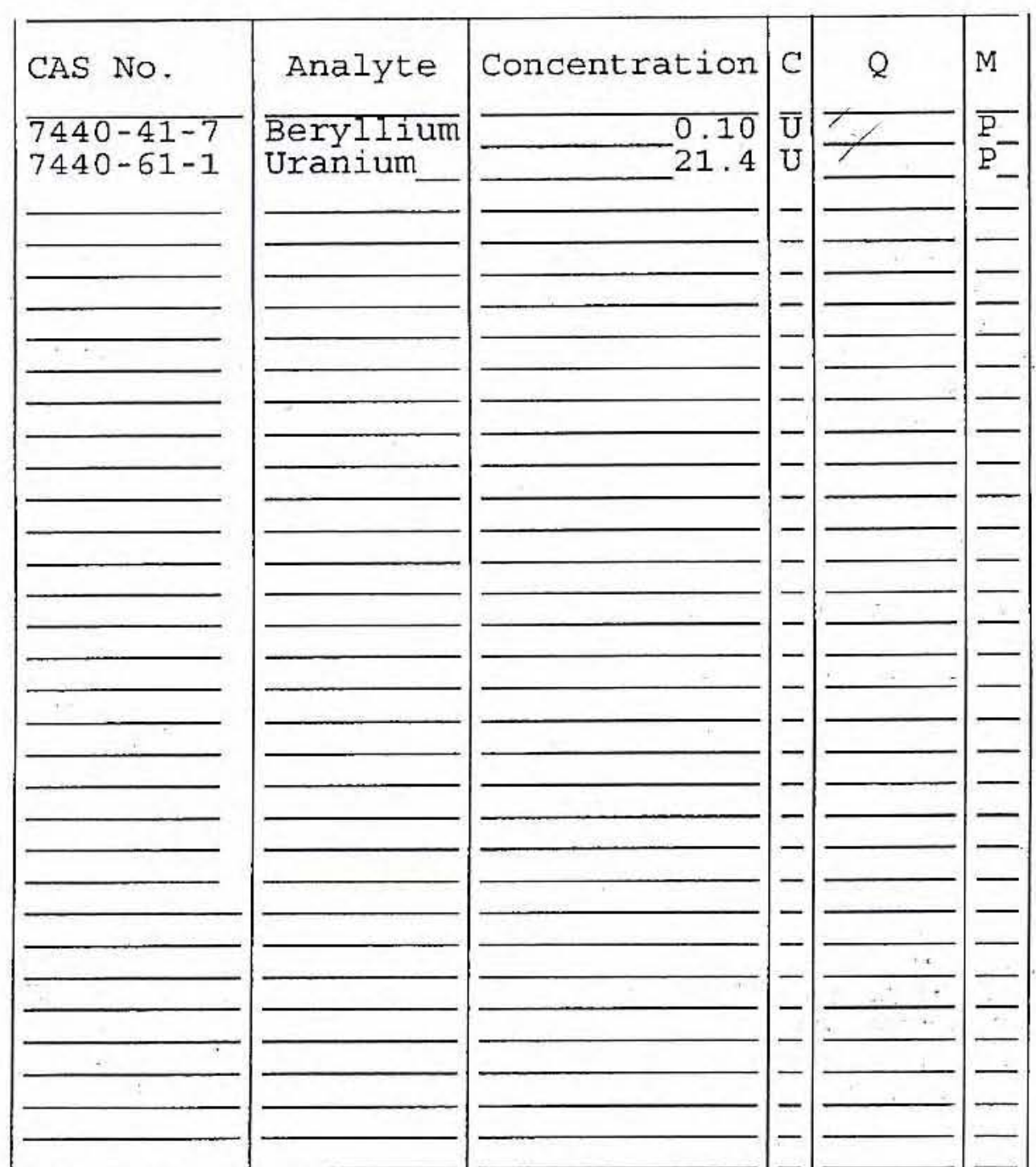

Color Before:

Color After:
Clarity Before: Clarity After:
Texture:

Artifacts:

Comments:

MPDT-R1 


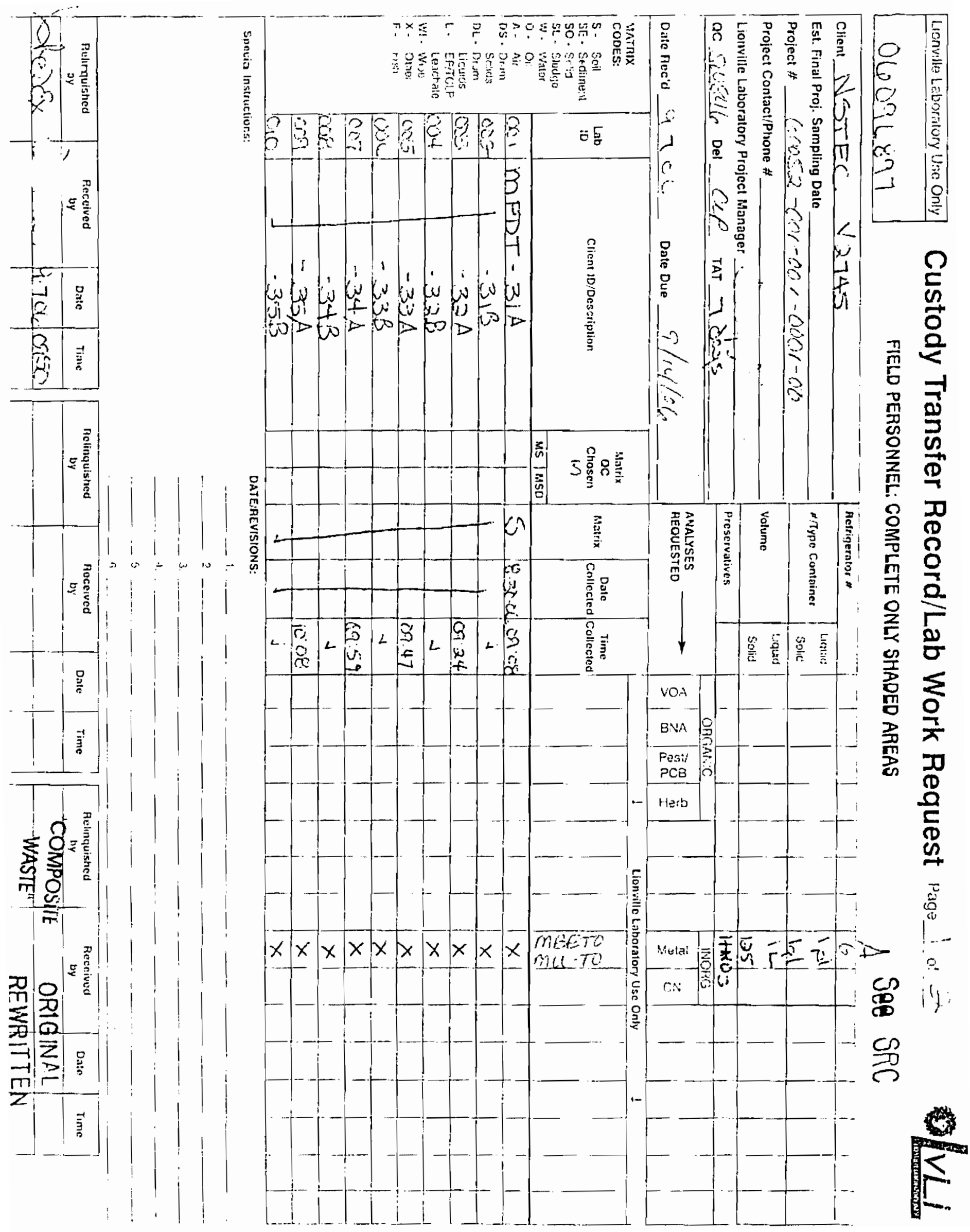




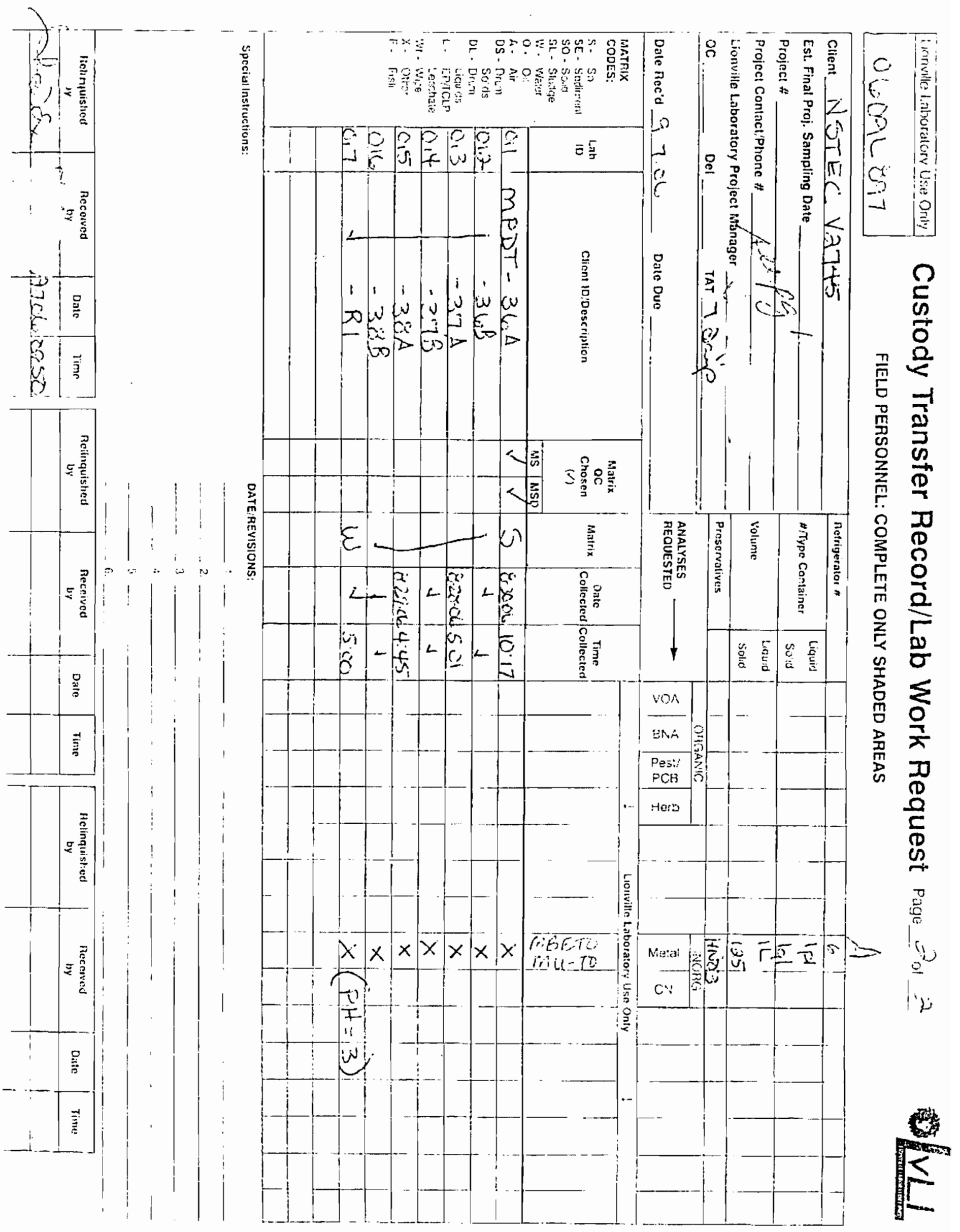




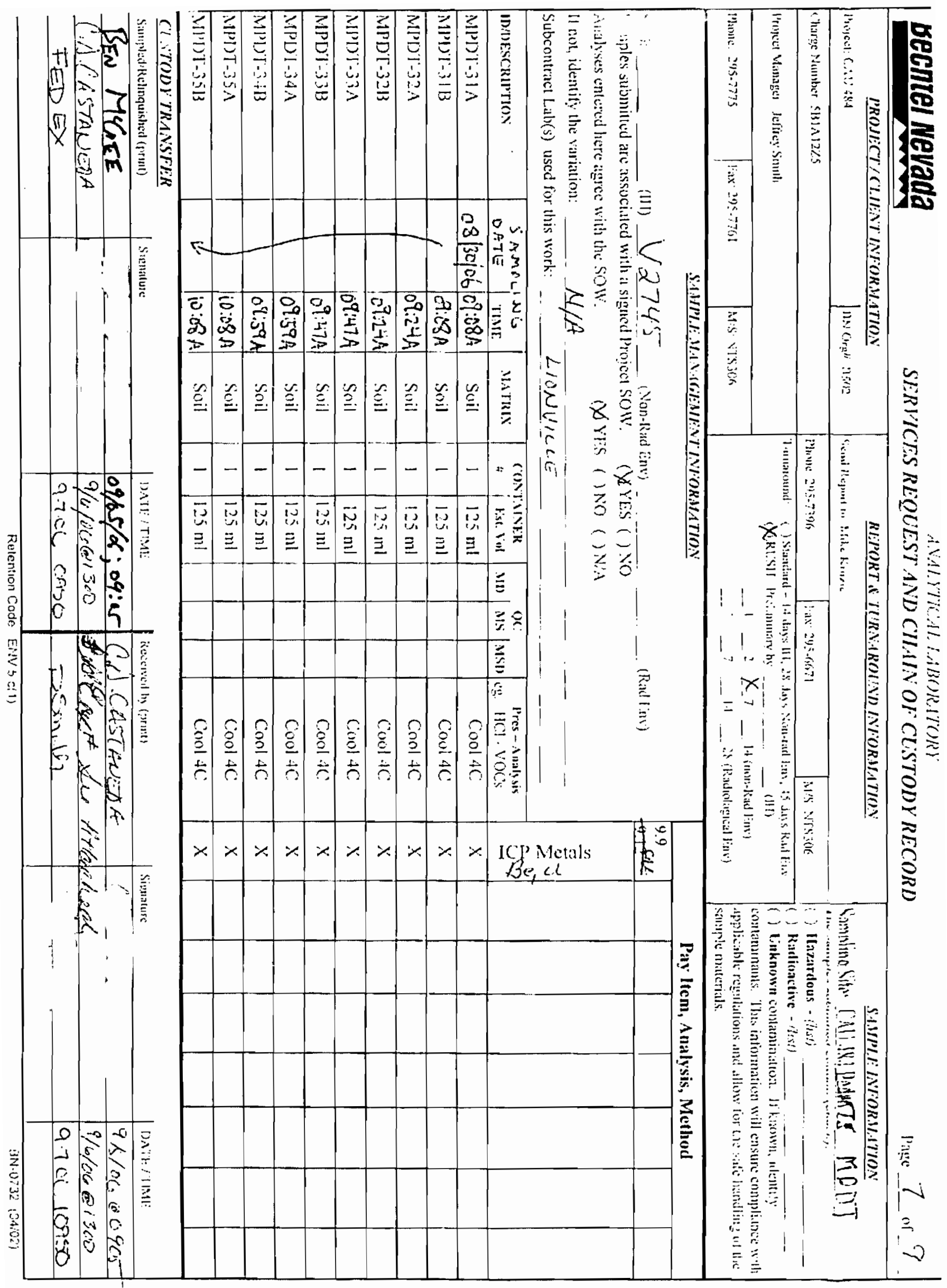




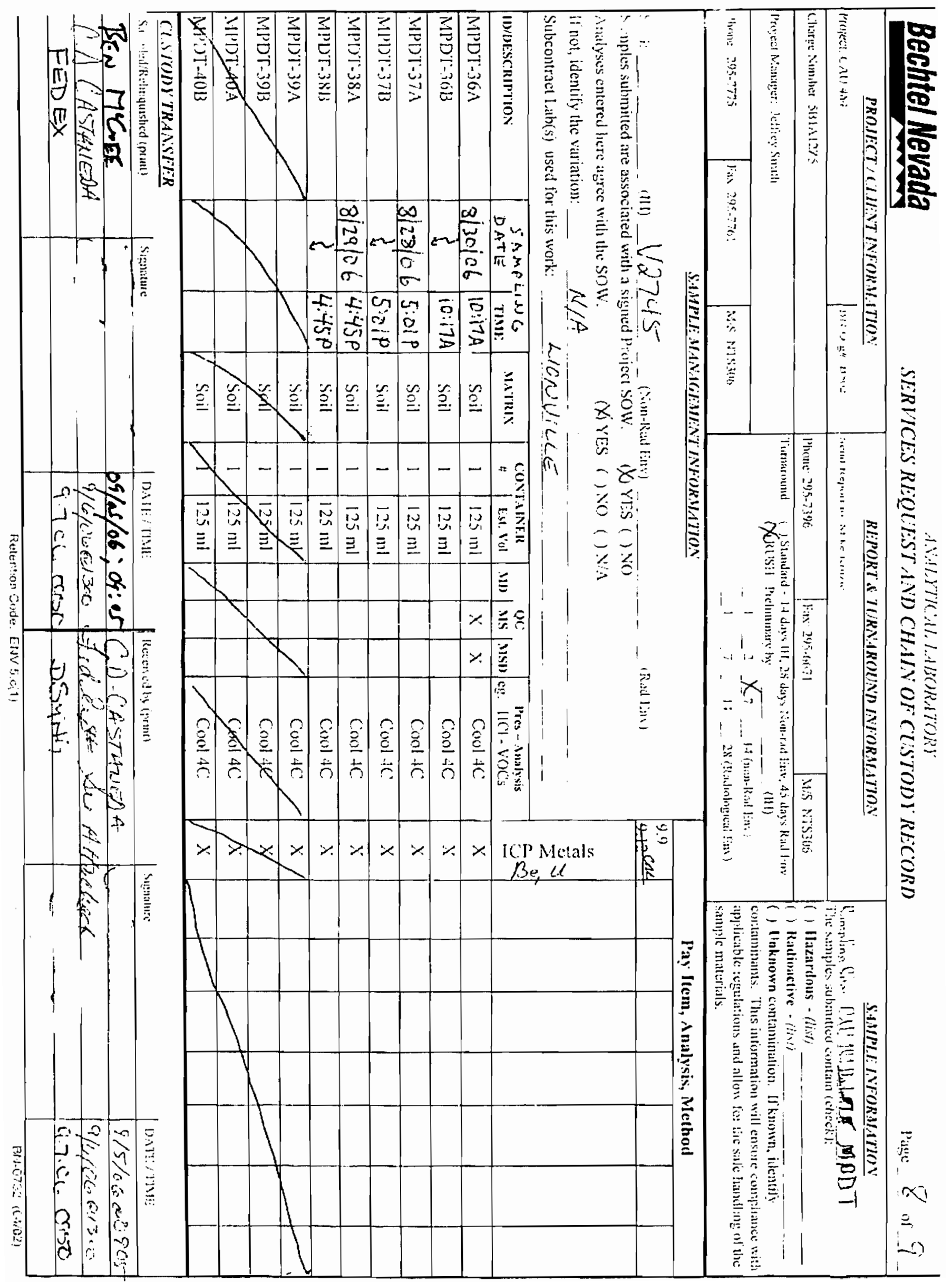




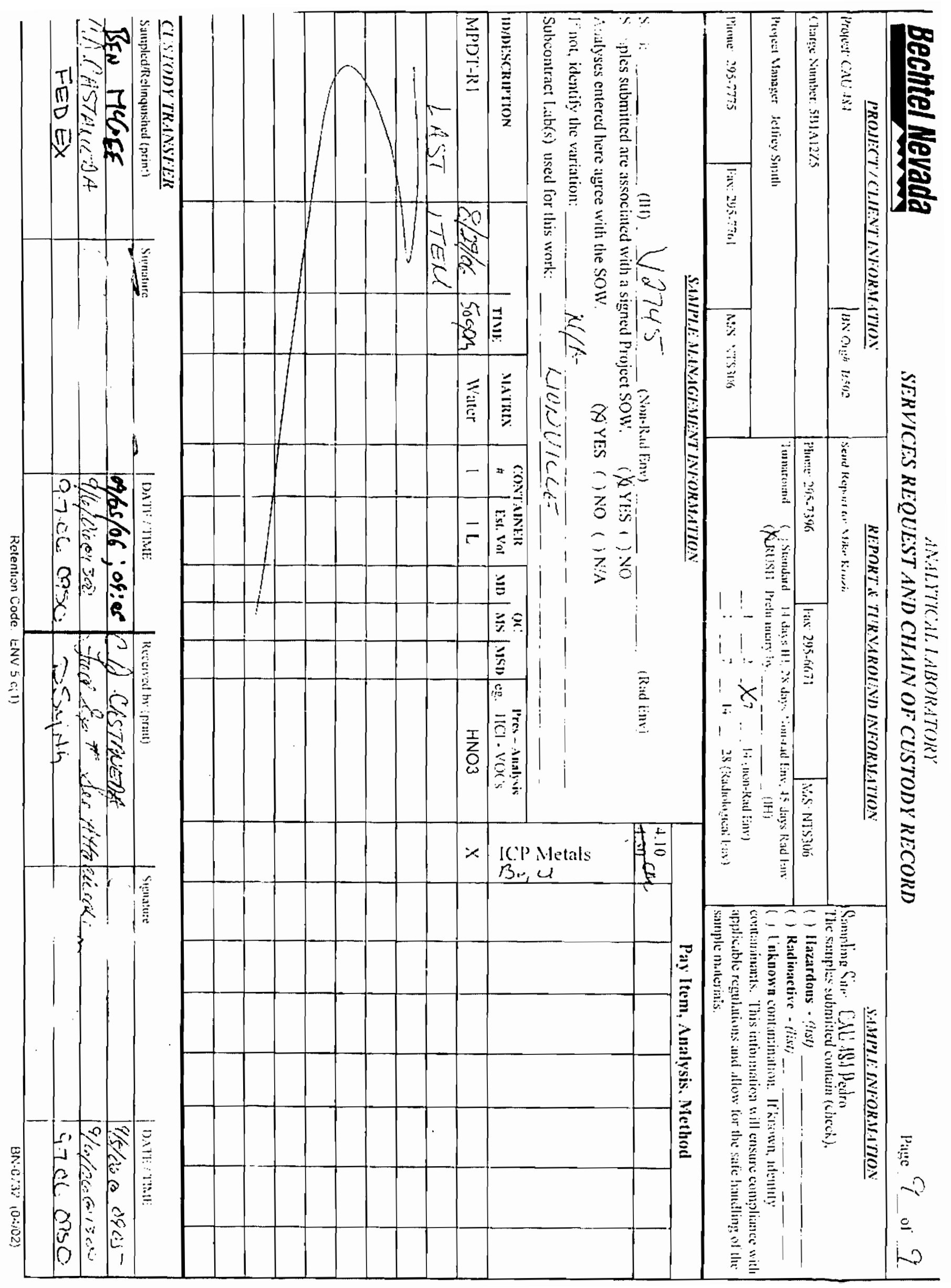




\title{
INVESTIGATION (GEOPROBE ${ }^{\circledR}$ ) SOIL SAMPLE ANALYTICAL RESULTS

\author{
CAS TA-52-004-TAAL \\ METAL PARTICLE DISPERSION TEST
}

\author{
Radiological Data
}




\section{Isotopic Uranium By Alpha Spectroscopy Sample Results Summary}

Client Name: National Sercurity Technologies, LLC Client Project Name: CAU 484 Client Project Number: V2746
Laboratory Name: Paragon Analytics

PAl Work Order: 0609032
Page: 1 of 7

Reported on: Tuesday, September 12, 2006 9:26:19 AM

\begin{tabular}{|c|c|c|c|c|c|c|c|c|c|c|}
\hline $\begin{array}{c}\text { Lab } \\
\text { Sample ID }\end{array}$ & Client Sample ID & $\begin{array}{l}\text { Sample } \\
\text { Type }\end{array}$ & Nuclide & Result +/- $2 \mathrm{~s}$ TPU & MDC & Units & Matrix & Prep Batch & $\begin{array}{c}\text { Date } \\
\text { Analyzed }\end{array}$ & Flags \\
\hline $0609032-1$ & MPDT-01A & Sample & U-233/234 & $1.44 E+00+/-6.42 E-01$ & $3.34 E-01$ & pCiig & SOIL & ASO60908-1 & $9 / 11 / 2006$ & LT \\
\hline $0609032-1$ & MPDT-01A & Sample & U-235 & $2.08 \mathrm{E}-01+/-2.51 \mathrm{E}-01$ & 2.93E-01 & pCiig & SOIL & AS060908-1 & $9 / 11 / 2006$ & $u$ \\
\hline $0609032-1$ & MPDT-01A & Sample & $\mathrm{U}-238$ & $1.26 \mathrm{E}+00+/-5.96 \mathrm{E}-01$ & $3.11 \mathrm{E}-01$ & pCilg & SOIL & AS060908-1 & $9 / 11 / 2006$ & LT \\
\hline $0609032-2$ & MPDT-01B & Sample & U-233/234 & $1.75 \mathrm{E}+00+/-6.22 \mathrm{E}-01$ & $3.04 E-01$ & $\mathrm{pCi} / \mathrm{g}$ & SOIL & AS060908-1 & $9 / 11 / 2006$ & LT \\
\hline $0609032-2$ & MPDT-01B & Sample & U-235 & 4.57E-02+/- 1.75E-01 & 2.06E-0 & $\mathrm{pCi} / \mathrm{g}$ & SOIL & AS060908-1 & $9 / 11 / 2006$ & $\mathrm{U}$ \\
\hline $0609032-2$ & MPDT-01B & Sample & $\mathrm{U}-238$ & $1.55 \mathrm{E}+00+1-5.77 \mathrm{E}-01$ & $2.73 E-01$ & $\mathrm{pCi} / \mathrm{g}$ & SOIL & AS060908-1 & $9 / 11 / 2006$ & LT \\
\hline $0609032-3$ & MPDT-02A & Sample & U-233/234 & $1.35 E+00+/-5.30 E-01$ & 2.35E-01 & pCiig & SOIL & AS060908-1 & $9 / 11 / 2006$ & LT \\
\hline $0609032-3$ & MPDT-02A & Sample & U-235 & $2.01 \mathrm{E}-01+/-2.04 \mathrm{E}-01$ & 1.36E-01 & pCiig & SOIL & AS060908-1 & $9 / 11 / 2006$ & LT \\
\hline $0609032-3$ & MPDT-02A & Sample & U-238 & $1.62 \mathrm{E}+00+/-5.88 \mathrm{E}-01$ & $2.00 \mathrm{E} \cdot 0.1$ & $\mathrm{pClig}$ & SOIL & AS060908-1 & $9 / 11 / 2006$ & LT \\
\hline
\end{tabular}

\section{Comments:}

Data Package ID: UR0609032-1

Qualifiers/Flags:

$U$ - Result is less than the sample specific MDC

LT - Result is less than Requested MDC, greater than sample specific MDC.

$Y 1$ - Chemical Yield is in control at 100.110\%. Quantitative Yield is assumed,

Y2 - Chemical Yield outside delautl limits.

M- The requested MDC was not met.

(4) The requested MDC was not met, but the reported activity is greater than the reported MDC

\section{Abbreviations:}

TPU - Total Propagated Uncertainty (see PAI SOP 743)

MDC - Minimum Delectable Concentration (see PAI SOP 709)

BDL - Below Detection Limit

\section{8}




\section{Isotopic Uranium By Alpha Spectroscopy Sample Results Summary}

Client Name: National Sercurity Technologies, LLC Client Project Name: CAU 484 Client Project Number: V2746
Laboratory Name: Paragon Analytics PAI Work Order: 0609032
Page: 2 of 7

Reported on: Tuesday, September 12, 2006 9:26:20 AM

\begin{tabular}{|c|c|c|c|c|c|c|c|c|c|c|}
\hline $\begin{array}{l}\text { Lab } \\
\text { Sample ID }\end{array}$ & Client Sample ID & $\begin{array}{c}\text { Sample } \\
\text { Type }\end{array}$ & Nuclide & Result $+/-2$ s TPU & MDC & Units & Matrix & Prep Batch & $\begin{array}{c}\text { Date } \\
\text { Analyzed }\end{array}$ & Flags \\
\hline $0609032-4$ & MPDT-02B & Sample & $U-233 / 234$ & $1.46 \mathrm{E}+00+1-5.70 \mathrm{E}-01$ & $1.88 \mathrm{E}-01$ & pClig & SOIL & AS060908-1 & $9 / 11 / 2006$ & LT \\
\hline $0609032-4$ & MPDT-02B & Sample & U-235 & $5.39 \mathrm{E}-02+/-1.88 \mathrm{E}-01$ & $1.46 \mathrm{E}-01$ & $\mathrm{pCi} / \mathrm{g}$ & SOIL & AS060908-1 & $9 / 11 / 2006$ & $u$ \\
\hline $0609032-4$ & MPDT-02B & Sample & U-238 & $1.60 \mathrm{E}+00+1-6.02 \mathrm{E}-01$ & $1.88 \mathrm{E}-01$ & $\mathrm{pCi} / \mathrm{g}$ & SOIL & AS060908-1 & $9 / 11 / 2006$ & LT \\
\hline $0609032-5$ & MPDT-03A & Sample & U-233/234 & $1.29 \mathrm{E}+00+/-5.14 \mathrm{E}-01$ & $2.16 \mathrm{E}-01$ & $\mathrm{pCi} / \mathrm{g}$ & SOIL & AS060908-1 & $9 / 11 / 2006$ & LT \\
\hline 0609032.5 & MPDT-03A & Sample & U-235 & $1.94 \mathrm{E}-01+/-2.01 \mathrm{E}-01$ & 2.03E-01 & $\mathrm{pCi} g$ & SOIL & AS060908-1 & $9 / 11 / 2006$ & $U$ \\
\hline $0609032-5$ & MPDT-03A & Sample & U.238 & $1.50 \mathrm{E}+00+/-5.61 \mathrm{E}-01$ & $2.32 \mathrm{E}-01$ & pCirg & SOIL & AS060908-1 & $9 / 11 / 2006$ & LT \\
\hline $0609032-6$ & MPDT-03B & Sample & $U-233 / 234$ & $1.49 \mathrm{E}+00+/-5.45 \mathrm{E}-01$ & $1.09 \mathrm{E}-01$ & $\mathrm{pCi} / \mathrm{g}$ & SOIL & AS060908-1 & $9 / 11 / 2006$ & LT \\
\hline $0609032 \cdot 6$ & MPDT-03B & Sample & U.235 & $9.04 \mathrm{E}-02+/-1.65 \mathrm{E}-01$ & $1.94 \mathrm{E}-01$ & $\mathrm{pCi} / \mathrm{g}$ & SOIL & AS060908-1 & $9 / 11 / 2006$ & $u$ \\
\hline $0609032-6$ & MPDT-03B & Sample & U.238 & $9.59 \mathrm{E}-01+/-4.24 \mathrm{E}-01$ & $1.89 E-01$ & $\mathrm{pCi} / \mathrm{g}$ & SOIL & AS060908-1 & $9 / 11 / 2006$ & LT \\
\hline
\end{tabular}

\section{Comments:}

Data Package ID: UR0609032-1

Qualifiers/Flags:

$U$-Resutt is less than the sample specific MOC

LT - Result is less than Requested MDC, greater than sample specific MDC.

$\mathrm{Y1}$ - Chemical Yield is in control at $100.110 \%$. Quantitative Yield is assumed

Y2 - Chemical Yield outside default limits.

M-The requested MDC was not met.

MI. The requested MDC was not met, but the reported activity is greater than the reported MDC.

Date Printed: Tuesday, September 12, 2006

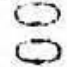

Or

\section{Abbreviations:}

TPU - Total Propagated Uncertainty (see PAI SOP 743)

MDC - Minimum Detectable Concentration (see PAI SOP 709)

BDL - Below Detection Limit 


\section{Isotopic Uranium By Alpha Spectroscopy Sample Results Summary}

Client Name: National Sercurity Technologies, LLC Client Project Name: CAU 484 Client Project Number: V2746
Laboratory Name: Paragon Analytics

PAI Work Order: 0609032
Page: 3 of 7

Reported on: Tuesday, September 12, 2006 9:26:20 AM

\begin{tabular}{|c|c|c|c|c|c|c|c|c|c|c|}
\hline $\begin{array}{c}\text { Lab } \\
\text { Sample ID }\end{array}$ & Client Sample ID & $\begin{array}{c}\text { Sample } \\
\text { Type }\end{array}$ & Nuclide & Result + $/-2$ s TPU & MDC & Units & Matrix & Prep Batch & $\begin{array}{c}\text { Date } \\
\text { Analyzed }\end{array}$ & Flags \\
\hline $0609032-7$ & MPDT-04A & Sample & U.233/234 & $1.61 \mathrm{E}+00+/-5.77 \mathrm{E}-01$ & $3.04 \mathrm{E}-01$ & $\mathrm{pCi} / \mathrm{g}$ & SOIL & AS060908-1 & $9 / 11 / 2006$ & LT \\
\hline $0609032-7$ & MPDT-04A & Sample & U-235 & $-1.28 \mathrm{E}-02+1-1.65 \mathrm{E}-01$ & $2.43 \mathrm{E}-01$ & $\mathrm{pClig}$ & SOIL & AS060908-1 & $9 / 11 / 2006$ & $u$ \\
\hline $0609032-7$ & MPDT.04A & Sample & $\mathrm{U}-238$ & $1.32 \mathrm{E}+00+1-5.09 \mathrm{E}-01$ & $1,89 \mathrm{E}-01$ & pCilg & SOIL & AS060908-1 & $9 / 11 / 2006$ & LT \\
\hline $0609032-8$ & MPDT-04B & Sample & U-233/234 & $1.79 \mathrm{E}+00+1-6.23 \mathrm{E}-01$ & $2.31 E \cdot 01$ & pCilg & SOIL & AS060908-1 & $9 / 11 / 2006$ & LT \\
\hline $0609032-8$ & MPDT-04B & Sample & $\mathrm{U}-235$ & $3.91 \mathrm{E}-01+/-2.87 \mathrm{E}-01$ & $2.03 \mathrm{E}-01$ & $\mathrm{pCi} / \mathrm{g}$ & SOIL & AS060908-1 & $9 / 11 / 2006$ & LT \\
\hline $0609032-8$ & MPDT-04B & Sample & $U-238$ & $1.22 \mathrm{E}+00+/-4.94 \mathrm{E}-01$ & $1.73 E-01$ & $\mathrm{pCi} / \mathrm{g}$ & SOIL & AS060908.1 & $9 / 11 / 2006$ & LT \\
\hline $0609032-9$ & MPDT-05A & Sample & U-233/234 & $1.59 E+00+/-5.77 E-01$ & $2.76 \mathrm{E}-01$ & pCiig & SOIL & AS060908-1 & $9 / 11 / 2006$ & $\mathrm{LT}$ \\
\hline $0609032-9$ & MPDT-05A & Sample & U-235 & $9.75 \mathrm{E}-02+/-1.70 \mathrm{E}-01$ & $1.32 \mathrm{E}-01$ & pCirg & SOIL & AS060908-1 & $9 / 11 / 2006$ & u \\
\hline $0609032-9$ & MPDT-05A & Sample & $\mathrm{U}-238$ & 8.97E-01+/-4.15E-01 & $2.28 \mathrm{E}-01$ & $\mathrm{pCl} / \mathrm{g}$ & SOIL & ASO60908-1 & $9 / 11 / 2006$ & LT \\
\hline
\end{tabular}

\section{Comments:}

Data Package ID: UR0609032-1

\section{Qualifiers/Flags:}

$U$ - Result is less than the sample specific MDC.

LT - Result is less than Requested MDC, greater than sample specific MDC.

Y1 - Chemical Yield is in control at 100-110\%. Quantitative Yield is assumed.

Y2 - Chemical Yield outside default limits.

$M$ - The requested MDC was not mot.

(W.)- The requested MDC was not met, but the reporled activity is greater than the reported MDC
Abbreviations:

TPU - Total Propagated Uncertainty (see PAI SOP 743)

MDC - Minimum Detectable Concentration (see PAI SOP 709 )

BDL - Below Detection Limit 


\section{Isotopic Uranium By Alpha Spectroscopy Sample Results Summary}

Client Name: National Sercurity Technologies, LLC Client Project Name: CAU 484 Client Project Number: V2746
Laboratory Name: Paragon Analytics

PAI Work Order: 0609032
Page: 4 of 7

Reported on: Tuesday, September 12, 2006 9:26:20 AM

\begin{tabular}{|c|c|c|c|c|c|c|c|c|c|c|}
\hline $\begin{array}{c}\text { Lab } \\
\text { Sample ID }\end{array}$ & Client Sample ID & $\begin{array}{c}\text { Sample } \\
\text { Type }\end{array}$ & Nuclide & Result +1- 2 s TPU & MDC & Units & Matrix & Prep Batch & $\begin{array}{c}\text { Date } \\
\text { Analyzed }\end{array}$ & Flags \\
\hline $0609032-10$ & MPDT-05B & Sample & U-233/234 & $1.36 \mathrm{E}+00+/-5.13 \mathrm{E}-01$ & $2.75 E-01$ & $\mathrm{pCi} i \mathrm{~g}$ & SOIL & AS060908-1 & $9 / 11 / 2006$ & LT \\
\hline $0609032-10$ & MPDT-05B & Sample & U-235 & $1.70 \mathrm{E}-01+/-1.84 \mathrm{E}-01$ & $2.33 \mathrm{E}-01$ & $\mathrm{pCi} / \mathrm{g}$ & SOIL & AS060908-1 & $9 / 11 / 2006$ & $u$ \\
\hline $0609032-10$ & MPDT-05B & Sample & U-238 & $1.34 \mathrm{E}+00+/-5.05 \mathrm{E}-01$ & $2.25 \mathrm{E}-01$ & $\mathrm{pCi} / \mathrm{g}$ & SOIL & AS060908-1 & $9 / 11 / 2006$ & LT \\
\hline $0609032-11$ & MPDT-06A & Sample & $U-233 / 234$ & $1.15 E+00+/-4.73 E-01$ & $1.69 \mathrm{E}-01$ & pCilg & SOIL & ASO60908-1 & $9 / 11 / 2006$ & LT \\
\hline $0609032-11$ & MPDT-06A & Sample & U-235 & $1.94 \mathrm{E}-01+/-1.96 \mathrm{E}-01$ & $1.31 \mathrm{E}-01$ & $\mathrm{pCi} / \mathrm{g}$ & SOIL & AS060908-1 & $9 / 11 / 2006$ & LT \\
\hline $0609032-11$ & MPDT-06A & Sample & U-238 & 8.77E-01+/- 4.07E-01 & $1.93 \mathrm{E}-01$ & pCilg & SOIL & AS060908-1 & 9/11/2006 & LT \\
\hline $0609032-12$ & MPDT-06B & Sample & U-233/234 & $1.62 \mathrm{E}+00+1-5.84 \mathrm{E}-01$ & $2.74 \mathrm{E}-01$ & pCi/g & SOIL. & AS060908-1 & $9 / 11 / 2006$ & LT \\
\hline $0609032-12$ & MPDT-06B & Sample & U-235 & $1.77 \mathrm{E}-01+/-1.97 \mathrm{E}-01$ & 2.67E-01 & $\mathrm{pCi} / \mathrm{g}$ & SOIL & ASO60908-1 & $9 / 11 / 2006$ & $u$ \\
\hline $0609032-12$ & MPDT-06B & Sample & U-238 & $1.76 \mathrm{E}+00+1-6.11 \mathrm{E}-01$ & $2.27 E-01$ & $\mathrm{pCi} / \mathrm{g}$ & SOIL & AS060908-1 & $9 / 11 / 2006$ & LT \\
\hline
\end{tabular}

Comments:

Data Package ID: UR0609032-1

\section{Qualifiers/Flags:}

$U$-Result is less than the sample specific MDC.

LT - Result is less than Requested MDC, greater than sample specific MDC.

Y1 - Chemical Yield is in control at $100.110 \%$, Quantitative Yield is assurned.

Y2 - Chernical Yield outside default limits.

The requested MDC was not met:

14. The requested $M O C$ was not met, but the reported activity is greater than the reparted MOC
Dgुfe Printed: Tuesday, September 12, 2006

$\varnothing$

$-3$

\section{Abbreviations:}

TPU - Totat Propagated Uncertainty (see PAI SOP 743)

MDC - Minimum Detectable Concentration (see PAI SOP 709)

BDL - Below Detection Limi 


\section{Isotopic Uranium By Alpha Spectroscopy Sample Results Summary}

Client Name: National Sercurity Technologies, LLC Client Project Name: CAU 484 Client Project Number: V2746
Laboratory Name: Paragon Analytics

PAI Work Order: 0609032
Page: 5 of 7

Reported on: Tuesday, September 12, 2006 9:26:20 AM

\begin{tabular}{|c|c|c|c|c|c|c|c|c|c|c|}
\hline $\begin{array}{c}\text { Lab } \\
\text { Sample ID }\end{array}$ & Client Sample ID & $\begin{array}{c}\text { Sample } \\
\text { Type }\end{array}$ & Nuclide & Result $+1-2 \mathrm{~s}$ TPU & MDC & Units & Matrix & Prep Batch & $\begin{array}{c}\text { Date } \\
\text { Analyzed }\end{array}$ & Flags \\
\hline $0609032-13$ & MPDT-07A & Sample & $U-233 / 234$ & $1.22 E+00+/-4.75 E-01$ & $1.96 \mathrm{E}-01$ & $\mathrm{pCi} / \mathrm{g}$ & SOIL & AS060908-1 & $9 / 11 / 2006$ & LT \\
\hline $0609032-13$ & MPDT-07A & Sample & U-235 & $3.70 E-02+/-1.57 E-01$ & 2.11E-01 & $\mathrm{pCl} / \mathrm{g}$ & SOIL & AS060908-1 & $9 / 11 / 2006$ & u \\
\hline $0609032-13$ & MPDT-07A & Sample & U-238 & $9.17 \mathrm{E}-01+/-4.03 \mathrm{E}-01$ & $1.57 \mathrm{E}-01$ & $\mathrm{pCi} / \mathrm{g}$ & SOIL & AS060908-1 & $9 / 11 / 2006$ & $\mathrm{LT}$ \\
\hline $0609032 \cdot 14$ & MPDT-07B & Sample & U.233/234 & $1.59 E+00+/-5.77 E-01$ & $2.54 E-01$ & pCiig & SOIL & AS060908-1 & $9 / 11 / 2006$ & LT \\
\hline $0609032-14$ & MPDT-07B & Sample & U-235 & $8.42 \mathrm{E}-02+/-1.70 \mathrm{E}-01$ & $2.50 \mathrm{E}-01$ & $\mathrm{pCi} / \mathrm{g}$ & SOIL & AS060908-1 & $9 / 11 / 2006$ & $u$ \\
\hline $0609032-14$ & MPDT-07B & Sample & U-238 & $1.07 \mathrm{E}+00+/-4.56 \mathrm{E}-01$ & 2.12E-01 & pCisg & SOIL & AS060908-1 & $9 / 11 / 2006$ & LT \\
\hline $0609032-15$ & MPDT-08A & Sample & $U-233 / 234$ & $2.17 E+00+/-7.80 E-01$ & $3.01 \mathrm{E}-01$ & $\mathrm{pCi} / \mathrm{g}$ & SOIL & AS060908-1 & $9 / 11 / 2006$ & \\
\hline $0609032-15$ & MPDT-08A & Sample & U-235 & $1.23 \mathrm{E}-01+/-2.25 \mathrm{E}-01$ & $2.64 \mathrm{E}-01$ & $\mathrm{pCi} / \mathrm{g}$ & SOIL & AS060908-1 & $9 / 11 / 2006$ & $u$ \\
\hline $0609032-15$ & MPDT-08A & Sample & U.238 & $1.13 E+00+/-5.36 E-01$ & $3.01 \mathrm{E}-01$ & $\mathrm{pC} i / \mathrm{g}$ & SOIL & AS060908-1 & $9 / 11 / 2006$ & LT \\
\hline
\end{tabular}

Comments:

Data Package ID: UR0609032-1

\section{Qualifiers/Flags:}

$U$ - Result is less than the sample specific MDC

LT - Result is less than Requested MDC, greater than sample specific MDC

Y1 - Chemical Yield is in contol at 100-110\%. Quantitative Yield is assumed

Y2 - Chemicat Yield outside delault limits.

$M$ - The requested $M D C$ was not met

M3 - The requested MDC was not met, but the reporled activity is greater than the reported MDC.
Date Printed: Tuesday, September 12, 2006

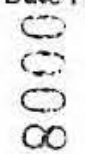

\section{Abbreviations:}

TPU - Tolal Propagated Uncertainty (see PAI SOP 743)

MDC - Minimum Detectable Concentration (see PAI SOP 709)

BDL - Below Dstection Limit 


\section{Isotopic Uranium By Alpha Spectroscopy Sample Results Summary}

Client Name: National Sercurity Technologies, LLC Client Project Name: CAU 484 Client Project Number: V2746

\section{Laboratory Name: Paragon Analytics \\ PAI Work Order: 0609032}

Page: 6 of 7

Reported on: Tuesday, September 12, 2006 9:26:20 AM

\begin{tabular}{|c|c|c|c|c|c|c|c|c|c|c|}
\hline $\begin{array}{c}\text { Lab } \\
\text { Sample ID }\end{array}$ & Client Sample ID & $\begin{array}{l}\text { Sample } \\
\text { Type }\end{array}$ & Nuclide & Result +/- 2 s TPU & MDC & Units & Matrix & Prep Batch & $\begin{array}{c}\text { Date } \\
\text { Analyzed }\end{array}$ & Flags \\
\hline $0609032-16$ & MPDT-08B & Sample & U.233/234 & $1.42 E+00+/-5.48 E-01$ & $3.89 E-01$ & pCíg & SOIL & AS060908-1 & $9 / 11 / 2006$ & LT \\
\hline $0609032-16$ & MPDT-08B & Sample & U-235 & $3.23 \mathrm{E}-01+/-2.60 \mathrm{E}-01$ & $2.46 \mathrm{E}-01$ & $\mathrm{pClig}$ & SOIL & ASO60908-1 & $9 / 11 / 2006$ & LT \\
\hline $0609032-16$ & MPDT-08B & Sample & $\mathrm{U}-238$ & $1.55 \mathrm{E}+00+/-5.68 \mathrm{E}-01$ & 2.99E-01 & pCiig & SOIL & AS060908-1 & $9 / 11 / 2006$ & LT \\
\hline $0609032-17$ & MPDT-09A & Sample & U-233/234 & $1.22 \mathrm{E}+00+/-5.16 \mathrm{E}-01$ & $3.30 \mathrm{E}-01$ & $\mathrm{pCi} / \mathrm{g}$ & SOIL & AS060908-1 & $9 / 11 / 2006$ & LT \\
\hline $0609032-17$ & MPDT-09A & Sample & U-235 & $1.01 \mathrm{E}-01+/-1.85 \mathrm{E}-01$ & 2.17E-01 & pCilg & SOIL & AS060908-1 & $9 / 11 / 2006$ & $u$ \\
\hline $0609032-17$ & MPDT-09A & Sample & $\mathrm{U}-238$ & $1.23 E+00+/-5.17 E-01$ & $2.88 \mathrm{E}-01$ & $\mathrm{pCi} / \mathrm{g}$ & SOIL & AS060908-1 & $9 / 11 / 2006$ & LT \\
\hline $0609032-18$ & MPDT-09B & Sample & $U-233 / 234$ & $1.56 \mathrm{E}+00+/-5.94 \mathrm{E}-01$ & $2.78 \mathrm{E}-01$ & $\mathrm{pCl} / \mathrm{g}$ & SOIL & AS060908-1 & $9 / 11 / 2006$ & LT \\
\hline $0609032-18$ & MPDT-09B & Sample & U-235 & $5.34 \mathrm{E}-02+/-1.86 \mathrm{E}-01$ & $1.45 \mathrm{E}-01$ & $\mathrm{pCl} / \mathrm{g}$ & SOIL & AS060908-1 & $9 / 11 / 2006$ & $u$ \\
\hline $0609032-18$ & MPDT-09B & Sample & $\mathrm{U}-238$ & $1.67 E+00+/-6.15 E-01$ & $2.33 E-01$ & $\mathrm{pCi} / \mathrm{g}$ & SOIL & AS060908-1 & $9 / 11 / 2006$ & LT \\
\hline
\end{tabular}

Comments:

Data Package ID: UR0609032-1

\section{Qualifiers/Flags:}

$U$ - Result is less than the sample specific MDC

LT - Result is less than Requested MDC, greater than sample specific MDC

$\mathrm{Y}_{1}$ - Chemical Yield is in control at $100-110 \%$. Quantitative Yield is assumed

Y2 - Chemical Yield outside default limits.

$M$ CIm requested $M D C$ was not me:

$M{ }^{2}-T$ tye requested MDC was not met, but the reported activity is greater than the reported MDC.

\section{Abbreviations:}

TPU - Total Propagated Uncertainty (see PAI SOP 743)

MDC - Minimum Detectable Concentration (see PAI SOP 709)

BDL - Below Detection Limit

Dato-Printed: Tuesday, September 12, 2006

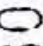

co

Version: $5.422 \mathrm{~A}$ 


\section{Isotopic Uranium By Alpha Spectroscopy Sample Results Summary}

Client Name: National Sercurity Technologies, LLC Client Project Name: CAU 484 Client Project Number: V2746

\section{Laboratory Name: Paragon Analytics}

PAI Work Order: 0609032
Page: 7 of 7

Reported on: Tuesday, September 12, 2006 9:26:20 AM

\begin{tabular}{|c|c|c|c|c|c|c|c|c|c|c|}
\hline $\begin{array}{c}\text { Lab } \\
\text { Sample ID }\end{array}$ & Client Sample ID & $\begin{array}{l}\text { Sample } \\
\text { Type }\end{array}$ & Nuclide & Result +/- 2 s TPU & MDC & Units & Matrix & Prep Batch & $\begin{array}{c}\text { Date } \\
\text { Analyzed }\end{array}$ & Flags \\
\hline $0609032-19$ & MPDT-10A & Sample & U-233/234 & $1.23 E+00+/-5.01 E-01$ & $1.75 \mathrm{E}-01$ & $\mathrm{pCi} / \mathrm{g}$ & SOIL & AS060908-1 & $9 / 11 / 2006$ & LT \\
\hline $0609032-19$ & MPDT-10A & Sample & U-235 & $0 E+00+/-1.75 E-01$ & $1.36 \mathrm{E}-01$ & $\mathrm{pCi} / \mathrm{g}$ & SOIL & AS060908-1 & $9 / 11 / 2006$ & $u$ \\
\hline $0609032-19$ & MPDT-10A & Sample & U-238 & $1.53 E+00+/-5.69 E-01$ & $2.00 E-01$ & $\mathrm{pCi} / \mathrm{g}$ & SOIL & AS060908-1 & $9 / 11 / 2006$ & LT \\
\hline $0609032-20$ & MPDT-10B & Sample & U-233/234 & $9.40 \mathrm{E}-01+1-4.33 \mathrm{E}-01$ & $2.22 \mathrm{E}-01$ & $\mathrm{pCi} / \mathrm{g}$ & SOIL & AS060908-1 & $9 / 11 / 2006$ & LT \\
\hline $0609032-20$ & MPDT-10B & Sample & U.235 & 4.63E-02 +/- $1.77 \mathrm{E}-01$ & $2.09 \mathrm{E}-01$ & pCilg & SOIL & ASO60908-1 & $9 / 11 / 2006$ & $U$ \\
\hline $0609032-20$ & MPDT-10B & Sample & U-238 & $1.20 \mathrm{E}+00+/-4.97 \mathrm{E}-01$ & 2.38E-01 & $\mathrm{pCi} / \mathrm{g}$ & SOIL & AS060908-1 & $9 / 11 / 2006$ & LT \\
\hline
\end{tabular}

\section{Comments:}

\section{Data Package ID: UR0609032-1}

\section{Qualifiers/Flags:}

$U$-Result is less than the sample specific MDC.

LT - Result is less than Requested MDC. greater than sample specific MDC

$Y 1$ - Chemical Yield is in control at 100-110\%. Quantitative Yield is assumed

Y2 - Chemical Yield outside defaut limits.

$M$. The requested $M D C$ was not met.

MYC The requested MDC was not met, but the reported activity is greater than the reported MDC 


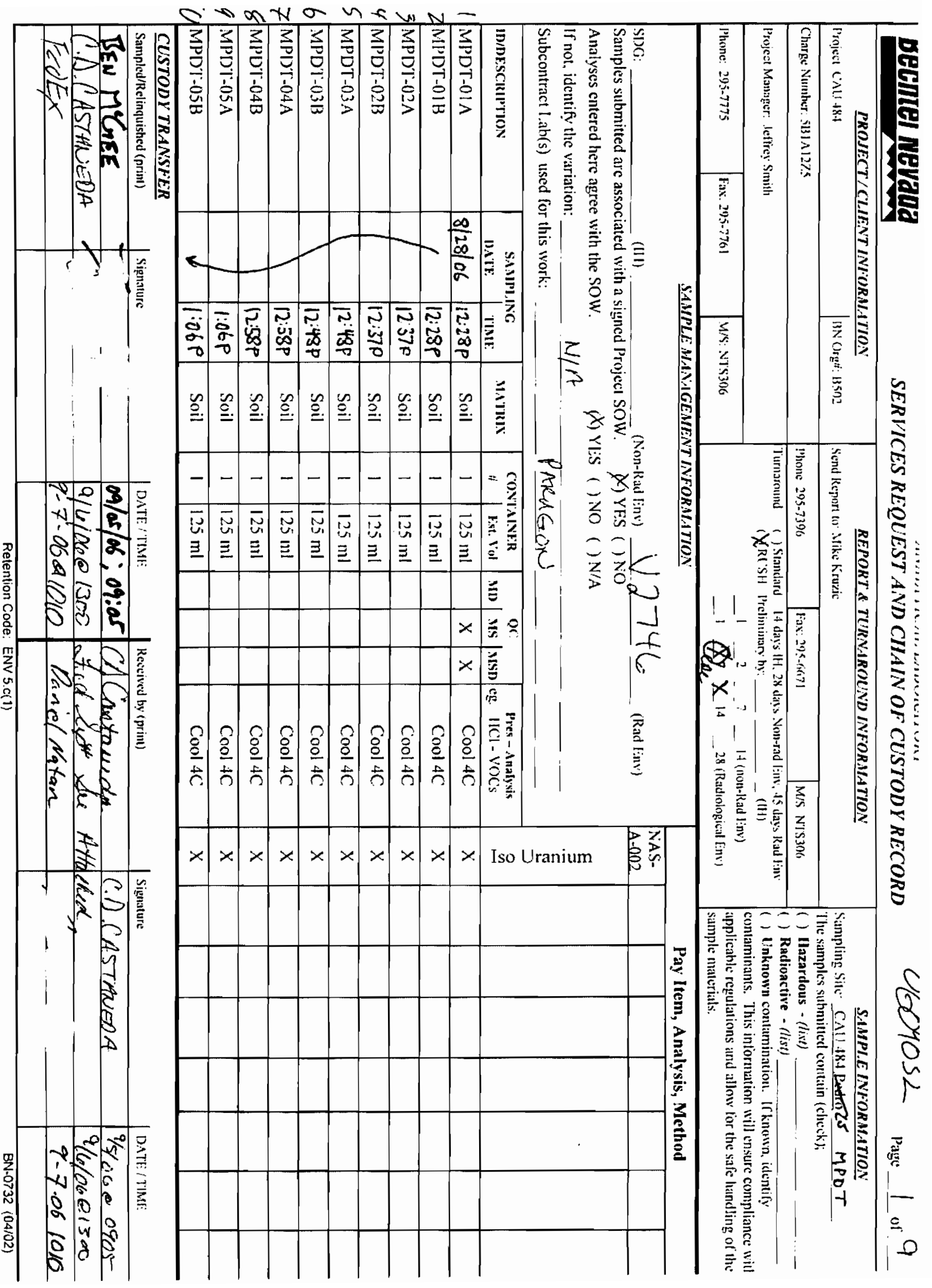




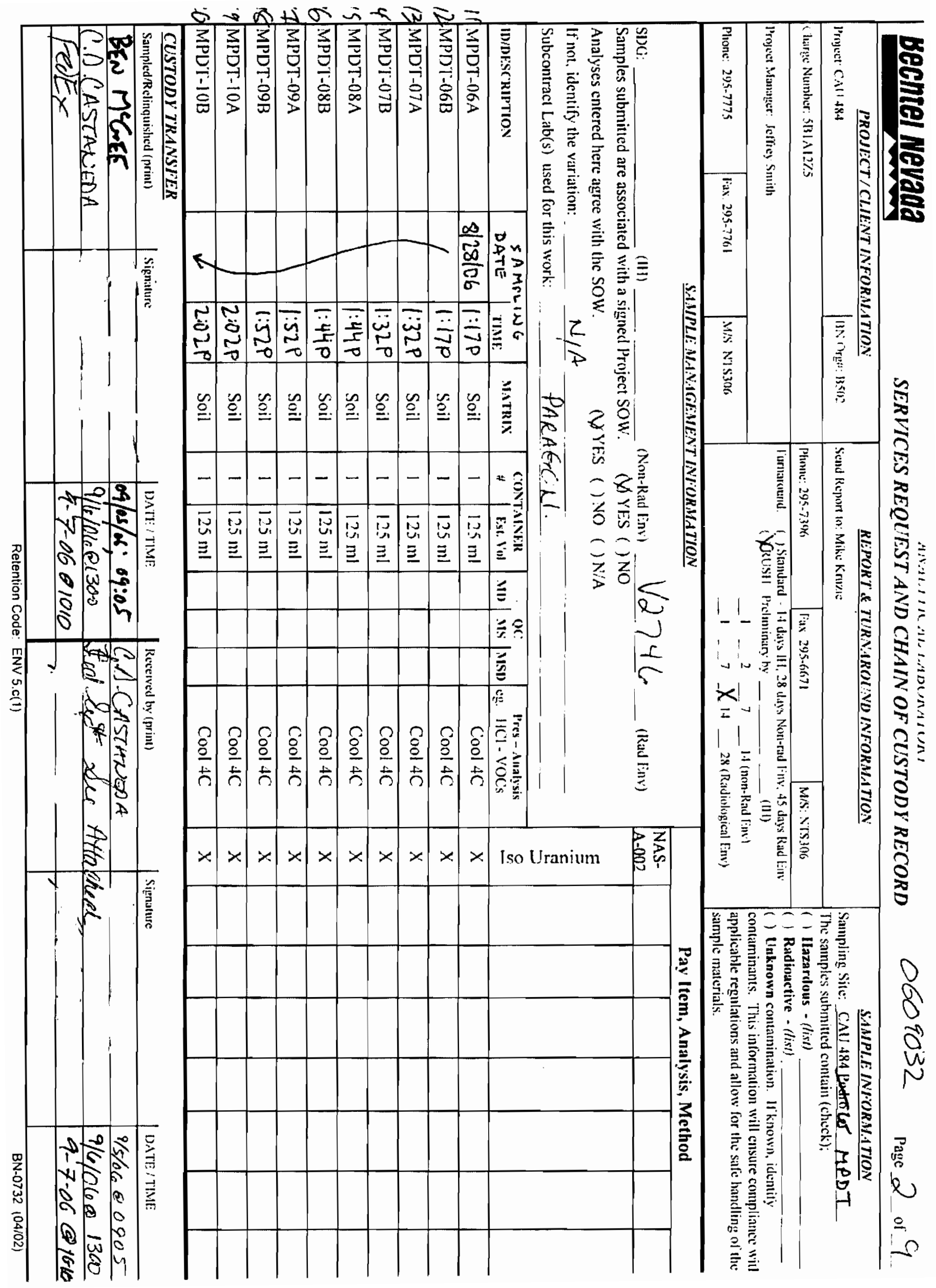




\section{Isotopic Uranium By Alpha Spectroscopy Sample Results Summary}

Client Name: National Sercurity Technologies, LLC Client Project Name: CAU 484 Client Project Number: V2746
Laboratory Name: Paragon Analytics

PAl Work Order: 0609033
Page: 1 of 7

Reported on: Tuesday, September 12,2006 10:57:21 AM

\begin{tabular}{|c|c|c|c|c|c|c|c|c|c|c|}
\hline $\begin{array}{c}\text { Lab } \\
\text { Sample ID }\end{array}$ & Client Sample ID & $\begin{array}{l}\text { Sample } \\
\text { Type }\end{array}$ & Nuclide & Result $+/-2$ s TPU & MDC & Units & Matrix & Prep Batch & $\begin{array}{c}\text { Date } \\
\text { Analyzed }\end{array}$ & Flags \\
\hline $0609033-1$ & MPDT-11A & Sample & U-233/234 & $1.93 \mathrm{E}+00+/-6.80 \mathrm{E}-01$ & $2.56 \mathrm{E}-01$ & $\mathrm{pCi} / \mathrm{g}$ & SOIL & AS060908-2 & $9 / 11 / 2006$ & LT \\
\hline $0609033-1$ & MPDT-11A & Sample & U.235 & $1.04 \mathrm{E}-01+/-1.91 \mathrm{E}-01$ & $2.24 \mathrm{E}-01$ & pCilg & SOIL & AS060908-2 & $9 / 11 / 2006$ & u \\
\hline $0609033-1$ & MPDT-11A & Sample & $U-238$ & $1.53 \mathrm{E}+00+/-5.89 \mathrm{E}-01$ & $1.91 \mathrm{E}-01$ & $\mathrm{pCi} / \mathrm{g}$ & SOIL & AS060908-2 & $9 / 11 / 2006$ & LT \\
\hline $0609033-2$ & MPDT-11B & Sample & U-233/234 & $1.27 \mathrm{E}+00+1-4.88 \mathrm{E}-01$ & $2,43 E-01$ & $\mathrm{pCl} / \mathrm{g}$ & SOIL & AS060908-2 & $9 / 11 / 2006$ & LT \\
\hline $0609033-2$ & MPDT-11B & Sample & $\mathrm{U}-235$ & $4.47 \mathrm{E}-02+/-1.56 \mathrm{E}-01$ & $1.21 \mathrm{E}-01$ & $\mathrm{pCl} / \mathrm{g}$ & SOIL & AS060908-2 & $9 / 11 / 2006$ & $u$ \\
\hline $0609033-2$ & MPDT-11B & Sample & U-238 & $1.09 \mathrm{E}+00+1-4.45 \mathrm{E}-01$ & $1.95 \mathrm{E}-01$ & $\mathrm{pCi} / \mathrm{g}$ & SOIL & AS060908-2 & $9 / 11 / 2006$ & LT \\
\hline 0609033-3 & MPDT-12A & Sample & $U-233 / 234$ & $1.76 \mathrm{E}+00+1-6.22 \mathrm{E}-01$ & $2.94 \mathrm{E}-01$ & $\mathrm{pCi} / \mathrm{g}$ & SOIL & AS060908-2 & $9 / 11 / 2006$ & LT \\
\hline $0609033-3$ & MPDT-12A & Sample & U-235 & 1.87E-01 +/-2.04E-01 & $2.57 \mathrm{E}-01$ & $\mathrm{pCi} / \mathrm{g}$ & SOIL & AS060908-2 & $9 / 11 / 2006$ & $\mathrm{u}$ \\
\hline $0609033-3$ & MPDT-12A & Sample & U-238 & $9.66 \mathrm{E}-01+1-4.39 \mathrm{E}-01$ & $2.35 \mathrm{E}-01$ & $\mathrm{pCl} / \mathrm{g}$ & SOIL & AS060908-2 & $9 / 11 / 2006$ & LT \\
\hline
\end{tabular}

\section{Comments:}

\section{Data Package ID: UR0609033-1}

\section{Qualifiers/Flags:}

$\mathrm{U}$-Result is less than the sample specific MDC.

LT - Result is less than Requested MDC, greater than sample speciric MDC.

$Y 1$ - Chemical Yield is in control at 100.110\%. Quantitative Yield is assumed.

Y2 - Chemical Yield outside default limits.

$M$. The requested MDC was not met.

${ }_{M 3}$ The requested MDC was not met, but the reported activity is greater than the reported MOC.

Daféprinted: Tuesday, September 12, 2006

\section{Abbreviations:}

TPU - Total Propagated Uncertainty (see PAI SOP 743)

MDC - Minimum Detectable Concentration (see PAI SOP 709)

BDL - Below Detection Lim 


\section{Isotopic Uranium By Alpha Spectroscopy Sample Results Summary}

Client Name: National Sercurity Technologies, LLC Client Project Name: CAU 484 Client Project Number: V2746
Laboratory Name: Paragon Analytics PAI Work Order: 0609033
Page: 2 of 7

Reported on: Tuesday, September 12,2006 10:57:21 AM

\begin{tabular}{|c|c|c|c|c|c|c|c|c|c|c|}
\hline $\begin{array}{c}\text { Lab } \\
\text { Sample ID }\end{array}$ & Client Sample ID & $\begin{array}{c}\text { Sample } \\
\text { Type }\end{array}$ & Nuclide & Result $+1-2$ s TPU & MDC & Units & Matrix & Prep Batch & $\begin{array}{c}\text { Date } \\
\text { Analyzed }\end{array}$ & Flags \\
\hline $0609033-4$ & MPDT-12B & Sample & U-233/234 & $1.54 E+00+/-5.63 E-01$ & $1.71 \mathrm{E}-01$ & $\mathrm{pCi} / \mathrm{g}$ & SOIL & AS060908-2 & $9 / 11 / 2006$ & LT \\
\hline $0609033-4$ & MPDT-12B & Sample & U.235 & $0 \mathrm{E}+00+/-1.70 \mathrm{E}-01$ & $1.33 E-01$ & $\mathrm{pCl} / \mathrm{g}$ & SOIL & AS060908-2 & $9 / 11 / 2006$ & $u$ \\
\hline $0609033-4$ & MPDT-12B & Sample & U-238 & 1.37E+00+/-5.26E-01 & $1.95 \mathrm{E}-01$ & pCi/g & SOIL & AS060908-2 & $9 / 11 / 2006$ & LT \\
\hline $0609033-5$ & MPDT-13A & Sample & U-233/234 & $1.52 \mathrm{E}+00+/-5.64 \mathrm{E}-01$ & $2.67 \mathrm{E}-01$ & $\mathrm{pCi} / \mathrm{g}$ & SOIL & AS060908-2 & $9 / 11 / 2006$ & LT \\
\hline $0609033-5$ & MPDT-13A & Sample & $U-235$ & $7.62 \mathrm{E}-02+/-1.71 \mathrm{E}-01$ & $2.86 \mathrm{E}-01$ & $\mathrm{pCl} / \mathrm{g}$ & SOIL & AS060908-2 & $9 / 11 / 2006$ & $u$ \\
\hline $0609033-5$ & MPDT-13A & Sample & $\mathrm{U}-238$ & $9.46 \mathrm{E}-01+/-4.29 \mathrm{E}-01$ & $2.30 E-01$ & $\mathrm{pCi} / \mathrm{g}$ & SOIL & AS060908-2 & $9 / 11 / 2006$ & LT \\
\hline $0609033-6$ & MPDT-13B & Sample & U-233/234 & $1.90 \mathrm{E}+00+1-6.43 \mathrm{E}-01$ & $2,13 E-01$ & $\mathrm{pCi} / \mathrm{g}$ & SOIL & AS060908-2 & $9 / 11 / 2006$ & LT \\
\hline 0609033-6 & MPDT-13B & Sample & $U-235$ & $8.92 \mathrm{E}-02+/-1.71 \mathrm{E}-01$ & $2.29 \mathrm{E}-01$ & $\mathrm{pCi} / \mathrm{g}$ & SOIL & AS060908-2 & $9 / 11 / 2006$ & $u$ \\
\hline 0609033.6 & MPDT-13B & Sample & $U-238$ & $1.66 \mathrm{E}+00+/-5.91 \mathrm{E}-01$ & $1.71 \mathrm{E}-01$ & $\mathrm{pCi} / \mathrm{g}$ & SOIL & AS060908-2 & $9 / 11 / 2006$ & LT \\
\hline
\end{tabular}

\section{Comments:}

\section{Data Package ID: UR0609033-1}

\section{Qualifiers/Flags:}

U - Result is less than the sample specific MDC.

LT - Result is less than Requested MDC, greater than sample specific MDC

$\mathrm{Y1}$. Chemical Yield is in control at 100-110\%. Quantitative Yield is assumed.

Y2 . Chemical Yeld outside detault limits.

$M$ - The requested $M D C$ was not met.

M3 The requested MDC was not met, but the reported activity is greater than the reported MDC 


\section{Isotopic Uranium By Alpha Spectroscopy Sample Results Summary}

Client Name: National SercurityTechnologies, LLC Client Project Name: CAU 484

Client Project Number: V2746
Laboratory Name: Paragon Analytics

PAI Work Order: 0609033
Page: 3 of 7

Reported on: Tuesday, September 12,2006 10:57:21 AM

\begin{tabular}{|c|c|c|c|c|c|c|c|c|c|c|}
\hline $\begin{array}{c}\text { Lab } \\
\text { Sample ID }\end{array}$ & Client Sample ID & $\begin{array}{c}\text { Sample } \\
\text { Type }\end{array}$ & Nuclide & Result $+/-2$ s TPU & MDC & Units & Matrix & Prep Batch & $\begin{array}{c}\text { Date } \\
\text { Analyzed }\end{array}$ & Flags \\
\hline $0609033-7$ & MPDT-14A & Sample & U-233/234 & $1.25 \mathrm{E}+00+1-4.89 \mathrm{E}-01$ & $2.30 \mathrm{E}-01$ & $\mathrm{pCi} / \mathrm{g}$ & SOIL & AS060908-2 & $9 / 11 / 2006$ & $\overline{\mathrm{LT}}$ \\
\hline $0609033-7$ & MPDT-14A & Sample & $\mathrm{U}-235$ & $7.61 \mathrm{E}-02+/-1.62 \mathrm{E}-01$ & $2.55 E-01$ & $\mathrm{pCi} / \mathrm{g}$ & SOIL & AS060908-2 & $9 / 11 / 2006$ & $u$ \\
\hline $0609033-7$ & MPDT-14A & Sample & $\mathrm{U}-238$ & $1.06 \mathrm{E}+00+1-4.44 \mathrm{E}-01$ & $2.02 E-01$ & $\mathrm{pCi} / \mathrm{g}$ & SOIL & AS060908-2 & $9 / 11 / 2006$ & LT \\
\hline $0609033-8$ & MPDT-14B & Sample & U-233/234 & $1.61 \mathrm{E}+00+/-5.81 \mathrm{E}-01$ & $3.52 \mathrm{E}-01$ & $\mathrm{pCi} / \mathrm{g}$ & SOIL & AS060908-2 & $9 / 12 / 2006$ & LT \\
\hline $0609033-8$ & MPDT-14B & Sample & U-235 & $7.30 \mathrm{E}-02+/-1.64 \mathrm{E}-01$ & $2.74 \mathrm{E}-01$ & $\mathrm{pCi} / \mathrm{g}$ & SOIL & AS060908-2 & $9 / 12 / 2006$ & $\mathrm{u}$ \\
\hline $0609033-8$ & MPDT-14B & Sample & U-238 & $1.15 \mathrm{E}+00+/-4.69 \mathrm{E}-01$ & $2.20 \mathrm{E}-01$ & $\mathrm{pCi} / \mathrm{g}$ & SOIL & AS060908-2 & $9 / 12 / 2006$ & LT \\
\hline $0609033-9$ & MPDT-15A & Sample & $U-233 / 234$ & $1.13 \mathrm{E}+00+/-4.68 \mathrm{E}-01$ & $2.56 \mathrm{E}-01$ & $\mathrm{pCl} / \mathrm{g}$ & SOIL & ASO60908-2 & $9 / 12 / 2006$ & LT \\
\hline $0609033-9$ & MPDT-15A & Sample & U.235 & $0 E+00+/-1.64 E-01$ & $1.27 \mathrm{E}-01$ & $\mathrm{pCi} / \mathrm{g}$ & SOIL & AS060908-2 & $9 / 12 / 2006$ & U \\
\hline 0609033-9 & MPDT-15A & Sample & U-238 & $1.27 E+00+1-4.97 E-01$ & $2.05 \mathrm{E}-01$ & $\mathrm{pCi} / \mathrm{g}$ & SOIL & AS060908-2 & $9 / 12 / 2006$ & LT \\
\hline
\end{tabular}

\section{Comments:}

\section{Data Package ID: UR0609033-1}

\section{Qualifiers/Flags:}

$U$ - Result is less than the sample specific MDC

LT - Result is less than Requested MDC, greater than sample specific MDC

Y1 - Chemical Yield is in control at $100.110 \%$. Quantitative Yield is assumed

Y2. Chemical Yield outside default limits.

$M$ CIIT requested MDC was not met.

$M 3 C$ Crequested $M D C$ was not met, but the reported activity is greater than the reported MDC. 


\section{Isotopic Uranium By Alpha Spectroscopy Sample Results Summary}

Client Name: National SercurityTechnologies, LLC Client Project Name: CAU 484 Client Project Number: V2746
Laboratory Name: Paragon Analytics PAI Work Order: 0609033
Page: 4 of 7

Reported on: Tuesday, September 12,2006 10:57:21 AM

\begin{tabular}{|c|c|c|c|c|c|c|c|c|c|c|}
\hline $\begin{array}{c}\text { Lab } \\
\text { Sample ID }\end{array}$ & Client Sample ID & $\begin{array}{l}\text { Sample } \\
\text { Type }\end{array}$ & Nuclide & Result +/- 2 s TPU & MDC & Units & Matrix & Prep Batch & $\begin{array}{c}\text { Date } \\
\text { Analyzed }\end{array}$ & Flags \\
\hline $0609033-10$ & MPDT-15B & Sample & U-233/234 & $1.31 \mathrm{E}+00+/-5.12 \mathrm{E}-01$ & $2.27 E-01$ & $\mathrm{pCi} / \mathrm{g}$ & SOIL & AS060908-2 & $9 / 12 / 2006$ & LT \\
\hline $0609033-10$ & MPDT-15B & Sample & U-235 & $1.90 \mathrm{E}-01+l-1.97 \mathrm{E}-01$ & $1.99 \mathrm{E}-01$ & $\mathrm{pCi} / \mathrm{g}$ & SOIL & AS060908-2 & $9 / 12 / 2006$ & $\mathrm{u}$ \\
\hline $0609033-10$ & MPDT-15B & Sample & U-238 & $1.48 \mathrm{E}+00+/-5.50 \mathrm{E}-01$ & $1.69 \mathrm{E}-01$ & $\mathrm{pCl} / \mathrm{g}$ & SOIL & AS060908-2 & $9 / 12 / 2006$ & LT \\
\hline $0609033-11$ & MPDT-16A & Sample & $U-233 / 234$ & $1.19 \mathrm{E}+00+1-4.93 \mathrm{E}-01$ & $2.80 \mathrm{E}-01$ & $\mathrm{pCl} / \mathrm{g}$ & SOIL & AS060908-2 & $9 / 12 / 2006$ & LT \\
\hline $0609033-11$ & MPDT-16A & Sample & $U-235$ & 1.17E-01+/-1.74E-01 & 3.17E-01 & $\mathrm{pCi} / \mathrm{g}$ & SOIL & AS060908-2 & $9 / 12 / 2006$ & u \\
\hline $0609033-11$ & MPDT-16A & Sample & $\mathrm{U}-238$ & $1.20 \mathrm{E}+00+/-4.94 \mathrm{E}-01$ & $2.46 \mathrm{E}-01$ & $\mathrm{pCi} / \mathrm{g}$ & SOIL & AS060908-2 & $9 / 12 / 2006$ & LT \\
\hline $0609033-12$ & MPDT-16B & Sample & $\mathrm{U}-233 / 234$ & $1.74 \mathrm{E}+00+/-5.96 \mathrm{E}-01$ & $2.40 \mathrm{E}-01$ & $\mathrm{pCi} / \mathrm{g}$ & SOIL & AS060908-2 & $9 / 12 / 2006$ & LT \\
\hline $0609033-12$ & MPDT-16B & Sample & U.235 & $1.72 \mathrm{E}-01+/-1.87 \mathrm{E}-01$ & $2.36 \mathrm{E}-01$ & $\mathrm{pCl} / \mathrm{g}$ & SOIL & AS060908-2 & $9 / 12 / 2006$ & $u$ \\
\hline 0609033-12 & MPDT-16B & Sample & U.238 & $1.67 \mathrm{E}+00+/-5.80 \mathrm{E}-01$ & $2.16 \mathrm{E}-01$ & $\mathrm{pCi} / \mathrm{g}$ & SOIL & AS060908-2 & $9 / 12 / 2006$ & LT \\
\hline
\end{tabular}

\section{Comments:}

\section{Data Package ID: UR0609033-1}
Qualifiers/Flags:
$U$ - Result is tess than the sample specific MDC.
LT - Result is less than Requested MDC, greater than sample specific MDC.
$Y_{1}$ - Chemical Yield is in control at 100-110\%. Quantitative Yield is assumed.
Y2 - Ghemical Yield outside defaull limits.
M. Fin requested MDC was not met.
M3 - In requested MDC was not met, but the reported activity is greater than the reported MDC 


\section{Isotopic Uranium By Alpha Spectroscopy Sample Results Summary}

Client Name: National Sercurity Technologies, LLC Client Project Name: CAU 484 Client Project Number: V2746
Laboratory Name: Paragon Analytics

PAl Work Order: 0609033
Page: 5 of 7

Reported on: Tuesday, September 12,2006 10:57:21 AM

\begin{tabular}{|c|c|c|c|c|c|c|c|c|c|c|}
\hline $\begin{array}{c}\text { Lab } \\
\text { Sample ID }\end{array}$ & Client Sample ID & $\begin{array}{c}\text { Sample } \\
\text { Type }\end{array}$ & Nuclide & Result $+1-2 \mathrm{~s}$ TPU & MDC & Units & Matrix & Prep Batch & $\begin{array}{c}\text { Date } \\
\text { Analyzed }\end{array}$ & Flags \\
\hline $0609033-13$ & MPDT-17A & Sample & U-233/234 & $1.36 \mathrm{E}+00+/-5.34 \mathrm{E}-01$ & $2.20 \mathrm{E}-01$ & $\mathrm{pCi} / \mathrm{g}$ & SOIL & ASO60908-2 & $9 / 12 / 2006$ & LT \\
\hline $0609033-13$ & MPDT-17A & Sample & U-235 & $9.66 \mathrm{E}-02+/-1.77 \mathrm{E}-01$ & $2.08 \mathrm{E}-01$ & $\mathrm{pCi} / \mathrm{g}$ & SOIL & AS060908-2 & $9 / 12 / 2006$ & U \\
\hline $0609033-13$ & MPDT-17A & Sample & U.238 & $1.67 \mathrm{E}+00+1-6.01 \mathrm{E}-01$ & $2.01 \mathrm{E}-01$ & $\mathrm{pCi} / \mathrm{g}$ & SOIL & AS060908-2 & $9 / 12 / 2006$ & LT \\
\hline $0609033-14$ & MPDT-17B & Sample & U-233/234 & $1.60 E+00+1-6.47 E-01$ & $2.96 \mathrm{E}-01$ & $\mathrm{pCi} / \mathrm{g}$ & SOIL & AS060908-2 & $9 / 12 / 2006$ & LT \\
\hline $0609033-14$ & MPDT-17B & Sample & U-235 & $1.84 \mathrm{E}-01+/-2.23 \mathrm{E}-01$ & $2.60 \mathrm{E}-01$ & $\mathrm{pCl} / \mathrm{g}$ & SOIL & AS060908-2 & $9 / 12 / 2006$ & U \\
\hline 0609033-14 & MPDT-17B & Sample & $U-238$ & $9.55 \mathrm{E}-01+1-4.84 \mathrm{E}-01$ & $2.76 \mathrm{E}-01$ & $\mathrm{pCi} / \mathrm{g}$ & SOIL & ASO60908-2 & $9 / 12 / 2006$ & LT \\
\hline $0609033-15$ & MPDT-18A & Sample & $U-233 / 234$ & $1.66 \mathrm{E}+00+/-6.11 \mathrm{E}-01$ & $4.08 \mathrm{E}-01$ & $\mathrm{pCi} / \mathrm{g}$ & SOIL & AS060908-2 & $9 / 12 / 2006$ & LT \\
\hline $0609033-15$ & MPDT-18A & Sample & U-235 & $2.88 \mathrm{E}-01+/-2.51 \mathrm{E}-01$ & $2.58 \mathrm{E}-01$ & $\mathrm{pCi} / \mathrm{g}$ & SOIL & AS060908-2 & $9 / 12 / 2006$ & LT \\
\hline $0609033 \cdot 15$ & MPDT-18A & Sample & $U-238$ & $9.42 E-01+/-4.39 E-01$ & $3.14 \mathrm{E}-01$ & $\mathrm{pCi} / \mathrm{g}$ & SOIL & AS060908-2 & $9 / 12 / 2006$ & LT \\
\hline
\end{tabular}

\section{Comments:}

Data Package ID: UR0609033-1

\section{Qualifiers/Flags:}

$U$ - Result is less than the sample specific MDC.

LT - Result is less than Requested MDC, greater than sample specific MDC

Y1. Chemical Yield is in control at 100-110\%, Quantitative Yield is assumed

$Y_{2}$. Chemical Yield outside default limits.

CDe requested MDC was nol met.

15e requested MDC was not met, but the reported activity is greater than the reported MDC. 


\section{Isotopic Uranium By Alpha Spectroscopy Sample Results Summary}

Client Name: National Sercurity Technologies, LLC Client Project Name: CAU 484 Client Project Number: V2746
Laboratory Name: Paragon Analytics PAl Work Order: 0609033
Page: 6 of 7

Reported on: Tuesday, September 12,2006 10:57:21 AM

\begin{tabular}{|c|c|c|c|c|c|c|c|c|c|c|}
\hline $\begin{array}{c}\text { Lab } \\
\text { Sample ID }\end{array}$ & Client Sample ID & $\begin{array}{l}\text { Sample } \\
\text { Type }\end{array}$ & Nuclide & Result $+1-2$ s TPU & MDC & Units & Matrix & Prep Batch & $\begin{array}{c}\text { Date } \\
\text { Analyzed }\end{array}$ & Flags \\
\hline 0609033-16 & MPDT-18B & Sample & U.233/234 & $1.78 \mathrm{E}+00+/-6.39 \mathrm{E}-01$ & $3.26 \mathrm{E}-01$ & $\mathrm{pCilg}$ & SOIL & AS060908-2 & $9 / 12 / 2006$ & LT \\
\hline $0609033-16$ & MPDT-18B & Sample & U-235 & $2.04 \mathrm{E}-01+/-2.12 \mathrm{E}-01$ & $2.15 E-01$ & $\mathrm{pCl} / \mathrm{g}$ & SOIL & AS060908-2 & $9 / 12 / 2006$ & $\mathrm{u}$ \\
\hline $0609033-16$ & MPDT-18B & Sample & $\mathrm{U}-238$ & $1.35 E+00+/-5.42 E-01$ & $2.85 E-01$ & $\mathrm{pCl} / \mathrm{g}$ & SOIL & AS060908-2 & $9 / 12 / 2006$ & LT \\
\hline $0609033-17$ & MPDT-19A & Sample & $U-233 / 234$ & $1.05 E+00+1-4.45 E-01$ & $2.18 \mathrm{E}-01$ & $\mathrm{pCi} / \mathrm{g}$ & SOIL & AS060908-2 & $9 / 12 / 2006$ & LT \\
\hline $0609033-17$ & MPDT-19A & Sample & U-235 & $9.31 \mathrm{E}-02+1-1.63 \mathrm{E}-01$ & $1.26 \mathrm{E}-01$ & pCilg & SOIL & AS060908-2 & $9 / 12 / 2006$ & $u$ \\
\hline $0609033-17$ & MPDT-19A & Sample & $\mathrm{U}-238$ & $1.70 \mathrm{E}+00+/-5.86 \mathrm{E}-01$ & $1.85 \mathrm{E}-01$ & pCi/g & SOIL & AS060908-2 & $9 / 12 / 2006$ & LT \\
\hline $0609033-18$ & MPDT-19B & Sample & U-233/234 & $1.67 \mathrm{E}+00+/-5.93 \mathrm{E}-01$ & 1.71E-01 & $\mathrm{pCi} / \mathrm{g}$ & SOIL & AS060908-2 & $9 / 12 / 2006$ & LT \\
\hline $0609033-18$ & MPDT-19B & Sample & U-235 & $9.82 \mathrm{E}-02+/-1.72 \mathrm{E}-01$ & 1.33E-01 & $\mathrm{pCi} / \mathrm{g}$ & SOIL & AS060908-2 & $9 / 12 / 2006$ & u \\
\hline $0609033-18$ & MPDT-19B & Sample & U-238 & $8.73 \mathrm{E}-01+/-4.08 \mathrm{E}-01$ & $1.71 \mathrm{E}-01$ & $\mathrm{pCli} / \mathrm{g}$ & SOIL & ASO60908-2 & $9 / 12 / 2006$ & LT \\
\hline
\end{tabular}

Comments:

\section{Data Package ID: UR0609033-1}

\section{Qualifiers/Flags:}

$U$ - Result is tess than the sample specific MDC.

LT - Result is less than Requested MOC, greater than sample specific MOC.

Y1 - Chemical Yield is in control at $100-110 \%$. Quantitative Yield is assumed

Y2 - Chemical Yield outside defaut limits.

The requested MDC was not met.

The requested MDC was not met, but the reported activity is greater than the reported MDC

\section{Abbreviations:}

TPU - Total Propagated Uncerlainty (see PAI SOP 743)

MDC - Minimurn Detectable Concentration (see PAI SOP 709)

$B D L$ - Below Detection Limit
DatePrinted: Tuesday, September 12,2006

OO 


\section{Isotopic Uranium By Alpha Spectroscopy Sample Results Summary}

Client Name: National Sercurity Technologies, LLC Client Project Name: CAU 484 Client Project Number: V2746

\author{
Laboratory Name: Paragon Analytics \\ PAl Work Order: 0609033
}

Page: 7 of 7

Reported on: Tuesday, September 12, 2006 10:57:21 AM

\begin{tabular}{|c|c|c|c|c|c|c|c|c|c|c|}
\hline $\begin{array}{c}\text { Lab } \\
\text { Sample ID }\end{array}$ & Client Sample ID & $\begin{array}{l}\text { Sample } \\
\text { Type }\end{array}$ & Nuclide & Result $+/-2 \mathrm{~s}$ TPU & MDC & Units & Matrix & Prep Batch & $\begin{array}{c}\text { Date } \\
\text { Analyzed }\end{array}$ & Flags \\
\hline $0509033-19$ & MPDT-20A & Sample & U-233/234 & $1.41 \mathrm{E}+00+/-5.60 \mathrm{E}-01$ & $2.35 \mathrm{E}-01$ & $\mathrm{pCi} / \mathrm{g}$ & SOIL. & AS060908.2 & $9 / 12 / 2006$ & $\overline{L T}$ \\
\hline $0609033-19$ & MPDT-20A & Sample & U-235 & $4.91 \mathrm{E}-02+/-1.88 \mathrm{E}-01$ & $2.21 \mathrm{E}-01$ & $\mathrm{pCi} / \mathrm{g}$ & SOIL & AS060908-2 & $9 / 12 / 2005$ & $u$ \\
\hline $0609033-19$ & MPDT-20A & Sample & U-238 & $1.18 \mathrm{E}+00+/-5.06 \mathrm{E}-01$ & $2.52 E-01$ & $\mathrm{pCi} / \mathrm{g}$ & SOIL & AS060908-2 & $9 / 12 / 2006$ & LT \\
\hline $0609033-20$ & MPDT-20B & Sample & U-233/234 & $2.18 \mathrm{E}+00+1-7.36 \mathrm{E}-01$ & $1.29 \mathrm{E}-01$ & $\mathrm{pCi} / \mathrm{g}$ & SOIL & AS060908-2 & $9 / 12 / 2006$ & \\
\hline $0609033-20$ & MPDT-20B & Sample & U-235 & $3.86 \mathrm{E}-01+/-3.02 \mathrm{E}-01$ & $2.29 \mathrm{E}-01$ & $\mathrm{pCi} / \mathrm{g}$ & SOIL & AS060908-2 & $9 / 12 / 2006$ & LT \\
\hline $0609033-20$ & MPDT-20B & Sample & $U-238$ & $1.08 E+00+/-4.89 E-01$ & $2.22 \mathrm{E}-01$ & $\mathrm{pCi} / \mathrm{g}$ & SOIL & AS060908-2 & $9 / 12 / 2006$ & LT \\
\hline
\end{tabular}

\section{Comments:}

\section{Data Package ID: UR0609033-1}

\section{Qualifiers/Flags:}

$U$-Result is less than the sample specific MDC.

LT - Result is less than Requested MDC, greater than sample specific MDC.

Y1 - Chemical Yield is in control at 100-110\%. Quantitative Yield is assumed.

Y2 - Chemical Yield outside default limits.

M.T. requested MDC was not met.

15. he requested MDC was not met, but the reporled activity is greater than the reported MDC 


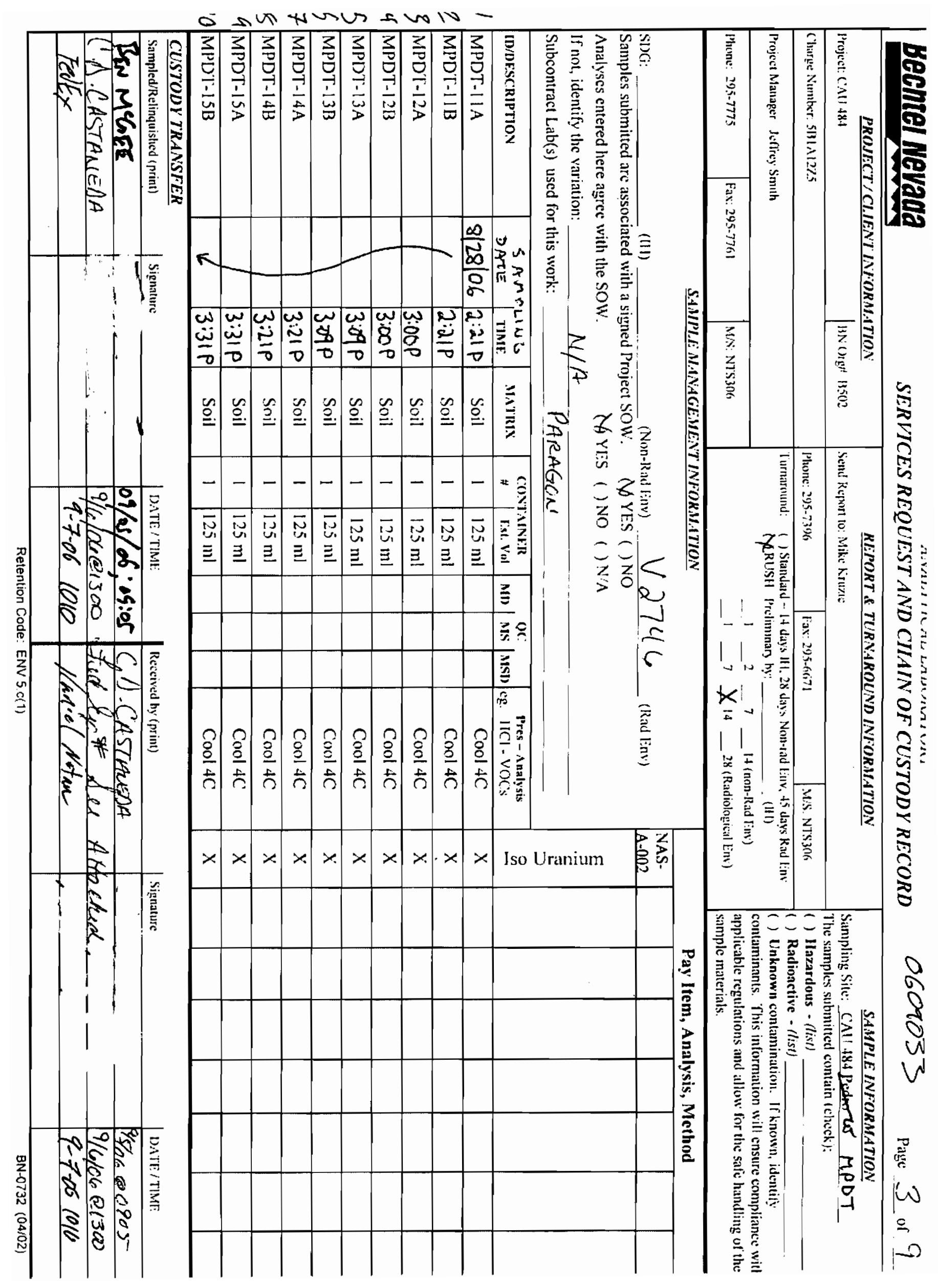




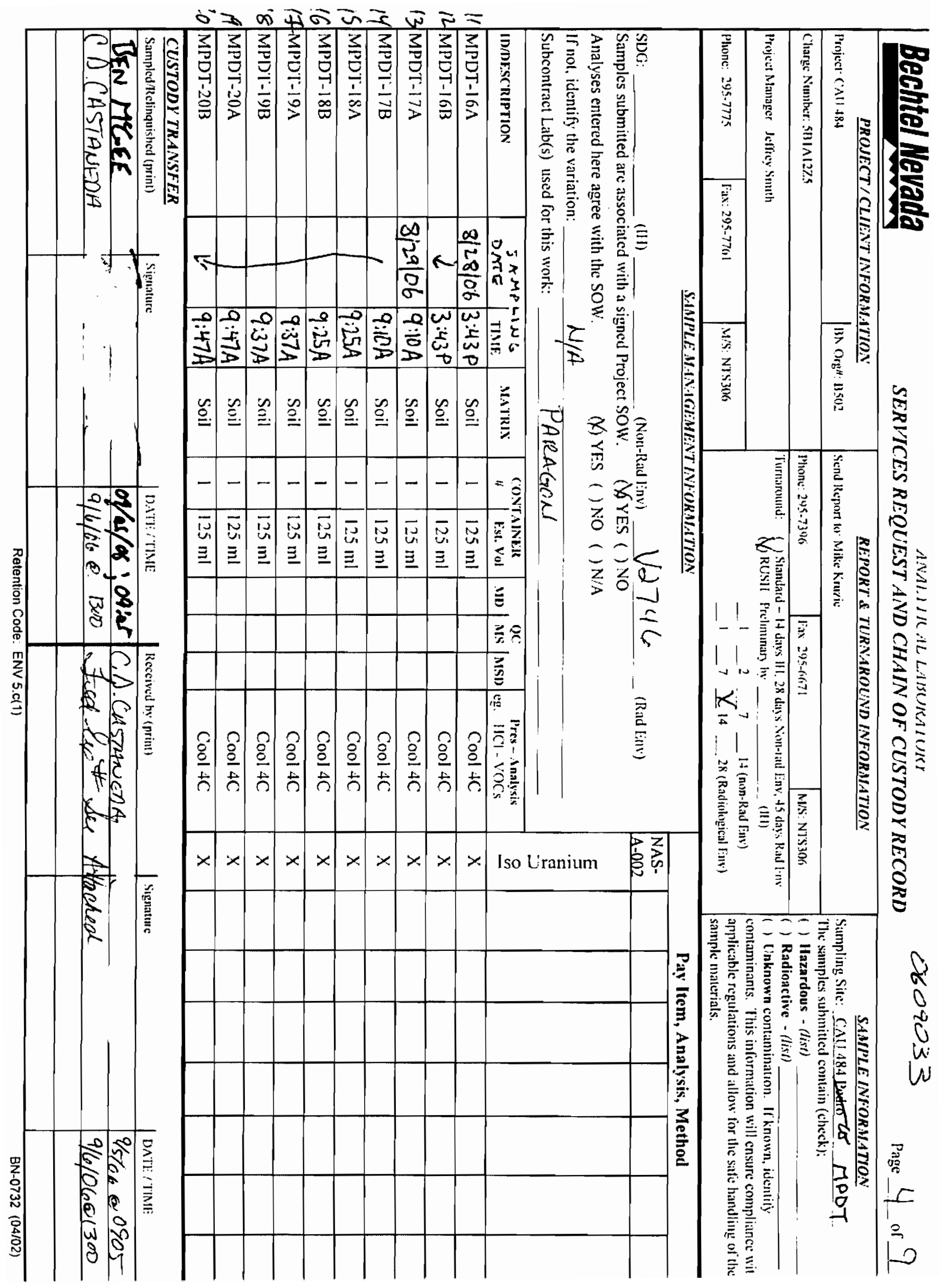




\section{Isotopic Uranium By Alpha Spectroscopy Sample Results Summary}

Client Name: National Sercurity Technologies, LLC Client Project Name: CAU 484

Client Project Number: V2746

\author{
Laboratory Name: Paragon Analytics \\ PAl Work Order: 0609034
}

Page: 1 of 7

Reported on: Tuesday, September 12,2006 1:26:57 PM

\begin{tabular}{|c|c|c|c|c|c|c|c|c|c|c|}
\hline $\begin{array}{c}\text { Lab } \\
\text { Sample ID }\end{array}$ & Client Sample ID & $\begin{array}{c}\text { Sample } \\
\text { Type }\end{array}$ & Nuclide & Result $+/-2$ s TPU & MDC & Units & Matrix & Prep Batch & $\begin{array}{c}\text { Date } \\
\text { Analyzed }\end{array}$ & Flags \\
\hline 0609034-1 & MPDT-21A & Sample & $\mathrm{U}-233 / 234$ & $9.28 \mathrm{E}-01+/-4.10 \mathrm{E}-01$ & $2.54 \mathrm{E}-01$ & $\mathrm{pCli} / \mathrm{g}$ & SOIL & AS060908-3 & $9 / 12 / 2006$ & LT \\
\hline $0609034-1$ & MPDT-21A & Sample & U-235 & $2.70 \mathrm{E}-01+1-2.24 \mathrm{E}-01$ & $1.22 \mathrm{E}-01$ & $\mathrm{pCi} / \mathrm{g}$ & SOIL & AS060908-3 & $9 / 12 / 2006$ & LT \\
\hline $0609034-1$ & MPDT-21A & Sample & $\mathrm{U}-238$ & $1.63 E+00+/-5.65 E-01$ & $2.10 \mathrm{E}-01$ & $\mathrm{pCi} / \mathrm{g}$ & SOIL & AS060908-3 & $9 / 12 / 2006$ & LT \\
\hline $0609034-2$ & MPDT-21B & Sample & U-233/234 & $1.84 \mathrm{E}+00+/-6.15 \mathrm{E}-01$ & $2.68 \mathrm{E}-01$ & $\mathrm{pCi} / \mathrm{g}$ & SOIL & AS060908-3 & $9 / 12 / 2006$ & LT \\
\hline $0609034-2$ & MPDT-21B & Sample & U-235 & $1.25 \mathrm{E}-01+/-1.61 \mathrm{E}-01$ & $2.35 \mathrm{E}-01$ & $\mathrm{pCi} / \mathrm{g}$ & SOIL & AS060908-3 & $9 / 12 / 2006$ & U \\
\hline $0609034-2$ & MPDT-21B & Sample & U-238 & $1.23 E+00+/-4.83 E-01$ & $2.14 \mathrm{E}-01$ & $\mathrm{pCi} / \mathrm{g}$ & SOIL & AS060908-3 & $9 / 12 / 2006$ & LT \\
\hline $0609034-3$ & MPDT-22A & Sample & U-233/234 & $1.30 \mathrm{E}+00+1-5.00 \mathrm{E}-01$ & $1.85 \mathrm{E}-01$ & $\mathrm{pCi} / \mathrm{g}$ & SOIL & AS060908-3 & $9 / 12 / 2006$ & LT \\
\hline $0609034-3$ & MPDT-22A & Sample & U-235 & $1.40 \mathrm{E}-01+/-1.64 \mathrm{E}-01$ & $1.26 \mathrm{E}-01$ & $\mathrm{pCi} / \mathrm{g}$ & SOIL & AS060908-3 & $9 / 12 / 2006$ & LT \\
\hline $0609034-3$ & MPDT-22A & Sample & U-238 & $1.03 \mathrm{E}+00+/-4.36 \mathrm{E}-01$ & $1.63 \mathrm{E}-01$ & $\mathrm{pCi} / \mathrm{g}$ & SOIL & AS060908-3 & $9 / 12 / 2006$ & LT \\
\hline
\end{tabular}

Comments:

\section{Data Package ID: UR0609034-1}

\section{Qualifiers/Flags:}

$U$-Result is less than the sample specific MDC.

$L T$ - Result is less than Requested MDC, greater than sample specific MDC

Y1 - Chemical Yield is in control at $100-110 \%$. Quantitative Yield is assumed.

YZ hernical Yield outside default limits.

Me requested $M D C$ was not met.

$\mathrm{M}^{3} \mathrm{~S}^{\text {the requested }} \mathrm{MDC}$ was not met, but the reported activity is greater than the reported MDC.

dgte Printed: Tuesday, September 12,2006
Abbreviations:

TPU - Total Propagated Uncertainty (see PAI SOP 743)

MDC - Minimum Detectable Concentration (see PAI SOP 709)

BDL - Below Detection Linit 


\section{Isotopic Uranium By Alpha Spectroscopy Sample Results Summary}

Client Name: National Sercurity Technologies, LLC Client Project Name: CAU 484

Client Project Number: V2746
Laboratory Name: Paragon Analytics

PAl Work Order: 0609034
Page: 2 of 7

Reported on: Tuesday, September 12,2006 1:26:57 PM

\begin{tabular}{|c|c|c|c|c|c|c|c|c|c|c|}
\hline $\begin{array}{c}\text { Lab } \\
\text { Sample ID }\end{array}$ & Client Sample ID & $\begin{array}{c}\text { Sample } \\
\text { Type }\end{array}$ & Nuclide & Result +/- 2 s TPU & MDC & Units & Matrix & Prep Batch & $\begin{array}{c}\text { Date } \\
\text { Analyzed }\end{array}$ & Flags \\
\hline $0609034-4$ & MPDT-22B & Sample & $\mathrm{U}-233 / 234$ & $1.42 \mathrm{E}+00+/-5.32 \mathrm{E}-01$ & $1.67 \mathrm{E}-01$ & $\mathrm{pCi} / \mathrm{g}$ & SOIL & ASO60908-3 & $9 / 12 / 2006$ & LT \\
\hline $0609034-4$ & MPDT-22B & Sample & U-235 & $9.55 E-02+/-1.67 E-01$ & $1.29 \mathrm{E}-01$ & pCi/g & SOIL & ASUG0908-3 & $9 / 12 / 2006$ & U \\
\hline $0609034-4$ & MPDT-22B & Sample & $U-238$ & $1.01 E+00+/-4.38 E-01$ & $1.90 \mathrm{E}-01$ & $\mathrm{pCi} / \mathrm{g}$ & SOIL & AS060908-3 & $9 / 12 / 2006$ & LT \\
\hline $0609034-5$ & MPDT-23A & Sample & U-233/234 & $1.92 \mathrm{E}+00+/-6.46 \mathrm{E}-01$ & $2.65 E-01$ & $\mathrm{pCi} / \mathrm{g}$ & SOIL & AS060908-3 & $9 / 12 / 2006$ & LT \\
\hline $0609034-5$ & MPDT-23A & Sample & U-235 & $2.68 \mathrm{E}-02+/-1.69 \mathrm{E}-01$ & $2.84 \mathrm{E}-01$ & $\mathrm{pCi} / \mathrm{g}$ & SOIL & ASO60908-3 & $9 / 12 / 2006$ & $\mathrm{u}$ \\
\hline $0609034-5$ & MPDT-23A & Sample & U.238 & $1.19 \mathrm{E}+00+1-4.85 \mathrm{E}-01$ & $2.28 \mathrm{E}-01$ & $\mathrm{pCil/g}$ & SOIL & AS060908-3 & $9 / 12 / 2006$ & LT \\
\hline 0609034.6 & MPDT-23B & Sample & U-233/234 & $1.28 \mathrm{E}+00+/-4.95 \mathrm{E}-01$ & $2.01 \mathrm{E}-01$ & pCiig & SOIL & AS060908-3 & $9 / 12 / 2006$ & LT \\
\hline $0609034-6$ & MPDT-23B & Sample & $U-235$ & $1.76 \mathrm{E}-01+/-1.87 \mathrm{E}-01$ & $2.16 \mathrm{E}-01$ & pCitg & SOIL & AS060908-3 & $9 / 12 / 2006$ & $u$ \\
\hline $0609034-6$ & MPDT-23B & Sample & U-238 & $1.37 \mathrm{E}+00+/-5.14 \mathrm{E}-01$ & $1.61 E-01$ & $\mathrm{pCling}$ & SOIL & AS060908-3 & $9 / 12 / 2006$ & LT \\
\hline
\end{tabular}

\section{Comments:}

\section{Data Package ID: UR0609034-1}

Qualifiers/Flags:

$U$ - Result is tess than the sample specific MDC.

LT - Result is less than Requested MDC, greater than sample specilic MDC.

Y1 - Chemical Yield is in control at 100-110\%. Quentitative Yieid is assumed.

Y2 - Chemical Yield outside deteult limits

$M$ - The requested $M D C$ was not.met.

The requested MDC was not met, but the reported activity is greater than the reported MDC

$\begin{array}{ll}0 \text { Tte Printed: Tuesday, September 12,2006 } & \text { Paragon Analytics } \\ \text { LIMS Version: } 5.422 \mathrm{~A}\end{array}$

Atbreviations:

TPU - Total Ptopagated Uncertainty (see PAI SOP 743

MDC - Minimum Detectable Concentration (see PAI SOP 709)

BDL - Below Detectim Limit 


\section{Isotopic Uranium By Alpha Spectroscopy Sample Results Summary}

Client Name: National Sercurity Technologies, LLC Client Project Name: CAU 484

Client Project Number: V2746
Laboratory Name: Paragon Analytics

PAI Work Order: 0609034
Page: 3 of 7

Reported on: Tuesday, September 12,2006 $1: 26: 57 \mathrm{PM}$

\begin{tabular}{|c|c|c|c|c|c|c|c|c|c|c|}
\hline $\begin{array}{c}\text { Lab } \\
\text { Sample ID }\end{array}$ & Client Sample ID & $\begin{array}{c}\text { Sample } \\
\text { Type }\end{array}$ & Nuclide & Result $+1-2 \mathrm{~s}$ TPU & MDC & Units & Matrix & Prep Batch & $\begin{array}{c}\text { Date } \\
\text { Analyzed }\end{array}$ & Flags \\
\hline $0609034-7$ & MPDT-24A & Sample & $U-233 / 234$ & $1.40 \mathrm{E}+00+1-5.29 \mathrm{E}-01$ & $2.36 \mathrm{E}-01$ & $\mathrm{pCi} / \mathrm{g}$ & SOIL & AS060908-3 & $9 / 12 / 2006$ & LT \\
\hline $0609034-7$ & MPDT-24A & Sample & U-235 & $3.04 \mathrm{E}-02+/-1.65 \mathrm{E}-01$ & $2.62 \mathrm{E}-01$ & $\mathrm{pC} i / \mathrm{g}$ & SOIL & AS060908-3 & $9 / 12 / 2006$ & $u$ \\
\hline $0609034-7$ & MPDT-24A & Sample & $\mathrm{U}-238$ & $1.32 \mathrm{E}+00+/-5.11 \mathrm{E}-01$ & $2.07 E-01$ & $\mathrm{pCi} / \mathrm{g}$ & SOIL & AS060908-3 & $9 / 12 / 2006$ & LT \\
\hline $0609034-8$ & MPDT-24B & Sample & U-233/234 & $1.32 E+00+/-5.23 E-01$ & 3.59E-01 & $\mathrm{pCi} / \mathrm{g}$ & SOIL. & AS060908-3 & $9 / 12 / 2006$ & LT \\
\hline $0609034-8$ & MPDT-24B & Sample & $U-235$ & $1.23 \mathrm{E}-01+1-1.69 \mathrm{E}-01$ & $2.80 \mathrm{E}-01$ & pCilg & SOIL & ASO60908-3 & $9 / 12 / 2006$ & $u$ \\
\hline $0609034-8$ & MPDT-24B & Sample & U-238 & $7.62 \mathrm{E}-01+/-3.77 \mathrm{E}-01$ & $2.25 \mathrm{E}-01$ & pCilg & SOIL & AS060908-3 & $9 / 12 / 2006$ & LT \\
\hline $0609034-9$ & MPDT-25A & Sample & U-233/234 & $1.53 E+00+/-5.84 E-01$ & $2.85 E-01$ & $\mathrm{pCi} / \mathrm{g}$ & SOIL & AS060908-3 & $9 / 12 / 2006$ & LT \\
\hline $0609034-9$ & MPDT-25A & Sample & $U-235$ & $0 \mathrm{E}+00+/-1.82 \mathrm{E} \cdot 01$ & $1.42 \mathrm{E}-01$ & pCilg & SOIL & AS060908-3 & $9 / 12 / 2006$ & $\mathrm{u}$ \\
\hline $0609034-9$ & MPDT-25A & Sample & $U-238$ & $1.55 \mathrm{E}+00+1-5.85 \mathrm{E}-01$ & $2.29 \mathrm{E}-01$ & $\mathrm{pCi} / \mathrm{g}$ & SOIL & AS060908-3 & $9 / 12 / 2006$ & LT \\
\hline
\end{tabular}

\section{Comments:}

\section{Data Package ID: UR0609034-1}

\section{Qualifiers/Flags:}

$U$ - Result is less than the sample specific MDC.

LT - Result is less than Requested MDC, greater than sample specific MDC.

$Y 1$. Chemical Yield is in control at $100-110 \%$. Quantitative Yield is assumed.

Y2 - Chemical Yield outside default limits.

$M$ - The requested MDC was not met.

The requested MDC was not met, but the reporled activity is greater than the reported MOC

$\begin{array}{lc}\text { Uate Printed: Tuesday, September 12,2006 } & \text { Paragon Analytics } \\ 0 & \text { L.MS Version: } 5.422 \mathrm{~A}\end{array}$

Abbreviations:

TPU - Total Propagated Uncertainty (see PAI SOP 743)

MDC - Minimum Deteclabie Concentration (see PAI SOP 709)

BDL. - Below Detection Limit 


\section{Isotopic Uranium By Alpha Spectroscopy Sample Results Summary}

Client Name: National Sercurity Technologies, LLC Client Project Name: CAU 484 Client Project Number: V2746

\author{
Laboratory Name: Paragon Analytics \\ PAl Work Order: 0609034
}

Page: 4 of 7

Reported on: Tuesday, September 12,2006 $1: 26: 57 \mathrm{PM}$

\begin{tabular}{|c|c|c|c|c|c|c|c|c|c|c|}
\hline $\begin{array}{c}\text { Lab } \\
\text { Sample ID }\end{array}$ & Client Sample ID & $\begin{array}{l}\text { Sample } \\
\text { Type }\end{array}$ & Nuclide & Result +1- 2 s TPU & MDC & Units & Matrix & Prep Batch & $\begin{array}{c}\text { Date } \\
\text { Analyzed }\end{array}$ & Flags \\
\hline $0609034-10$ & MPDT-25B & Sample & U-233/234 & $1.09 \mathrm{E}+00+1-4.62 \mathrm{E}-01$ & $2.26 \mathrm{E}-01$ & pCiig & SOIL & ASO60908-3 & $9 / 12 / 2006$ & LT \\
\hline $0609034-10$ & MPDT-25B & Sample & $\mathrm{U}-235$ & $4.40 \mathrm{E}-02+i-1.68 \mathrm{E}-01$ & $1.98 \mathrm{E}-01$ & $\mathrm{pCi} / \mathrm{g}$ & SOIL & AS060908-3 & $9 / 12 / 2006$ & $u$ \\
\hline $0609034-10$ & MPDT-25B & Sample & $U-238$ & $1.06 \mathrm{E}+00+1-4.53 \mathrm{E}-01$ & $1.69 \mathrm{E} \cdot 0 \mathrm{i}$ & $\mathrm{pCi} / \mathrm{g}$ & SOIL & AS060908-3 & $9 / 12 / 2006$ & LT \\
\hline $0609034-11$ & MPDT-26A & Sample & U-233/234 & $1.08 \mathrm{E}+00+1-4.47 \mathrm{E}-01$ & $2.55 \mathrm{E}-01$ & $\mathrm{pCi} / \mathrm{g}$ & SOIL & ASO60908-3 & $9 / 12 / 2006$ & LT \\
\hline $0609034-11$ & MPDT-26A & Sample & $U \cdot 235$ & $1.66 \mathrm{E}-02+1-1.57 \mathrm{E}-01$ & $2.88 \mathrm{E}-01$ & $\mathrm{pCi} / \mathrm{g}$ & SOIL. & AS060908-3 & $9 / 12 / 2006$ & $u$ \\
\hline $0609034-11$ & MPDT-26A & Sample & $\mathrm{U}-238$ & $1.09 \mathrm{E}+00+/-4.48 \mathrm{E}-01$ & $2.23 \mathrm{E}-01$ & $\mathrm{pCi} / \mathrm{g}$ & SOIL & AS060908-3 & $9 / 12 / 2006$ & LT \\
\hline $0609034-12$ & MPDT-26B & Sample & U-233/234 & $1.71 \mathrm{E}+00+/-5.95 \mathrm{E}-01$ & $2.21 E-01$ & $\mathrm{pClig}$ & SOIL & AS060908-3 & $9 / 12 / 2006$ & LT \\
\hline $0609034-12$ & MPDT-26B & Sample & $U-235$ & $8.60 \mathrm{E}-02+1-1.65 \mathrm{E}-01$ & $2.21 \mathrm{E}-01$ & $\mathrm{pCi} / \mathrm{g}$ & SOIL & ASO60908-3 & $9 / 12 / 2006$ & u \\
\hline $0609034-12$ & MPDT-26B & Sample & U. 238 & $1.11 \mathrm{E}+00+1-4.61 \mathrm{E}-01$ & $2.21 E-01$ & pCirg & SOIL & AS060908-3 & $9 / 12 / 2006$ & LT \\
\hline
\end{tabular}

\section{Comments:}

\section{Data Package ID: UR0609034-1}

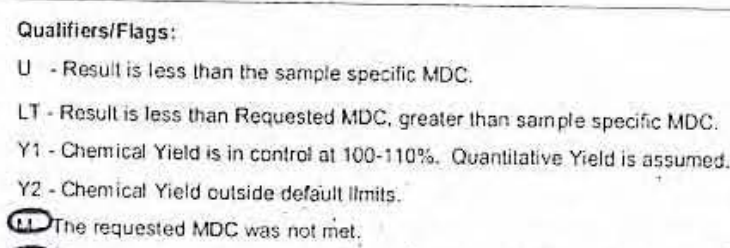

$U$ - Result is less than the sample specific MDC.

LT - Result is less than Requested MDC, greater than sample specific MDC.

$Y_{1}$ - Chernical Yield is in control at 100-110\%. Quantitative Yield is assumed.

Y2 - Chemical Yield outside defauth litrits

(1) The requested MDC was not riet.

613. The requested MDC was not mel, but the reported activity is greater than the reported MDC

$\begin{array}{lc}\text { Qate Printed: Tuesday, September 12,2006 } & \text { Paragon Analytics } \\ \text { LIMS Version: } 5.422 \mathrm{~A}\end{array}$

Abbreviations:

TPU - Total Propsgated Uncertainty (see PAI SOP 743)

MDC - Minimum Detectable Concentration (see PAI SOP 709)

BDL - Below Detection Limit 


\section{Isotopic Uranium By Alpha Spectroscopy Sample Results Summary}

Client Name: National SercurityTechnologies, LLC Client Project Name: CAU 484

Client Project Number: V2746
Laboratory Name: Paragon Analytics PAl Work Order: 0609034
Page: 5 of 7

Reported on: Tuesday, September 12,2006 1:26:57 PM

\begin{tabular}{|c|c|c|c|c|c|c|c|c|c|c|}
\hline $\begin{array}{c}\text { Lab } \\
\text { Sample ID }\end{array}$ & Client Sample ID & $\begin{array}{c}\text { Sample } \\
\text { Type }\end{array}$ & Nuclide & Result $+/-2$ s TPU & MDC & Units & Matrix & Prep Batch & $\begin{array}{c}\text { Date } \\
\text { Analyzed }\end{array}$ & Flags \\
\hline $0609034-13$ & MPDT-27A & Sample & U-233/234 & $1.66 \mathrm{E}+00+/-6.07 \mathrm{E}-01$ & $2.26 \mathrm{E}-01$ & pCilg & SOIL & AS060908-3 & $9 / 12 / 2006$ & LT \\
\hline $0609034-13$ & MPDT-27A & Sample & U-235 & $4.72 E-02+/-1.81 E-01$ & 2.13E-01 & $\mathrm{pCi} / \mathrm{g}$ & SOIL & AS060908-3 & $9 / 12 / 2006$ & $u$ \\
\hline $0609034-13$ & MPDT-27A & Sample & U.238 & $1.45 \mathrm{E}+00+/-5.59 \mathrm{E}-01$ & $2.07 E-01$ & $\mathrm{pCi} / \mathrm{g}$ & SOIL & AS060908-3 & $9 / 12 / 2006$ & LT \\
\hline $0609034-14$ & MPDT-27B & Sample & U-233/234 & $1.10 E+00+/-5.11 E-01$ & $2.80 \mathrm{E}-01$ & $\mathrm{pClig}$ & SOIL & AS060908-3 & $9 / 12 / 2006$ & LT \\
\hline $0609034-14$ & MPDT-27B & Sample & U.235 & $2.34 \mathrm{E}-01+/-2,43 \mathrm{E}-01$ & $2.46 \mathrm{E}-01$ & $\mathrm{pCl} / \mathrm{g}$ & SOIL & AS060908-3 & $9 / 12 / 2006$ & $u$ \\
\hline $0609034-14$ & MPDT-27B & Sample & $U-238$ & $1.05 \mathrm{E}+00+1-4.98 \mathrm{E}-01$ & 2.80 E-01 & pCilg & SOIL & AS060908-3 & $9 / 12 / 2006$ & LT \\
\hline $0609034-15$ & MPDT-28A & Sample & U-233/234 & $1.58 \mathrm{E}+00+1-5.74 \mathrm{E}-01$ & $3.79 \mathrm{E}-01$ & $\mathrm{pCi} / \mathrm{g}$ & SOIL & AS060908-3 & $9 / 12 / 2006$ & LT \\
\hline $0609034-15$ & MPDT-28A & Sample & $\mathrm{U}-235$ & $1.74 \mathrm{E}-01+/-1.89 \mathrm{E}-01$ & 2.39E-01 & $\mathrm{pCi} / \mathrm{g}$ & SOIL & AS060908-3 & $9 / 12 / 2006$ & U \\
\hline $0609034-15$ & MPDT-28A & Sample & $U \cdot 238$ & $1.43 E+00+1-5.35 E-01$ & $2.91 \mathrm{E}-01$ & $\mathrm{pCi} / \mathrm{g}$ & SOIL. & ASO60908-3 & $9 / 12 / 2006$ & LT \\
\hline
\end{tabular}

\section{Comments:}

\section{Data Package ID: UR0609034-1}

\section{Qualifiers/Flags:}

$U$ - Result is less than the sample specific MDC.

LT - Result is less than Requested MDC, greater than sample specific MDC.

$Y 1$. Chemical Yield is in control at 100-110\%, Quantitative Yield is assumed.

Y2 - Chemical Yield outside default imrits.

M- T

M3 - Trequested MDC was not mel, but the reported activity is greater than the reporled MDC.

\section{Abbreviations:}

TPU - Total Propagated Uncertainty (see PAI SOP 743)

MDC - Minimum Detectabte Concentration (see PAI SOP 709)

BDL - Below Delection Lim 


\section{Isotopic Uranium By Alpha Spectroscopy Sample Results Summary}

Client Name: National SercurityTechnologies, LLC Client Project Name: CAU 484 Client Project Number: V2746
Laboratory Name: Paragon Analytics

PAl Work Order: 0609034
Page: 6 of 7

Reported on: Tuesday, September 12,2006 1:26:57 PM

\begin{tabular}{|c|c|c|c|c|c|c|c|c|c|c|}
\hline $\begin{array}{c}\text { Lab } \\
\text { Sample ID }\end{array}$ & Client Sample ID & $\begin{array}{l}\text { Sample } \\
\text { Type }\end{array}$ & Nuclide & Result $+/-2 \mathrm{~s}$ TPU & MDC & Units & Matrix & Prep Batch & $\begin{array}{c}\text { Date } \\
\text { Analyzed }\end{array}$ & Flags \\
\hline $0609034-16$ & MPDT-28B & Sample & $U-233 / 234$ & $1.50 \mathrm{E}+00+/-5.53 \mathrm{E}-01$ & $2.87 E-01$ & $\mathrm{pCi} / \mathrm{g}$ & SOIL & ASO60908-3 & $9 / 12 / 2006$ & LT \\
\hline $0609034-16$ & MPDT-28B & Sample & $U-235$ & $1.38 \mathrm{E}-01+/-1.67 \mathrm{E}-01$ & 1.95E-01 & pCirg & SOIL & ASO60908-3 & $9 / 12 / 2006$ & $u$ \\
\hline $0609034-16$ & MPDT-28B & Sample & $\mathrm{U}-238$ & $1.02 E+00+1-4.44 E-01$ & $2.58 \mathrm{E}-01$ & $\mathrm{pCi} / \mathrm{g}$ & SOIL & AS060908-3 & $9 / 12 / 2006$ & LT \\
\hline $0609034-17$ & MPDT-29A & Sample & U.233/234 & $1.30 E+00+/-5.11 E-01$ & $2.26 \mathrm{E}-01$ & $\mathrm{pCli} / \mathrm{g}$ & SOIL & ASO60908-3 & $9 / 12 / 2006$ & LT \\
\hline $0609034-17$ & MPDT-29A & Sample & U-235 & $1.45 \mathrm{E}-01+/-1.70 \mathrm{E}-01$ & 1.31E-01 & $\mathrm{pCilg}$ & SOIL & ASU60908-3 & $9 / 12 / 2006$ & LT \\
\hline $0609034-17$ & MPDT-29A & Sample & U-238 & $1.47 \mathrm{E}+00+1-5.48 \mathrm{E}-01$ & $1.93 E-01$ & pCilg & SOIL & ASO60908-3 & $9 / 12 / 2006$ & LT \\
\hline $0609034-18$ & MPDT-29B & Sample & $\mathrm{U}-233 / 234$ & $1.67 \mathrm{E}+00+/-5.89 \mathrm{E}-01$ & $1.68 \mathrm{E}-01$ & $\mathrm{pCi} / \mathrm{g}$ & SOIL & ASO60908-3 & $9 / 12 / 2006$ & LT \\
\hline $0609034-18$ & MPDT-29B & Sample & U-235 & $1.44 \mathrm{E}-01+/-1.69 \mathrm{E}-01$ & $1.30 \mathrm{E}-01$ & $\mathrm{pCi} / \mathrm{g}$ & SOIL & AS060908-3 & $9 / 12 / 2006$ & LT \\
\hline $0609034-18$ & MPDT-29B & Sample & U-238 & $1.55 \mathrm{E}+00+/-5.63 \mathrm{E}-01$ & $1.68 \mathrm{E}-01$ & $\mathrm{pCli} / \mathrm{g}$ & SOIL & AS060908-3 & $9 / 12 / 2006$ & LT \\
\hline
\end{tabular}

\section{Comments:}

Data Package ID: UR0609034-1

\footnotetext{
Qualifiers/Flags:

U - Result is less than the sample specific MDC.

LT - Result is less than Requested MDC, greater than sample specific MOC.

Y1 - Chemical Yield is in control at $100-110 \%$. Quantitative Yield is assumed

Y2 - Chemical Yield outside default limits.

(1) The requested MDC was not miet.
}

43. The requested MDC was not met, but the reported activily is greater than the reported MDC.

Abbreviations:

TPU - Total Propagated Uncertainty (see PAI SOP 743)

MDC - Minimum Detectable Concentration (see PAI SOP 709)

BDL - Below Detection Limit 


\section{Isotopic Uranium By Alpha Spectroscopy Sample Results Summary}

Client Name: National Sercurity Technologies, LLC Client Project Name: CAU 484 Client Project Number: V2746
Laboratory Name: Paragon Analytics

PAl Work Order: 0609034
Page: 7 of 7

Reported on: Tuesday, September 12,2006 1:26:58 PM

\begin{tabular}{|c|c|c|c|c|c|c|c|c|c|c|}
\hline $\begin{array}{c}\text { Lab } \\
\text { Sample ID }\end{array}$ & Client Sample ID & $\begin{array}{c}\text { Sample } \\
\text { Type }\end{array}$ & Nuclide & Resuit $+/-2$ s TPU & MDC & Units & Matrix & Prep Batch & $\begin{array}{c}\text { Date } \\
\text { Analyzed }\end{array}$ & Flags \\
\hline $0609034-19$ & MPDT-30A & Sample & U-233/234 & $1.36 \mathrm{E}+00+/-5.14 \mathrm{E}-01$ & 2.01E-01 & pCirg & SOIL. & AS060908-3 & $9 / 12 / 2006$ & $\overline{L T}$ \\
\hline $0609034-19$ & MPDT-30A & Sampie & U-235 & $8.83 \mathrm{E}-02+1-1.61 \mathrm{E}-01$ & $1.90 \mathrm{E} \cdot 01$ & pCirg & SOIL & ASO60908-3 & $9 / 12 / 2006$ & $u$ \\
\hline $0609034-19$ & MPDT-30A & Sample & $\mathrm{U}-238$ & $1.60 E+00+/-5.65 E-01$ & $2.16 \mathrm{E}-01$ & pCiig & SOIL & ASO60908-3 & $9 / 12 / 2006$ & LT \\
\hline $0609034-20$ & MPDT-30B & Sample & U. $233 / 234$ & 1.16E+00+/-4.76E-01 & $1.12 \mathrm{E}-01$ & pCilg & SOIL & ASO60908-3 & $9 / 12 / 2006$ & LT \\
\hline $0609034-20$ & MPDT-30B & Sample & $\mathrm{U}-235$ & $1.42 \mathrm{E}-01+/-1.71 \mathrm{E}-01$ & $2.00 E-01$ & $\mathrm{pCi} / \mathrm{g}$ & SOIL & AS060908-3 & $9 / 12 / 2006$ & $u$ \\
\hline $0609034-20$ & MPDT $-30 B$ & Sample & $U-238$ & $1.15 E+00+1-4.76 E-01$ & $1.94 \mathrm{E}-01$ & $\mathrm{pClig}$ & SOIL & AS060908-3 & $9 / 12 / 2006$ & LT \\
\hline
\end{tabular}

\section{Comments:}

\section{Data Package ID: UR0609034-1}

Qualifiers/Flags:

$U$-Result is less than the sampte specific MDC

LT - Result is less than Requested MDC, greater than sample specific MDC.

$Y 1$. Chemical Yield is in control at $100-110 \%$. Quantitative Yield is assumed

Y2 - Chernical Yield outside default limits

M- Cho requested MDC was not met.

${ }^{M 3}$ requested MDC was not met, but the reported activity is greater than the reported MOC
Abbreviations:

TPU - Total Propagated Uncertainly (see PAI SOP 743)

MDC - Mnimum Detectable Concentration (see PAI SOP 709)

BDL - Below Detection Limit 


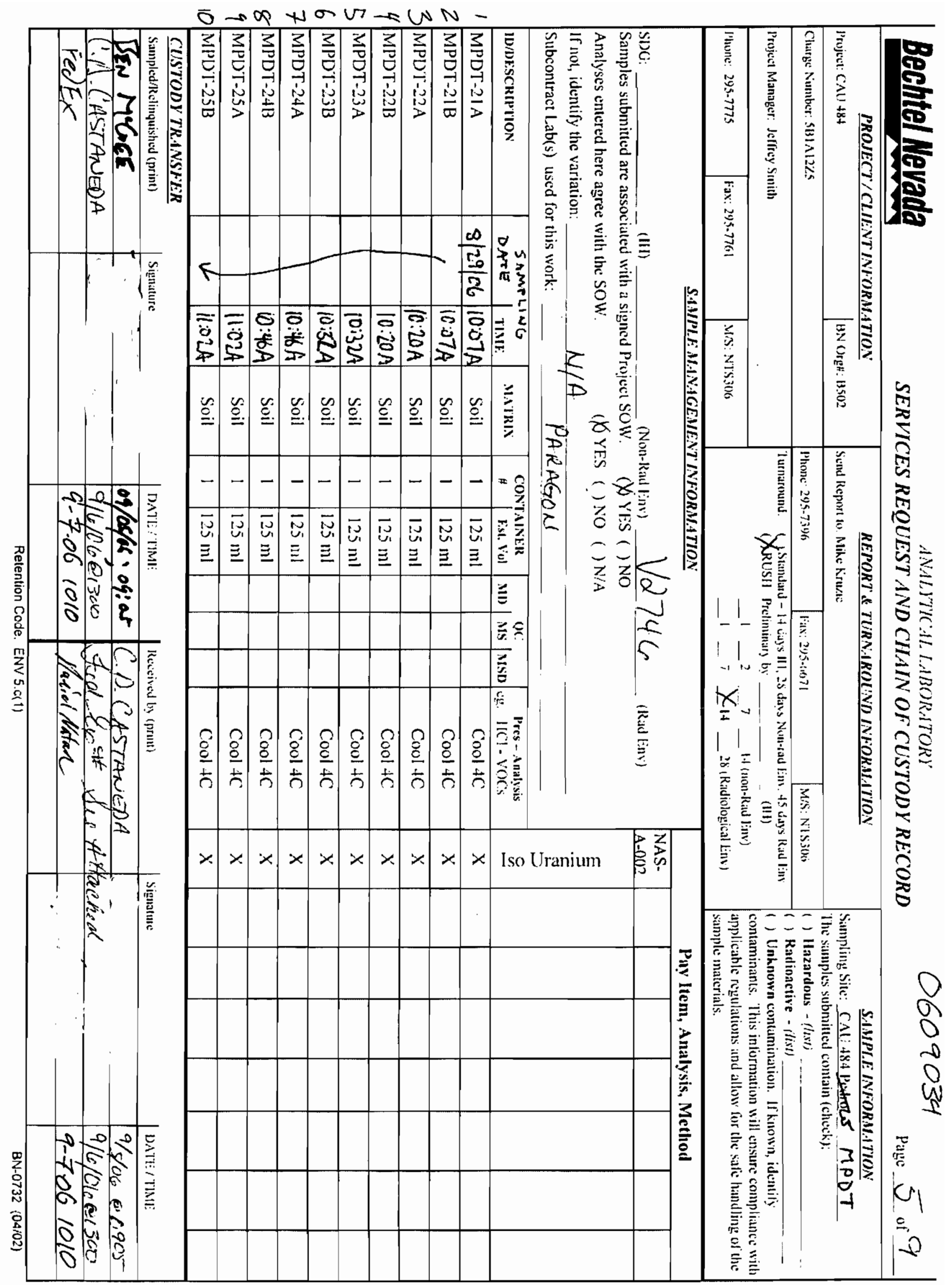




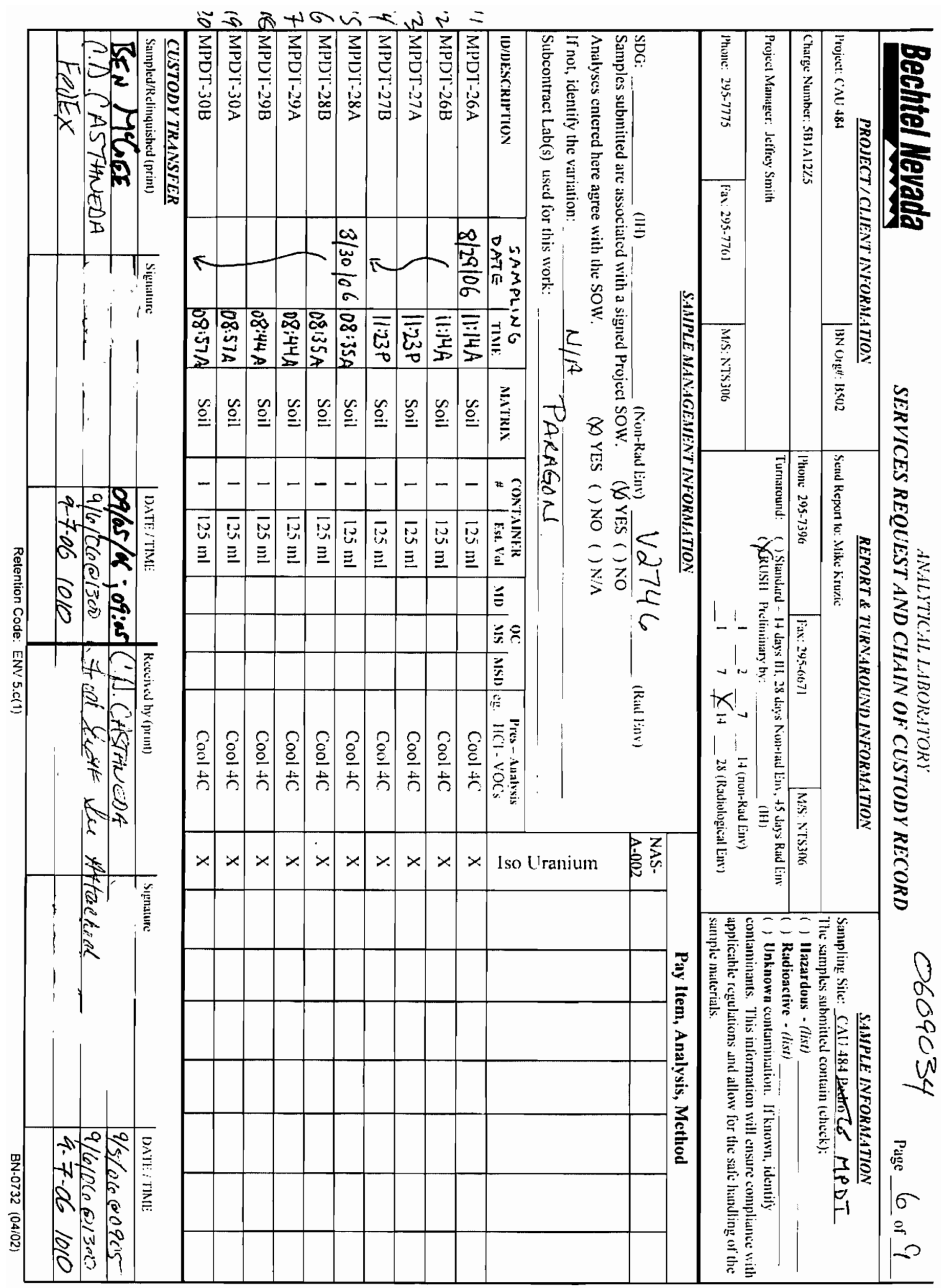




\section{Isotopic Uranium By Alpha Spectroscopy Sample Results Summary}

Client Name: National Sercurity Technologies, LLC Client Project Name: CAU 484 Client Project Number: V2746
Laboratory Name: Paragon Analytics

PAI Work Order: 0609036
Page: 1 of 6

Reported on: Friday, September 15, 2006 2:33:43 PM

\begin{tabular}{|c|c|c|c|c|c|c|c|c|c|c|}
\hline $\begin{array}{c}\text { Lab } \\
\text { Sample ID }\end{array}$ & Client Sample ID & $\begin{array}{c}\text { Sample } \\
\text { Type }\end{array}$ & Nuclide & Result + $/-2$ s TPU & MDC & Units & Matrix & Prep Batch & $\begin{array}{c}\text { Date } \\
\text { Analyzed }\end{array}$ & Flags \\
\hline $0609036-1$ & MPDT-31A & Sample & $U-233 / 234$ & $1.59 \mathrm{E}+00+/-5.63 \mathrm{E}-01$ & $2.67 \mathrm{E}-01$ & $\mathrm{pCi} / \mathrm{g}$ & SOIL & AS060908-4 & $9 / 12 / 2006$ & LT \\
\hline $0609036-1$ & MPDT-31A & Sample & U-235 & $7.87 E-02+/-1.59 E-01$ & 2.33E-01 & $\mathrm{pCi} / \mathrm{g}$ & SOIL & AS060908-4 & $9 / 12 / 2006$ & $u$ \\
\hline $0609036-1$ & MPDT-31A & Sample & U-238 & $1.15 E+00+/-4.62 E-01$ & $2.13 E-01$ & $\mathrm{pCi} / \mathrm{g}$ & SOIL & AS060908-4 & $9 / 12 / 2006$ & LT \\
\hline $0609036-2$ & MPDT-31B & Sample & $\mathrm{U}-233 / 234$ & $1.77 \mathrm{E}+00+/-5.86 \mathrm{E}-01$ & $1.52 \mathrm{E}-01$ & pCi'g & SOIL & AS060908-4 & $9 / 12 / 2006$ & LT \\
\hline $0609036-2$ & MPDT-31B & Sample & U-235 & $1.31 \mathrm{E}-01+/-1.53 \mathrm{E}-01$ & $1.18 \mathrm{E}-01$ & $\mathrm{pCi} / \mathrm{g}$ & SOIL & AS060908-4 & $9 / 12 / 2006$ & LT \\
\hline $0609036-2$ & MPDT-31B & Sample & U-238 & $1.33 E+00+1-4.92 E-01$ & $1.73 \mathrm{E}-01$ & $\mathrm{pCi} / \mathrm{g}$ & SOIL & AS060908-4 & $9 / 12 / 2006$ & LT \\
\hline $0609036-3$ & MPDT-32A & Sample & U-233/234 & $1.21 \mathrm{E}+00+1-4.76 \mathrm{E}-01$ & $2.46 \mathrm{E}-01$ & $\mathrm{pCi} / \mathrm{g}$ & SOIL & AS060908-4 & $9 / 12 / 2006$ & LT \\
\hline $0609036-3$ & MPDT-32A & Sample & U-235 & $7.01 \mathrm{E}-02+/-1.58 \mathrm{E}-01$ & $2.64 \mathrm{E}-01$ & pCilg & SOIL & AS060908-4 & $9 / 12 / 2006$ & $u$ \\
\hline $0609036-3$ & MPDT-32A & Sample & $\mathrm{U}-238$ & $1.22 \mathrm{E}+00+/-4.77 \mathrm{E}-01$ & $2.12 E-01$ & pCilg & SOIL & AS060908-4 & $9 / 12 / 2006$ & LT \\
\hline
\end{tabular}

Comments:

\section{Data Package ID: UR0609036-1}

Qualifiers/Flags:

$U$-Result is less than the sample specific MDC.

LT - Result is less than Requested MDC, greater than sample specific MDC.

Y1 . Chemical Yield is in control at $100-110 \%$. Quantitative Yield is assumed.

Y2 - Chenical Yield outside default limits.

$M$ - The requested MDC was not met.

M3. The requested MDC was not met, but the reported activity is greater than the reported MDC.
Abbreviations:

TPU - Total Propagated Uncerteinty (see PAI SOP 743)

MDC - Minimum Detectable Concentration (see PAI SOP 709)

BDL. - Beiow Detection Limit 


\section{Isotopic Uranium By Alpha Spectroscopy Sample Results Summary}

Client Name: National Sercurity Technologies, LLC Client Project Name: CAU 484 Client Project Number: V2746
Laboratory Name: Paragon Analytics

PAI Work Order: 0609036
Page: 2 of 6

Reported on: Friday, September 15, 2006 2:33:44 PM

\begin{tabular}{|c|c|c|c|c|c|c|c|c|c|c|}
\hline $\begin{array}{c}\text { Lab } \\
\text { Sample ID }\end{array}$ & Client Sample ID & $\begin{array}{c}\text { Sample } \\
\text { Type }\end{array}$ & Nuclide & Result +1- 2 s TPU & MDC & Units & Matrix & Prep Batch & $\begin{array}{c}\text { Date } \\
\text { Analyzed }\end{array}$ & Flags \\
\hline $0609036-4$ & MPDT-32B & Sample & $U-233 / 234$ & $1.45 \mathrm{E}+00+/-5.28 \mathrm{E}-01$ & $1.80 \mathrm{E}-01$ & $\mathrm{pCl} / \mathrm{g}$ & SOIL & AS060908-4 & $9 / 12 / 2006$ & LT \\
\hline $0609036-4$ & MPDT-32B & Sample & U-235 & $3.71 \mathrm{E}-02+/-1.57 \mathrm{E}-01$ & $2.12 \mathrm{E}-01$ & $\mathrm{pCi} / \mathrm{g}$ & SOIL & ASO60908-4 & $9 / 12 / 2006$ & u \\
\hline $0609036-4$ & MPDT-32B & Sample & U-238 & $1.50 E+00+/-5.37 E-01$ & $1.04 \mathrm{E}-01$ & $\mathrm{pCi} / \mathrm{g}$ & SOIL & AS060908-4 & $9 / 12 / 2006$ & LT \\
\hline $0609036-5$ & MPDT-33A & Sample & U-233/234 & $1.52 \mathrm{E}+00+/-5.40 \mathrm{E}-01$ & 2.19E-01 & $\mathrm{pCi} / \mathrm{g}$ & SOIL & AS060908-4 & $9 / 12 / 2006$ & LT \\
\hline $0609036-5$ & MPDT-33A & Sample & U-235 & $7.25 \mathrm{E}-02+/-1.54 \mathrm{E}-01$ & $2,43 \mathrm{E}-01$ & $\mathrm{pCi} / \mathrm{g}$ & SOIL & AS060908-4 & $9 / 12 / 2006$ & $u$ \\
\hline $0609036-5$ & MPDT-33A & Sample & U-238 & $1.12 \mathrm{E}+00+1-4.49 \mathrm{E}-01$ & $1.93 \mathrm{E}-01$ & $\mathrm{pCi} / \mathrm{g}$ & SOIL & AS060908-4 & $9 / 12 / 2006$ & LT \\
\hline $0609036-6$ & MPDT-33B & Sample & U-233/234 & $1.28 \mathrm{E}+00+/-5.42 \mathrm{E}-01$ & $2.65 \mathrm{E}-01$ & $\mathrm{pCi} / \mathrm{g}$ & SOIL & AS060908-4 & $9 / 12 / 2006$ & LT \\
\hline $0609036 \cdot 6$ & MPDT-33B & Sample & U-235 & 1.65E-01+/-1.99E-01 & $2.32 \mathrm{E}-01$ & $\mathrm{pCi} / \mathrm{g}$ & SOIL & AS060908-4 & $9 / 12 / 2006$ & U \\
\hline $0609036-6$ & MPDT-33B & Sample & U-238 & $1.48 \mathrm{E}+00+1-5.88 \mathrm{E}-01$ & $2.47 E-01$ & pCilg & SOIL & AS060908-4 & $9 / 12 / 2006$ & LT \\
\hline
\end{tabular}

\section{Comments:}

Data Package ID: UR0609036-1

Qualifiers/Flags:

$U$-Result is less than the sample specific MDC

LT - Result is less than Requested MDC, greater than sample specific MDC.

$Y_{1}$ - Chemical Yield is in control at 100-110\%. Quantitative Yield is assumed.

Y2 - Chemical Yield outside default limits.

$M$ - The requested $M D C$ was not met.

M3 - The requested MDC was not met, but the reported activity is greater than the reported MDC.
Abbreviations:

TPU - Total Propagated Uncertainty (see PAI SOP 743)

MOC - Minimum Detectable Concentration (see PAI SOP 709)

BDL - Betow Detectian Limit

\begin{tabular}{|c|c|c|}
\hline Date & $\begin{array}{c}\text { Paragon Analytics } \\
\text { LIMS Version: } 5.422 \mathrm{~A}\end{array}$ & Page 2 of 6 \\
\hline
\end{tabular}




\section{Isotopic Uranium By Alpha Spectroscopy Sample Results Summary}

Client Name: National Sercurity Technologies, LLC Client Project Name: CAU 484 Client Project Number: V2746
Laboratory Name: Paragon Analytics PAI Work Order: 0609036
Page: 3 of 6

Reported on: Friday, September 15, 2006 2:33:44 PM

\begin{tabular}{|c|c|c|c|c|c|c|c|c|c|c|}
\hline $\begin{array}{l}\text { Lab } \\
\text { Sample ID }\end{array}$ & Client Sample ID & $\begin{array}{l}\text { Sample } \\
\text { Type }\end{array}$ & Nuclide & Result +/- 2 s TPU & MDC & Units & Matrix & Prep Batch & $\begin{array}{c}\text { Date } \\
\text { Analyzed }\end{array}$ & Flags \\
\hline $0609036-7$ & MPDT-34A & Sample & U-233/234 & $1.33 E+00+1-5.43 E-01$ & 4.13E-01 & $\mathrm{pCi} / \mathrm{g}$ & SOIL & AS060908-4 & $9 / 12 / 2006$ & LT \\
\hline $0609036-7$ & MPDT-34A & Sample & $\mathrm{U}-235$ & 1.39E-01+/-1.78E-01 & $2.61 \mathrm{E}-01$ & $\mathrm{pCi} / \mathrm{g}$ & SOIL & AS060908-4 & $9 / 12 / 2006$ & $u$ \\
\hline $0609036-7$ & MPDT-34A & Sample & $\mathrm{U}-238$ & $1.34 \mathrm{E}+00+/-5.36 \mathrm{E}-01$ & $3.17 \mathrm{E}-01$ & $\mathrm{pCi} / \mathrm{g}$ & SOIL & AS $060908-4$ & $9 / 12 / 2006$ & LT \\
\hline $0609036-8$ & MPDT-34B & Sample & $U-233 / 234$ & $1.81 \mathrm{E}+00+1-6.25 \mathrm{E}-01$ & $3.02 \mathrm{E}-01$ & $\mathrm{pCi} / \mathrm{g}$ & SOIL & AS060908-4 & $9 / 12 / 2006$ & LT \\
\hline $0609036-8$ & MPDT-34B & Sample & $U-235$ & $9.24 E-02+1-1.69 E-01$ & $1.99 \mathrm{E}-01$ & $\mathrm{pCi} / \mathrm{g}$ & SOIL & AS060908-4 & $9 / 12 / 2006$ & $u$ \\
\hline $0609036-8$ & MPDT-34B & Sample & U-238 & $1.37 \mathrm{E}+00+1-5.29 \mathrm{E}-01$ & $2.63 \mathrm{E}-01$ & $\mathrm{pCi} / \mathrm{g}$ & SOIL & AS060908-4 & $9 / 12 / 2006$ & LT \\
\hline $0609036-9$ & MPDT-35A & Sample & U-233/234 & $1.62 \mathrm{E}+00+1-6.03 \mathrm{E}-01$ & $1.85 \mathrm{E}-01$ & $\mathrm{pCi} / \mathrm{g}$ & SOIL & AS060908-4 & $9 / 12 / 2006$ & LT \\
\hline $0609036-9$ & MPDT-35A & Sample & U.235 & $0 \mathrm{E}+00+/-1.85 \mathrm{E}-01$ & $1.44 \mathrm{E}-01$ & $\mathrm{pCi} / \mathrm{g}$ & SOIL & AS060908-4 & $9 / 12 / 2006$ & u \\
\hline $0609036-9$ & MPDT-35A & Sample & $\mathrm{U}-238$ & $1.49 \mathrm{E}+00+/-5.73 \mathrm{E}-01$ & $1.86 \mathrm{E}-01$ & $\mathrm{pCi} / \mathrm{g}$ & SOIL & AS060908-4 & $9 / 12 / 2006$ & LT \\
\hline
\end{tabular}

\section{Comments:}

Data Package ID: UR0609036-1

\section{Qualifiers/Flags:}

$U$-Result is less than the sample specific MDC.

LT - Result is less than Requested MDC, greater than sample specific MDC.

Y1 - Chemical Yield is in control at 100-110\%. Quantitative Yield is assumed.

Y2-Chemical Yield outside default lirrits.

$M$ - The requested $M D C$ was not met.

$M 3$ - The requested MDC was not met, but the reported activity is greater than the reported MDC,
Abbreviations:

TPU - Total Propagated Uncertainty (see PAI SOP 743)

MDC - Minimum Detectable Concentration (see PAI SOP 709)

BOL - Below Detection Limit

Date Finfted: Friday, September 15, 2006 


\section{Isotopic Uranium By Alpha Spectroscopy Sample Results Summary}

Client Name: National Sercurity Technologies, LLC Client Project Name: CAU 484 Client Project Number: V2746
Laboratory Name: Paragon Analytics PAI Work Order: 0609036
Page: 4 of 6

Reported on: Friday. September 15, 2006 2:33:44 PM

\begin{tabular}{|c|c|c|c|c|c|c|c|c|c|c|}
\hline $\begin{array}{c}\text { Lab } \\
\text { Sample ID }\end{array}$ & Client Sample ID & $\begin{array}{c}\text { Sample } \\
\text { Type }\end{array}$ & Nuclide & Result $+1-2$ s TPU & MDC & Units & Matrix & Prep Batch & $\begin{array}{c}\text { Date } \\
\text { Analyzed }\end{array}$ & Flags \\
\hline $0609036-10$ & MPDT-35B & Sample & U-233/234 & $1.39 \mathrm{E}+00+/-5.32 \mathrm{E}-01$ & $2.12 \mathrm{E}-01$ & $\mathrm{pCl} / \mathrm{g}$ & SOIL & AS060908-4 & $9 / 12 / 2006$ & LT \\
\hline $0609036-10$ & MPDT-35B & Sample & $U-235$ & $-4.38 \mathrm{E}-03+/-1.69 \mathrm{E}-01$ & $2.00 \mathrm{E}-01$ & $\mathrm{pCi} / \mathrm{g}$ & SOIL. & AS060908-4 & $9 / 12 / 2006$ & $\mathrm{U}$ \\
\hline $0609036-10$ & MPDT-35B & Sample & U-238 & $1.06 \mathrm{E}+00+/-4.55 \mathrm{E}-01$ & 2.27E-01 & $\mathrm{pCi} / \mathrm{g}$ & SOIL & AS060908-4 & $9 / 12 / 2006$ & LT \\
\hline $0609036-11$ & MPDT-36A & Sample & $U-233 / 234$ & $1.58 \mathrm{E}+00+/-5.72 \mathrm{E}-01$ & 1.13E-01 & $\mathrm{pCl} / \mathrm{g}$ & SOIL & AS060908-4 & $9 / 12 / 2006$ & LT \\
\hline $0609036-11$ & MPDT-36A & Sample & U-235 & $-4.40 \mathrm{E}-03+/-1.70 \mathrm{E}-01$ & $2.01 \mathrm{E}-01$ & $\mathrm{pCi} / \mathrm{g}$ & SOIL & AS060908.4 & $9 / 12 / 2006$ & $u$ \\
\hline $0609036-11$ & MPDT-36A & Sample & U-238 & $9.49 \mathrm{E}-01+1-4.27 \mathrm{E}-01$ & $1.95 \mathrm{E}-01$ & pCilg & SOIL & AS060908-4 & $9 / 12 / 2006$ & LT \\
\hline $0609036-12$ & MPDT $-36 \mathrm{~B}$ & Sample & U-2.33/234 & $1.96 \mathrm{E}+00+/-6.55 \mathrm{E}-01$ & $3.09 E-01$ & $\mathrm{pCi} / \mathrm{g}$ & SOIL & AS060908-4 & $9 / 12 / 2006$ & LT \\
\hline $0609036-12$ & MPDT-36B & Sample & U-235 & $1.32 \mathrm{E}-01+/-1.69 \mathrm{E}-01$ & $2.47 \mathrm{E}-01$ & $\mathrm{pCl} / \mathrm{g}$ & SOIL & AS060908-4 & $9 / 12 / 2006$ & $u$ \\
\hline $0609036-12$ & MPDT-36B & Sample & $\mathrm{U}-238$ & $1.34 \mathrm{E}+00+1-5.18 \mathrm{E}-01$ & $1.92 \mathrm{E}-01$ & $\mathrm{pCi} / \mathrm{g}$ & SOIL & AS060908-4 & $9 / 12 / 2006$ & LT \\
\hline
\end{tabular}

Comments:

\section{Data Package ID: UR0609036-1}

\section{Qualifiers/Flags:}

$U$-Result is less than the sample specific MDC.

LT - Result is less than Requested MDC, greater than sample specific MDC

$Y_{1}$ - Chemical Yield is in control at 100-110\%. Quantitative Yield is assurned.

Y2 - Chemical Yield outside default limits.

$M$ - The requested $M D C$ was not met

M3 - T Uested MDC was not met, but the reported activity is greater than the reported MDC.

\section{Abbreviations:}

TPU - Total Propagated Uncertainty (see PAI SOP 7.43)

MDC - Minimum Detectable Concentration (see PAI SOP 709)

BDL - Below Detection Limil

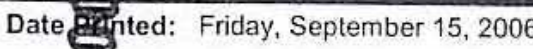




\section{Isotopic Uranium By Alpha Spectroscopy Sample Results Summary}

Client Name: National Sercurity Technologies, LLC Client Project Name: CAU 484 Client Project Number: V2746
Laboratory Name: Paragon Analytics

PAI Work Order: 0609036
Page: 5 of 6

Reported on: Friday, September 15, 2006 2:33:44 PM

\begin{tabular}{|c|c|c|c|c|c|c|c|c|c|c|}
\hline $\begin{array}{c}\text { Lab } \\
\text { Sample ID }\end{array}$ & Client Sample ID & $\begin{array}{l}\text { Sample } \\
\text { Type }\end{array}$ & Nuclide & Result +1- 2 s TPU & MDC & Units & Matrix & Prep Batch & $\begin{array}{c}\text { Date } \\
\text { Analyzed }\end{array}$ & Flags \\
\hline $0609036-13$ & MPDT-37A & Sample & U-233/234 & $1.35 E+00+/-5.24 E-01$ & $2.28 \mathrm{E}-01$ & $\mathrm{pCi} / \mathrm{g}$ & SOIL & AS060908-4 & $9 / 12 / 2006$ & LT \\
\hline 0609036-13 & MPDT-37A & Sample & U-235 & $9.31 \mathrm{E}-02+/-1.70 \mathrm{E}-01$ & $2.09 \mathrm{E}-01$ & $\mathrm{pCi} / \mathrm{g}$ & SOIL & AS060908-4 & $9 / 12 / 2006$ & $u$ \\
\hline $0609036-13$ & MPDT-37A & Sample & U-238 & $1.49 E+00+/-5.53 E-01$ & $1.70 E-01$ & $\mathrm{pCi} / \mathrm{g}$ & SOIL & AS060908.4 & $9 / 12 / 2006$ & LT \\
\hline $0609036-14$ & MPDT-37B & Sample & U-233/234 & $1.43 \mathrm{E}+00+/-5.31 \mathrm{E}-01$ & $2.62 \mathrm{E}-01$ & $\mathrm{pCi} / \mathrm{g}$ & SOIL & AS060908-4 & $9 / 12 / 2006$ & LT \\
\hline $0609036-14$ & MPDT-37B & Sample & U-235 & $9.25 \mathrm{E}-02+/-1.62 \mathrm{E}-01$ & $1.25 \mathrm{E}-01$ & $\mathrm{pCli} / \mathrm{g}$ & SOIL & ASO60908-4 & $9 / 12 / 2006$ & $u$ \\
\hline $0609036-14$ & MPDT-37B & Sample & U-238 & $1.32 E+00+/-5.06 E-01$ & $2.16 \mathrm{E}-01$ & $\mathrm{pCi} / \mathrm{g}$ & SOIL & AS060908-4 & 9/12/2006 & LT \\
\hline $0609036-15$ & MPDT-38A & Sample & U-233/234 & $1.47 E+00+/-5.29 E-01$ & $2.58 \mathrm{E}-01$ & $\mathrm{pCi} / \mathrm{g}$ & SOIL & AS060908-4 & $9 / 12 / 2006$ & LT \\
\hline $0609036-15$ & MPDT-38A & Sample & $\mathrm{U}-235$ & $3.22 \mathrm{E}-02+/-1.53 \mathrm{E}-01$ & $2.26 \mathrm{E}-01$ & $\mathrm{pCi} / \mathrm{g}$ & SOIL & AS060908-4 & $9 / 12 / 2006$ & $u$ \\
\hline $0609036-15$ & MPDT-38A & Sample & $\mathrm{U}-238$ & $9.96 \mathrm{E}-01+/-4.21 \mathrm{E}-01$ & $2.19 \mathrm{E}-01$ & $\mathrm{pCi} / \mathrm{g}$ & SOIL & AS060908-4 & $9 / 12 / 2006$ & LT \\
\hline
\end{tabular}

Comments:

Data Package ID: UR0609036-1

Qualifiers/Flags:

U - Result is less than the sample specific MDC.

LT - Result is less than Requested MDC, greater than sample specific MOC

Y1 - Chemical Yield is in control at $100-110 \%$. Quantitative Yield is assumed.

Y2 - Chernical Yield outside default limits.

$M$ - The raguesled MDC was not met

M3 - Th

Date kfthted: Friday, September 15, 2006

8

6
Abbreviations:

TPU - Tolal Propagated Uncertainty (see PAI SOP 743)

MDC - Minimum Detectable Concentration (see PAI SOP 709)

BDL - Below Detection Lim: 


\section{Isotopic Uranium By Alpha Spectroscopy Sample Results Summary}

Client Name: National Sercurity Technolagies, LLC Client Project Name: CAU 484 Client Project Number: V2746

\section{Laboratory Name: Paragon Analytics \\ PAI Work Order: 0609036}

Page: 6 of 6

Reported on: Friday, Seplember 15, 2006 2:33:44 PM

\begin{tabular}{|c|c|c|c|c|c|c|c|c|c|c|}
\hline $\begin{array}{c}\text { Lab } \\
\text { Sample ID }\end{array}$ & Client Sample ID & $\begin{array}{c}\text { Sample } \\
\text { Type }\end{array}$ & Nuclide & Result $+/-2$ s TPU & MDC & Units & Matrix & Prep Batch & $\begin{array}{c}\text { Date } \\
\text { Analyzed }\end{array}$ & Flags \\
\hline $0609036-16$ & MPDT-38B & Sample & U-233/234 & $1.67 \mathrm{E}+00+1-5.95 \mathrm{E}-01$ & $1.96 \mathrm{E}-01$ & $\mathrm{pCi} / \mathrm{g}$ & SOIL & AS060908-4 & $9 / 12 / 2006$ & $\overline{L T}$ \\
\hline $0609036-16$ & MPDT-38B & Sample & U-235 & $1.48 \mathrm{E}-01+/-1.73 \mathrm{E}-01$ & $1.34 \mathrm{E}-01$ & $\mathrm{pCi} / \mathrm{g}$ & SOIL & AS060908-4 & $9 / 12 / 2006$ & LT \\
\hline $0609036-16$ & MPDT-38B & Sample & U.238 & $1.46 \mathrm{E}+00+1-5.50 \mathrm{E}-01$ & $1.72 \mathrm{E}-01$ & $\mathrm{pCi} / \mathrm{g}$ & SOIL & ASO60908-4 & $9 / 12 / 2006$ & LT \\
\hline $0609036-17$ & MPDT-R1 & Sample & U.233/234 & $9.77 \mathrm{E}-02+1-1.09 \mathrm{E}-01$ & $1.73 E-01$ & $\mathrm{pCi} / \mathrm{l}$ & WATER & AS060908-7 & $9 / 12 / 2006$ & Y2,U,M \\
\hline $0609036-17$ & MPDT-R1 & Sample & U.235 & $1.77 \mathrm{E}-02+/-6.14 \mathrm{E}-02$ & $1.16 \mathrm{E}-01$ & $\mathrm{pCi} / \mathrm{l}$ & WATER & AS060908-7 & $9 / 12 / 2006$ & $\mathrm{Y} 2, \mathrm{U}, \mathrm{M}$ \\
\hline $0609036-17$ & MPDT-R1 & Sample & $U-238$ & $7.51 \mathrm{E}-02+/-6.54 \mathrm{E}-02$ & $9.03 E-02$ & $\mathrm{pCi} / \mathrm{l}$ & WATER & AS060908-7 & $9 / 12 / 2006$ & YZ,U,M \\
\hline
\end{tabular}

\section{Comments:}

\section{Data Package ID: UR0609036-1}

\section{Qualifiers/Flags:}

$U$ - Result is less than the sample specific MDC

LT - Result is less than Requested MDC, greater than sample specific MDC.

$Y 1$ - Chemical Yield is in control at $100-110 \%$ Quantitative Yield is assumed

Y2 - Chemical Yield outside default limits.

$M$ - The requested $M D C$ was not $m e t$.

M3 - The requested MDC was not met, but the reported activity is greater than the reported MOC

\section{Abbreviations:}

TPU - Total Propagated Uncertainty (see PAI SOP 743)

MOC - Minimum Detectable Concentration (see PAI SOP 709)

BOL. Below Detection Lim

\section{8}




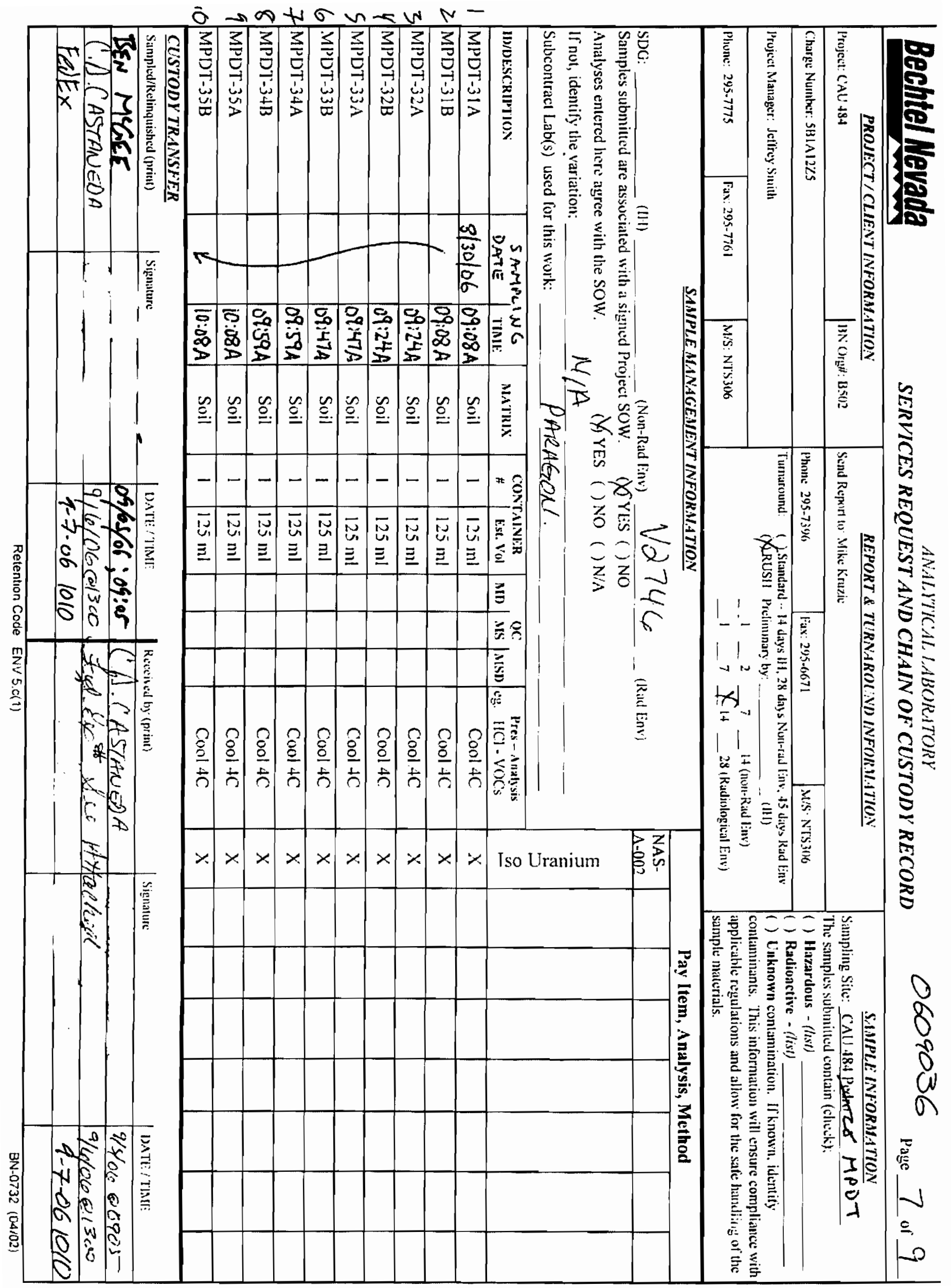




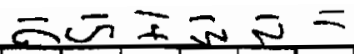

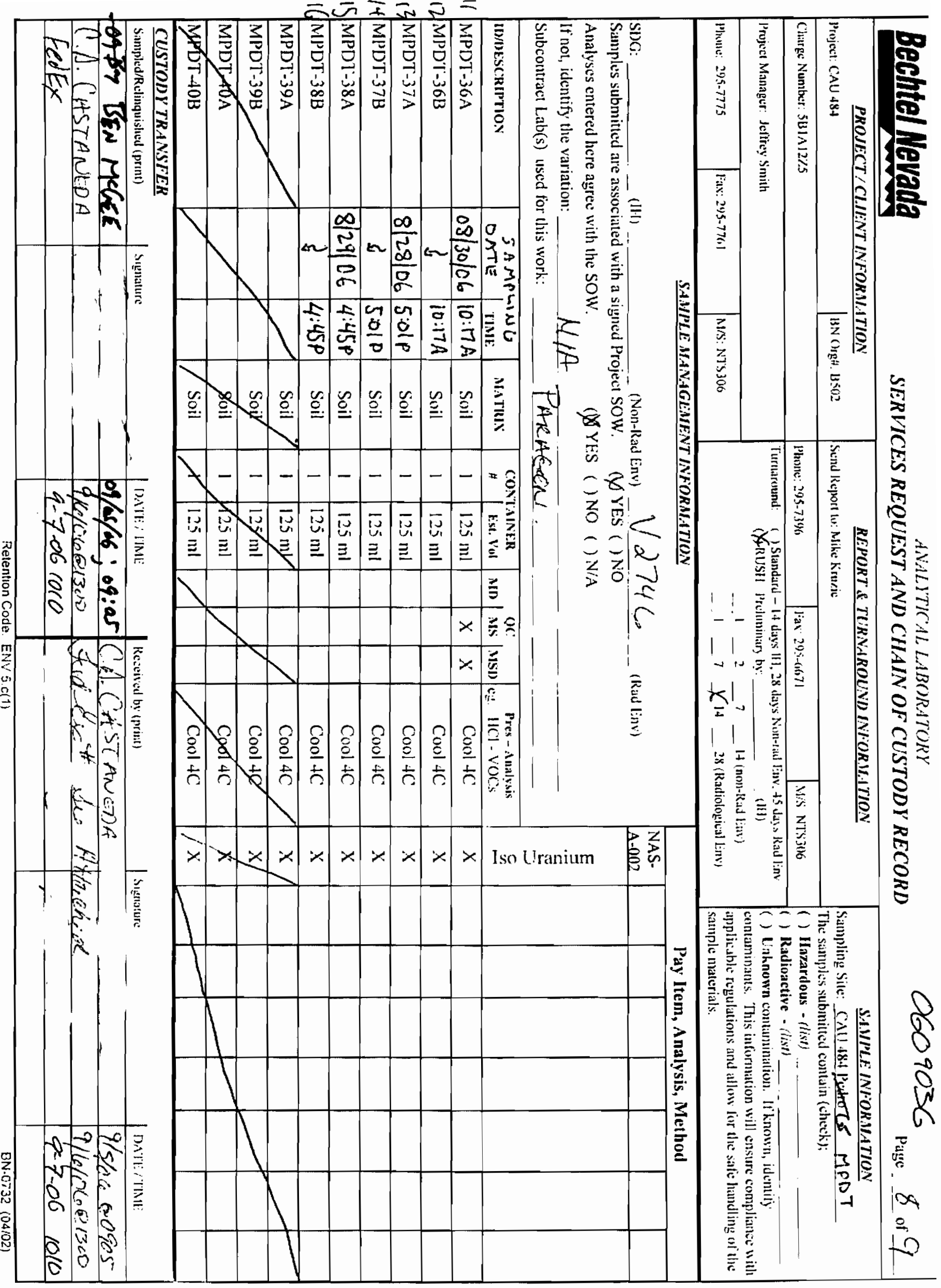




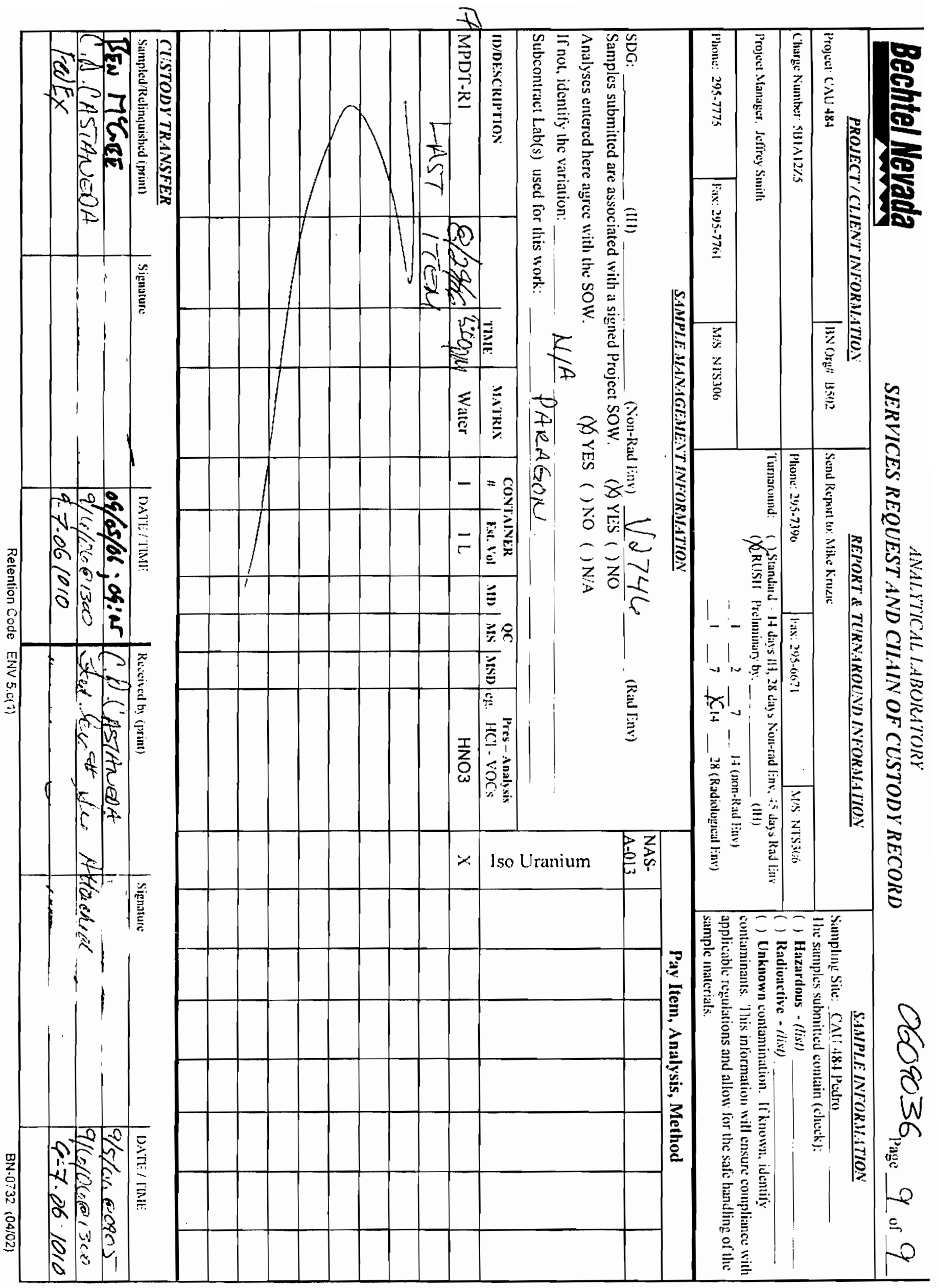

